\title{
SIENA: Efficient Compilation of Selective Protein Binding Site Ensembles
}

\section{Supporting Information}

\author{
Stefan Bietz and Matthias Rarey* \\ University of Hamburg, Center for Bioinformatics (ZBH), Bundesstrasse 43, 20146 \\ Hamburg \\ E-mail: rarey@zbh.uni-hamburg.de
}

*To whom correspondence should be addressed 


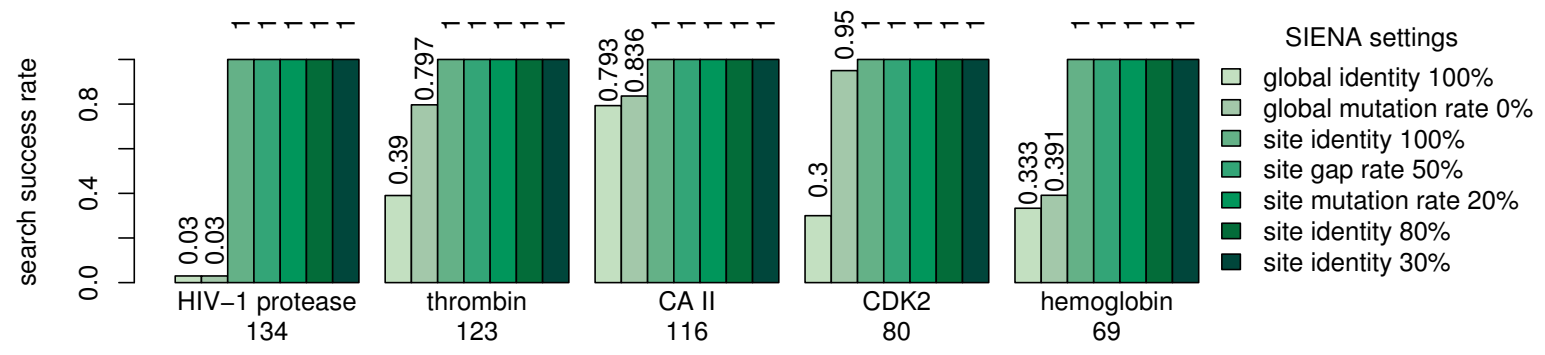

a)
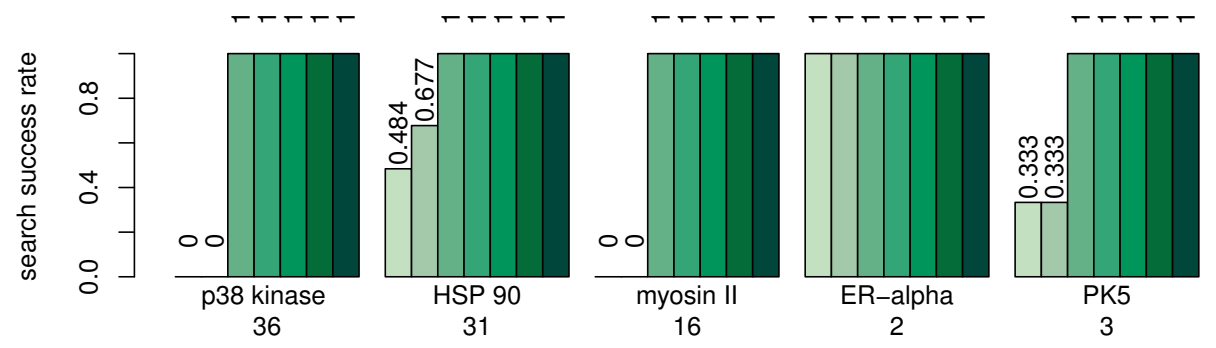

SIENA settings

$\square$ global identity $100 \%$

$\square$ global mutation rate $0 \%$

$\square$ site identity $100 \%$

$\square$ site gap rate $50 \%$

$\square$ site mutation rate $20 \%$

$\square$ site identity $80 \%$

- site identity $30 \%$

b)

Figure S1: Portion of structures that are identified by SIENA with different settings for (a) the five most frequent and (b) the five most flexible Astex Non-native targets. The reference sets are defined by the Astex Non-native Set. Their sizes are indicated by the number below the protein names. Two entries (2NMW and 2C5P) were substituted by their superseding entries (3OXC and 2WEV). One HSP 90 entry (2CDD) has been removed from the PDB and was therefore also removed from the reference set. 


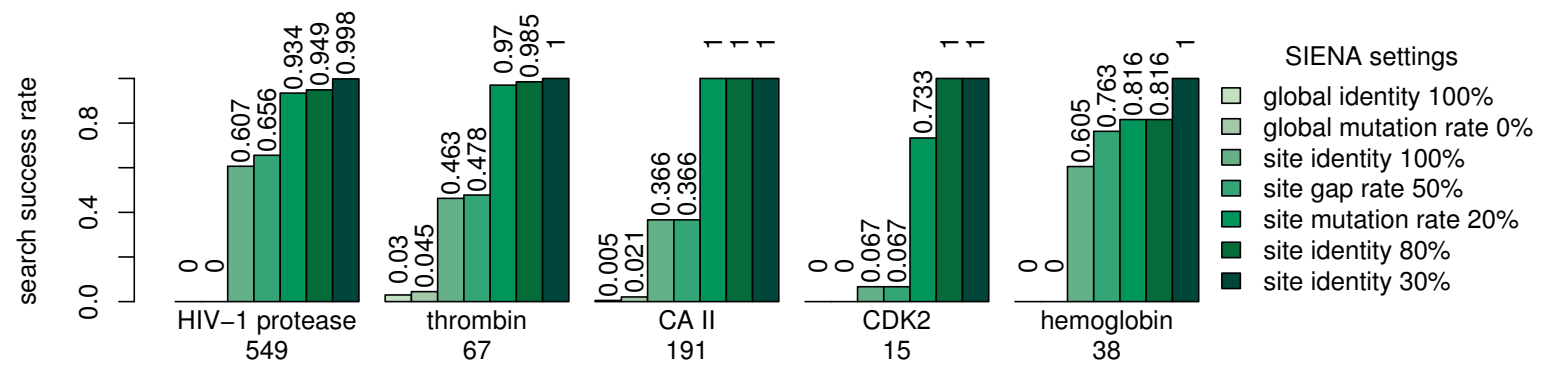

a)
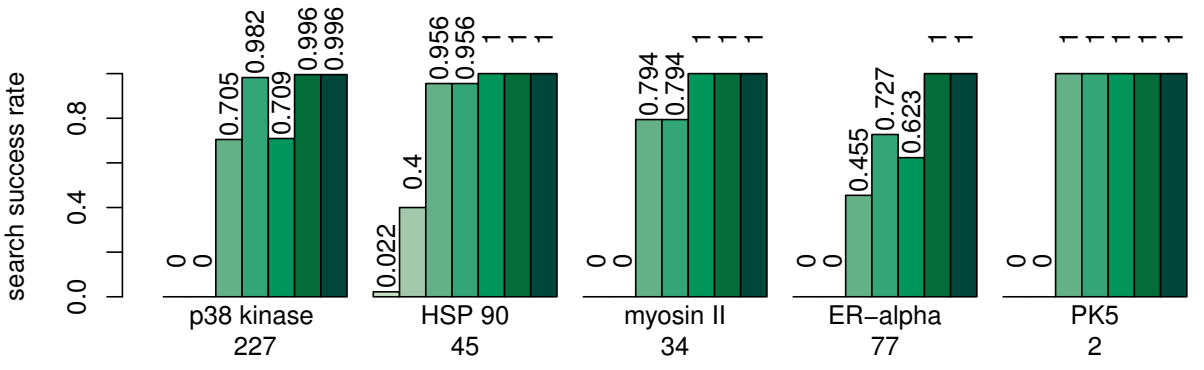

SIENA settings

$\square$ global identity $100 \%$

$\square$ global mutation rate $0 \%$

$\square$ site identity $100 \%$

$\square$ site gap rate $50 \%$

$\square$ site mutation rate $20 \%$

site identity $80 \%$

- site identity $30 \%$

b)

Figure S2: Portion of structures that are identified by SIENA with different settings for (a) the five most frequent and (b) the five most flexible Astex Non-native targets. The reference sets are defined by PDB BLAST searches using a chain identity of $90 \%$. Their sizes are indicated by the number below the protein names. Results that were also obtained with PDB BLAST searches using a chain identity of $100 \%$ were removed from the reference sets. 


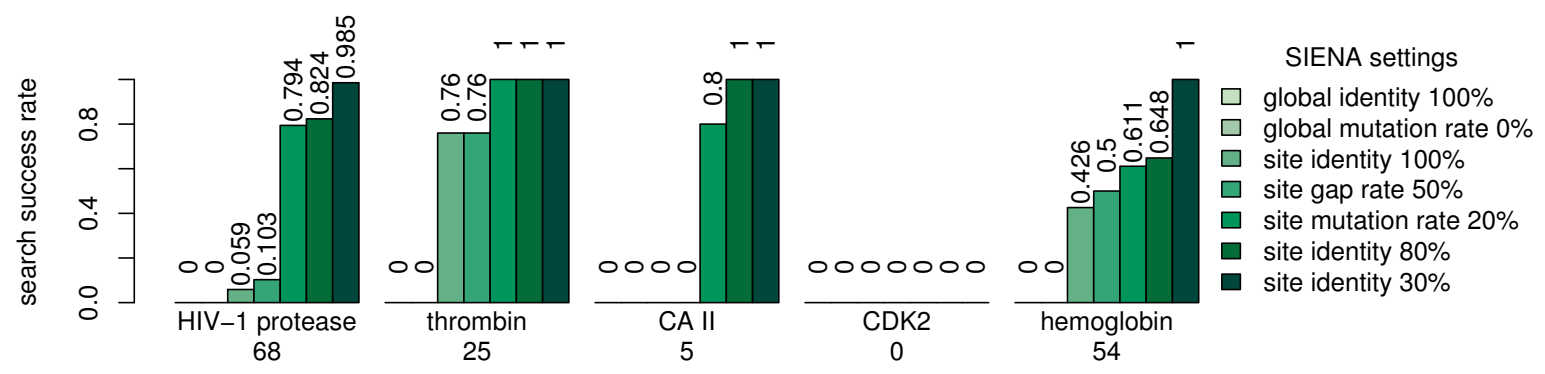

a)
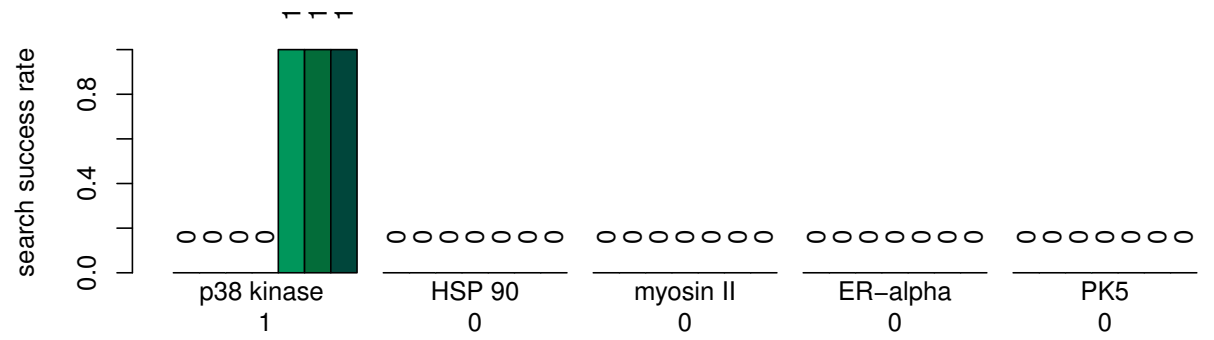

SIENA settings

$\square$ global identity $100 \%$

$\square$ global mutation rate $0 \%$

$\square$ site identity $100 \%$

$\square$ site gap rate $50 \%$

$\square$ site mutation rate $20 \%$

site identity $80 \%$

site identity $30 \%$

b)

Figure S3: Portion of structures that are identified by SIENA with different settings for (a) the five most frequent and (b) the five most flexible Astex Non-native targets. The reference sets are defined by PDB BLAST searches using a chain identity of $80 \%$. Their sizes are indicated by the number below the protein names. Results that were also obtained with PDB BLAST searches using a chain identity of $90 \%$ were removed from the reference sets. 

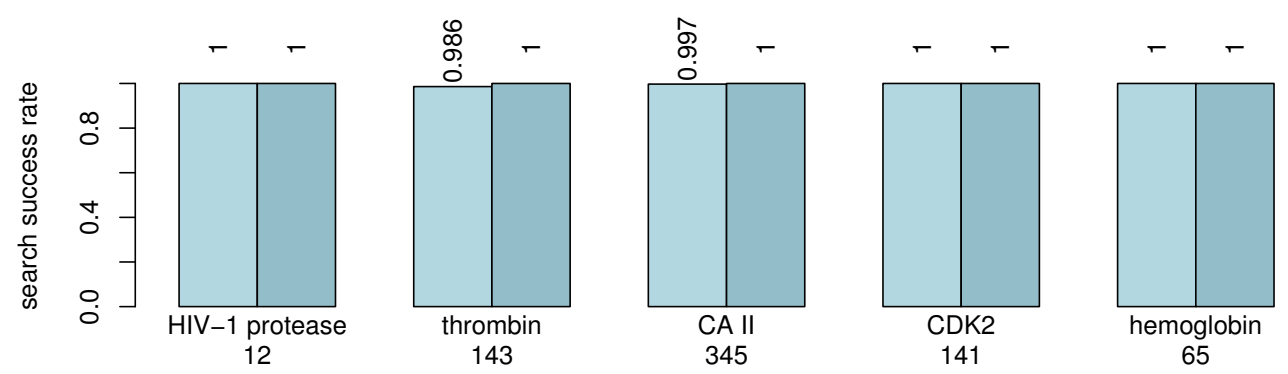

BLAST chain identity

$\square \quad 100 \%$

$\square 90 \%$

a)
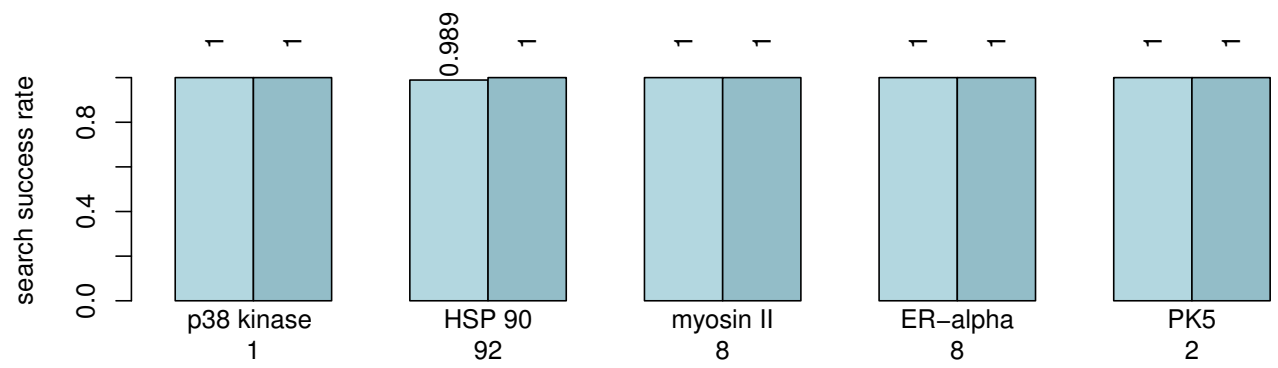

BLAST chain identity

$\square 100 \%$

$\square \quad 90 \%$

b)

Figure S4: Portion of structures that are identified by BLAST with different settings for (a) the five most frequent and (b) the five most flexible Astex Non-native targets. The reference sets are defined by SIENA searches using a global identity of $100 \%$. Their sizes are indicated by the number below the protein names. 

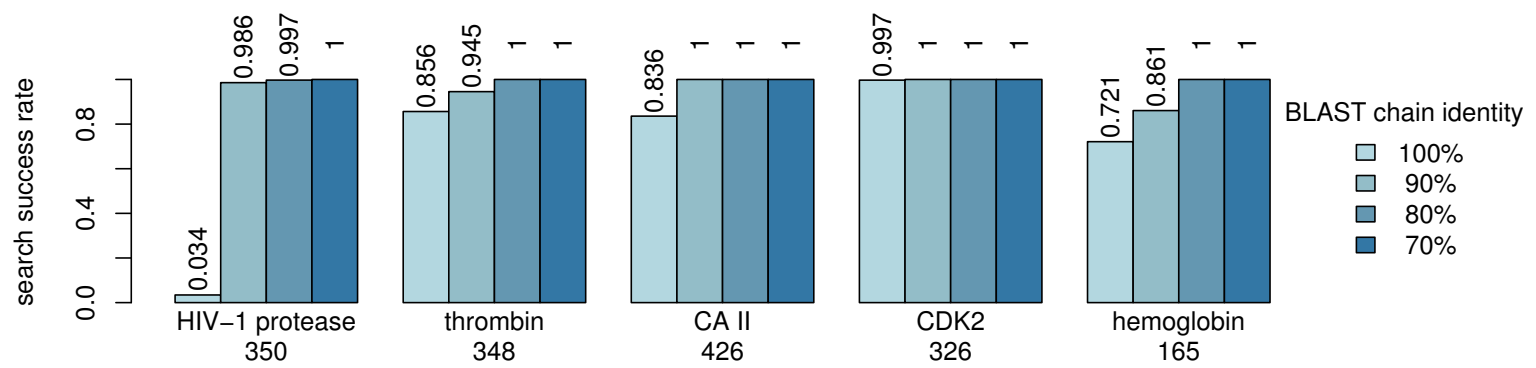

a)
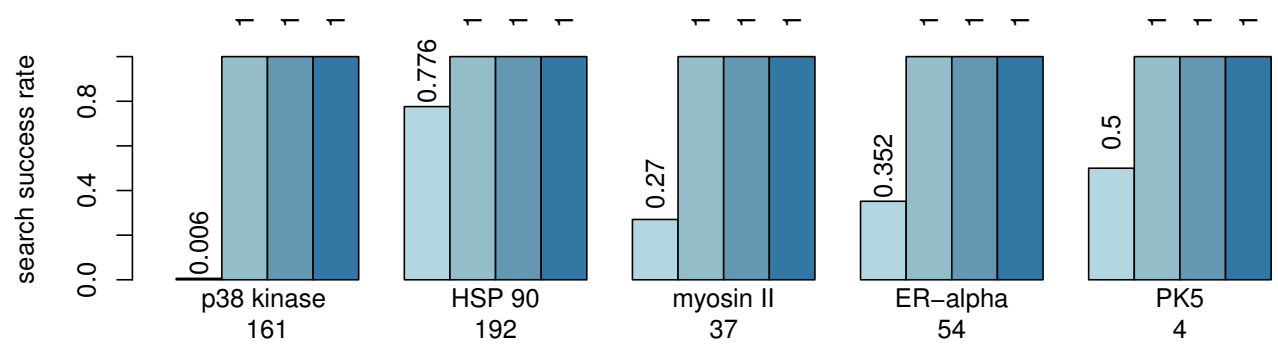

BLAST chain identity

$\square 100 \%$

$\square \quad 90 \%$

$\square 80 \%$

$\square 70 \%$

b)

Figure S5: Portion of structures that are identified by BLAST with different settings for (a) the five most frequent and (b) the five most flexible Astex Non-native targets. The reference sets are defined by SIENA searches using a site identity of 100\%. Their sizes are indicated by the number below the protein names. 

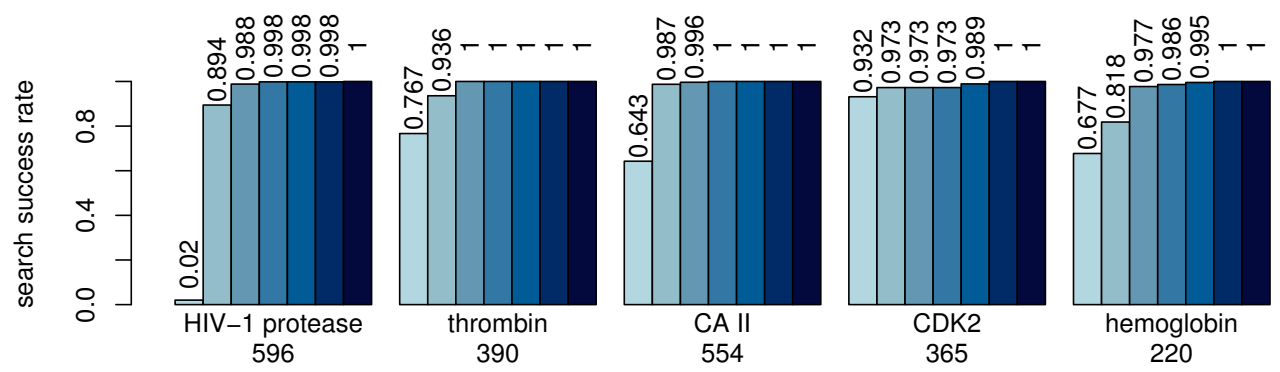

BLAST chain identity

$$
\begin{aligned}
& \square \quad 100 \% \\
& \square \quad 90 \% \\
& \square \quad 80 \% \\
& \square \quad 70 \% \\
& \square \quad 60 \% \\
& \square \quad 50 \% \\
& \square \quad 40 \%
\end{aligned}
$$

a)
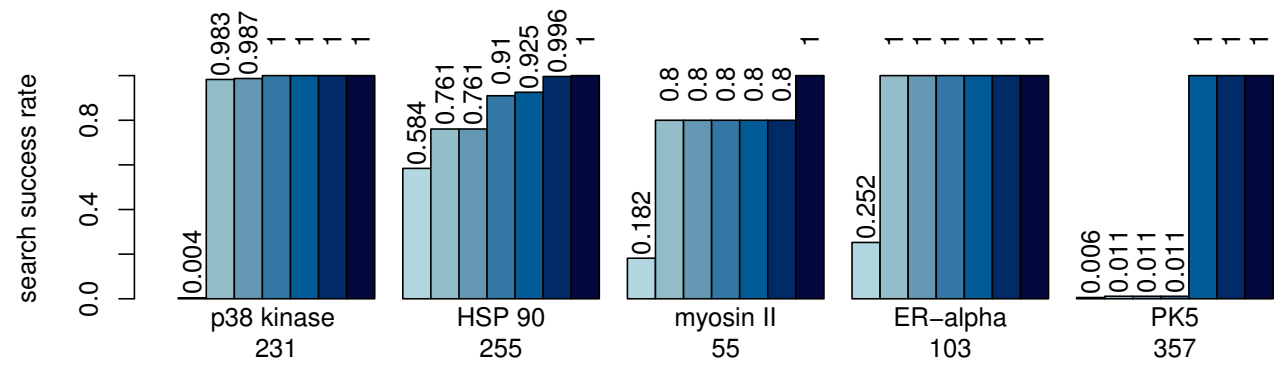

BLAST chain identity

$\square 100 \%$

$\square 90 \%$

$\square \quad 80 \%$

$\square 70 \%$

ㅁ $60 \%$

- $50 \%$

- $40 \%$

b)

Figure S6: Portion of structures that are identified by BLAST with different settings for (a) the five most frequent and (b) the five most flexible Astex Non-native targets. The reference sets are defined by SIENA searches using a site identity of $80 \%$. Their sizes are indicated by the number below the protein names. 


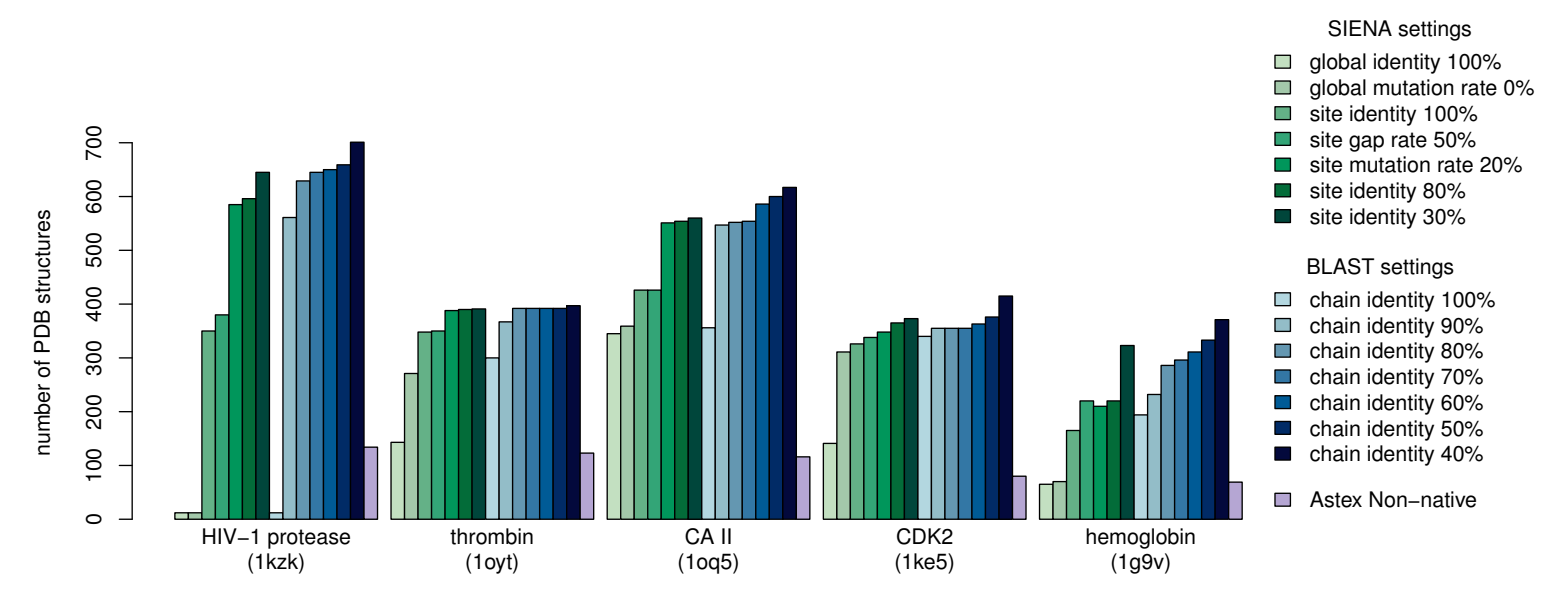

a)

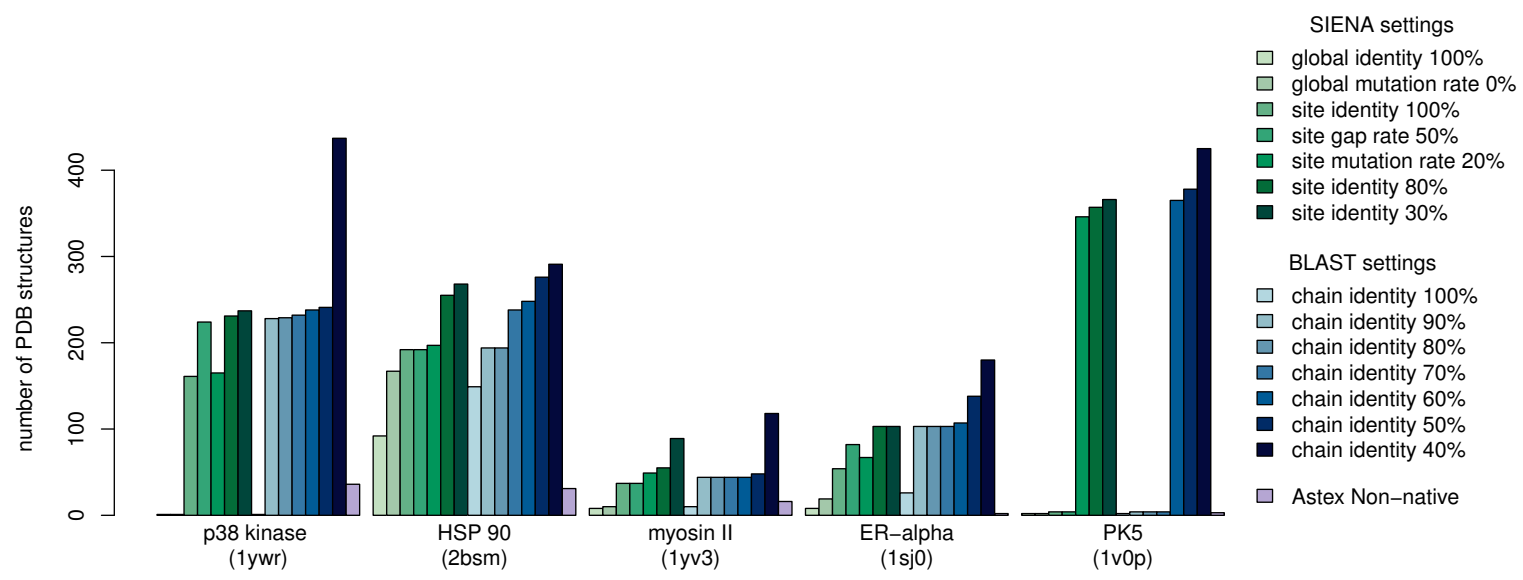

b)

Figure S7: Number of PDB structures that are contained in the Astex Non-native Set and in the ensembles generated by BLAST and SIENA for (a) the five most frequent and (b) the five most flexible Astex Non-native targets. 

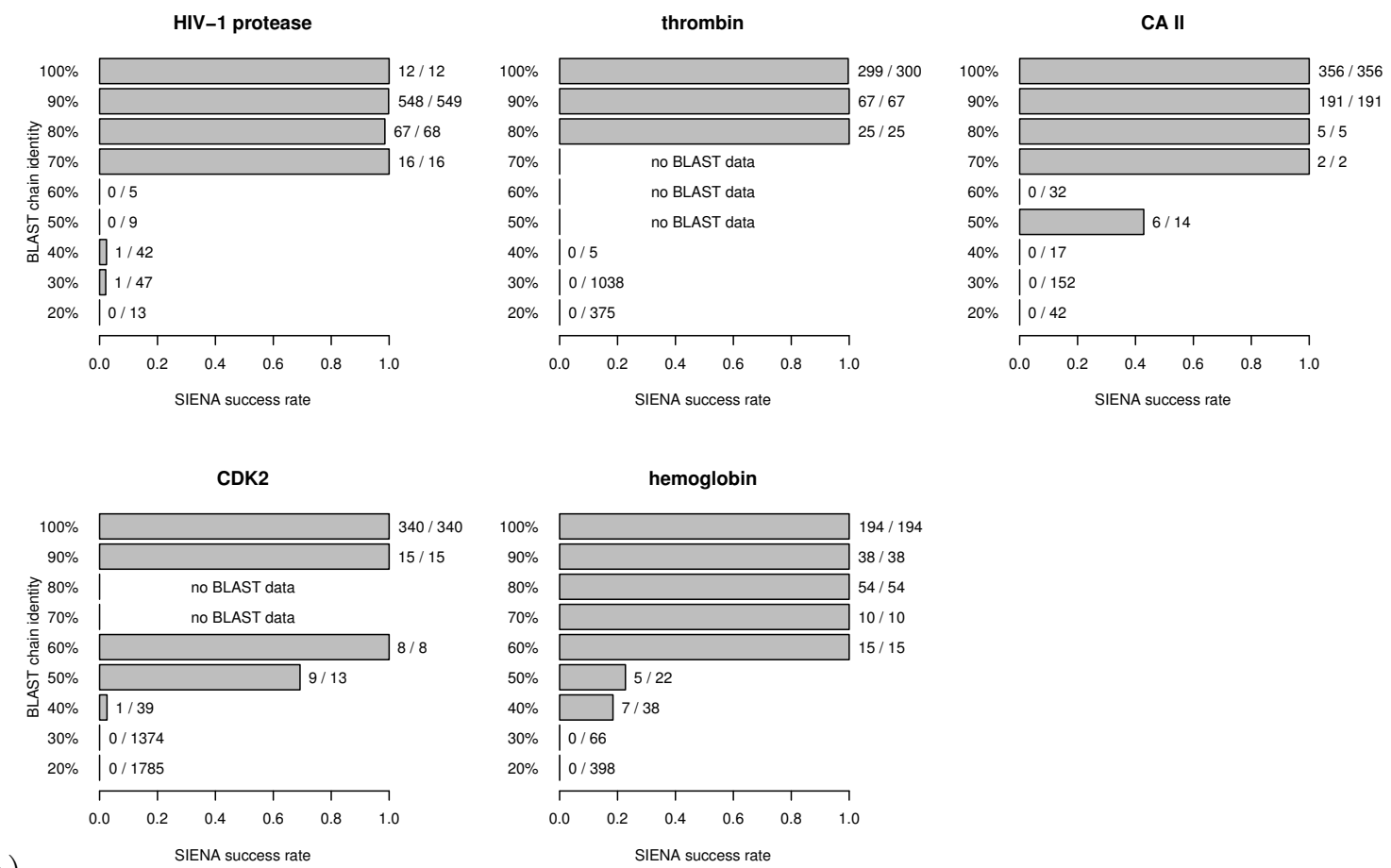

a)
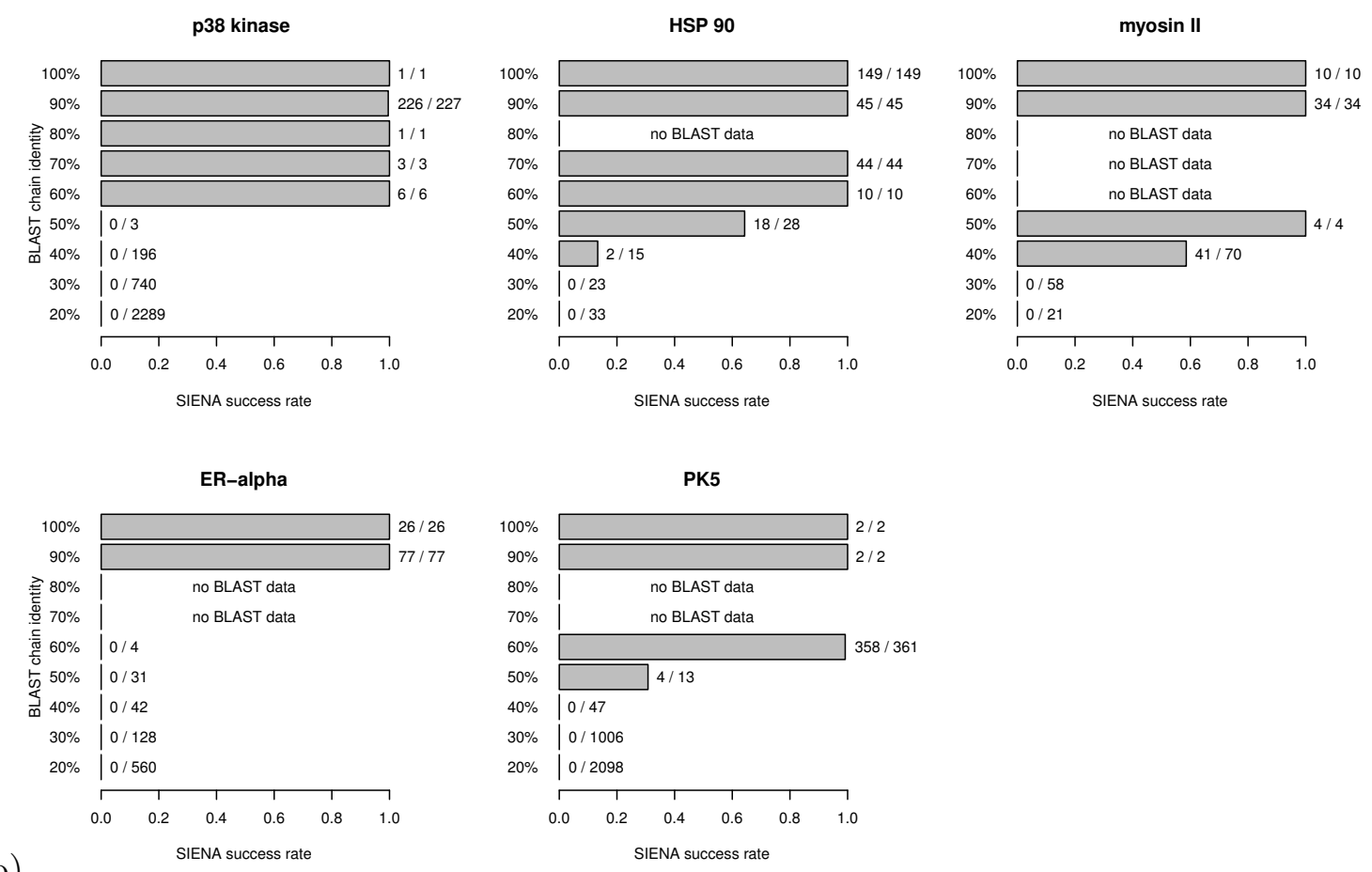

Figure S8: Portion of structures that are identified by SIENA using the $30 \%$ site identity threshold for (a) the five most frequent and (b) the five most flexible Astex Non-native targets. For each target, different reference sets were generated with PDB BLAST searches using varying chain identity thresholds. Each reference set contains only those structures which were not yet detected at the respectively higher identity thresholds. 

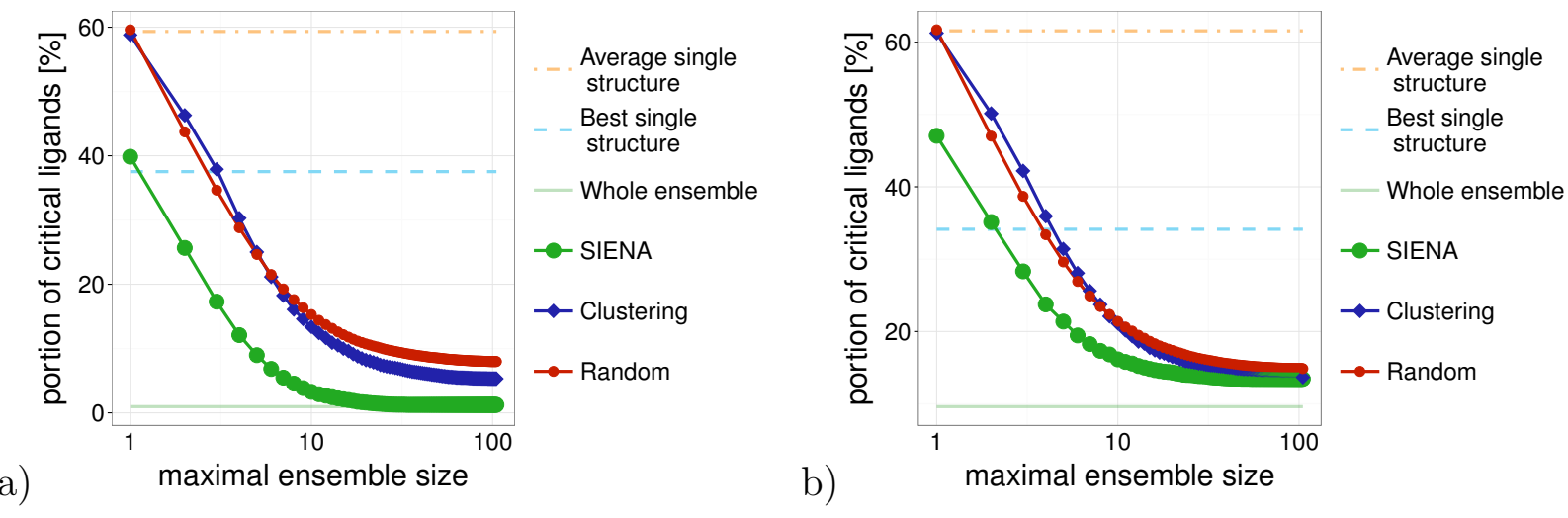

Figure S9: Portion of critical ligands of all NBSE targets as a function of the maximal number of structures in a reduced ensemble (maximal ensemble size). As reference values, the average and best performance of single structures are depicted. Additionally, the optimal ensemble performance is given by the performance of the non-reduced training set ensemble. (a) Results for all training sets (b) Results for all test sets.

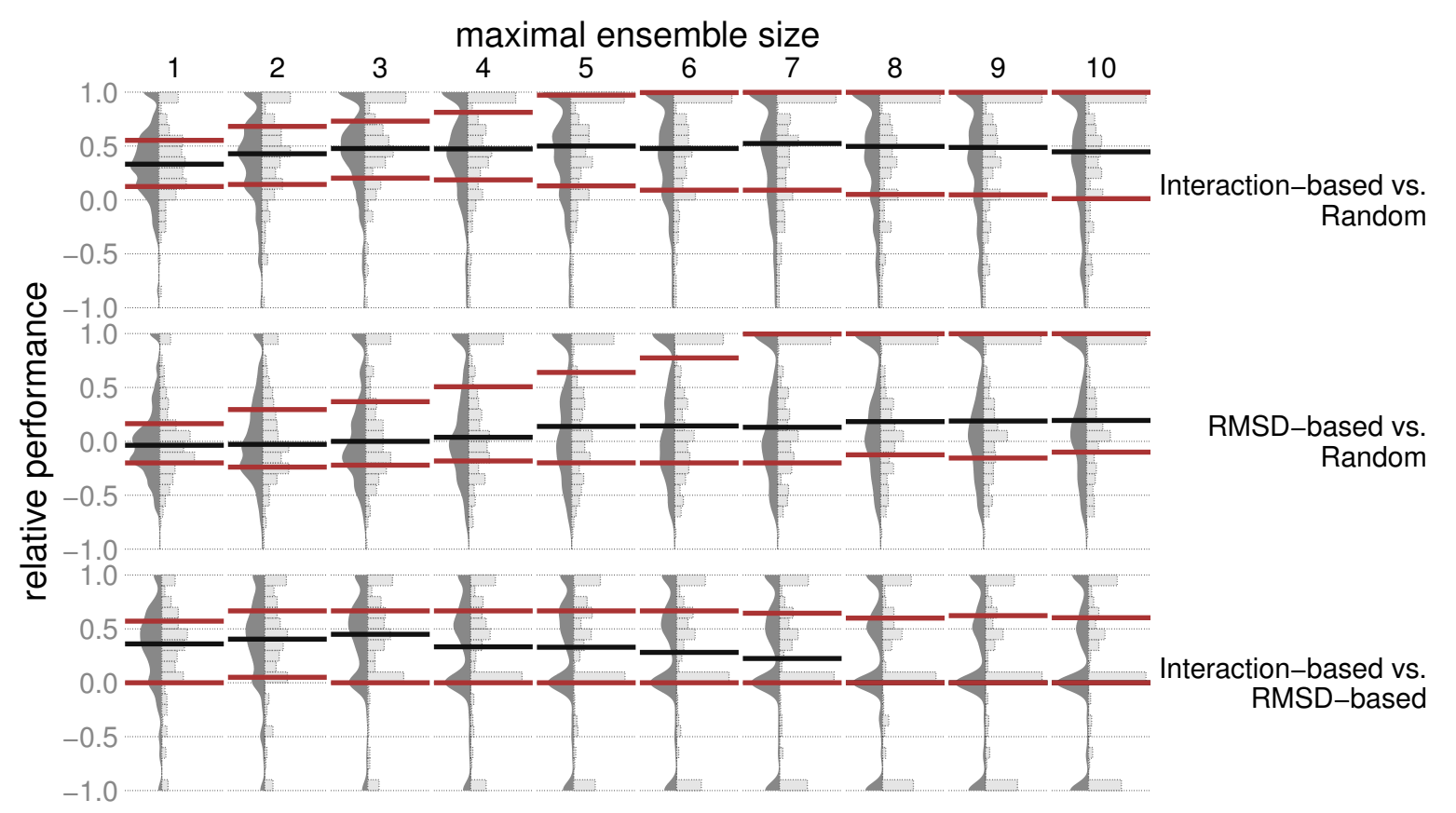

Figure S10: Relative performance of different ensemble reduction approaches as a function of the maximal number of structures in a reduced ensemble (maximal ensemble size). The depicted data represents the result from the test set evaluation. The distribution of the relative performance across all NBSE targets is depicted as an approximated density on the left-hand side and in form of a histograms on the right-hand side of each subfigure. The black and red bars indicate the median values and the $25 \%$ and $75 \%$ quantiles, respectively. Note: Figure 5 depicts data for different ensemble sizes. 


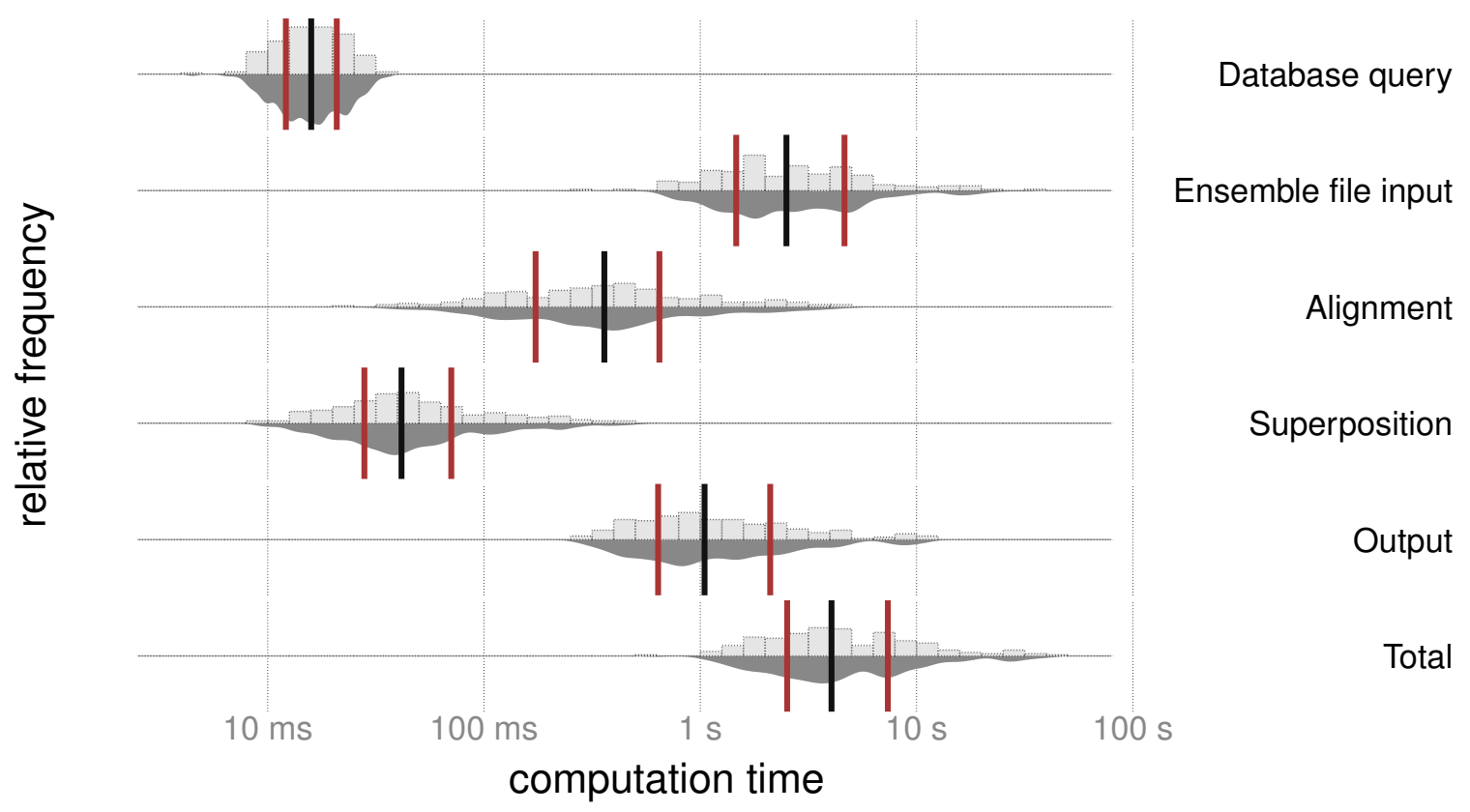

Figure S11: Computation times for all major steps in the SIENA's ensemble generation process for non-reduced ensembles from the NBSE set. The distribution of the computation times across all NBSE targets is depicted as an approximated density on the lower half and in form of a histogram on the upper half of each subfigure. The black and red bars indicate the median values and the $25 \%$ and $75 \%$ quantiles, respectively. 


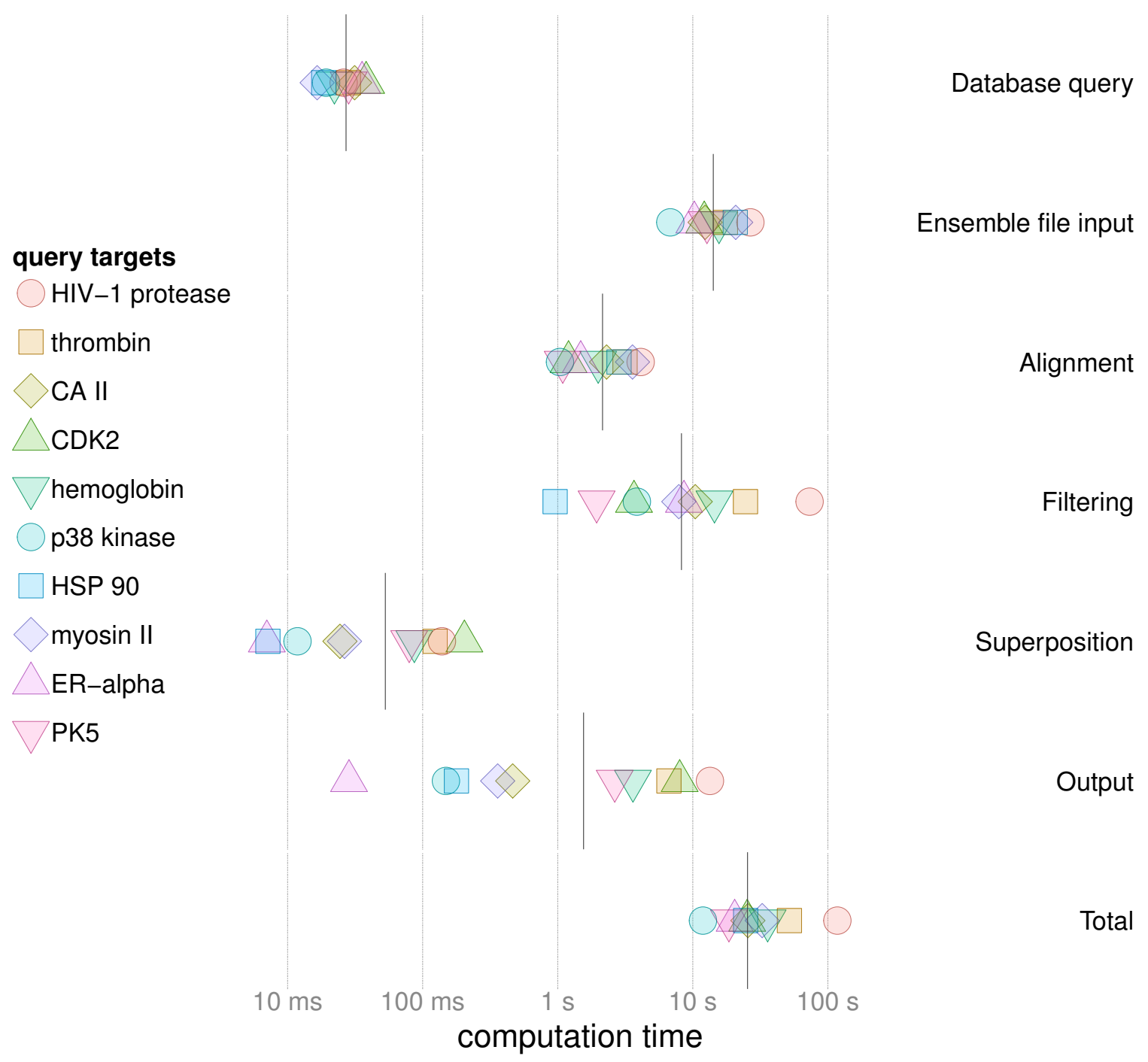

Figure S12: Computation times for all major steps in the SIENA's ensemble generation process for 10 target from the Astex Diverse set using the $100 \%$ global identity setting. 


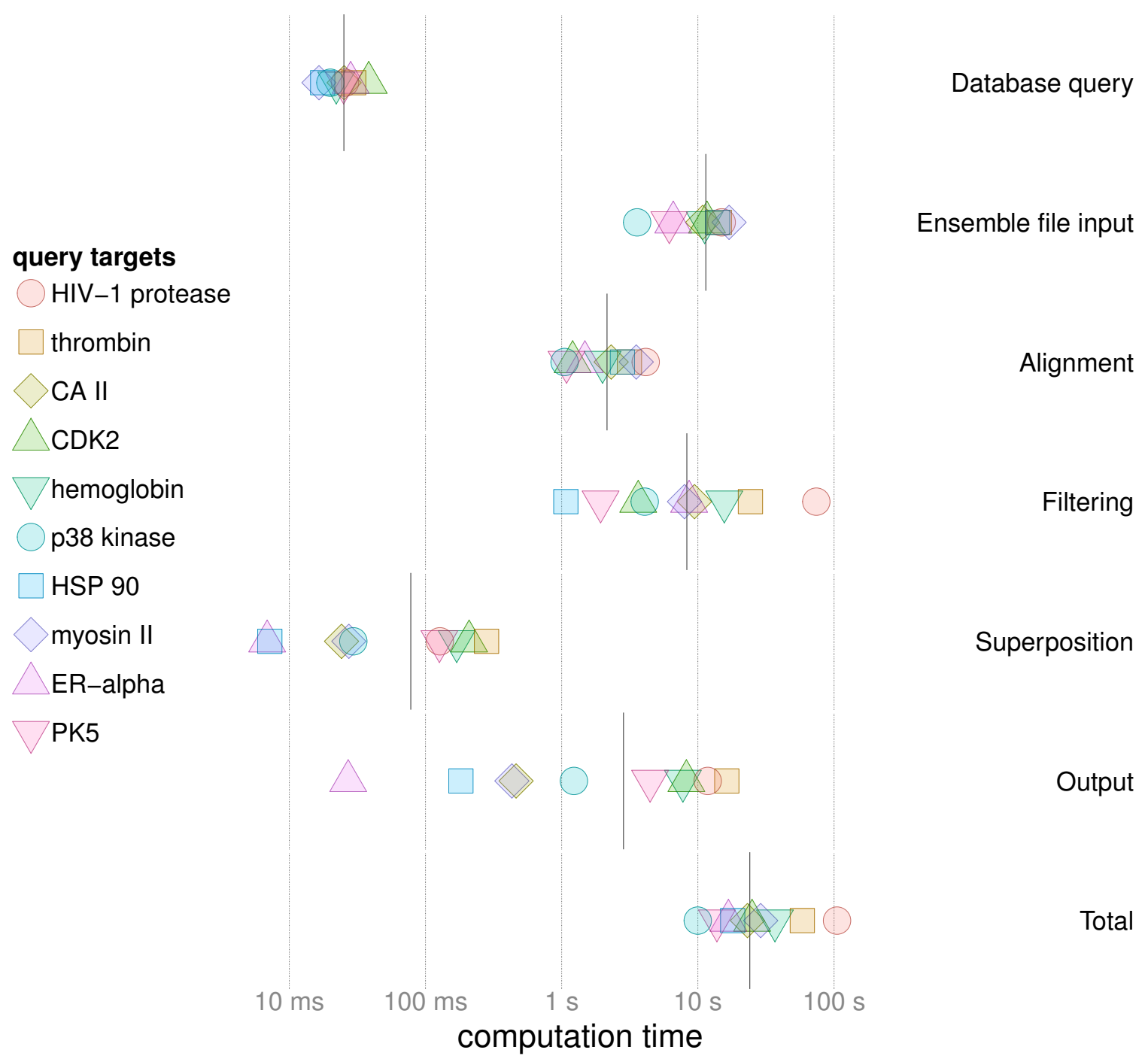

Figure S13: Computation times for all major steps in the SIENA's ensemble generation process for 10 target from the Astex Diverse set using the $0 \%$ global mutation rate setting. 


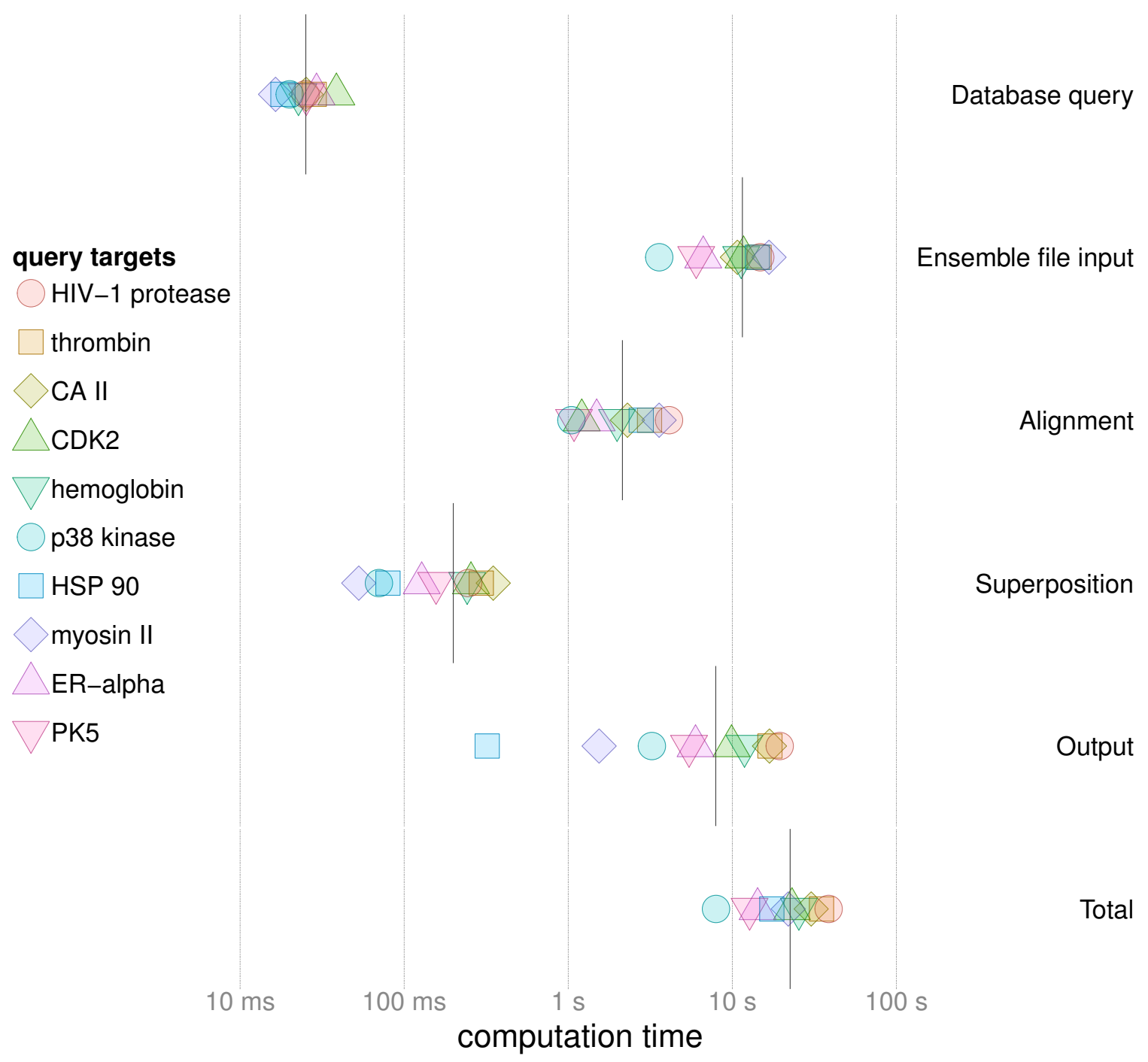

Figure S14: Computation times for all major steps in the SIENA's ensemble generation process for 10 target from the Astex Diverse set using the 100\% site identity setting. 


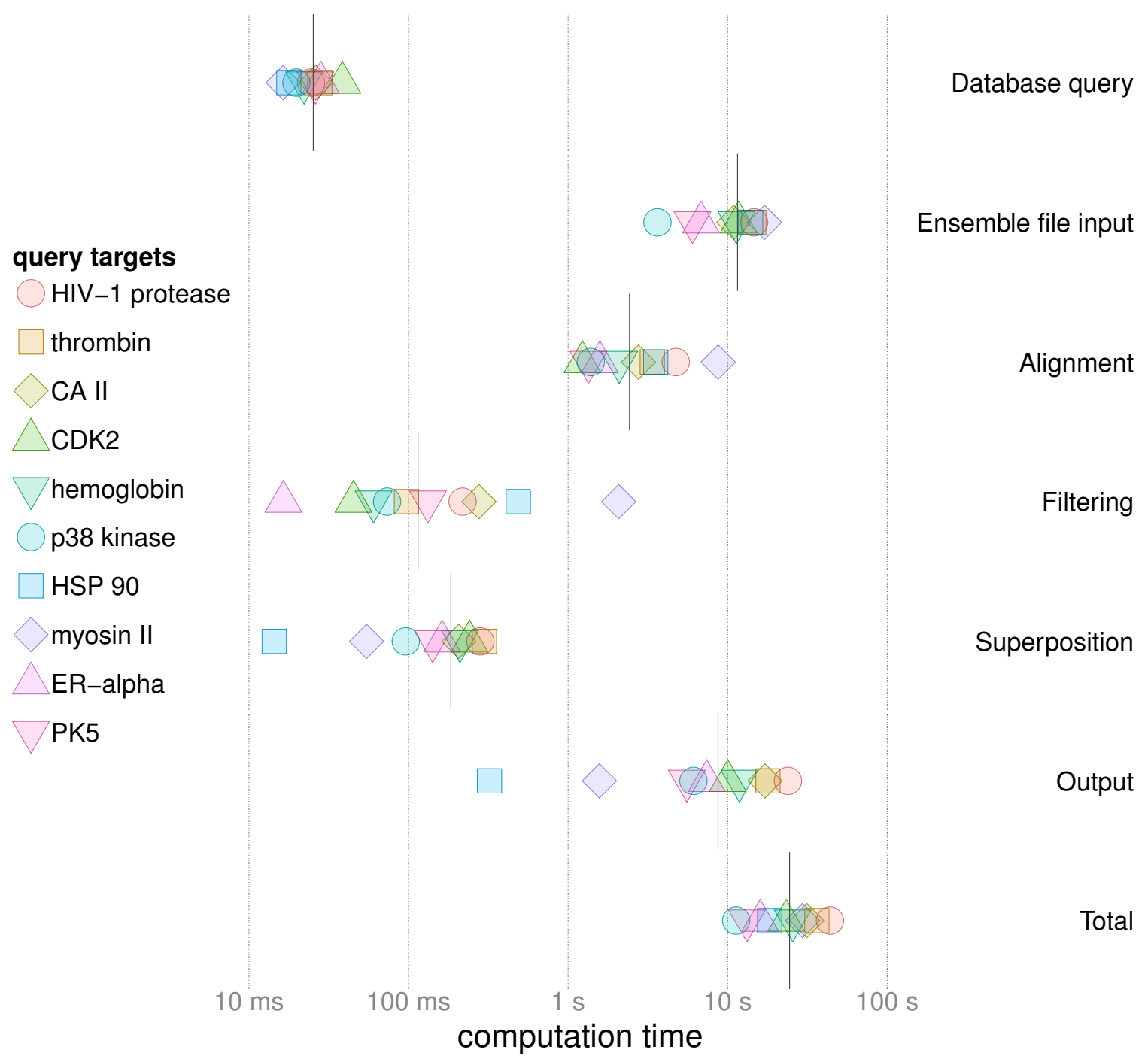

Figure S15: Computation times for all major steps in the SIENA's ensemble generation process for 10 target from the Astex Diverse set using the $50 \%$ site gap rate setting. 


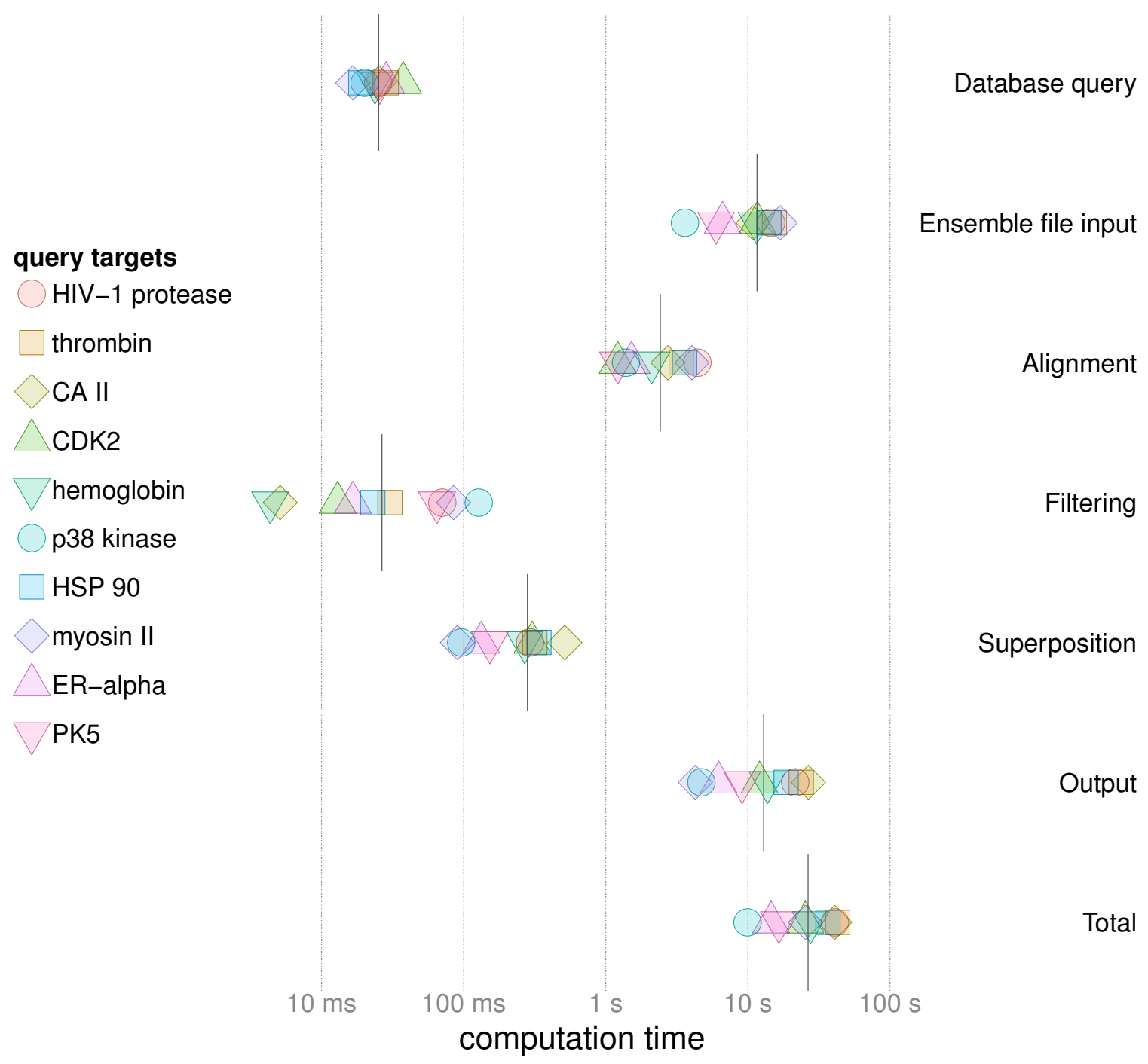

Figure S16: Computation times for all major steps in the SIENA's ensemble generation process for 10 target from the Astex Diverse set using the 20\% site mutation rate setting. 


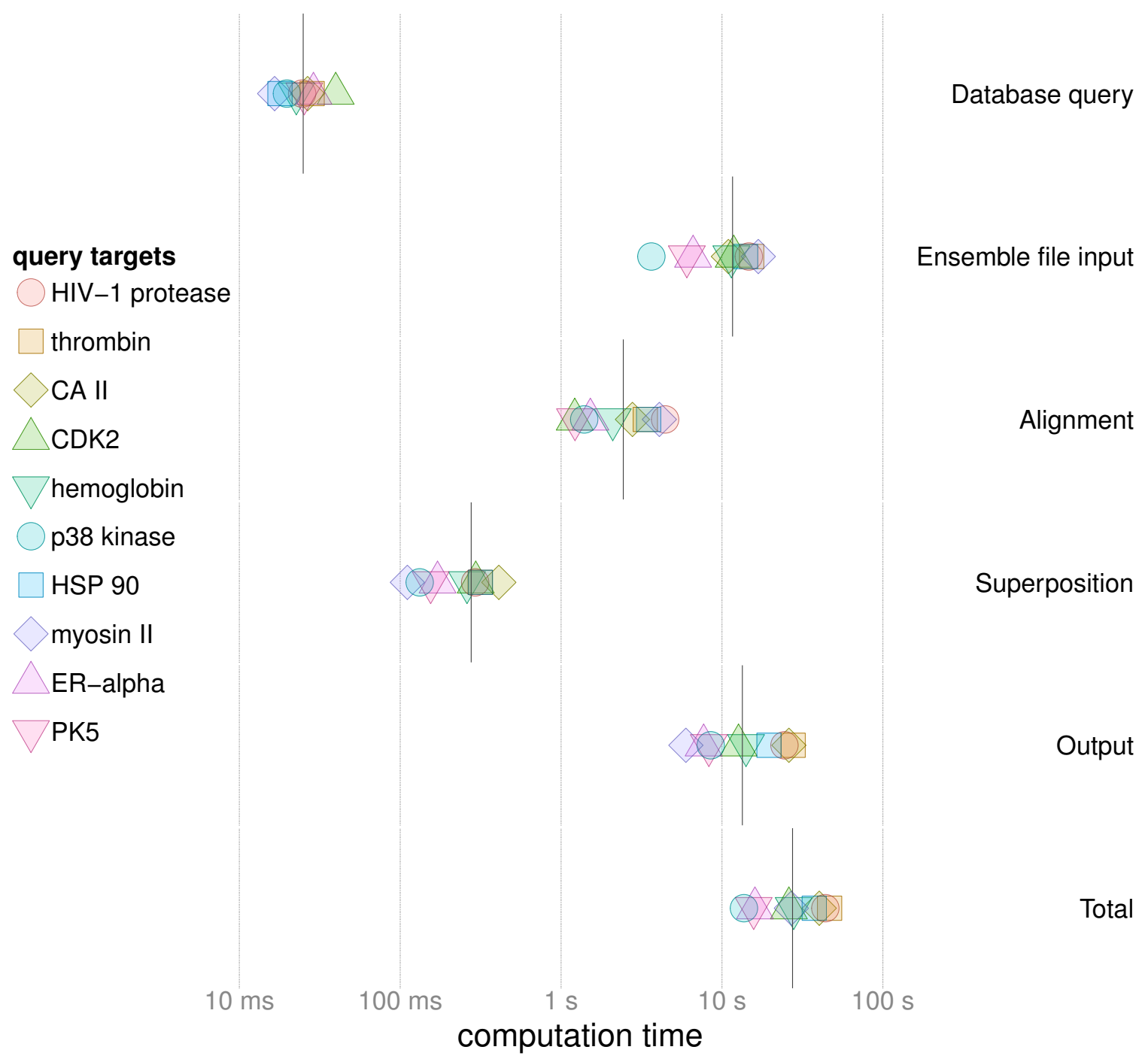

Figure S17: Computation times for all major steps in the SIENA's ensemble generation process for 10 target from the Astex Diverse set using the $80 \%$ site identity setting. 


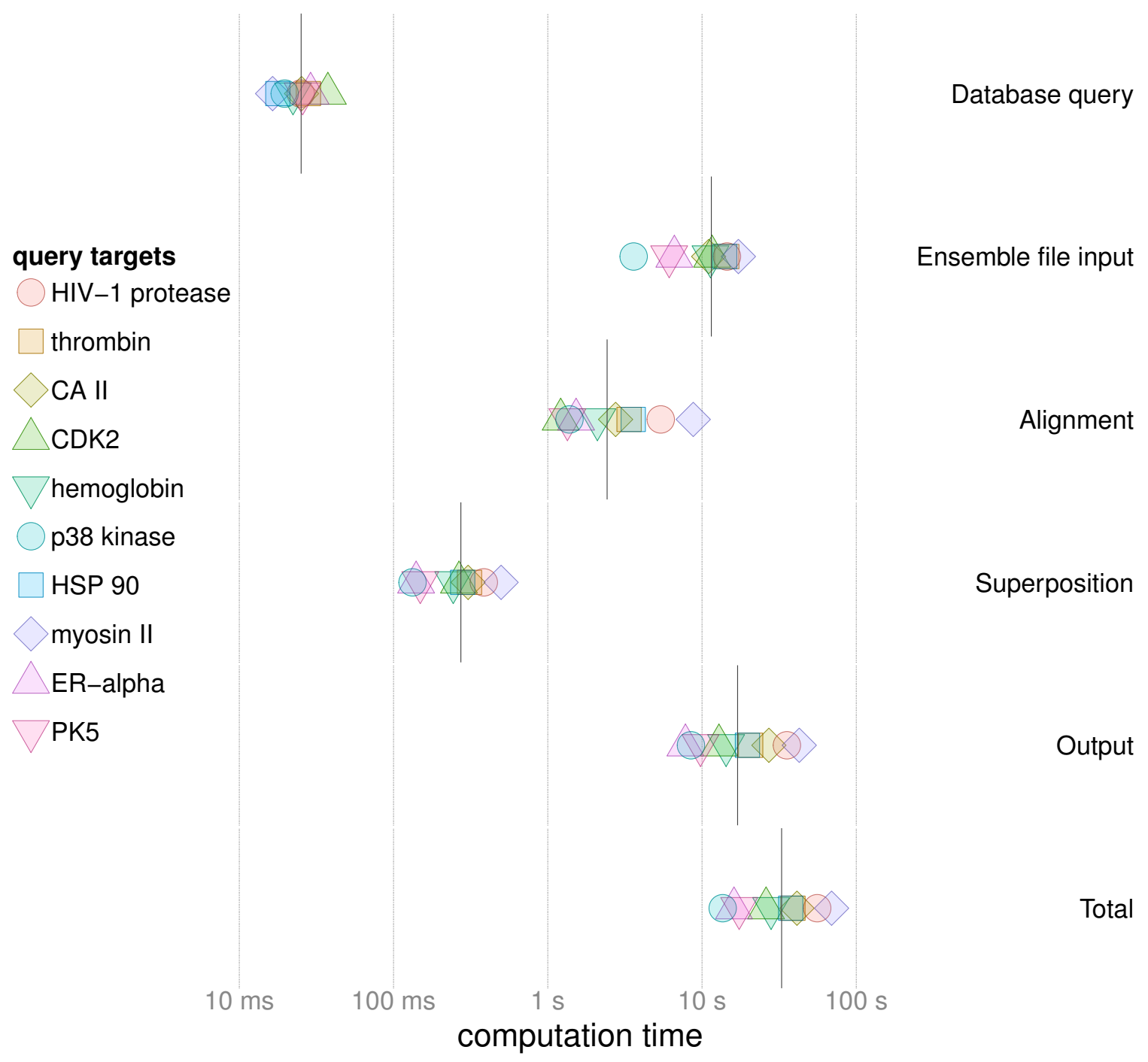

Figure S18: Computation times for all major steps in the SIENA's ensemble generation process for 10 target from the Astex Diverse set using the $30 \%$ site identity setting. 
Table S1: List of molecules which are not considered as ligands. Molecules are indicated by PDB identifiers.

Ligand HET codes: 0HH, 10A, 12H, 12P, 13S, 140, 144, 15P, 16A, 16D, 192, 1AB, 1AC, 1AN, 1BO, 1BP, 1CB, 1CM, 1GN, 1LU, 1MA, 1MC, 1MR, 1MZ, 1PE, 1PG, 202, 20S, 217, 233, 24T, 25T, 26D, 2AC, 2AF, 2AP, 2BM, 2BR, 2CH, 2CM, 2EZ, 2FU, 2HA, 2HP, 2IB, 2IM, 2KT, 2ME, 2MG, 2MP, 2MZ, 2NO, 2OS, 2PA, 2PC, 2PE, 2PN, 2PO, 34A, 3AP, 3BB, 3BR, 3CH, 3CL, 3CN, 3EP, 3FA, 3GR, 3HL, 3MC, 3MF, 3MO, 3MP, 3MT, 3NP, 3OH, 3OL, 3PH, 3PO, 3PP, 3PY, 3TR, 4AP, 4AX, 4CB, 4HA, 4IP, 4MV, 4MZ, 4PA, 5AN, 5BR, 5IP, 5MC, 5MP, 5MU, 6NA, 6PC, 749, 7MG, 9CS, A2G, A3B, A48, A5P, A6P, $\mathrm{AAB}, \mathrm{AAC}, \mathrm{AAE}, \mathrm{ABA}, \mathrm{ABH}, \mathrm{ABN}, \mathrm{ABU}, \mathrm{AC} 0, \mathrm{AC} 5, \mathrm{ACA}, \mathrm{ACD}, \mathrm{ACE}, \mathrm{ACM}, \mathrm{ACN}$, ACR, ACT, ACY, ADA, ADE, ADM, ADP, AE3, AEM, AFB, AG2, AGA, AGU, AHG, AHI, AHR, AI2, AIB, AIO, AJ3, AKB, AKR, ALA, ALG, ALQ, AMB, AMC, AML, AMT, AMV, ANL, AOA, APB, ARE, ARF, ARS, ART, AS, AS2, ASN, ASP, AST, ATJ, ATO, ATQ, AZI, B2A, B2F, B2I, B2V, BAL, BAM, BBU, BCD, BCT, BDB, BDD, BDP, BEM, BEN, BEO, BEQ, BET, BEZ, BGC, BGL, BGX, BH1, BHH, BHL, BIB, BJH, BJI, BJP, BLA, BLE, BLV, BLY, BMA, BME,BME, BML, BMM, BMT, BNG, BNO, BNS, BNZ, BO3, BO4, BOC, BOG, BOM, BOR, BPH, BR5, BRB, BRC, BRJ, BRP, BTB, BTL, BTN, BU1, BU2, BU3, BUA, BUB, BUQ, BVC, BVF, BVG, BZB, BZF, BZI, BZP, C21, C2A, C2B, C2N, C8E, CA1, CAB, CAC, CAD, CAM, CAN, CAP, CAQ, CAS, CAT, CCM, CCN, CCP, CDL, CE1, CE8, CE9, CEF, CEJ, CEP, CEQ, CFA, CFQ, CFT, CGU, CH2, CHM, CHT, CHX, CIG, CIR, CIT, CKP, CLB, CLD, CLL, CLX, CM6, CME, CMP, CMS, CMT, CNH, CNN, CO2, CO3, COI, COM, CP, CP4, CPS, CRD, CRN, CRS, CRT, CSD, CSO, CSS, CSW, CTB, CTR, CTT, CVB, CXB, CXE, CXF, CXL, CXM, CYC, CYH, CYI, CYS, CYT, D12, D1D, D2P, DA1, DAL, DAO, DAS, DAV, DBP, DCE, DCY, DDQ, DEM, DEN, DEP, DER, DFP, DFX, DG6, DGA, DGG, DGL, DGY, DHA, DHD, DHK, DHM, DHS, DIA, DIB, DIO, DLE, DM1, DMF, DMG, DMN, DMR, DMS, DOD, DP3, DP4, DPE, DPF, DPJ, DPN, DPO, DPR, DRN, DSG, DSN, DSS, DTD, DTH, DTI, DTL, DTO, DTT, 
DTU, DTV, DUC, DVA, DXE, DXX, DZZ, EAP, EDO, EEE, EFS, EGC, EGD, ELA, EMM, ENC, EOH, EPE, EPO, ESA, ETA, ETF, ETI, ETM, ETN, ETP, ETX, ETY, F09, F6P, FA1, FA6, FAC, FAG, FBA, FCA, FCB, FCL, FCN, FFP, FLA, FLC, FLM, FMN, FMS, FMT, FNG, FOA, FOP, FOR, FPI, FPN, FPO, FPR, FPY, FRU, FU2, FUC, FUM, FUX, FX3, G16, G1P, G3P, G4D, G4S, G6P, GAG, GAI, GAL, GAQ, GAU, GB, GBD, GBL, GC4, GCO, GCU, GDM, GEG, GER, GG6, GLA, GLC, GLL, GLN, GLO, GLR, GLS, GLU, GLV, GLY, GM1, GOA, GOL, GPM, GSC, GSH, GUA, GVE, GVH, GYP, GZZ, H01, H02, H2O, H2S, H2U, H4B, HAE, HAI, HAV, HBA, HBR, HBS, HCA, HCS, HDA, HDS, HE2, HE4, HED, HEQ, HEX, HEZ, HGU, HHO, HIO, HIU, HLE, HLT, HMC, HMF, HMN, HOA, HOH, HOZ, HP6, HPA, HPH, HPN, HPY, HSE, HSL, HSM, HSW, HT, HTO, HTS, HXA, HY1, HYA, HYF, HYP, I, I3P, I4P, IAP, IAS, IBO, IBS, IBZ, ICN, ICP, ICT, IDH, IDM, IDR, IDS, IHG, IHP, ILE, IMD, IMR, IND, IOB, IOL, IOM, IP5, IPA, IPH, IPM, IPU, ISP, ISU, ITU, IUR, IVA, IZC, JEF, KCX, KDF, KIV, KMT, KOS, KPH, L1P, L2P, L3P, L4P, LAC, LAF, LAR, LAT, LBT, LCP, LDA, LDM, LDY, LEA, LEN, LEU, LG3, LG4, LG5, LG6, LGV, LI1, LIO, LIS, LLP, LMT, LMU, LNK, LNL, LPC, LPG, LTL, LVG, LXP, LYS, LYT, M1N, M1P, M2G, M2M, M6D, M6P, MAE, MAH, MAK, MAL, MAN, MAS, MAV, MAW, MBD, MBN, MBR, MBT, MBV, MCR, MCT, MD2, MDD, ME2, MEC, MED, MEE, MES, MET, MEV, MEZ, MFU, MG8, MGO, MGX, MHN, MHO, MIC, MLA, MLE, MLI, MLM, MLP, MLR, MLT, MMA, MMP, MMQ, MMZ, MNA, MNC, MOH, MOR, MPA, MPC, MPD, MPG, MPI, MPJ, MPO, MR3, MRC, MRD, MRY, MSE, MSF, MSM, MTG, MTL, MUR, MVA, MVL, MXE, MYR, MYS, N2O, N2P, N8E, NAG, NAK, NBE, NBF, NBN, NBT, NBU, NBZ, NCA, NCM, ND4, NDG, NEH, NEN, NEQ, NET, NGA, NGS, NH2, NH3, NH4, NHE, NHV, NHY, NIO, NIS, NLE, NME, NMH, PGE, , NO, NO2, NO3, NOE, NOY, NPB, NPN, NPY, NS1, NS5, NSM, NT, NTA, NTB, NTC, NTJ, NTN, NVA, NVI, NXA, O, OAA, OC9, OCA, OCT, OCY, ODS, OMC, OMG, OPE, ORN, OSM, OXD, OXE, OXL, OXM, OXN, OXP, OXQ, P1R, P2O, P33, P4C, P4G, P6G, PAE, PAH, PAM, PBA, PBC, PBR, PCA, PCR, PCT, PCZ, PDO, PDT, PE3, PE4, PE5, 
PE6, PE7, PE9, PEA, PED, PEG, PEL, PEO, PEU, PG0, PG3, PG4, PG5, PG6, PGA, PGE, PGH, PGO, PGR, PH1, PHB, PHN, PHO, PHS, PHZ, PI, PID, PIH, PIM, PIN, PIP, PIS, PLD, PLM, PLP, PMP, PNZ, PO2, PO4, POA, POL, PON, POP, PPB, PPF, PPI, PPK, PPV, PRI, PRO, PS5, PSE, PSL, PSU, PTD, PTL, PTR, PUB, PUT, PXY, PY7, PYC, PYD, PYE, PYF, PYG, PYL, PYM, PYQ, PYR, PYS, PYT, PYZ, PZO, QPS, QV4, R5P, RAF, RCL, RCO, REA, RET, RGI, RIB, RIP, RNS, RNT, RPD, RPL, RUB, S0H, SAR, SAT, SB1, SBD, SBE, SBL, SBO, SBT, SCC, SCH, SCN, SCS, SDS, SE, SE4, SEP, SER, SES, SFO, SGL, SGM, SGN, SHF, SHO, SHV, SIA, SIF, SIN, SLE, SLF, SM2, SM3, SM4, SMB, SMC, SO2, SO3, SO4, SO4, SOA, SOR, SPA, SPH, SPM, SPN, SPO, SQU, SRB, SRD, SRT, SS1, SS2, STA, SUC, SUF, SUM, T1A, T42, TAM, TAR, TAS, TAU, TBU, TC4, TCB, TCZ, TDA, TDR, TEO, TF4, TFA, TFB, THE, THJ, THP, THR, TLA, TMA, TME, TMT, TMZ, TOU, TP5, TPO, TRA, TRC, TRD, TRE, TRI, TRS, TRT, TSM, TSZ, TTN, TWT, TYI, TYS, TZC, TZE, TZL, TZZ, U10, UNA, UND, UNK, UPL, URA, URE, URF, URP, UVW, V35, V36, VA1, VAL, VIG, VSO, VX, VXA, WBU, WZ1, WZ2, XAP, XDL, XDN, XIF, XLS, XPE, XUL, XYD, XYH, XYL, XYP, XYS, YAN, YG, YRR, ASG, DCL, DKA, MBG, MH6, PC1, PHQ, RAM, T32, 2LU, 3MG, 4SC, 6CT, 9MR, ACI, AGL, B1F, B4G, BCG, BRM, BZO, CBI, CBM, CBX, CBZ, CEC, CH3, CM5, CMO, CRY, CYA, DIS, DOM, DOX, DUM, EHN, EOX, ETD, ETO, FLO, FUB, FX1, G2I, GAC, GCM, GCS, GLB, GLD, GS1, GTE, H1M, HP3, HPG, HS2, HYD, ILG, IOH, IPS, KDA, KDB, KDD, KDE, KDR, KFG, KO2, KO4, MAB, MCB, MCE, MTO, MTT, NMO, NYT, OBD, OHE, OMB, OME, OTE, OXA, OXO, OXY, OXZ, PAR, PER, PPM, PSS, PVL, QPU, SBU, SEO, SFN, SGC, SOH, SOM, SUL, TMN, TPH, UNF, UNL, X4S, X5S, YT3.

Ion HET codes: $3 \mathrm{CO}, 4 \mathrm{MO}, 6 \mathrm{MO}, \mathrm{AG}, \mathrm{AL}, \mathrm{AR}, \mathrm{AU}, \mathrm{AU} 3, \mathrm{BA}, \mathrm{BR}, \mathrm{BR}, \mathrm{CA}, \mathrm{CD}$, CE, CL, CMO, CO, CR, CS, CU, CU1, CYN, EU, EU3, F, FE, FE2, GA, GD, GD3, HDZ, HG, HO, HO3, IOD, IR3, K, KR, LA, LI, LU, MG, MN, NA, NI, OS4, OXY, PB, PD, PER, PO3, PR, PT, RB, SM, SR, SX, TB, TL, U1, VO4, W, XE, YB, YT3, ZN. 
Table S2: Applied SIENA settings

\begin{tabular}{|c|c|c|}
\hline setting & description & applied parameters \\
\hline global identity $100 \%$ & $\begin{array}{l}\text { Generates a global alignment and removes all } \\
\text { matches which do not contain the complete } \\
\text { query structure. }\end{array}$ & $\begin{array}{l}\text { identical global } \\
\text { sequence = true; }\end{array}$ \\
\hline global mutation rate $0 \%$ & $\begin{array}{l}\text { Generates a global alignment and removes all } \\
\text { matches that exhibit mutations anywhere in } \\
\text { the structure or missing residues in the bind- } \\
\text { ing site. Gaps are only accepted in outside } \\
\text { of the binding site. }\end{array}$ & $\begin{array}{l}\text { no global } \\
\text { mutations = true; }\end{array}$ \\
\hline site identity $100 \%$ & $\begin{array}{l}\text { Generates a binding site alignment and re- } \\
\text { moves all matches that do not have exactly } \\
\text { the same sequence as the query binding site. }\end{array}$ & - (default setting $)$ \\
\hline site gap rate $50 \%$ & $\begin{array}{l}\text { Generates a binding site alignment and re- } \\
\text { moves all matches that have binding site mu- } \\
\text { tations or more than } 50 \% \text { missing residues in } \\
\text { the binding site. }\end{array}$ & $\begin{array}{l}\text { site identity }=0.5 ; \\
\text { maximal number of } \\
\text { mutations }=0 ;\end{array}$ \\
\hline site mutation rate $20 \%$ & $\begin{array}{l}\text { Generates a binding site alignment and re- } \\
\text { moves all matches that have any binding site } \\
\text { gaps or more than } 20 \% \text { of residues with de- } \\
\text { viating residue type in the binding site. }\end{array}$ & $\begin{array}{l}\text { site identity }=0.8 \\
\text { site coverage }=1.0\end{array}$ \\
\hline site identity $80 \%$ & $\begin{array}{l}\text { Generates a binding site alignment and re- } \\
\text { moves all matches that have less than } 80 \% \\
\text { identical binding site residues. }\end{array}$ & site identity $=0.8$ \\
\hline site identity $30 \%$ & $\begin{array}{l}\text { Generates a binding site alignment and re- } \\
\text { moves all matches that have less than } 30 \% \\
\text { identical binding site residues. }\end{array}$ & site identity $=0.3$ \\
\hline
\end{tabular}


Table S3: Structures with modified residues from ensemble generated with BLAST using a $100 \%$ chain identity query.

\begin{tabular}{llll}
\hline target & modification & residue position & PDB codes \\
\hline CDK2 & sulfinoalanine & CYS 177 & 1GZ8, 1W8C, 2R3I, 2R3J, 2R3K, 2R3L, \\
& & & 2R3M, 2R3N, 2R3O, 2R3P, 2R3Q, 4NJ3 \\
CDK2 & sulfoalanine & CYS 177 & 4ERW \\
CDK2 & lysine Nz-carboxylic acid & LYS 33 & 1H01, 1OIR \\
CDK2 & acetyllysine & LYS 33 & 4RJ3 \\
CDK2 & phosphotyrosine & TYR 15 & 2CJM \\
CDK2 & phosphothreonine & THR 160 & 2CJM \\
CA II & acetyllysine & LYS 112 & 4ZWI
\end{tabular}


Table S4: PDB codes and number of unique ligands for all targets in the NBSE set.

\begin{tabular}{|c|c|c|c|c|c|}
\hline & PDB code & number of unique ligands & 37 & $2 \mathrm{C} 6 \mathrm{C}$ & 40 \\
\hline 1 & $1 \mathrm{I} 9 \mathrm{M}$ & 280 & 38 & 3IOD & 39 \\
\hline 2 & $3 \mathrm{INE}$ & 259 & 39 & $2 \mathrm{~B} 7 \mathrm{~A}$ & 39 \\
\hline 3 & $2 \mathrm{VTJ}$ & 256 & 40 & $1 \mathrm{KMV}$ & 36 \\
\hline 4 & $3 \mathrm{OIH}$ & 211 & 41 & $1 \mathrm{~N} 0 \mathrm{~T}$ & 36 \\
\hline 5 & $1 \mathrm{AF} 8$ & 211 & 42 & $2 \mathrm{NP} 8$ & 36 \\
\hline & & 170 & 43 & $3 \mathrm{VT} 8$ & 36 \\
\hline 6 & $10 Y 7$ & 166 & 44 & 1DAW & 34 \\
\hline 7 & $1 \mathrm{D} 1 \mathrm{~V}$ & 157 & 45 & $2 \mathrm{FLR}$ & 34 \\
\hline 8 & $1 \mathrm{KV} 2$ & 151 & 46 & 3ZWS & 33 \\
\hline 9 & $3 \mathrm{UH} 4$ & 117 & 47 & $1 \mathrm{YOU}$ & 33 \\
\hline 10 & $1 \mathrm{XKB}$ & 100 & & & 35 \\
\hline 11 & $3 \mathrm{U} 5 \mathrm{~J}$ & 99 & 48 & $3 \mathrm{CHP}$ & 33 \\
\hline 12 & $2 \mathrm{CGU}$ & 94 & 49 & 1IE8 & 31 \\
\hline 13 & $3 \mathrm{R} 8 \mathrm{~A}$ & 83 & 50 & $4 \mathrm{M} 6 \mathrm{Q}$ & 30 \\
\hline 14 & 4J03 & 77 & 51 & 1UT6 & 29 \\
\hline 15 & $2 \mathrm{AZR}$ & 75 & 52 & $1 \mathrm{O} 1 \mathrm{~T}$ & 29 \\
\hline 16 & $2 \mathrm{I} 0 \mathrm{~J}$ & 72 & 53 & $2 \mathrm{ZI} 5$ & 29 \\
\hline 17 & $2 \mathrm{O} 65$ & 66 & 54 & 3TUD & 29 \\
\hline 18 & $3 \mathrm{ZSO}$ & 63 & 55 & $4 \mathrm{NRA}$ & 29 \\
\hline 19 & 4LM0 & 62 & 56 & $1 \mathrm{ODN}$ & 28 \\
\hline 20 & $1 \mathrm{EKO}$ & 52 & 57 & $3 \mathrm{R} 7 \mathrm{M}$ & 28 \\
\hline 21 & 1OWE & 51 & 58 & 1UU3 & 28 \\
\hline 22 & $3 \mathrm{~W} 1 \mathrm{~T}$ & 50 & 59 & $2 \mathrm{EVC}$ & 28 \\
\hline 23 & $1 \mathrm{~K} 4 \mathrm{G}$ & 49 & 60 & $2 \mathrm{G} 8 \mathrm{~N}$ & 28 \\
\hline 24 & $2 \mathrm{JC} 0$ & 48 & 61 & $4 \mathrm{FJP}$ & 28 \\
\hline 25 & 3BWJ & 47 & 62 & 2B37 & 27 \\
\hline 26 & $2 \mathrm{RFN}$ & 47 & 63 & 1XGI & 27 \\
\hline 27 & $2 \mathrm{C} 7 \mathrm{~V}$ & 46 & 64 & $3 \mathrm{LBK}$ & 27 \\
\hline 28 & 2BUC & 45 & 65 & $1 \mathrm{OF} 1$ & 26 \\
\hline 29 & $1 \mathrm{TBB}$ & 44 & 66 & $3 \mathrm{AMY}$ & 26 \\
\hline 30 & $1 \mathrm{QF0}$ & 43 & 67 & 3LP2 & 25 \\
\hline 31 & $3 \mathrm{SA} 0$ & 43 & 68 & $2 \mathrm{OLI}$ & 24 \\
\hline 32 & $1 \mathrm{GVT}$ & 41 & 69 & $1 \mathrm{QKN}$ & 24 \\
\hline 33 & $3 \mathrm{OXI}$ & 41 & 70 & $1 \mathrm{QPE}$ & 24 \\
\hline 34 & $2 \mathrm{BZS}$ & 40 & 71 & $2 \mathrm{XPA}$ & 24 \\
\hline 35 & 1RMZ & 40 & 72 & $3 \mathrm{ZCW}$ & 23 \\
\hline
\end{tabular}




\begin{tabular}{|c|c|c|}
\hline & PDB code & number of unique ligands \\
\hline 73 & $3 \mathrm{SOR}$ & 23 \\
\hline 74 & $3 \mathrm{U} 8 \mathrm{~F}$ & 23 \\
\hline 75 & $4 \mathrm{~F} 63$ & 22 \\
\hline 76 & $109 \mathrm{U}$ & 22 \\
\hline 77 & $4 \mathrm{~B} 7 \mathrm{Z}$ & 22 \\
\hline 78 & 2PJA & 22 \\
\hline 79 & $3 \mathrm{~A} 7 \mathrm{~J}$ & 22 \\
\hline 80 & $3 \mathrm{~F} 0 \mathrm{~B}$ & 22 \\
\hline 81 & 1QSS & 21 \\
\hline 82 & 3ТН8 & 21 \\
\hline 83 & $2 \mathrm{XNV}$ & 21 \\
\hline 84 & $4 \mathrm{E} 5 \mathrm{~F}$ & 21 \\
\hline 85 & $2 \mathrm{JNP}$ & 20 \\
\hline 86 & $1 \mathrm{VSO}$ & 20 \\
\hline 87 & $3 \mathrm{TFQ}$ & 20 \\
\hline 88 & $1 \mathrm{ZXC}$ & 20 \\
\hline 89 & 3EN6 & 20 \\
\hline 90 & $2 \mathrm{XP} 2$ & 20 \\
\hline 91 & $3 \mathrm{~V} 8 \mathrm{~T}$ & 20 \\
\hline 92 & $4 \mathrm{ALI}$ & 20 \\
\hline 93 & 1BGQ & 19 \\
\hline 94 & 1DAJ & 19 \\
\hline 95 & 2Q1L & 19 \\
\hline 96 & $1 \mathrm{E} 7 \mathrm{~V}$ & 19 \\
\hline 97 & $1 \mathrm{FPU}$ & 19 \\
\hline 98 & $1 \mathrm{SRF}$ & 19 \\
\hline 99 & $1 \mathrm{MP} 8$ & 19 \\
\hline 100 & 1TOW & 19 \\
\hline 101 & $2 \mathrm{CVD}$ & 19 \\
\hline 102 & $2 \mathrm{WSA}$ & 19 \\
\hline 103 & 3QQS & 19 \\
\hline 104 & 4MJP & 19 \\
\hline 105 & $4 \mathrm{MX} 9$ & 19 \\
\hline 106 & $4 \mathrm{LRR}$ & 18 \\
\hline 107 & $1 \mathrm{E} 3 \mathrm{G}$ & 18 \\
\hline 108 & $3 \mathrm{G} 5 \mathrm{H}$ & 18 \\
\hline 109 & $4 \mathrm{~A} 9 \mathrm{R}$ & 18 \\
\hline
\end{tabular}

\begin{tabular}{|c|c|c|}
\hline & PDB code & number of unique ligands \\
\hline 110 & $2 \mathrm{~J} 7 \mathrm{D}$ & 18 \\
\hline 111 & 2WQO & 18 \\
\hline 112 & $3 \mathrm{G} 1 \mathrm{O}$ & 18 \\
\hline 113 & 3PJ3 & 18 \\
\hline 114 & $1 \mathrm{M} 8 \mathrm{D}$ & 17 \\
\hline 115 & $3 \mathrm{D} 0 \mathrm{E}$ & 17 \\
\hline 116 & $1 \mathrm{P} 2 \mathrm{Y}$ & 17 \\
\hline 117 & $2 \mathrm{E} 92$ & 17 \\
\hline 118 & $2 \mathrm{HCT}$ & 17 \\
\hline 119 & 4DWB & 17 \\
\hline 120 & 2UW6 & 17 \\
\hline 121 & 2NQ6 & 17 \\
\hline 122 & $2 \mathrm{OPM}$ & 17 \\
\hline 123 & $2 \mathrm{ZJU}$ & 17 \\
\hline 124 & $3 \mathrm{KE} 8$ & 17 \\
\hline 125 & 4JFI & 17 \\
\hline 126 & $1 \mathrm{~K} 7 \mathrm{~F}$ & 16 \\
\hline 127 & $4 \mathrm{ASJ}$ & 16 \\
\hline 128 & $1 \mathrm{HII}$ & 16 \\
\hline 129 & 3BEJ & 16 \\
\hline 130 & $3 \mathrm{DNG}$ & 16 \\
\hline 131 & $1 \mathrm{UIO}$ & 15 \\
\hline 132 & $1 \mathrm{PK} 7$ & 15 \\
\hline 133 & $1 \mathrm{BX} 6$ & 15 \\
\hline 134 & $1 \mathrm{PXX}$ & 15 \\
\hline 135 & $1 \mathrm{Y} 6 \mathrm{~A}$ & 15 \\
\hline 136 & $1 \mathrm{Z} 6 \mathrm{P}$ & 15 \\
\hline 137 & $2 \mathrm{JFF}$ & 15 \\
\hline 138 & $2 \mathrm{~V} 58$ & 15 \\
\hline 139 & $3 \mathrm{ACX}$ & 15 \\
\hline 140 & $4 \mathrm{FPO}$ & 15 \\
\hline 141 & $1 \mathrm{~A} 28$ & 14 \\
\hline 142 & $1 \mathrm{AOE}$ & 14 \\
\hline 143 & 1UK1 & 14 \\
\hline 144 & 3Q30 & 14 \\
\hline 145 & $3 \mathrm{ET} 1$ & 14 \\
\hline 146 & $1 \mathrm{NNU}$ & 14 \\
\hline
\end{tabular}




\begin{tabular}{|c|c|c|}
\hline & PDB code & number of unique ligands \\
\hline 147 & $2 \mathrm{O} 1 \mathrm{Y}$ & 14 \\
\hline 148 & $3 \mathrm{G} 2 \mathrm{Y}$ & 14 \\
\hline 149 & $4 \mathrm{GFD}$ & 14 \\
\hline 150 & $4 \mathrm{MX} 1$ & 14 \\
\hline 151 & $1 \mathrm{GSS}$ & 13 \\
\hline 152 & $1 \mathrm{JOM}$ & 13 \\
\hline 153 & $1 \mathrm{~J} 4 \mathrm{H}$ & 13 \\
\hline 154 & $1 \mathrm{O} 6 \mathrm{I}$ & 13 \\
\hline 155 & $3 \mathrm{IK} 0$ & 13 \\
\hline 156 & $2 \mathrm{QK} 8$ & 13 \\
\hline 157 & $2 \mathrm{VES}$ & 13 \\
\hline 158 & $2 \mathrm{VWU}$ & 13 \\
\hline 159 & $2 \times 90$ & 13 \\
\hline 160 & $3 \mathrm{EBH}$ & 13 \\
\hline 161 & $3 \mathrm{EYG}$ & 13 \\
\hline 162 & $3 \mathrm{HXE}$ & 13 \\
\hline 163 & 3PCI & 13 \\
\hline 164 & 3PEC & 13 \\
\hline 165 & $3 \mathrm{TFU}$ & 13 \\
\hline 166 & $3 \mathrm{ZRC}$ & 13 \\
\hline 167 & $4 \mathrm{DKO}$ & 13 \\
\hline 168 & $1 \mathrm{~V} 48$ & 12 \\
\hline 169 & $1 \mathrm{D} 0 \mathrm{~S}$ & 12 \\
\hline 170 & $1 \mathrm{U} 0 \mathrm{Z}$ & 12 \\
\hline 171 & 1RD4 & 12 \\
\hline 172 & $2 \mathrm{QPK}$ & 12 \\
\hline 173 & $2 \mathrm{~W} 0 \mathrm{~V}$ & 12 \\
\hline 174 & $2 \mathrm{VKE}$ & 12 \\
\hline 175 & $2 \mathrm{ZSD}$ & 12 \\
\hline 176 & 3ID8 & 12 \\
\hline 177 & $4 \mathrm{C} 4 \mathrm{~F}$ & 12 \\
\hline 178 & $4 \mathrm{NB} 6$ & 12 \\
\hline 179 & 4HW2 & 12 \\
\hline 180 & $4 \mathrm{~A} 6 \mathrm{~L}$ & 12 \\
\hline 181 & $4 \mathrm{AJN}$ & 12 \\
\hline 182 & $4 \mathrm{LY} 9$ & 12 \\
\hline
\end{tabular}


Figure S19: The following figures illustrate the number of critical ligands for all individual NBSE targets as a function of the maximal number of structures in a reduced ensemble (maximal ensemble size). As reference values, the average and best performance of single structures is depicted. Additionally, the optimal ensemble performance is given by the performance of the non-reduced training set ensemble. An overall statistic on all targets is given by Figures 4 and S9. 
Training set

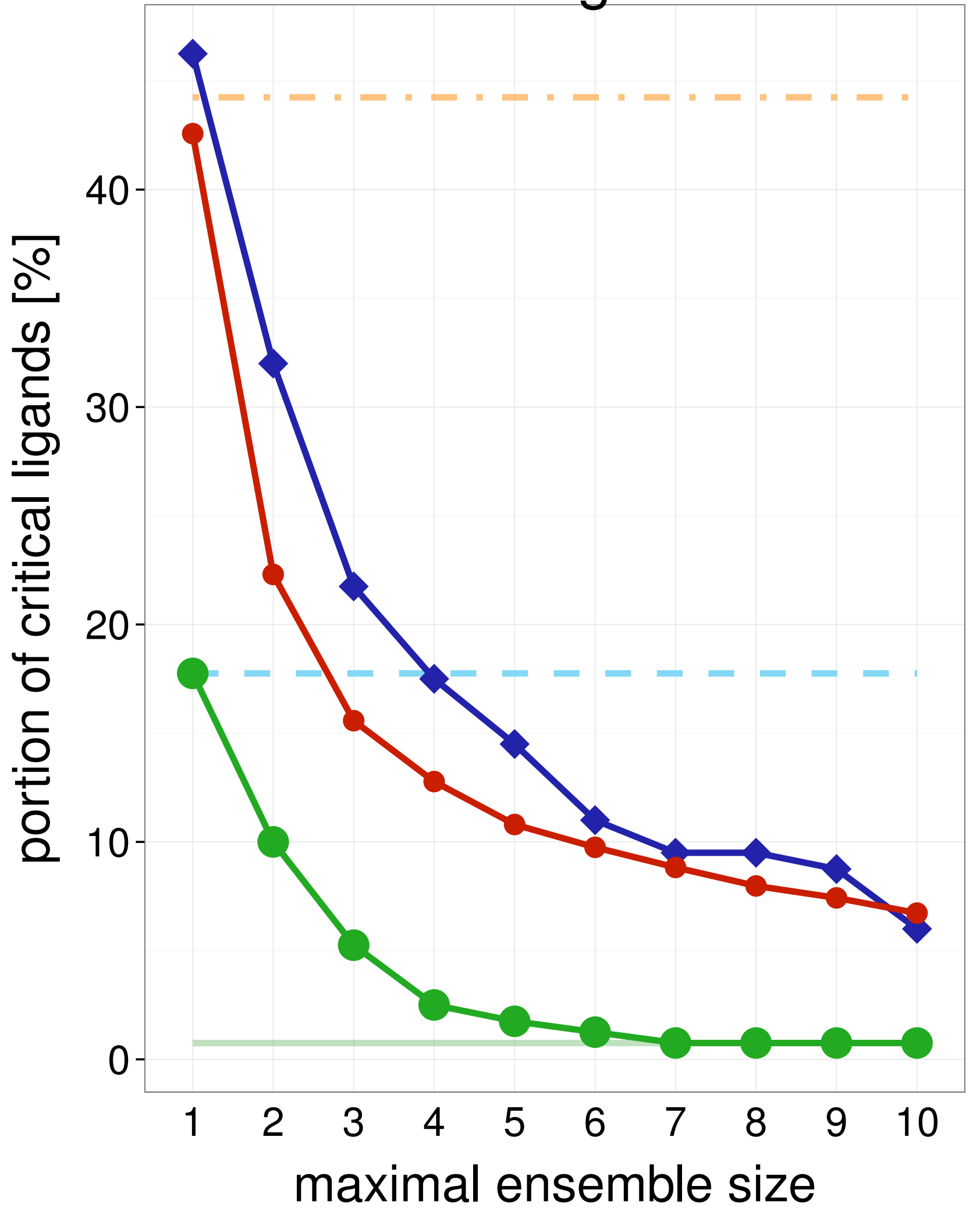

Test set

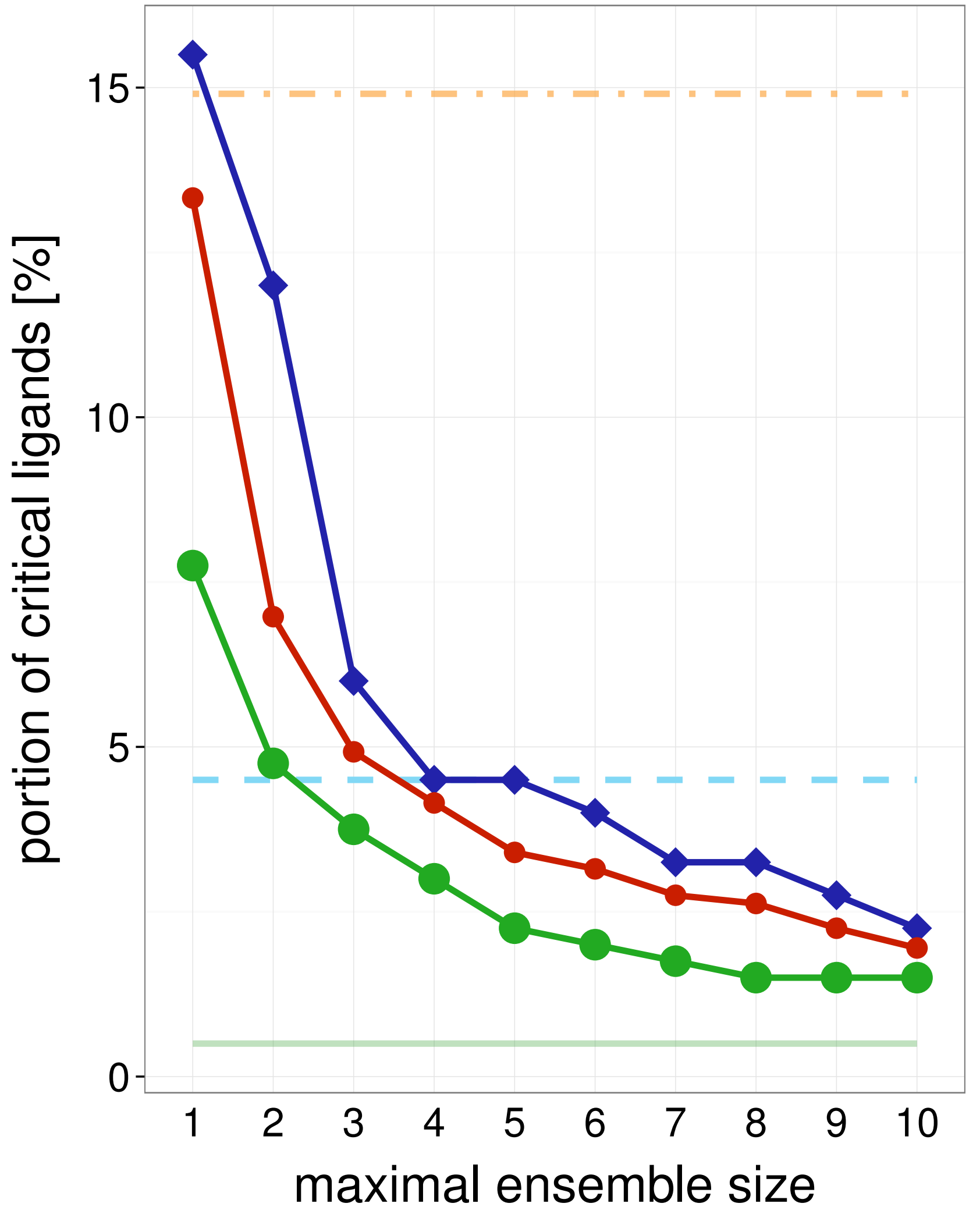

Average single structure

_ _ Best single structure

Whole ensemble

- SIENA

$\neg$ Clustering

$\multimap$ Random

Average single structure

_ _ Best single structure

Whole ensemble

- SIENA

$\smile$ Clustering

$\because$ Random
$119 M$

Training set

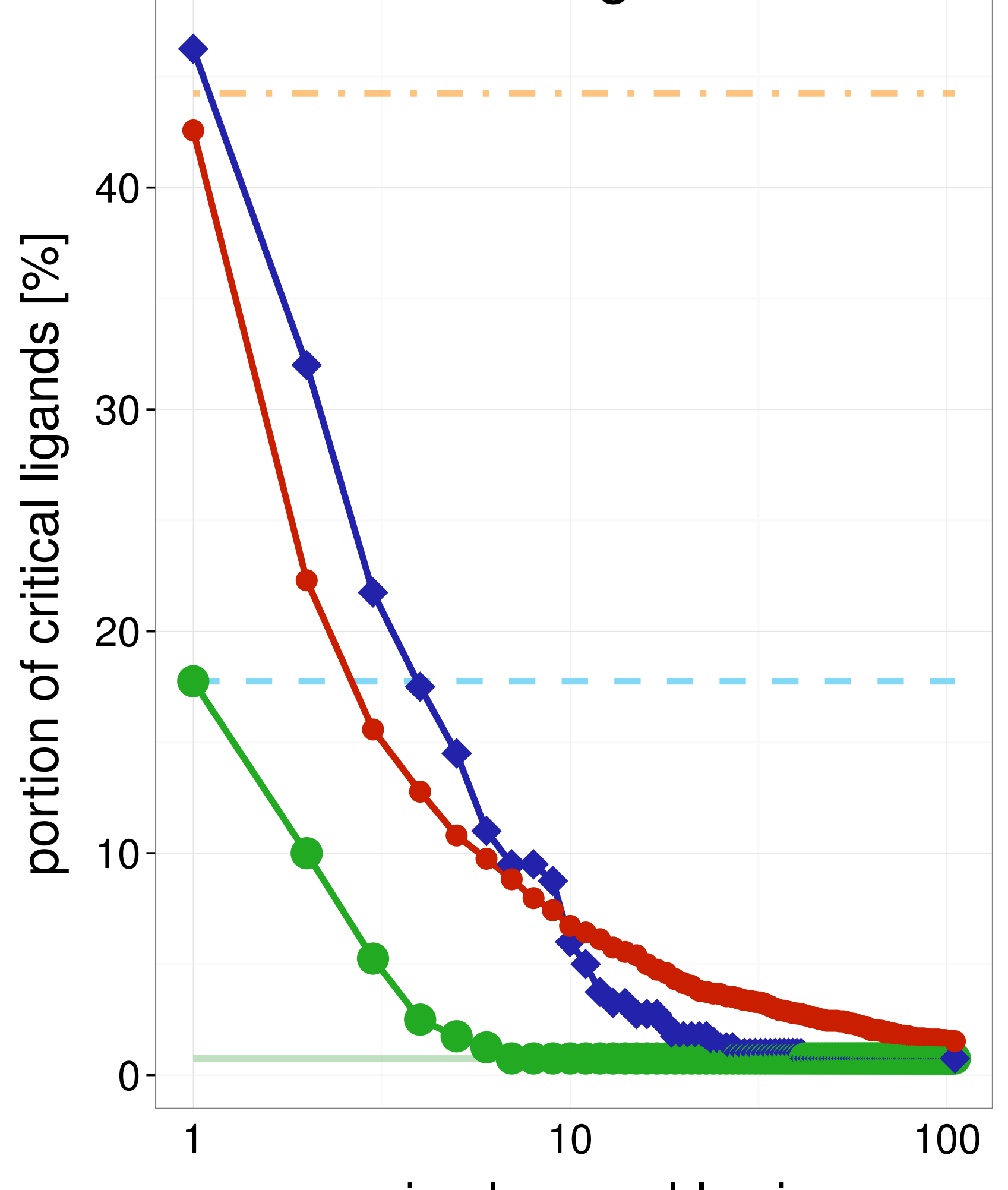

Average single structure

Best single structure

Whole ensemble

- SIENA

$\neg$ Clustering

- Random

maximal ensemble size

Test set

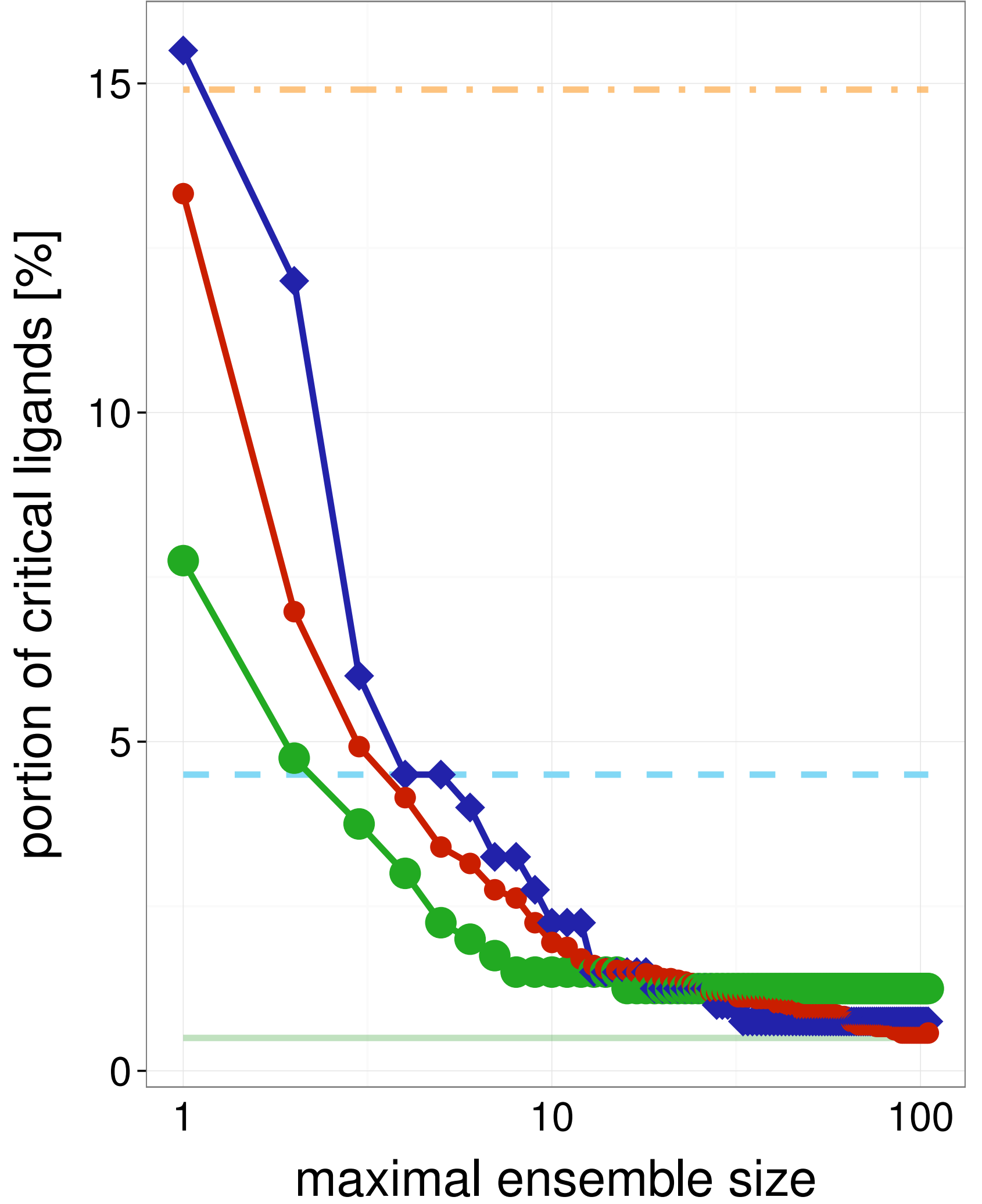

Average single structure

_ Best single structure

Whole ensemble

SIENA

$\sim$ Clustering

$\longrightarrow$ Random 


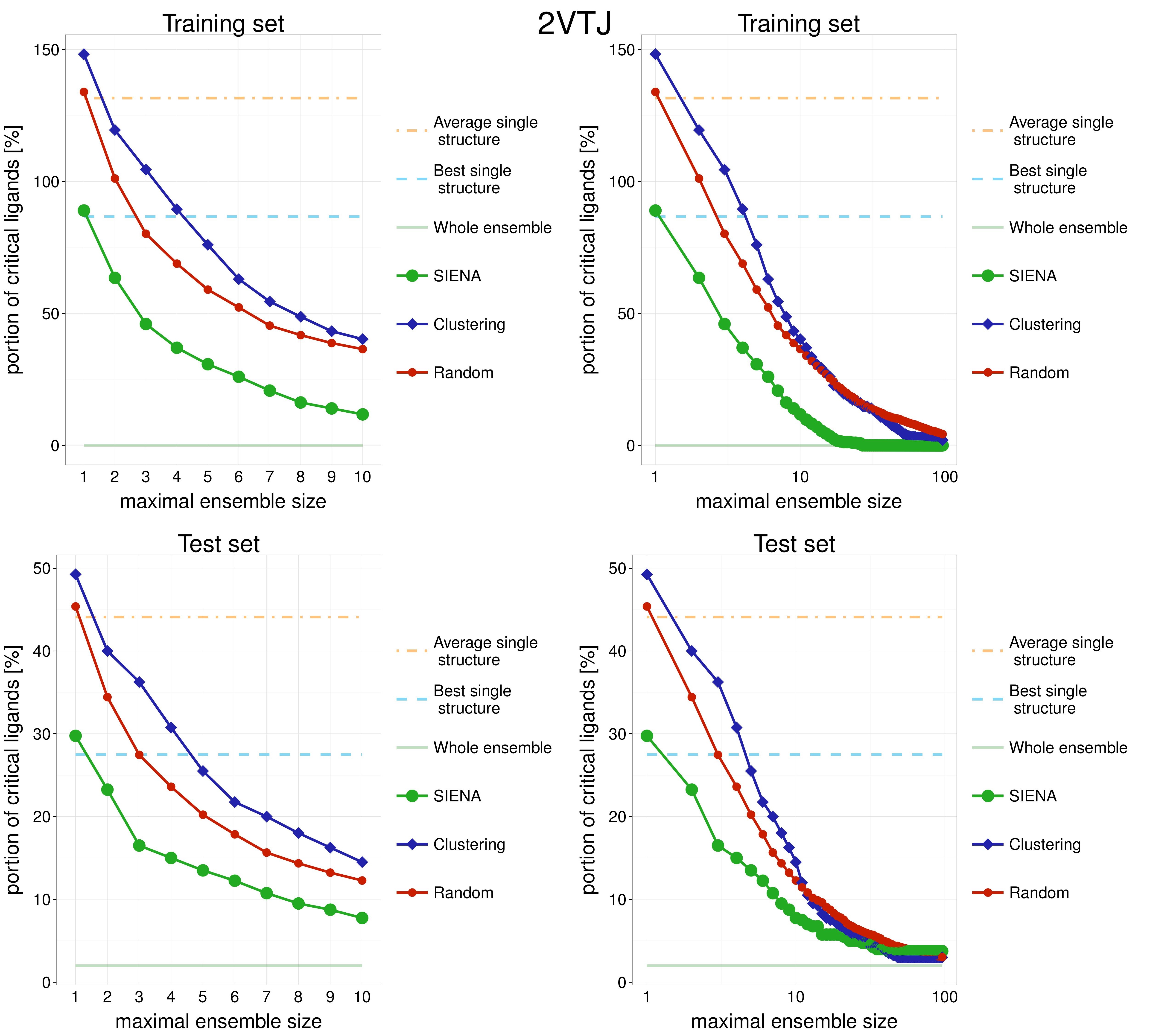




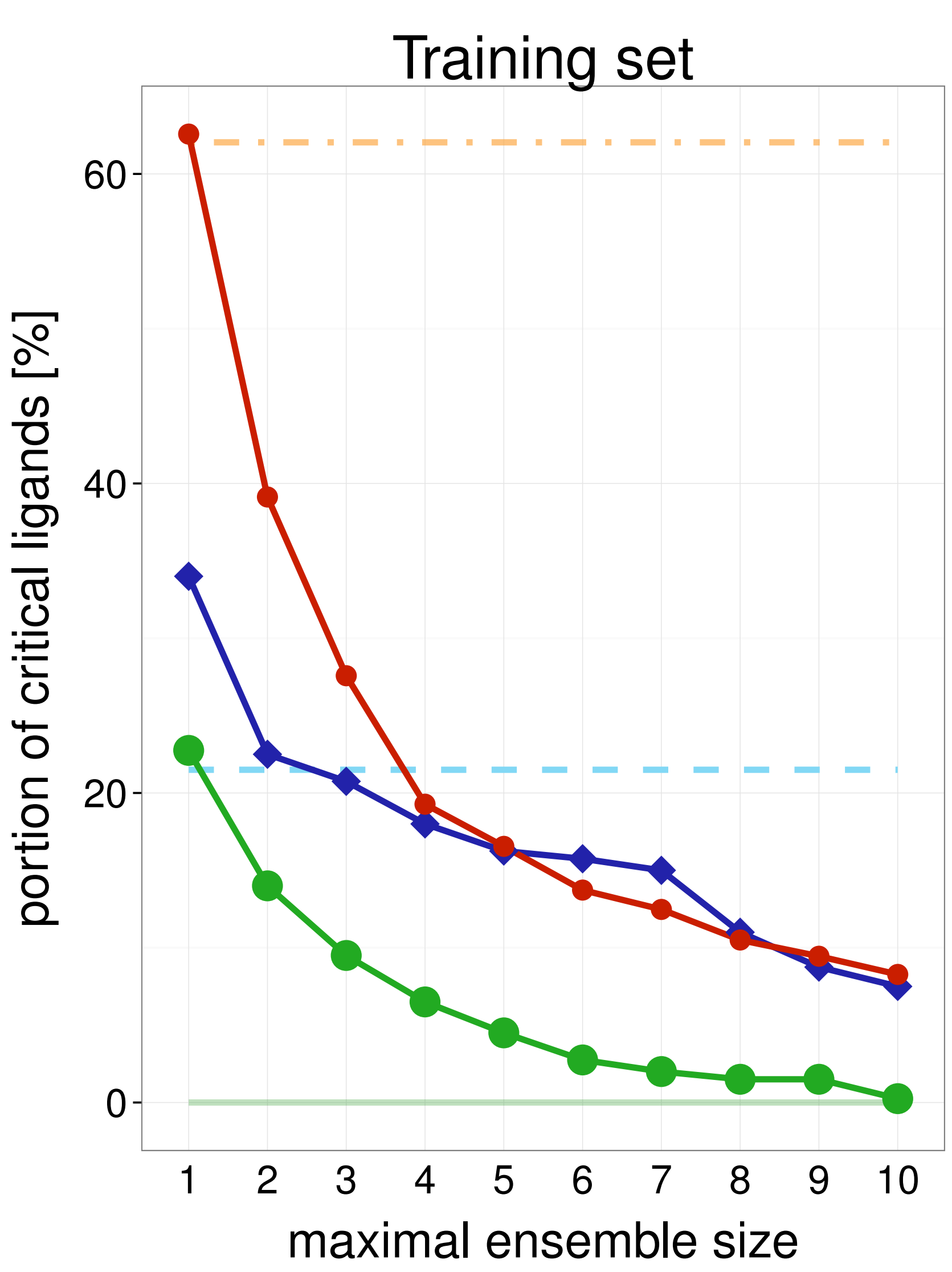

\section{Test set}

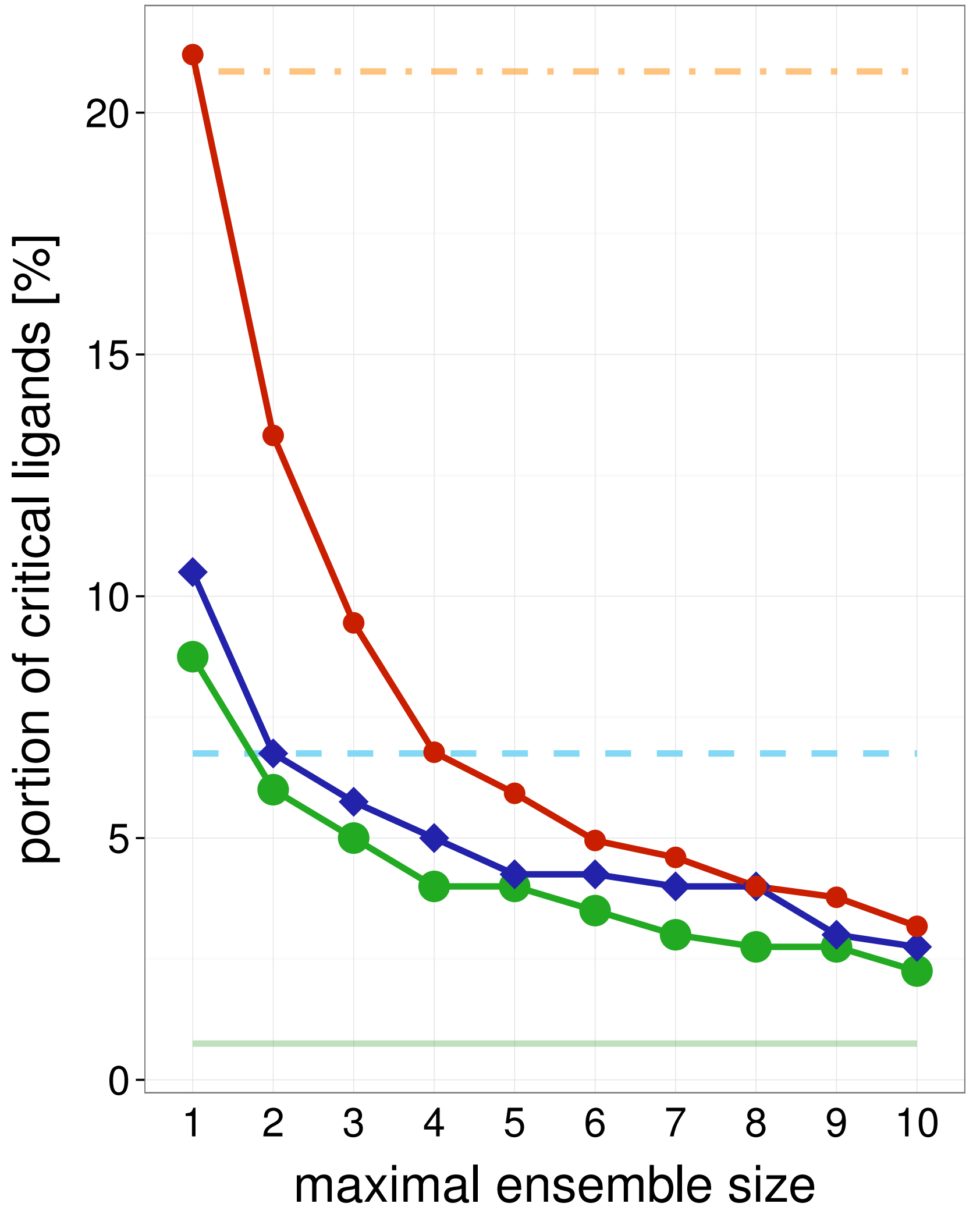

Average single structure

_ Best single structure

Whole ensemble

- SIENA

$\neg$ Clustering

$\multimap$ Random

Average single structure

_ _ Best single structure

Whole ensemble

- SIENA

$\smile$ Clustering

$\multimap$ Random

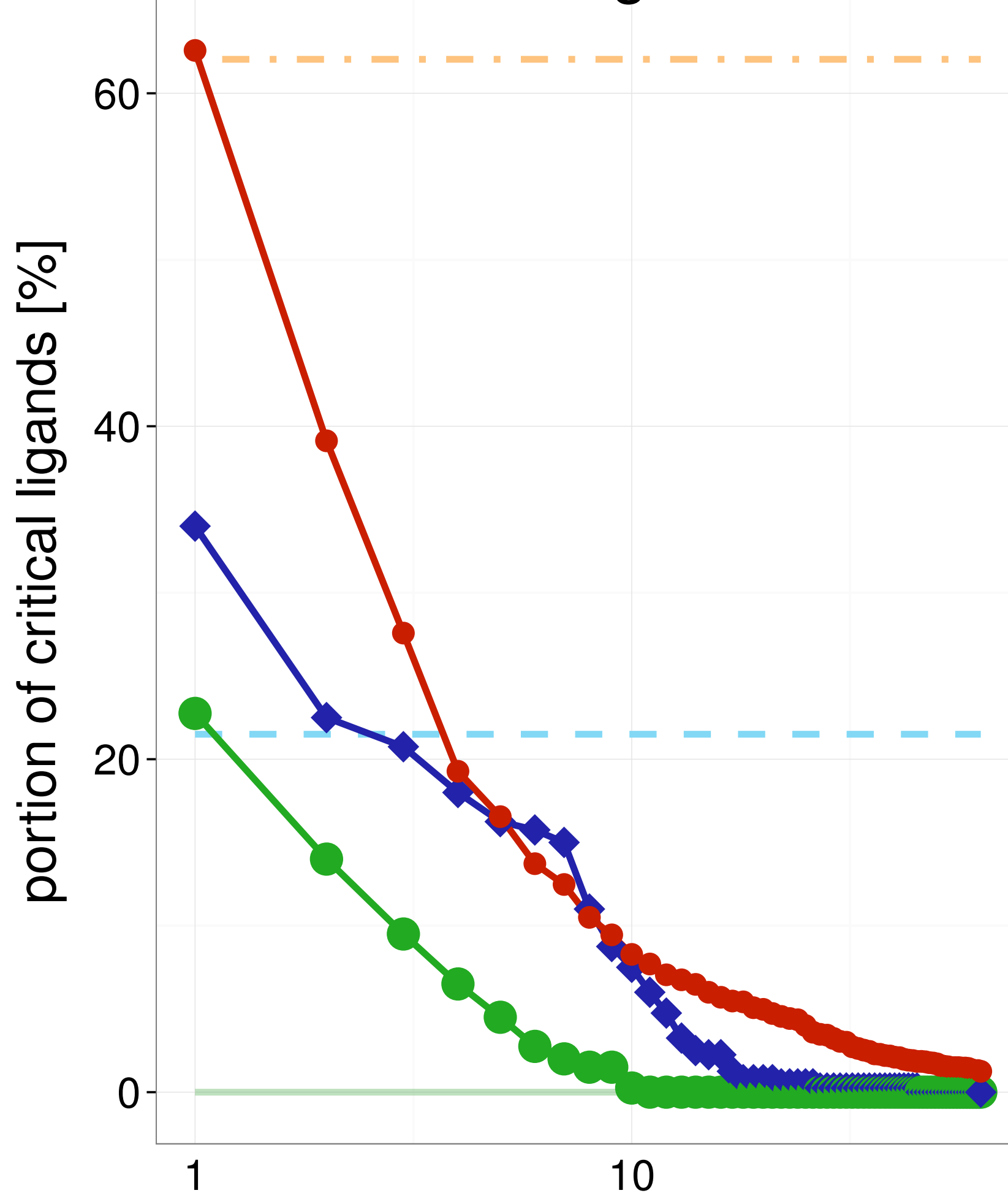

maximal ensemble size

Test set

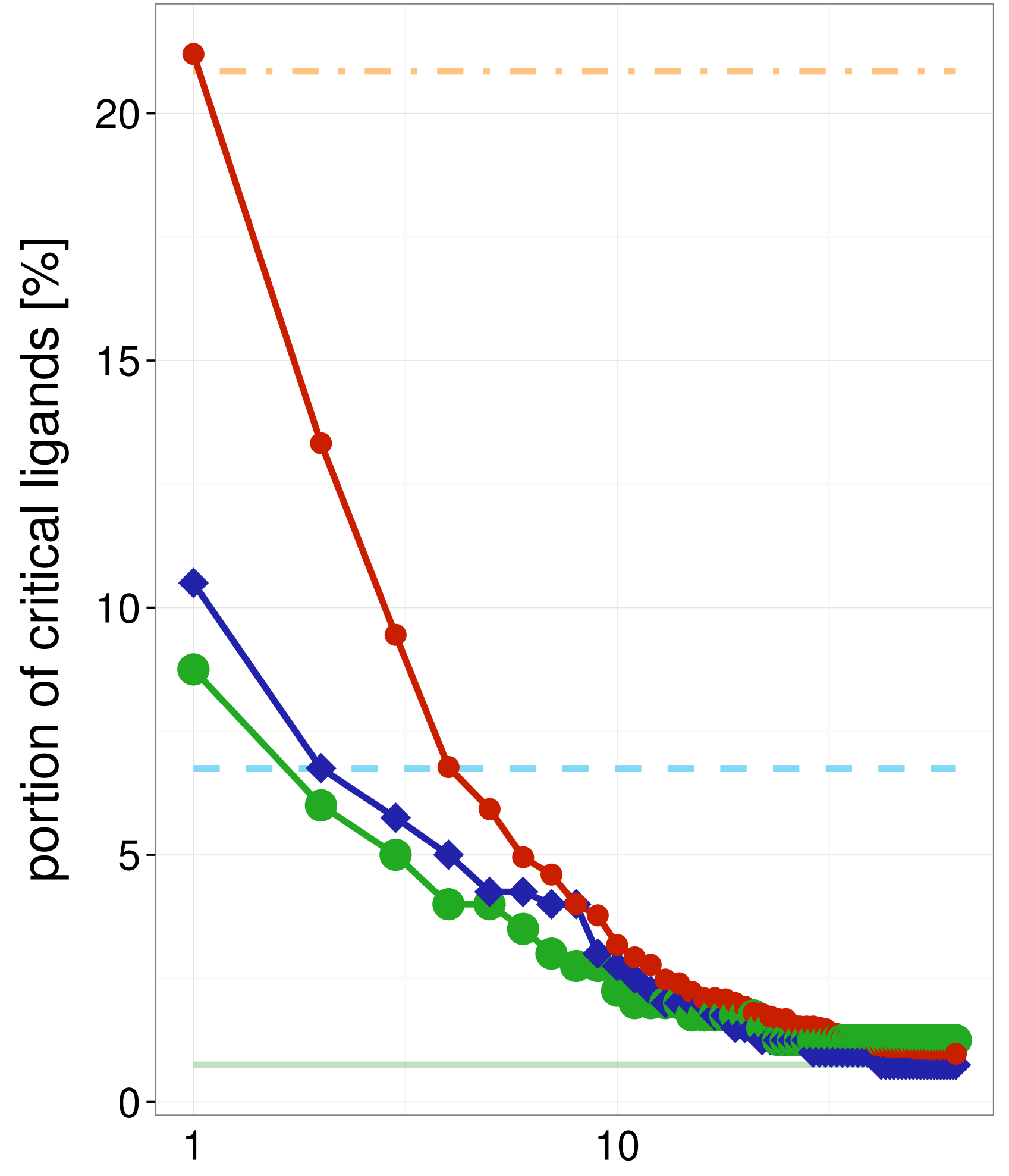

maximal ensemble size
Average single structure

_ _ Best single structure

Whole ensemble

SIENA

$\neg$ Clustering

$\longrightarrow$ Random 
Training set

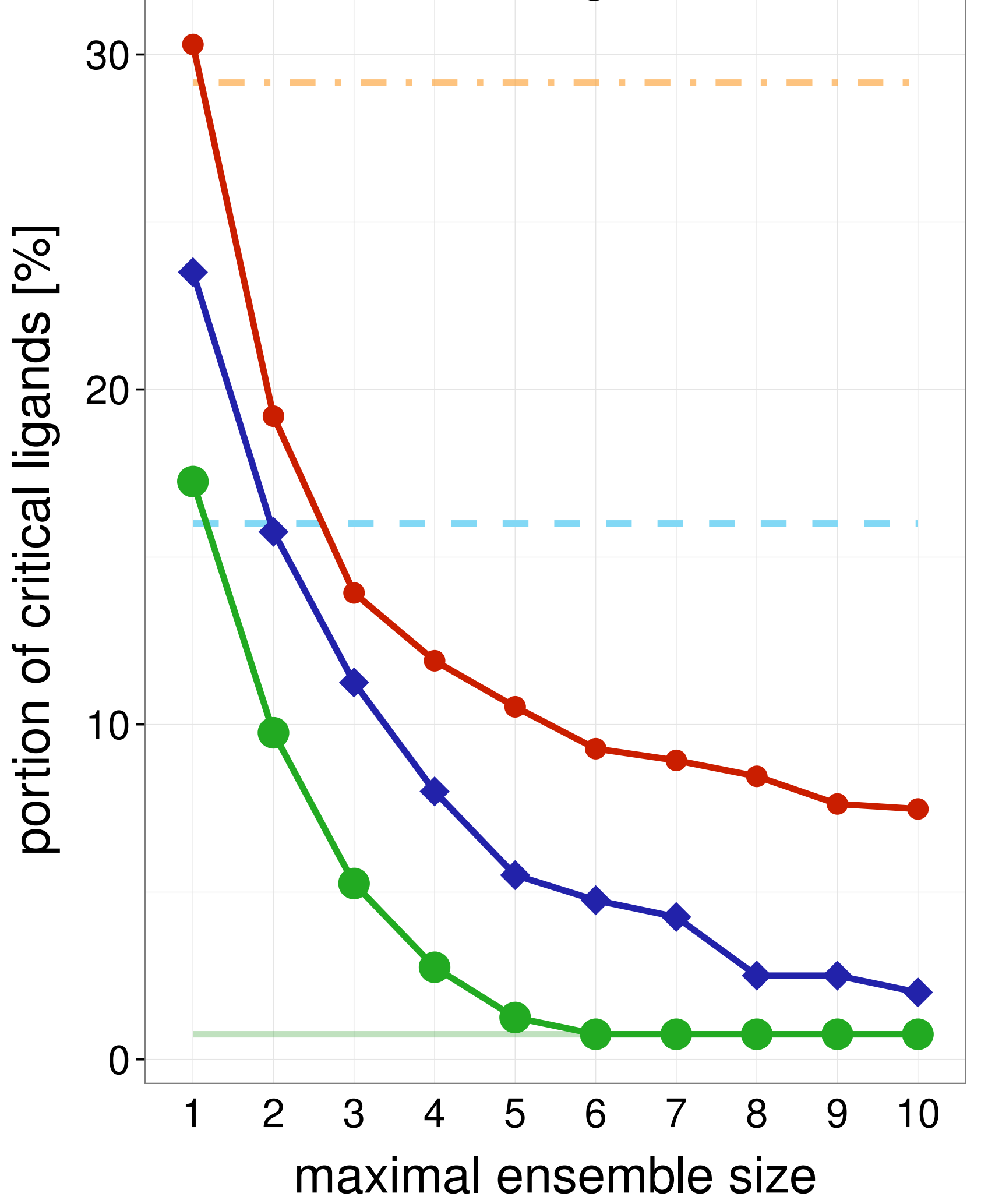

Test set

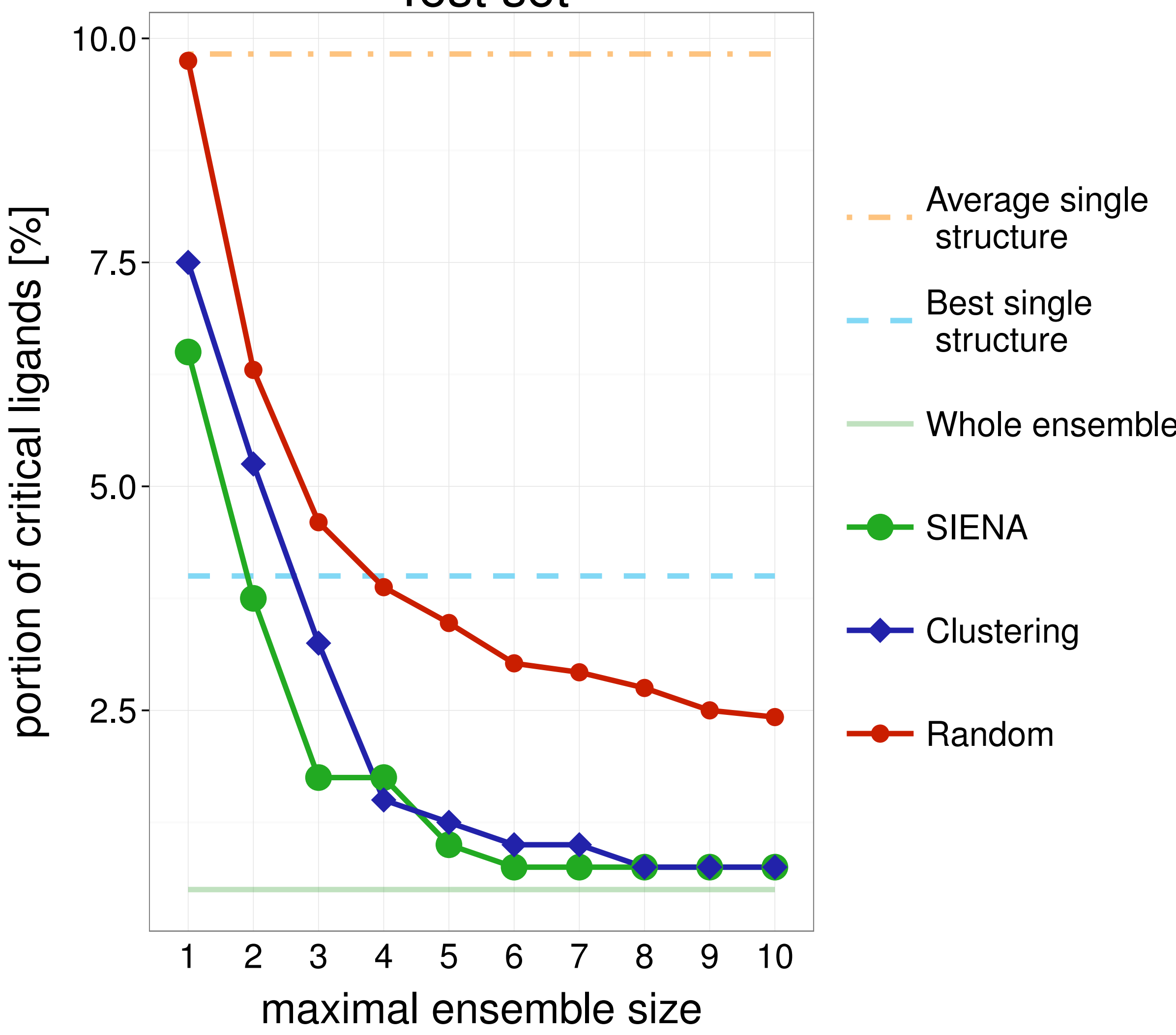

Average single structure

_ Best single structure

Whole ensemble

- SIENA

$\neg$ Clustering
1D1V $\quad$ Training set

$\multimap$ Random

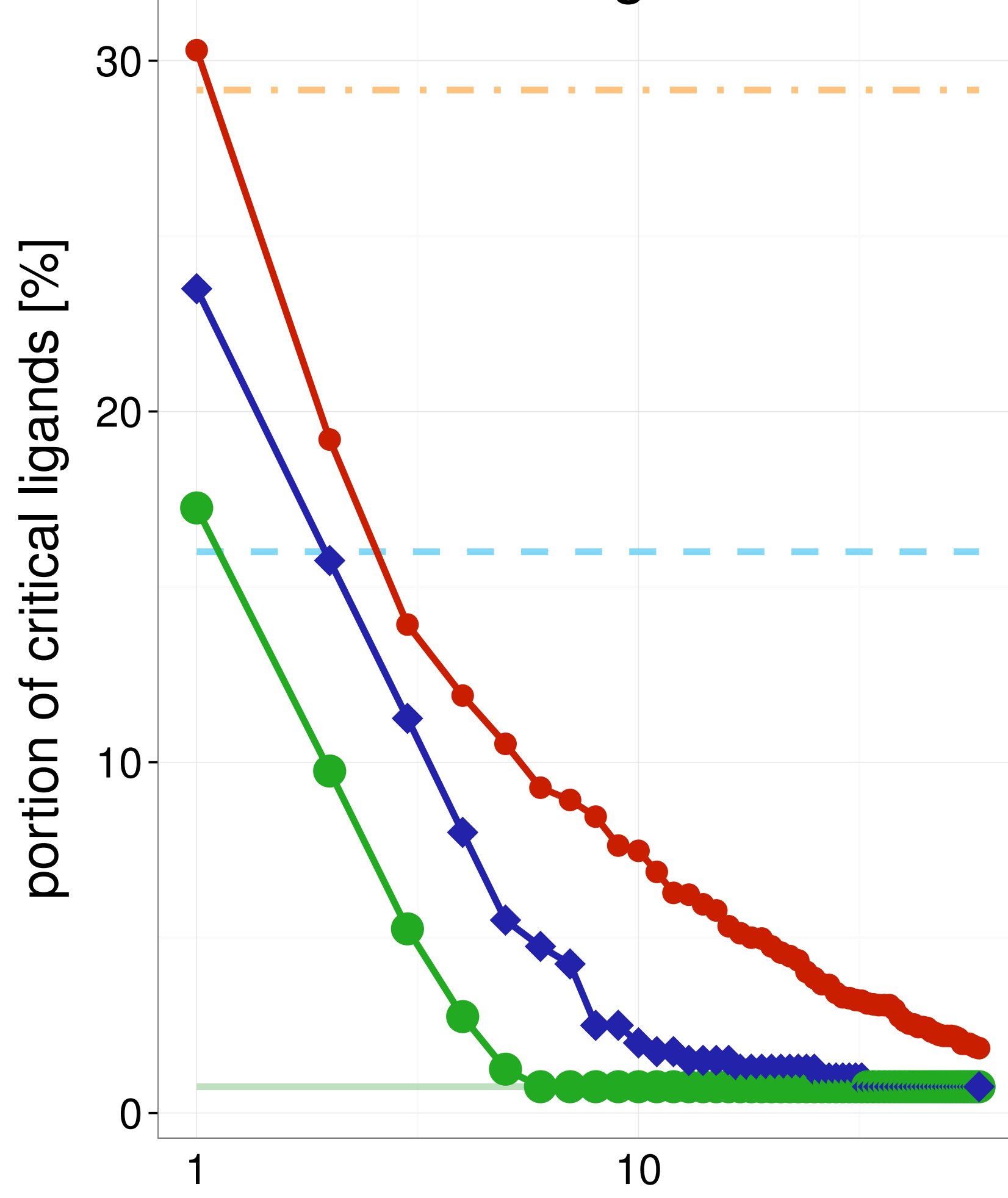

maximal ensemble size

Test set

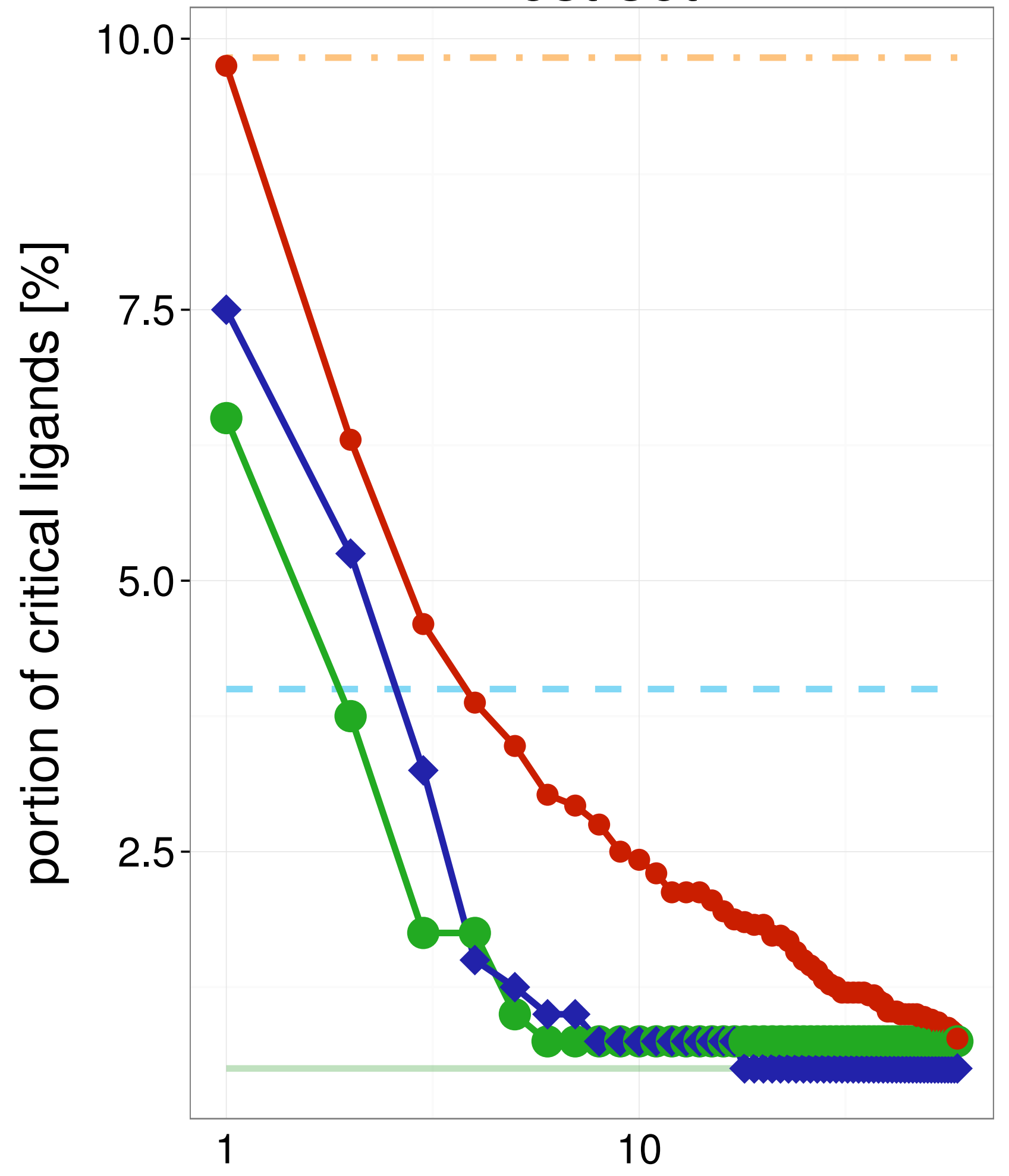

Average single structure

_ Best single structure

Whole ensemble

- SIENA

$\neg$ Clustering

$\multimap$ Random 
Training set

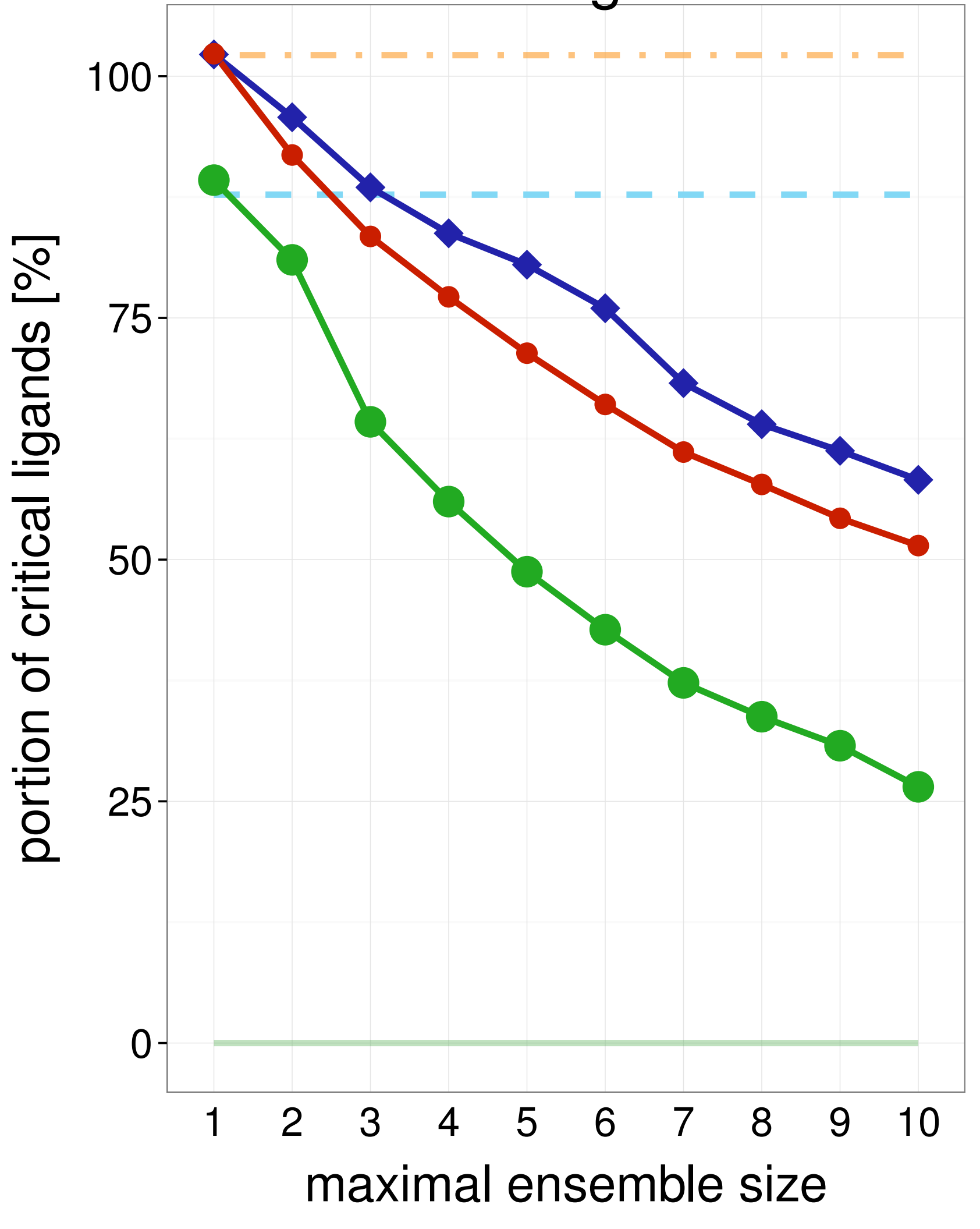

Test set

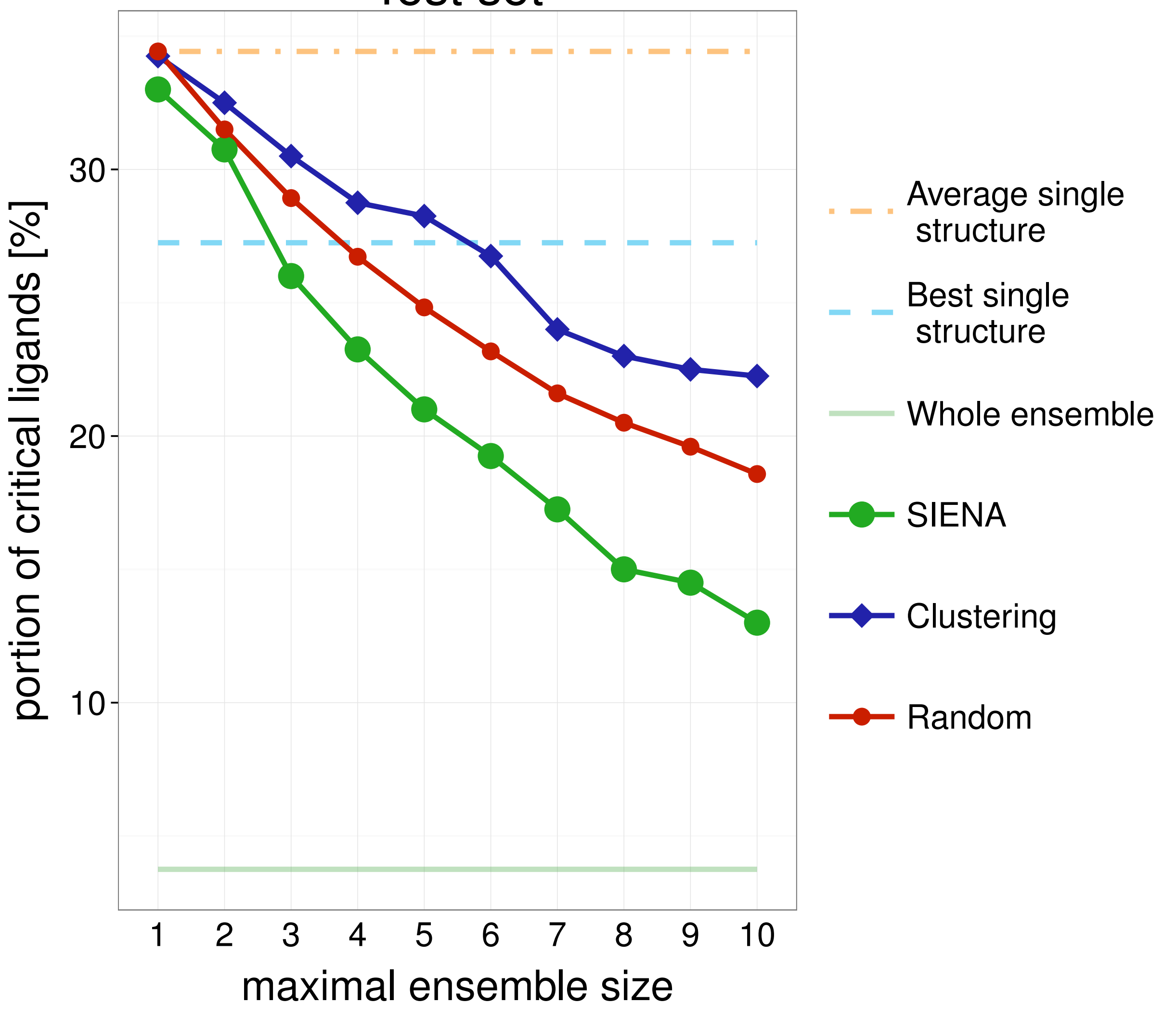

Average single structure

_ - Best single

structure

Whole ensemble

- SIENA

$\neg$ Clustering

$\multimap$ Random
Training set

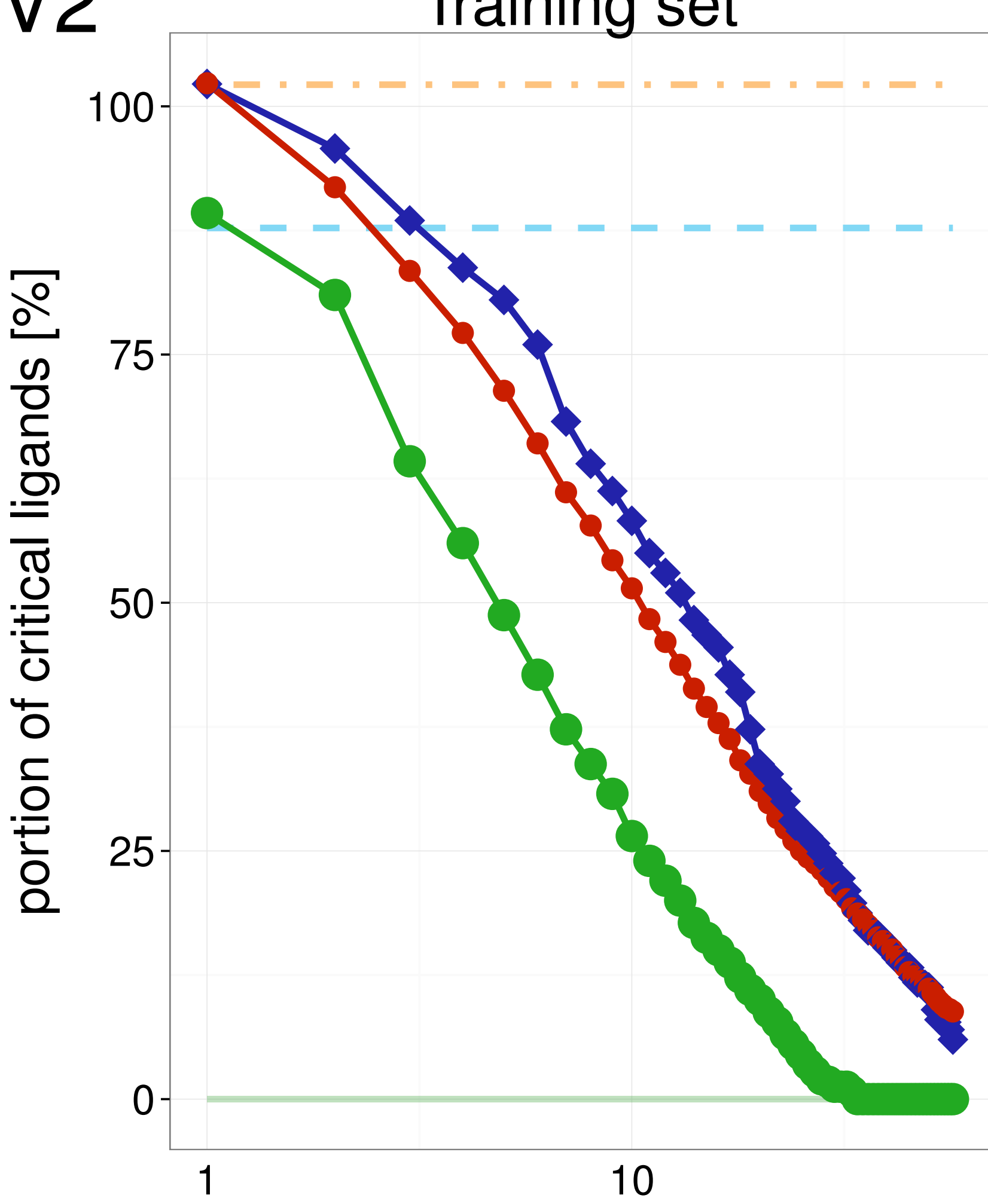

maximal ensemble size

\section{Test set}

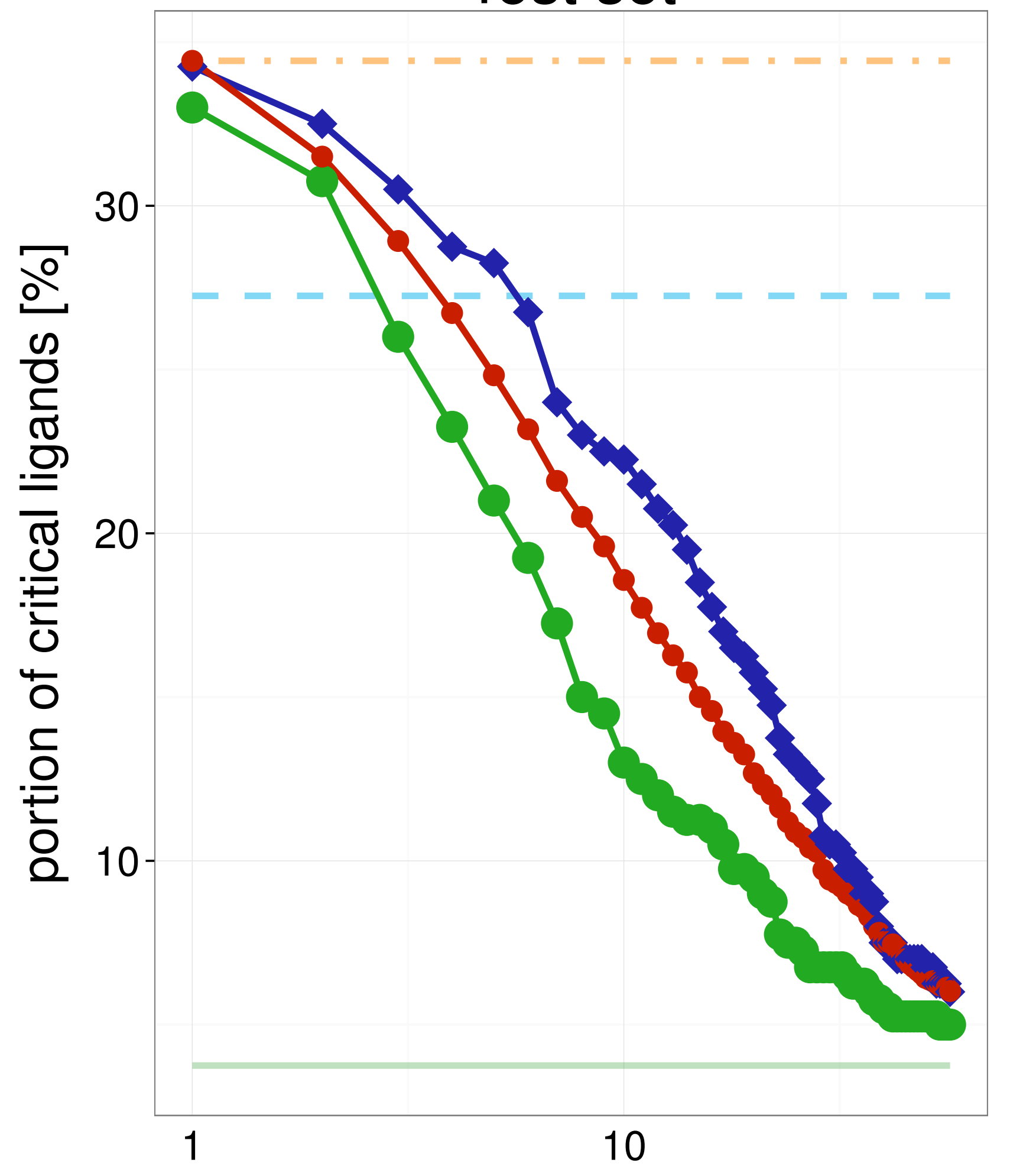

Average single structure

_ _ Best single structure

Whole ensemble

- SIENA

$\leadsto$ Clustering

$\multimap$ Random

maximal ensemble size 


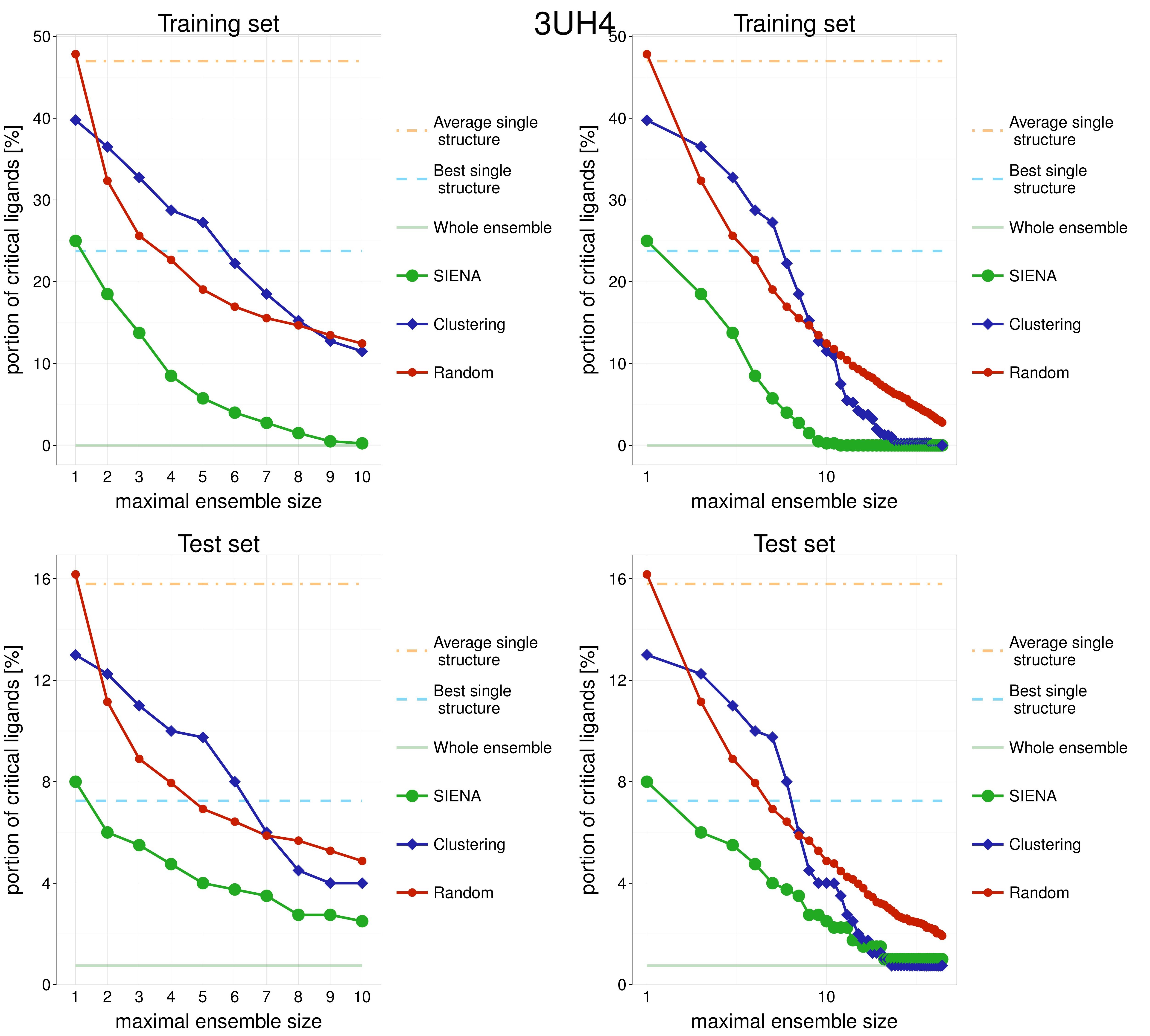




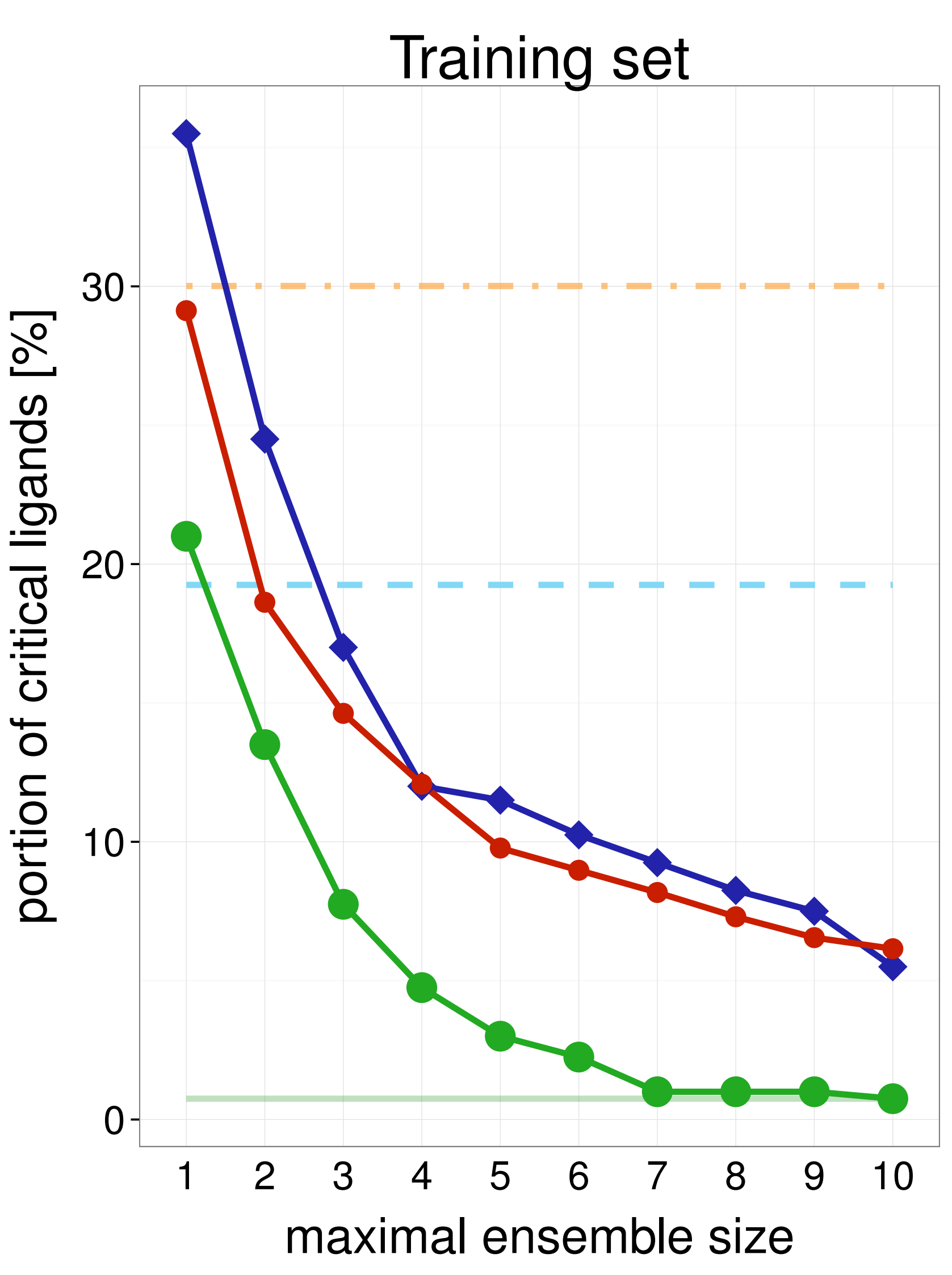

3U5J Training set
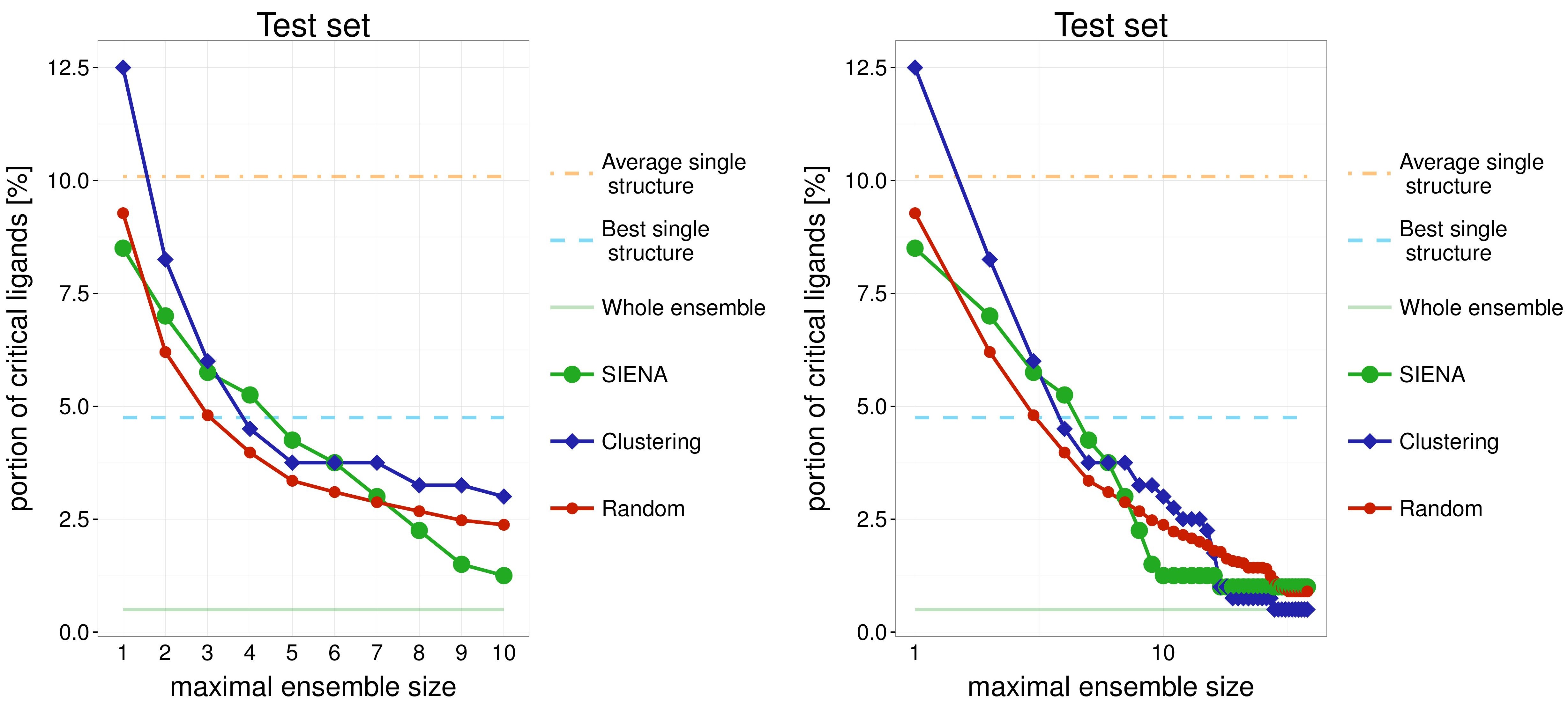


\section{Training set}

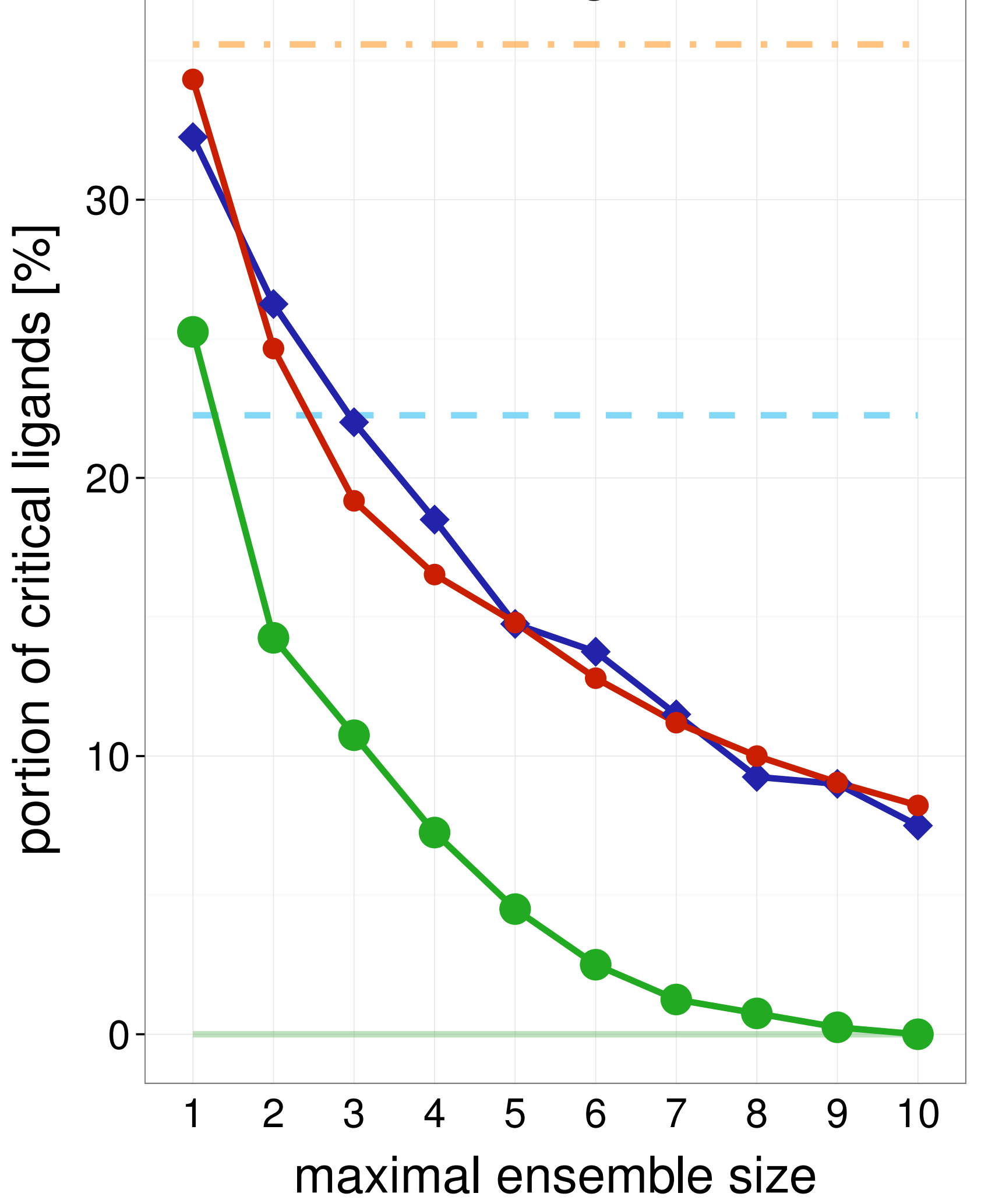

Test set

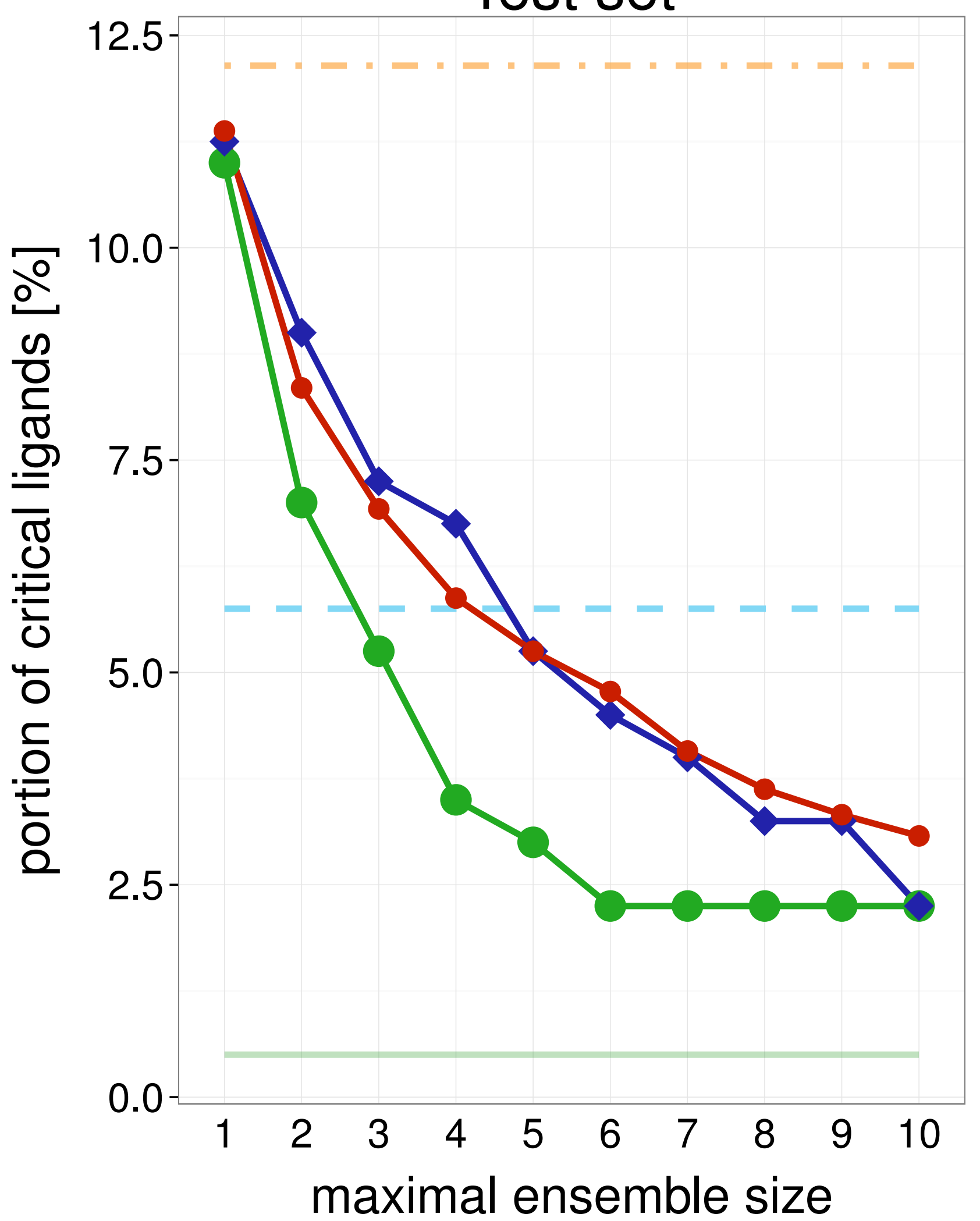

\section{CGU}

Training set

Average single structure

_ _ Best single

structure

Whole ensemble

- SIENA

$\neg$ Clustering

$\multimap$ Random

Average single structure

_ _ Best single structure

Whole ensemble

- SIENA

$\leadsto$ Clustering

$\multimap$ Random

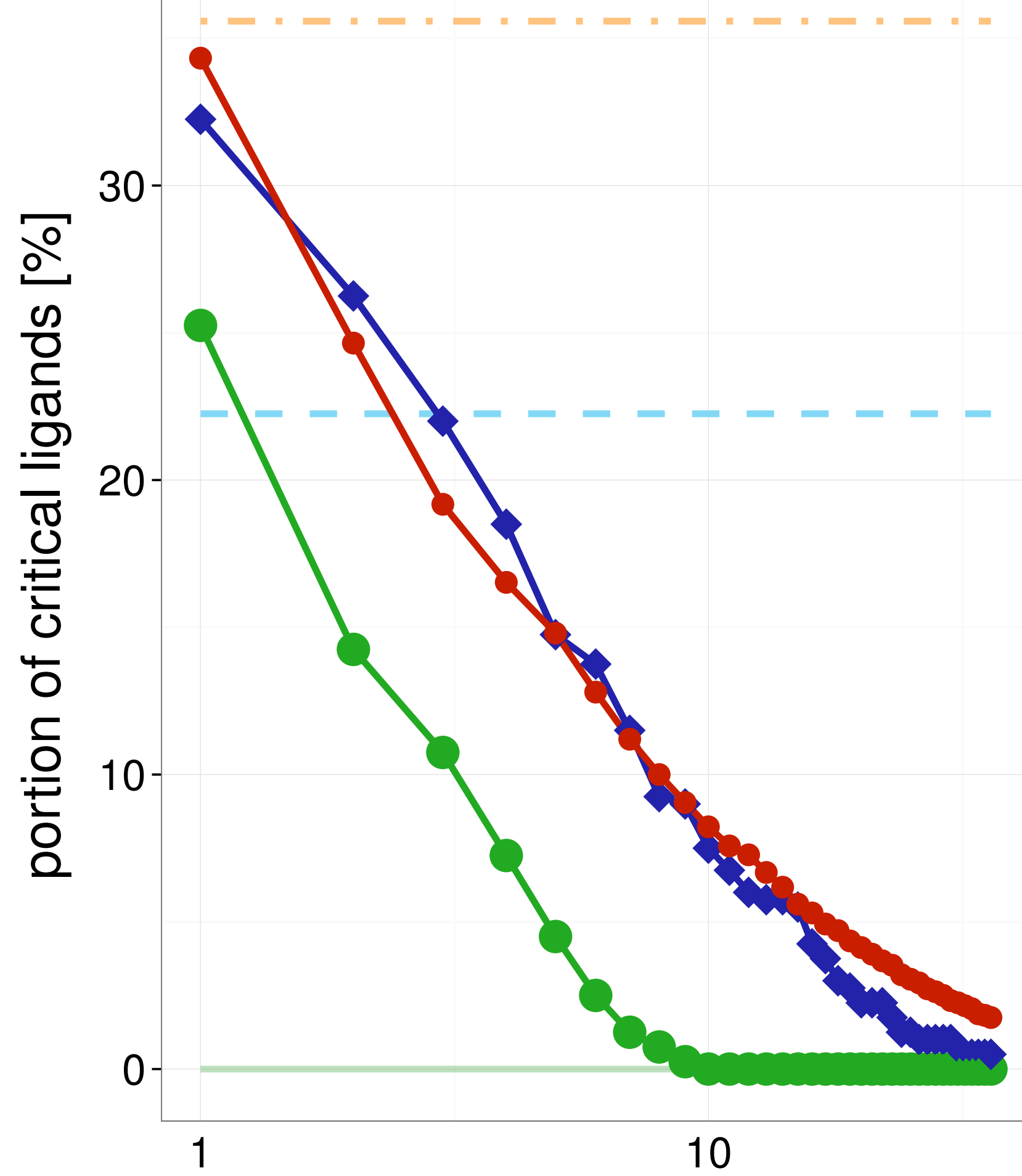

maximal ensemble size

Test set

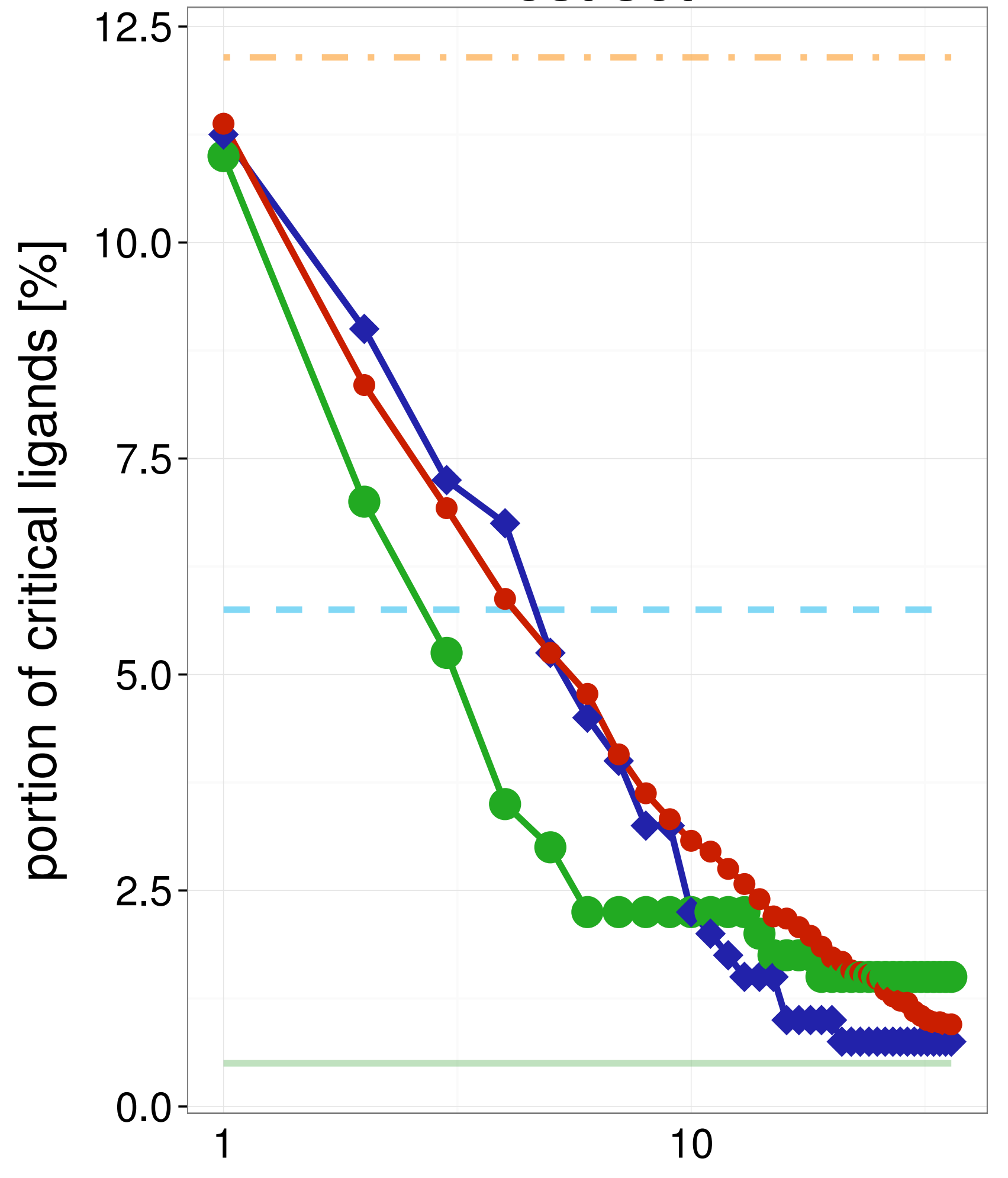

maximal ensemble size
Average single structure

- Best single structure

Whole ensemble

- SIENA

$\neg$ Clustering

$\multimap$ Random 
Training set

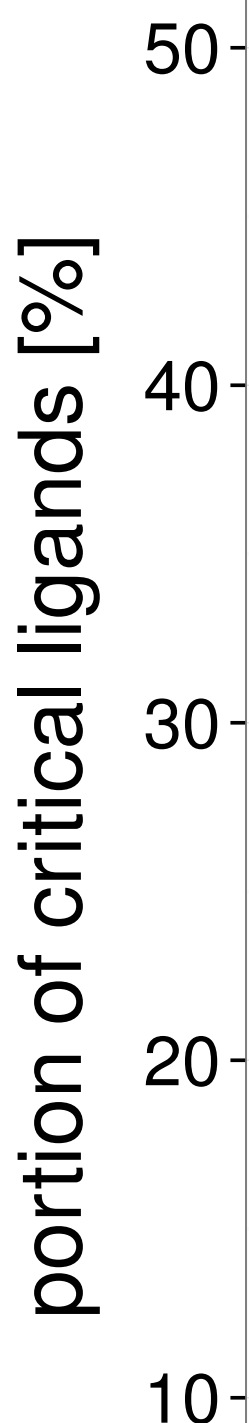

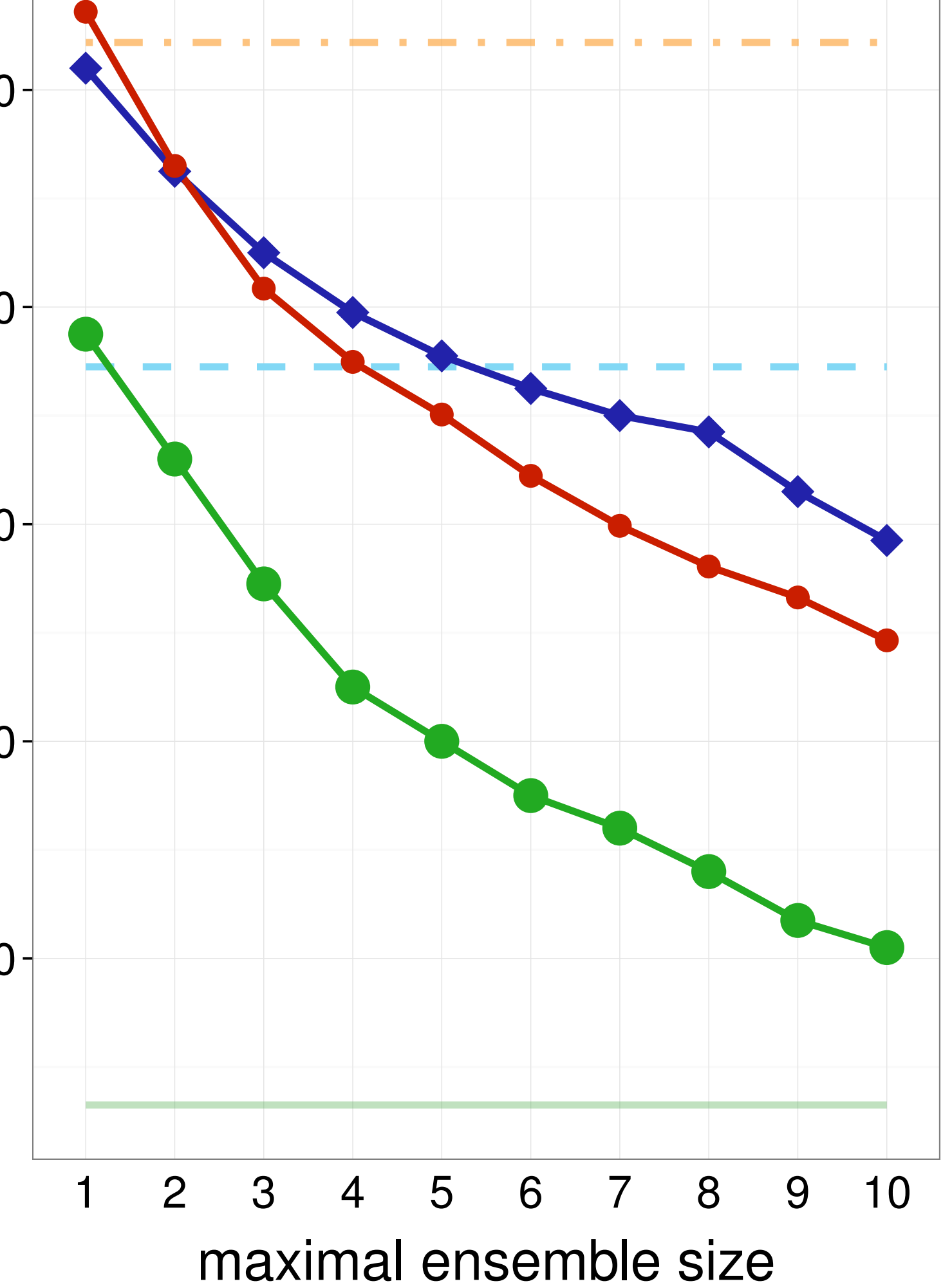

Test set

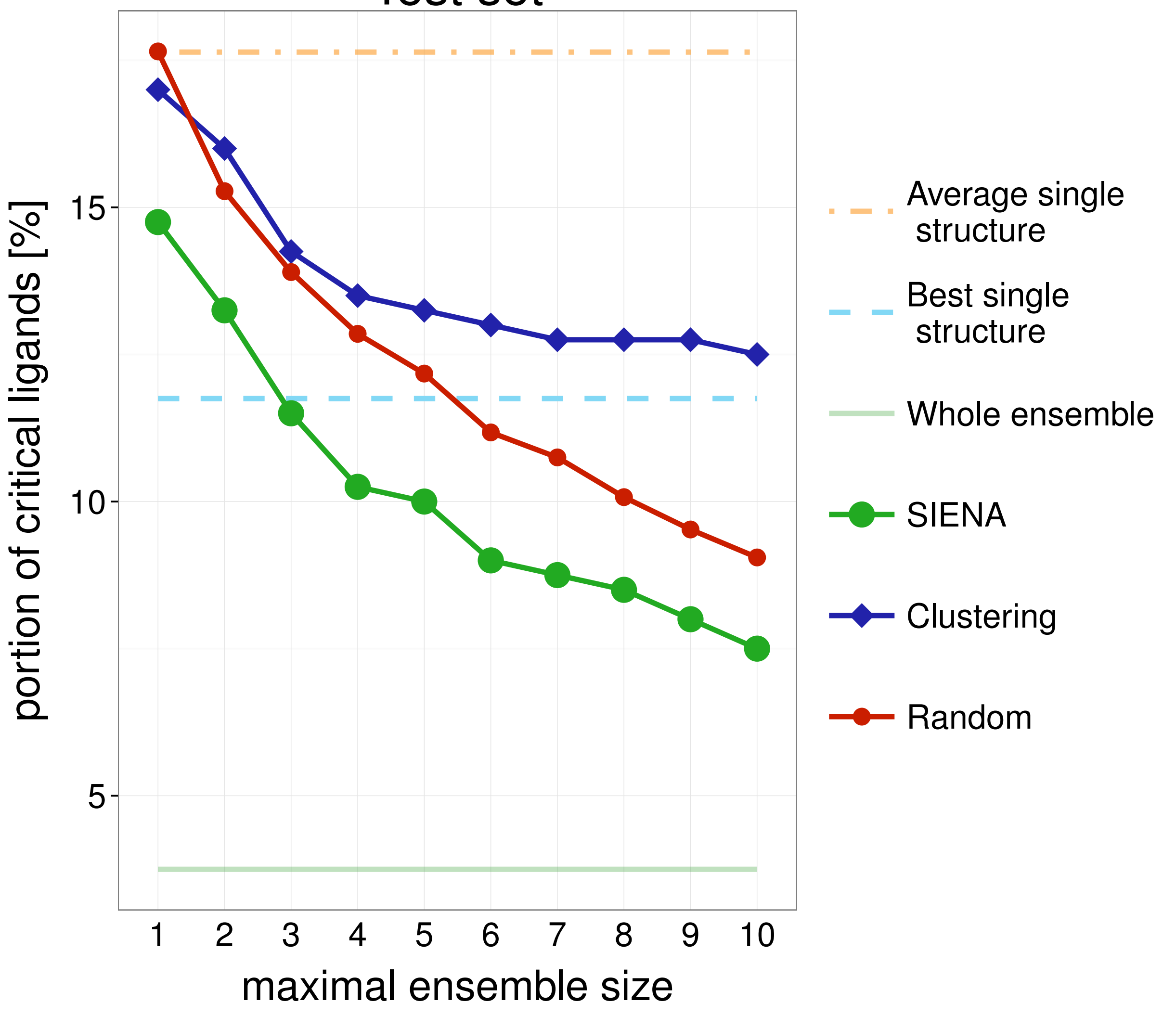

Average single structure

_ Best single structure

Whole ensemble

- SIENA

$\smile$ Clustering

$\multimap$ Random

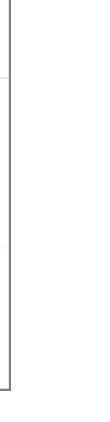

maximal ensemble size

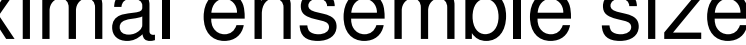

3R8A Training set

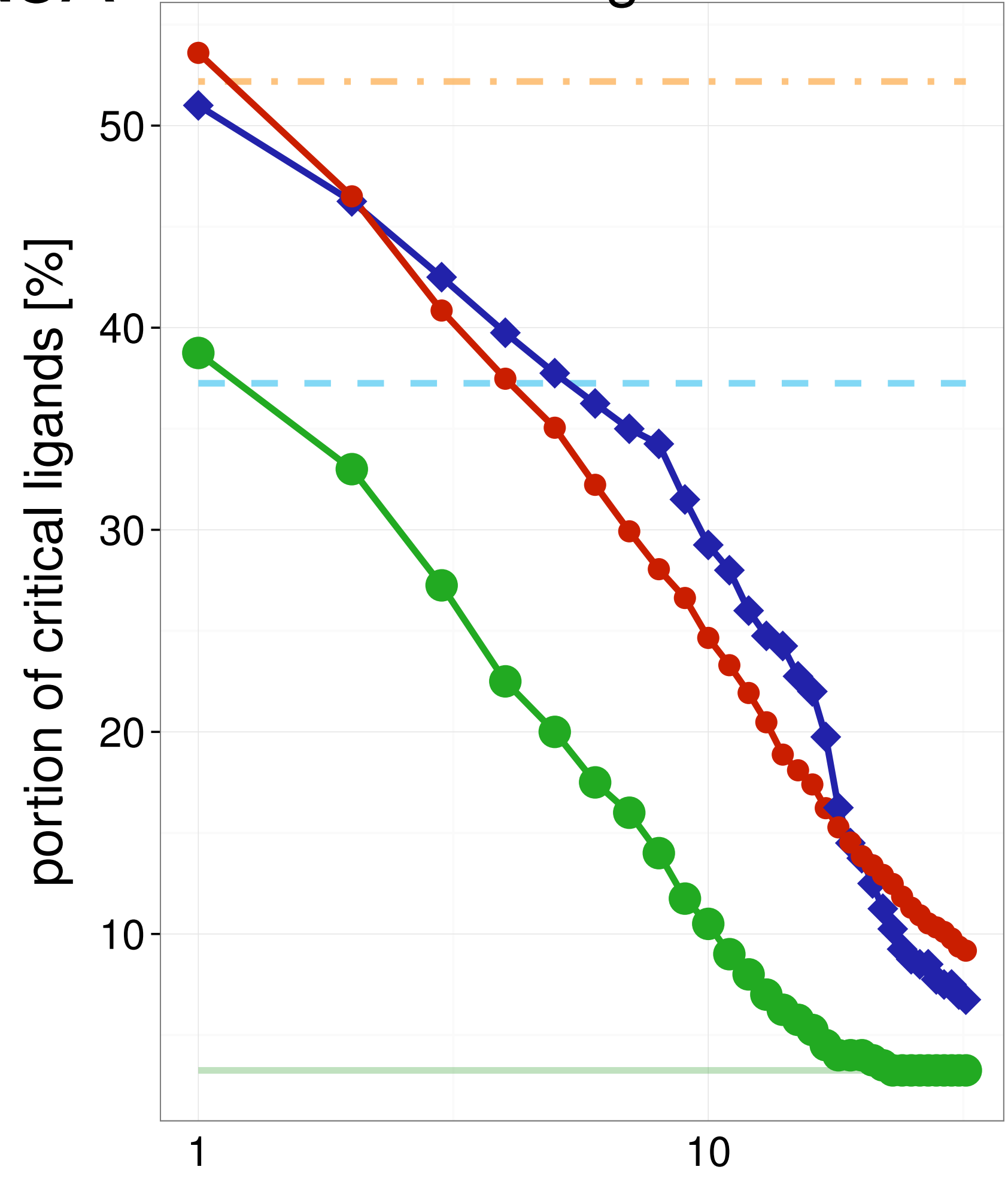

maximal ensemble size

\section{Test set}

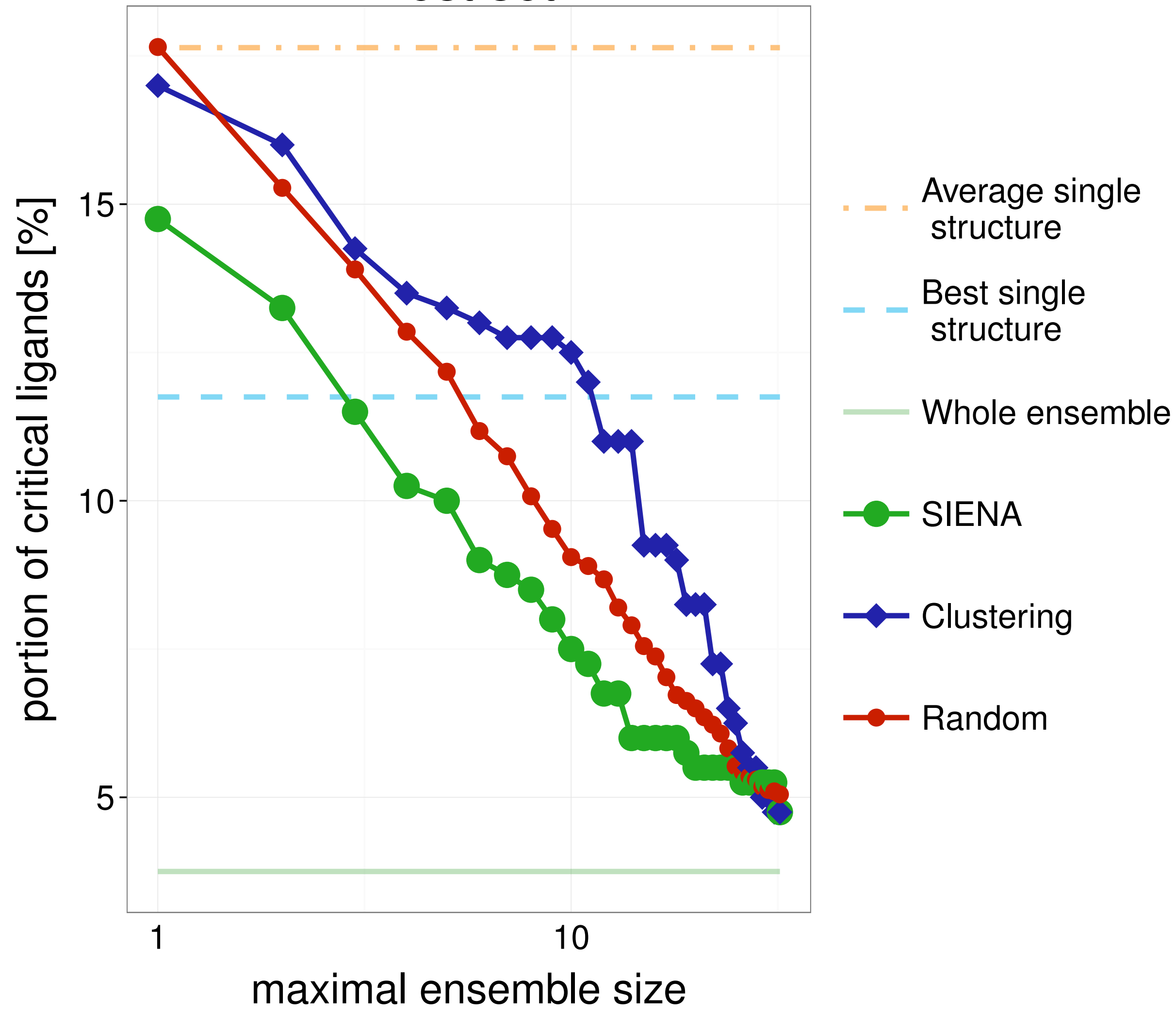




\section{Training set}

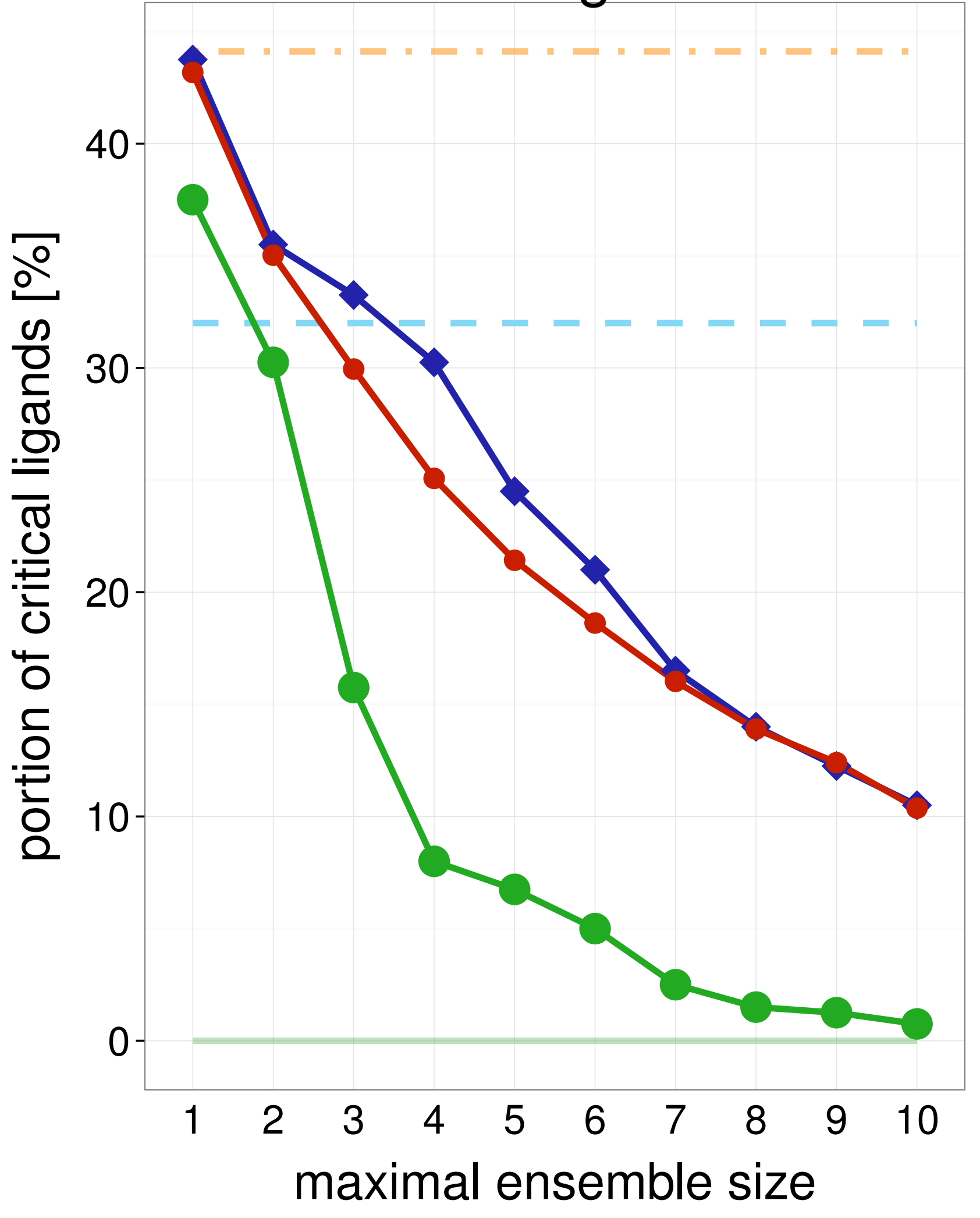

\section{Test set}

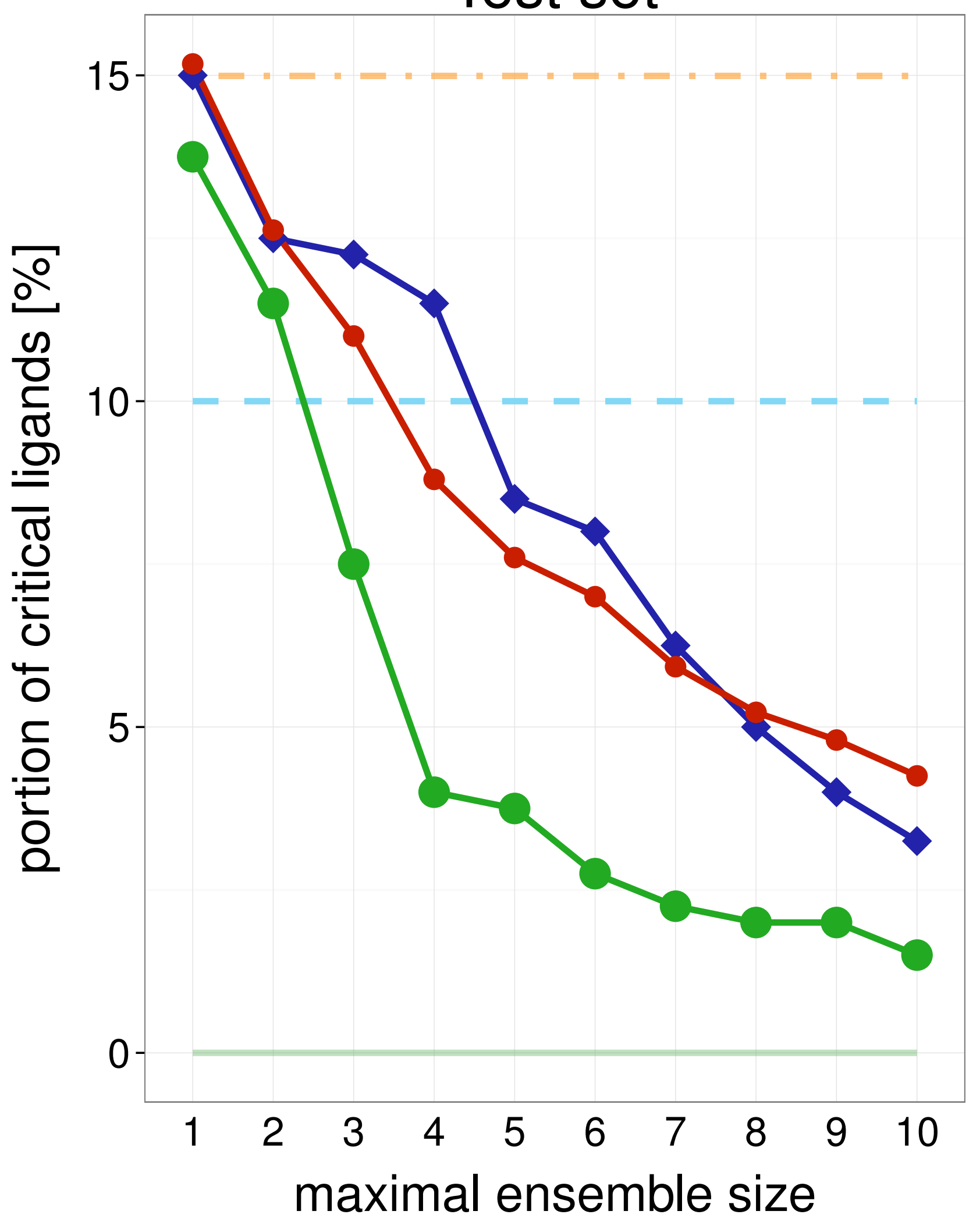

Average single structure

_ _ Best single structure

Whole ensemble

- SIENA

$\neg$ Clustering

$\longrightarrow$ Random

Average single structure

_ Best single structure

Whole ensemble

- SIENA

$\neg$ Clustering

$\multimap$ Random
Training set

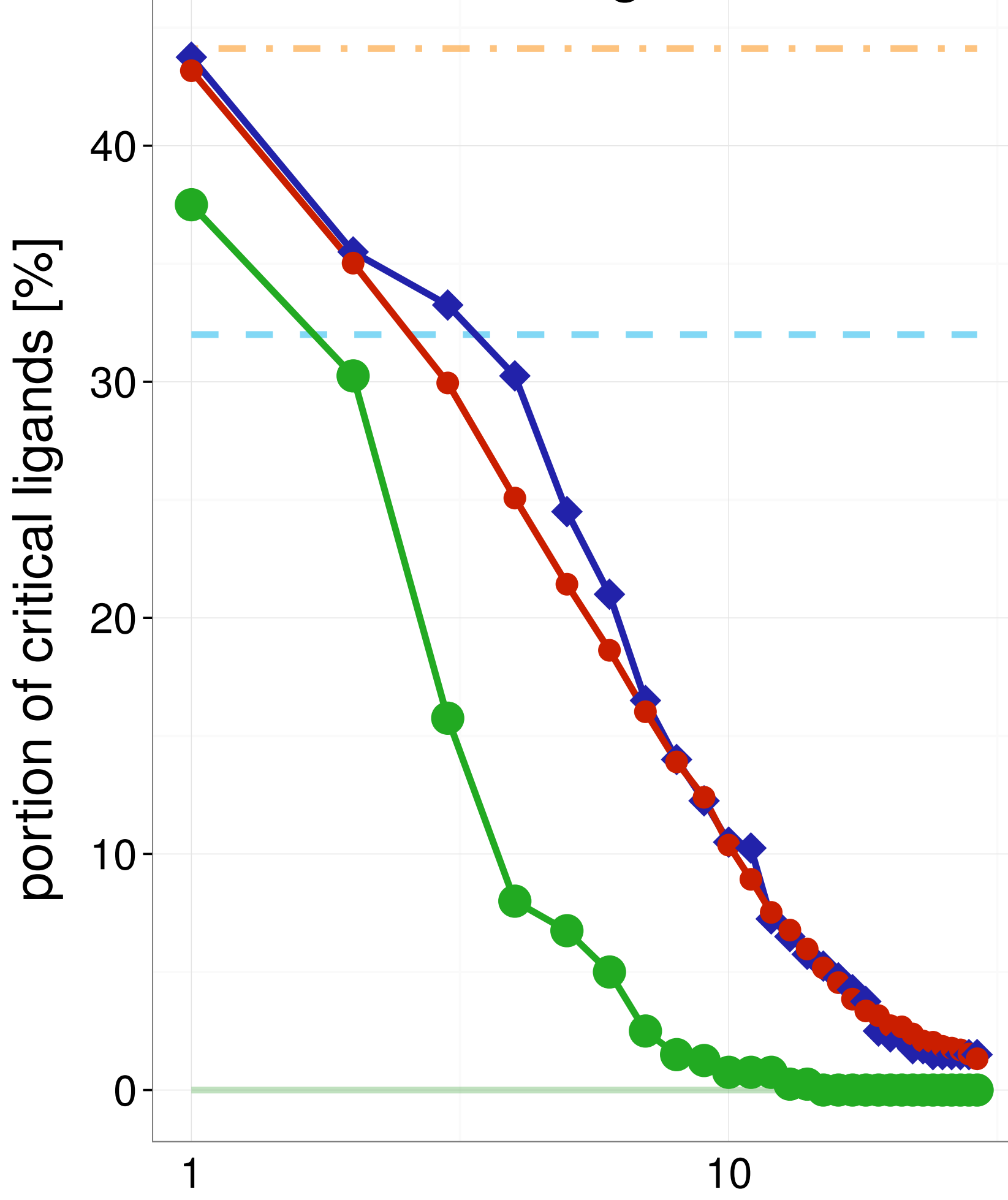

maximal ensemble size

Test set

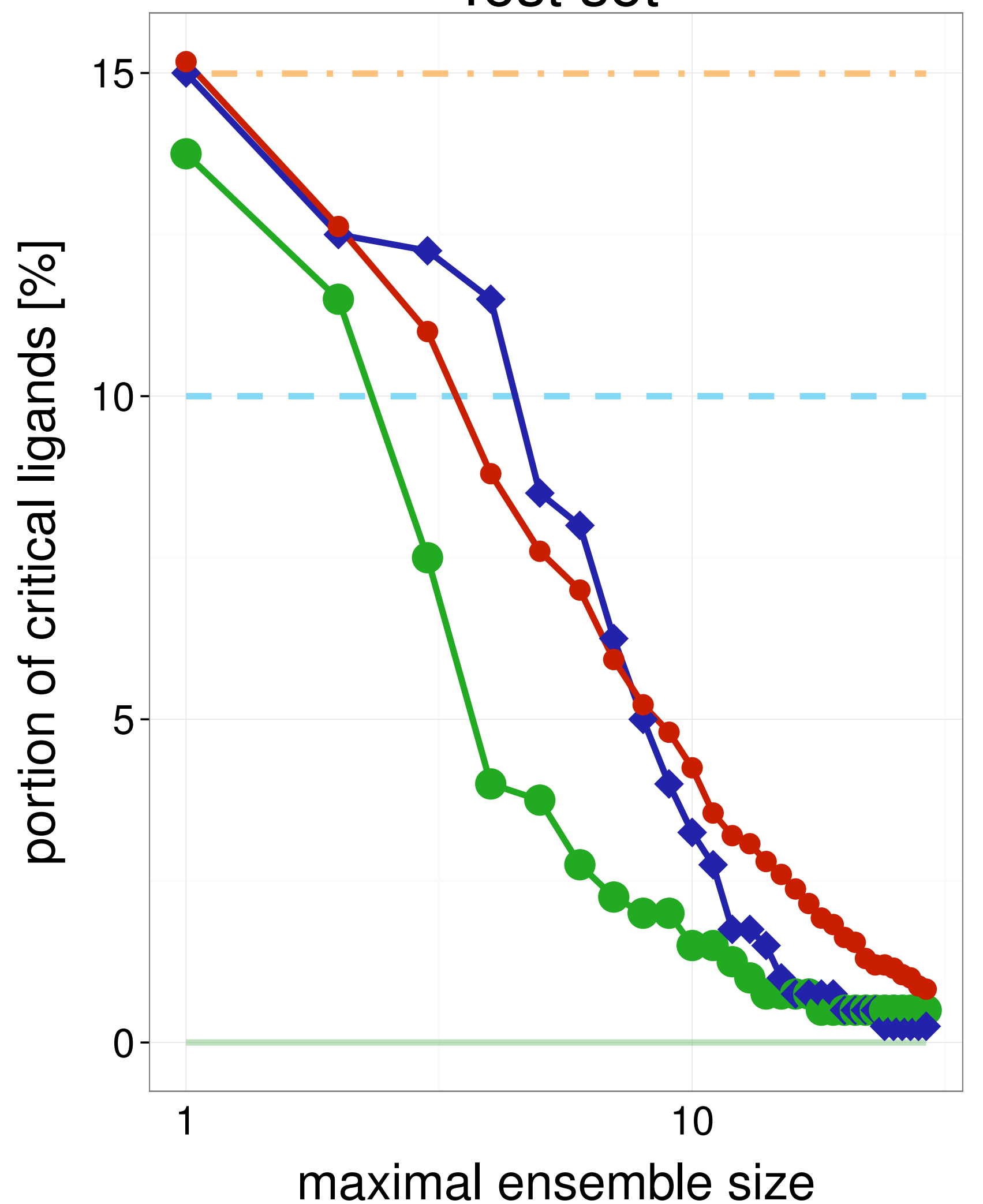

Average single structure

Best single structure

Whole ensemble

- SIENA

$\checkmark$ Clustering

$\multimap$ Random

Average single structure

Best single structure

Whole ensemble

- SIENA

$\sim$ Clustering

$\longrightarrow$ Random 


\section{Training set}

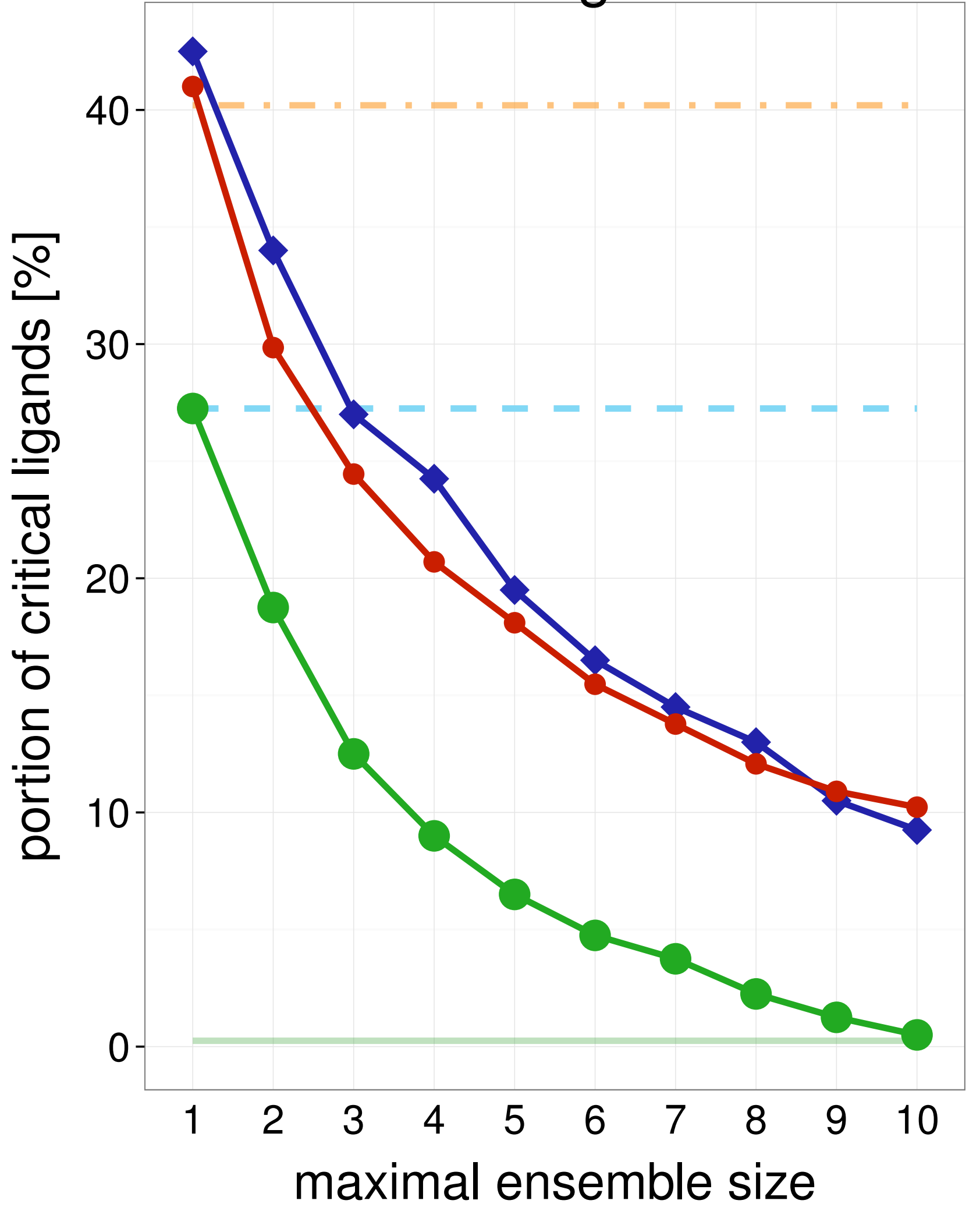

Test set

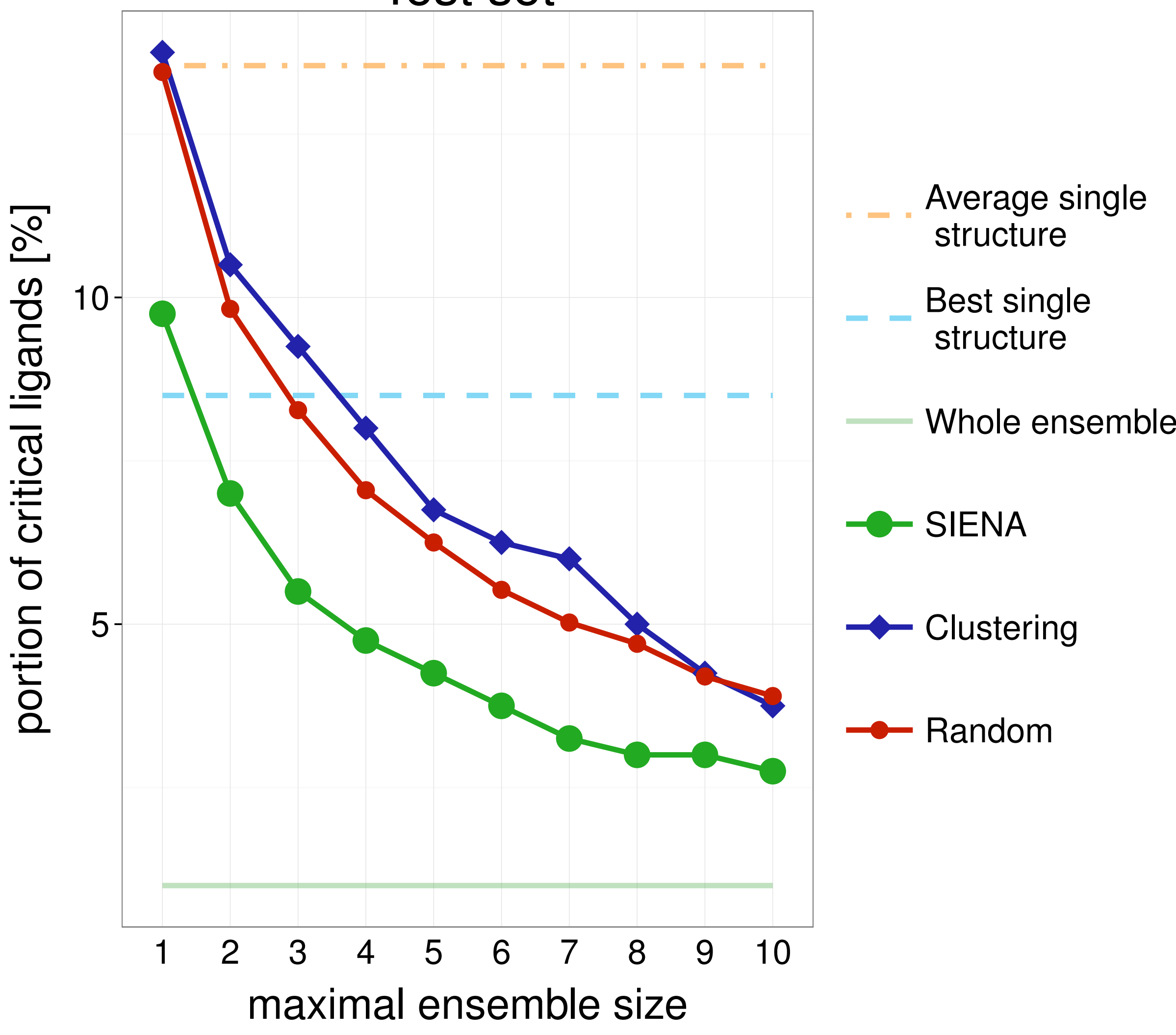

Average single structure

_ Best single structure

Whole ensemble

- SIENA

$\neg$ Clustering

$\multimap$ Random
Training set

0

1

maximal ensemble size

Test set

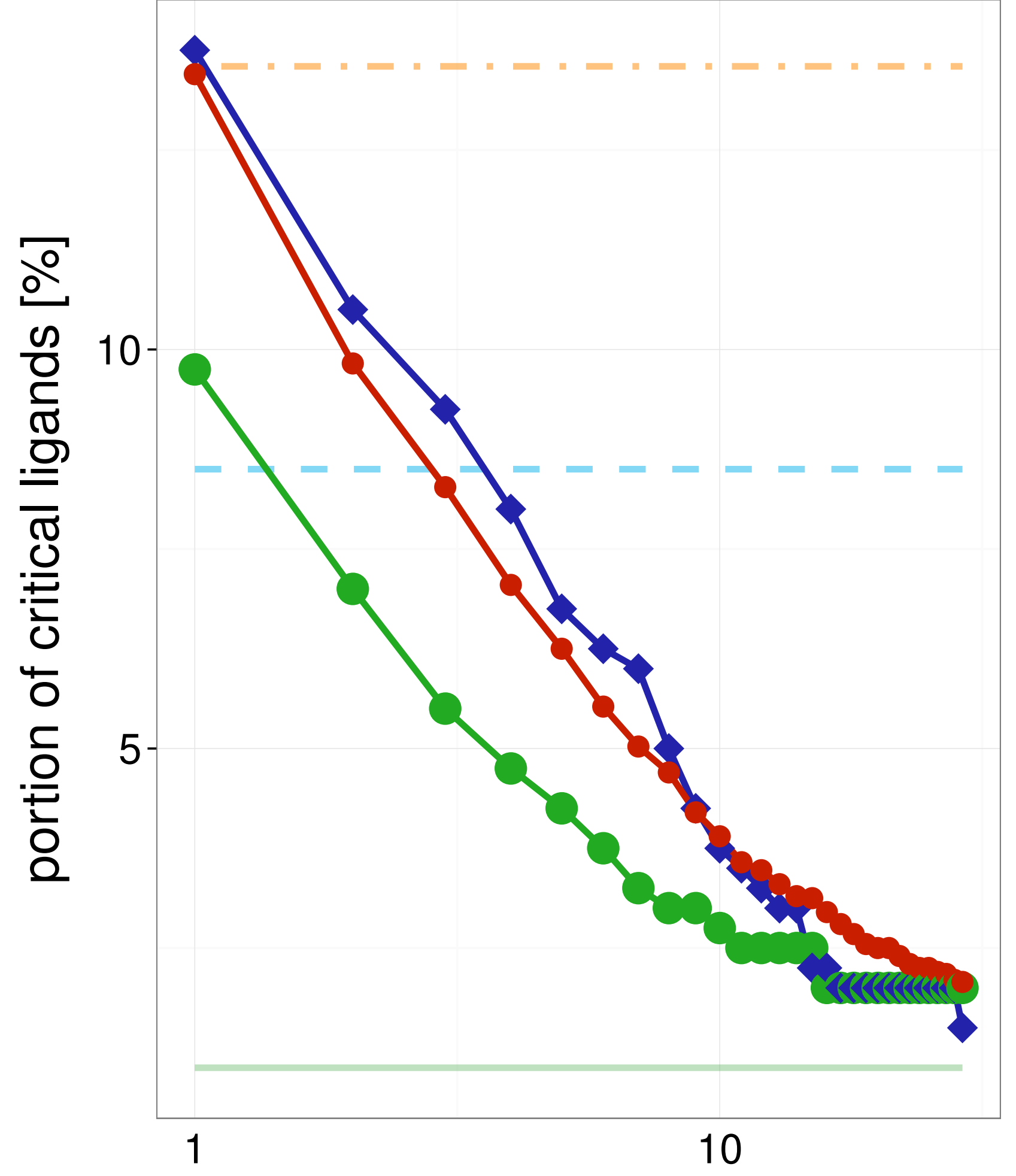

maximal ensemble size
Average single structure

Best single structure

Whole ensemble

- SIENA

$\checkmark$ Clustering

$\rightarrow$ Random

Average single structure

_ Best single structure

Whole ensemble

- SIENA

$\neg$ Clustering

$\multimap$ Random 


\section{Training set}

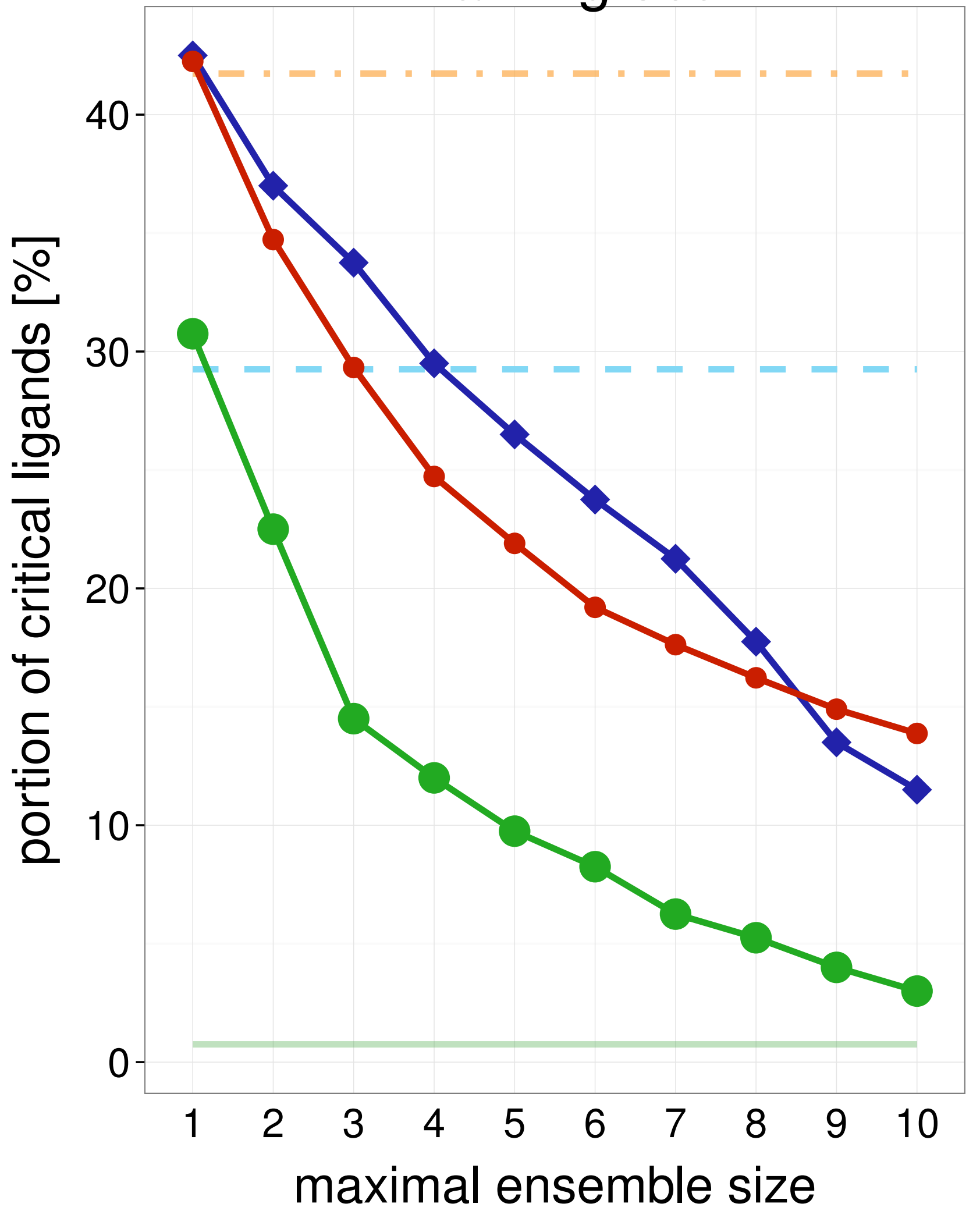

Test set

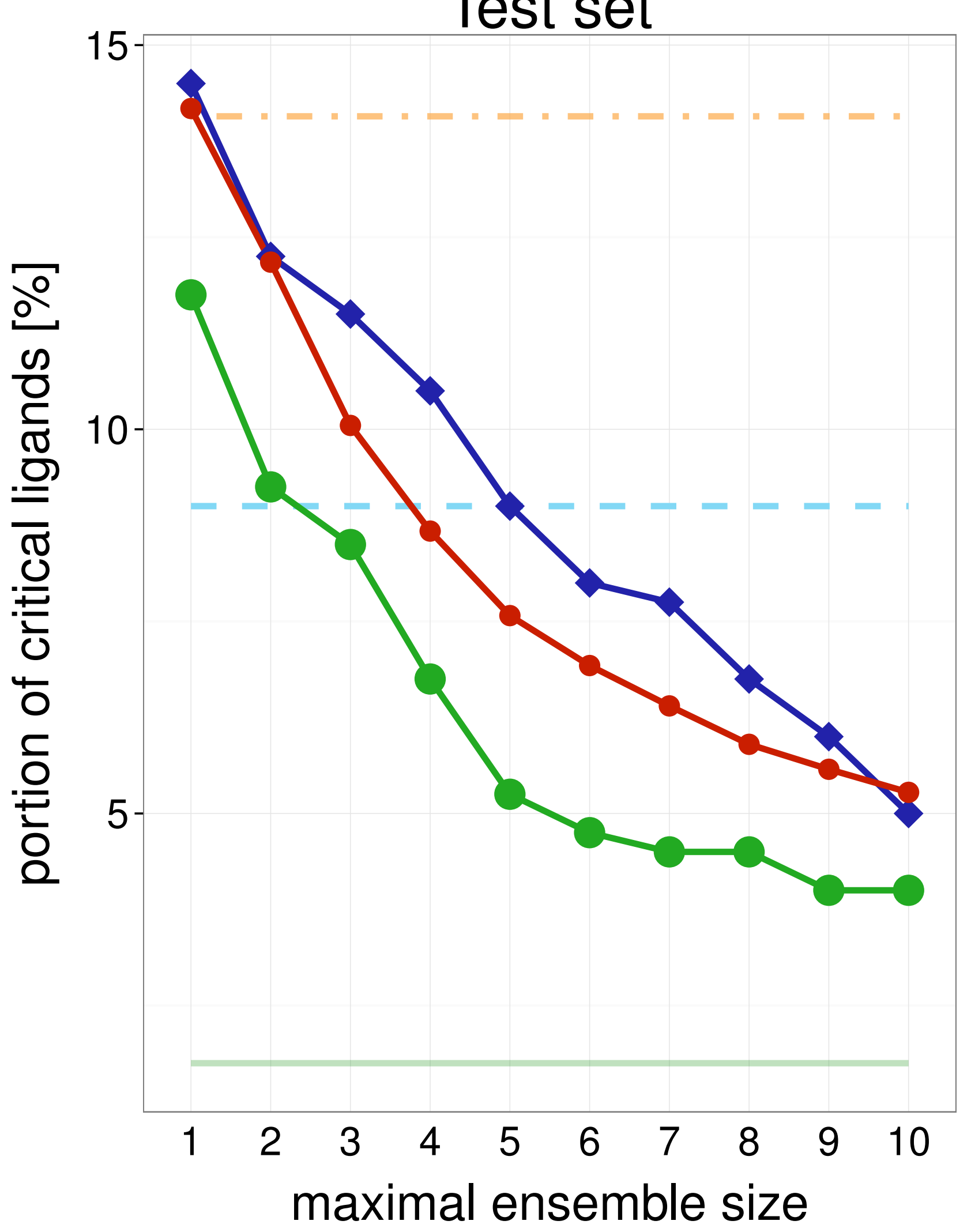

Average single structure

_ Best single structure

Whole ensemble

- SIENA

$\neg$ Clustering

$\multimap$ Random

Average single structure

_ _ Best single structure

Whole ensemble

- SIENA

$\sim$ Clustering

$\multimap$ Random
$210 \mathrm{~J}$

\section{Training set}

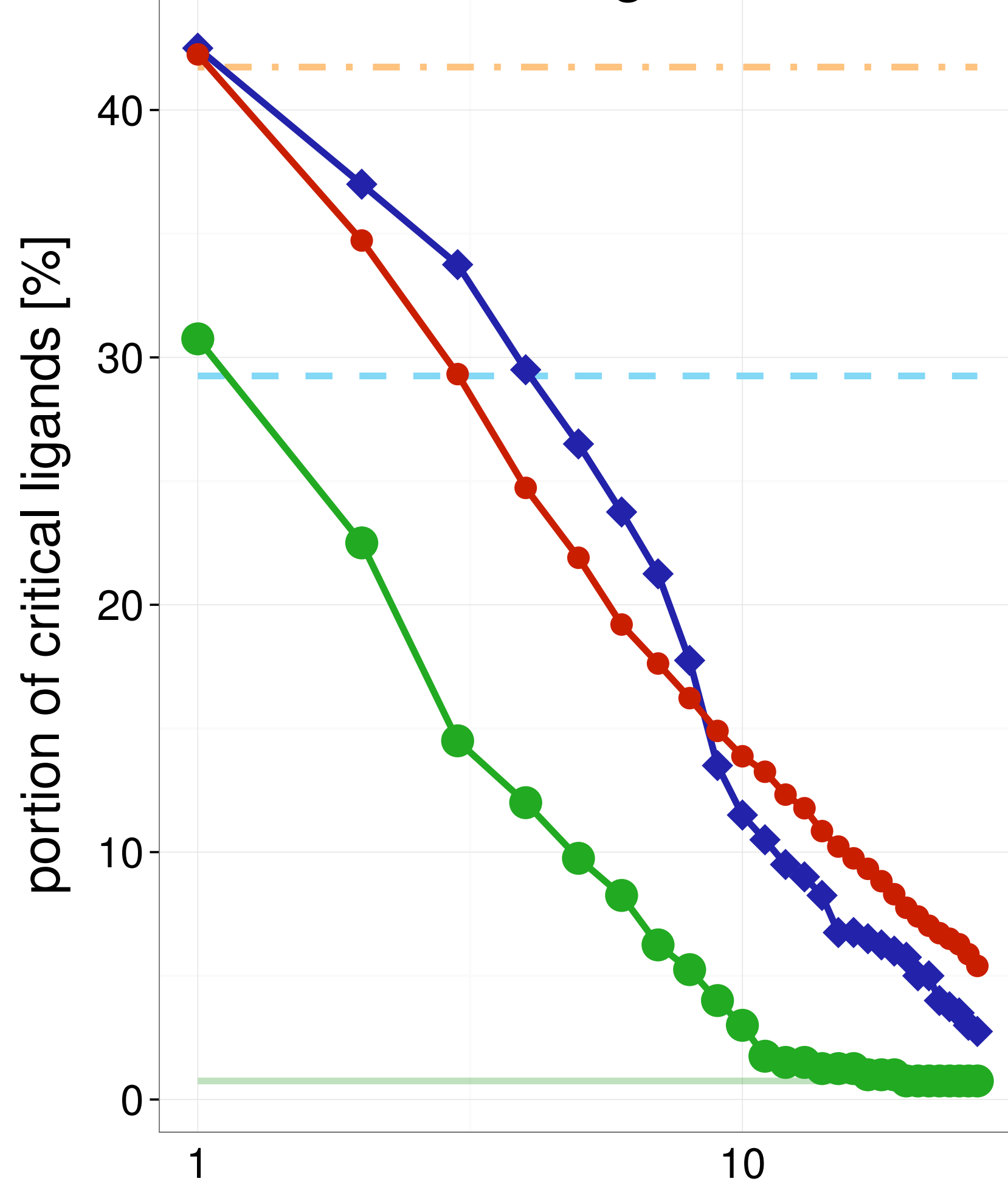

maximal ensemble size

\section{Test set}

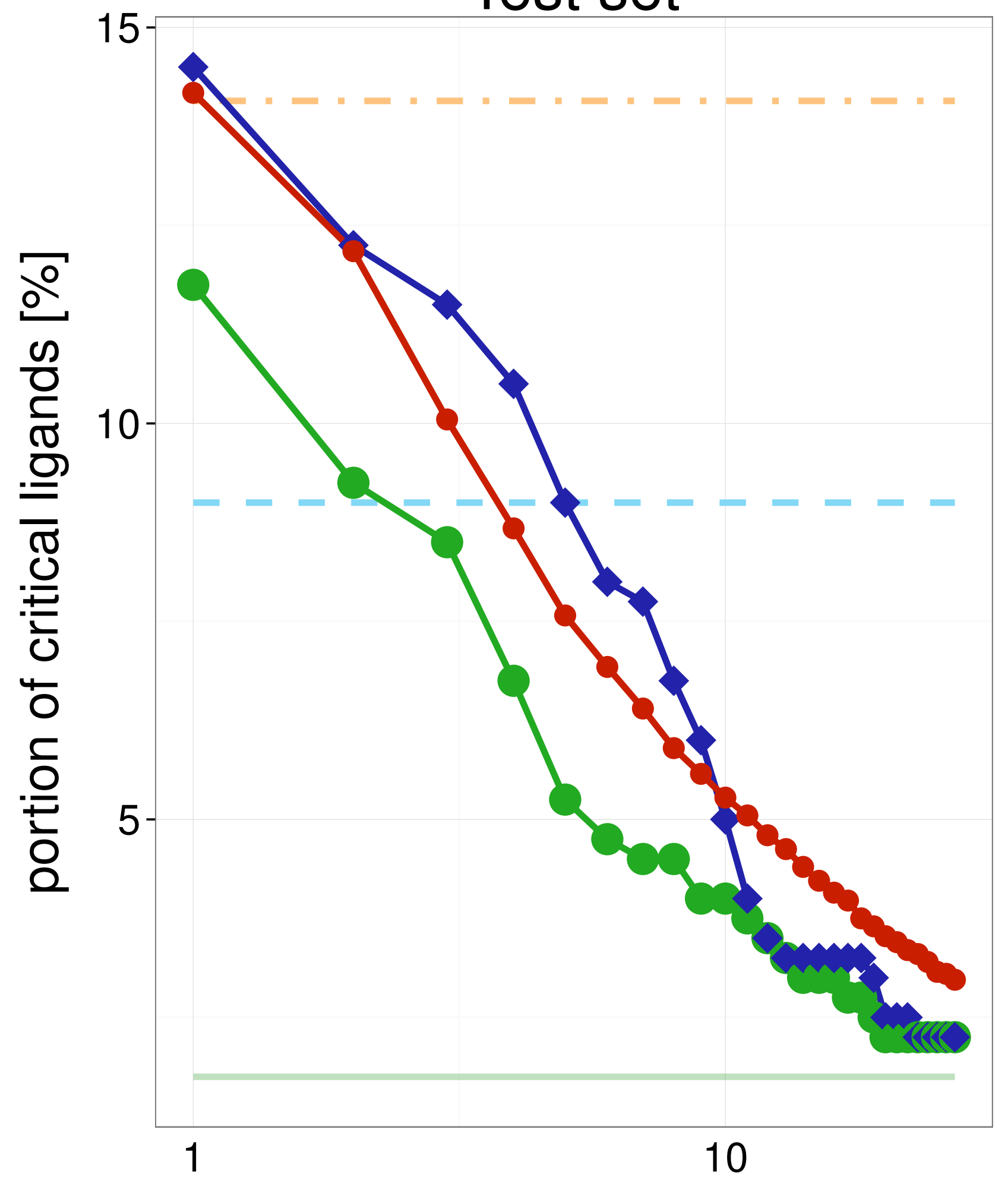

Average single structure

_ _ Best single structure

Whole ensemble

$\multimap-$ SIENA

$\leadsto$ Clustering

$\multimap$ Random

maximal ensemble size 


\section{Training set}

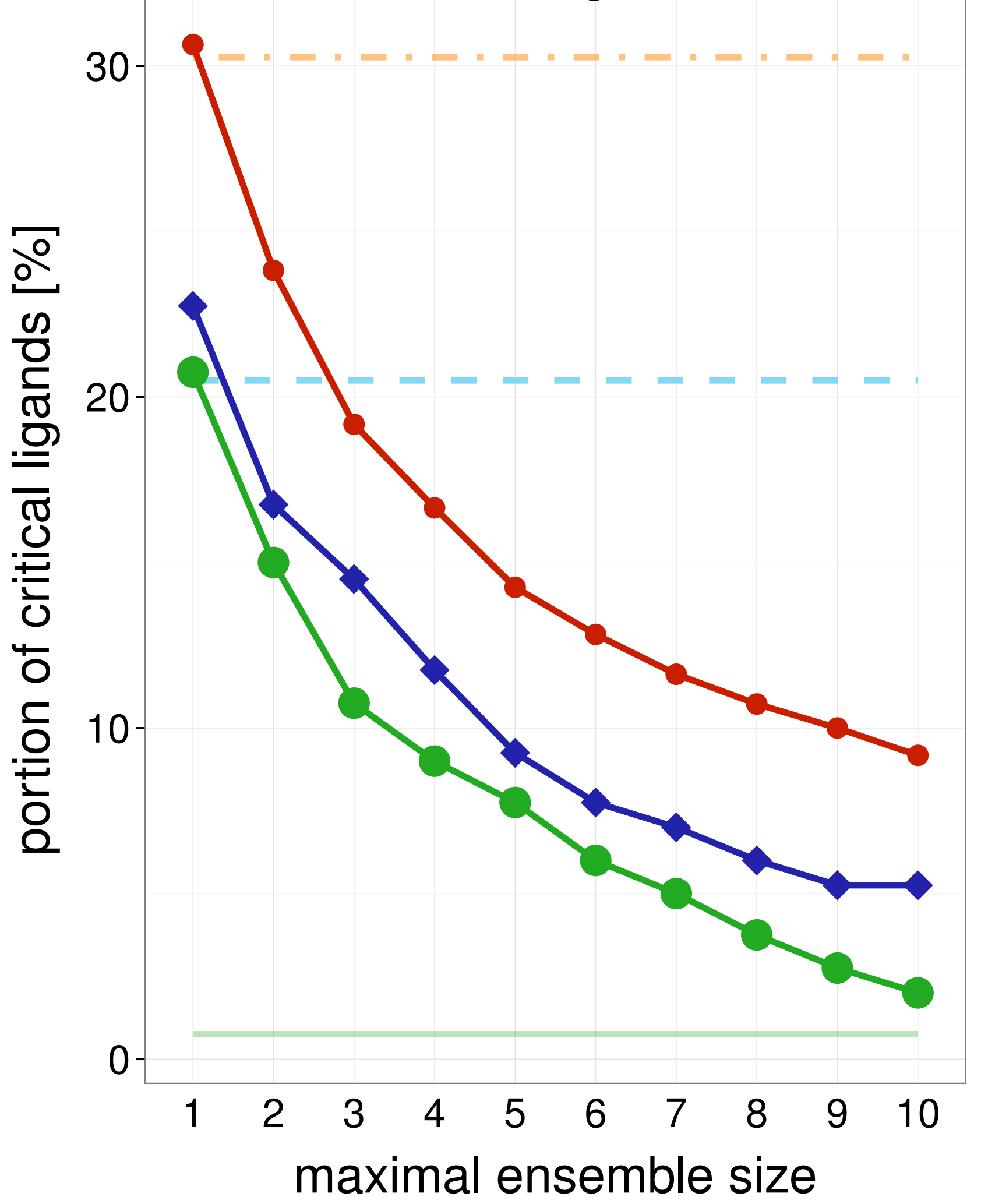

\section{Test set}

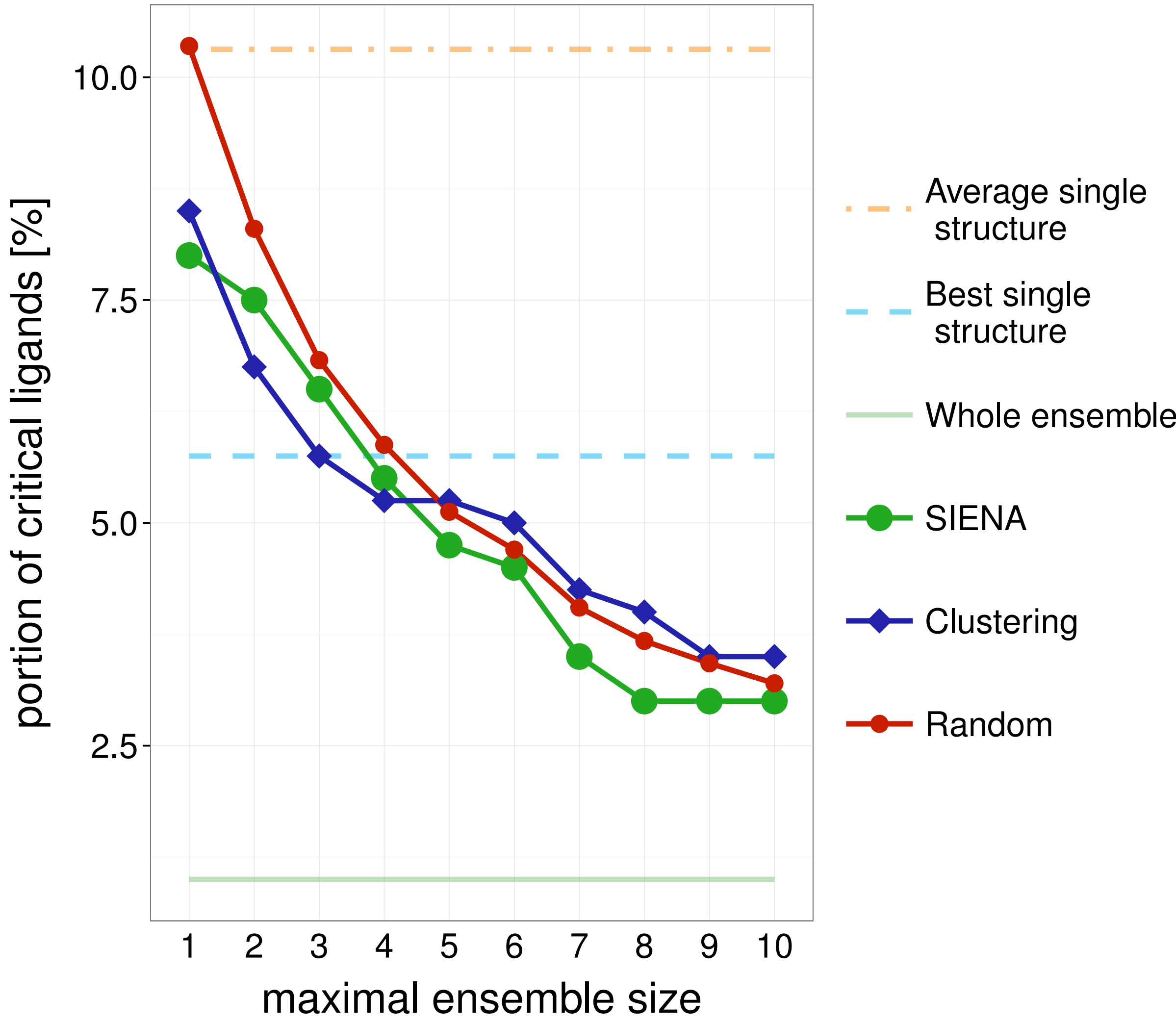

Training set

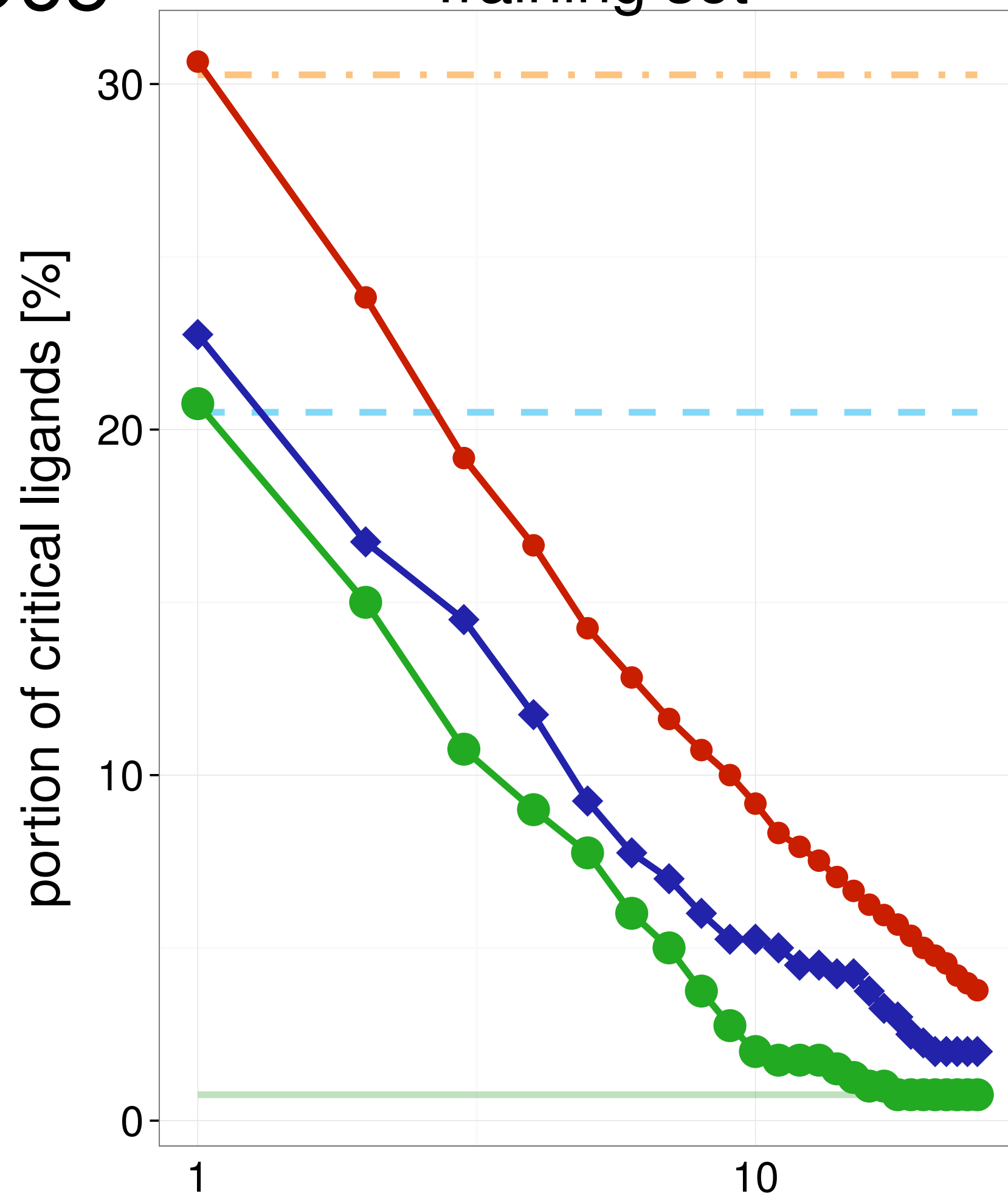

maximal ensemble size

Test set

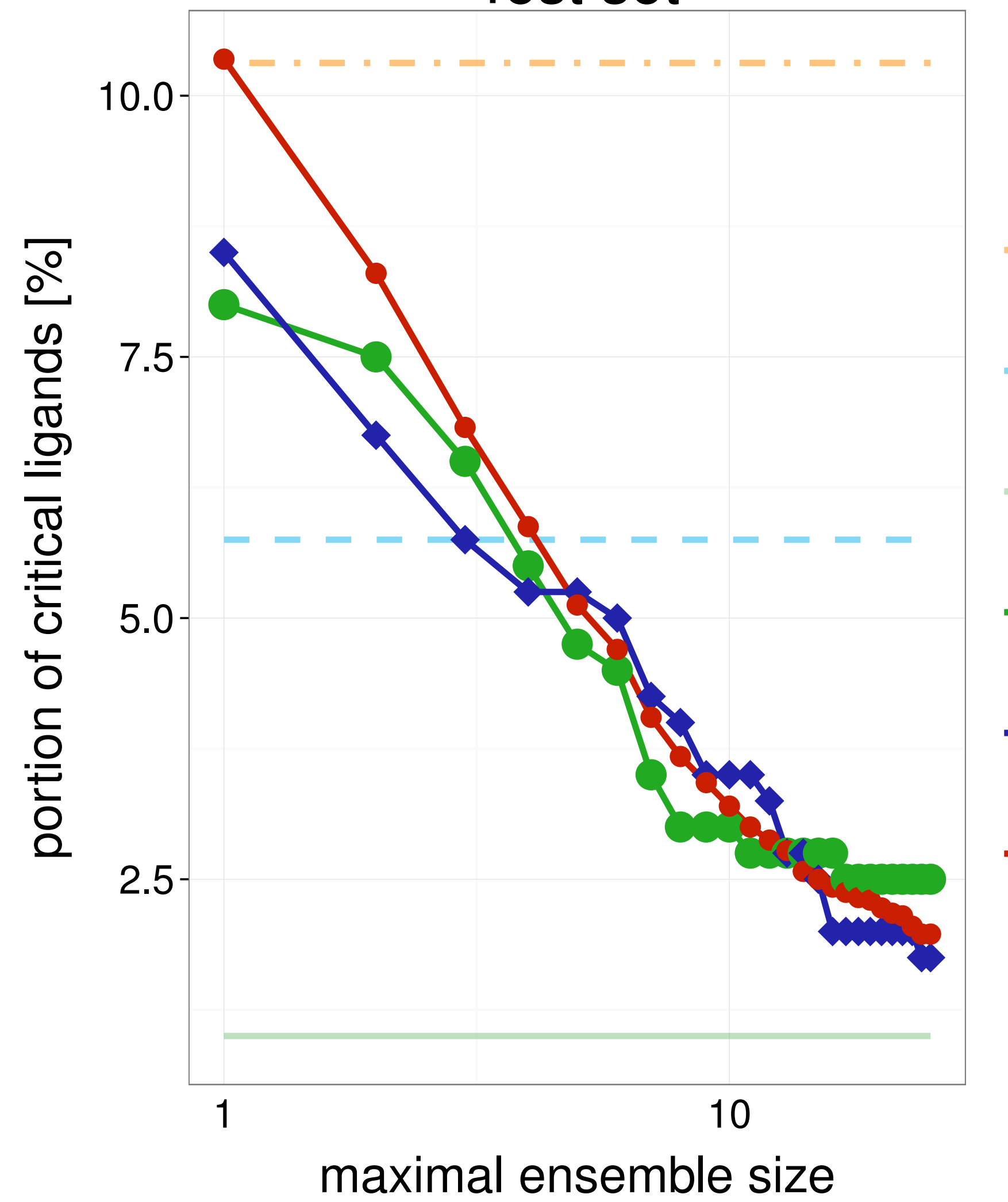

Average single structure

Best single structure

Whole ensemble

- SIENA

$\neg$ Clustering

$\multimap$ Random

Average single structure

_ _ Best single structure

Whole ensemble

- SIENA

$\neg$ Clustering

$\multimap$ Random 


\section{Training set}

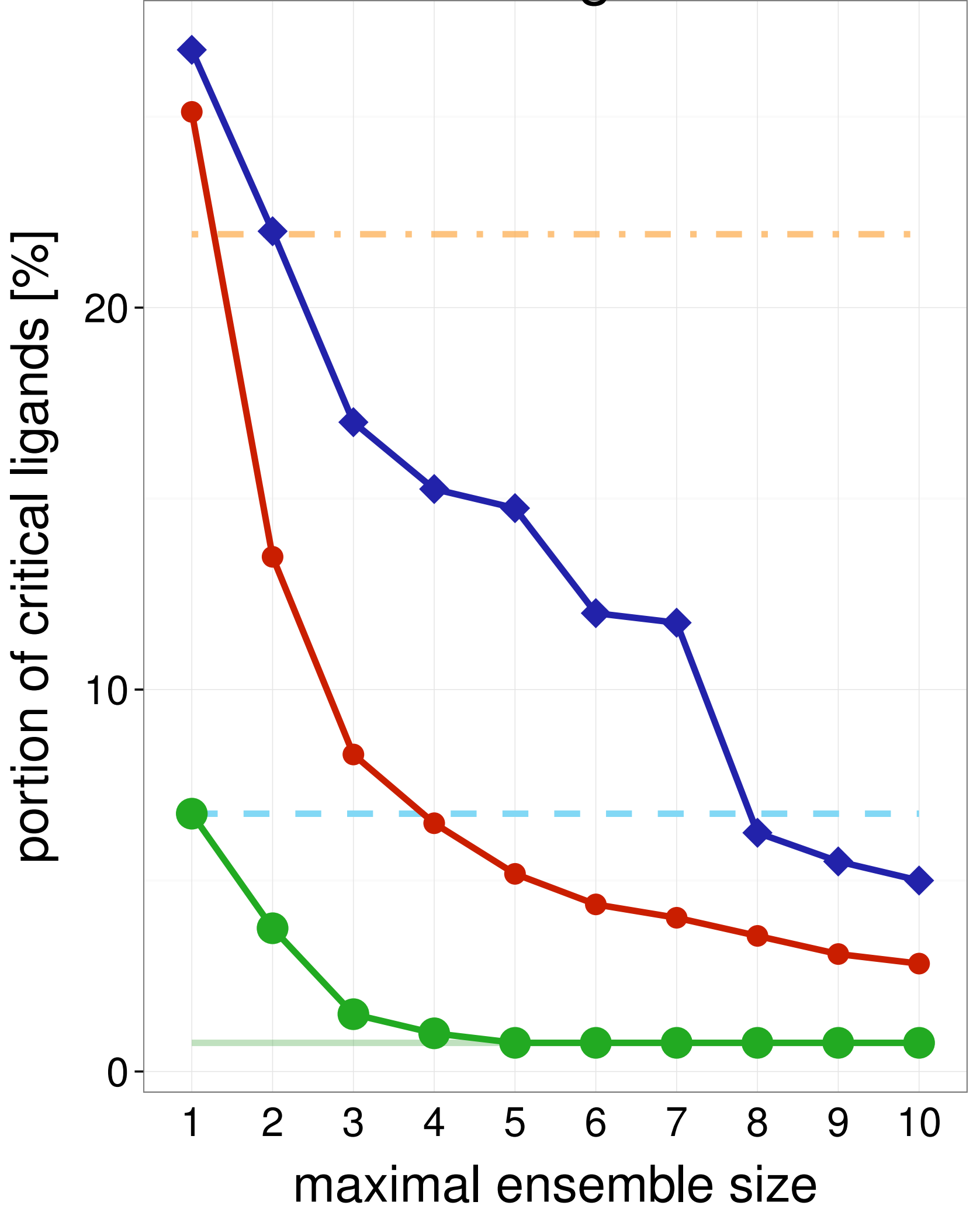

\section{Test set}

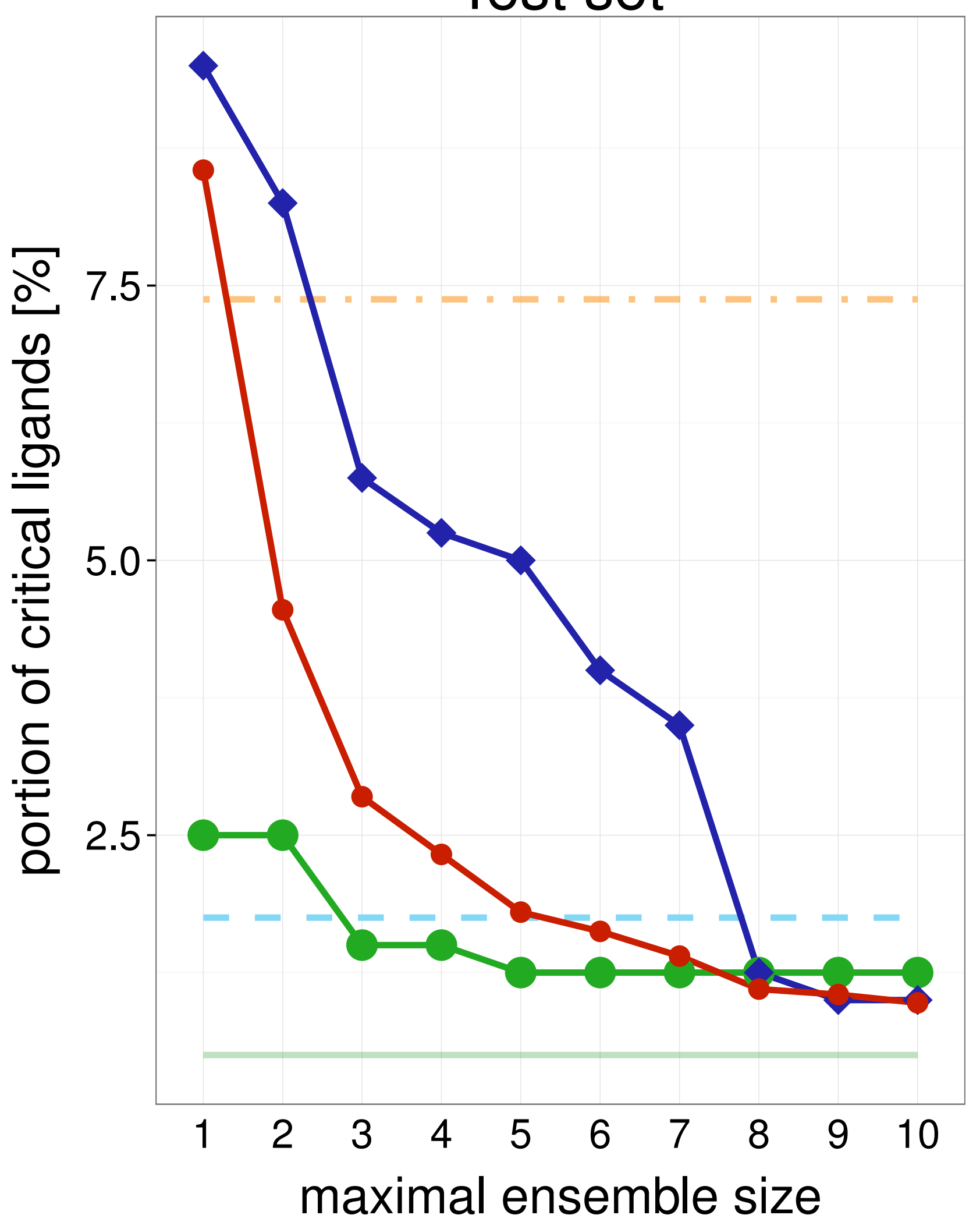

3ZSO

Training set

Average single structure

_ Best single

structure

Whole ensemble

- SIENA

$\neg$ Clustering

$\multimap$ Random

Average single structure

\section{_ Best single} structure

Whole ensemble

- SIENA

$\neg$ Clustering

$\because$ Random

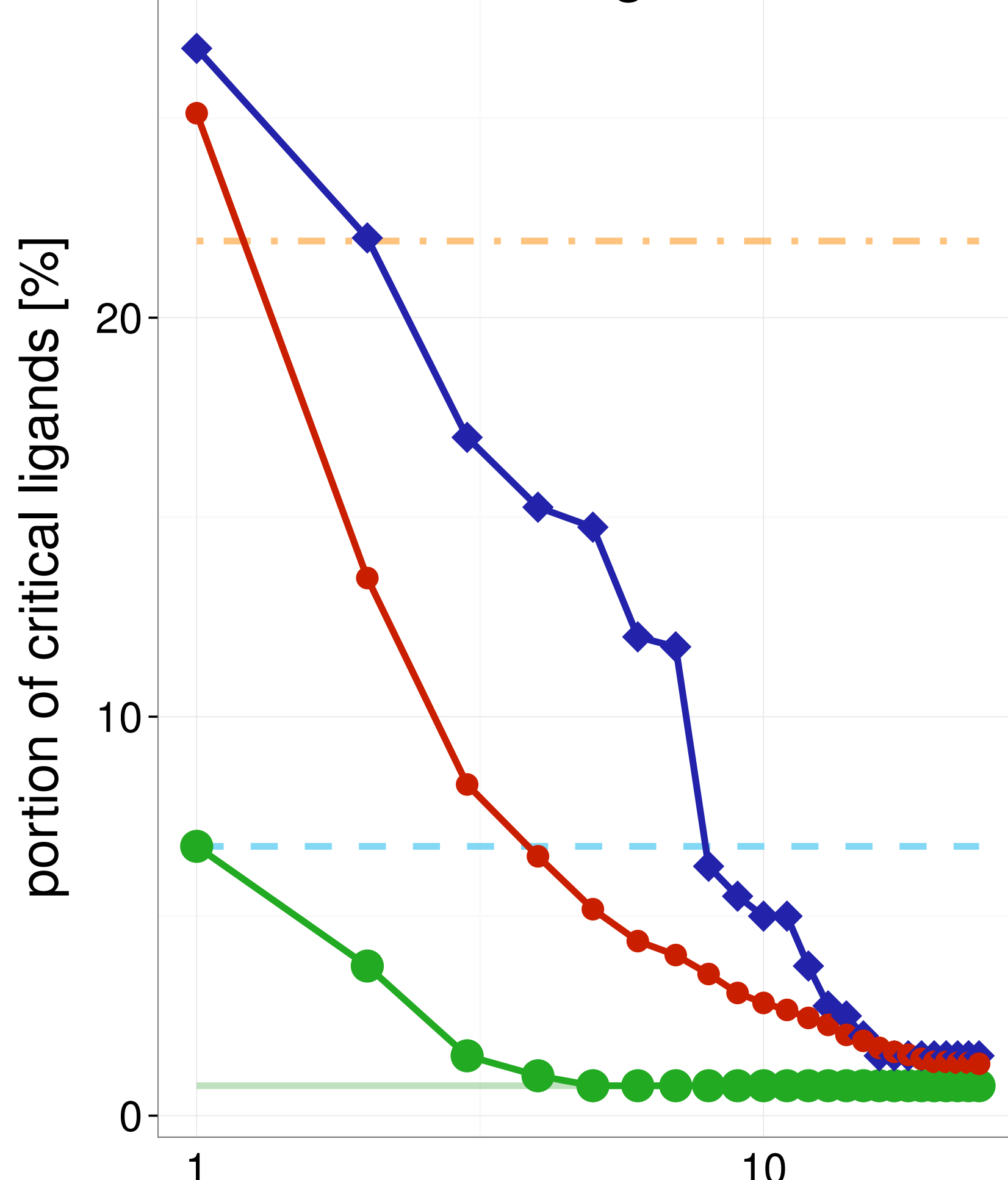

maximal ensemble size

Test set

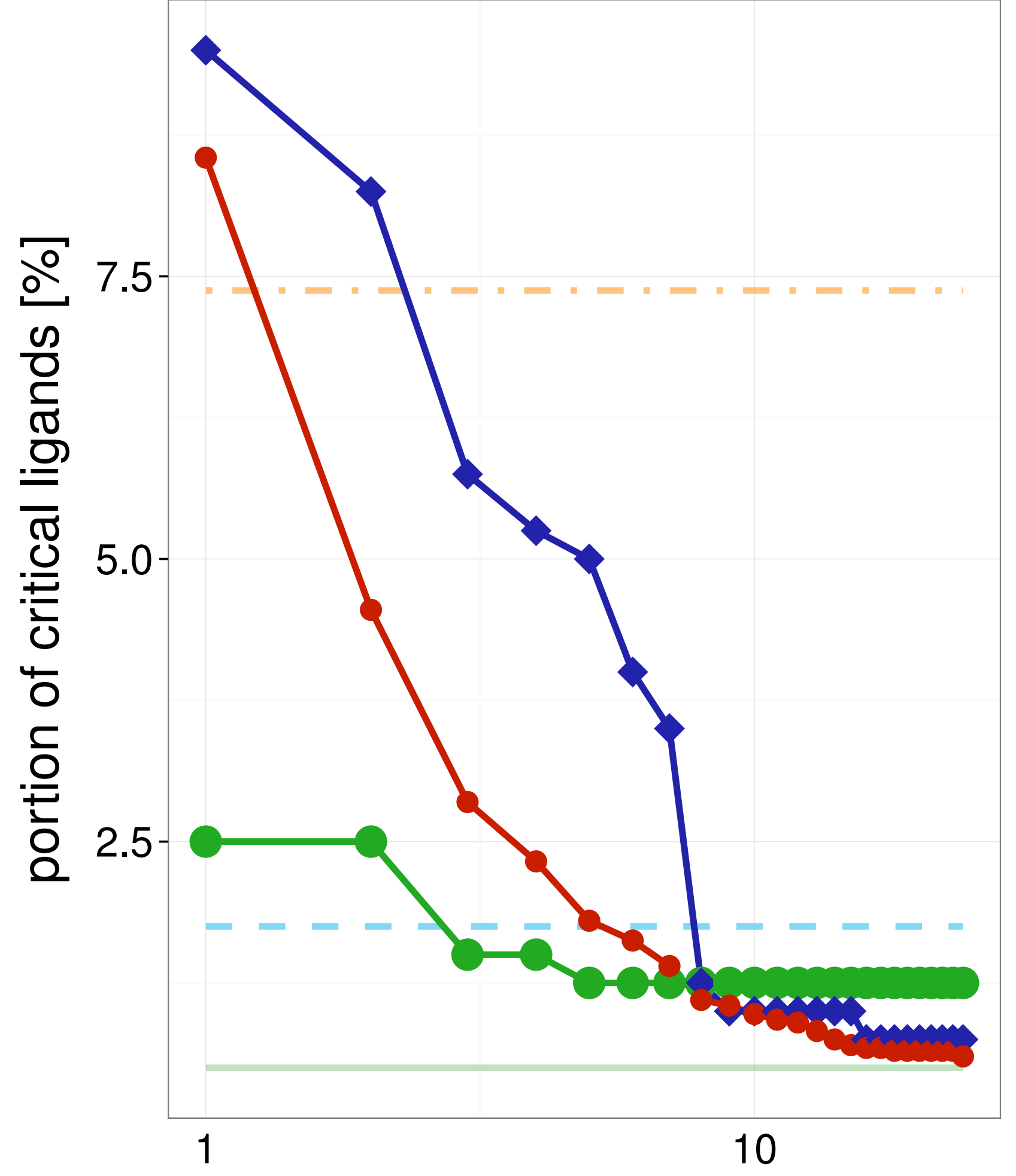

maximal ensemble size
Average single structure

_ Best single structure

Whole ensemble

- SIENA

$\neg$ Clustering

$\multimap$ Random 


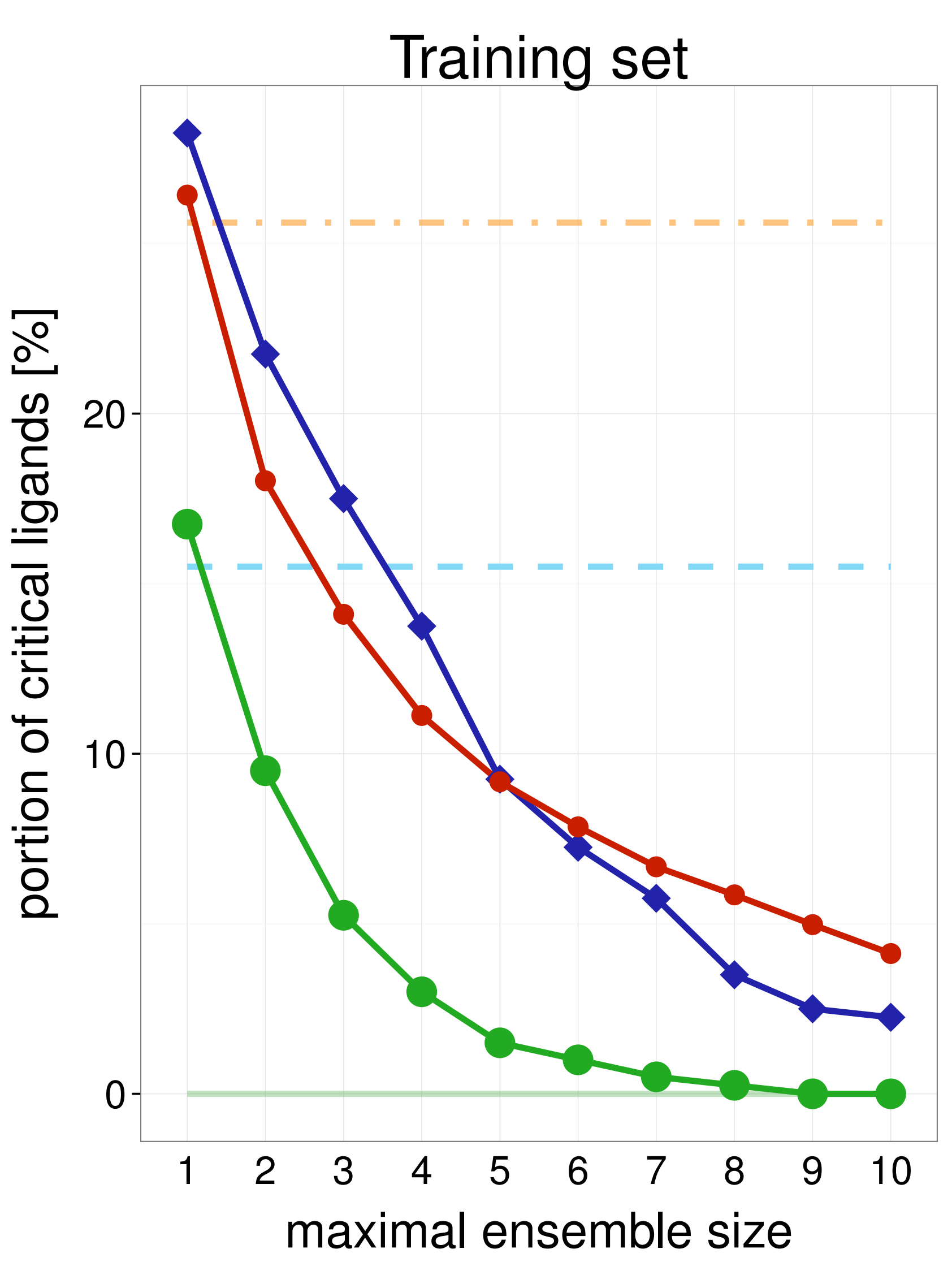

\section{Test set}

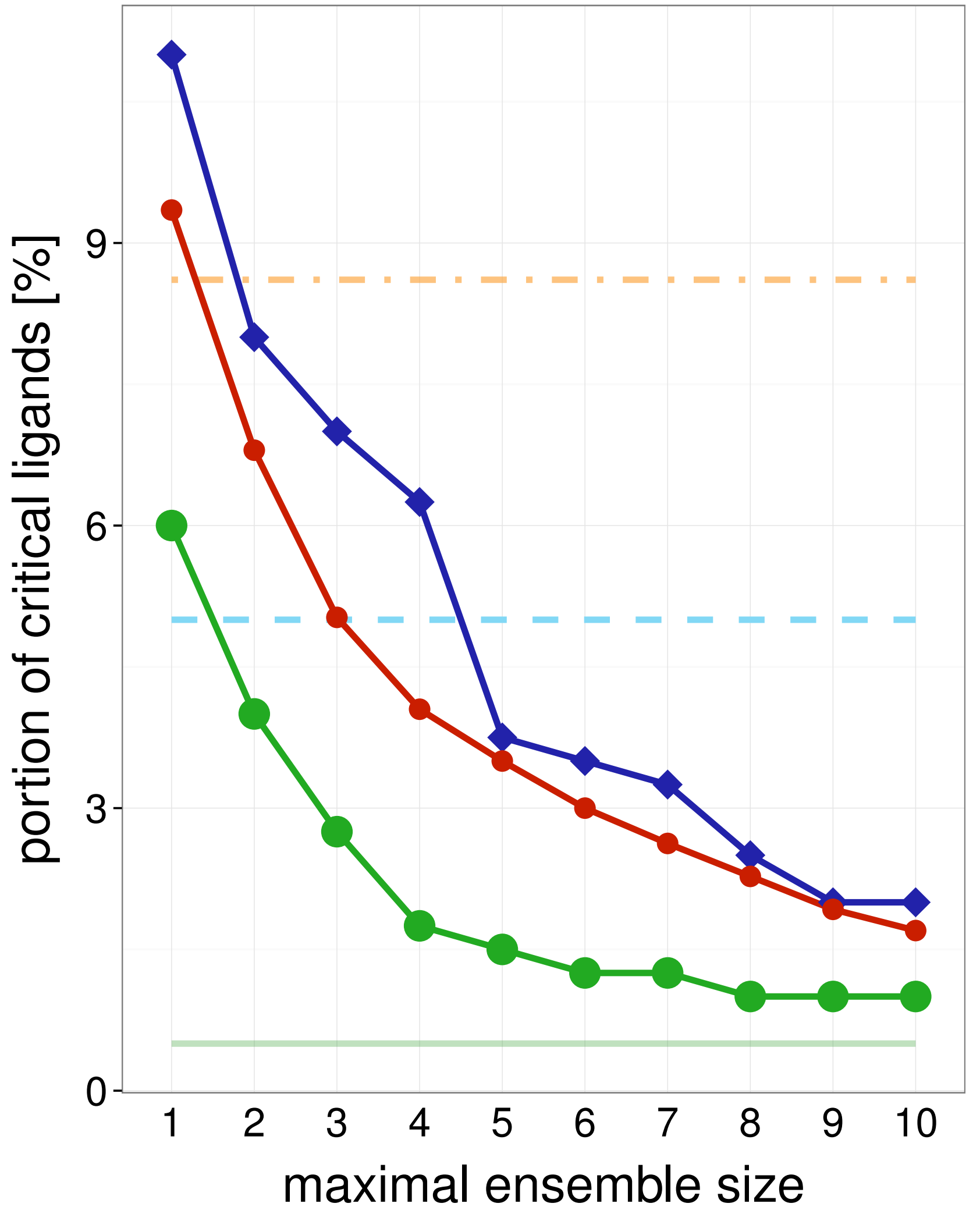

4LM0

Training set

Average single structure

_ Best single

structure

Whole ensemble

- SIENA

$\multimap$ Clustering

$\multimap$ Random

Average single structure

\section{_ Best single} structure

Whole ensemble

- SIENA

$\smile$ Clustering

$\multimap$ Random

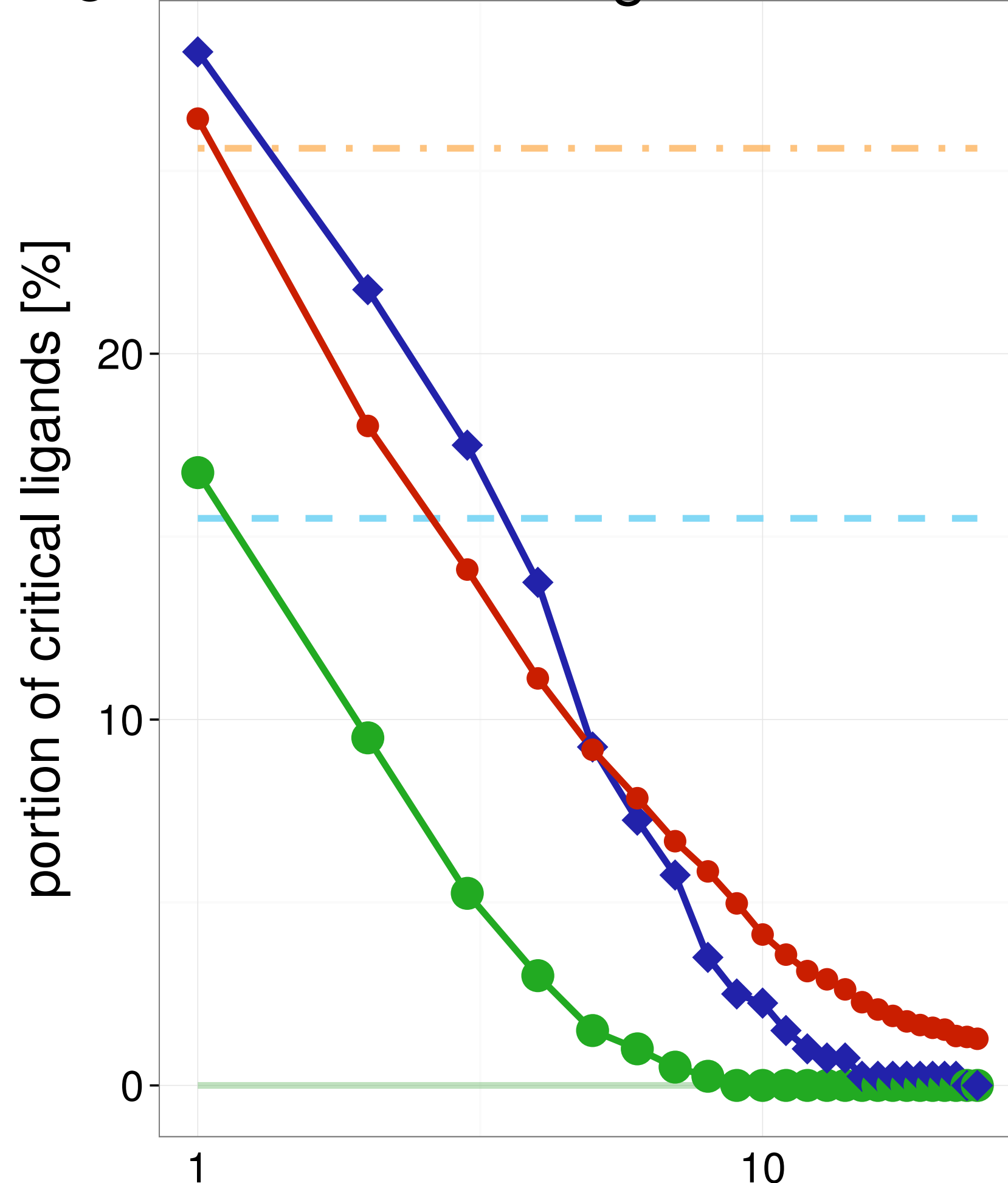

maximal ensemble size

Test set

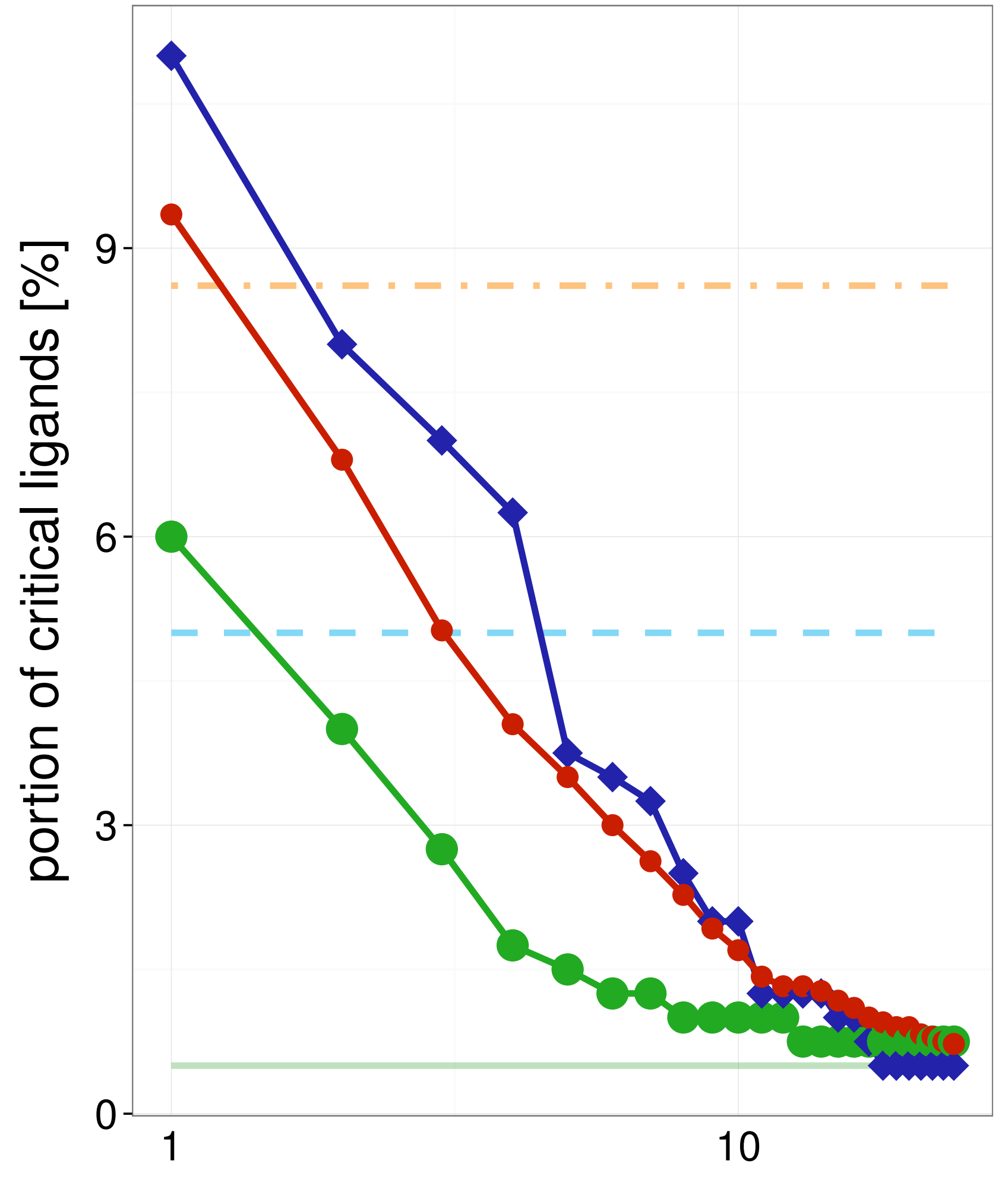

maximal ensemble size
Average single structure

_ Best single structure

Whole ensemble

- SIENA

$\neg$ Clustering

$\multimap$ Random 


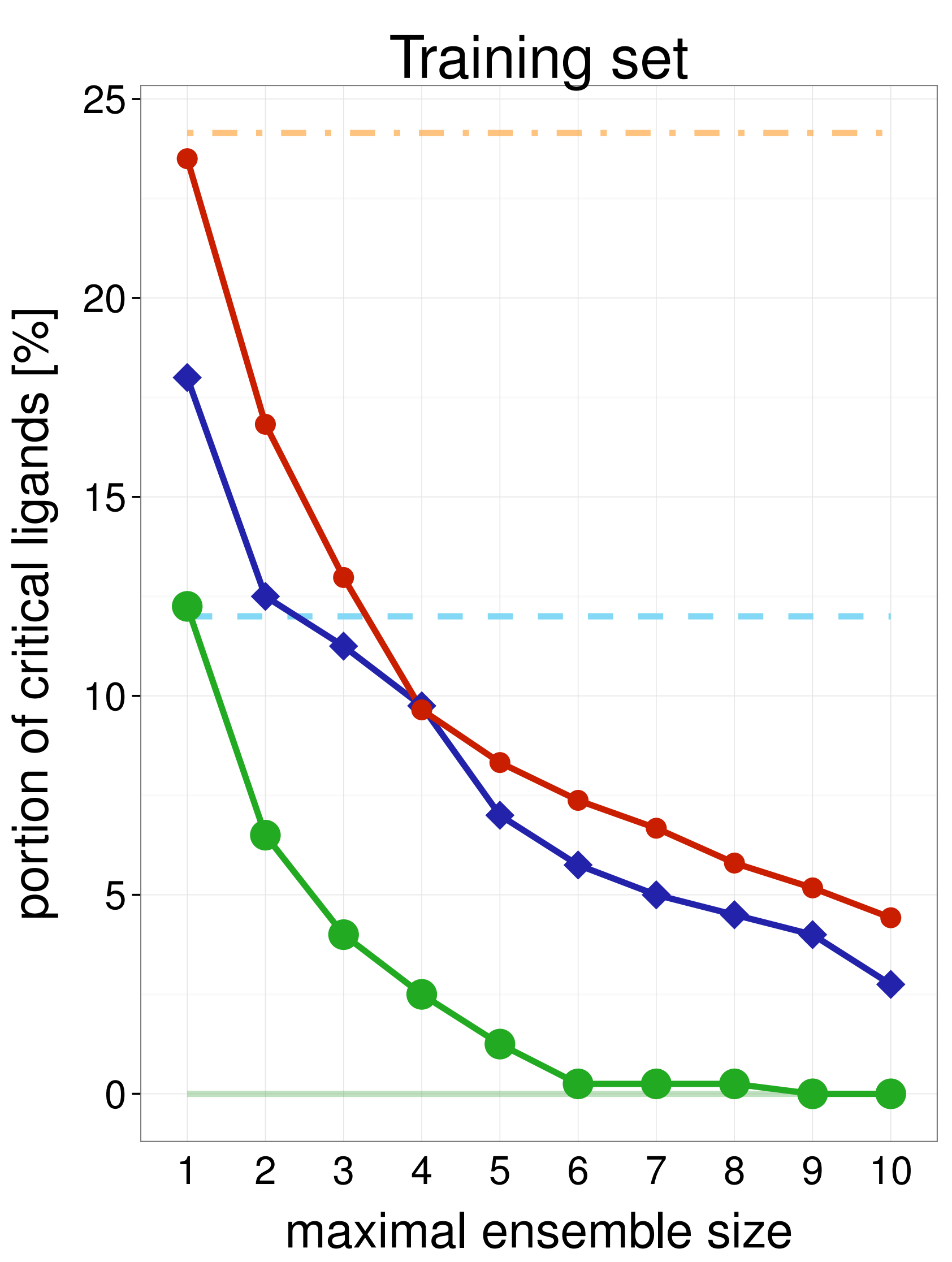

\section{Test set}

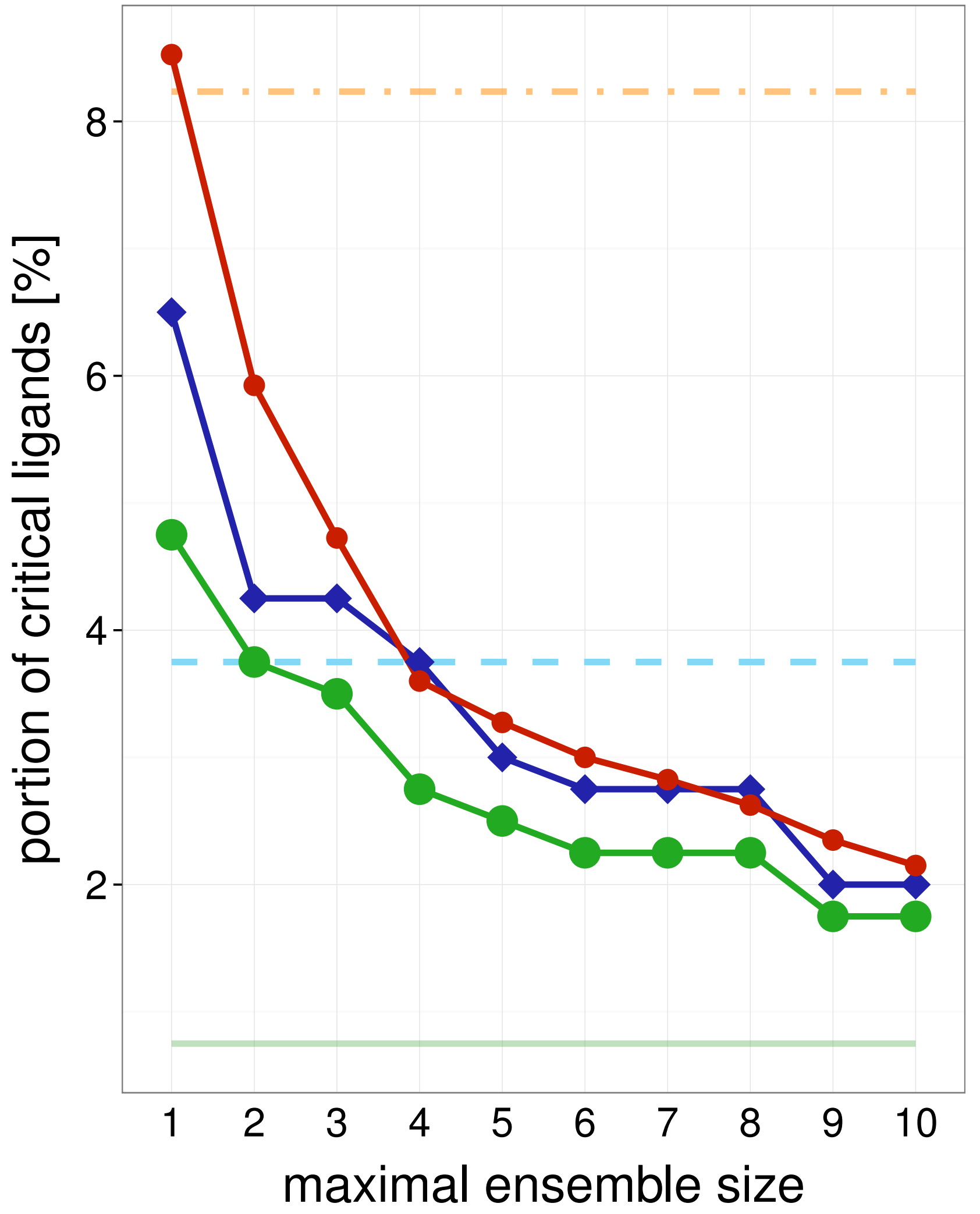

$1 \mathrm{EKO}_{25} \quad$ Training set

Average single structure

_ _ Best single structure

Whole ensemble

- SIENA

$\neg$ Clustering

$\rightarrow$ Random

Average single structure

_ _ Best single structure

Whole ensemble

- SIENA

$\neg$ Clustering

$\multimap$ Random

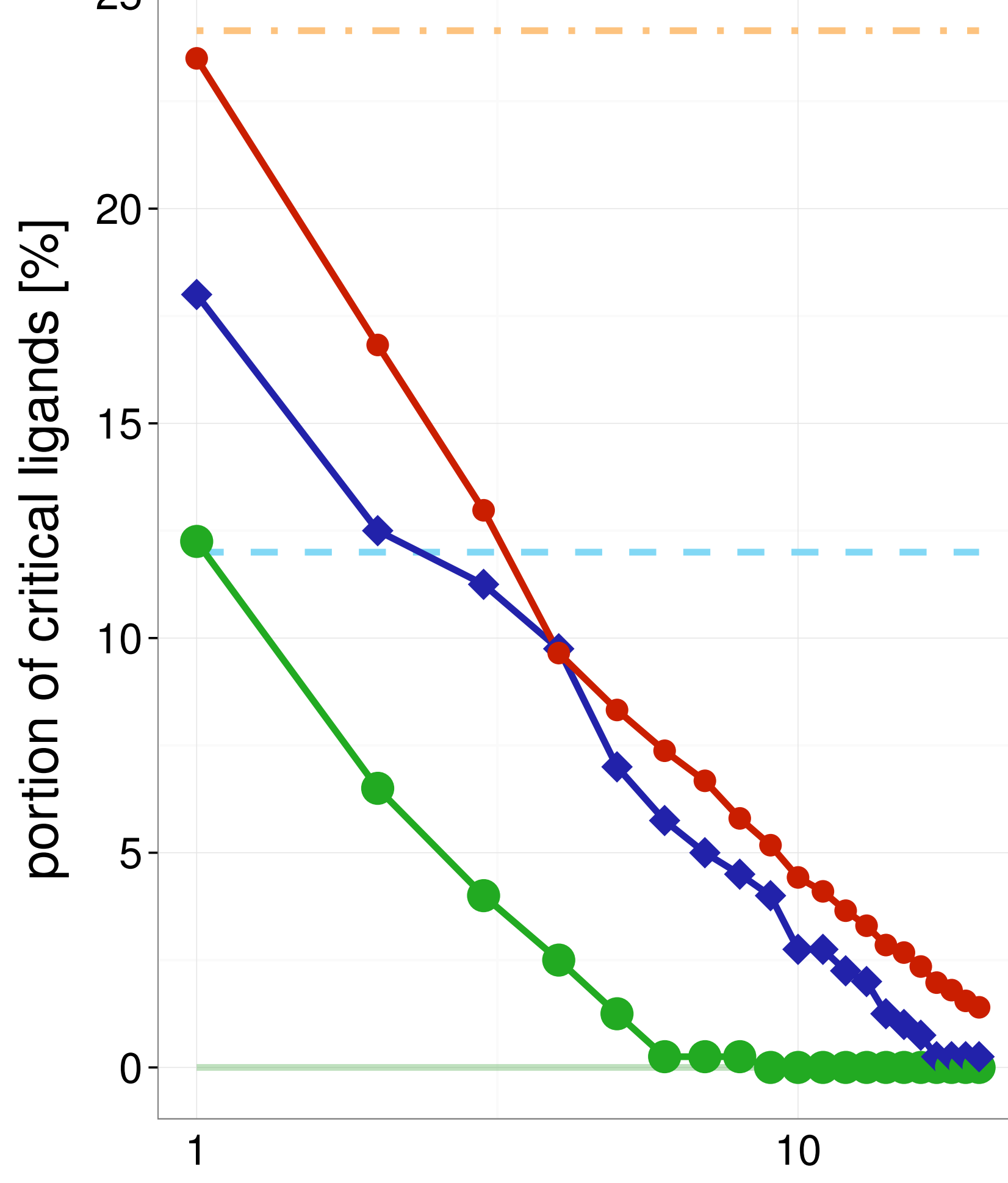

maximal ensemble size

Test set

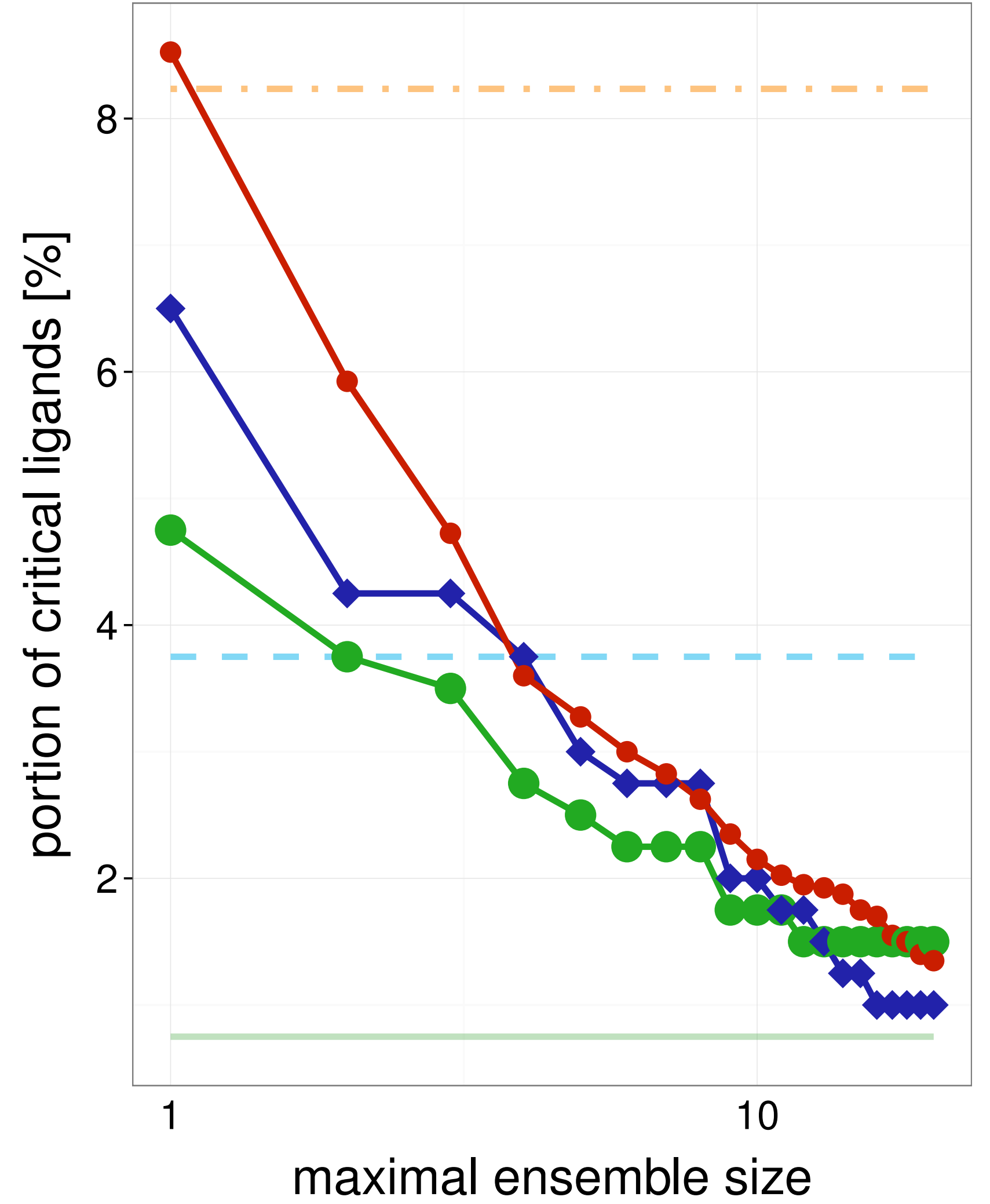

Average single structure

- Best single structure

Whole ensemble

- SIENA

$\neg$ Clustering

$\rightarrow$ Random

Average single structure

_ Best single structure

Whole ensemble

SIENA

$\neg$ Clustering

$\multimap$ Random 
Training set

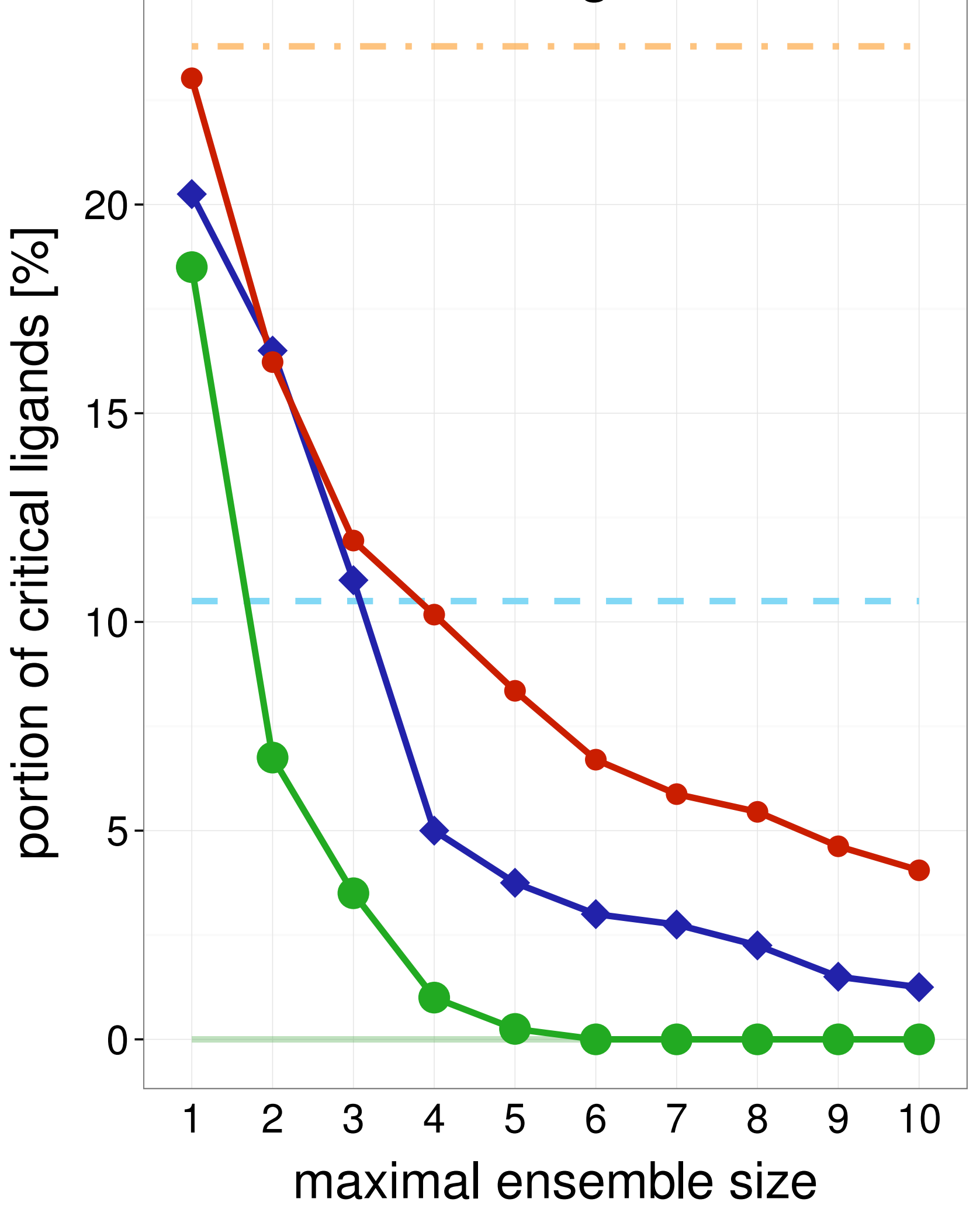

Test set

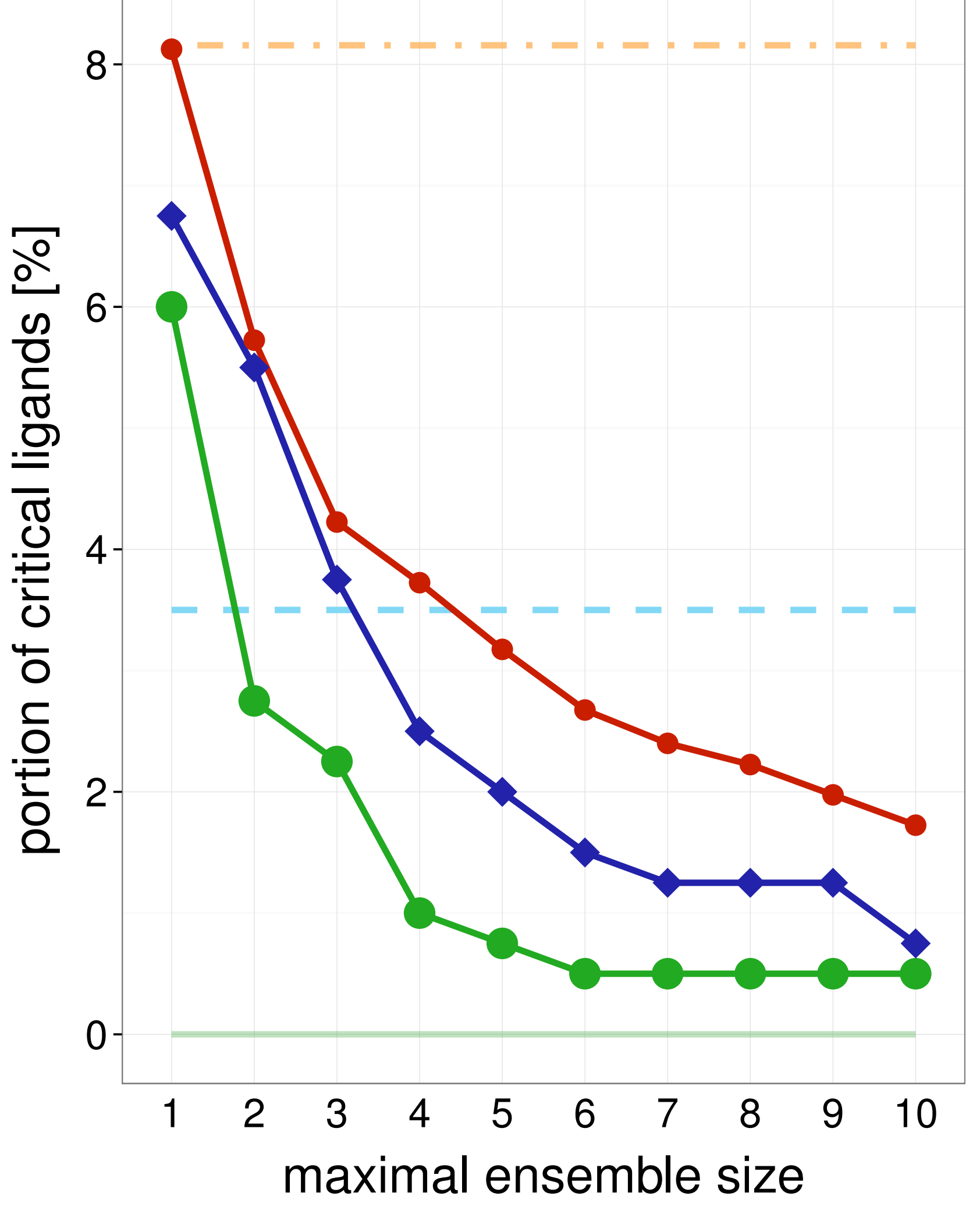

$1 \mathrm{~K} 4 \mathrm{G}$

\section{Training set}

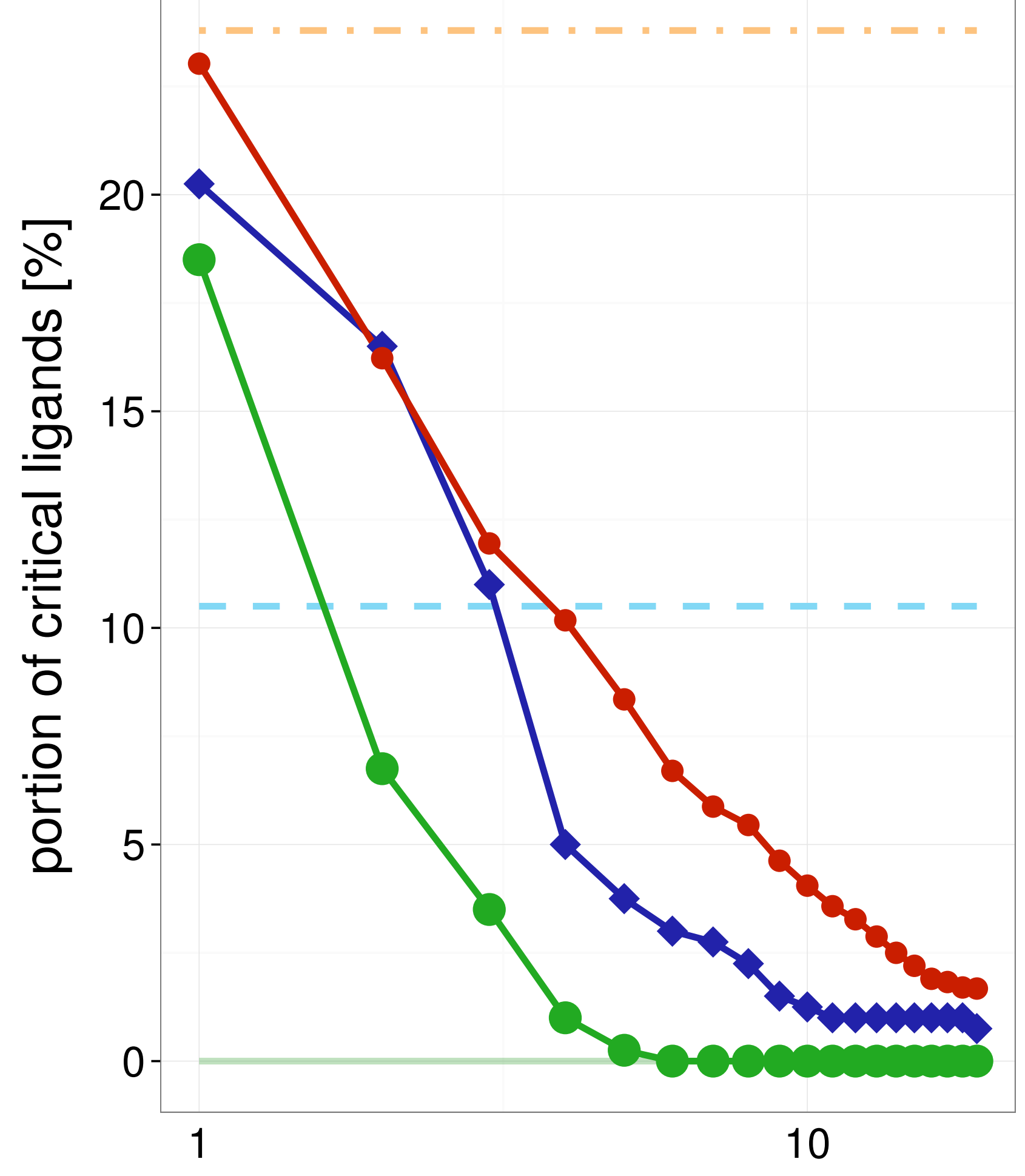

maximal ensemble size

\section{Test set}

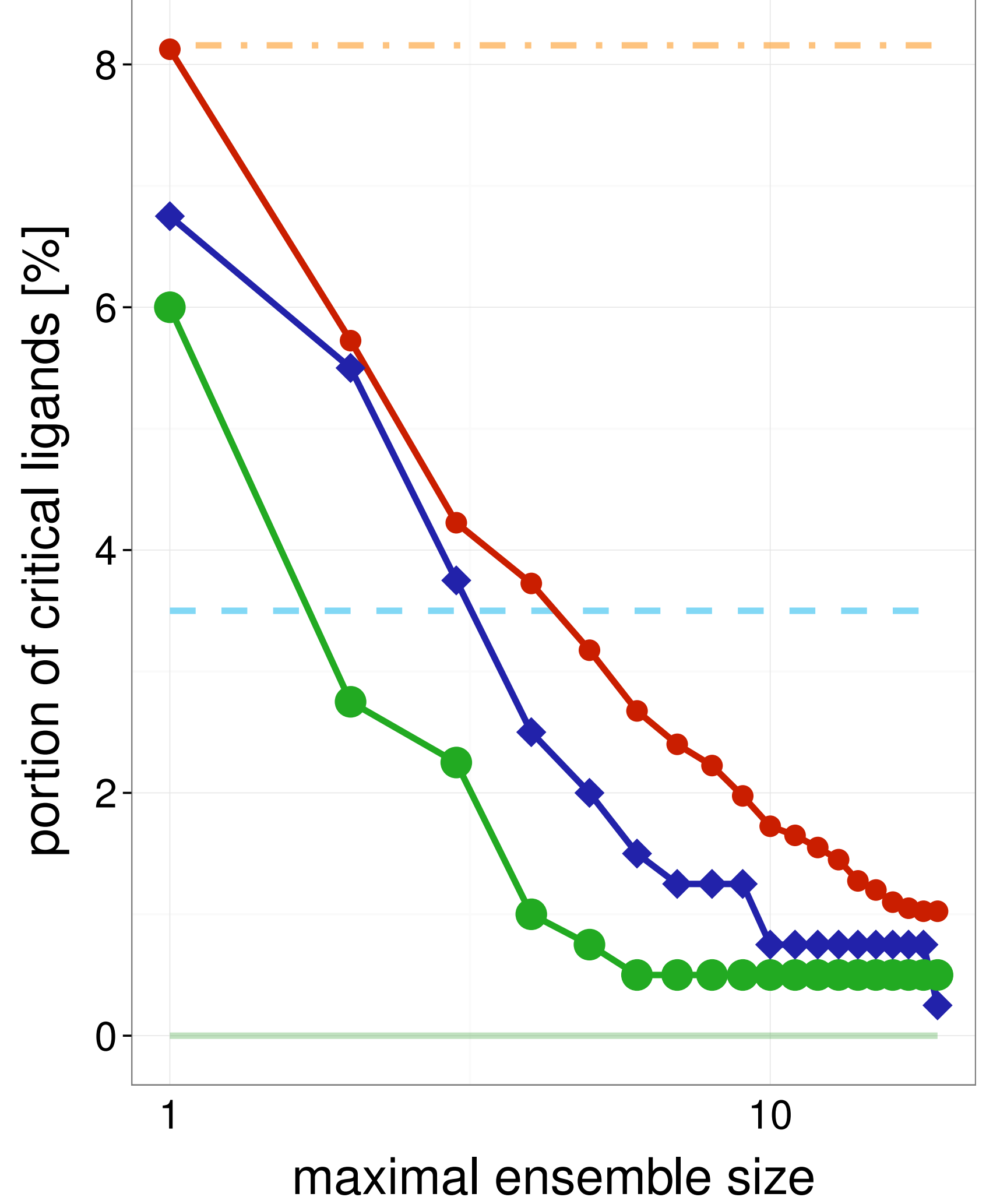

maximal ensemble size
Average single structure

- Best single structure

Whole ensemble

- SIENA

$\checkmark$ Clustering

$\multimap$ Random

Average single structure

Average single structure

_ Best single structure

Whole ensemble

- SIENA

$\smile$ Clustering

- Random
Best single structure

Whole ensemble

- SIENA

$\neg$ Clustering

$\multimap$ Random 


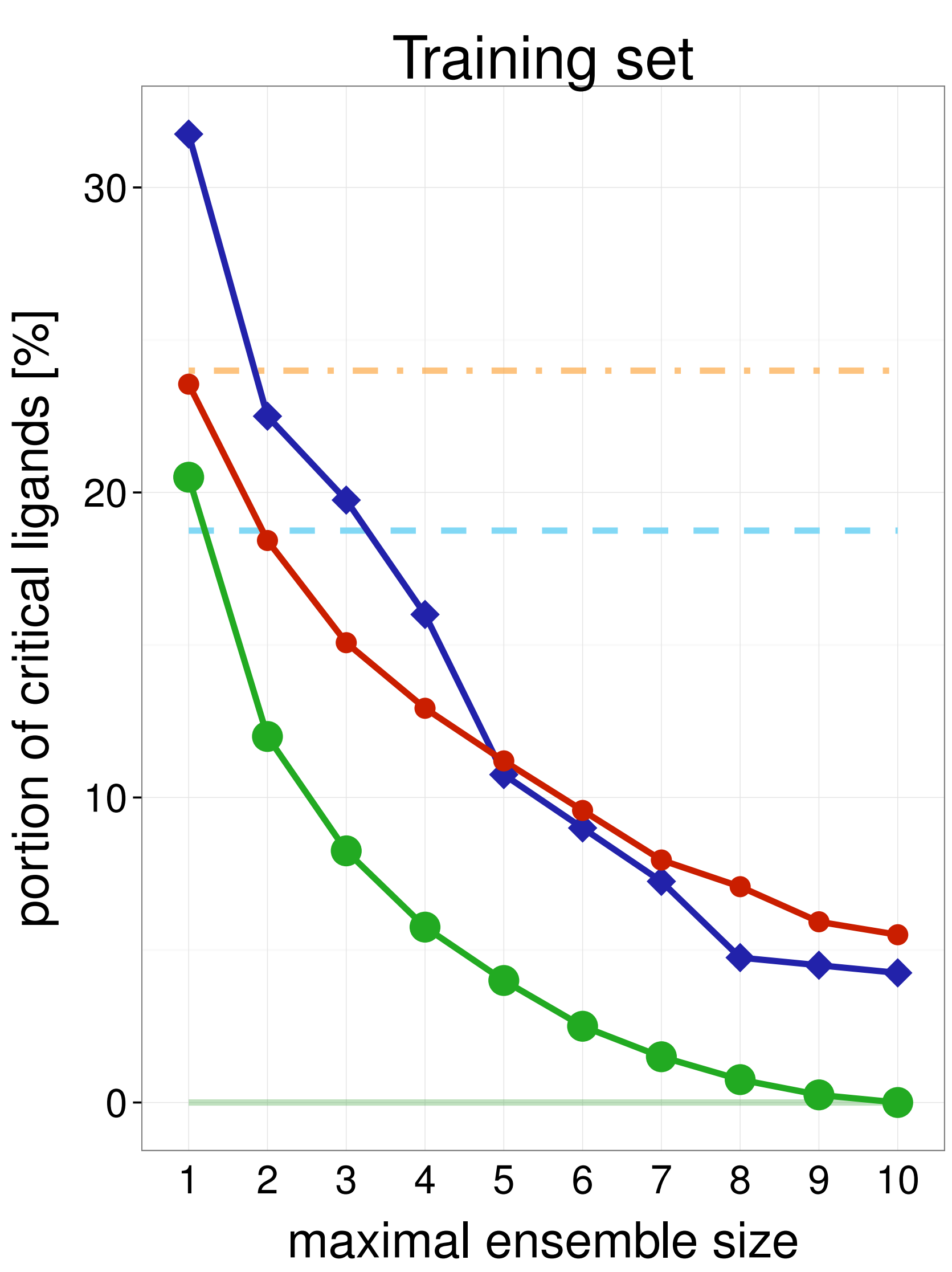

\section{Test set}

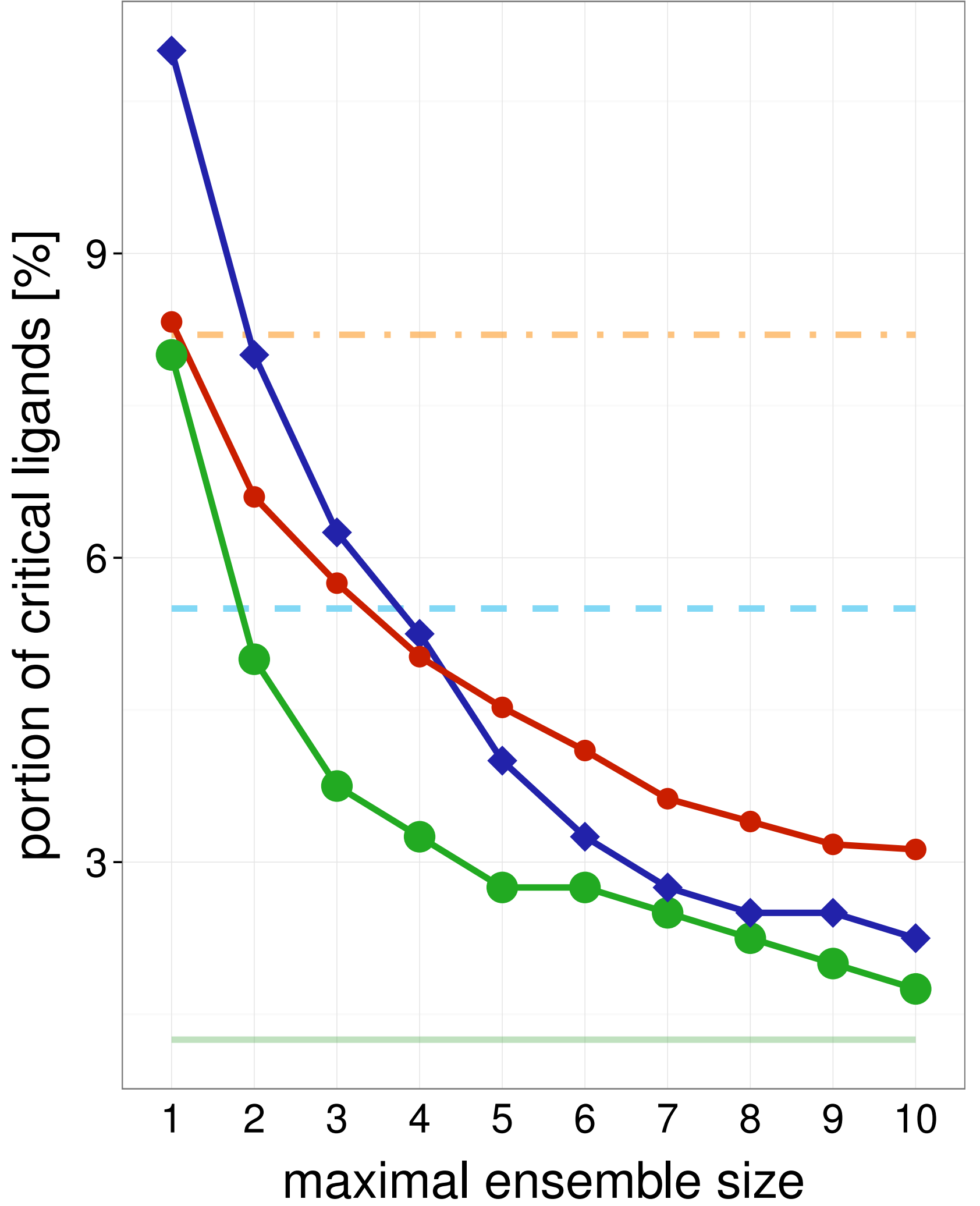

Average single structure

_ _ Best single structure

Whole ensemble

- SIENA

$\neg$ Clustering

$\longrightarrow$ Random

Average single structure

_ Best single structure

Whole ensemble

- SIENA

$\neg$ Clustering

$\because$ Random
Training set

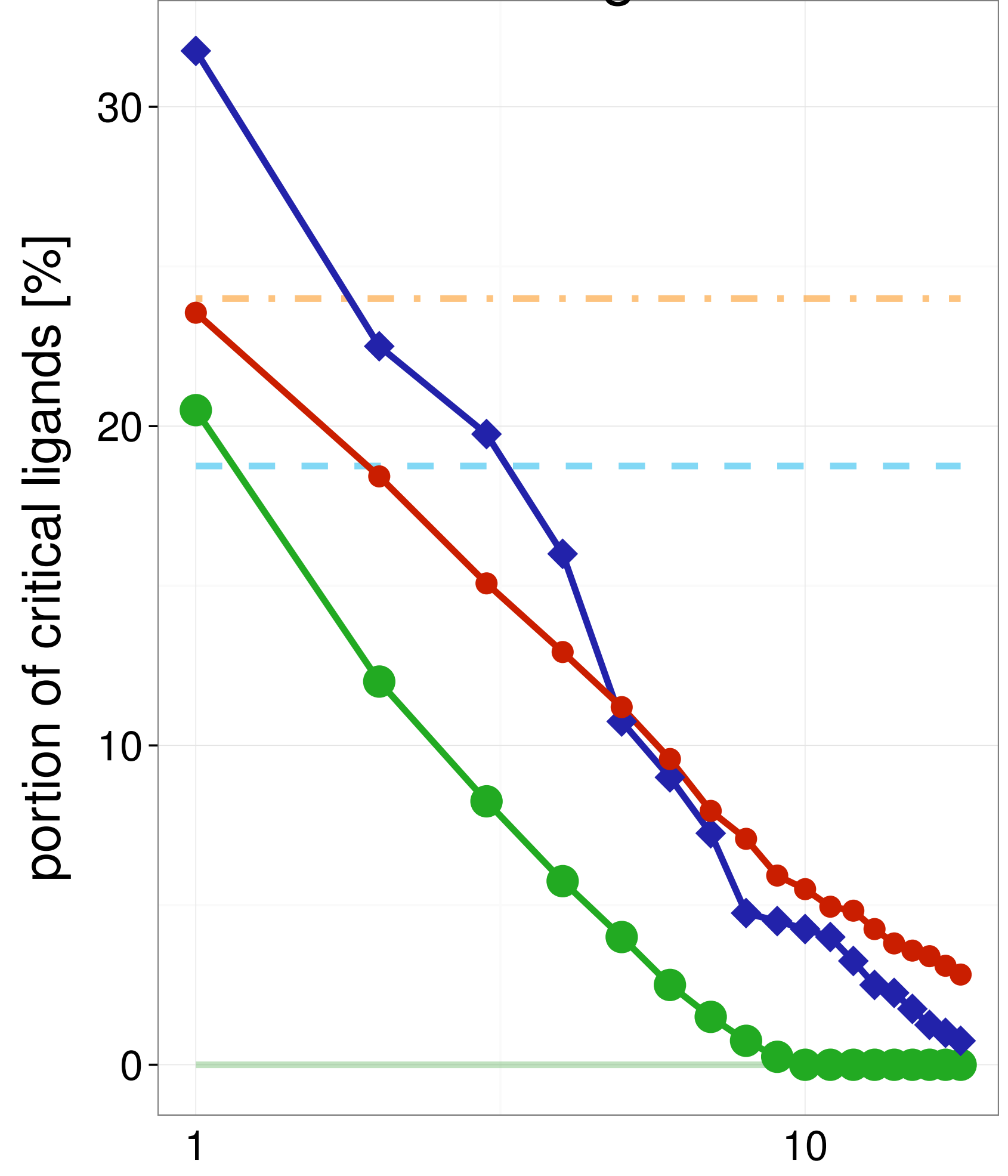

maximal ensemble size

Test set

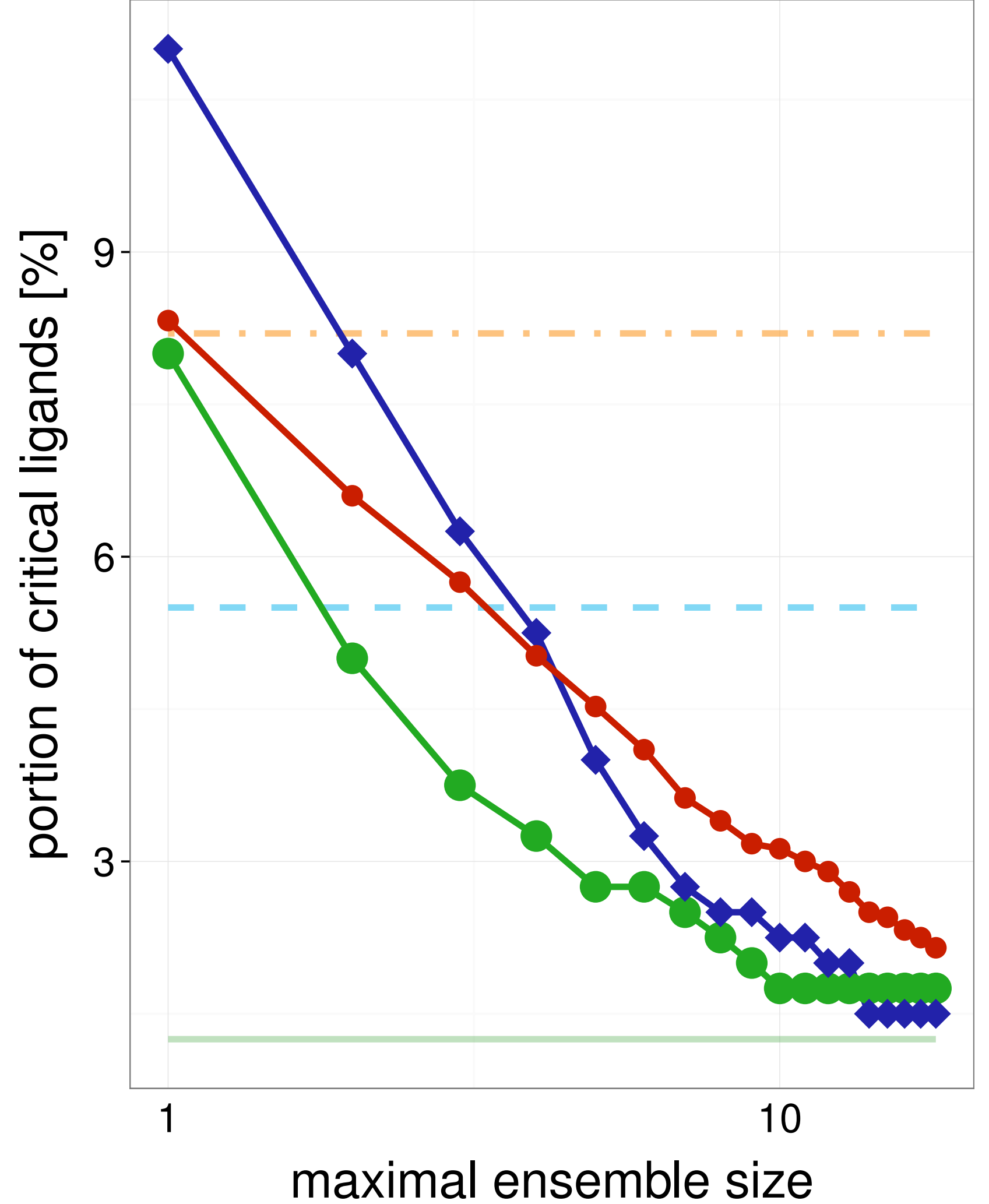

Average single structure

Best single structure

Whole ensemble

- SIENA

$\neg$ Clustering

$\rightarrow$ Random

Average single structure

Best single structure

Whole ensemble

- SIENA

$\neg$ Clustering

$\multimap$ Random 


\section{Training set}

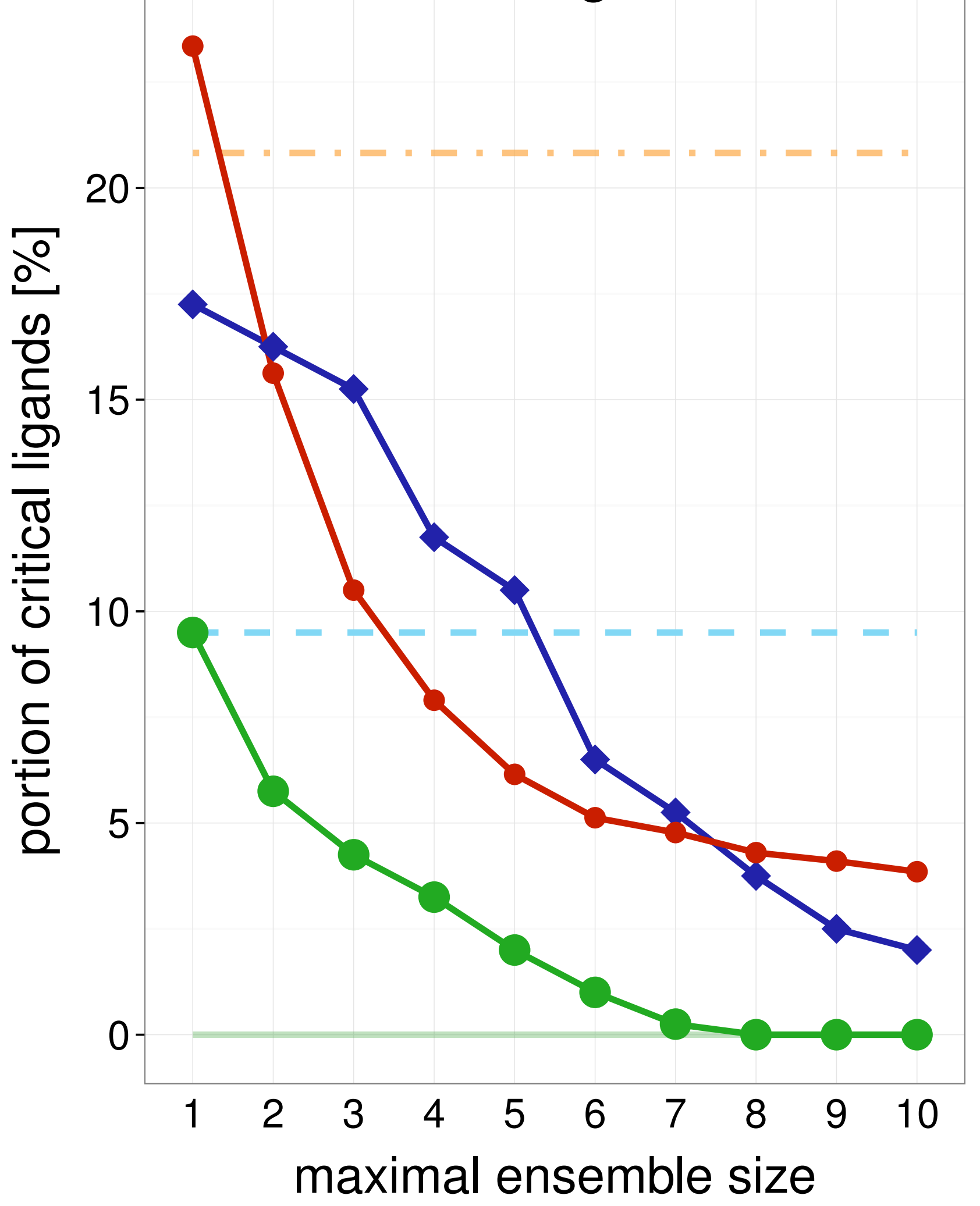

Test set

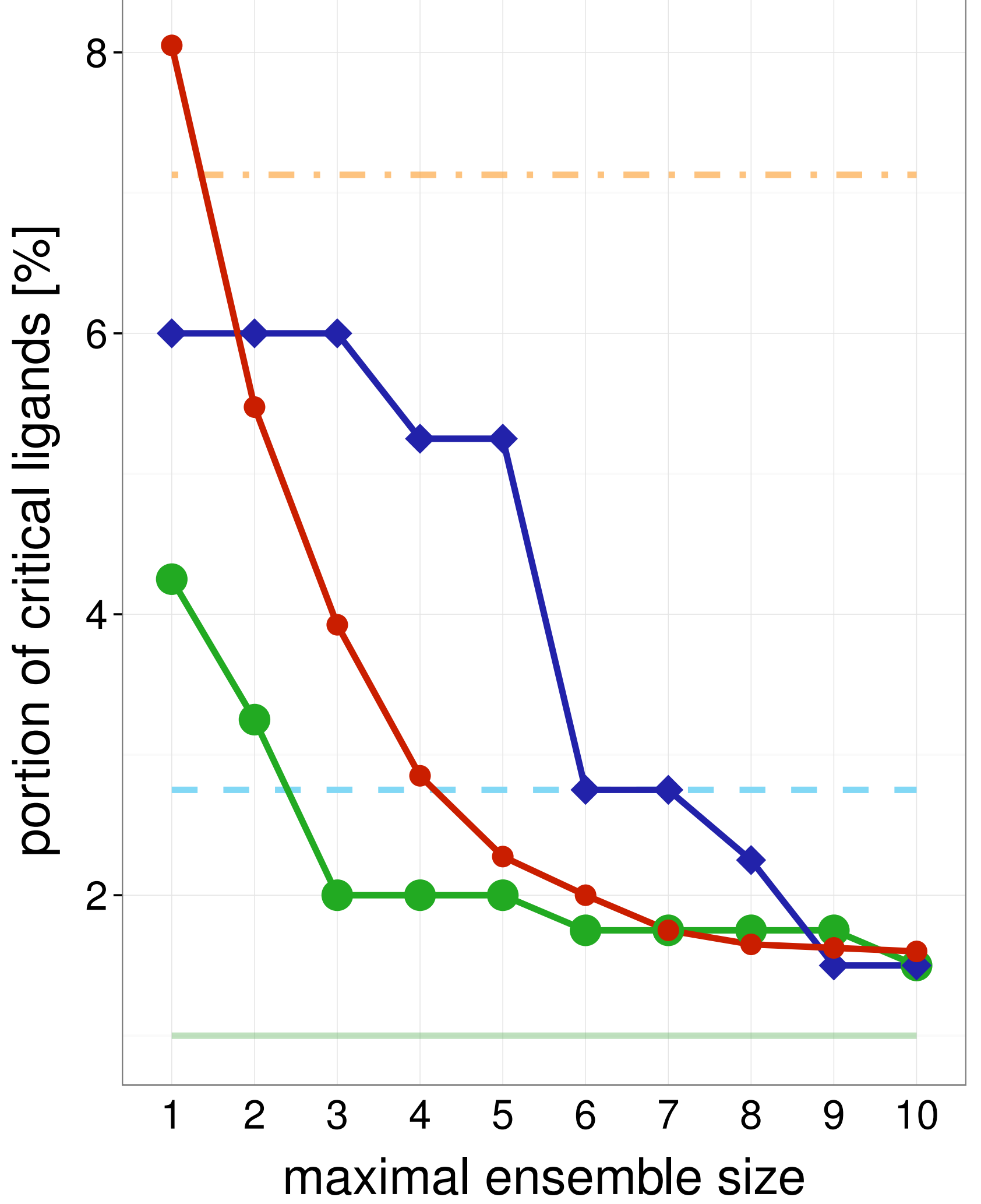

2JC0

Training set

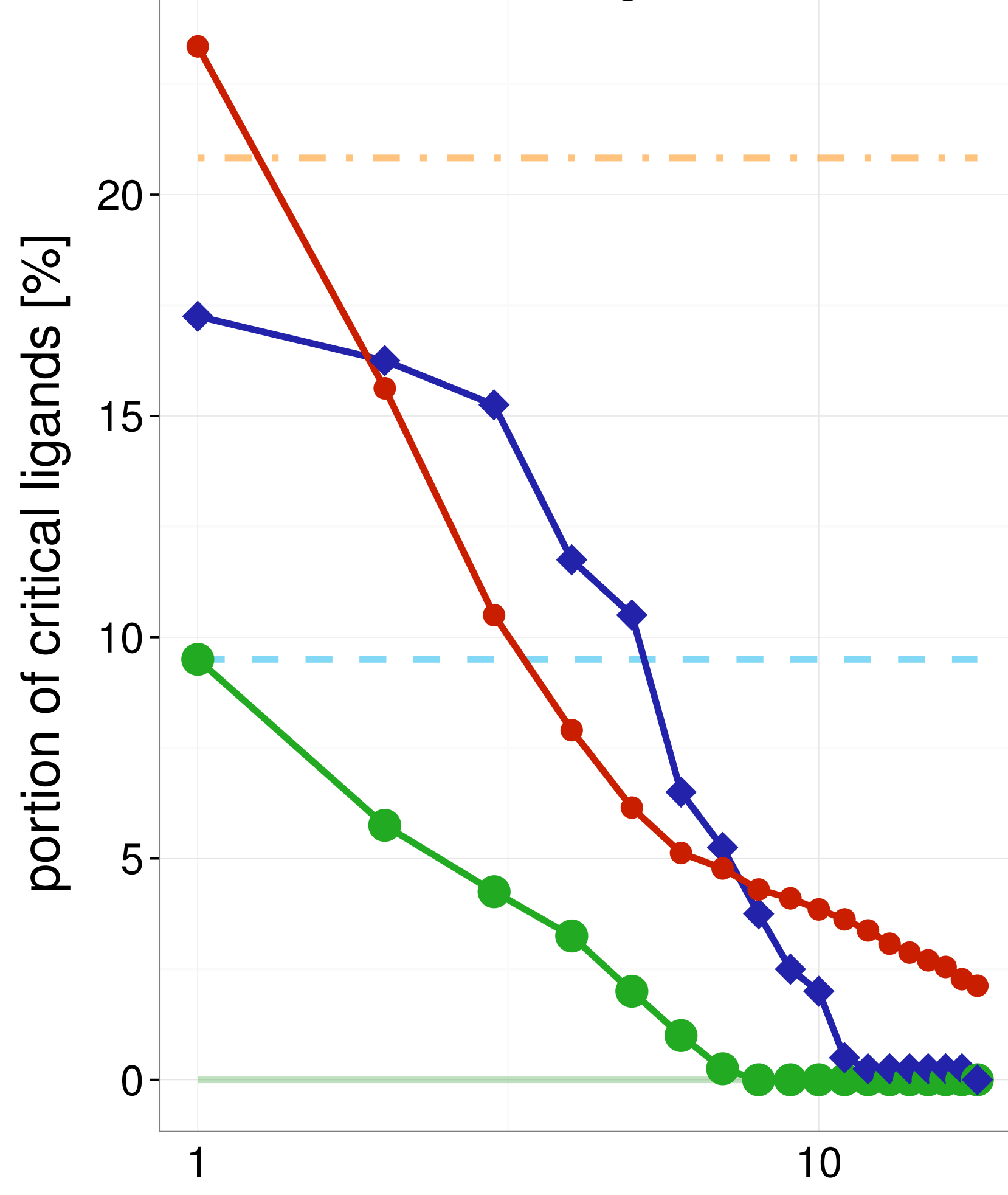

maximal ensemble size

\section{Test set}

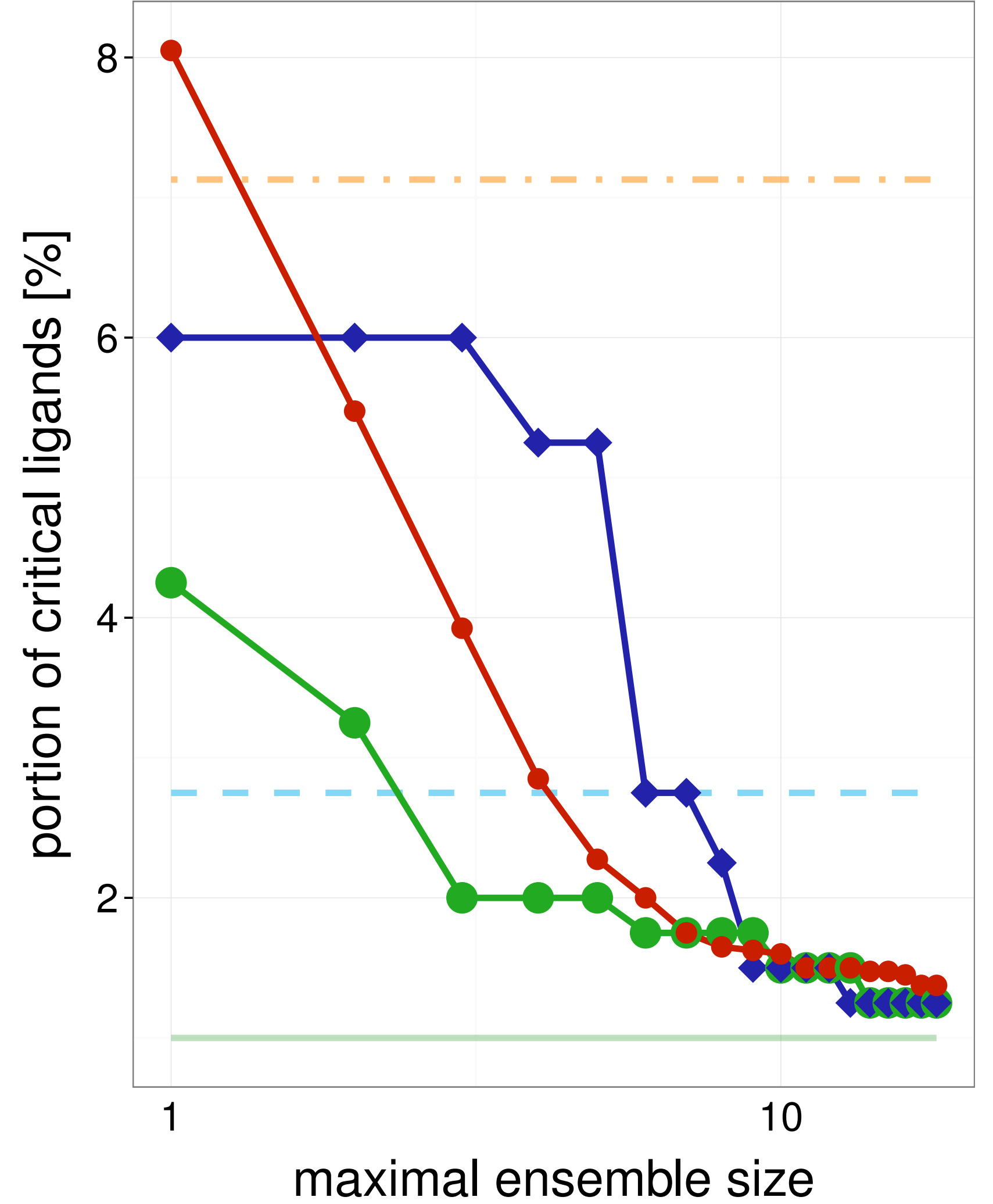

Average single structure

- Best single structure

Whole ensemble

- SIENA

$\smile$ Clustering

$\multimap$ Random

Average single structure

Average single structure

_ Best single structure

Whole ensemble

- SIENA

$\neg$ Clustering

- Random
Best single structure

Whole ensemble

- SIENA

$\neg$ Clustering

$\multimap$ Random 


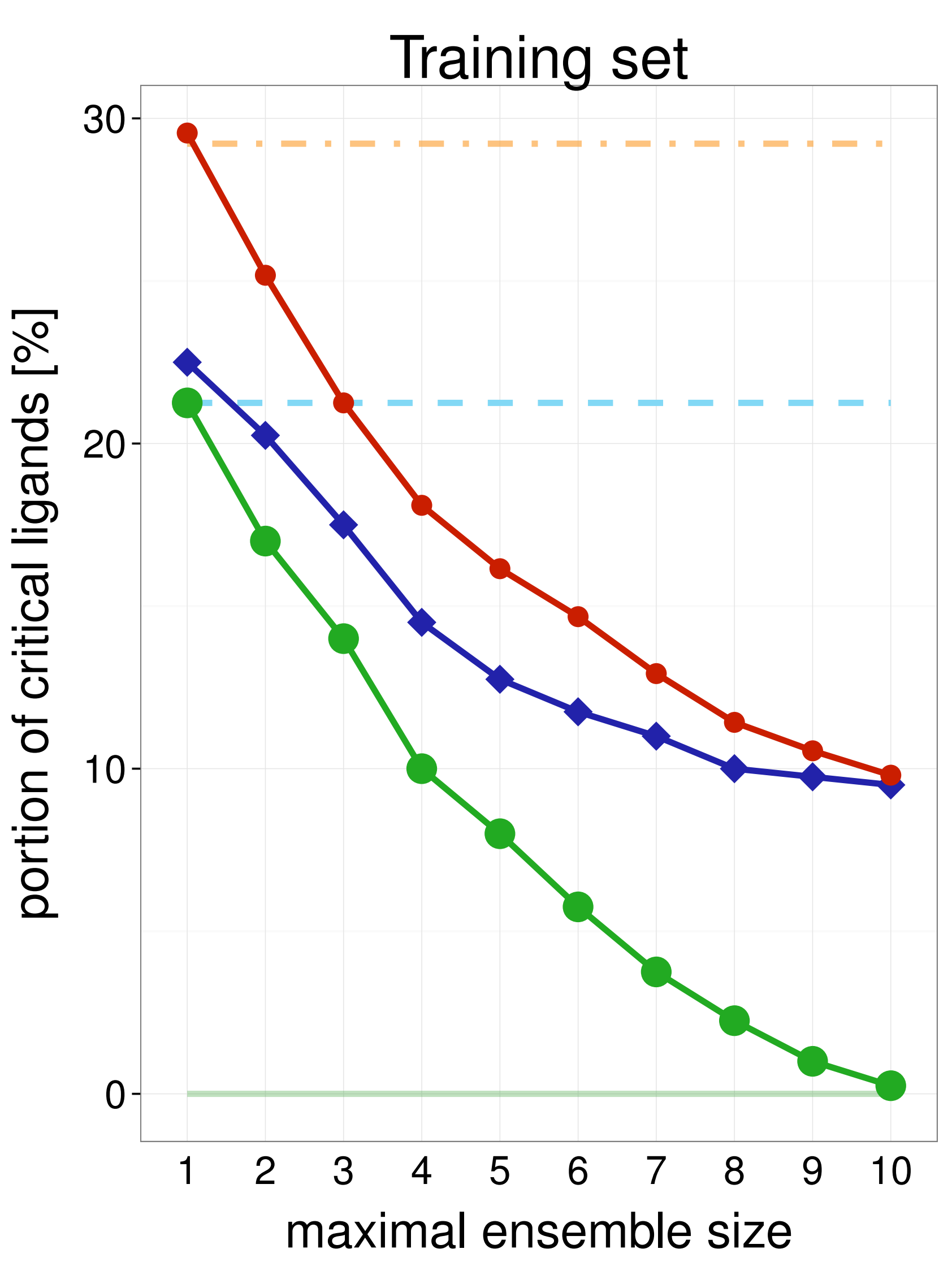

Test set

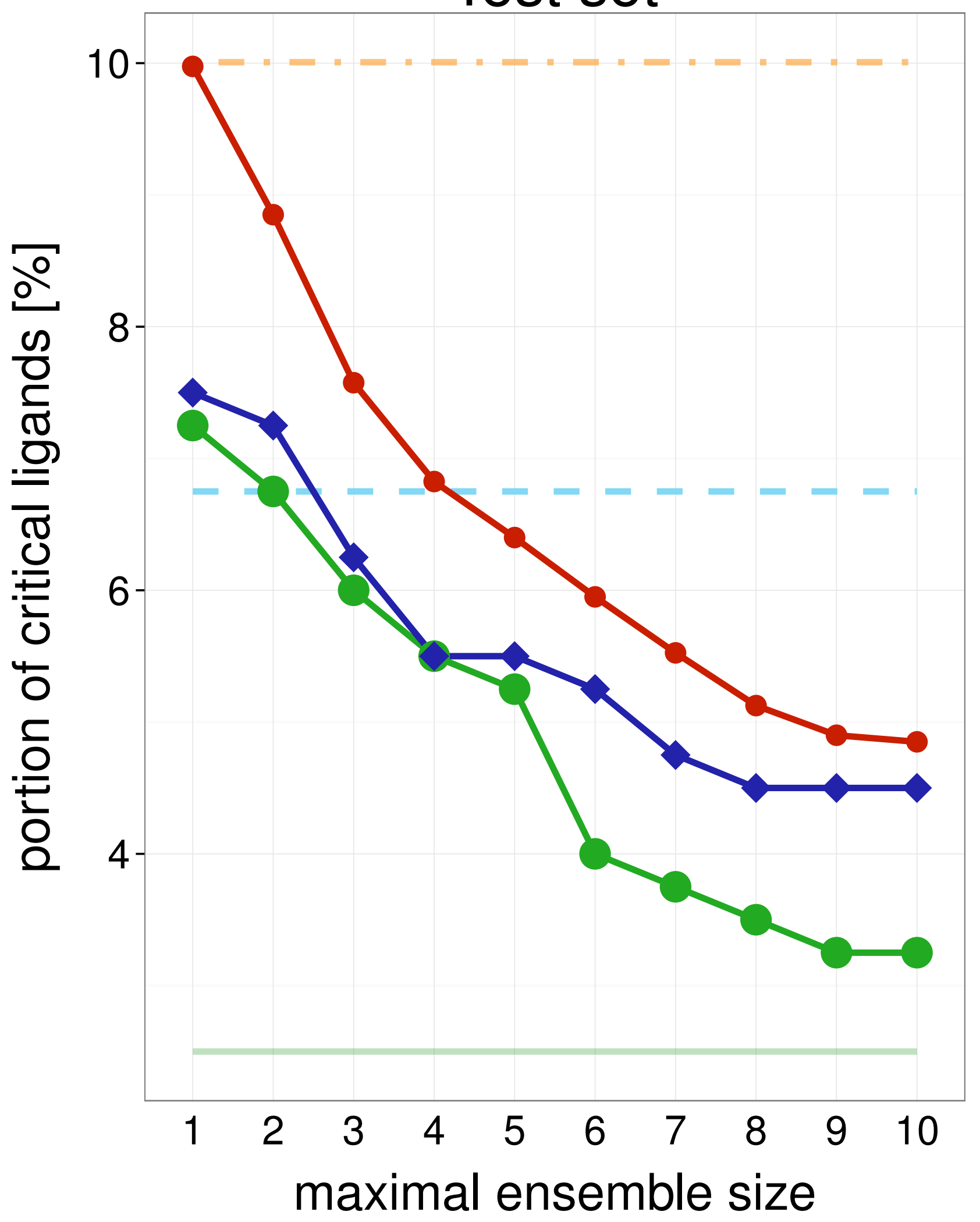

Average single structure

_ Best single structure

Whole ensemble

- SIENA

$\neg$ Clustering

$\multimap$ Random

Average single structure

_ Best single structure

Whole ensemble

- SIENA

$\neg$ Clustering

$\multimap$ Random
2RFN

Training set

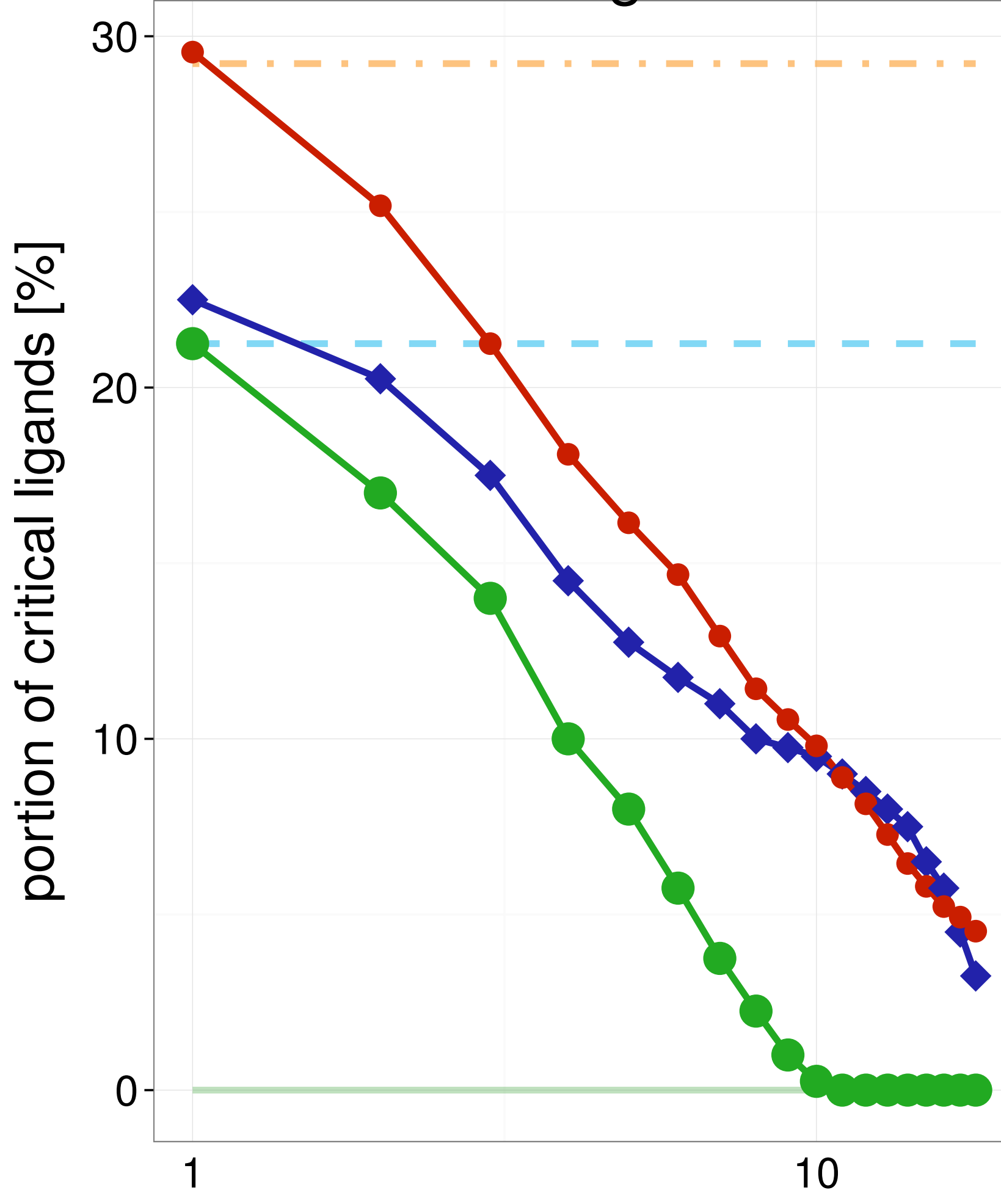

maximal ensemble size

\section{Test set}

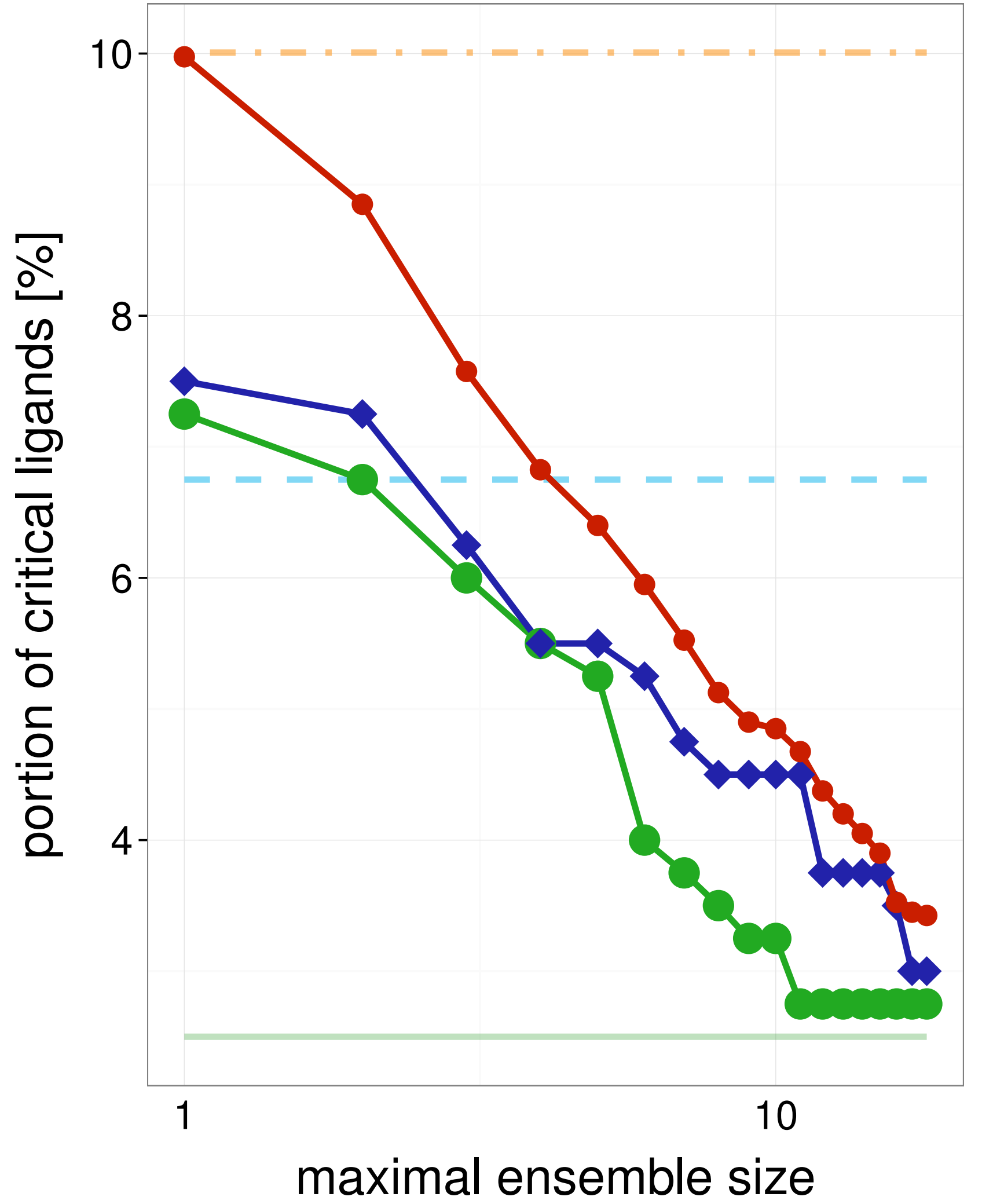

Average single structure

Best single structure

Whole ensemble

- - SIENA

$\checkmark$ Clustering

$\multimap$ Random

Average single structure

_ _ Best single structure

Whole ensemble

SIENA

$\neg$ Clustering

$\multimap$ Random 


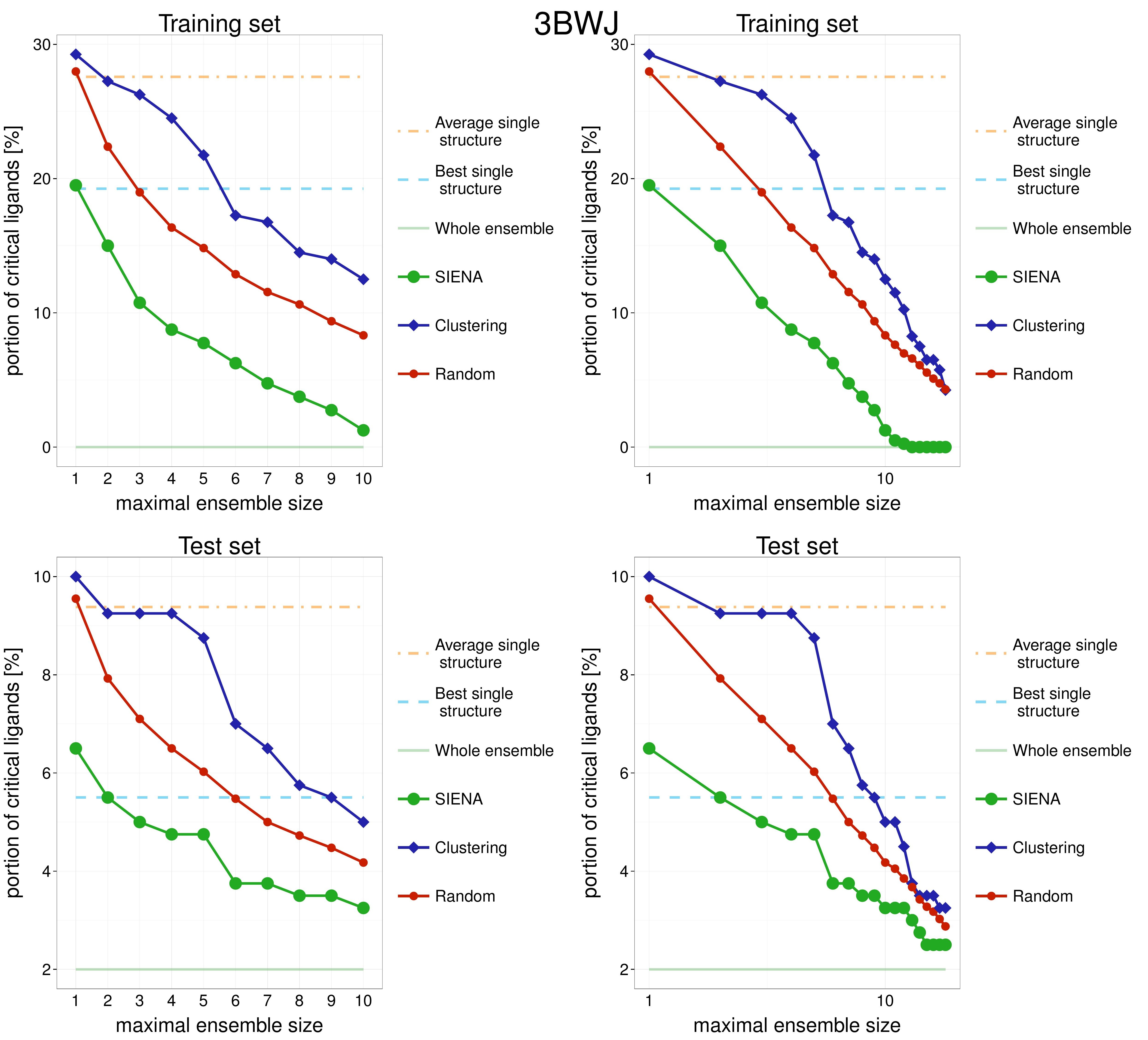


Training set

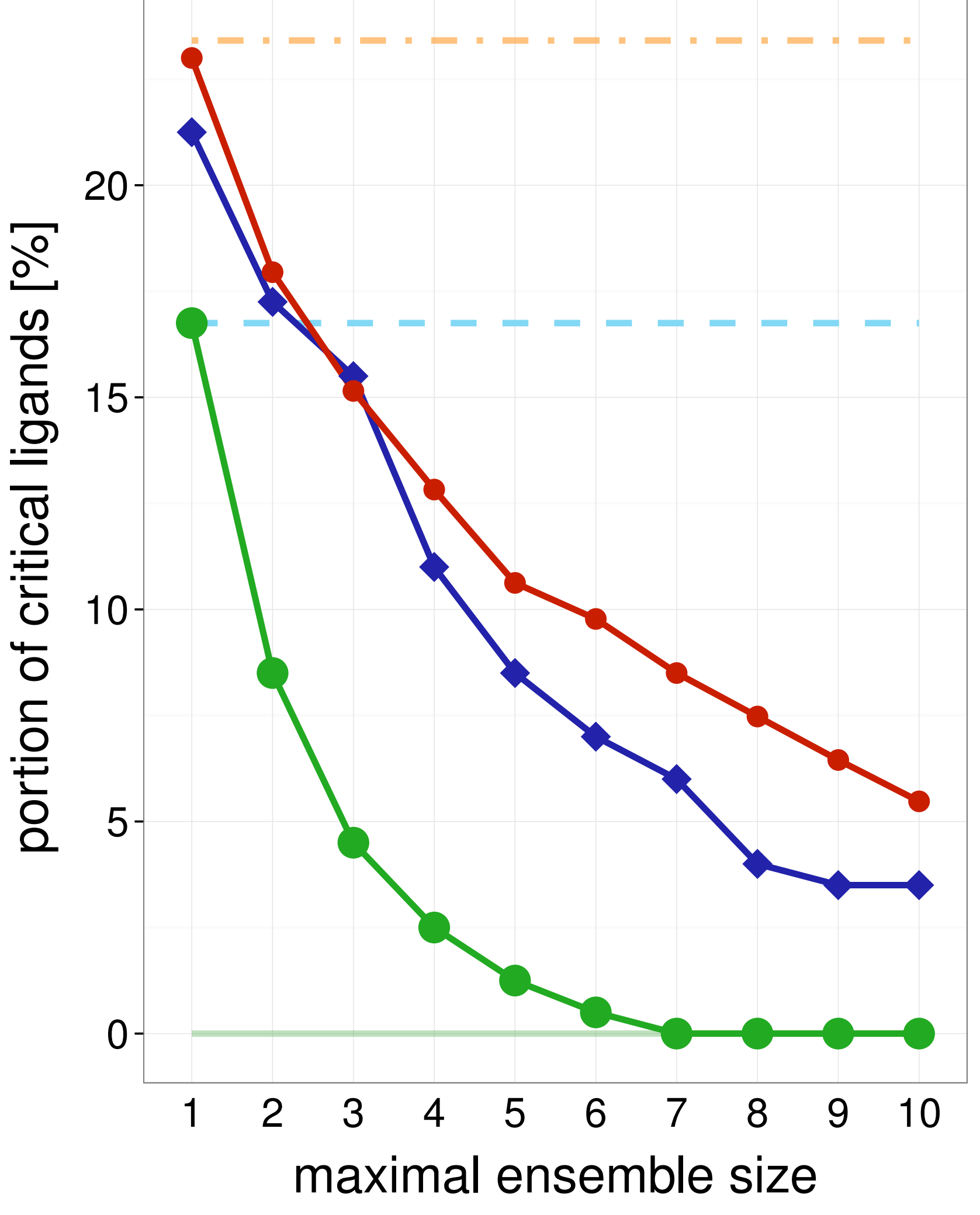

Test set

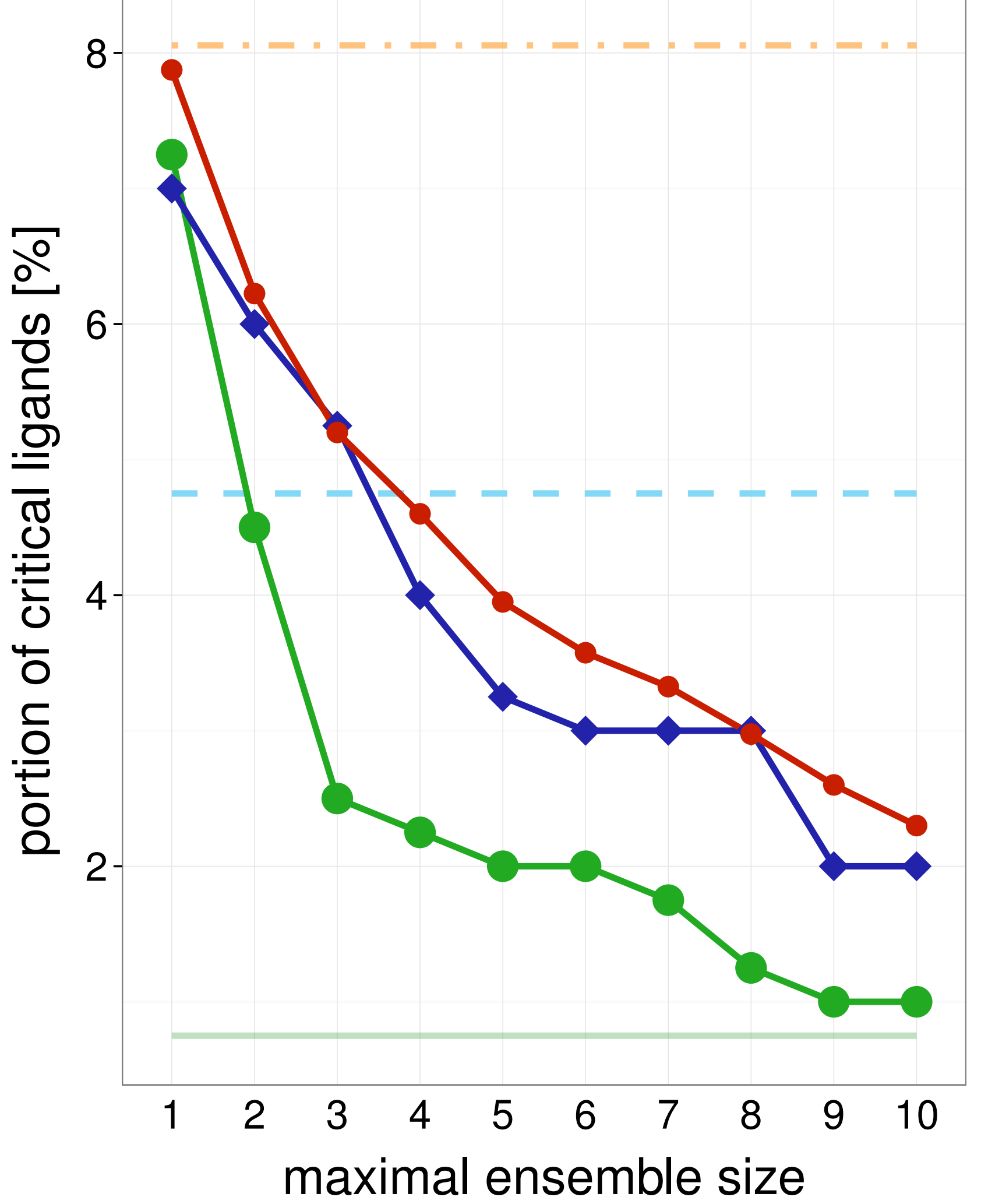

\section{Training set}

Average single structure

_ Best single structure

Whole ensemble

- SIENA

$\neg$ Clustering

$\multimap$ Random

Average single structure

_ _ Best single structure

Whole ensemble

- SIENA

$\leadsto$ Clustering

$\multimap$ Random

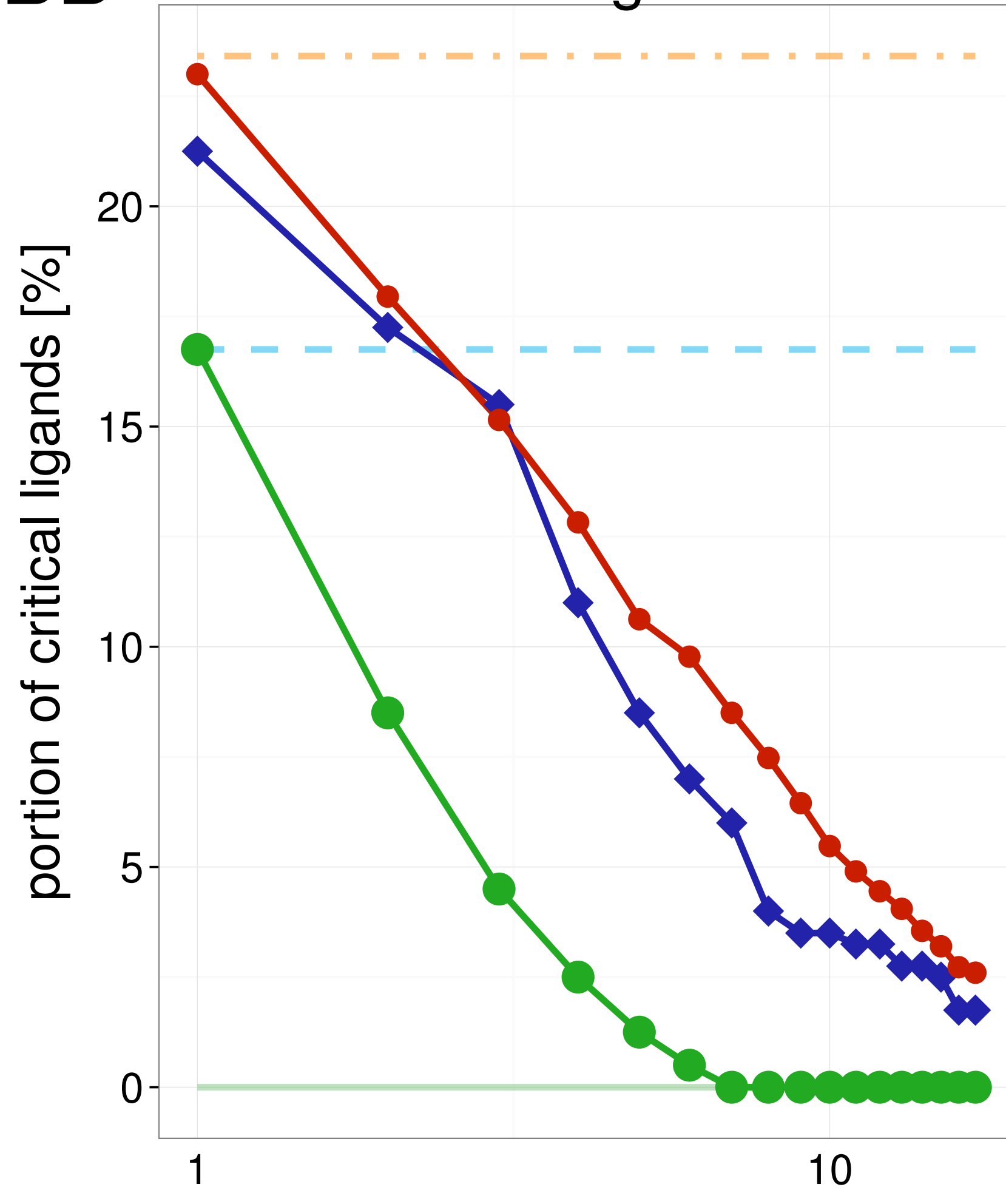

maximal ensemble size

\section{Test set}

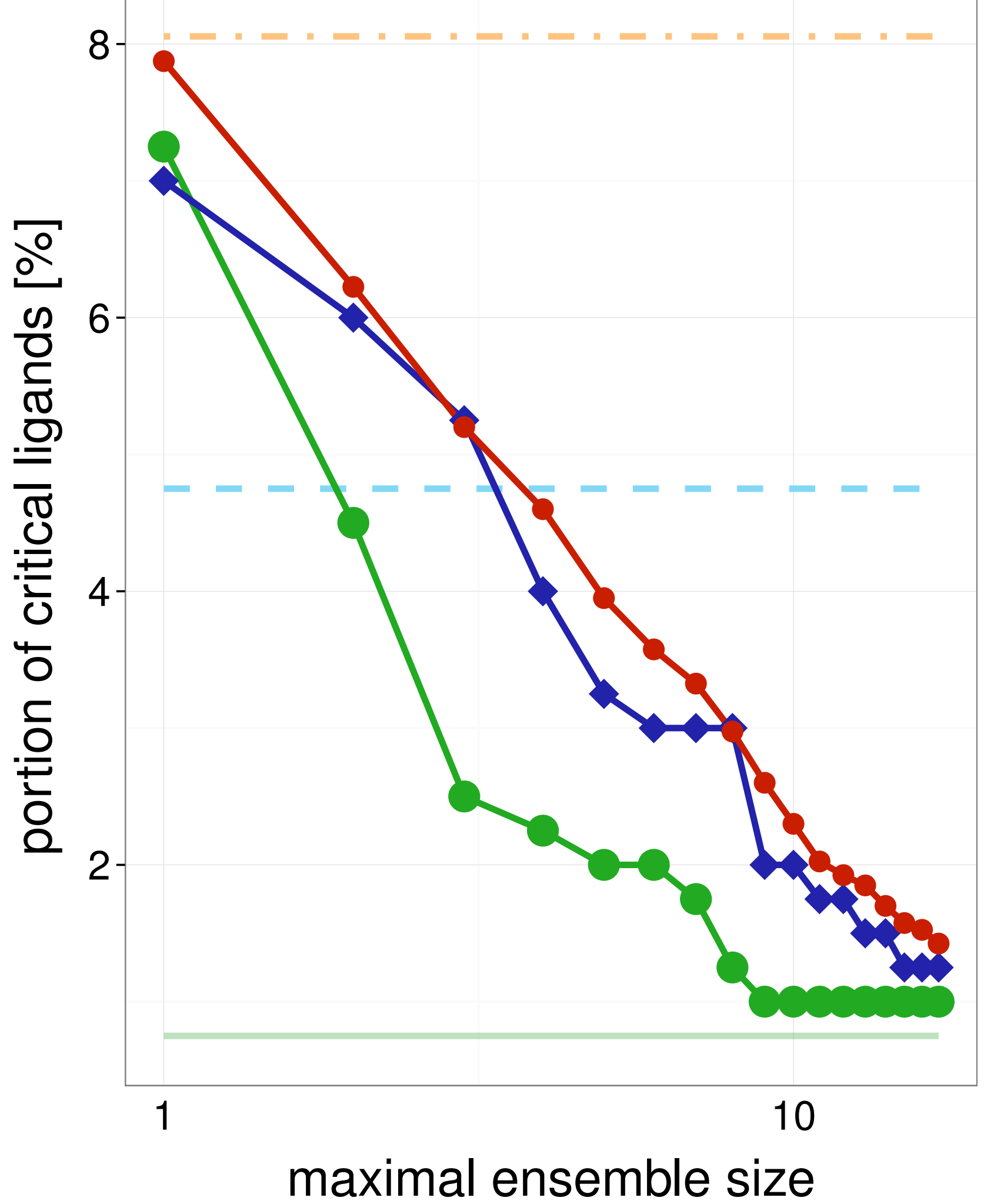

Average single structure

Best single structure

Whole ensemble

- SIENA

$\neg$ Clustering

$\multimap$ Random

Average single structure

_ Best single structure

Whole ensemble

- SIENA

$\neg$ Clustering

$\multimap$ Random 


\section{Training set}

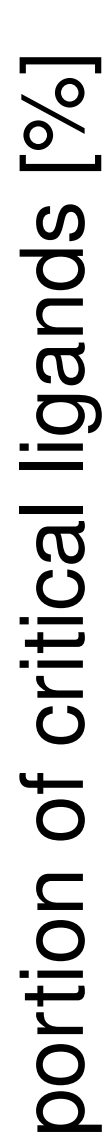

$0-$

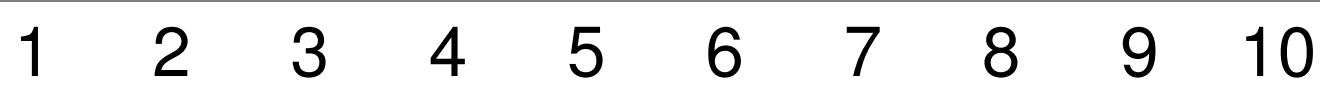

maximal ensemble size

Test set

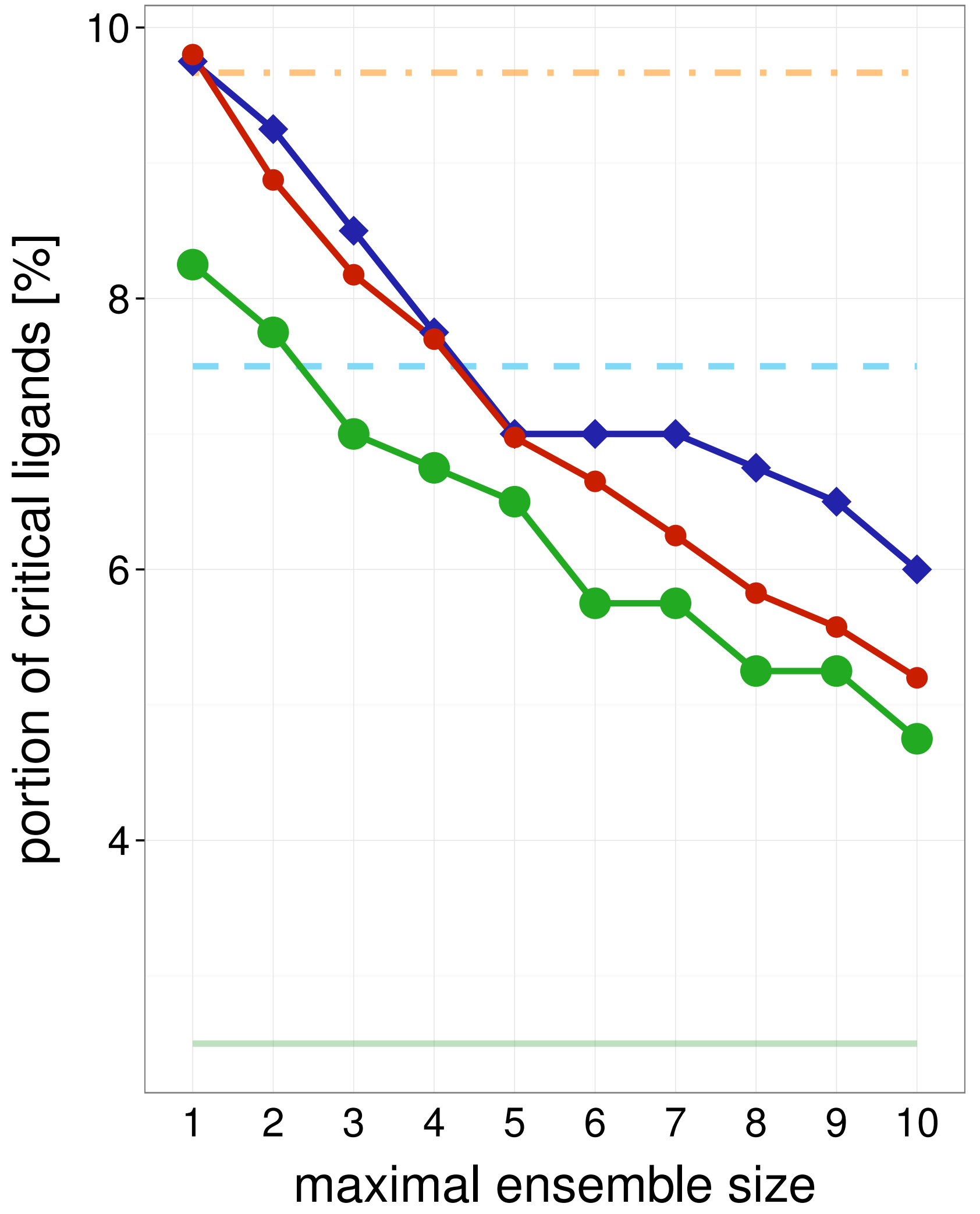

3SA0

Training set

Average single structure

_ Best single

structure

Whole ensemble

- SIENA

$\neg$ Clustering

$\longrightarrow$ Random

Average single structure

\section{_ Best single}

structure

Whole ensemble

- SIENA

$\neg$ Clustering

$\multimap$ Random

\section{Test set}

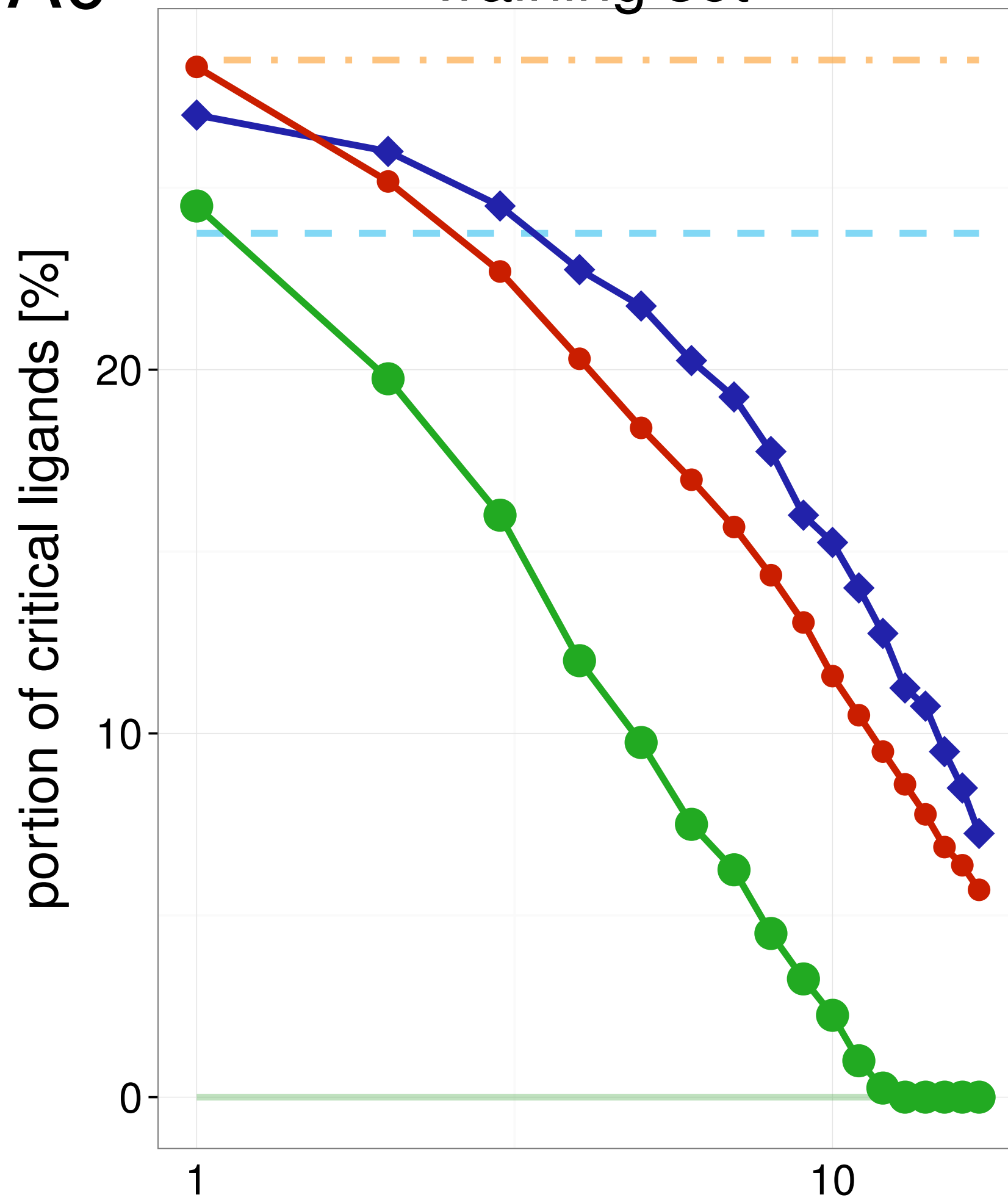

maximal ensemble size

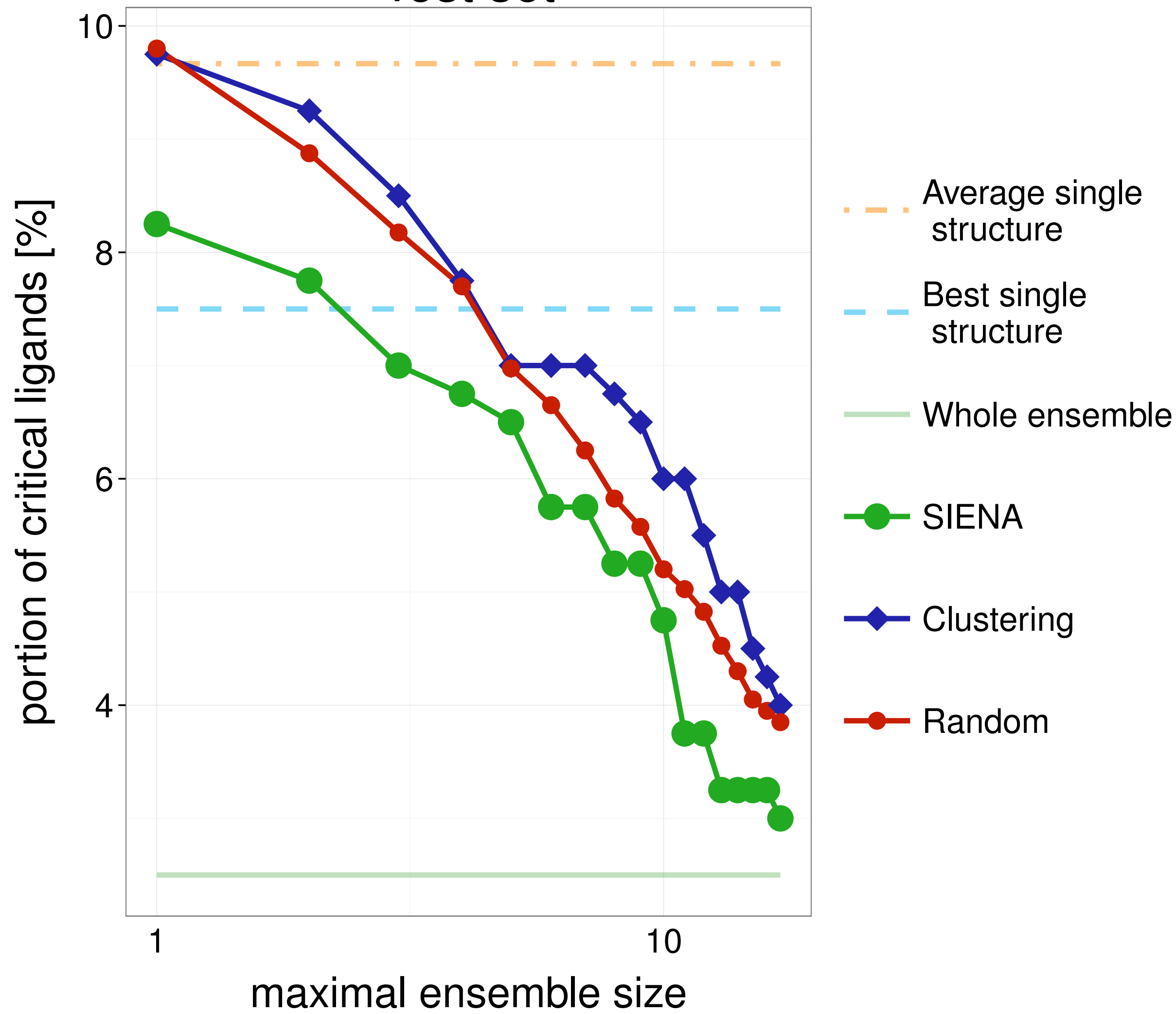


Training set

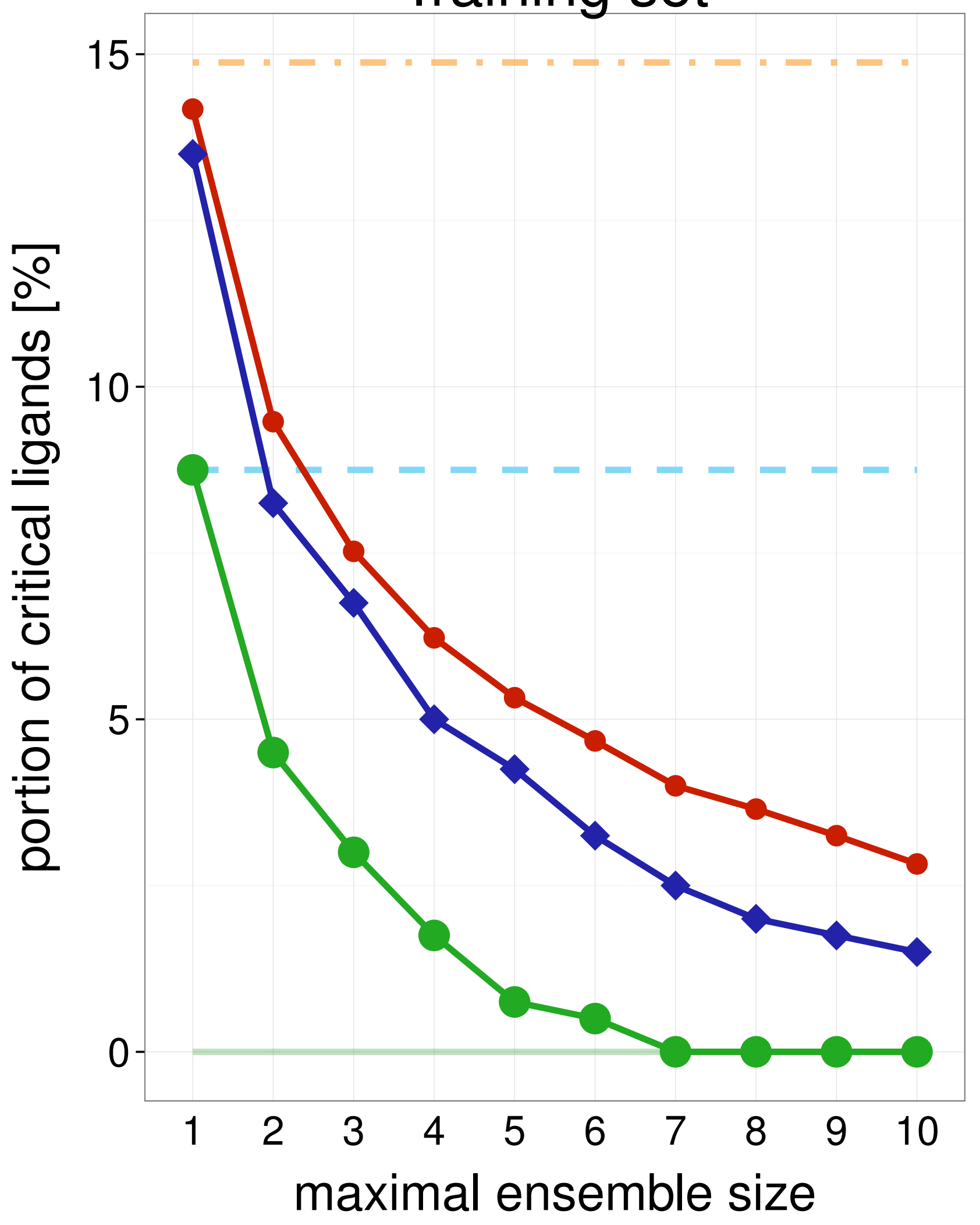

Test set

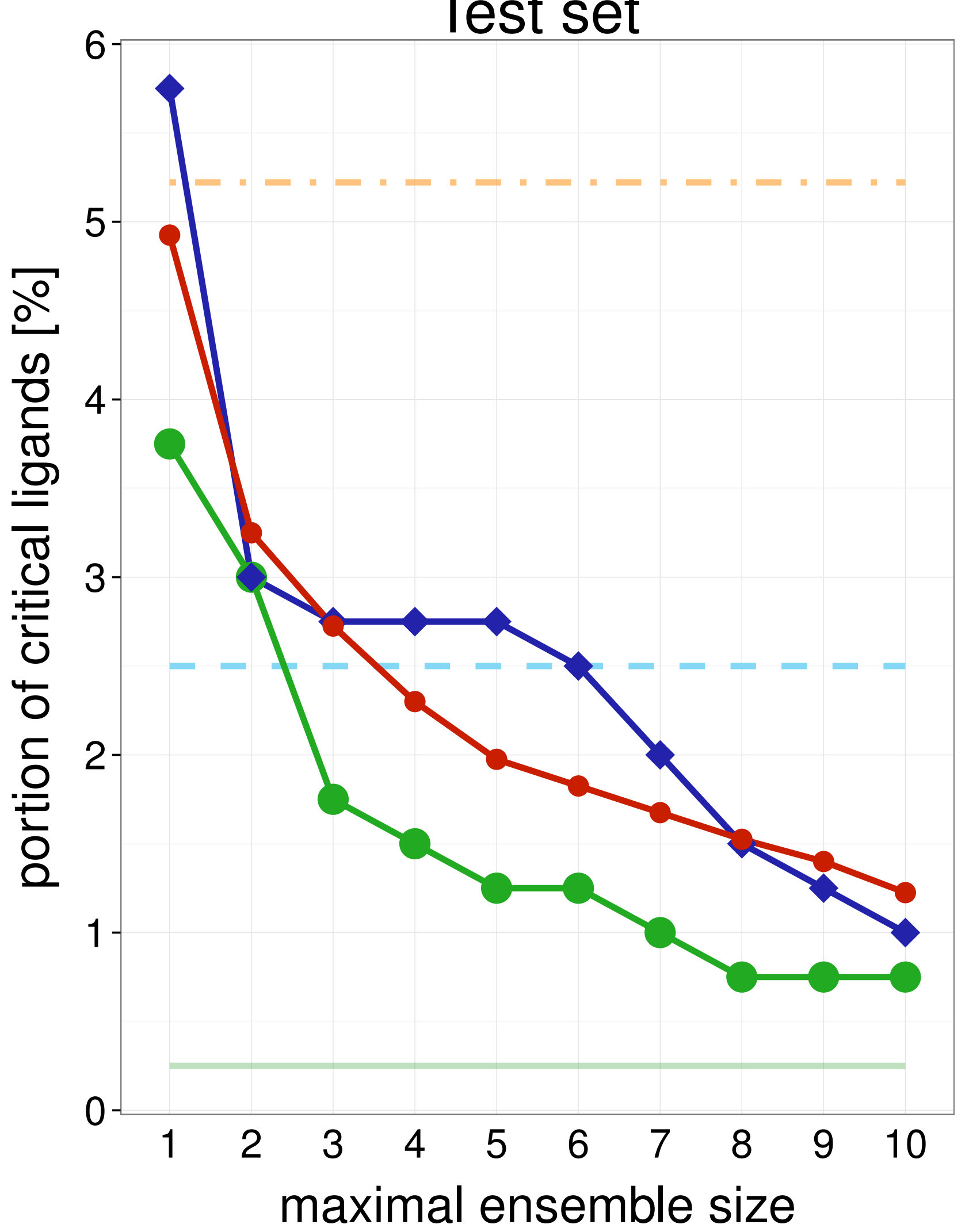

1GVT

Training set

Average single structure

_ Best single structure

Whole ensemble

$\multimap-$ SIENA

$\neg$ Clustering

- Random

Average single structure

_ _ Best single structure

Whole ensemble

- SIENA

$\smile$ Clustering

$\multimap$ Random
$15-$

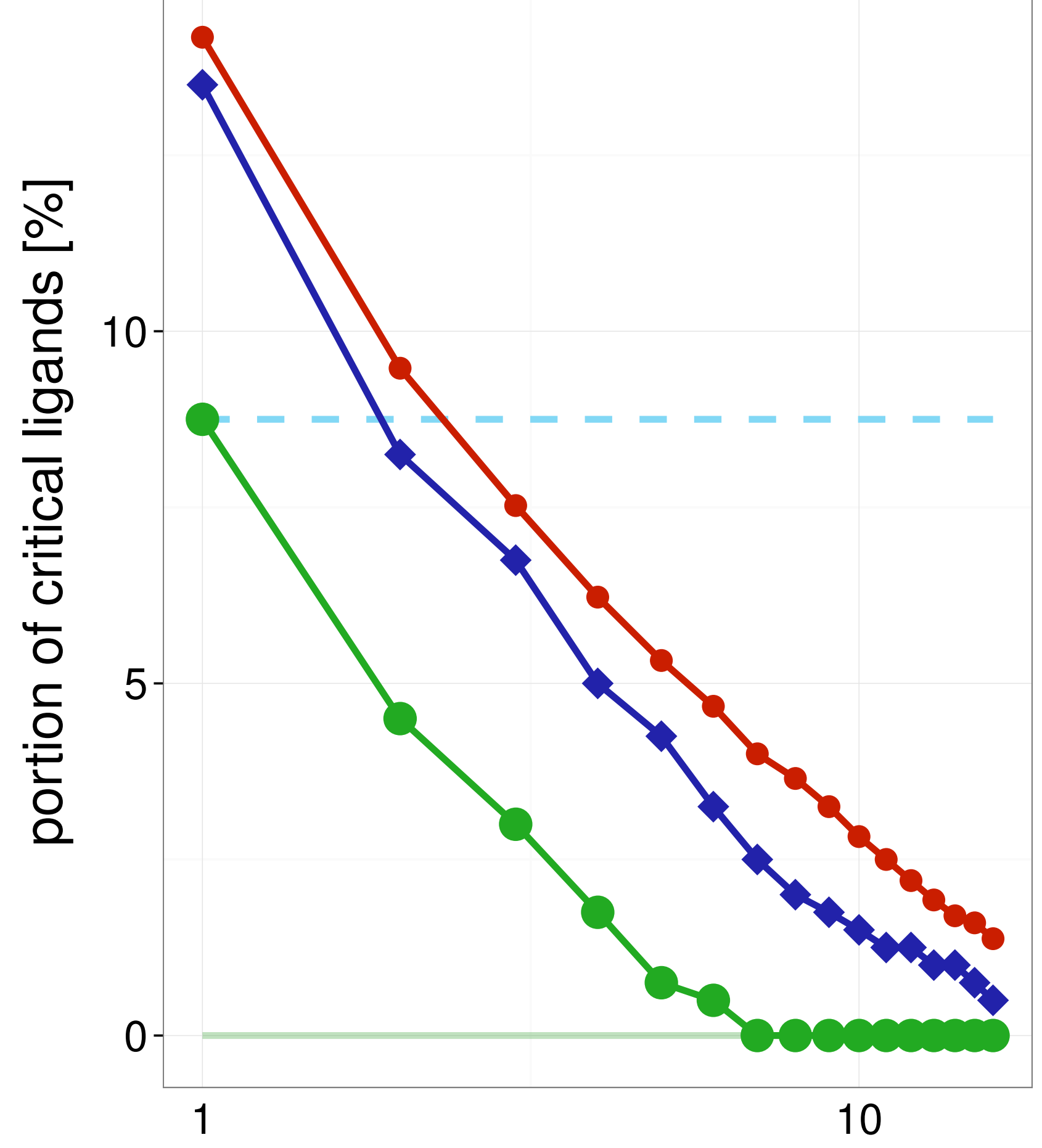

maximal ensemble size

Test set

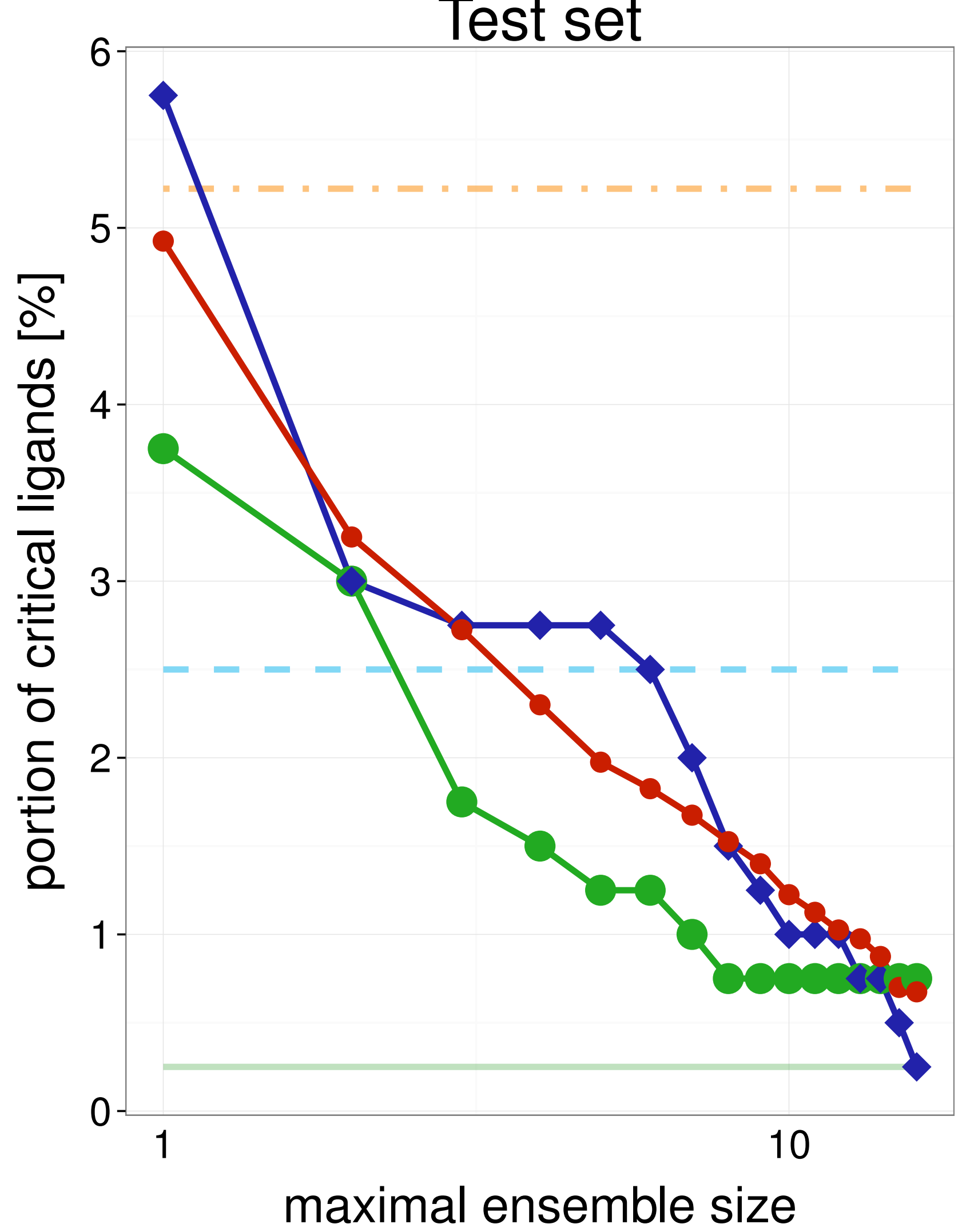

Average single structure

Best single structure

Whole ensemble

SIENA

$\multimap$ Clustering

$\multimap$ Random

Average single structure

Best single structure

Whole ensemble

SIENA

$\sim$ Clustering

$\multimap$ Random 
Training set

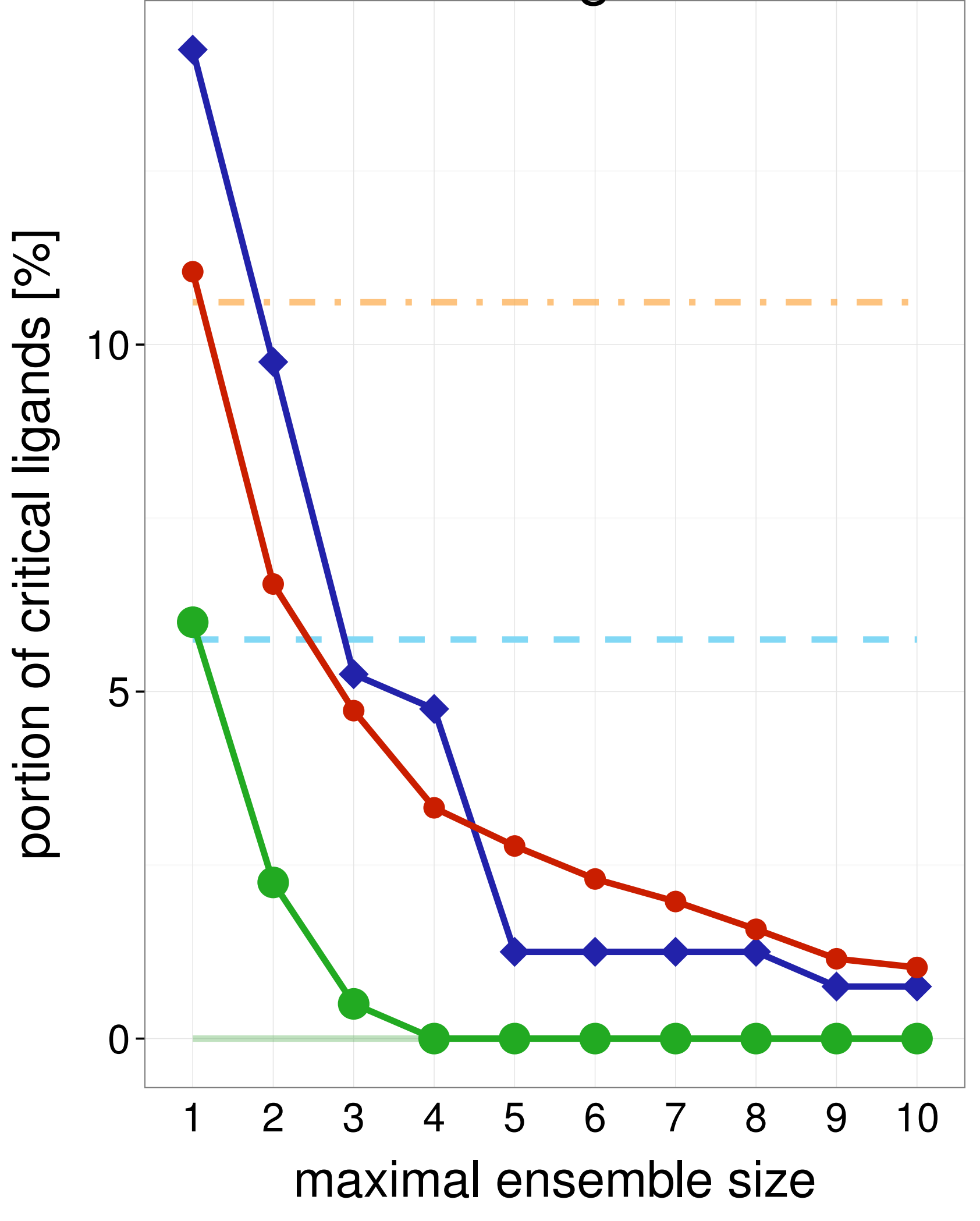

Test set

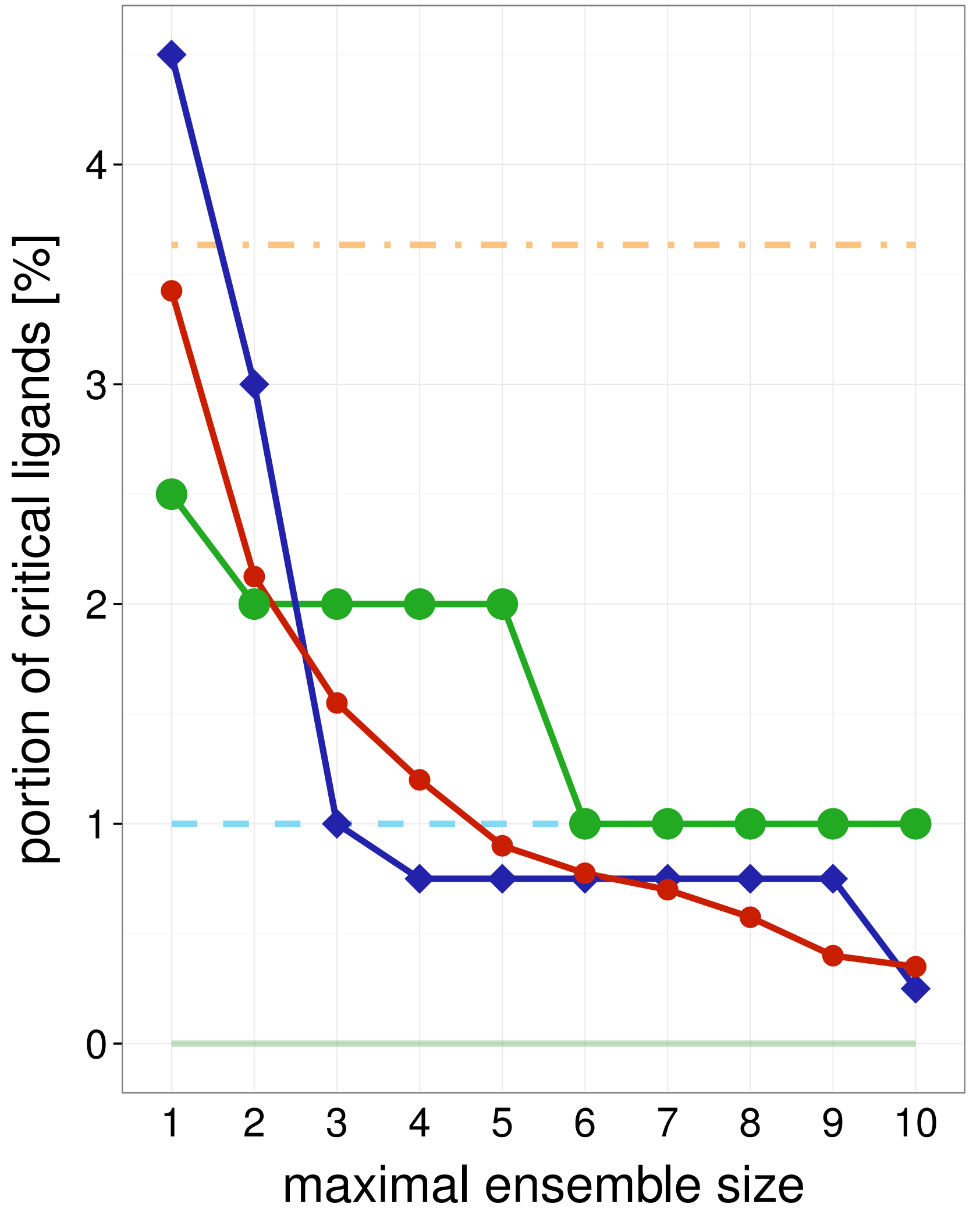

Average single structure

_ _ Best single structure

Whole ensemble

- SIENA

$\neg$ Clustering

$\multimap$ Random

Average single structure

_ Best single structure

Whole ensemble

- SIENA

$\neg$ Clustering

$\because$ Random
Training set

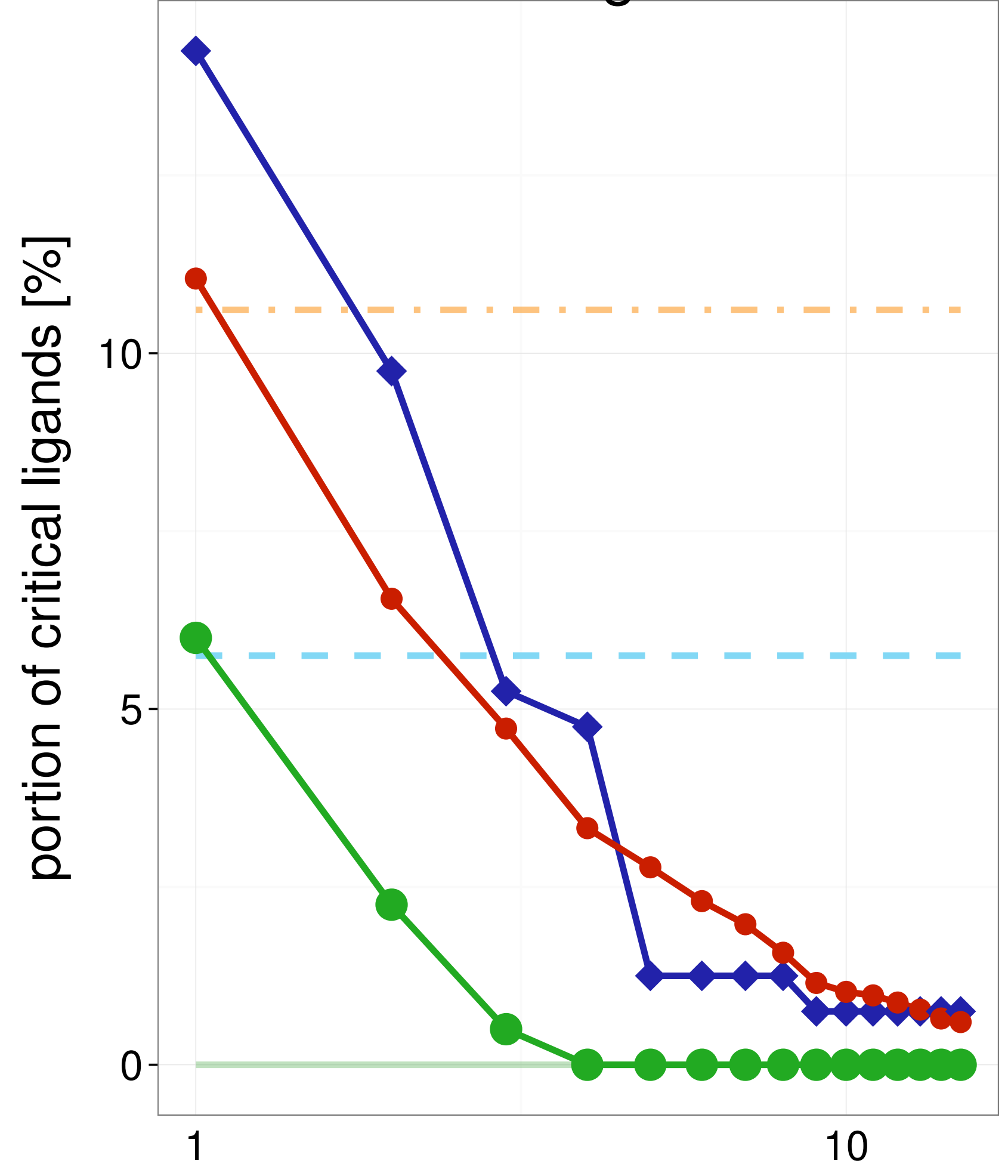

Average single structure

Best single structure

Whole ensemble

- SIENA

$\multimap$ Clustering

- Random

maximal ensemble size

Test set

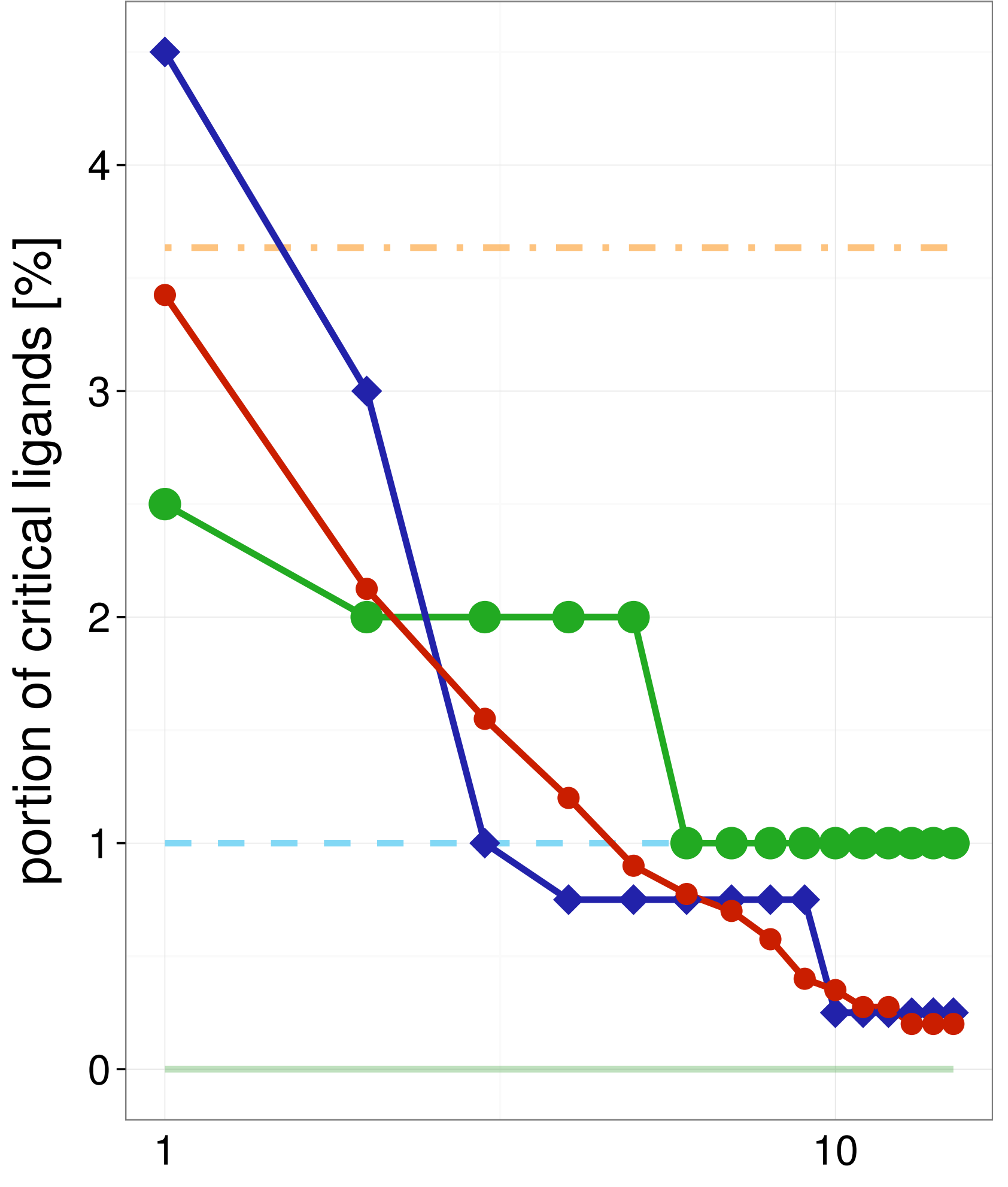

Average single structure

Best single structure

Whole ensemble

SIENA

$\sim$ Clustering

$\longrightarrow$ Random 


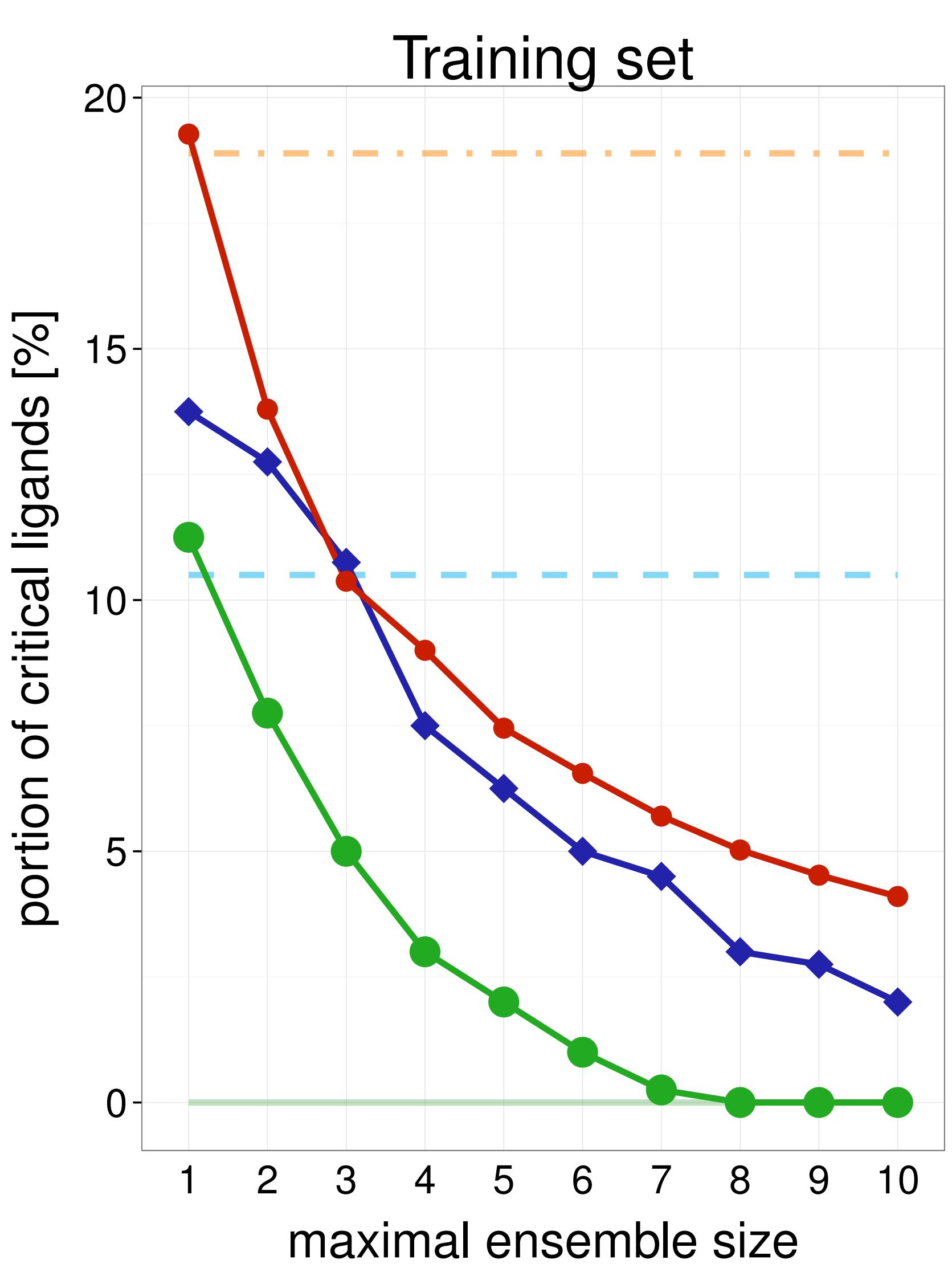

Test set

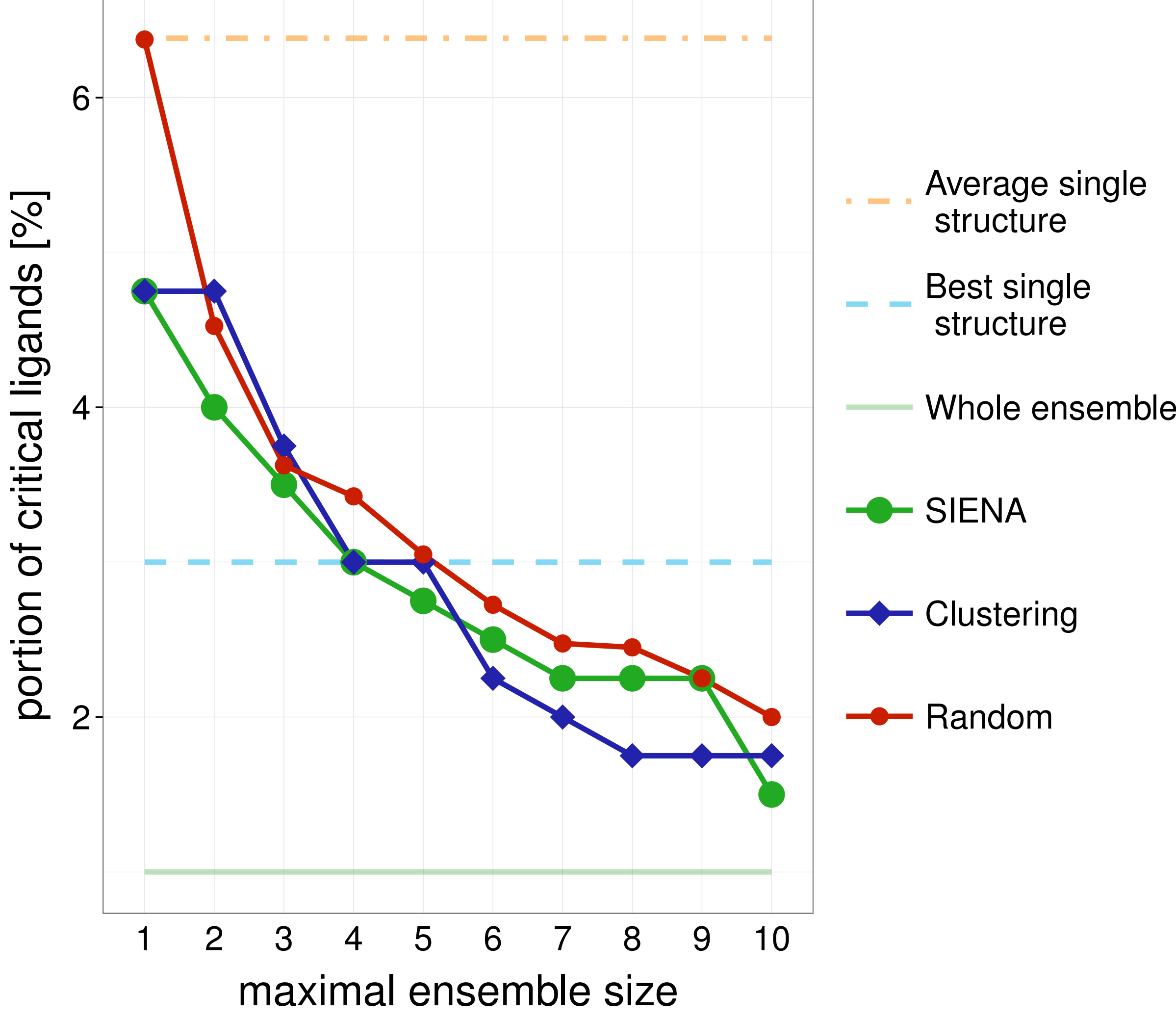

Average single structure

_ Best single structure

Whole ensemble

- SIENA

$\neg$ Clustering

$\multimap$ Random

$2 B 7 A_{20} \quad$ Training set 


\section{Training set}

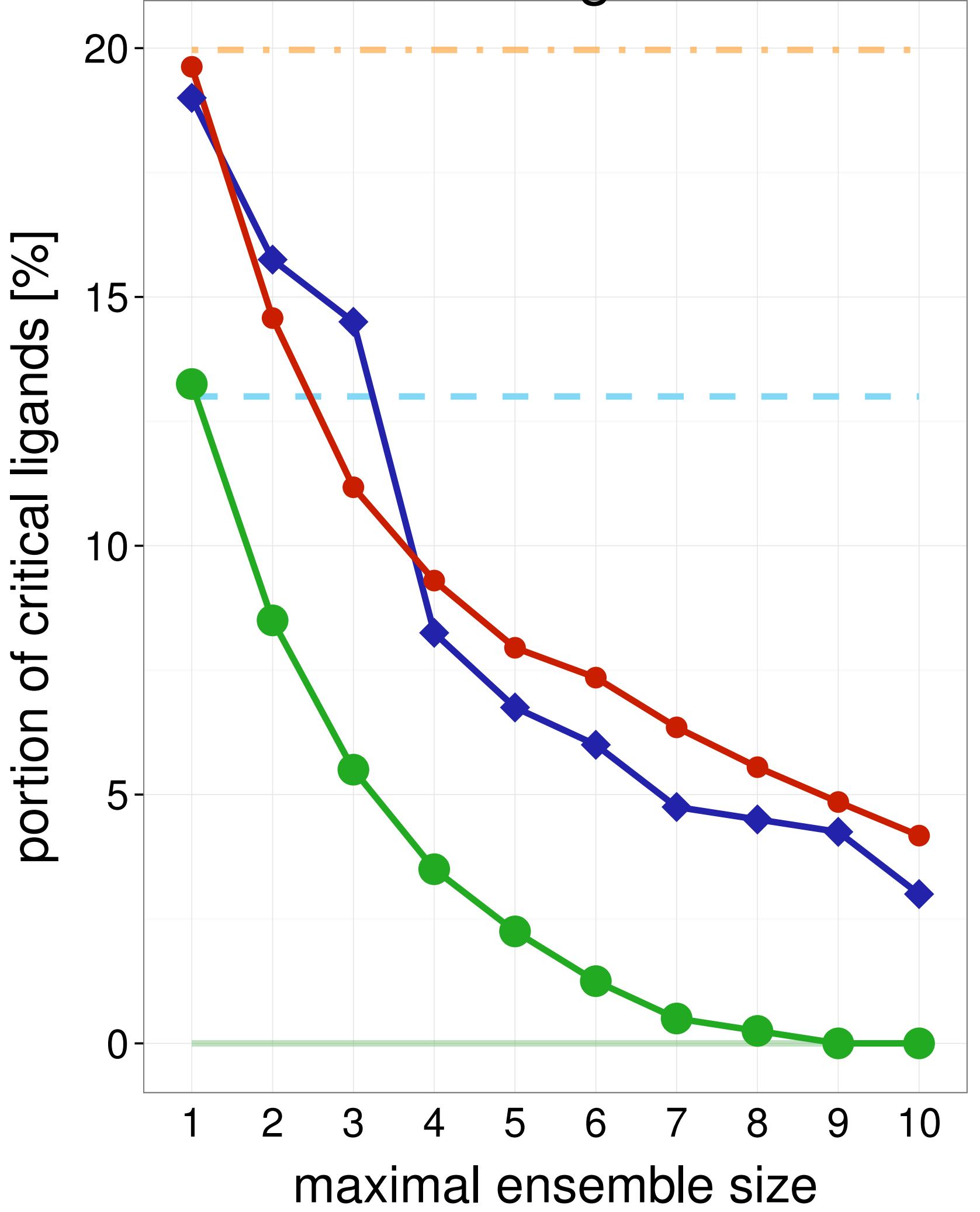

Test set

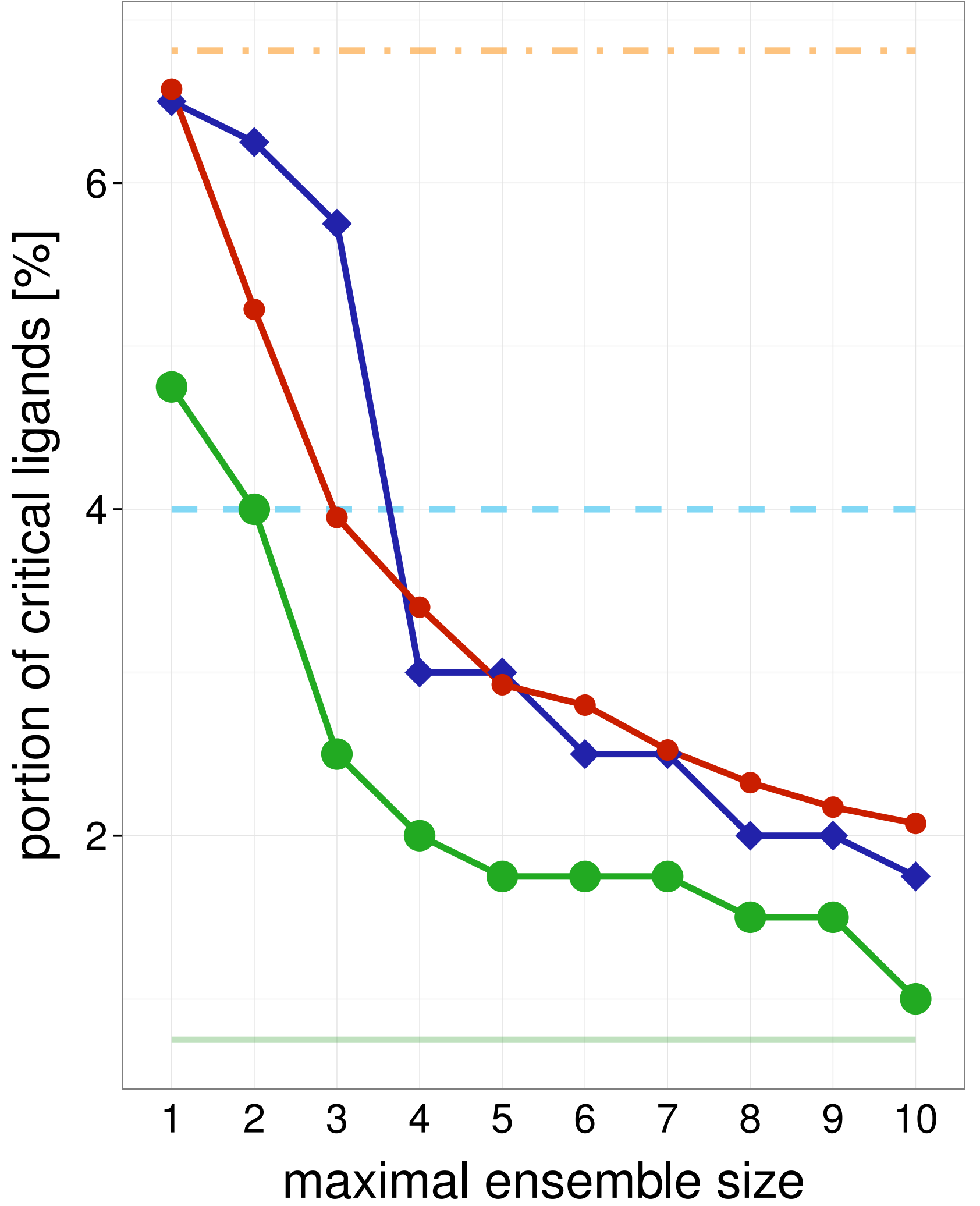

2BKS

Training set

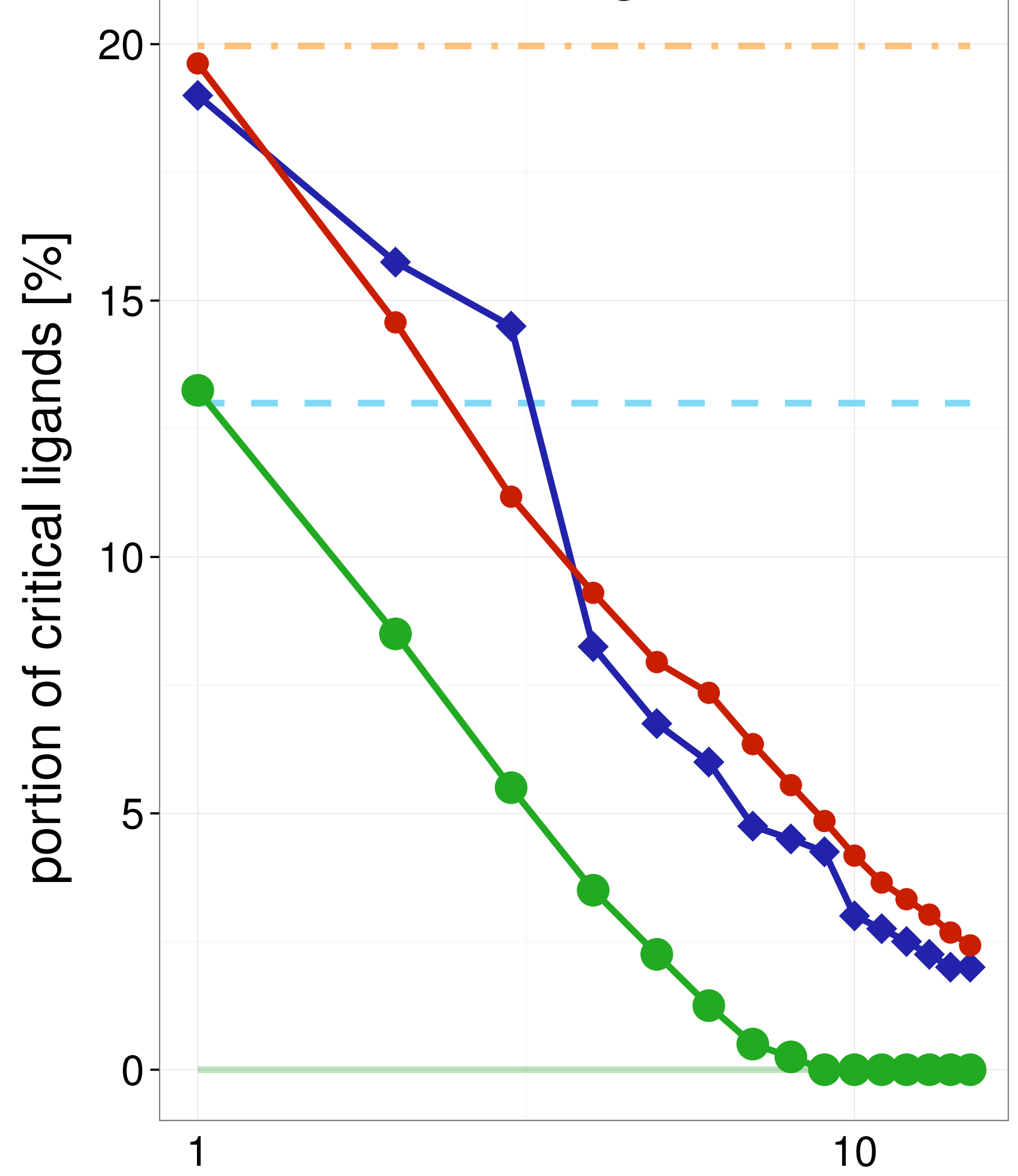

maximal ensemble size

Test set

Average single structure

\section{_ Best single} structure

Whole ensemble

- SIENA

$\neg$ Clustering

$\multimap$ Random

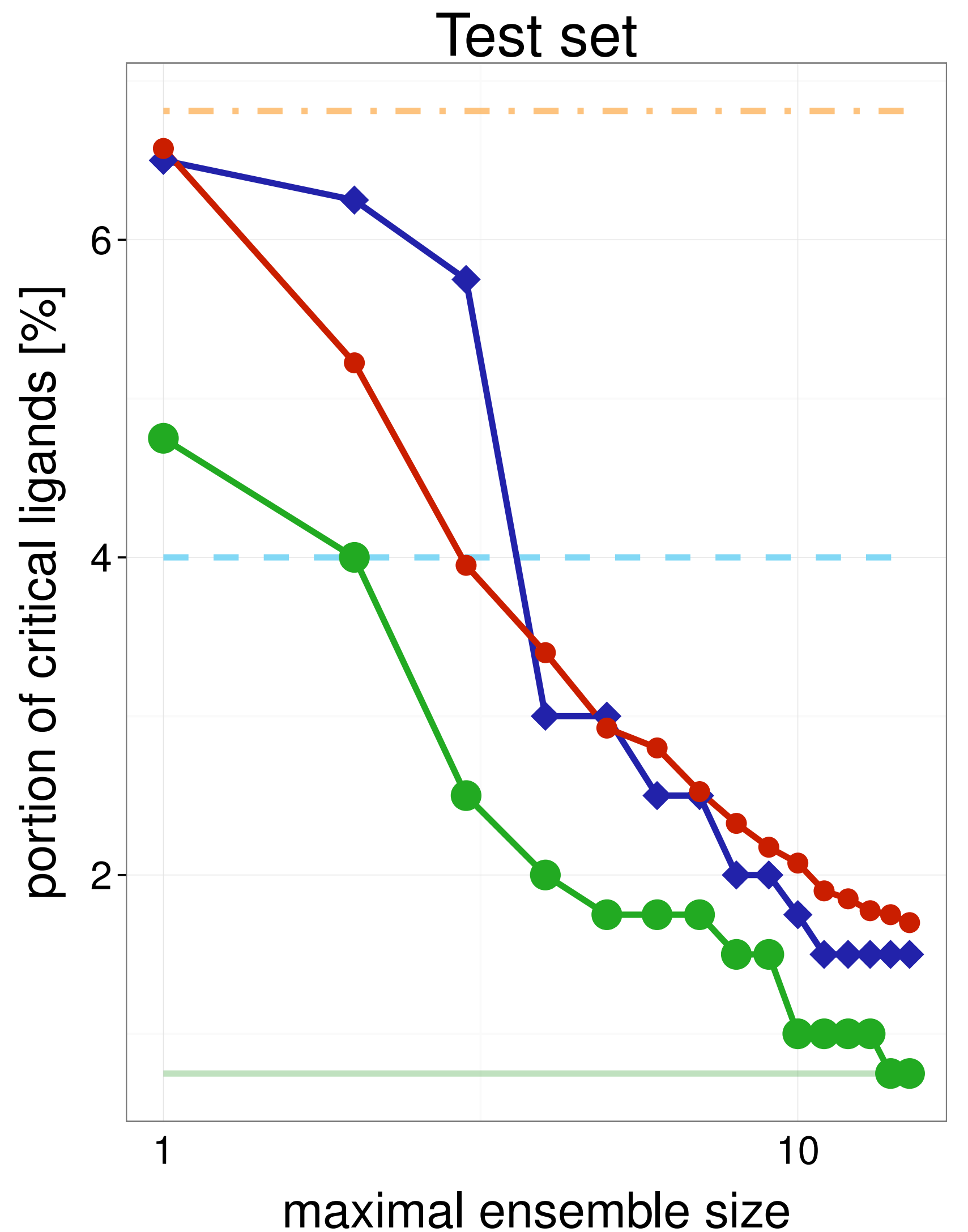

Average single structure

_ Best single structure

Whole ensemble

- SIENA

$\leadsto$ Clustering

$\multimap$ Random 


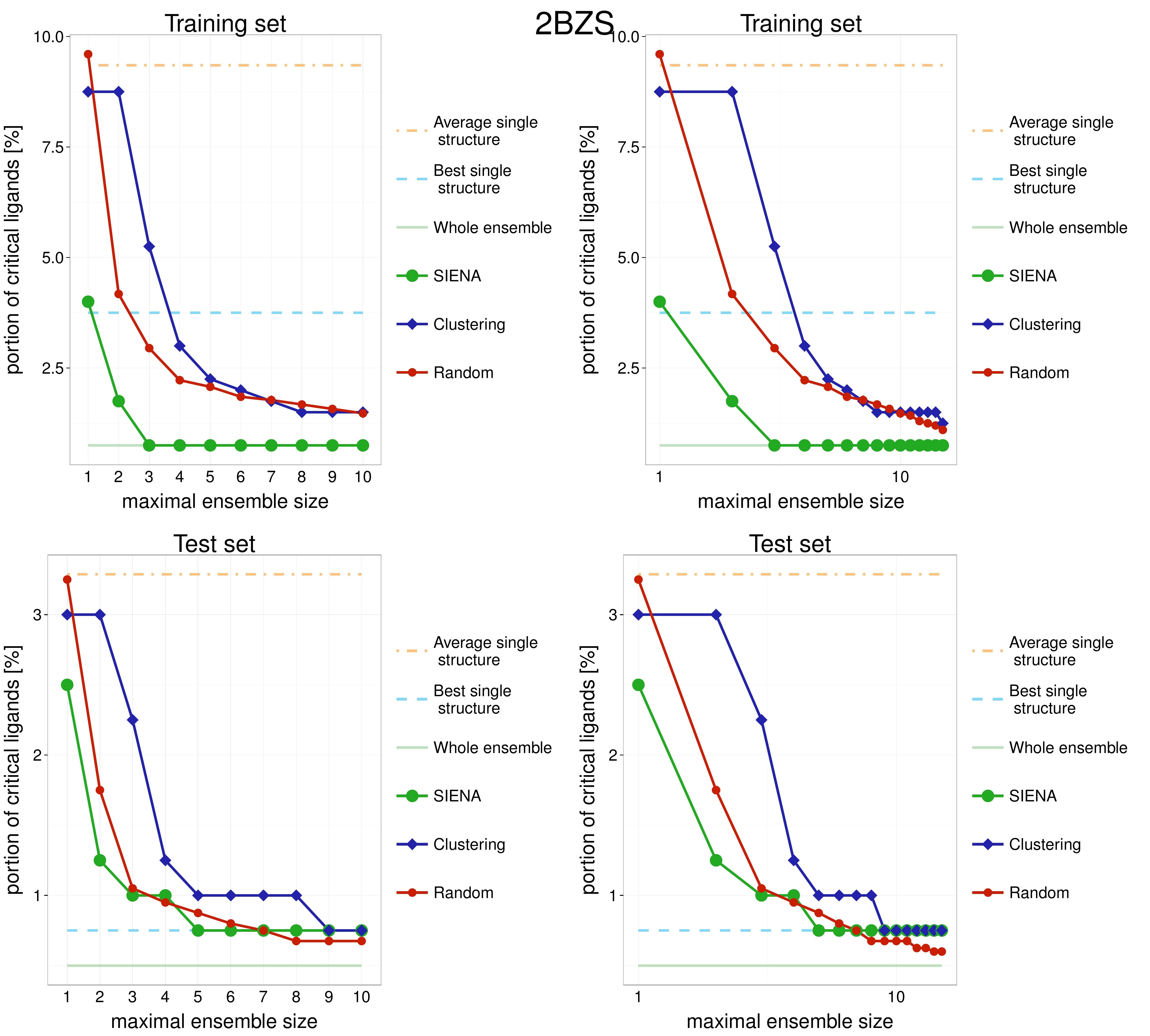




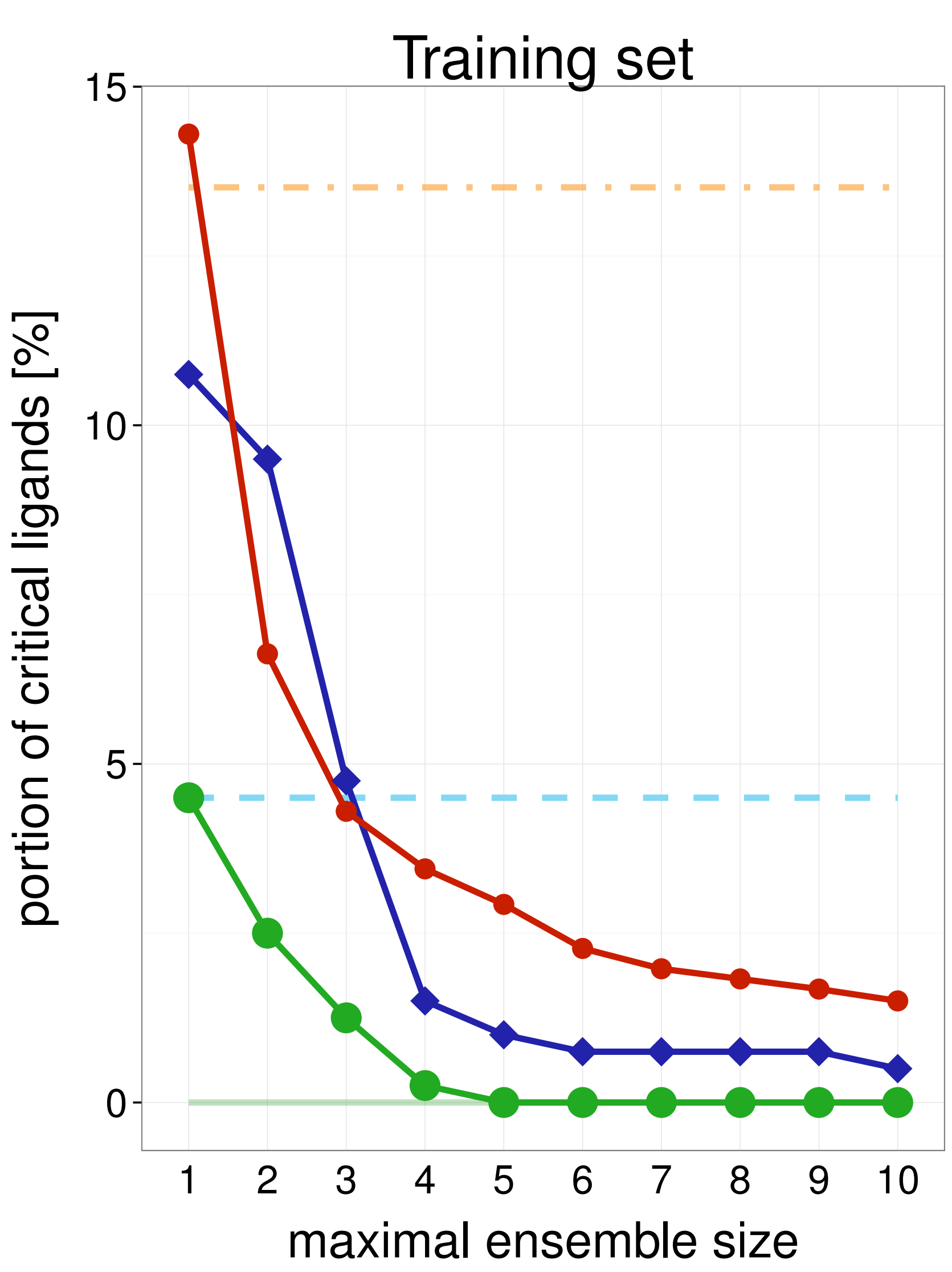

Test set

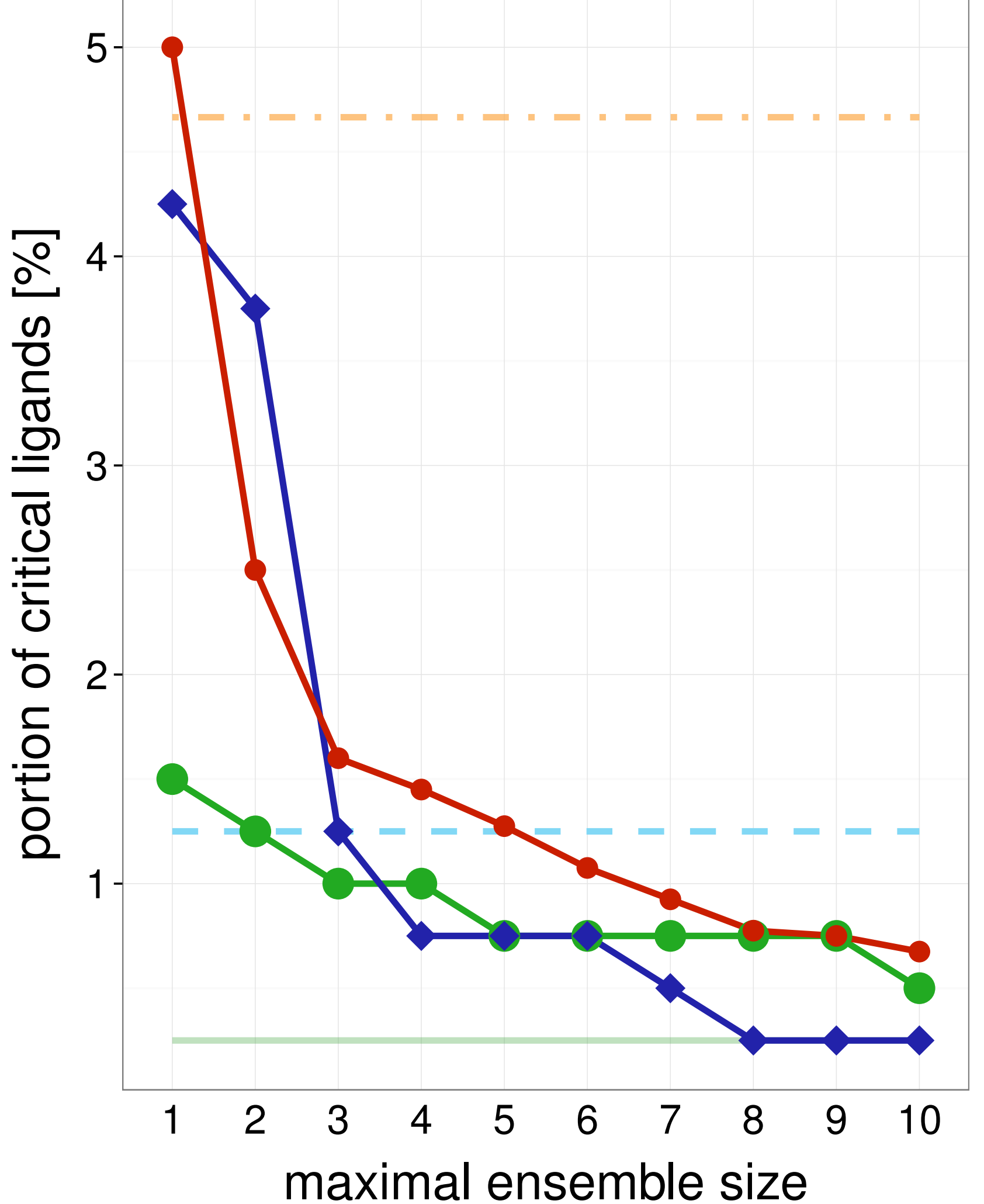

Average single structure

- Best single structure

Whole ensemble

- SIENA

$\neg$ Clustering

$\multimap$ Random

Average single structure

_ _ Best single structure

Whole ensemble

- SIENA

$\neg$ Clustering

$\multimap$ Random
$2 \mathrm{C} 6 \mathrm{G}_{5}$

Training set

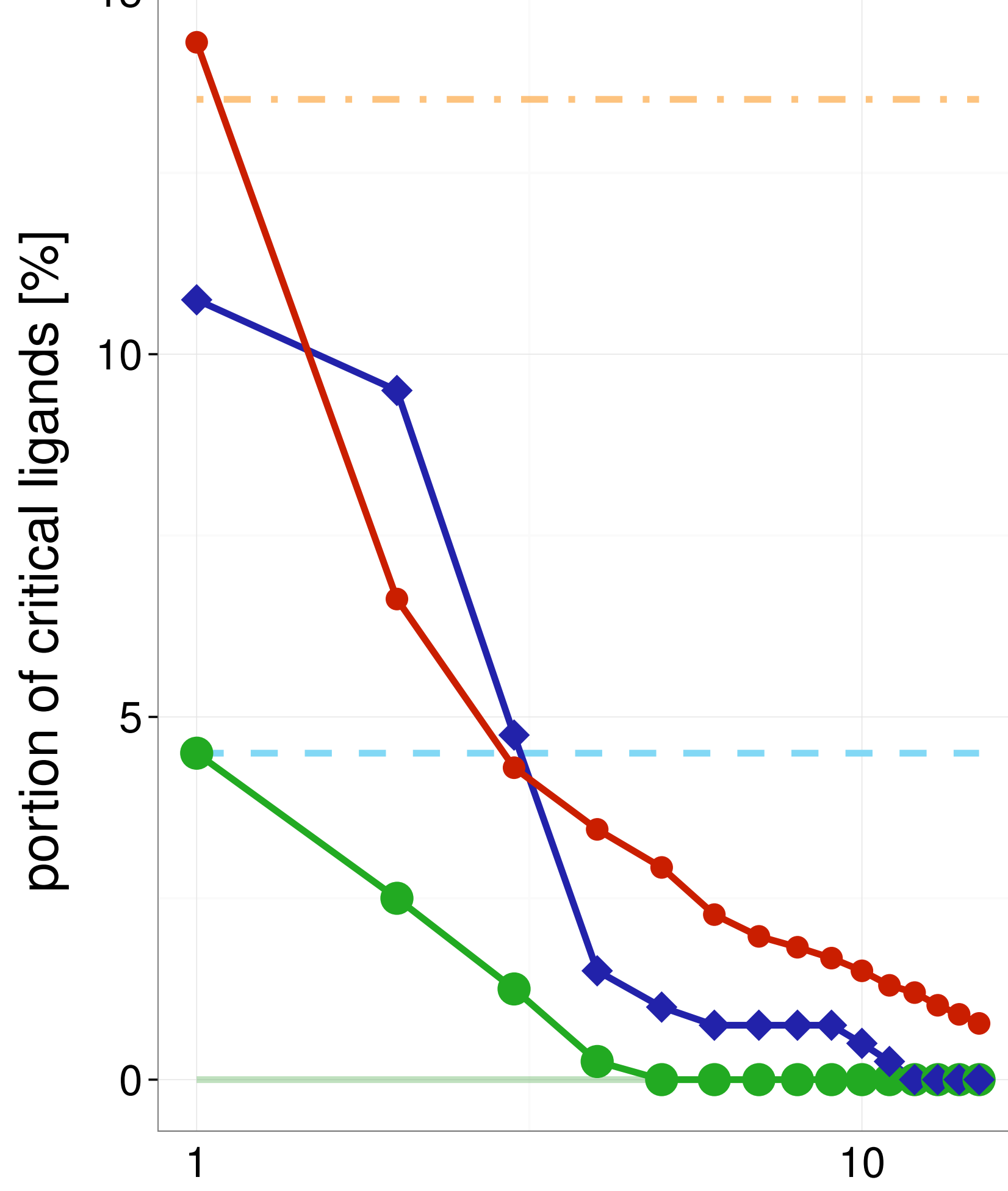

Average single structure

- Best single structure

Whole ensemble

- SIENA

$\leadsto$ Clustering

$\multimap$ Random

maximal ensemble size

Test set

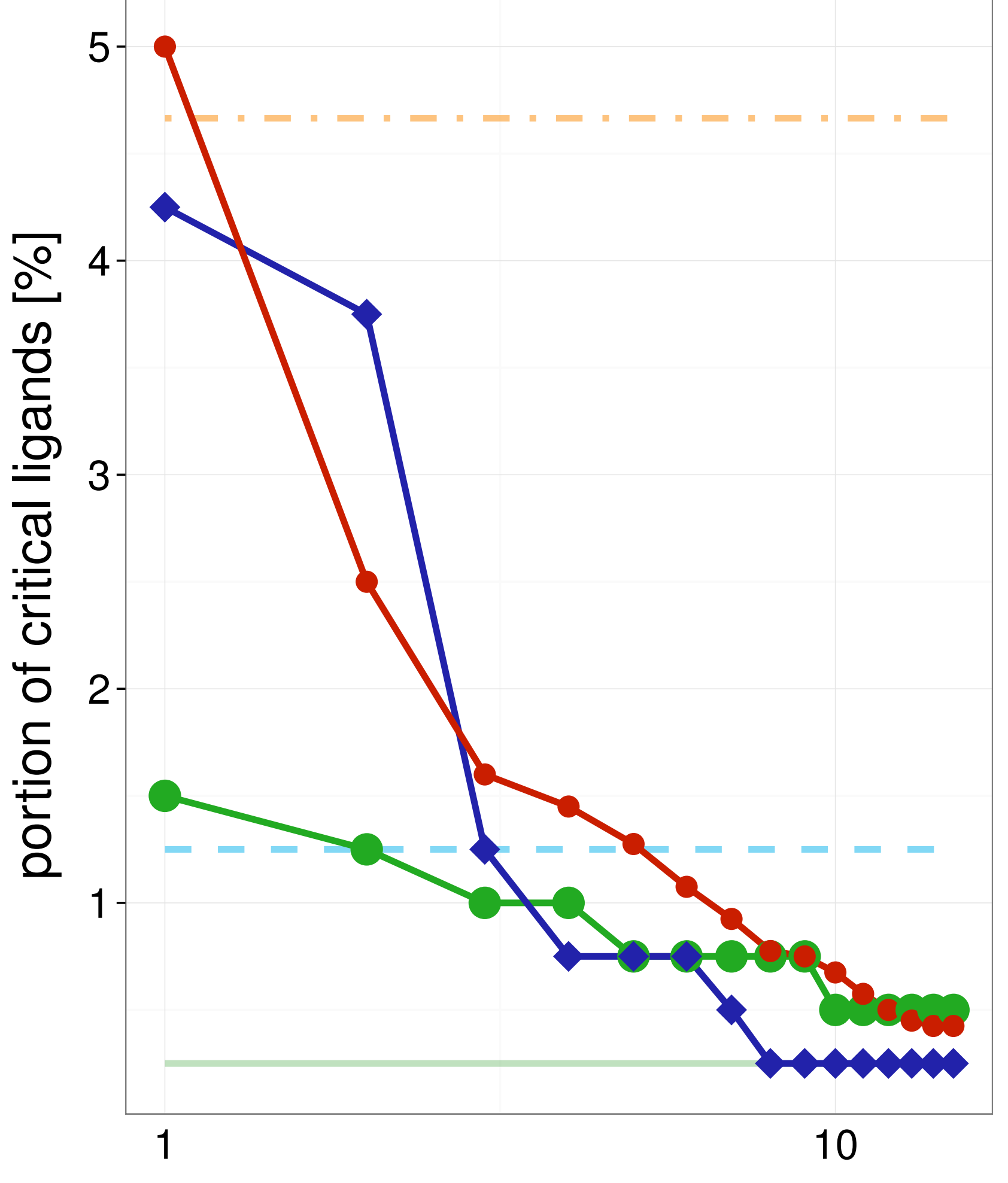

Average single structure

_ _ Best single structure

Whole ensemble

- SIENA

$\leadsto$ Clustering

$\longrightarrow$ Random 


\section{Training set}

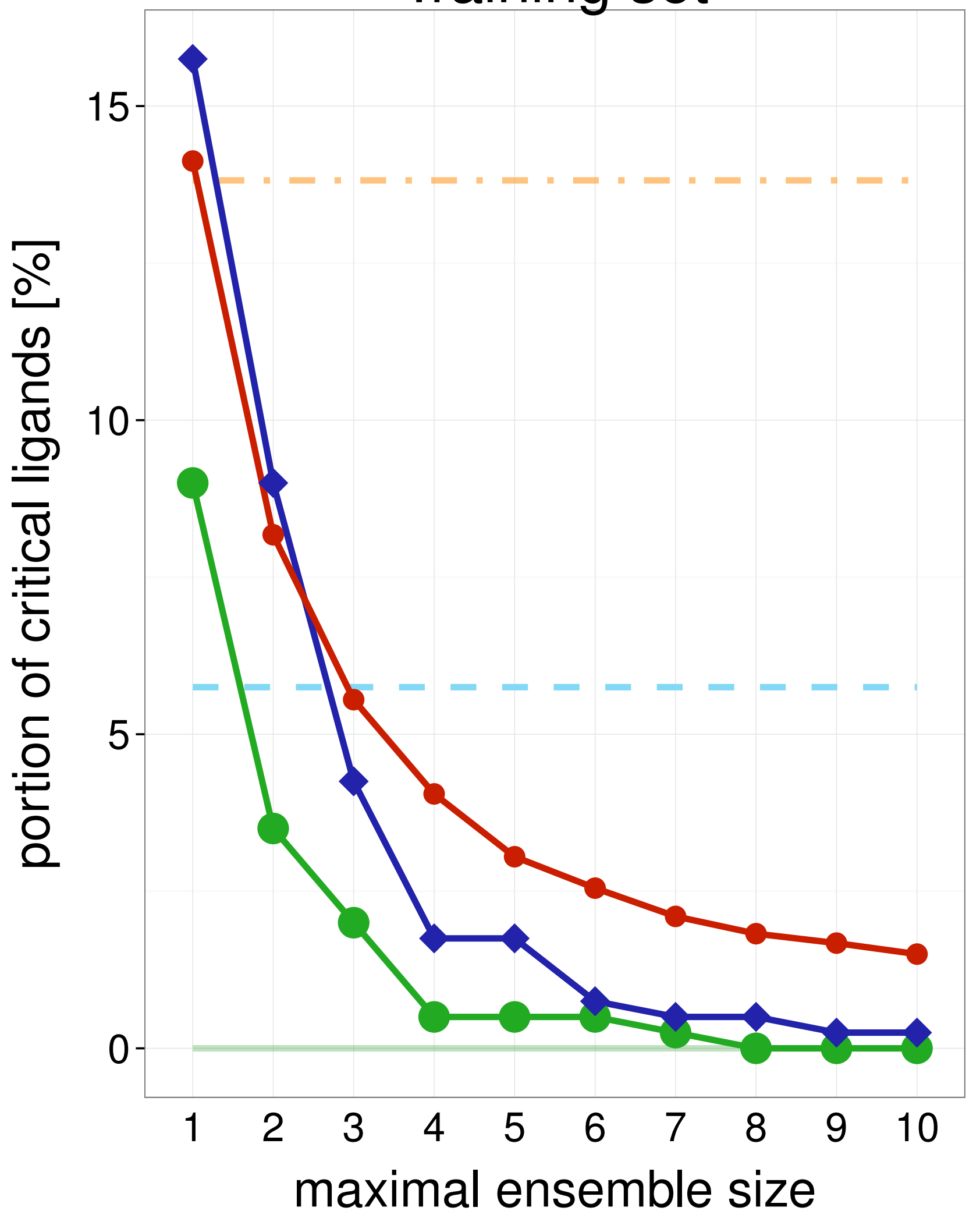

Test set

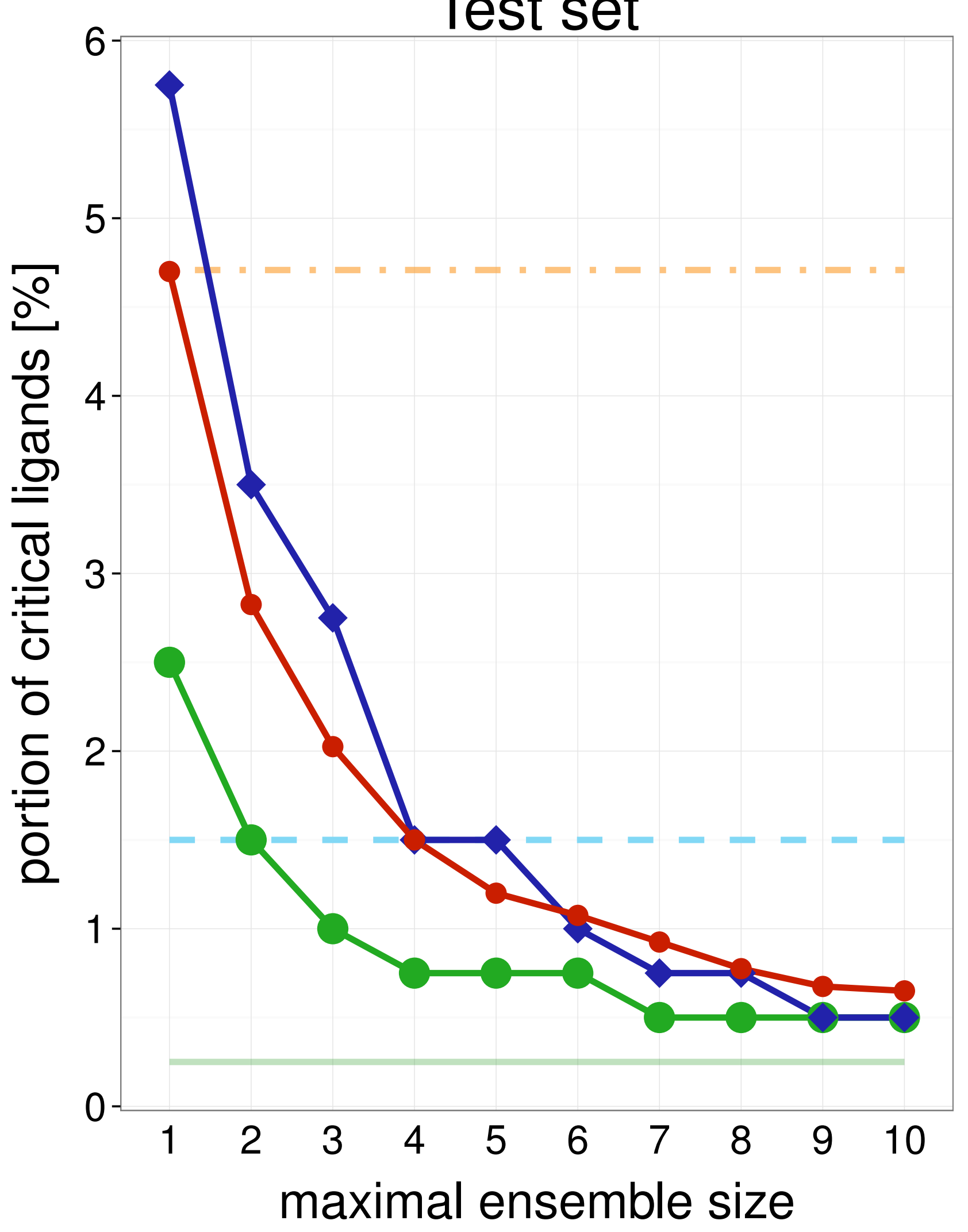

\section{IOD Training set}

Average single structure

_ Best single structure

Whole ensemble

- - SIENA

$\checkmark$ Clustering

$\multimap$ Random

Average single structure

_ Best single structure

Whole ensemble

- SIENA

$\sim$ Clustering

- Random

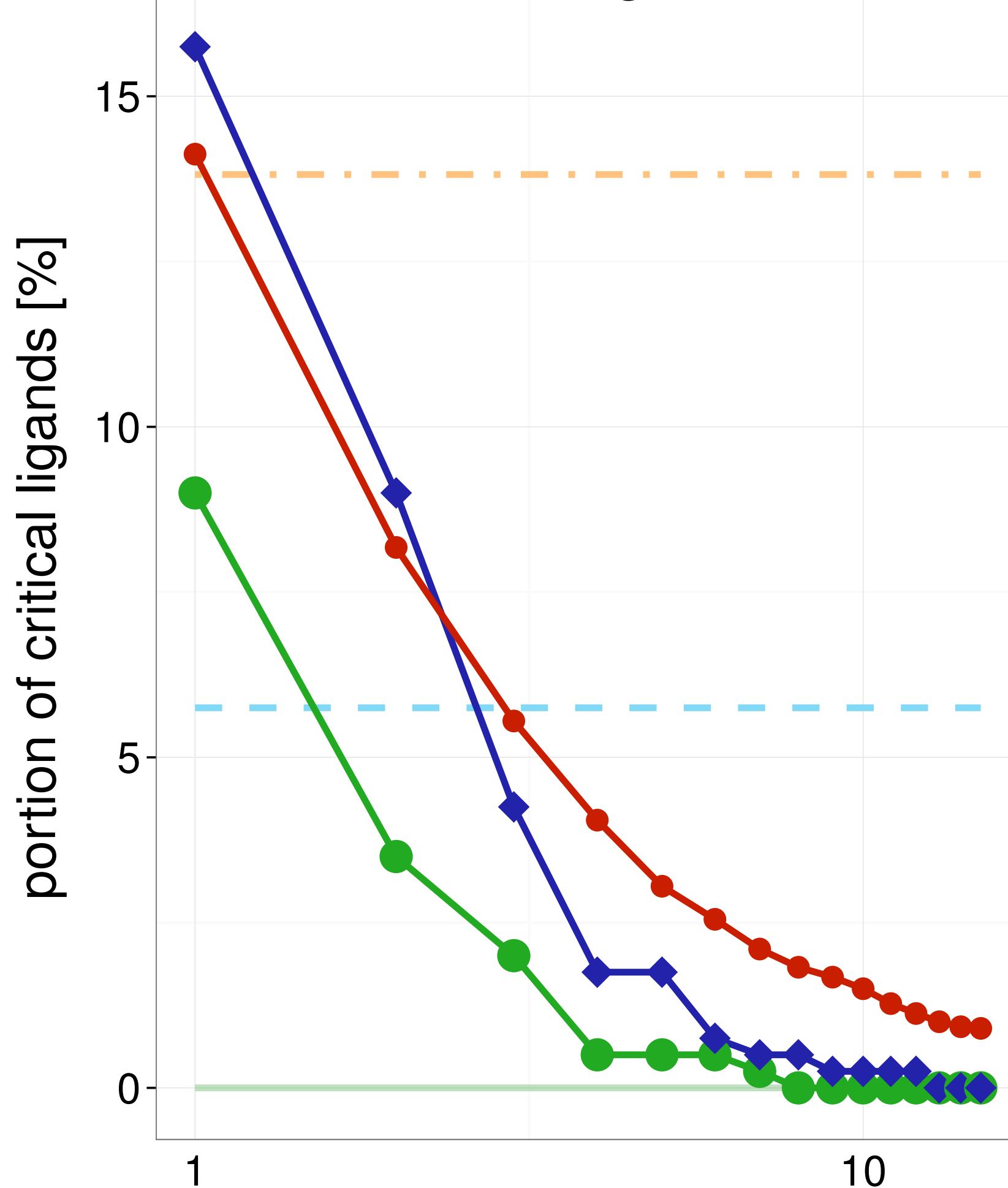

maximal ensemble size

Test set

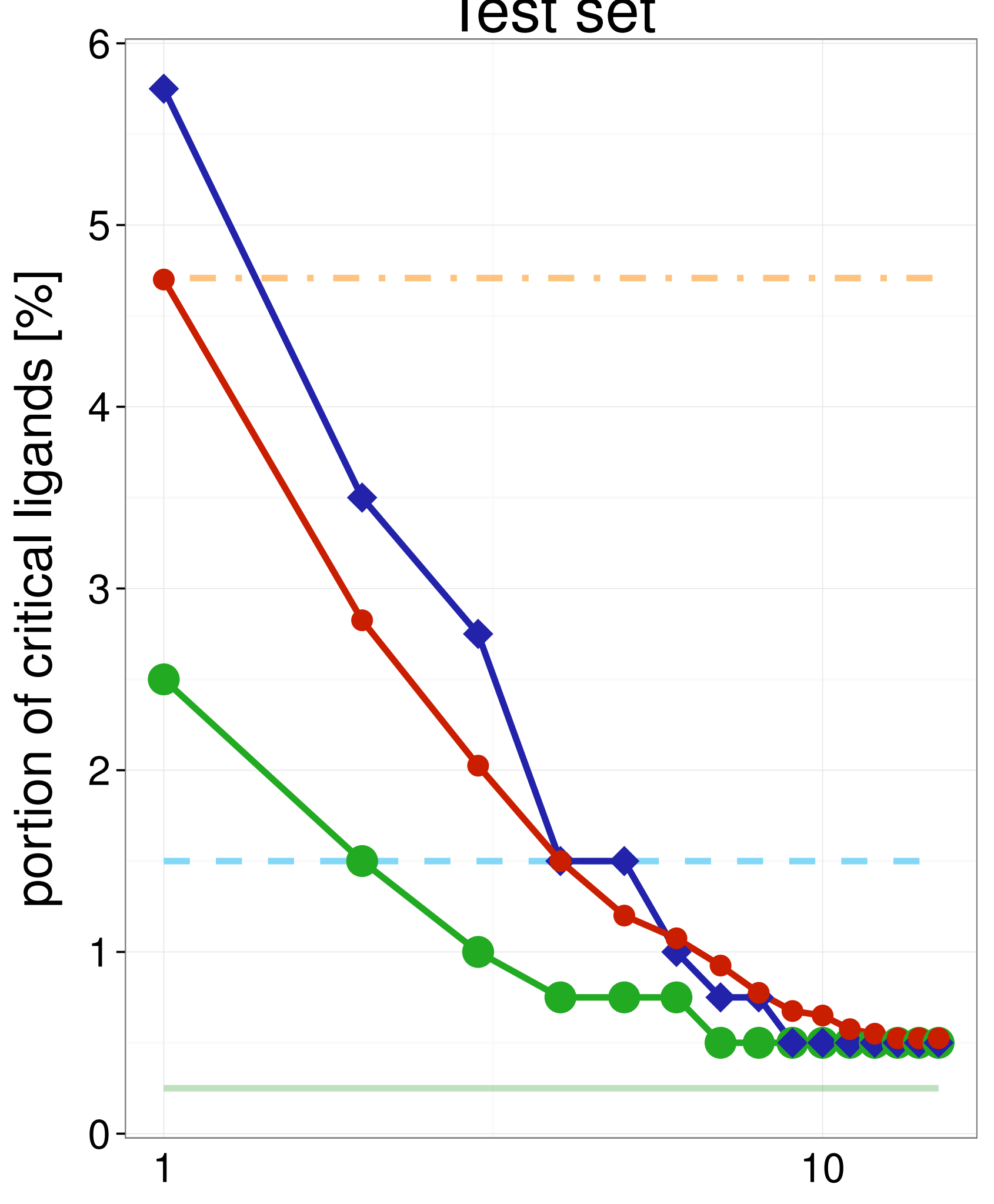

maximal ensemble size
Average single structure

Best single structure

Whole ensemble

SIENA

$\sim$ Clustering

$\multimap$ Random 


\section{Training set}

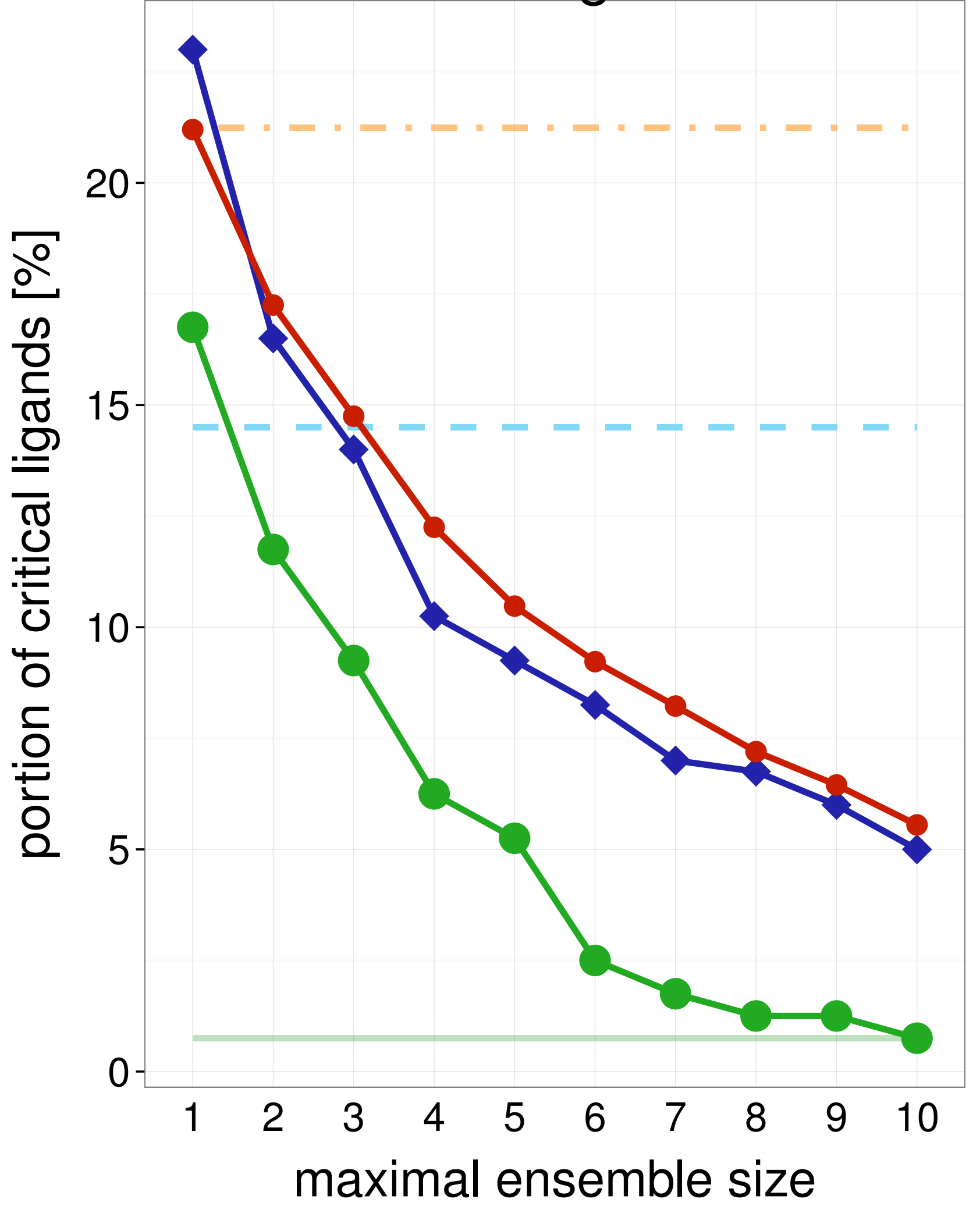

\section{Test set}

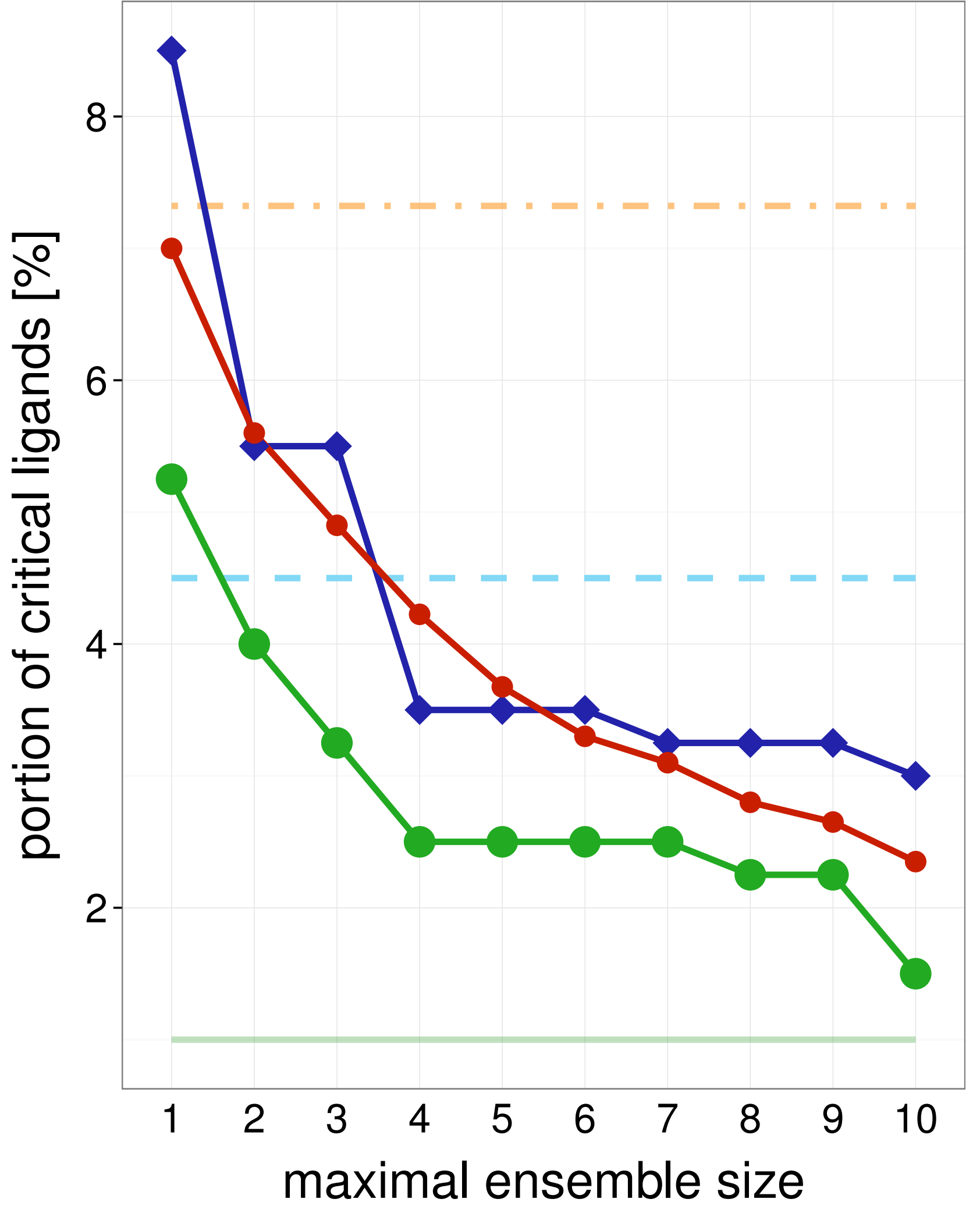

Training set

Average single structure

_ _ Best single structure

Whole ensemble

- SIENA

$\neg$ Clustering

$\longrightarrow$ Random

Average single structure

_ _ Best single structure

Whole ensemble

- SIENA

$\neg$ Clustering

$\multimap$ Random

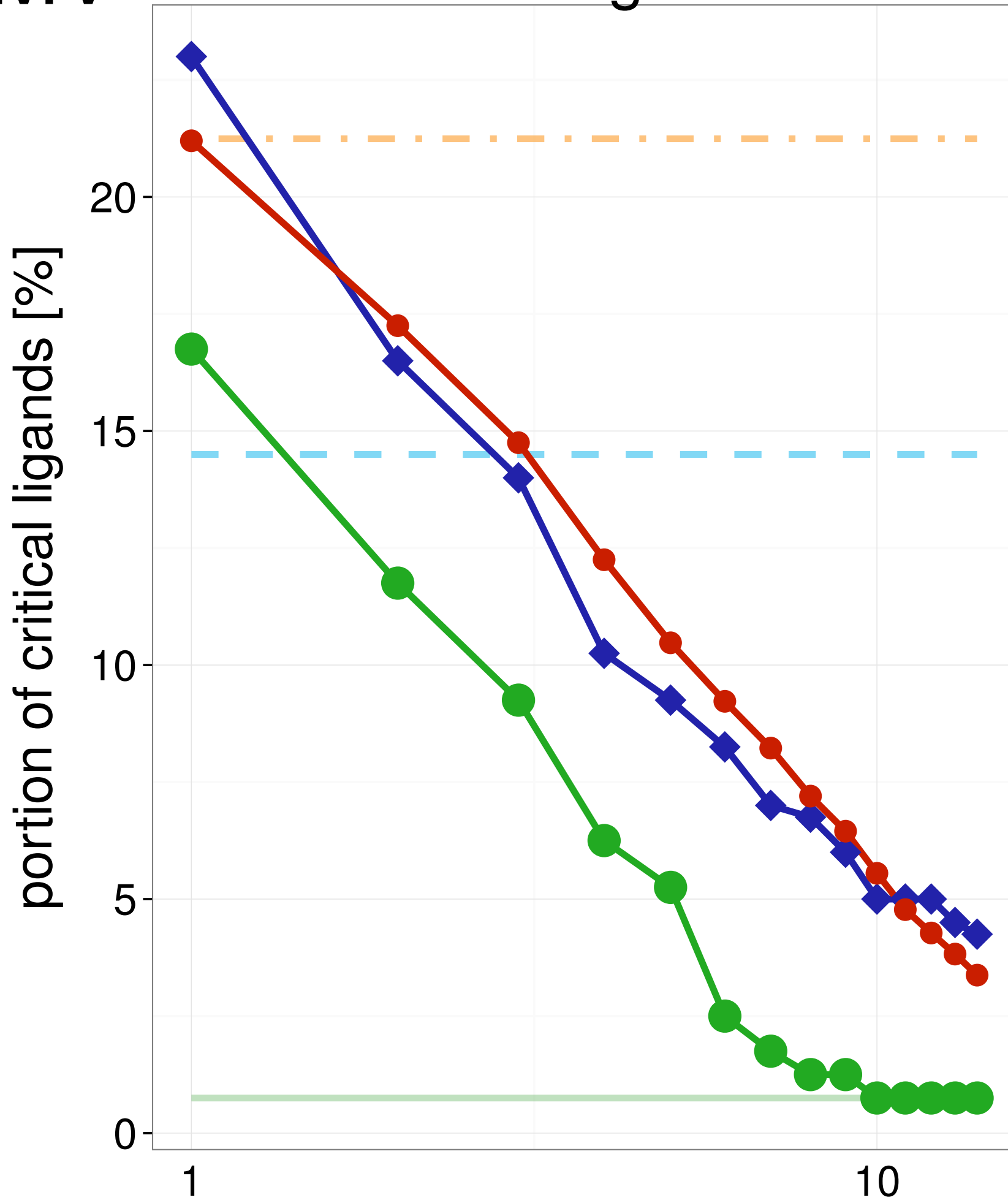

maximal ensemble size

Test set

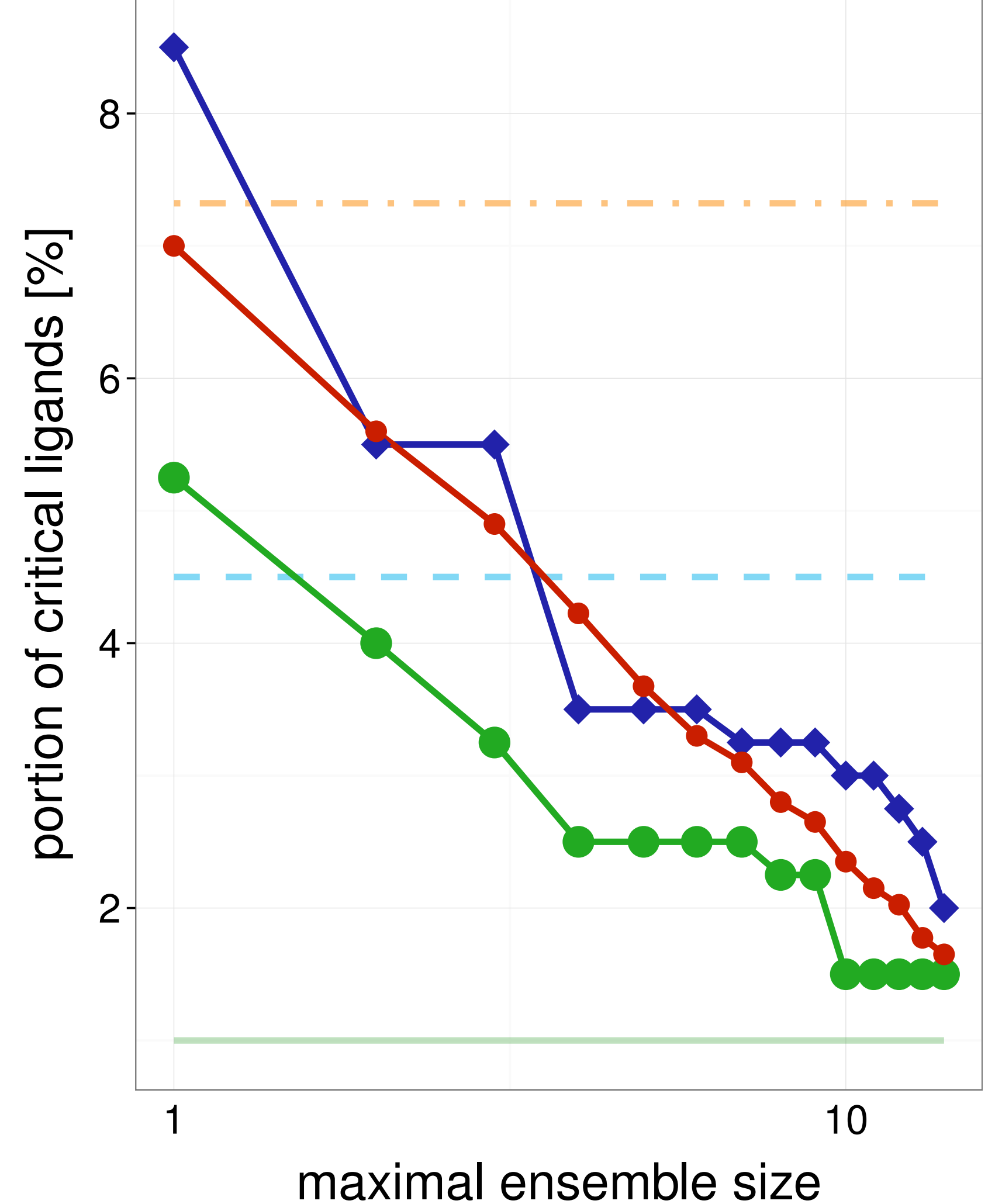

Average single structure

Best single structure

Whole ensemble

- SIENA

$\checkmark$ Clustering

$\multimap$ Random

Average single structure

- Best single structure

Whole ensemble

- SIENA

$\neg$ Clustering

$\multimap$ Random 


\section{Training set}

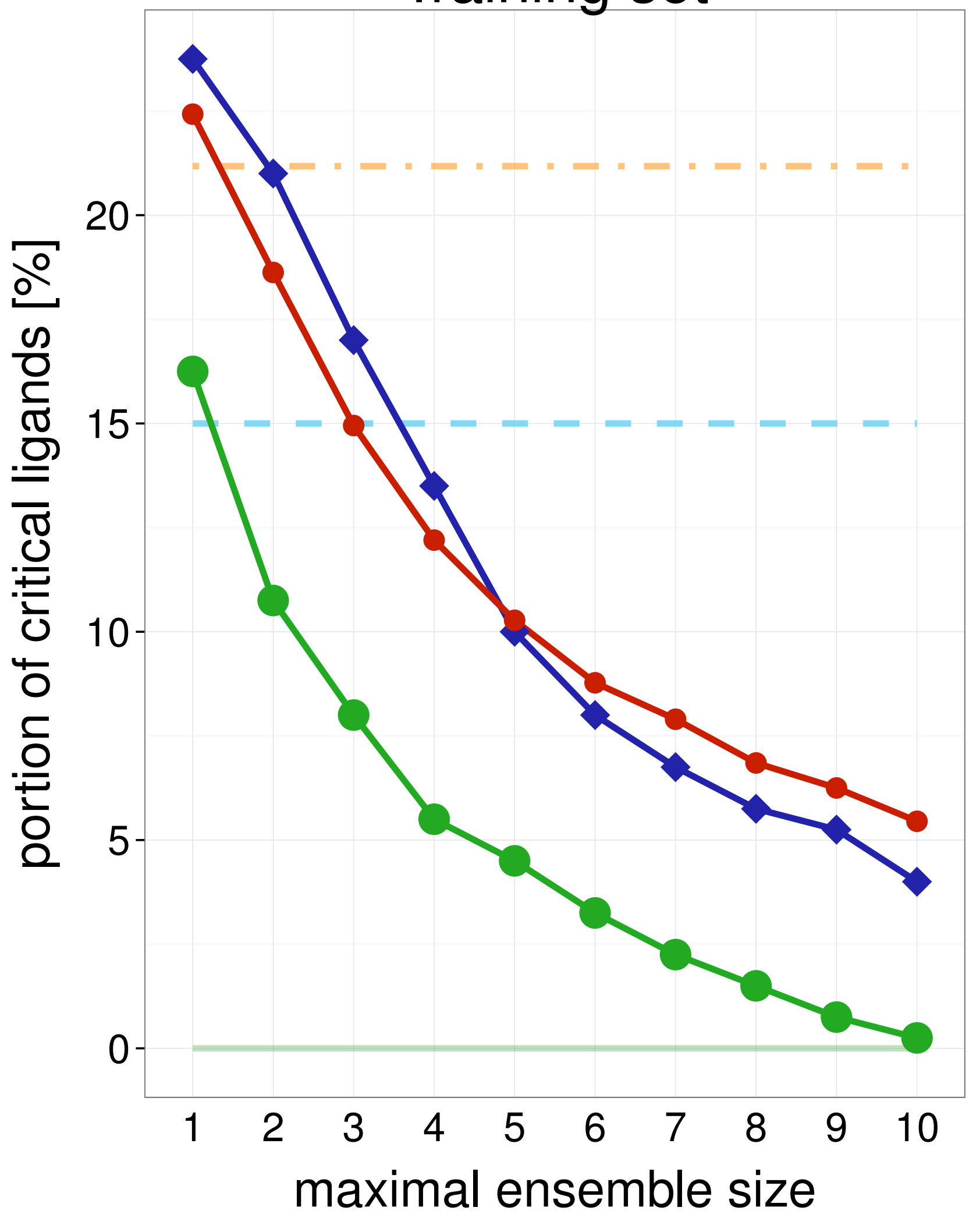

Test set

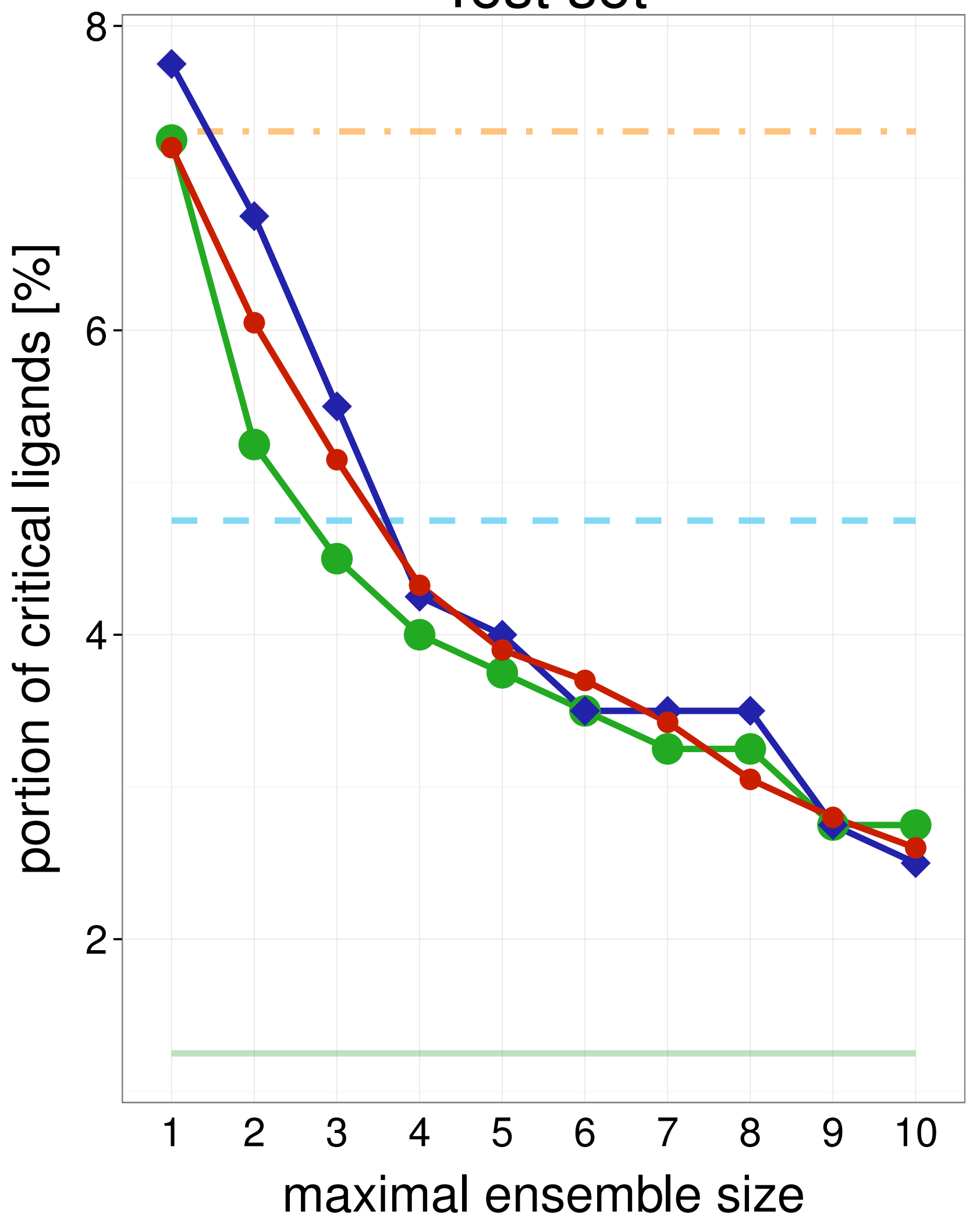

2NP8

Training set

Average single structure

_ _ Best single structure

Whole ensemble

- SIENA

$\neg$ Clustering

$\multimap$ Random

Average single structure

\section{_ Best single} structure

Whole ensemble

- SIENA

$\neg$ Clustering

$\multimap$ Random

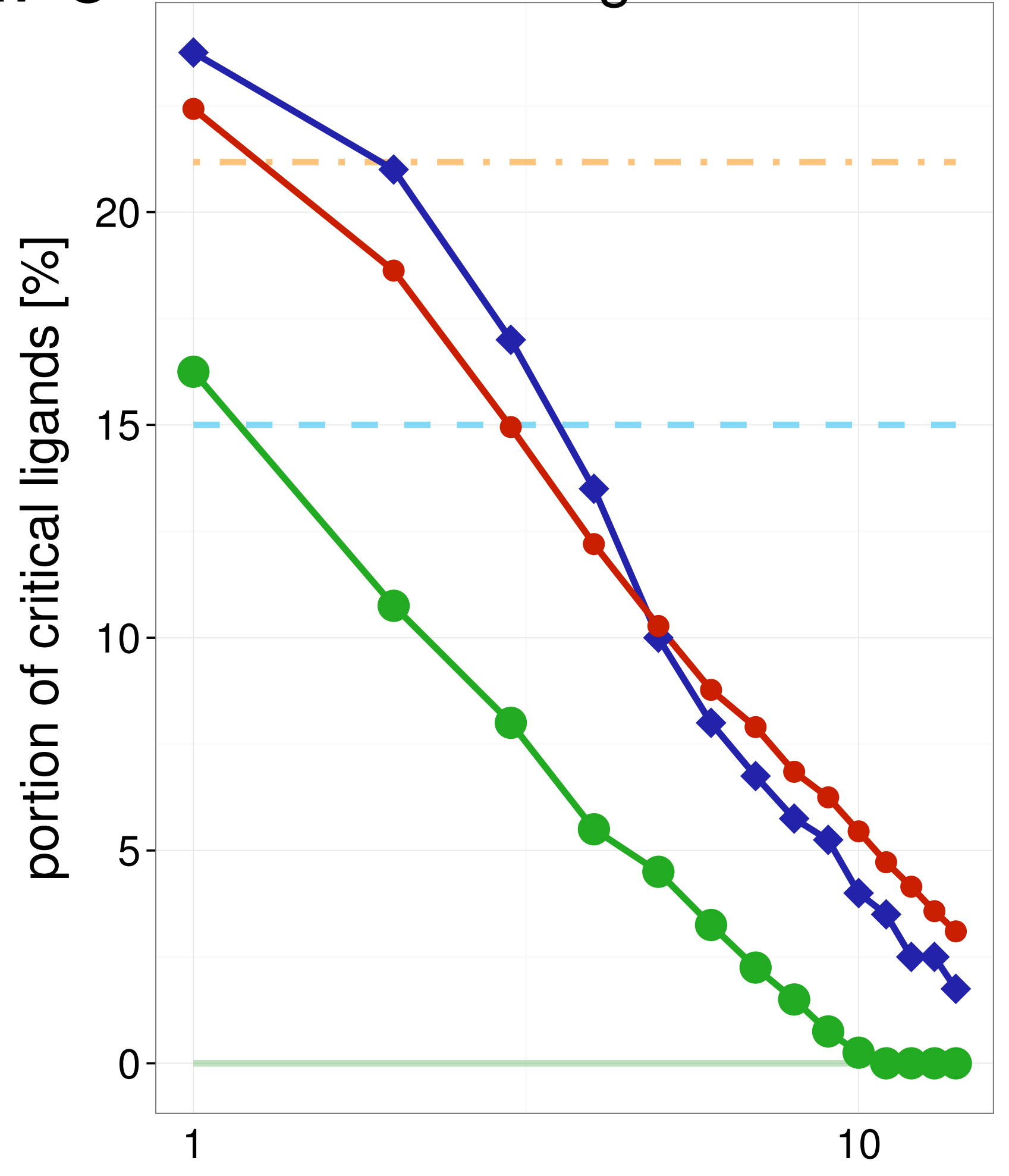

maximal ensemble size

Test set

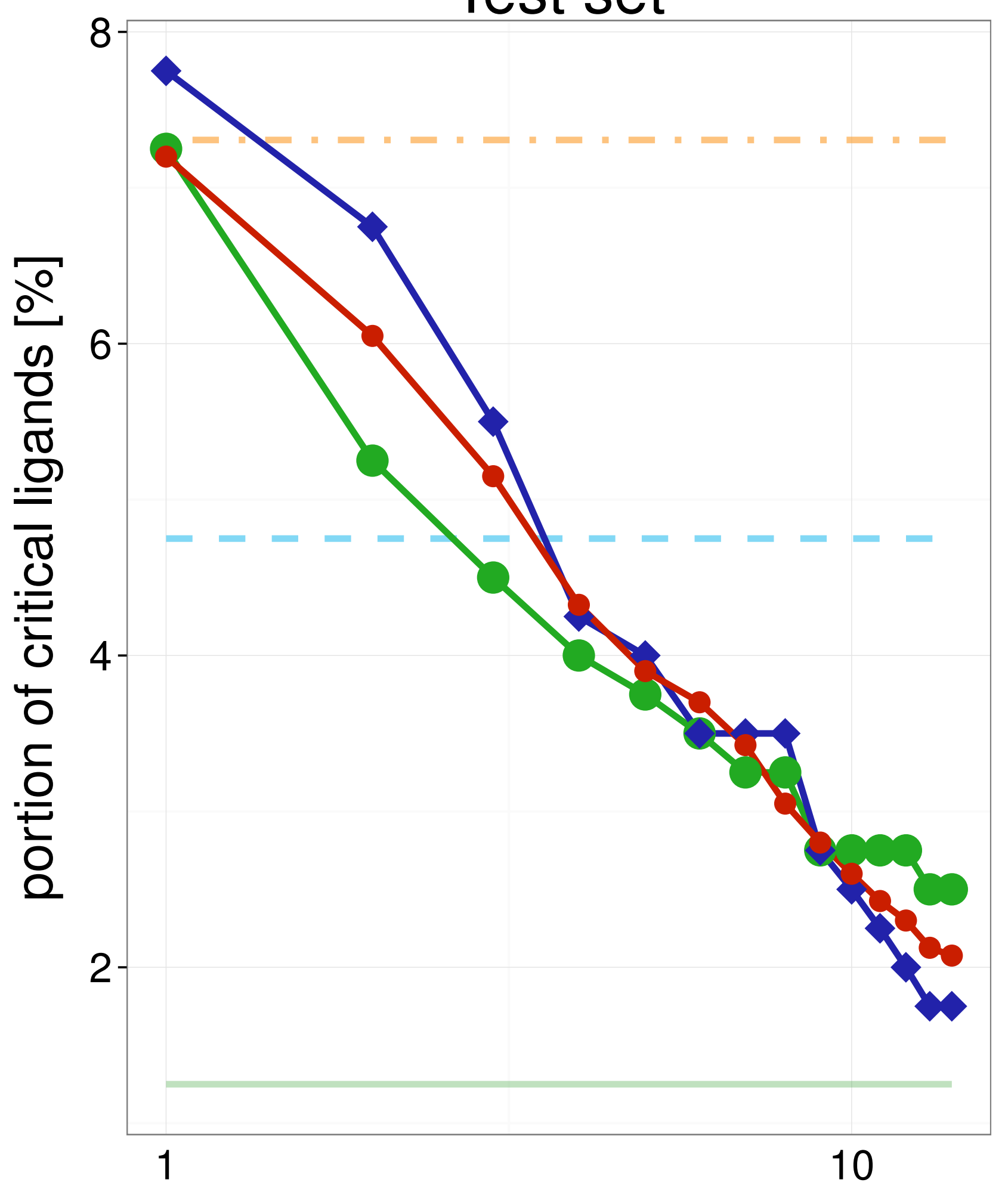

maximal ensemble size
Average single structure

_ Best single structure

Whole ensemble

SIENA

$\leadsto$ Clustering

$\multimap$ Random 
Training set

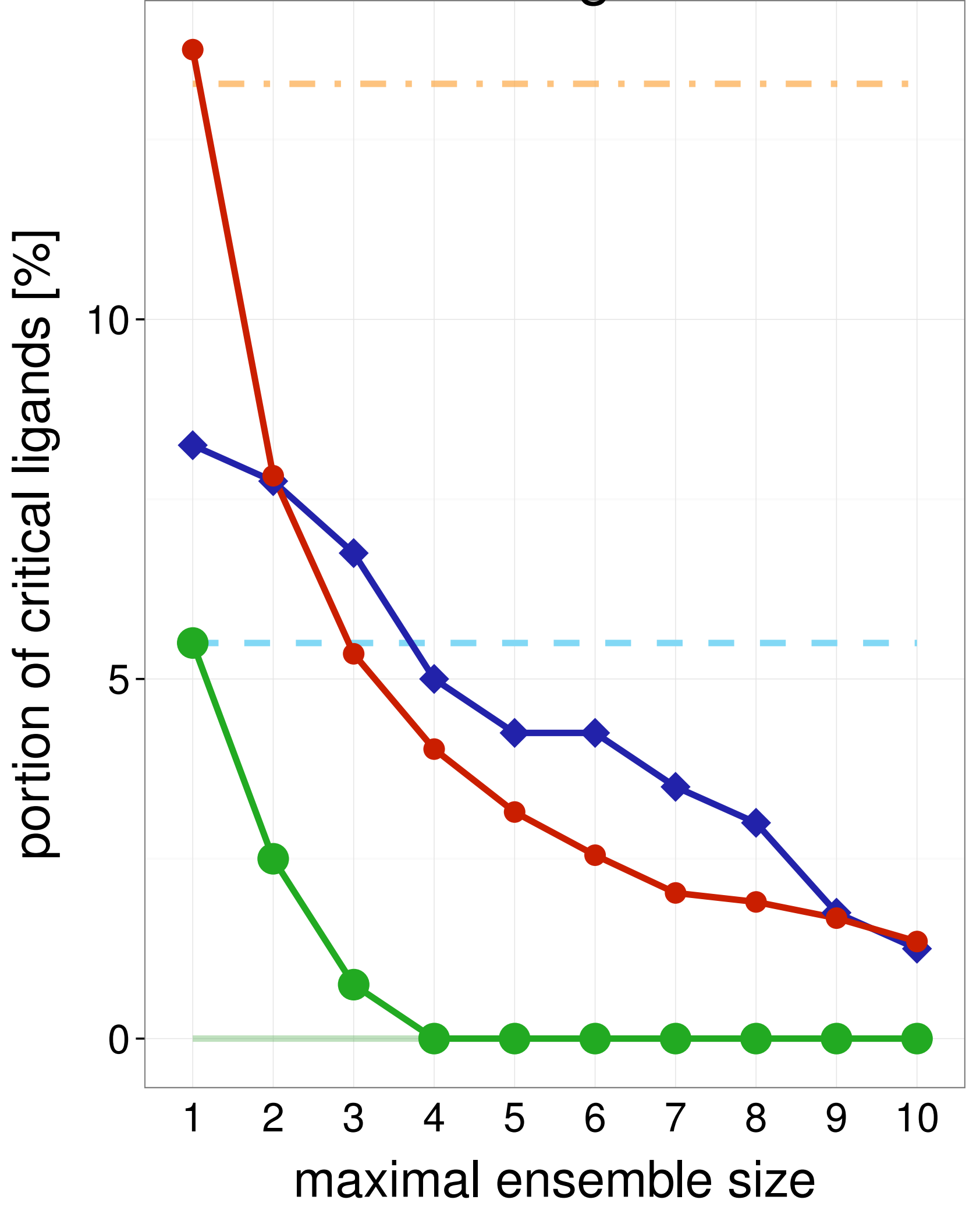

Test set

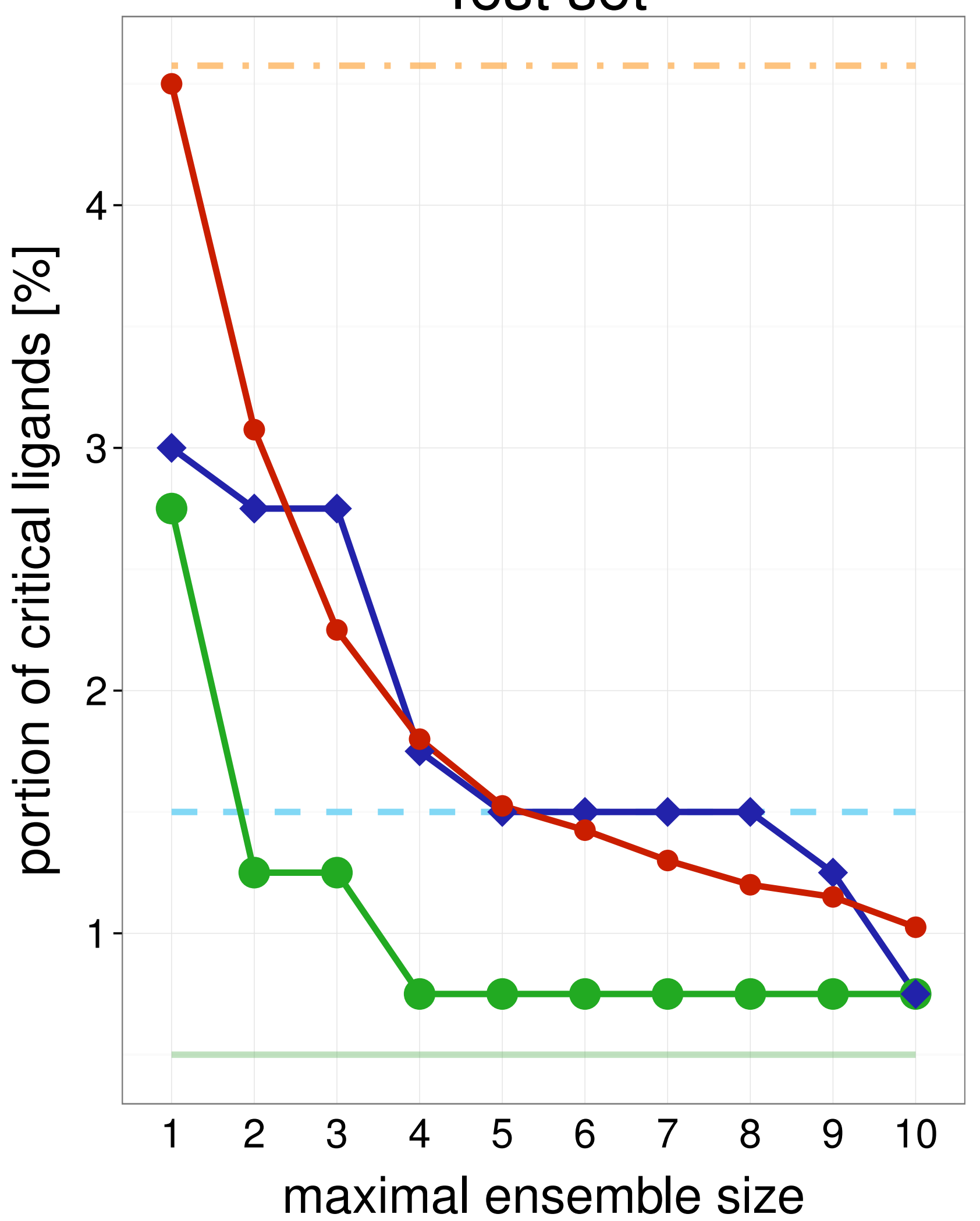

Average single structure

_ _ Best single structure

Whole ensemble

- SIENA

$\neg$ Clustering

$\rightarrow$ Random

Average single structure

_ Best single structure

Whole ensemble

- SIENA

$\neg$ Clustering

- Random

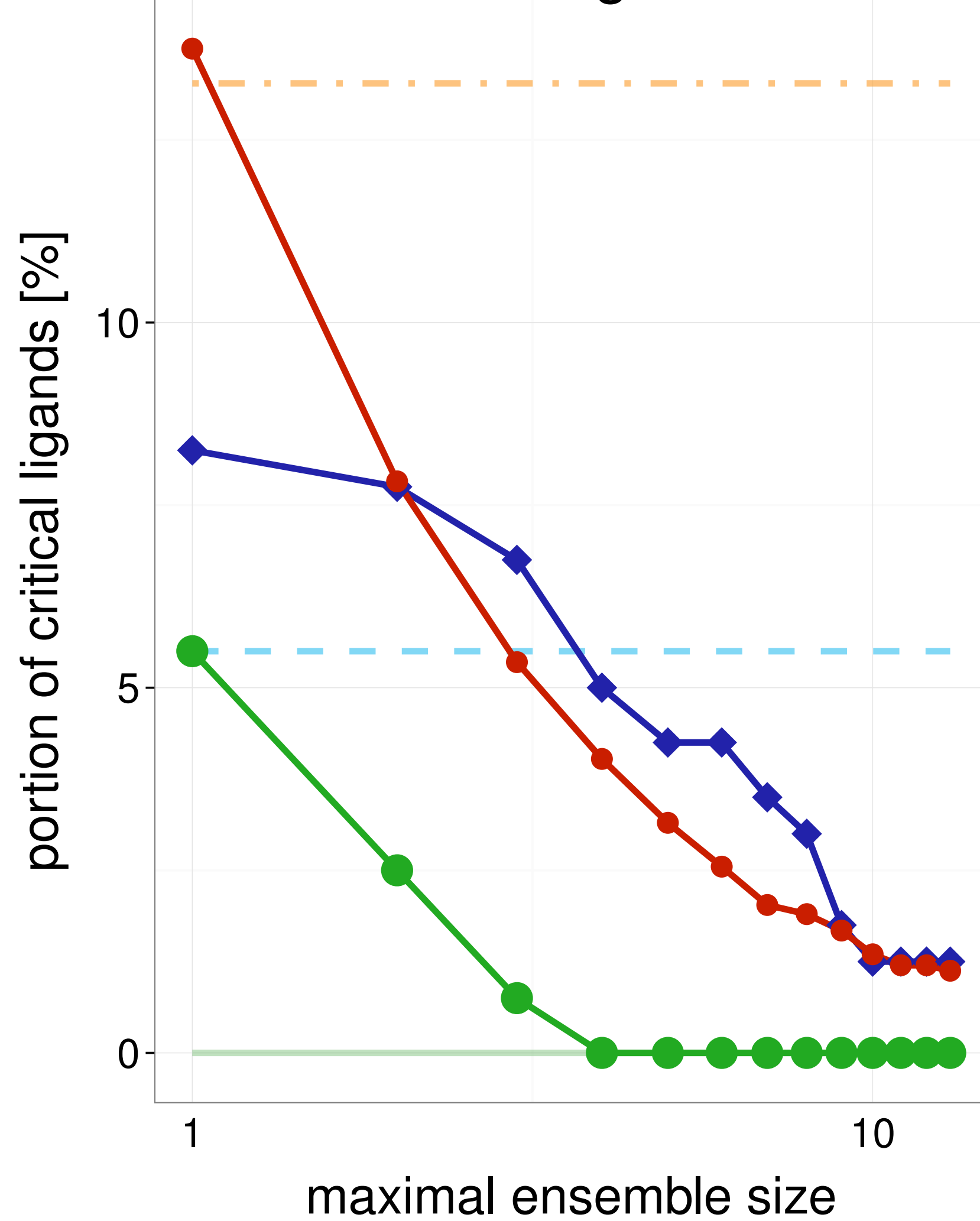

Average single structure

- Best single structure

Whole ensemble

- SIENA

$\checkmark$ Clustering

$\rightarrow$ Random

Test set

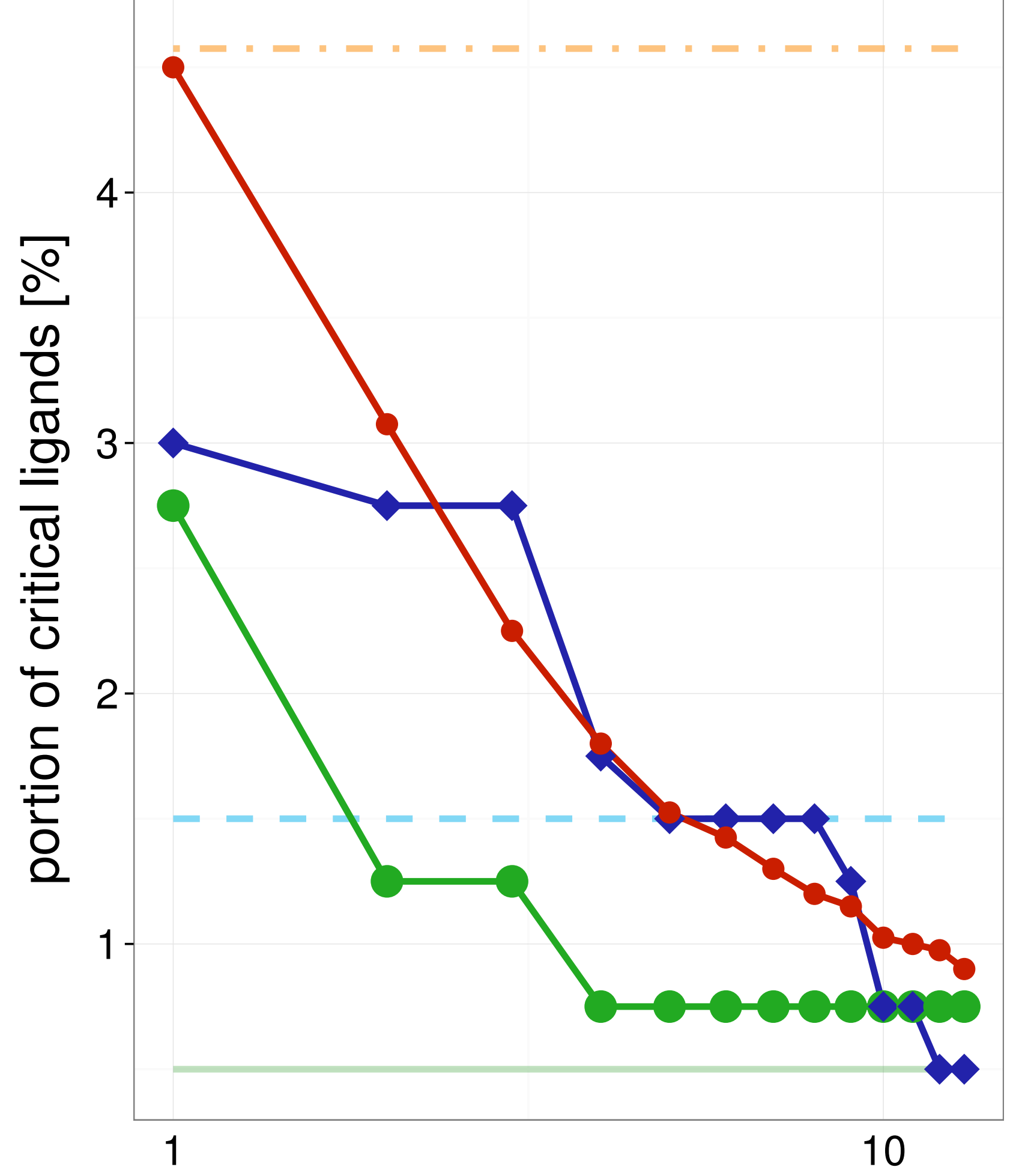

Average single structure

_ _ Best single structure

Whole ensemble

- SIENA

$\leadsto$ Clustering

$\longrightarrow$ Random 
Training set

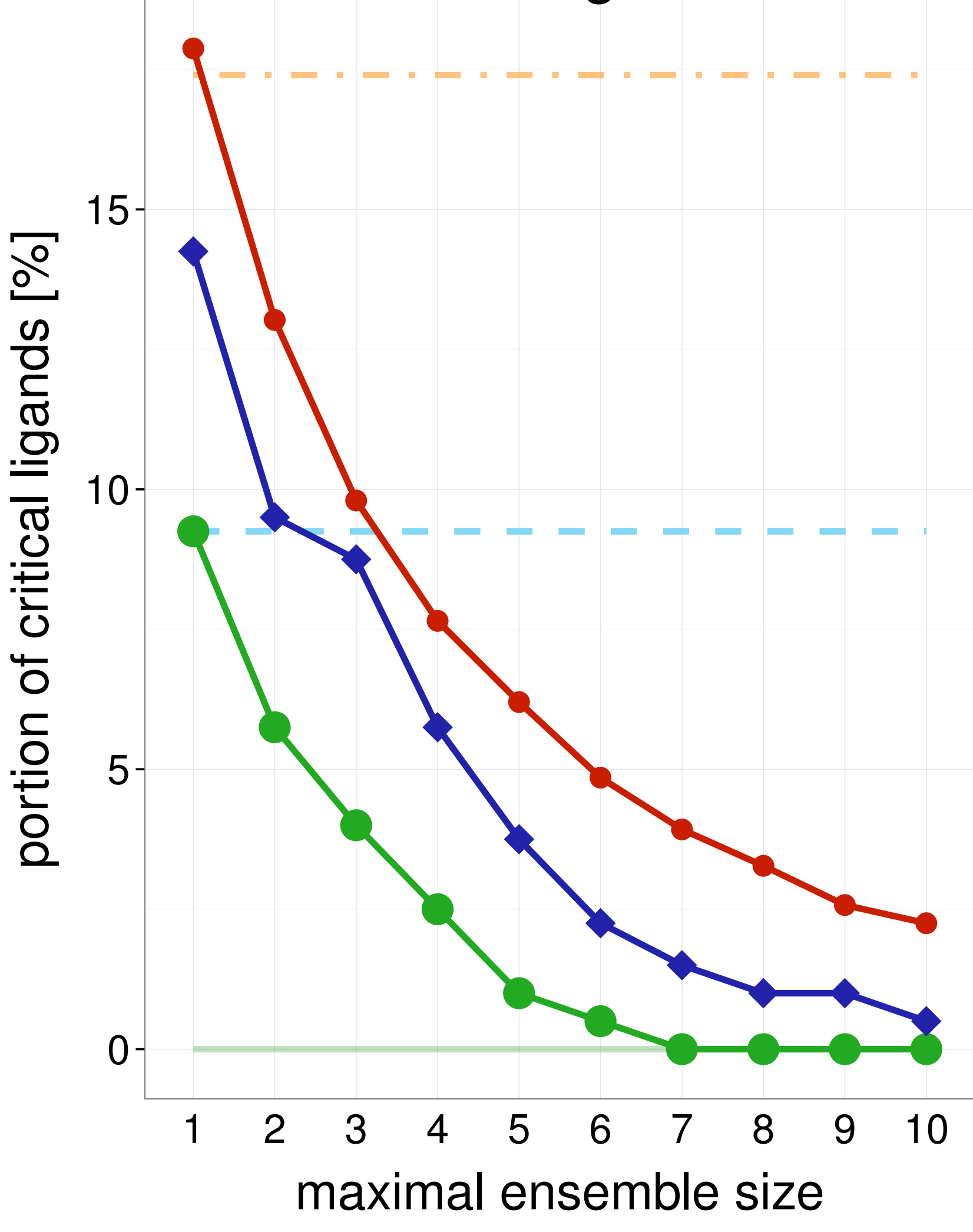

Test set

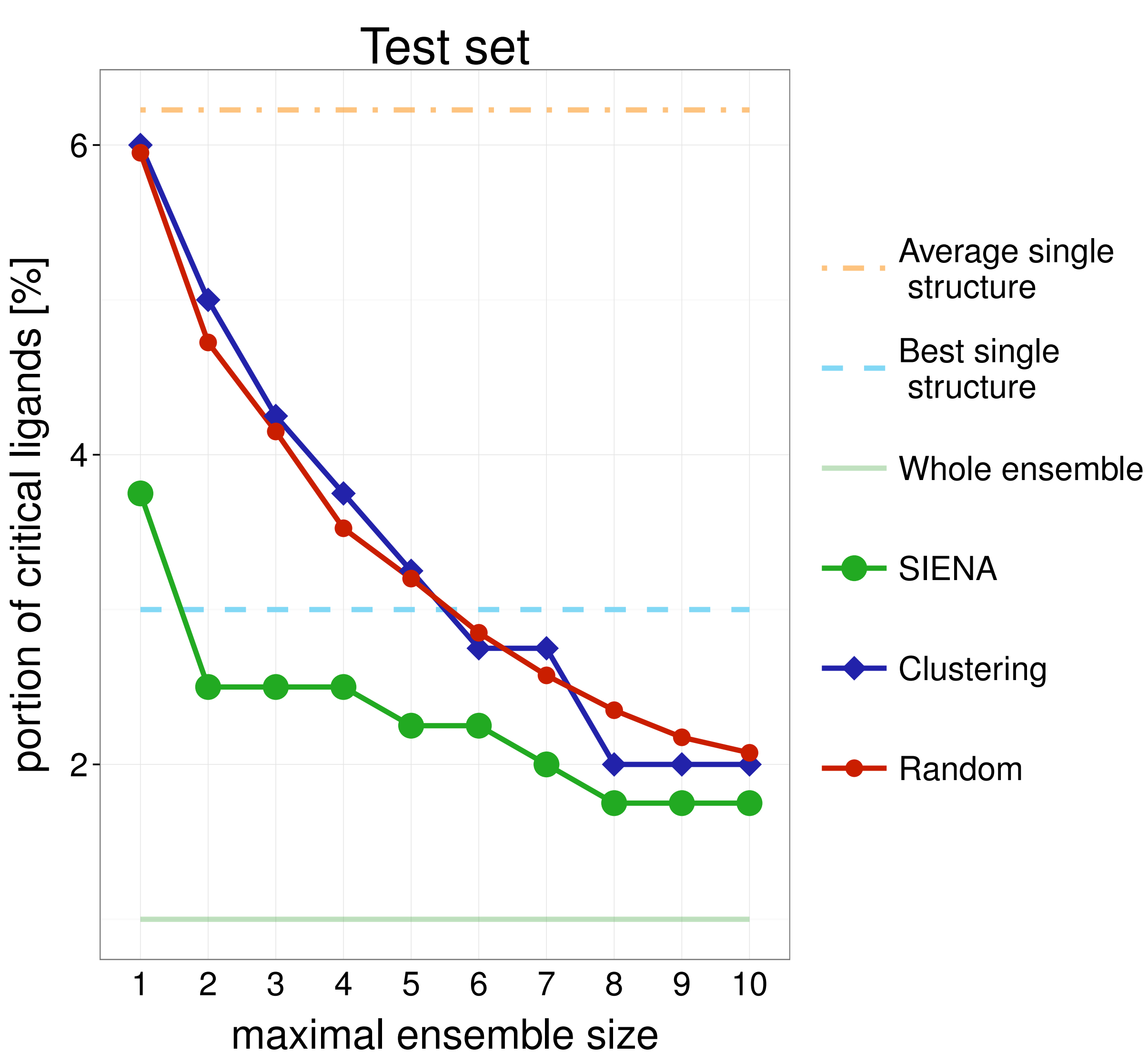

$1 Y O U \quad$ Training set

Average single structure

_ _ Best single structure

Whole ensemble

- SIENA

$\neg$ Clustering

$\multimap$ Random

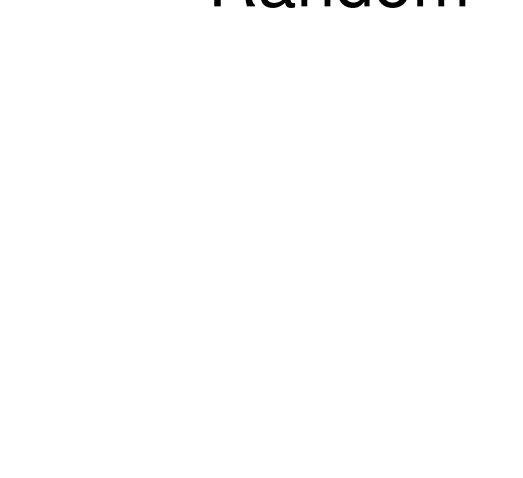

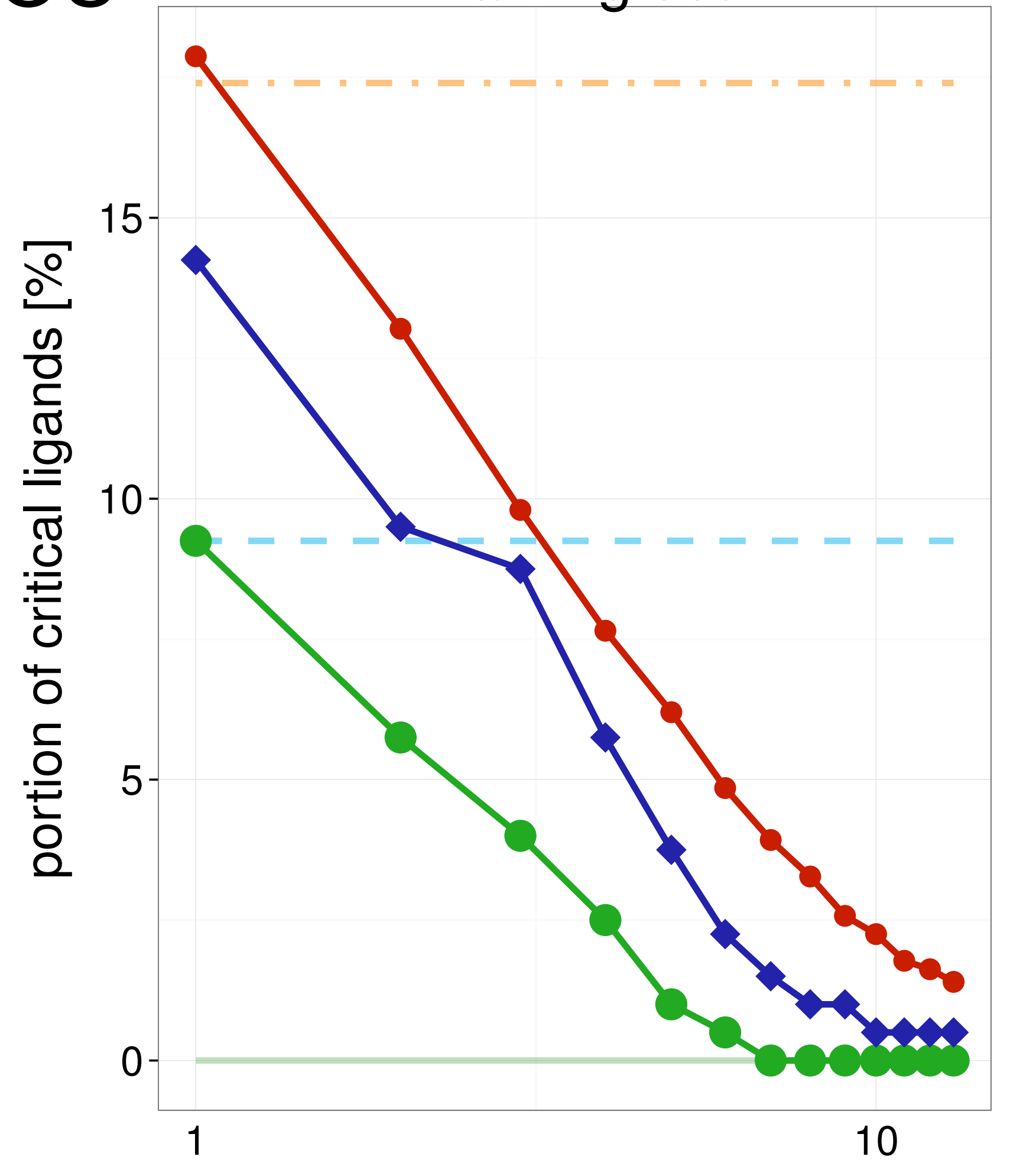

maximal ensemble size

Test set

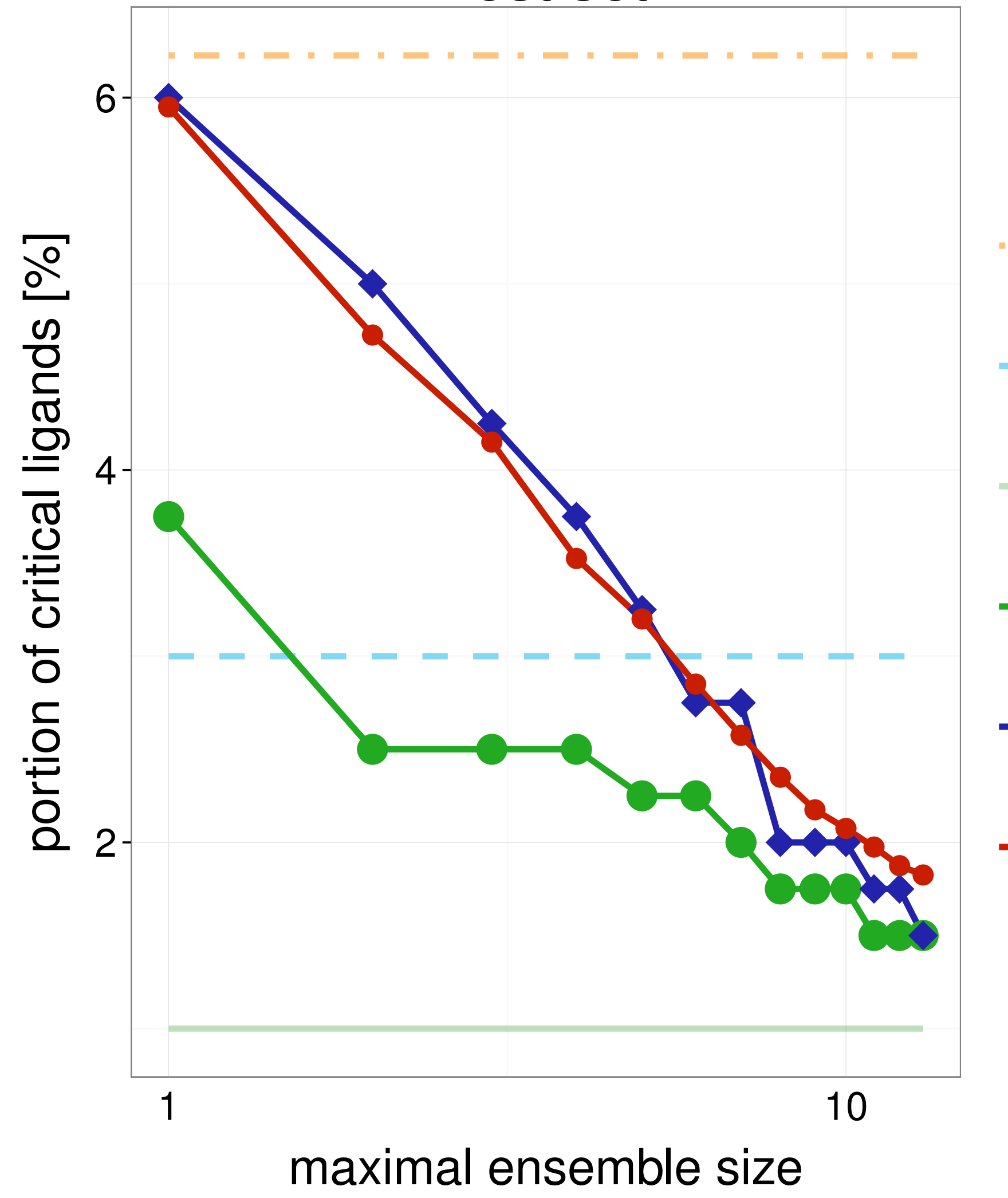

Average single structure

- Best single structure

Whole ensemble

- SIENA

$\checkmark$ Clustering

$\multimap$ Random

Average single structure

_ Best single structure

Whole ensemble

- SIENA

$\neg$ Clustering

$\multimap$ Random 


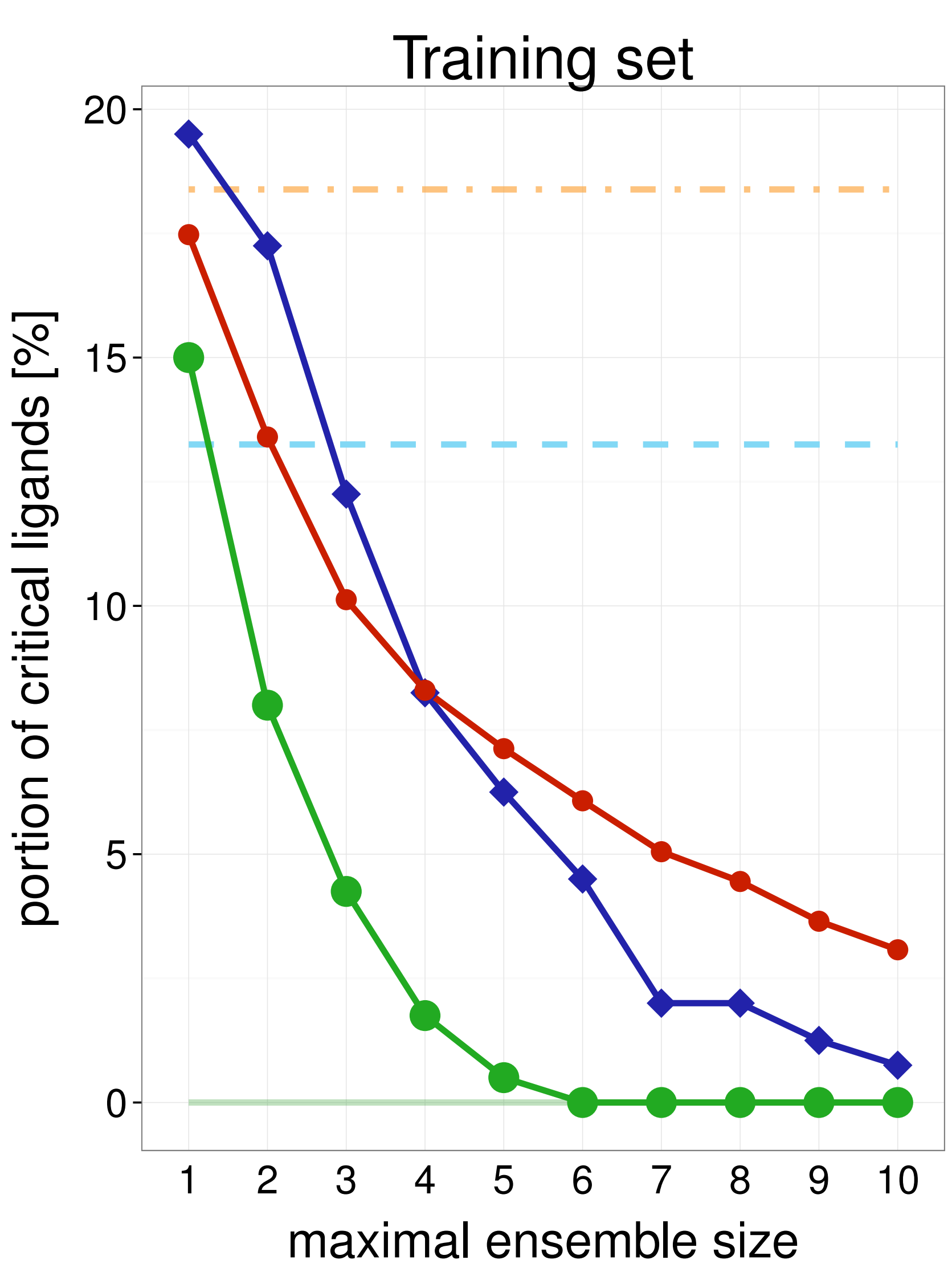

\section{Test set}

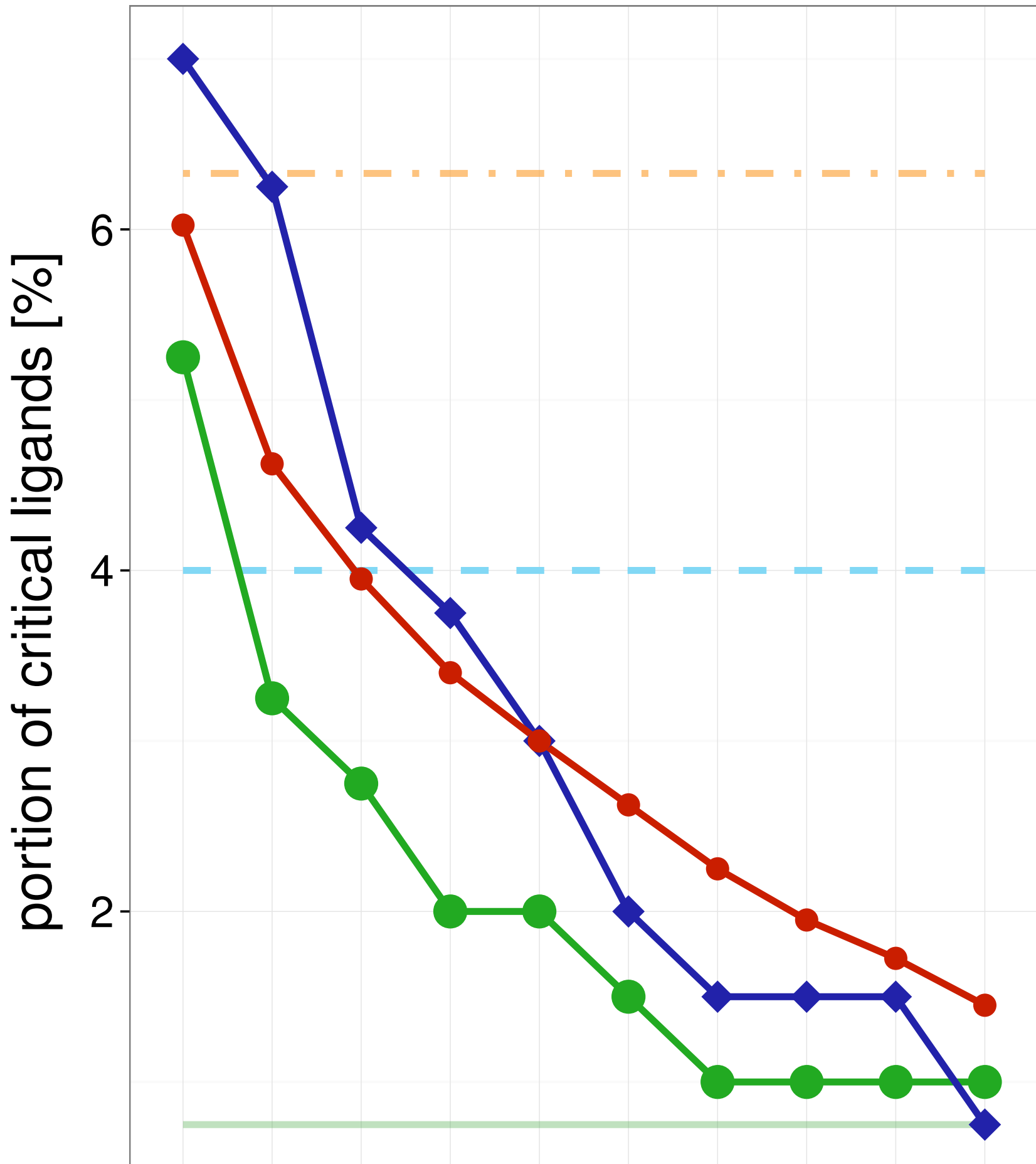

$\begin{array}{llllllllll}1 & 2 & 3 & 4 & 5 & 6 & 7 & 8 & 9 & 10\end{array}$ maximal ensemble size
2FLR

Training set

Average single structure

_ Best single structure

Whole ensemble

- SIENA

$\neg$ Clustering

$\rightarrow$ Random

Average single structure

_ Best single structure

Whole ensemble

- SIENA

$\neg$ Clustering

$\multimap$ Random

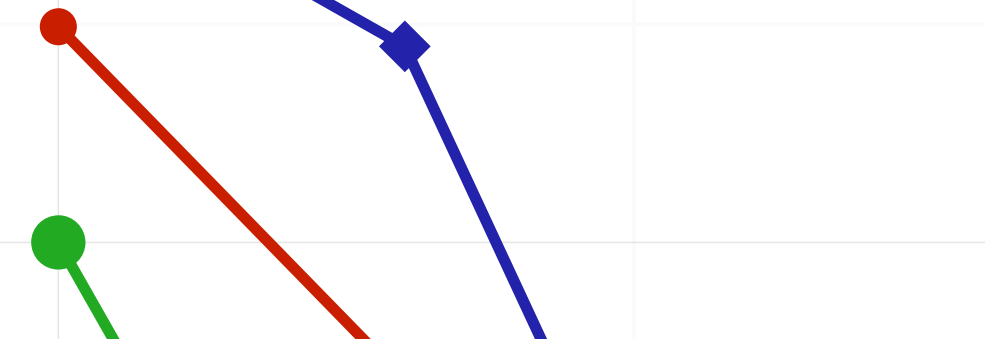

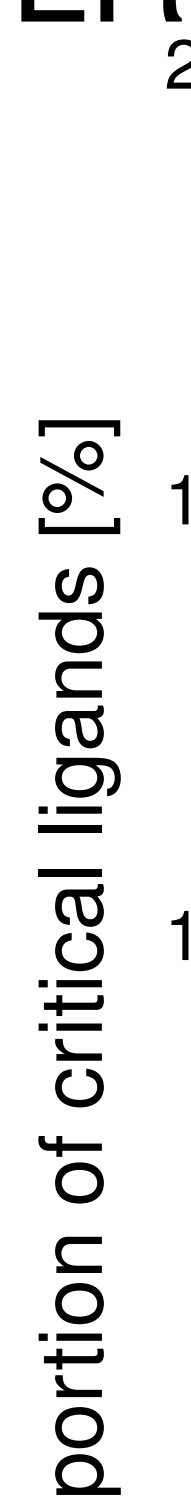
$20-$

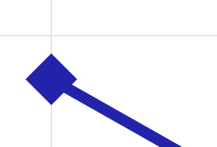

1

maximal ensemble size

Test set

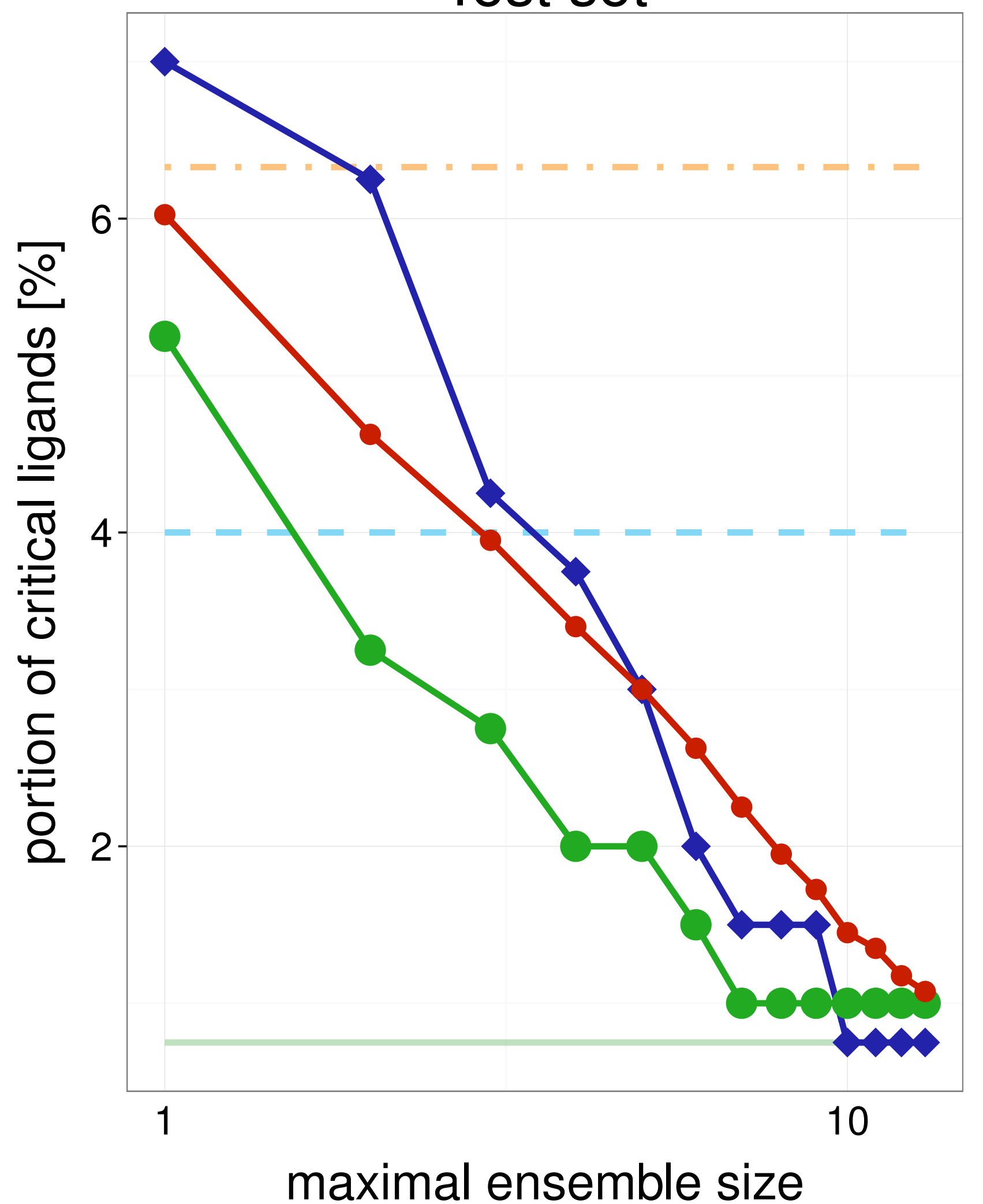

Average single structure

Best single structure

Whole ensemble

- SIENA

$\neg$ Clustering

$\multimap$ Random

Average single structure

_ _ Best single structure

Whole ensemble

- SIENA

$\neg$ Clustering

$\multimap$ Random 


\section{Training set}

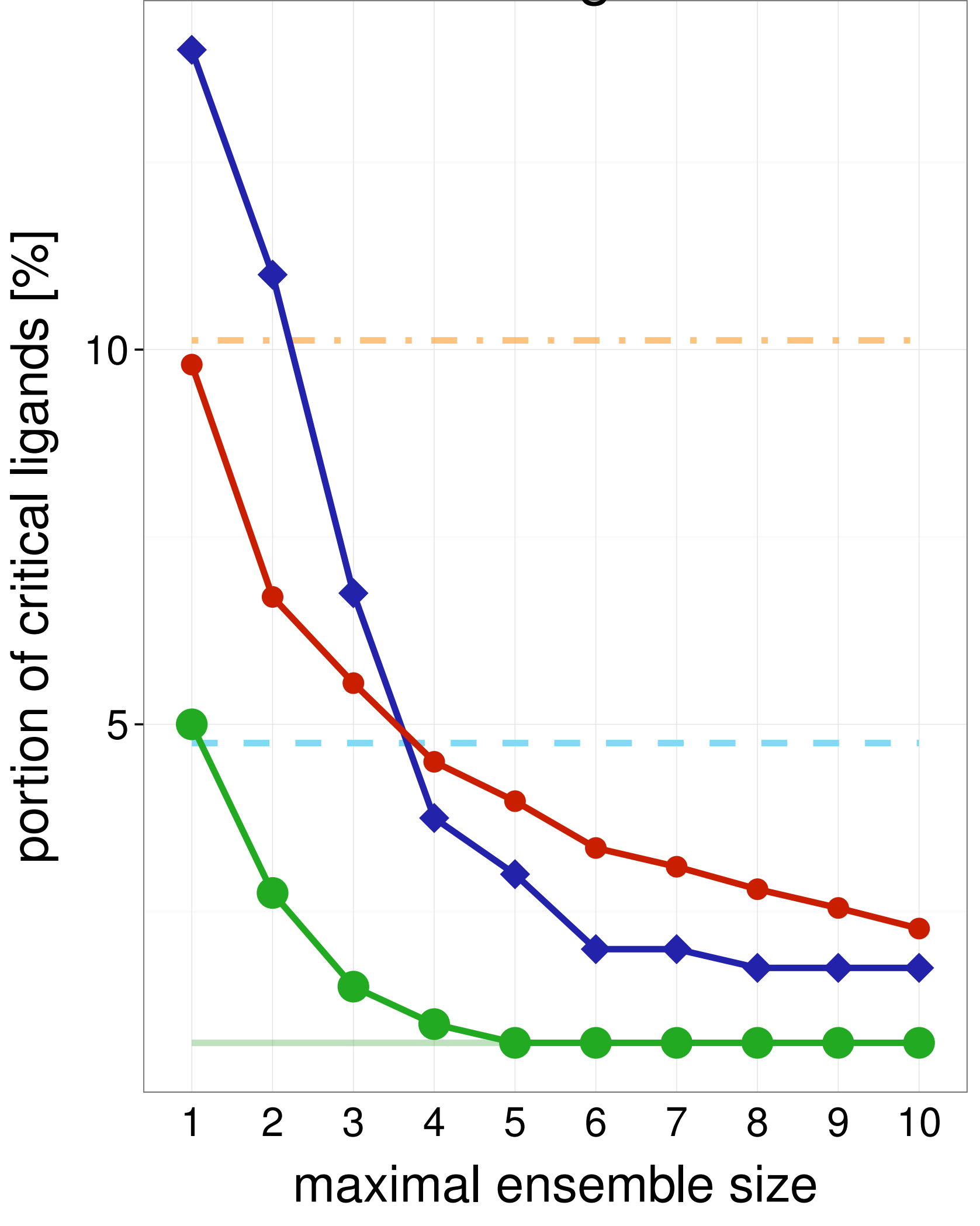

\section{Test set}

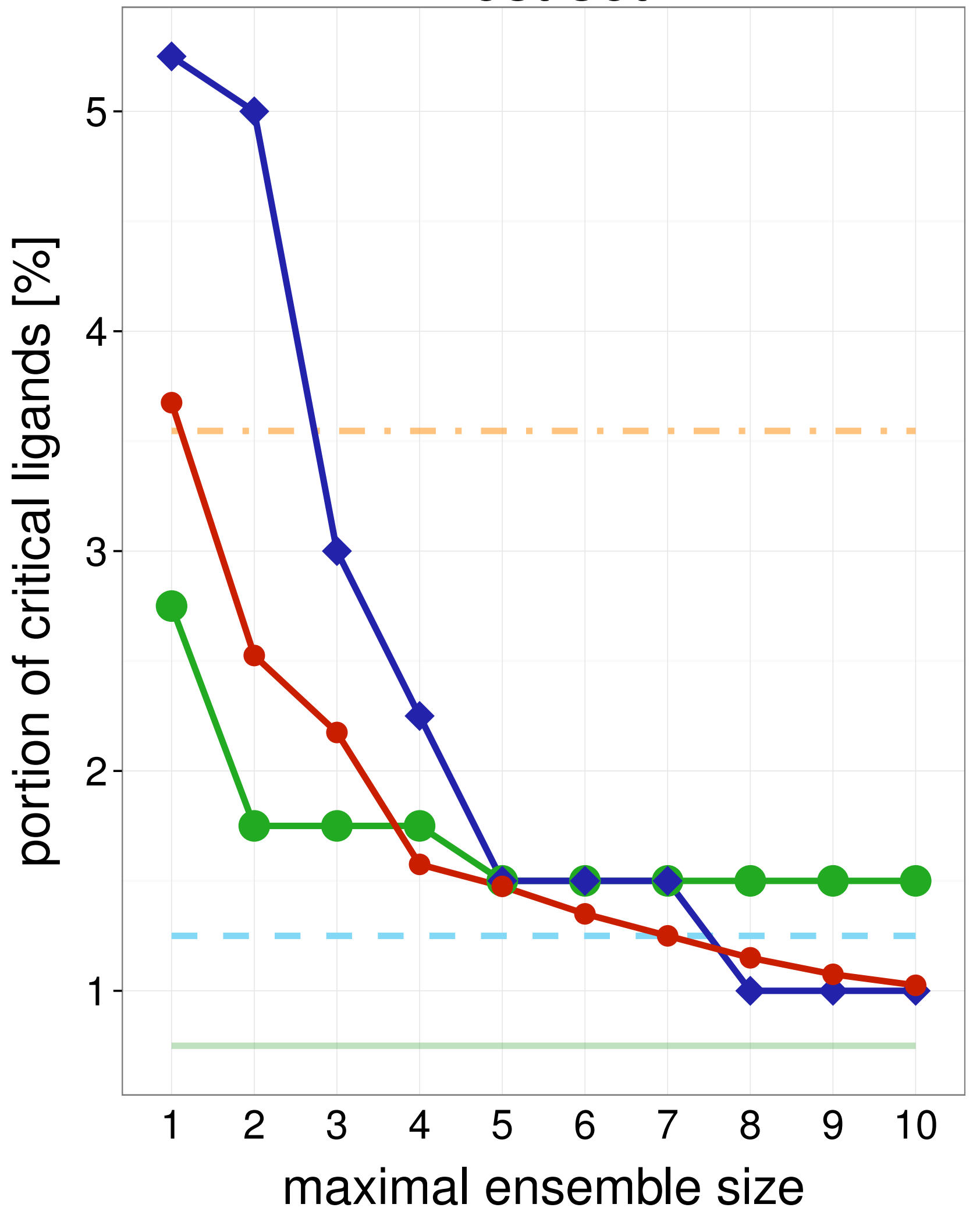

Average single structure

_ Best single structure

Whole ensemble

- SIENA

$\neg$ Clustering

$\rightarrow$ Random

Average single structure

_ Best single structure

Whole ensemble

- SIENA

$\smile$ Clustering

$\multimap$ Random
$3 \mathrm{CHP}$

Training set

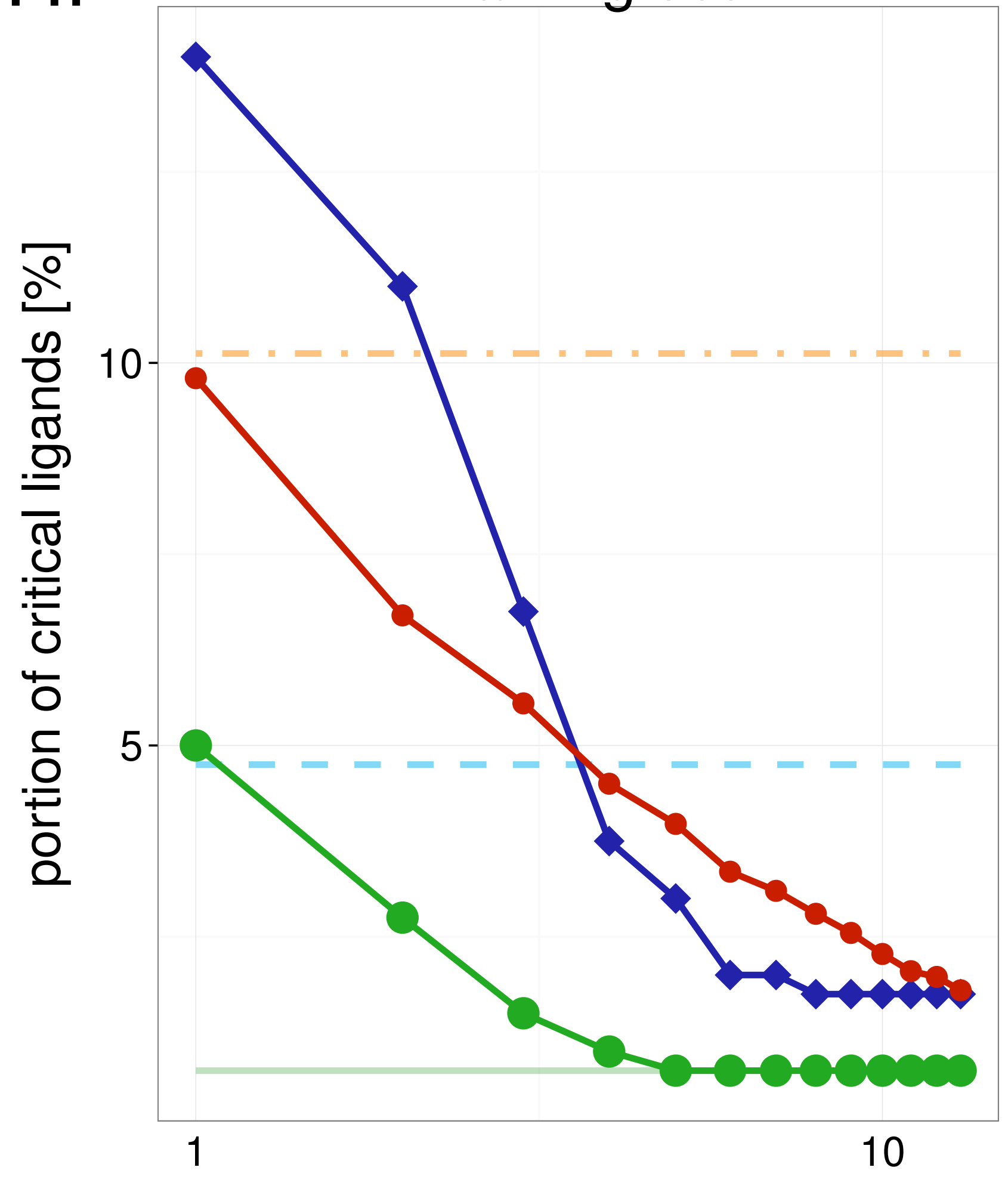

maximal ensemble size

Test set

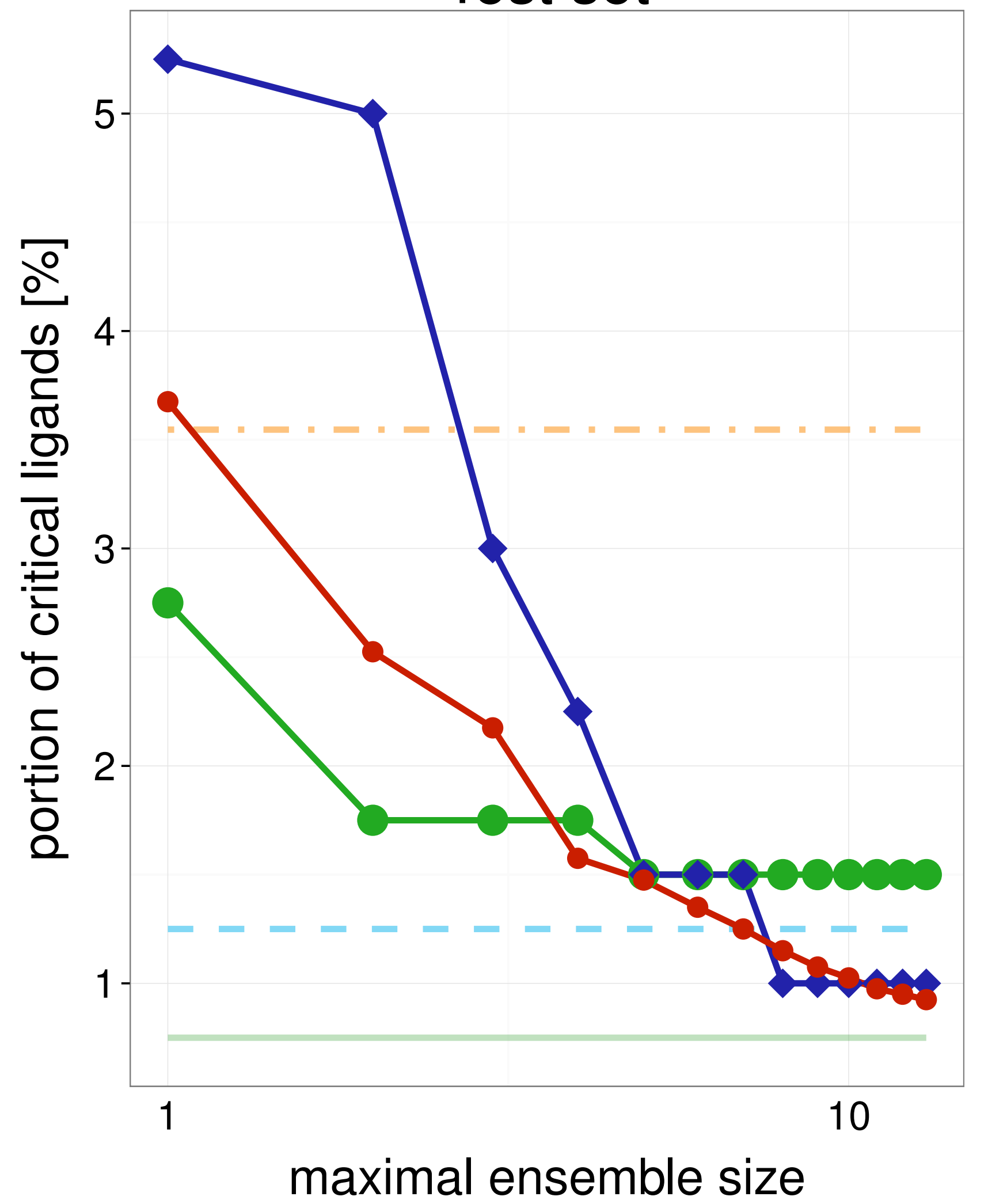

Average single structure

Best single structure

Whole ensemble

- SIENA

$\neg$ Clustering

$\multimap$ Random

Average single structure

_ Best single structure

Whole ensemble

SIENA

$\neg$ Clustering

$\longrightarrow$ Random 


\section{Training set}

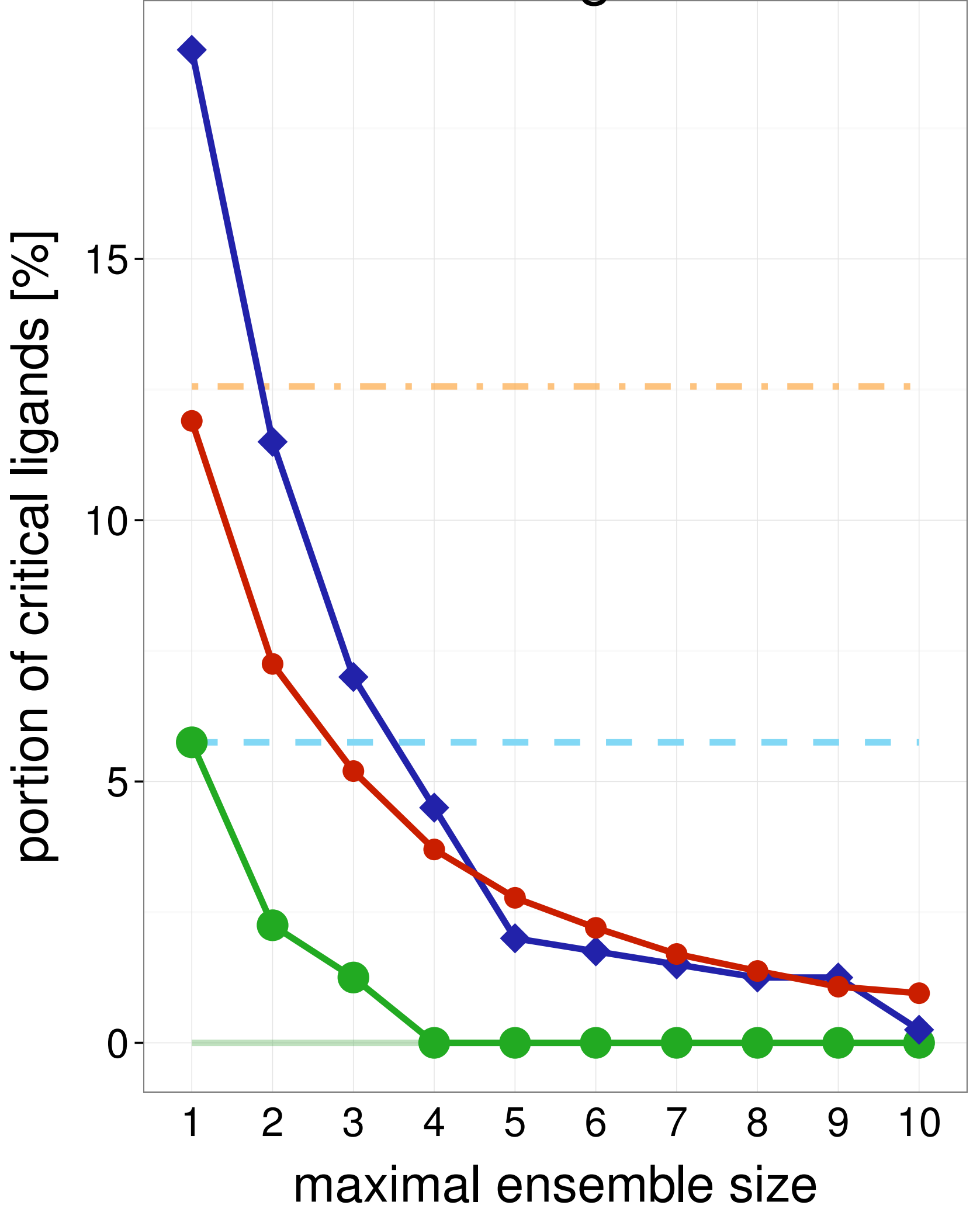

\section{Test set}

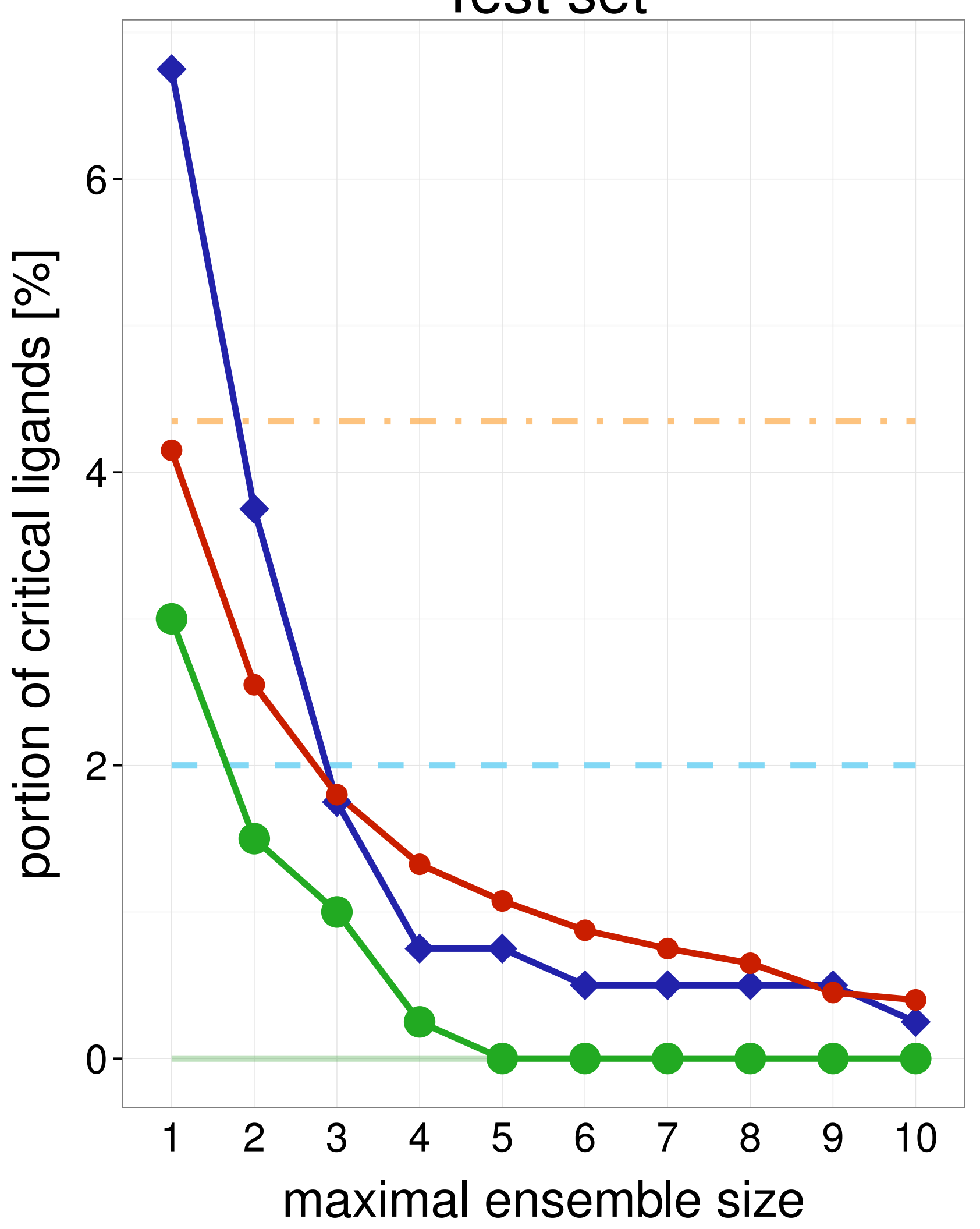

3ZWS

Training set

Average single structure

_ Best single structure

Whole ensemble

- SIENA

$\neg$ Clustering

$\multimap$ Random

Average single structure

_ _ Best single structure

Whole ensemble

- SIENA

$\neg$ Clustering

$\multimap$ Random

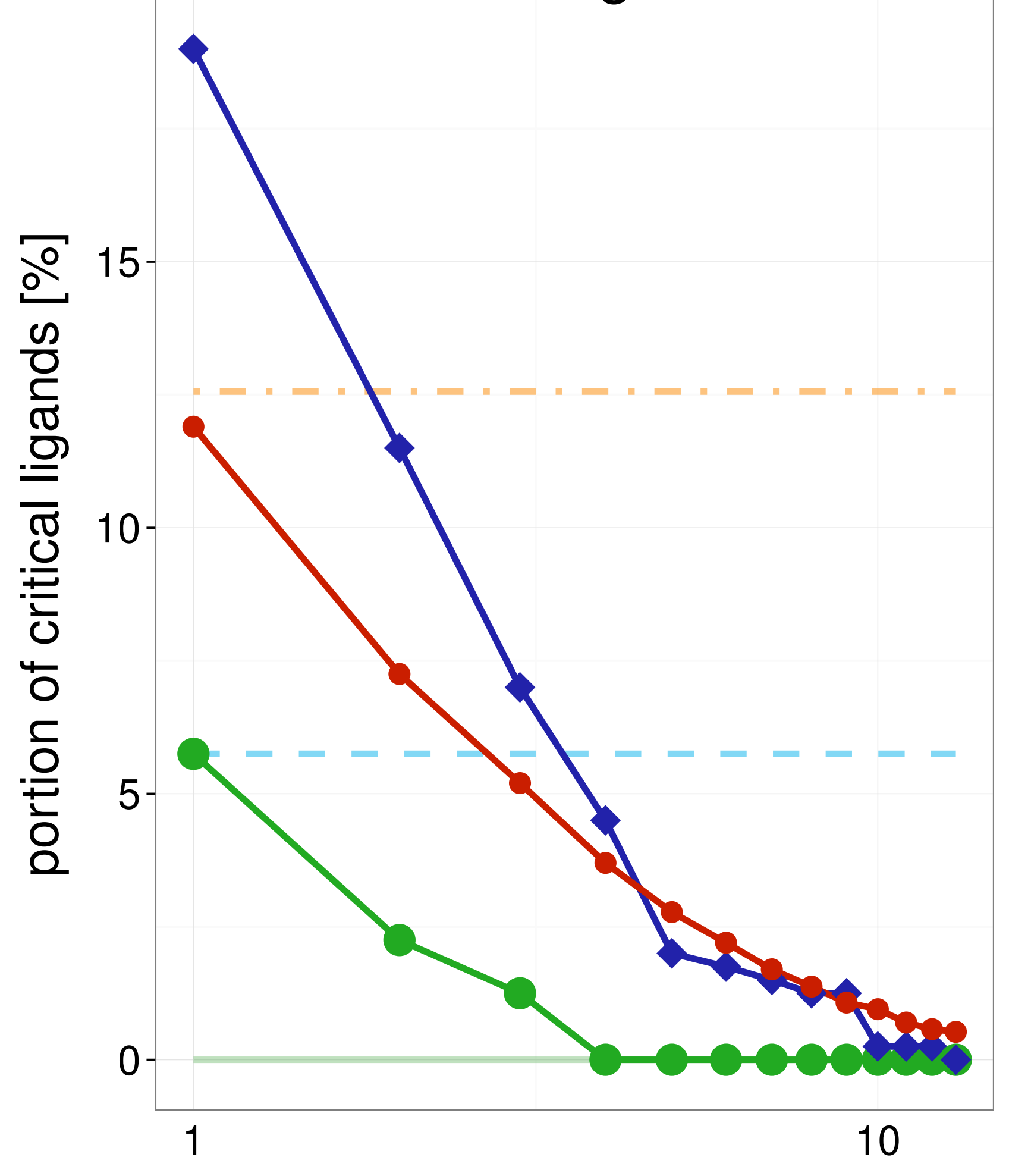

maximal ensemble size

Test set

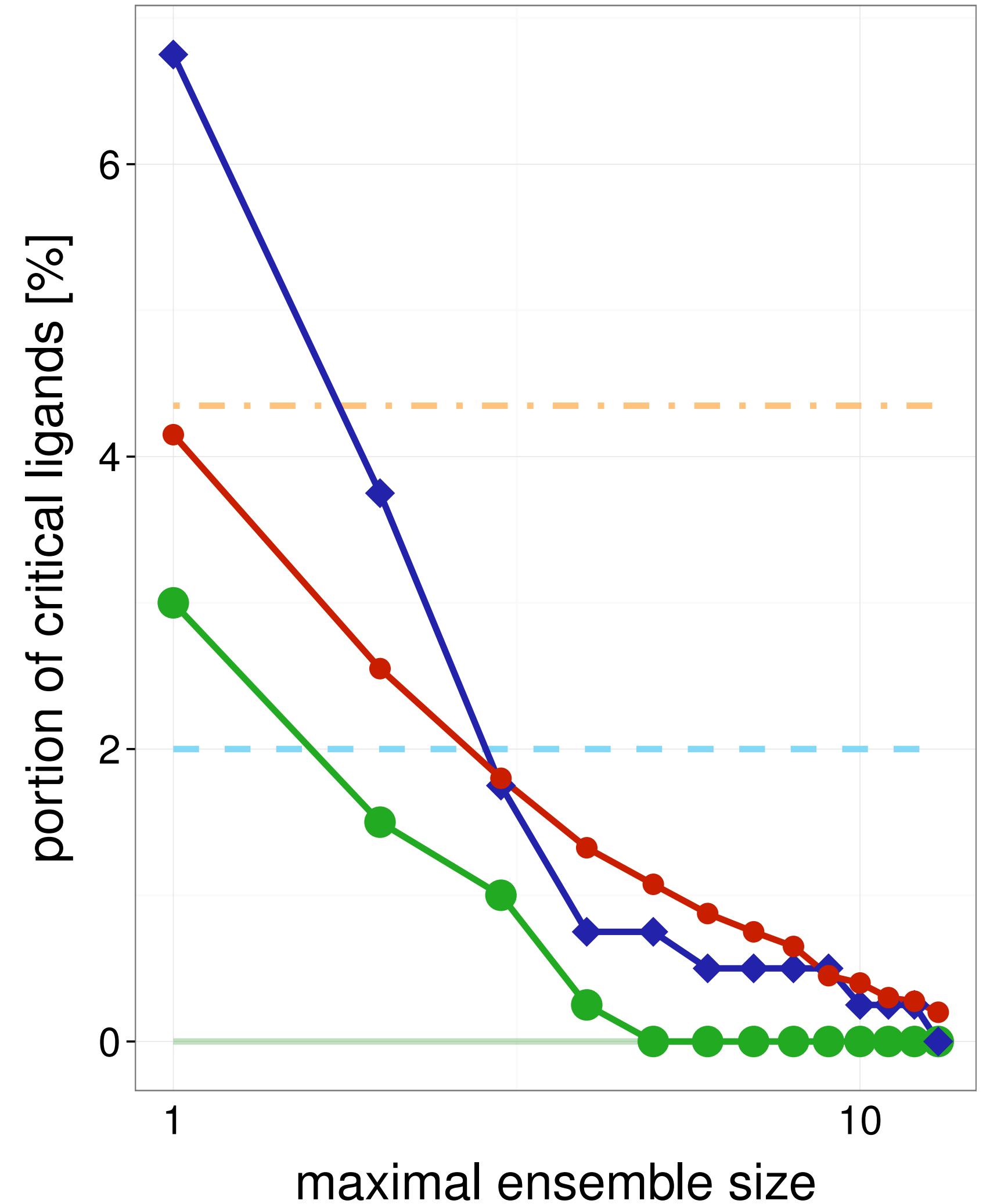

Average single structure

Best single structure

Whole ensemble

- SIENA

$\neg$ Clustering

- Random

Average single structure

_ Best single structure

Whole ensemble

SIENA

$\neg$ Clustering

$\longrightarrow$ Random 
Training set

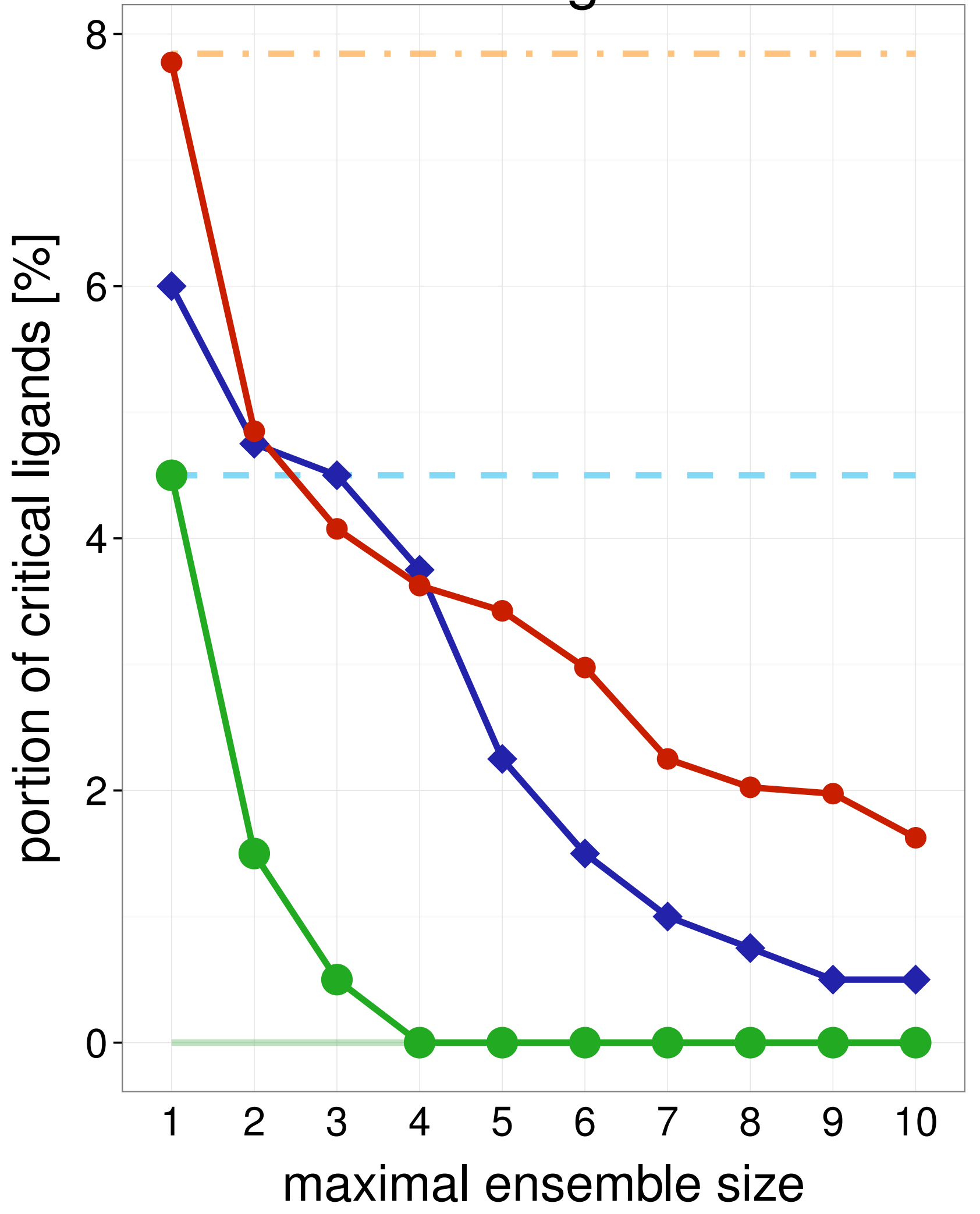

Test set

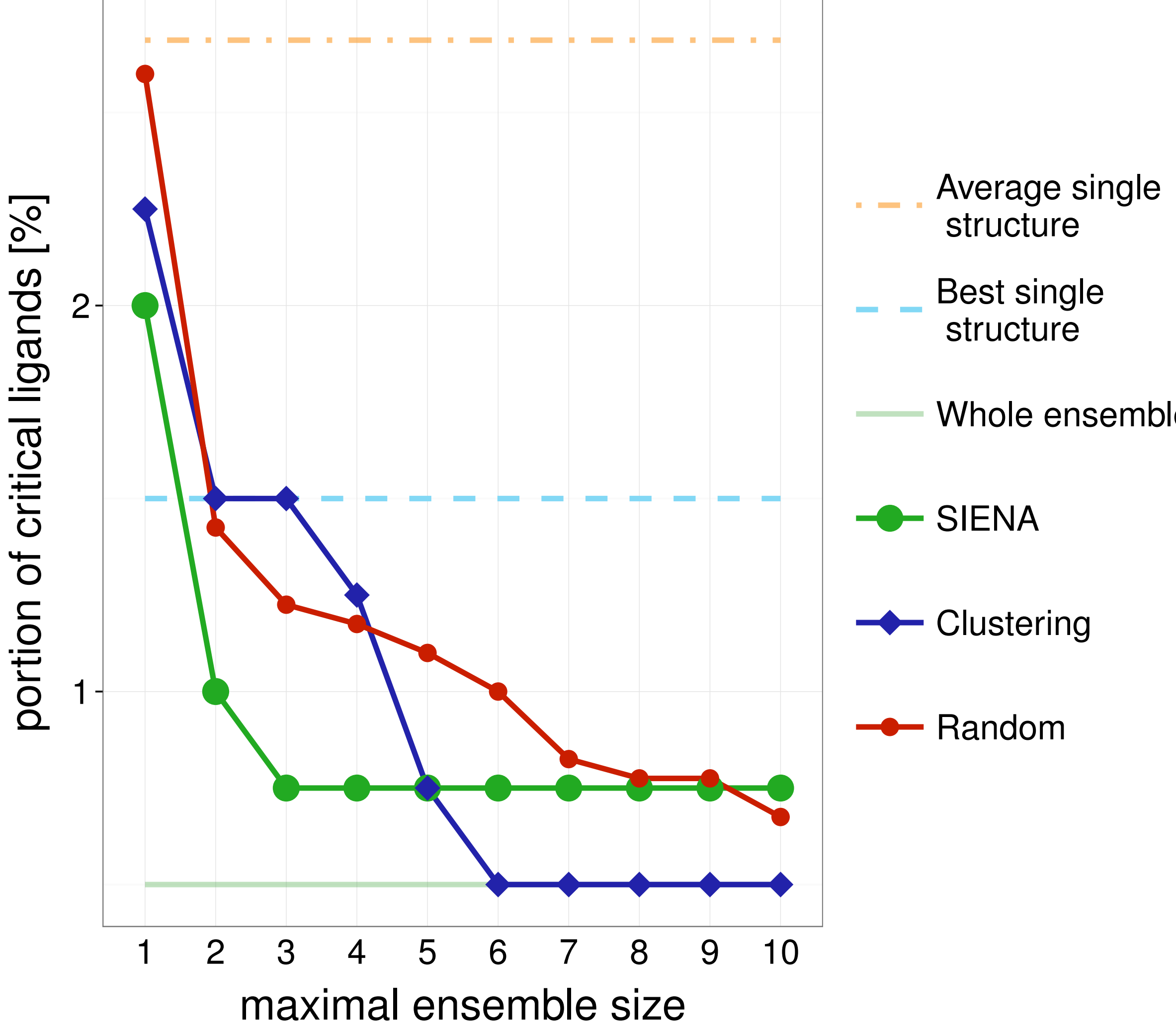

Average single structure

_ Best single structure

- SIENA

$\neg$ Clustering

$\multimap$ Random
1IE8

Whole ensemble

1

maximal ensemble size

Test set

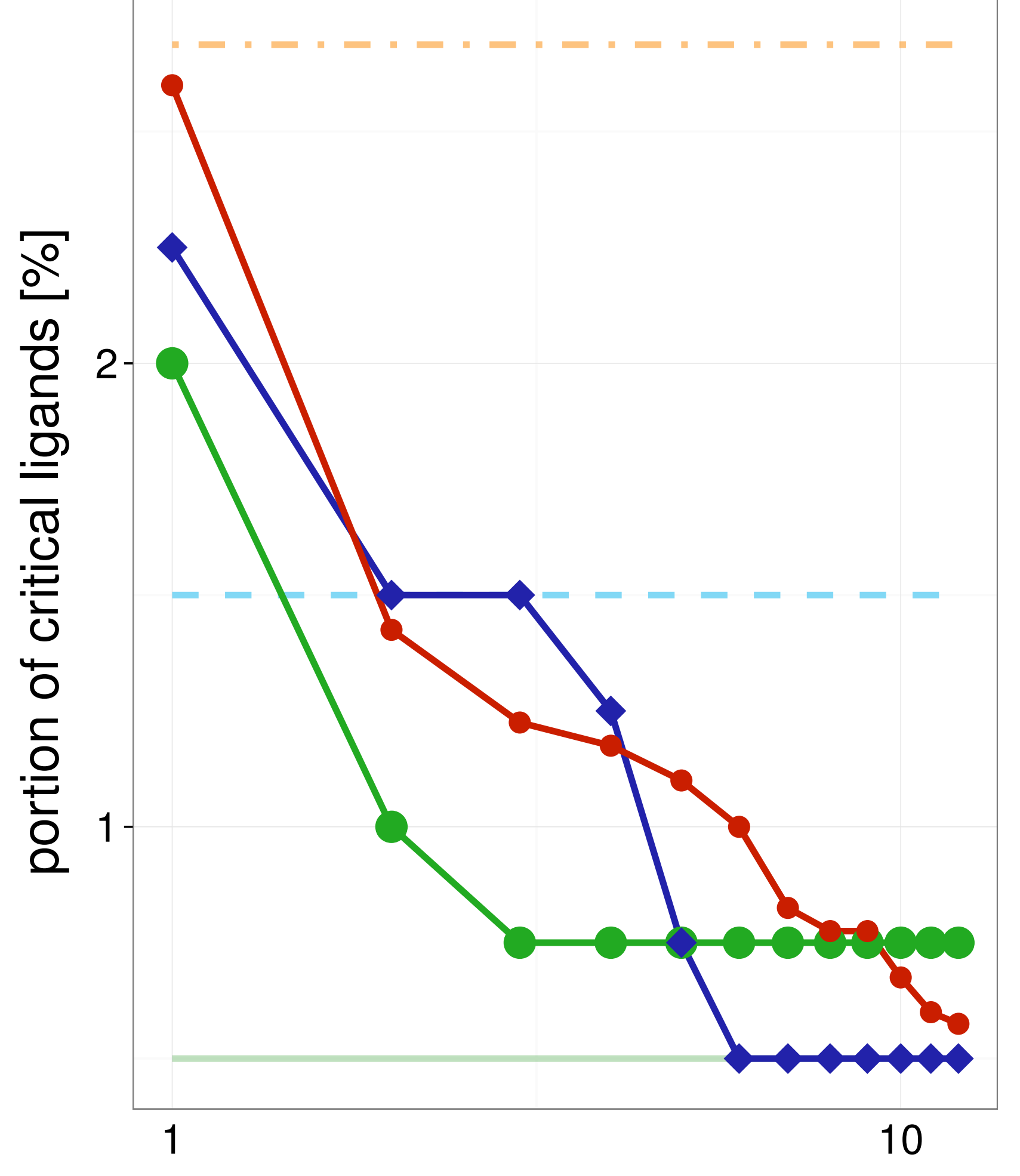

Average single structure

_ _ Best single structure

Whole ensemble

- SIENA

$\neg$ Clustering

$\multimap$ Random 
Training set

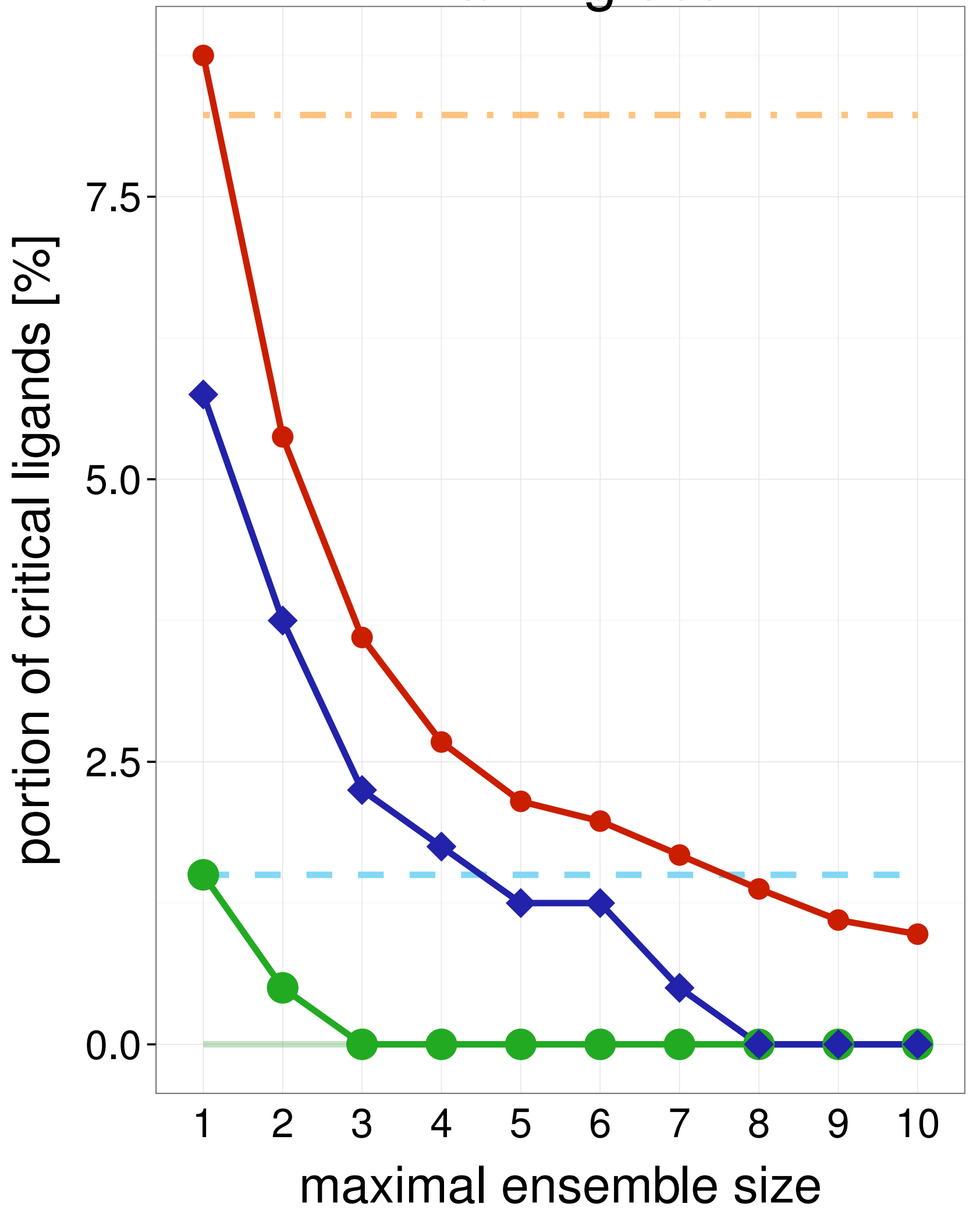

Test set

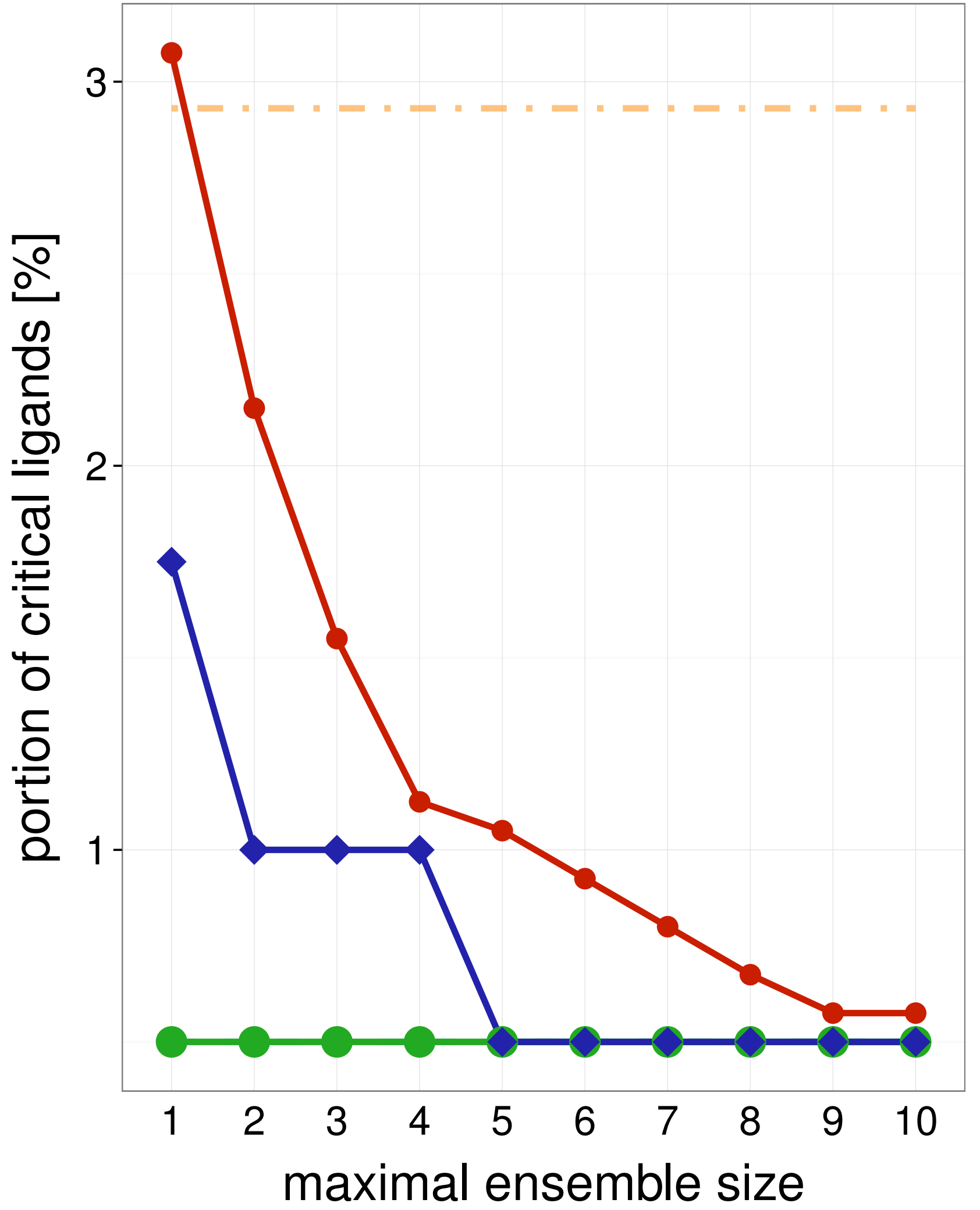

$4 M 6 Q$

Training set

Average single structure

_ _ Best single structure

Whole ensemble

- SIENA

$\smile$ Clustering

$\multimap$ Random

Average single structure

_ _ Best single structure

Whole ensemble

- SIENA

$\neg$ Clustering

$\multimap$ Random

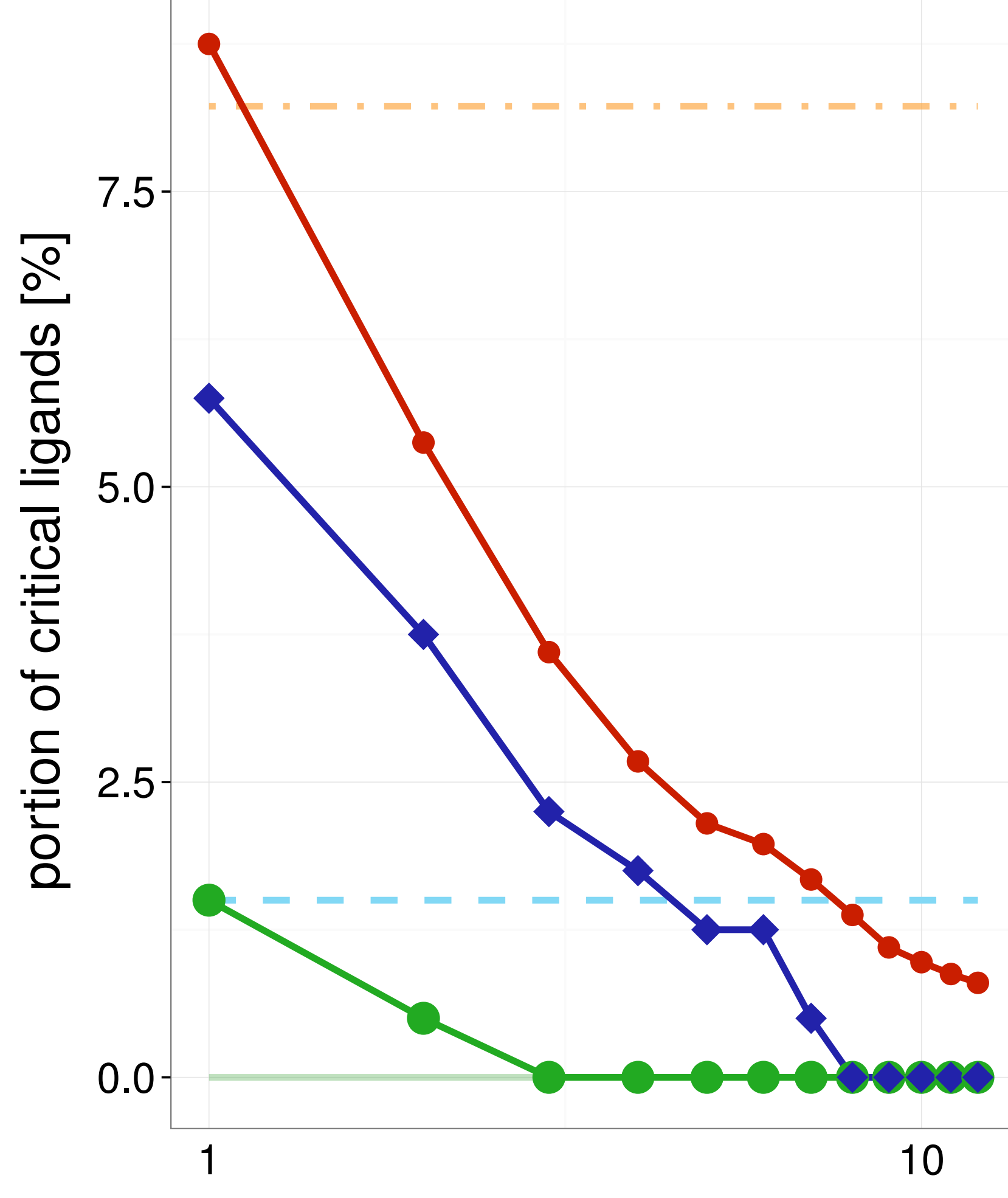

maximal ensemble size

Test set

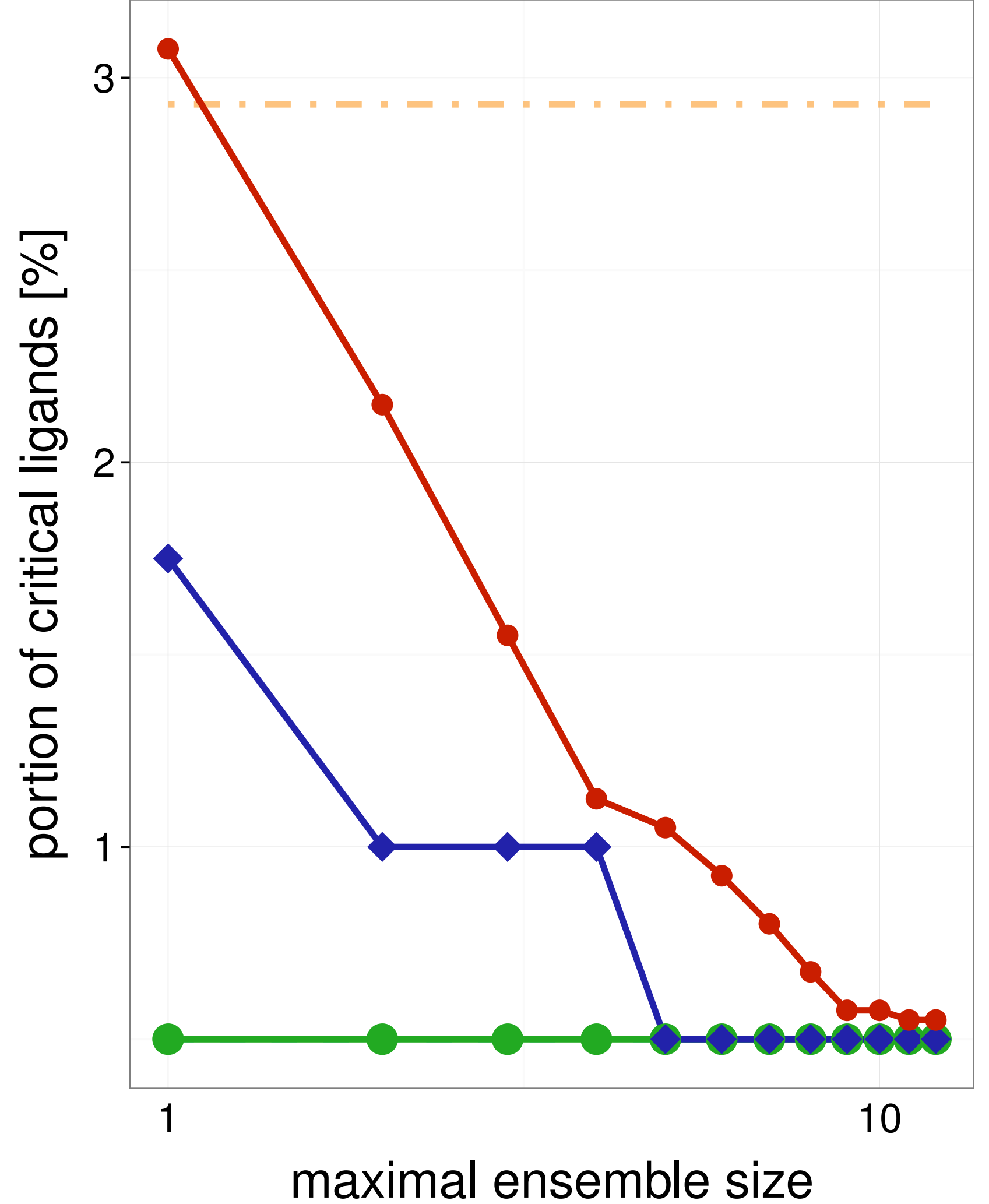

Average single structure

_ Best single structure

Whole ensemble

- SIENA

$\checkmark$ Clustering

$\multimap$ Random

Average single structure

_ Best single structure

Whole ensemble

- SIENA

$\leadsto$ Clustering

$\multimap$ Random 


\section{Training set}

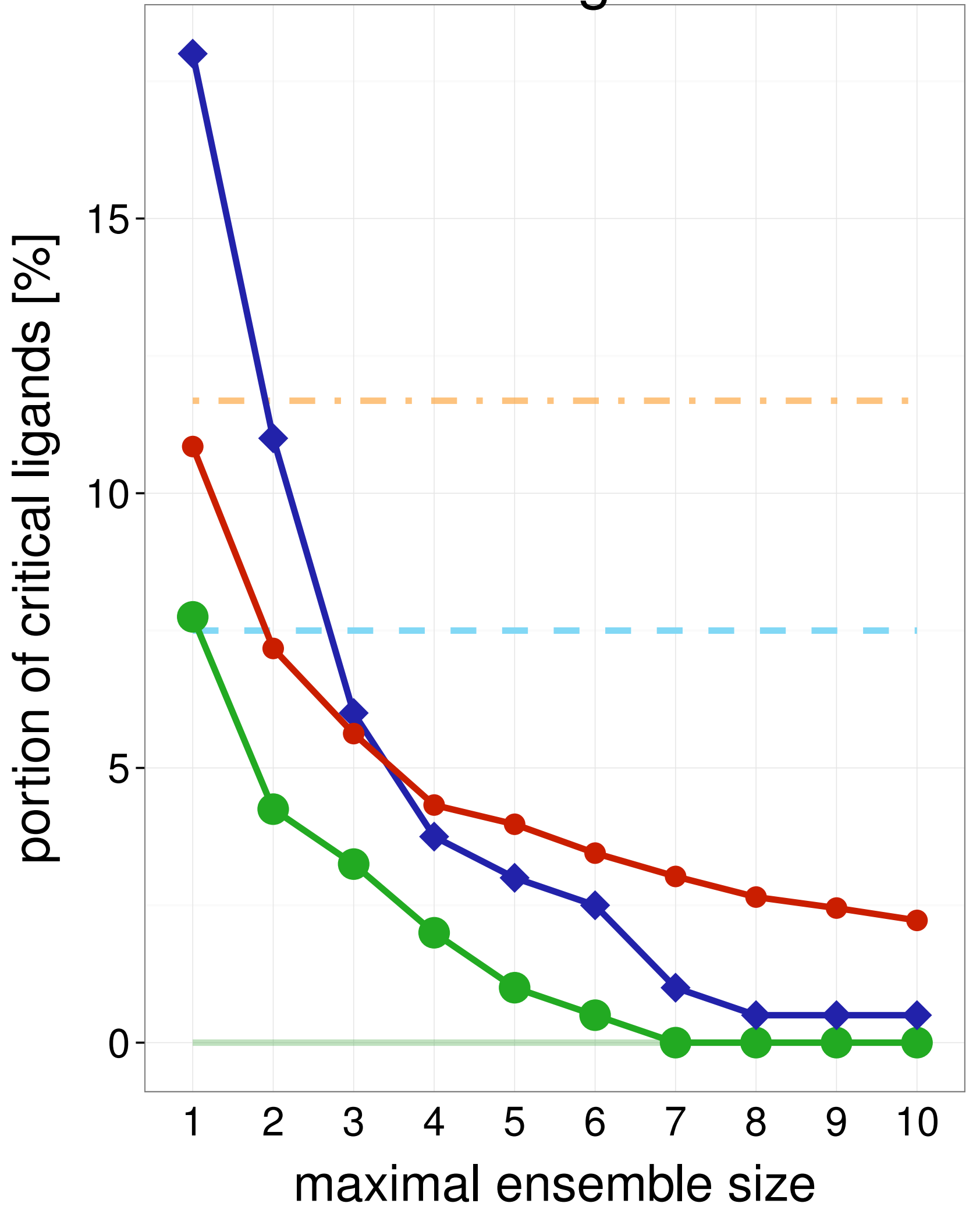

\section{Test set}

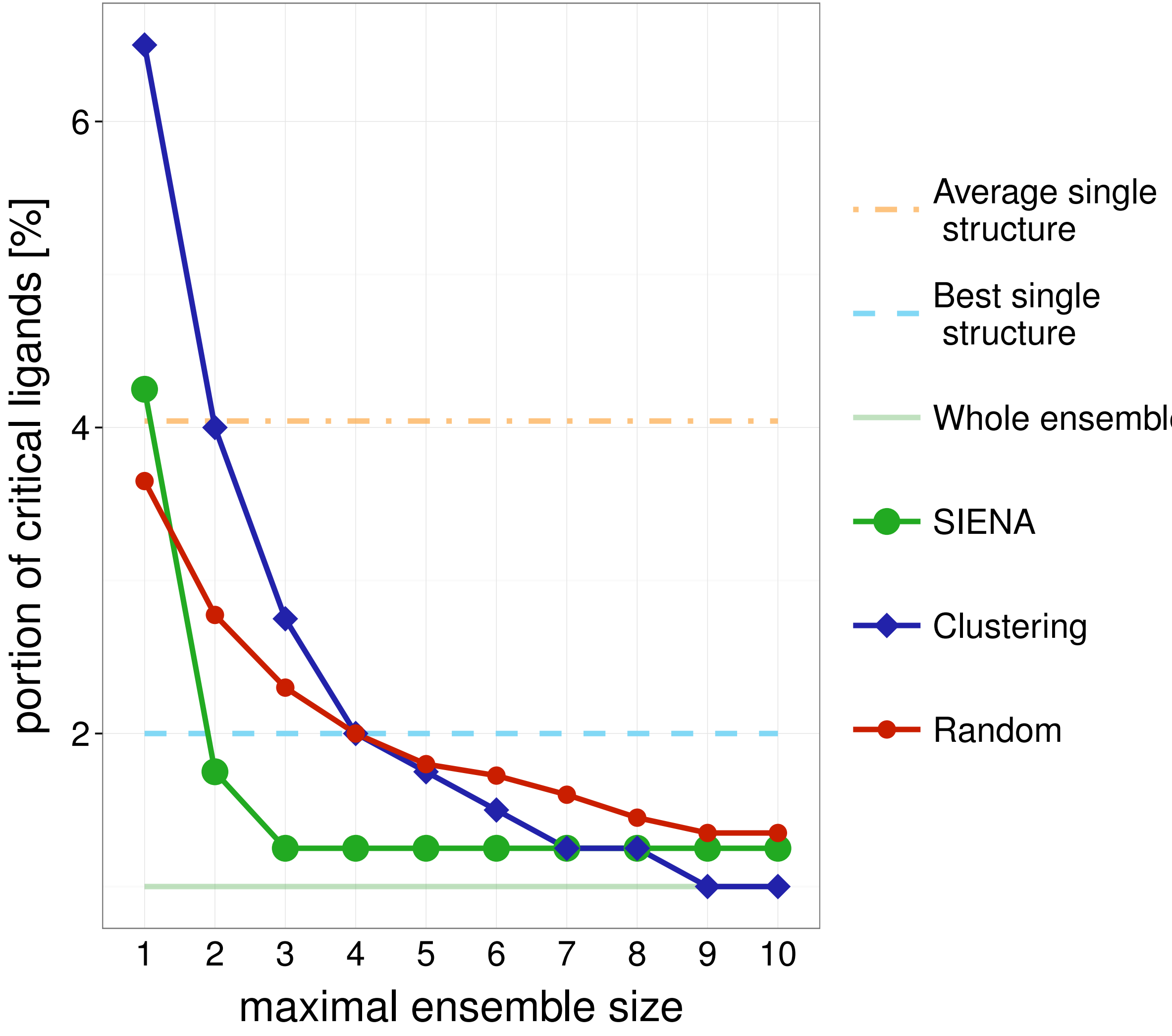

Average single structure

_ _ Best single structure

- SIENA

$\neg$ Clustering

$\multimap$ Random

Average single
$101 \mathrm{~T} \quad$ Training set

Whole ensemble

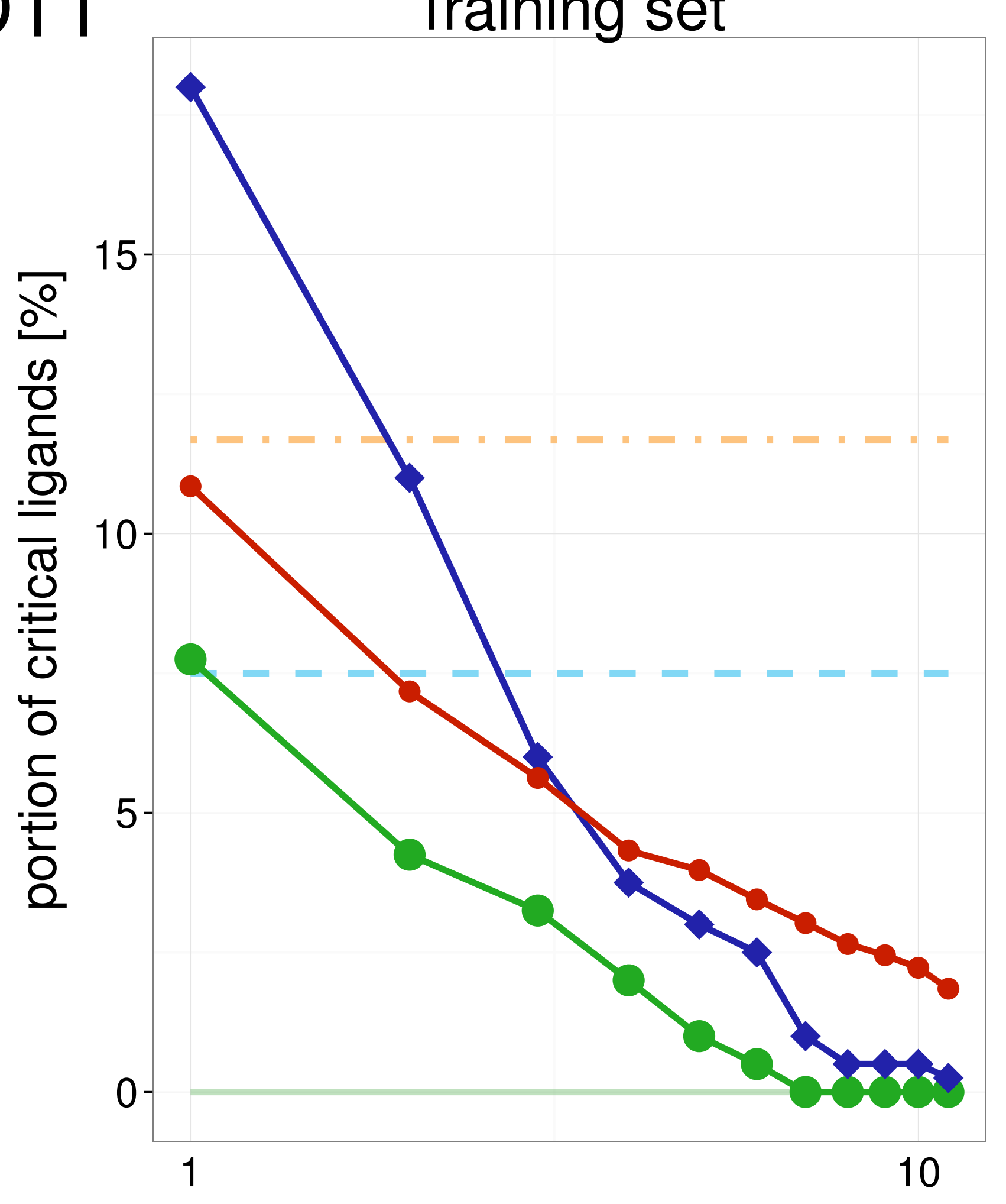

maximal ensemble size

Test set

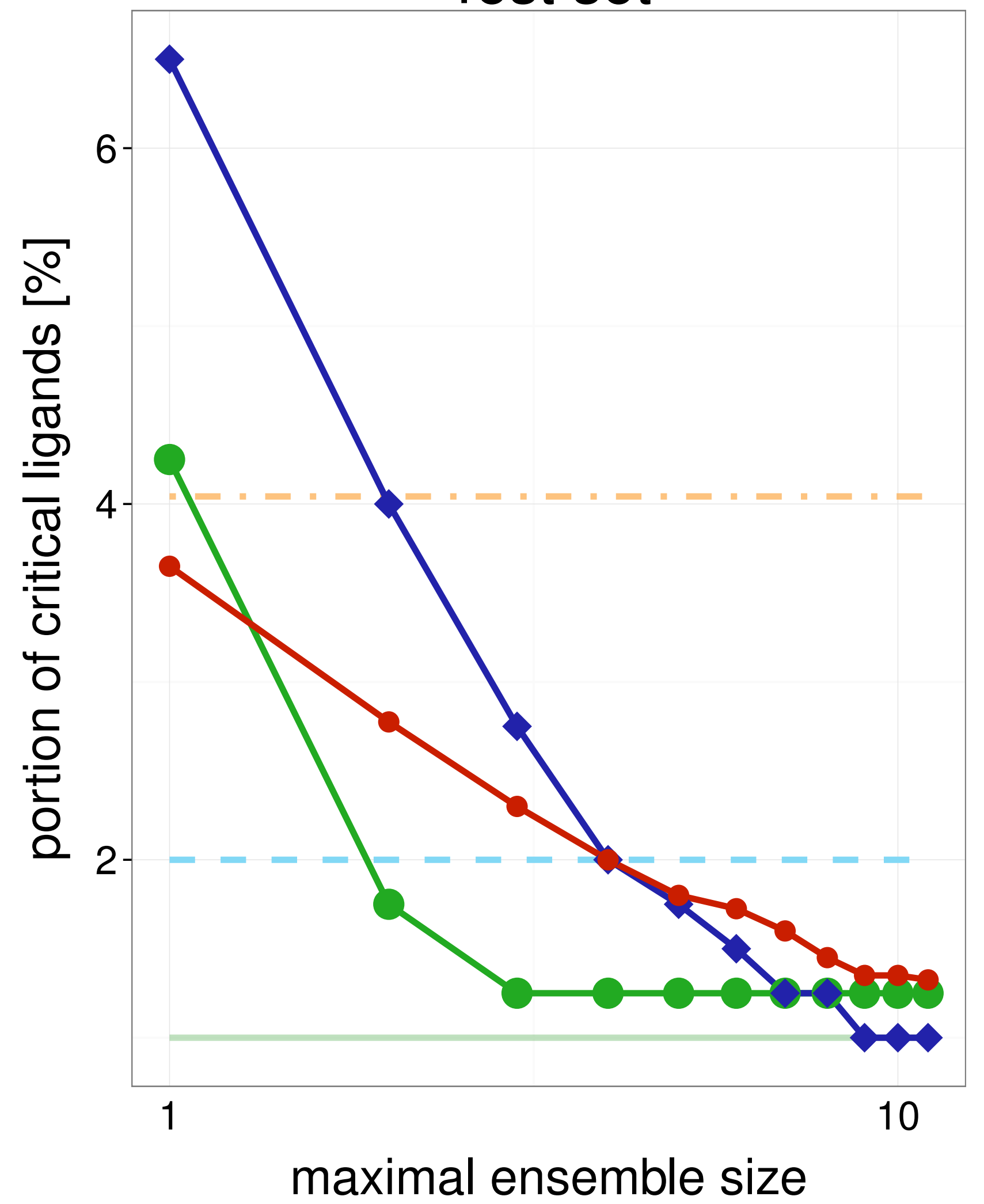

Average single structure

Best single structure

Whole ensemble

- SIENA

$\checkmark$ Clustering

$\multimap$ Random

Average single structure

_ _ Best single structure

Whole ensemble

SIENA

$\neg$ Clustering

$\longrightarrow$ Random 


\section{6 $\quad$ Training set}

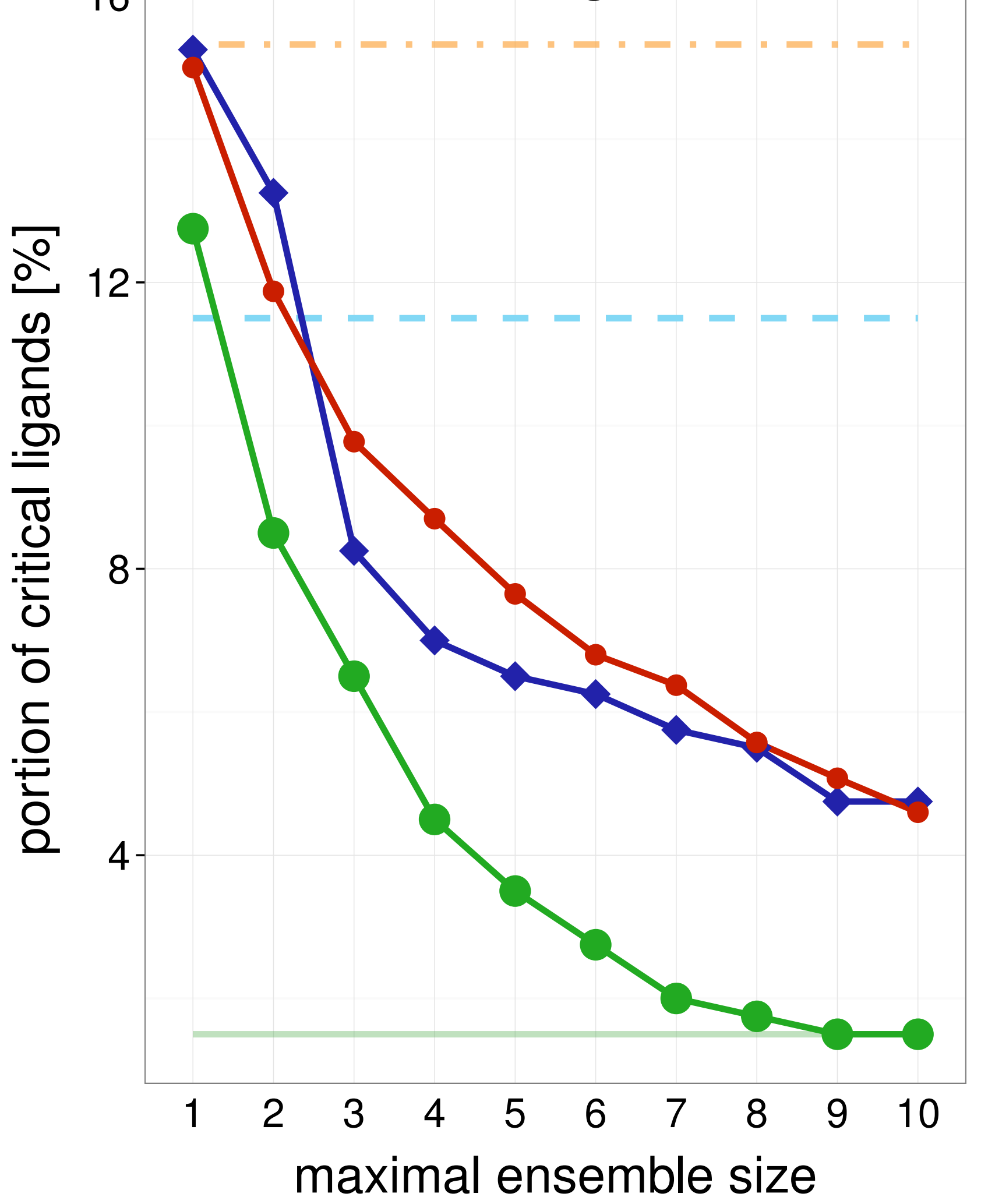

Test set

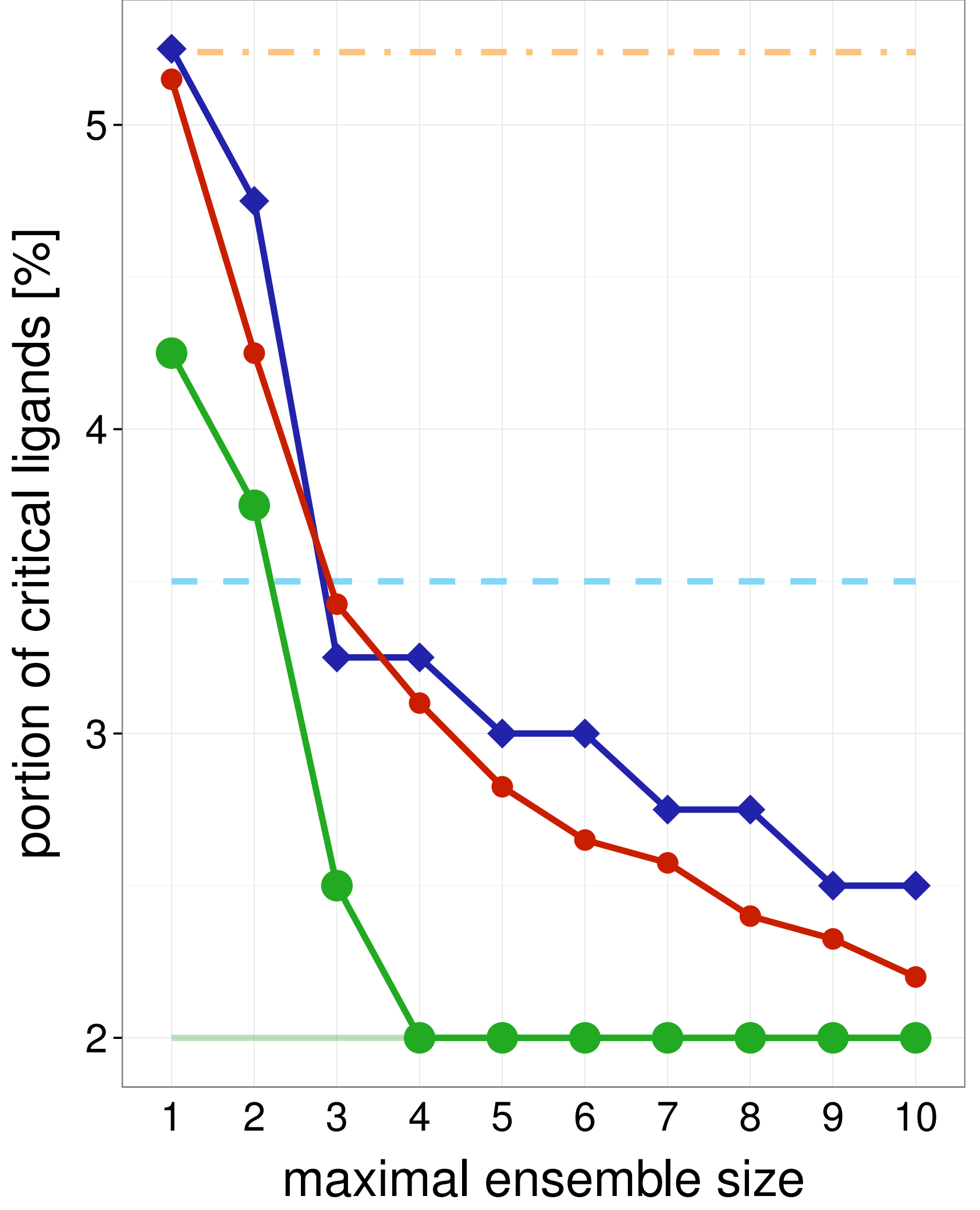

Average single structure

_ Best single structure

Whole ensemble

- SIENA

$\leadsto$ Clustering

$\rightarrow$ Random

Average single structure

_ Best single structure

Whole ensemble

- SIENA

$\checkmark$ Clustering

$\rightarrow$ Random
$1 \mathrm{UTG}_{16}$

Training set

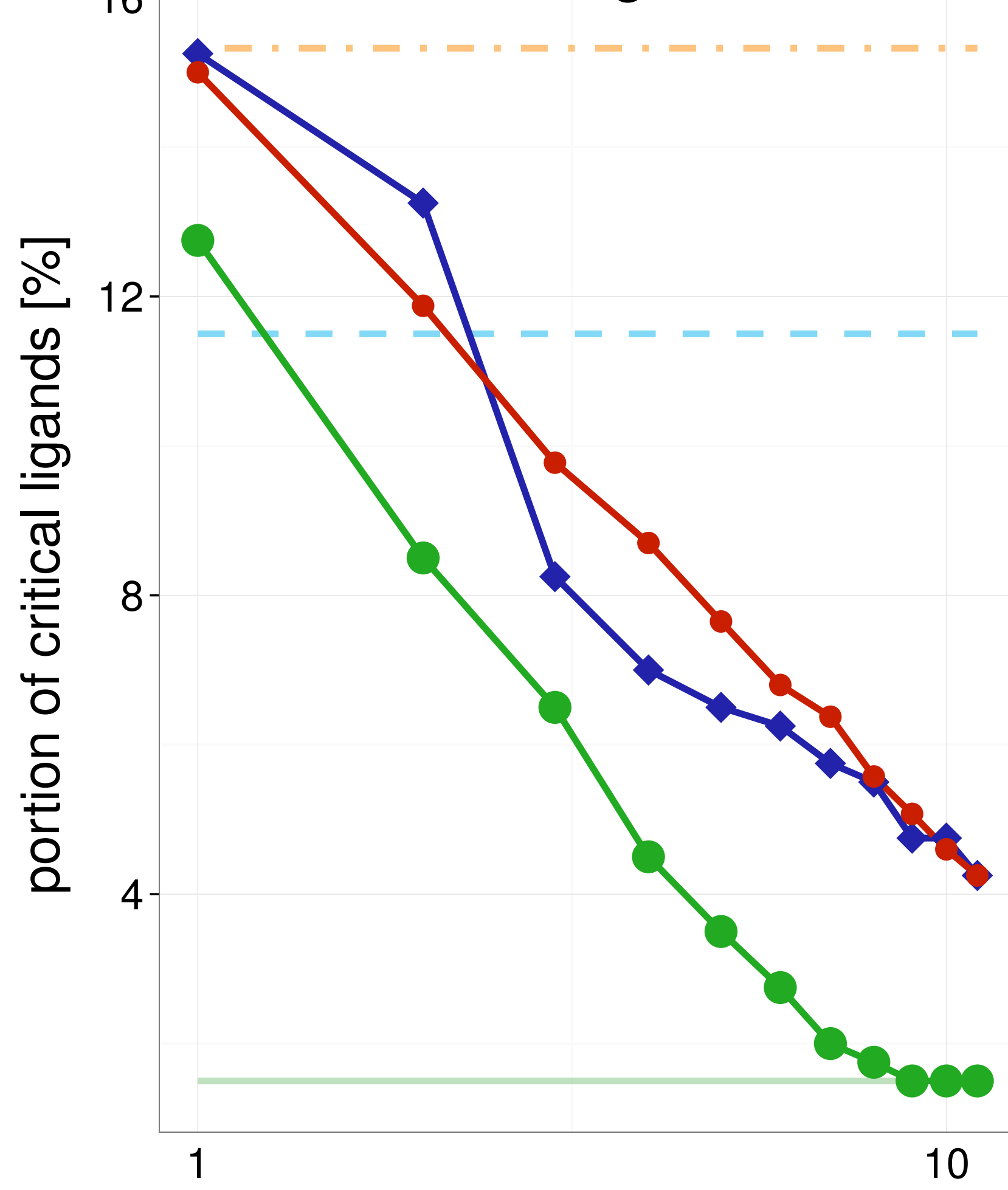

maximal ensemble size

Test set

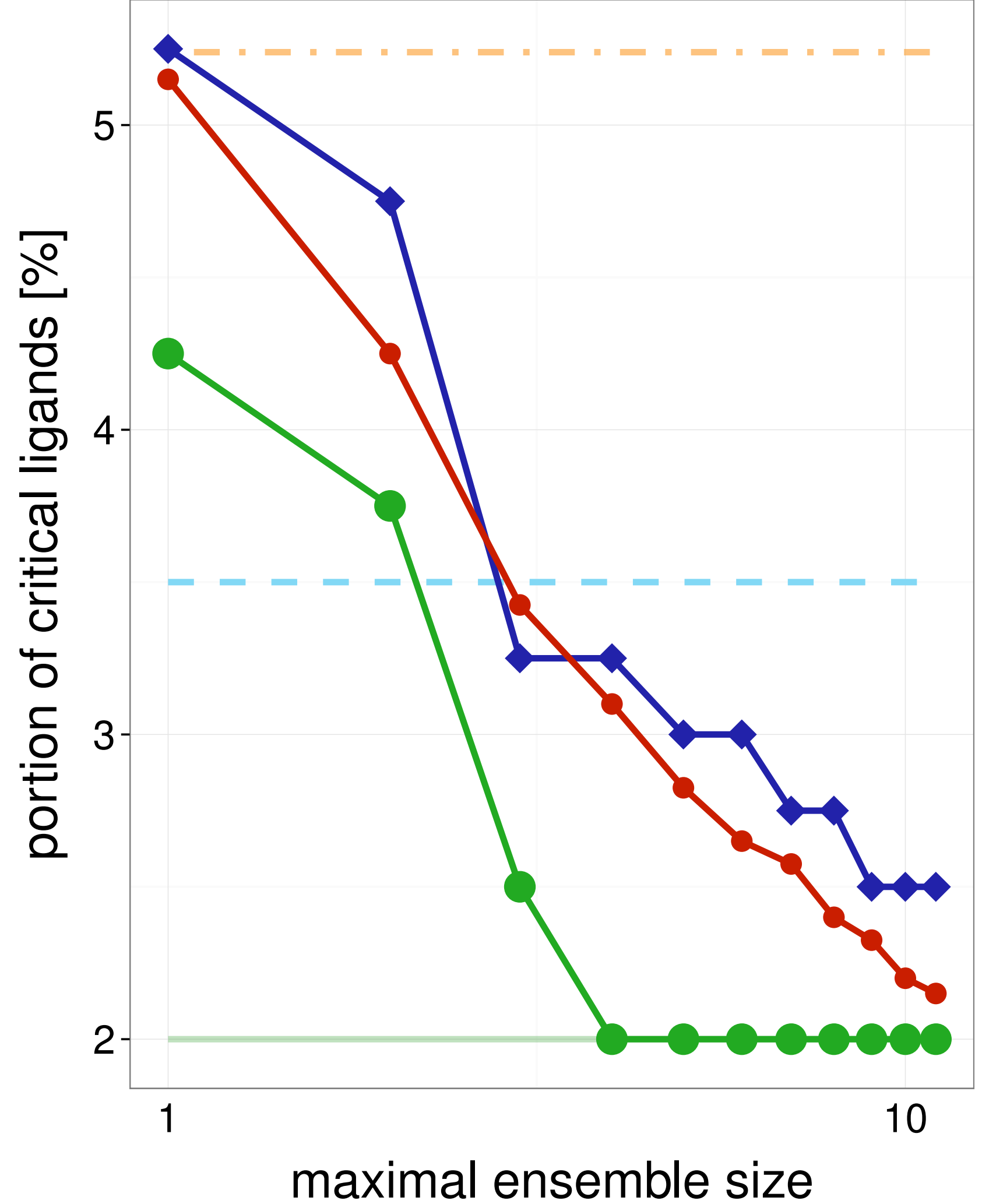

Average single structure

Best single structure

Whole ensemble

- SIENA

$\checkmark$ Clustering

$\rightarrow$ Random

Average single structure

_ Best single structure

Whole ensemble

- SIENA

$\neg$ Clustering

$\multimap$ Random 
Training set

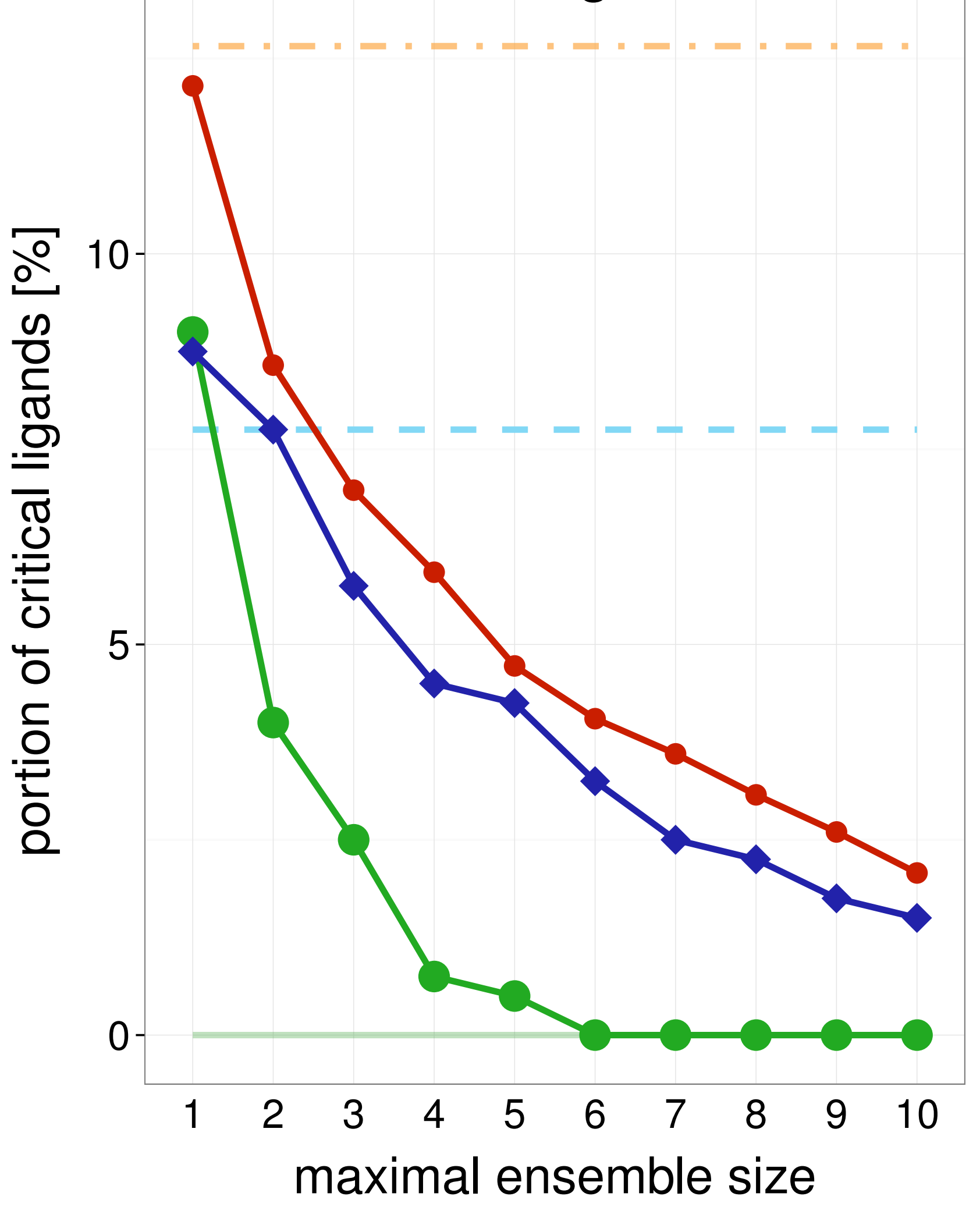

Test set

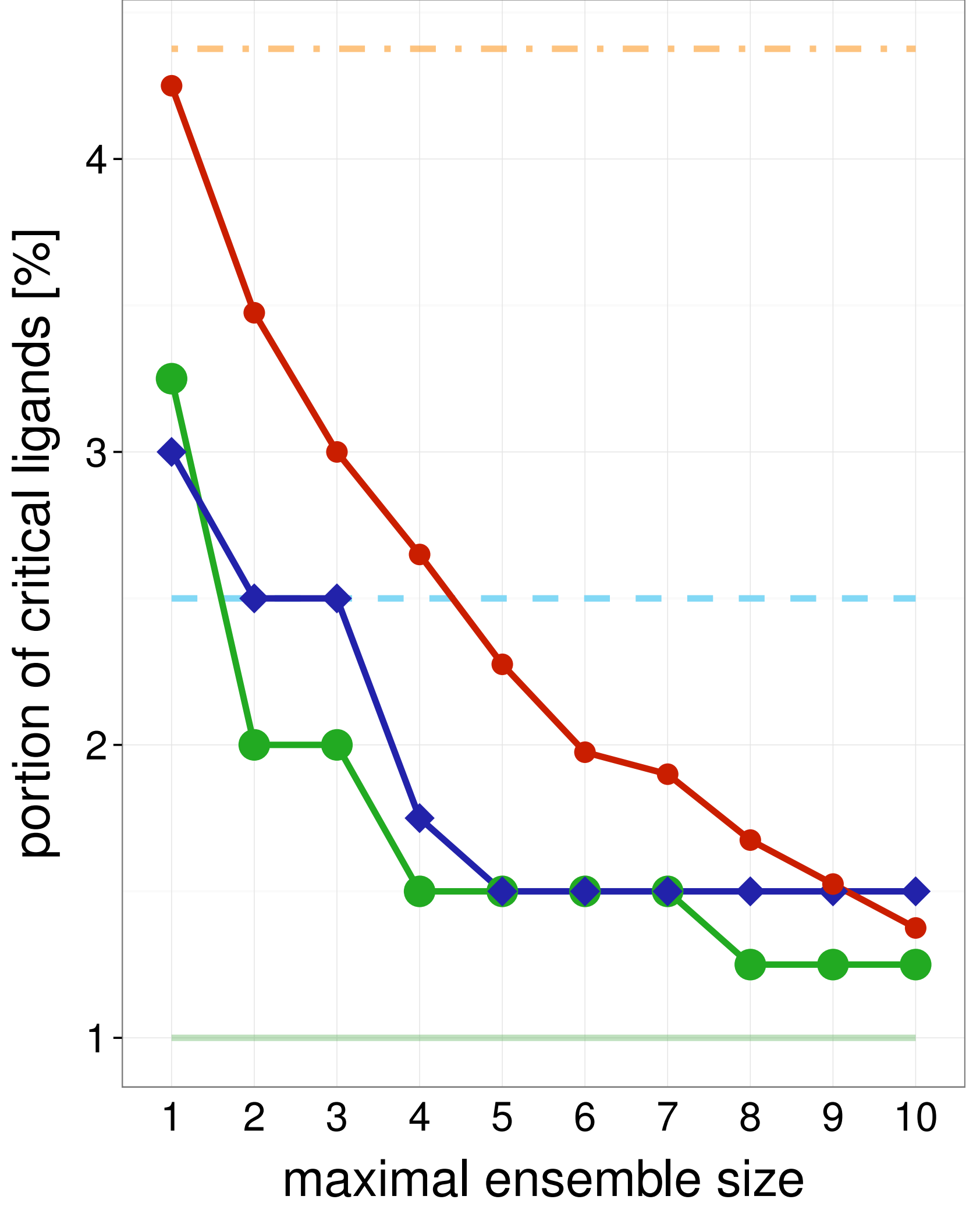

Average single structure

_ Best single structure

Whole ensemble

- SIENA

$\neg$ Clustering

$\multimap$ Random

Average single structure

_ Best single structure

Whole ensemble

- SIENA

$\neg$ Clustering

$\multimap$ Random
1UU3

Training set

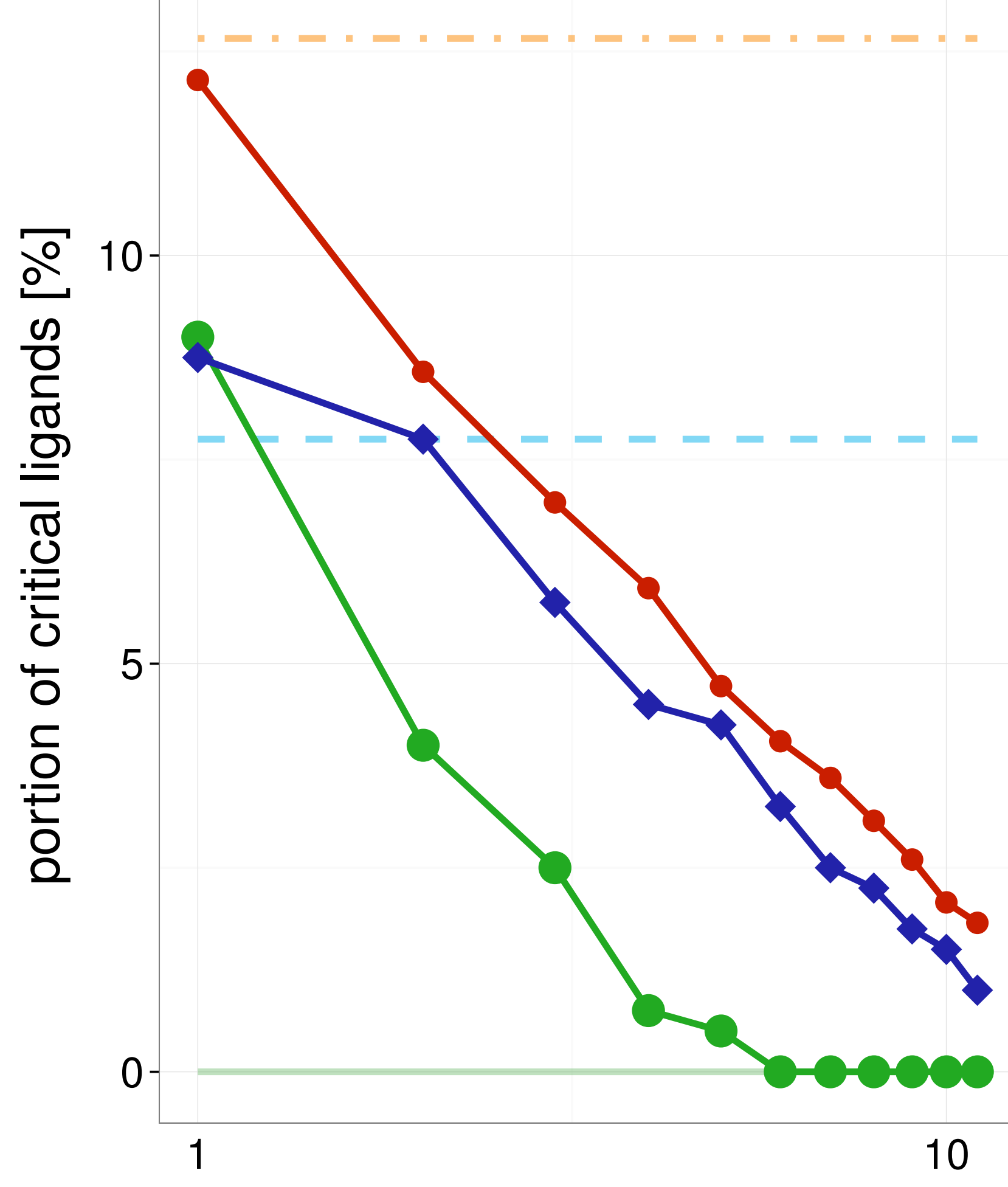

maximal ensemble size

Test set

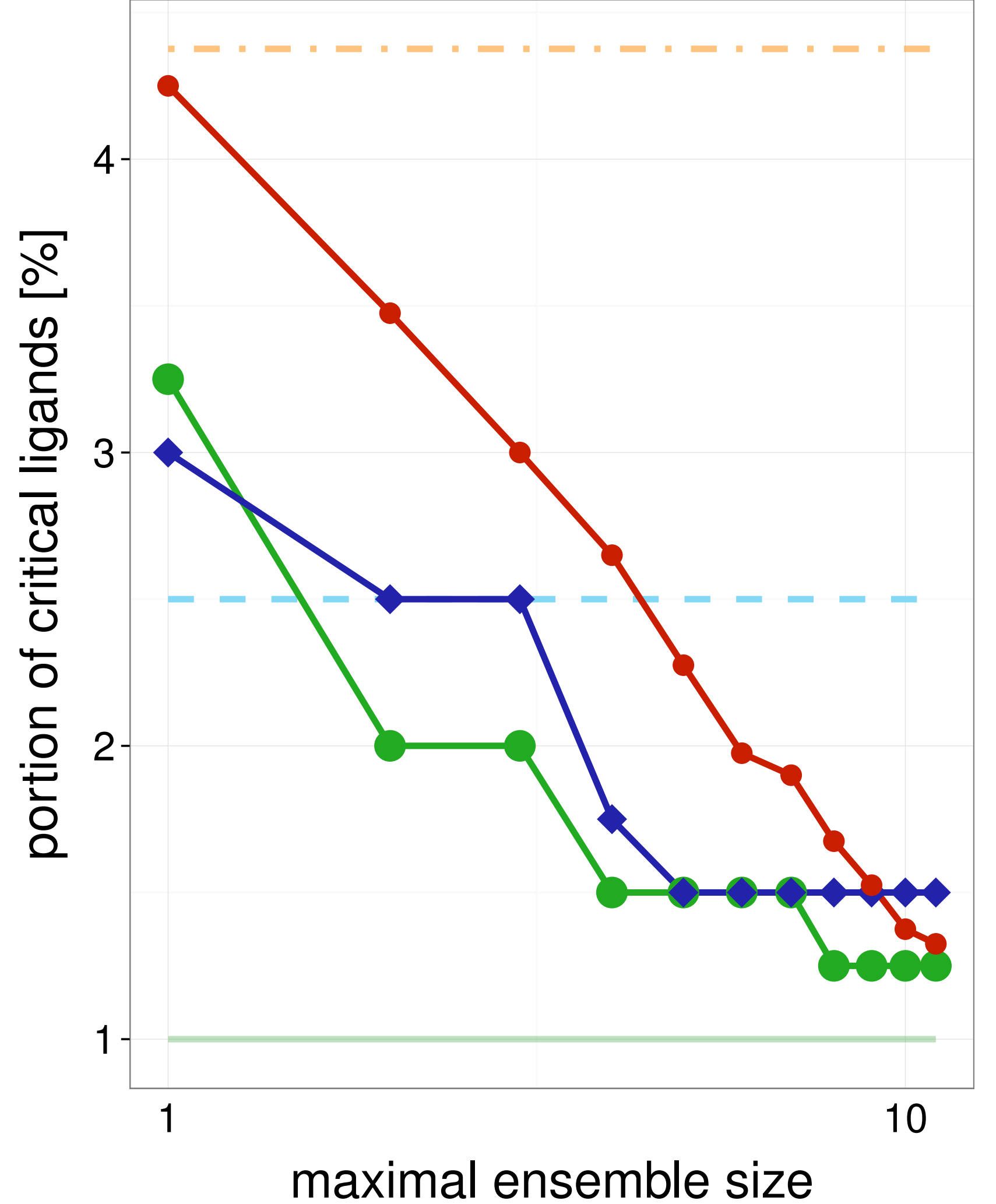

Average single structure

_ Best single structure

Whole ensemble

- SIENA

$\neg$ Clustering

$\multimap$ Random

Average single structure

- Best single structure

Whole ensemble

- SIENA

$\neg$ Clustering

$\multimap$ Random 
Training set

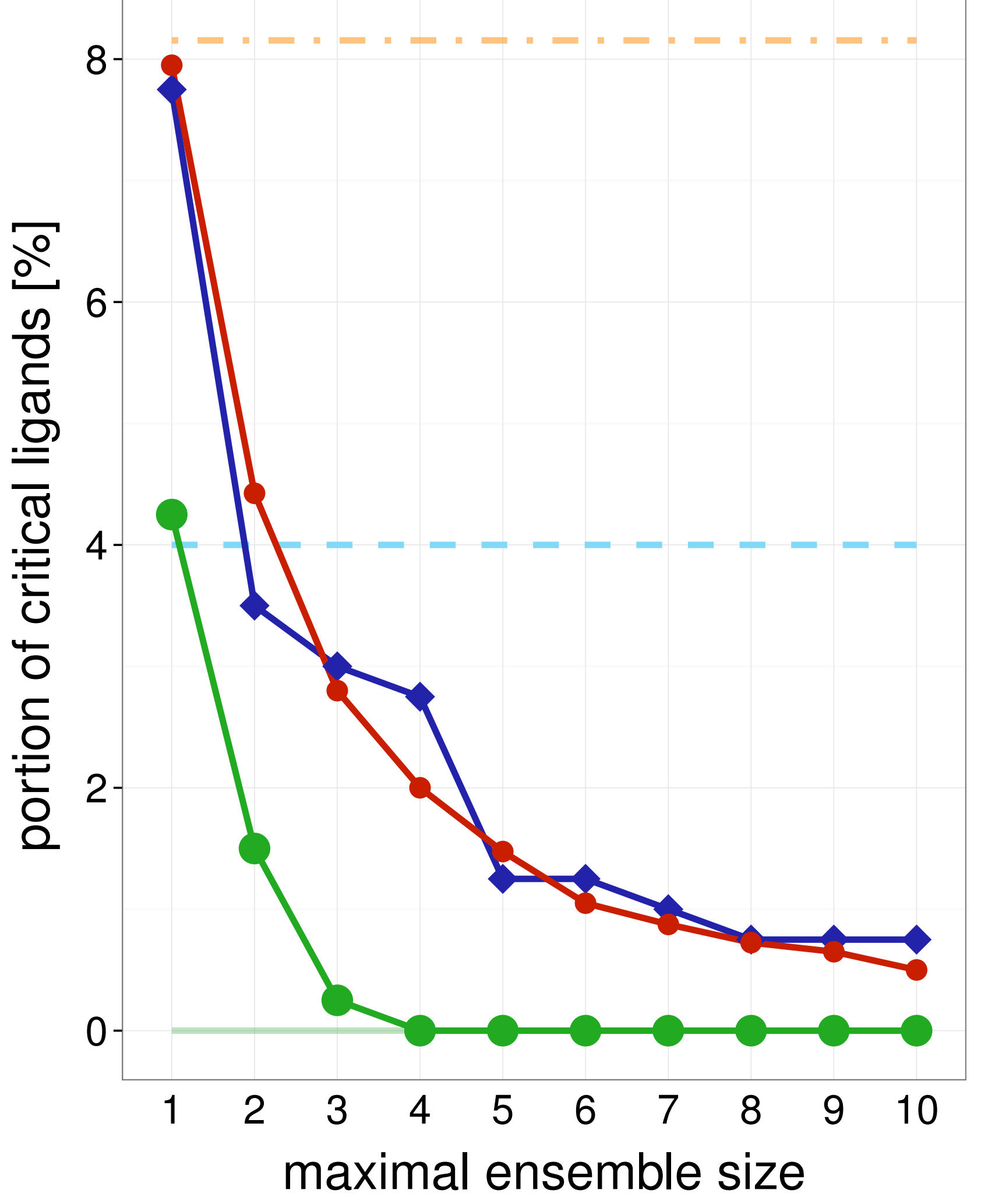

Test set

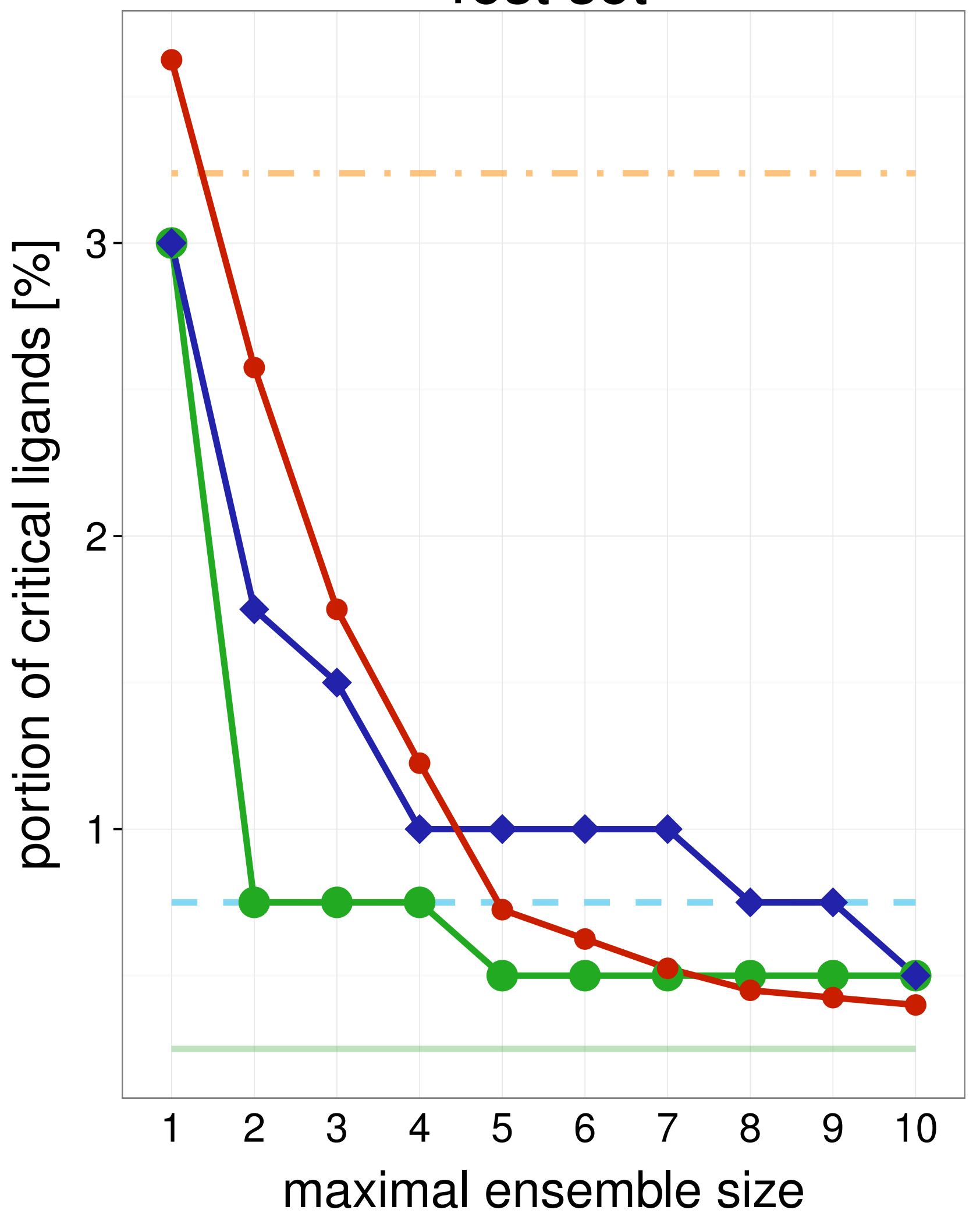

Average single structure

_ Best single structure

Whole ensemble

- SIENA

$\neg$ Clustering

$\multimap$ Random

Average single structure

_ _ Best single structure

Whole ensemble

- SIENA

$\neg$ Clustering

- Random
$1 \times \mathrm{GI}$

Training set

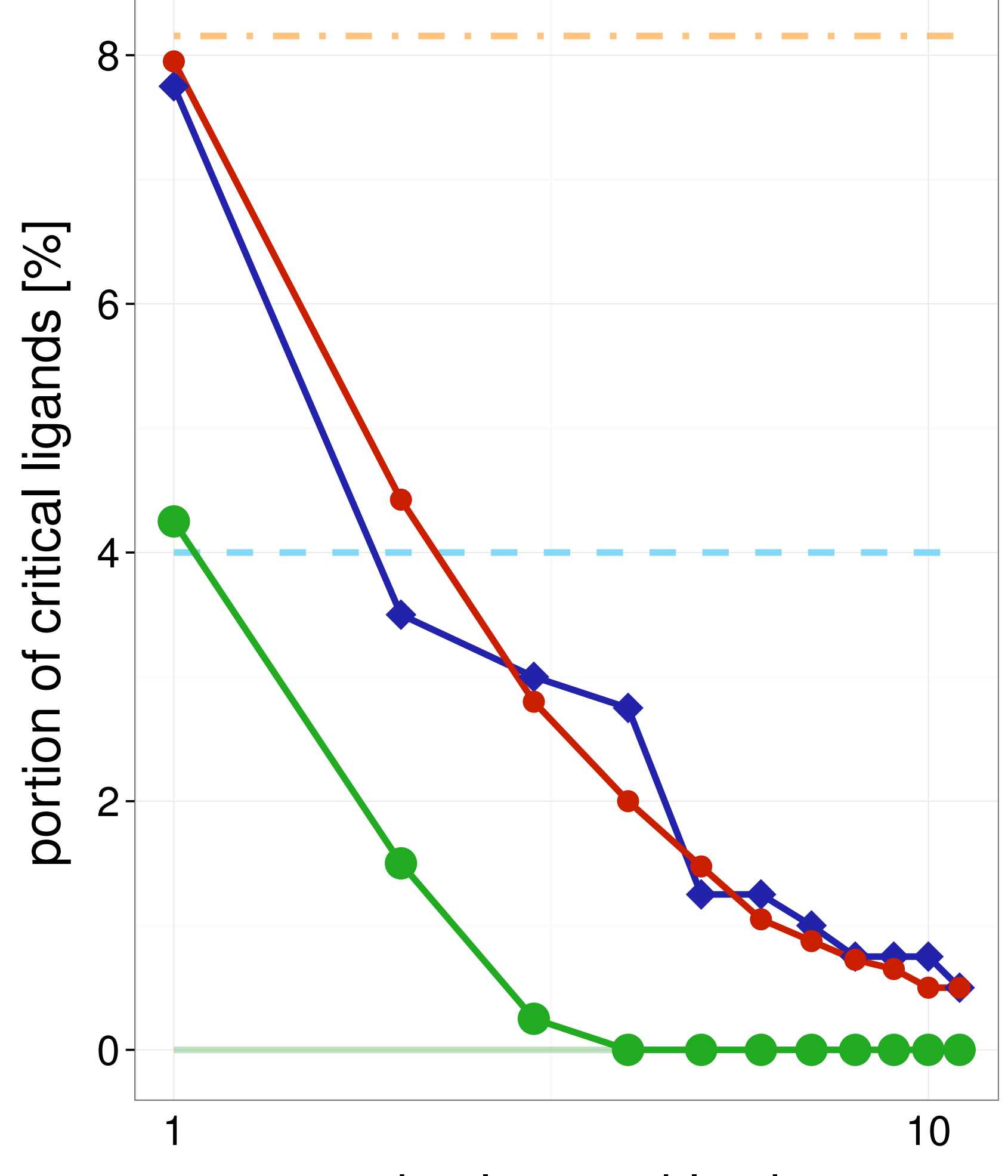

Average single structure

Best single structure

Whole ensemble

- SIENA

$\leadsto$ Clustering

$\because$ Random

maximal ensemble size

Test set

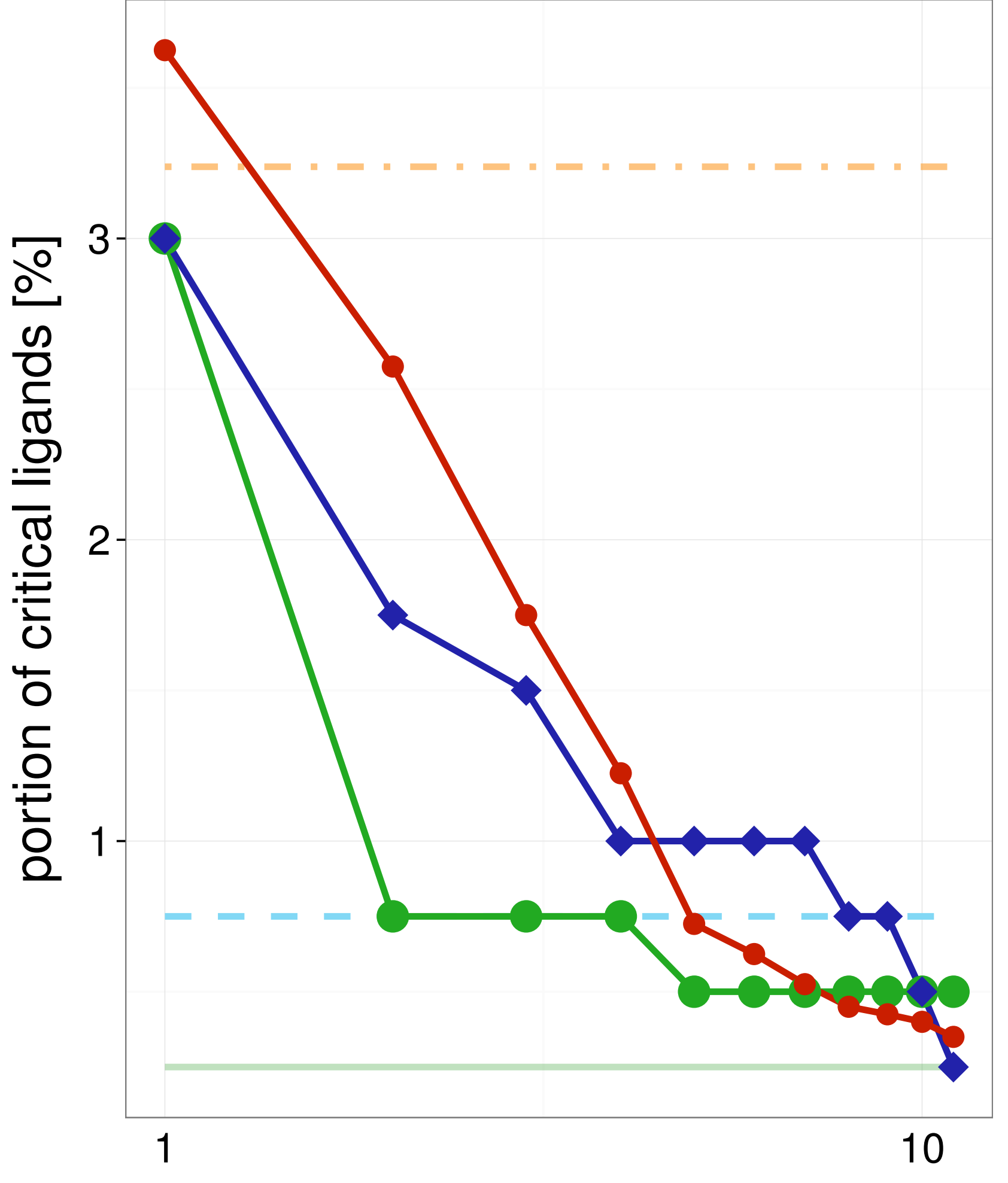

Average single structure

_ _ Best single structure

Whole ensemble

SIENA

$\sim$ Clustering

$\multimap$ Random 


\section{Training set}

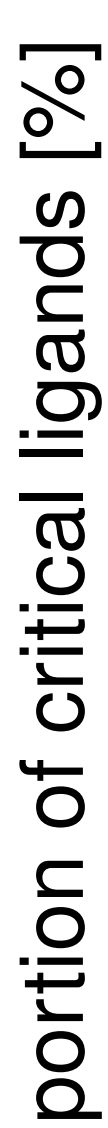

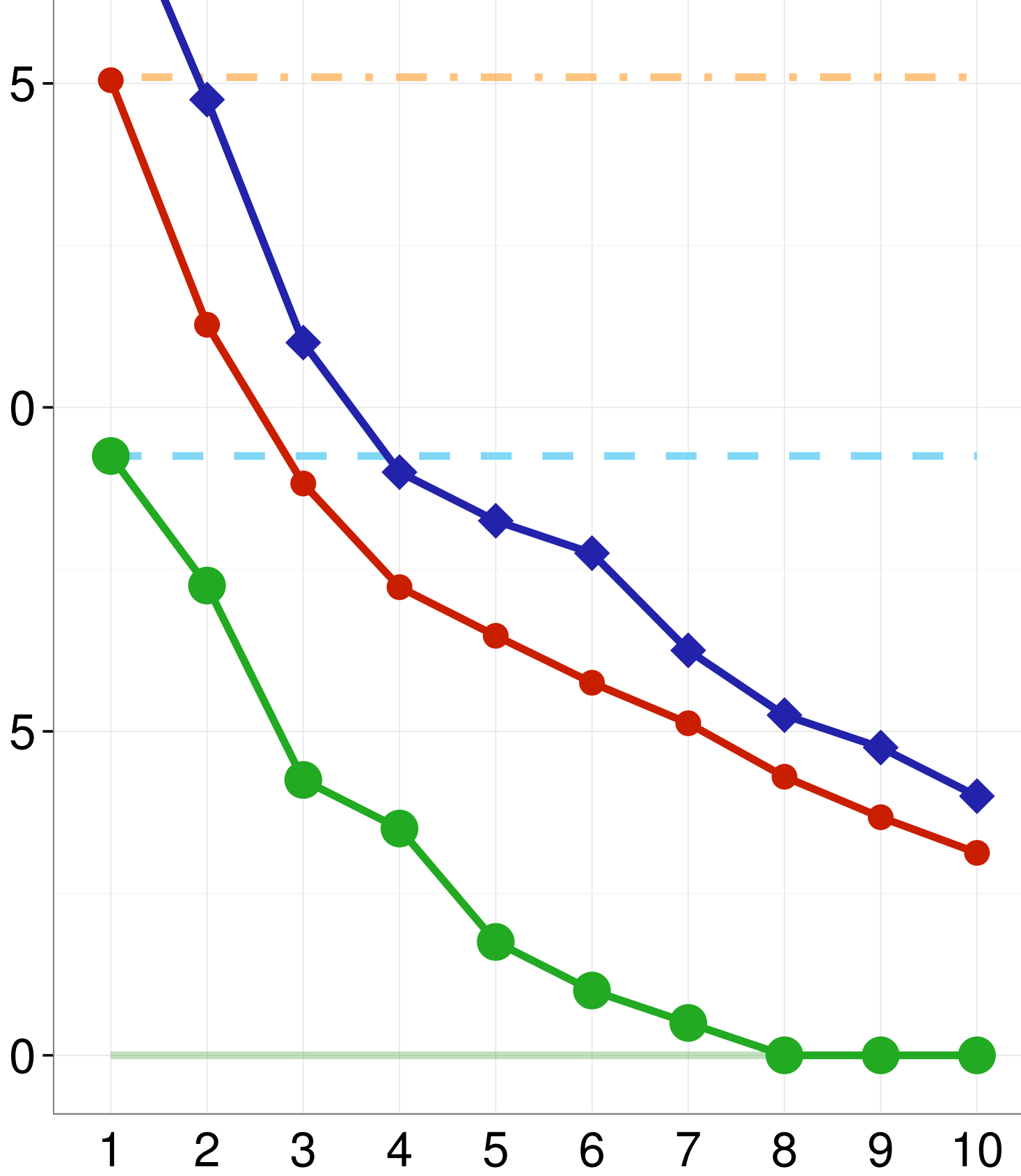

maximal ensemble size

Test set

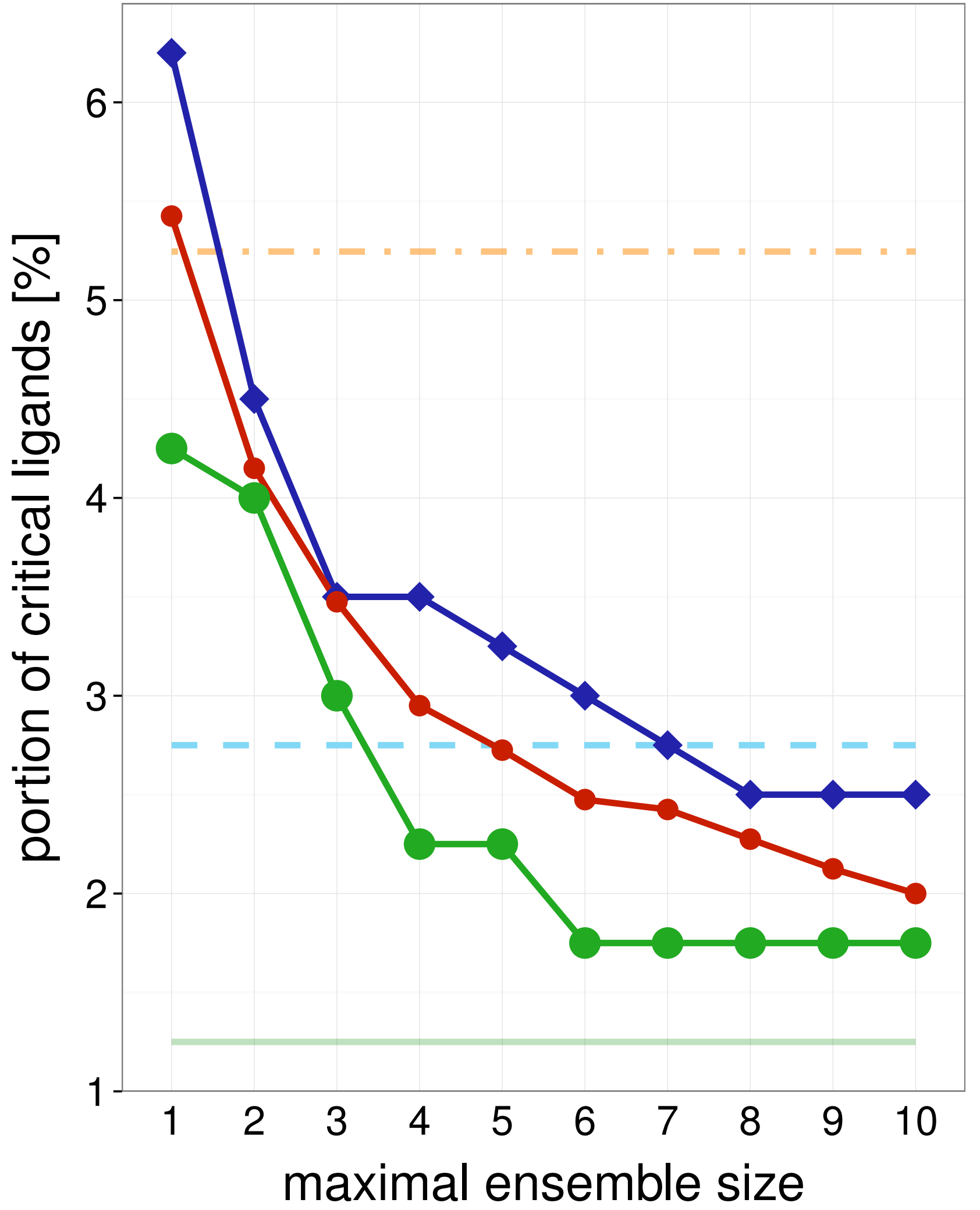

2EVC

Training set

Average single structure

- Best single structure

Whole ensemble

- SIENA

$\neg$ Clustering

$\multimap$ Random

Average single structure

_ Best single structure

Whole ensemble

- SIENA

$\neg$ Clustering

$\multimap$ Random

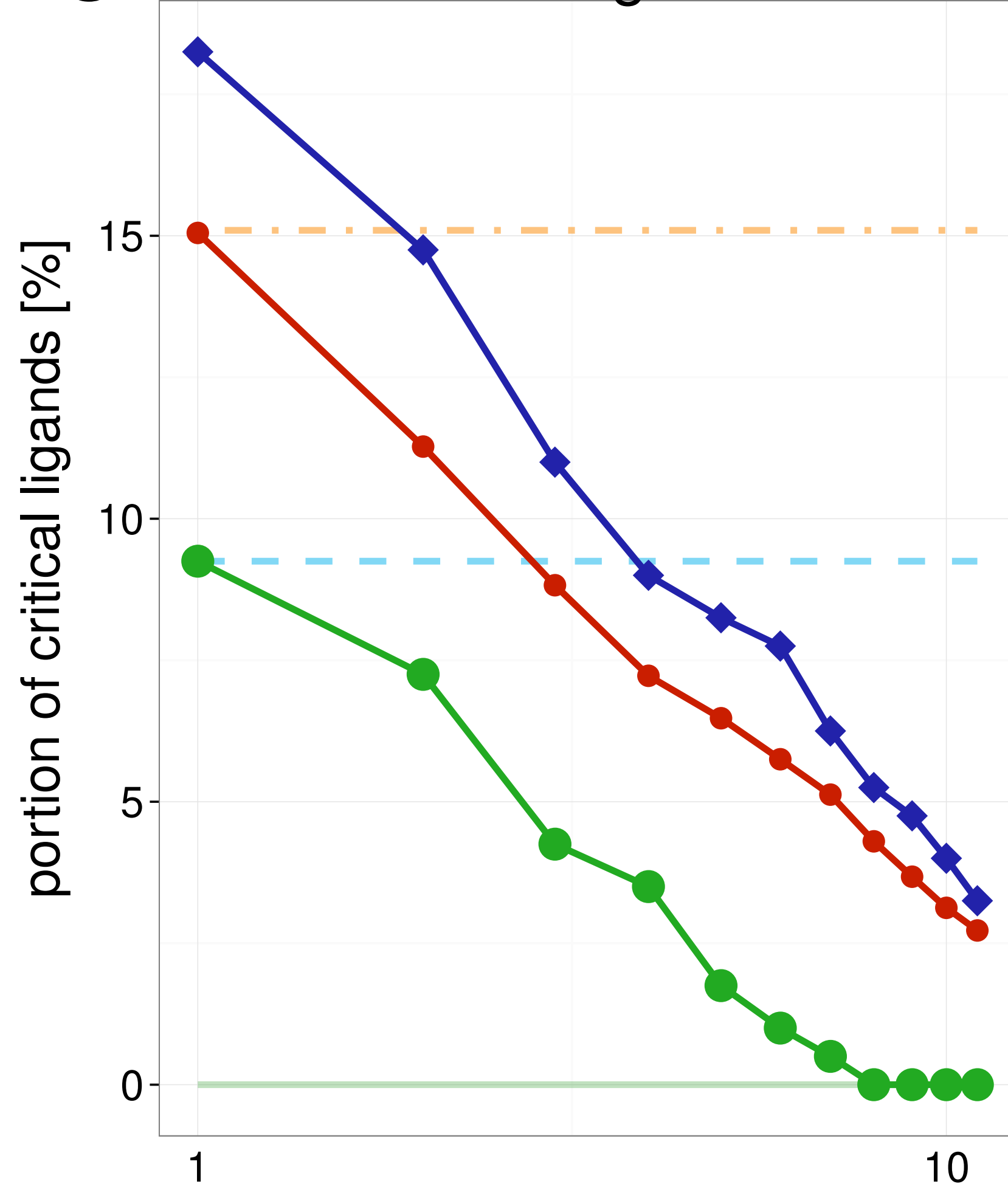

maximal ensemble size

Test set

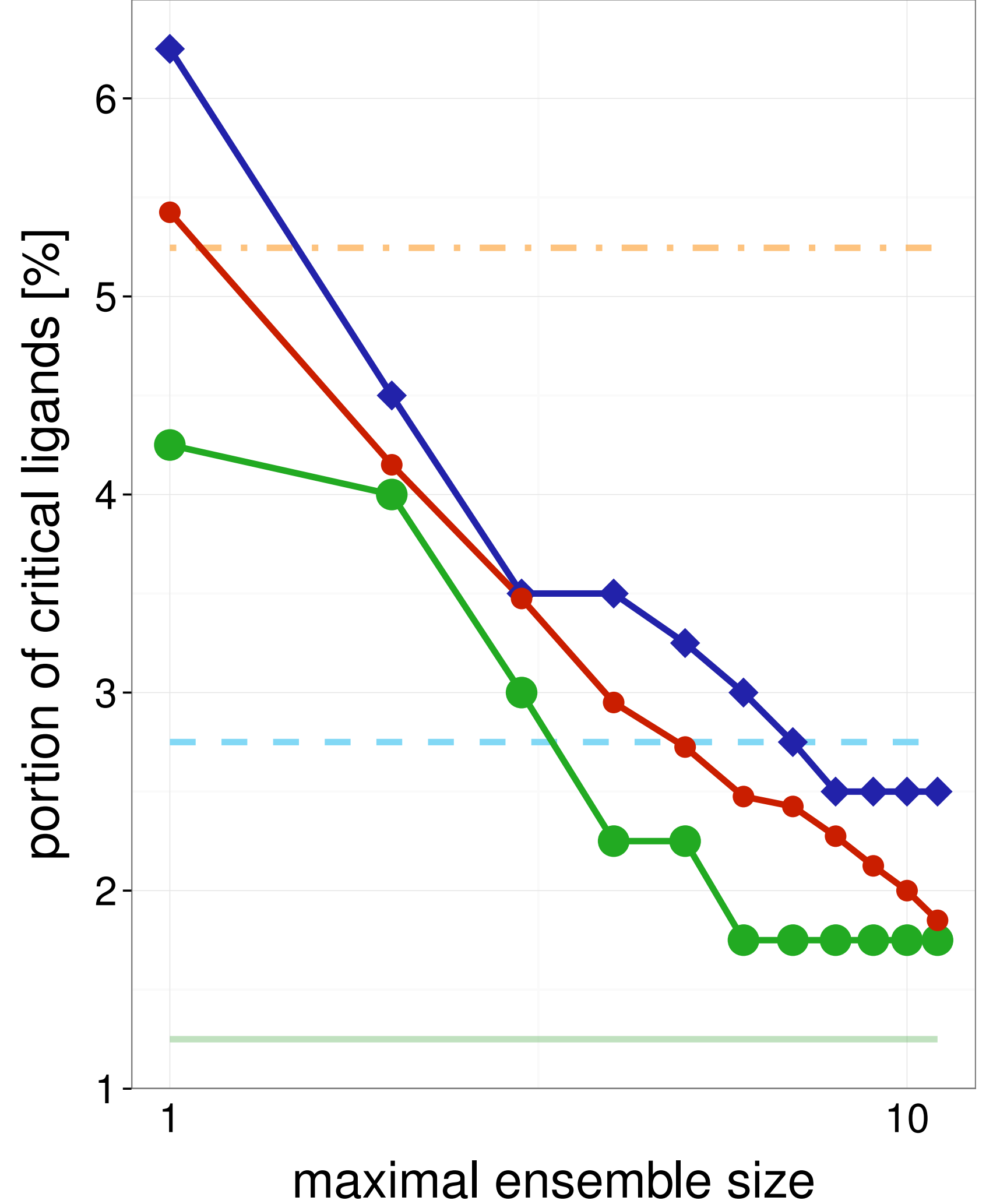

Average single structure

Best single structure

Whole ensemble

- SIENA

$\neg$ Clustering

$\multimap$ Random

Average single structure

Best single structure

Whole ensemble

SIENA

$\neg$ Clustering

$\multimap$ Random 
Training set

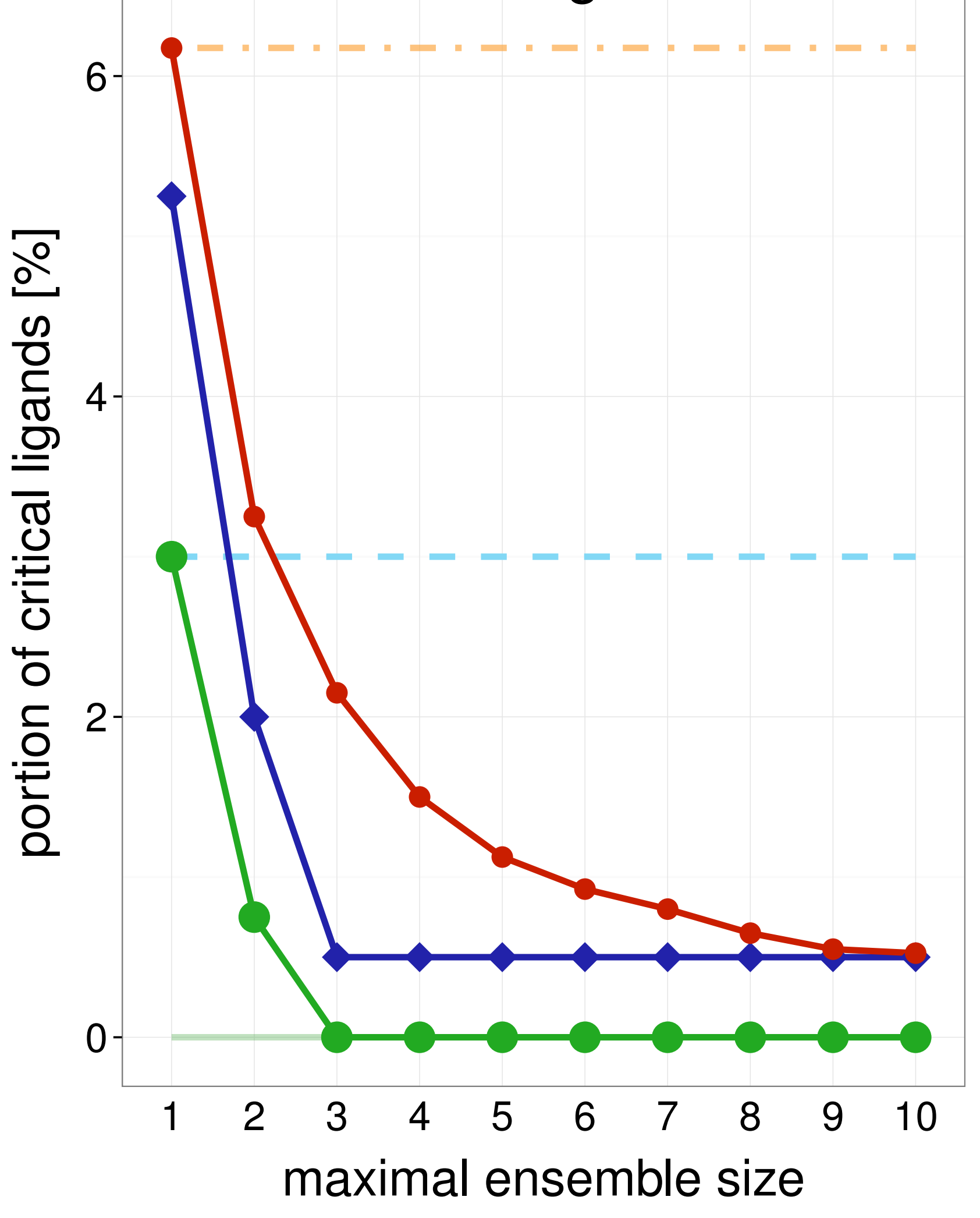

Test set

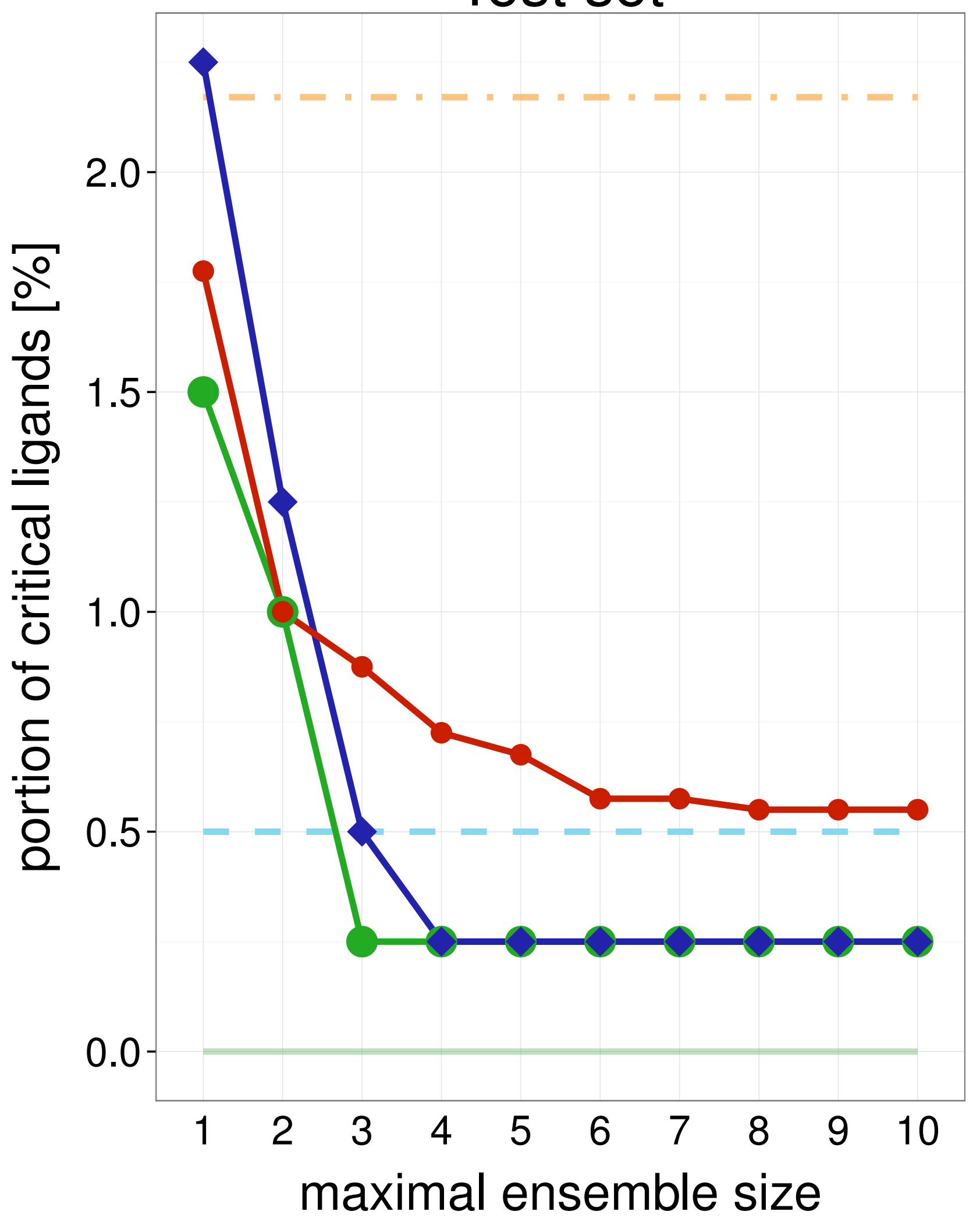

Average single structure

_ Best single structure

Whole ensemble

- SIENA

$\smile$ Clustering

$\multimap$ Random

Average single structure

_ Best single structure

Whole ensemble

- SIENA

$\neg$ Clustering

$\multimap$ Random

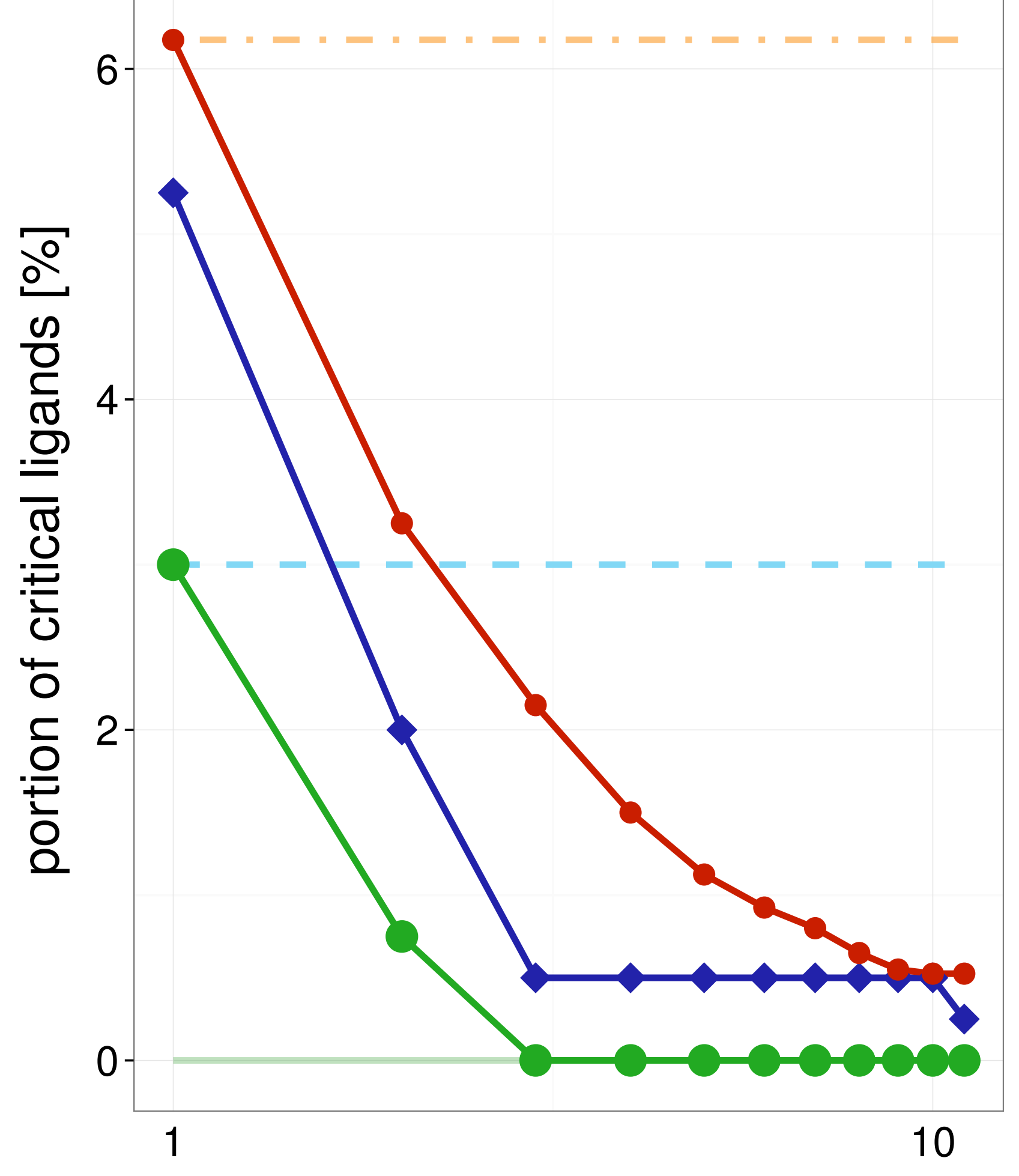

maximal ensemble size

Test set

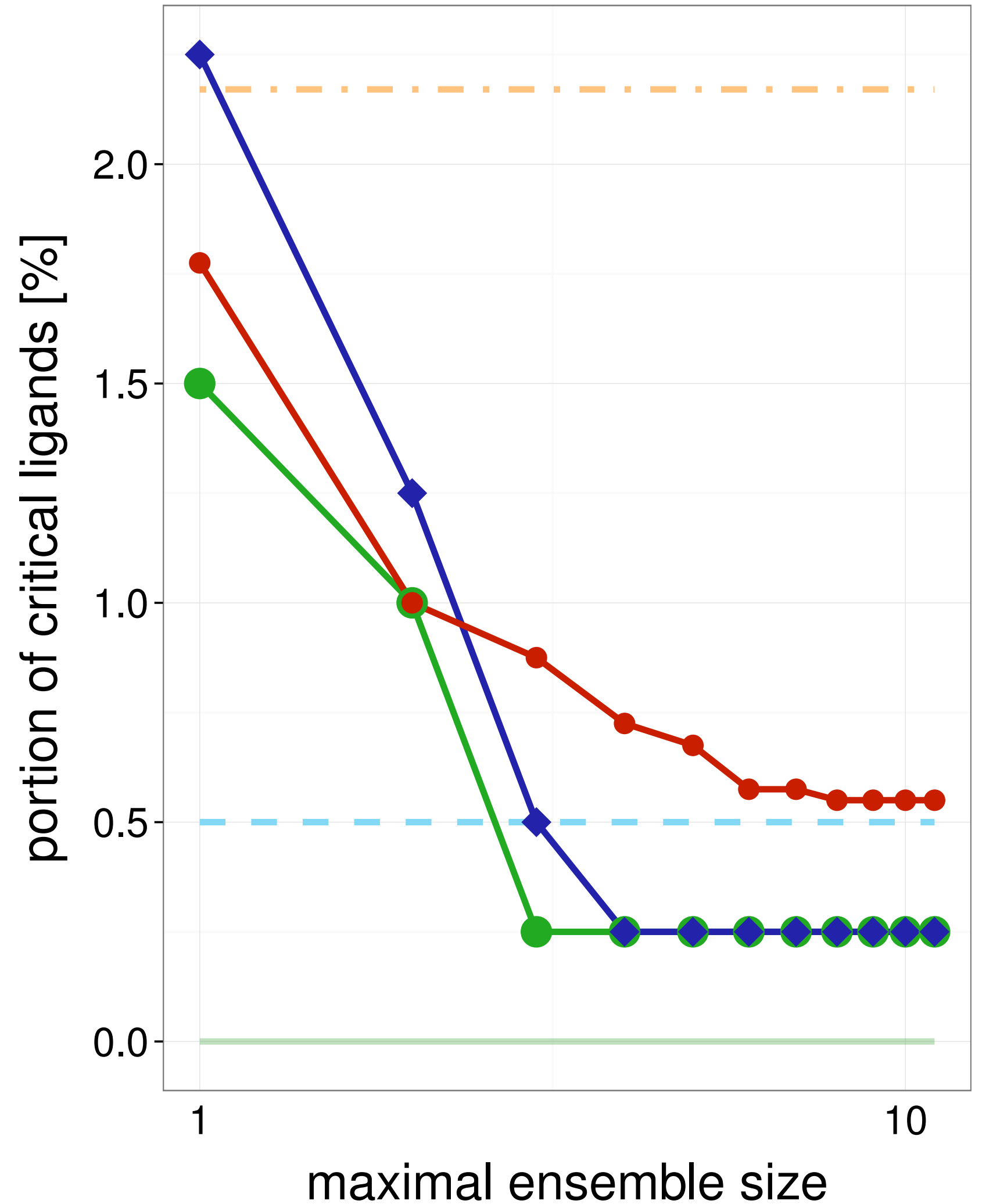

Average single structure

Best single structure

Whole ensemble

- SIENA

$\leadsto$ Clustering

$\multimap$ Random

Average single structure

Best single structure

Whole ensemble

SIENA

$\rightarrow$ Clustering

$\longrightarrow$ Random 
Training set

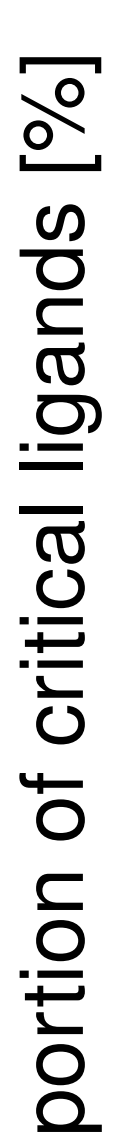

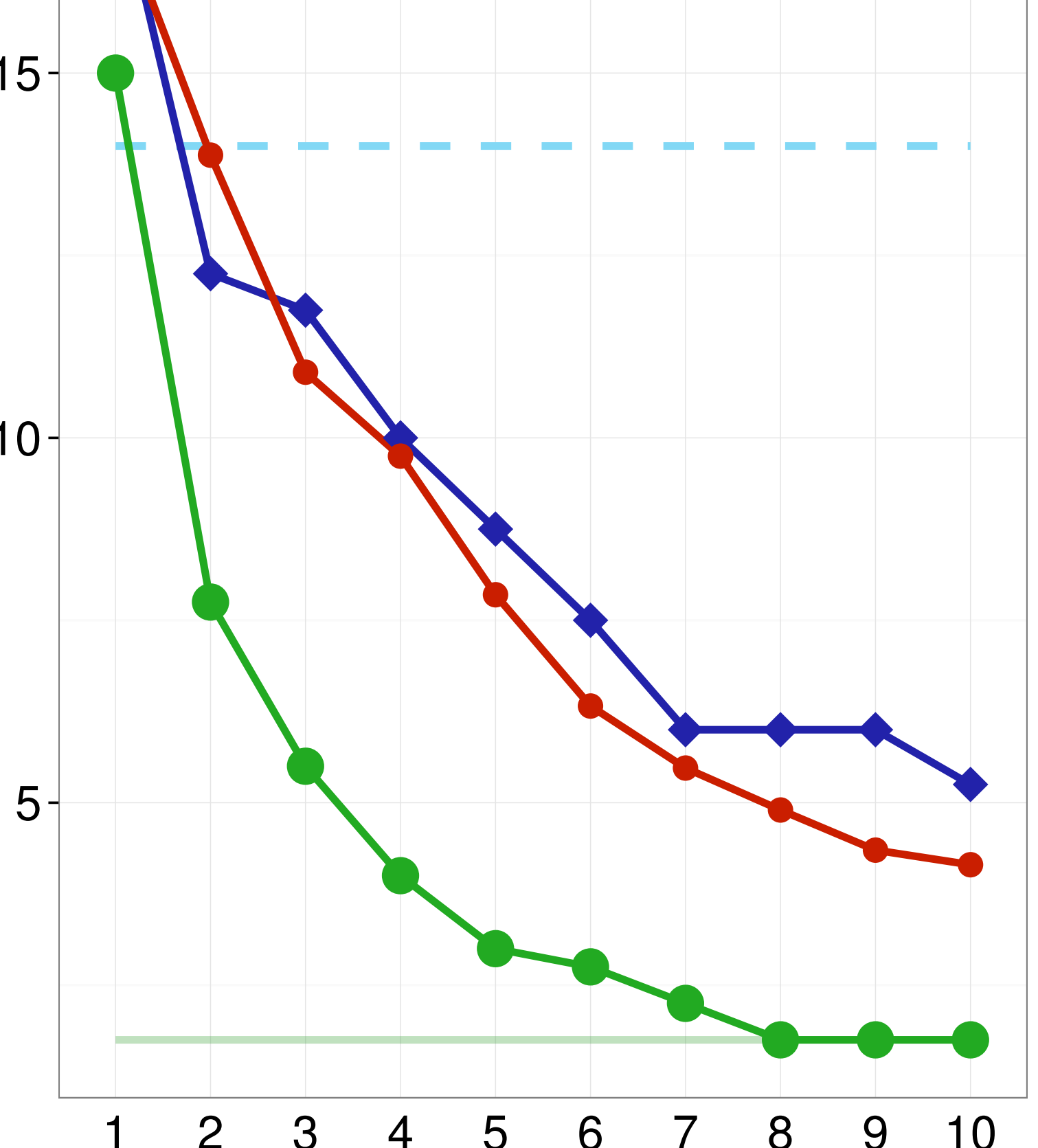

maximal ensemble size

Test set

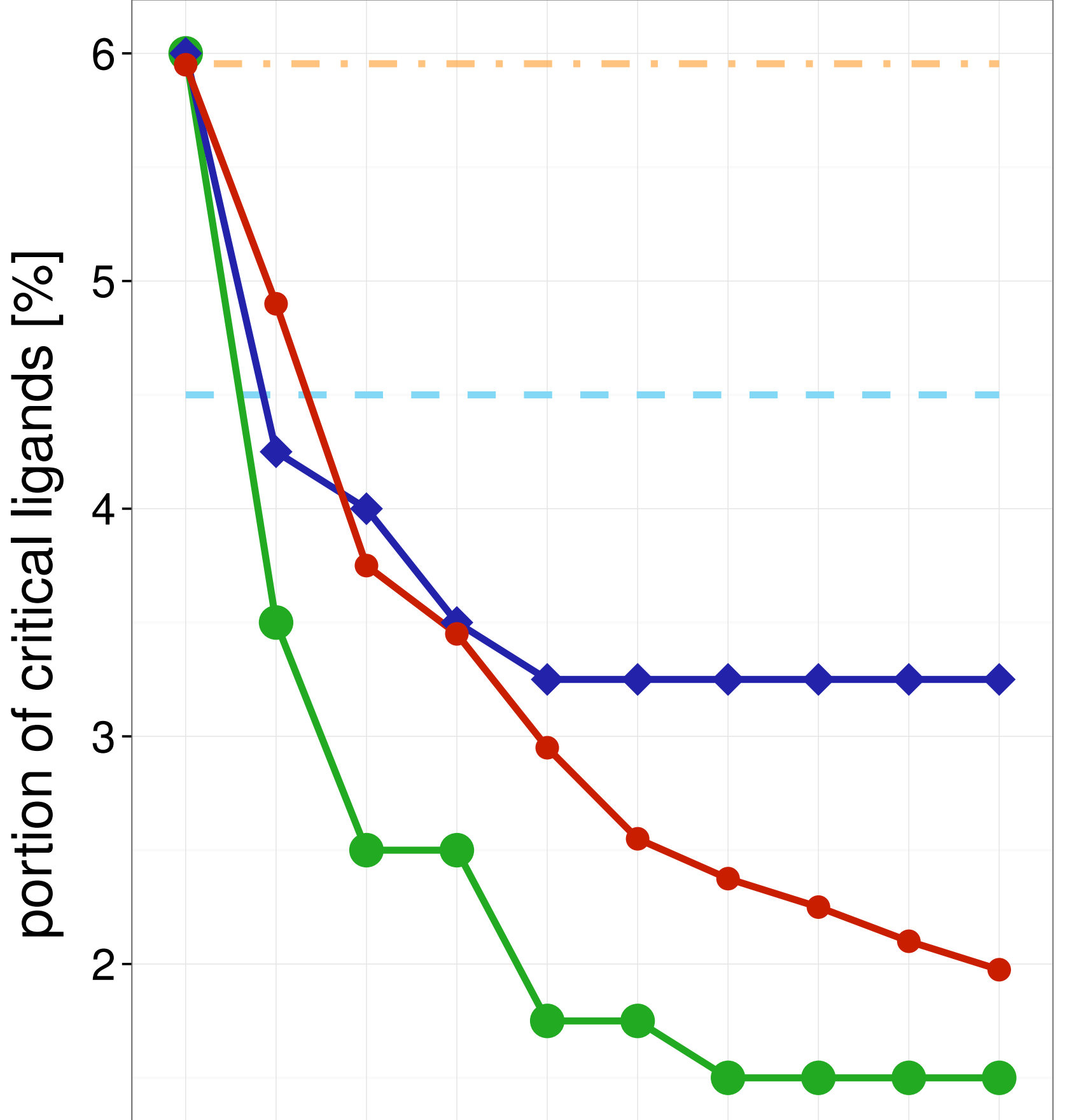

$\begin{array}{llllllllll}1 & 2 & 3 & 4 & 5 & 6 & 7 & 8 & 9 & 10\end{array}$ maximal ensemble size
$2 Z 15 \quad$ Training set

Average single structure

_ Best single

structure

Whole ensemble

- SIENA

$\neg$ Clustering

$\rightarrow$ Random

Average single structure

_ Best single structure

Whole ensemble

- SIENA

$\neg$ Clustering

$\multimap$ Random

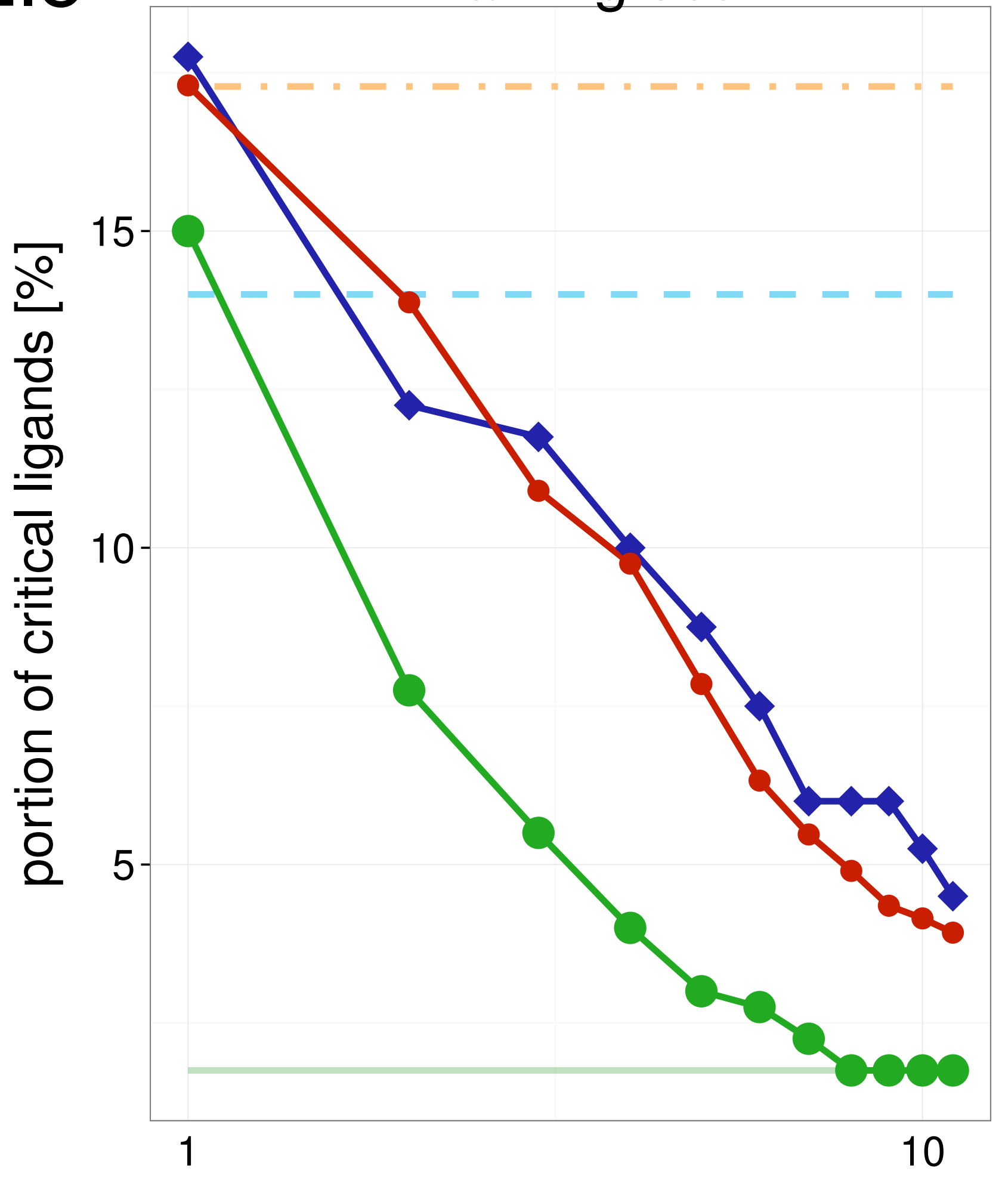

maximal ensemble size

Test set

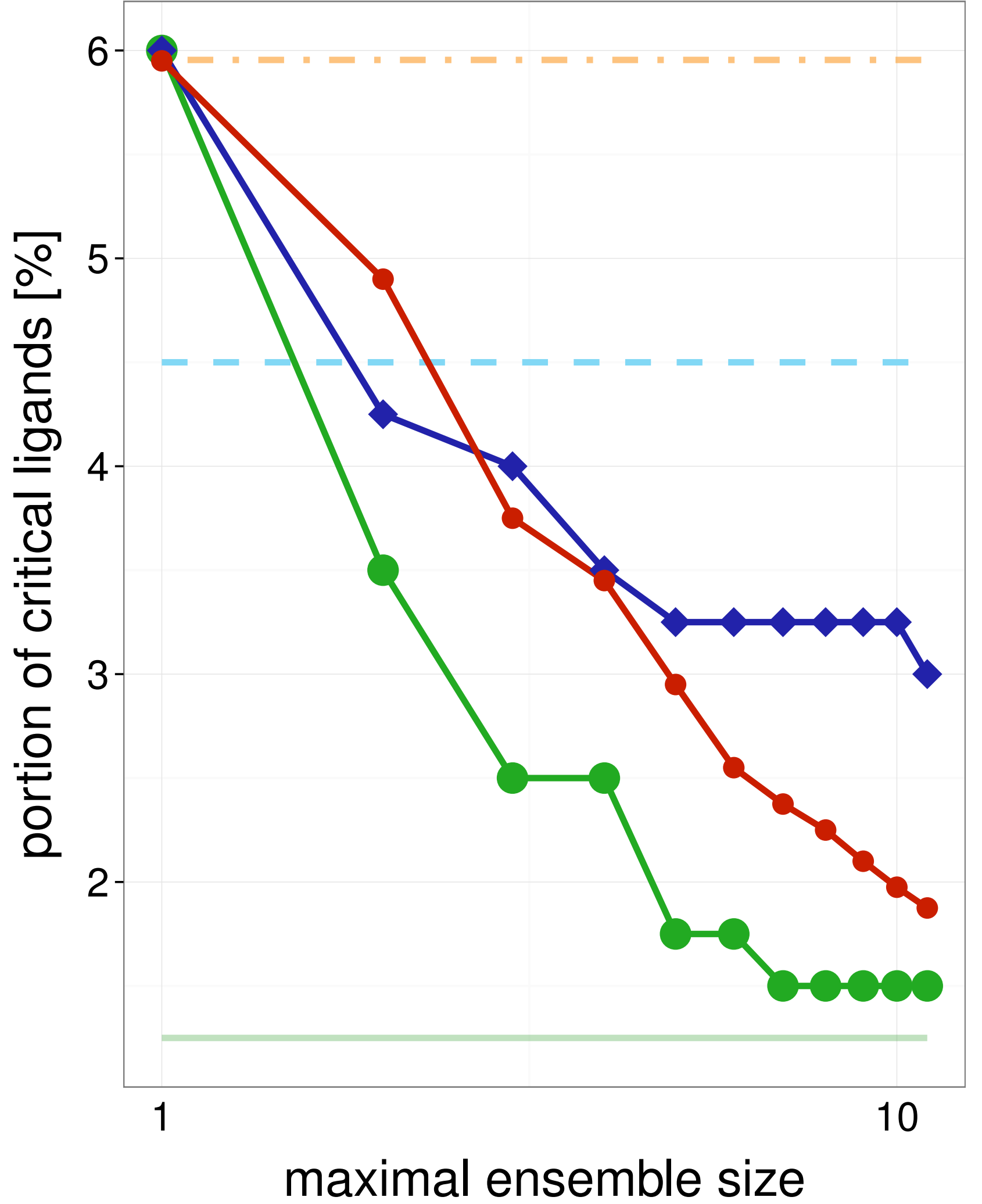

Average single structure

Best single structure

Whole ensemble

- SIENA

$\neg$ Clustering

$\rightarrow$ Random

Average single structure

Best single structure

Whole ensemble

- SIENA

$\neg$ Clustering

$\multimap$ Random 


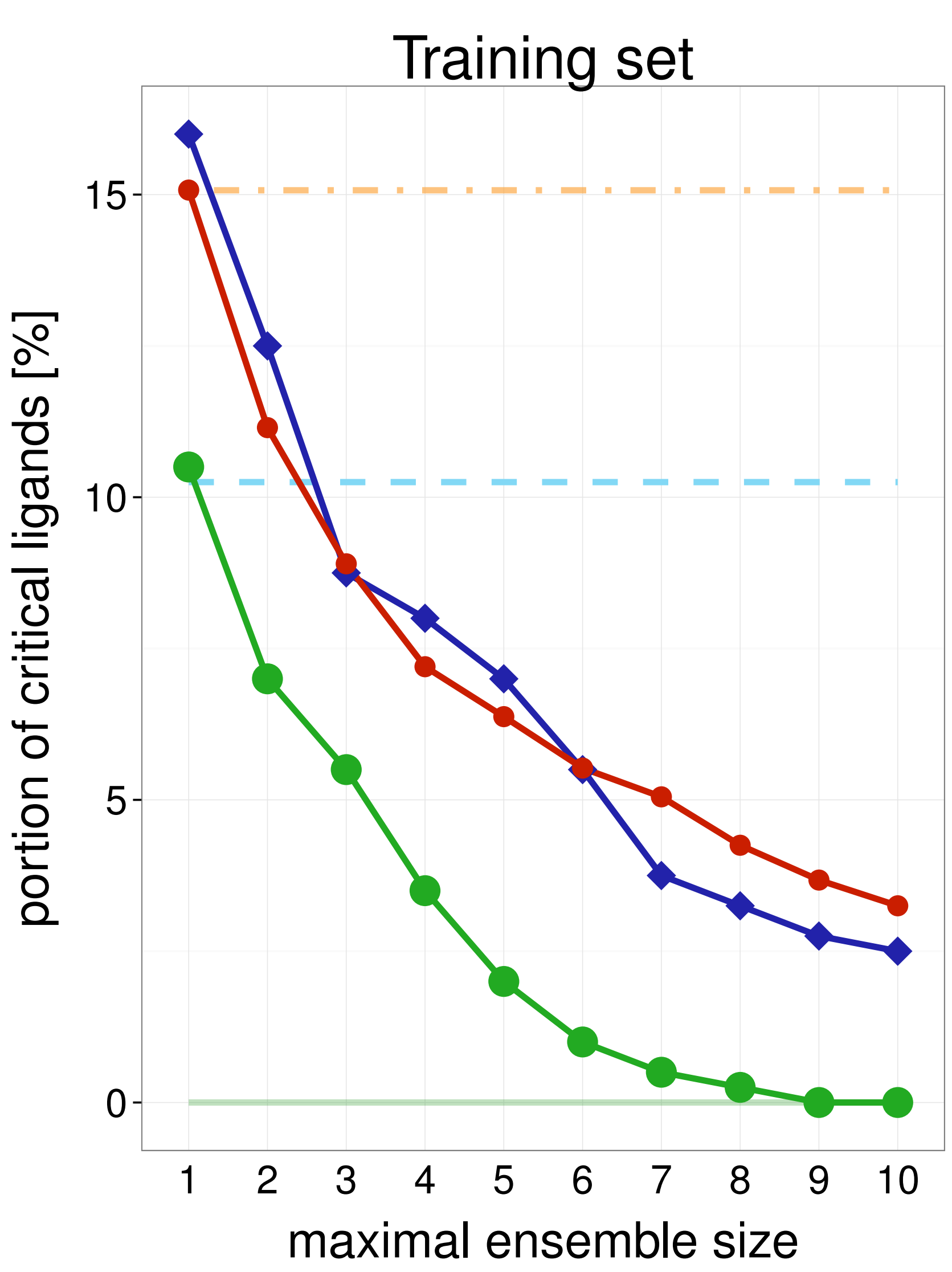

\section{Test set}

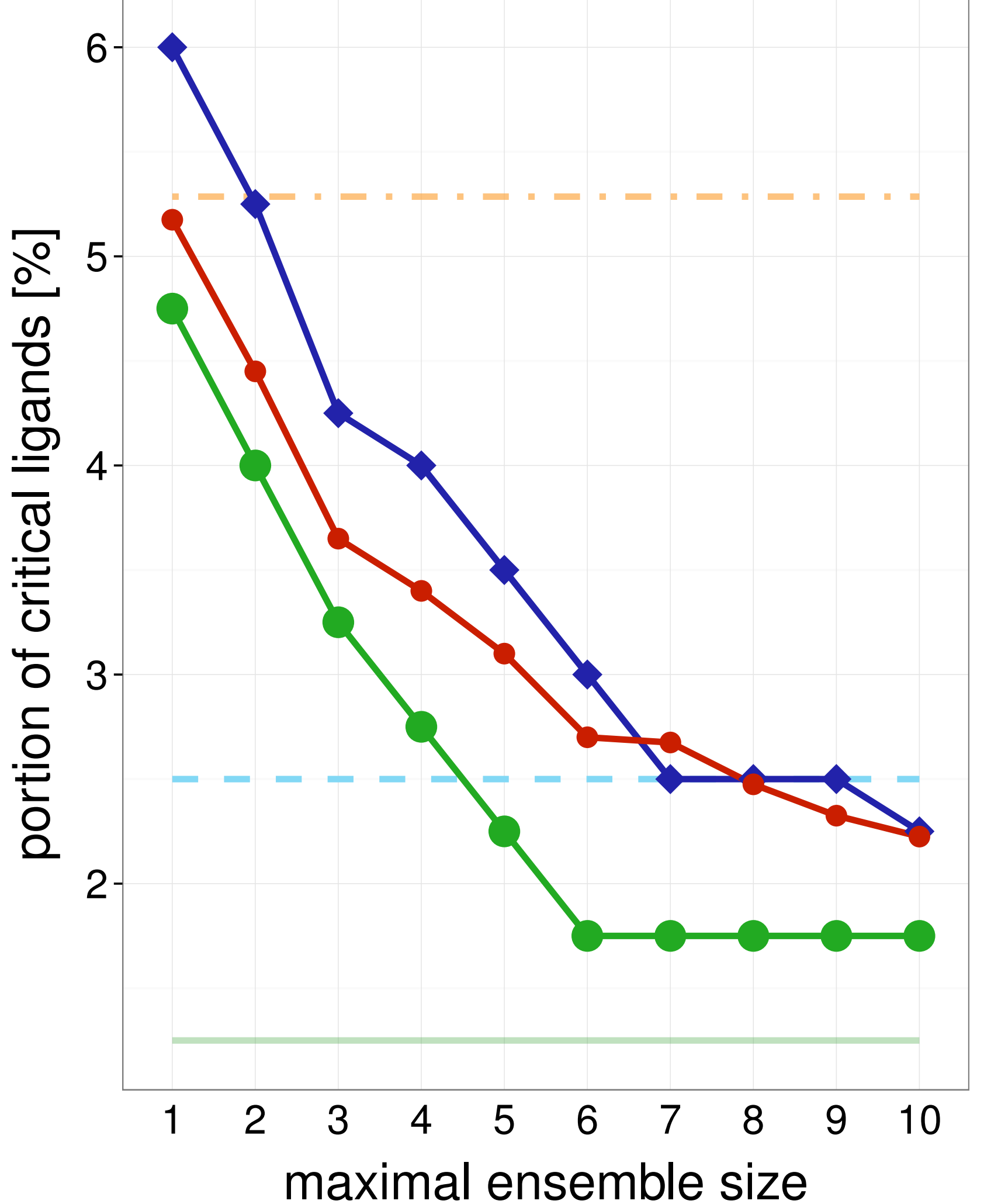

3LBK

Training set

Average single structure

_ Best single structure

Whole ensemble

- SIENA

$\neg$ Clustering

$\rightarrow$ Random

Average single structure

\section{_ Best single} structure

Whole ensemble

- SIENA

$\neg$ Clustering

$\multimap$ Random

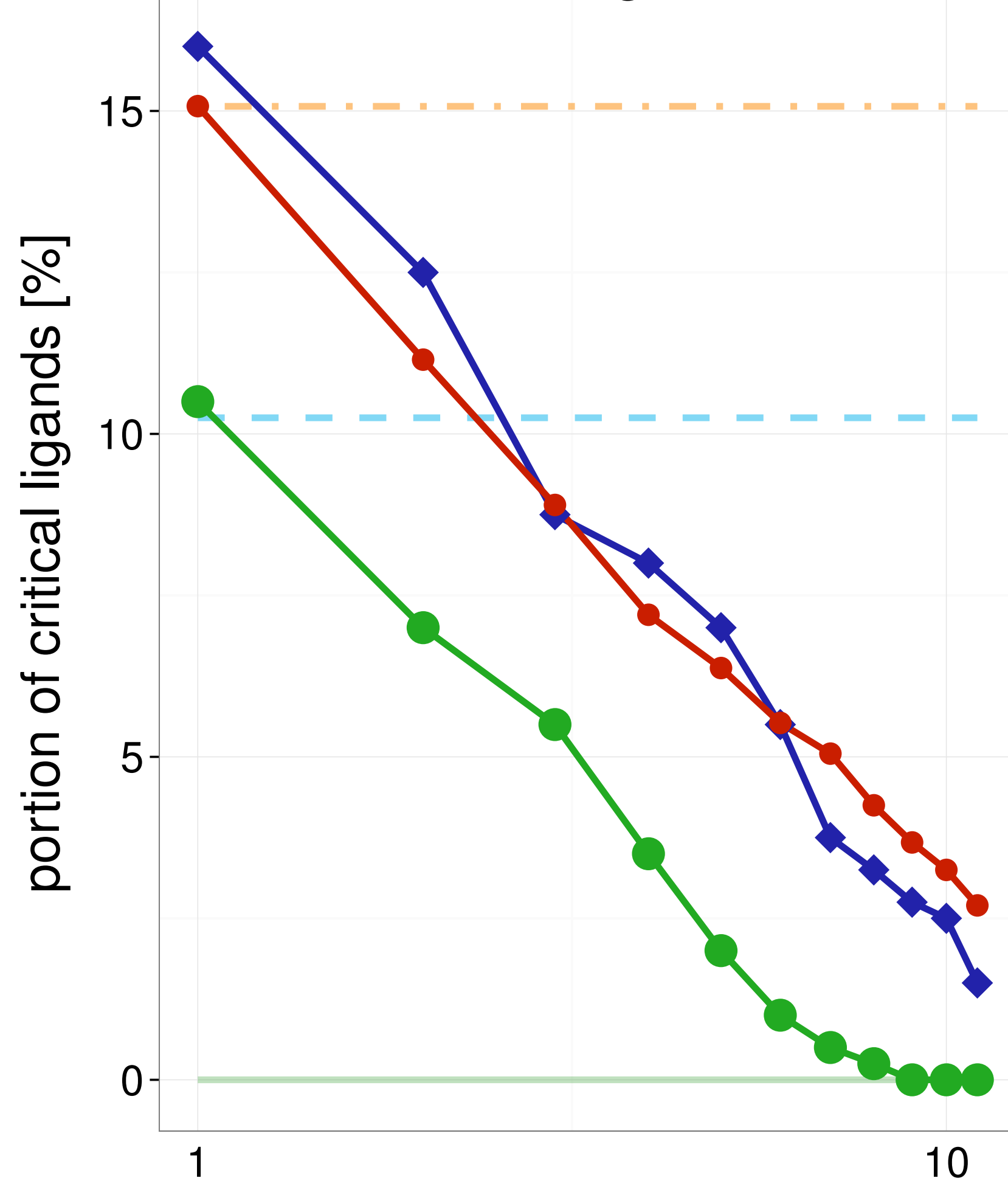

maximal ensemble size

Test set

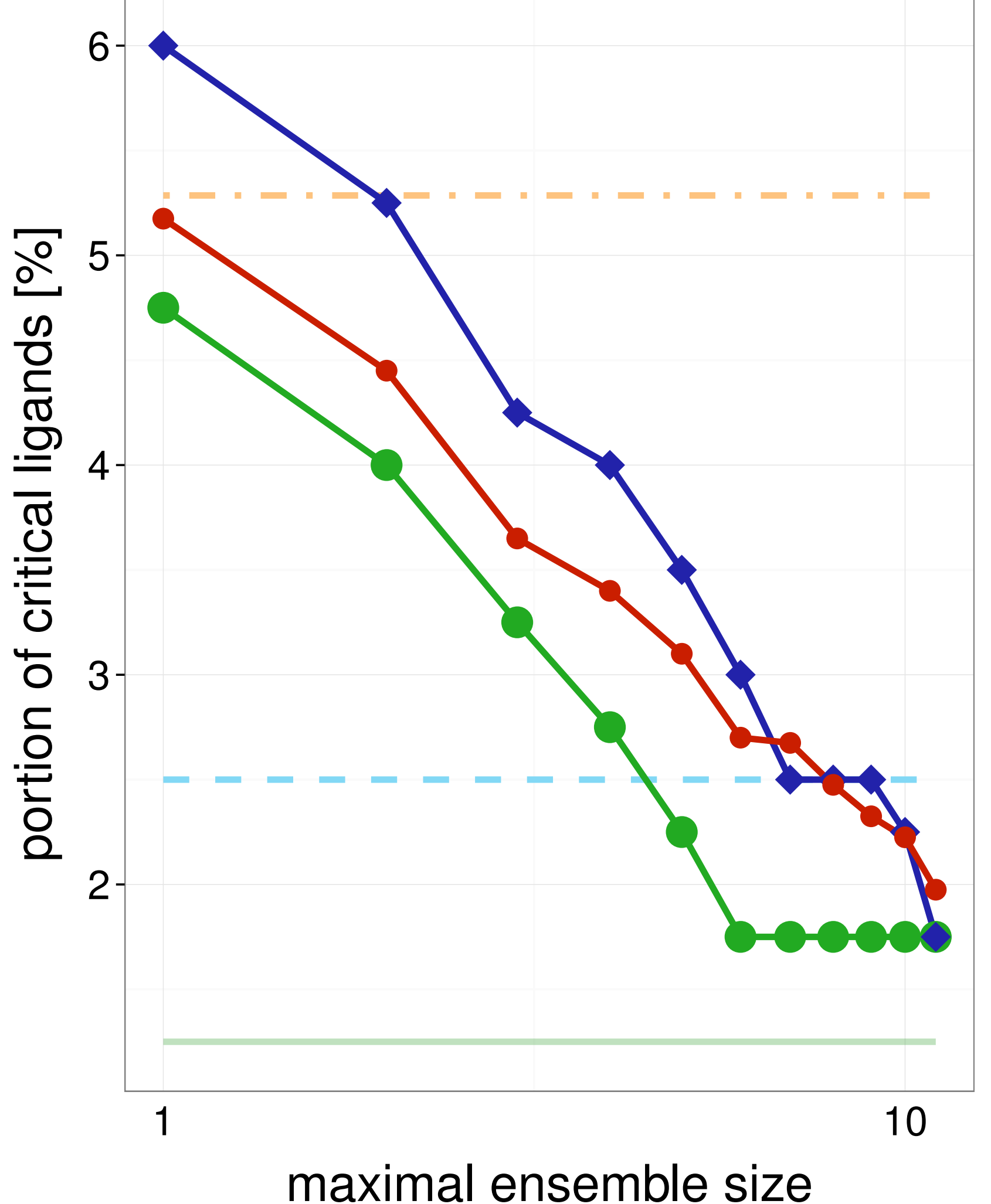

Average single structure

Best single structure

Whole ensemble

- SIENA

$\checkmark$ Clustering

$\longrightarrow$ Random

Average single structure

_ _ Best single structure

Whole ensemble

SIENA

$\neg$ Clustering

$\multimap$ Random 


\section{Training set}

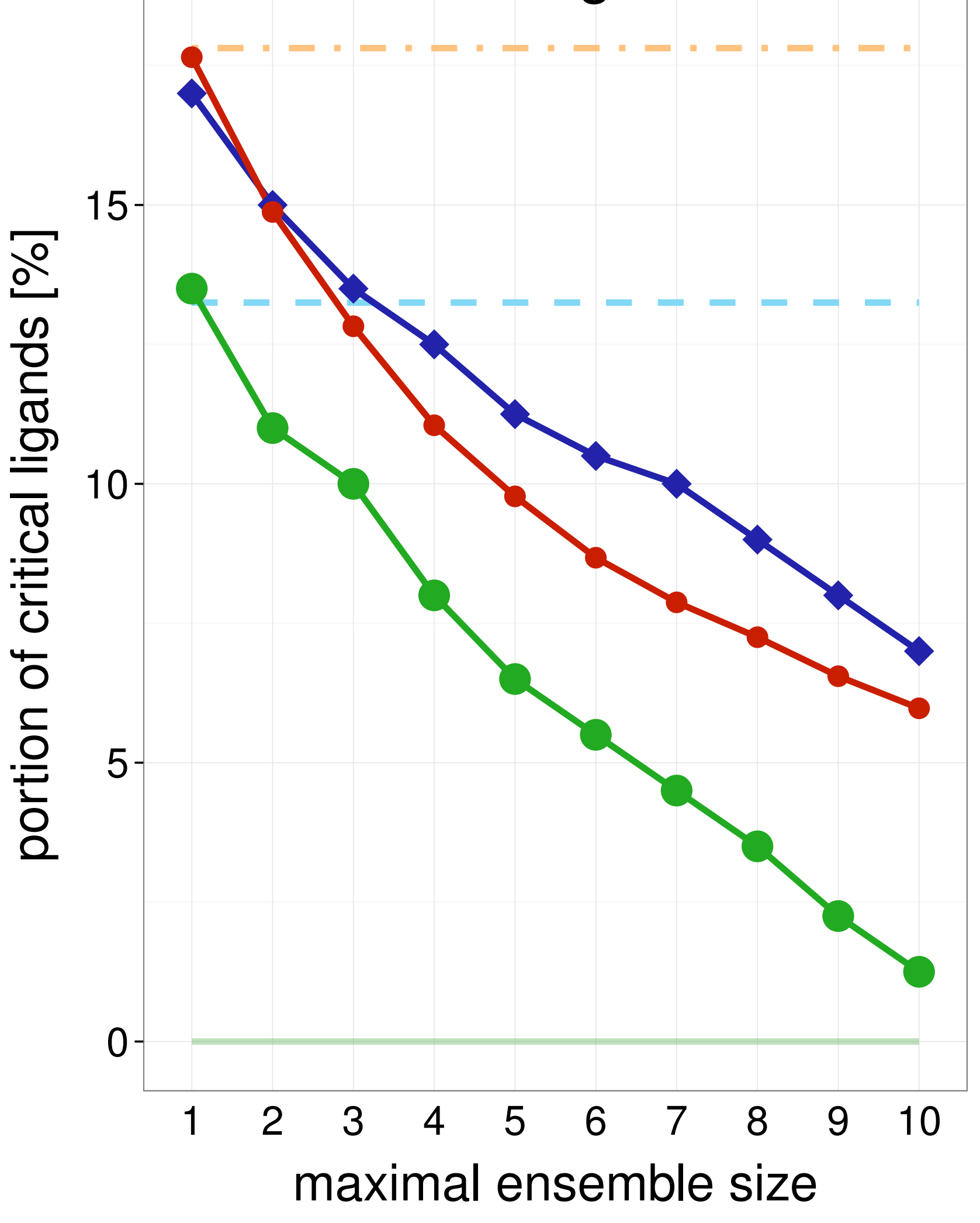

\section{Test set}

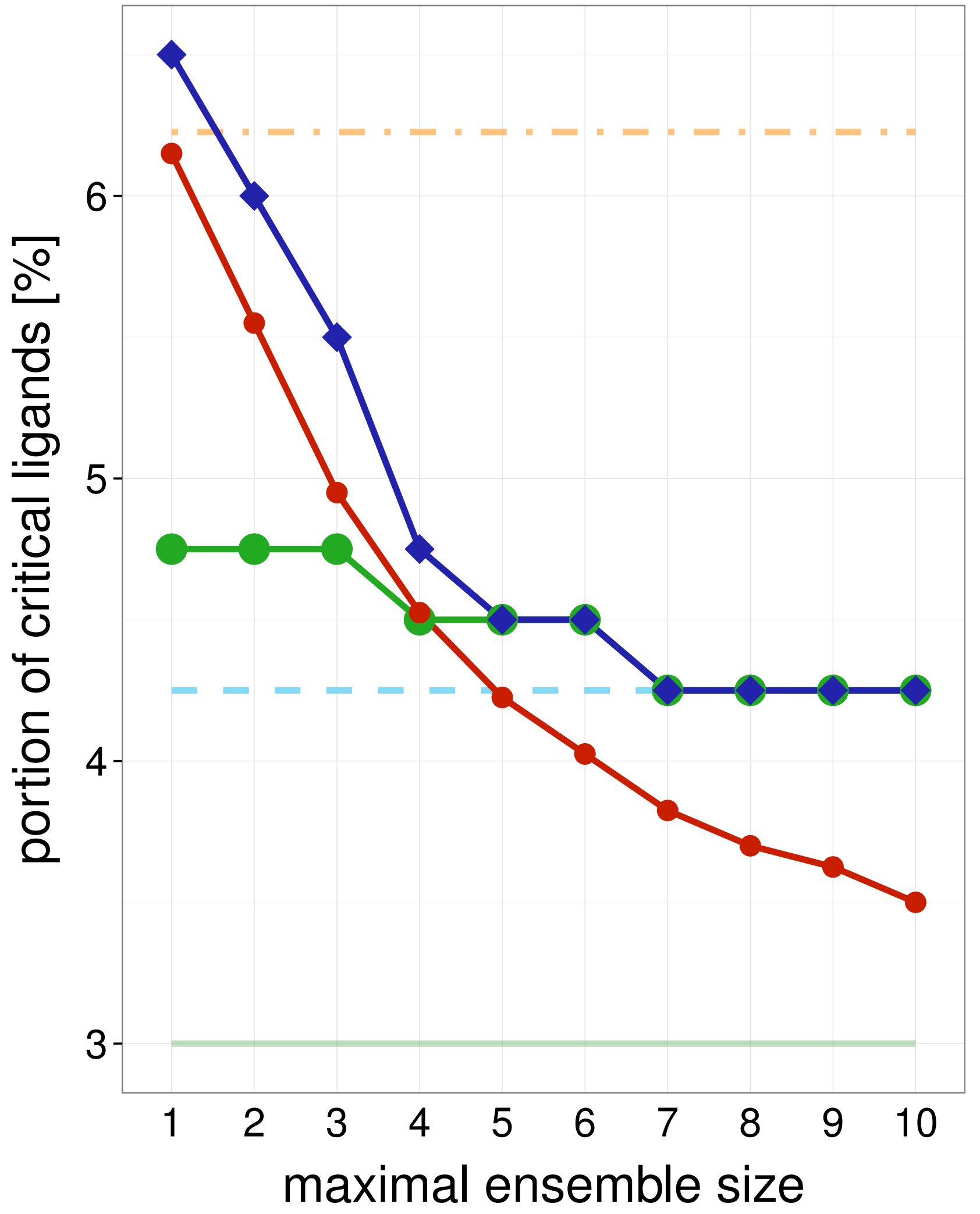

3R7M

Training set

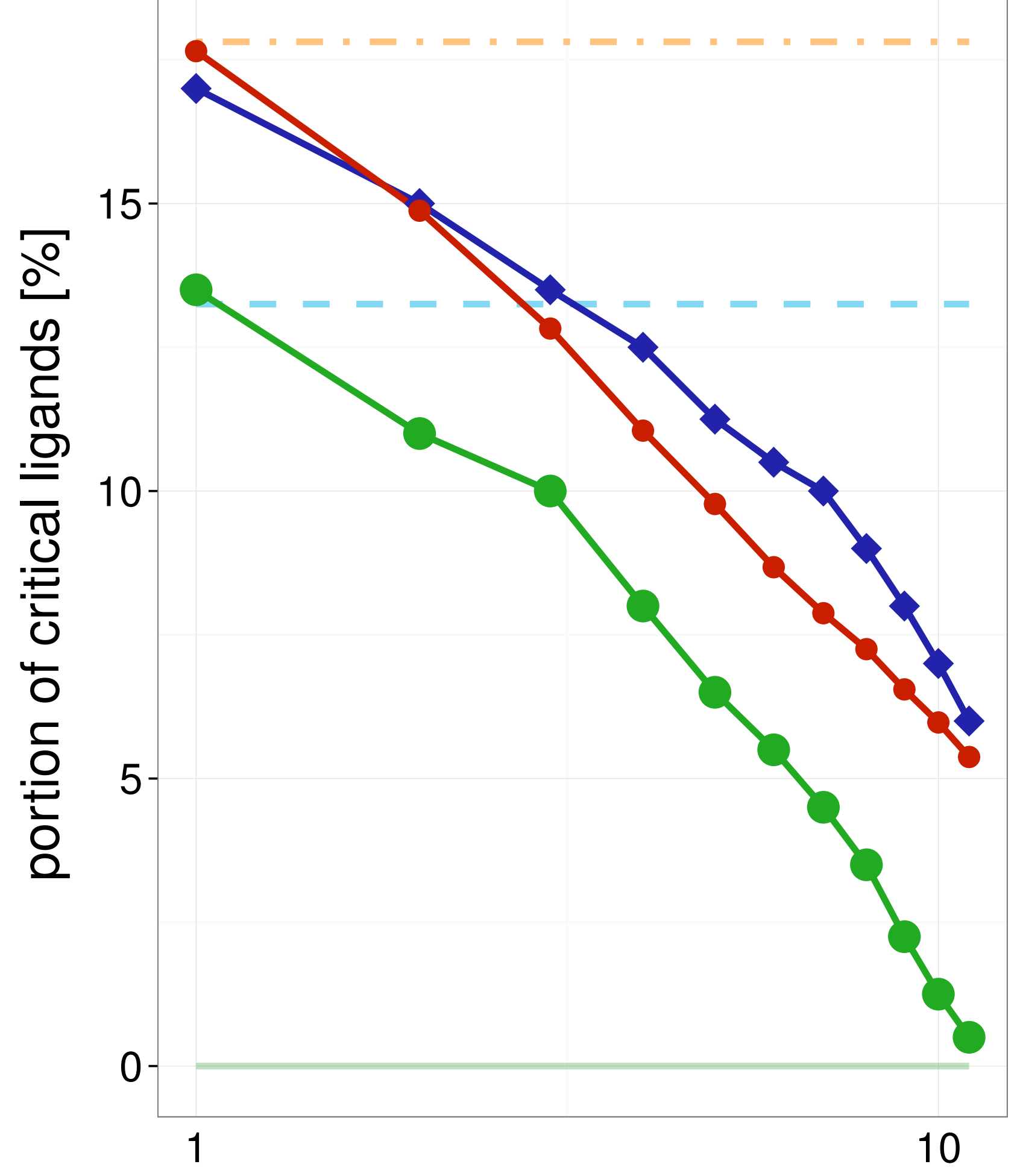

maximal ensemble size

\section{Test set}

Average single structure

_ _ Best single structure

Whole ensemble

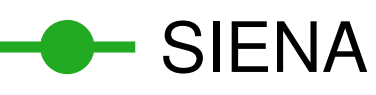

$\smile$ Clustering

$\multimap$ Random

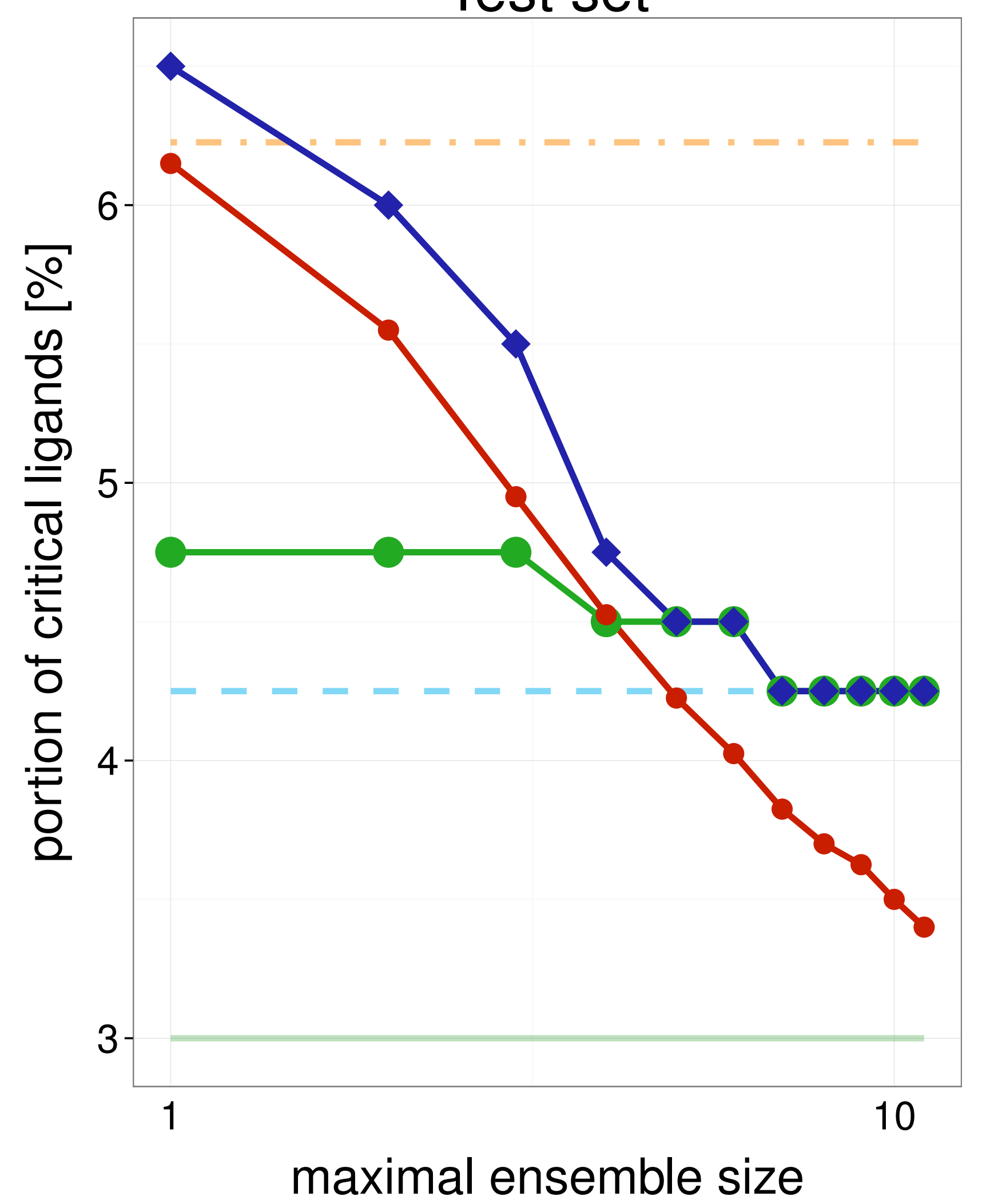

Average single structure

Best single structure

Whole ensemble

- SIENA

$\neg$ Clustering

$\longrightarrow$ Random 


\section{Training set}

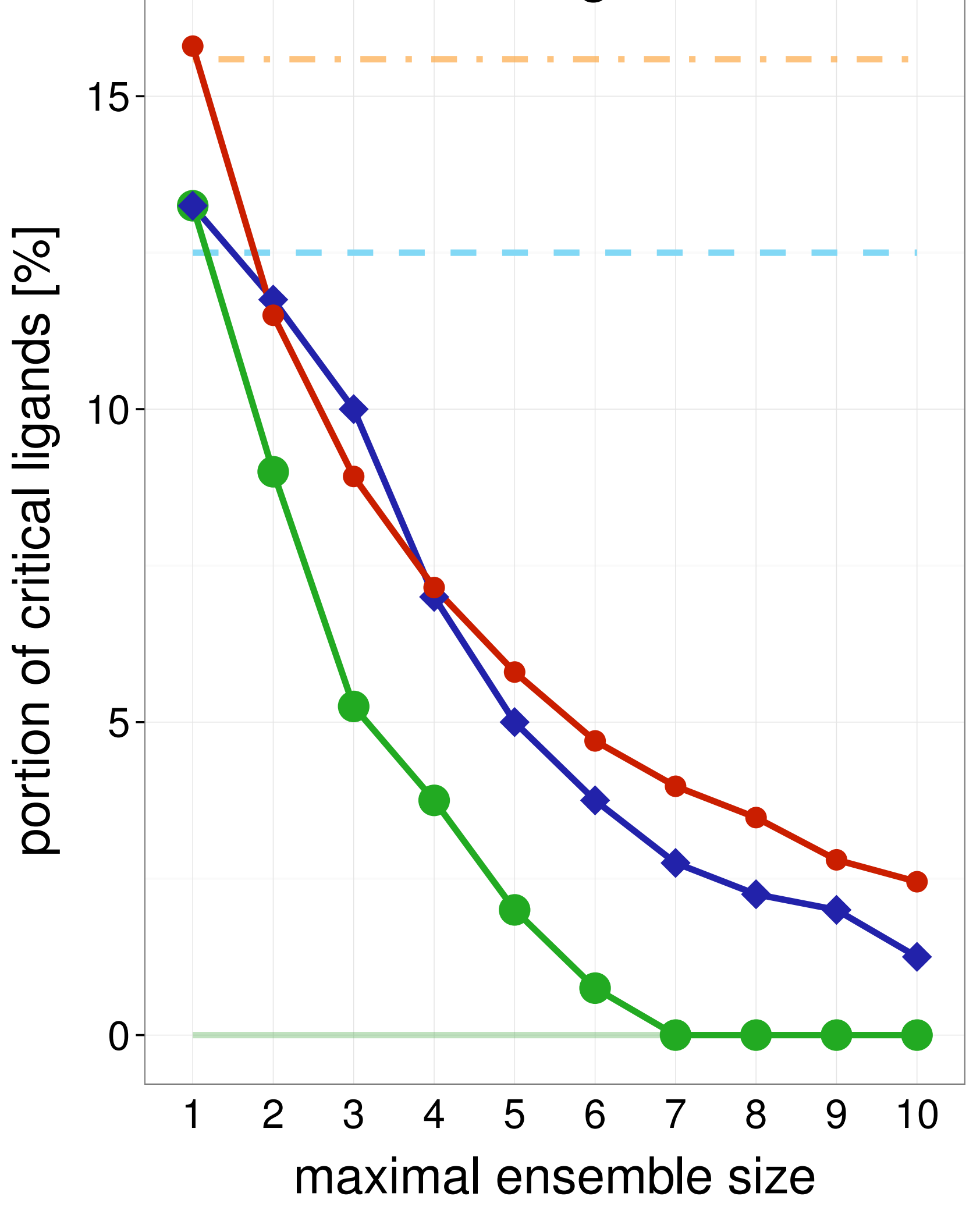

Test set

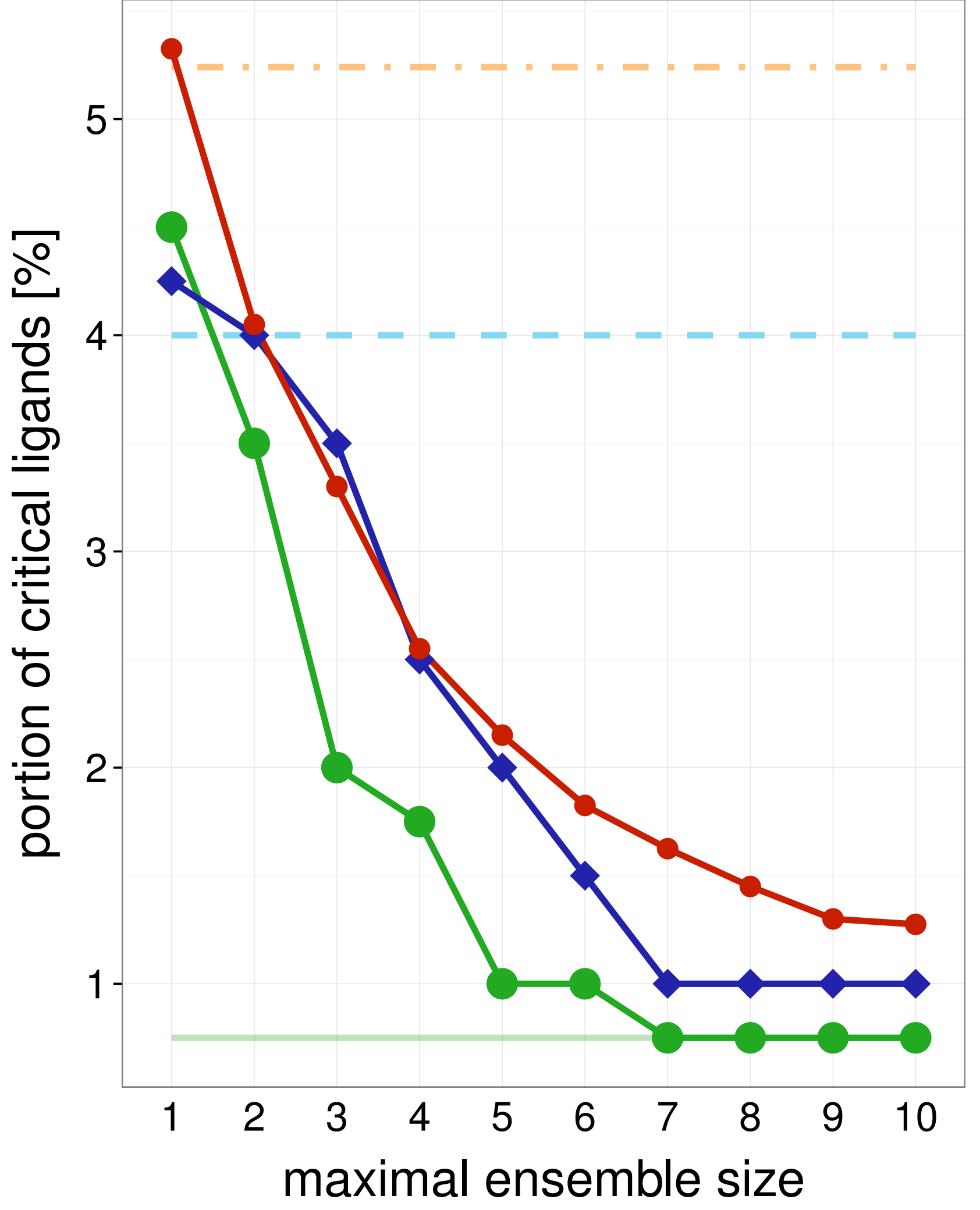

Average single structure

_ Best single structure

Whole ensemble

- SIENA

$\smile$ Clustering

$\multimap$ Random

Average single structure

_ _ Best single structure

Whole ensemble

- SIENA

$\smile$ Clustering

- Random
3TUD

Training set

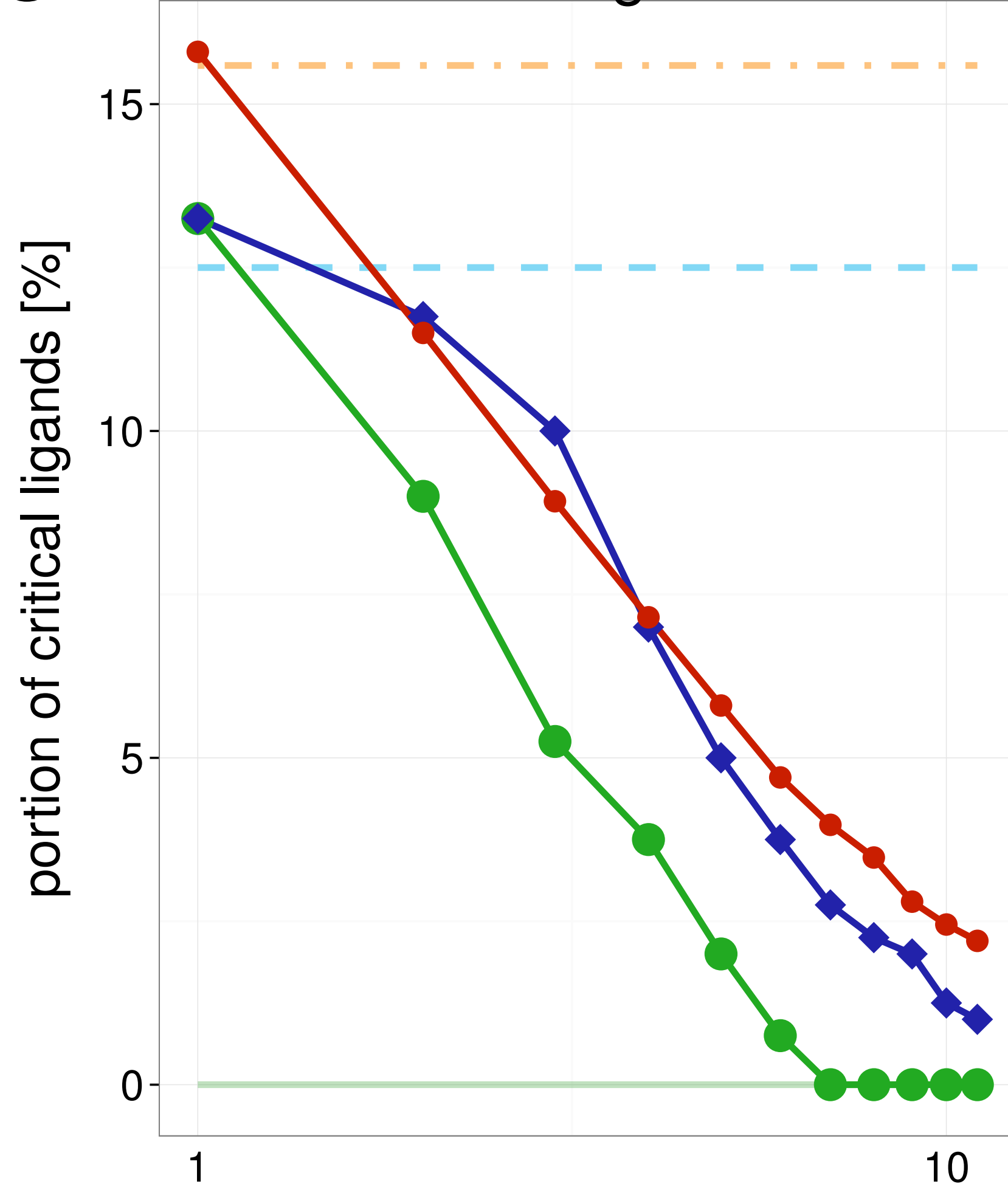

maximal ensemble size

\section{Test set}

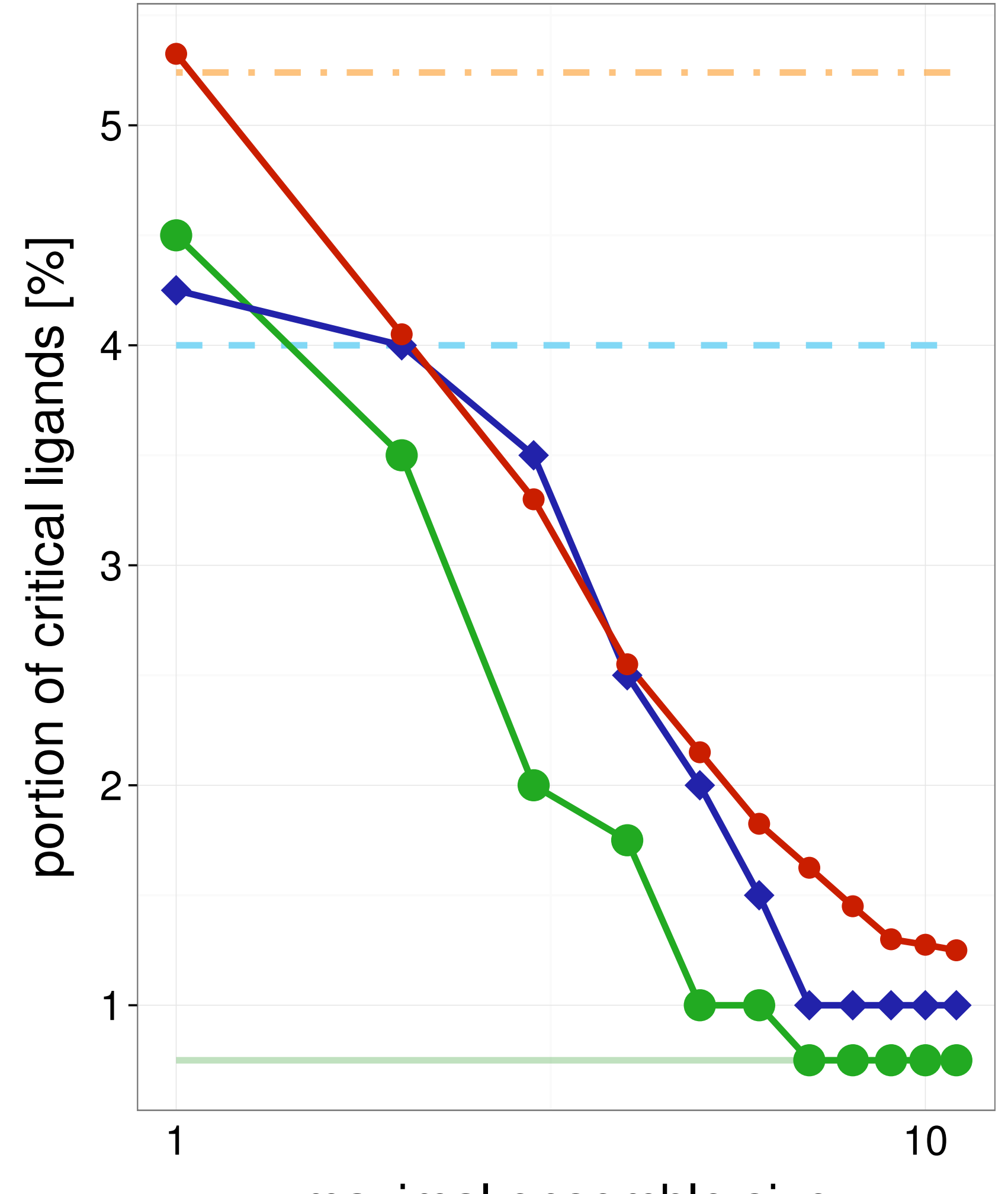

Average single structure

_ Best single structure

Whole ensemble

- SIENA

$\neg$ Clustering

$\multimap$ Random 
Training set

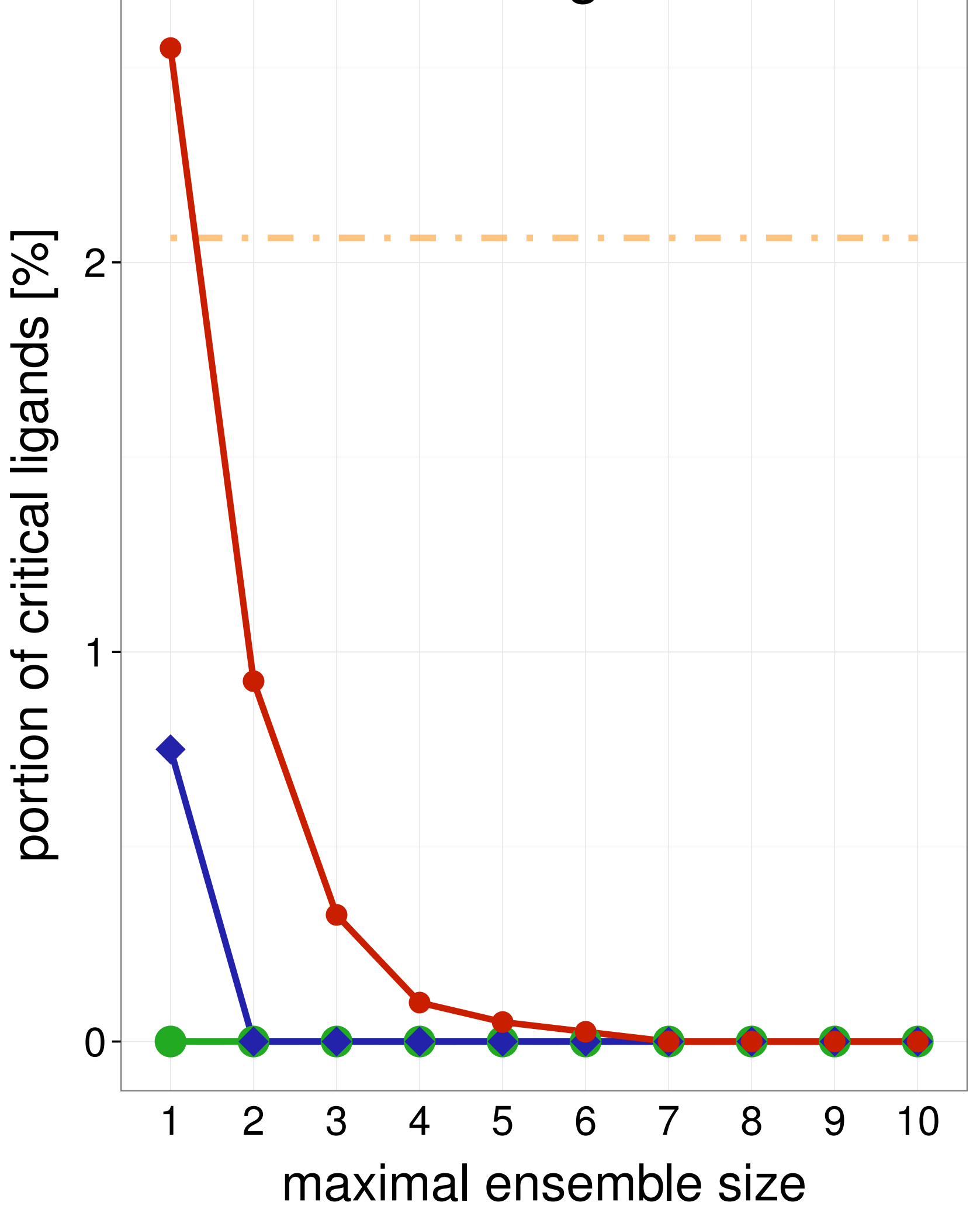

\section{Test set}

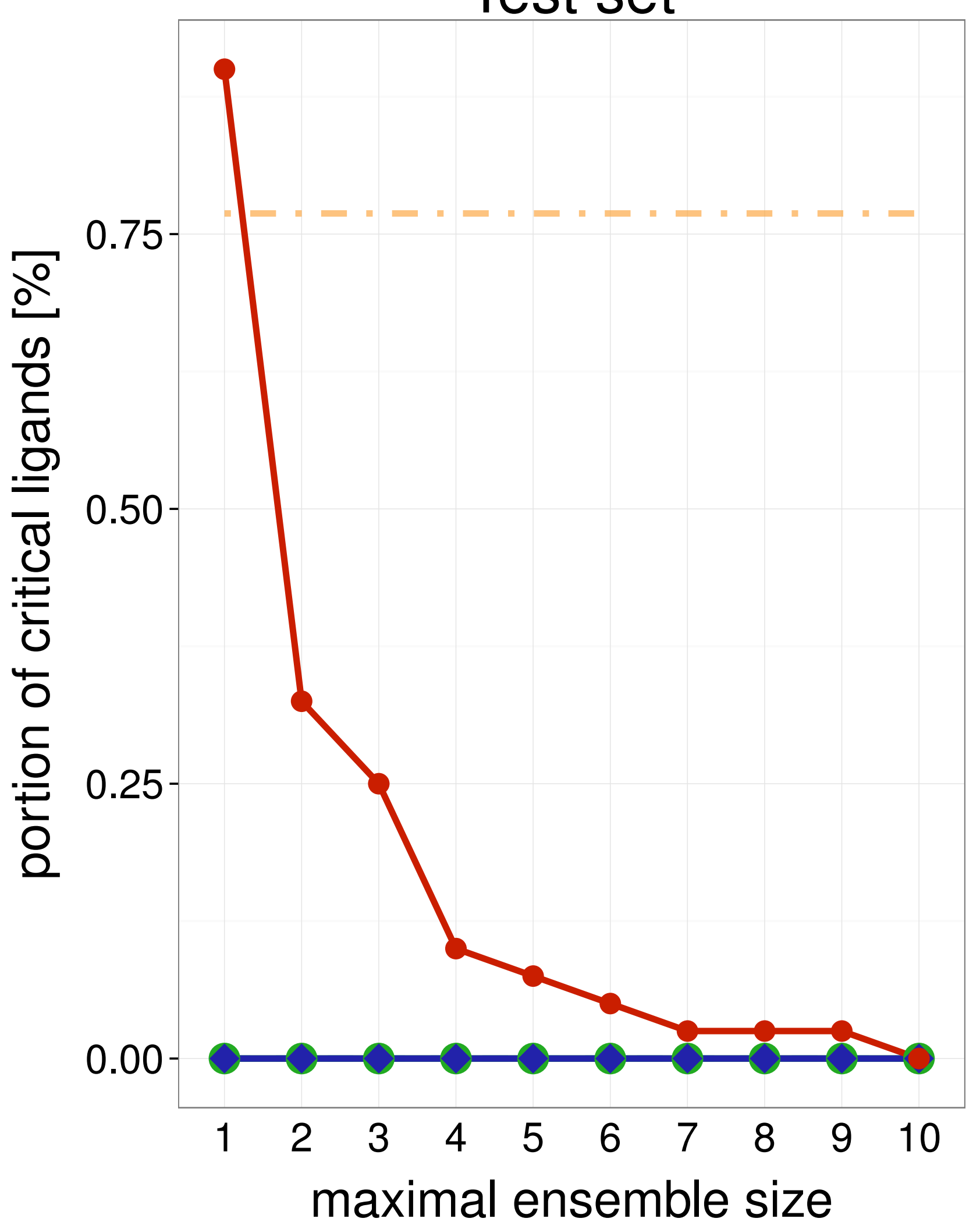

Average single structure

_ _ Best single structure

Whole ensemble

- SIENA

$\smile$ Clustering

$\multimap$ Random

Average single structure

_ Best single structure

Whole ensemble

- SIENA

$\neg$ Clustering

- Random

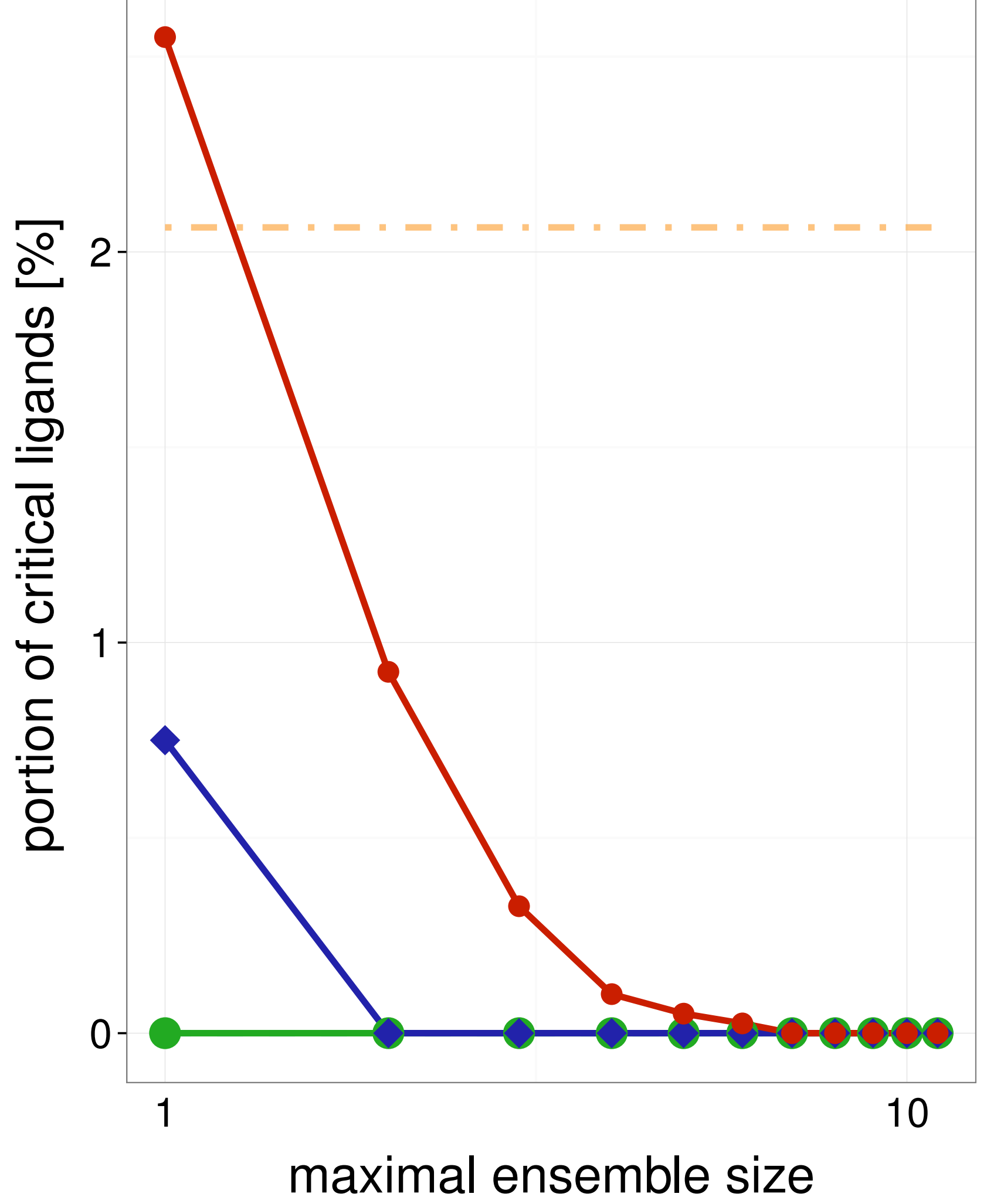

Average single structure

Best single structure

Whole ensemble

- SIENA

$\leadsto$ Clustering

$\multimap$ Random

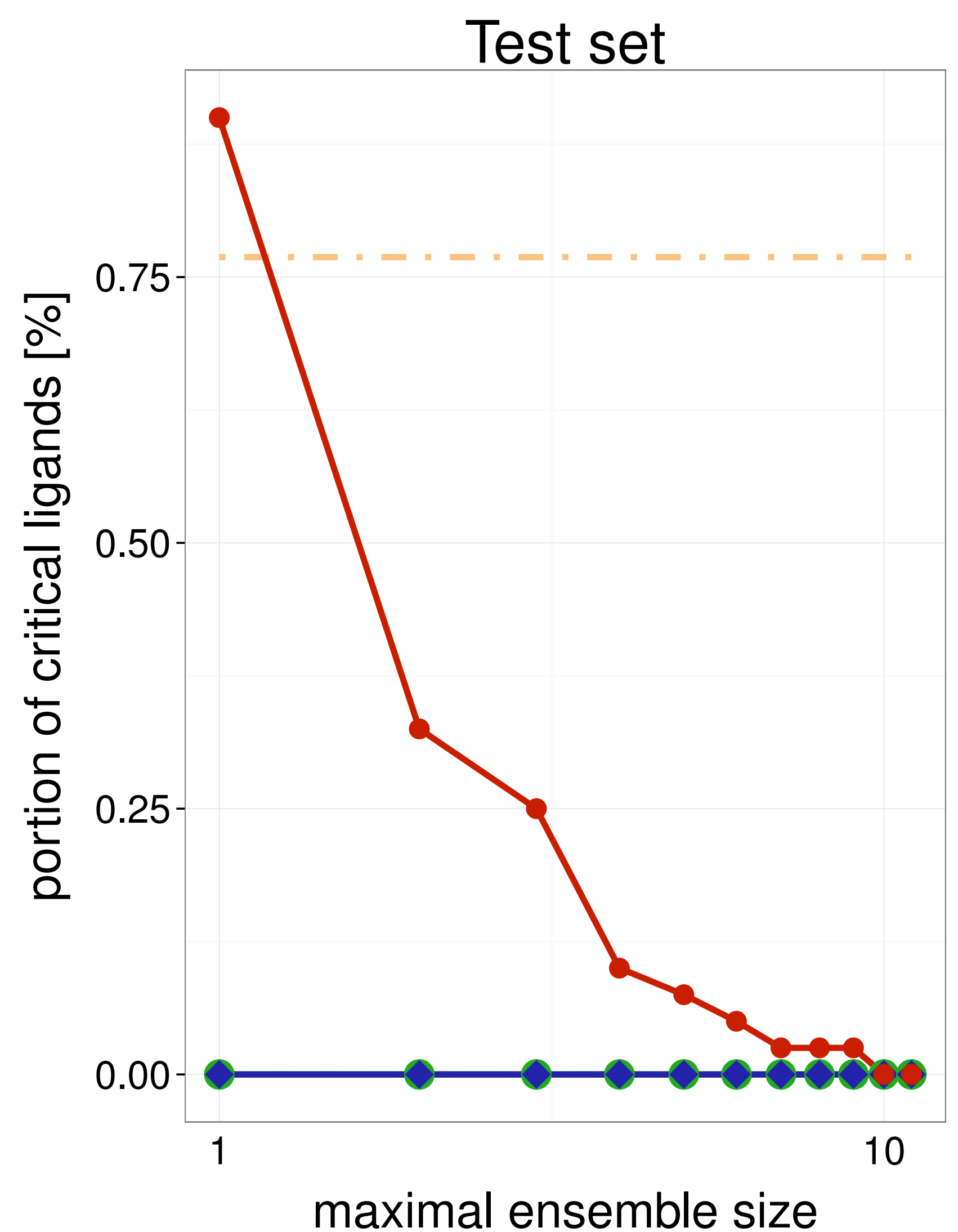

Average single structure

_ Best single structure

Whole ensemble

SIENA

$\leadsto$ Clustering

$\longrightarrow$ Random 


\section{Training set}

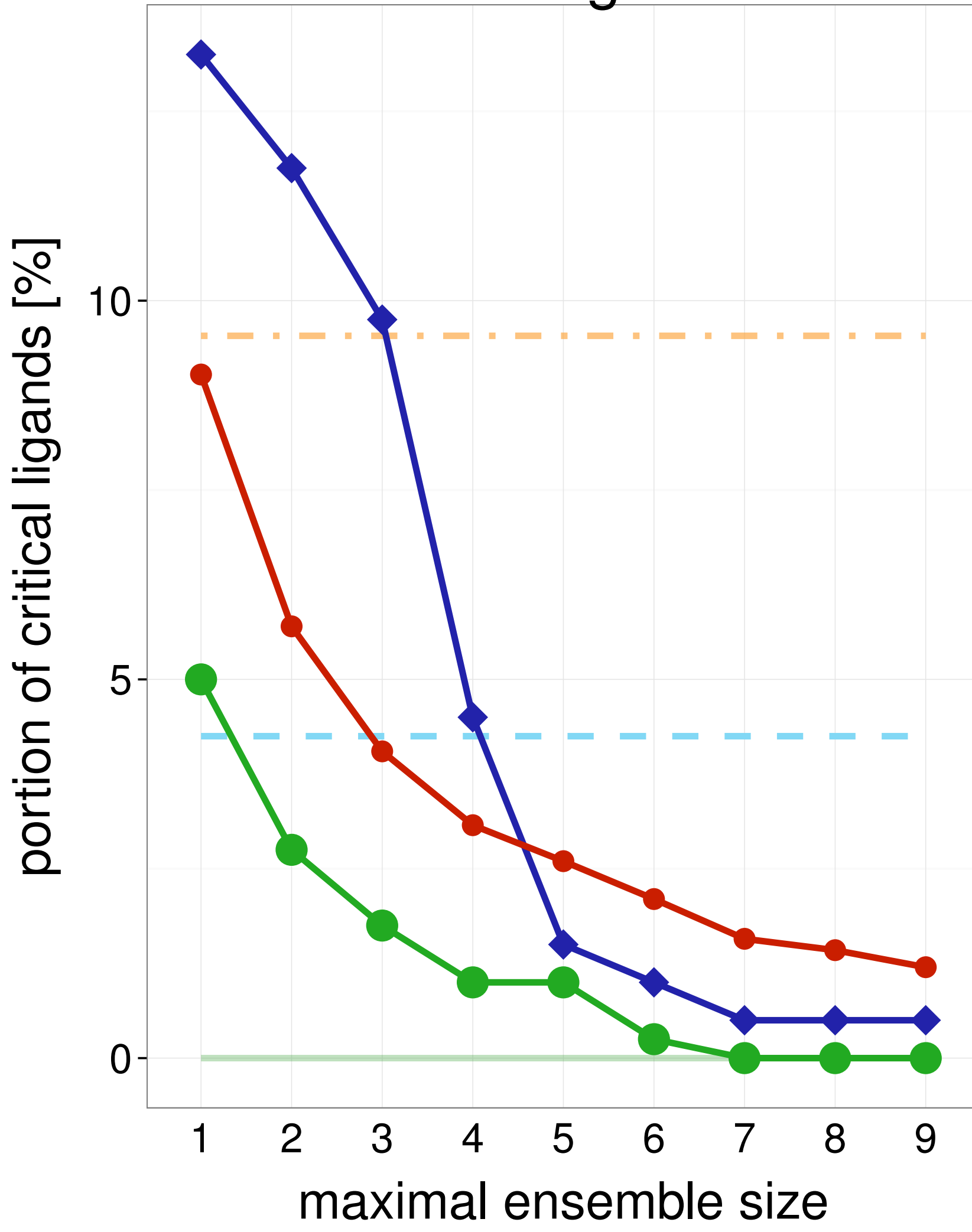

Test set

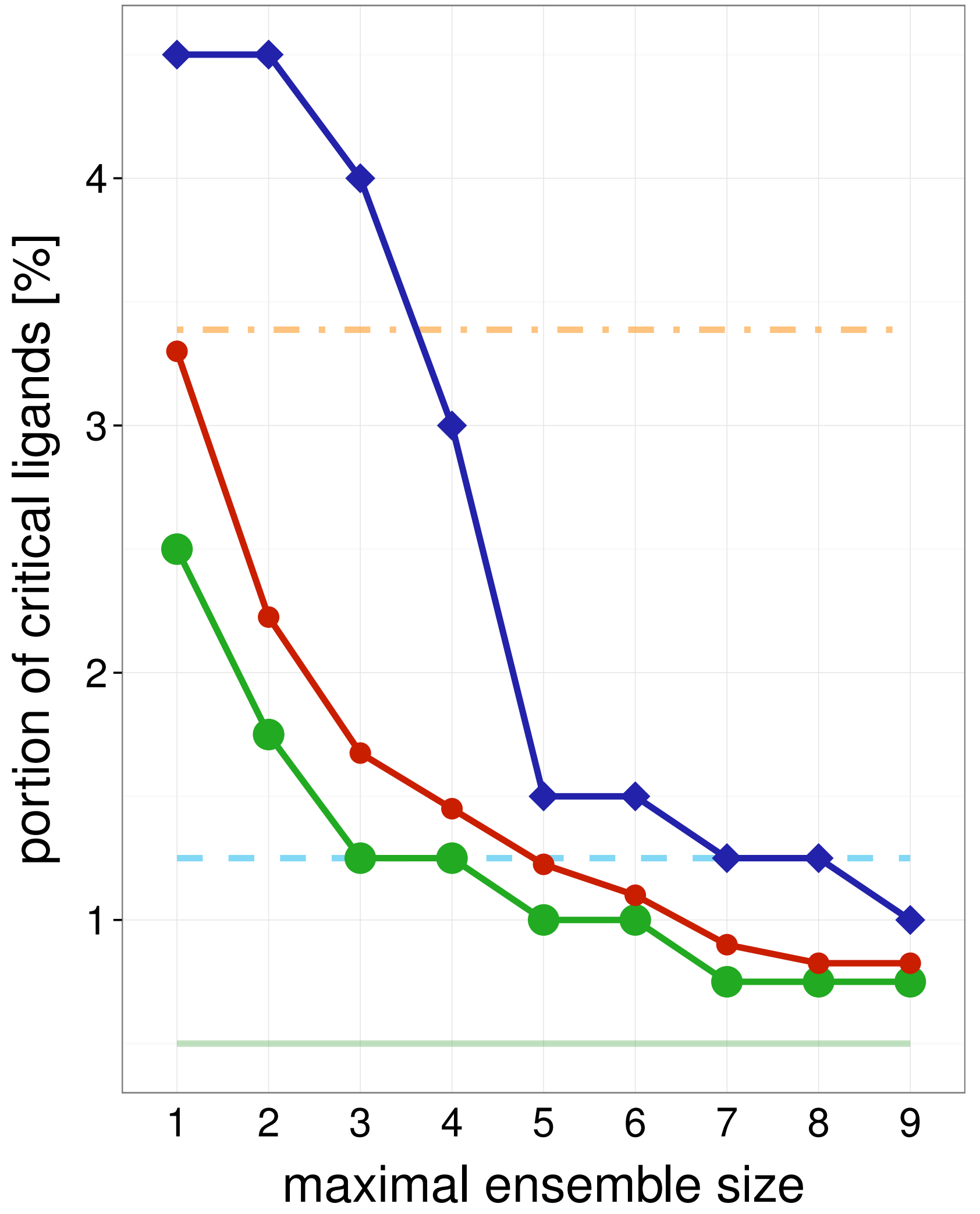

$1 \mathrm{QKN}$

Training set

Average single structure

_ Best single structure

Whole ensemble

- SIENA

$\neg$ Clustering

$\multimap$ Random

Average single structure

_ _ Best single structure

Whole ensemble

- SIENA

$\neg$ Clustering

$\multimap$ Random

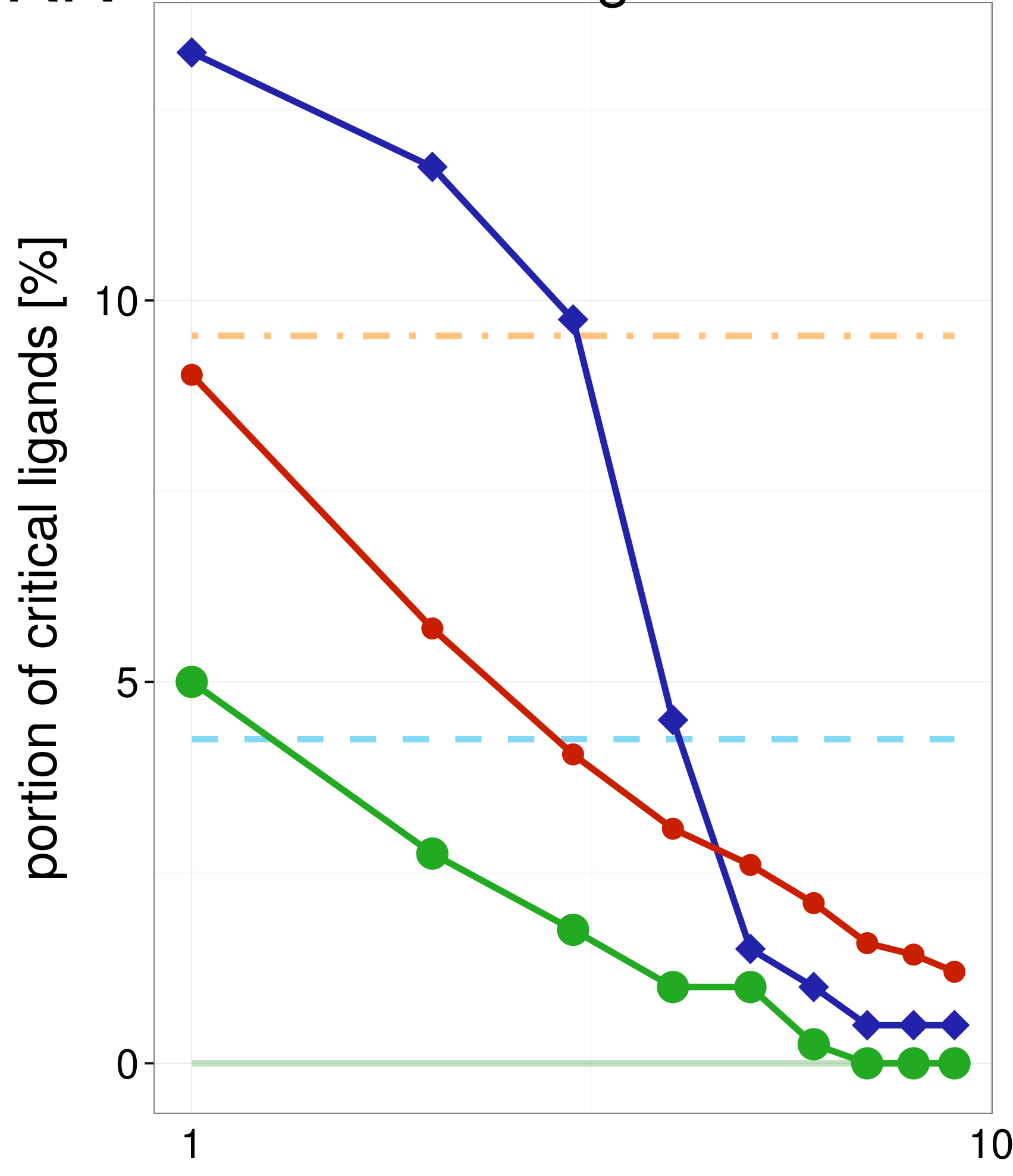

maximal ensemble size

\section{Test set}

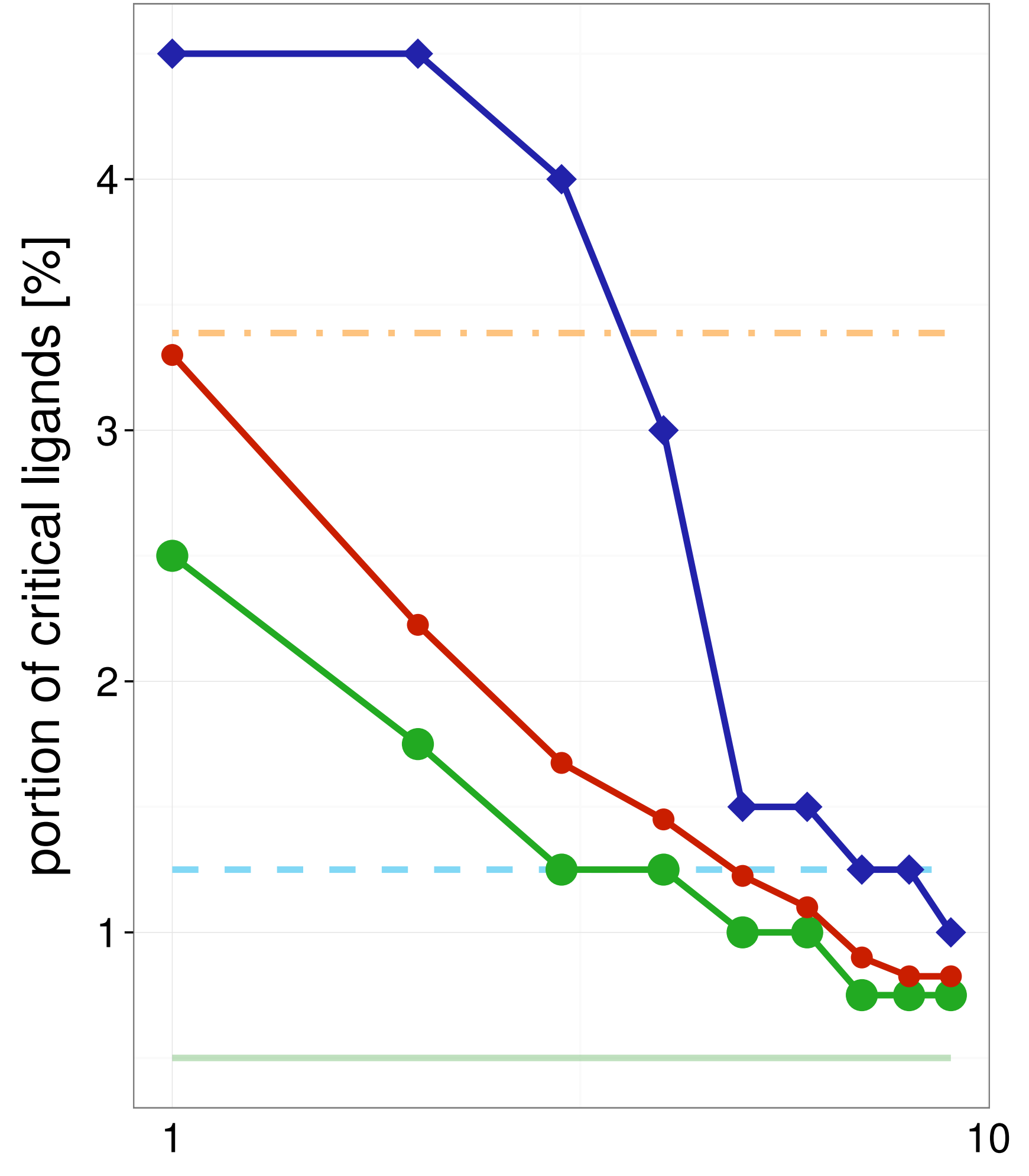

maximal ensemble size
Average single structure

_ Best single structure

Whole ensemble

- SIENA

$\smile$ Clustering

$\multimap$ Random 


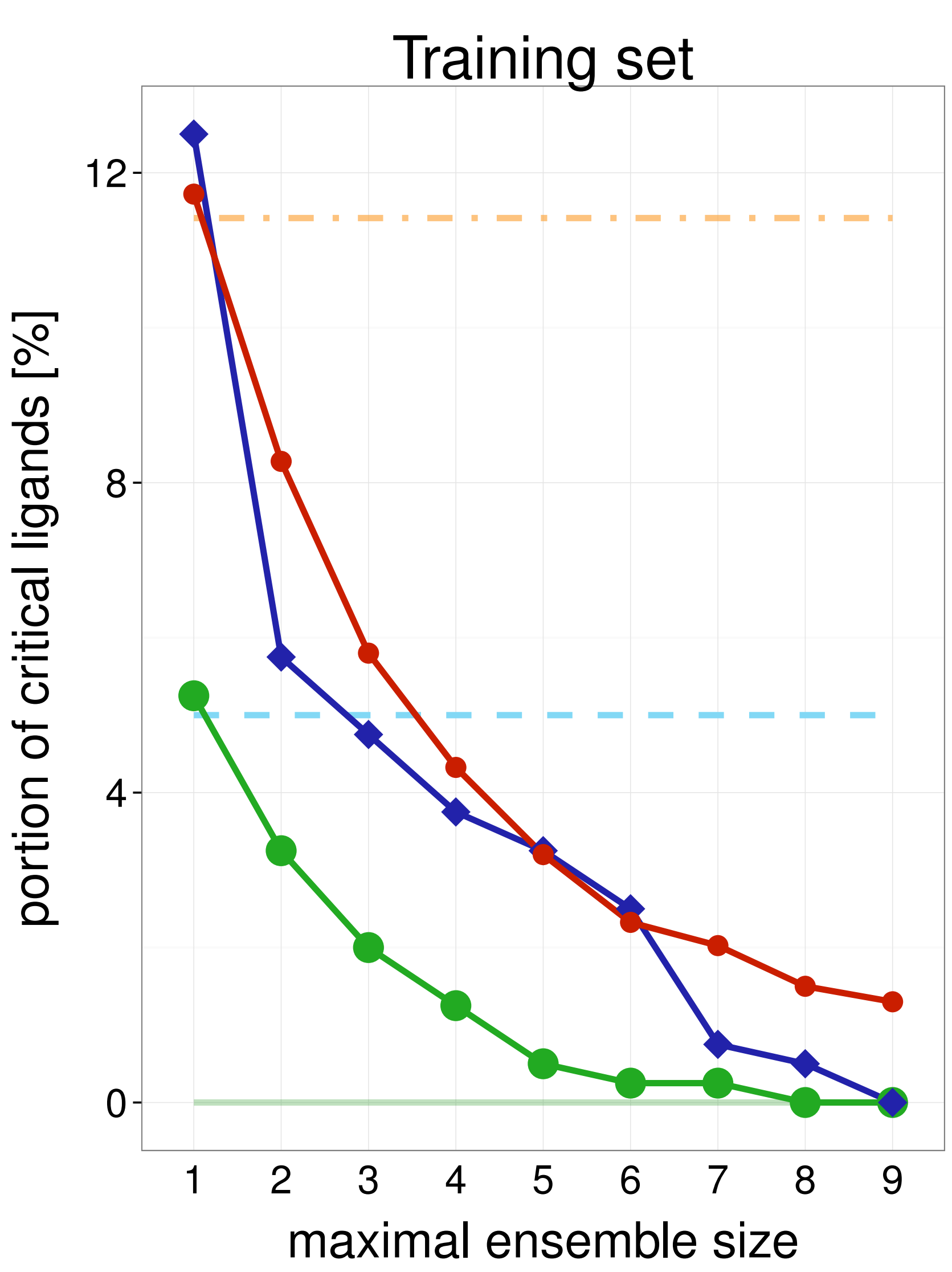

Test set

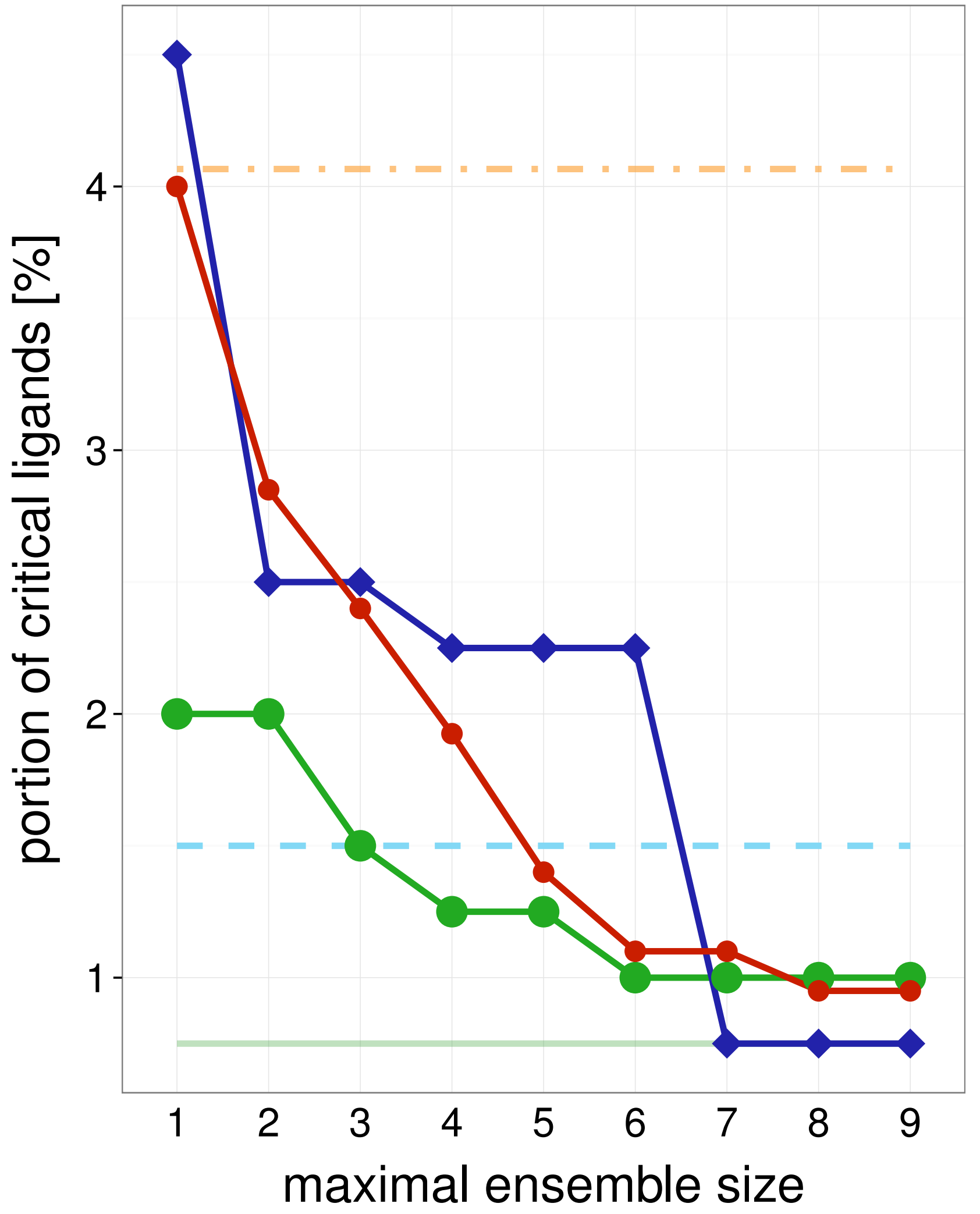

2PJA Training set

Average single structure

_ Best single

structure

Whole ensemble

- SIENA

$\smile$ Clustering

$\longrightarrow$ Random

Average single structure

_ Best single structure

Whole ensemble

- SIENA

$\neg$ Clustering

$\because$ Random

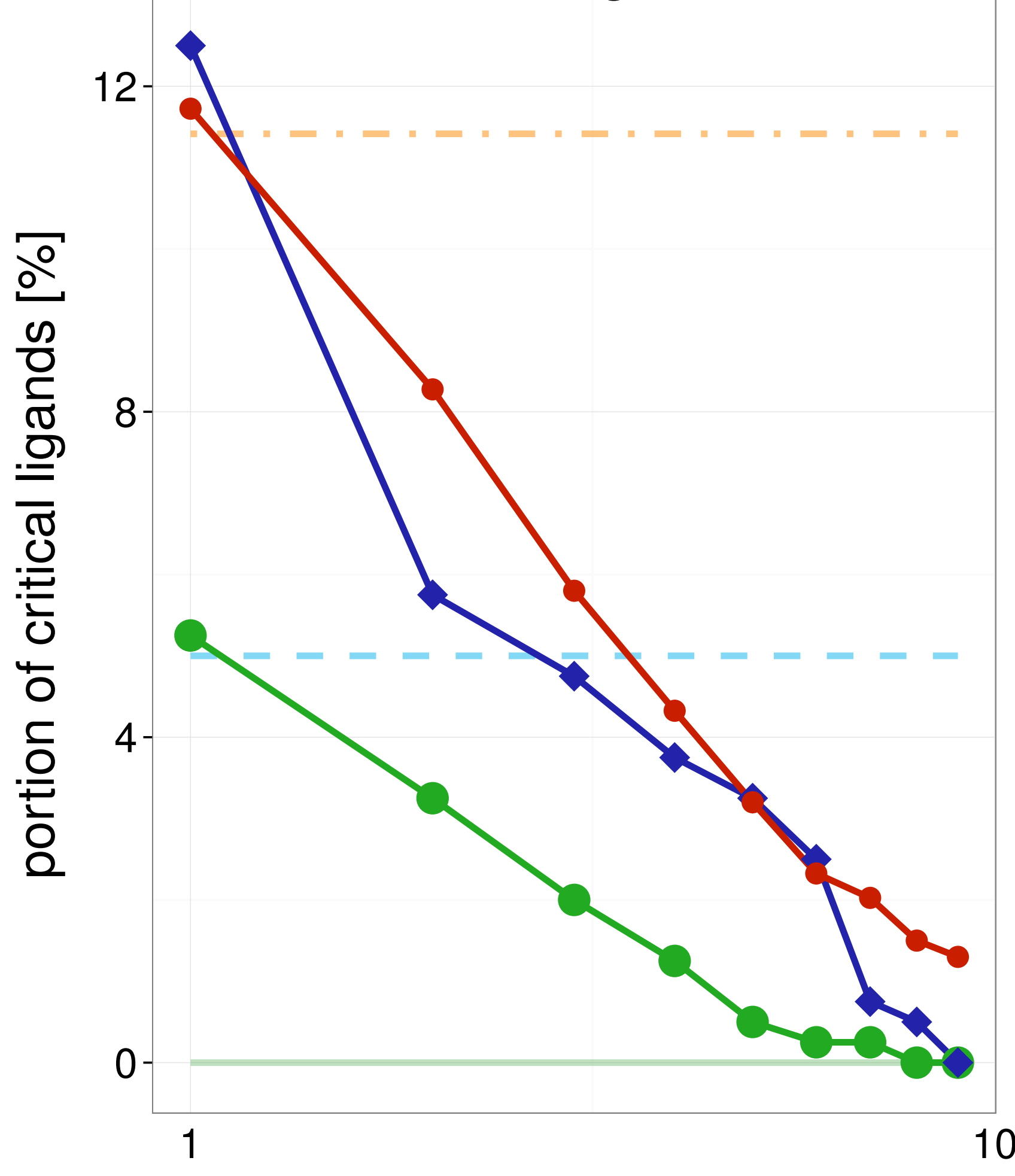

maximal ensemble size

Test set

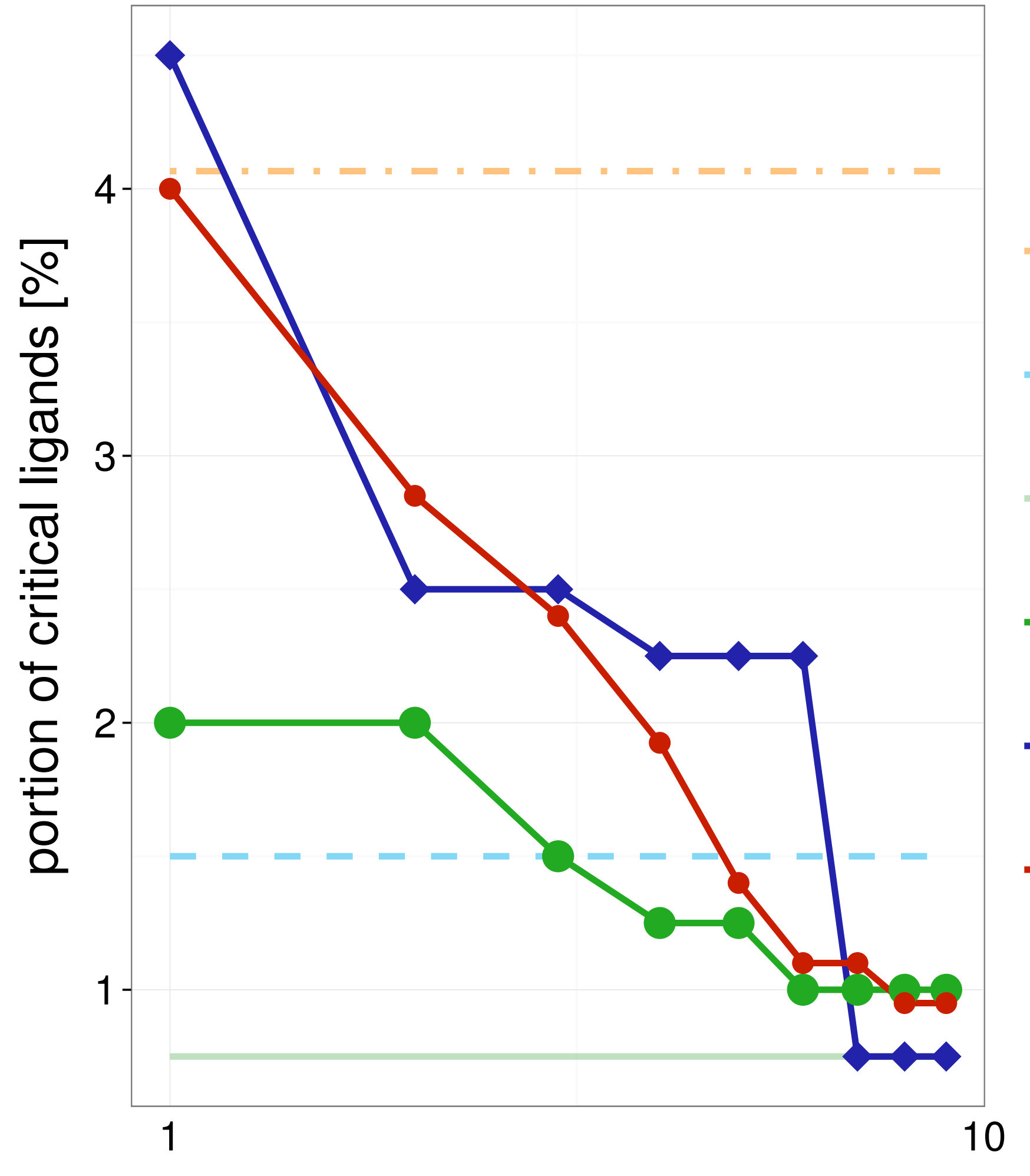

maximal ensemble size
Average single structure

_ Best single structure

Whole ensemble

SIENA

$\leadsto$ Clustering

$\multimap$ Random 
Training set

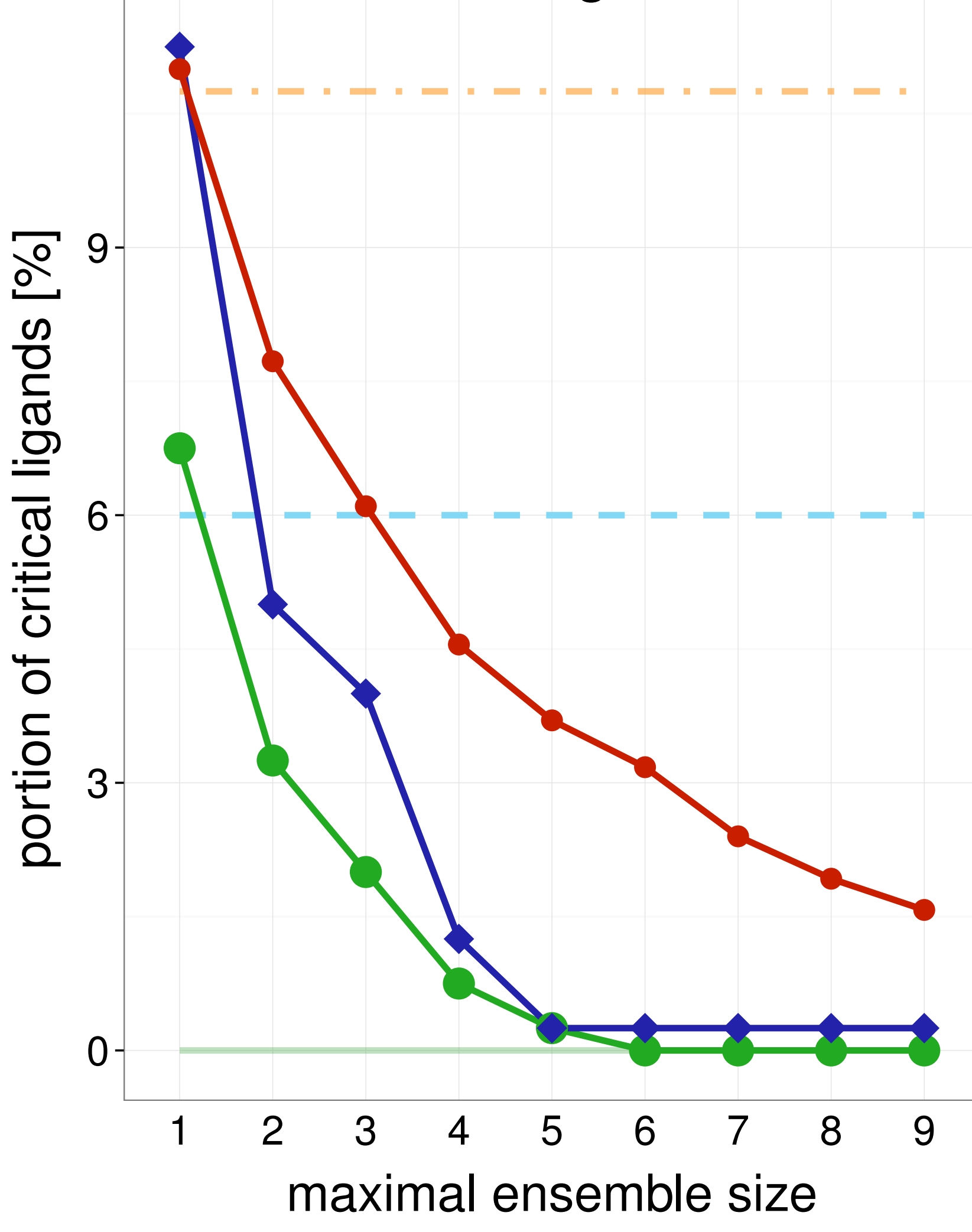

Test set

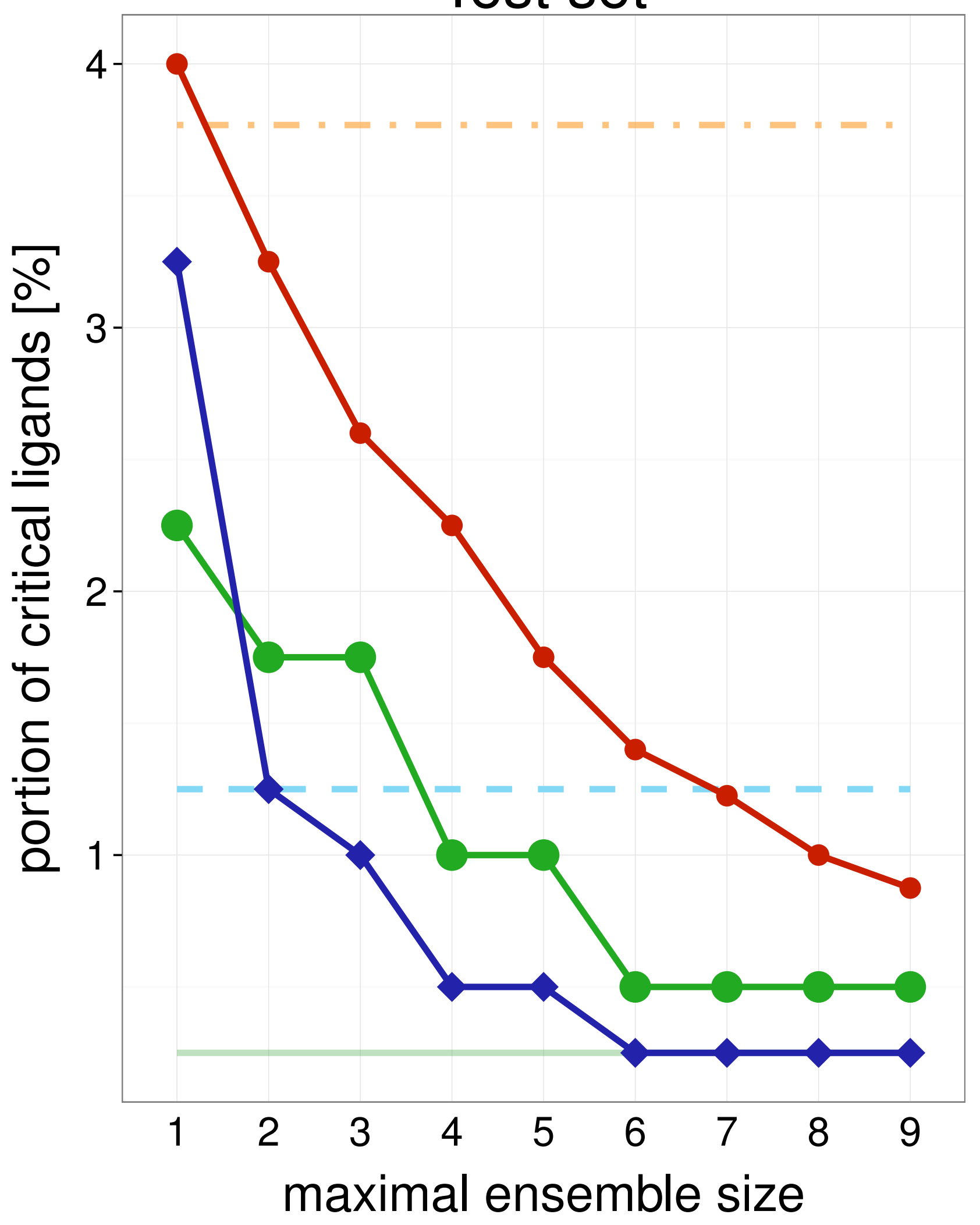

Average single structure

_ Best single structure

Whole ensemble

- SIENA

$\neg$ Clustering

- Random

Average single structure

_ Best single structure

Whole ensemble

- SIENA

$\smile$ Clustering

$\multimap$ Random

Test set

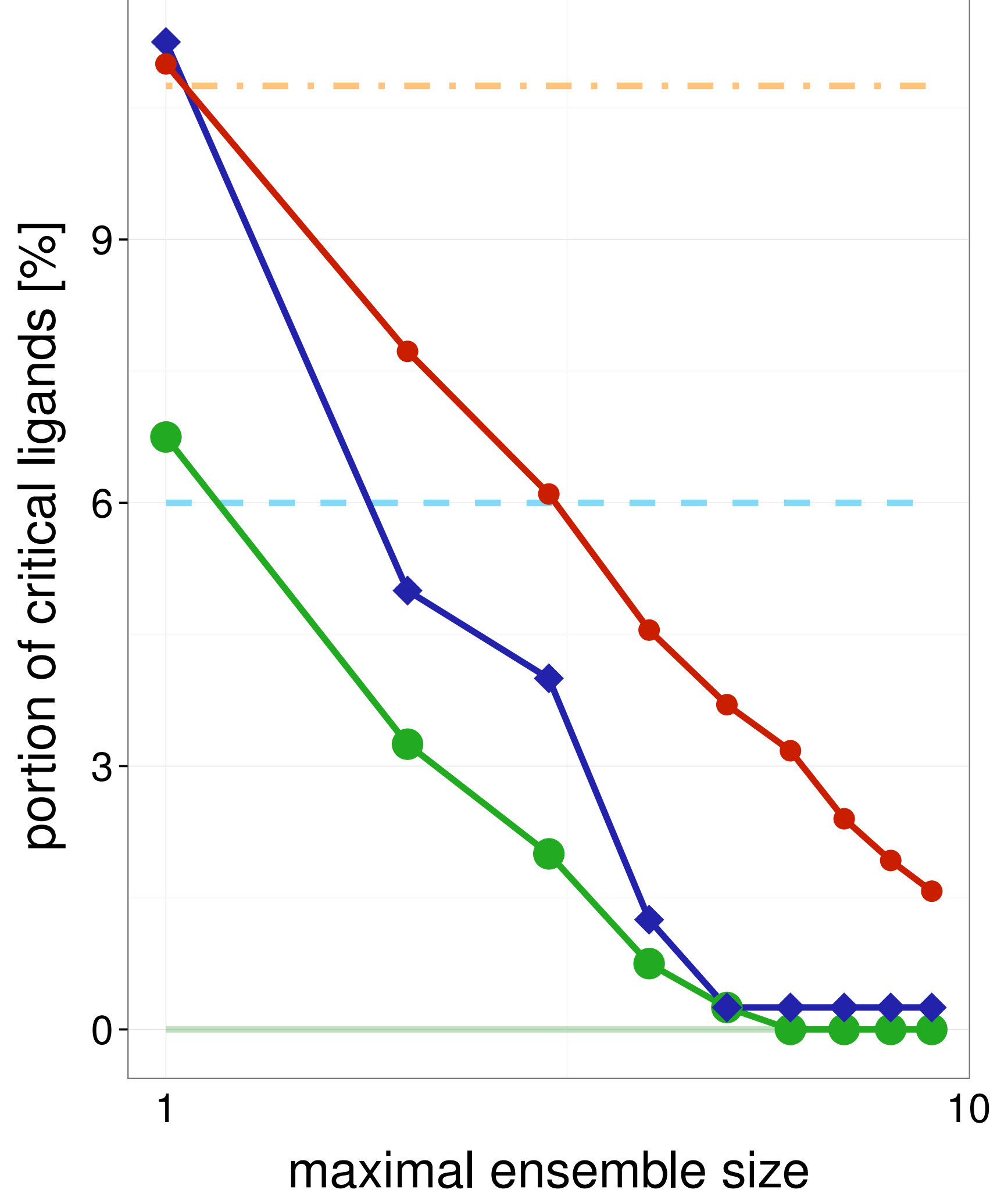

Average single structure

_ Best single structure

Whole ensemble

- SIENA

$\leadsto$ Clustering

- Random

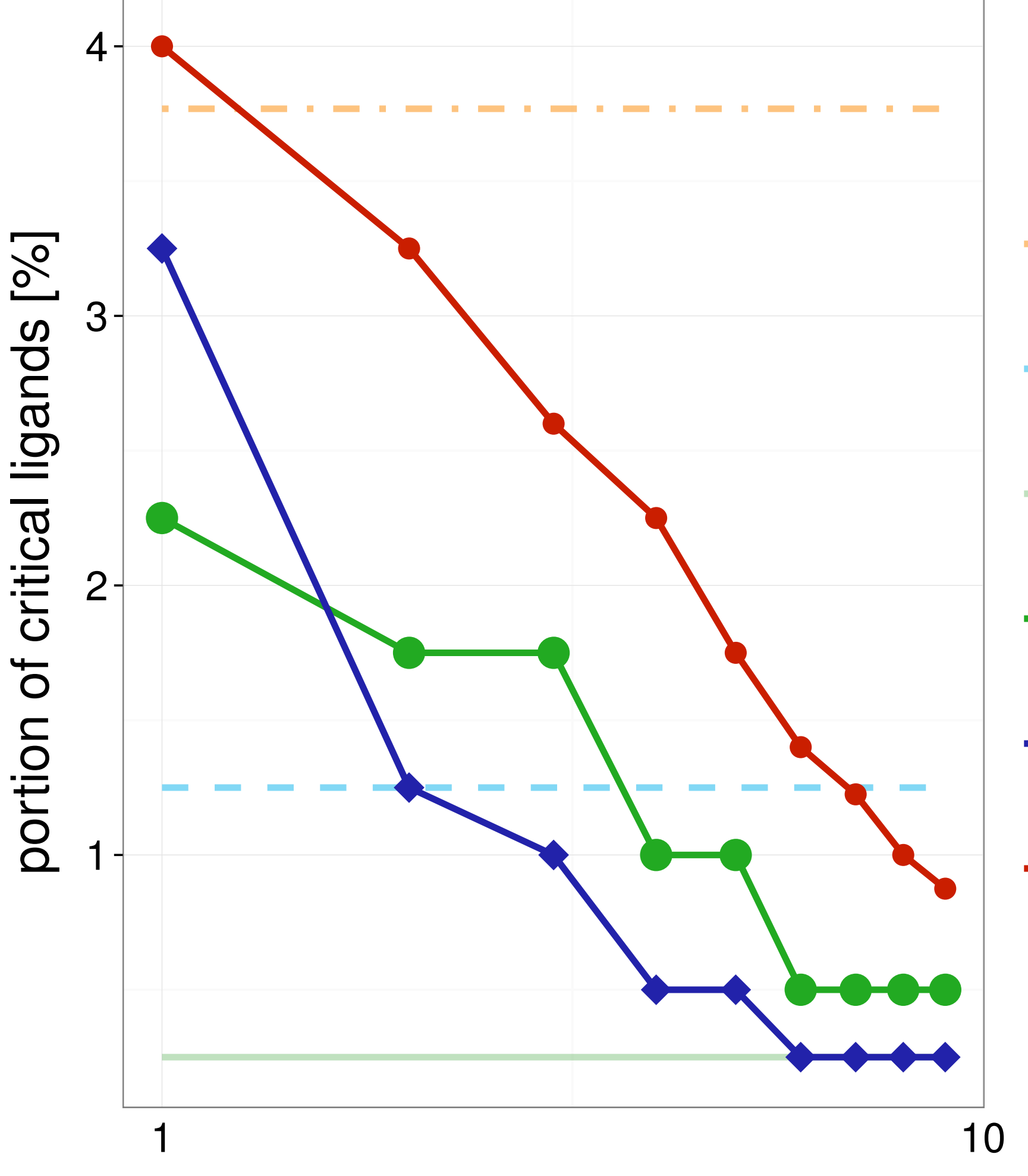

maximal ensemble size
Average single structure

_ Best single structure

Whole ensemble

SIENA

$\sim$ Clustering

$\multimap$ Random 


\section{Training set}
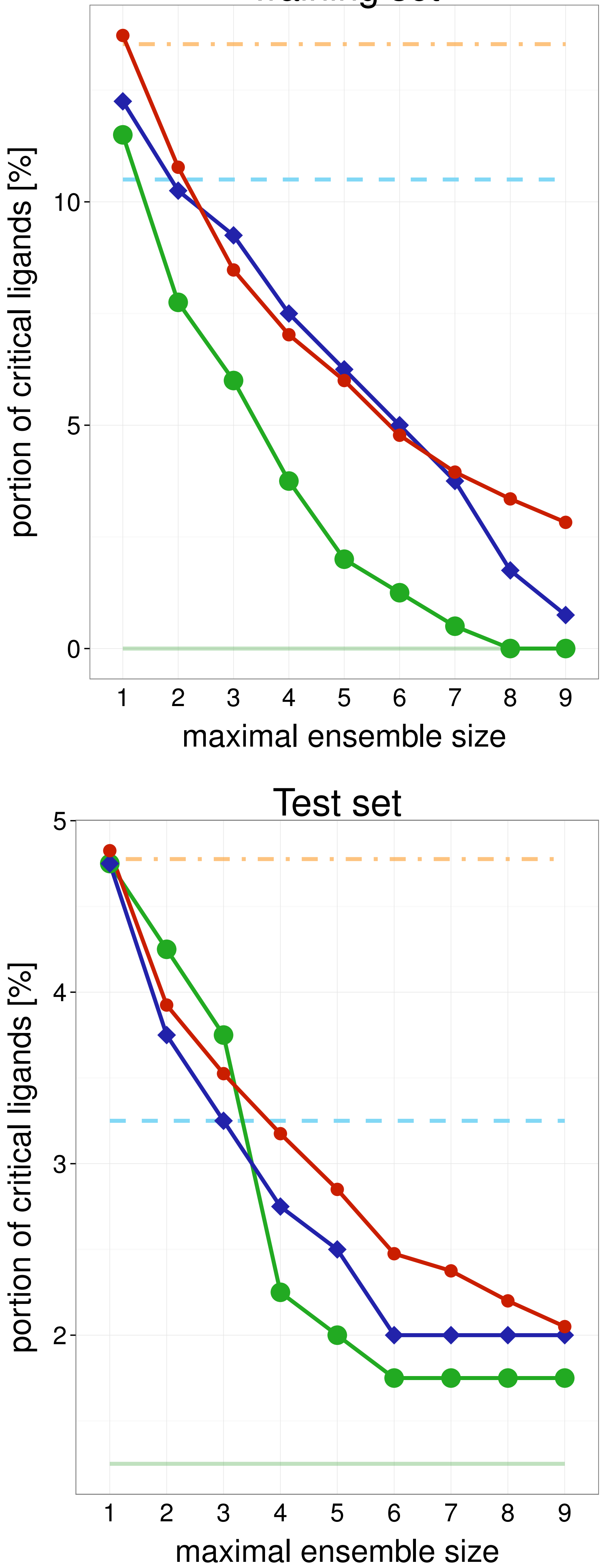

3A7J

Training set

Average single structure

_ Best single

structure

Whole ensemble

- SIENA

$\neg$ Clustering

$\multimap$ Random

Average single structure

\section{_ Best single} structure

Whole ensemble

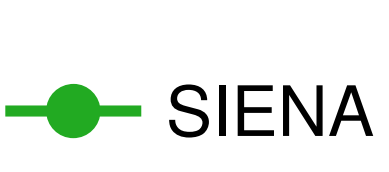

$\checkmark$ Clustering

- Random

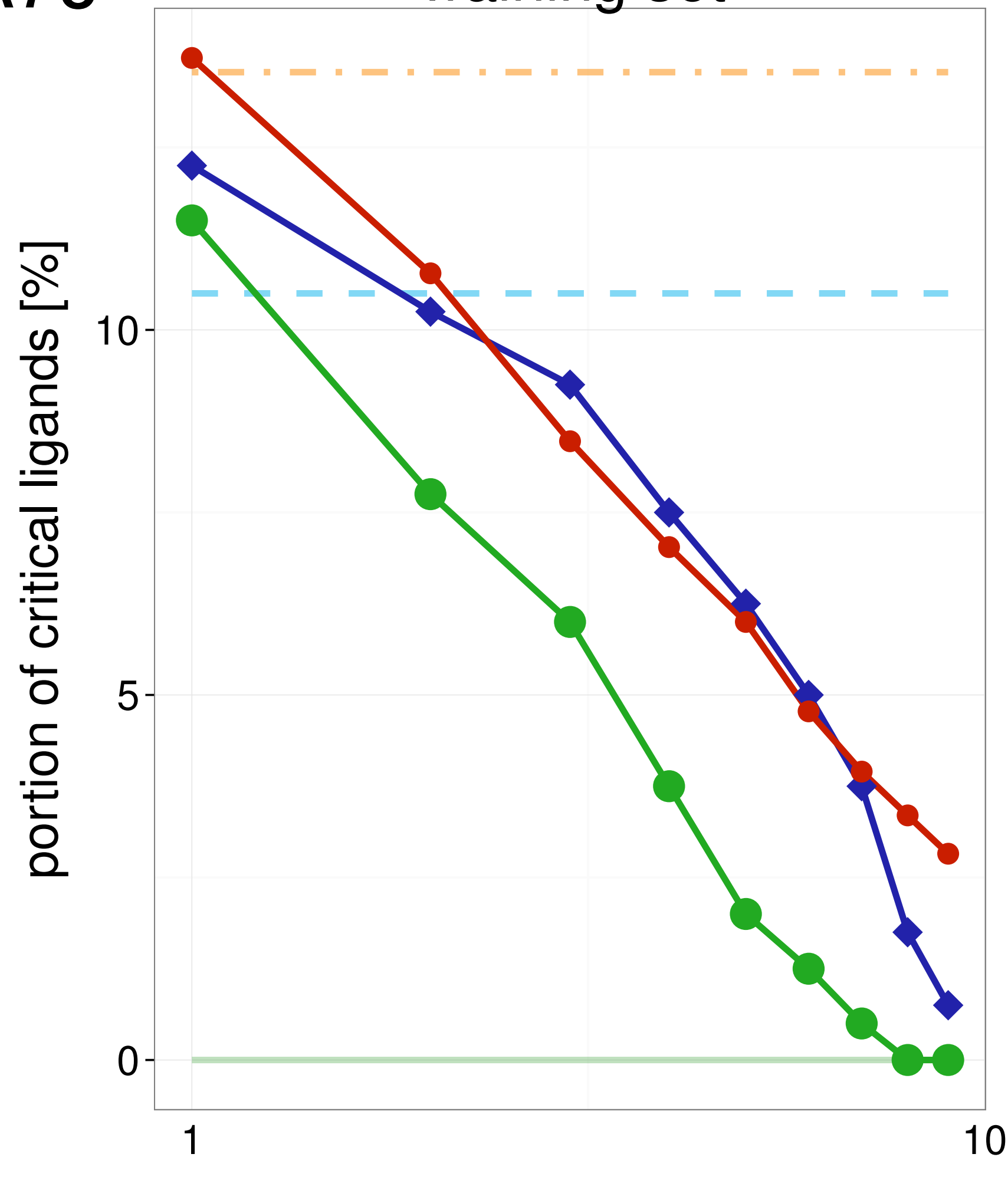

maximal ensemble size

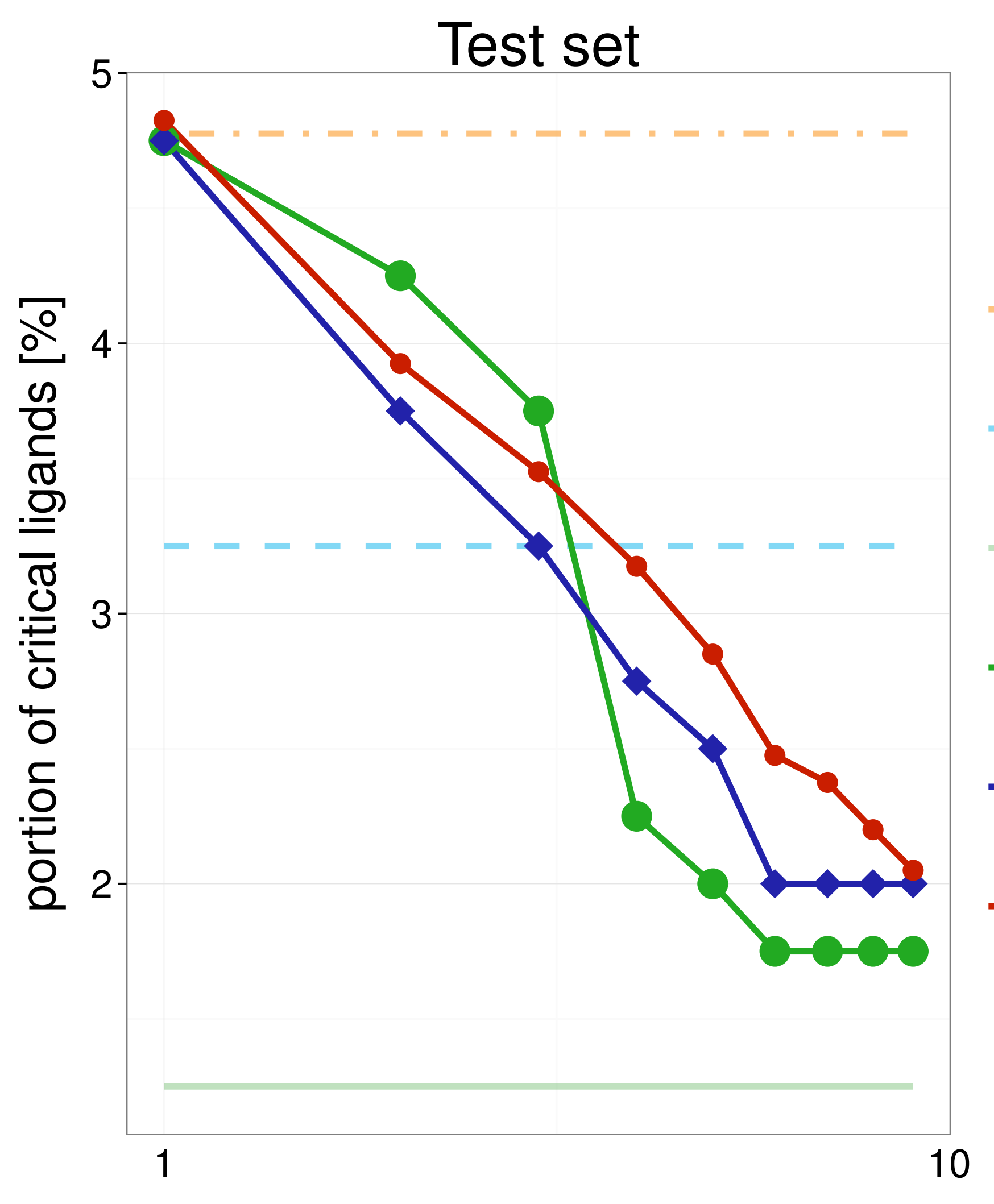

maximal ensemble size
Average single structure

_ _ Best single structure

Whole ensemble

- SIENA

$\checkmark$ Clustering

- Random 


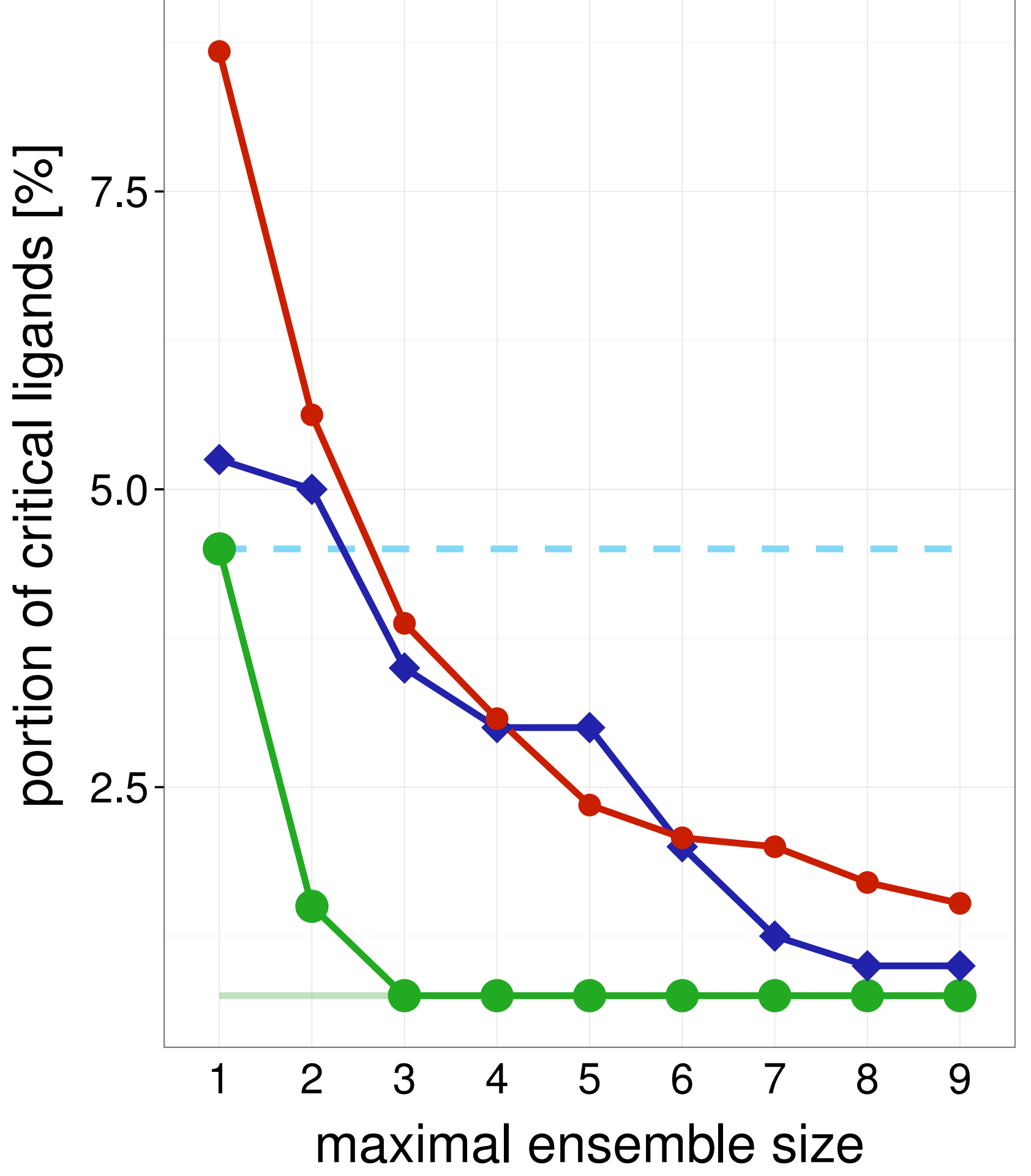

Test set

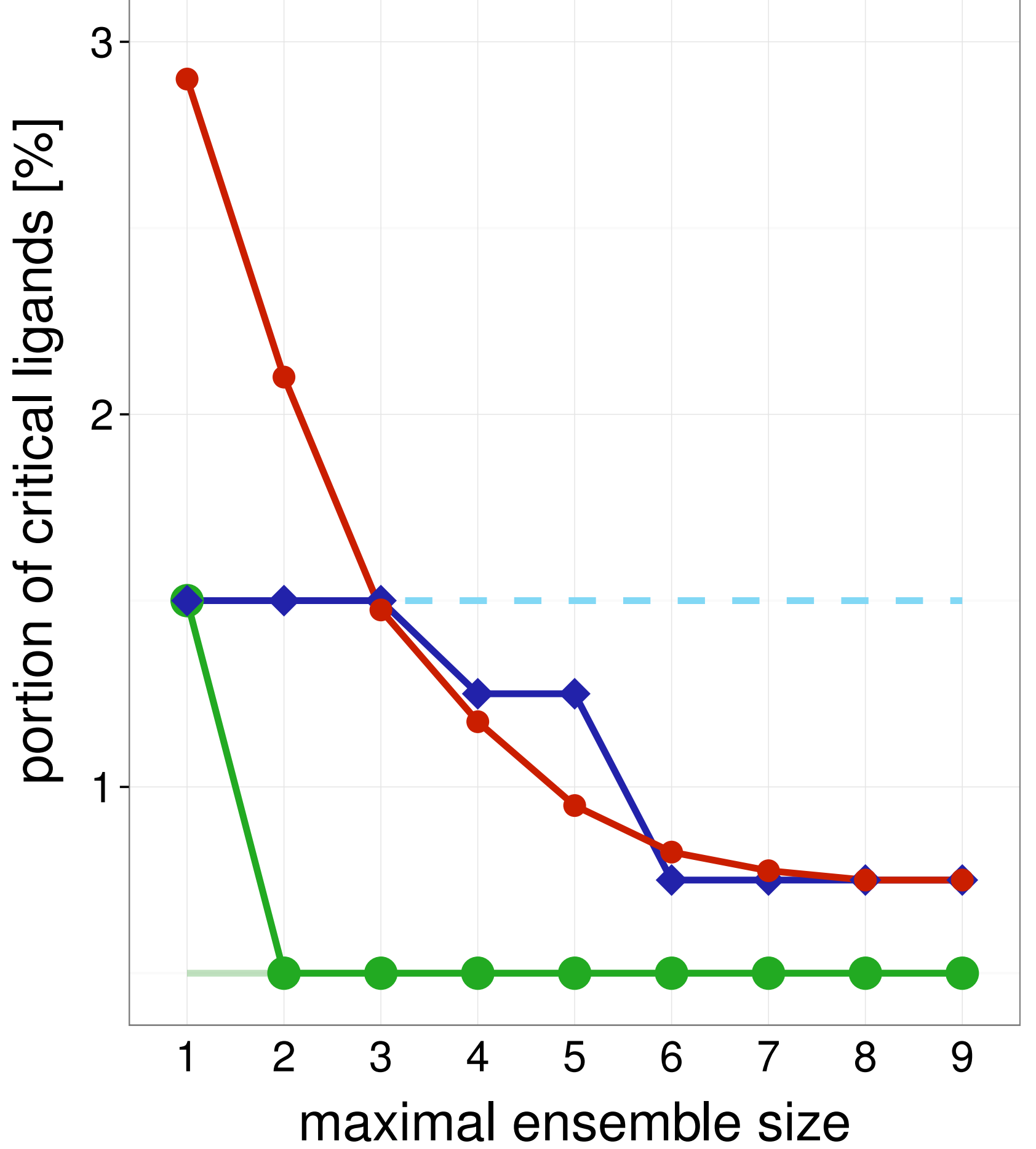

Average single structure

_ _ Best single structure

Whole ensemble

- SIENA

$\neg$ Clustering

$\multimap$ Random

Average single structure

_ Best single structure

Whole ensemble

- SIENA

$\smile$ Clustering

$\because$ Random

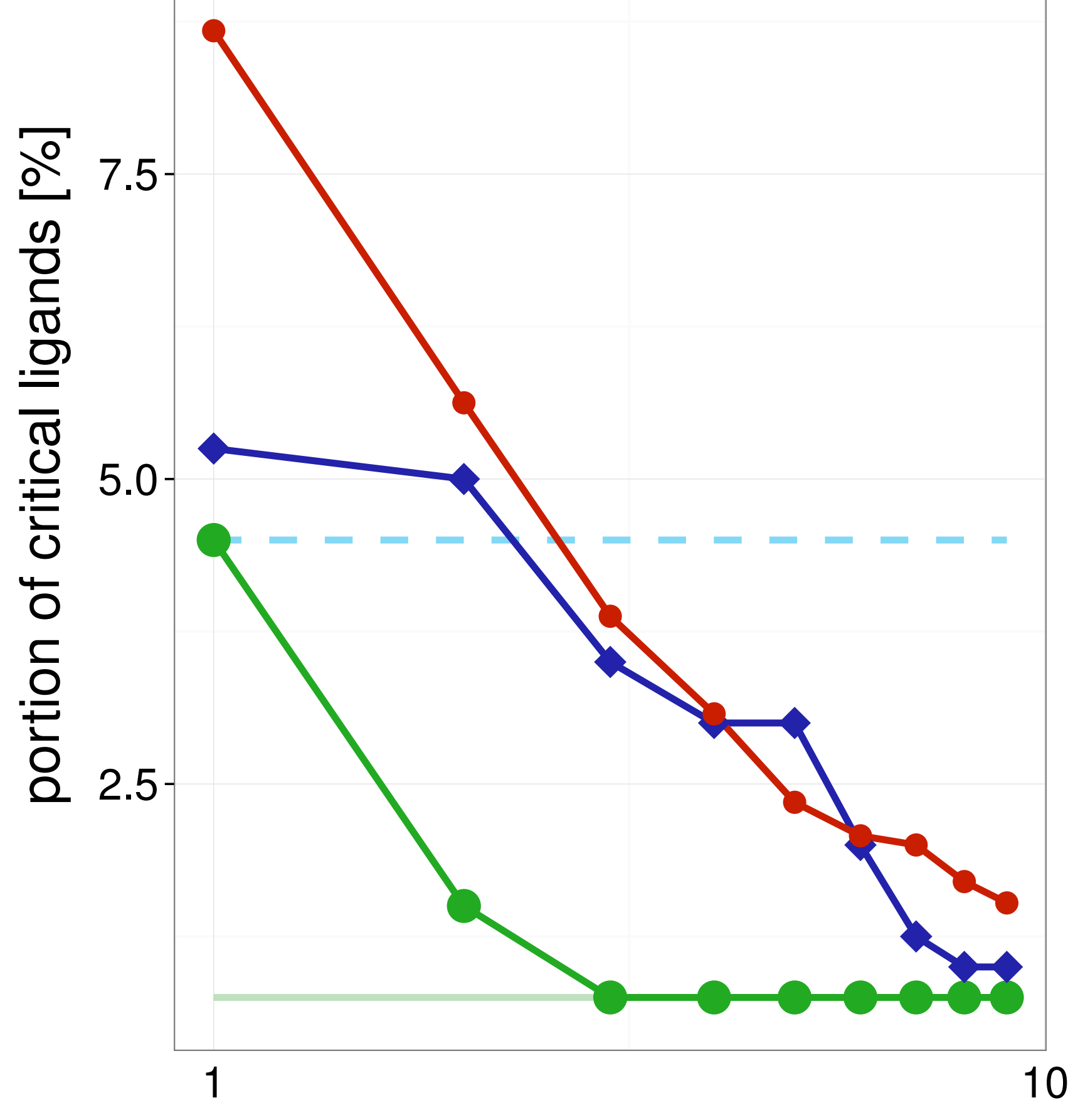

maximal ensemble size

Test set

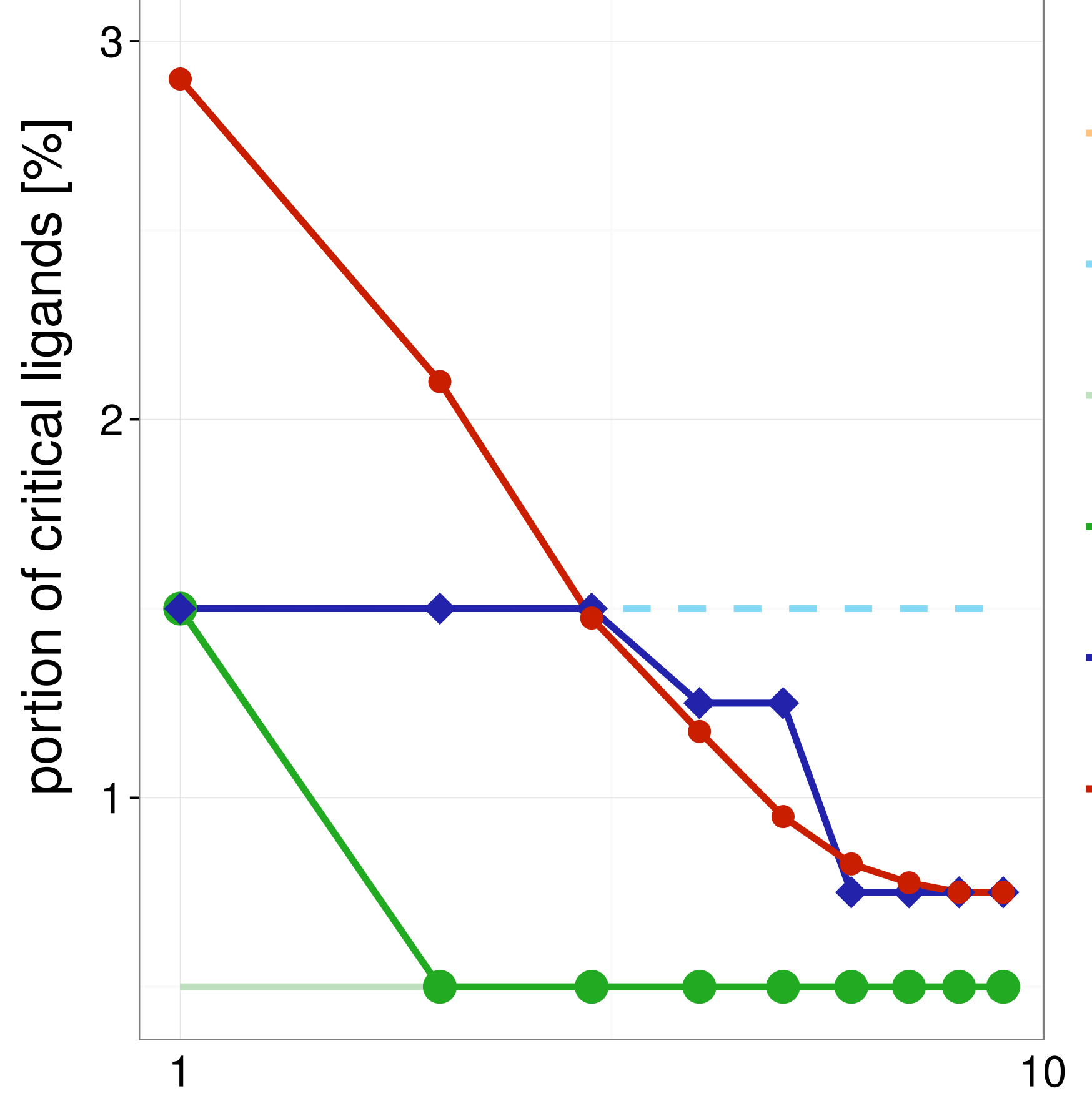

maximal ensemble size
Average single structure

_ Best single structure

Whole ensemble

SIENA

$\neg$ Clustering

$\multimap$ Random 


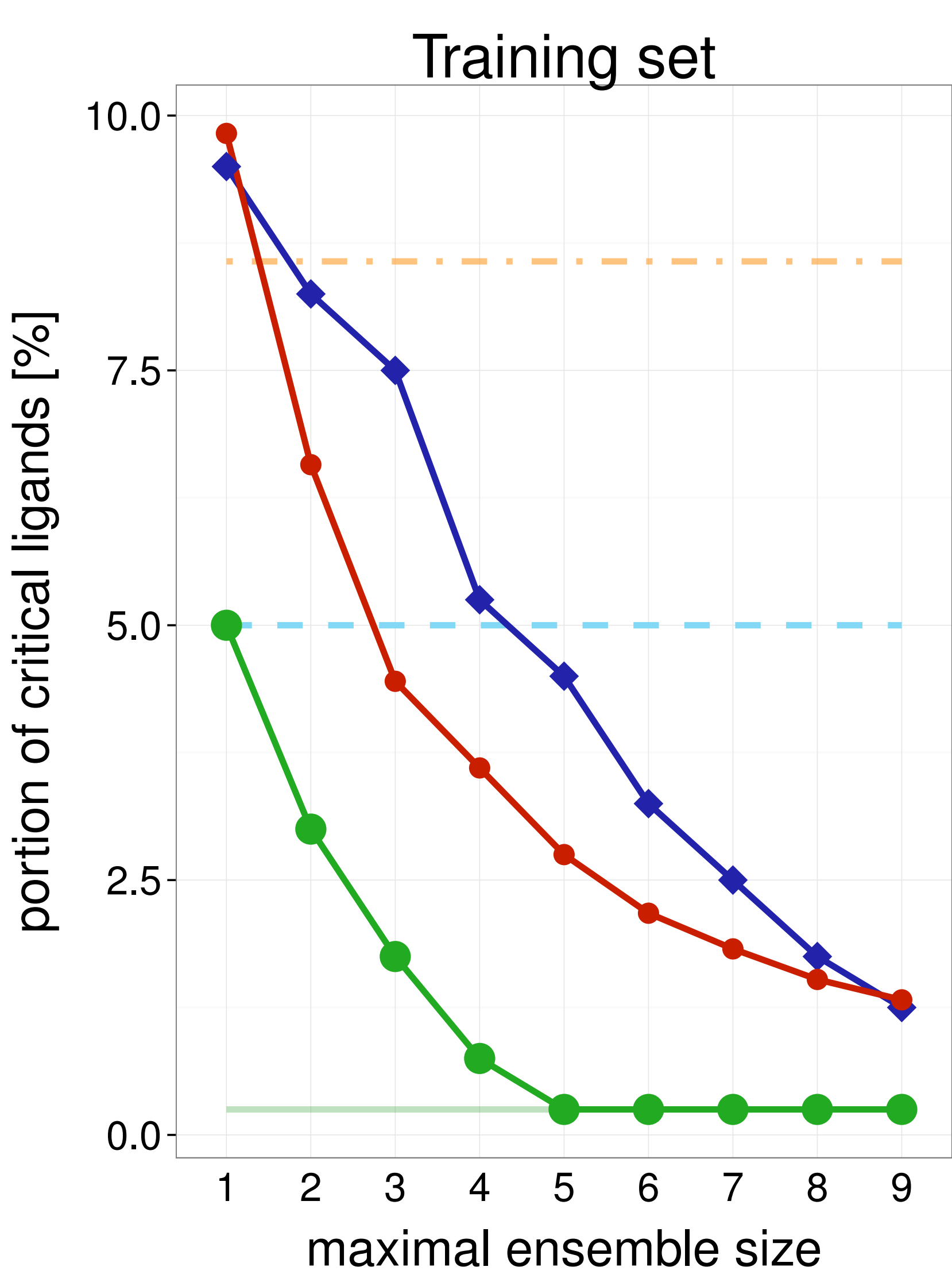

\section{Test set}

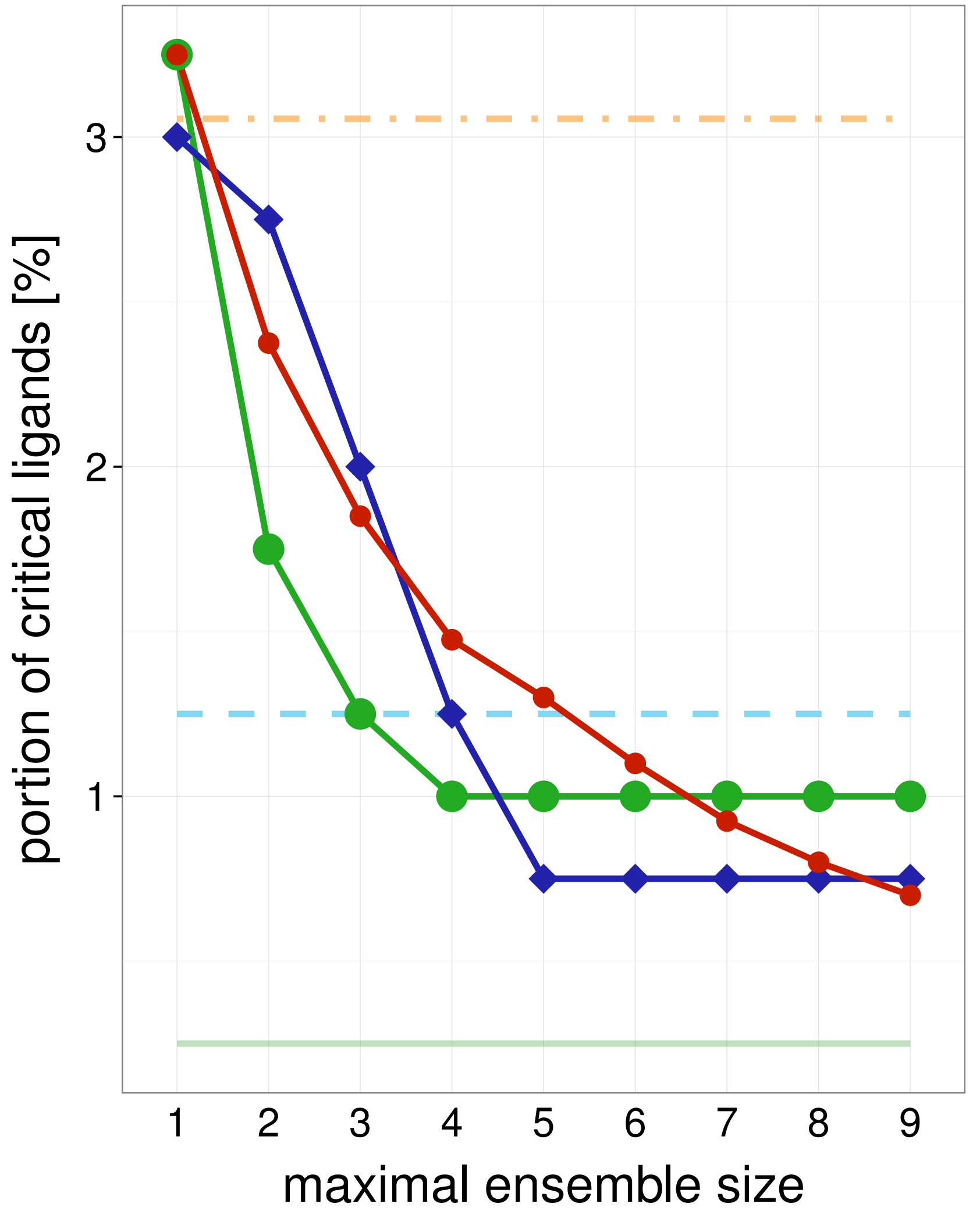

$3 \mathrm{U} 8 \mathrm{~F}$

Training set

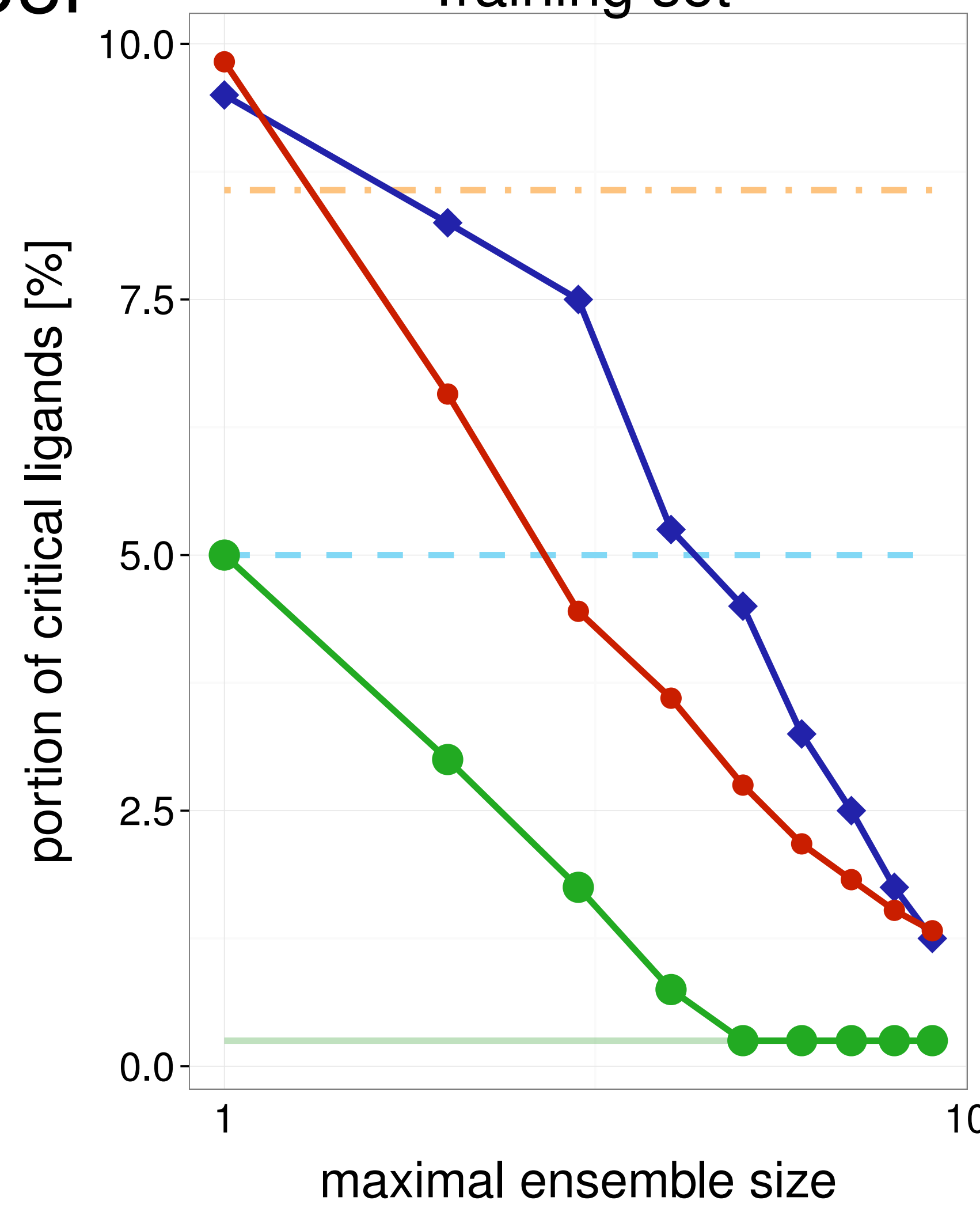

Average single structure

- Best single structure

Whole ensemble

Whole ensemble

- SIENA

$\multimap$ Clustering

$\multimap$ Random

Average single structure

_ Best single structure

Whole ensemble

- SIENA

$\neg$ Clustering

$\multimap$ Random
Average single structure

_ Best single structure

Whole ensemble

SIENA

$\sim$ Clustering

$\because$ Random

\section{Test set}

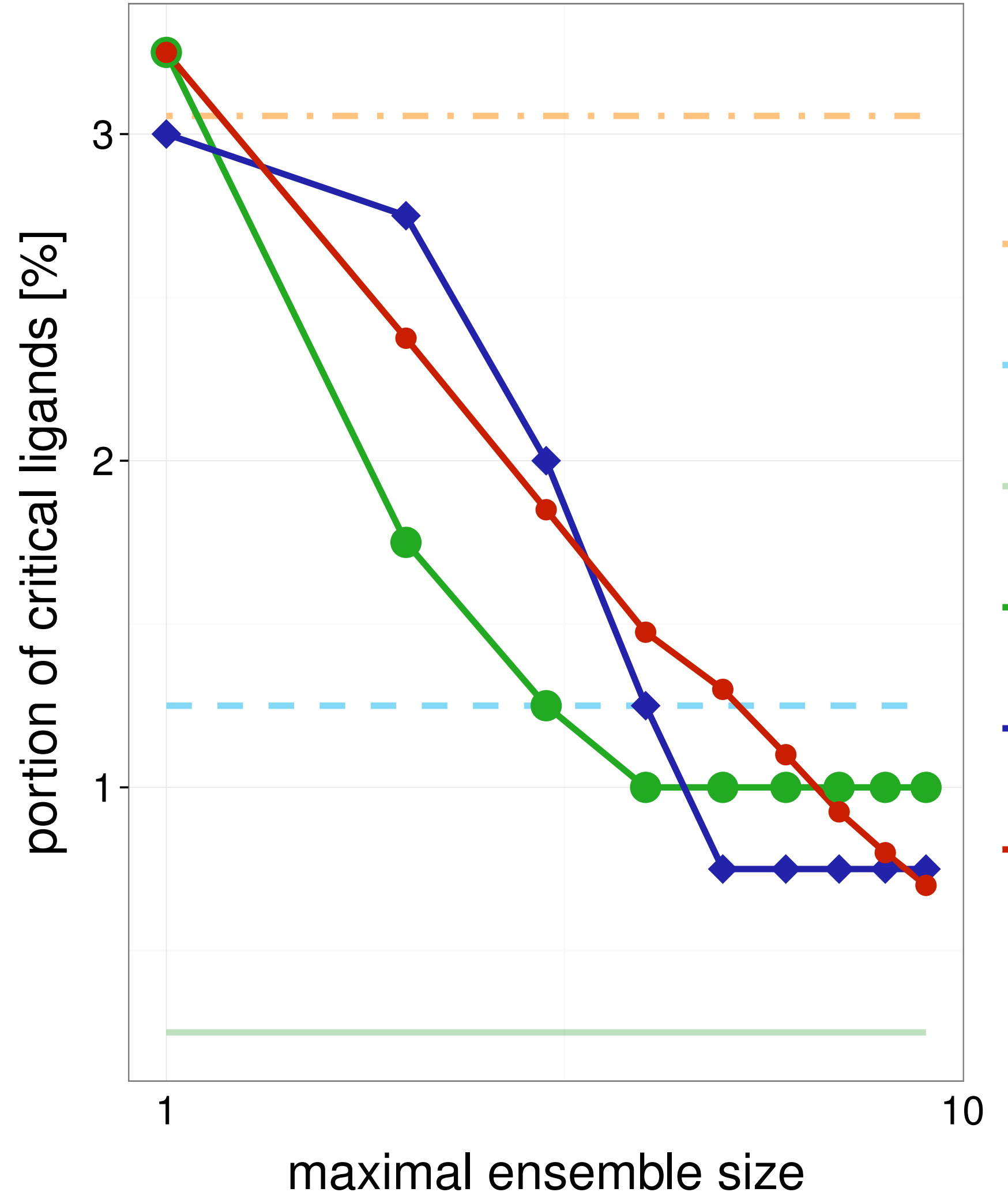




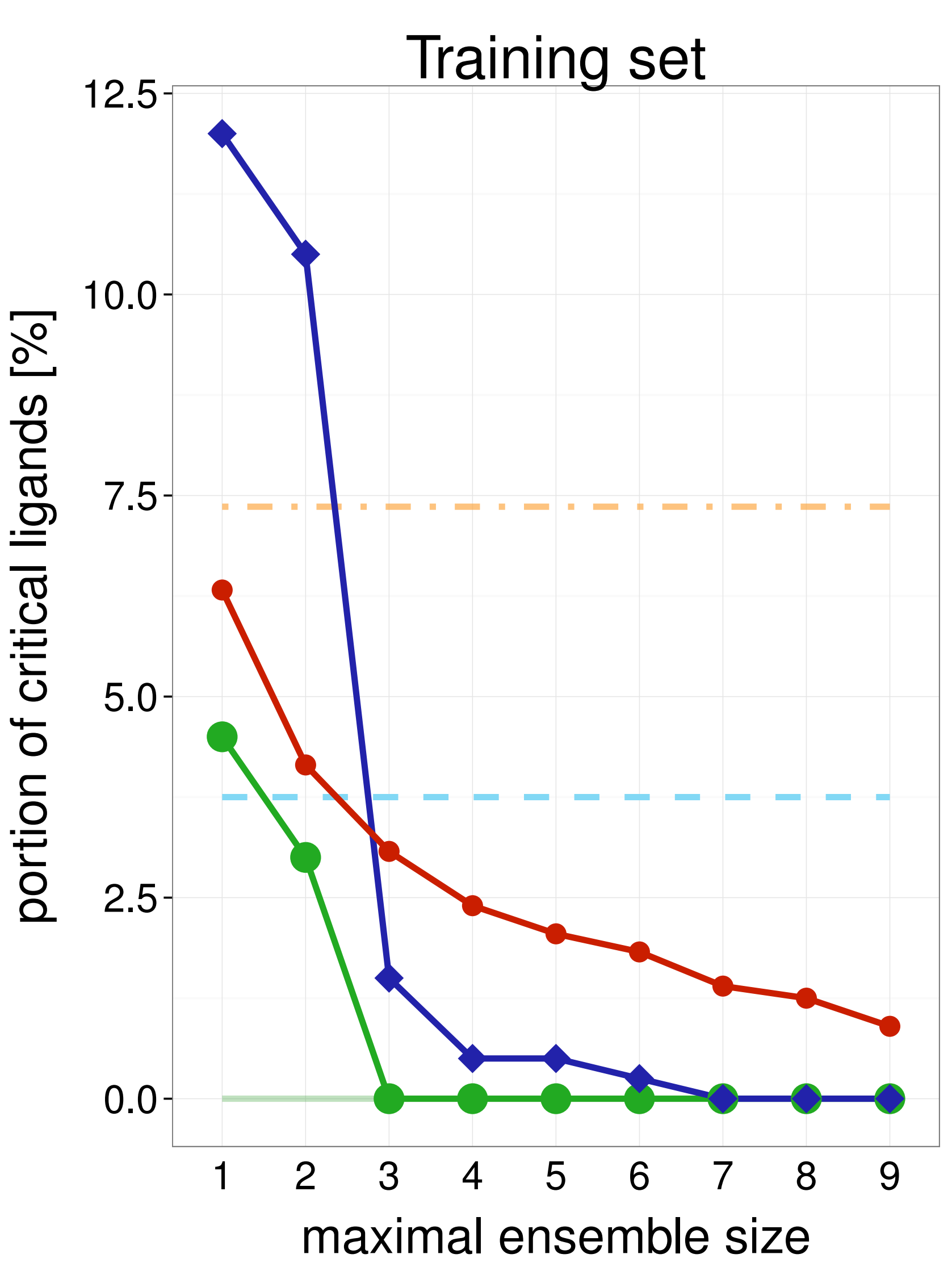

$3 \mathrm{ZCW}_{2.5} \quad$ Training set

Test set

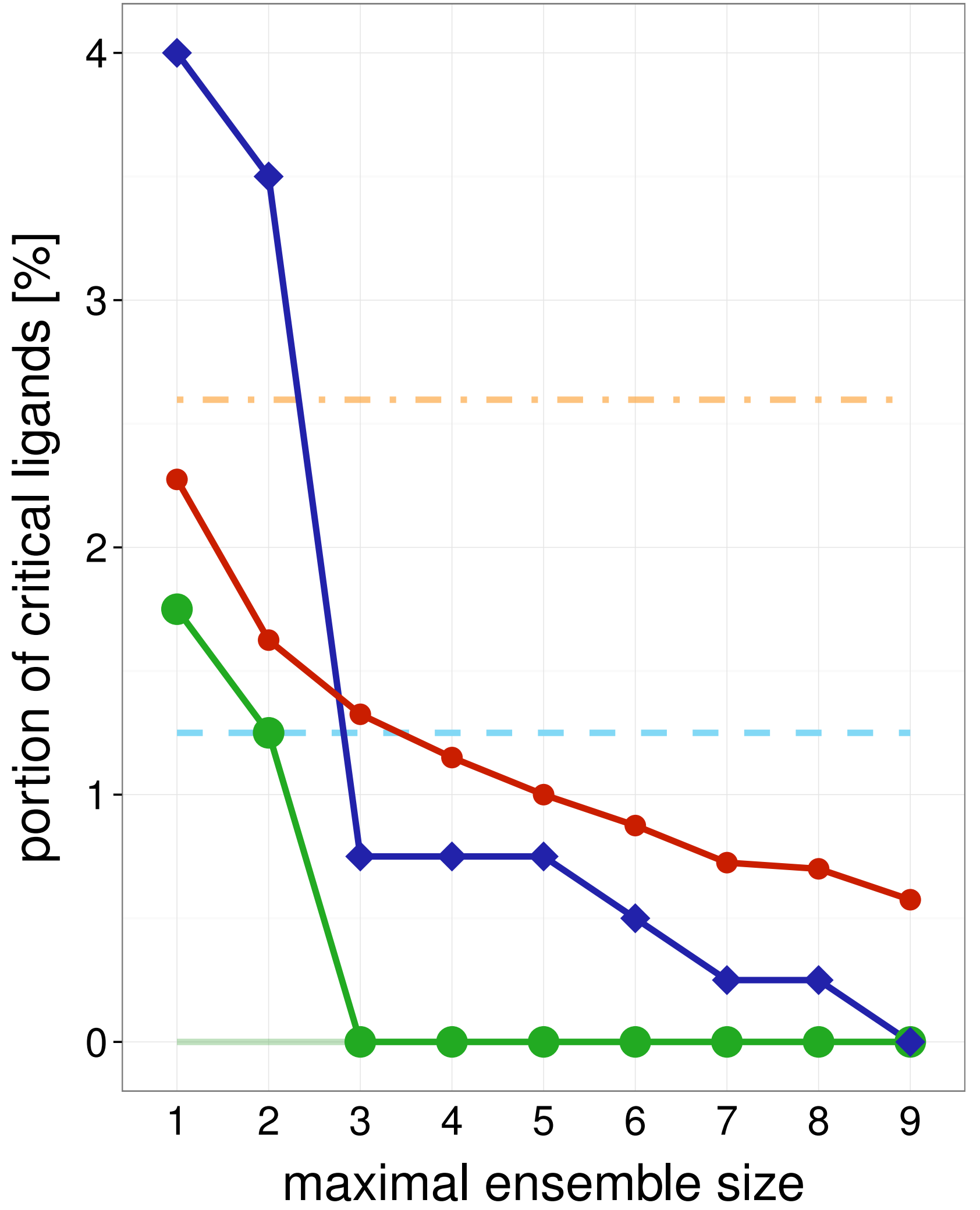

Average single structure

_ _ Best single structure

Whole ensemble

- SIENA

$\neg$ Clustering

$\multimap$ Random

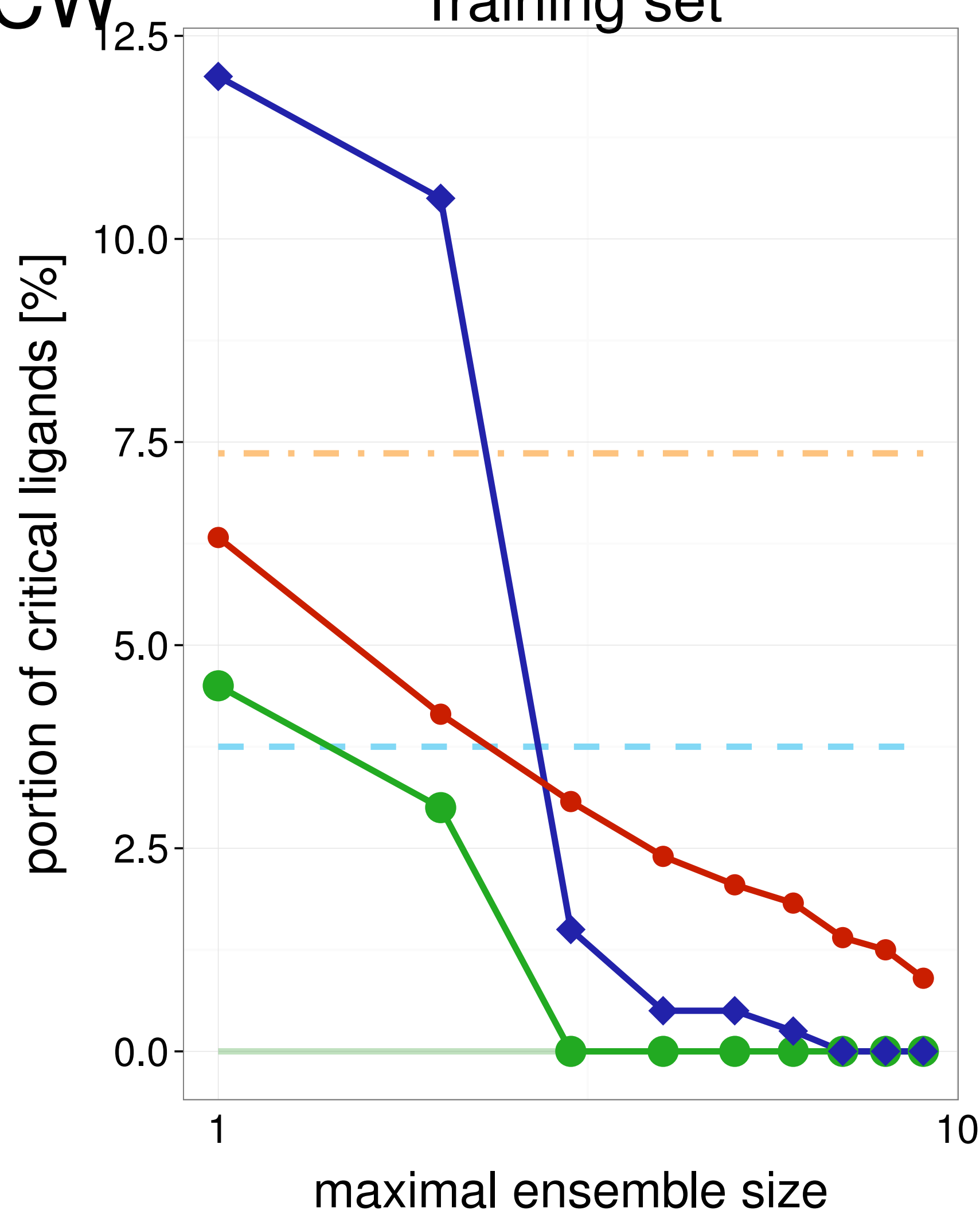

Average single structure

- Best single structure

Whole ensemble

- SIENA

$\checkmark$ Clustering

$\multimap$ Random

Test set

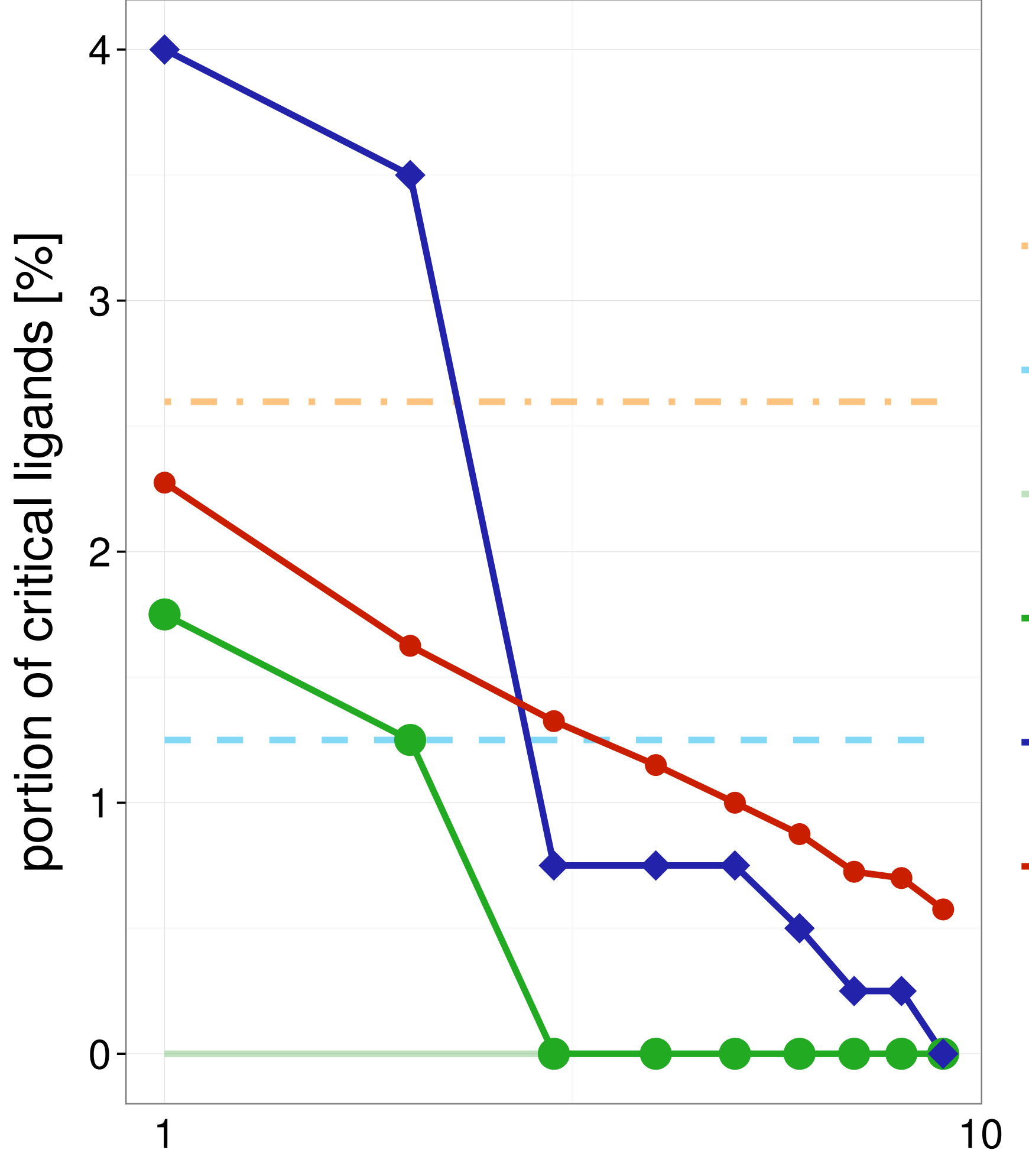

Average single structure

Best single structure

Whole ensemble

- SIENA

$\neg$ Clustering

$\multimap$ Random 

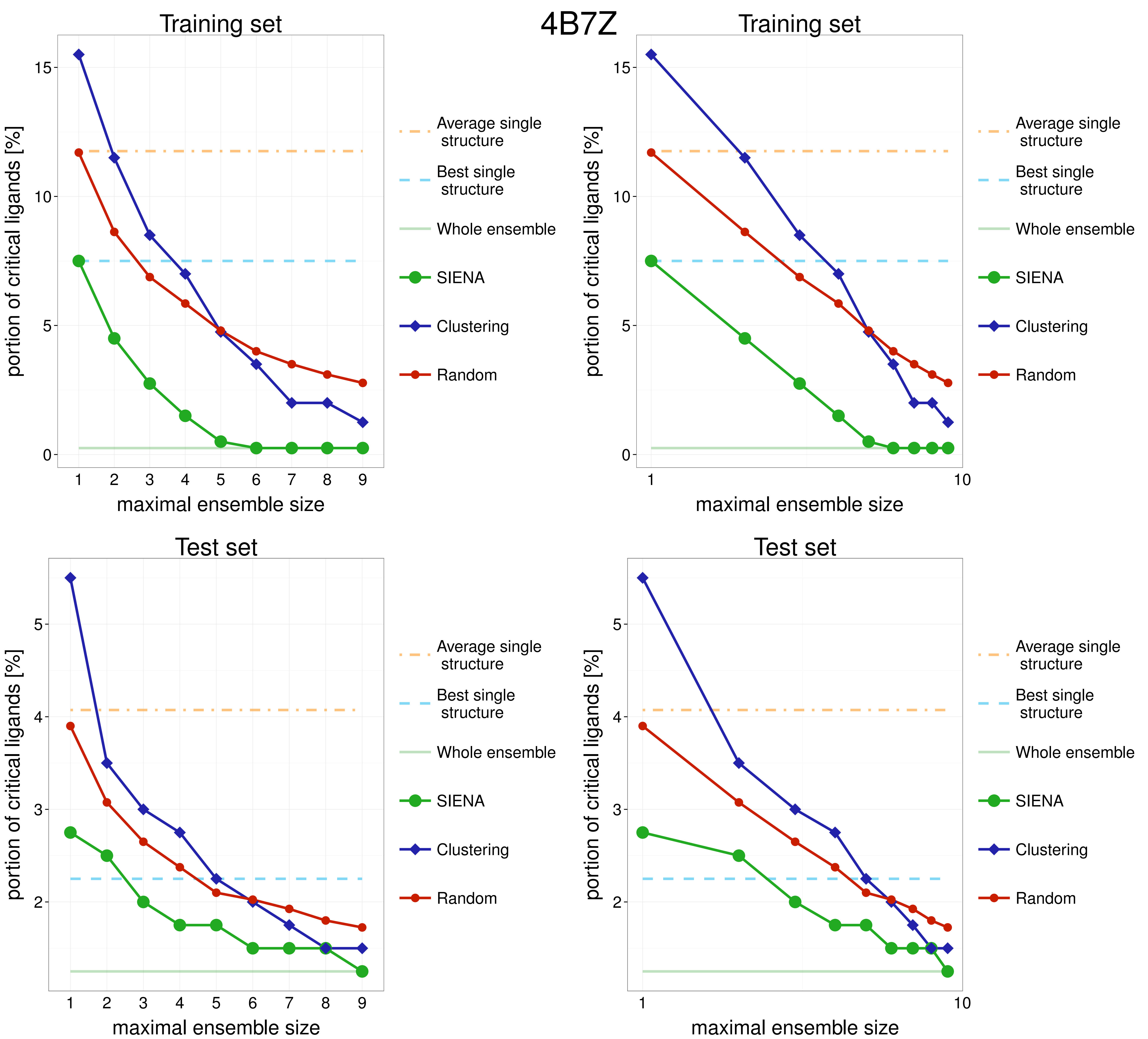
Training set

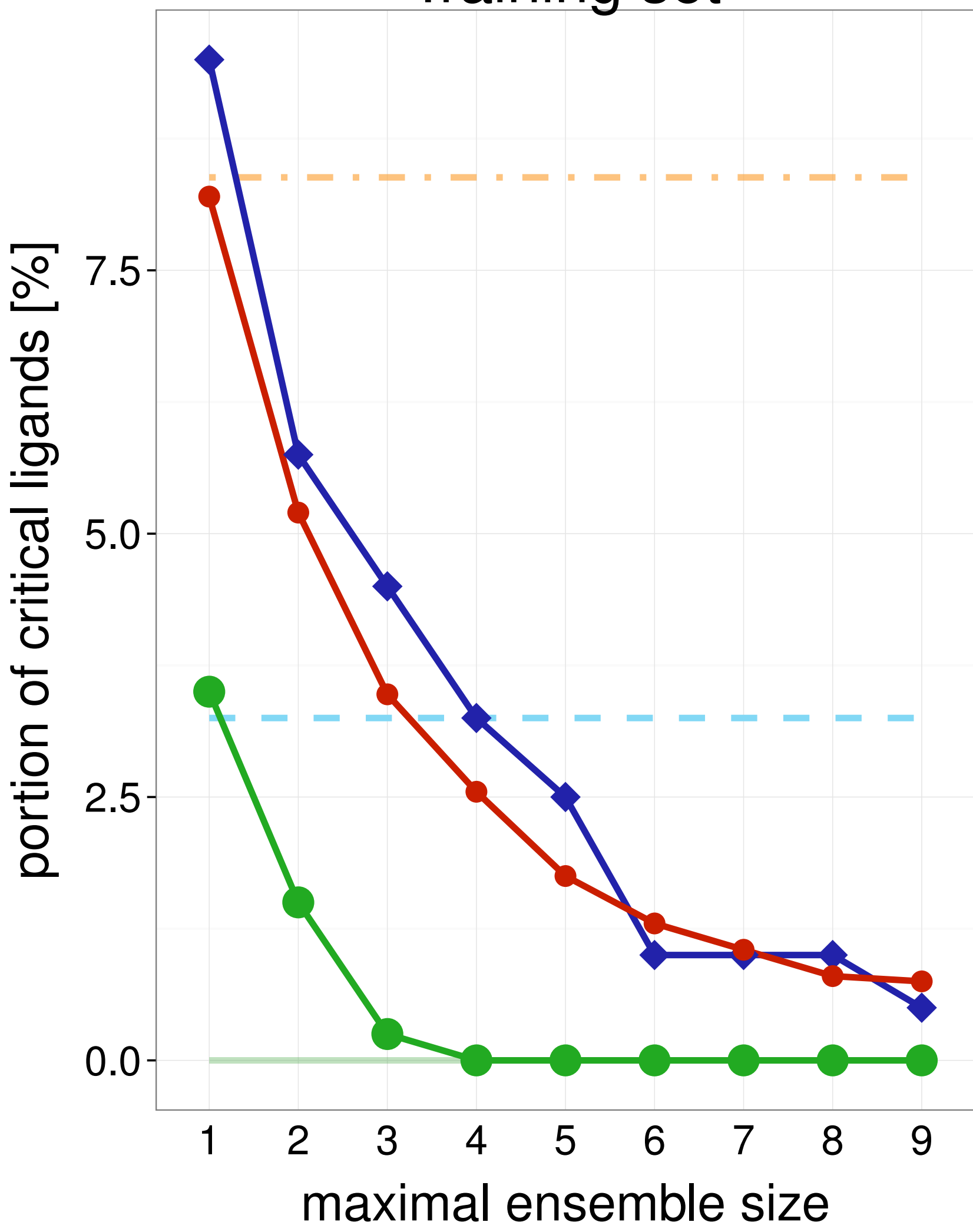

Test set

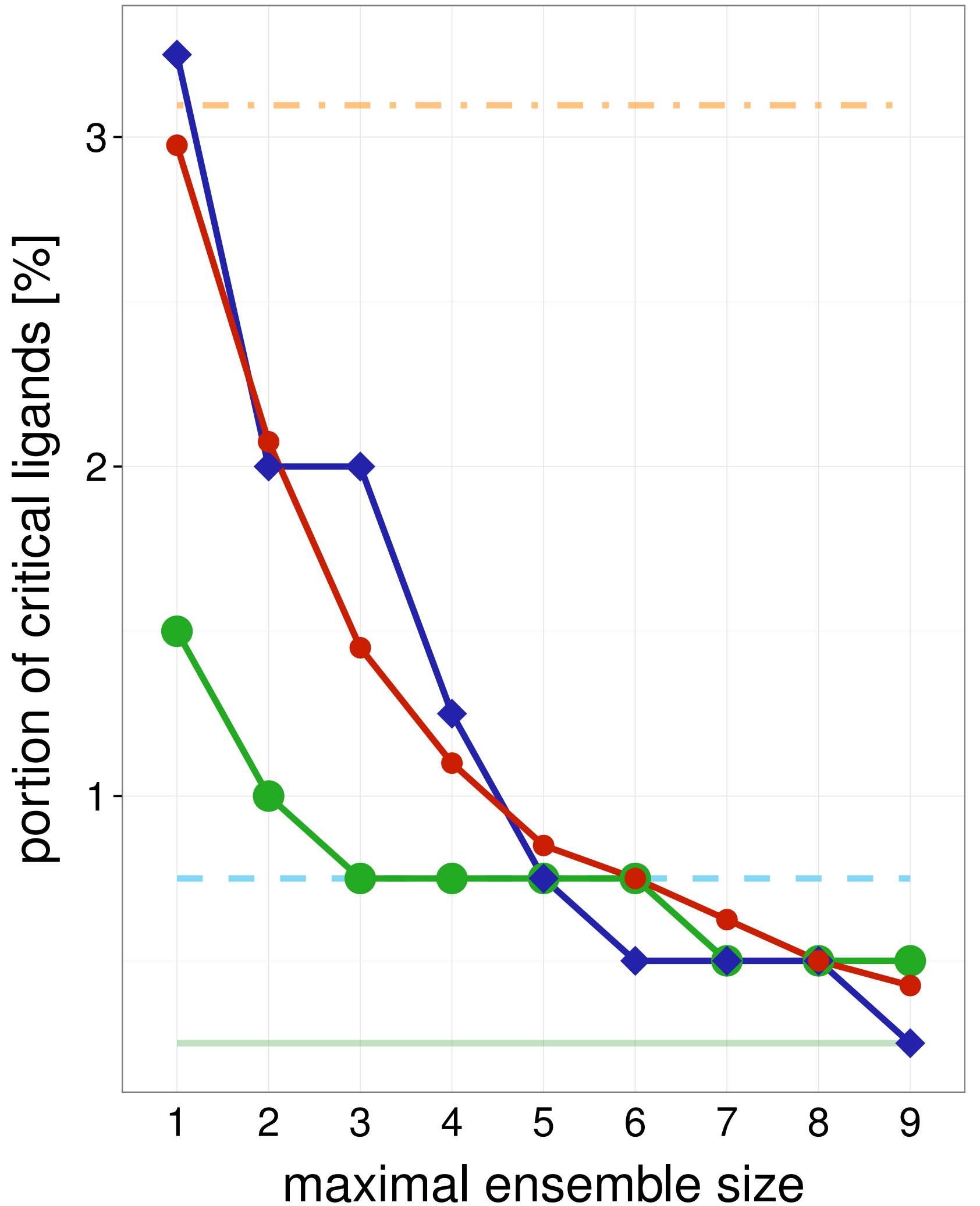

Average single structure

_ Best single structure

Whole ensemble

- SIENA

$\neg$ Clustering

$\multimap$ Random

Average single structure

_ _ Best single structure

Whole ensemble

- SIENA

$\checkmark$ Clustering

$\multimap$ Random
4F63

Training set

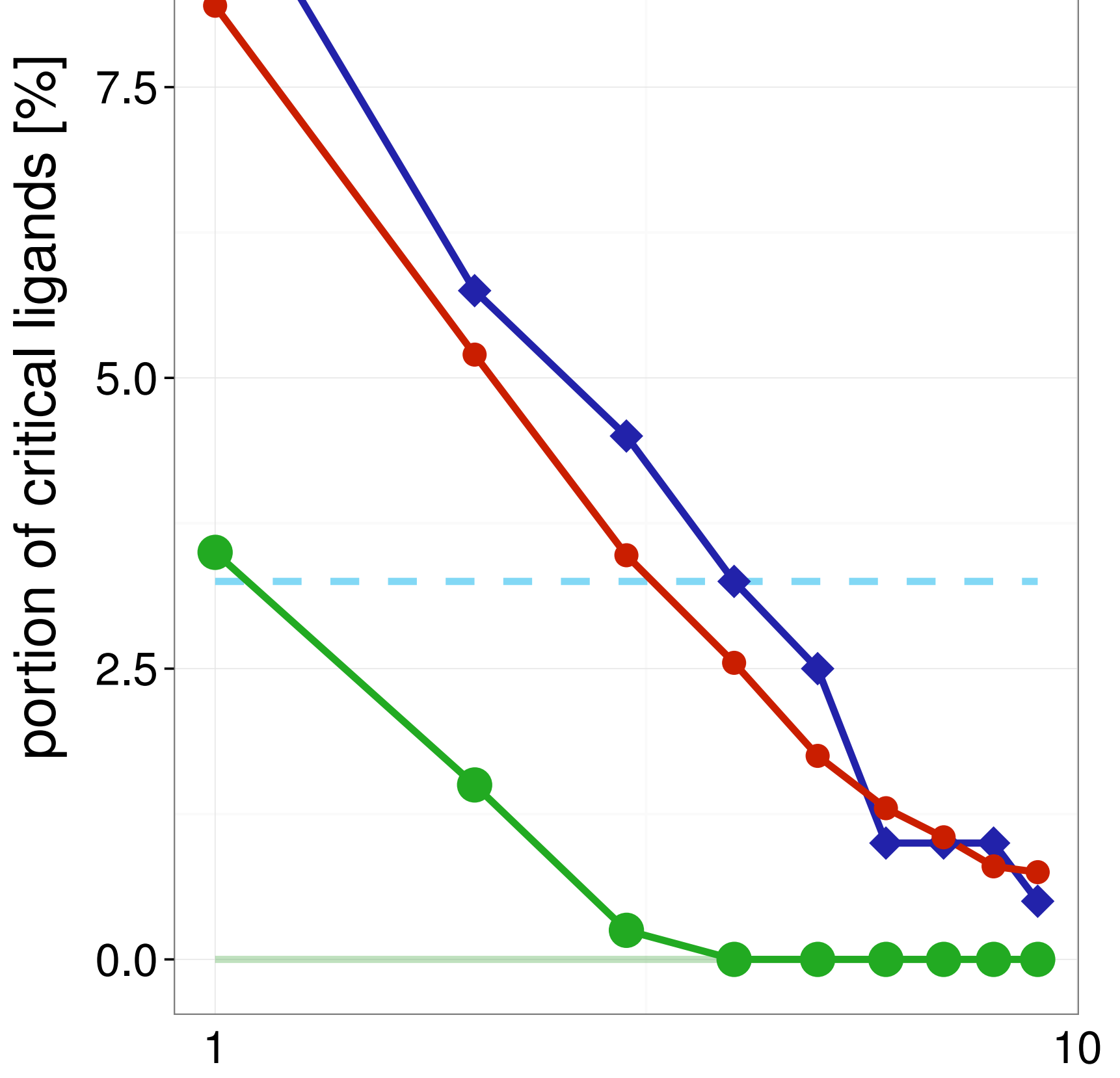

Average single structure

Best single structure

Whole ensemble

- SIENA

$\checkmark$ Clustering

- Random

maximal ensemble size

Test set

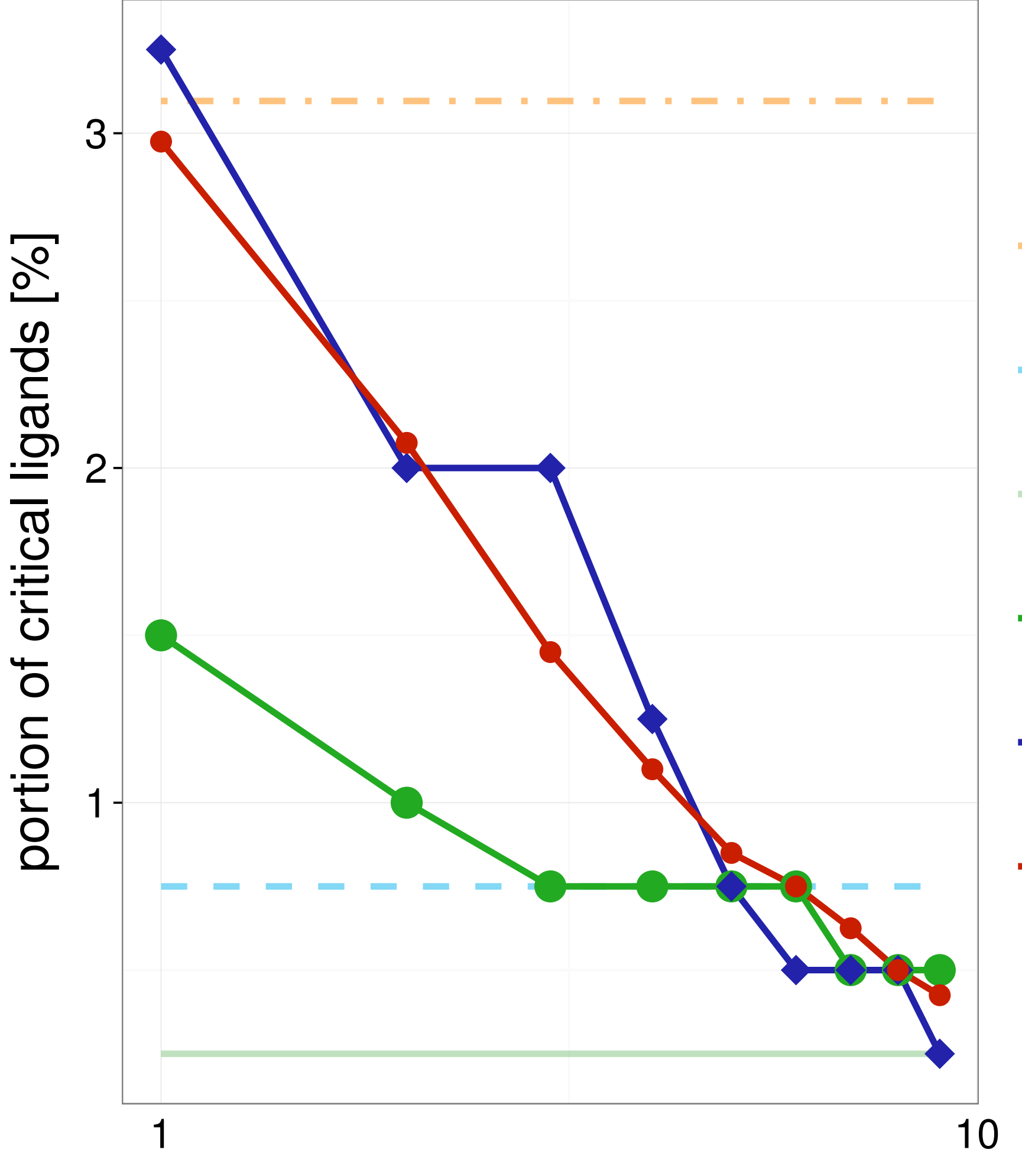

Average single structure

_ Best single structure

Whole ensemble

SIENA

$\sim$ Clustering

$\longrightarrow$ Random 
Training set

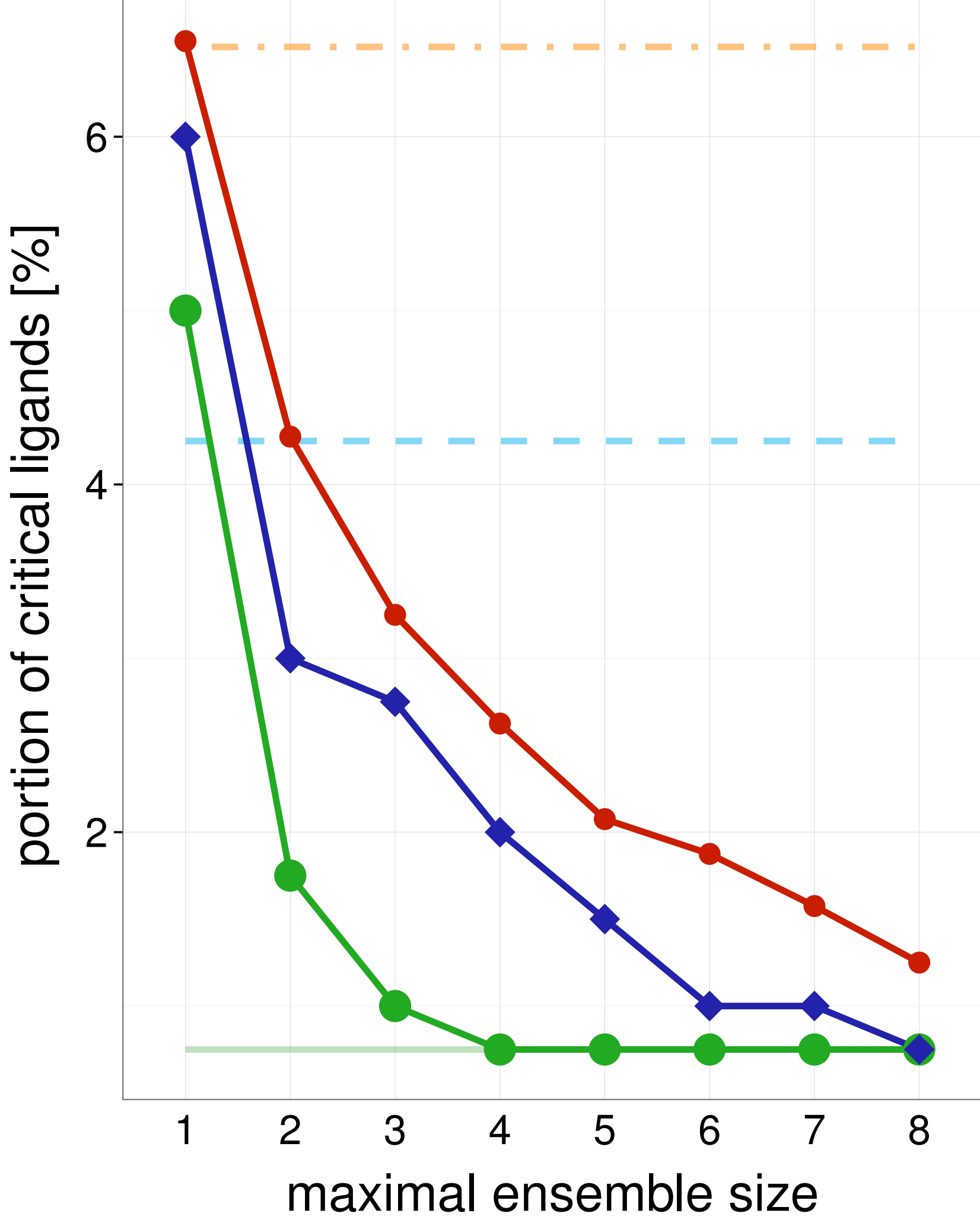

Test set

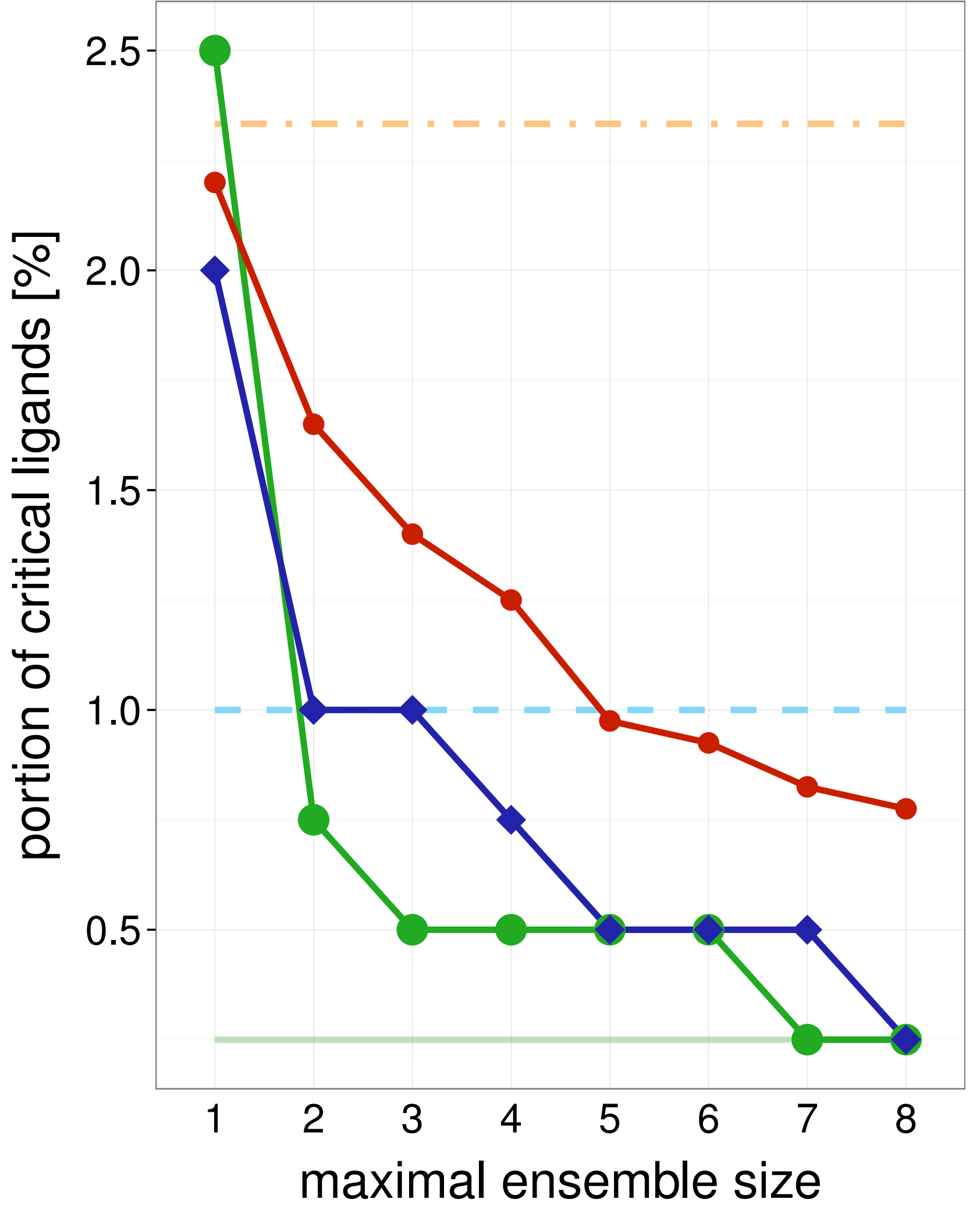

Average single structure

_ Best single structure

Whole ensemble

- SIENA

$\neg$ Clustering

$\multimap$ Random

Average single structure

_ _ Best single structure

Whole ensemble

- SIENA

$\neg$ Clustering

- Random
Training set

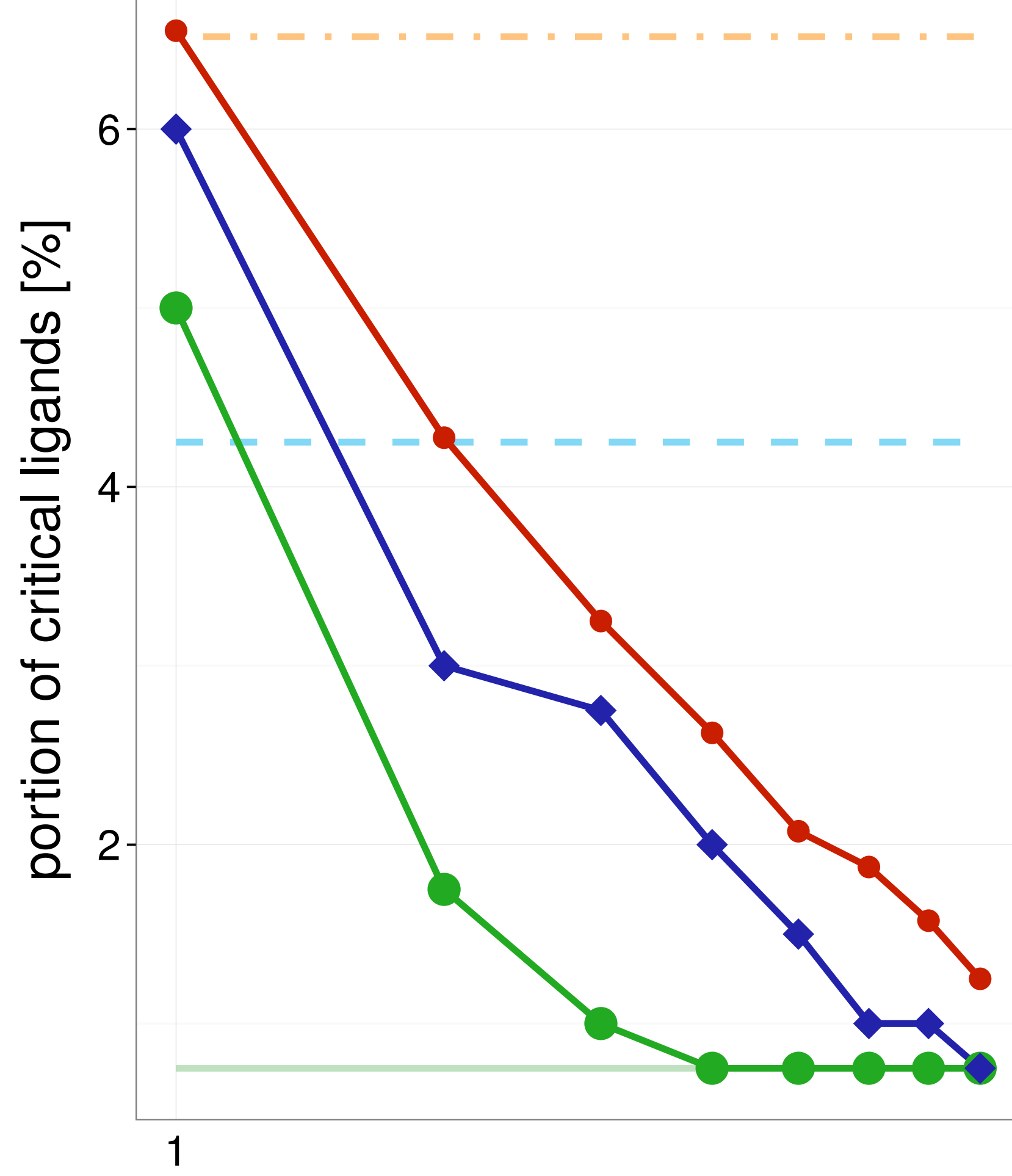

maximal ensemble size

Test set

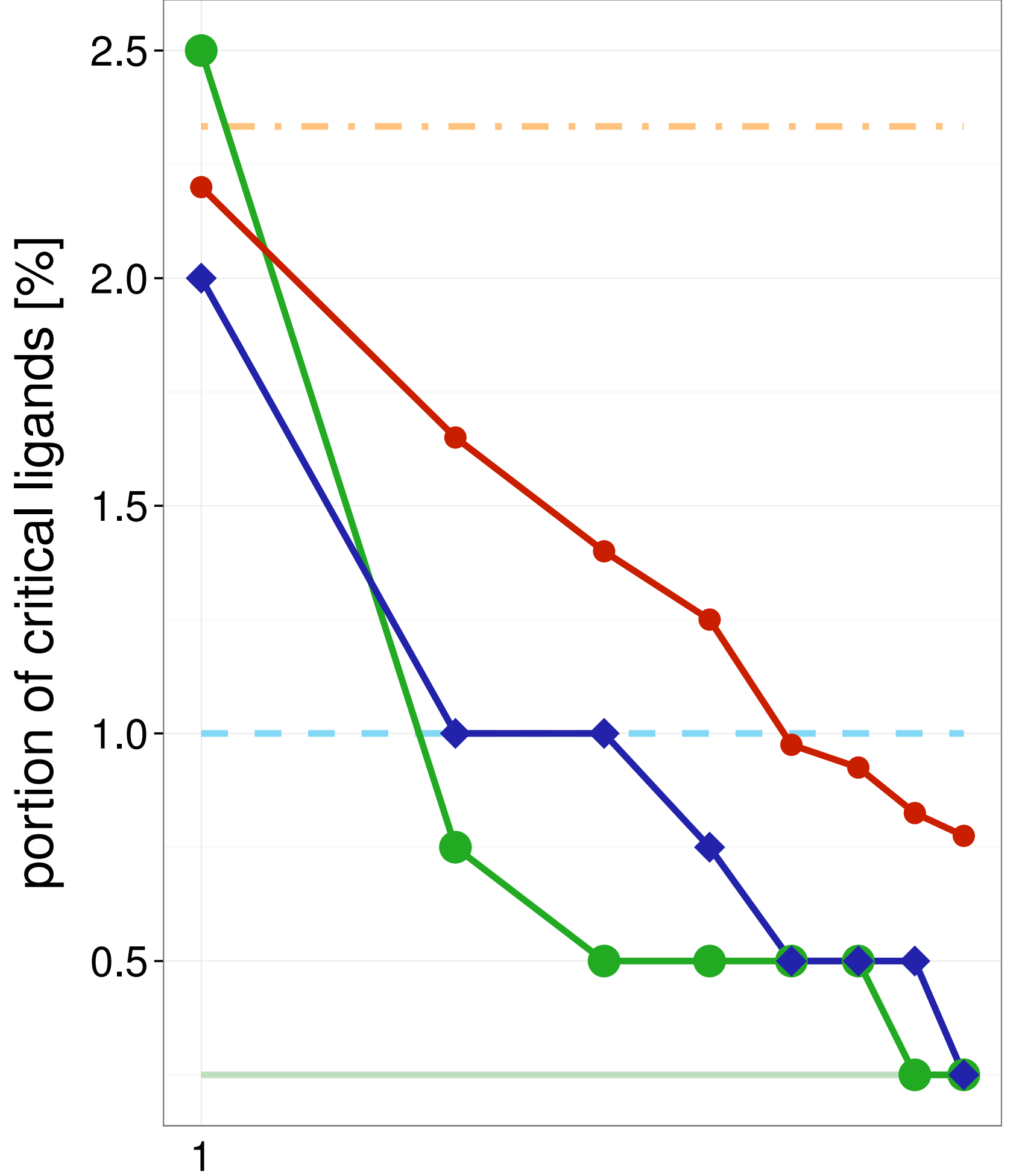

Average single structure

- Best single structure

Whole ensemble

SIENA

$\leadsto$ Clustering

$\longrightarrow$ Random 


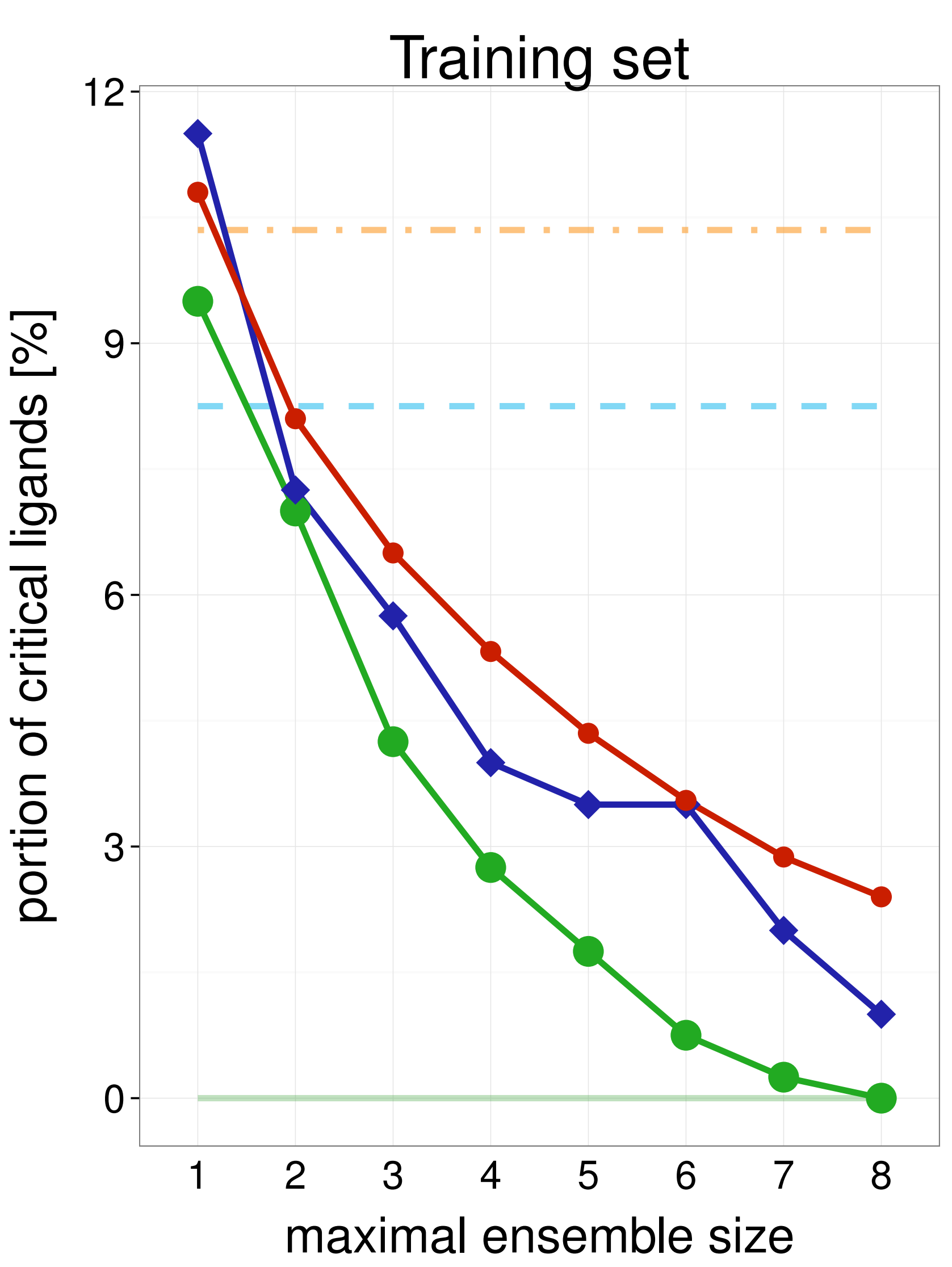

Test set

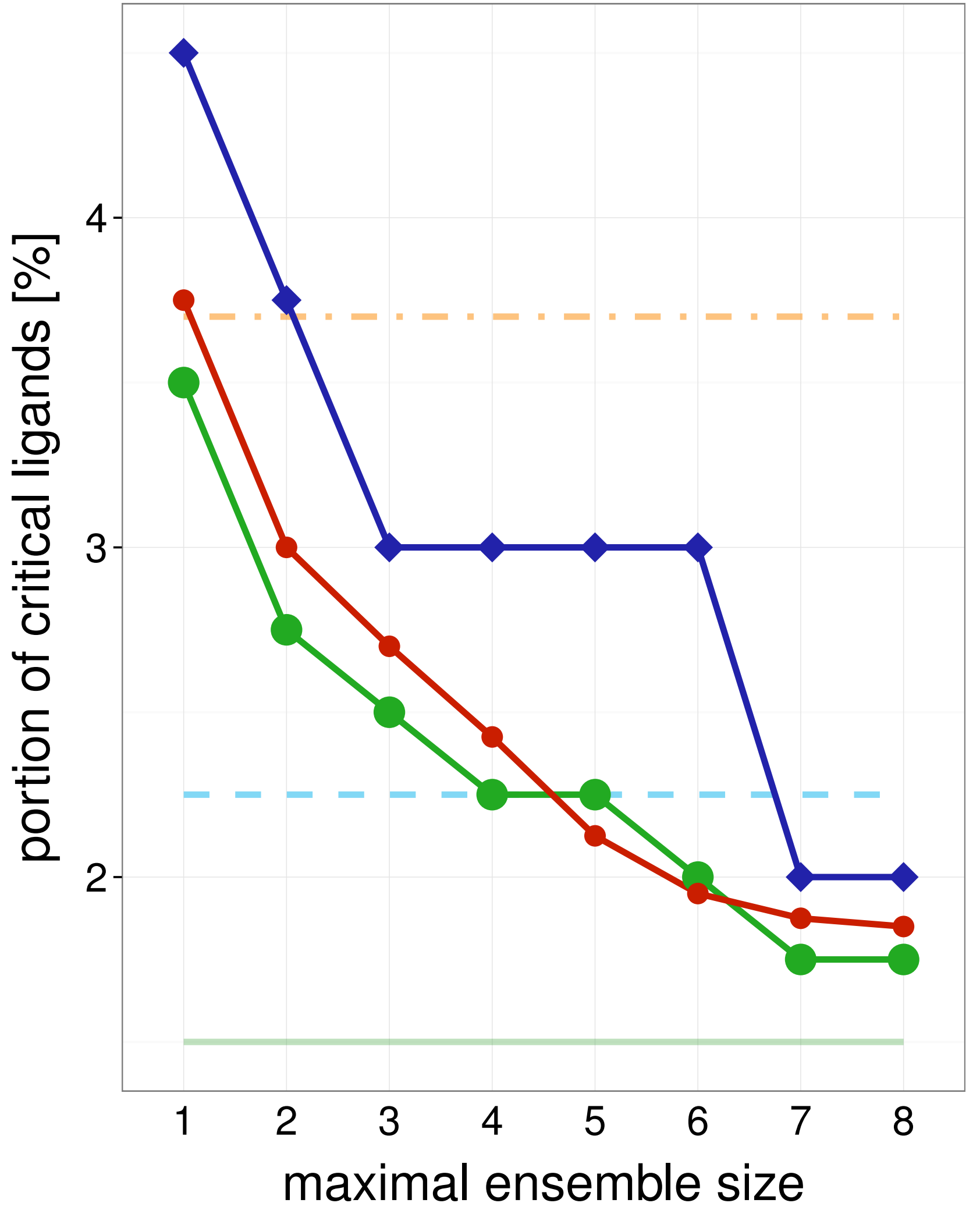

$1 E 7 V_{12} \quad$ Training set

Average single structure

_ Best single structure

Whole ensemble

- SIENA

$\neg$ Clustering

$\multimap$ Random

Average single structure

_ _ Best single structure

Whole ensemble

- SIENA

$\neg$ Clustering

- Random

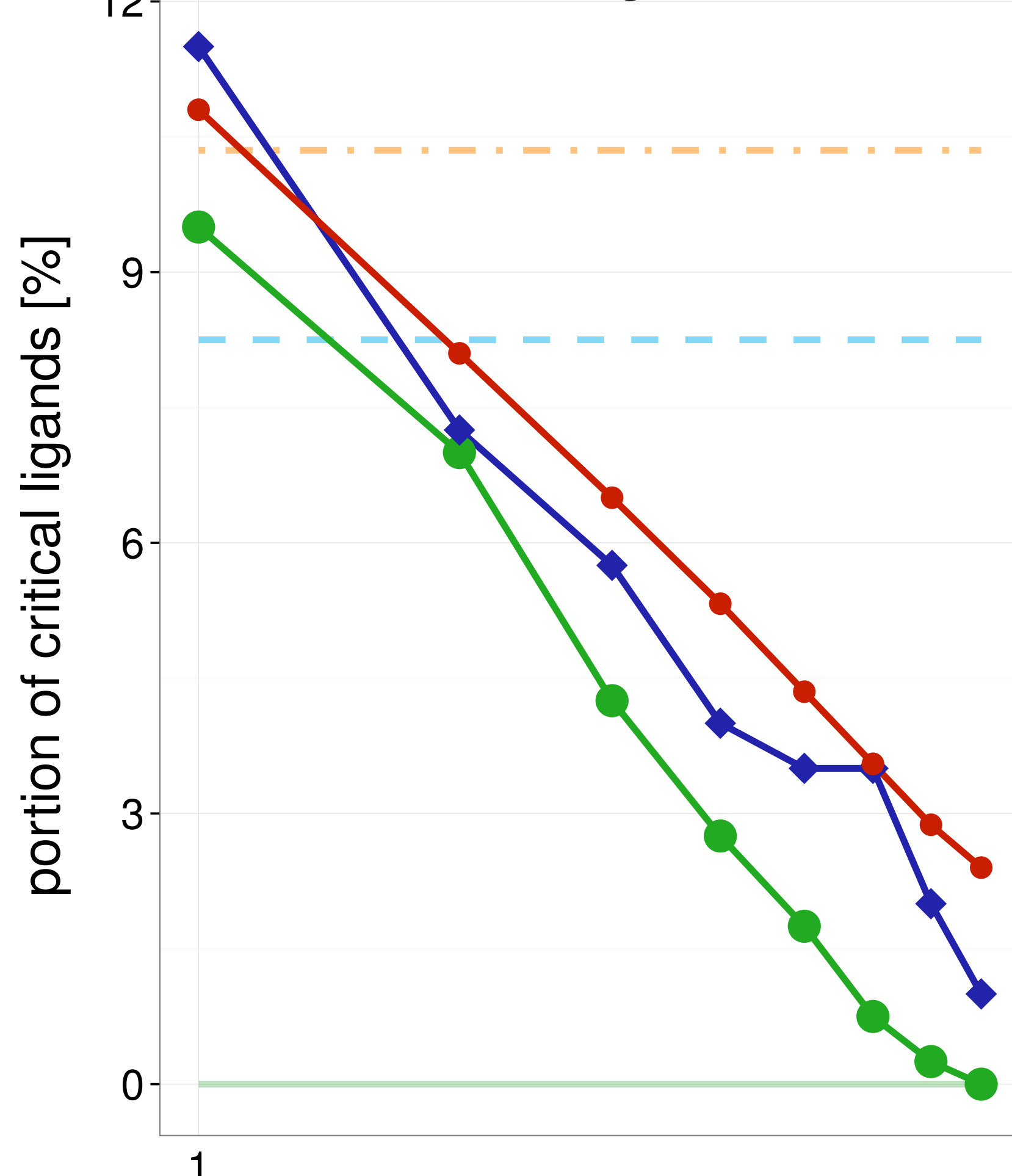

maximal ensemble size

Test set

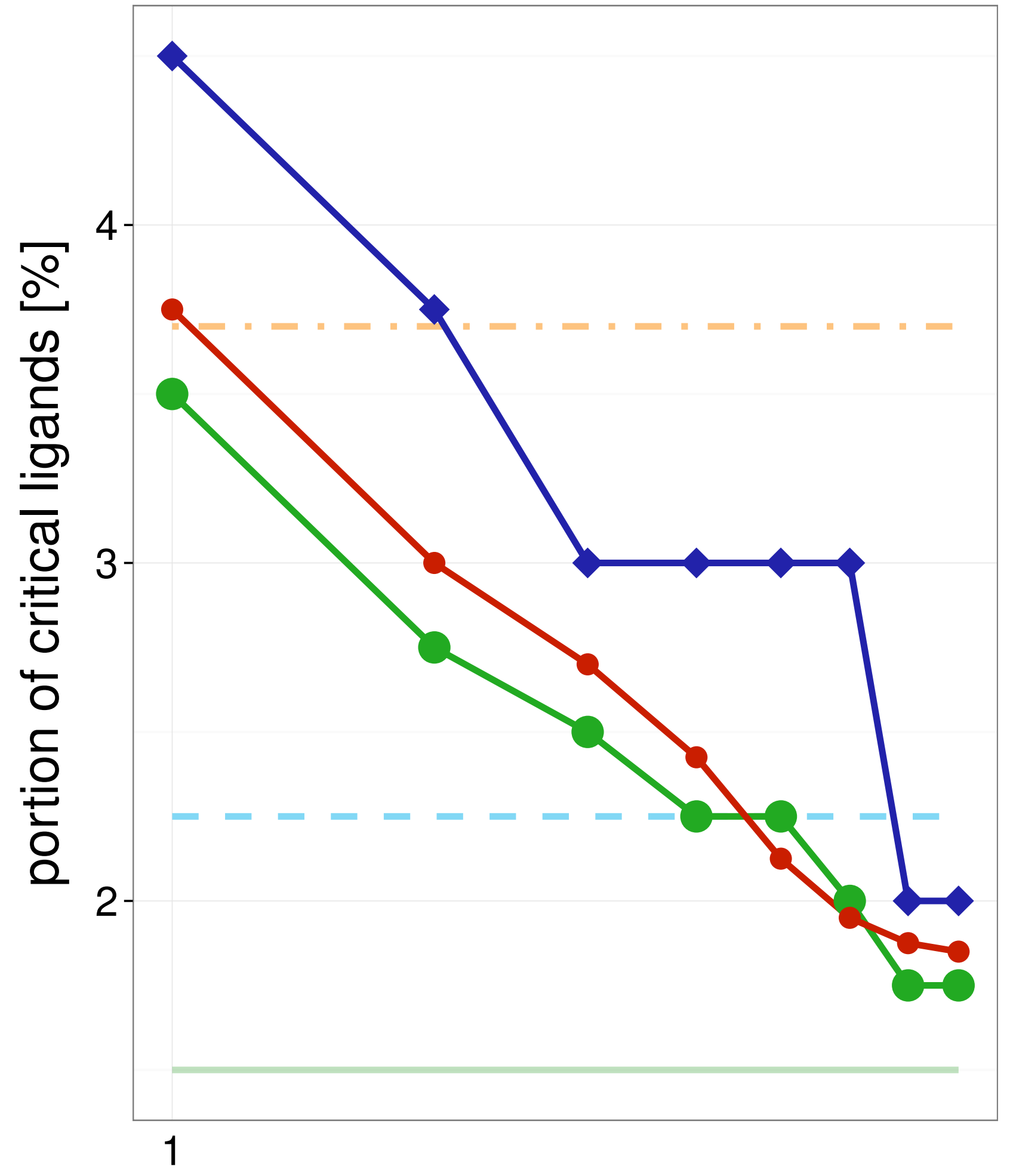

maximal ensemble size
Average single structure

_ Best single structure

Whole ensemble

SIENA

$\neg$ Clustering

$\because$ Random 


\section{Training set}

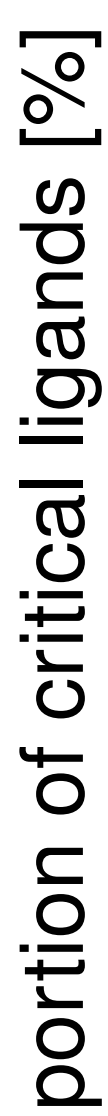

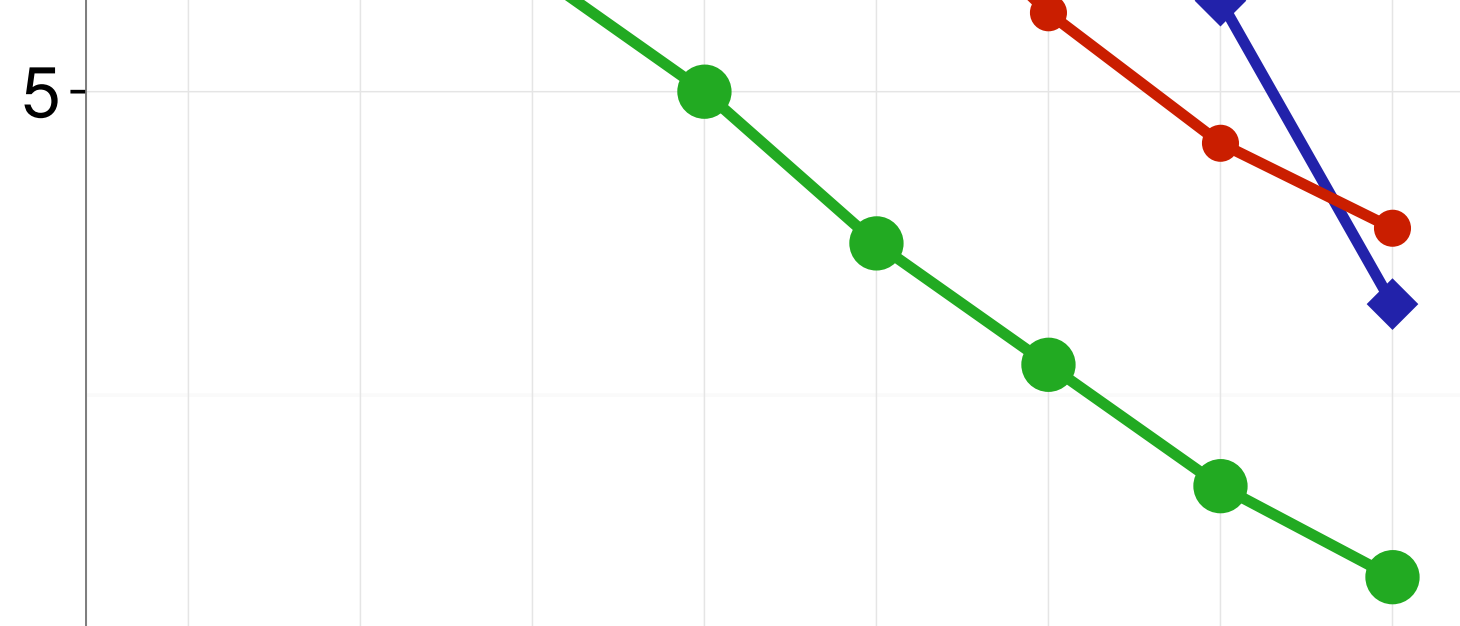

0

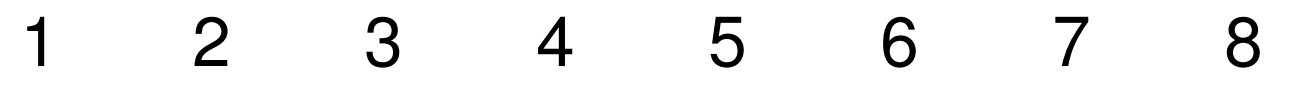

maximal ensemble size

Test set

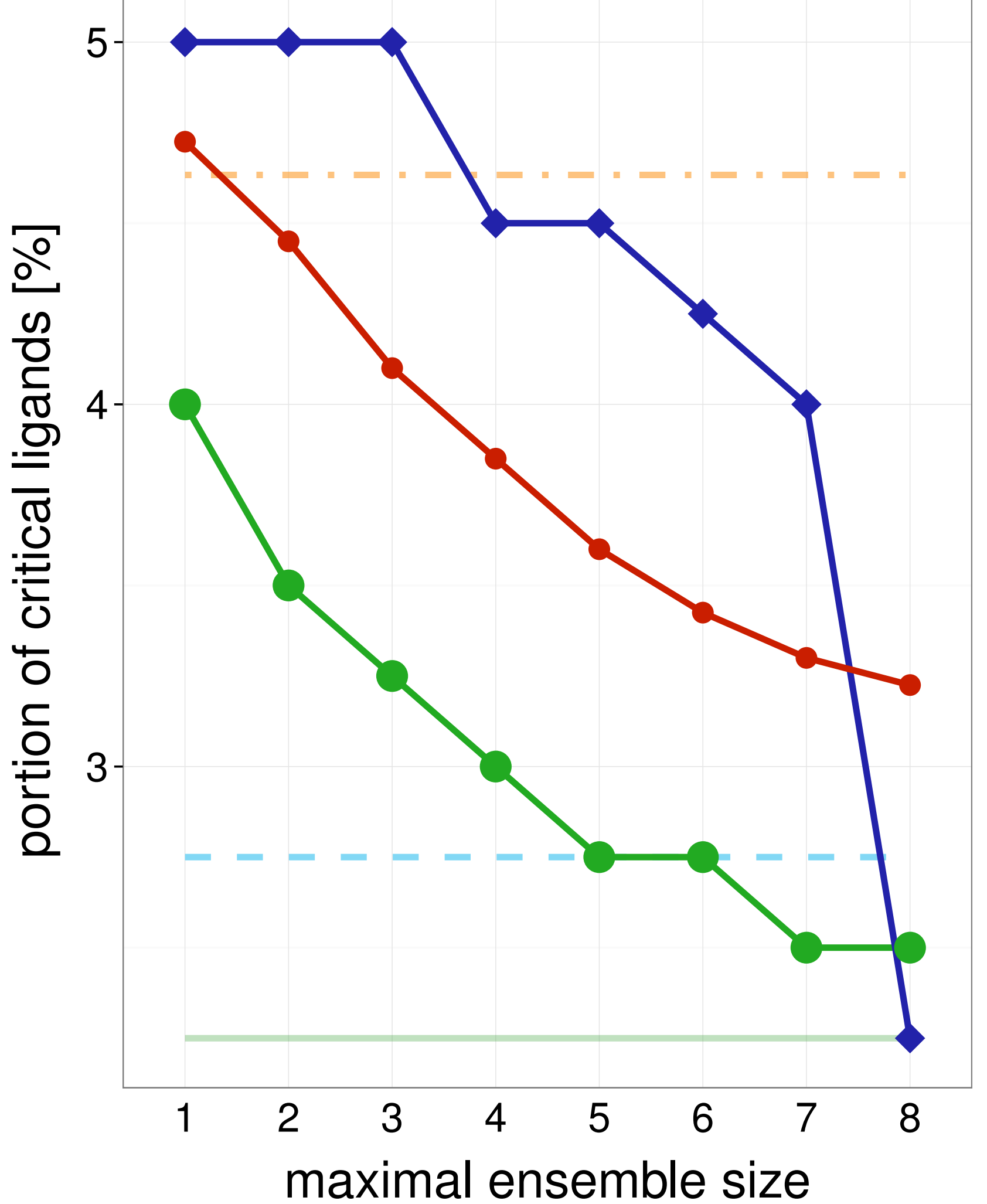

Average single structure

_ Best single structure

Whole ensemble

- SIENA

$\neg$ Clustering

$\multimap$ Random

Average single structure

_ _ Best single structure

Whole ensemble

- SIENA

$\neg$ Clustering

$\rightarrow$ Random
1FPU

Training set

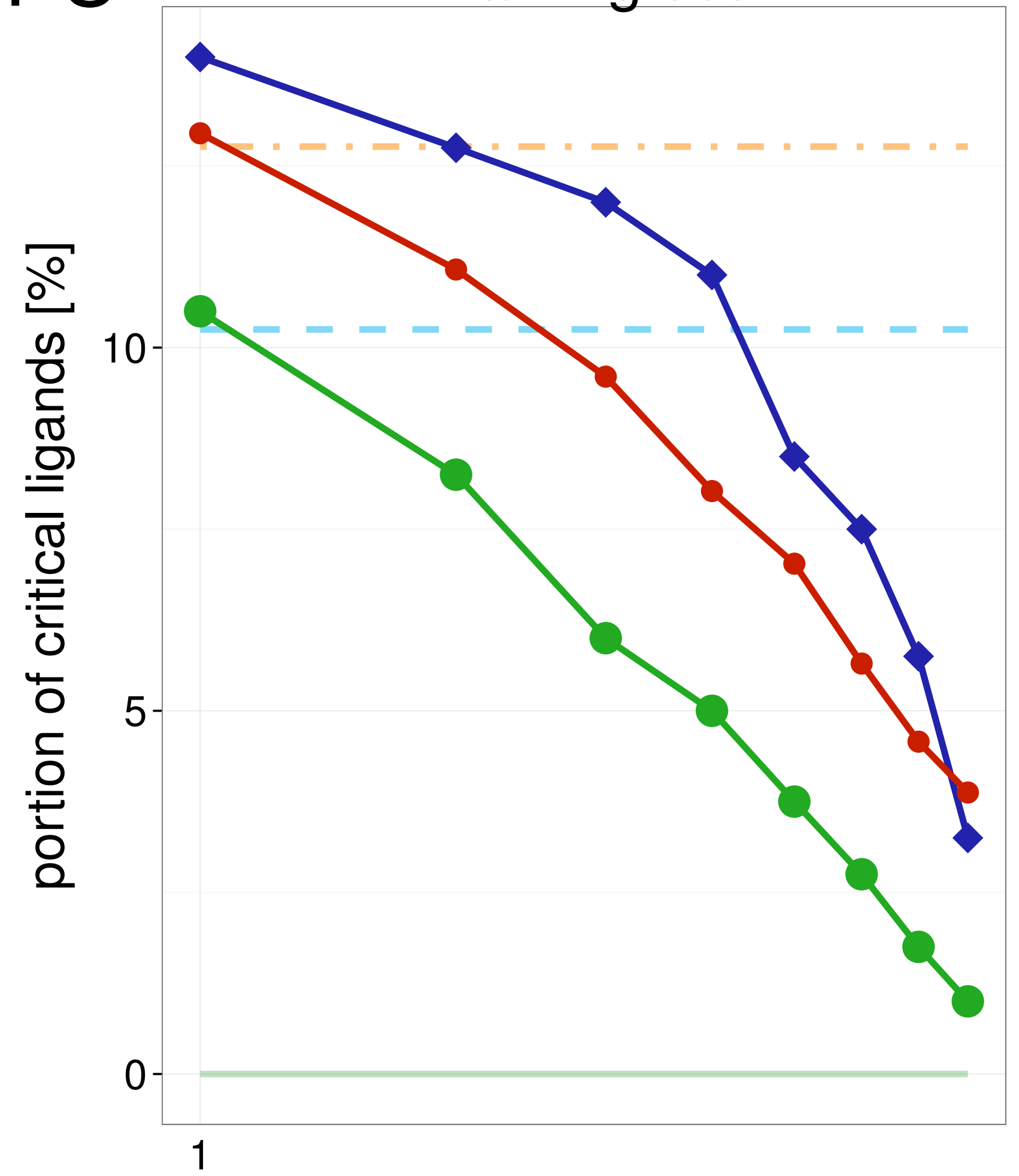

Average single structure

Best single structure

Whole ensemble

- SIENA

$\neg$ Clustering

$\multimap$ Random

maximal ensemble size

\section{Test set}

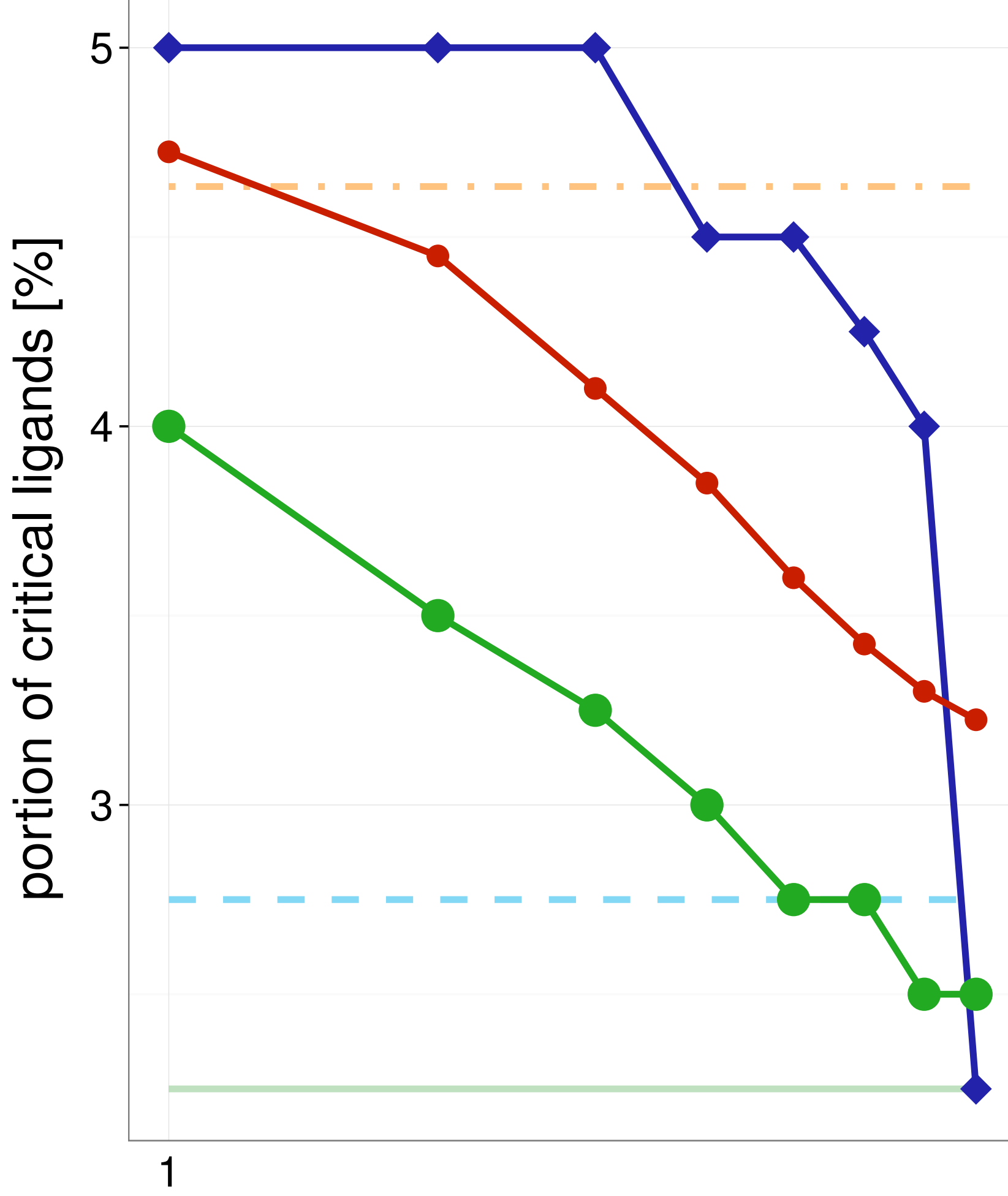

Average single structure

_ Best single structure

Whole ensemble

- SIENA

$\leadsto$ Clustering

$\multimap$ Random 


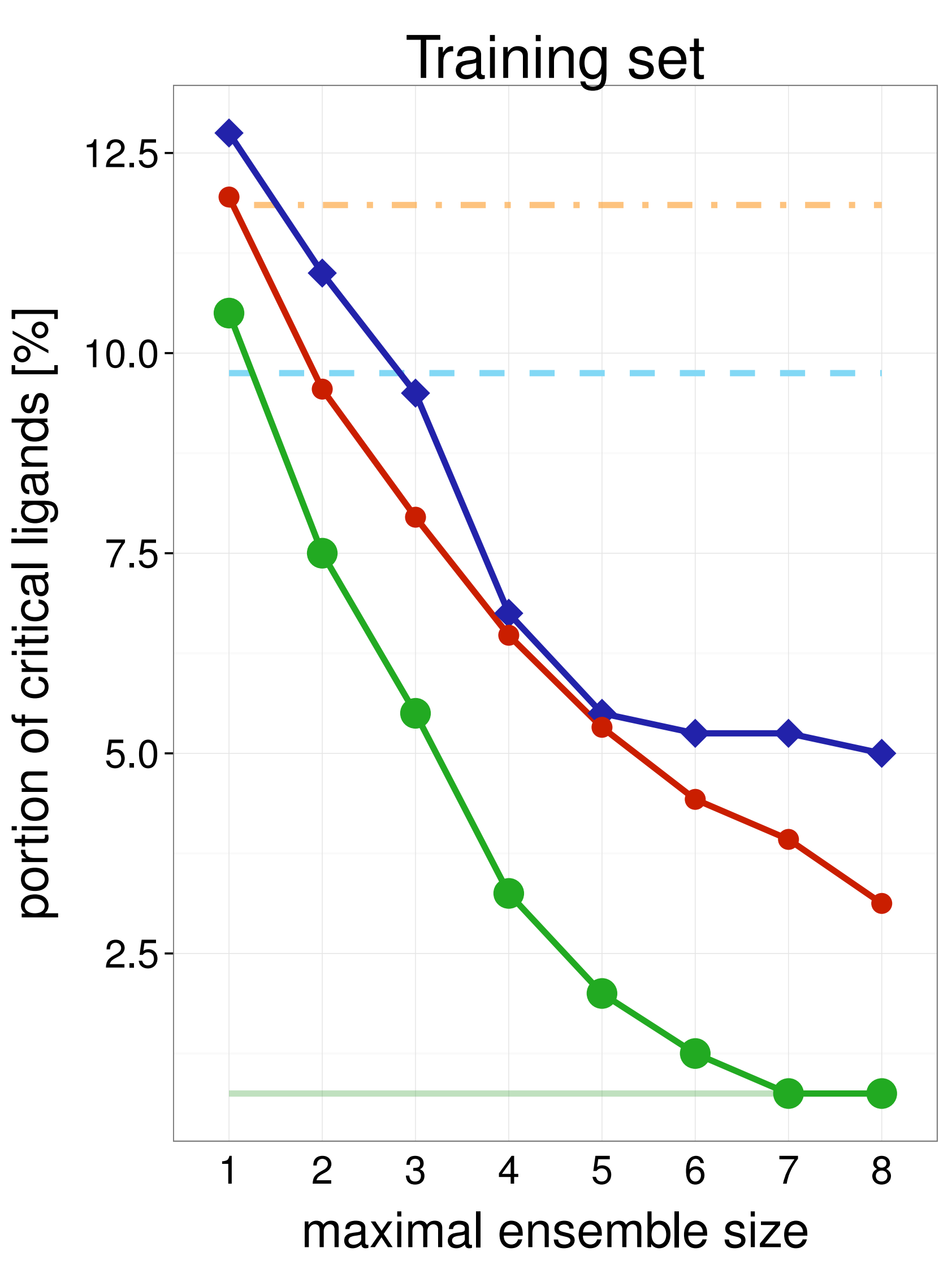

1MP8

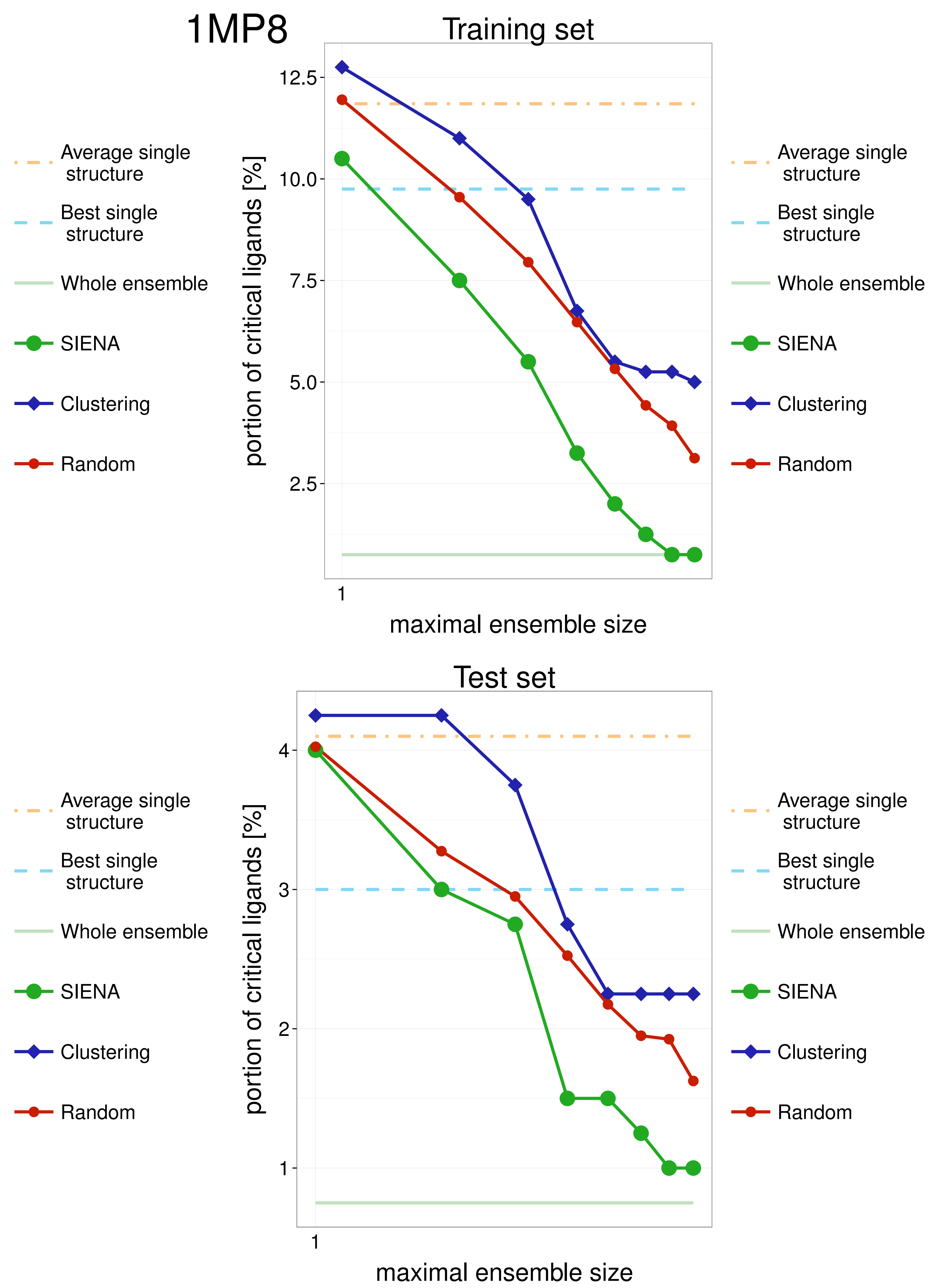


Training set

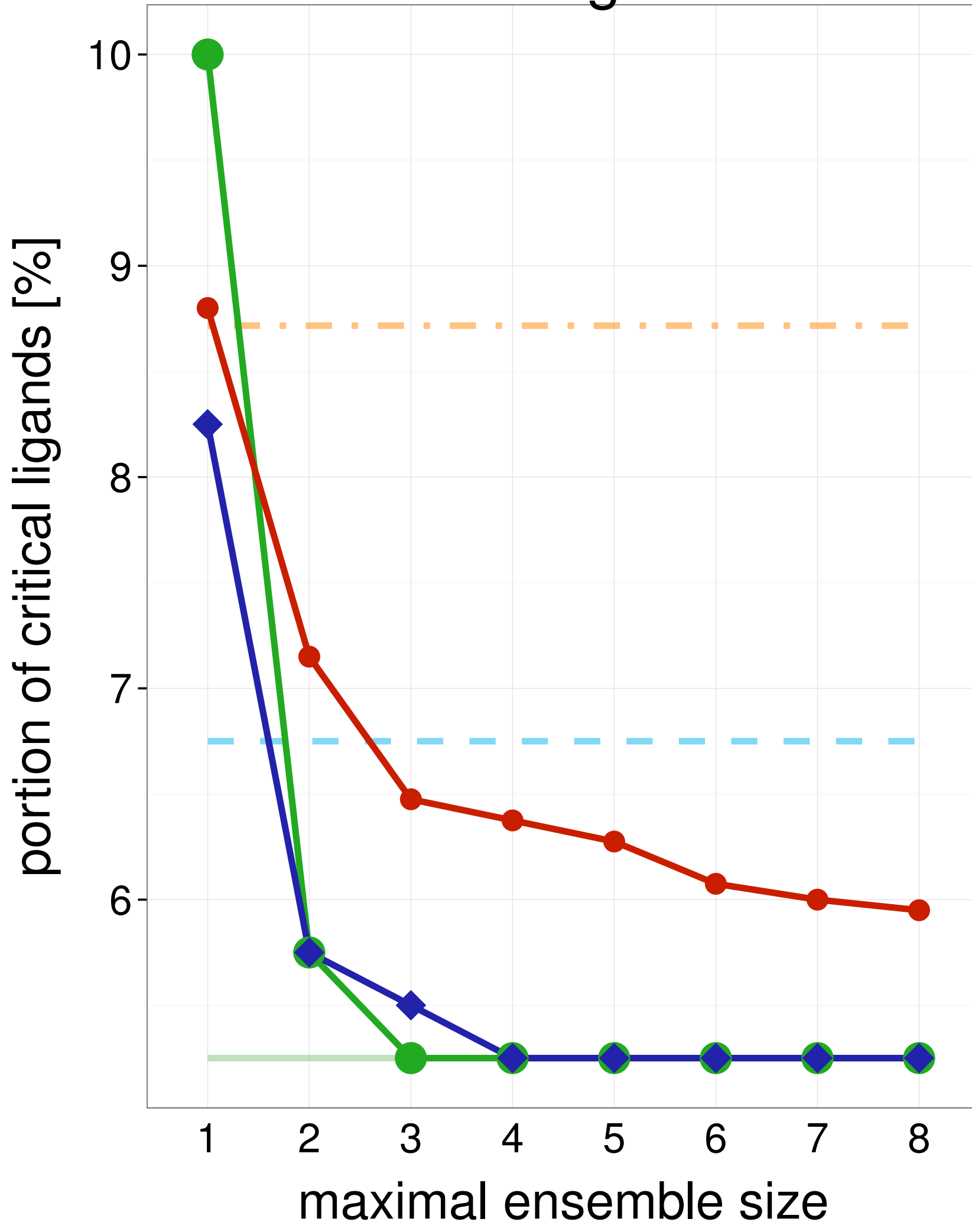

Test set

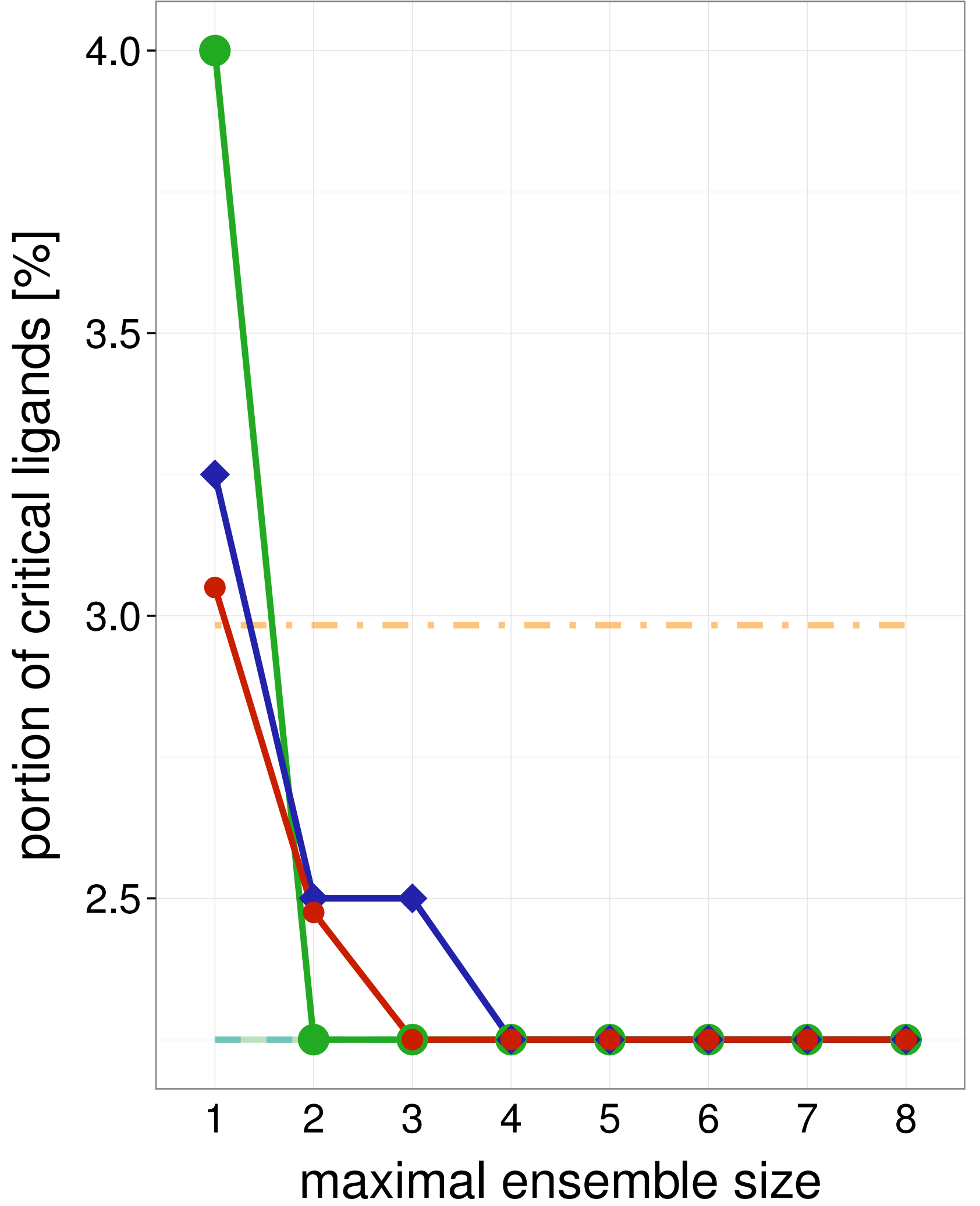

Average single structure

_ Best single structure

Whole ensemble

- SIENA

$\neg$ Clustering

$\multimap$ Random

Average single structure

_ _ Best single structure

Whole ensemble

- SIENA

$\leadsto$ Clustering

- Random
1SRF

Training set

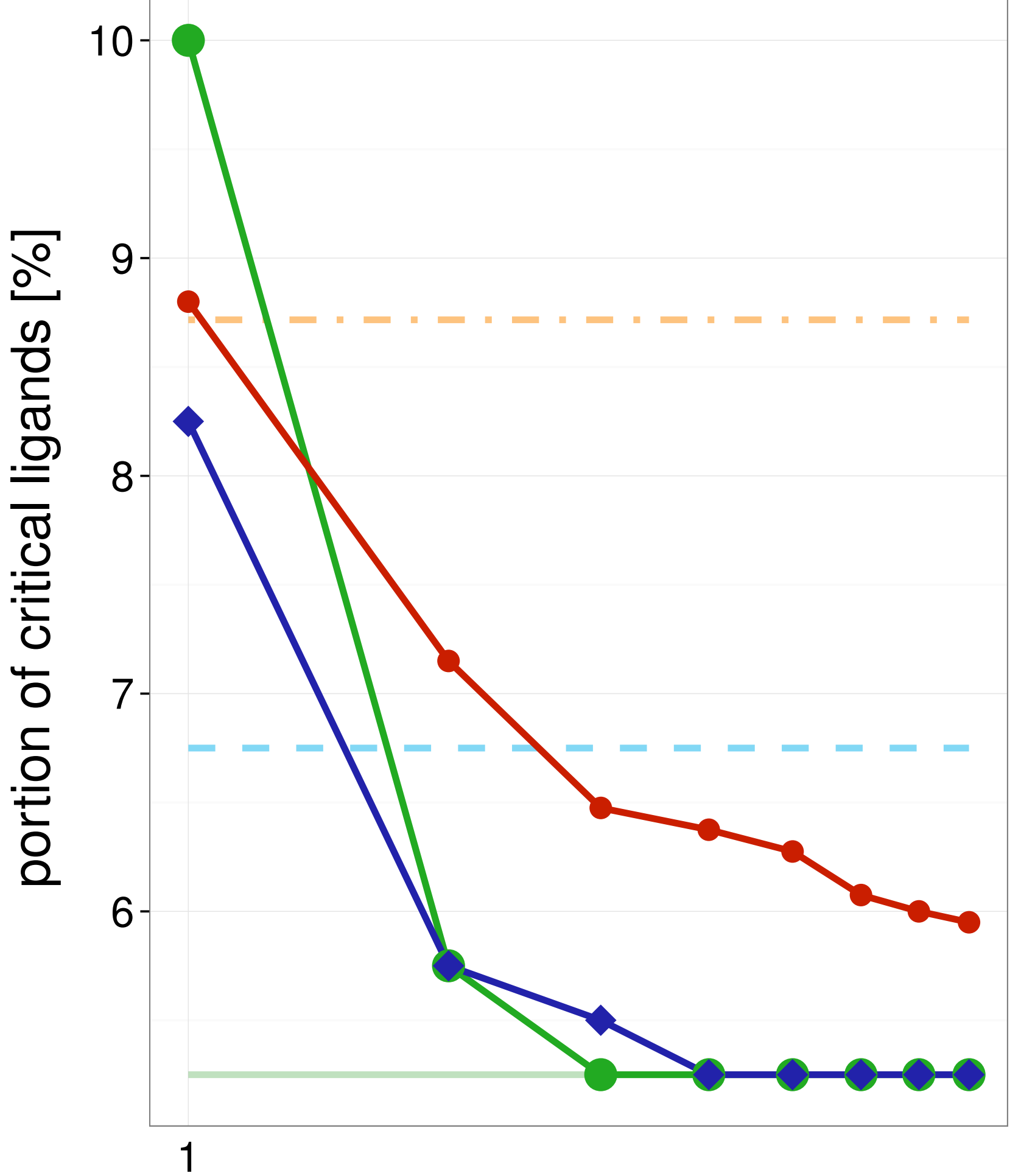

maximal ensemble size

Test set

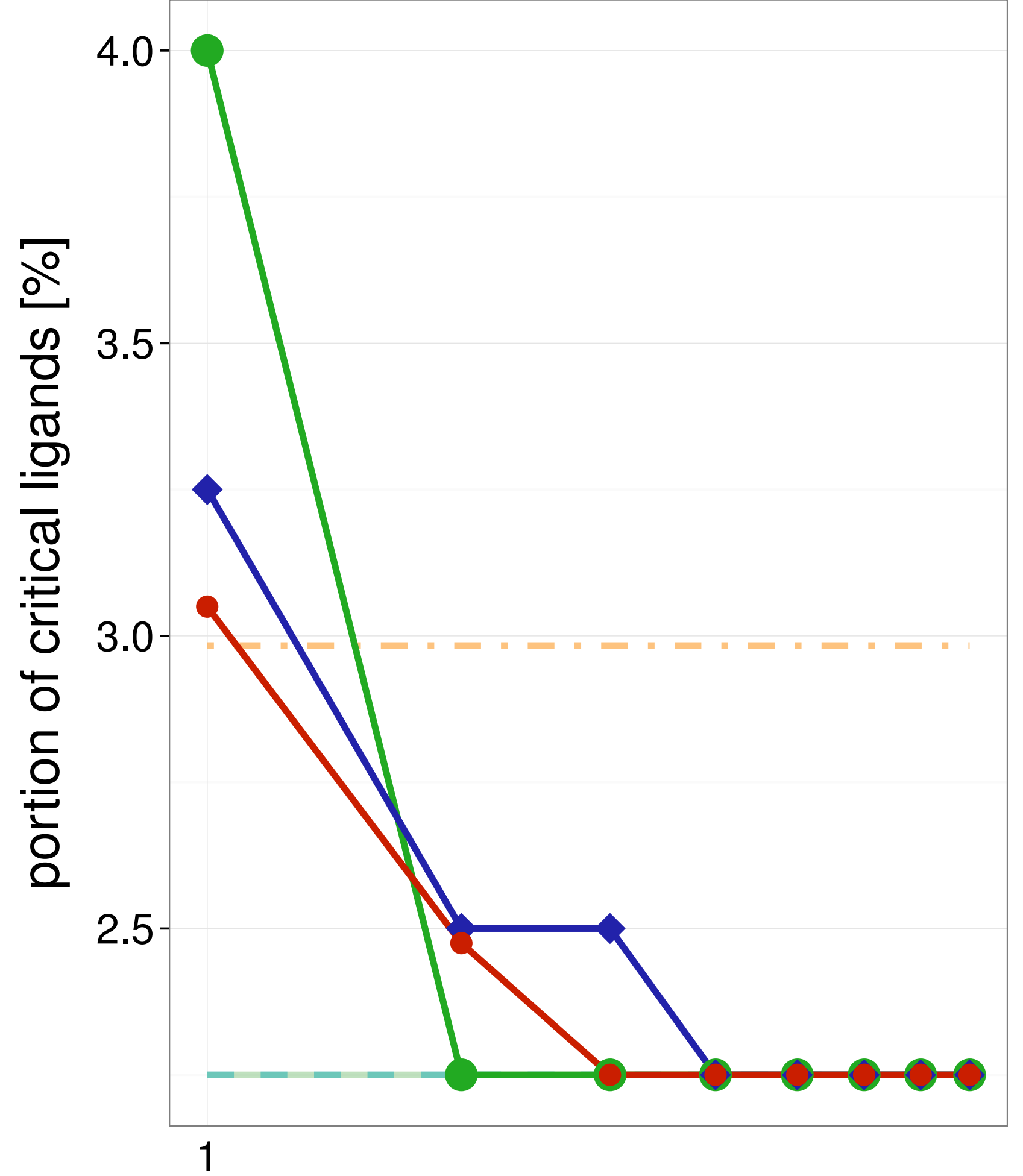

maximal ensemble size
Average single structure

_ _ Best single structure

Whole ensemble

SIENA

$\neg$ Clustering

$\multimap$ Random 
Training set

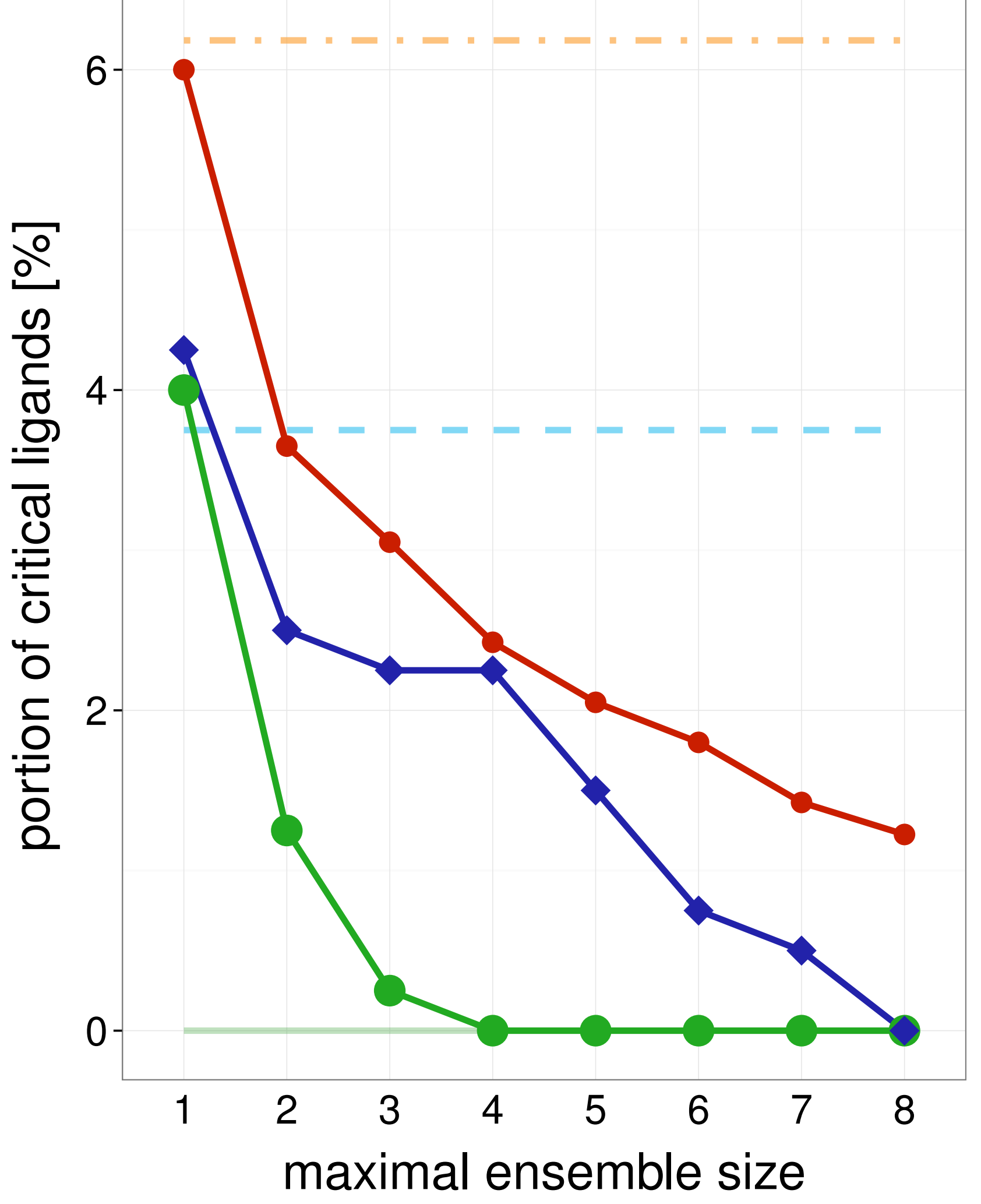

Test set

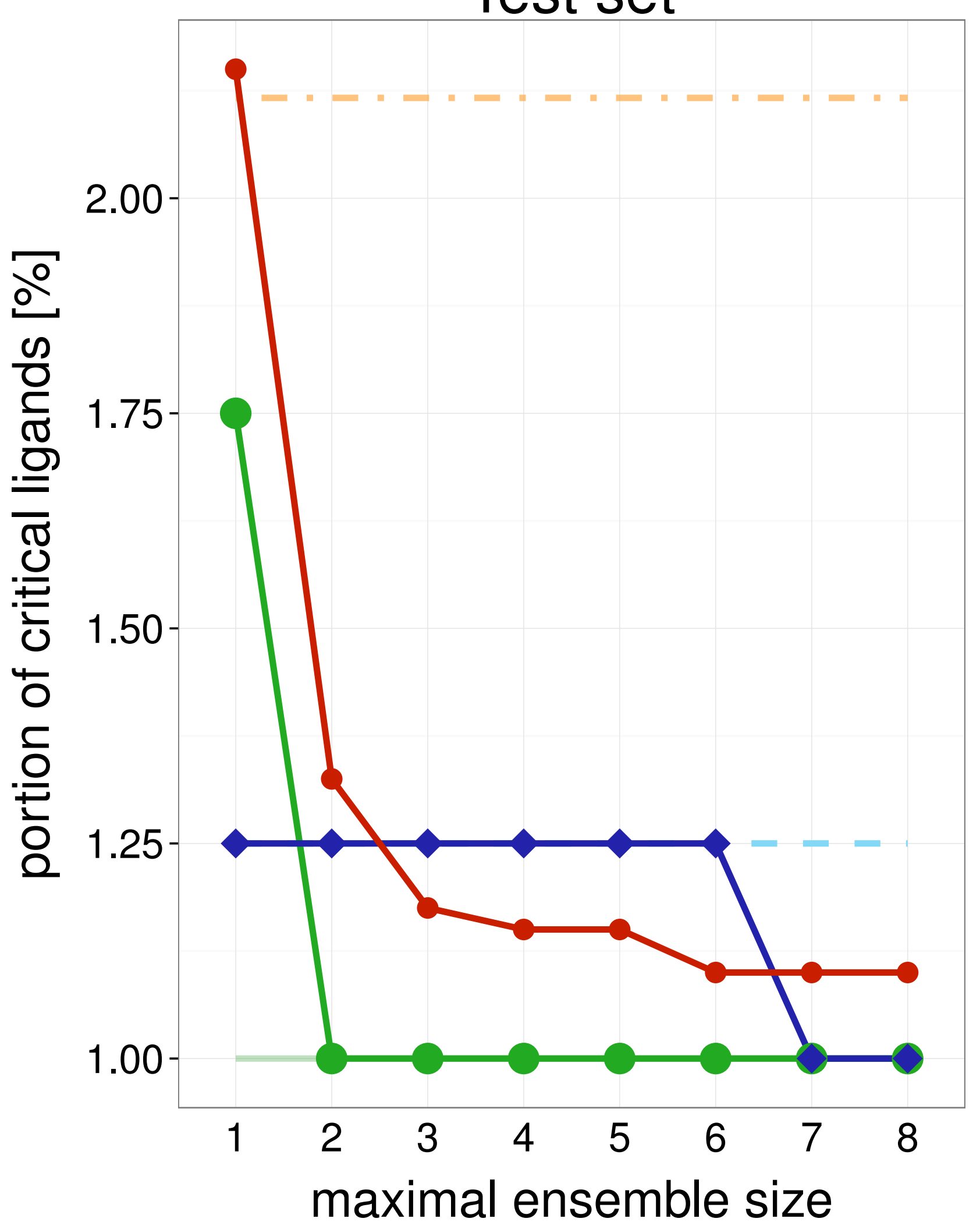

Average single structure

_ _ Best single structure

Whole ensemble

- SIENA

$\neg$ Clustering

$\multimap$ Random

Average single structure

_ _ Best single structure

Whole ensemble

- SIENA

$\smile$ Clustering

$\multimap$ Random
Training set

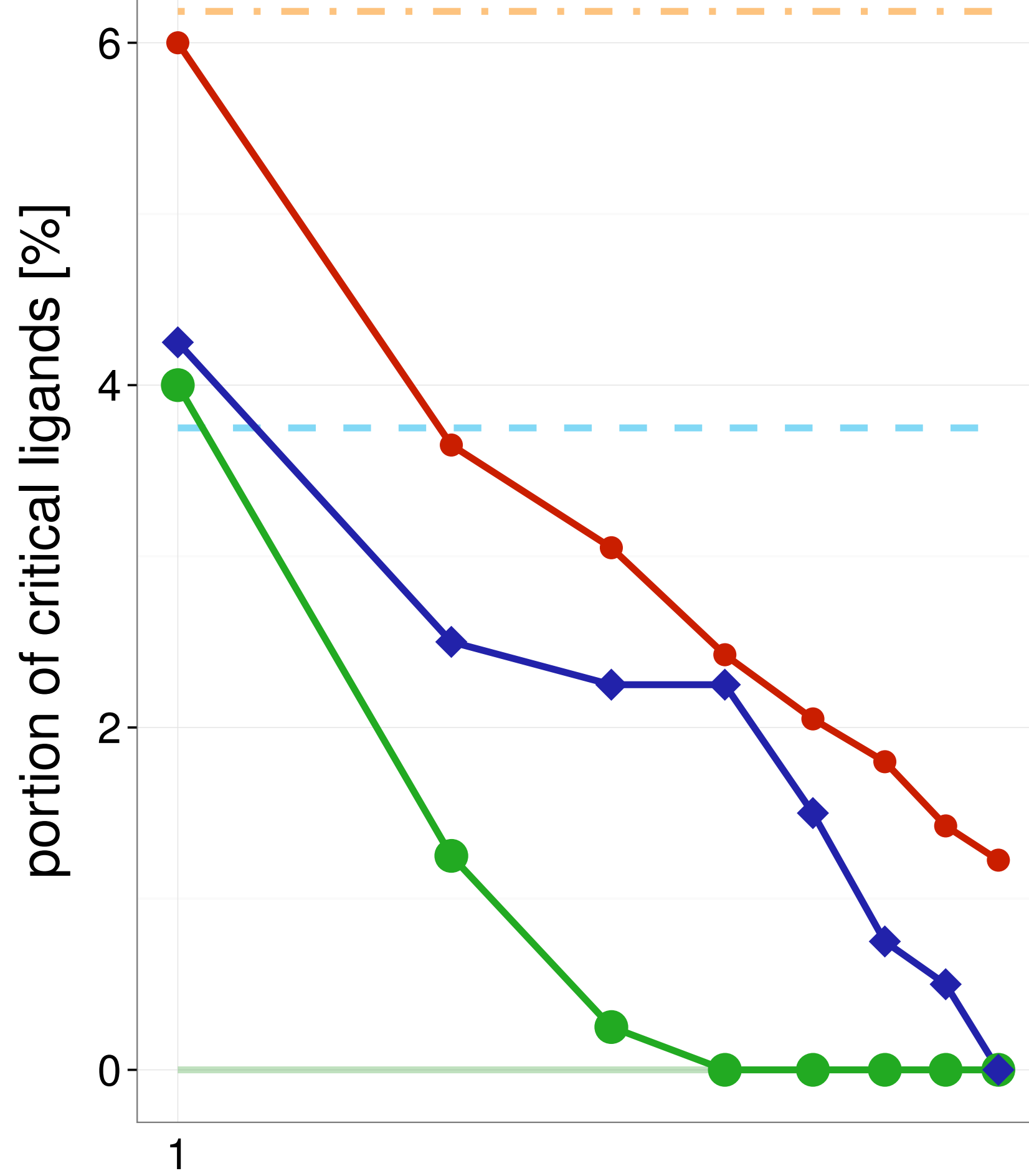

maximal ensemble size

Test set

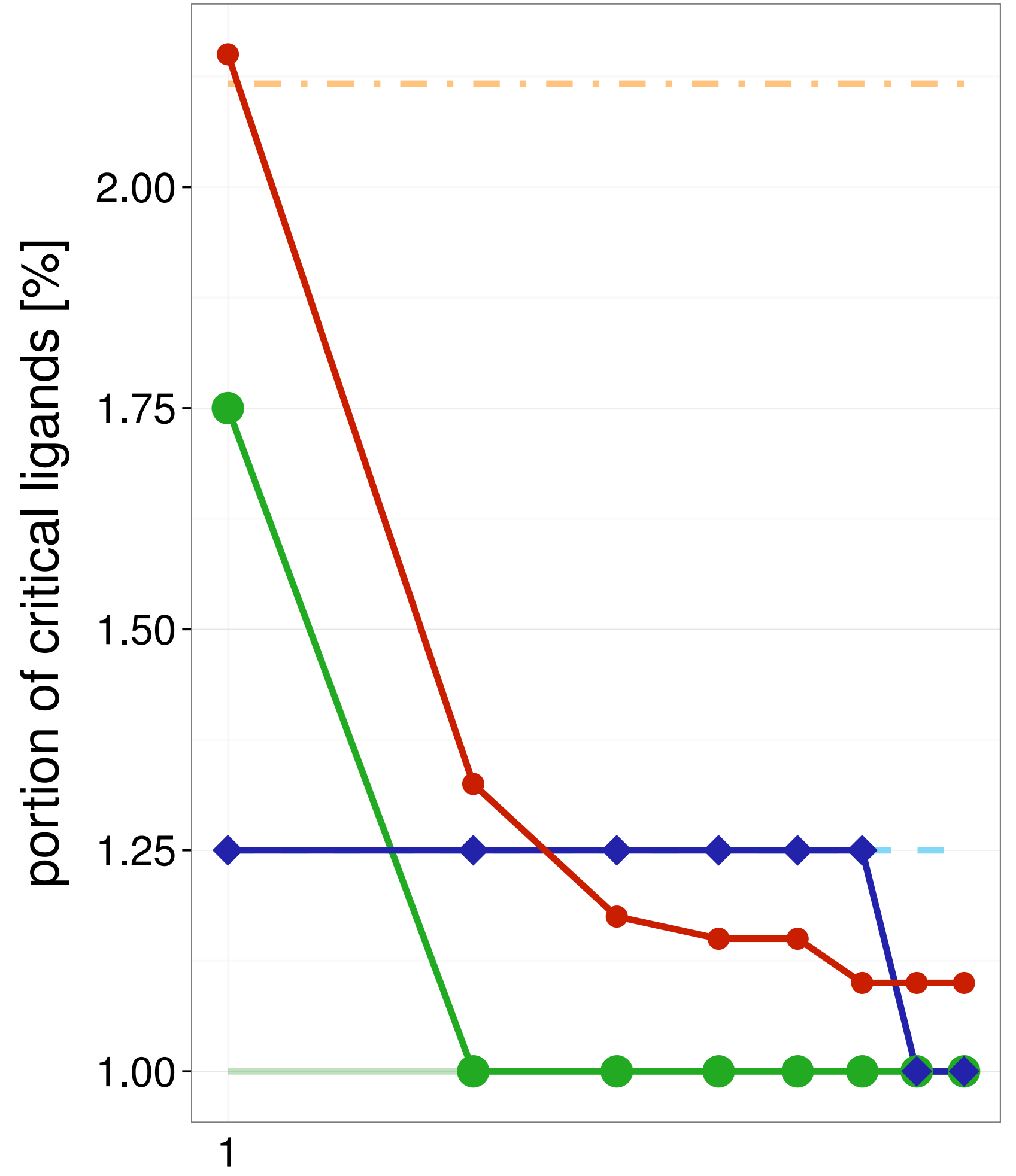

Average single structure

_ Best single structure

Whole ensemble

SIENA

$\leadsto$ Clustering

$\longrightarrow$ Random 


\section{Training set}

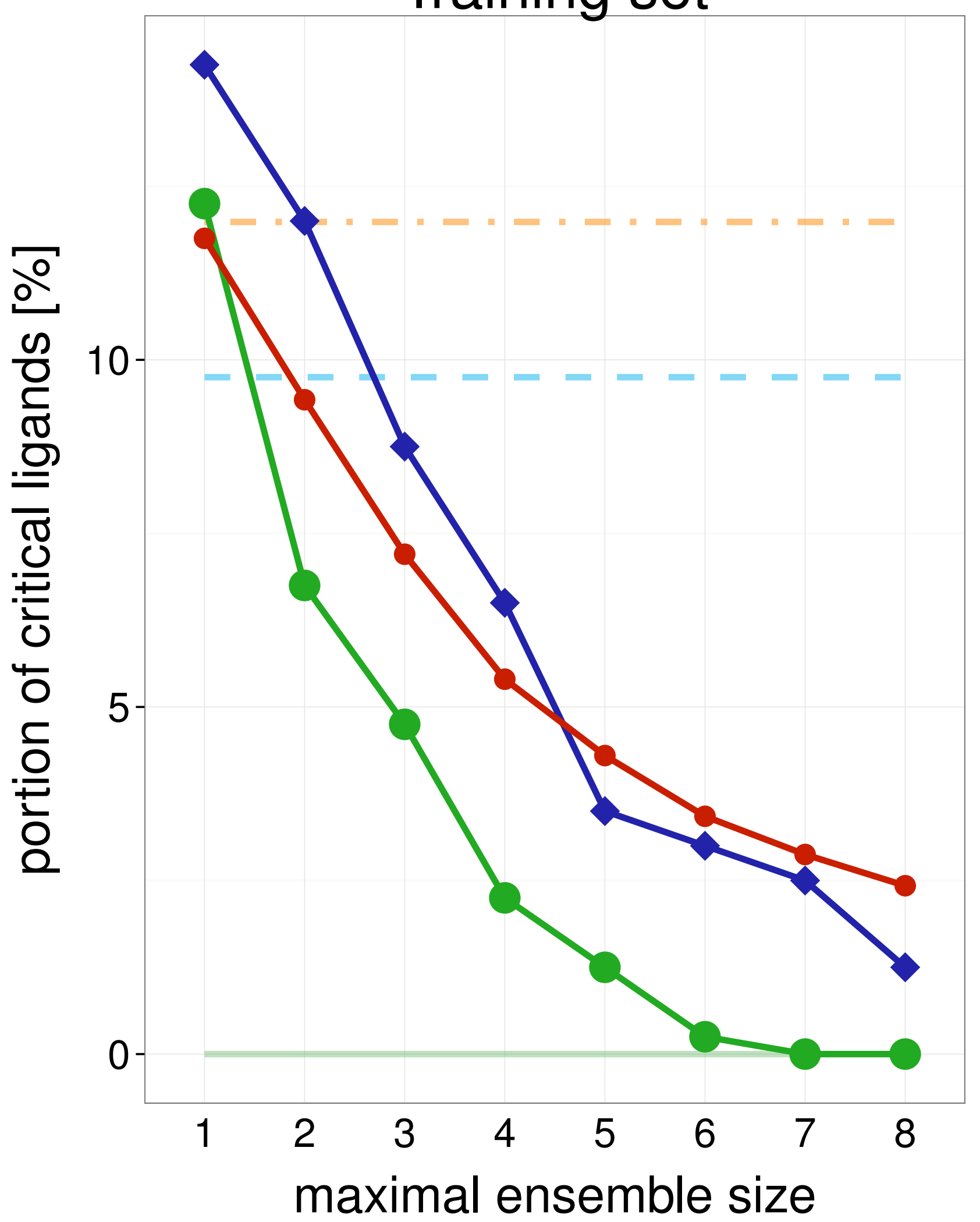

Test set

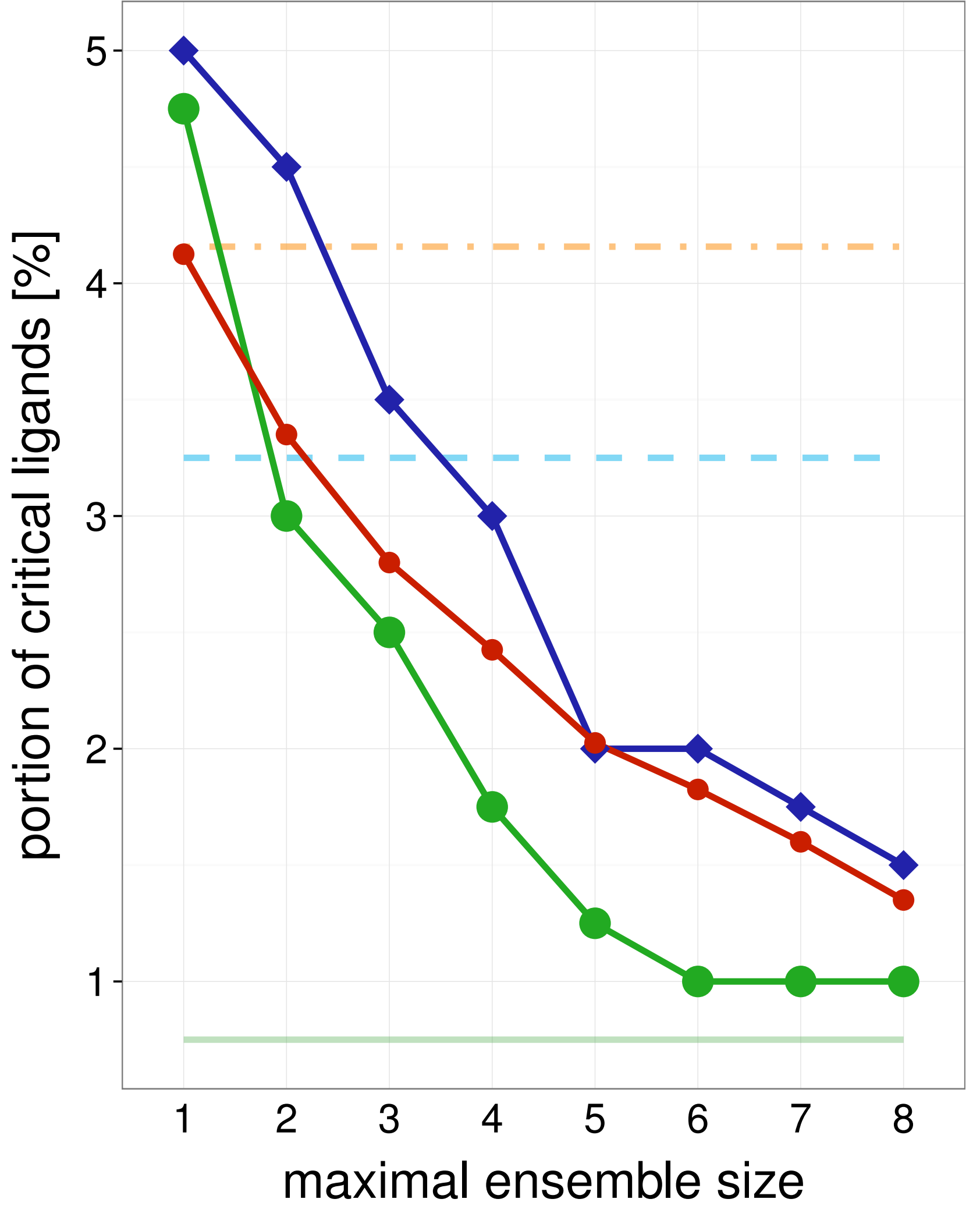

Average single structure

_ Best single structure

Whole ensemble

- SIENA

$\neg$ Clustering

$\multimap$ Random

Average single structure

_ _ Best single structure

Whole ensemble

- SIENA

$\smile$ Clustering

$\multimap$ Random
1VSO

Training set

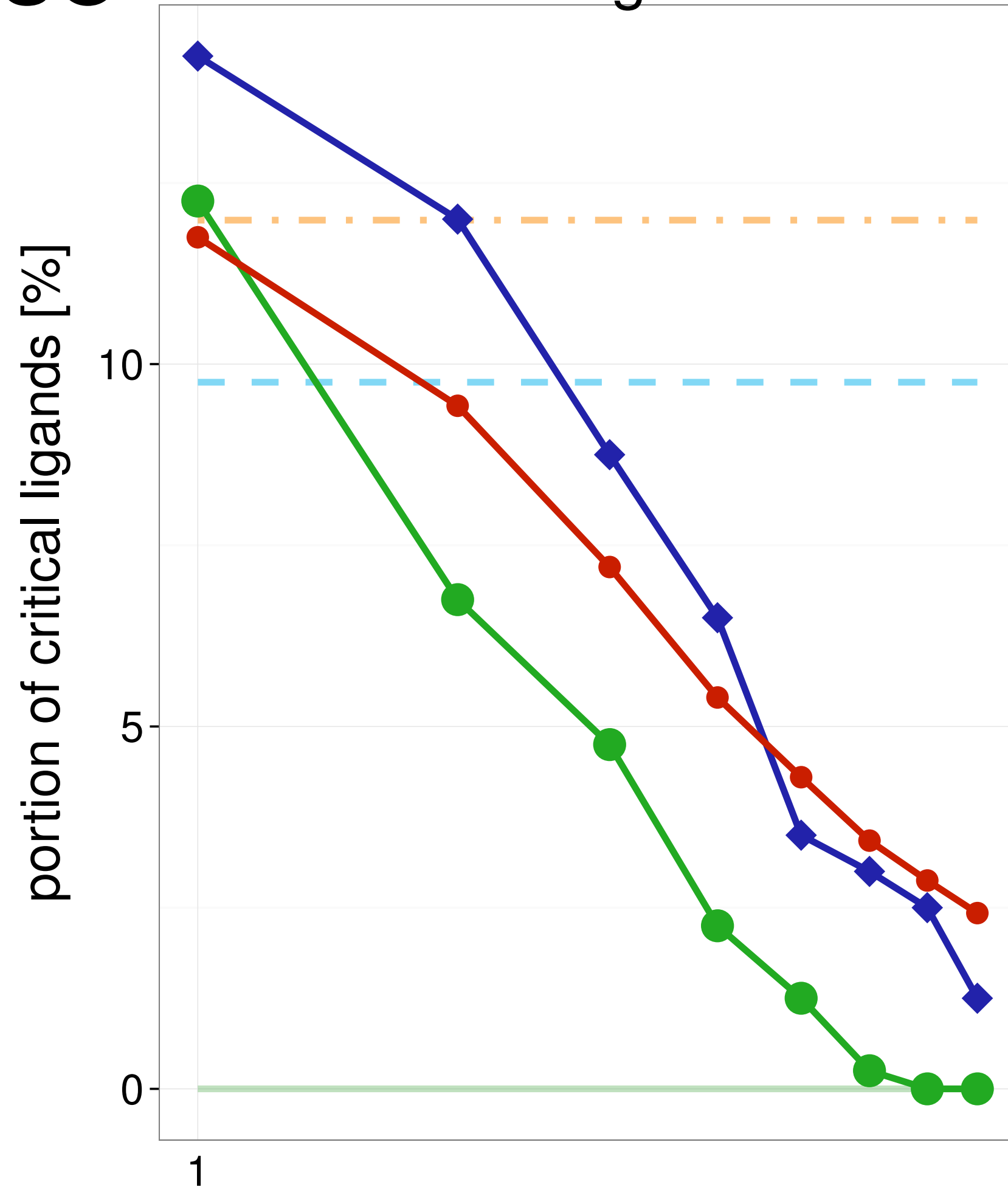

maximal ensemble size

Test set

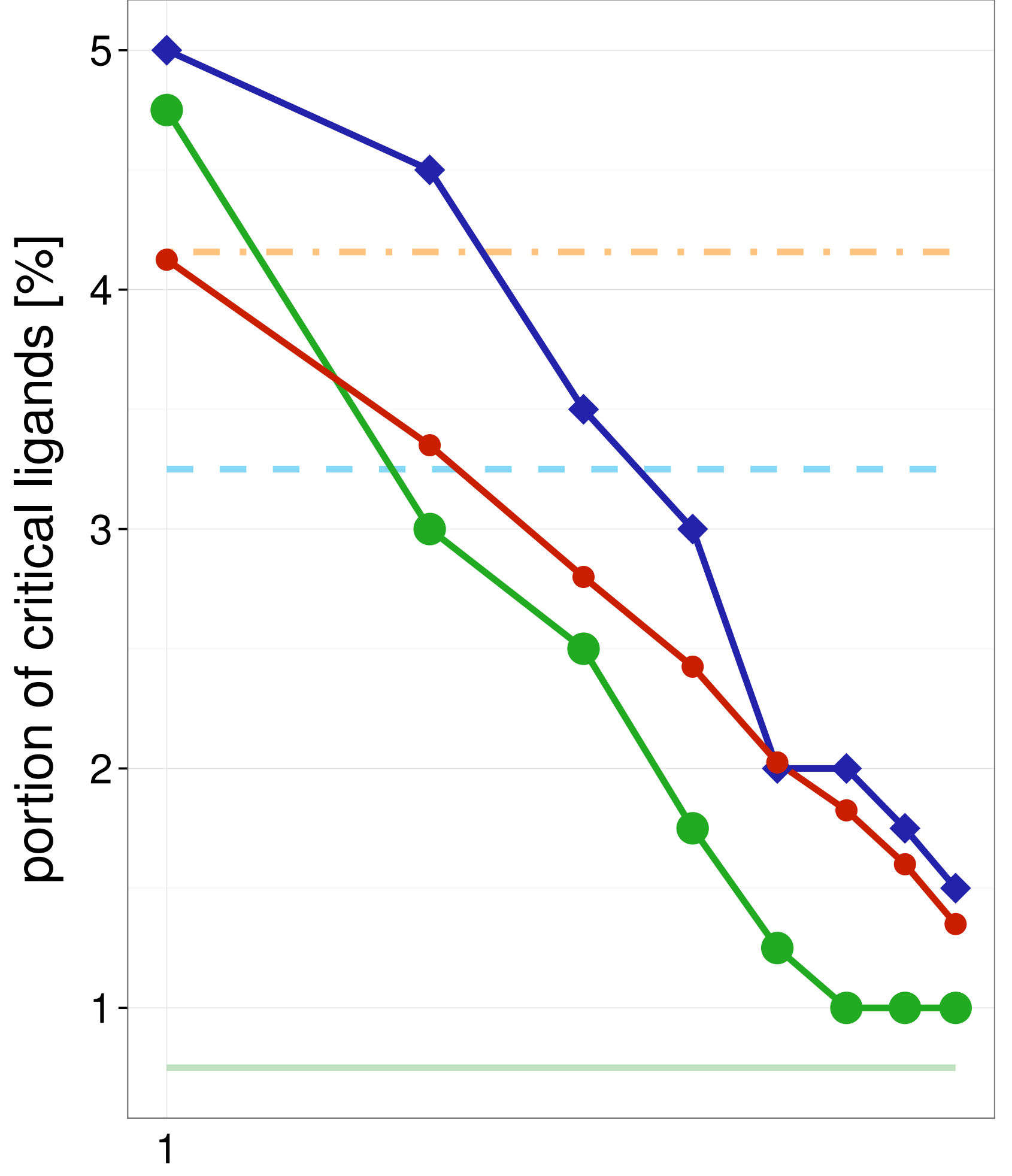

maximal ensemble size
Average single structure

_ Best single structure

Whole ensemble

SIENA

$\neg$ Clustering

$\multimap$ Random 
Training set

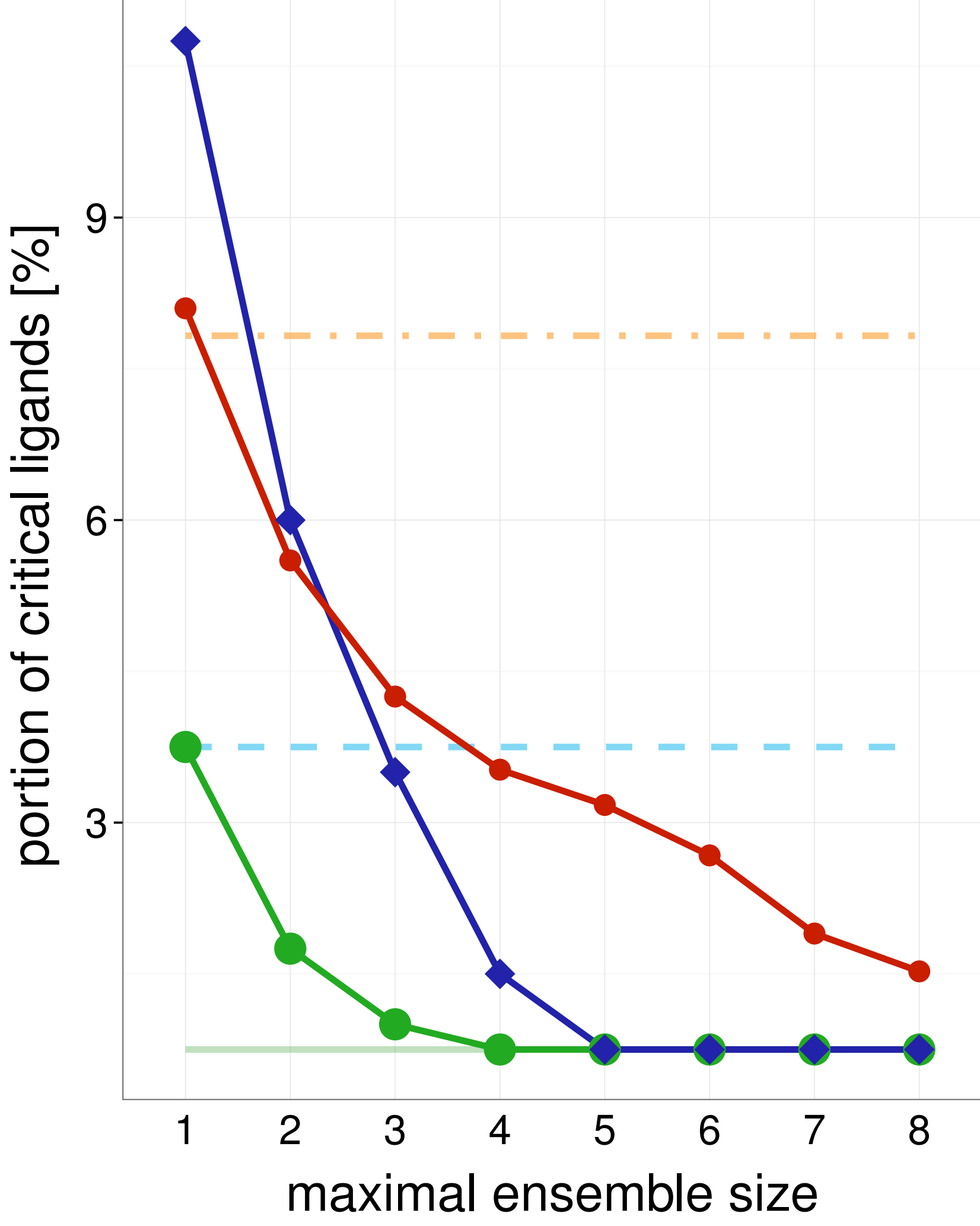

Test set

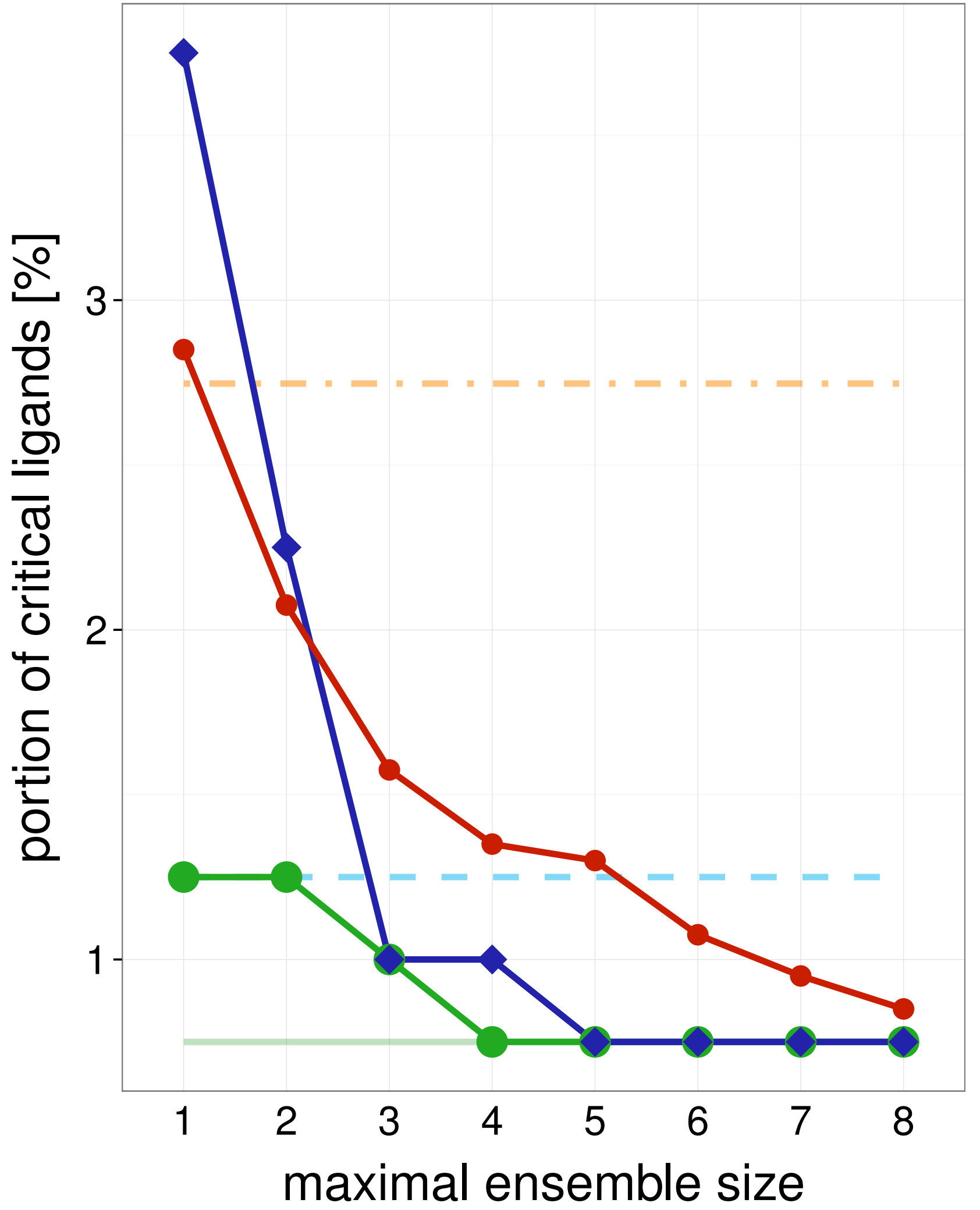

Average single structure

_ _ Best single structure

Whole ensemble

- SIENA

$\neg$ Clustering

$\multimap$ Random

Average single structure

_ _ Best single structure

Whole ensemble

- SIENA

$\neg$ Clustering

- Random
Training set

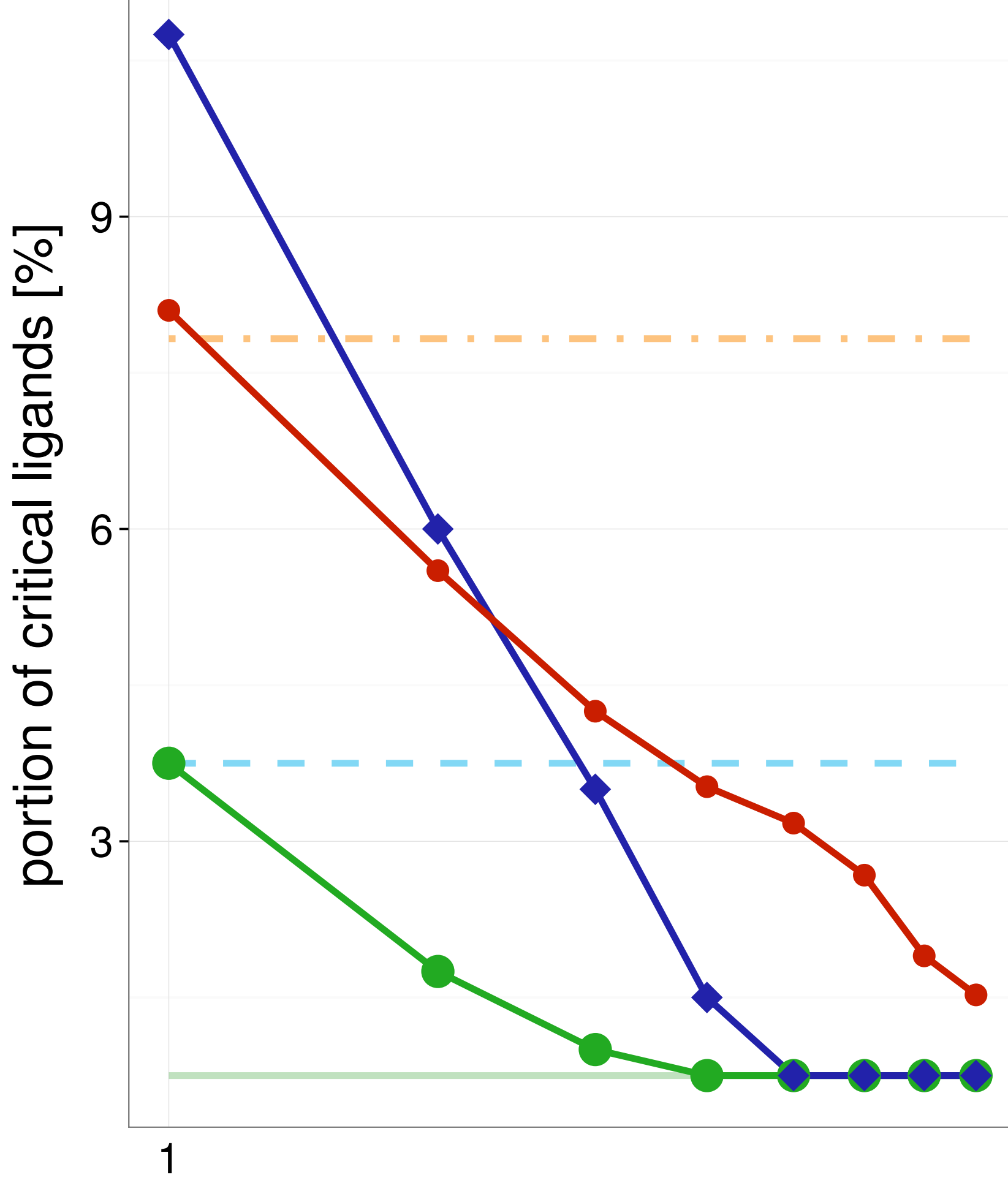

maximal ensemble size

Test set

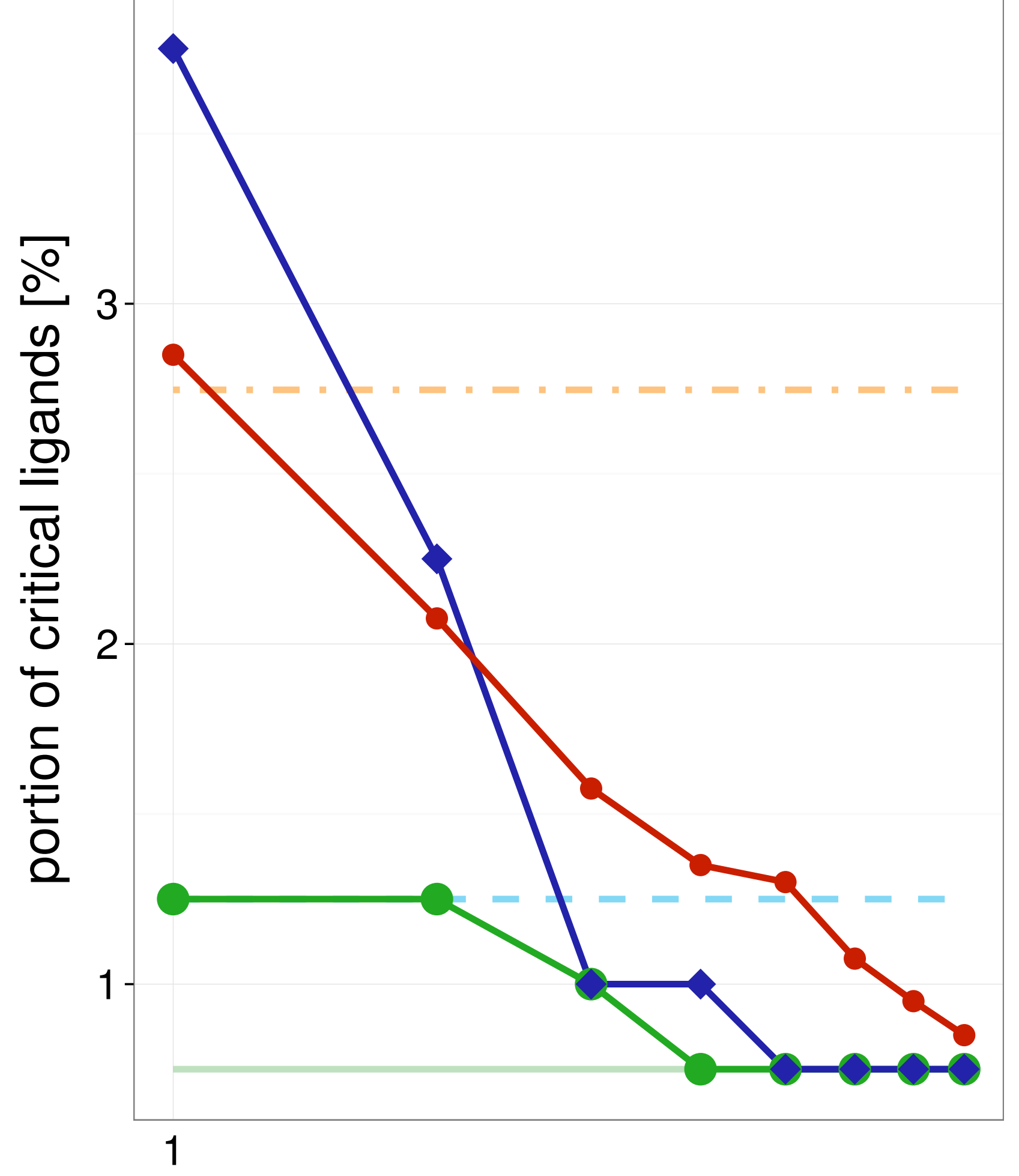

Average single structure

Best single structure

Whole ensemble

SIENA

$\sim$ Clustering

$\longrightarrow$ Random 


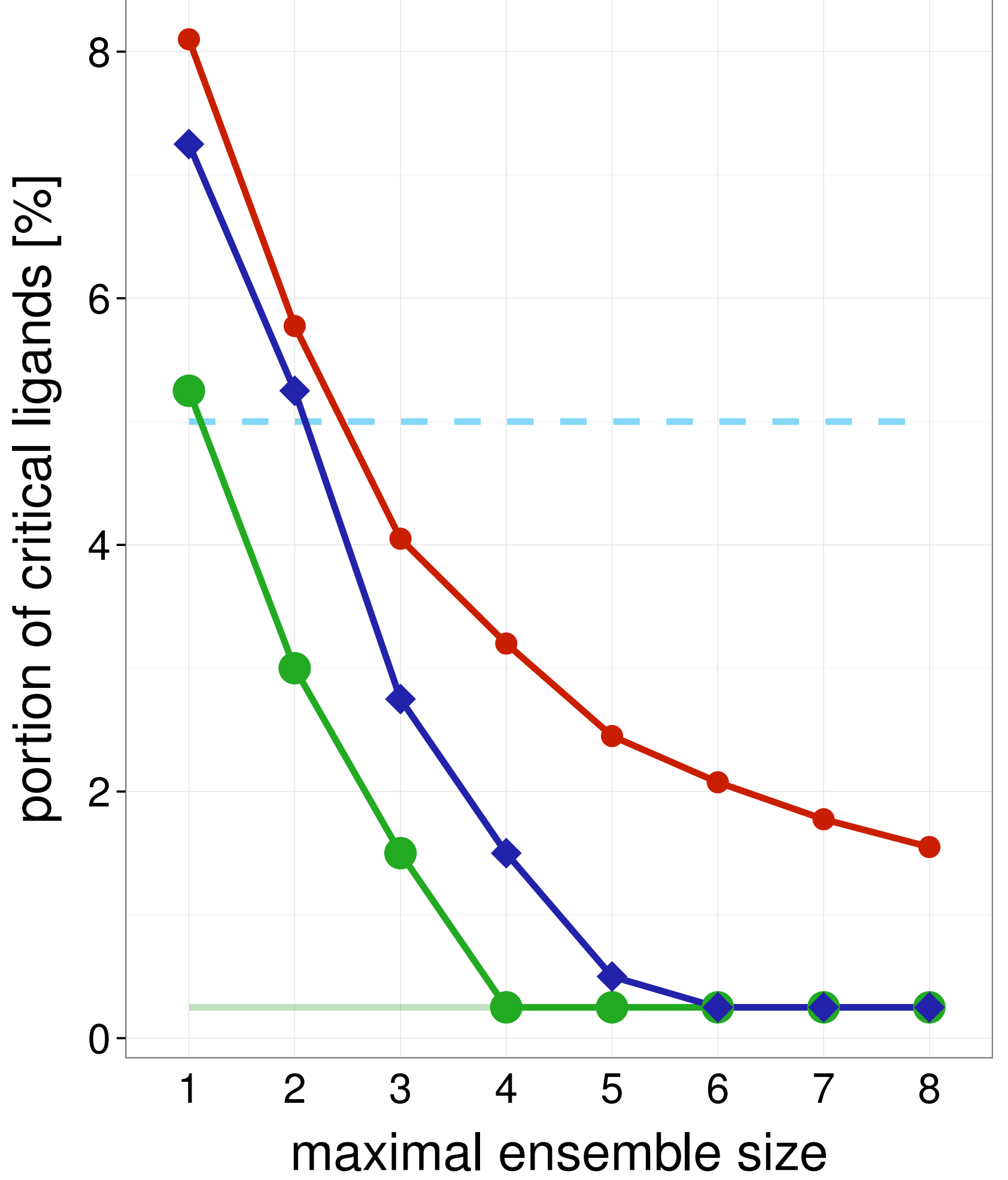

Test set

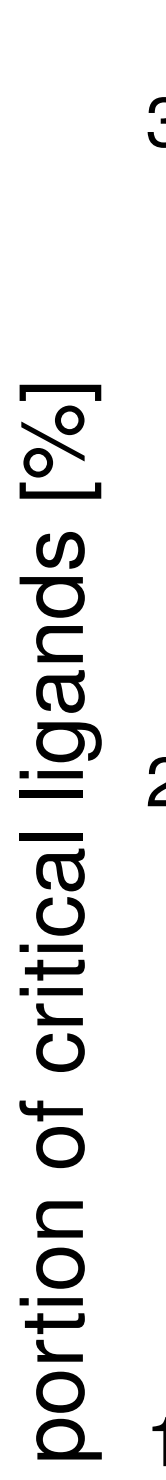

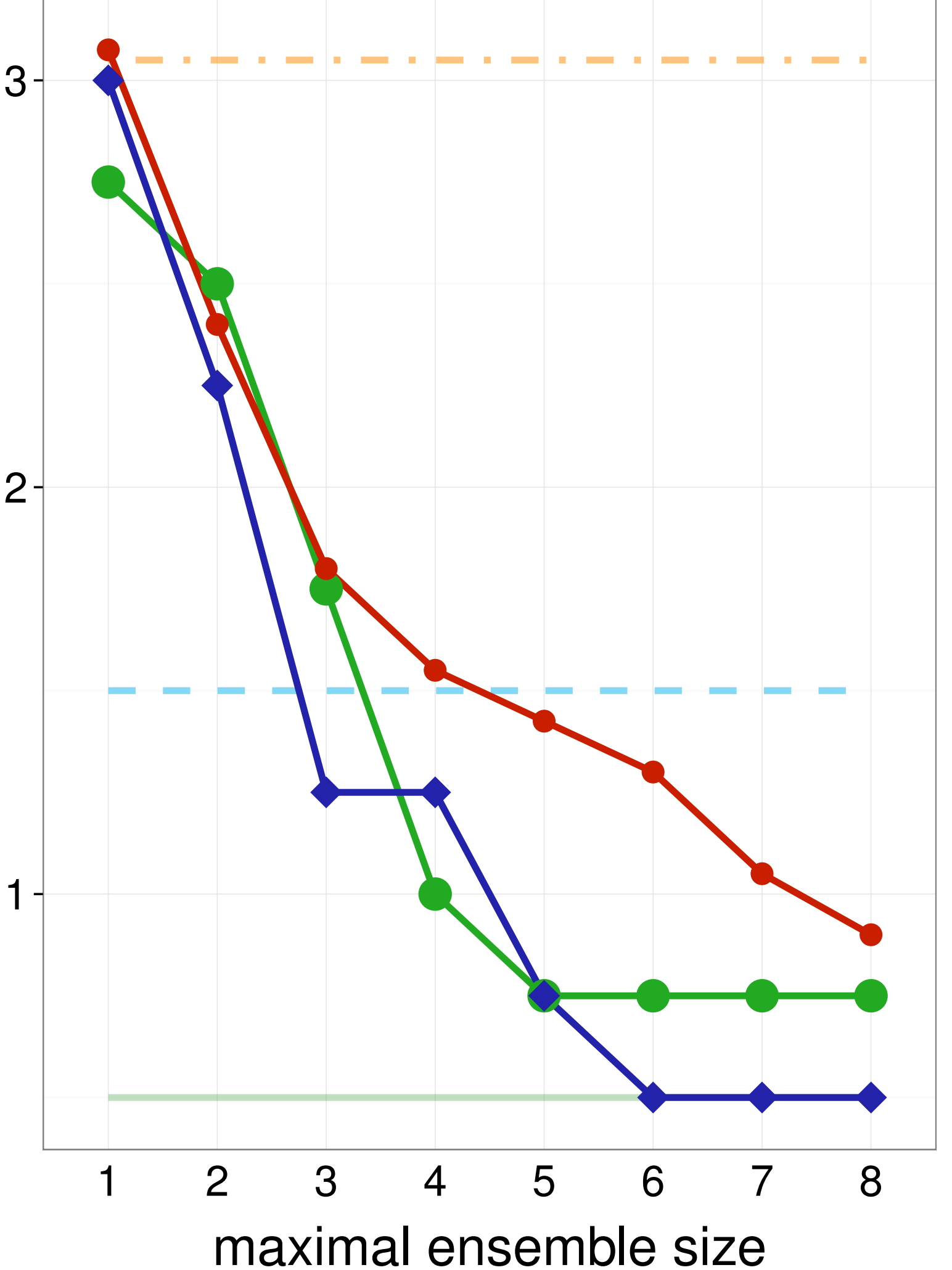

Average single structure

_ Best single structure

Whole ensemble

- SIENA

$\neg$ Clustering

$\multimap$ Random

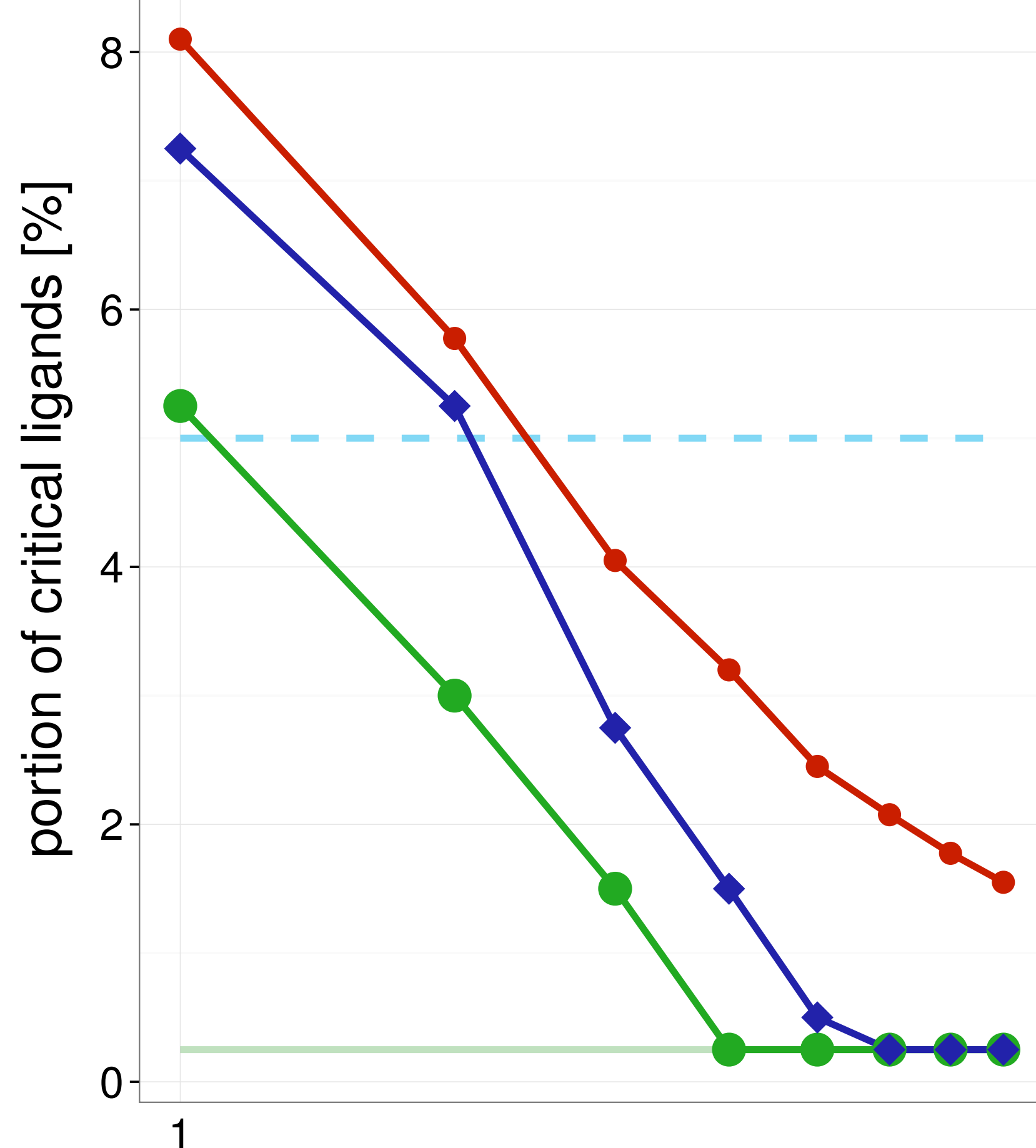

maximal ensemble size

Test set

Average single structure

_ _ Best single structure

Whole ensemble

- SIENA

$\leadsto$ Clustering

$\multimap$ Random

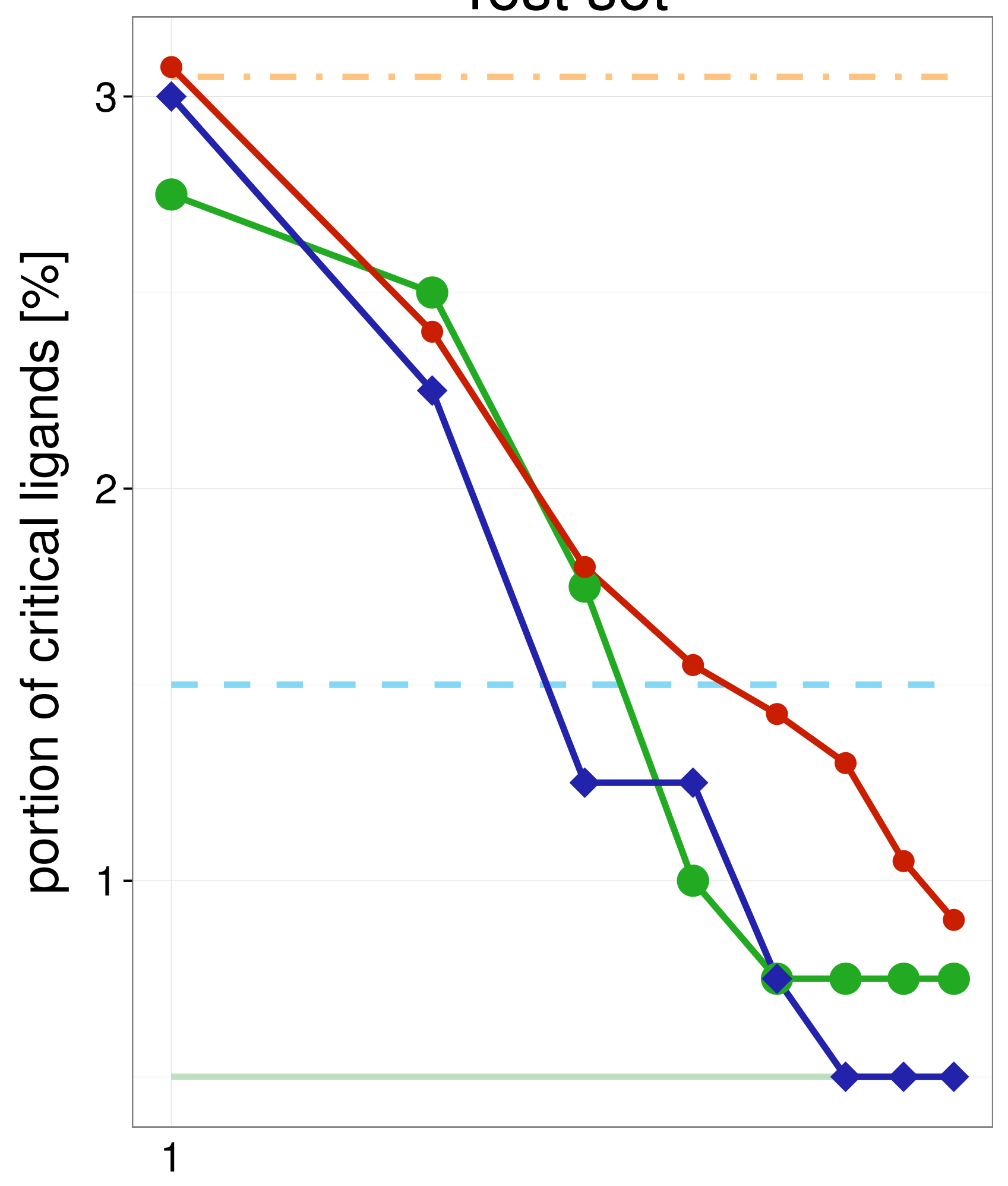

maximal ensemble size
Average single structure

_ Best single structure

Whole ensemble

SIENA

$\neg$ Clustering

$\multimap$ Random 
Training set

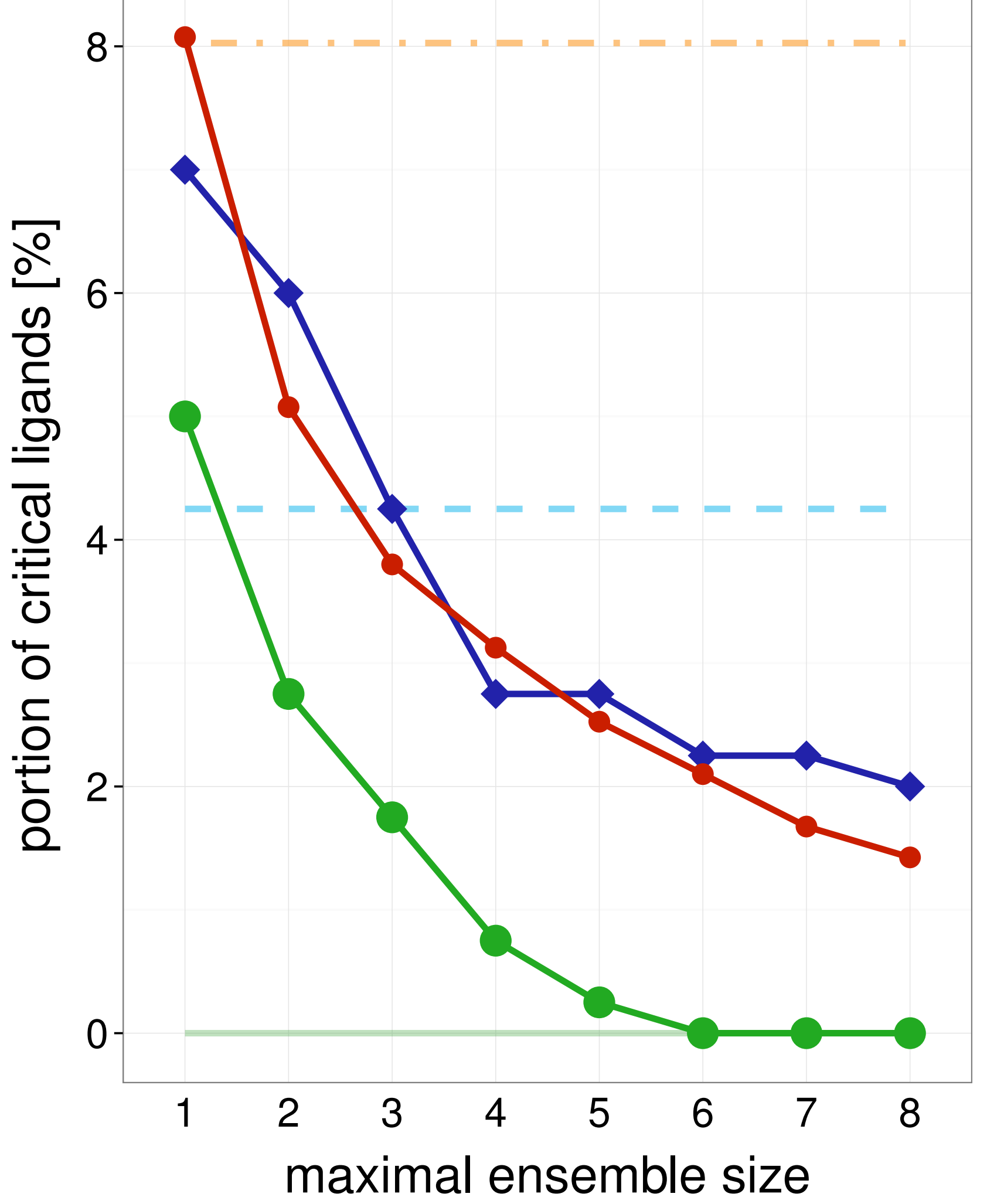

Test set

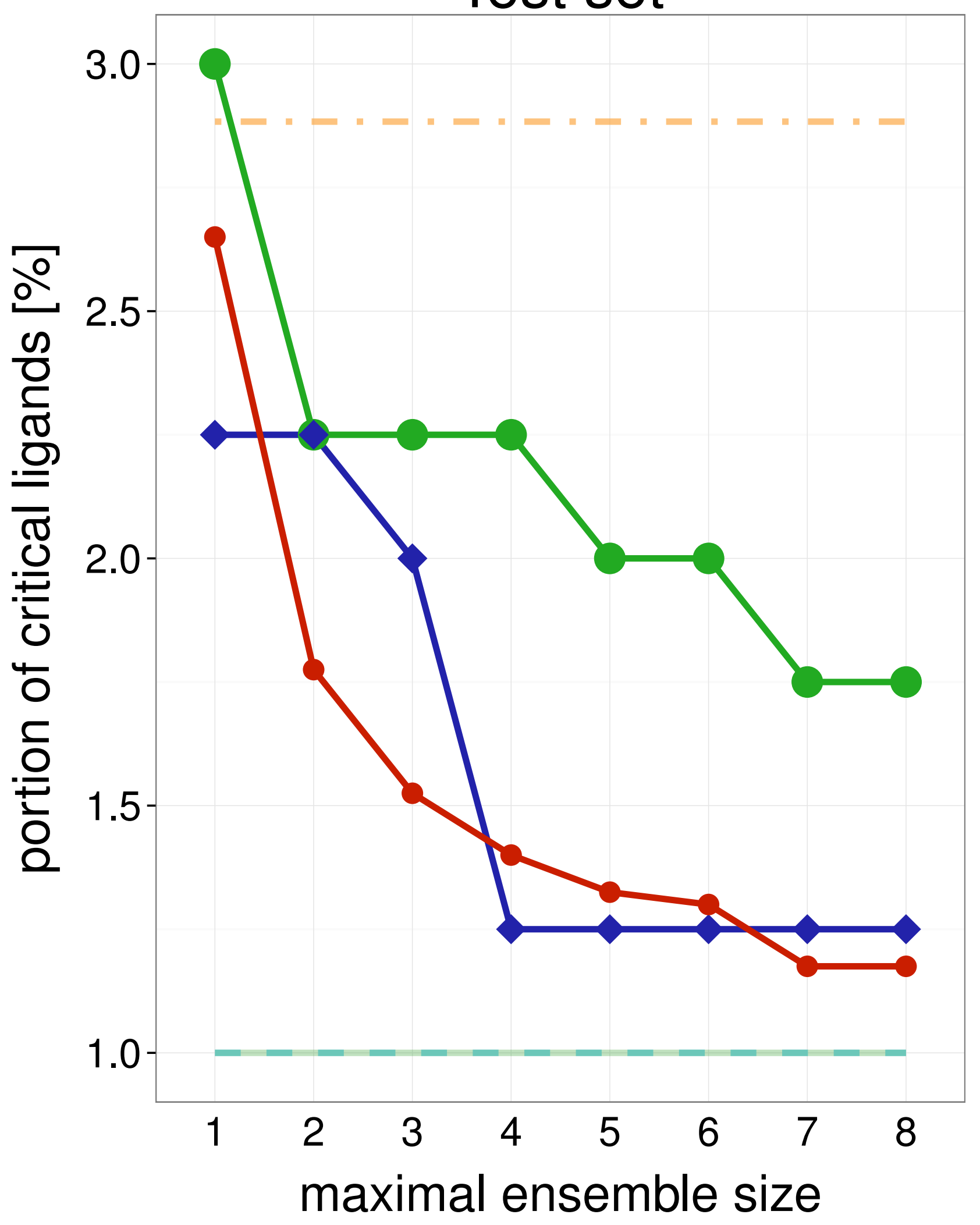

Average single structure

- Best single structure

Whole ensemble

- SIENA

$\neg$ Clustering

$\multimap$ Random

Average single structure

_ _ Best single structure

Whole ensemble

- SIENA

$\neg$ Clustering

$\multimap$ Random
Training set

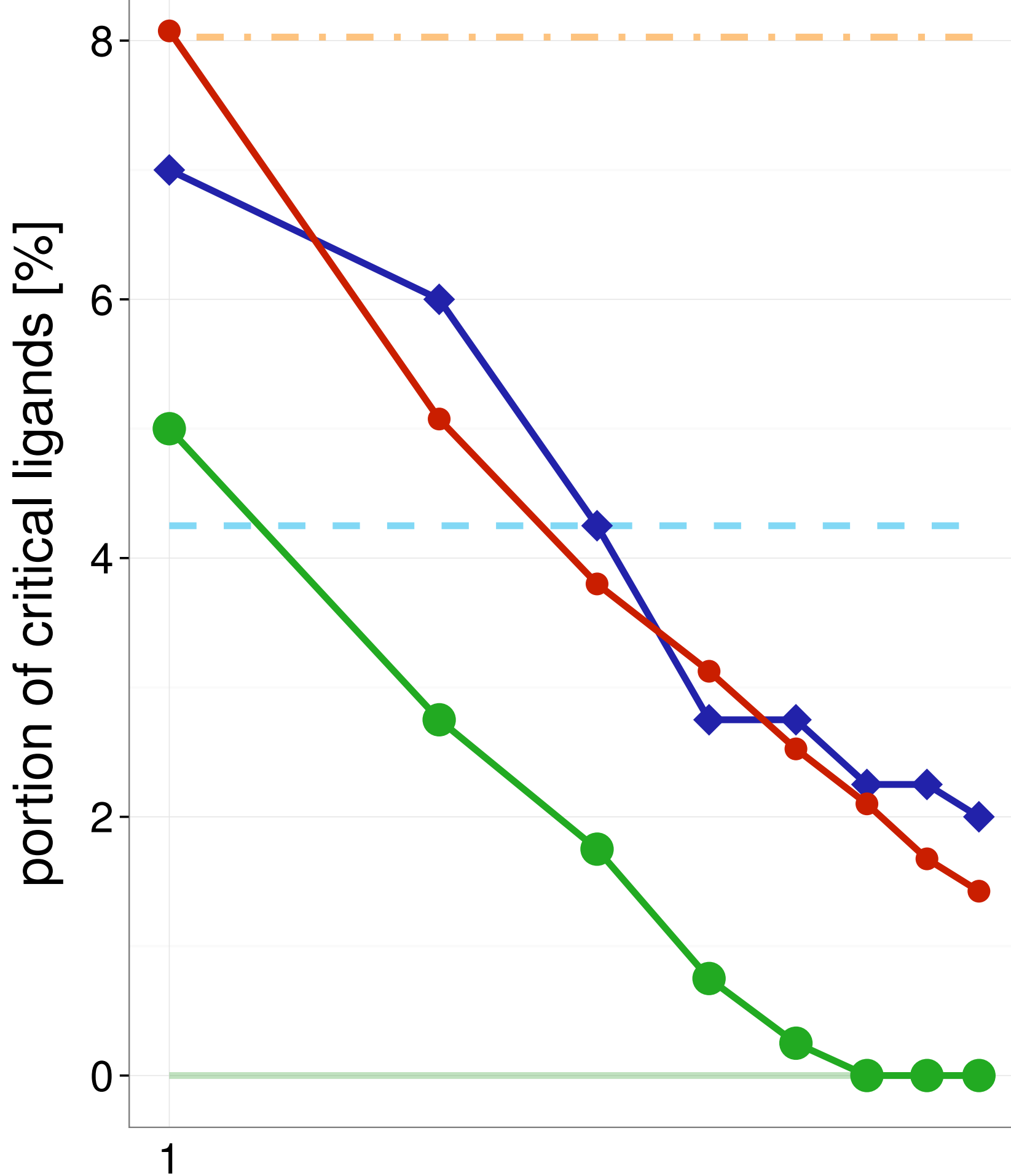

maximal ensemble size

Test set

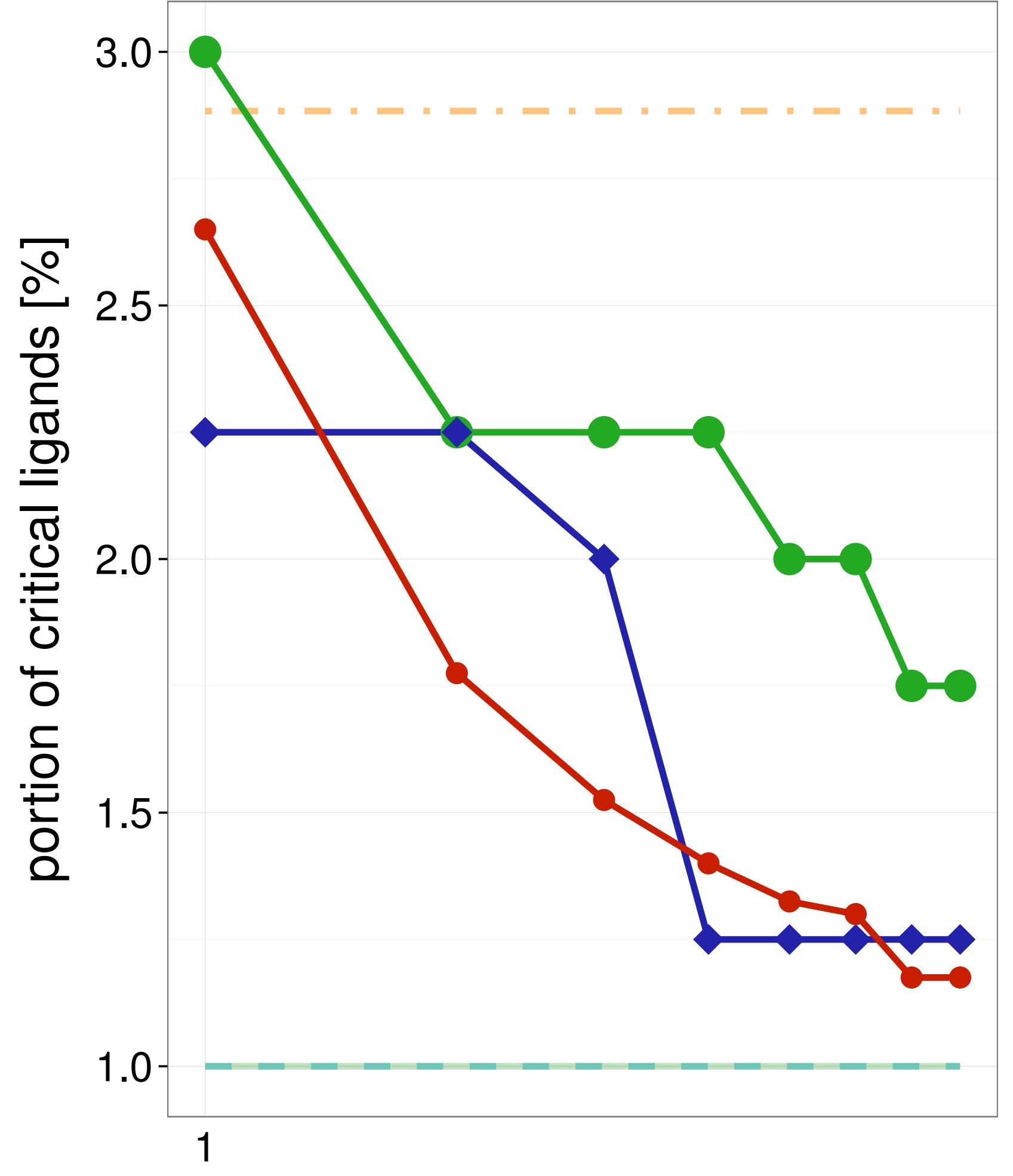

Average single structure

_ Best single structure

Whole ensemble

- SIENA

$\neg$ Clustering

$\longrightarrow$ Random 
Training set

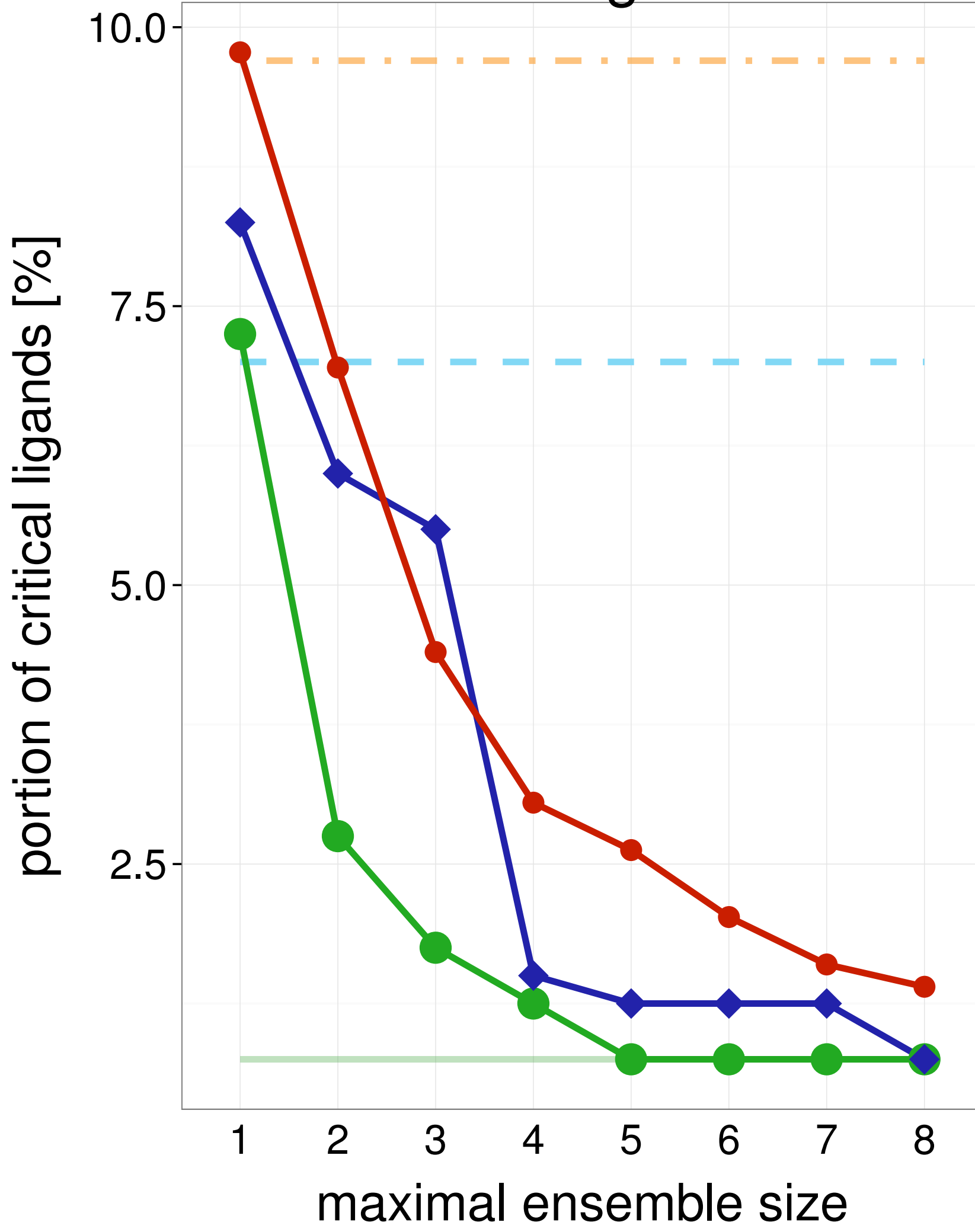

\section{Test set}

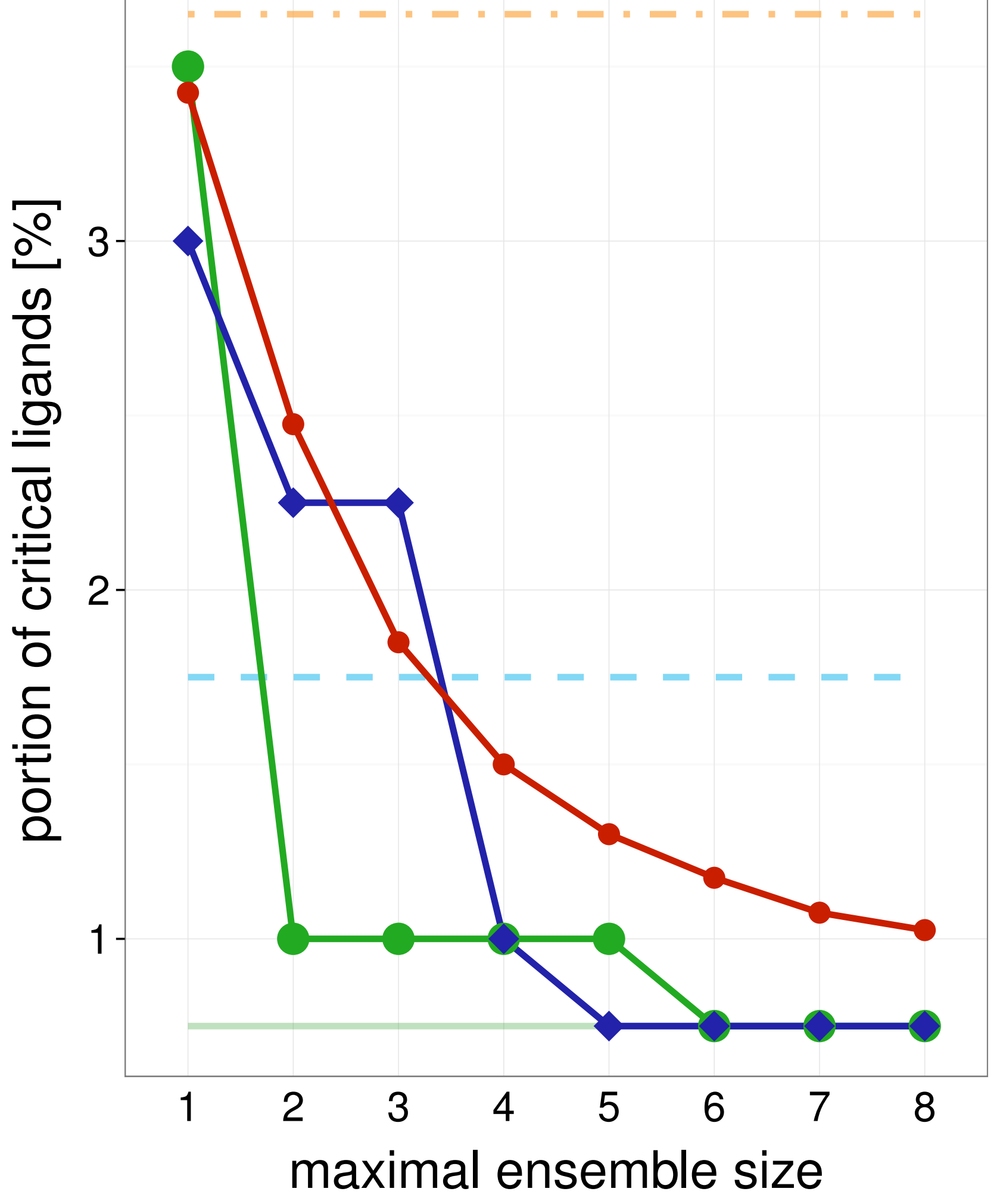

Average single structure

_ Best single structure

Whole ensemble

- SIENA

$\neg$ Clustering

$\rightarrow$ Random

Average single structure

_ _ Best single structure

Whole ensemble

- SIENA

$\neg$ Clustering

$\multimap$ Random
2WSA

Training set

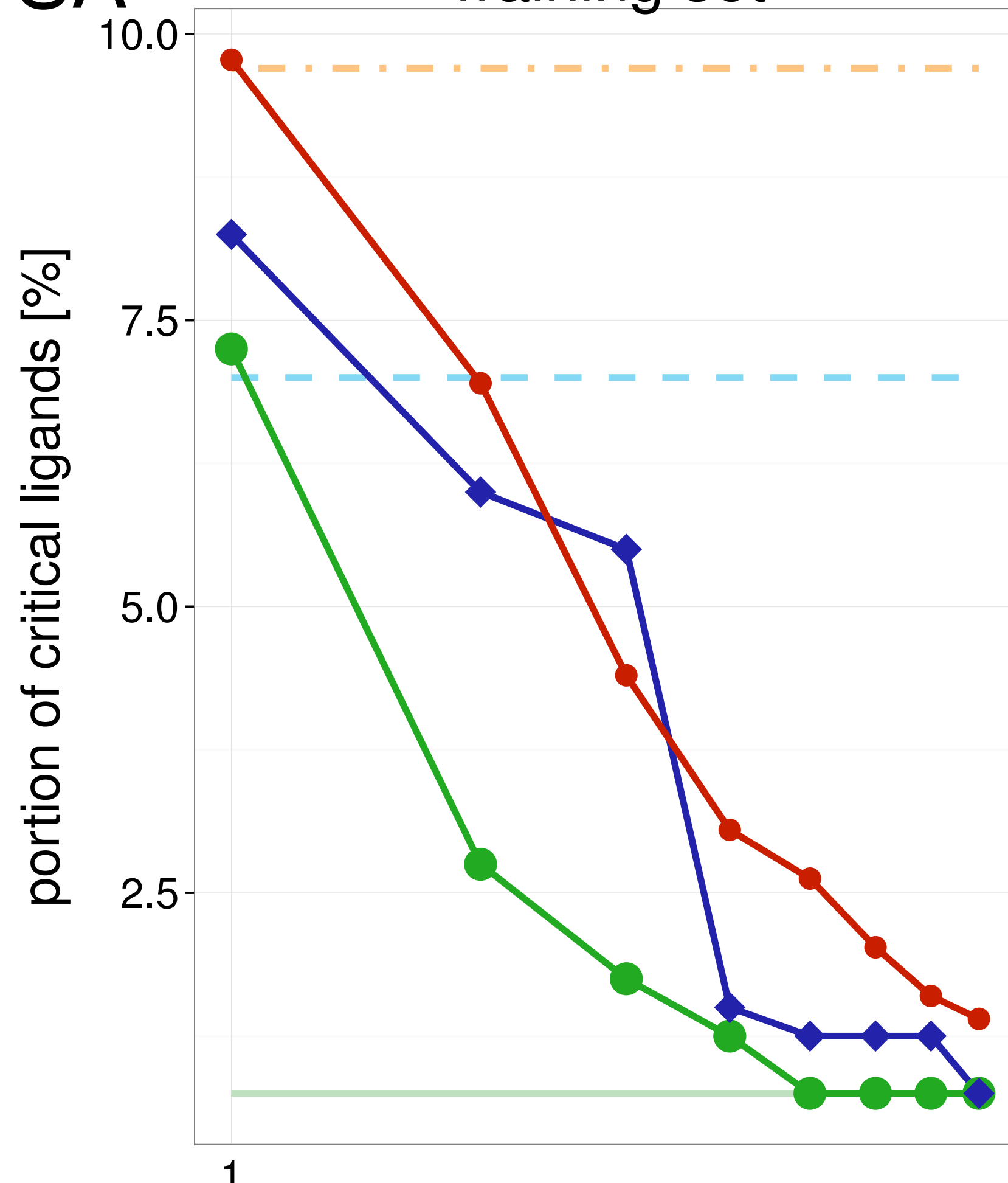

maximal ensemble size

Test set

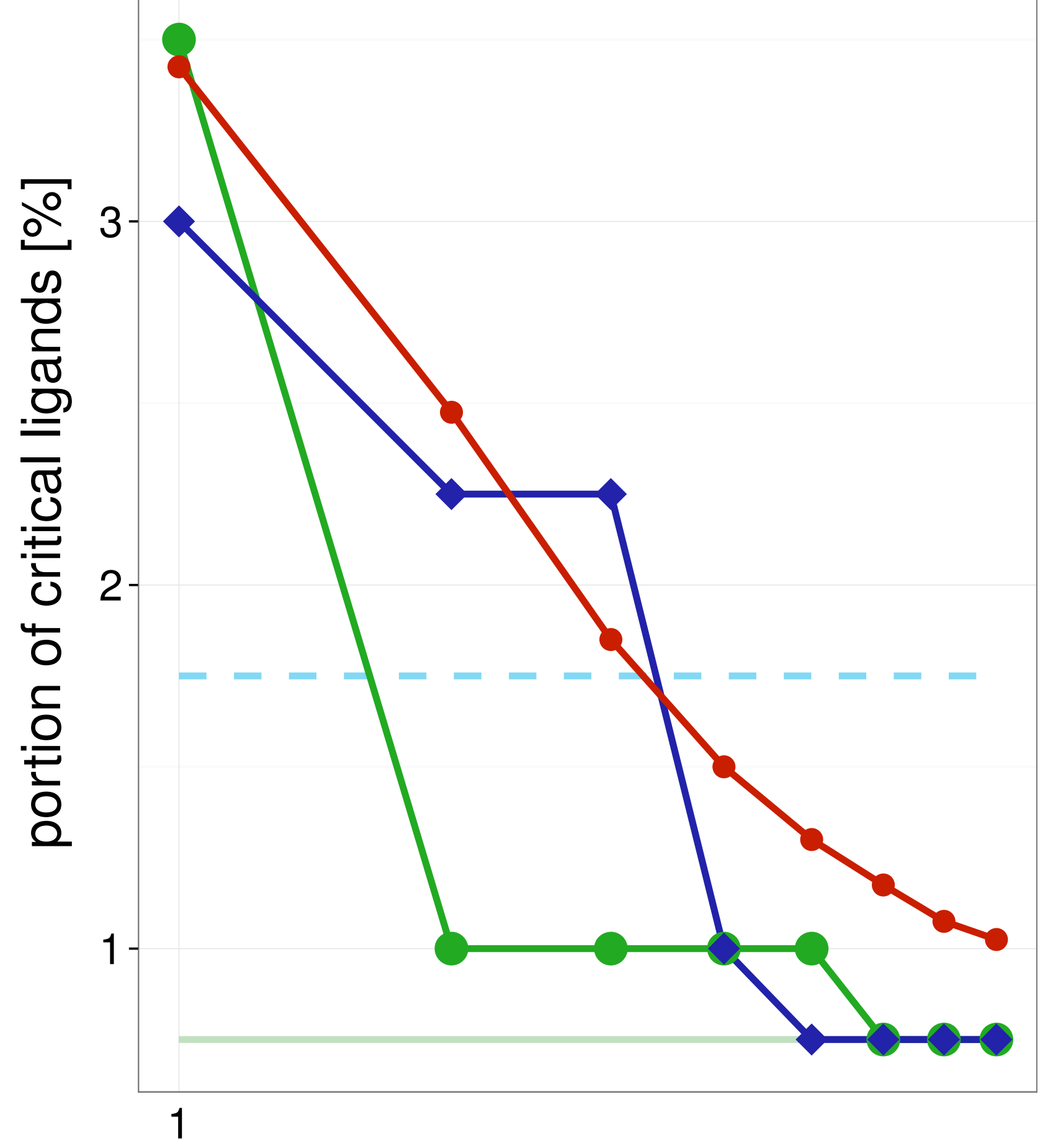

Average single structure

_ Best single structure

Whole ensemble

SIENA

$\leadsto$ Clustering

$\multimap$ Random 
Training set

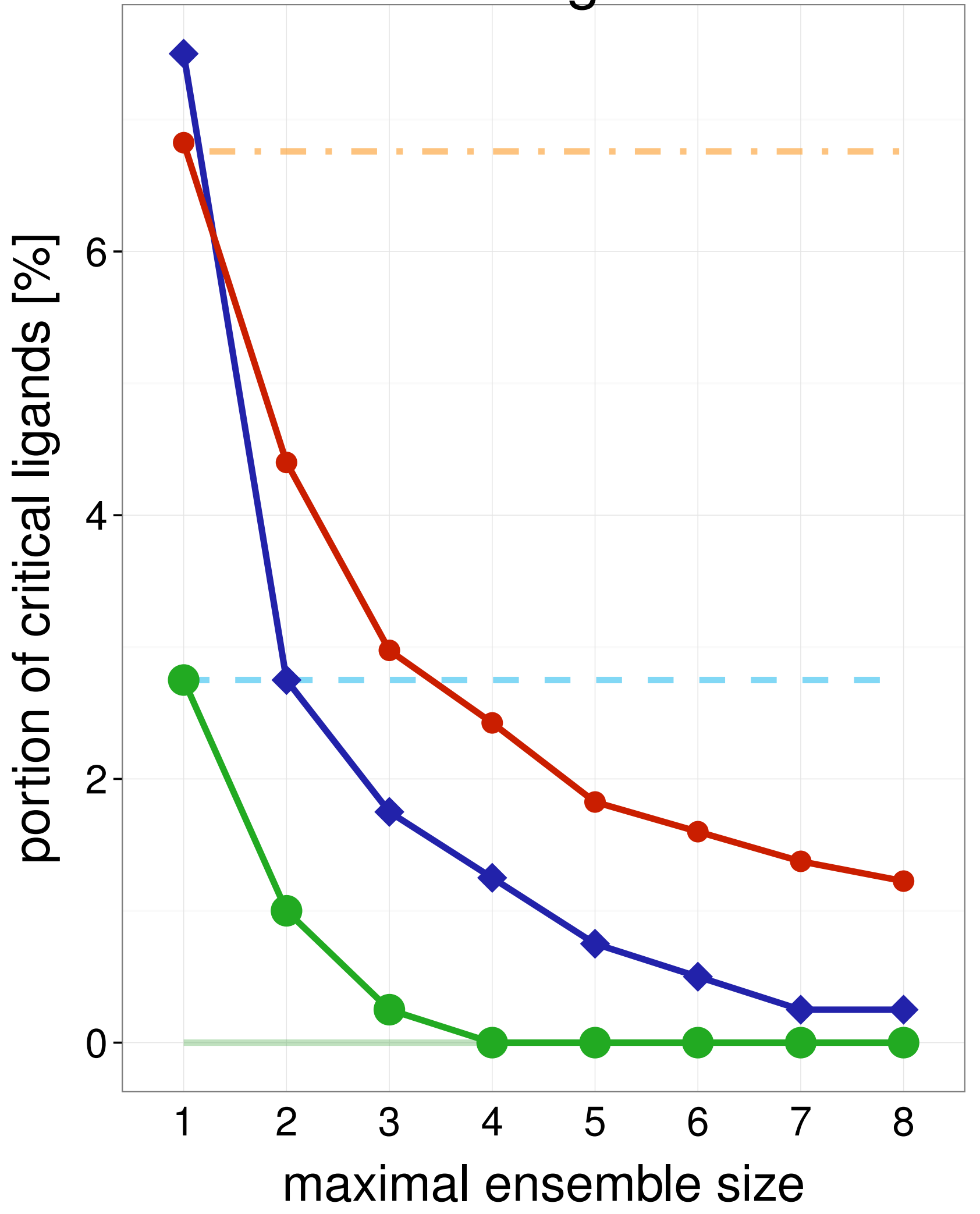

Test set

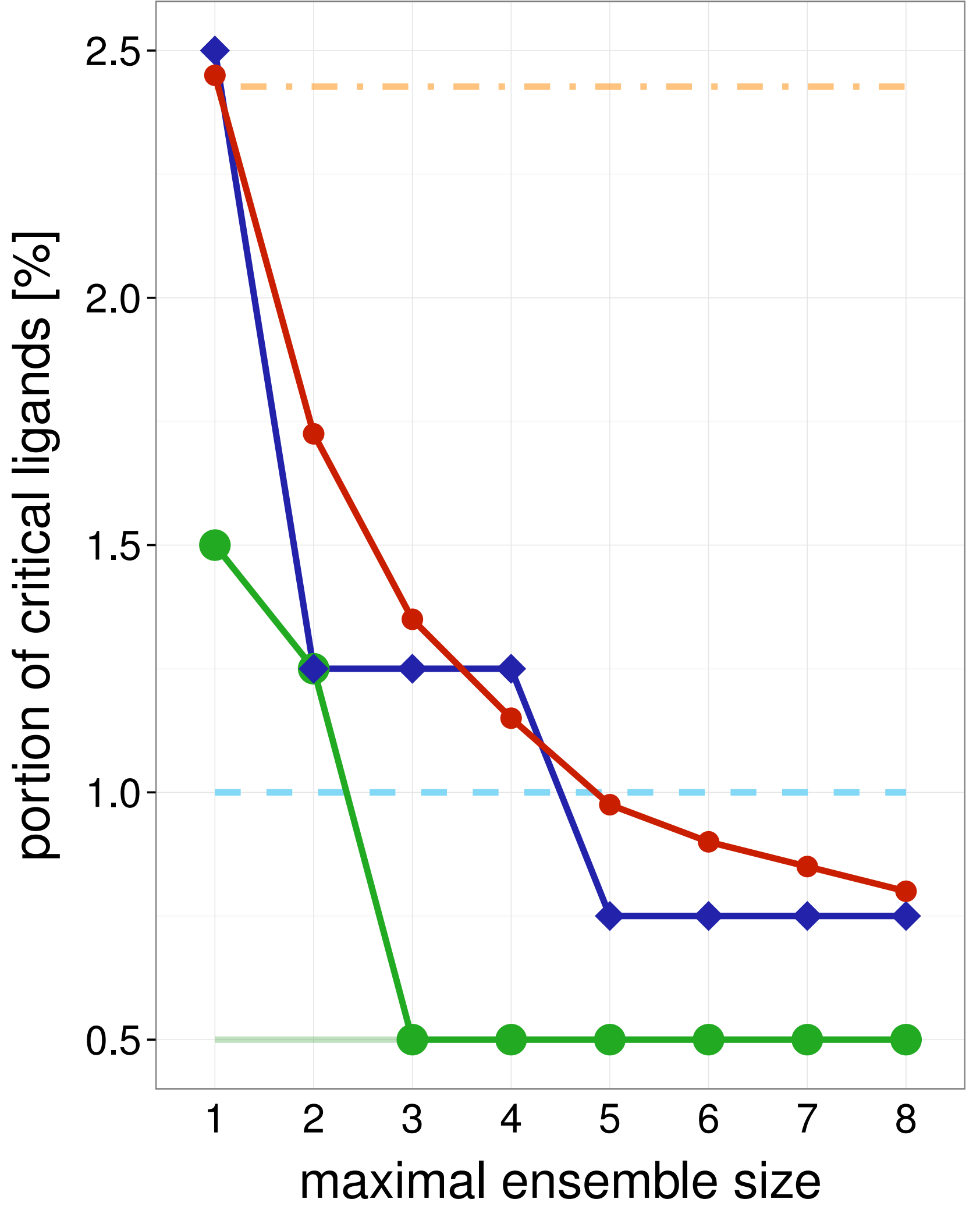

Average single structure

_ Best single structure

Whole ensemble

$\multimap$ SIENA

$\neg$ Clustering

$\multimap$ Random

Average single structure

_ Best single structure

Whole ensemble

- SIENA

$\neg$ Clustering

$\multimap$ Random

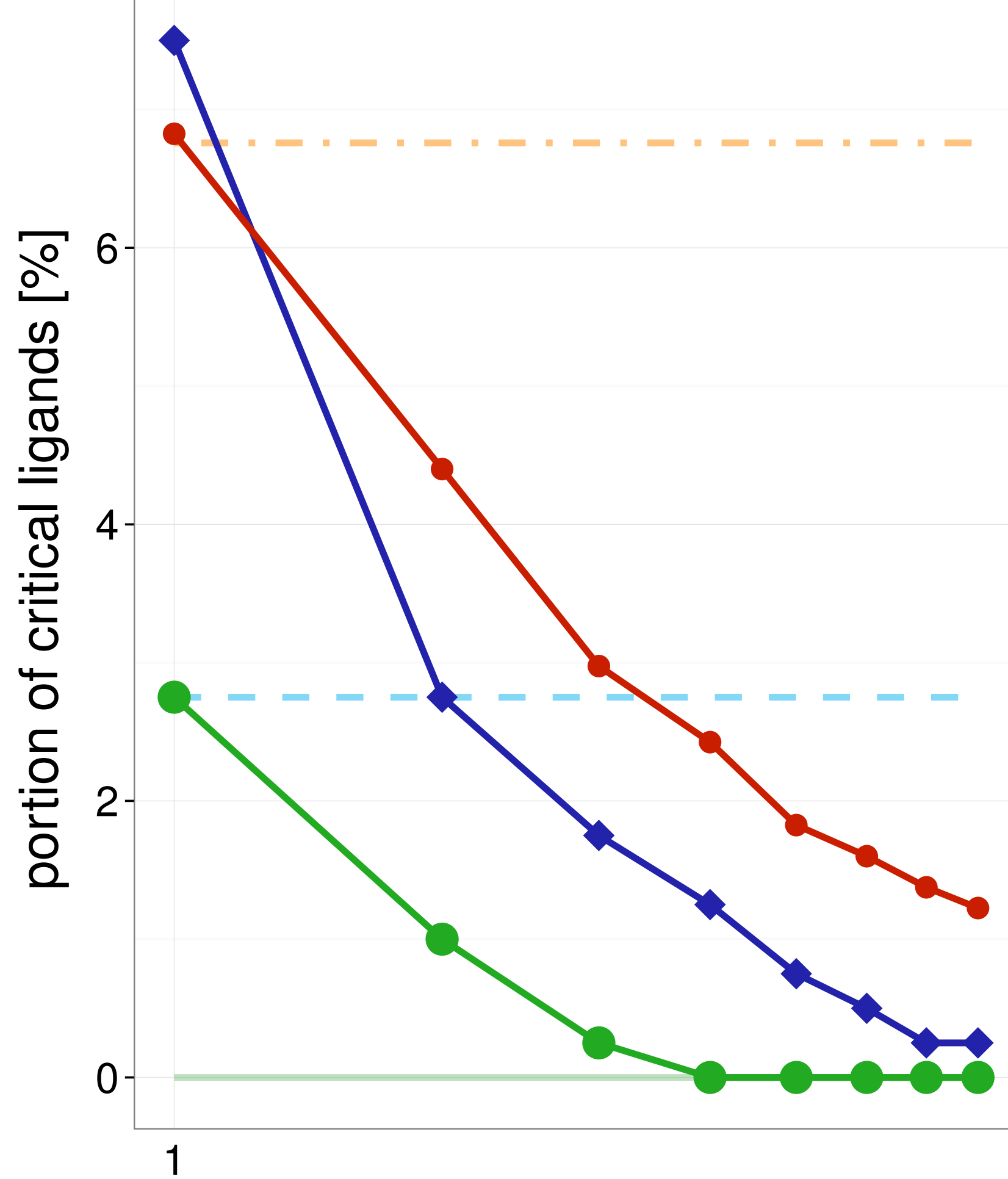

maximal ensemble size

Test set

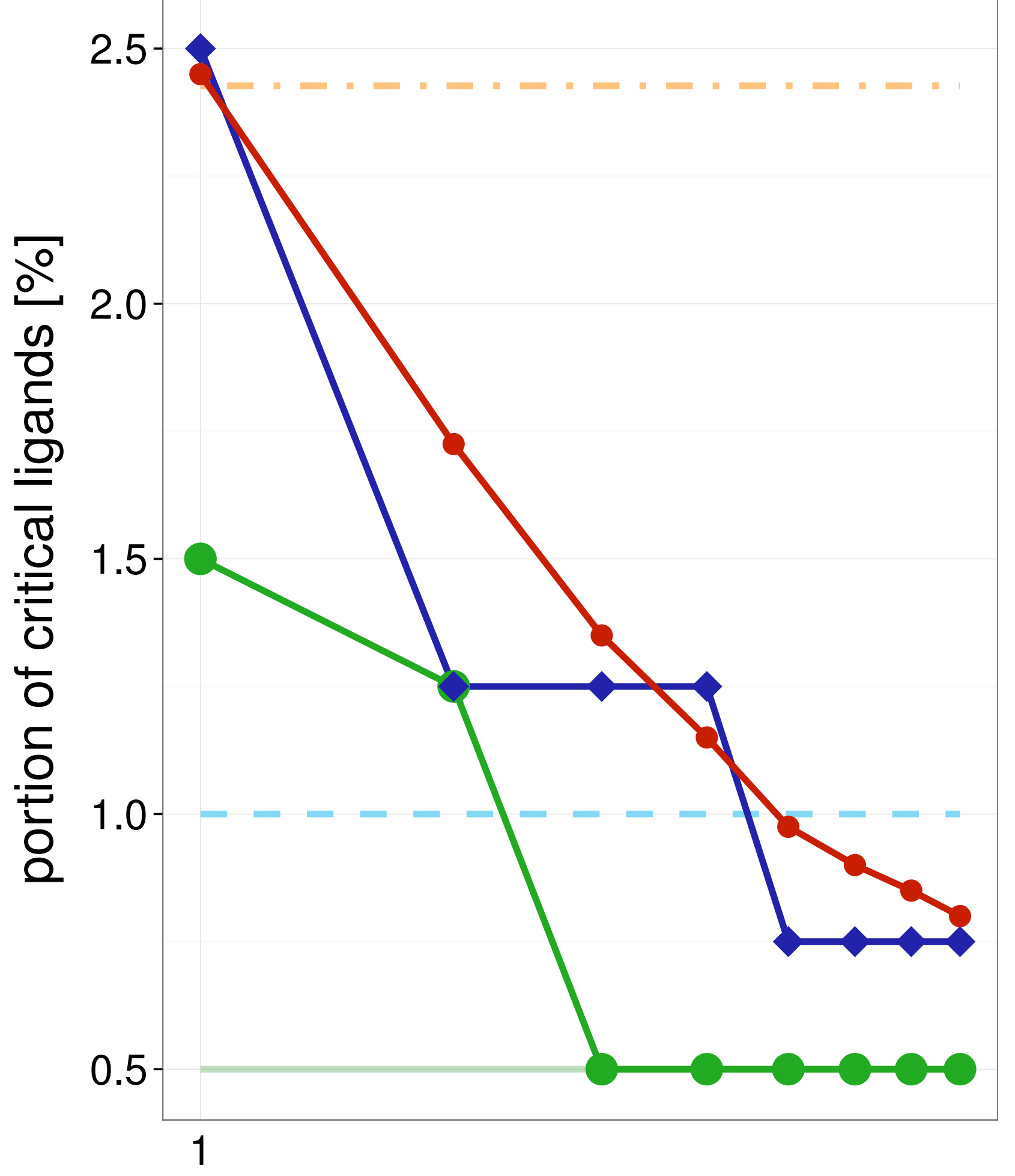

maximal ensemble size
Average single structure

_ Best single structure

Whole ensemble

SIENA

$\neg$ Clustering

$\rightarrow$ Random 


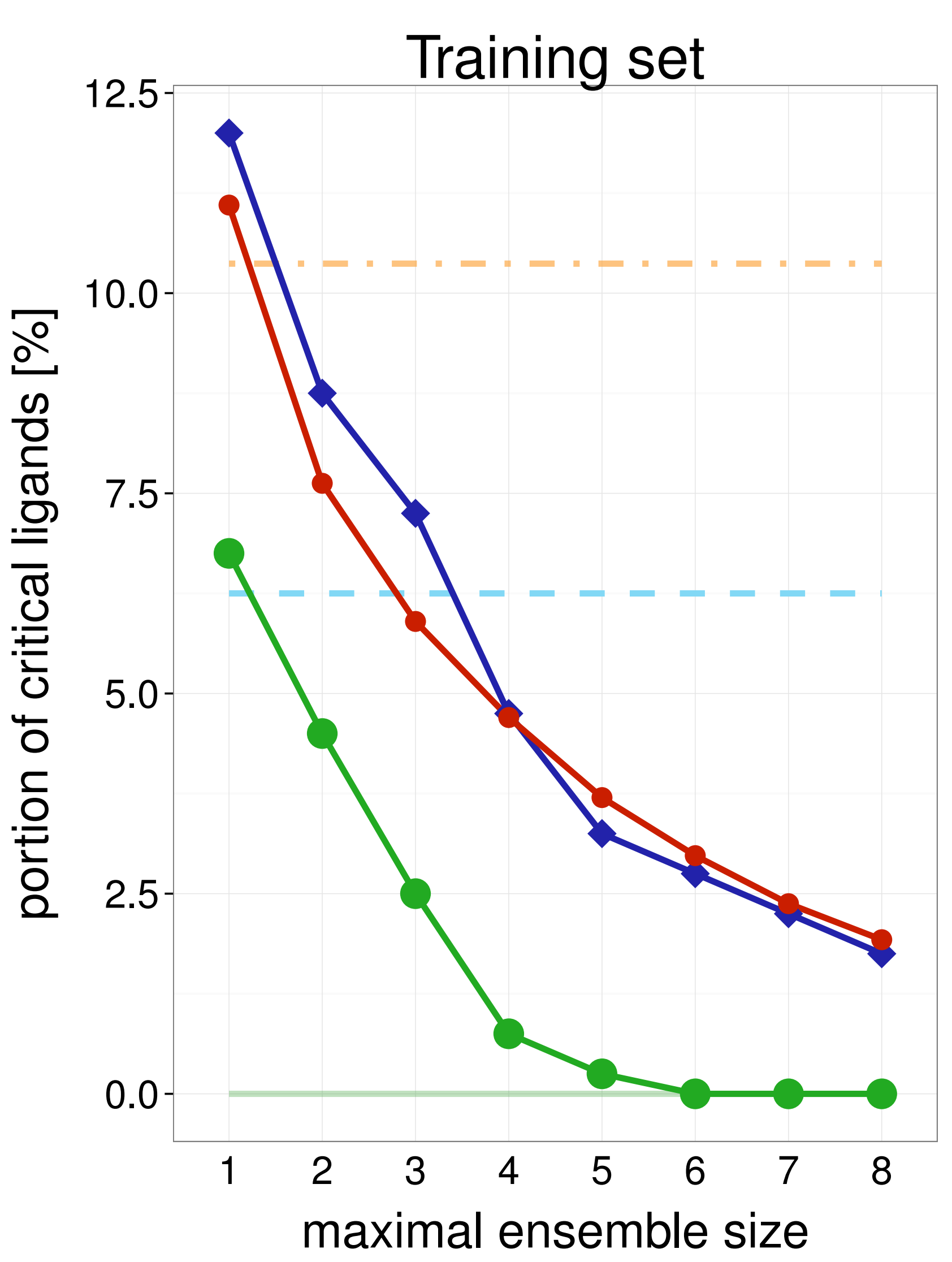

Test set

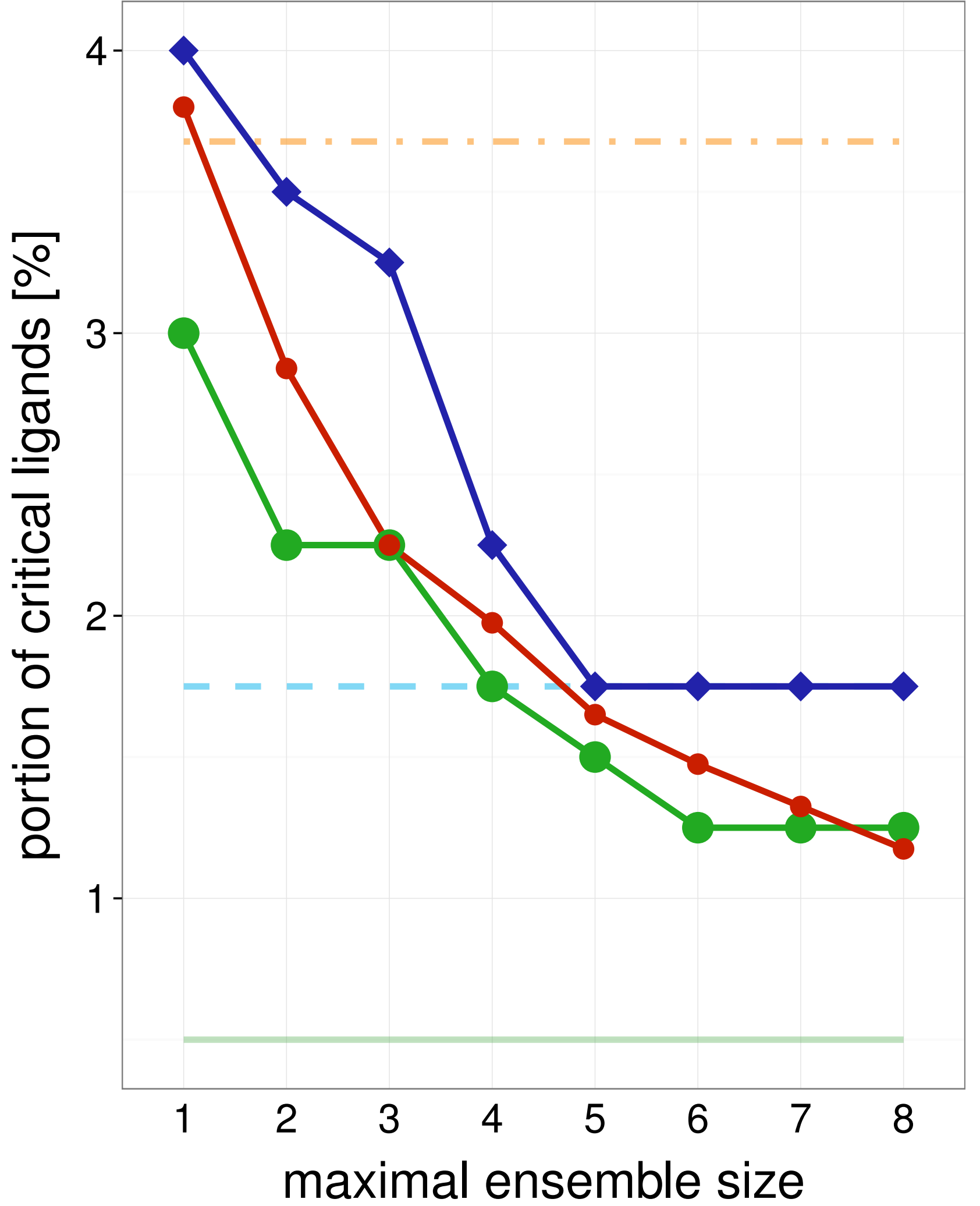

3EN6

Training set

Average single structure

_ Best single structure

Whole ensemble

- SIENA

$\neg$ Clustering

- Random

Average single structure

_ _ Best single structure

Whole ensemble

- SIENA

$\neg$ Clustering

$\longrightarrow$ Random

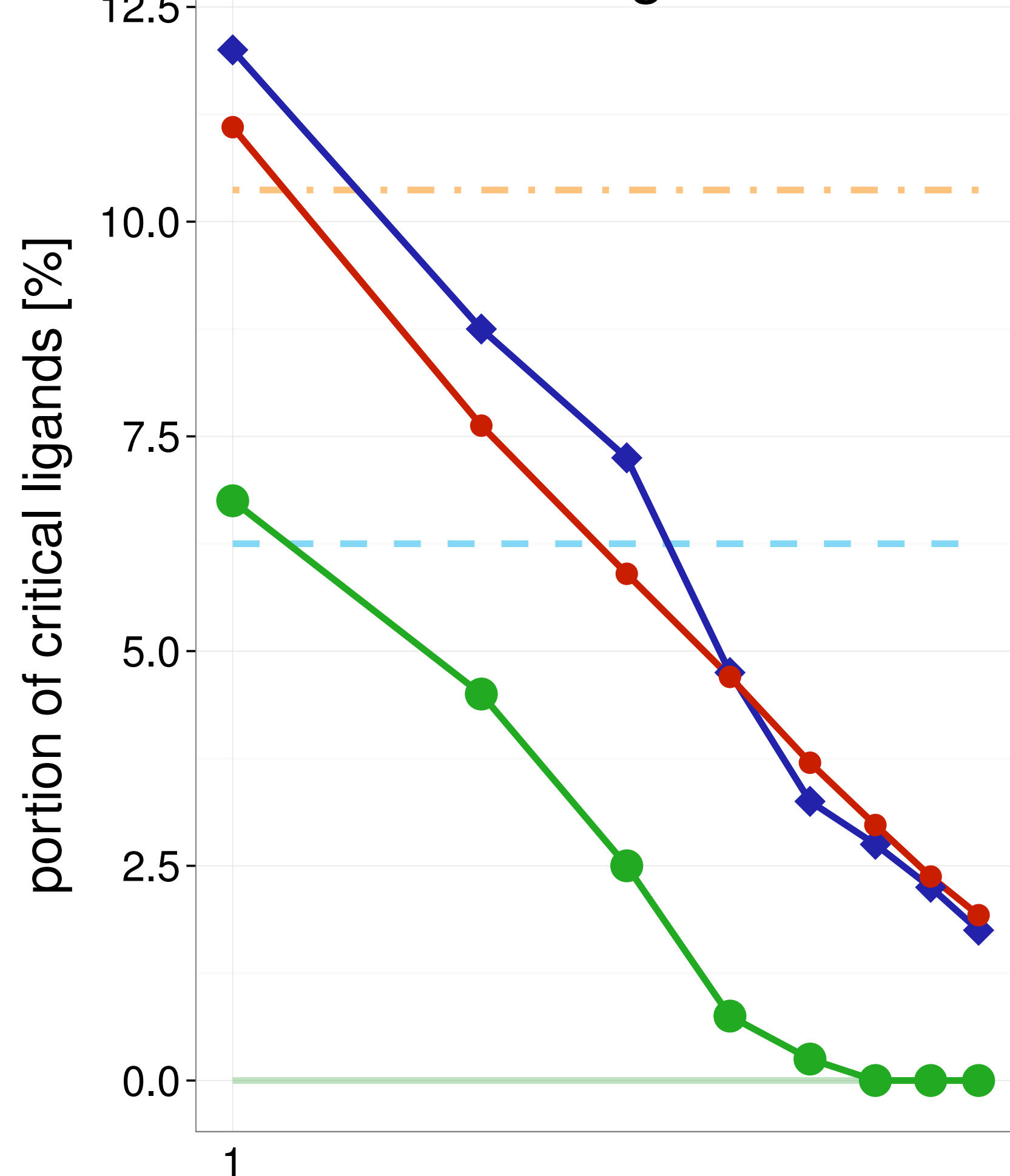

maximal ensemble size

Test set

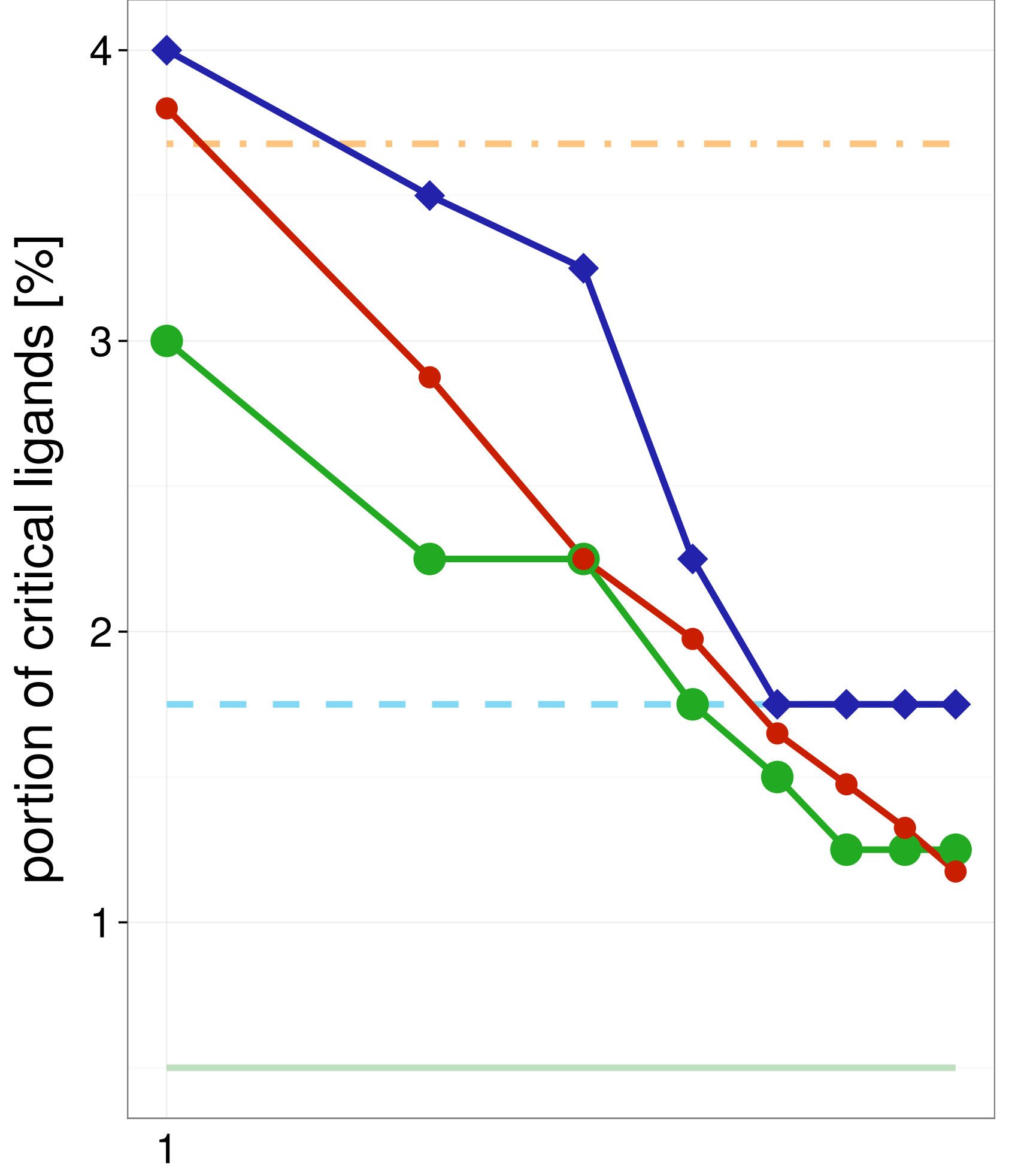

maximal ensemble size
Average single structure

_ Best single structure

Whole ensemble

SIENA

$\sim$ Clustering

$\multimap$ Random 


\section{Training set}

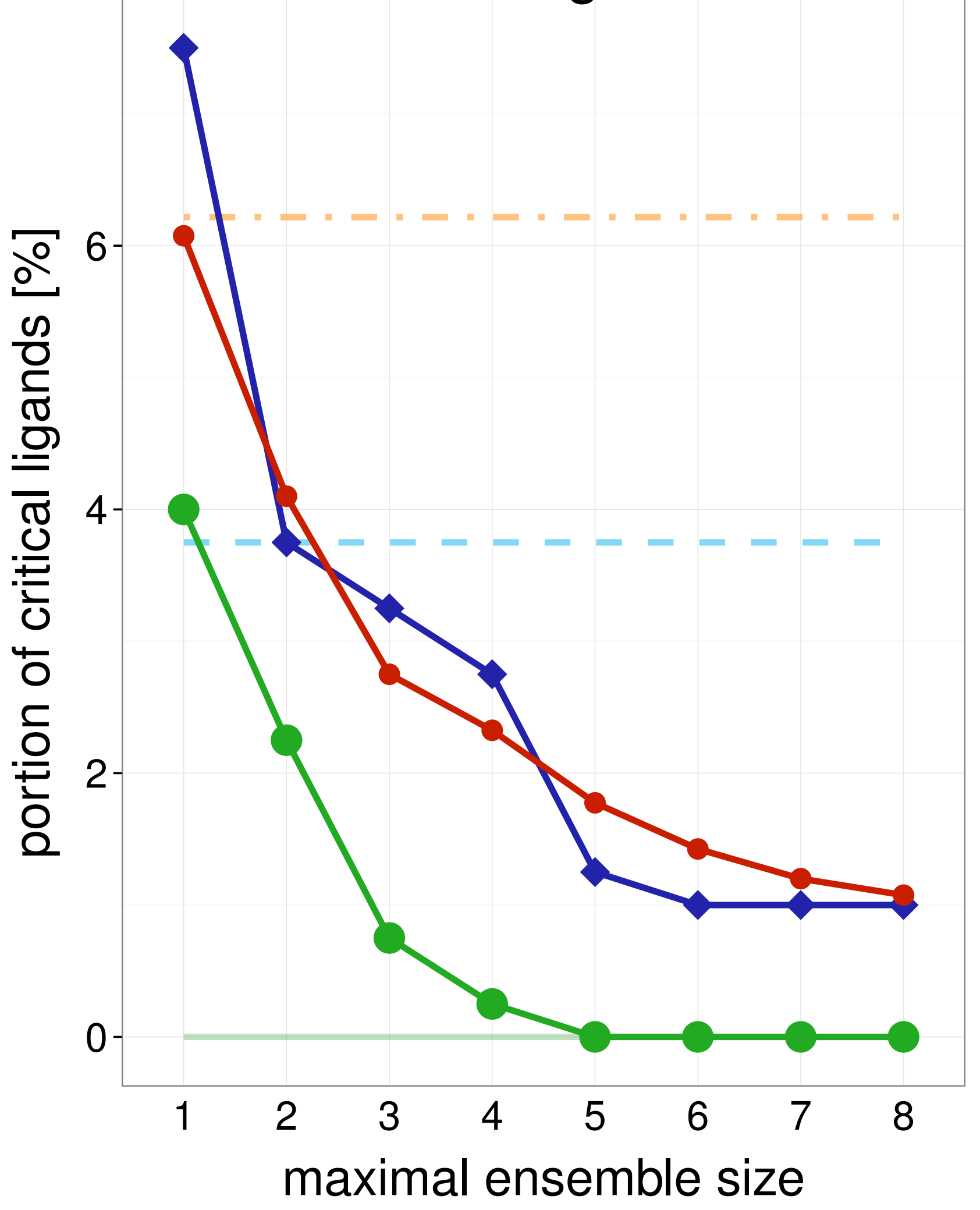

Test set

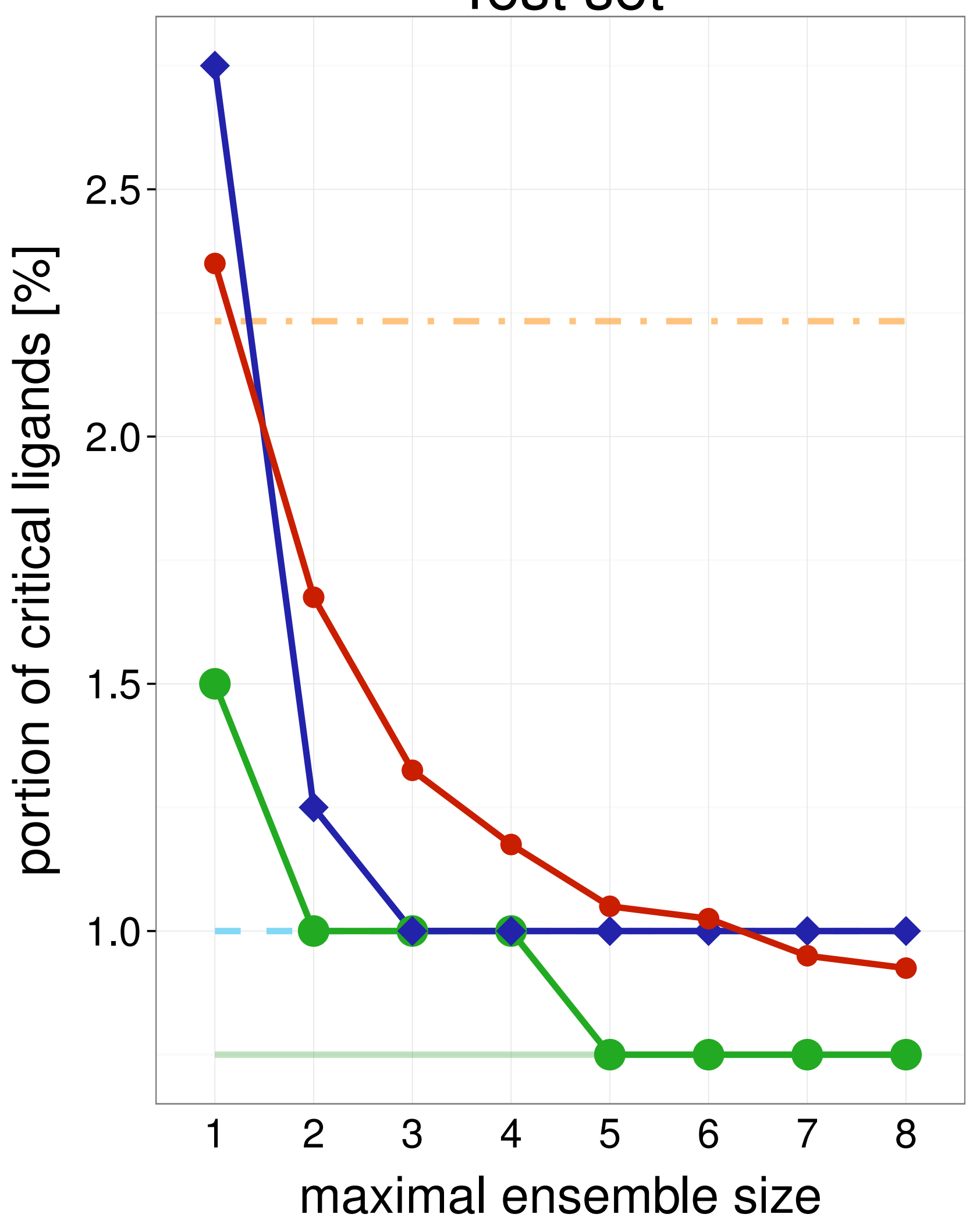

Average single structure

_ Best single structure

Whole ensemble

- SIENA

$\neg$ Clustering

$\multimap$ Random

Average single structure

\section{_ Best single} structure

Whole ensemble

- SIENA

$\smile$ Clustering

$\multimap$ Random

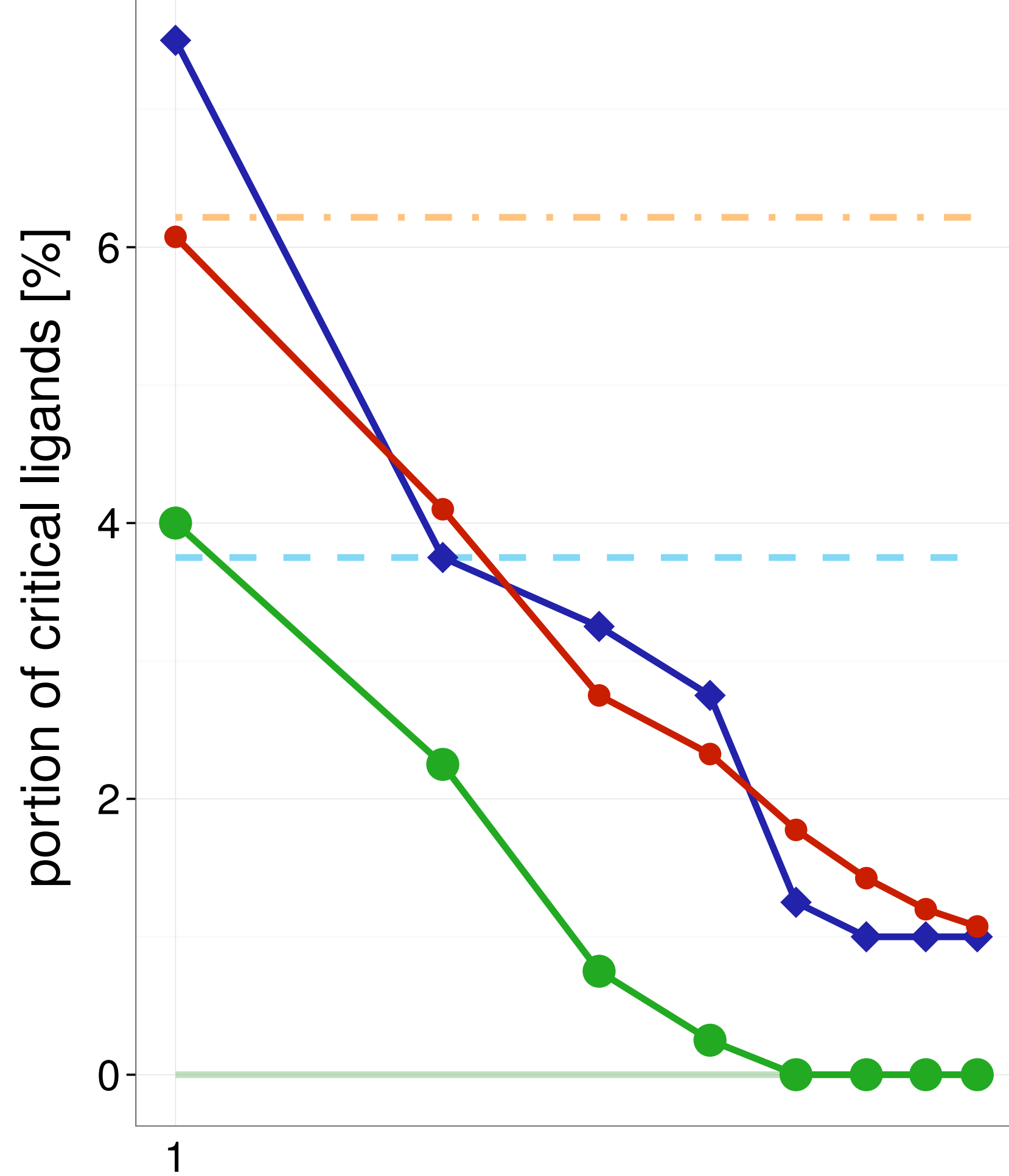

maximal ensemble size

Test set

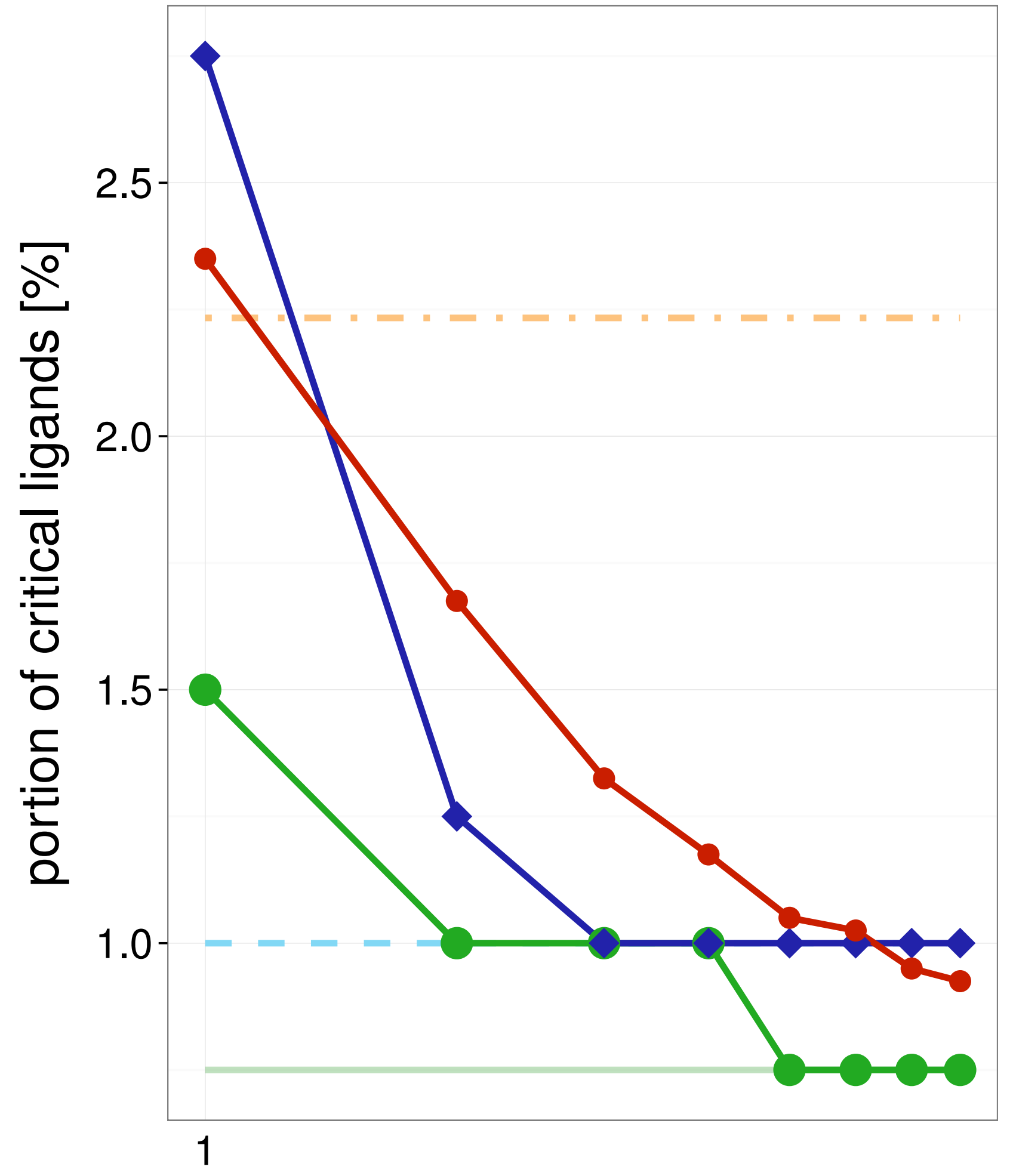

Average single structure

Best single structure

Whole ensemble

SIENA

$\sim$ Clustering

$\longrightarrow$ Random 


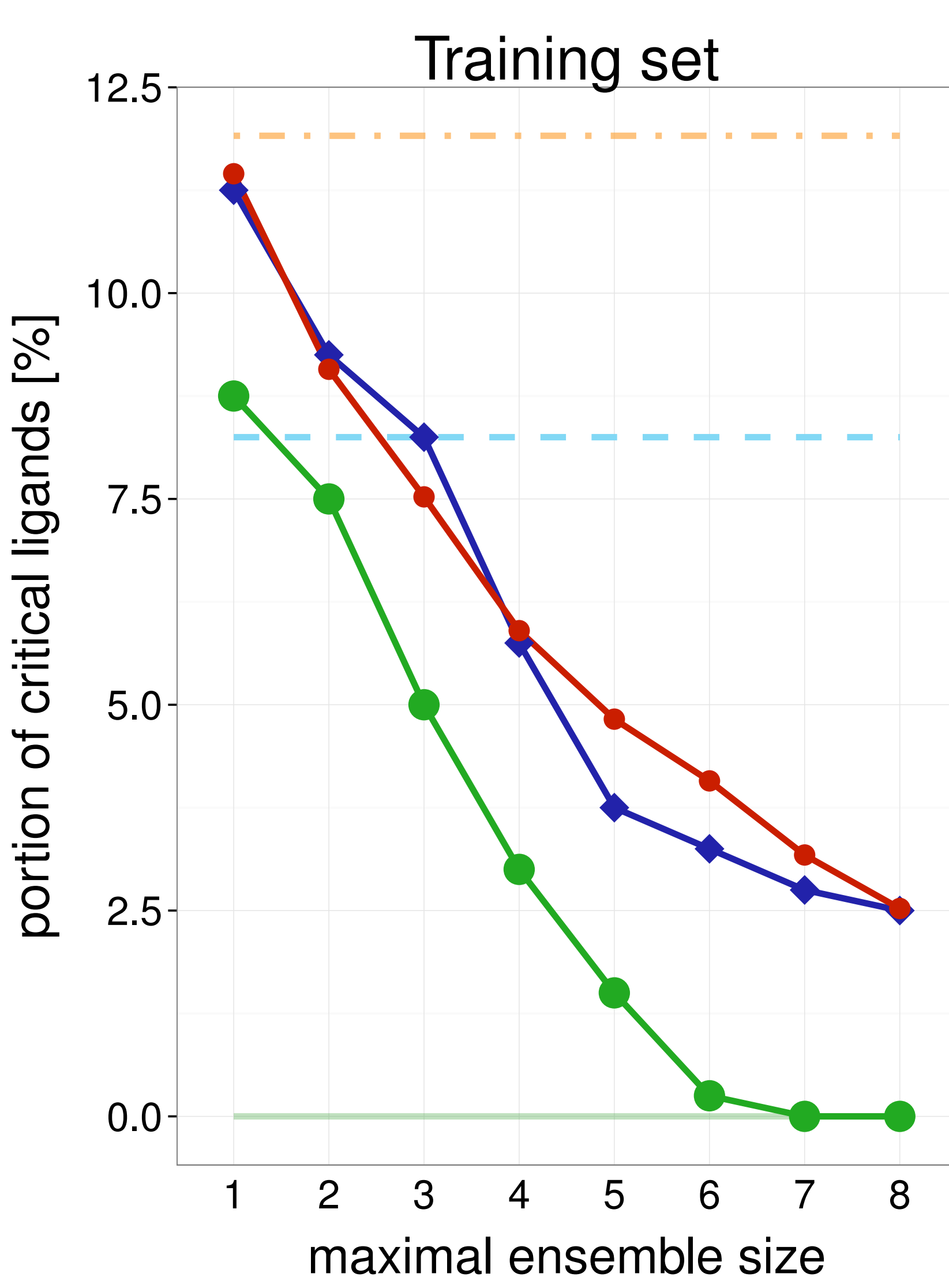

\section{Test set}

4

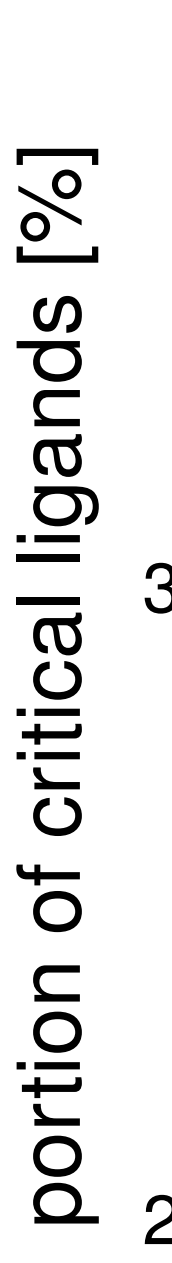

3

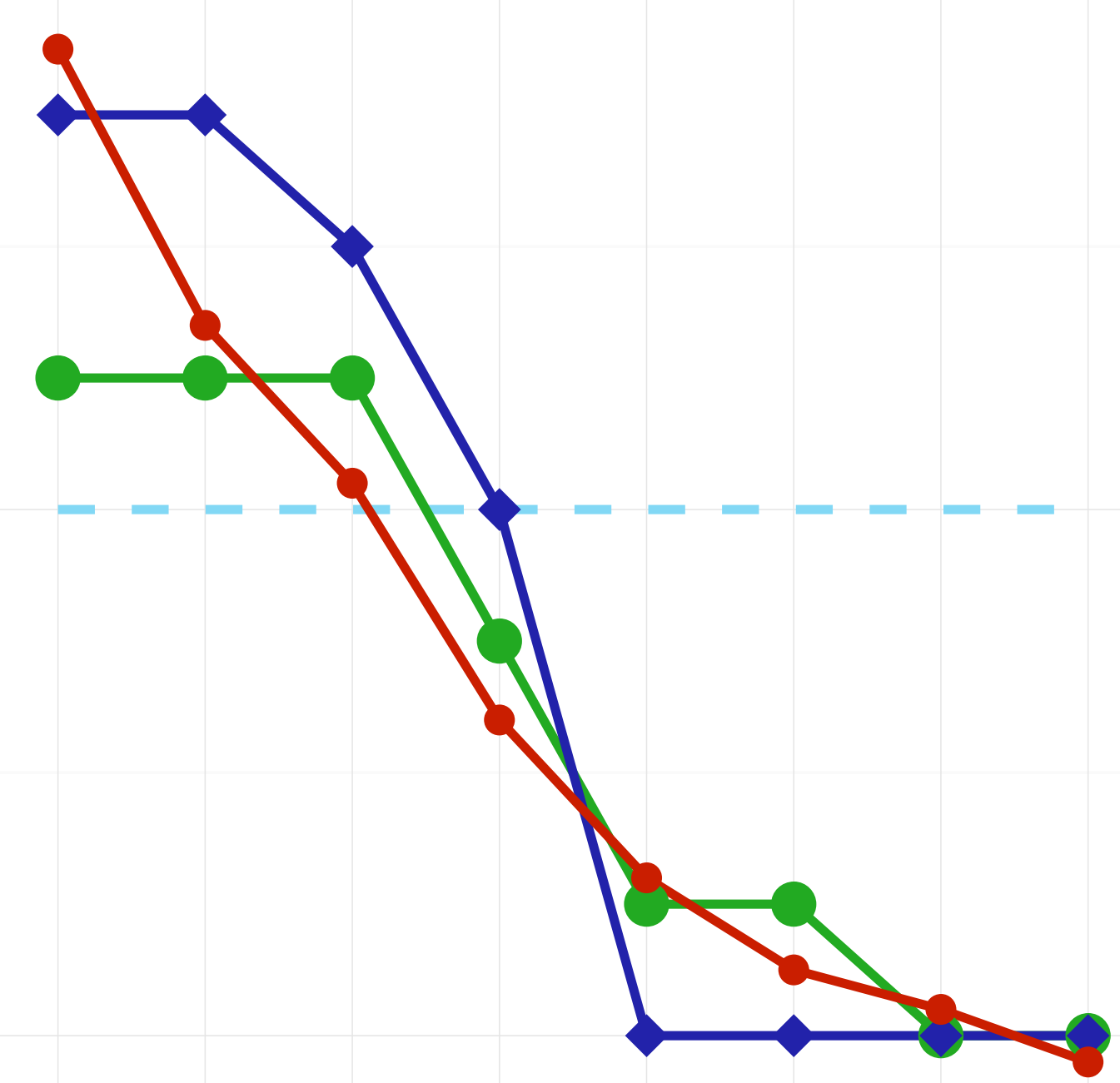

$3 T F Q_{2.5} \quad$ Training set

Average single structure

_ Best single structure

Whole ensemble

- SIENA

$\neg$ Clustering

$\multimap$ Random

Average single structure

_ _ Best single structure

Whole ensemble

1

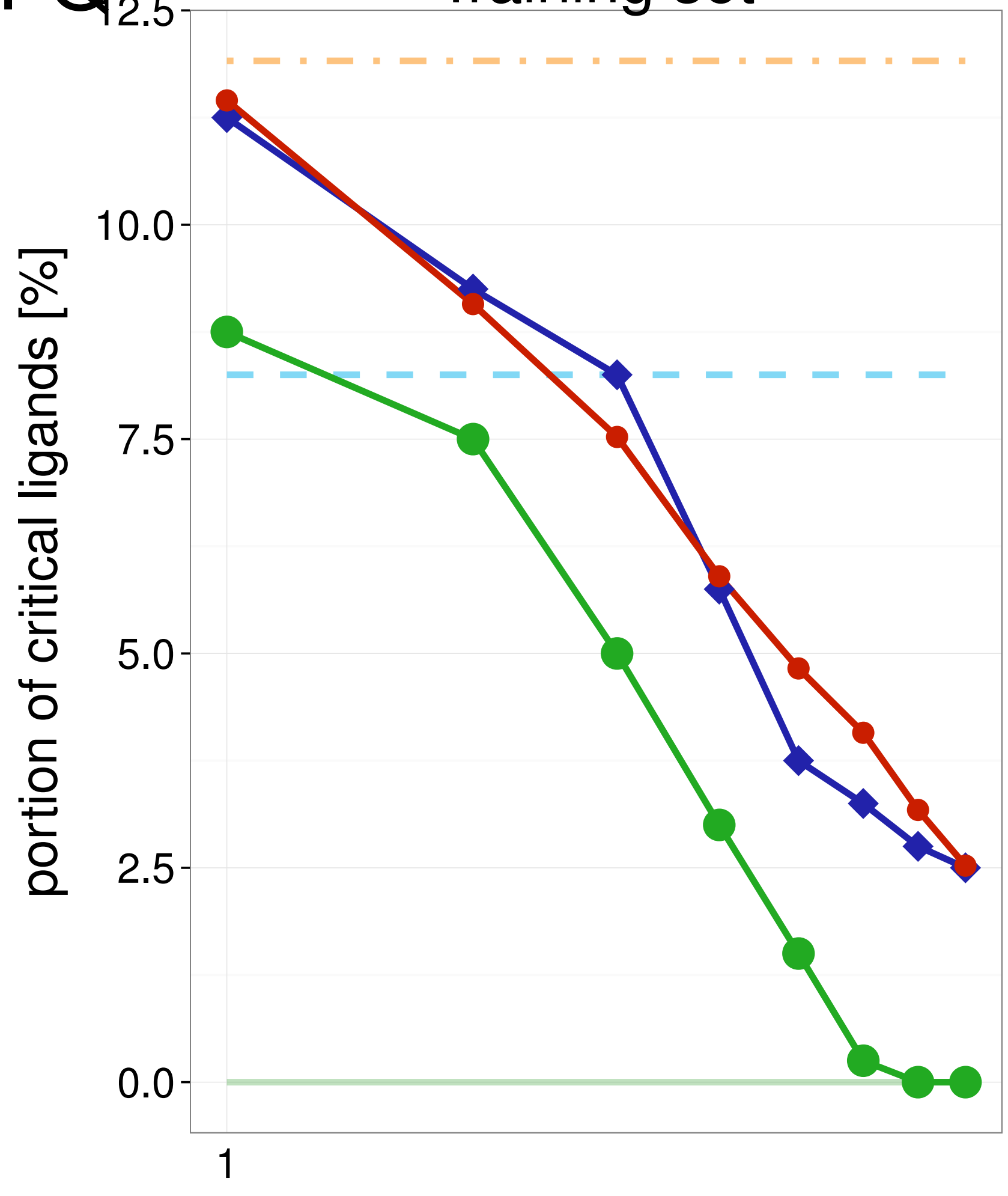

maximal ensemble size

Test set

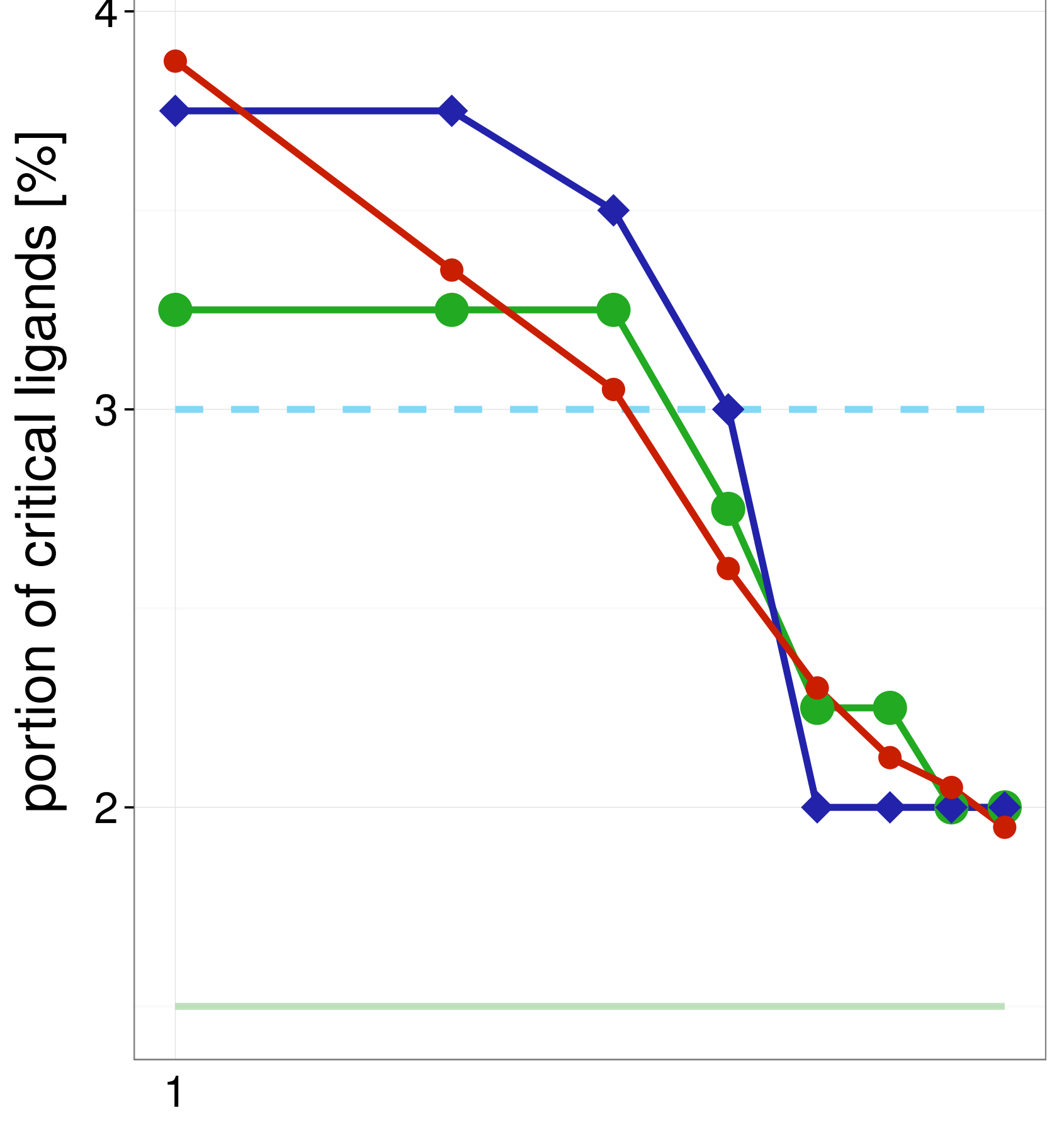

Average single structure

_ _ Best single structure

Whole ensemble

SIENA

$\neg$ Clustering

- Random 


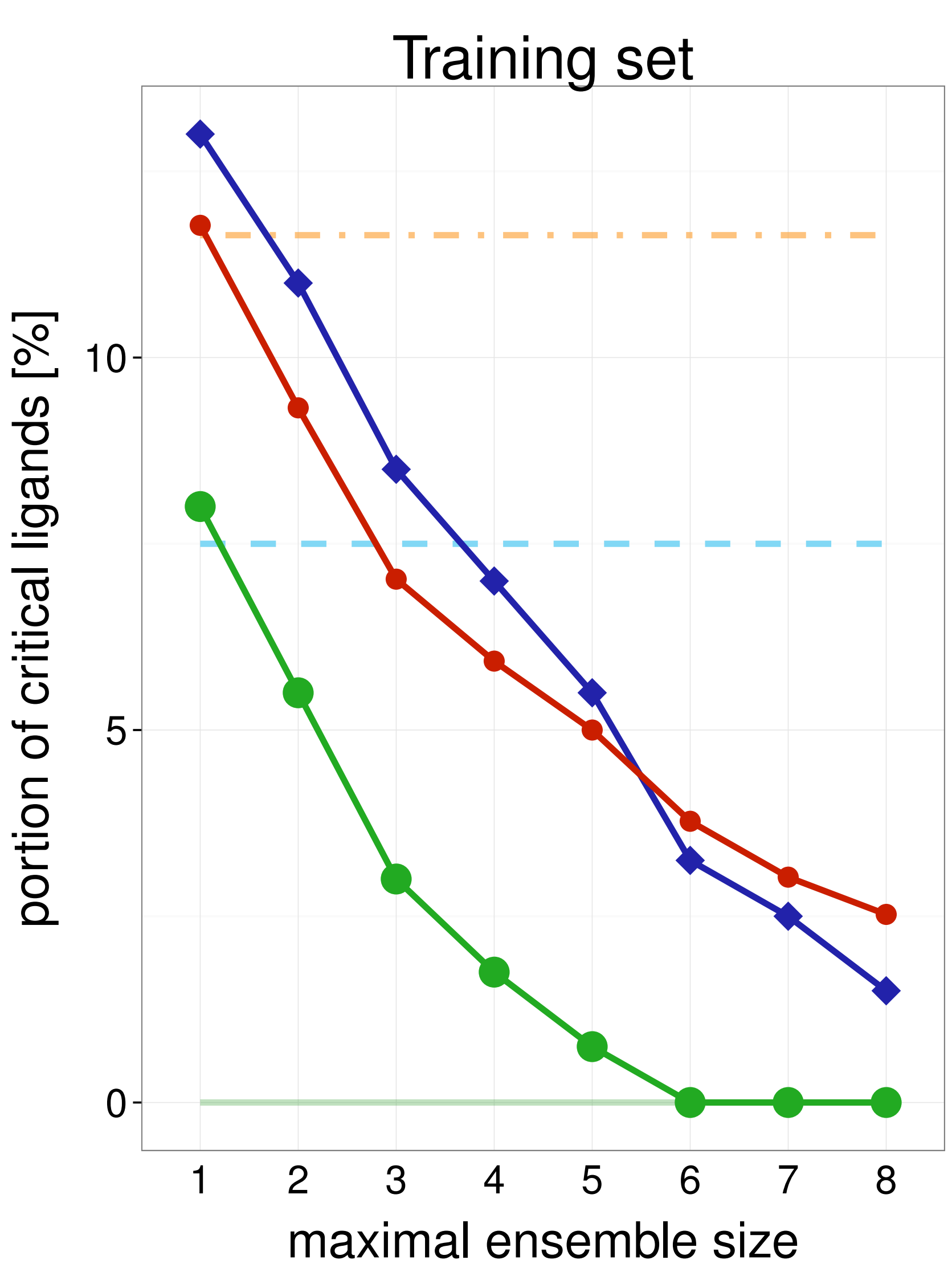

\section{Test set}

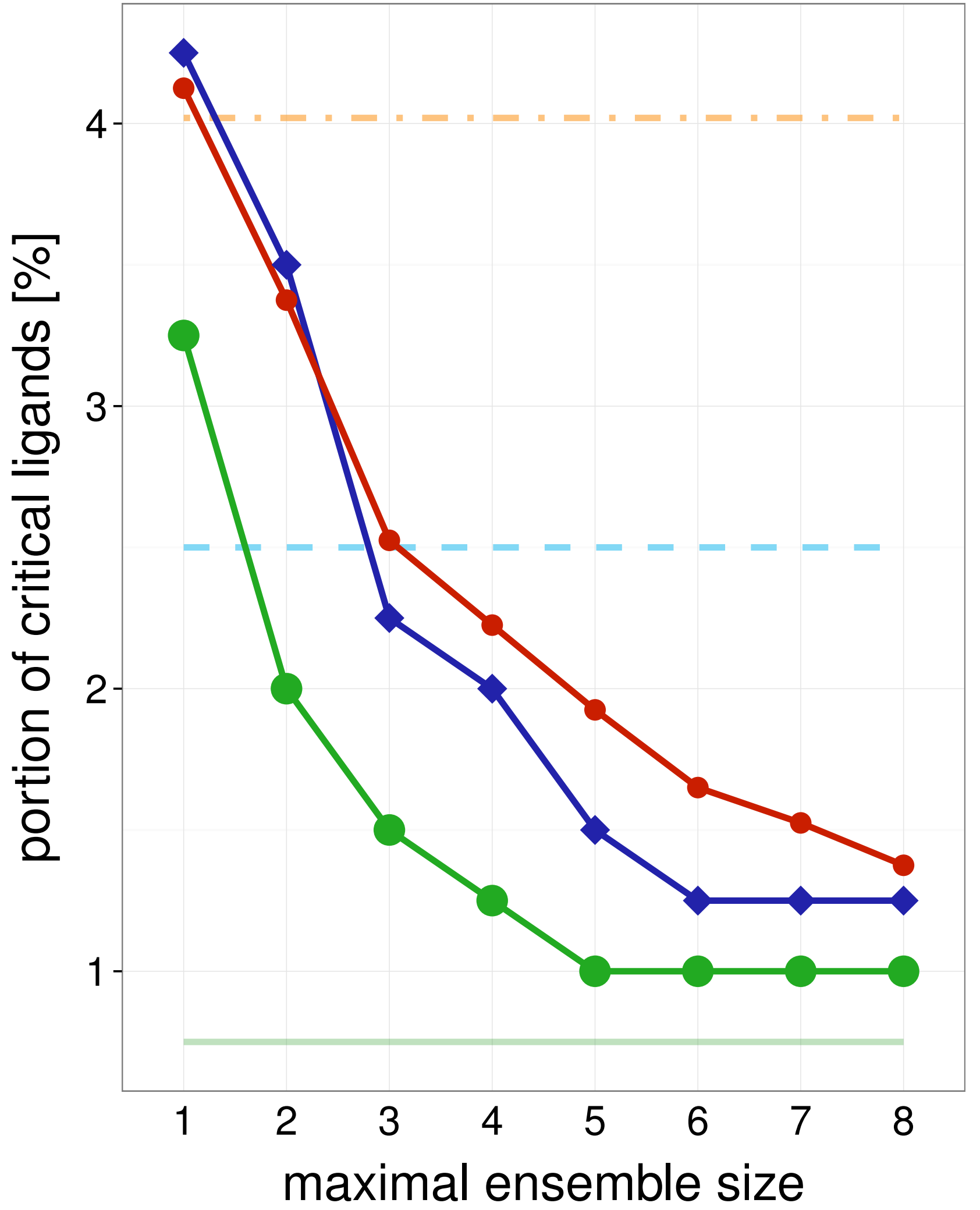

3V8T

Training set

Average single structure

_ Best single structure

Whole ensemble

- SIENA

$\neg$ Clustering

$\multimap$ Random

Average single structure

_ _ Best single structure

Whole ensemble

- SIENA

$\checkmark$ Clustering

$\multimap$ Random

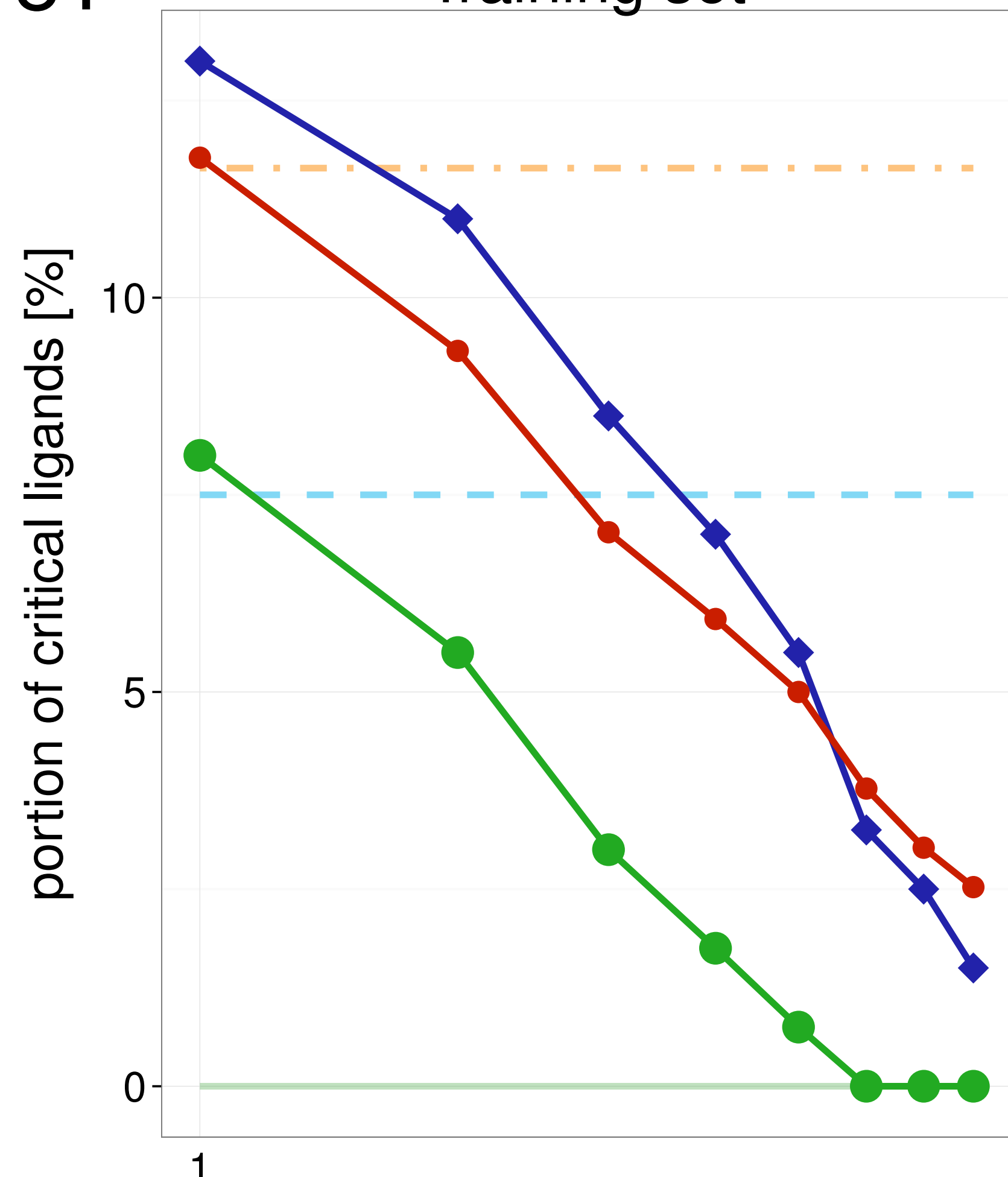

maximal ensemble size

Test set

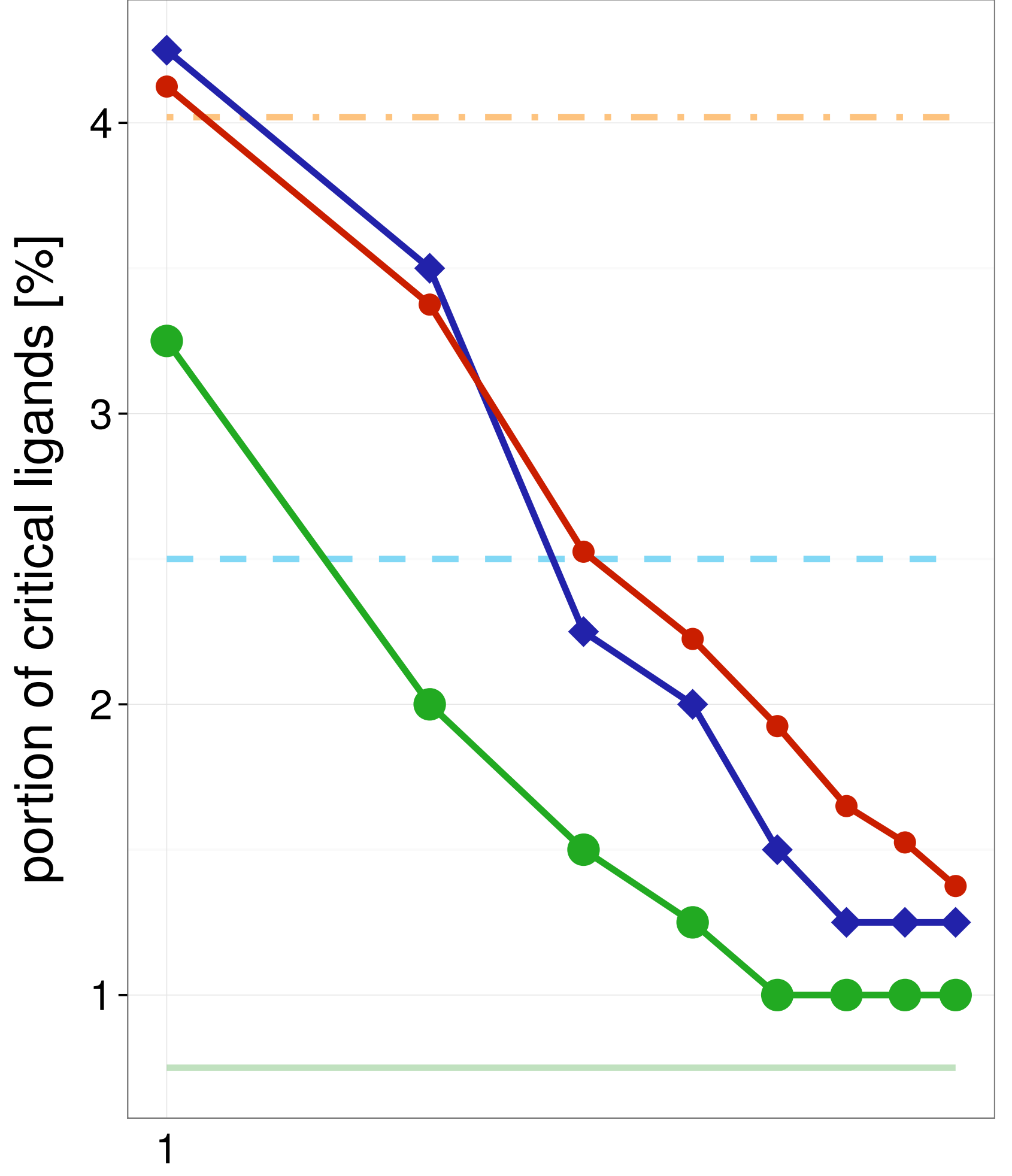

maximal ensemble size
Average single structure

_ Best single structure

Whole ensemble

- SIENA

$\neg$ Clustering

$\longrightarrow$ Random 


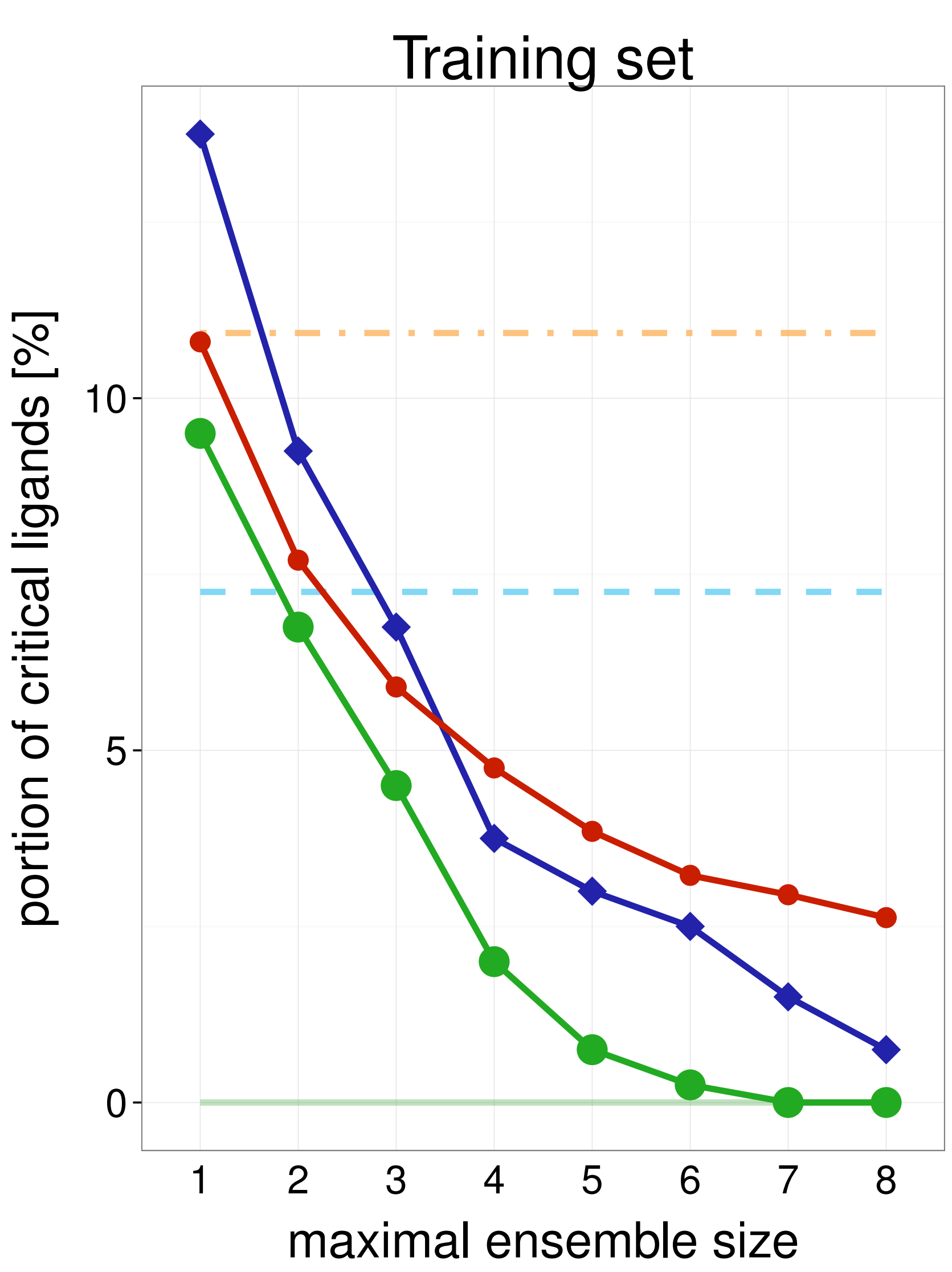

Test set

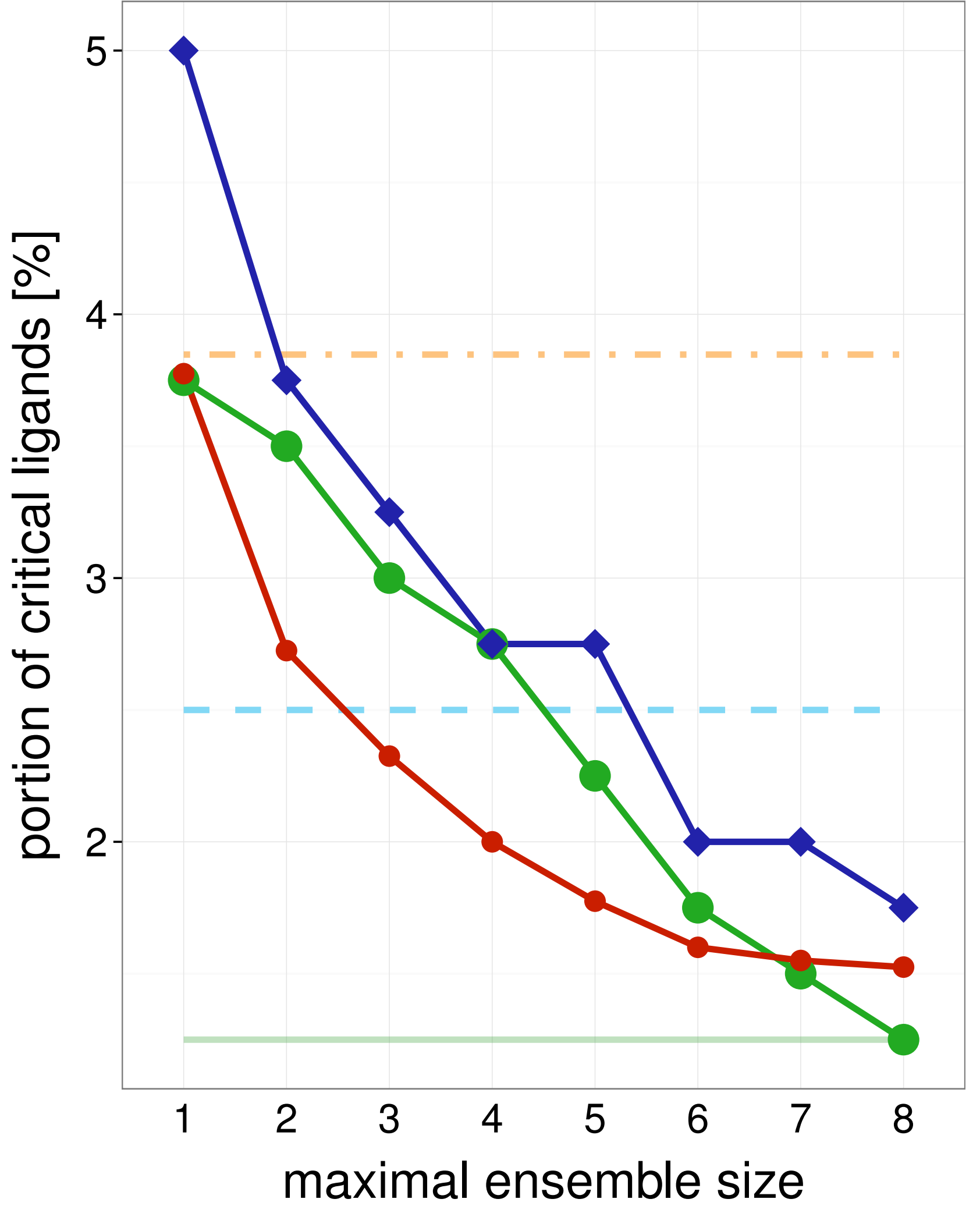

Average single structure

_ _ Best single structure

Whole ensemble

- SIENA

$\neg$ Clustering

$\multimap$ Random

Average single structure

_ _ Best single structure

Whole ensemble

- SIENA

$\checkmark$ Clustering

- Random
4E5F

Training set

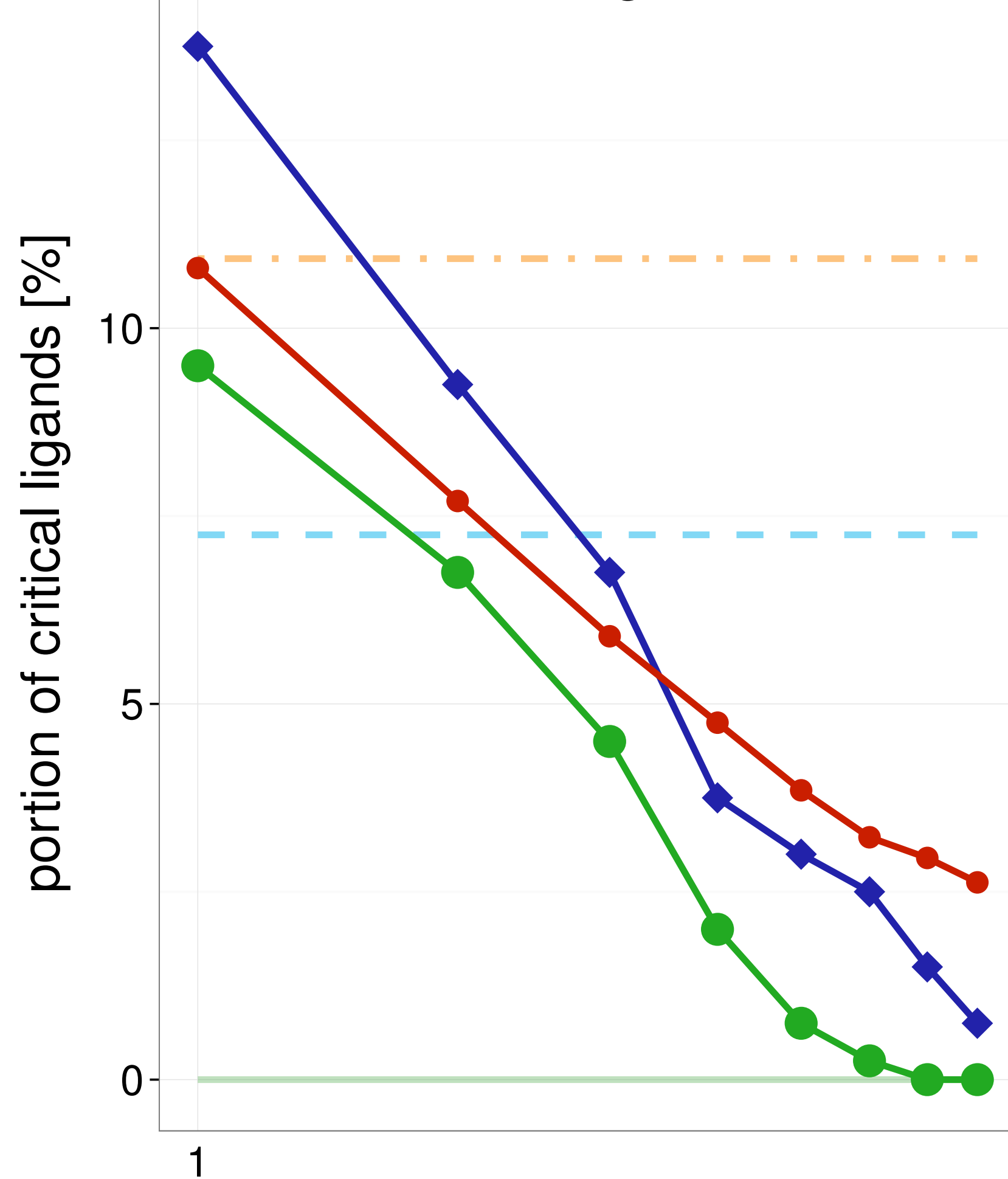

maximal ensemble size

Test set

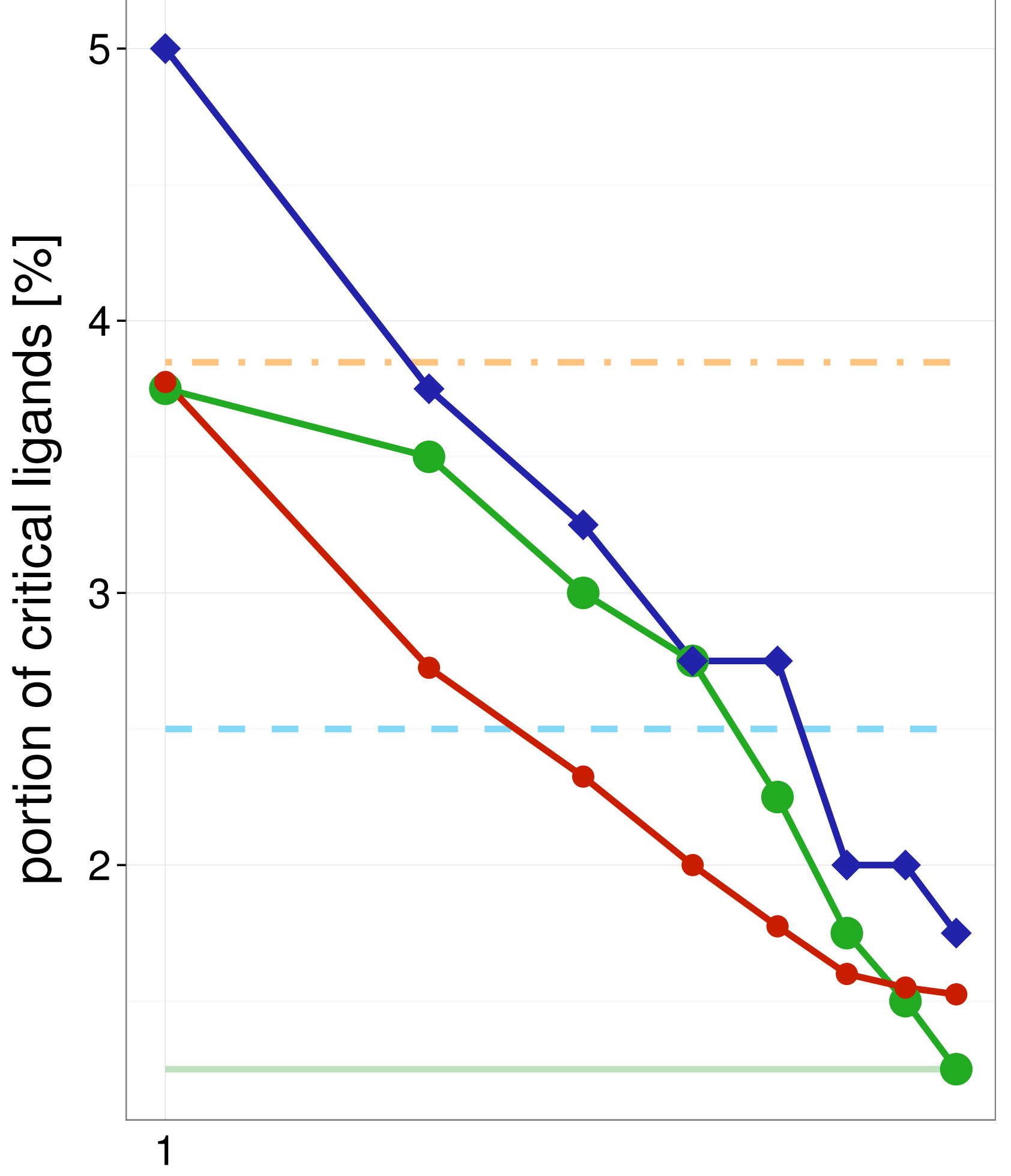

maximal ensemble size
Average single structure

_ Best single structure

Whole ensemble

SIENA

$\sim$ Clustering

$\multimap$ Random 
Training set

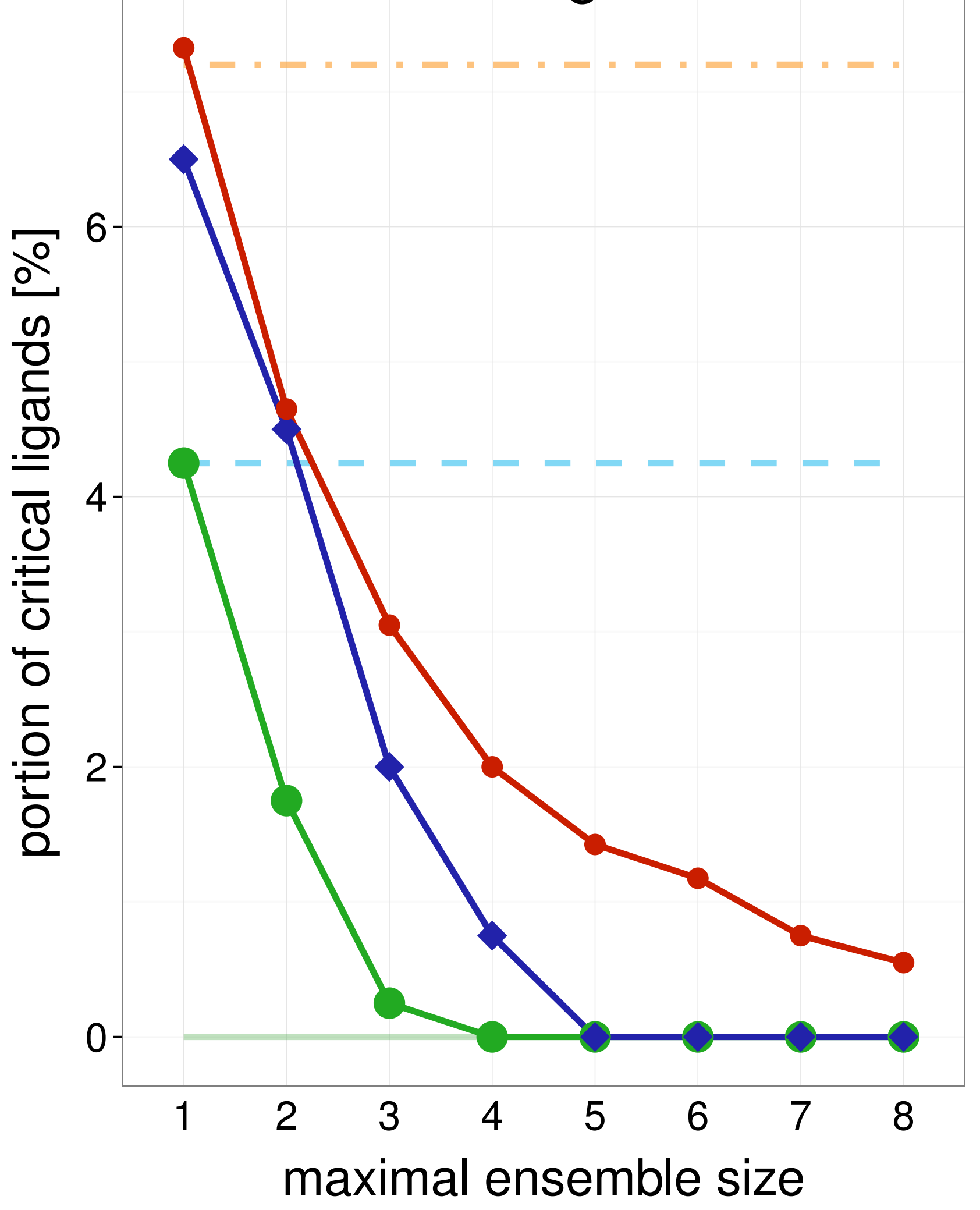

Test set

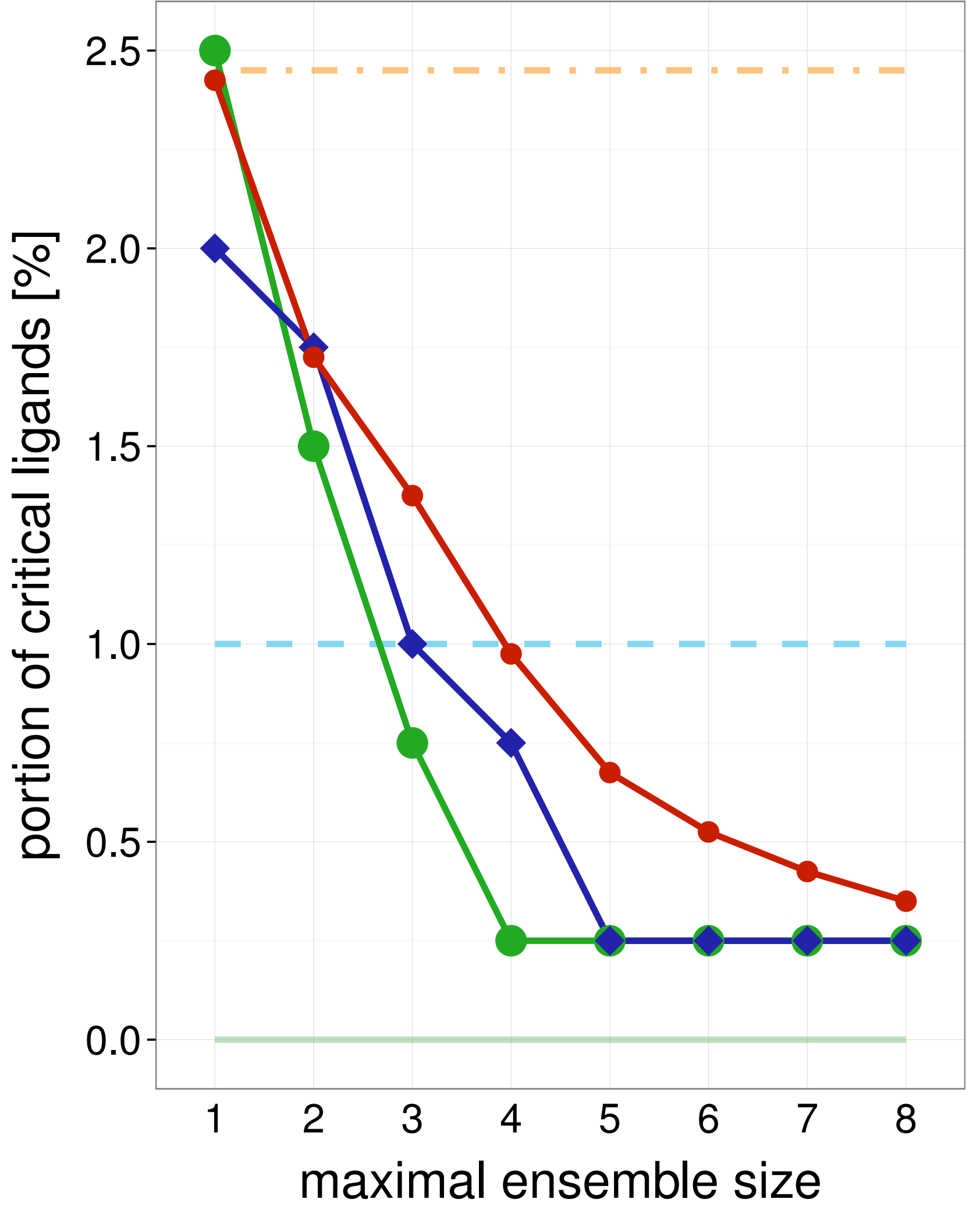

Average single structure

_ Best single structure

Whole ensemble

- SIENA

$\neg$ Clustering

$\multimap$ Random

Average single structure

_ _ Best single structure

Whole ensemble

- SIENA

$\sim$ Clustering

$\multimap$ Random

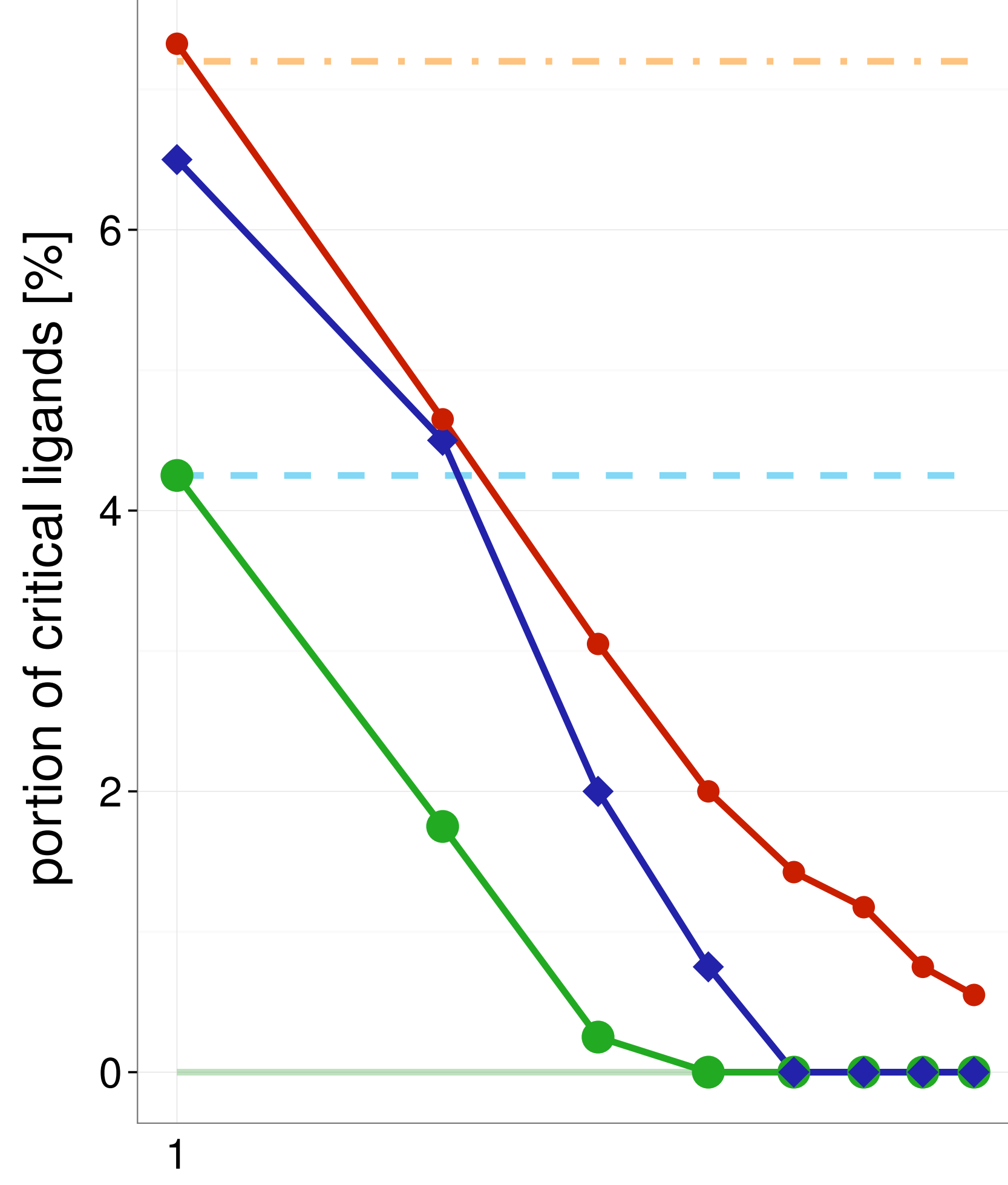

maximal ensemble size

Test set

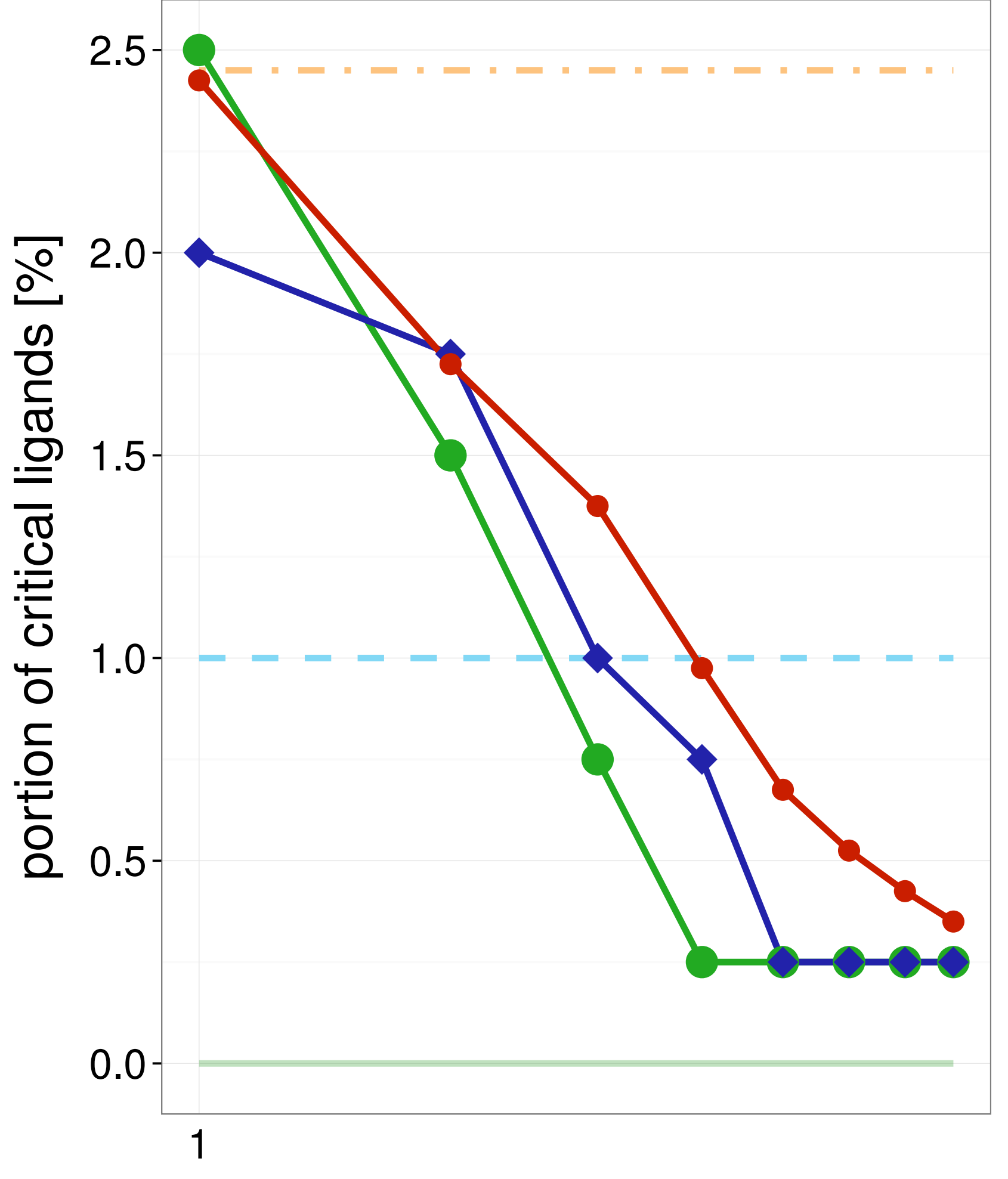

maximal ensemble size
Average single structure

_ Best single structure

Whole ensemble

SIENA

$\sim$ Clustering

$\multimap$ Random 


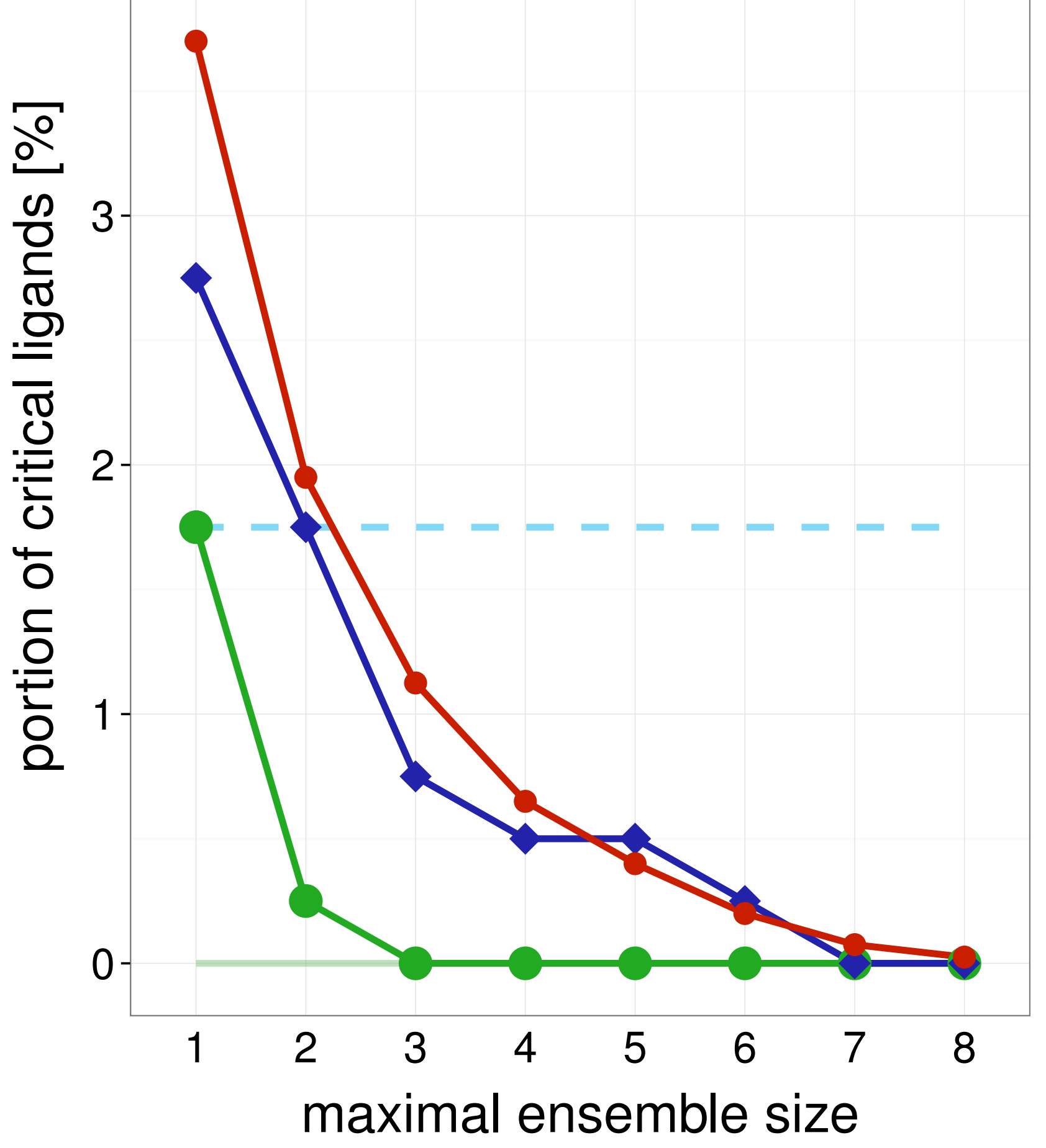

Average single structure

_ Best single structure

Whole ensemble

- SIENA

$\neg$ Clustering

$\multimap$ Random

Test set

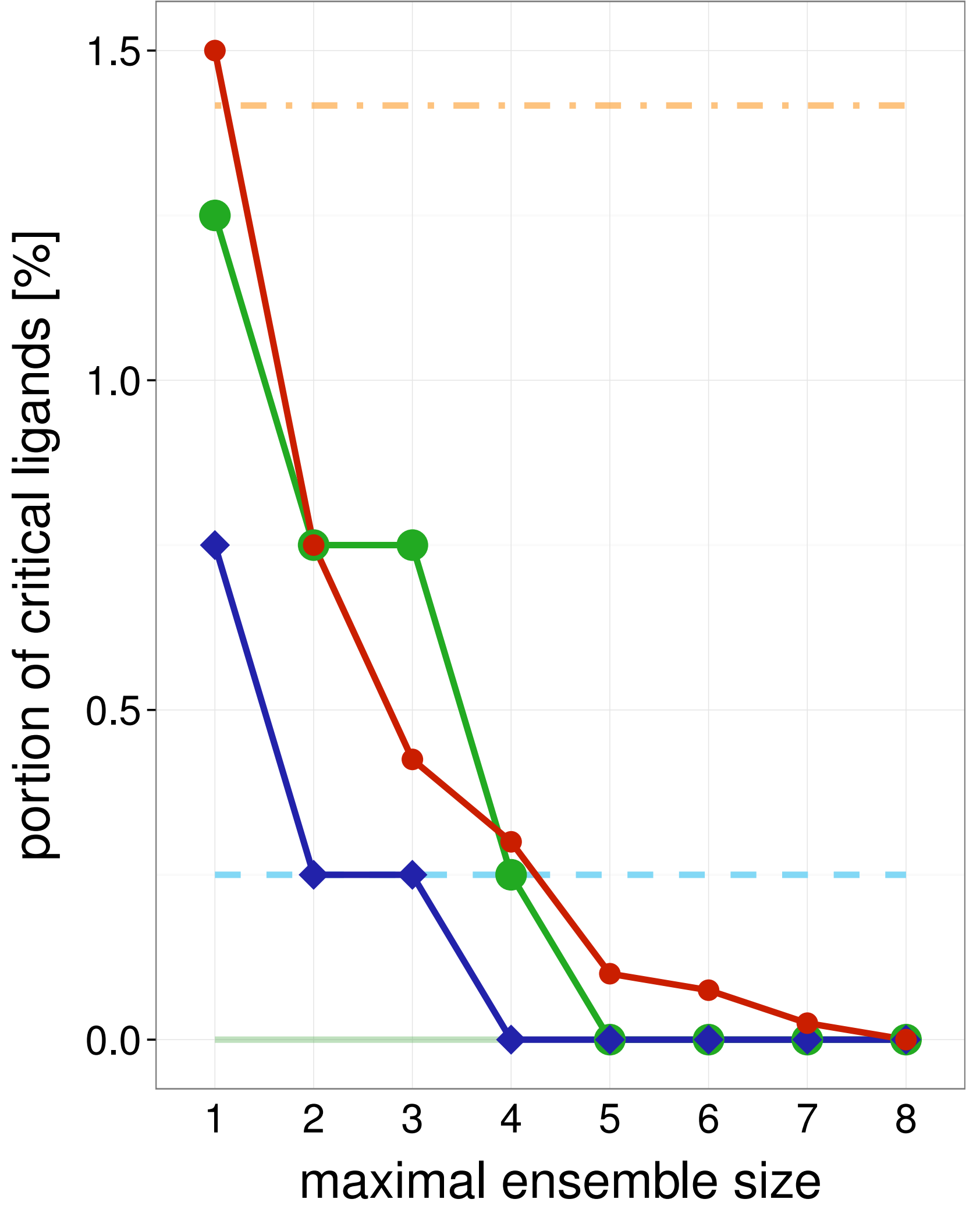

Average single structure

_ _ Best single structure

Whole ensemble

- SIENA

$\neg$ Clustering

$\multimap$ Random

maximal ensemble size

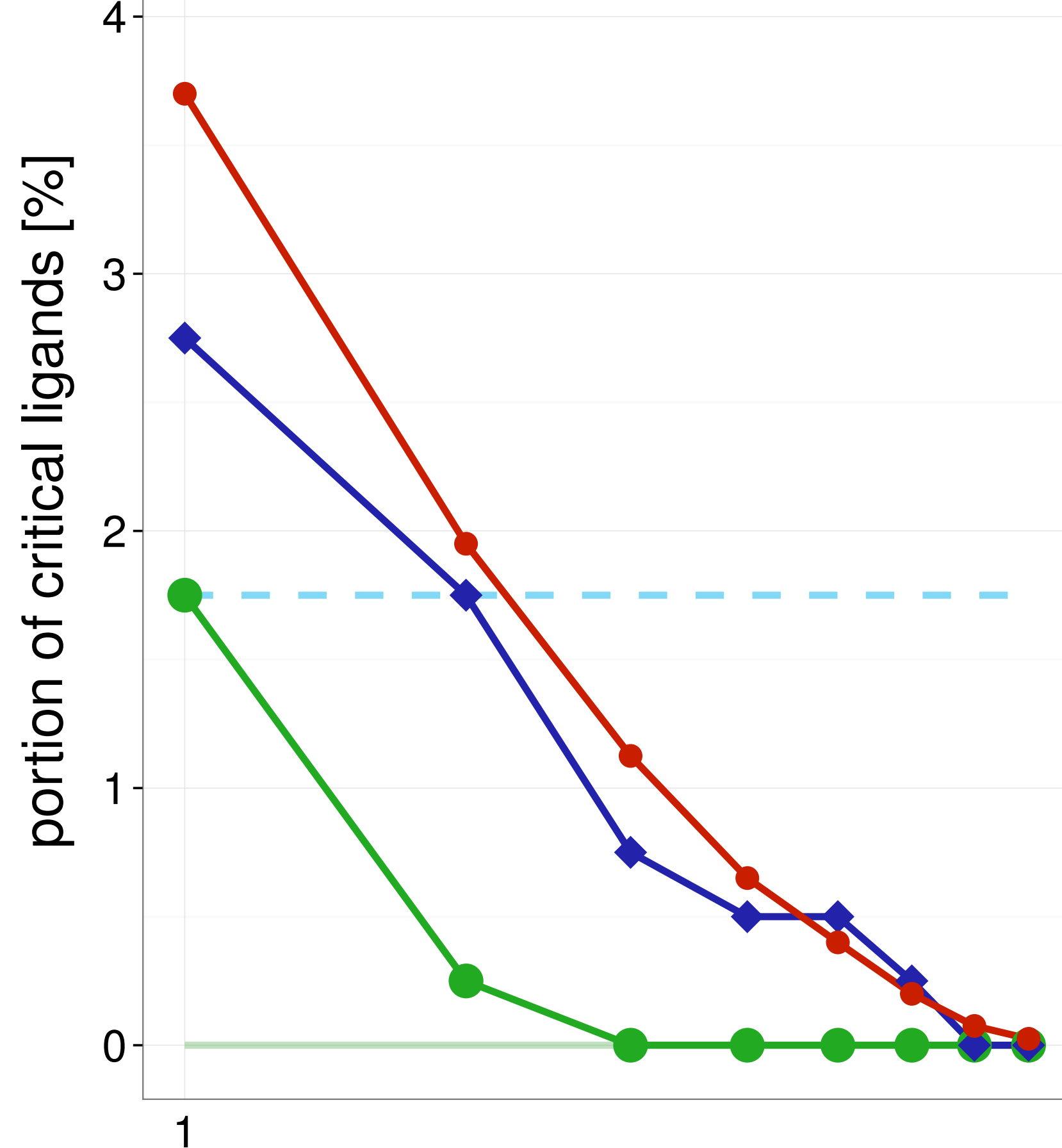

maximal ensemble size

Test set

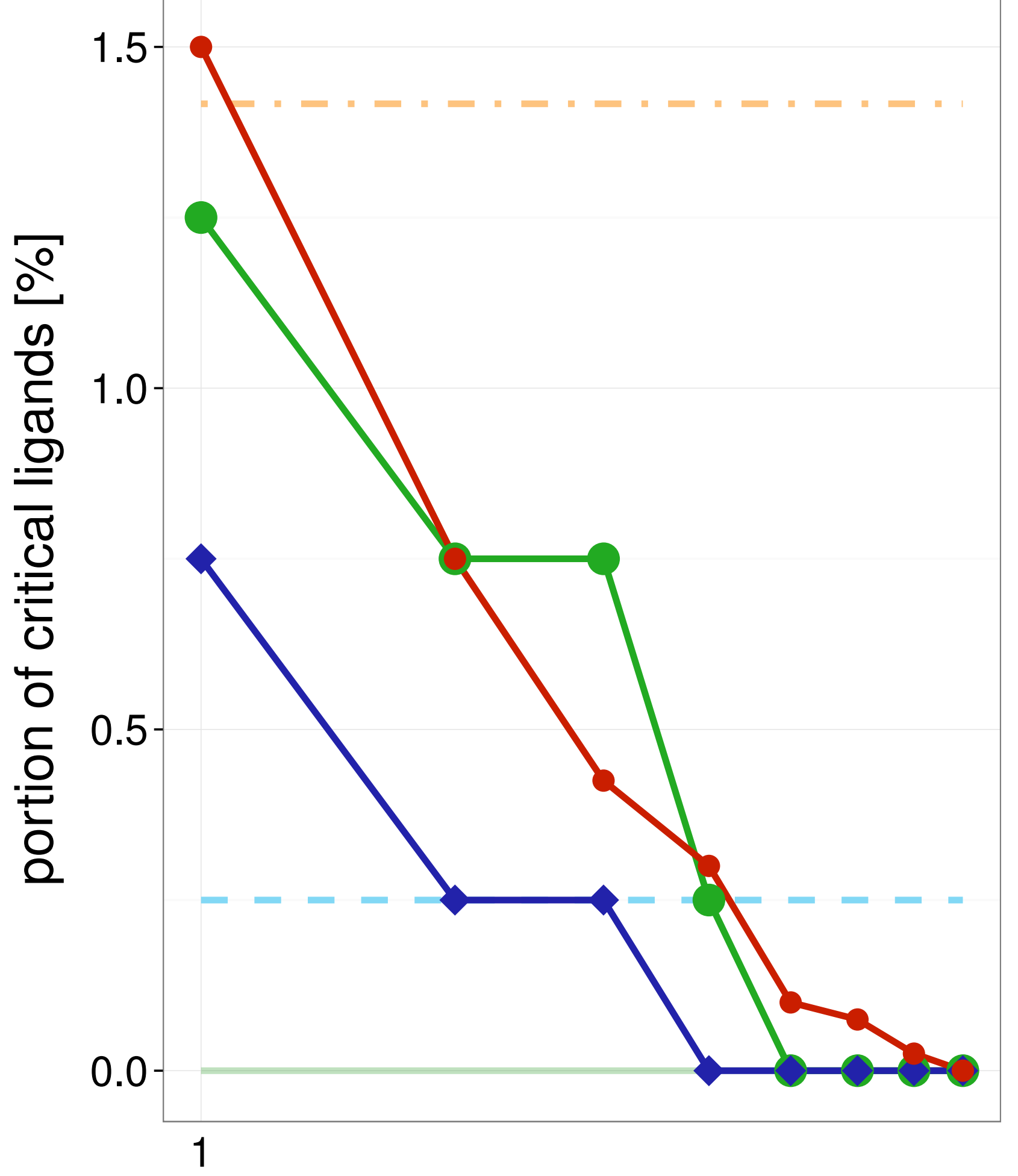

maximal ensemble size
Average single structure

_ Best single structure

Whole ensemble

SIENA

$\neg$ Clustering

$\longrightarrow$ Random 
Training set

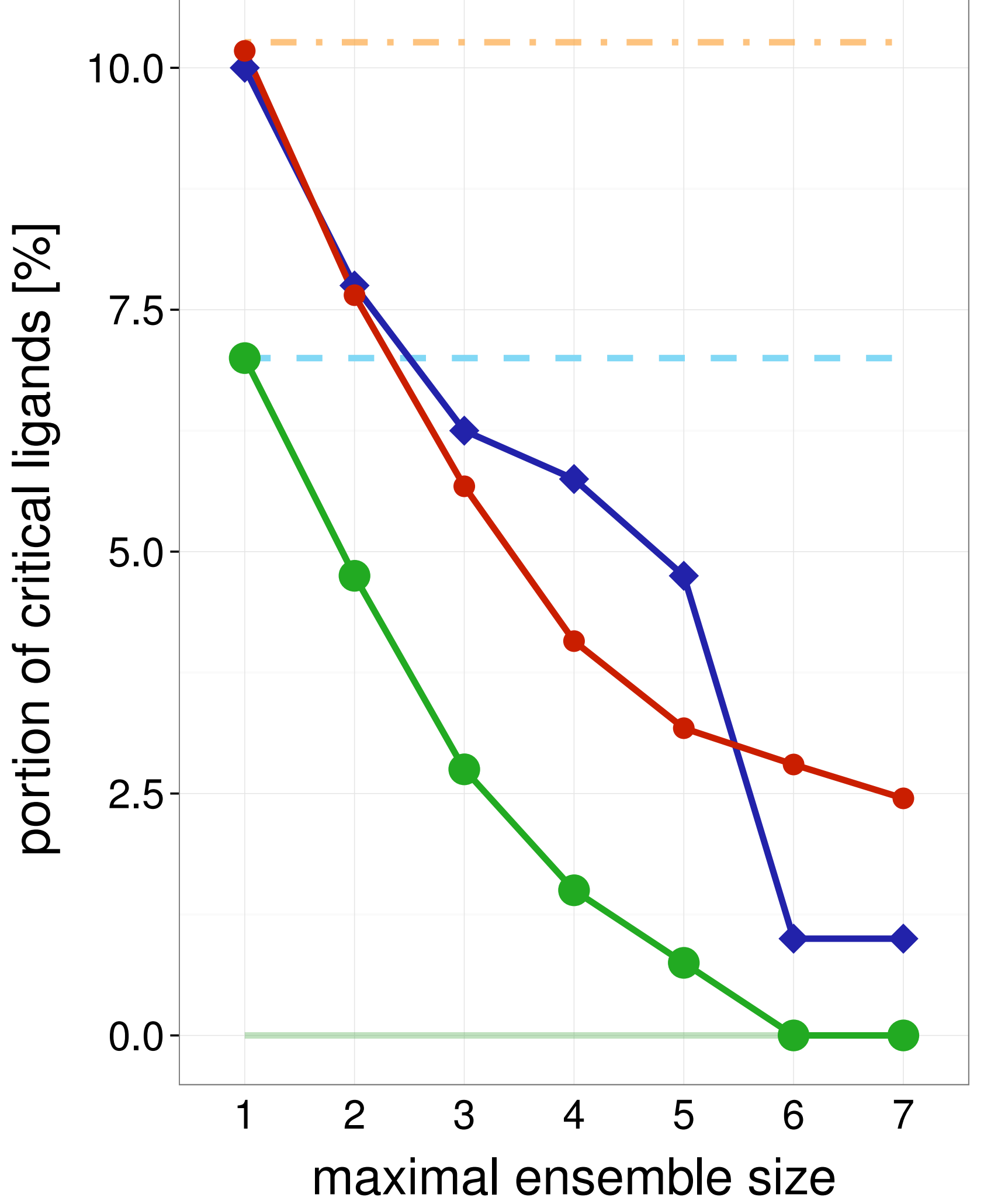

\section{Test set}

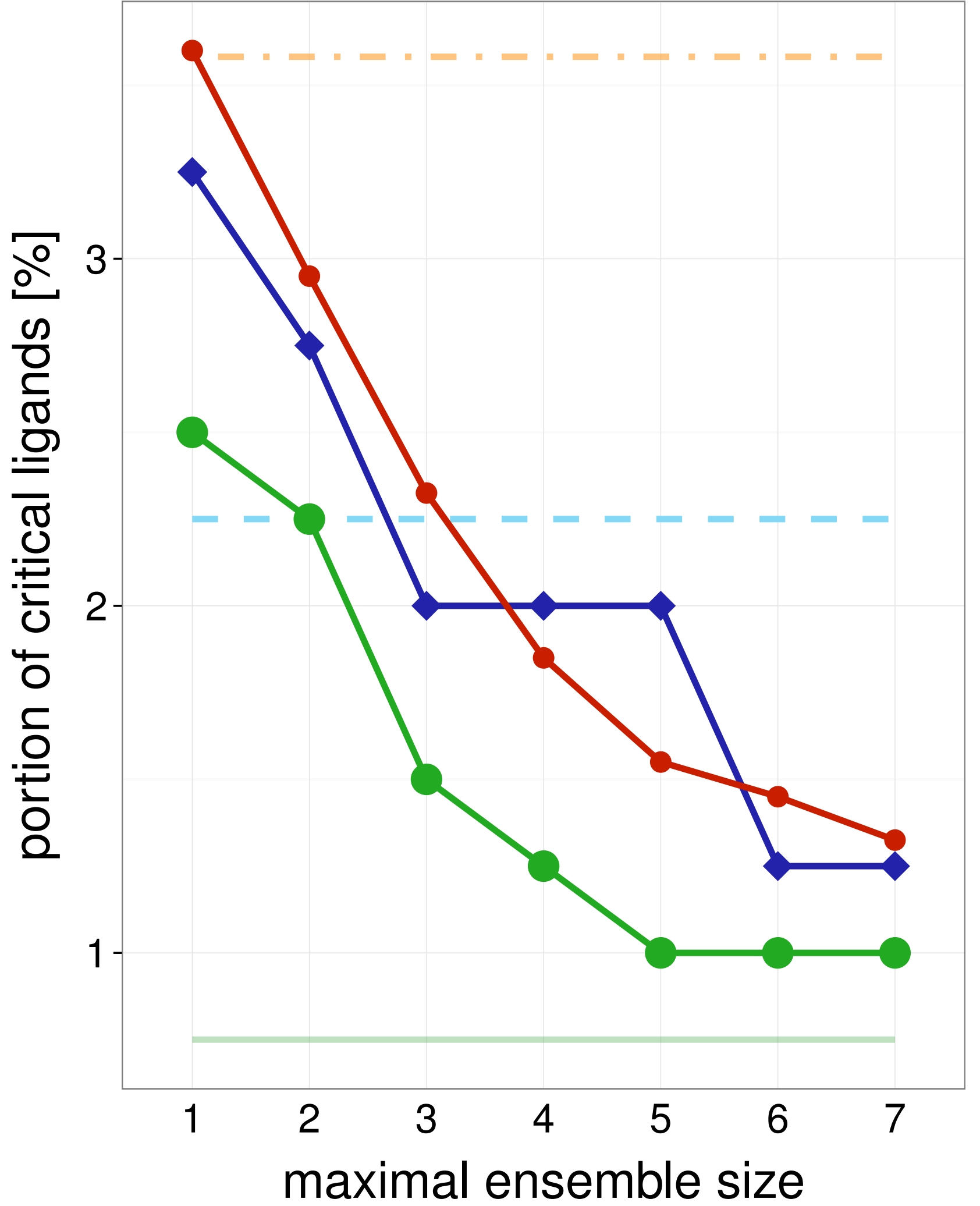

1E3G

Training set

Average single structure

_ Best single

structure

Whole ensemble

- SIENA

$\neg$ Clustering

$\rightarrow$ Random

Average single structure

_ Best single

structure

Whole ensemble

- SIENA

$\neg$ Clustering

$\longrightarrow$ Random

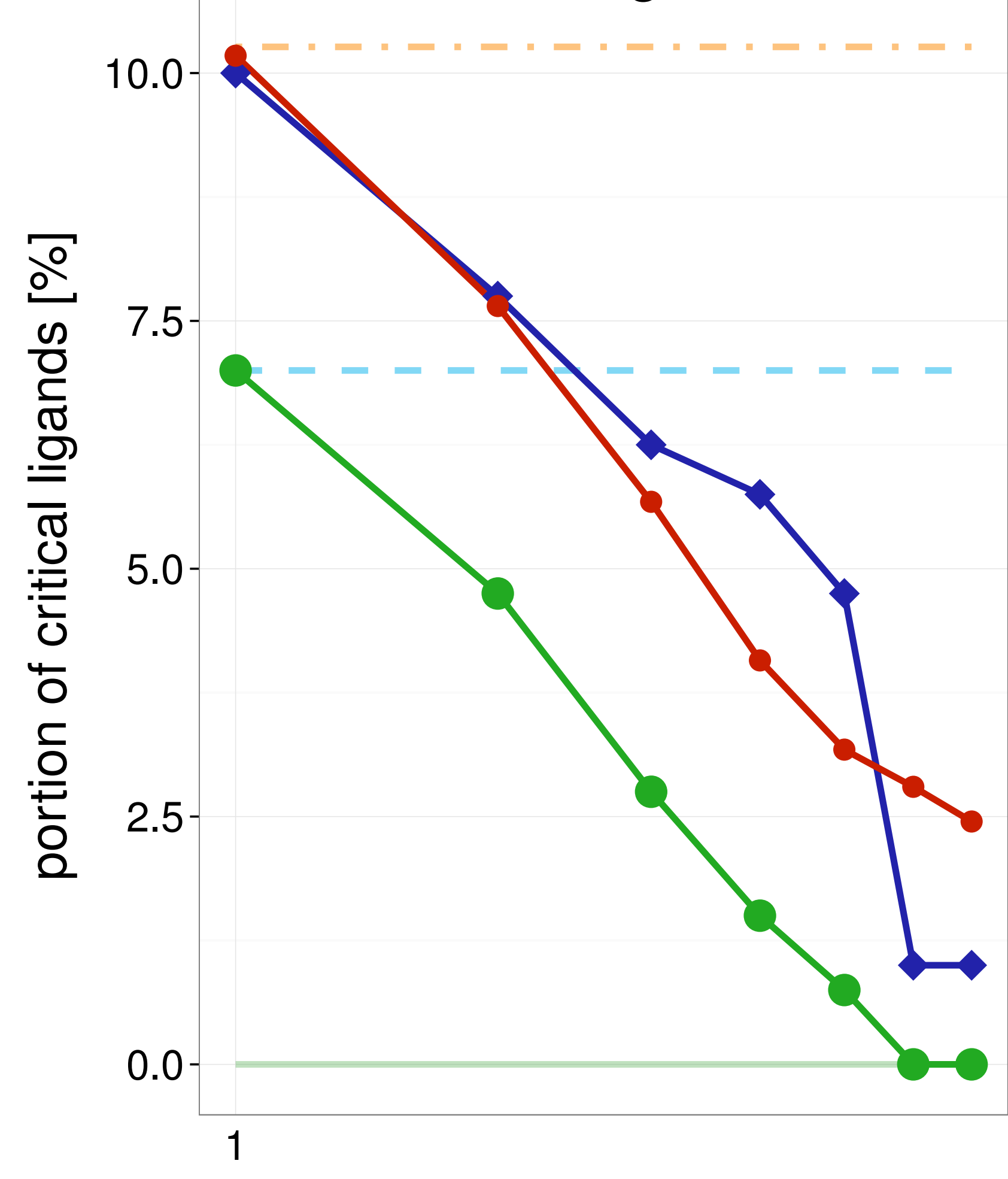

maximal ensemble size

\section{Test set}

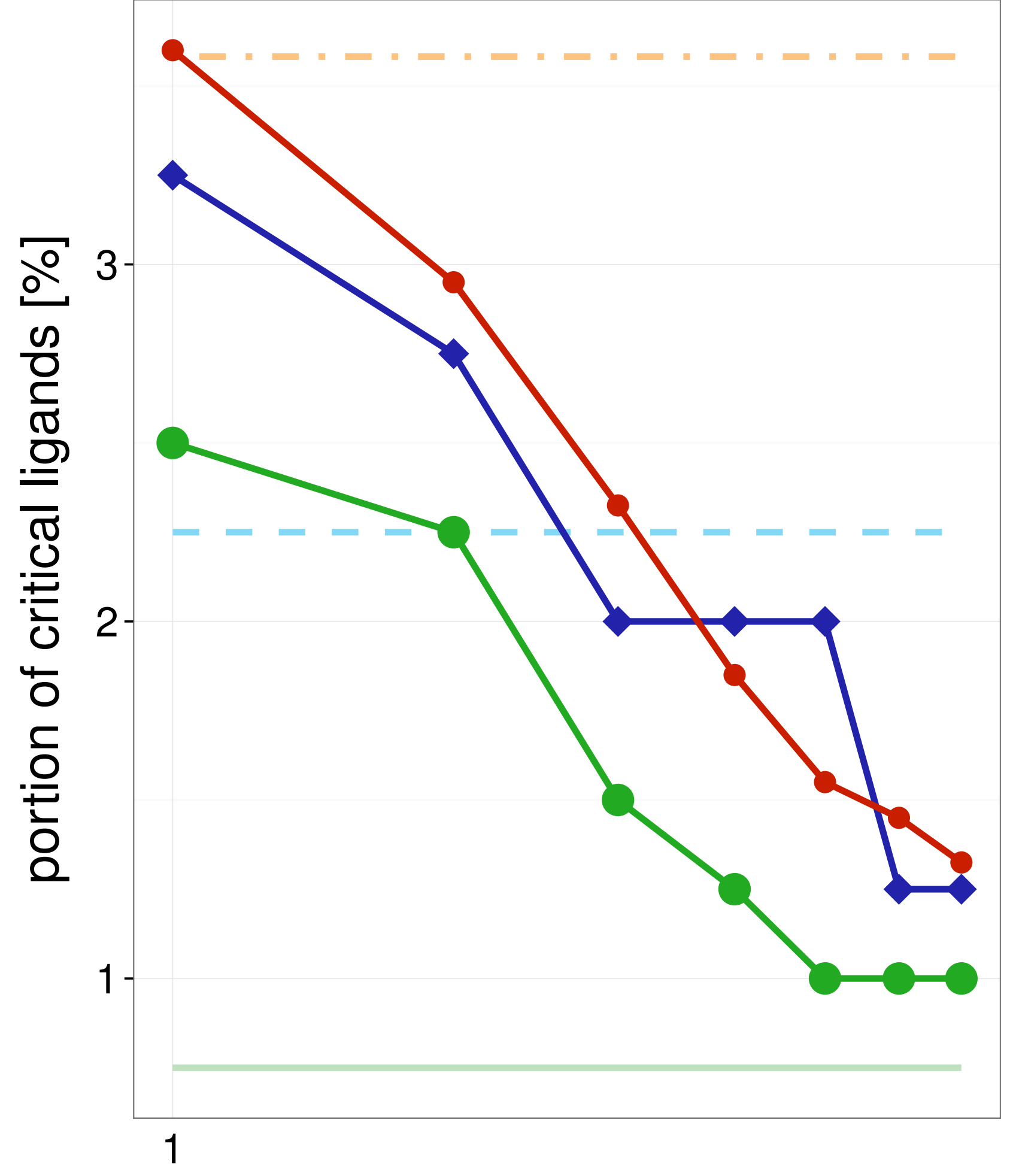

maximal ensemble size
Average single structure

_ Best single structure

Whole ensemble

SIENA

$\neg$ Clustering

- Random 
Training set

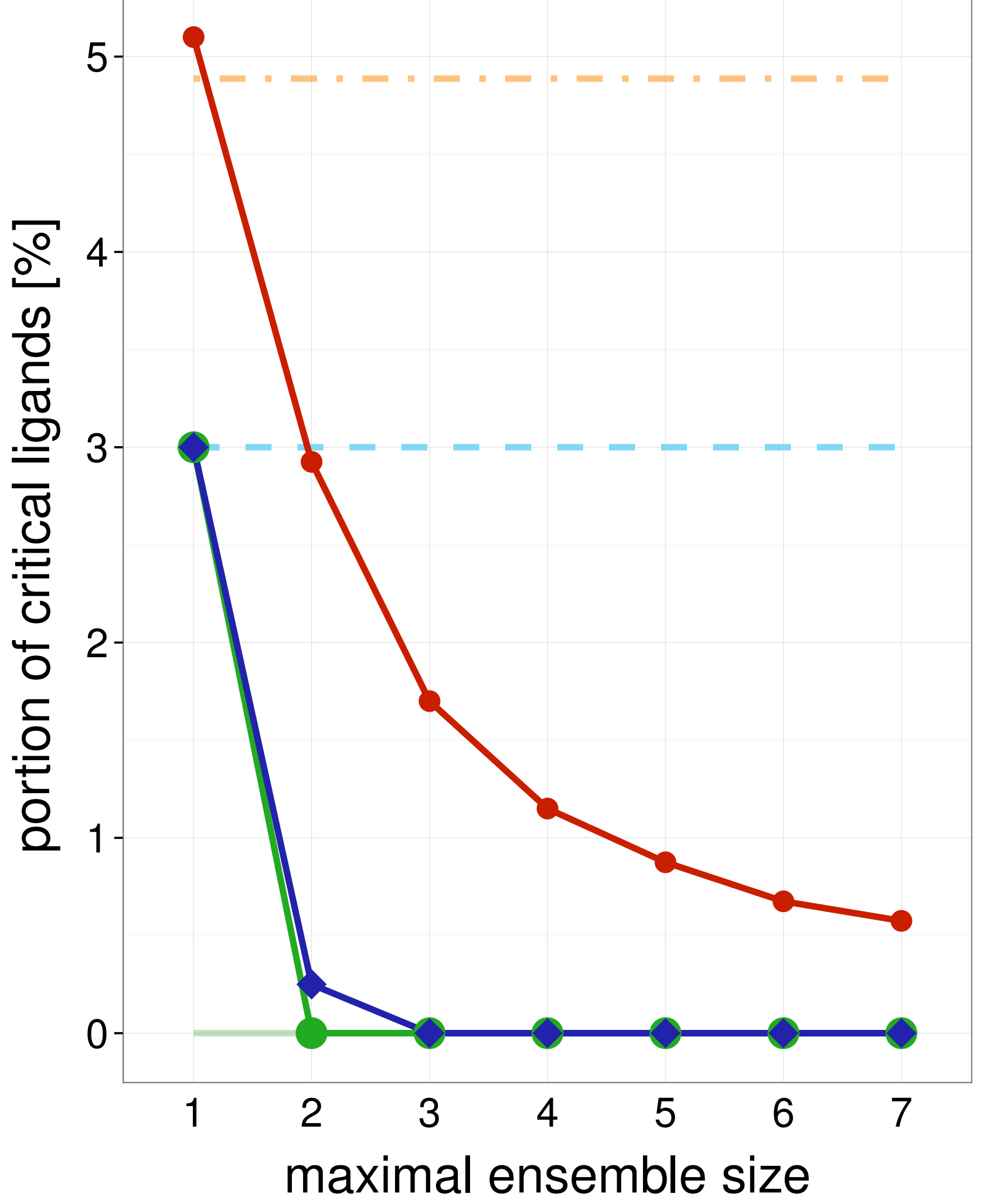

Test set

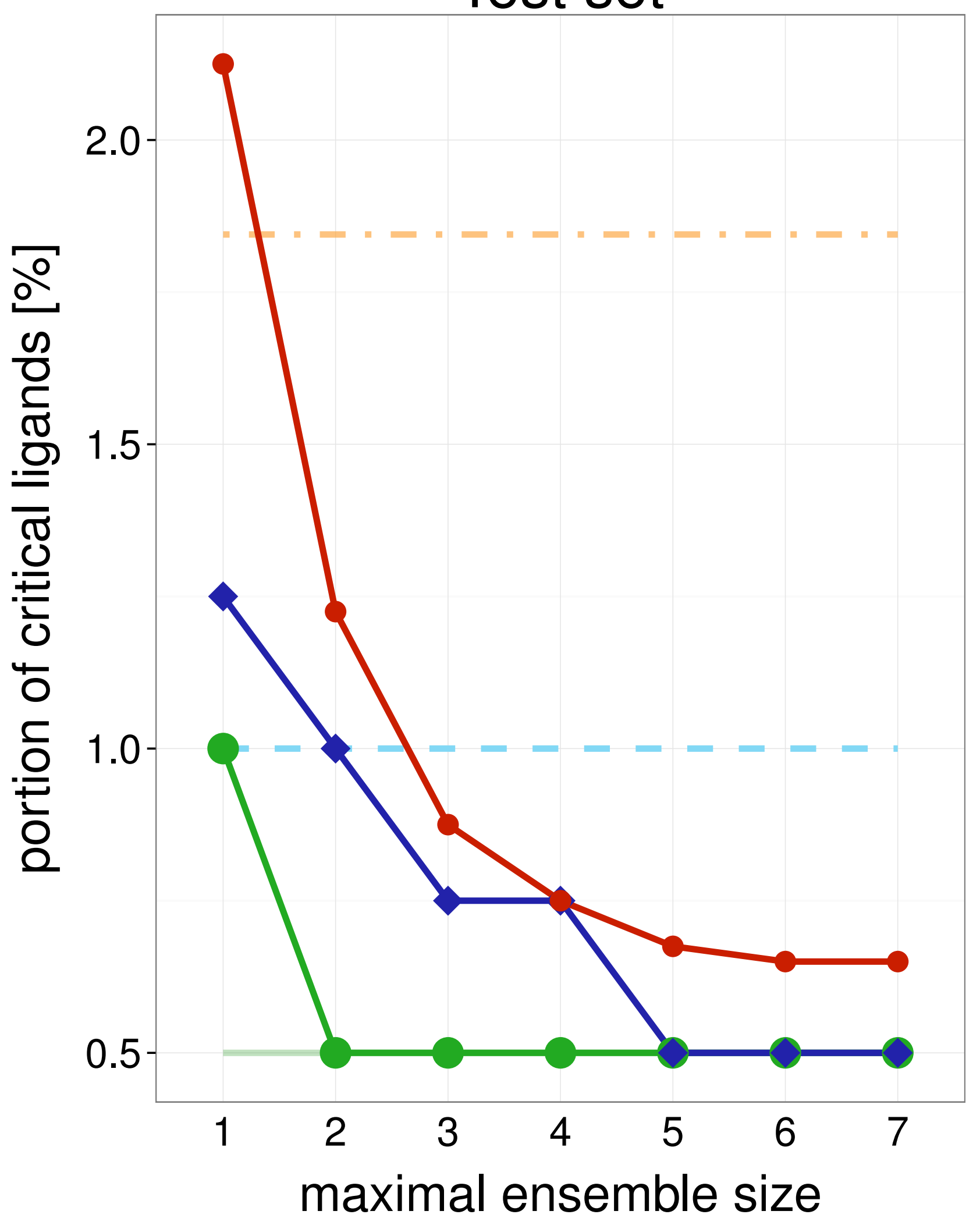

1M8D

Training set

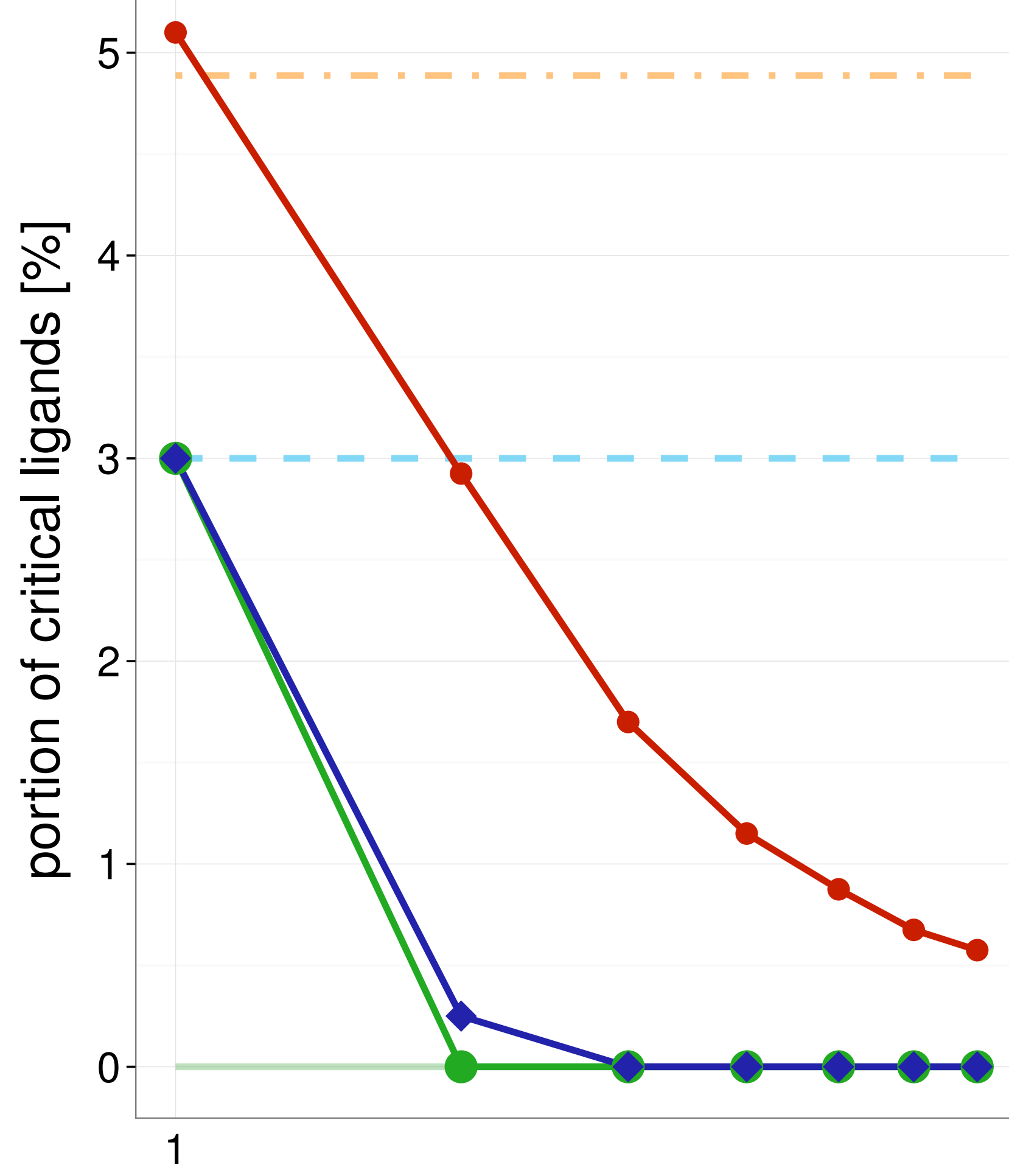

Average single structure

_ Best single structure

Whole ensemble

- SIENA

$\neg$ Clustering

$\multimap$ Random

Average single structure

_ _ Best single structure

Whole ensemble

- SIENA

$\neg$ Clustering

$\multimap$ Random
Average single structure

_ - Best single structure

Whole ensemble

SIENA

$\neg$ Clustering

$\multimap$ Random maximal ensemble size

Test set

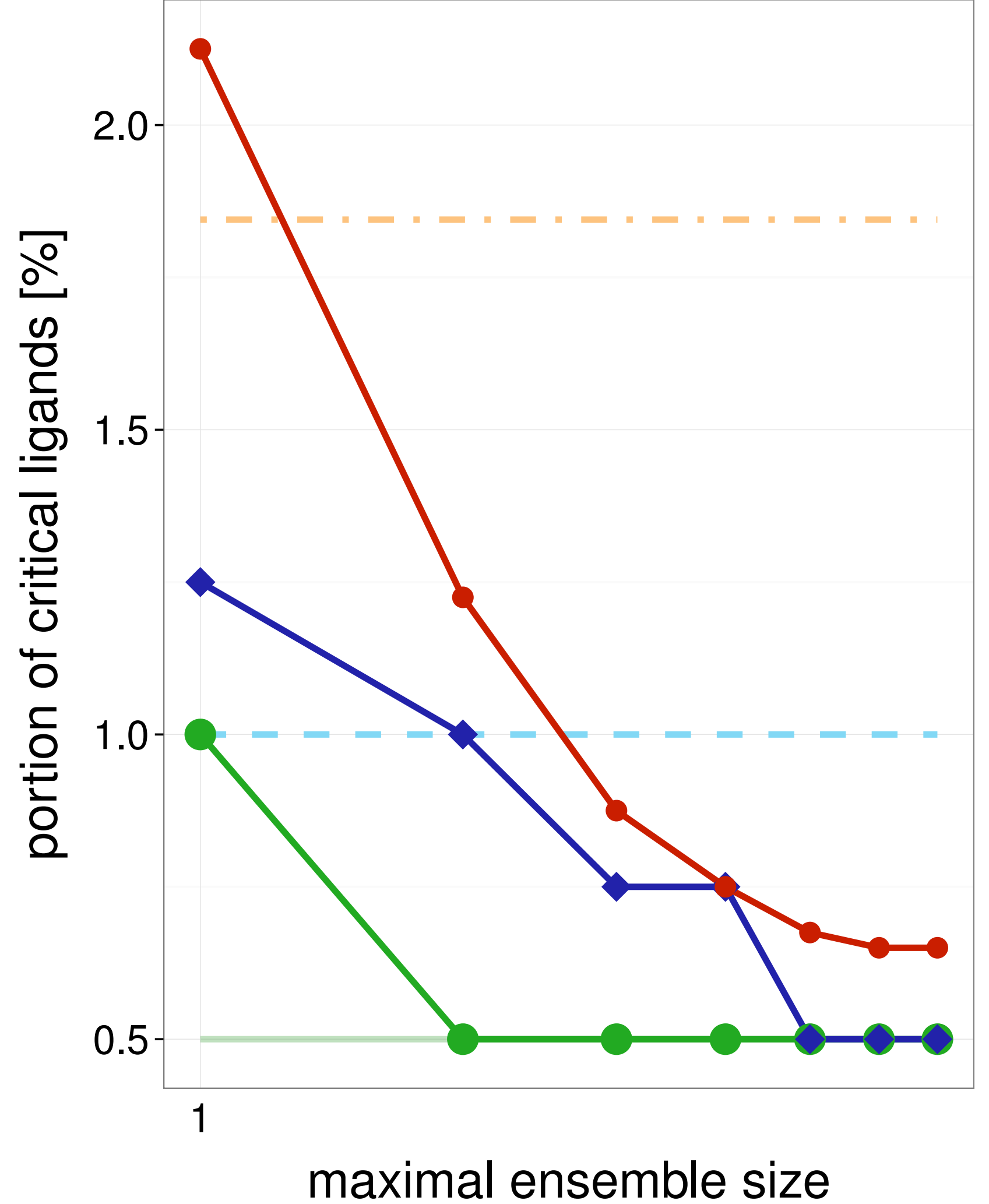




\section{Training set}

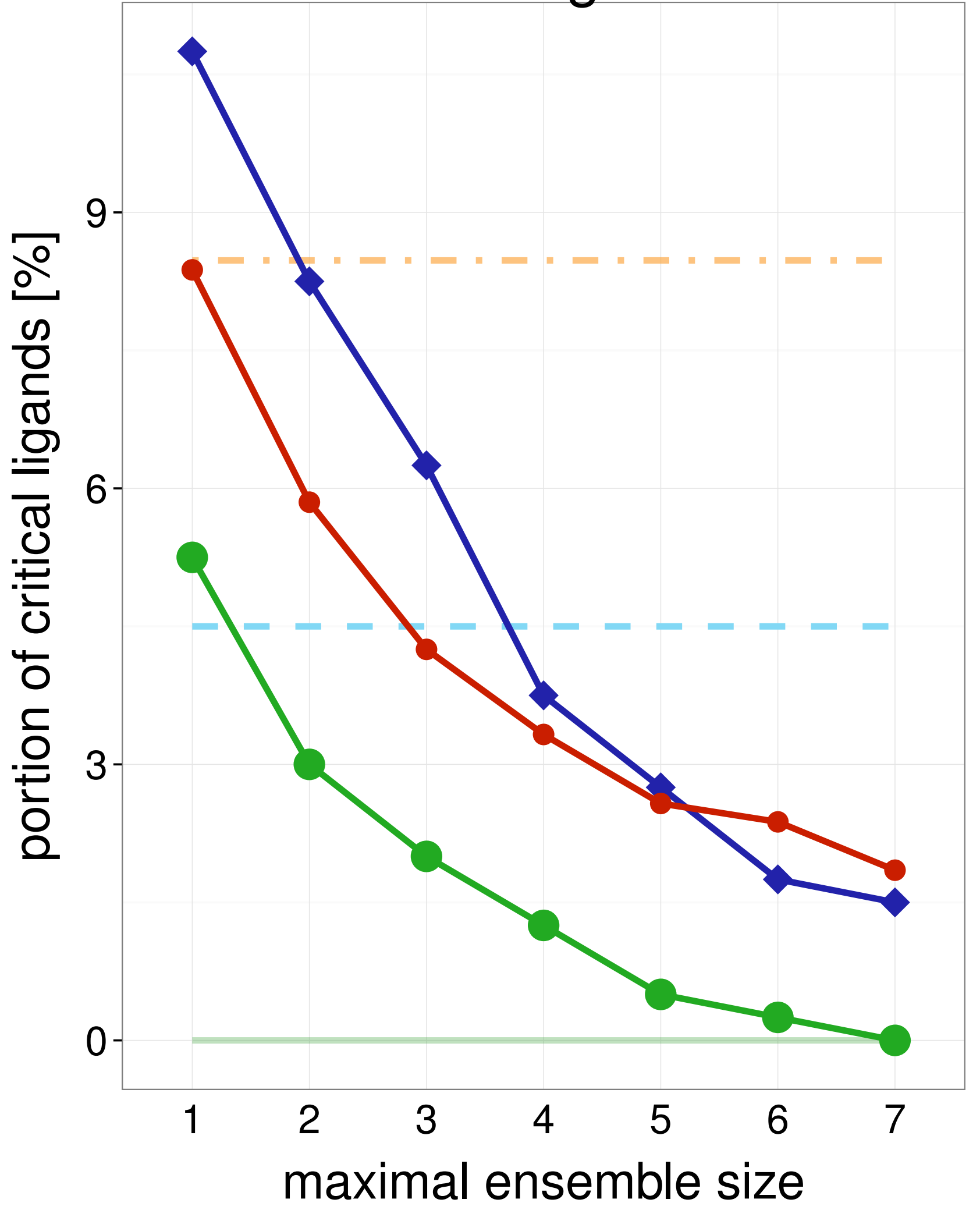

Test set

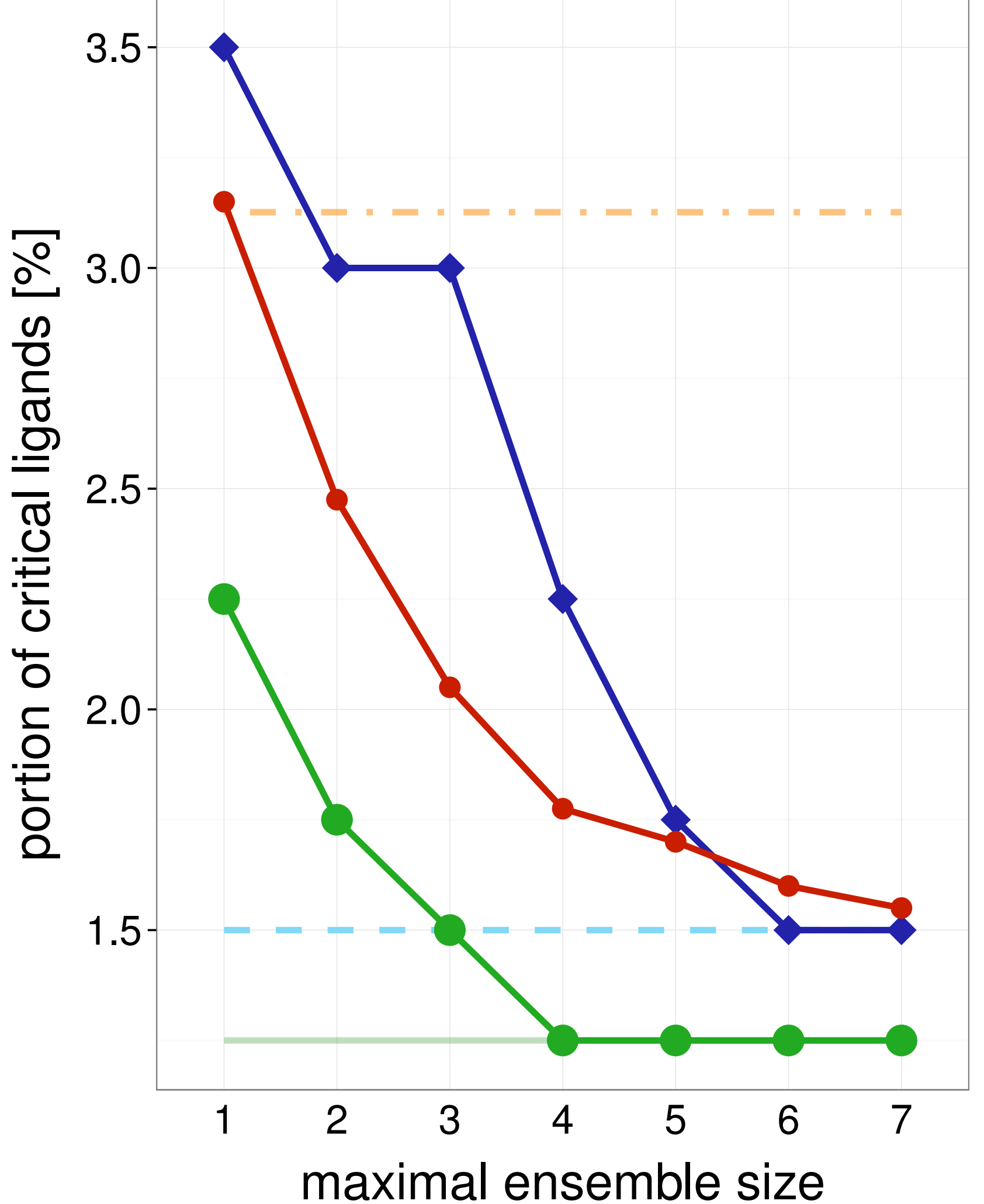

2E92

Training set

Average single structure

_ Best single structure

Whole ensemble

- SIENA

$\neg$ Clustering

$\multimap$ Random

Average single structure

_ _ Best single structure

Whole ensemble

- SIENA

$\neg$ Clustering

$\multimap$ Random

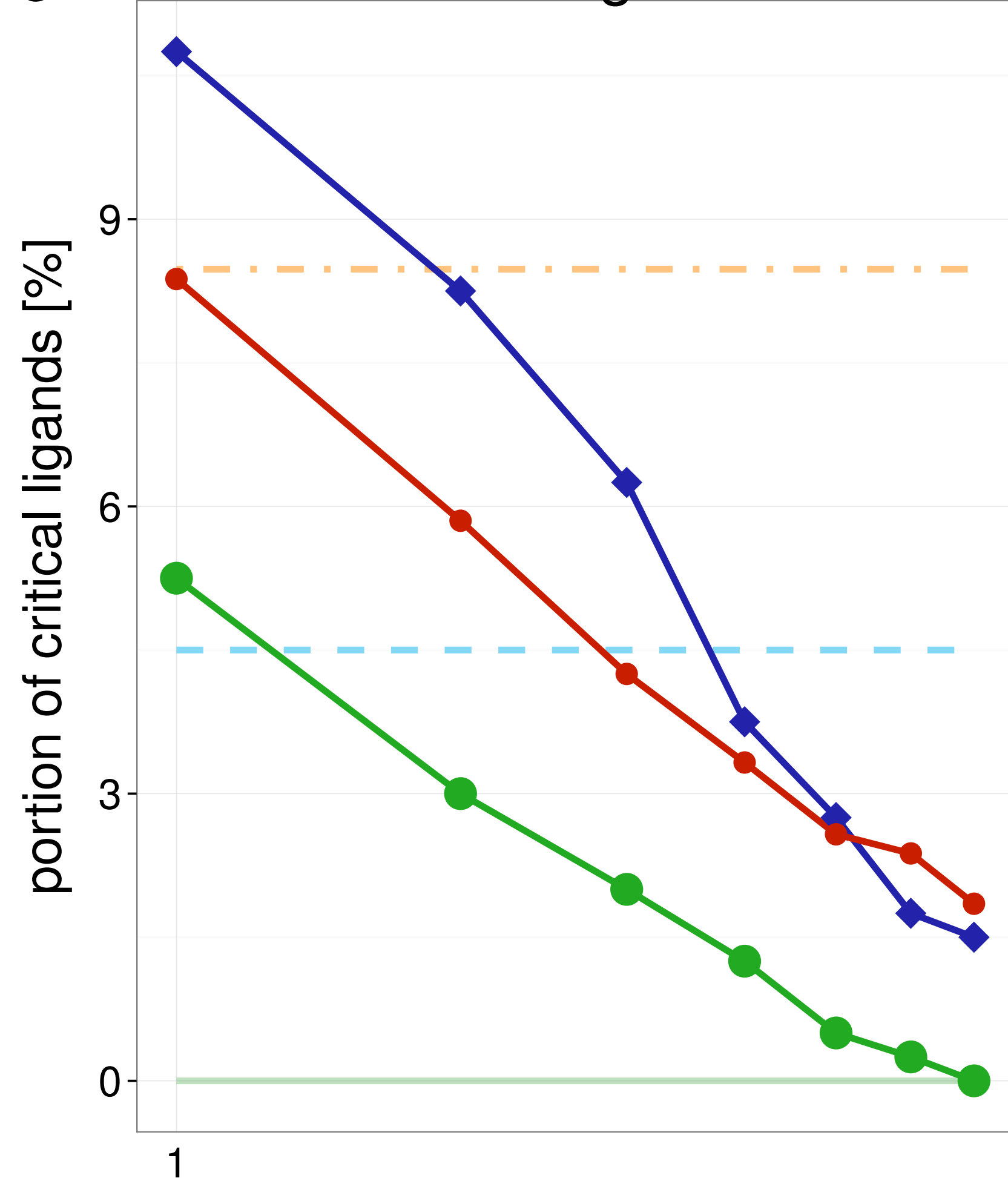

maximal ensemble size

Test set

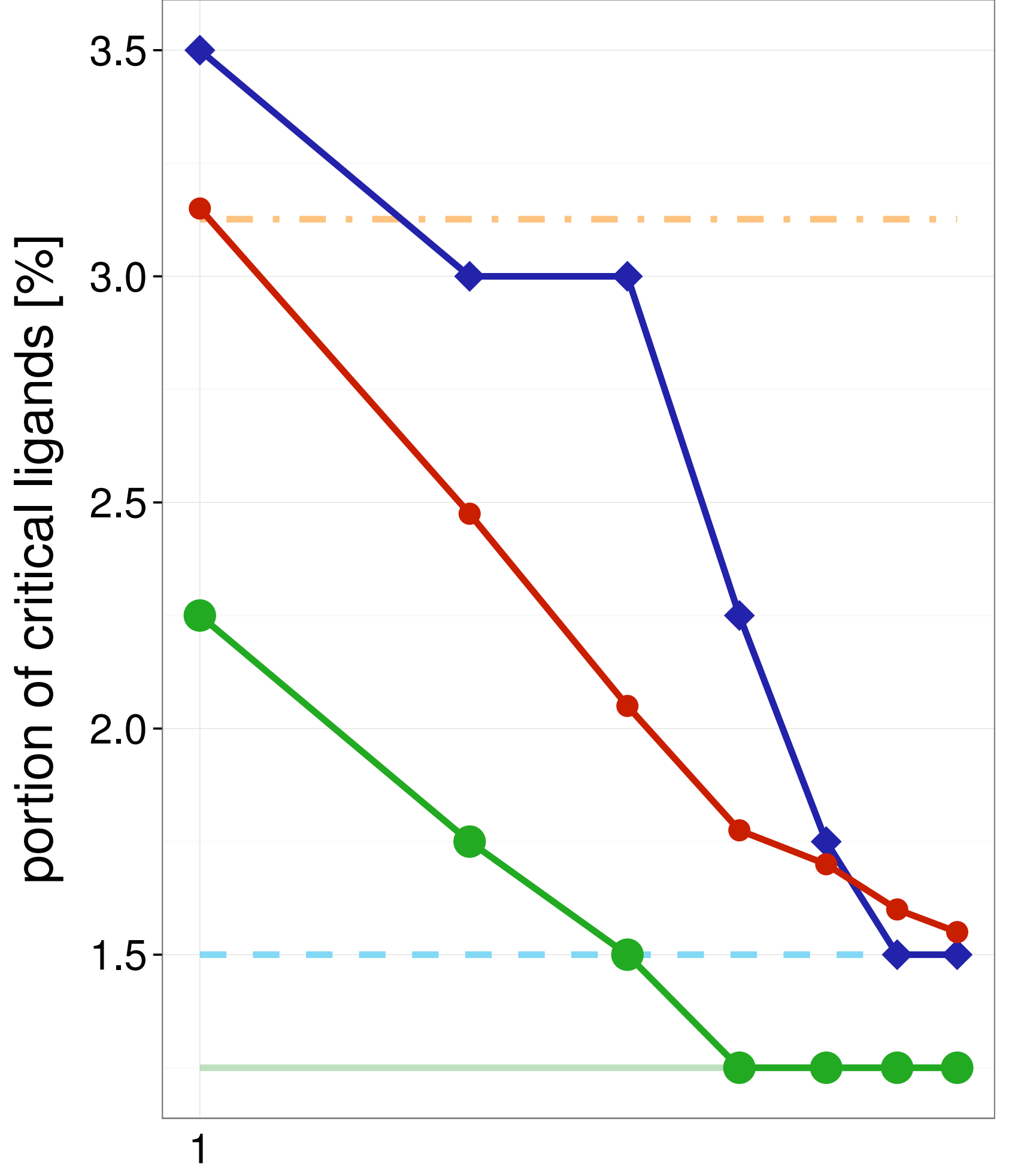

maximal ensemble size
Average single structure

_ - Best single structure

Whole ensemble

SIENA

$\leadsto$ Clustering

$\rightarrow$ Random 


\section{Training set}

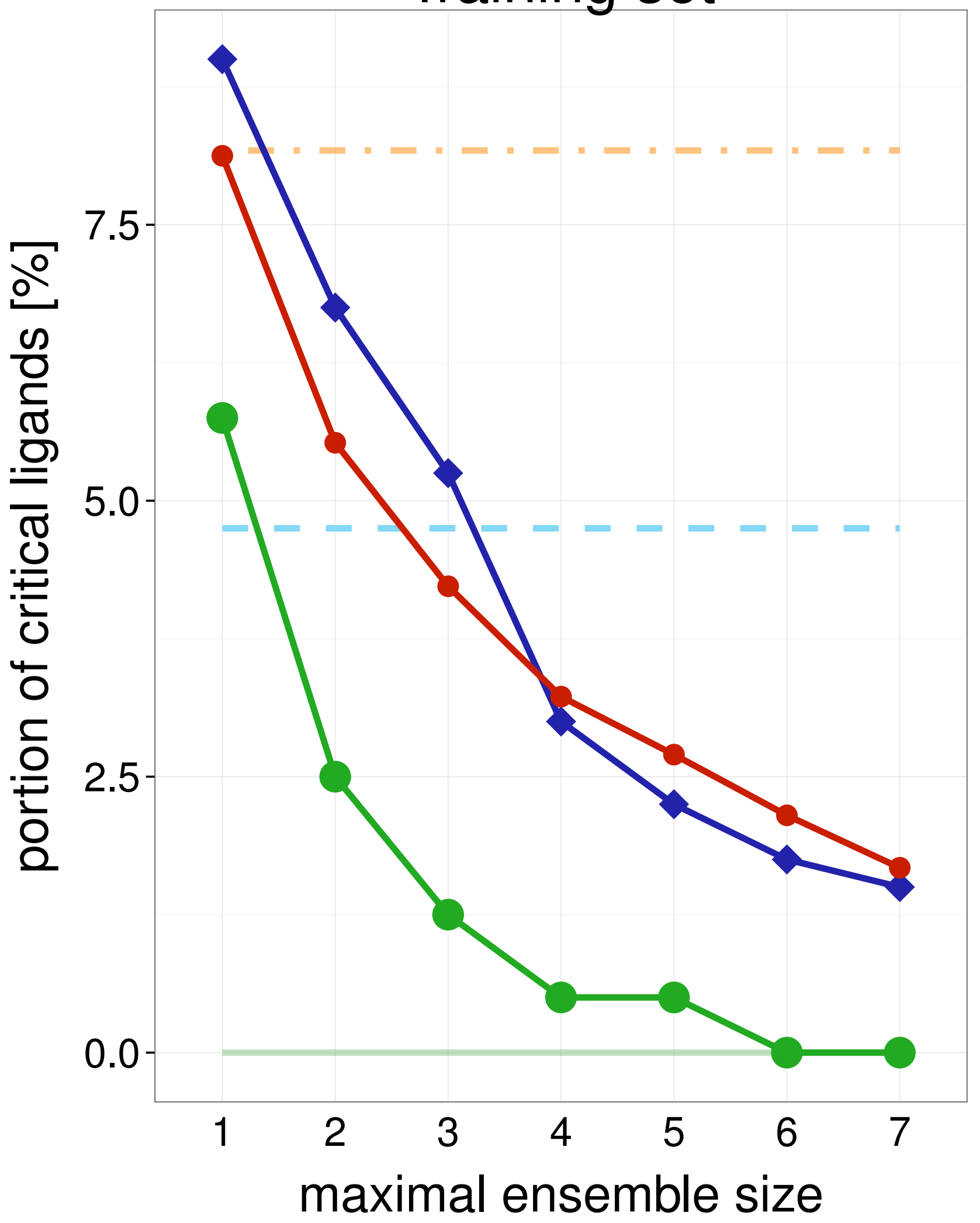

\section{Test set}

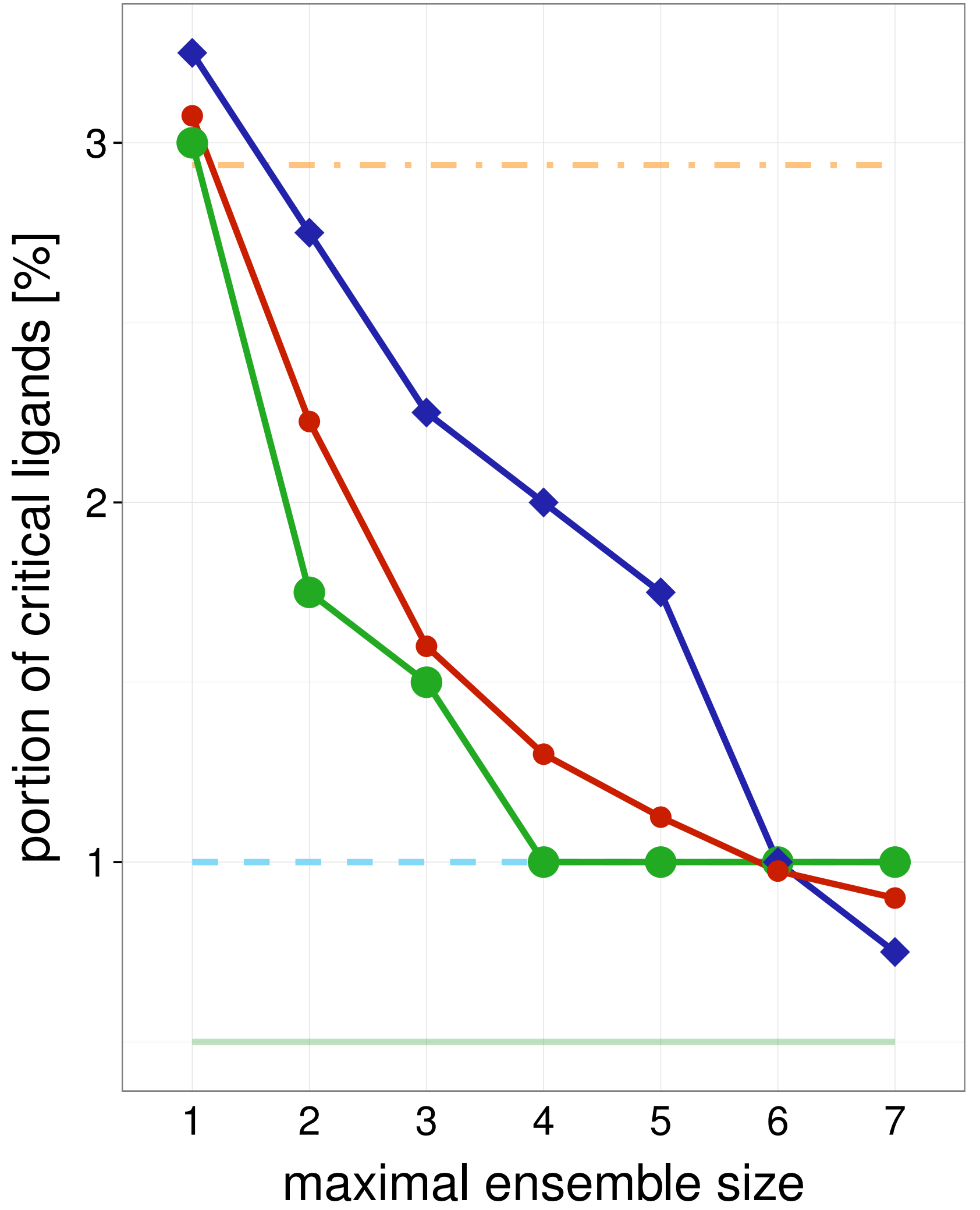

2HCT

Training set

Average single structure

- Best single structure

Whole ensemble

- SIENA

$\neg$ Clustering

$\longrightarrow$ Random

Average single structure

_ _ Best single structure

Whole ensemble

- SIENA

$\smile$ Clustering

$\multimap$ Random

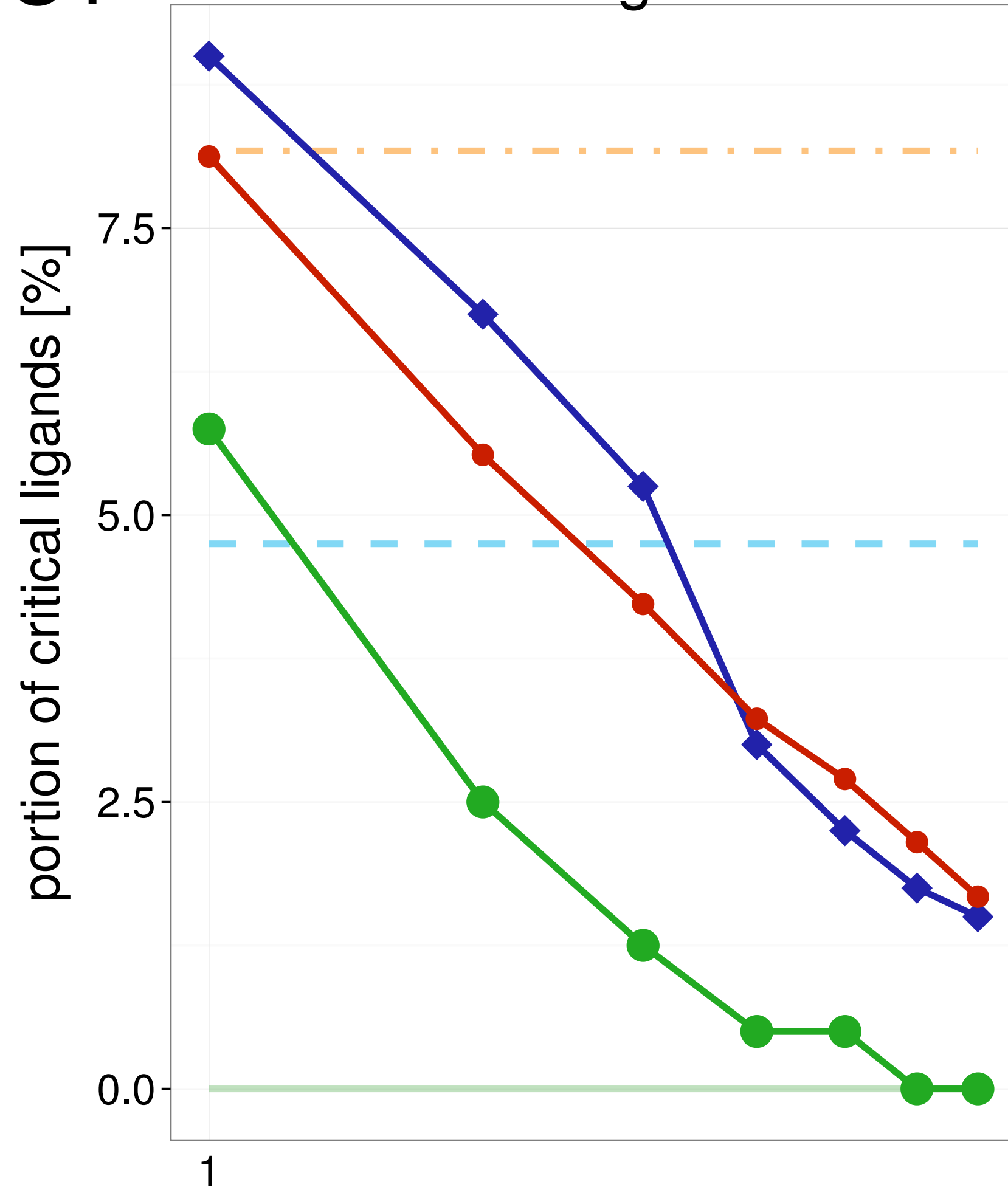

maximal ensemble size

Test set

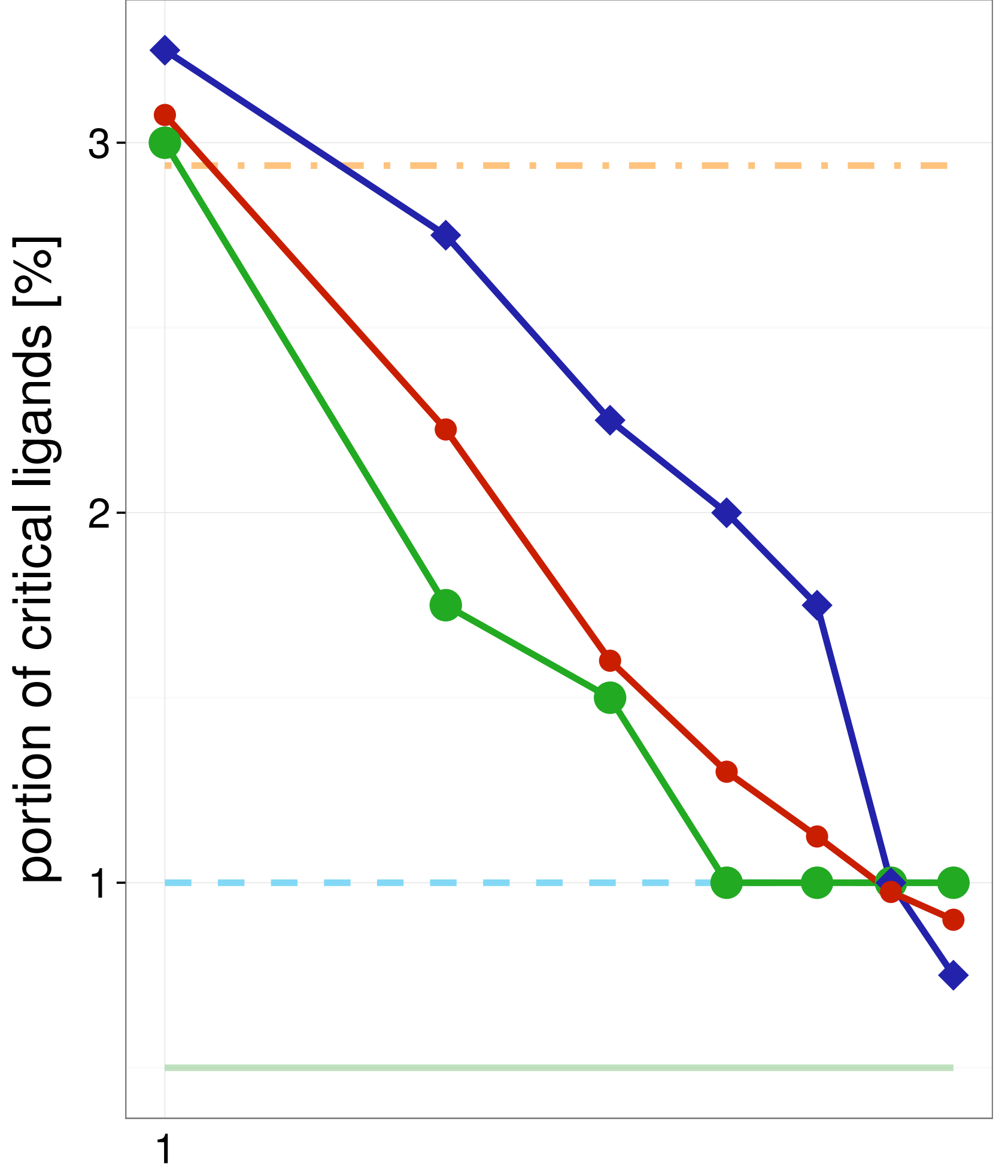

maximal ensemble size
Average single structure

_ _ Best single structure

Whole ensemble

SIENA

$\neg$ Clustering

$\multimap$ Random 
Training set

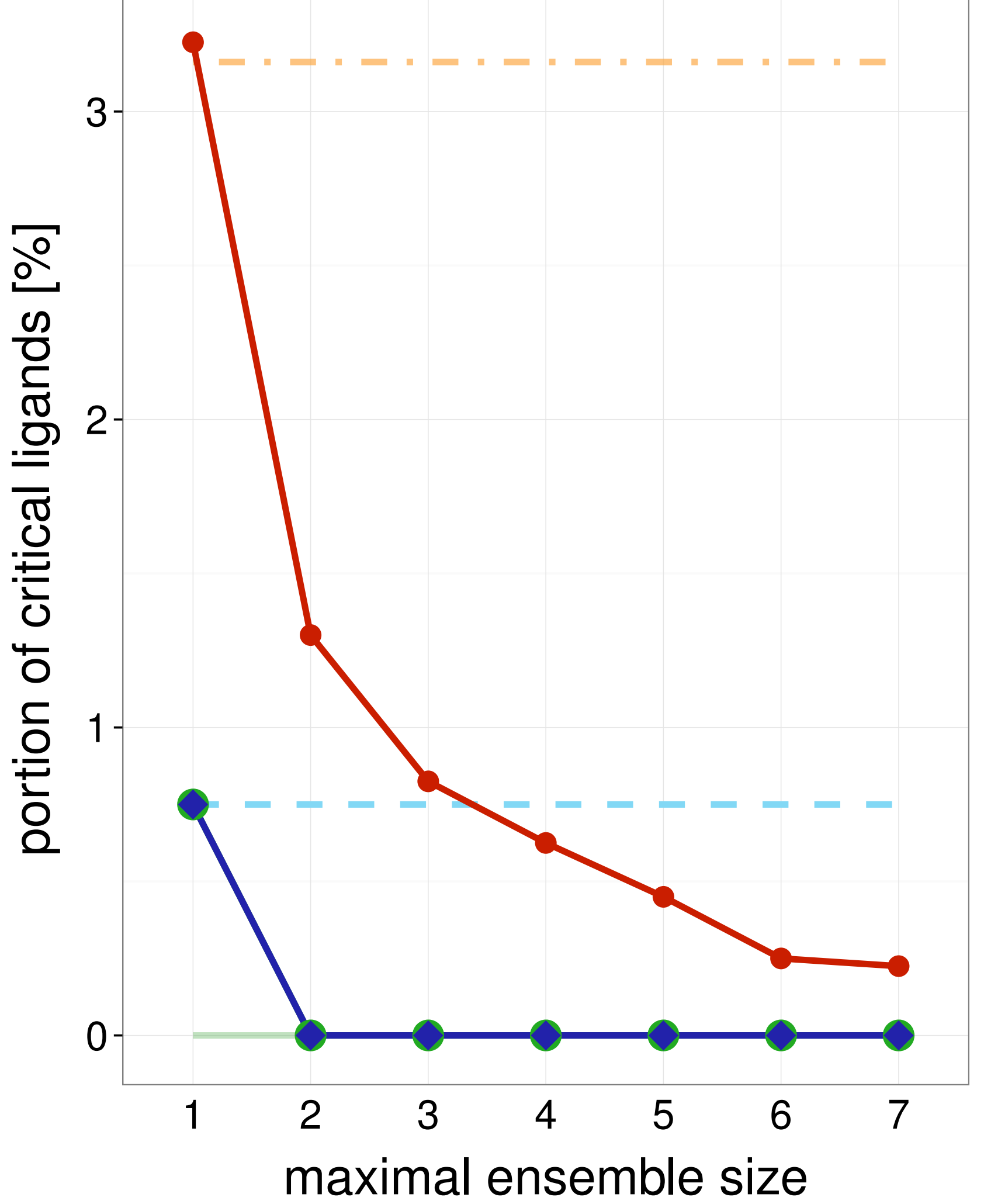

Test set

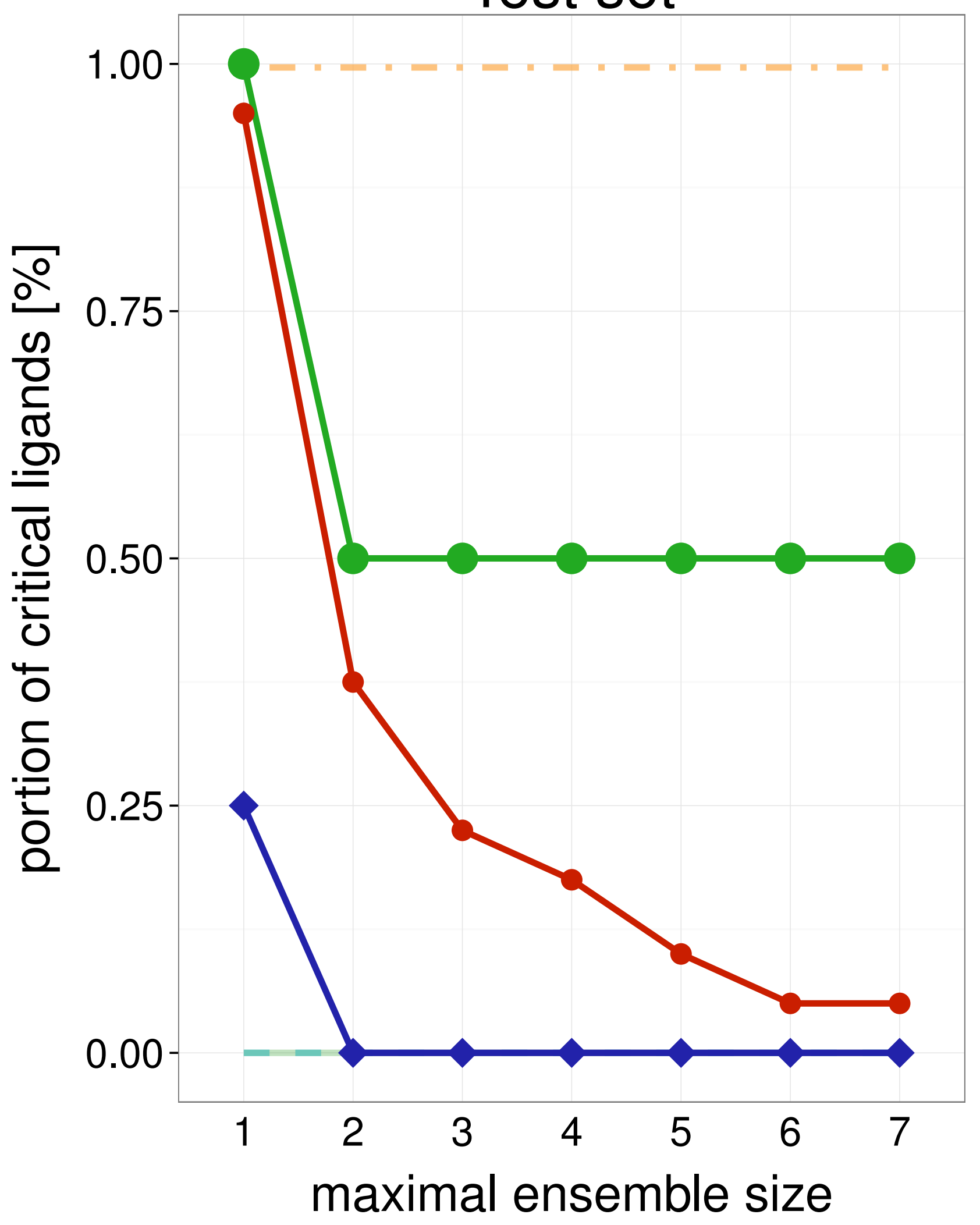

Average single structure

_ Best single structure

Whole ensemble

- SIENA

$\neg$ Clustering

$\multimap$ Random

Average single structure

_ _ Best single structure

Whole ensemble

- SIENA

$\smile$ Clustering

$\multimap$ Random
Training set

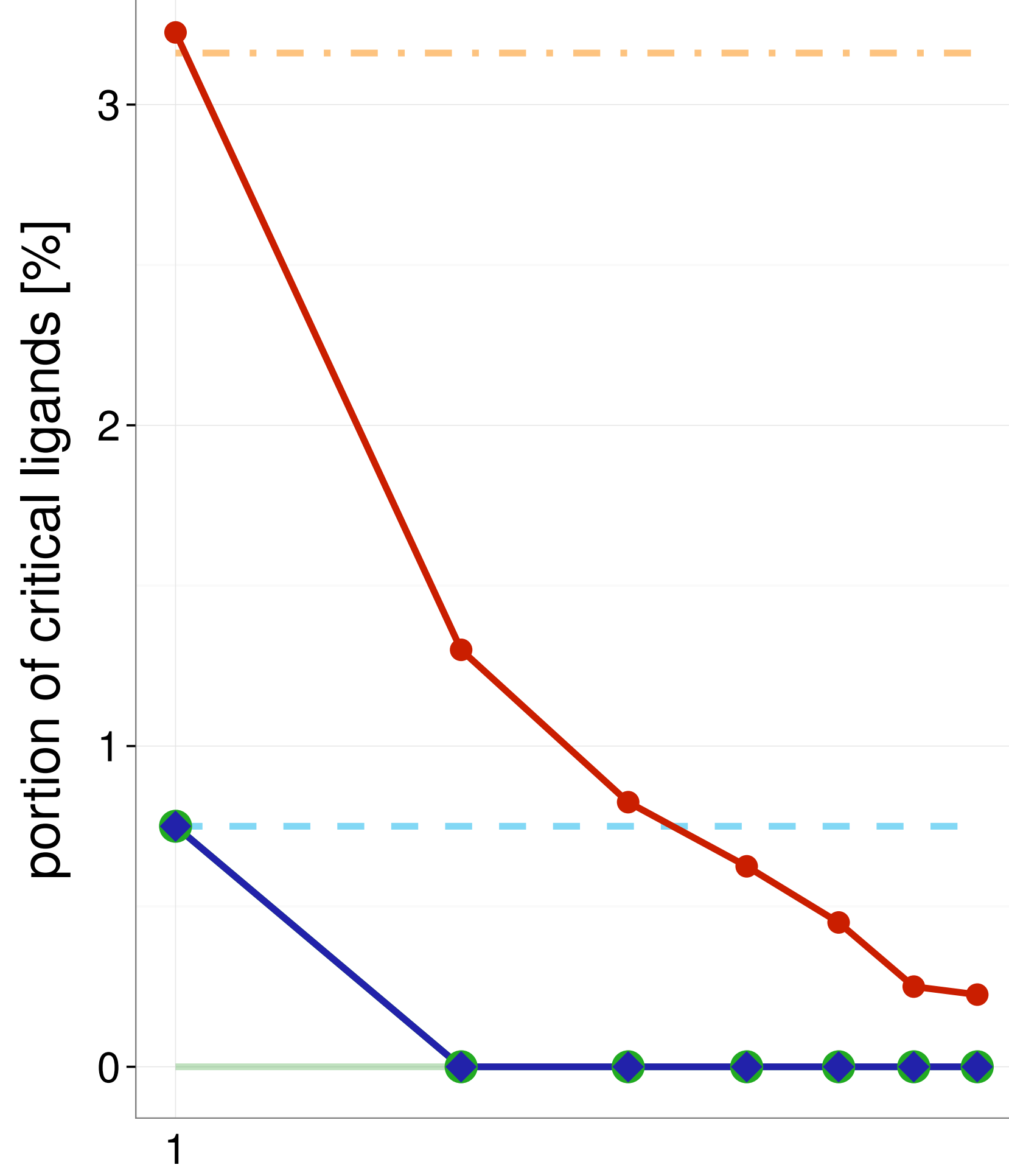

maximal ensemble size

Test set

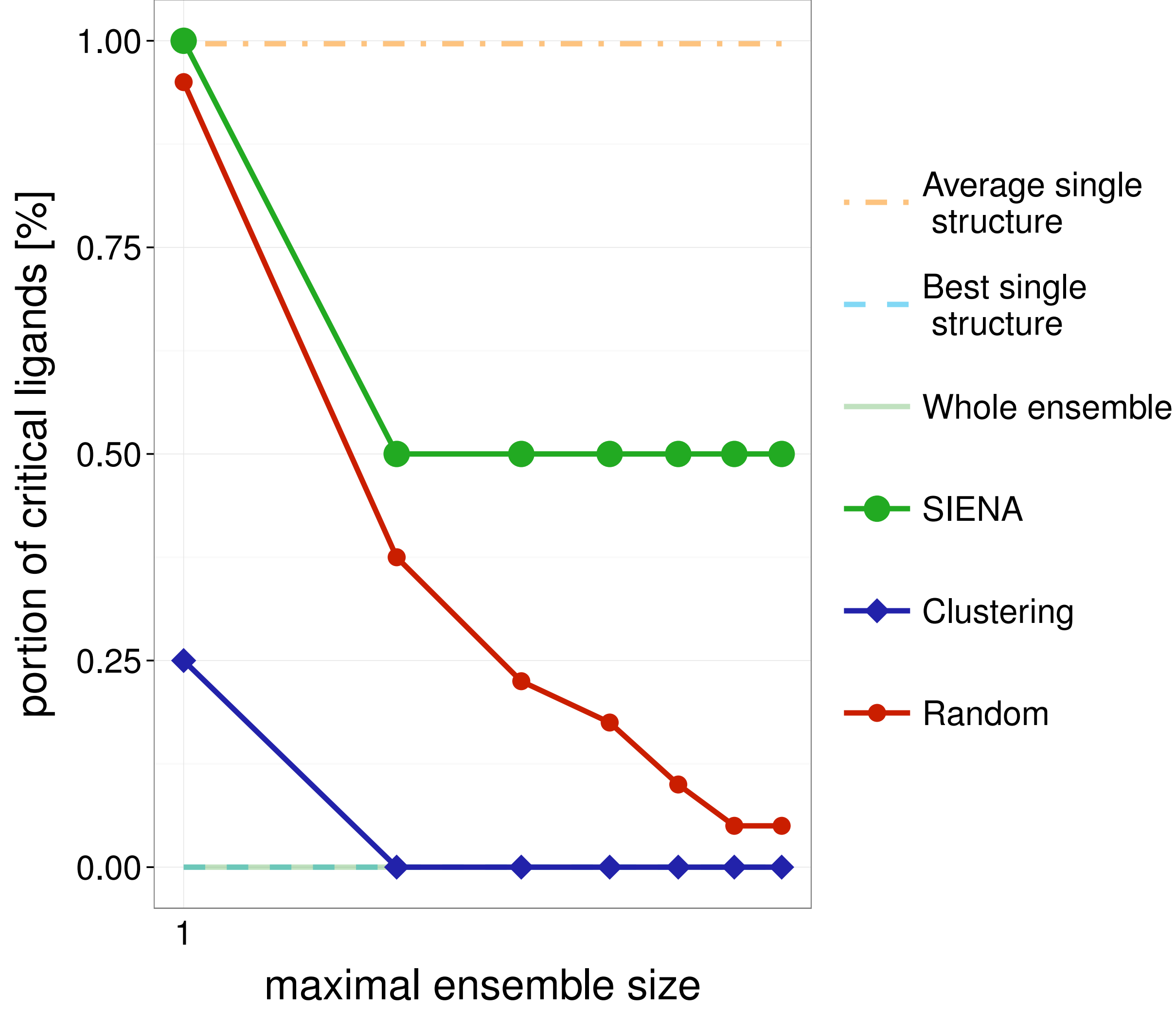


Training set

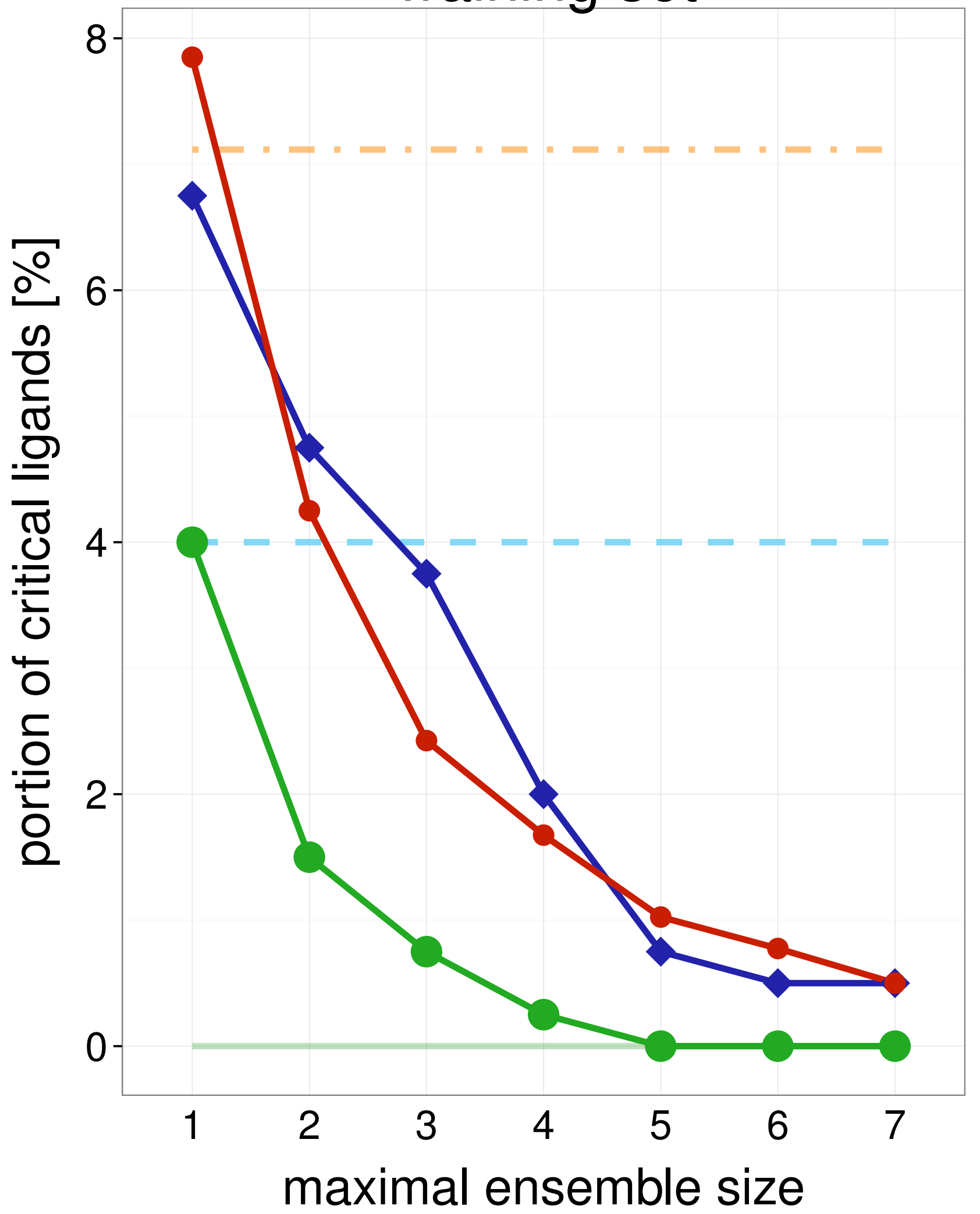

Test set

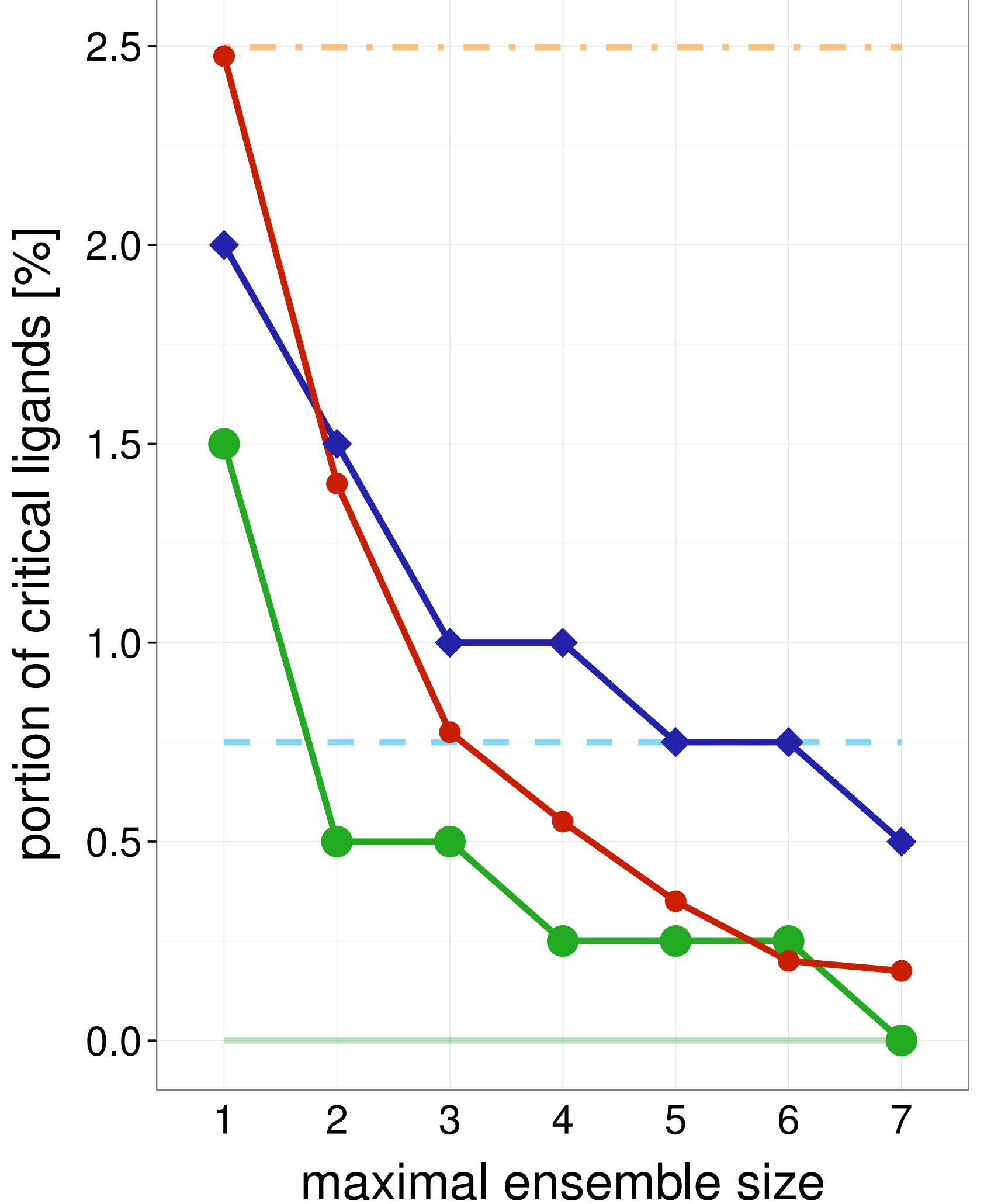

Average single structure

- Best single structure

Whole ensemble

- SIENA

$\smile$ Clustering

$\longrightarrow$ Random

Average single structure

_ Best single structure

Whole ensemble

- SIENA

$\smile$ Clustering

$\multimap$ Random
Training set

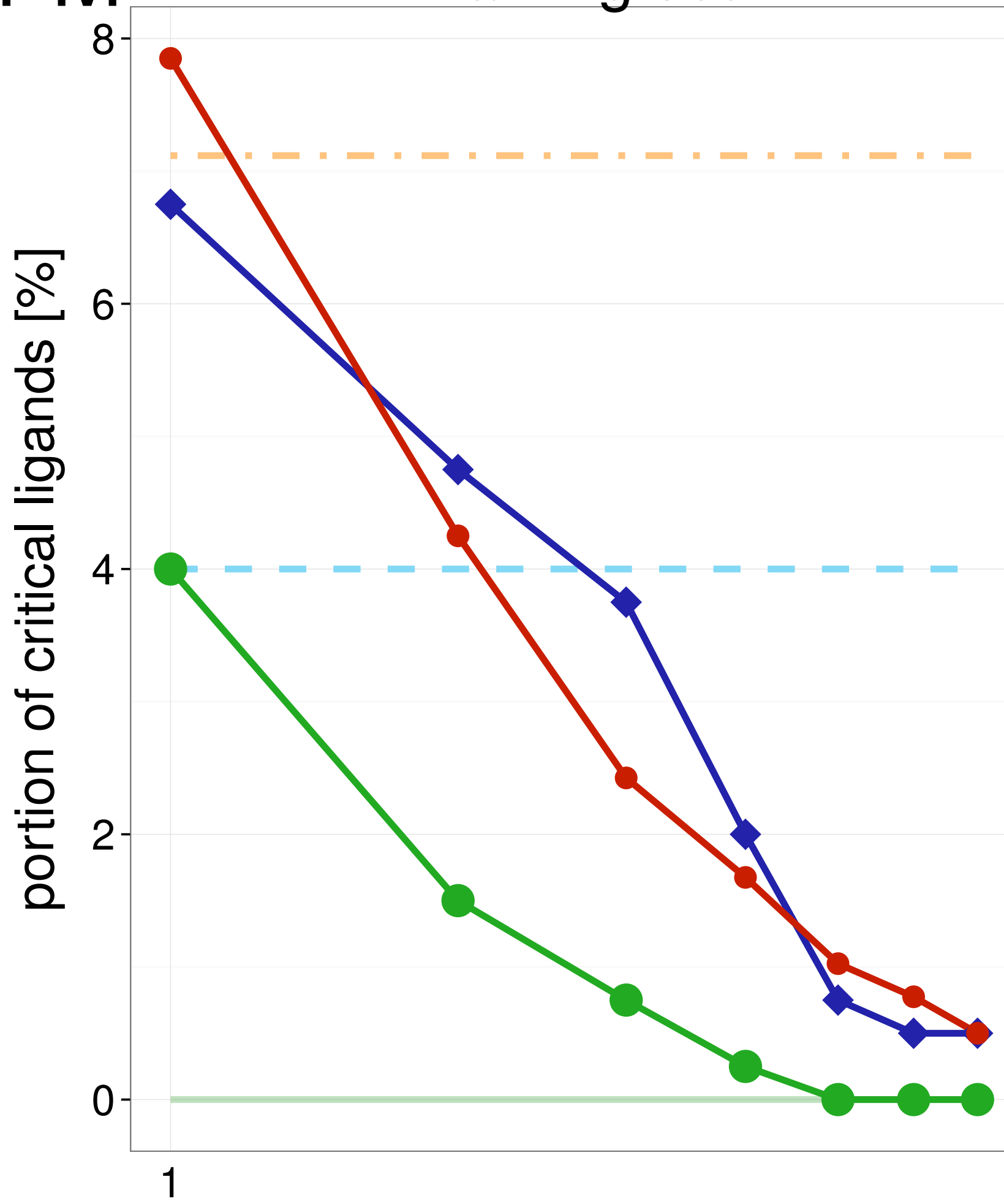

maximal ensemble size

Test set

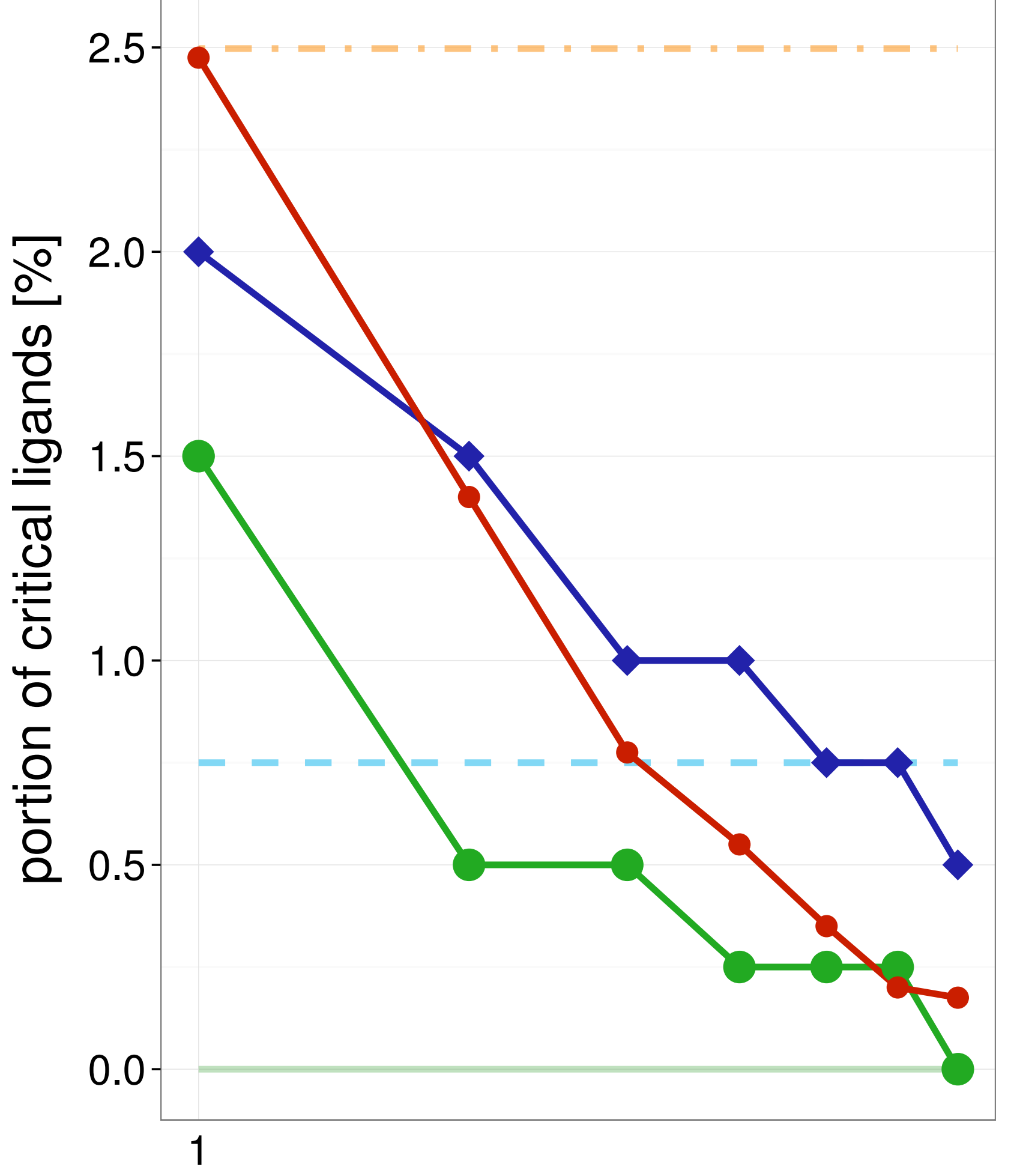

Average single structure

_ Best single structure

Whole ensemble

- SIENA

$\neg$ Clustering

$\multimap$ Random 


\section{Training set}

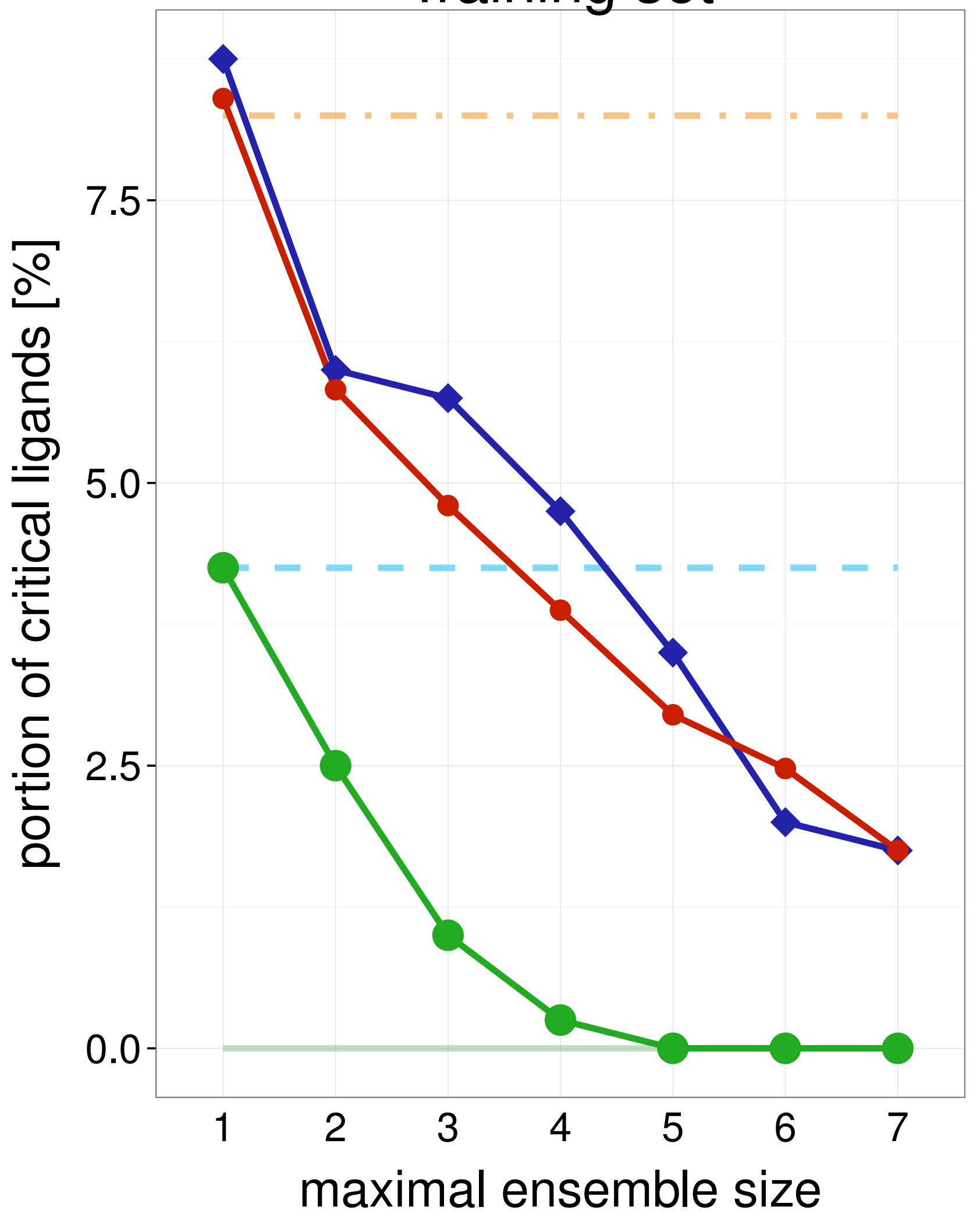

Test set

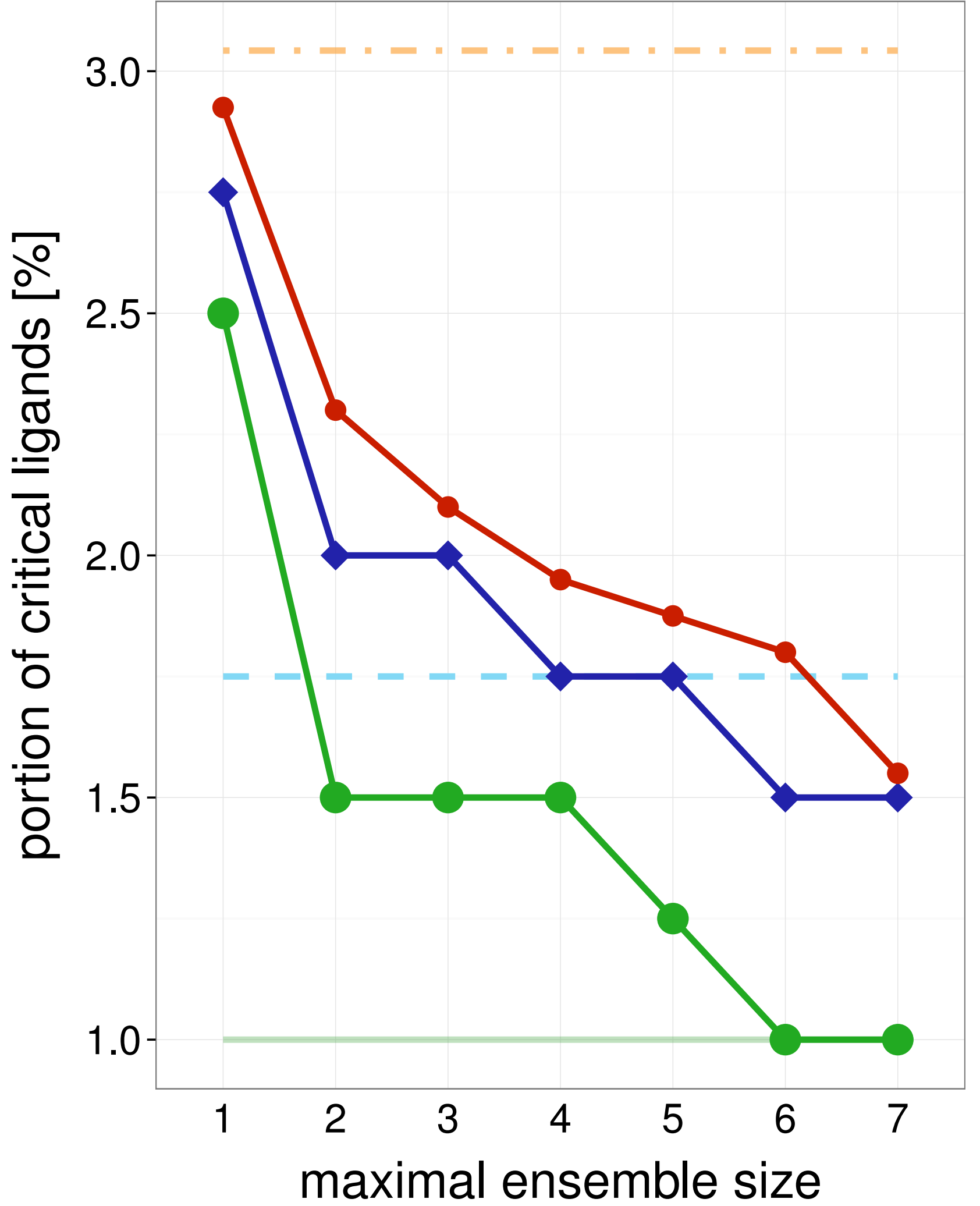

2UW6

Training set

Average single structure

_ Best single structure

Whole ensemble

- SIENA

$\neg$ Clustering

- Random

Average single structure

_ _ Best single structure

Whole ensemble

- SIENA

$\smile$ Clustering

$\because$ Random

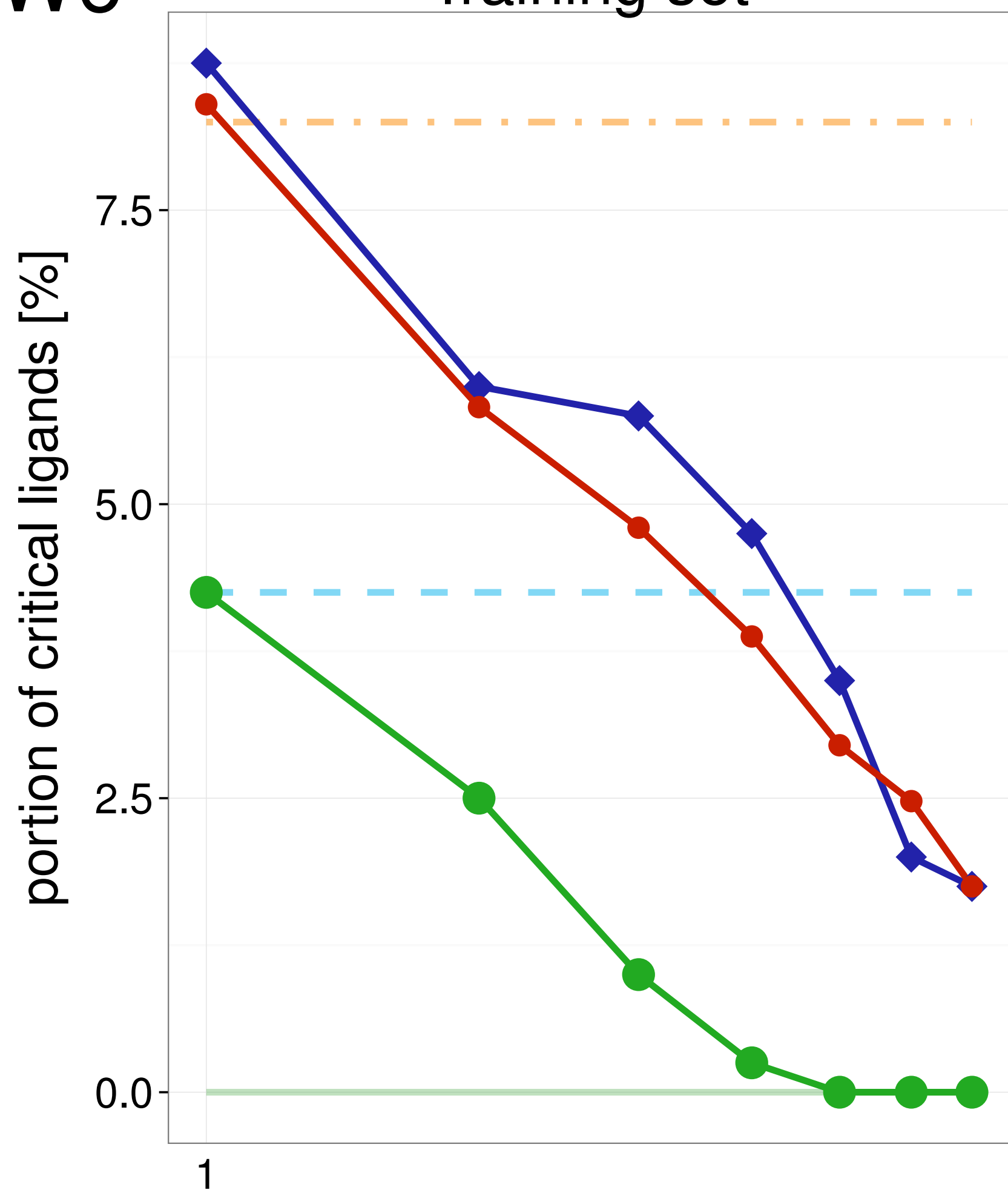

maximal ensemble size

Test set

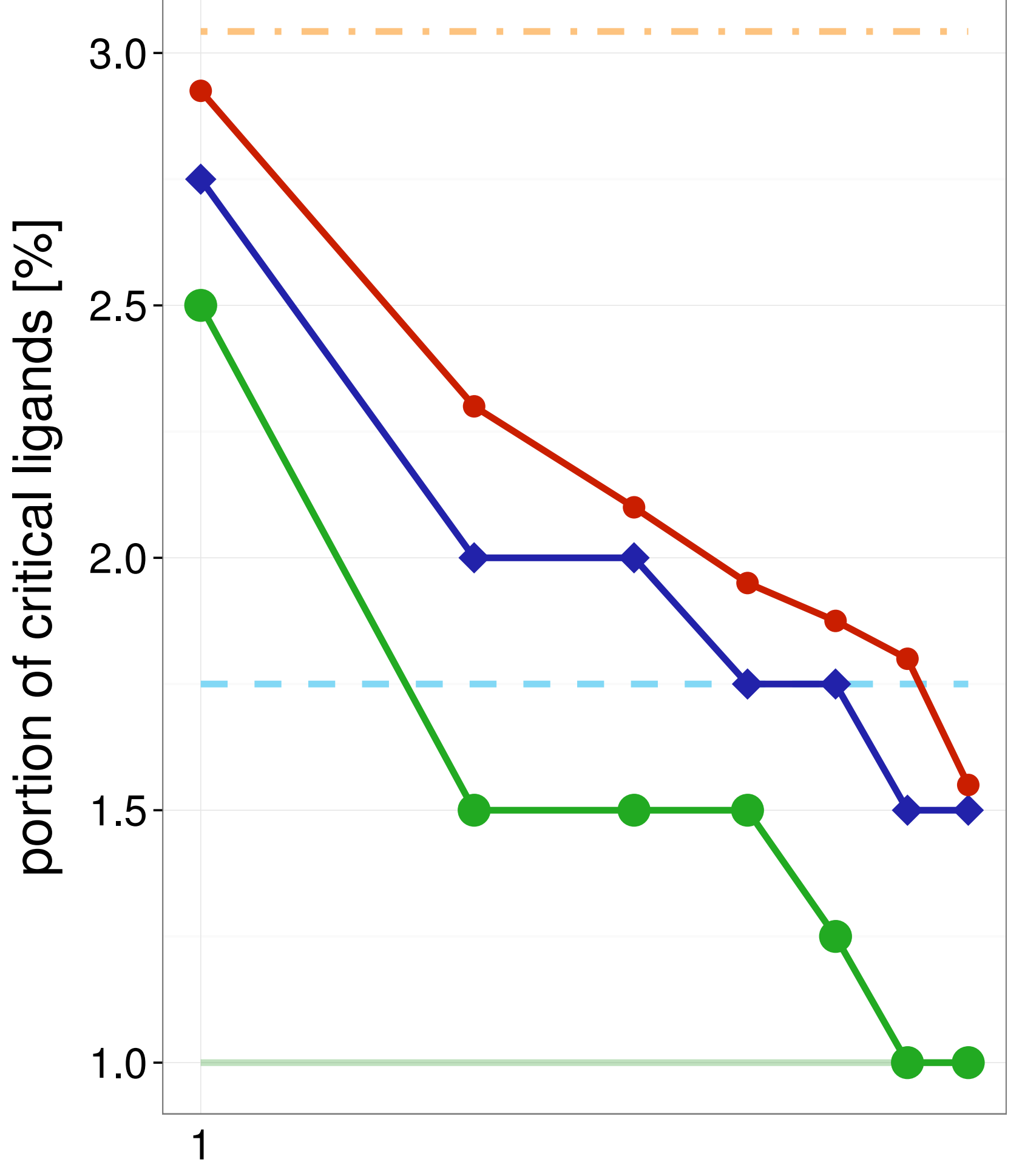

maximal ensemble size
Average single structure

_ _ Best single structure

Whole ensemble

SIENA

$\sim$ Clustering

$\multimap$ Random 
Training set

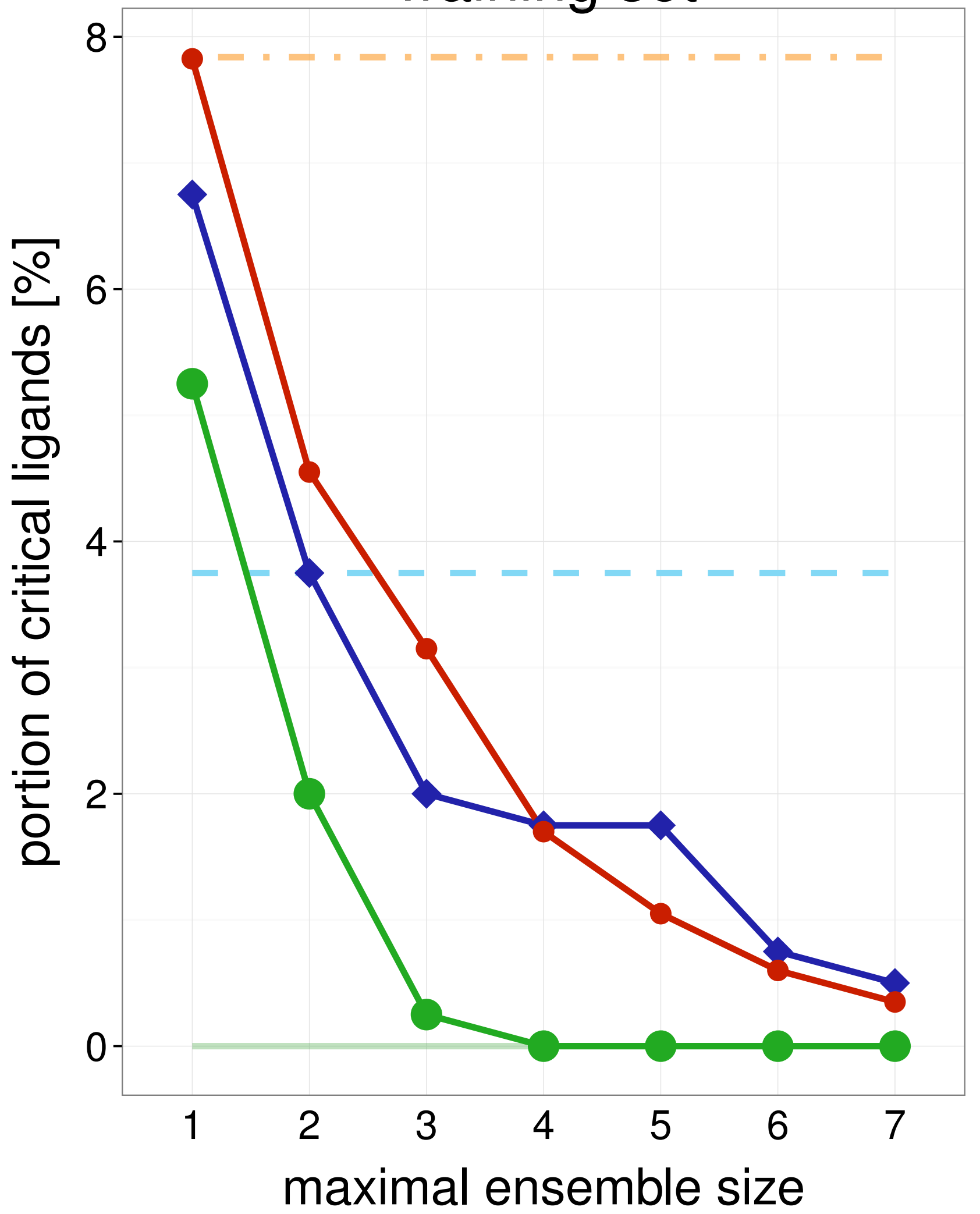

Test set

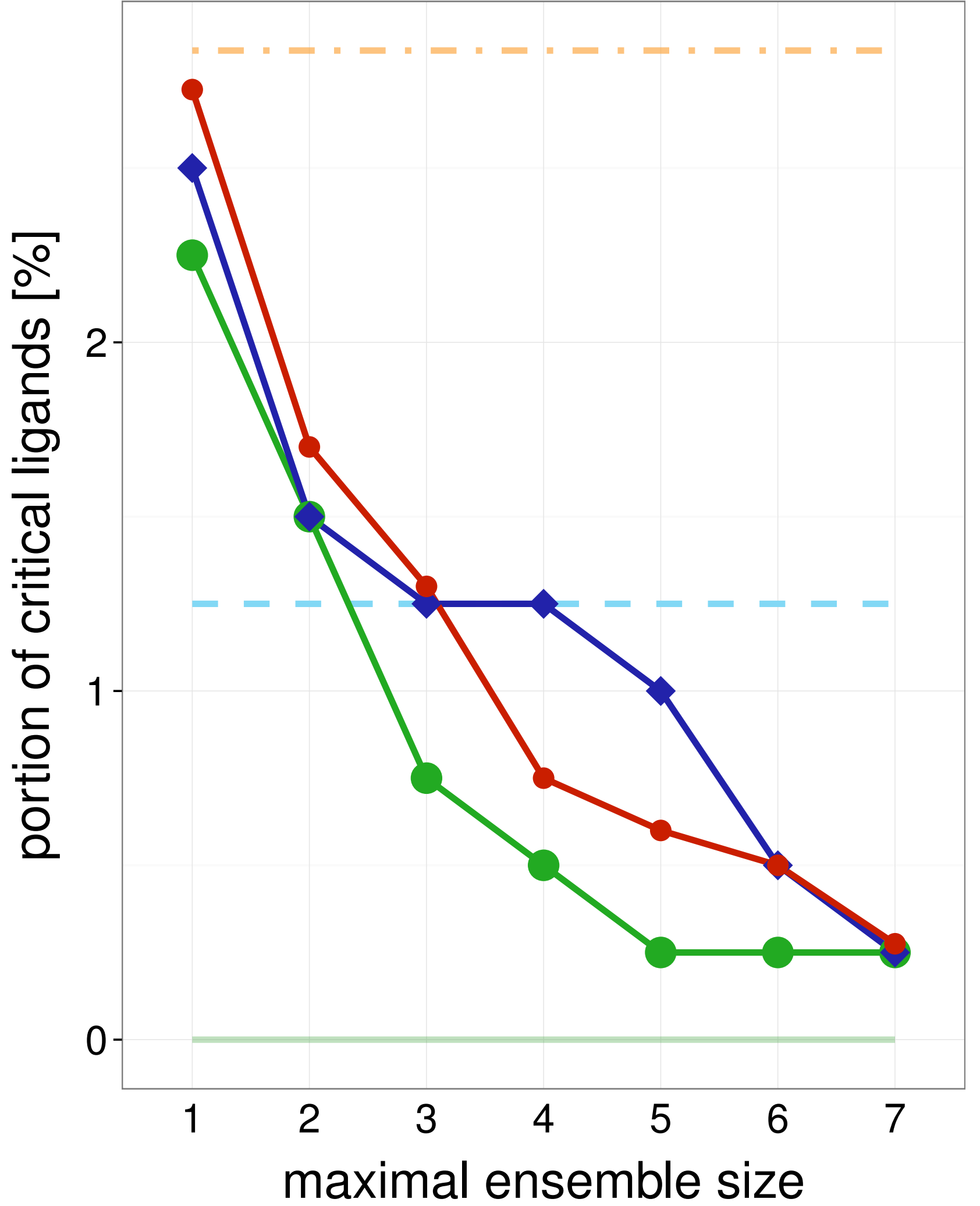

Average single structure

- Best single structure

Whole ensemble

- SIENA

$\neg$ Clustering

$\multimap$ Random

Average single structure

_ _ Best single structure

Whole ensemble

- SIENA

$\neg$ Clustering

- Random
$2 \mathrm{WQO}$

Training set

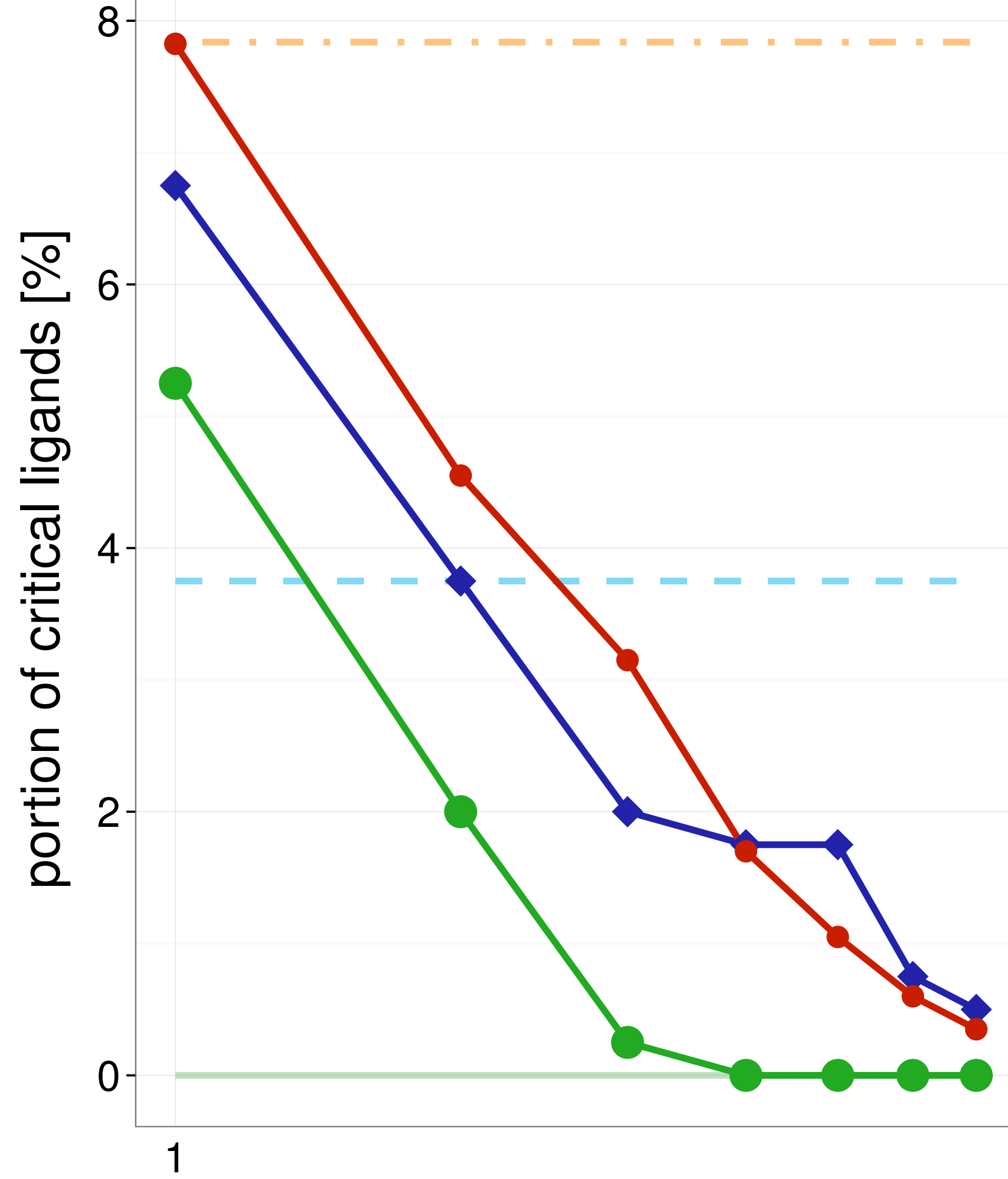

Average single structure

Best single structure

Whole ensemble

- SIENA

$\checkmark$ Clustering

$\because$ Random

maximal ensemble size

Test set

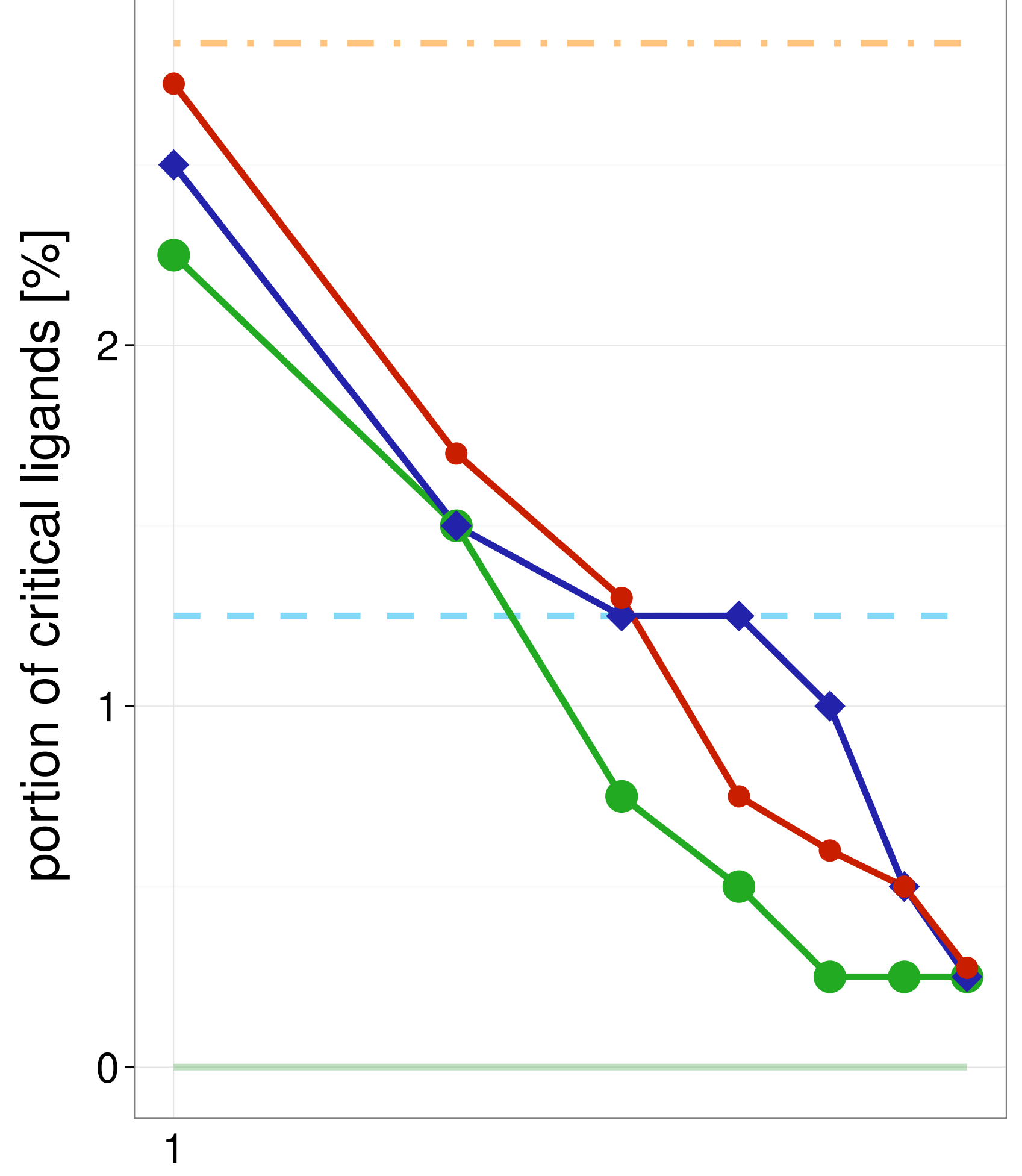

Average single structure

_ Best single structure

Whole ensemble

SIENA

$\neg$ Clustering

$\multimap$ Random 
Training set

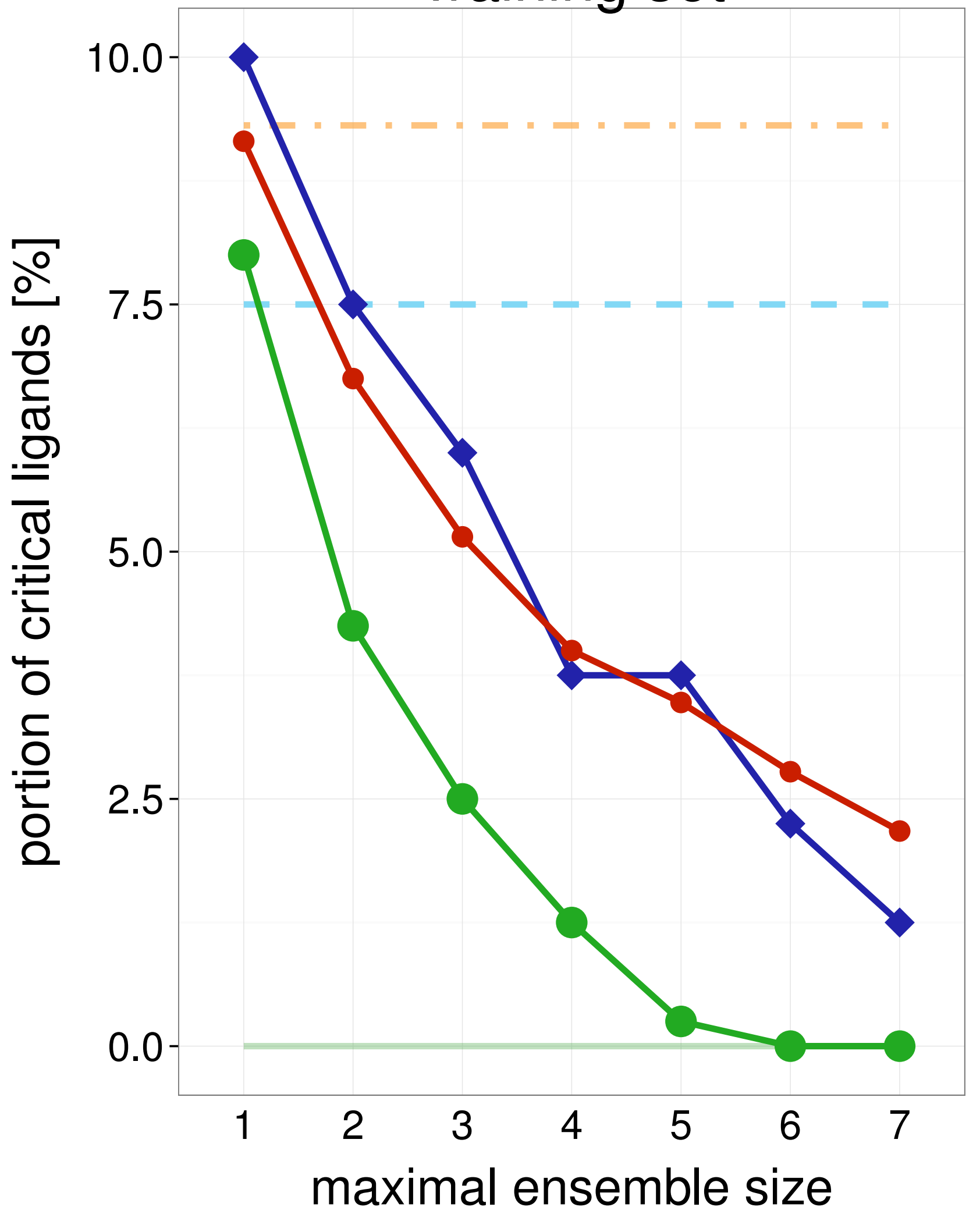

Test set

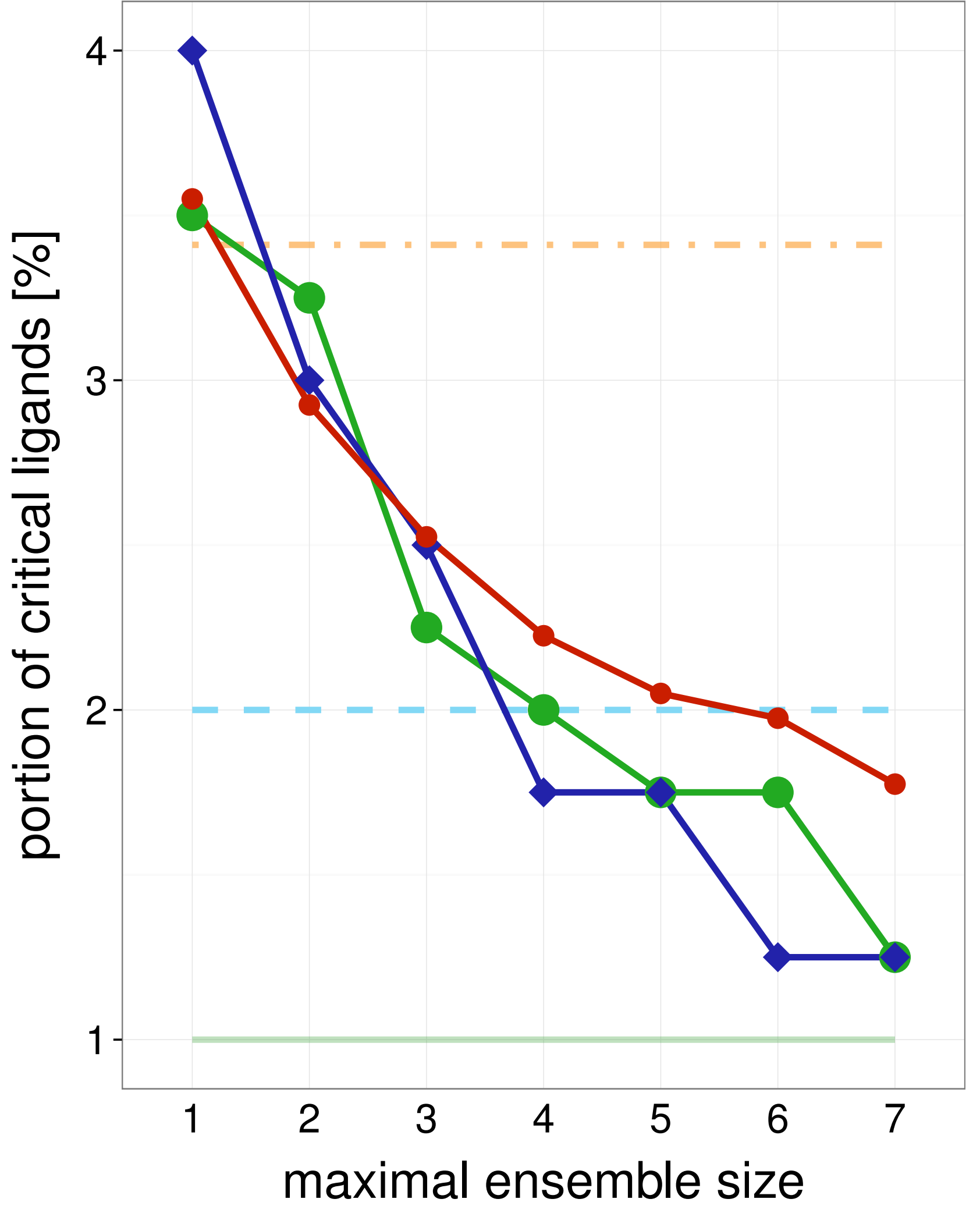

Average single structure

_ _ Best single structure

Whole ensemble

- SIENA

$\neg$ Clustering

$\multimap$ Random

Average single structure

_ Best single structure

Whole ensemble

- SIENA

$\neg$ Clustering

$\multimap$ Random
Training set

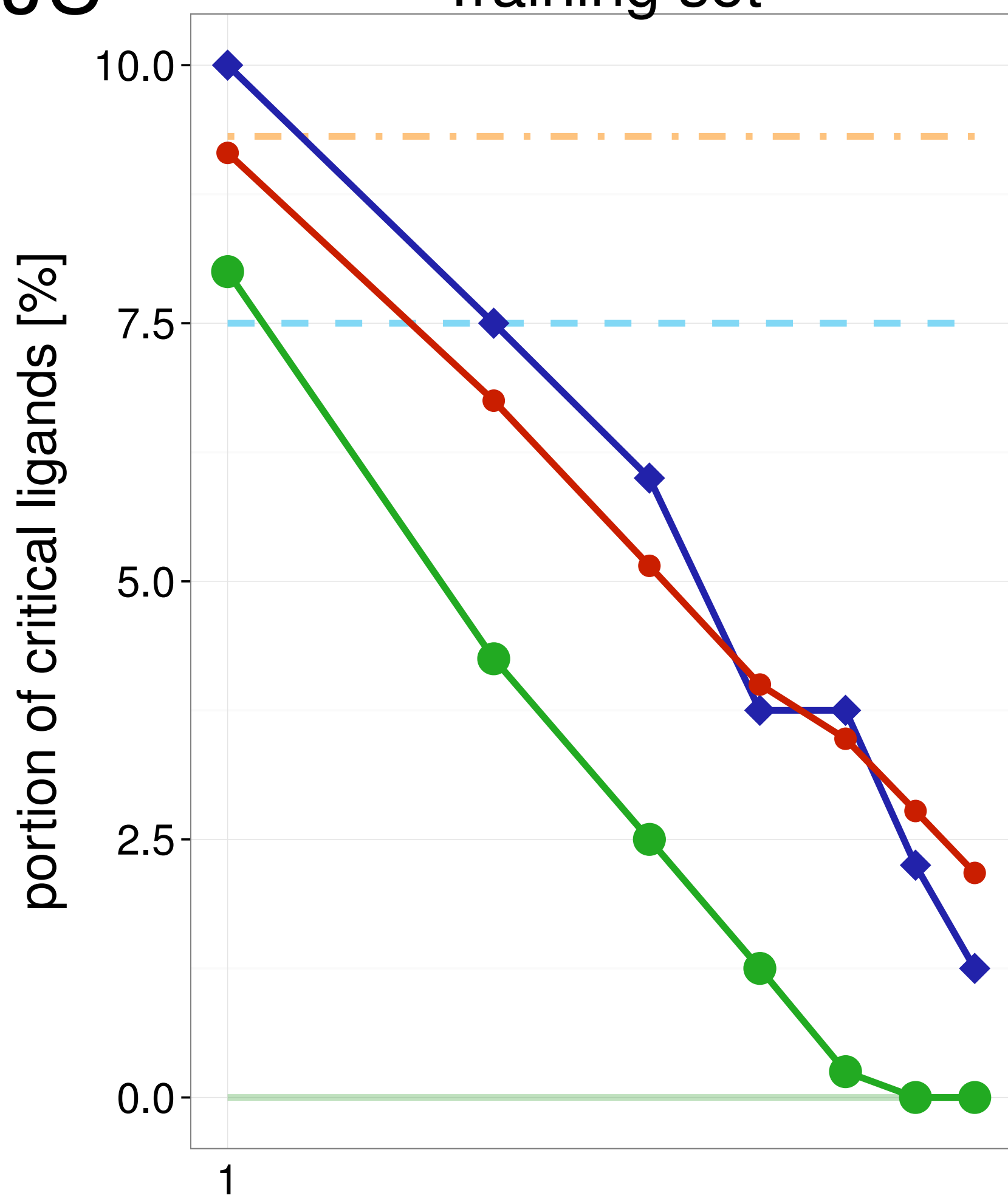

maximal ensemble size

Test set

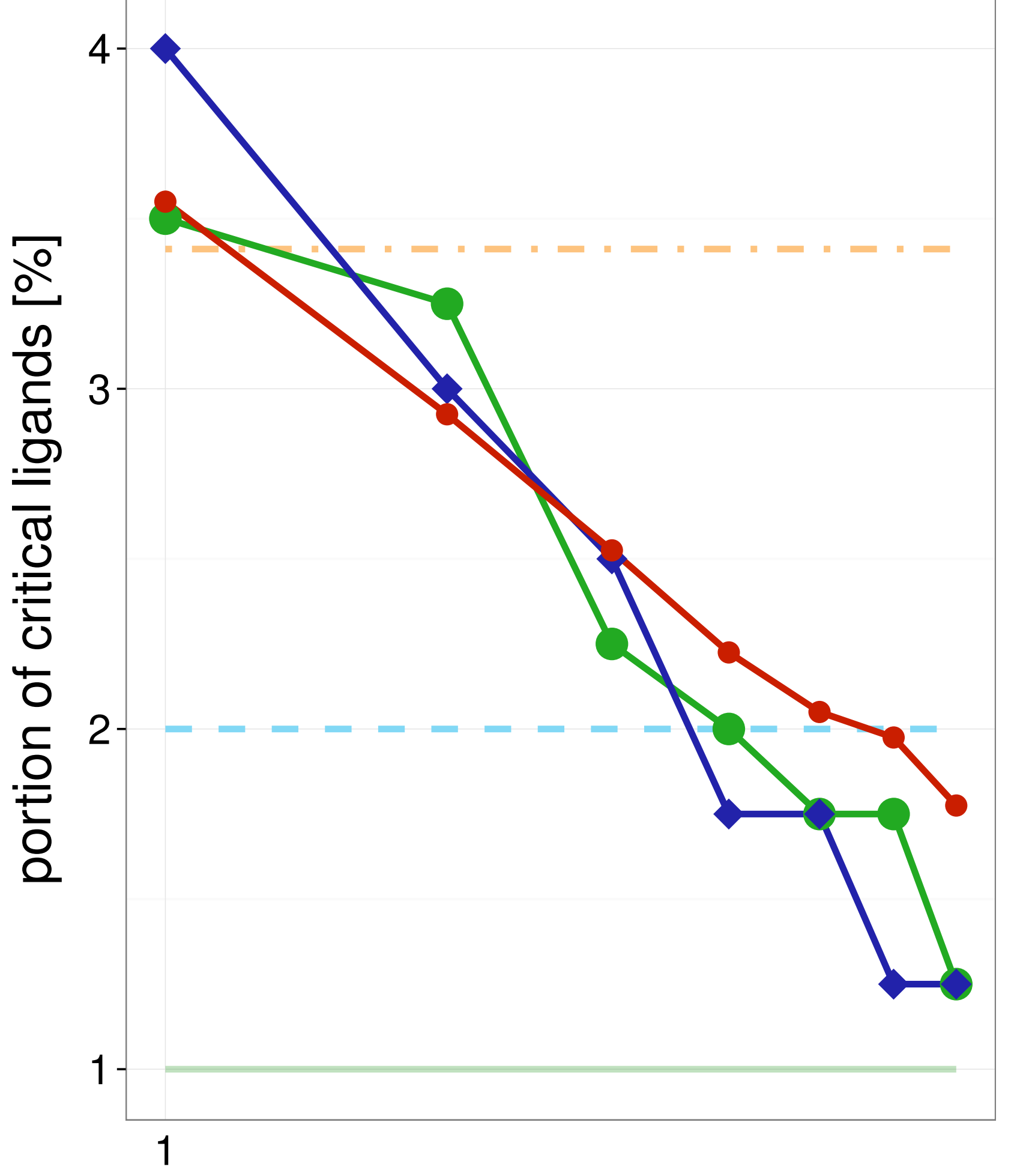

maximal ensemble size
Average single structure

- Best single structure

Whole ensemble

SIENA

$\neg$ Clustering

$\multimap$ Random 
Training set

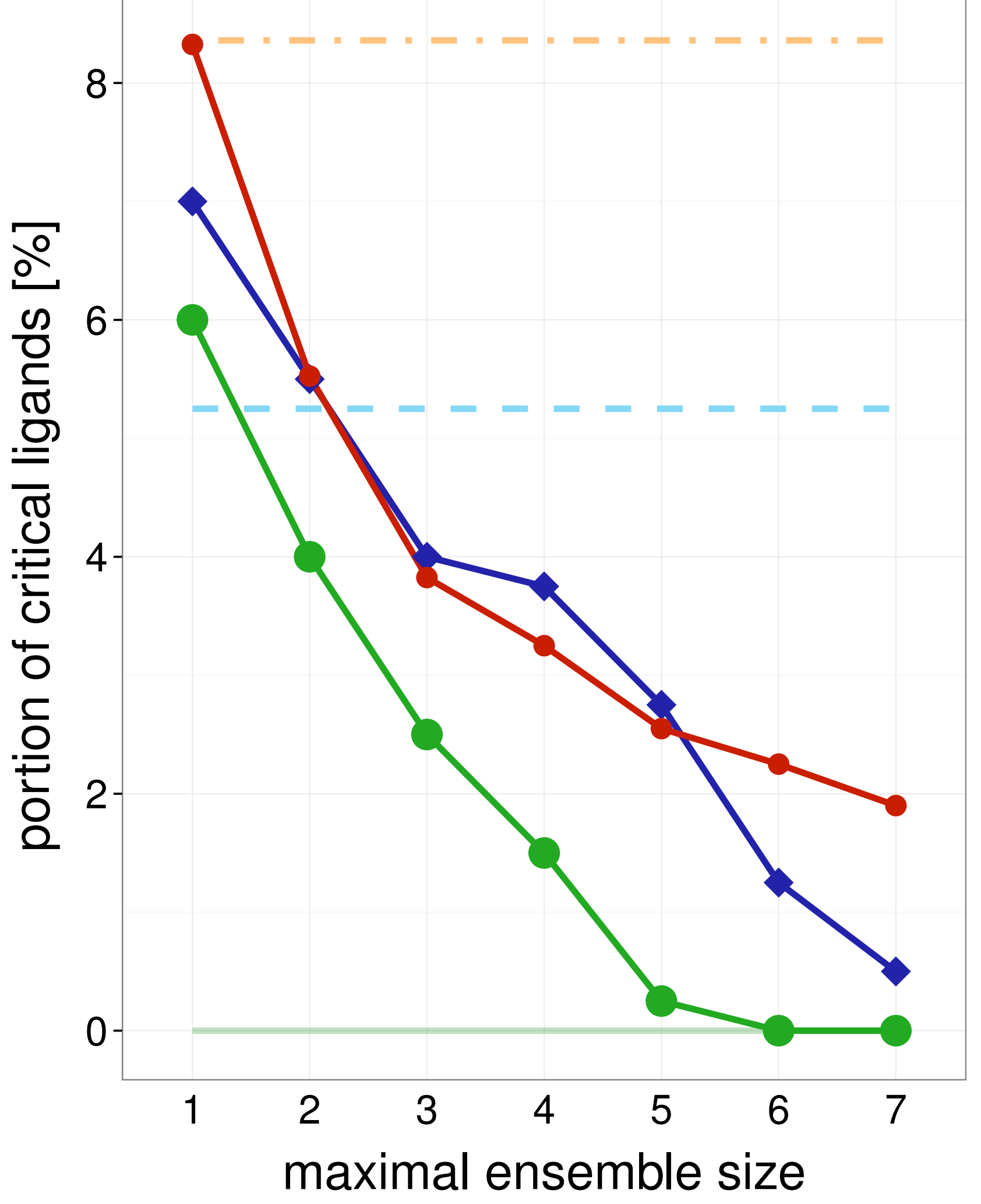

Test set

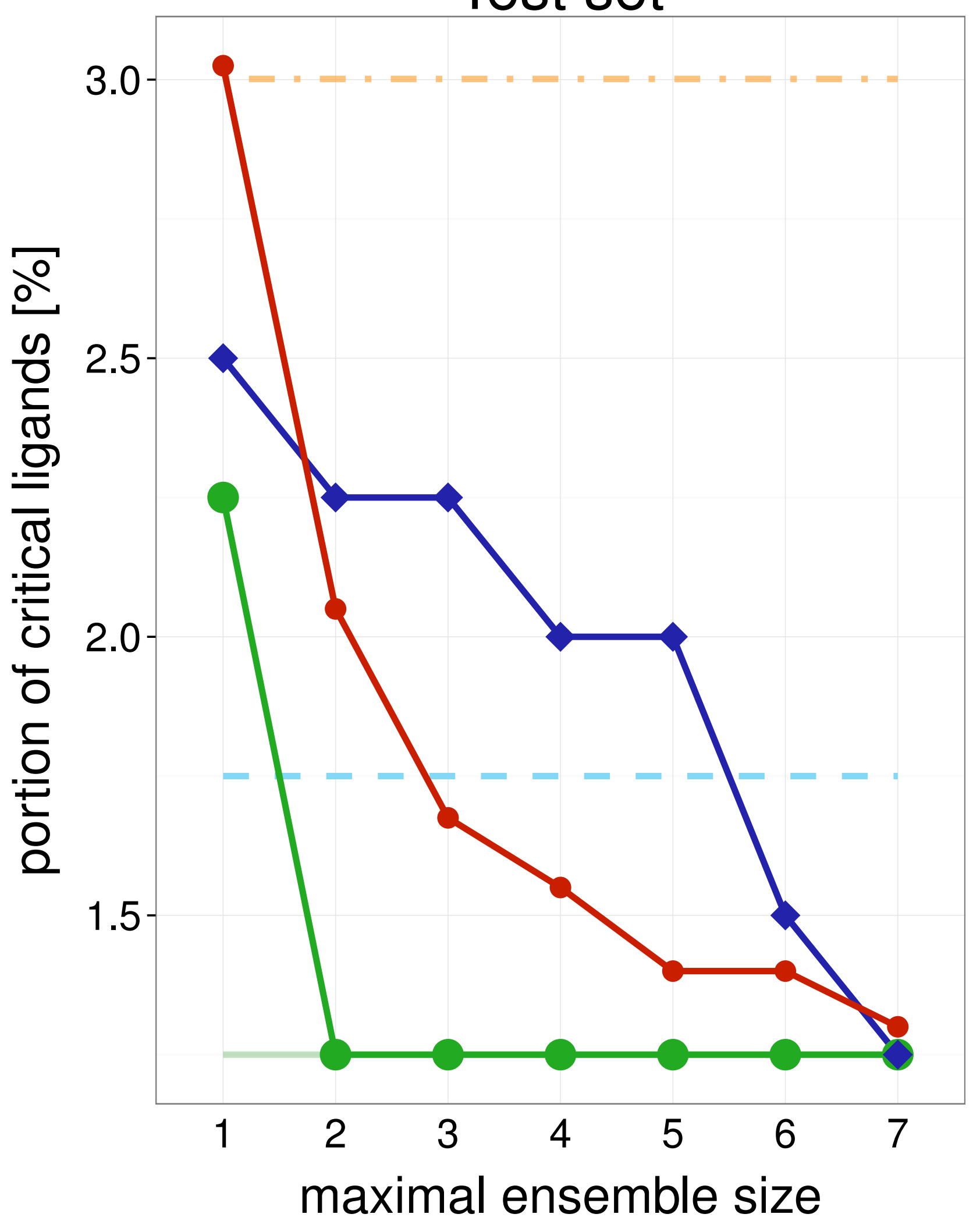

Average single structure

_ Best single structure

Whole ensemble

- SIENA

$\neg$ Clustering

$\multimap$ Random

Average single structure

_ _ Best single structure

Whole ensemble

- SIENA

$\neg$ Clustering

- Random
Training set

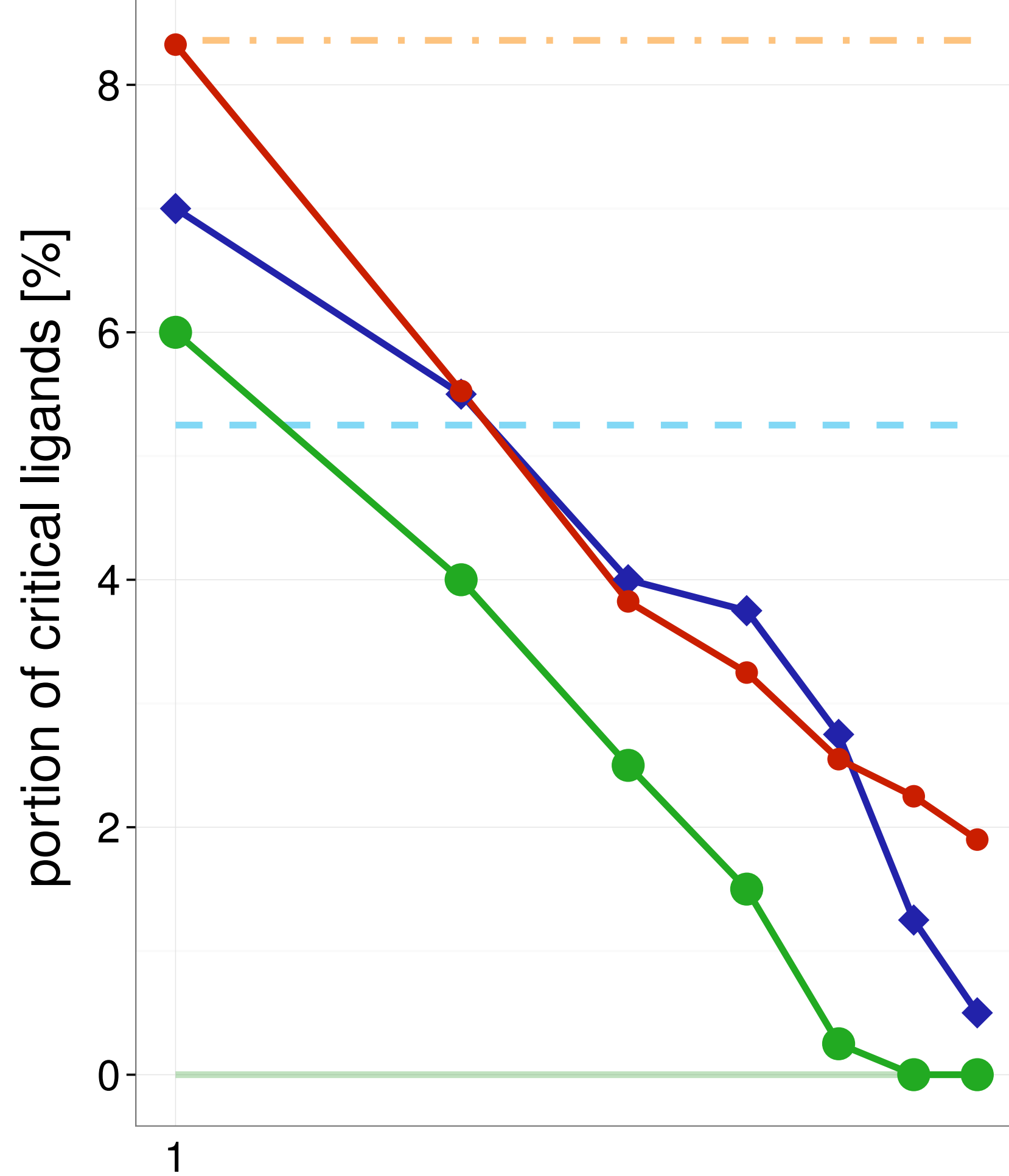

maximal ensemble size

Test set

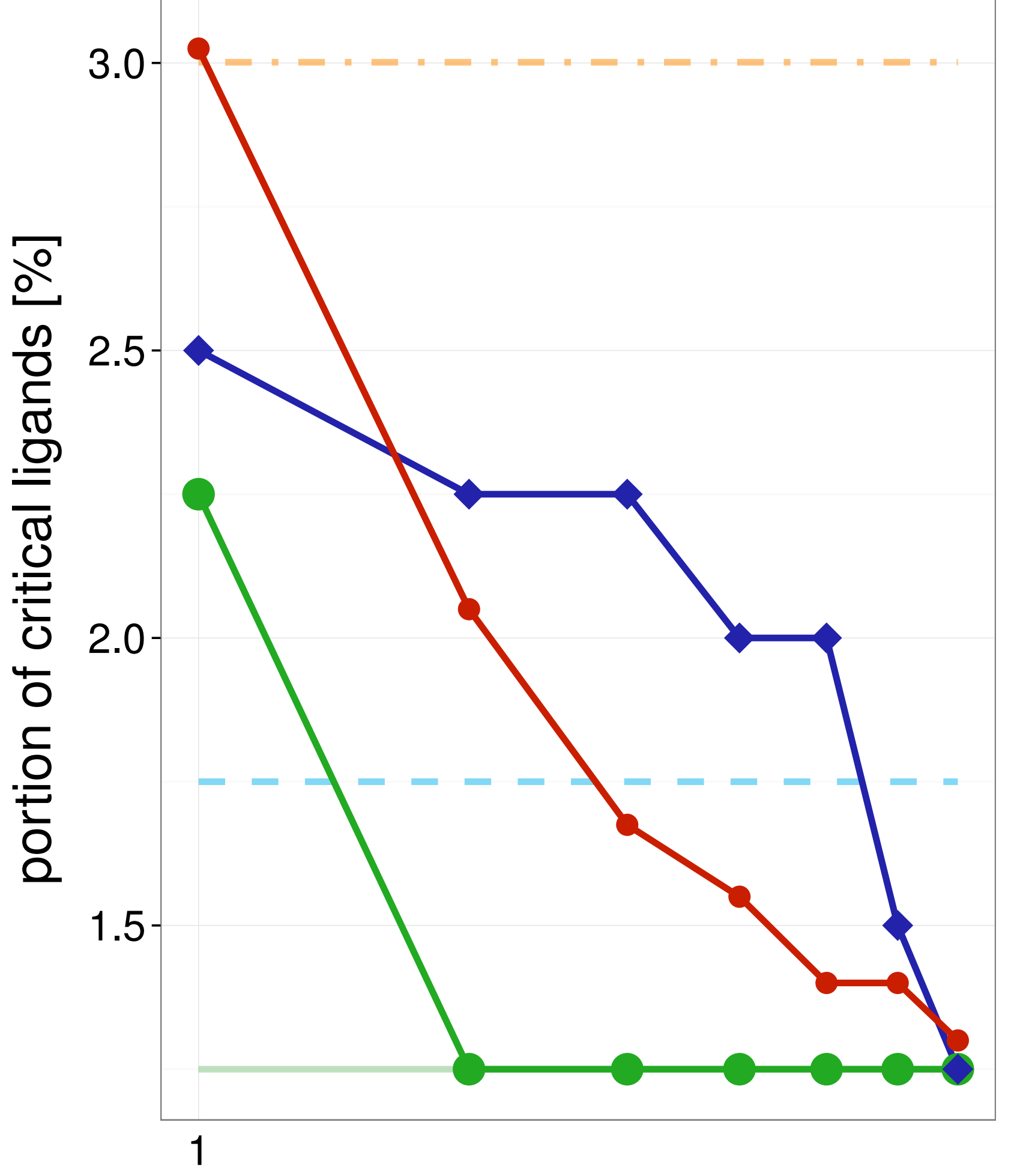

Average single structure

_ Best single structure

Whole ensemble

- SIENA

$\neg$ Clustering

$\rightarrow$ Random 
Training set

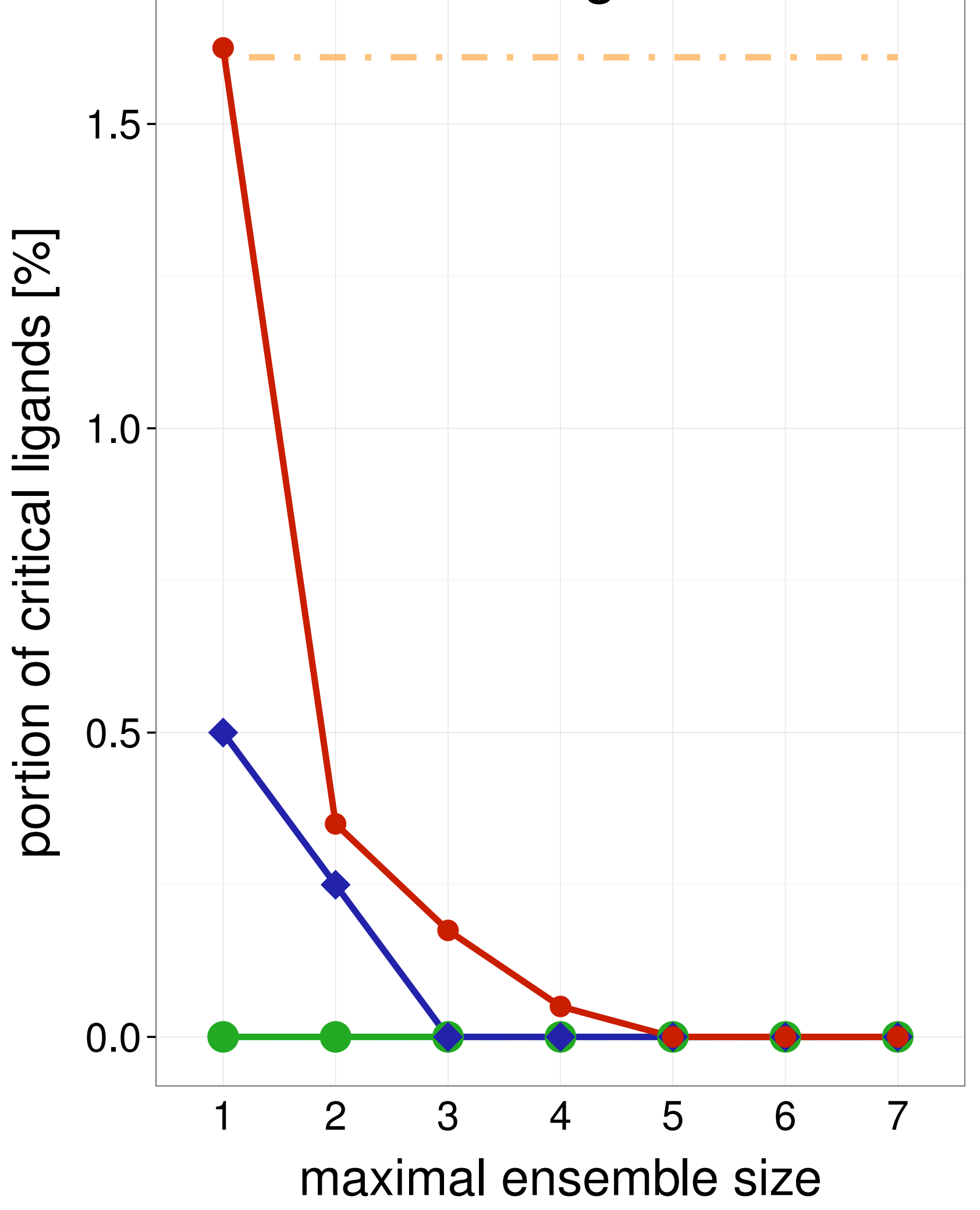

Test set

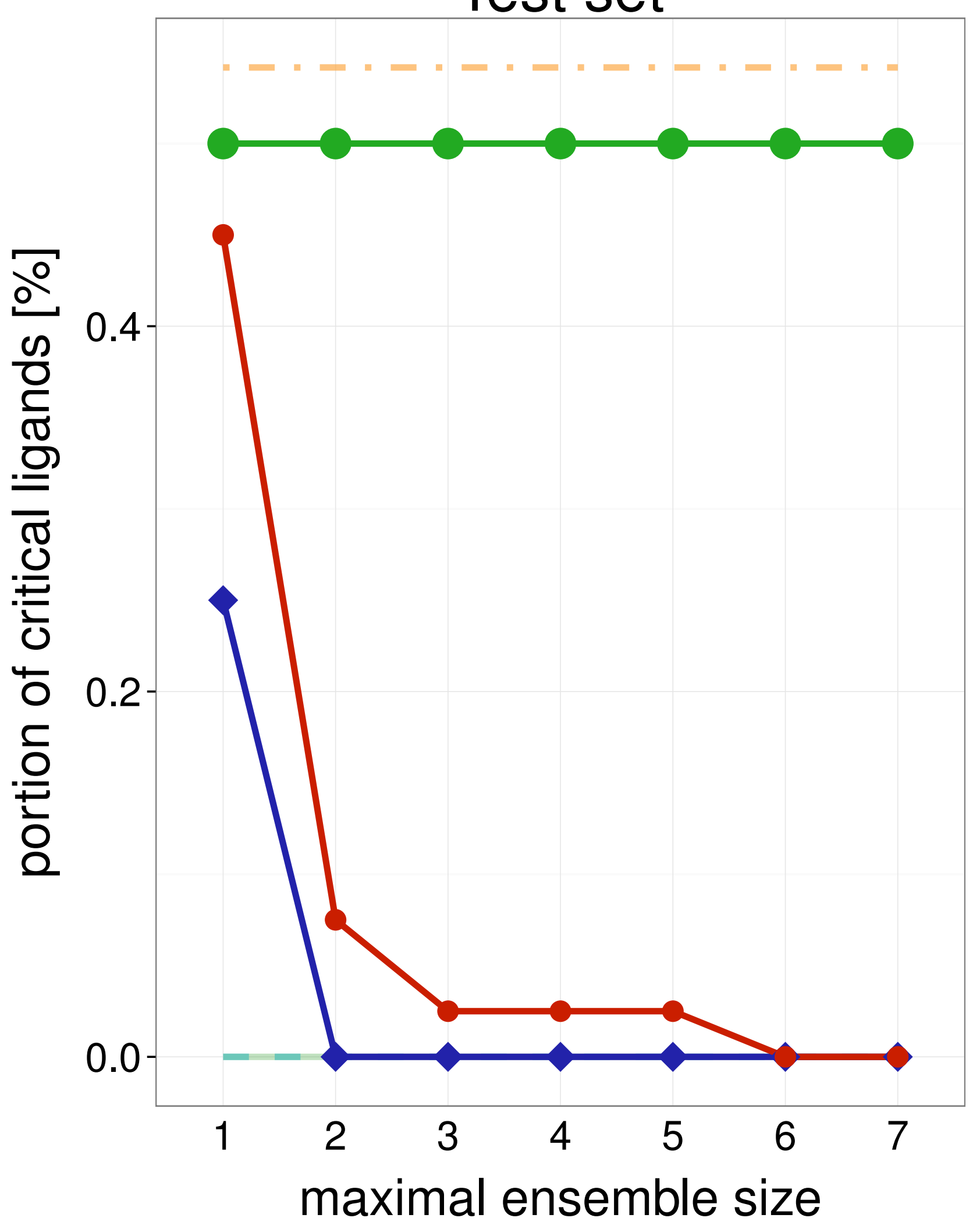

Average single structure

_ _ Best single structure

Whole ensemble

- SIENA

$\smile$ Clustering

$\multimap$ Random

Average single structure

_ _ Best single structure

Whole ensemble

- SIENA

$\neg$ Clustering

$\multimap$ Random
Training set

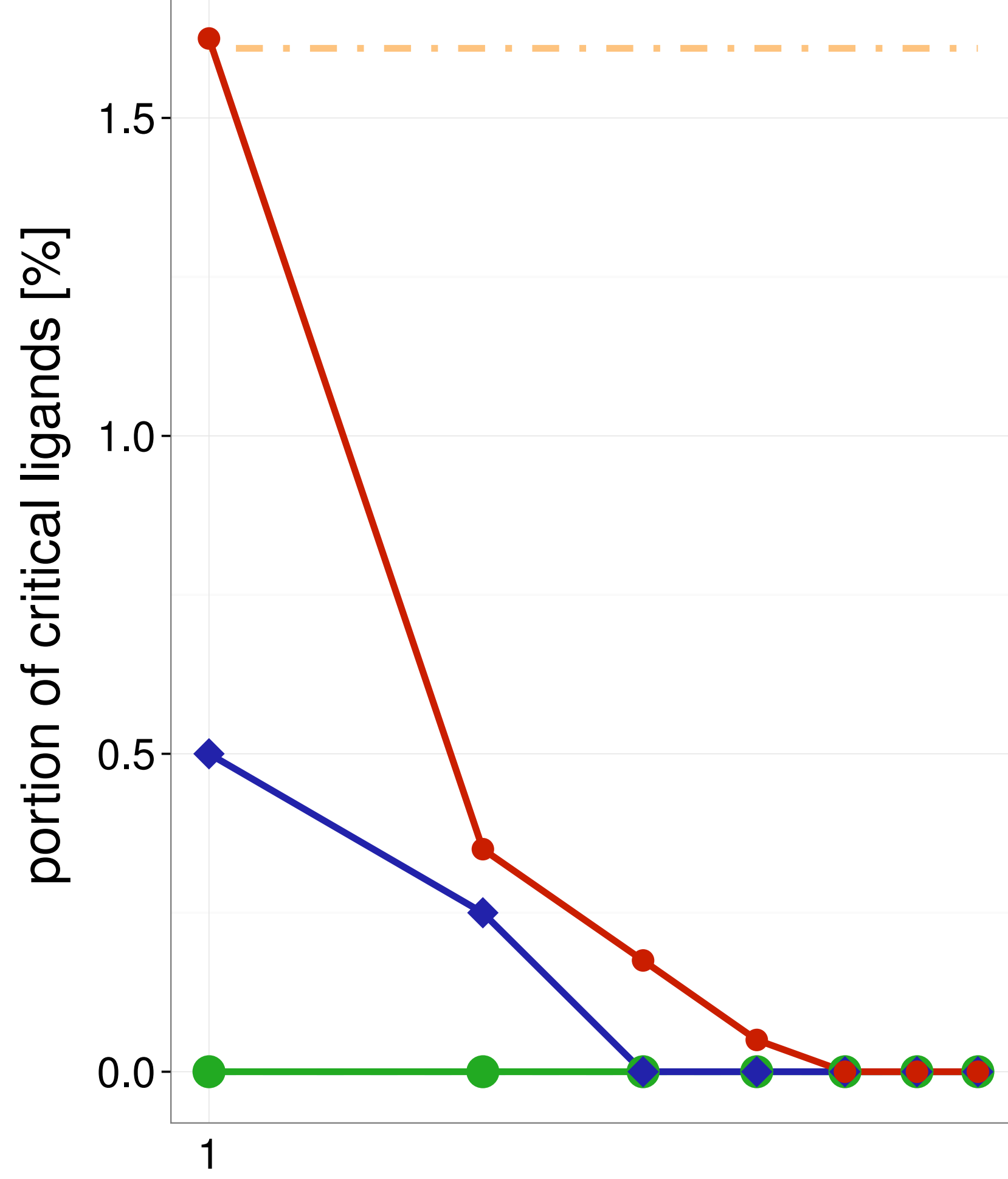

maximal ensemble size

Test set

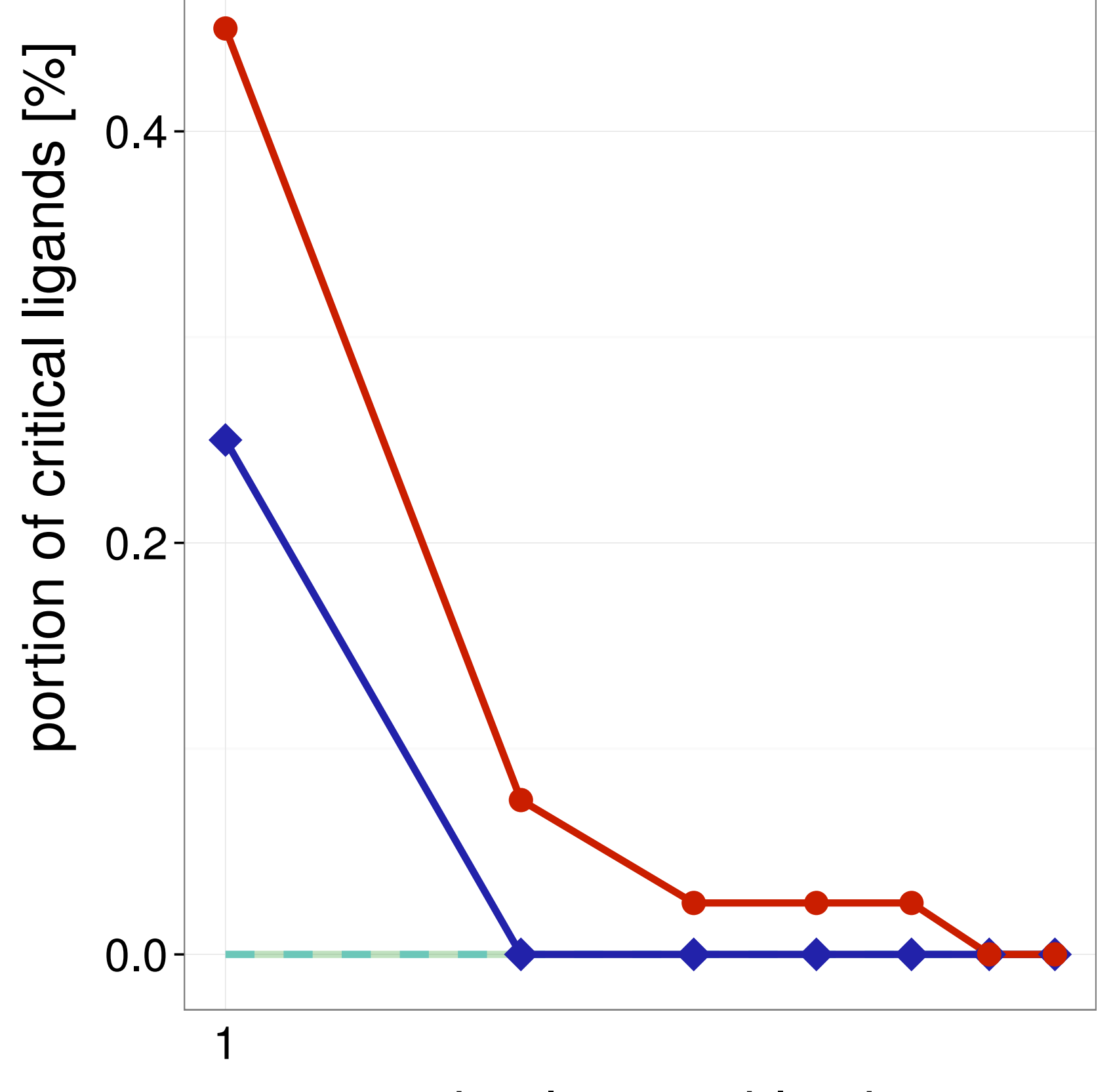

Average single structure

_ Best single structure

Whole ensemble

SIENA

$\neg$ Clustering

$\multimap$ Random 
Training set

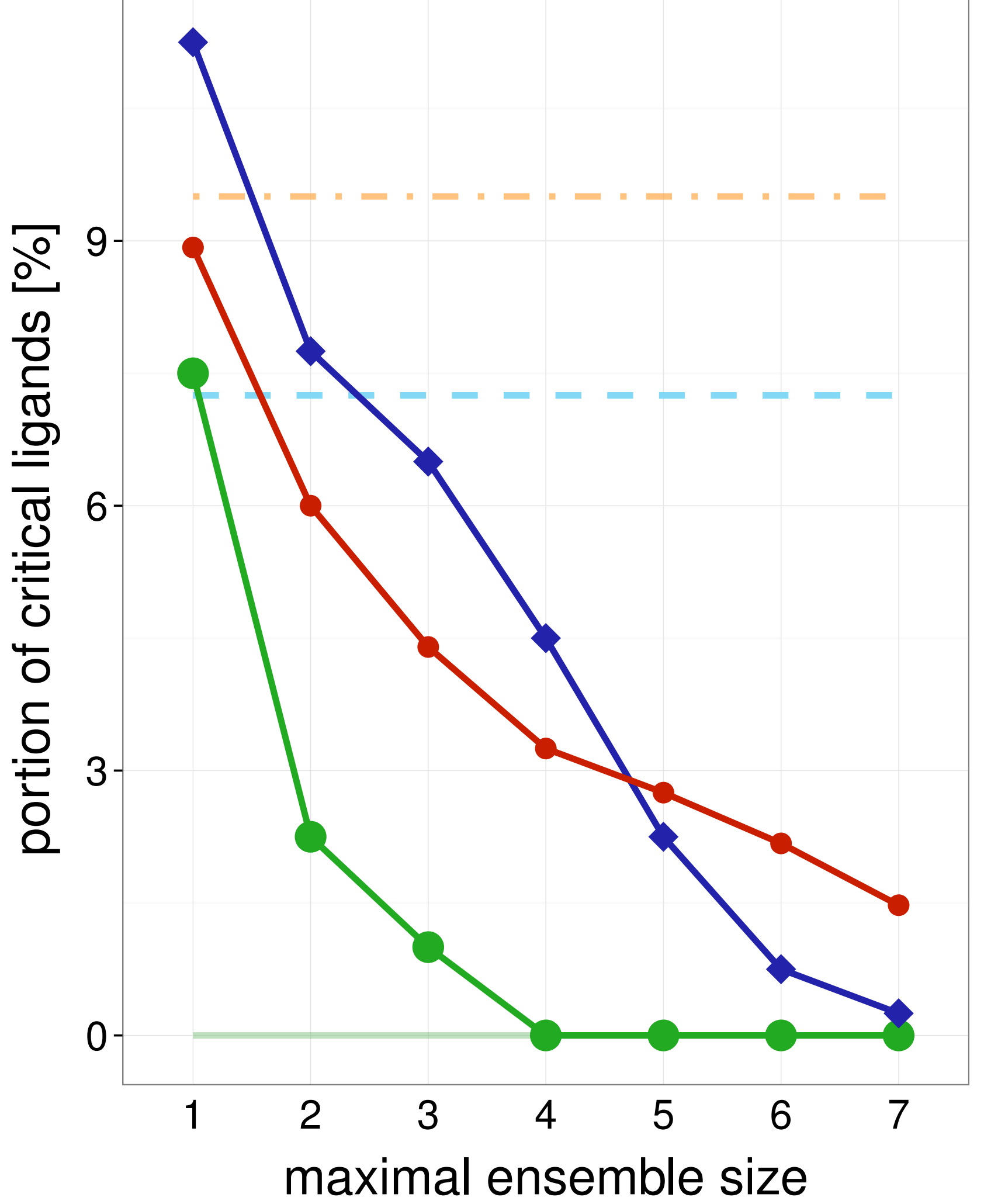

Test set

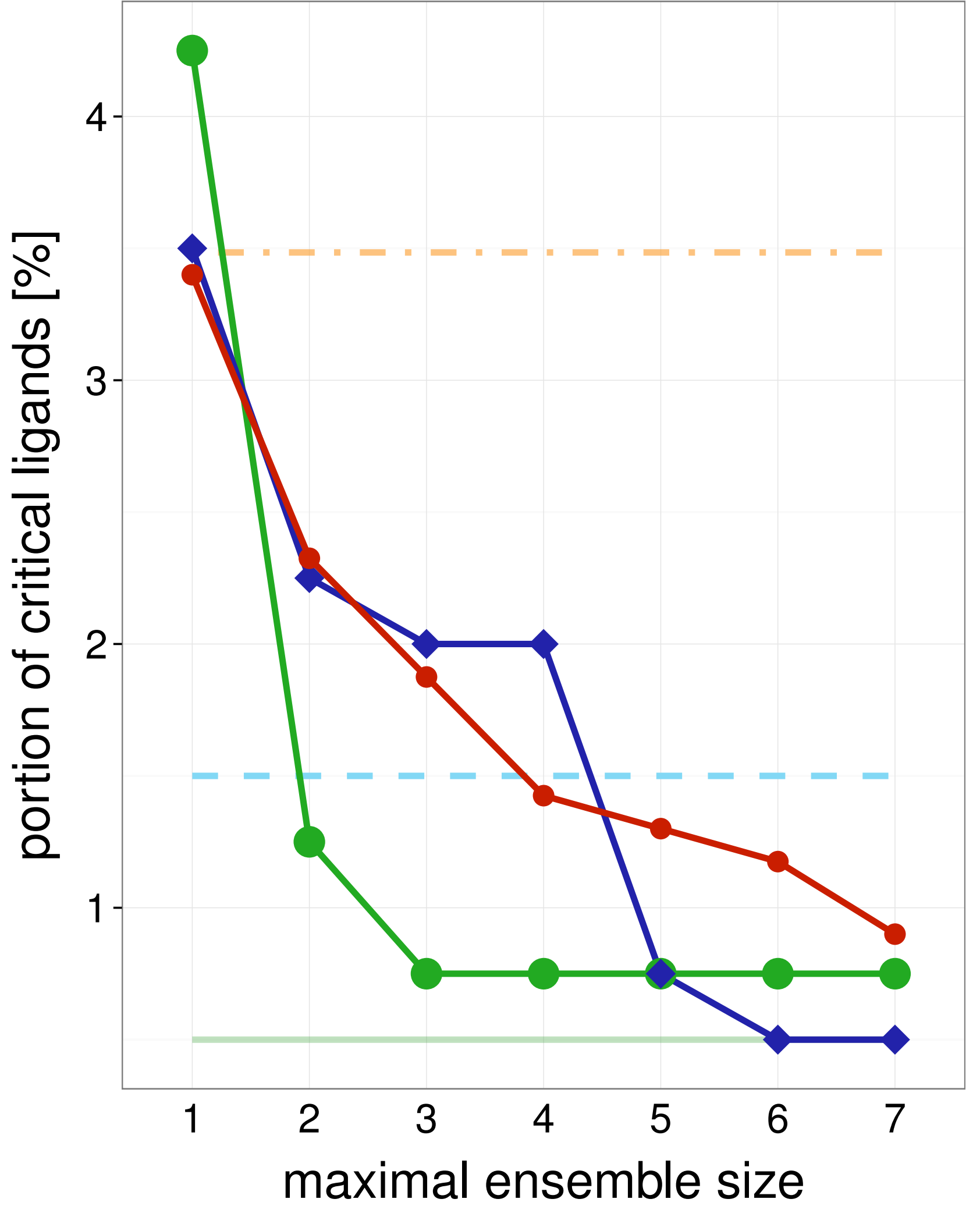

Average single structure

- Best single structure

Whole ensemble

- - SIENA

$\multimap$ Clustering

$\longrightarrow$ Random

Average single structure

_ _ Best single structure

Whole ensemble

- SIENA

$\neg$ Clustering

- Random

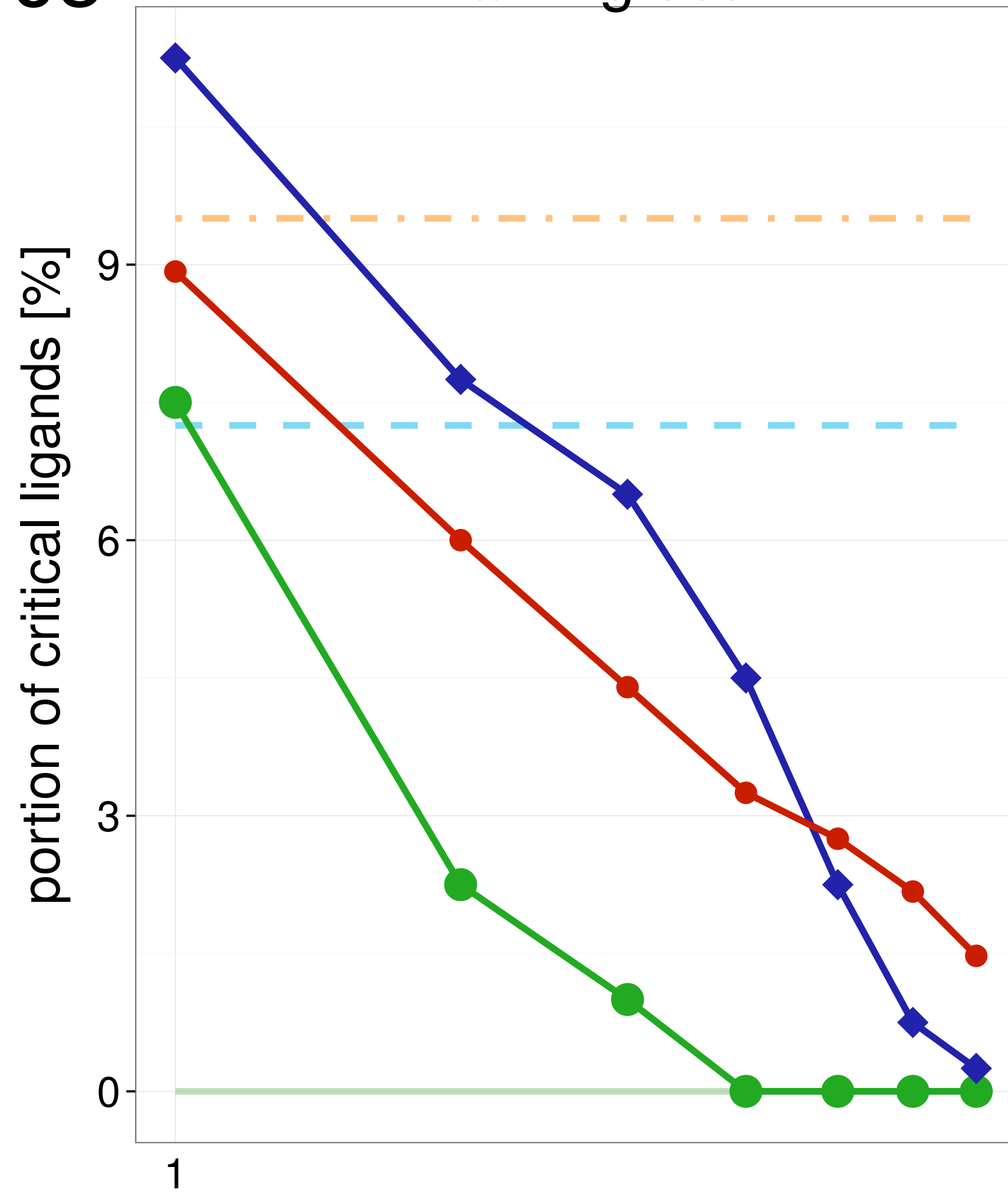

maximal ensemble size

Test set

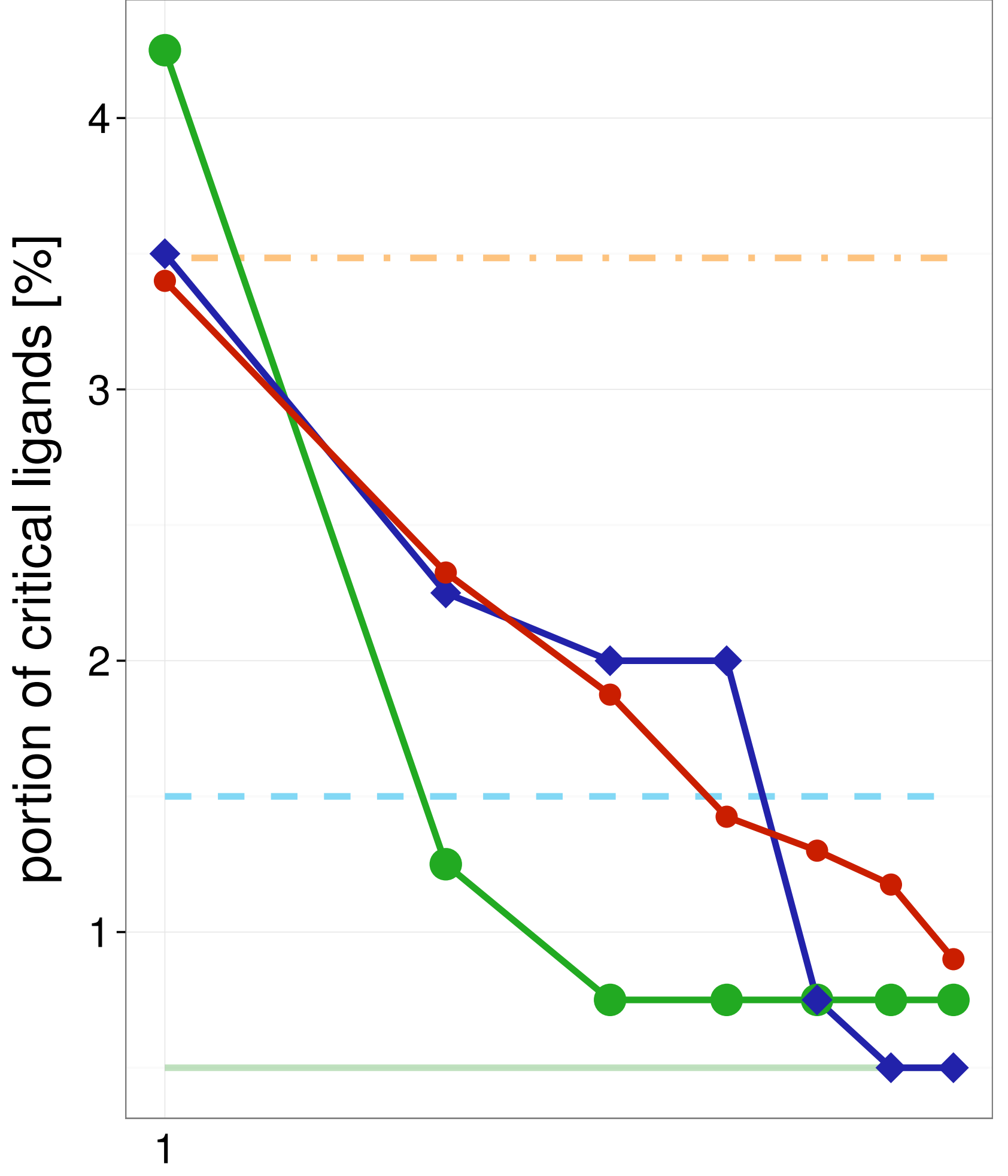

maximal ensemble size
Average single structure

Best single structure

Whole ensemble

SIENA

$\sim$ Clustering

$\longrightarrow$ Random 
Training set

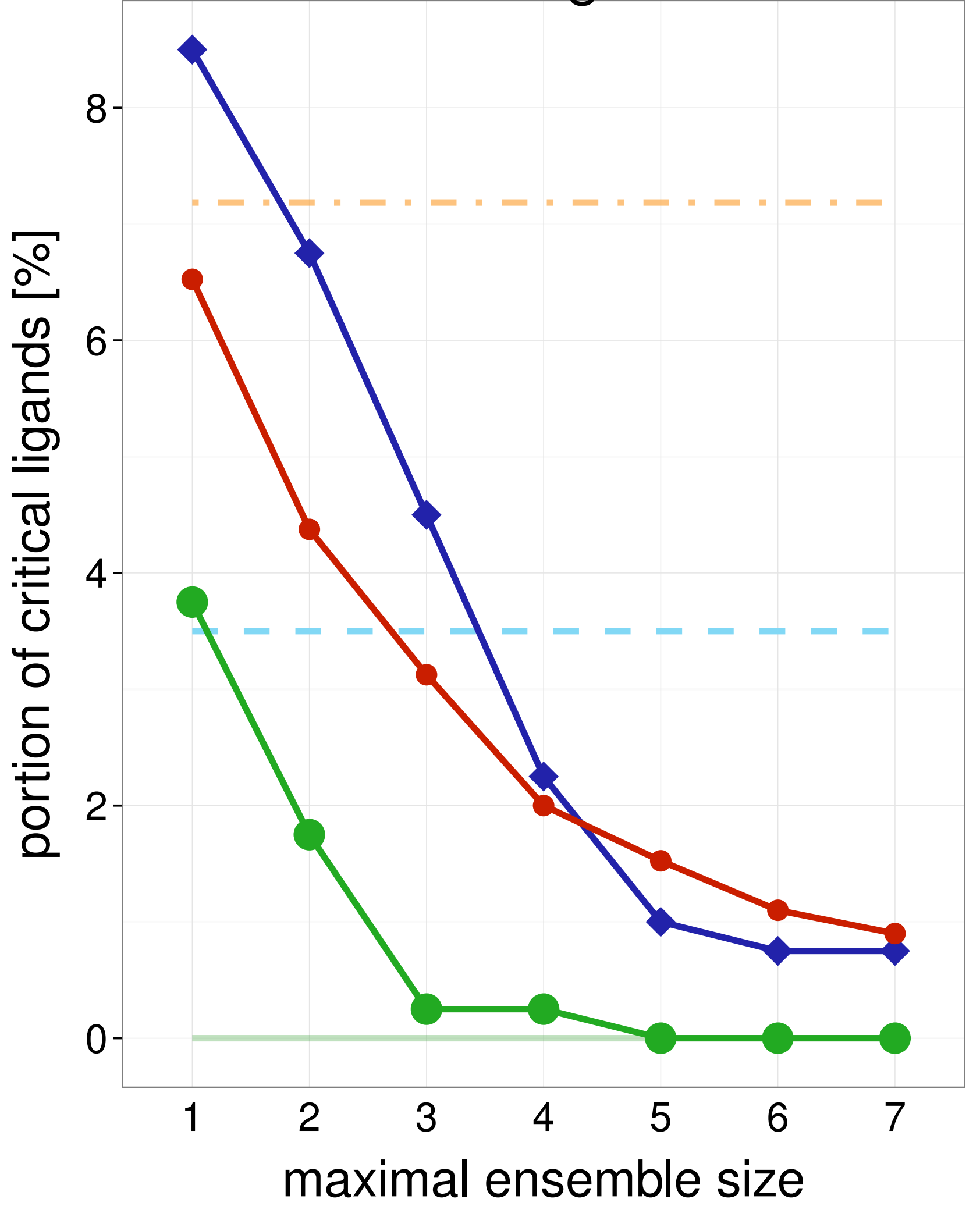

Test set

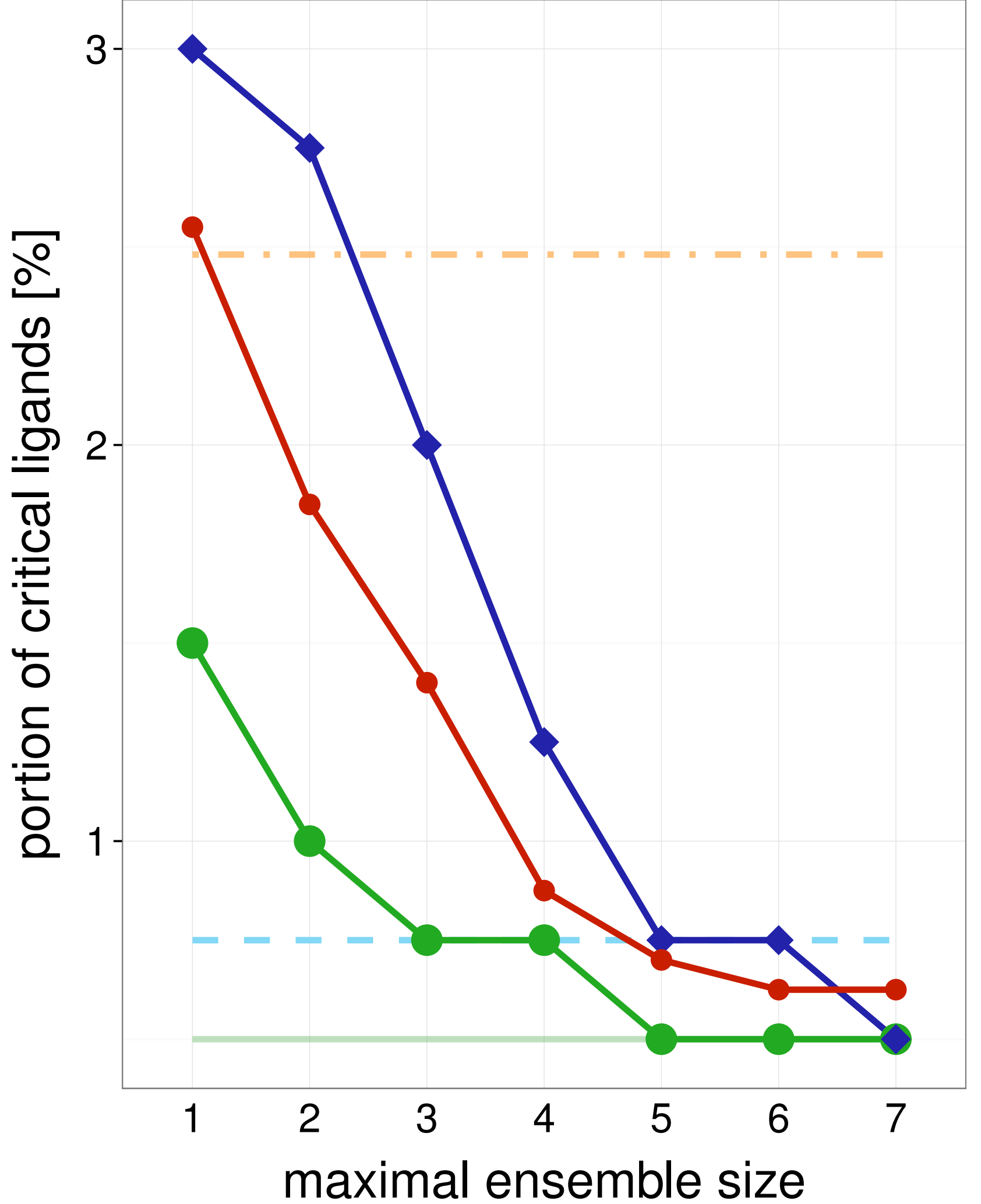

4DWB

Training set

Average single structure

_ Best single

structure

Whole ensemble

- SIENA

$\multimap$ Clustering

$\multimap$ Random

Average single structure

_ Best single structure

Whole ensemble

- SIENA

$\neg$ Clustering

$\multimap$ Random

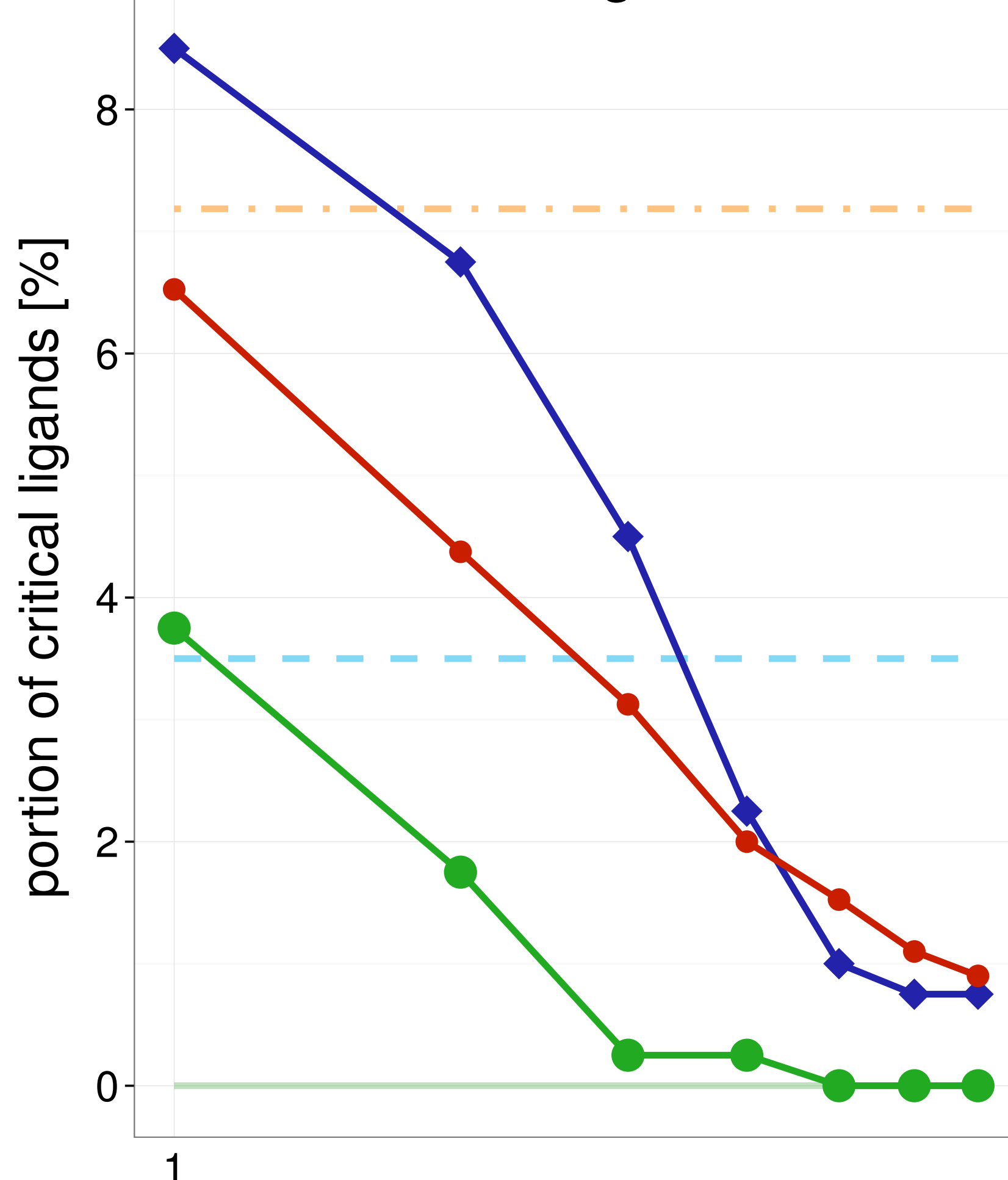

maximal ensemble size

Test set

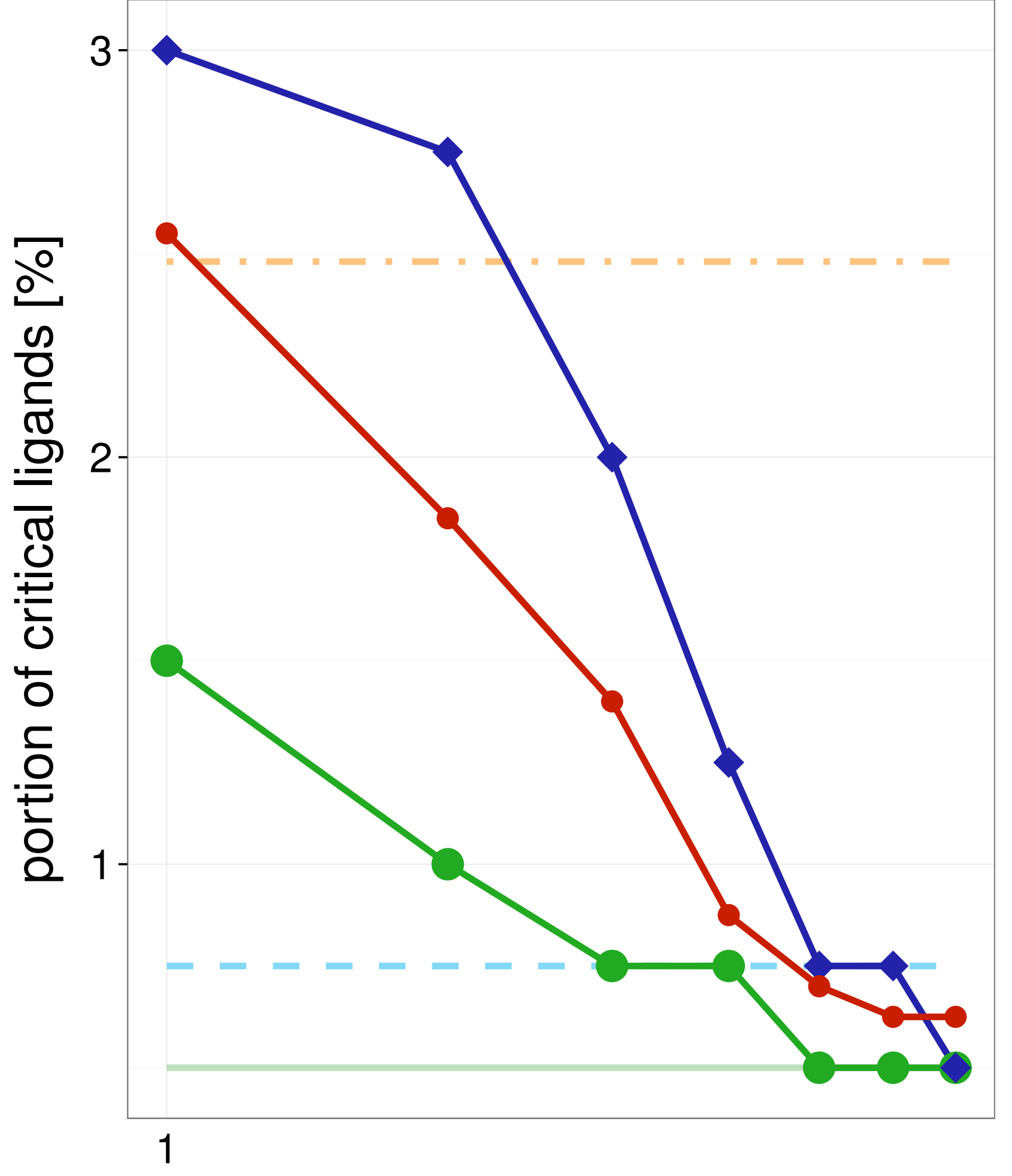

maximal ensemble size
Average single structure

_ Best single structure

Whole ensemble

SIENA

$\leadsto$ Clustering

$\multimap$ Random 


\section{Training set}

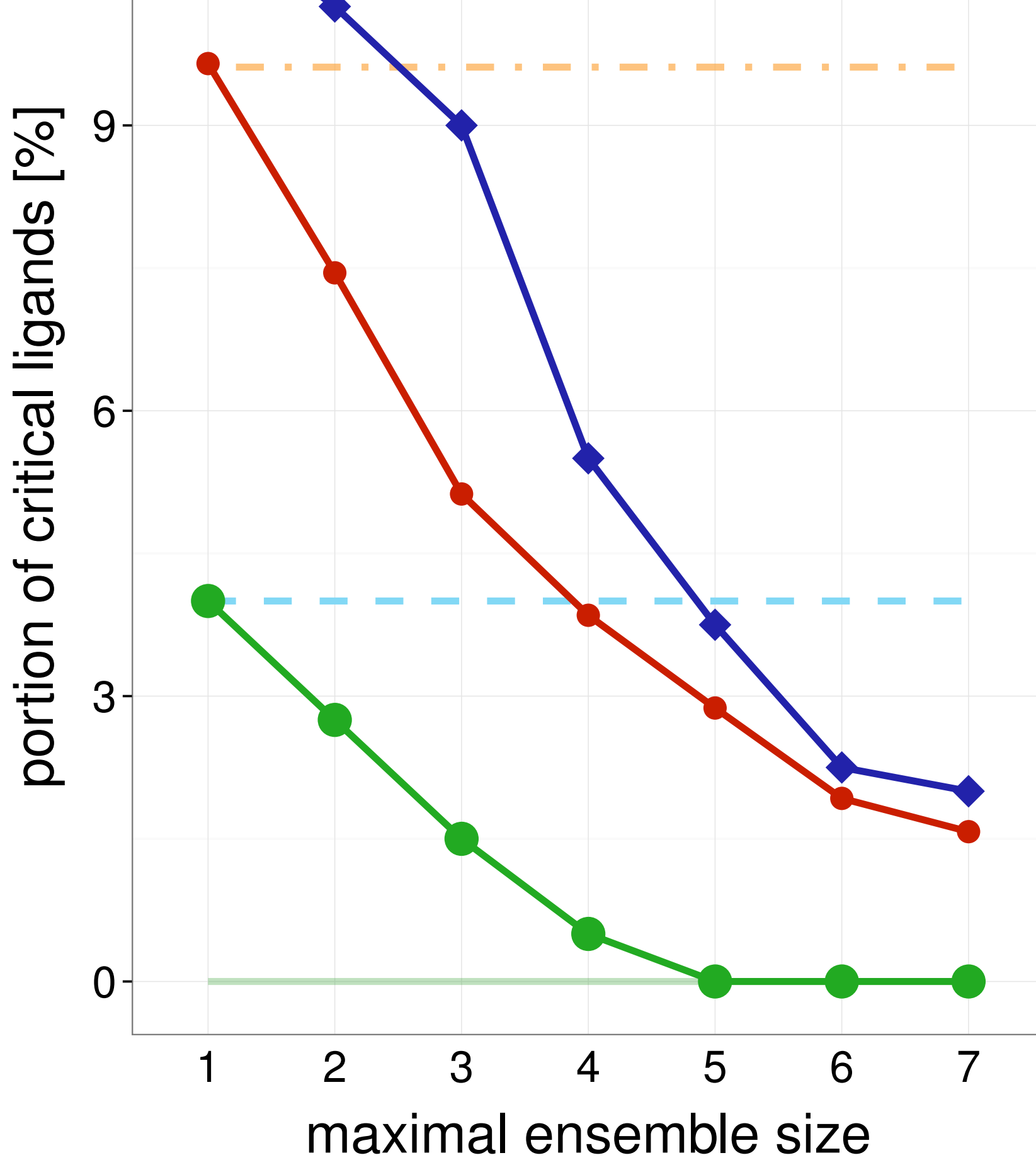

\section{Test set}

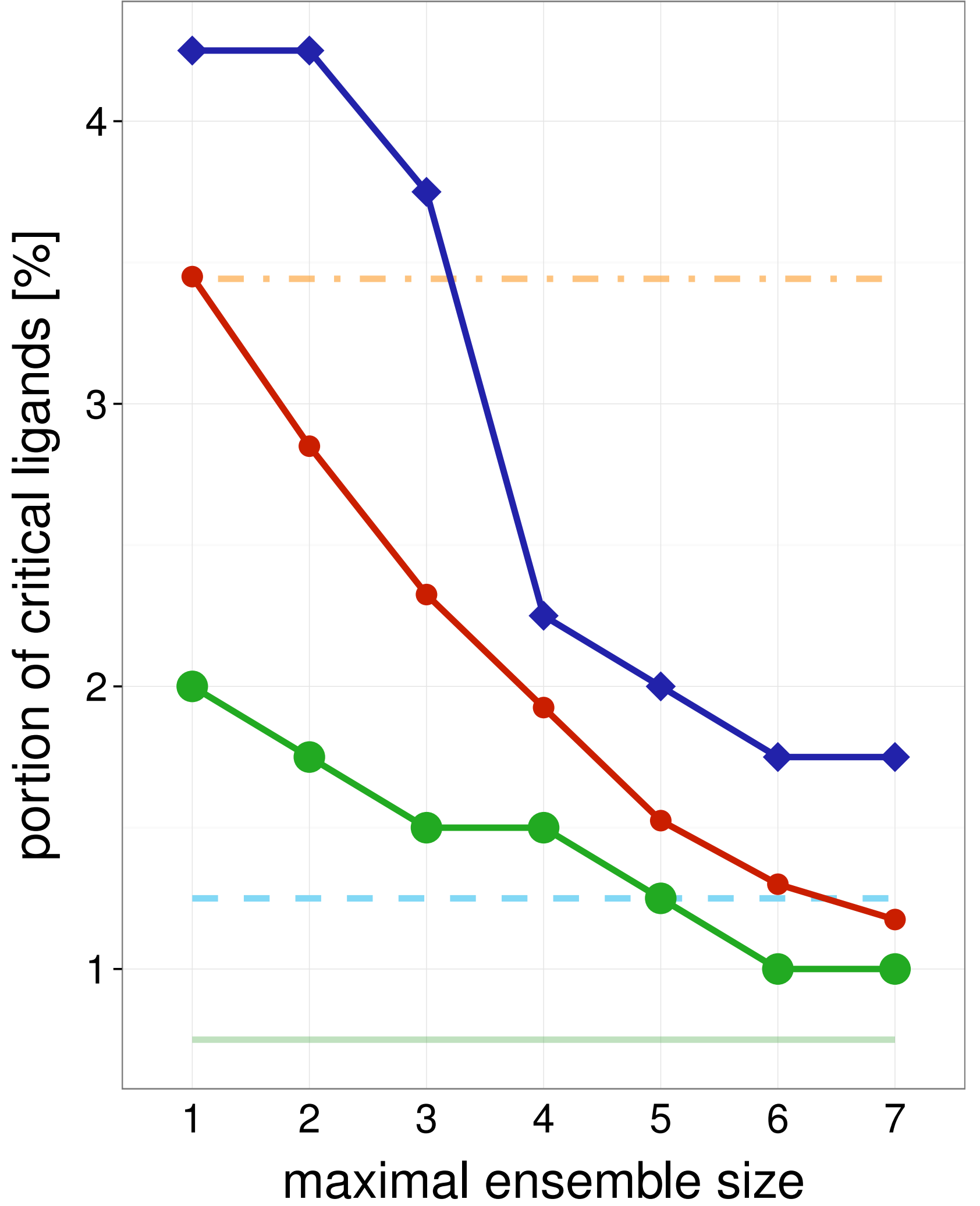

Average single structure

_ Best single structure

Whole ensemble

- SIENA

$\neg$ Clustering

- Random

Average single structure

_ Best single structure

Whole ensemble

- SIENA

$\smile$ Clustering

$\multimap$ Random

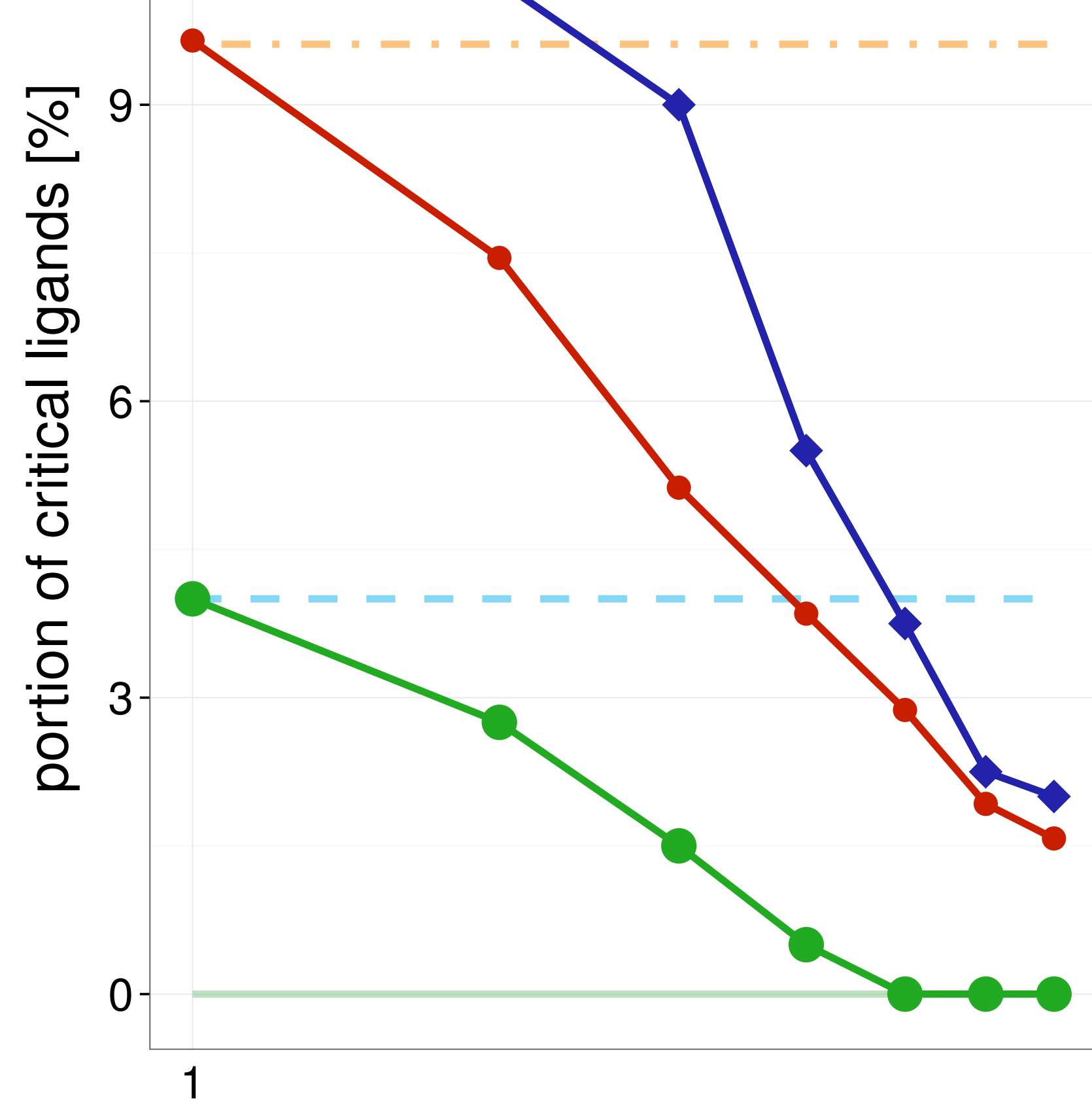

Average single structure

Best single structure

Whole ensemble

- SIENA

$\leadsto$ Clustering

- Random

maximal ensemble size

Test set

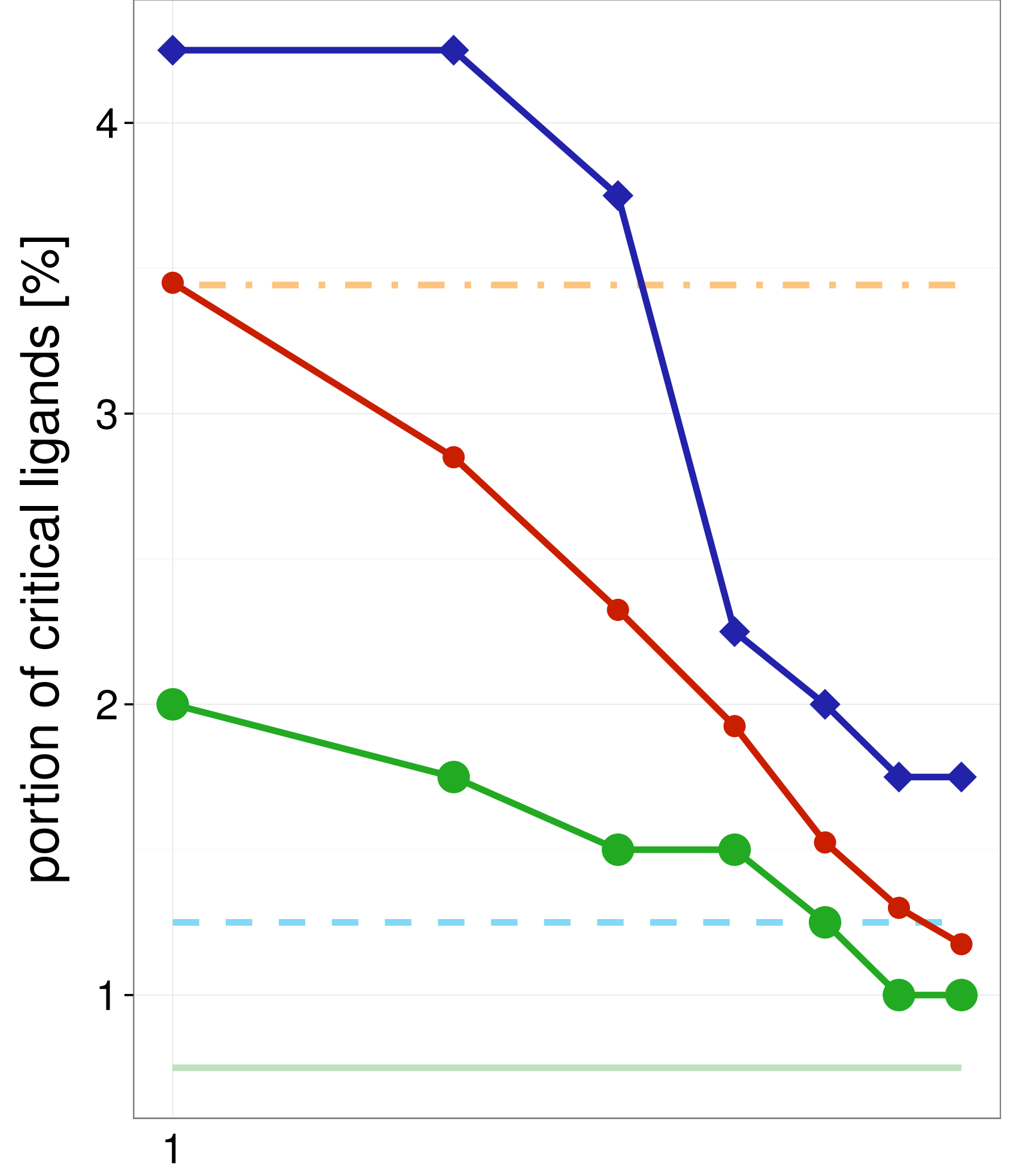

Average single structure

- Best single structure

Whole ensemble

SIENA

$\neg$ Clustering

$\multimap$ Random 


\section{Training set}

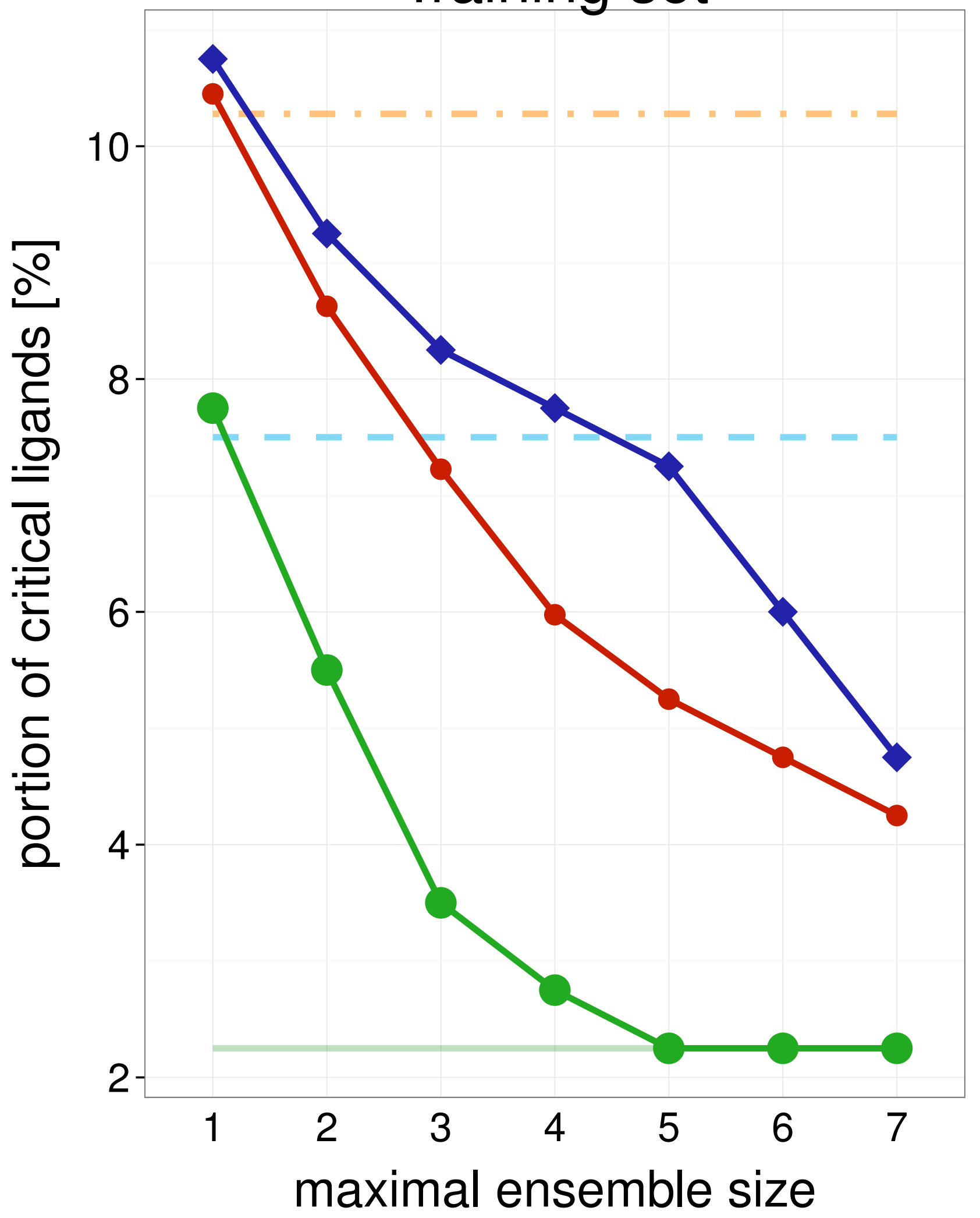

Test set

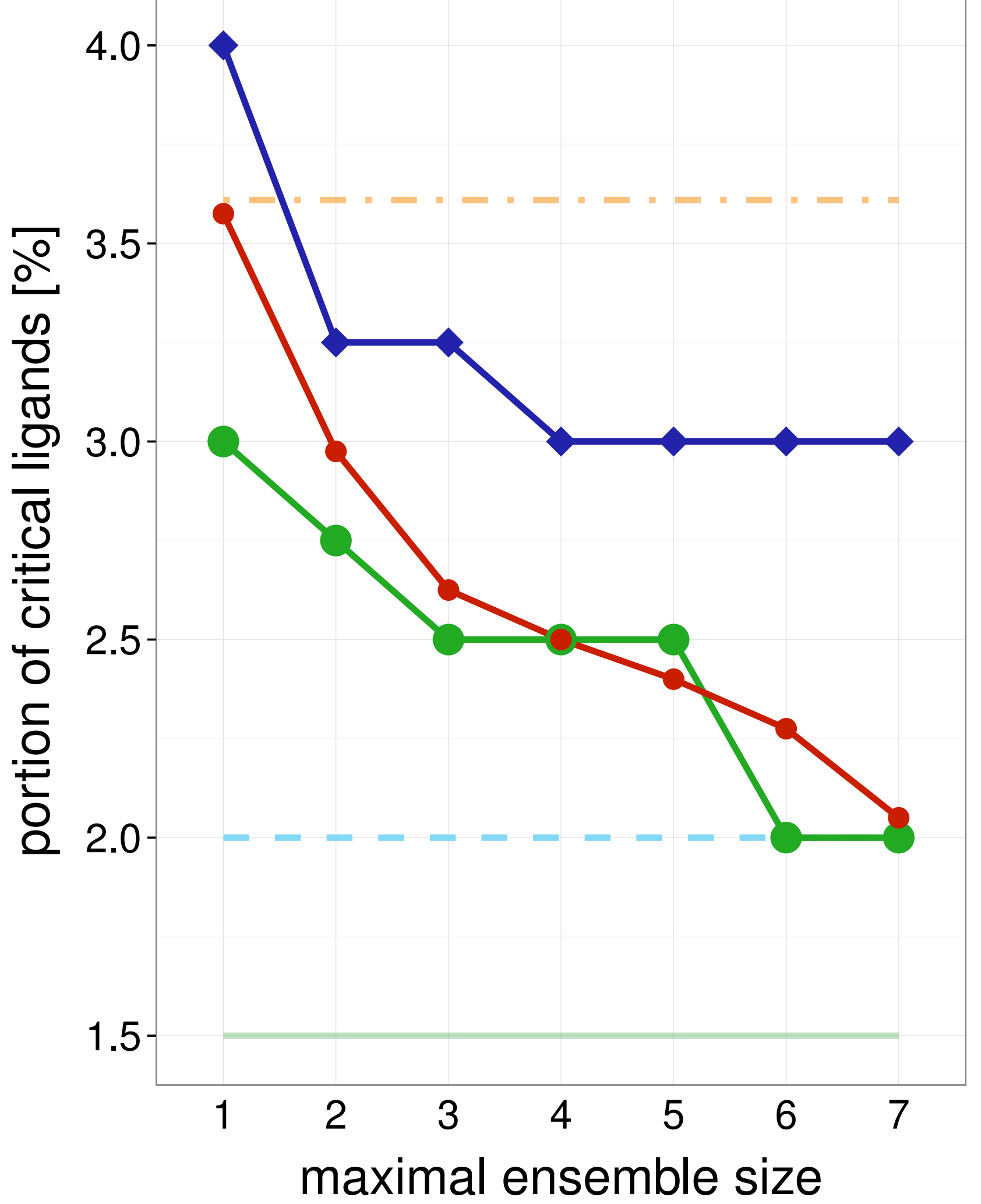

4LRR

Training set

Average single structure

_ Best single structure

Whole ensemble

- SIENA

$\smile$ Clustering

$\multimap$ Random

Average single structure

_ Best single structure

Whole ensemble

- SIENA

$\sim$ Clustering

$\multimap$ Random

Test set

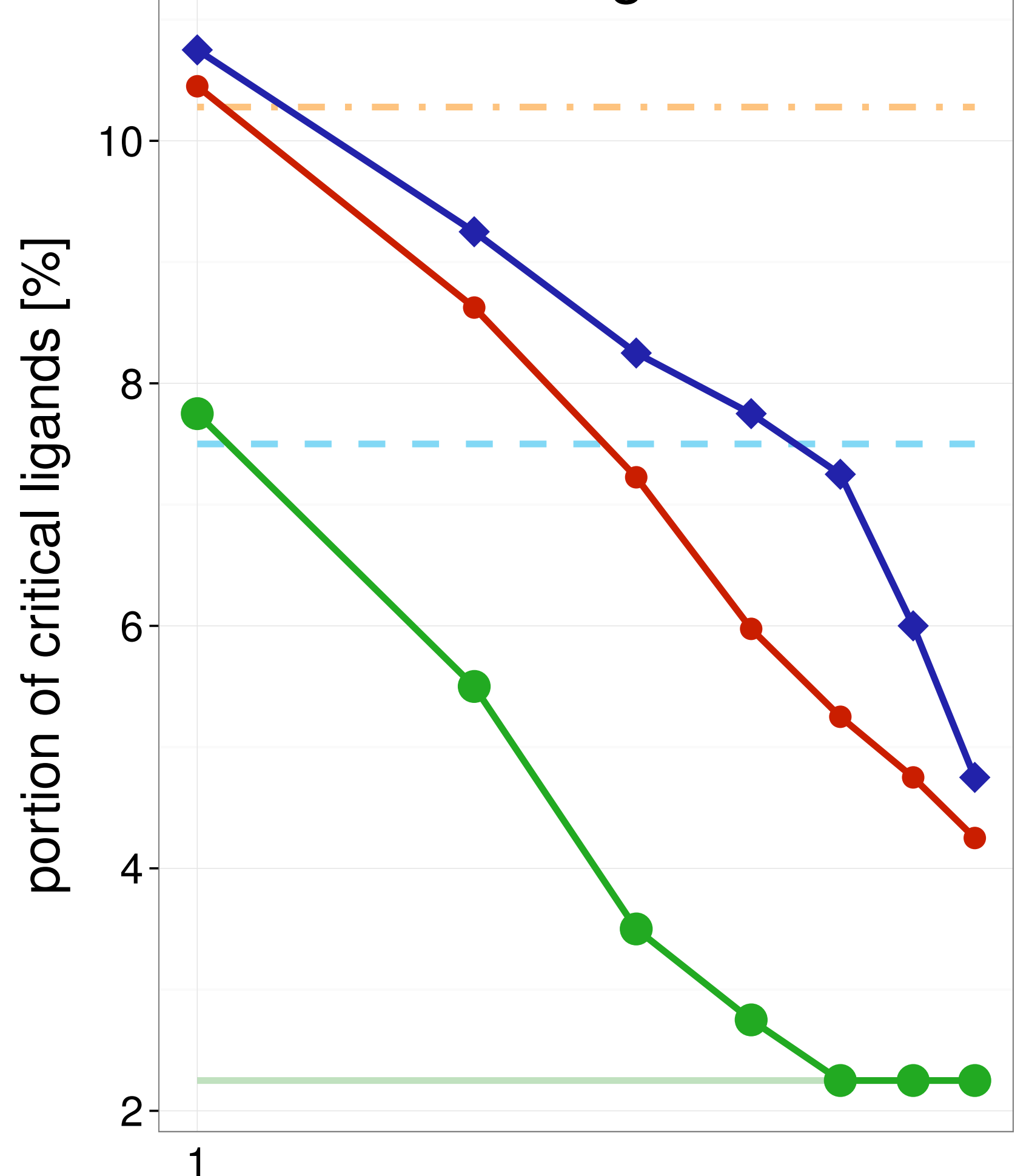

maximal ensemble size

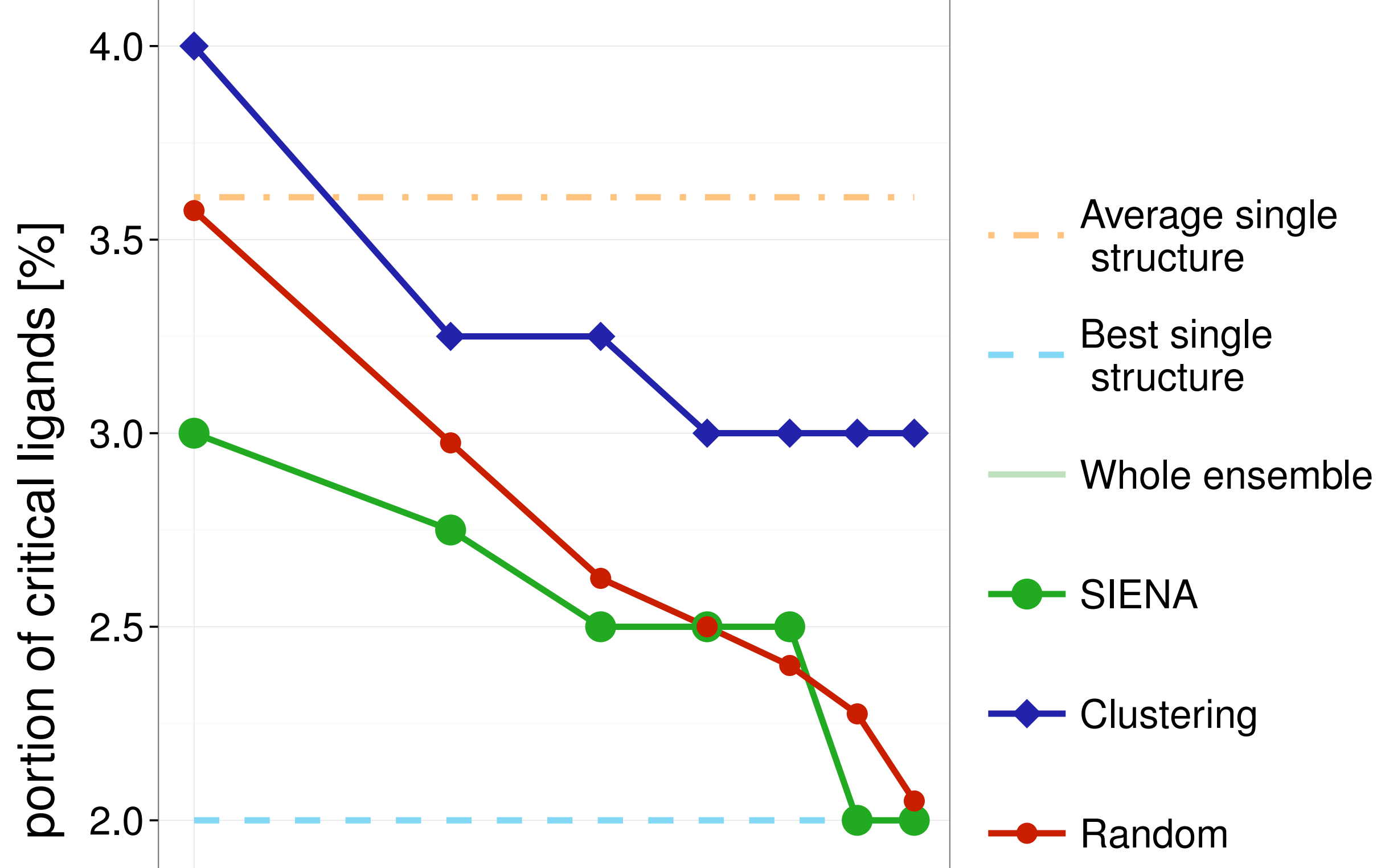

1.5

1
Average single structure

_ Best single structure

Whole ensemble

- SIENA

$\sim$ Clustering

$\multimap$ Random

Average single

Best single

Whole ensemble 
Training set

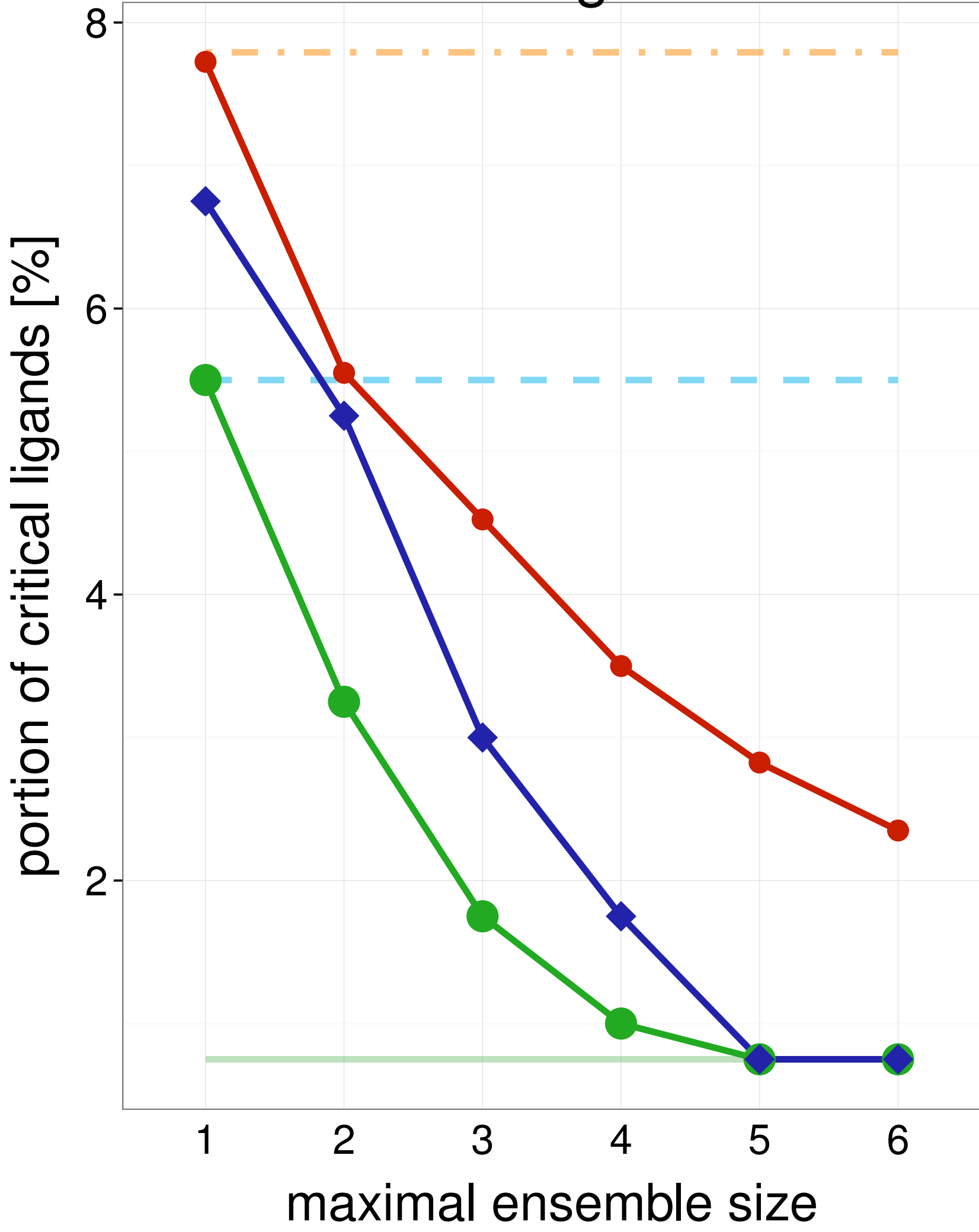

\section{Test set}

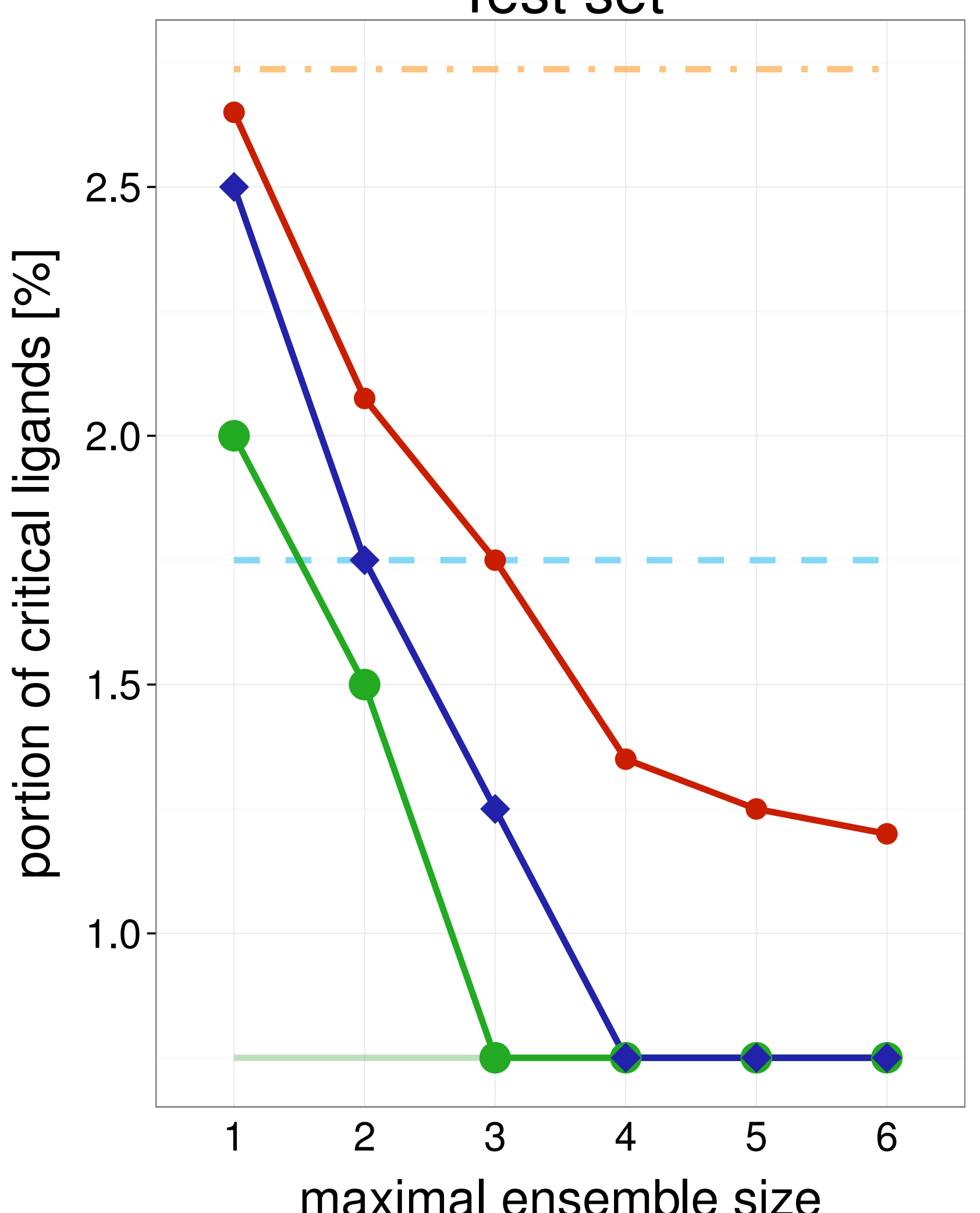

Average single structure

_ Best single structure

Whole ensemble

- SIENA

$\neg$ Clustering

$\multimap$ Random

Average single structure

_ _ Best single structure

Whole ensemble

- SIENA

$\checkmark$ Clustering

- Random

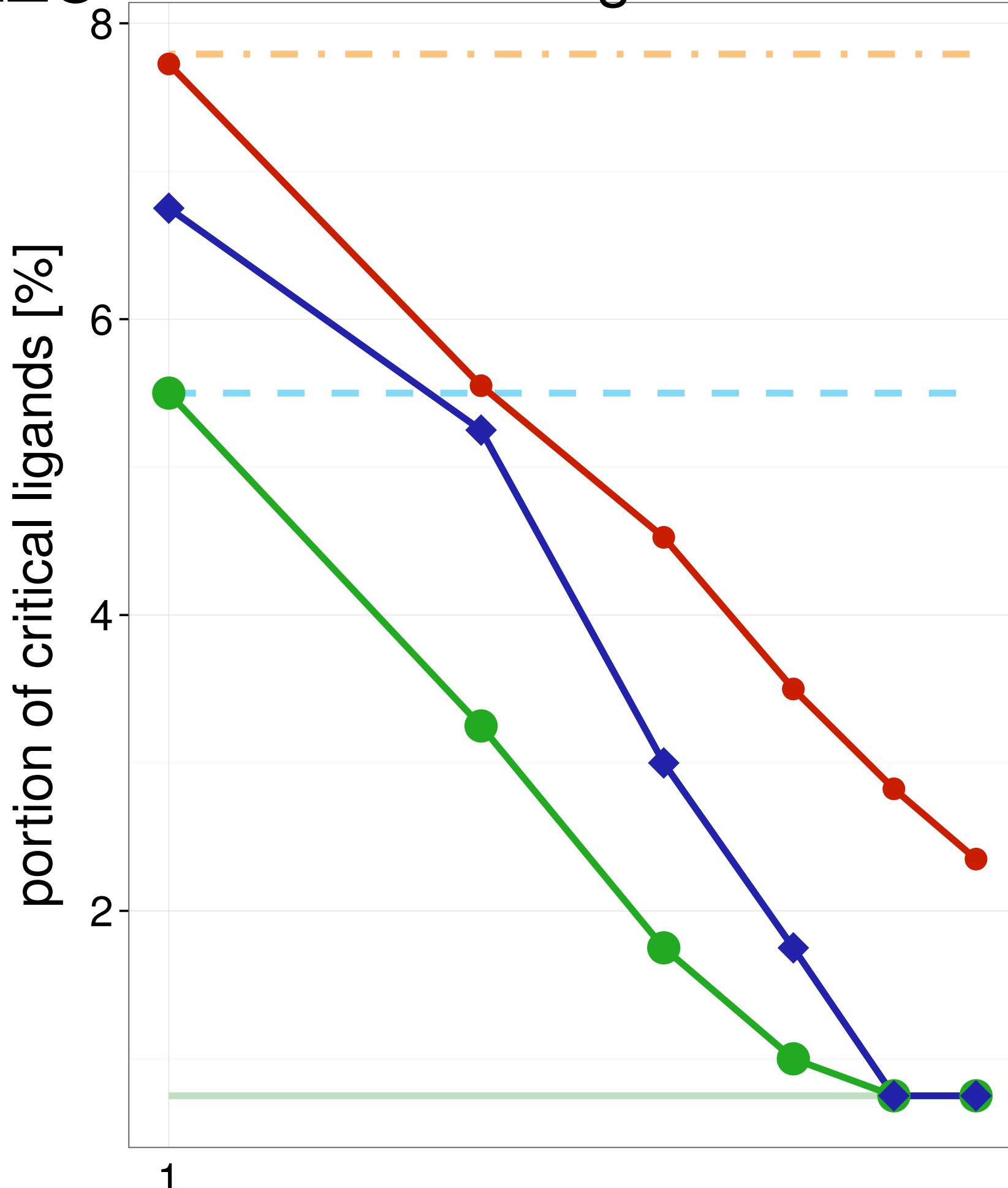

maximal ensemble size

\section{Test set}

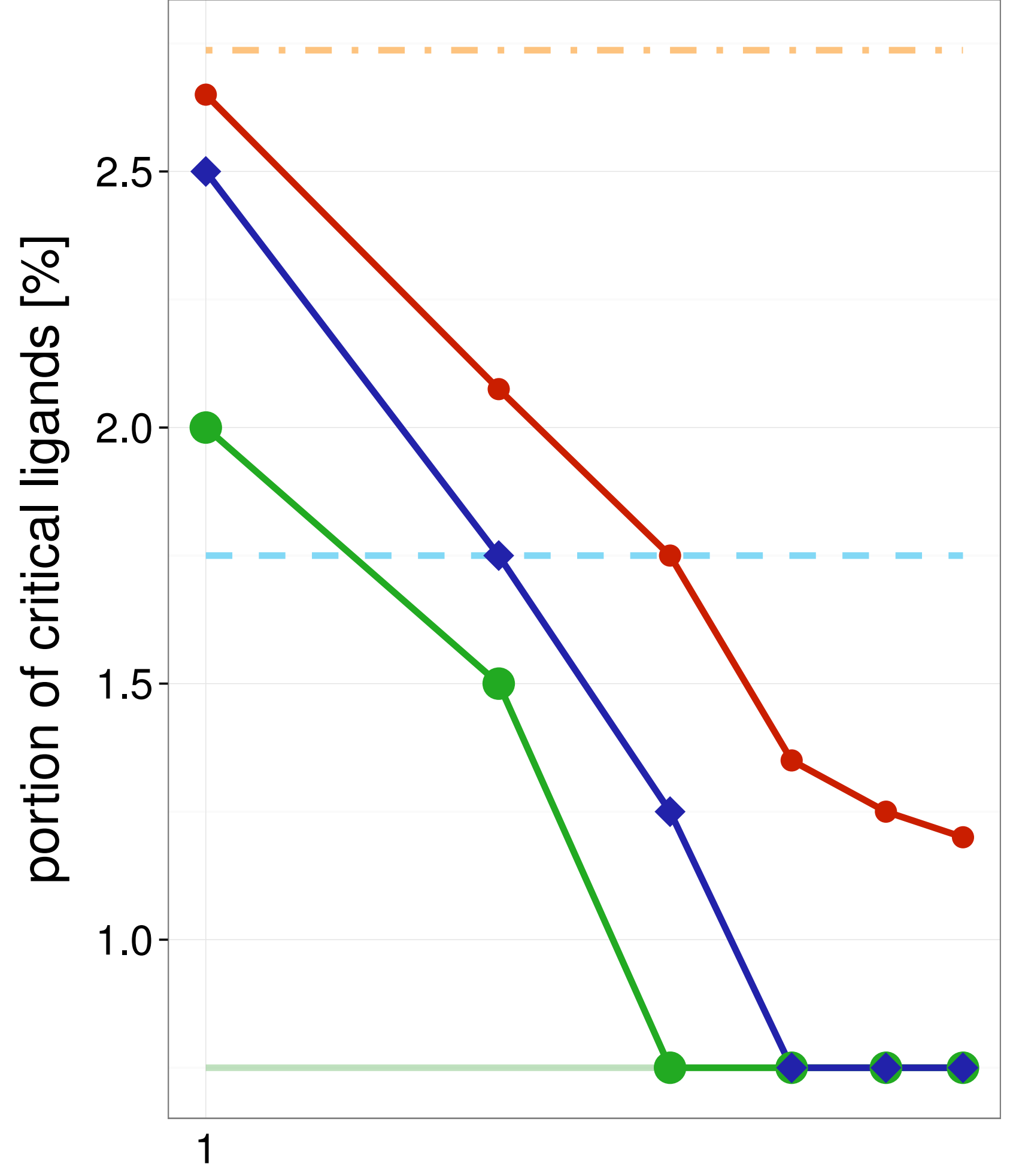

maximal ensemble size
Average single structure

_ _ Best single structure

Whole ensemble

SIENA

$\neg$ Clustering

$\multimap$ Random 
Training set

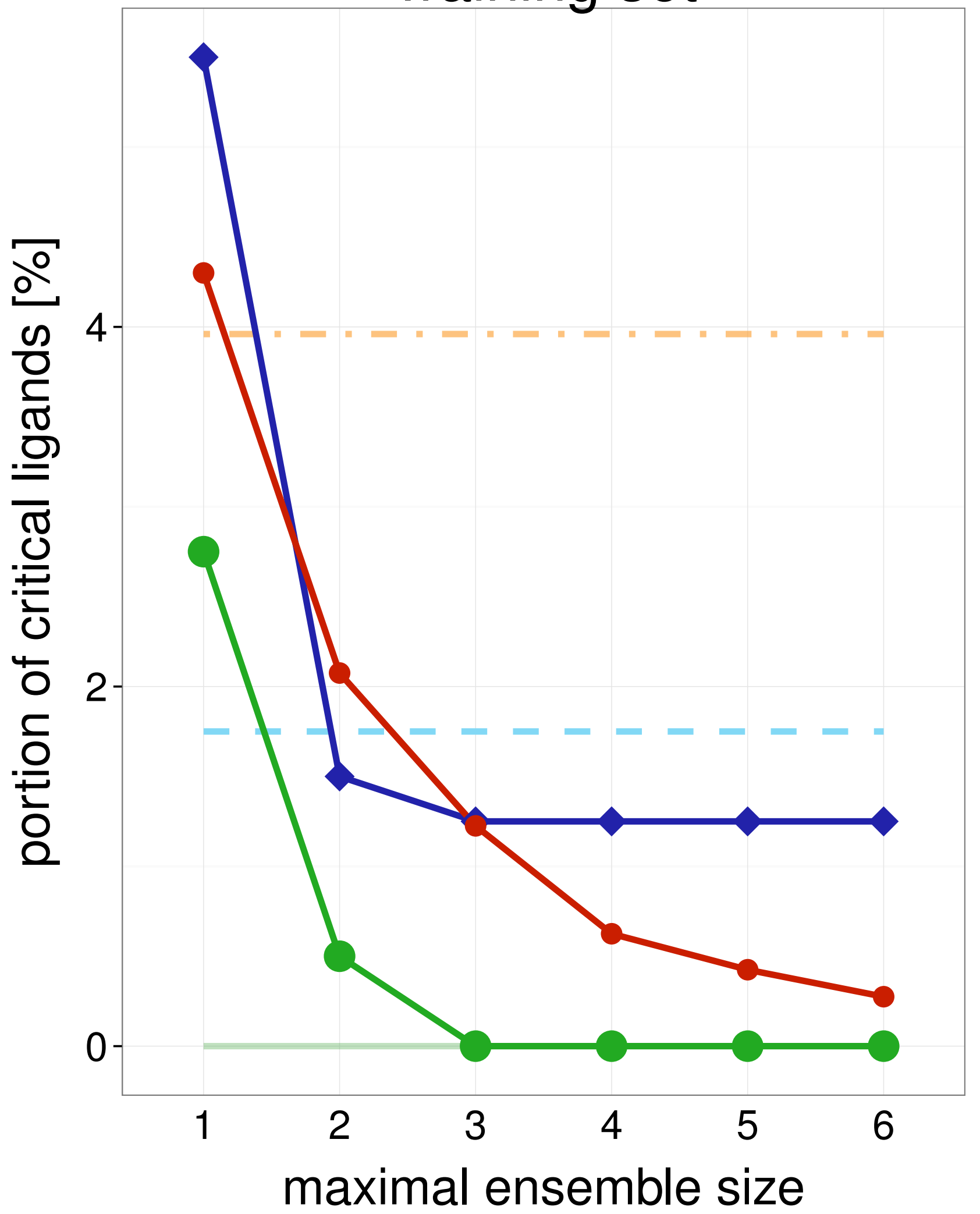

Test set

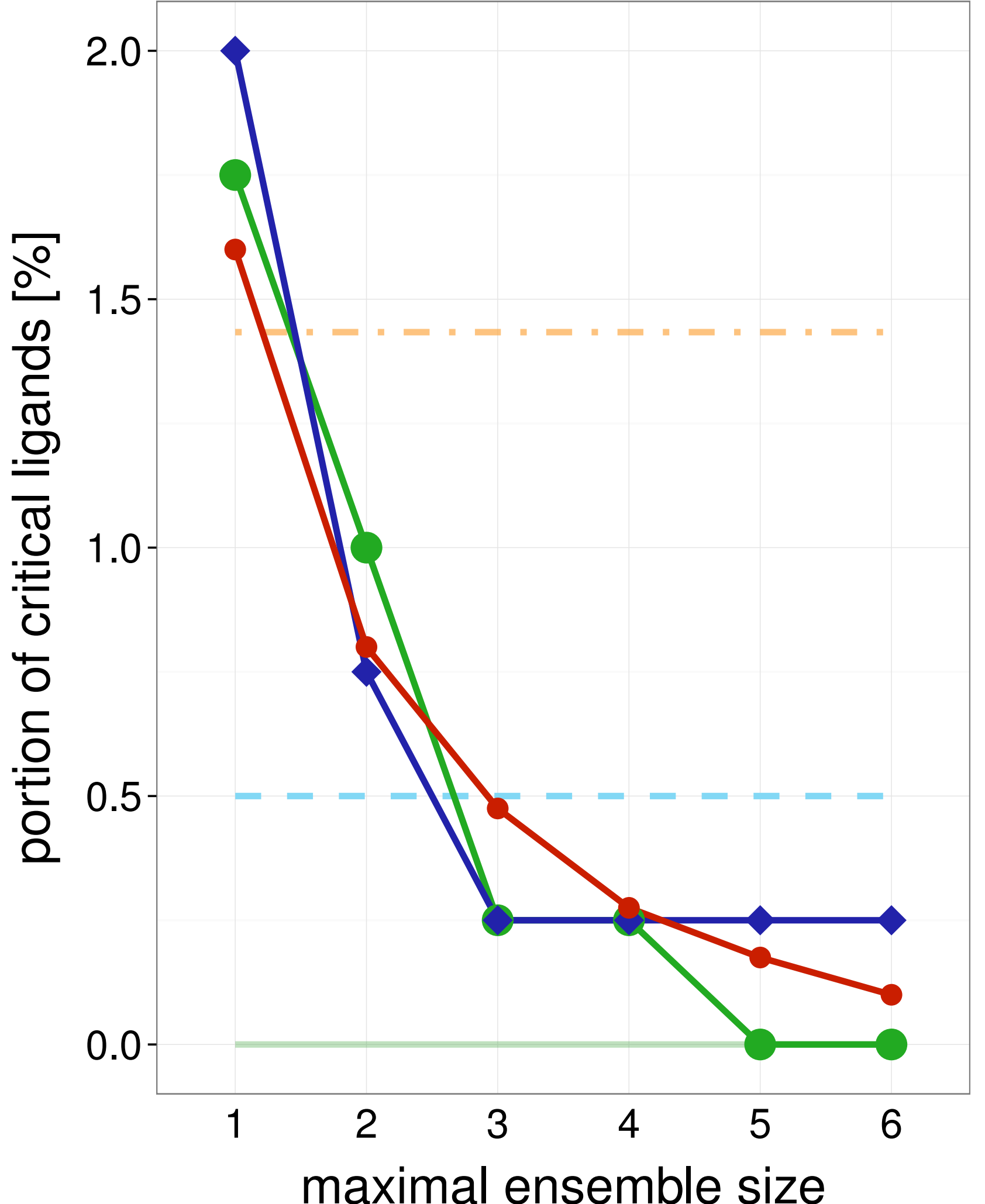

Average single structure

_ _ Best single structure

Whole ensemble

- SIENA

$\neg$ Clustering

$\multimap$ Random

Average single structure

_ Best single structure

Whole ensemble

- SIENA

$\neg$ Clustering

$\multimap$ Random

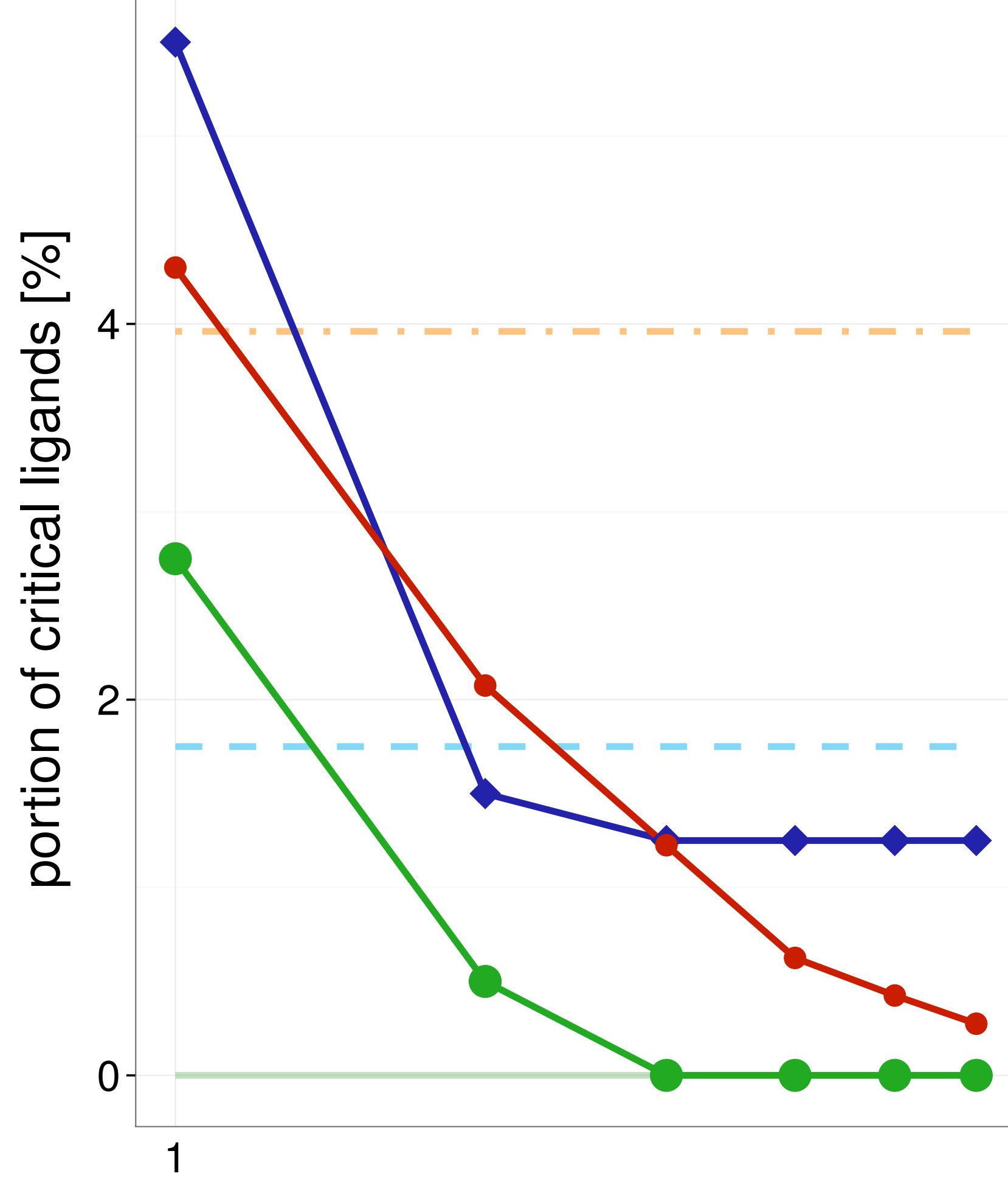

maximal ensemble size

Test set

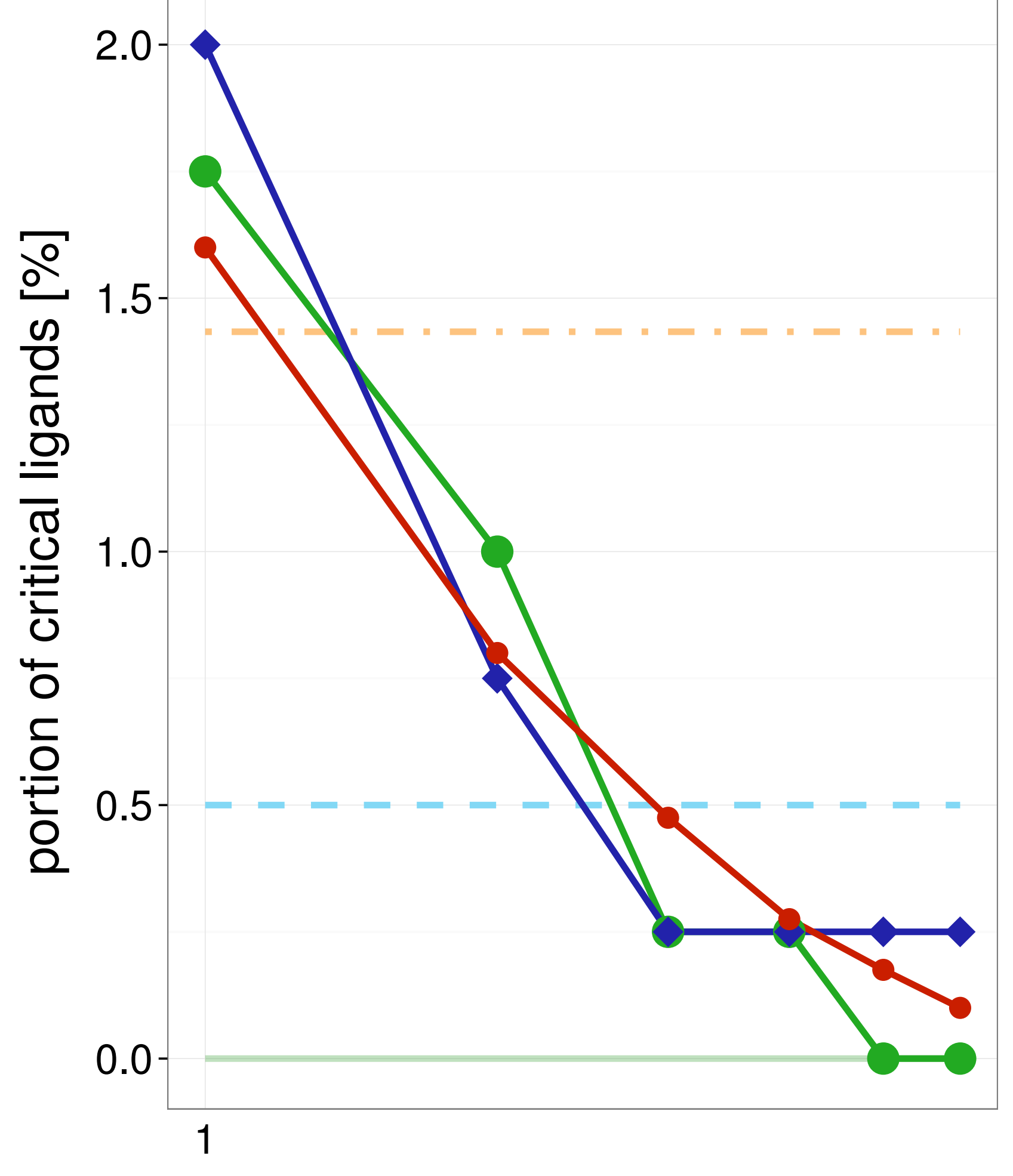

maximal ensemble size
Average single structure

_ Best single structure

Whole ensemble

SIENA

$\neg$ Clustering

$\longrightarrow$ Random 
Training set

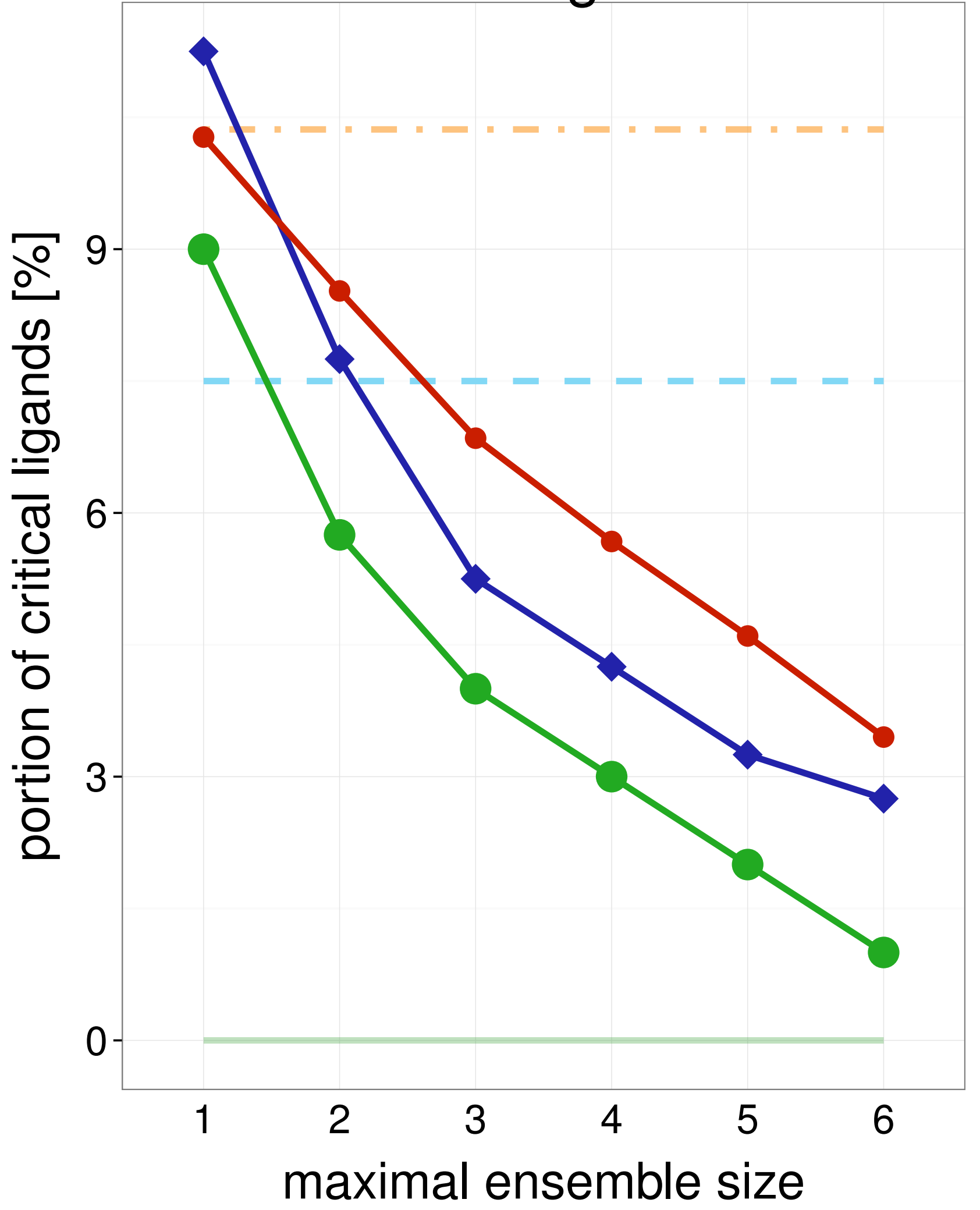

Test set

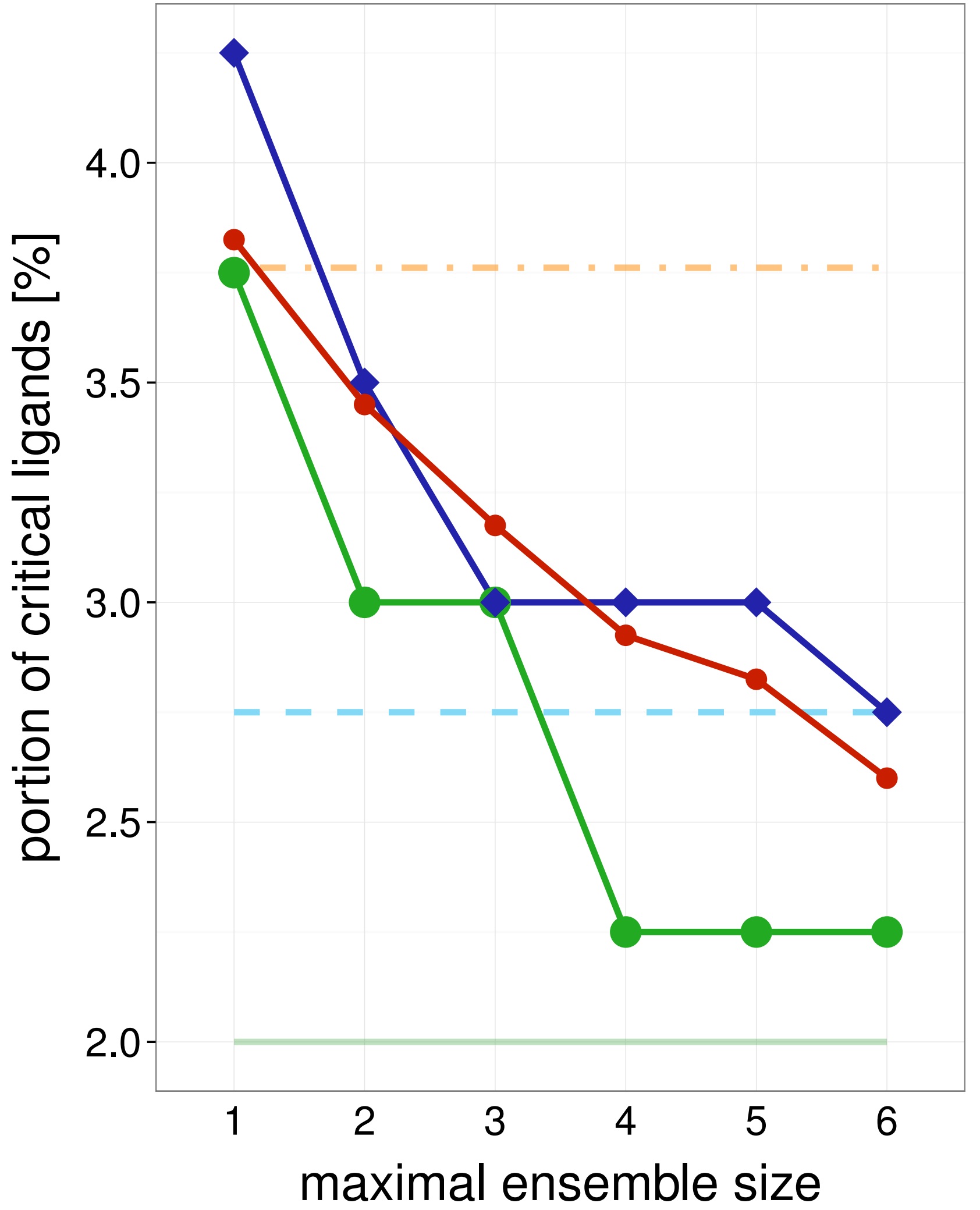

Average single structure

_ Best single structure

Whole ensemble

- SIENA

$\neg$ Clustering

$\multimap$ Random

Average single structure

_ _ Best single structure

Whole ensemble

- SIENA

$\neg$ Clustering

$\multimap$ Random
$1 \mathrm{HII}$

Training set

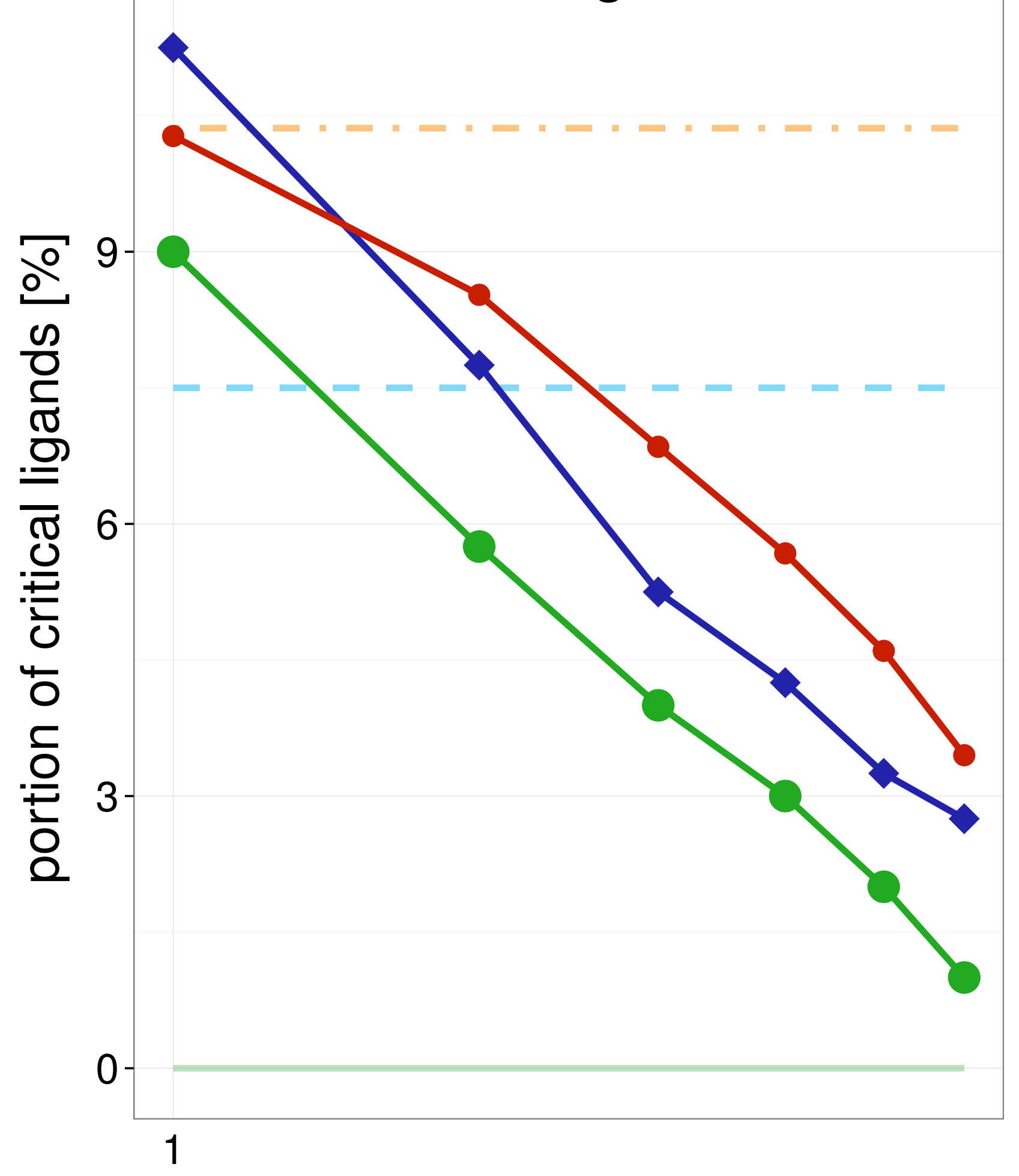

maximal ensemble size

Test set

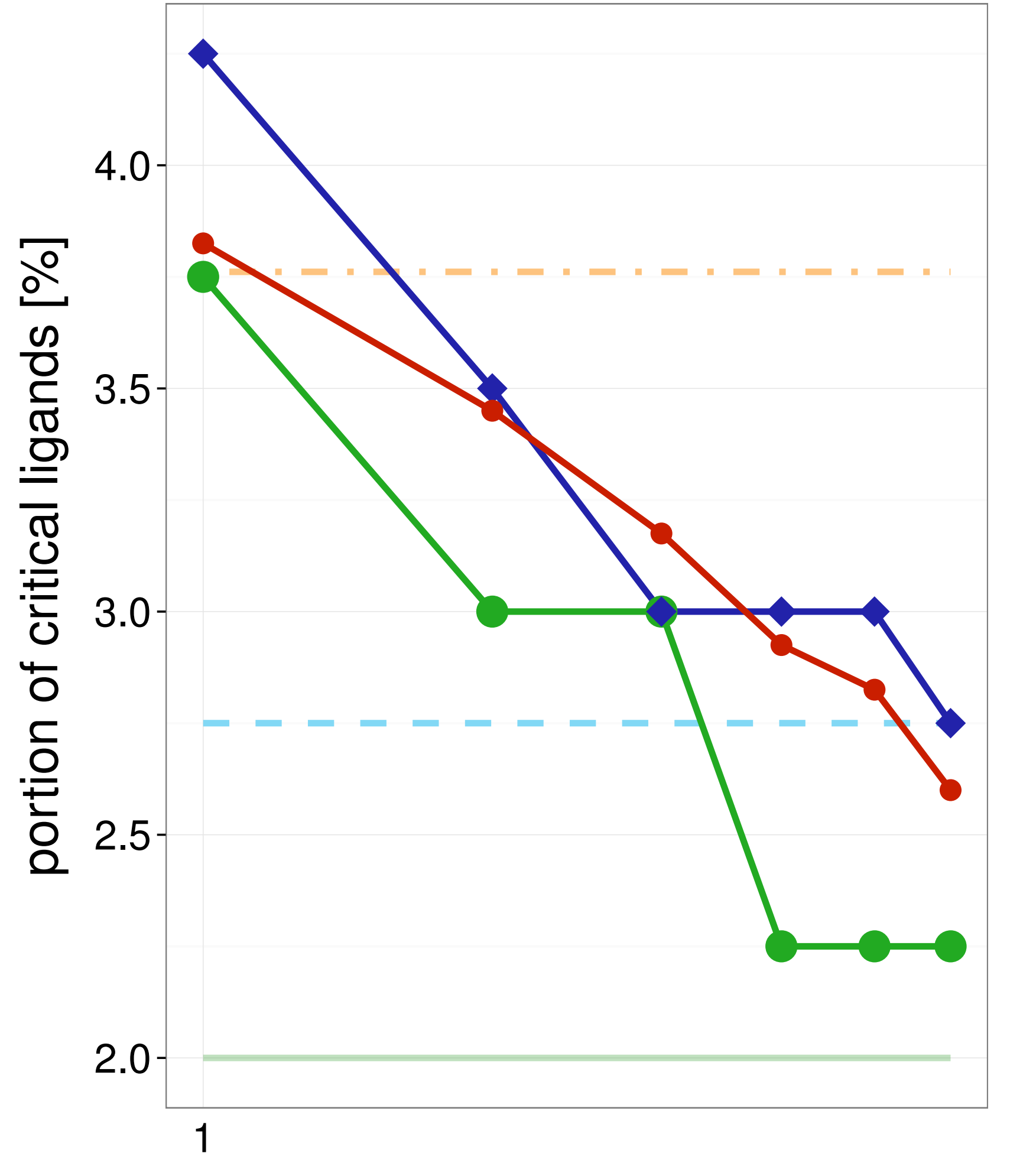

Average single structure

Best single structure

Whole ensemble

SIENA

$\neg$ Clustering

$\multimap$ Random 


\section{Training set}

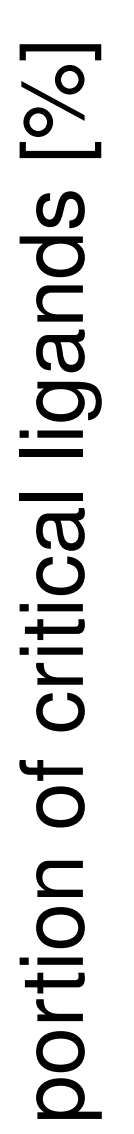

5.0

2.5

3.0

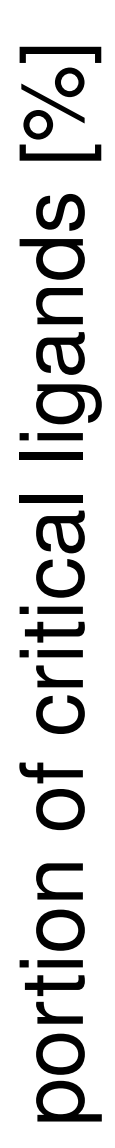

maximal ensemble size

maximal ensemble size

Test set

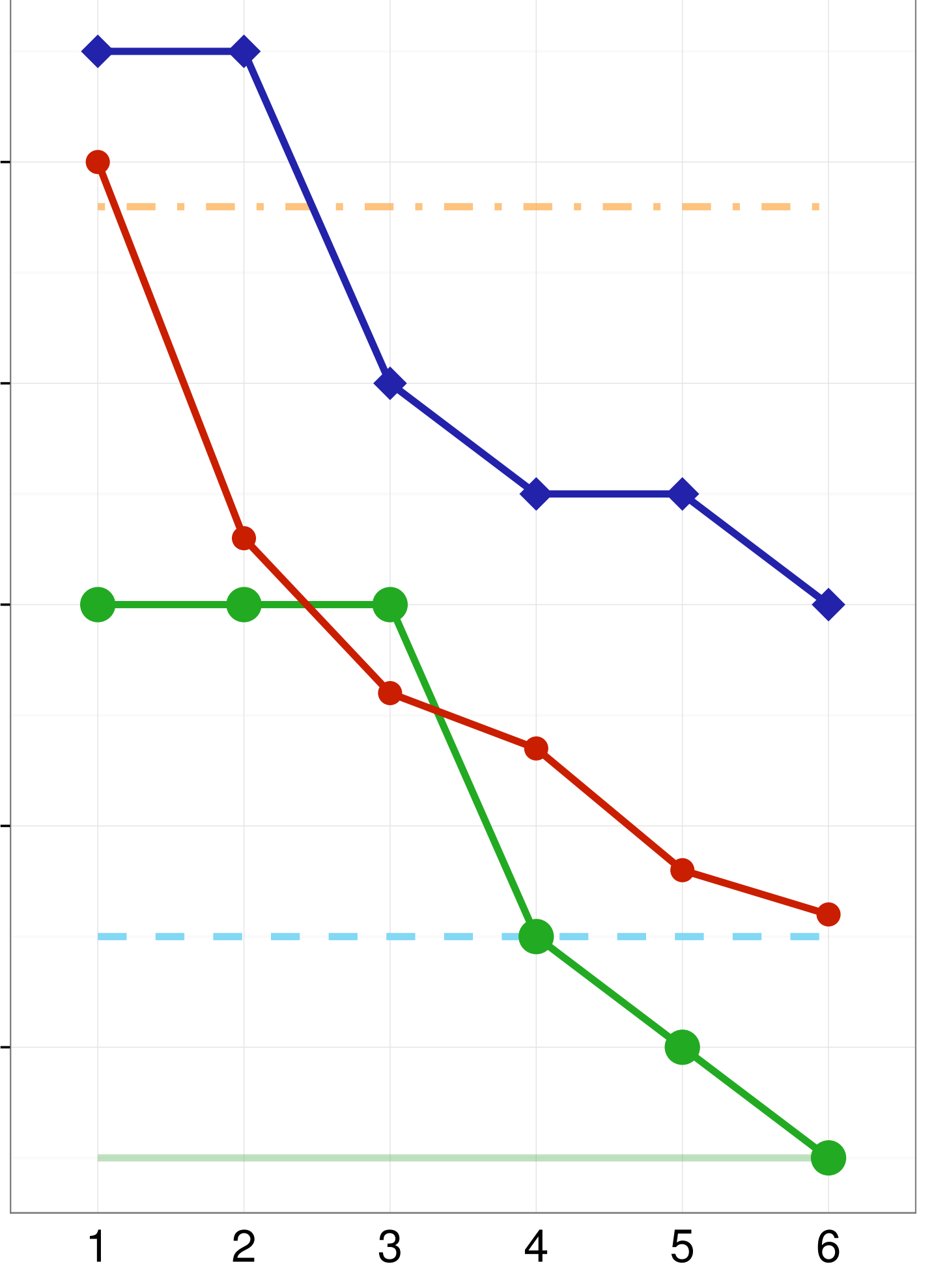

Training set

Average single structure

- Best single structure

Whole ensemble

- SIENA

$\neg$ Clustering

$\multimap$ Random

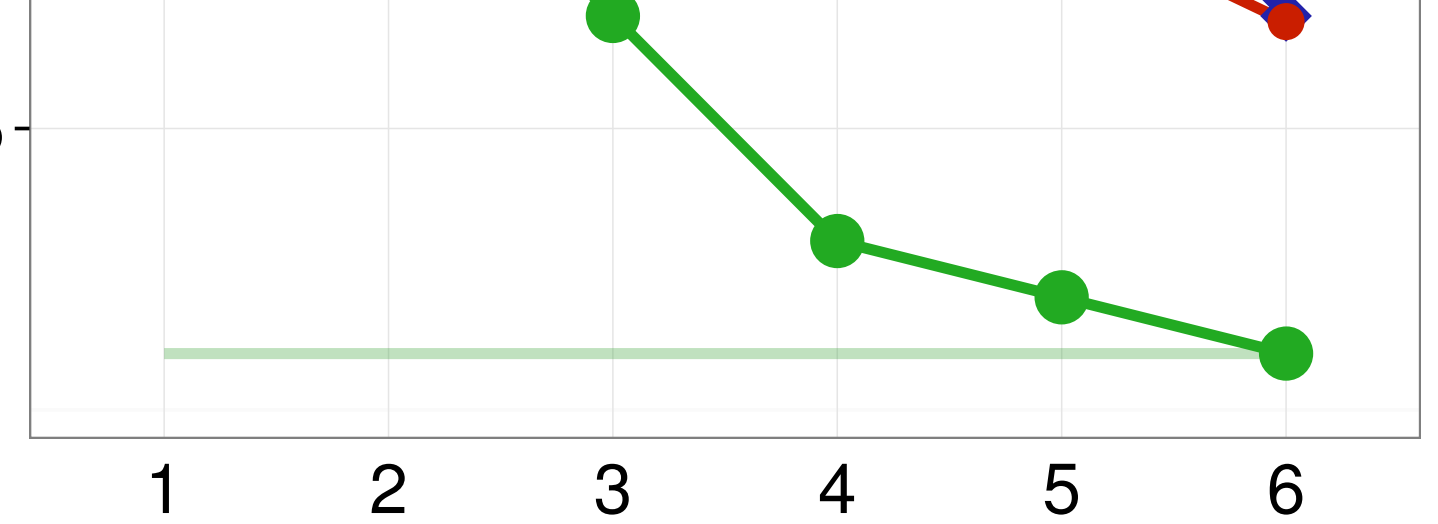

Average single structure

_ _ Best single structure

Whole ensemble

- SIENA

$\neg$ Clustering

$\multimap$ Random

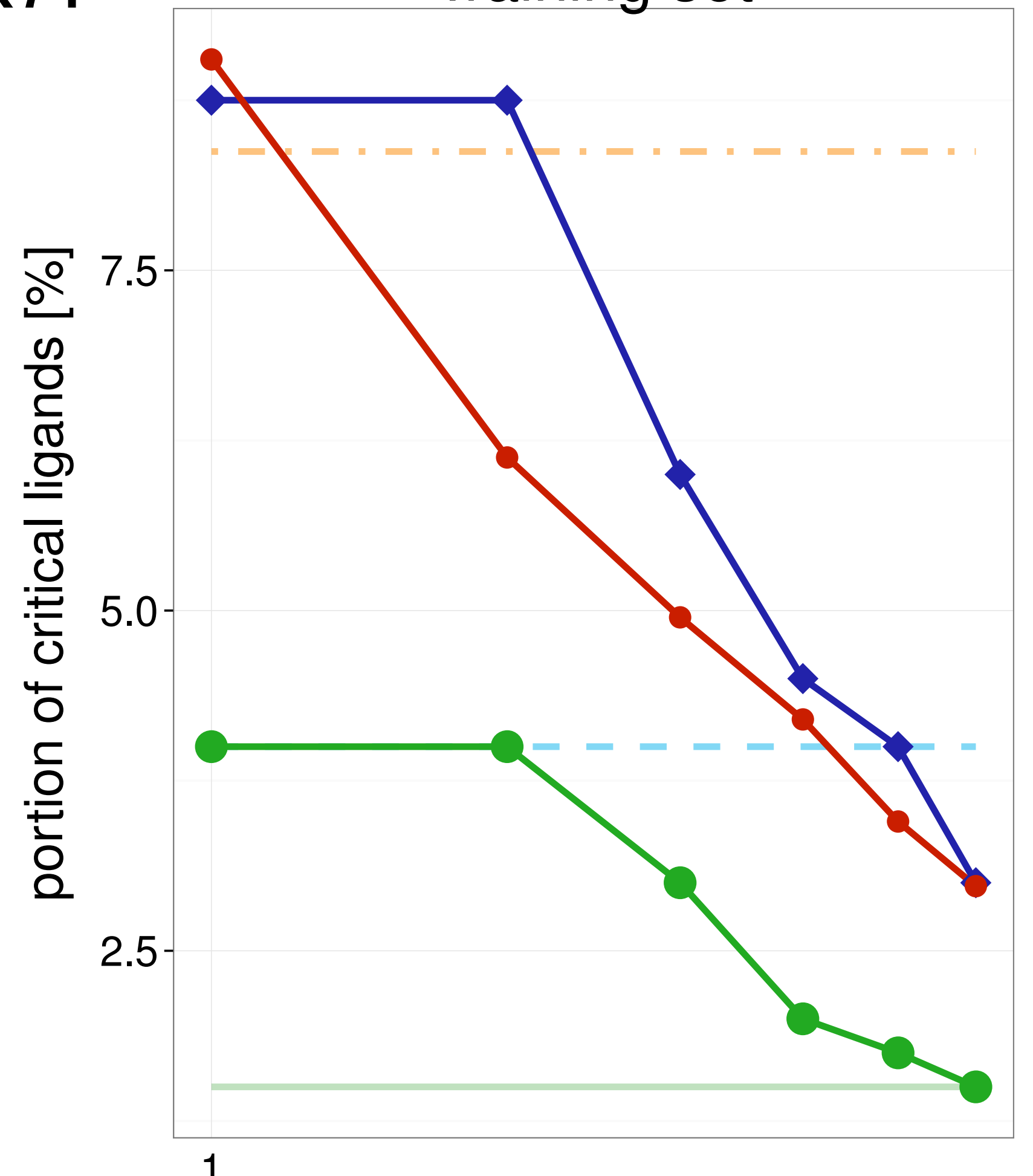

Average single structure

_ Best single structure

Whole ensemble

- SIENA

$\neg$ Clustering

$\rightarrow$ Random

maximal ensemble size

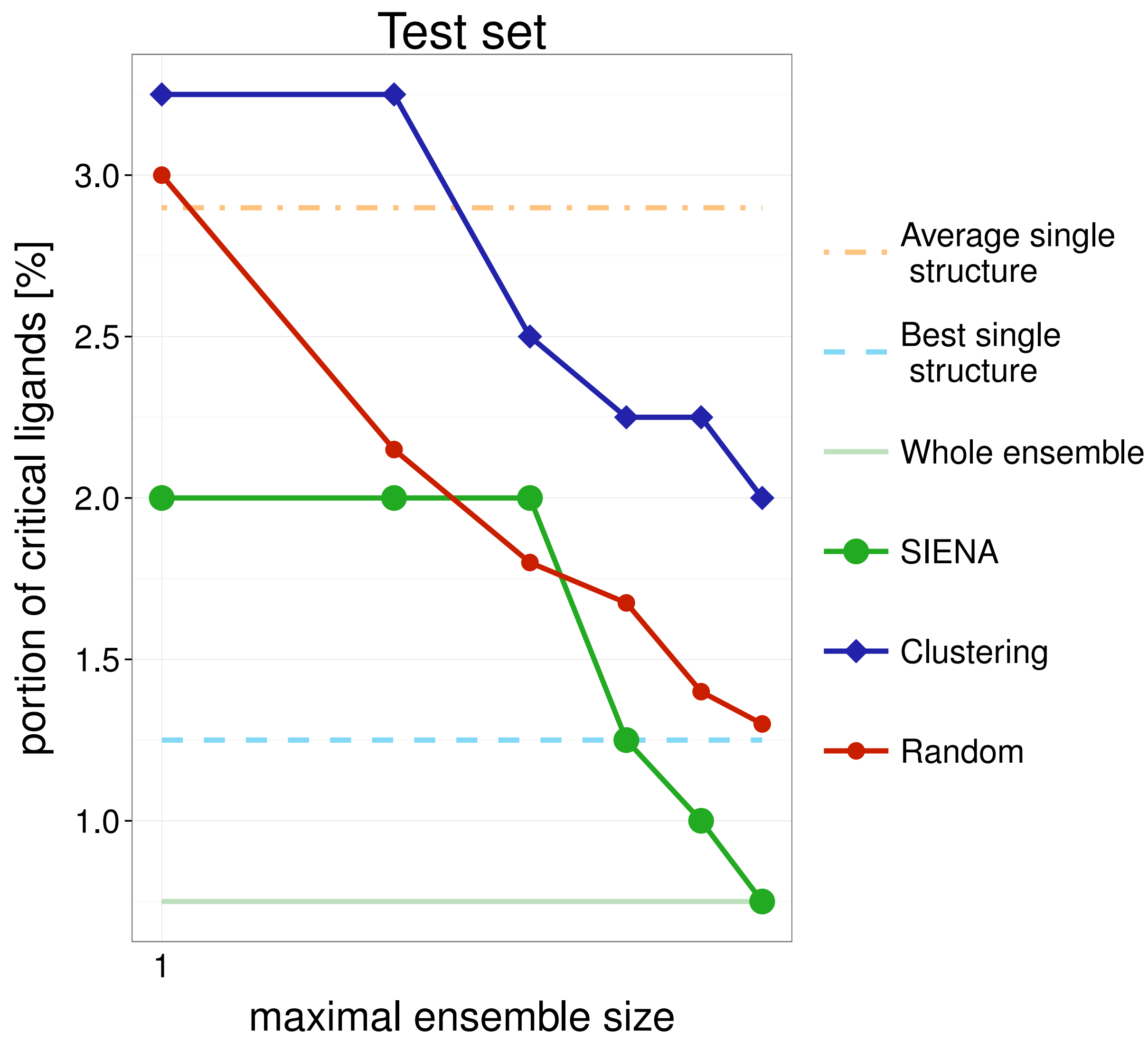


Training set

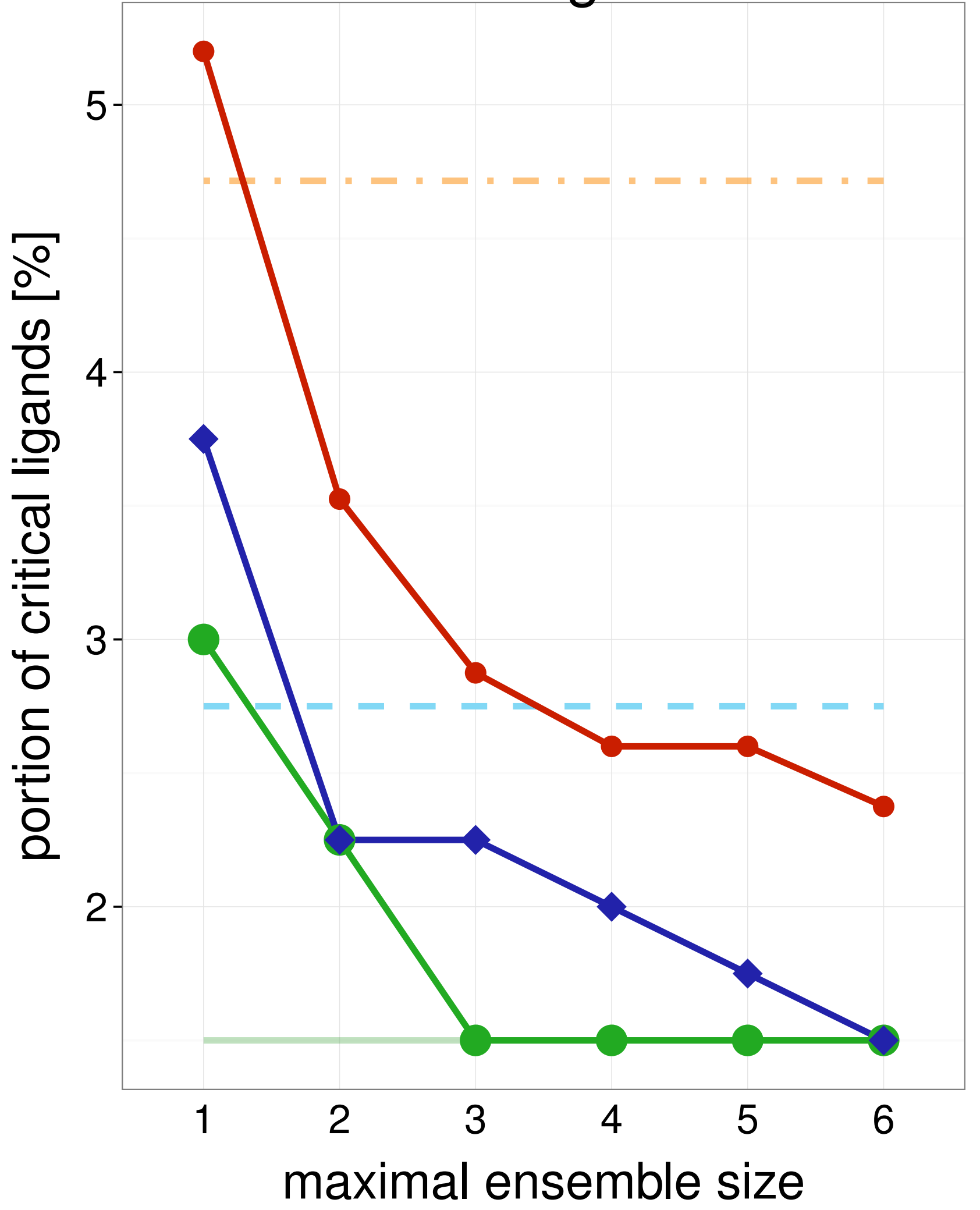

\section{Test set}

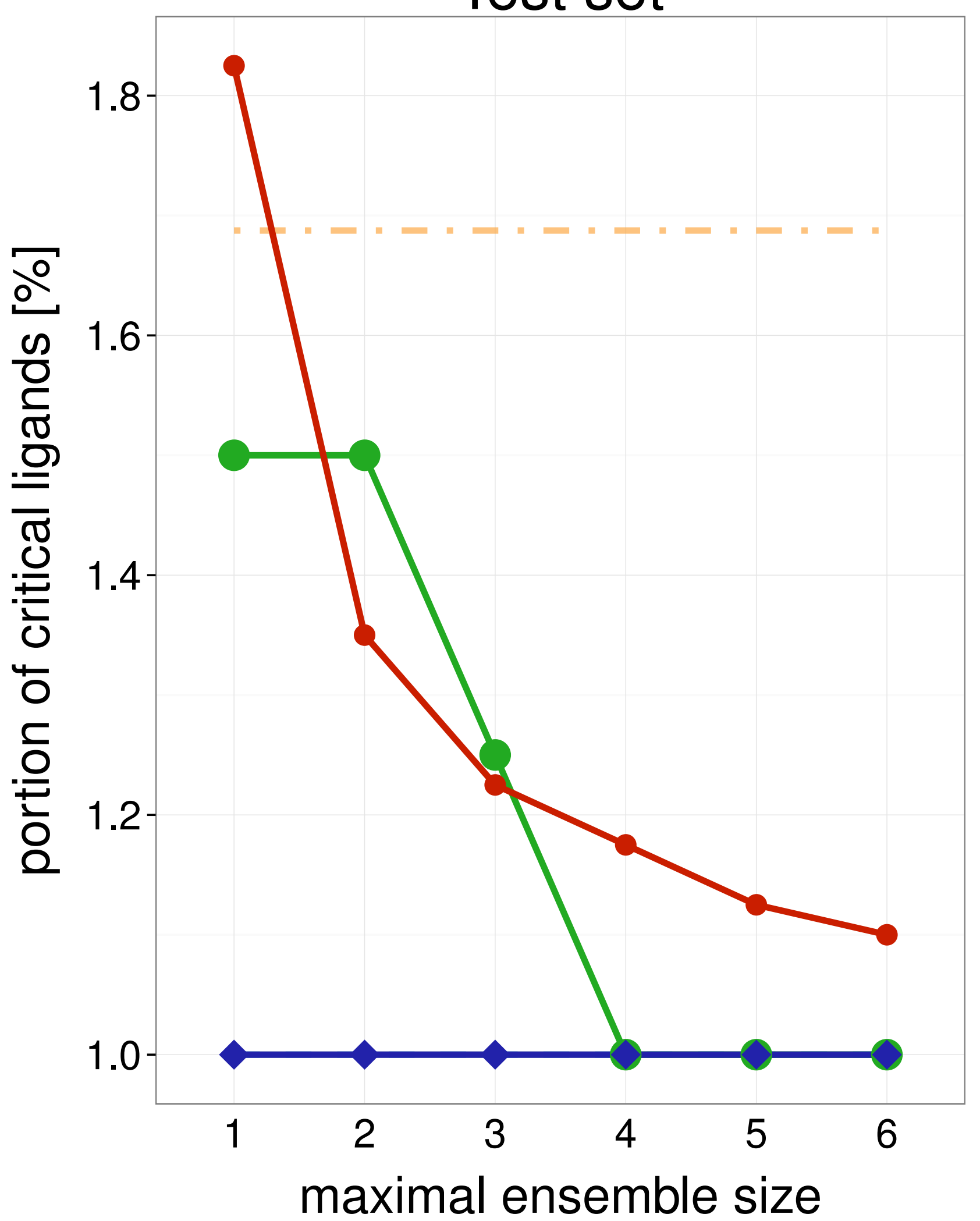

1NNU

Training set

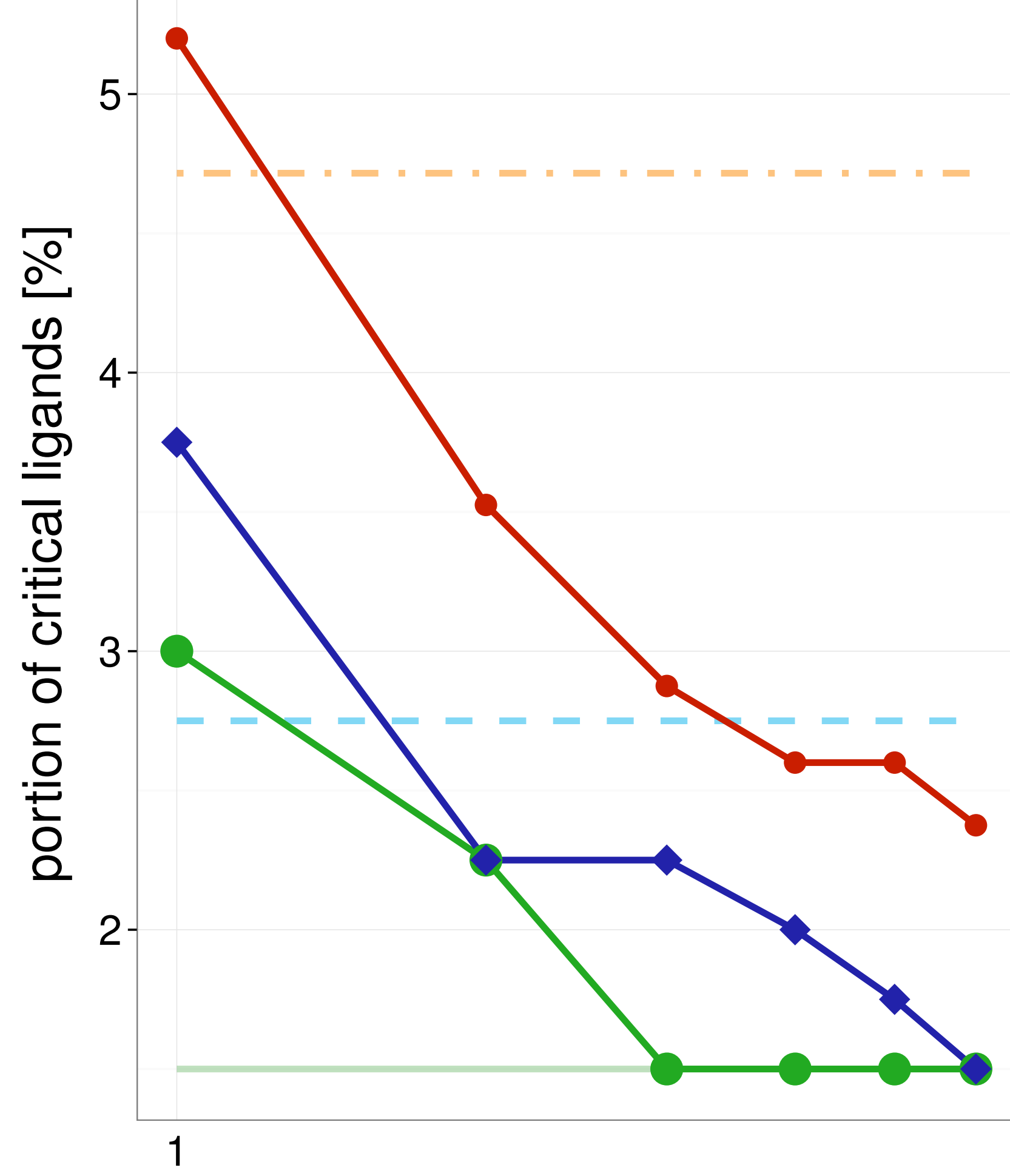

maximal ensemble size

Test set

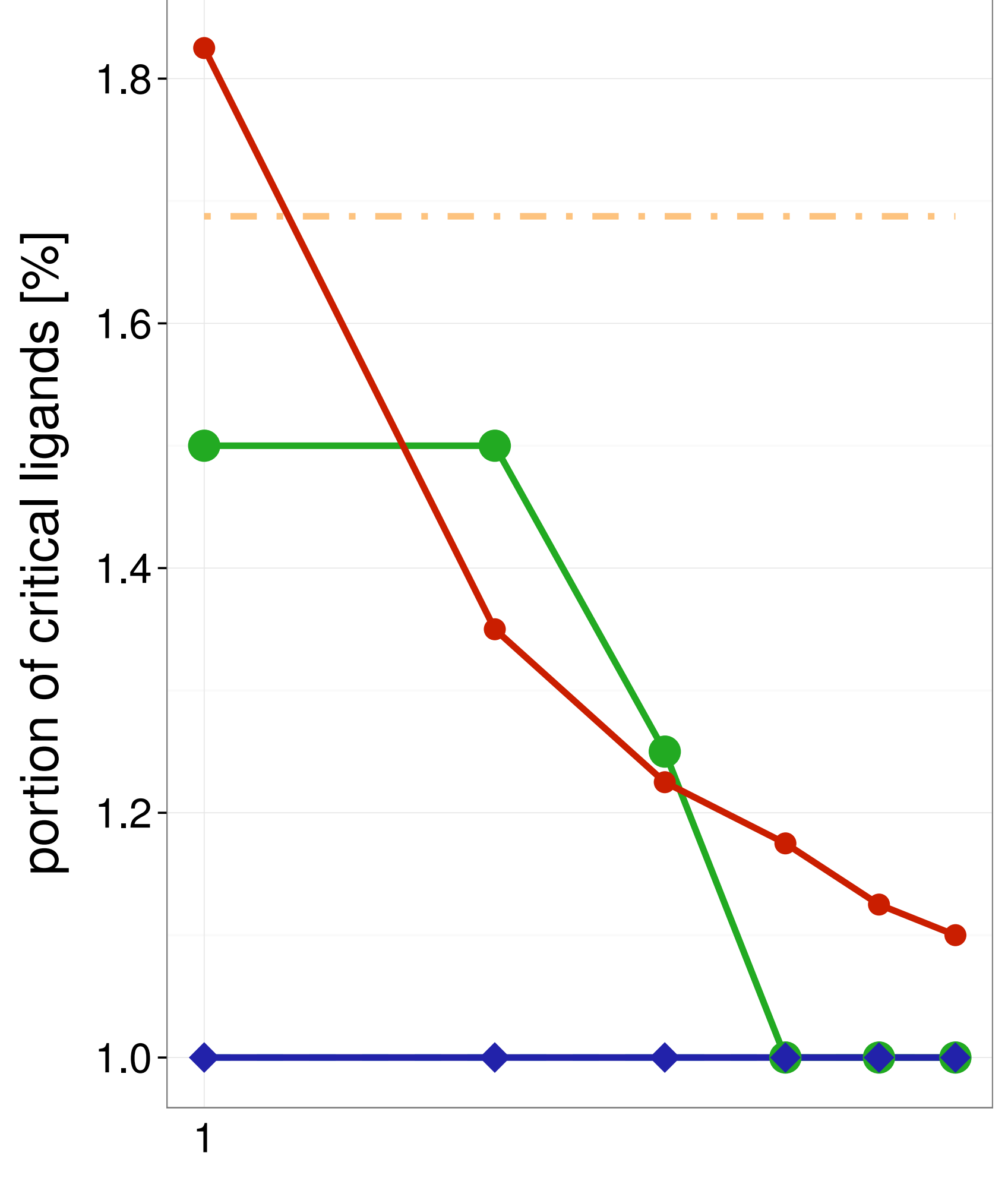

maximal ensemble size
Whole ensemble

- SIENA

$\neg$ Clustering

$\multimap$ Random
Average single structure

_ Best single structure

Whole ensemble

\section{structure}

Best single

structure

Whole ensemble

SIENA

$\neg$ Clustering

$\multimap$ Random

SIENA

$\neg$ Clustering

$\multimap$ Random 
Training set

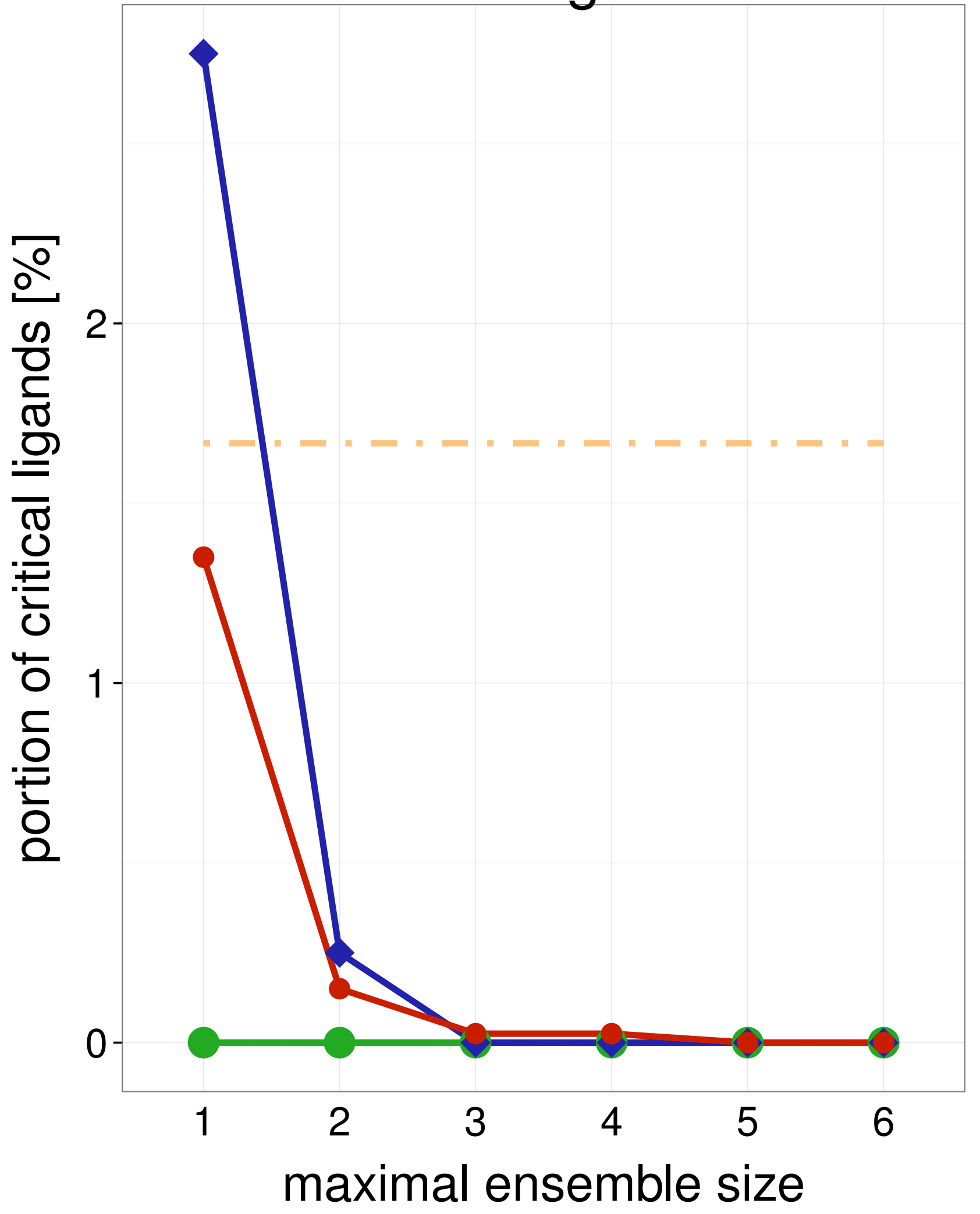

\section{Test set}

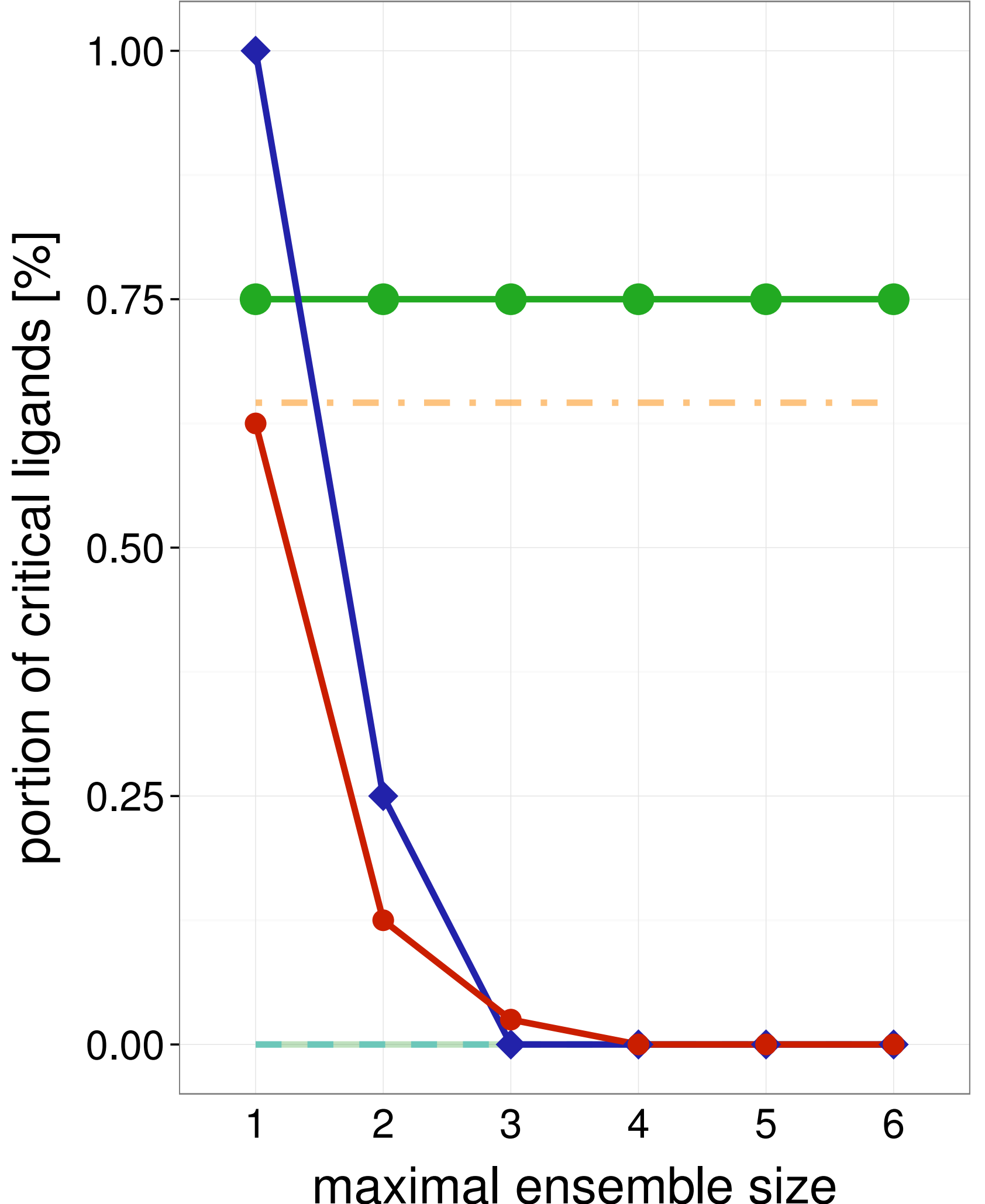

Average single structure

_ Best single structure

Whole ensemble

- SIENA

$\multimap$ Clustering

$\longrightarrow$ Random

Average single structure

_ _ Best single structure

Whole ensemble

- SIENA

$\neg$ Clustering

- Random
Training set

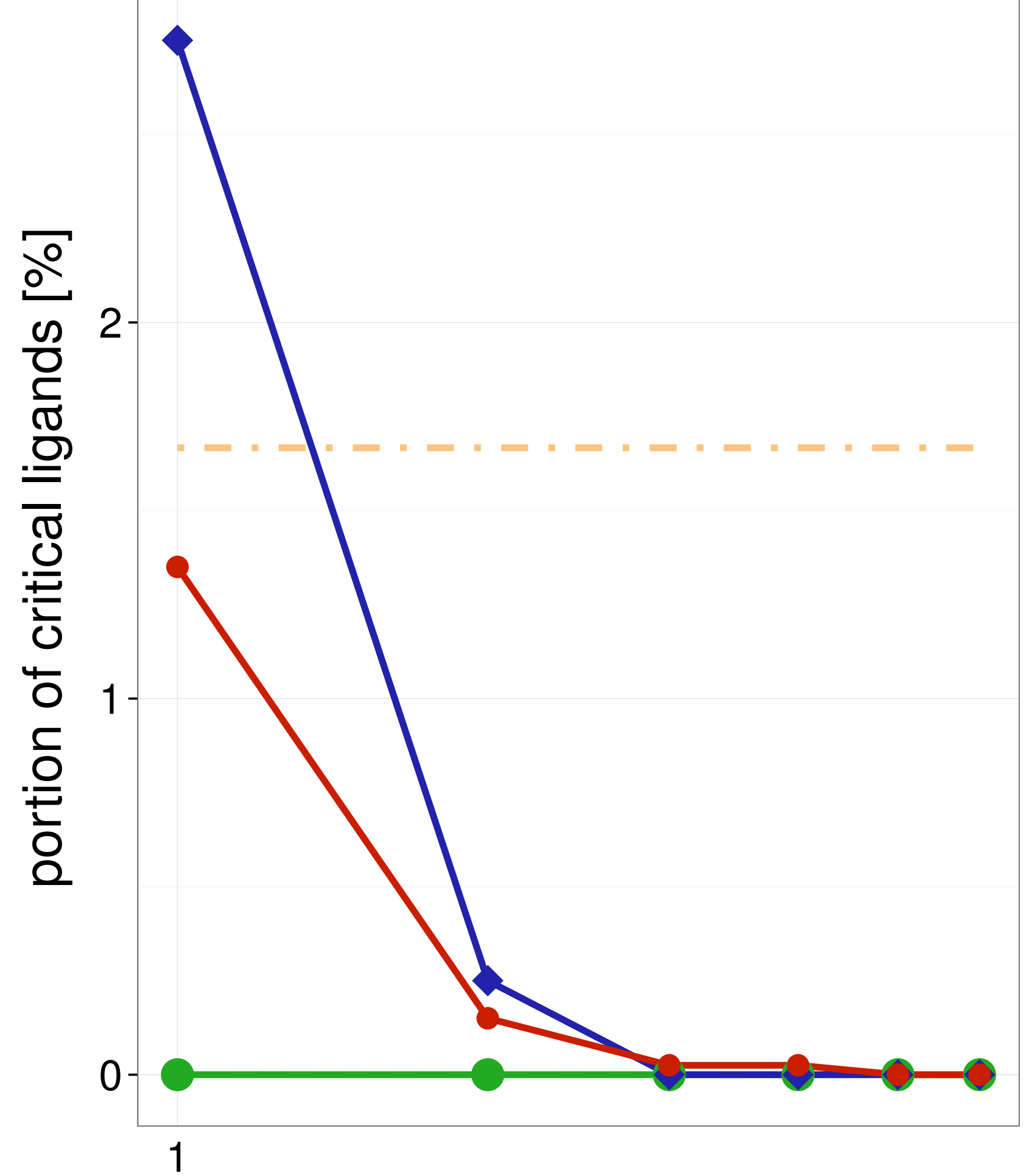

maximal ensemble size

Test set

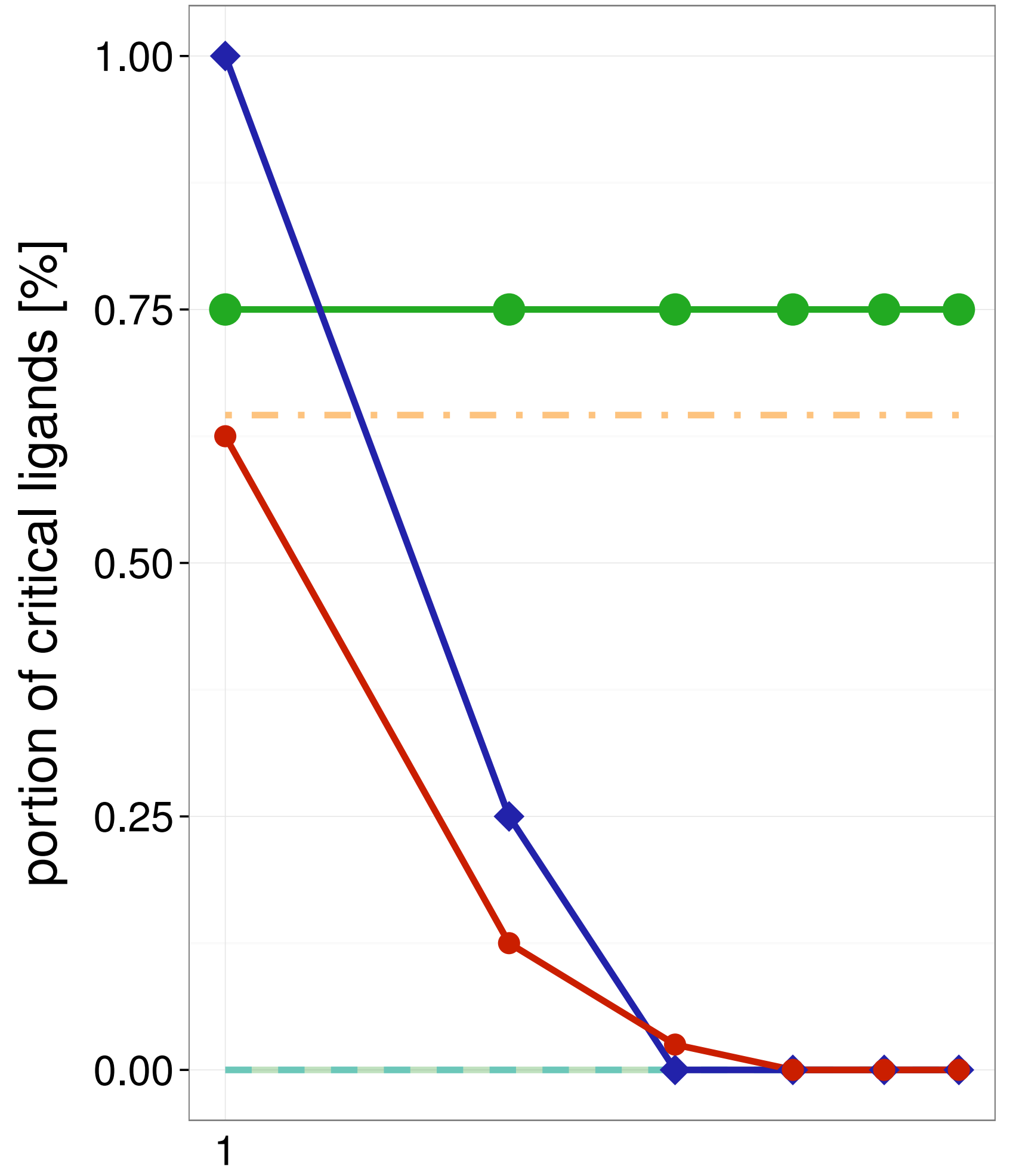

Average single structure

Best single structure

Whole ensemble

SIENA

$\neg$ Clustering

$\longrightarrow$ Random 
Training set

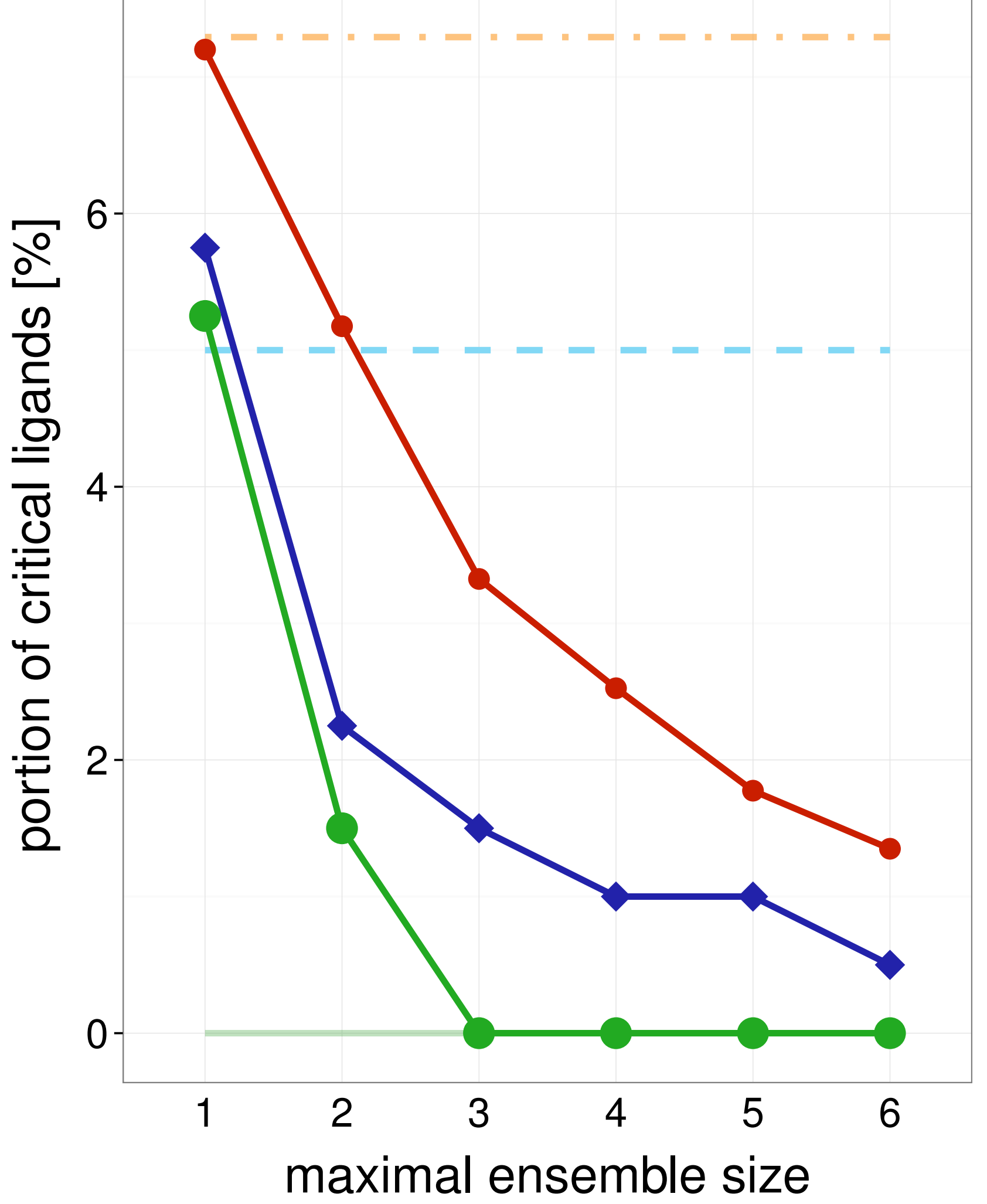

Test set

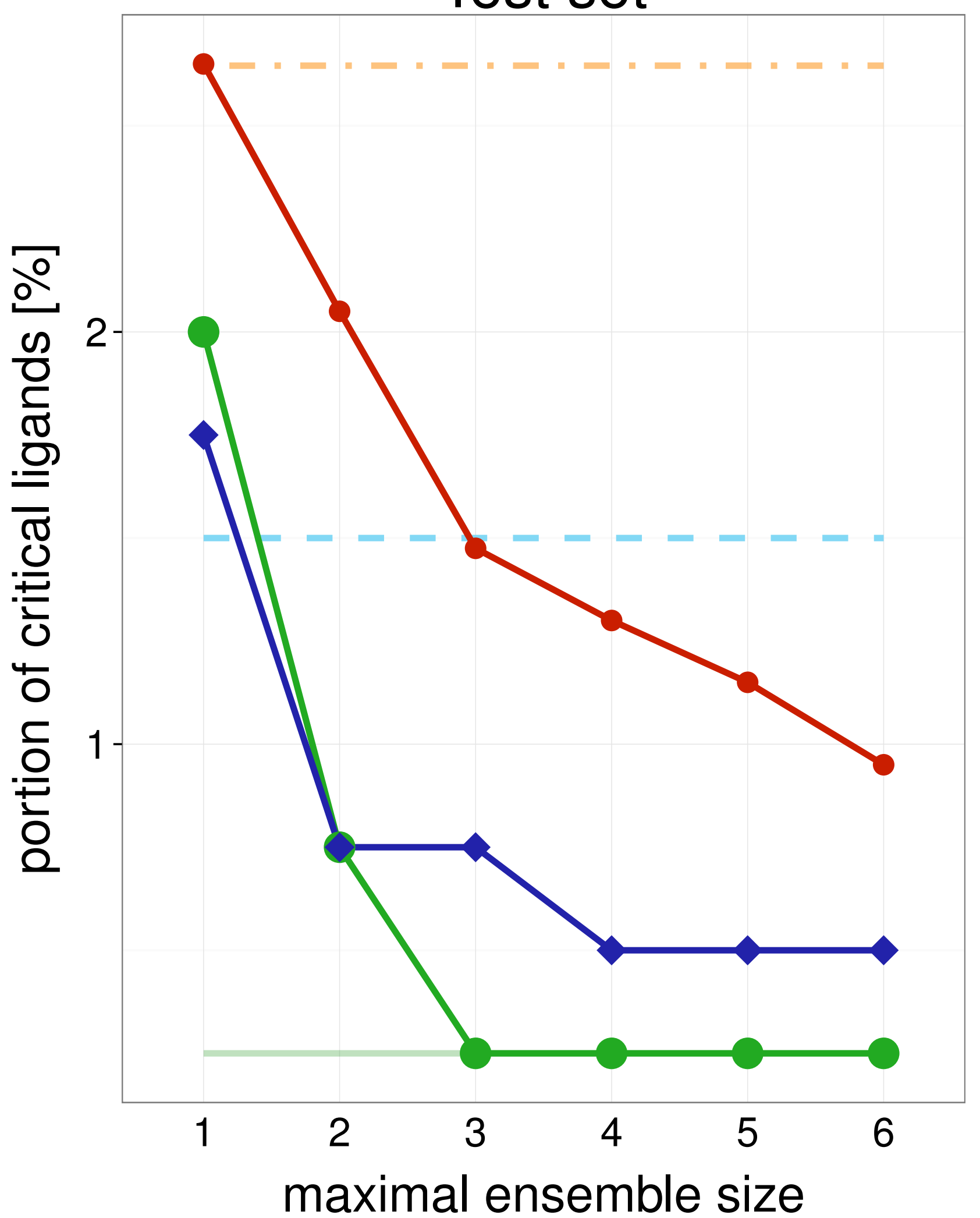

Average single structure

_ Best single structure

Whole ensemble

- SIENA

$\neg$ Clustering

$\multimap$ Random

Average single structure

_ Best single structure

Whole ensemble

- SIENA

$\neg$ Clustering

$\because$ Random

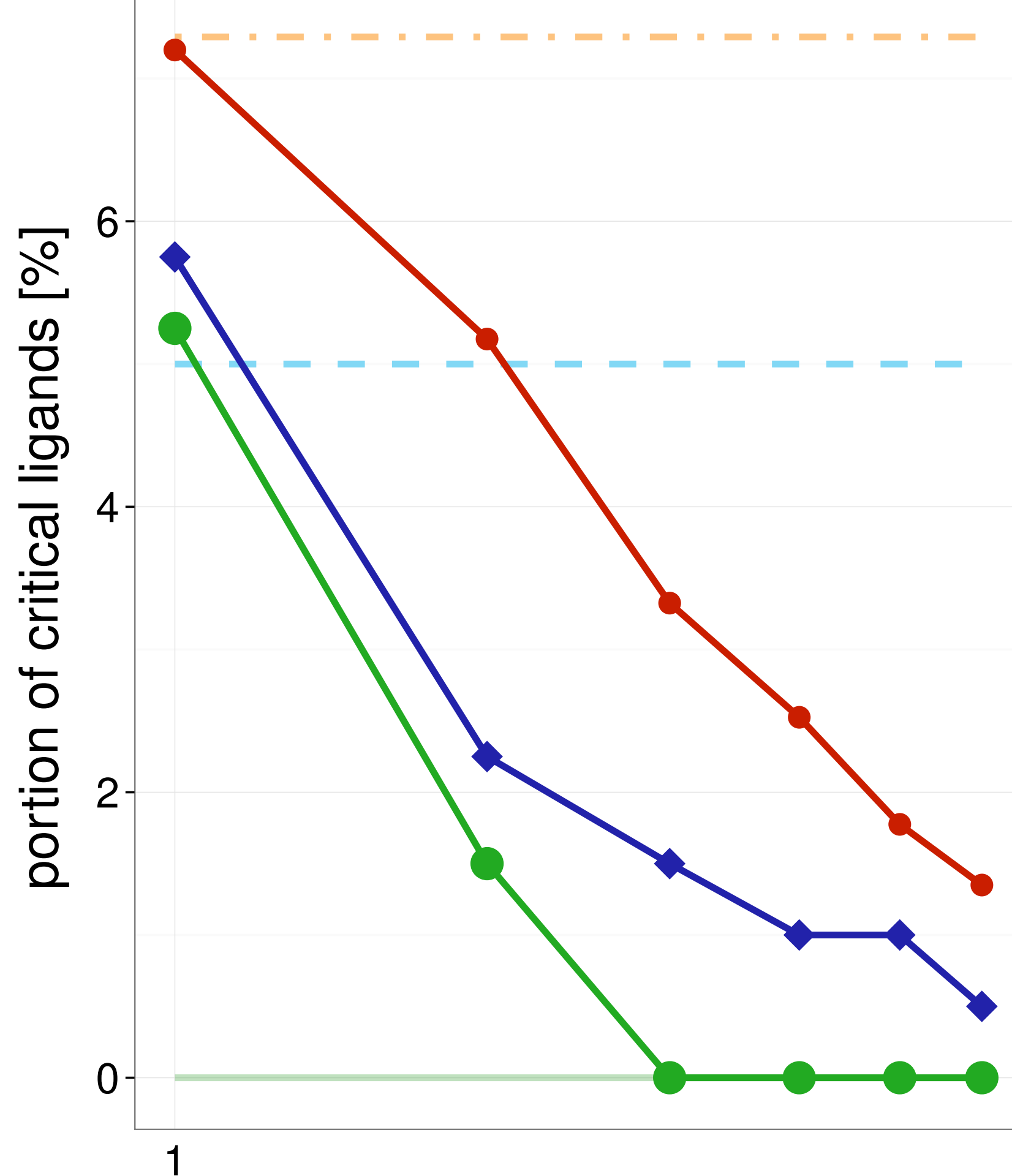

maximal ensemble size

Test set

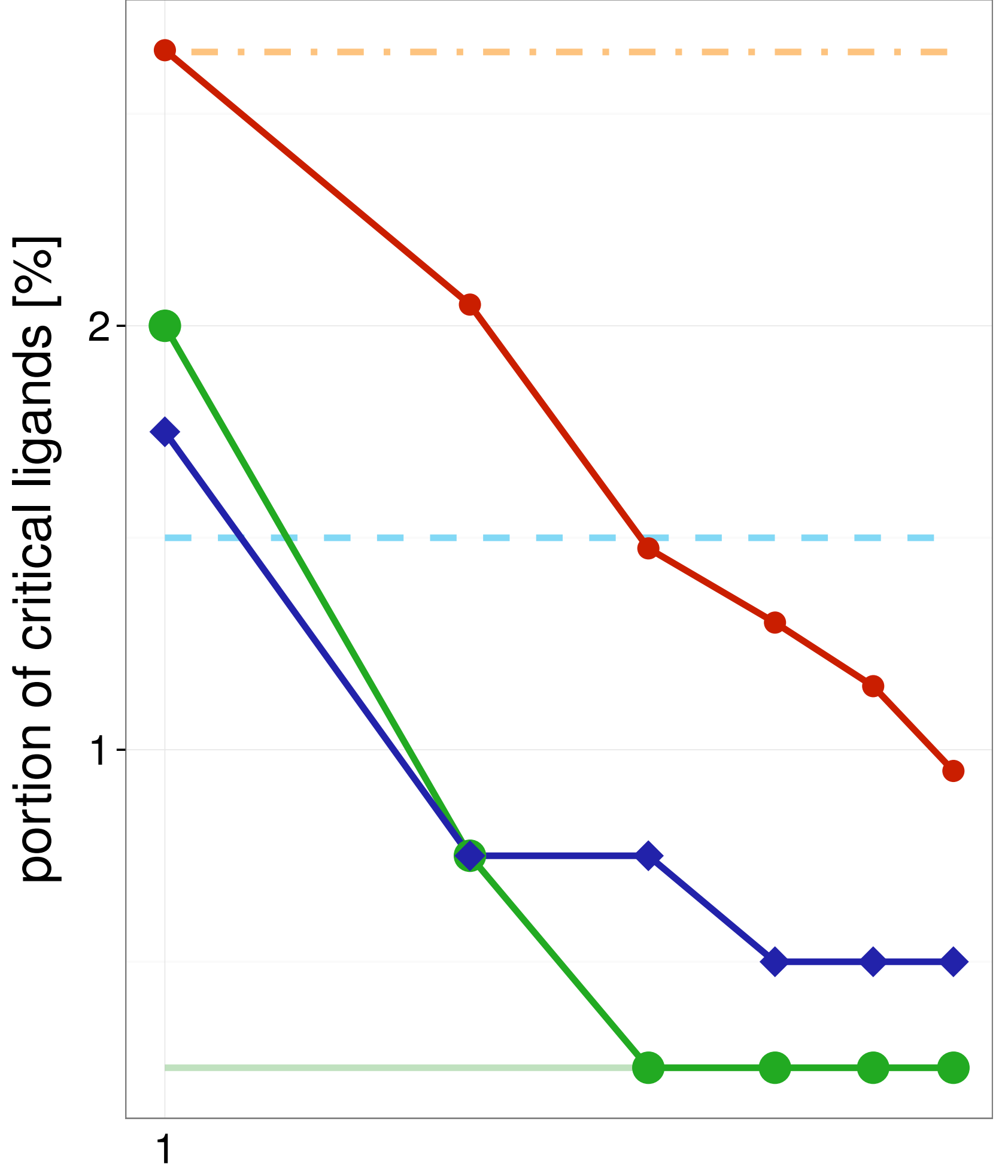

maximal ensemble size
Average single structure

_ Best single structure

Whole ensemble

SIENA

$\leadsto$ Clustering

$\multimap$ Random 
Training set

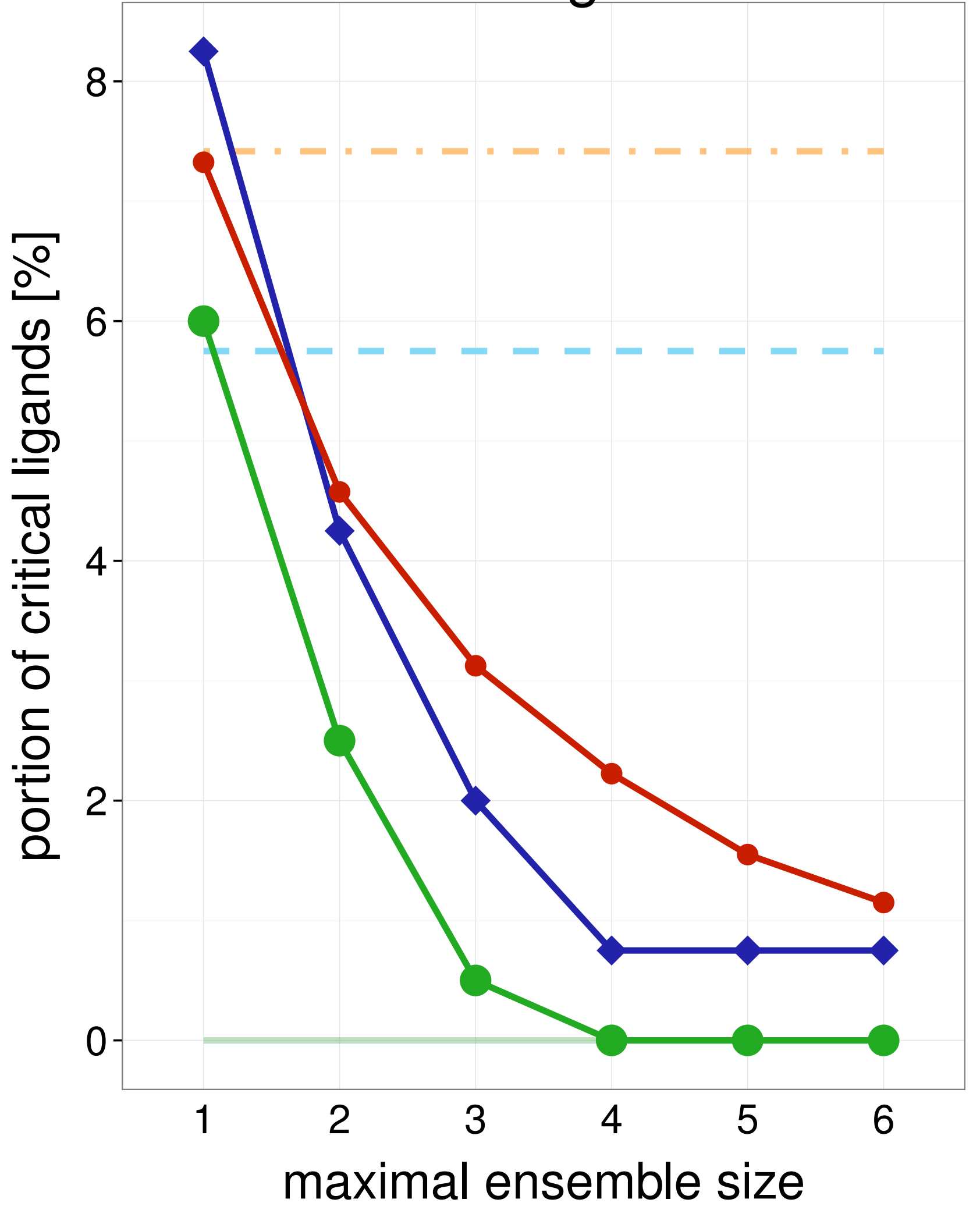

\section{Test set}

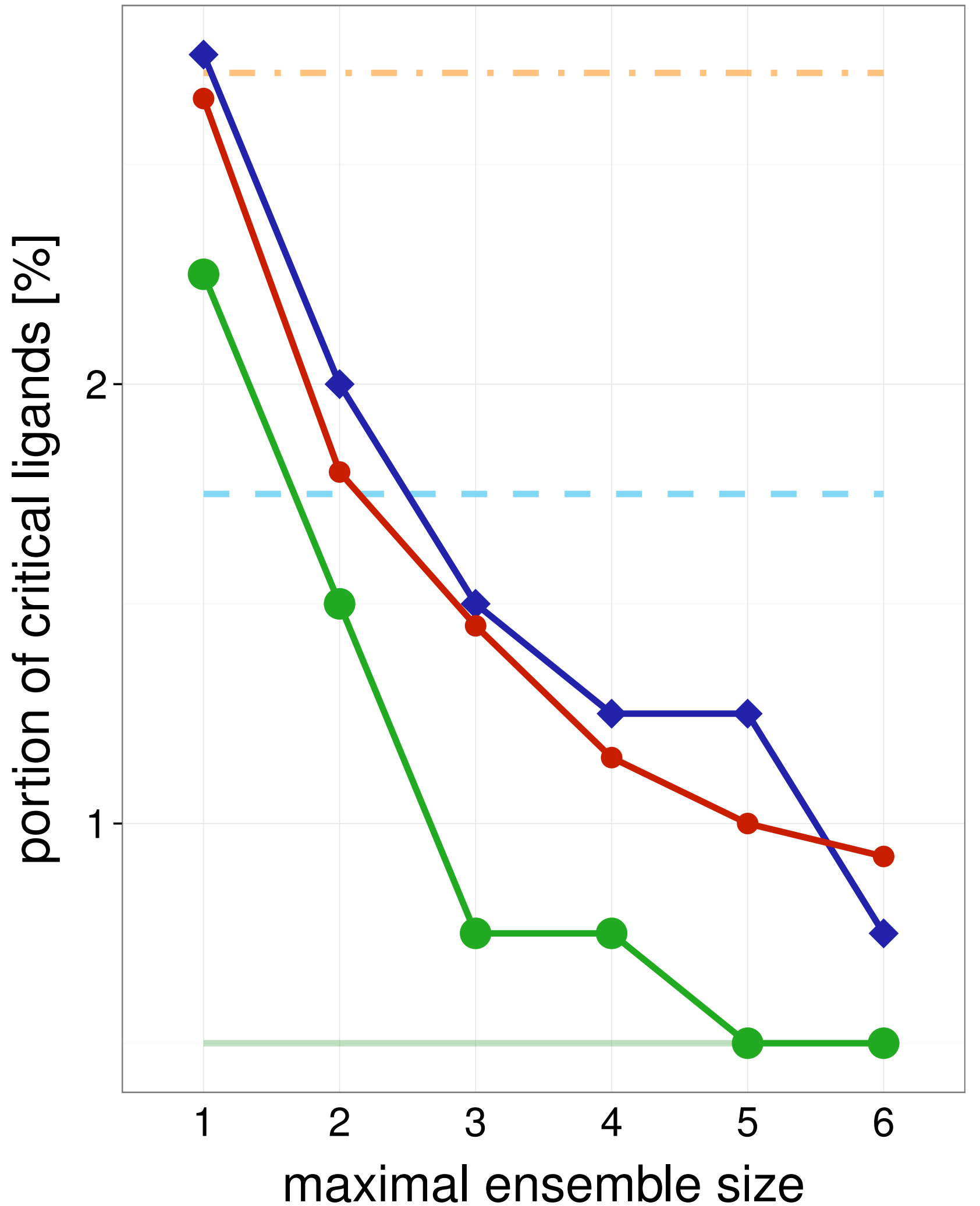

$1 U 10$

Training set

Average single structure

_ Best single structure

Whole ensemble

- SIENA

$\neg$ Clustering

$\multimap$ Random

Average single structure

_ Best single structure

Whole ensemble

- SIENA

$\neg$ Clustering

$\rightarrow$ Random

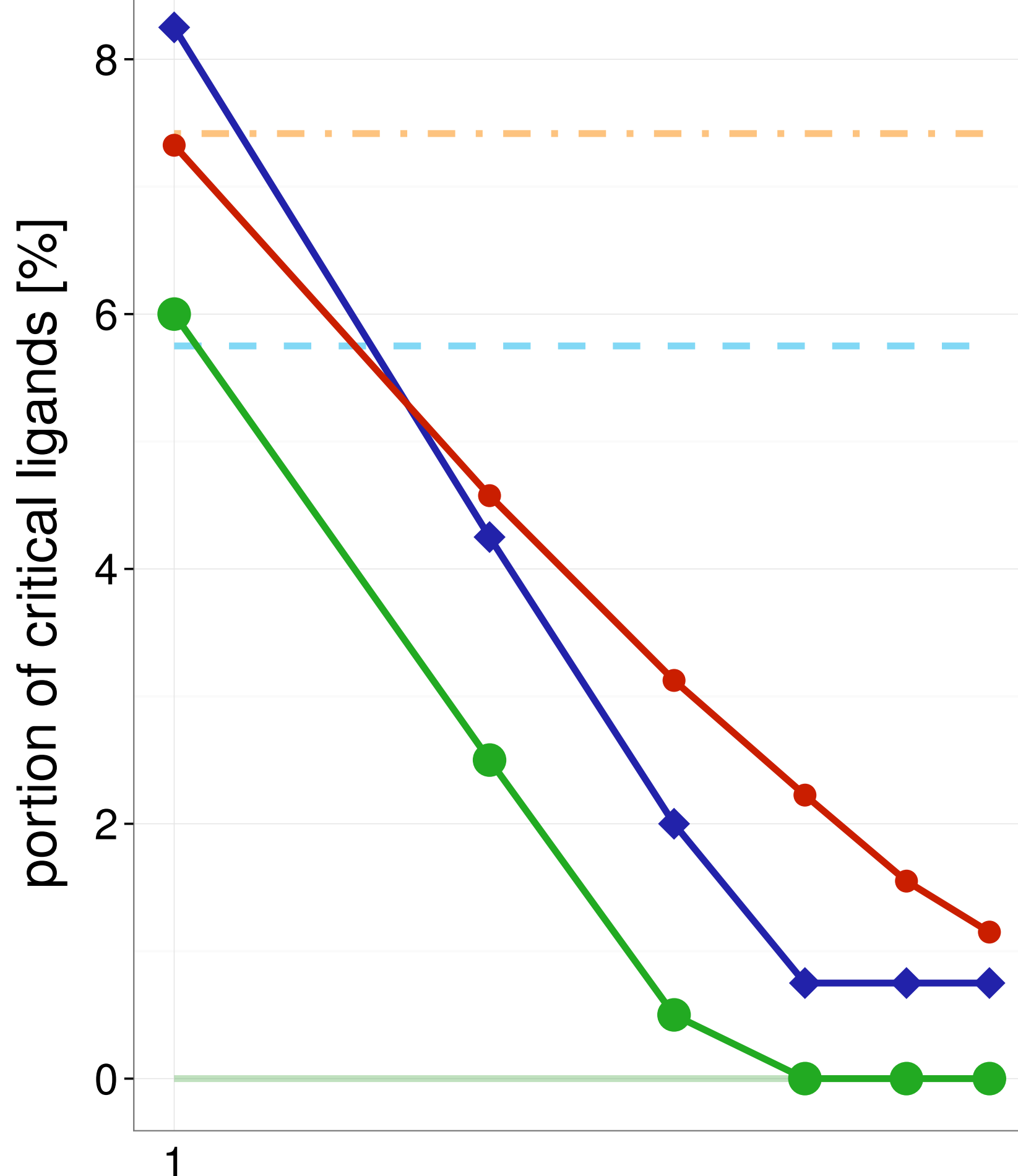

maximal ensemble size

Test set

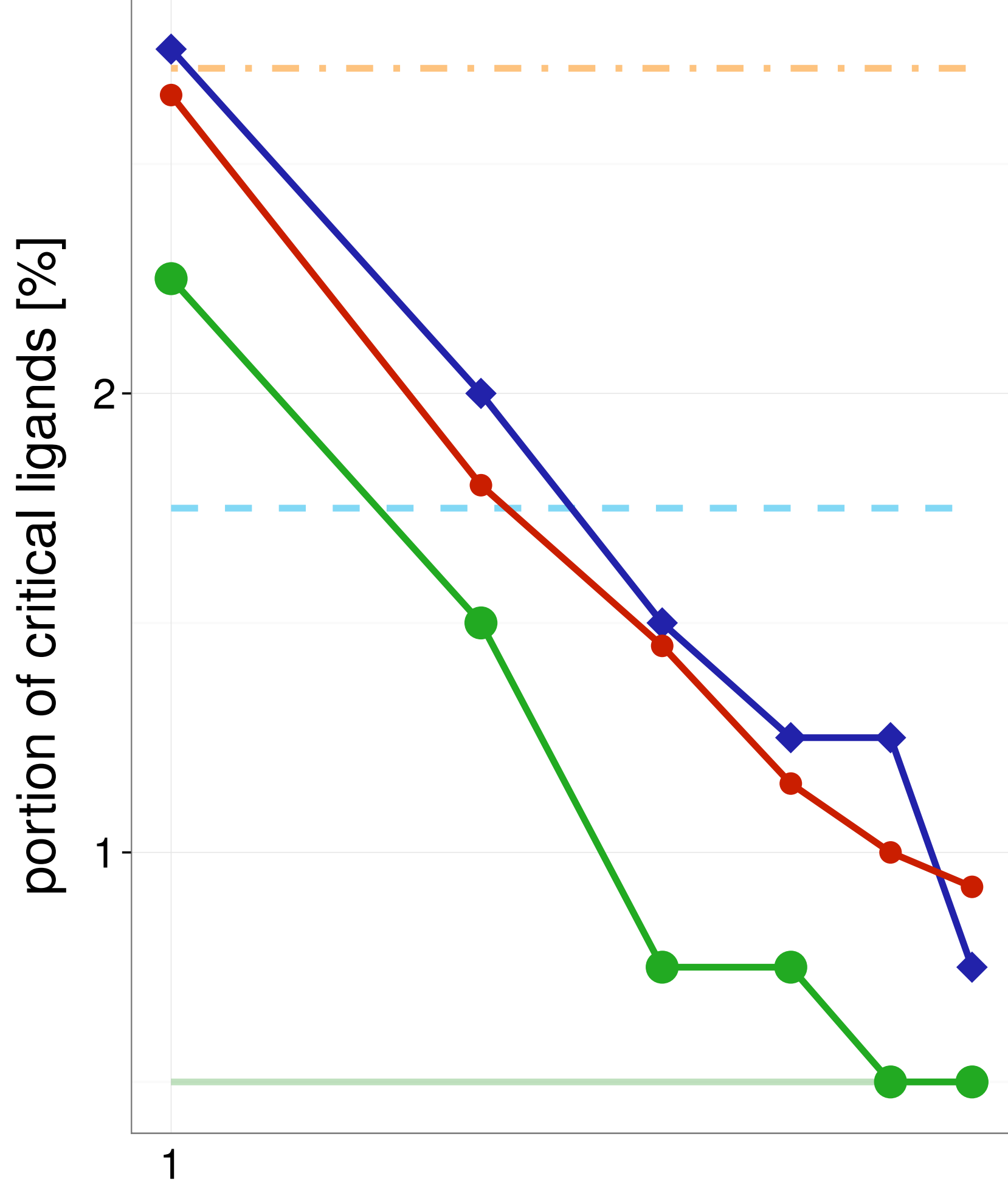

maximal ensemble size
Average single structure

_ Best single structure

Whole ensemble

SIENA

$\leadsto$ Clustering

$\multimap$ Random 
Training set

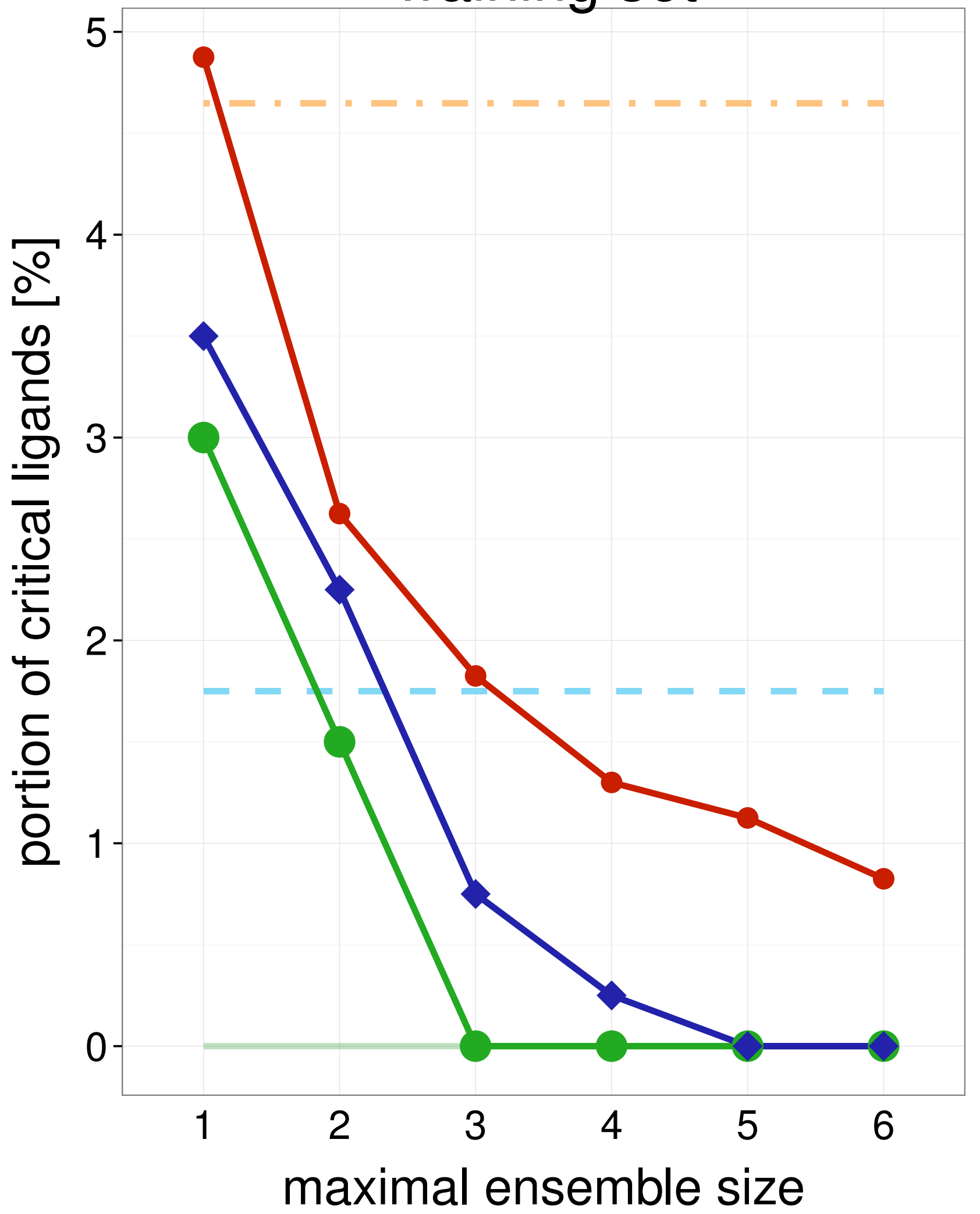

\section{Test set}

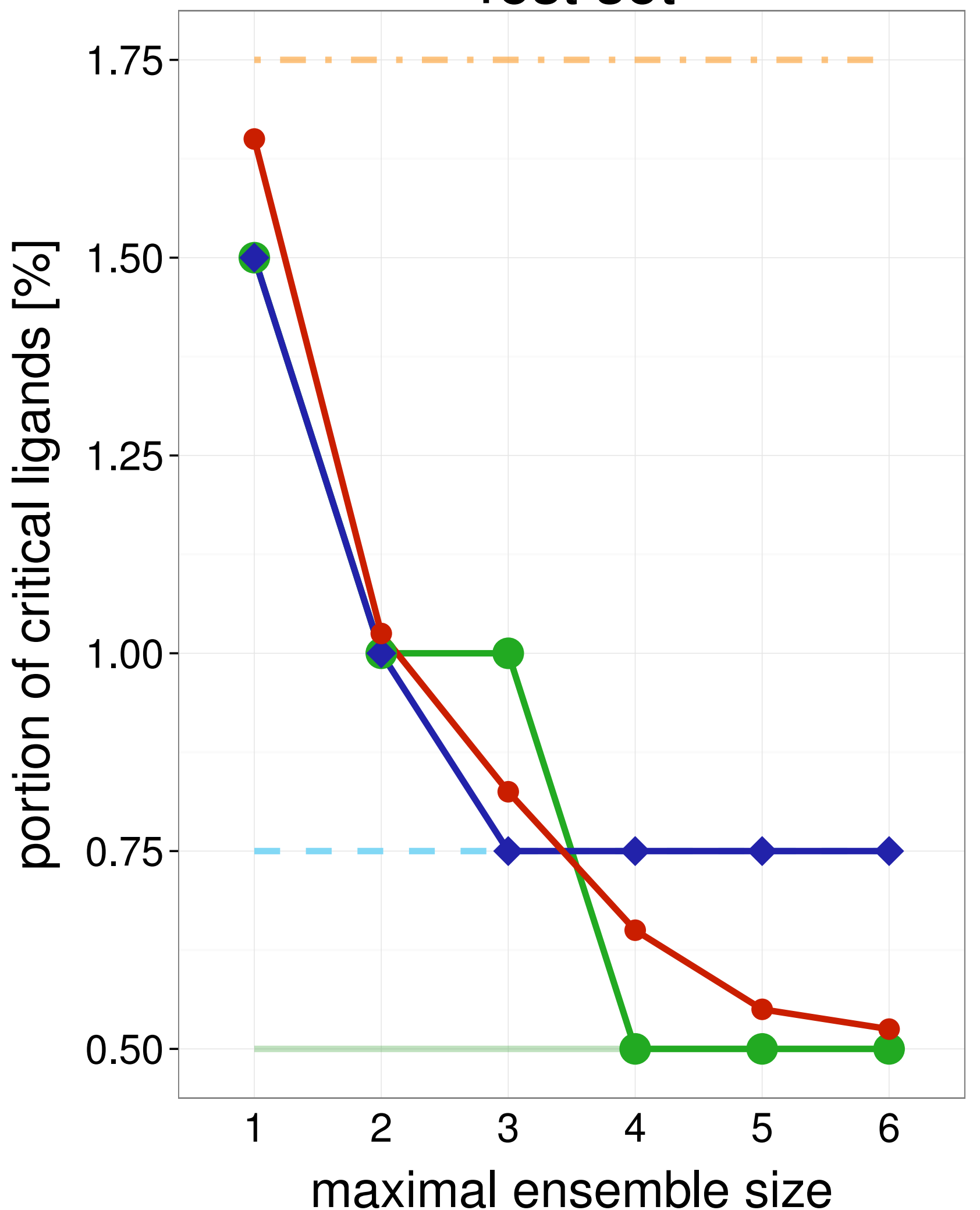

Average single structure

- Best single structure

Whole ensemble

- SIENA

$\neg$ Clustering

$\multimap$ Random

Average single structure

_ _ Best single structure

Whole ensemble

- SIENA

$\neg$ Clustering

$\multimap$ Random
1UK1

Training set

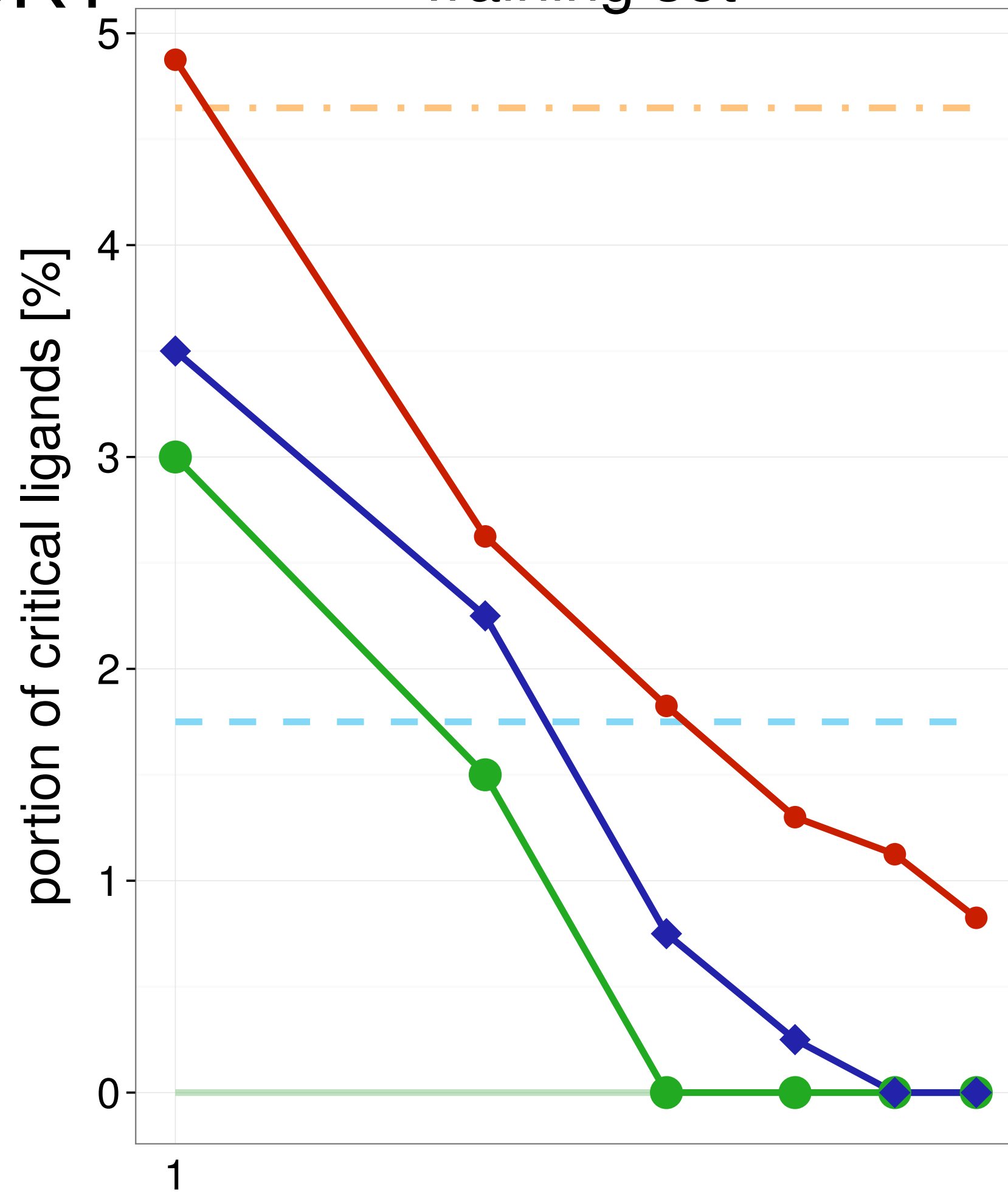

Average single structure

Best single structure

Whole ensemble

- SIENA

$\checkmark$ Clustering

- Random

maximal ensemble size

Test set

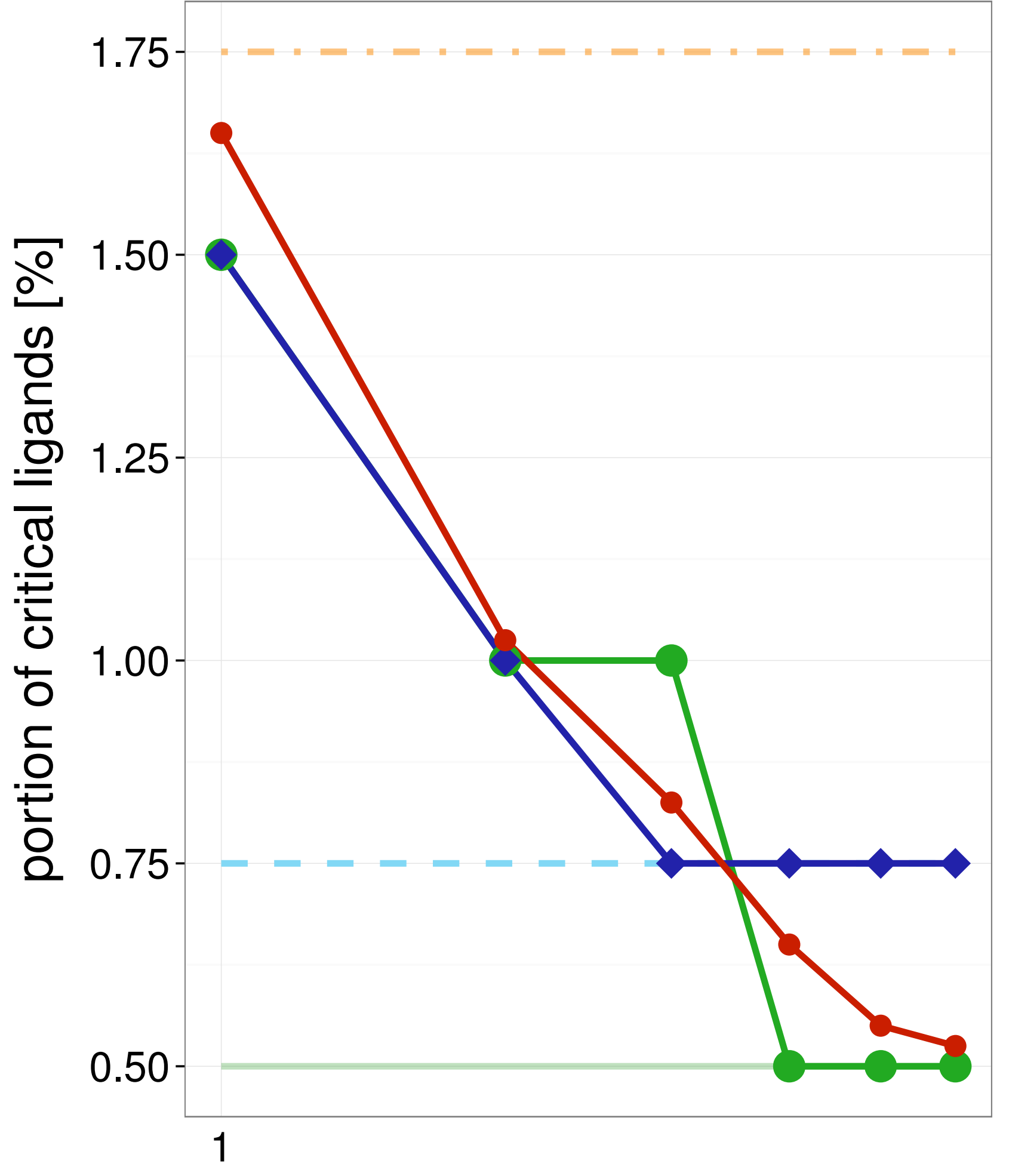

Average single structure

_ Best single structure

Whole ensemble

- SIENA

$\leadsto$ Clustering

$\multimap$ Random 
Training set

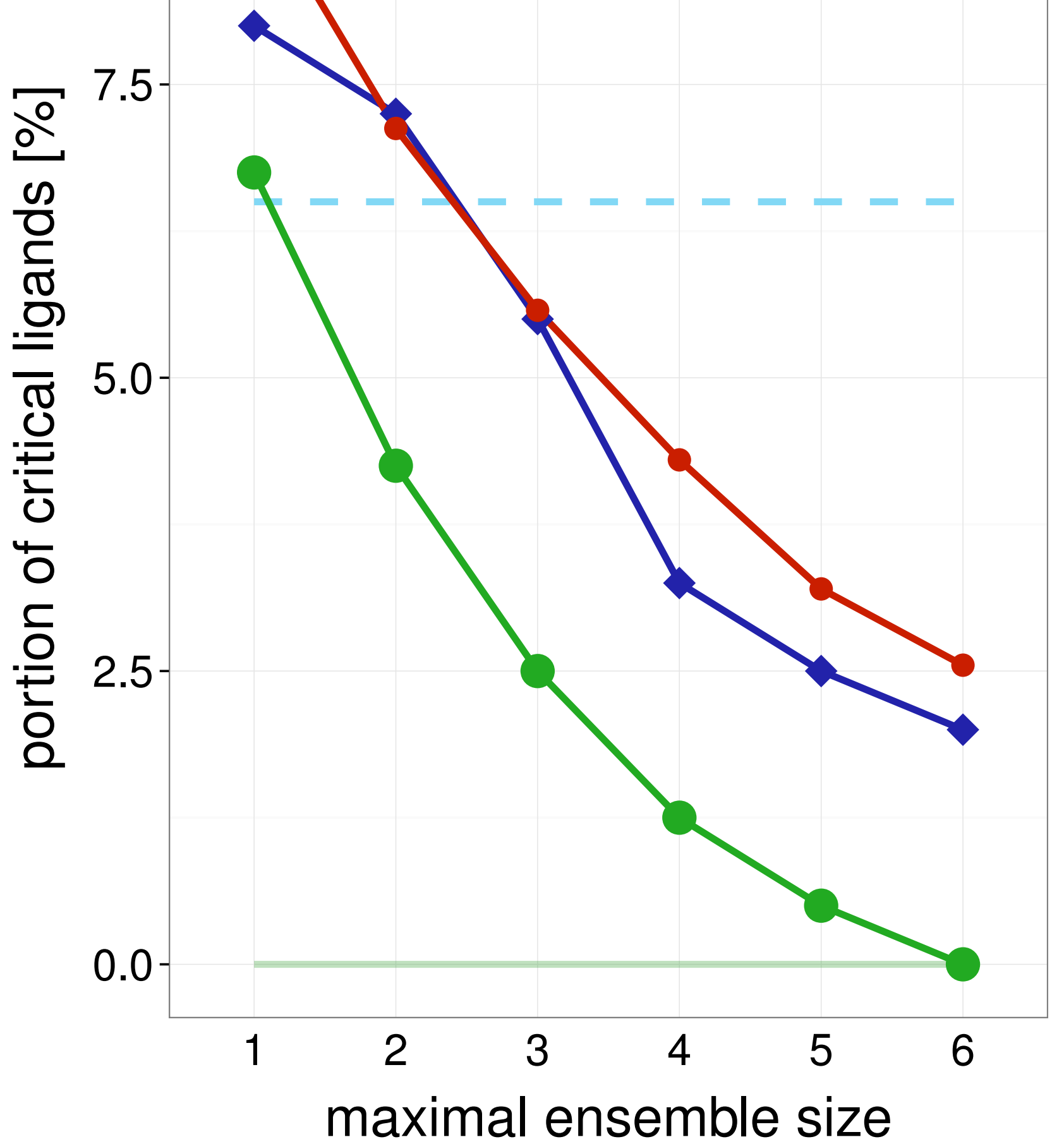

\section{Test set}

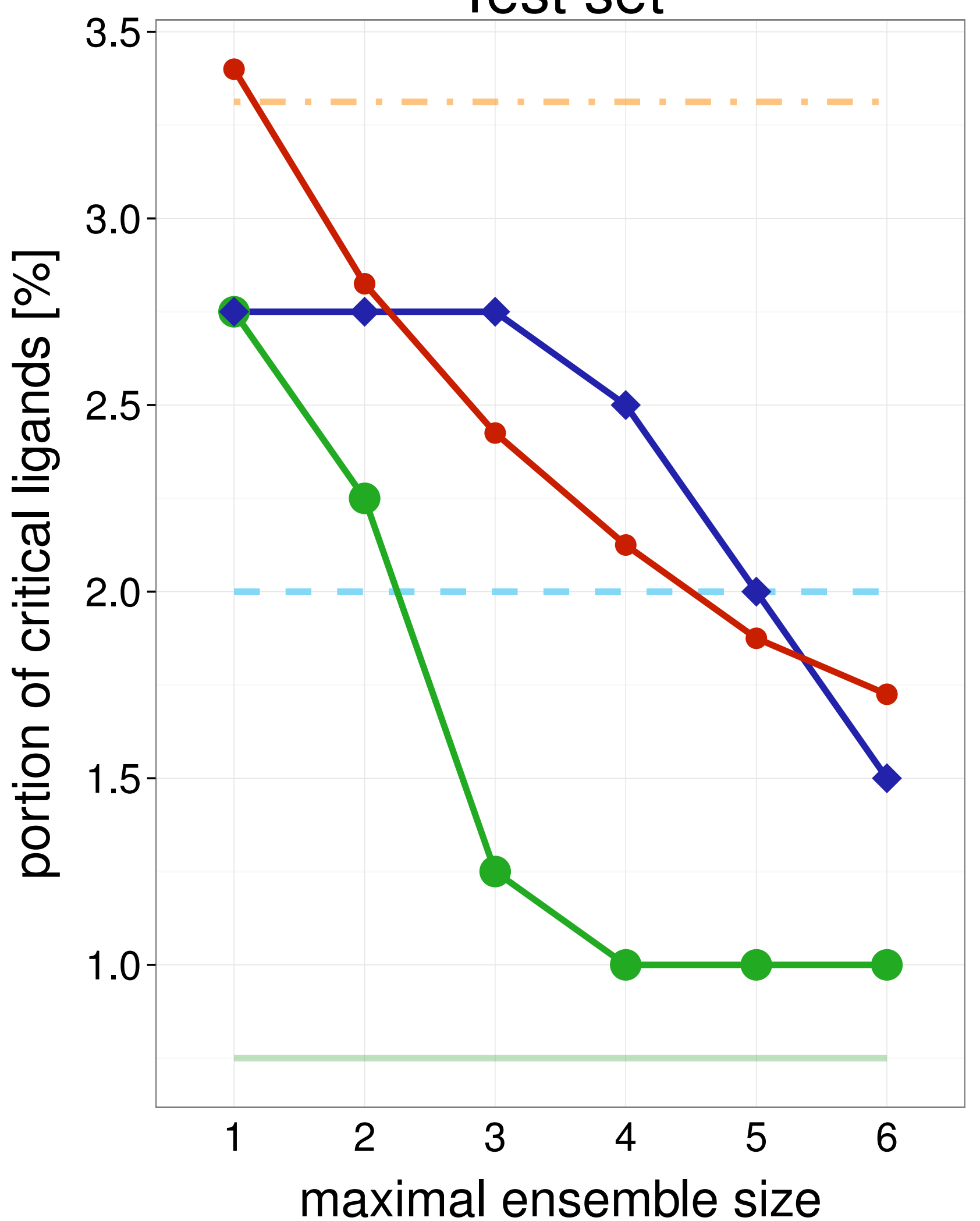

1Y6A

Training set

Average single structure

_ Best single structure

Whole ensemble

- SIENA

$\neg$ Clustering

$\multimap$ Random

Average single structure

_ _ Best single structure

Whole ensemble

- SIENA

$\neg$ Clustering

$\multimap$ Random

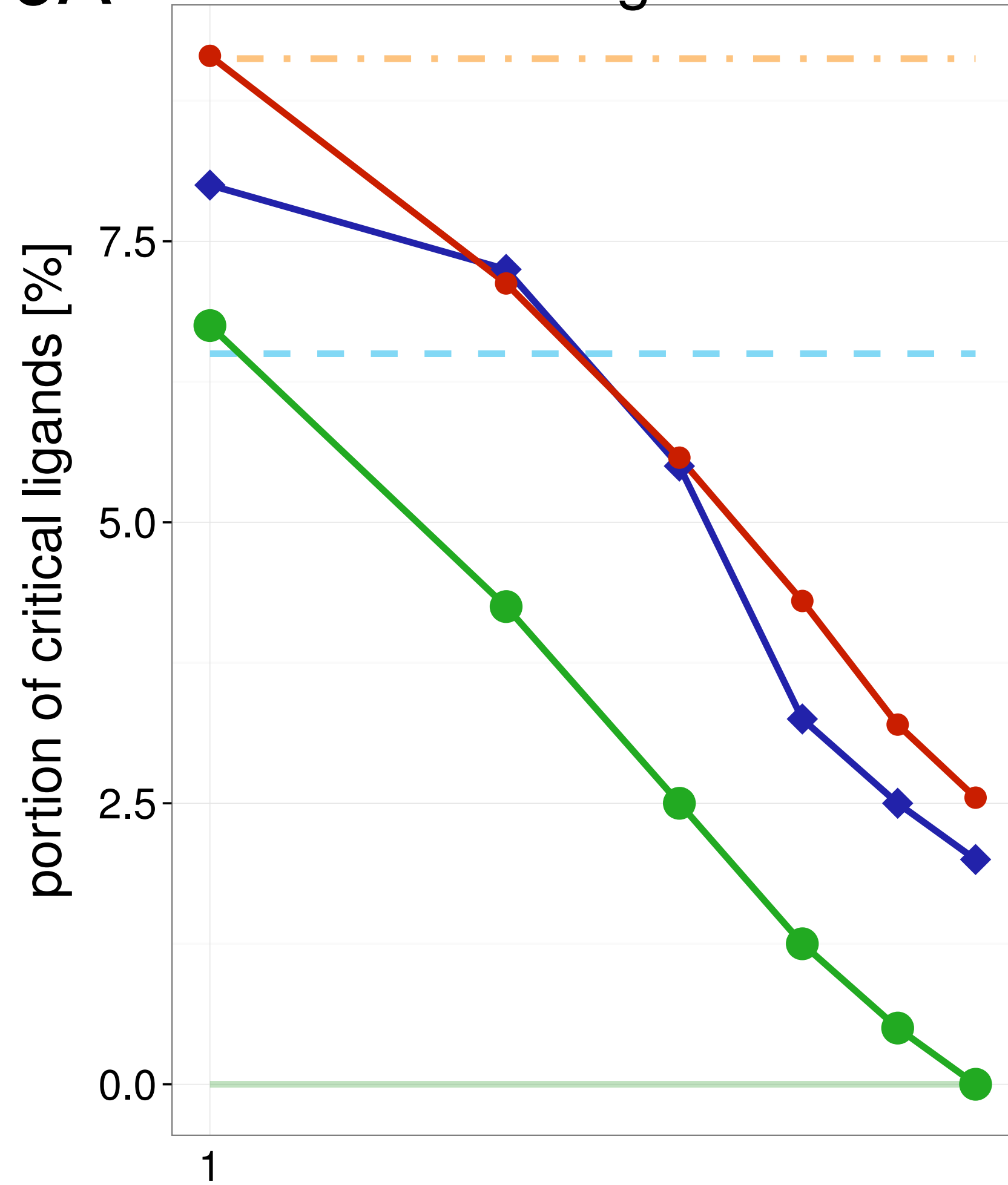

maximal ensemble size

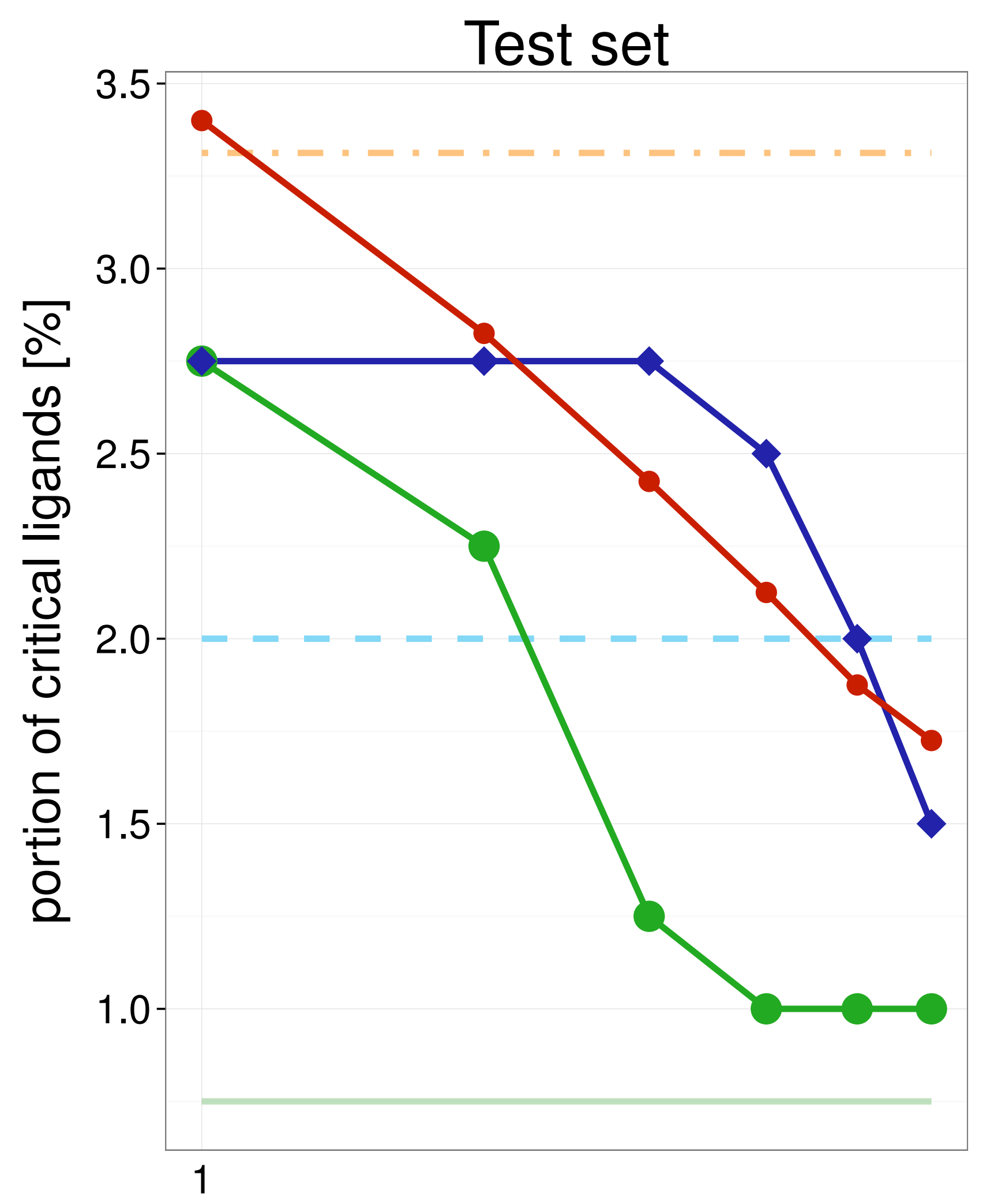

maximal ensemble size
Average single structure

_ Best single structure

Whole ensemble

- SIENA

$\neg$ Clustering

$\multimap$ Random 
Training set

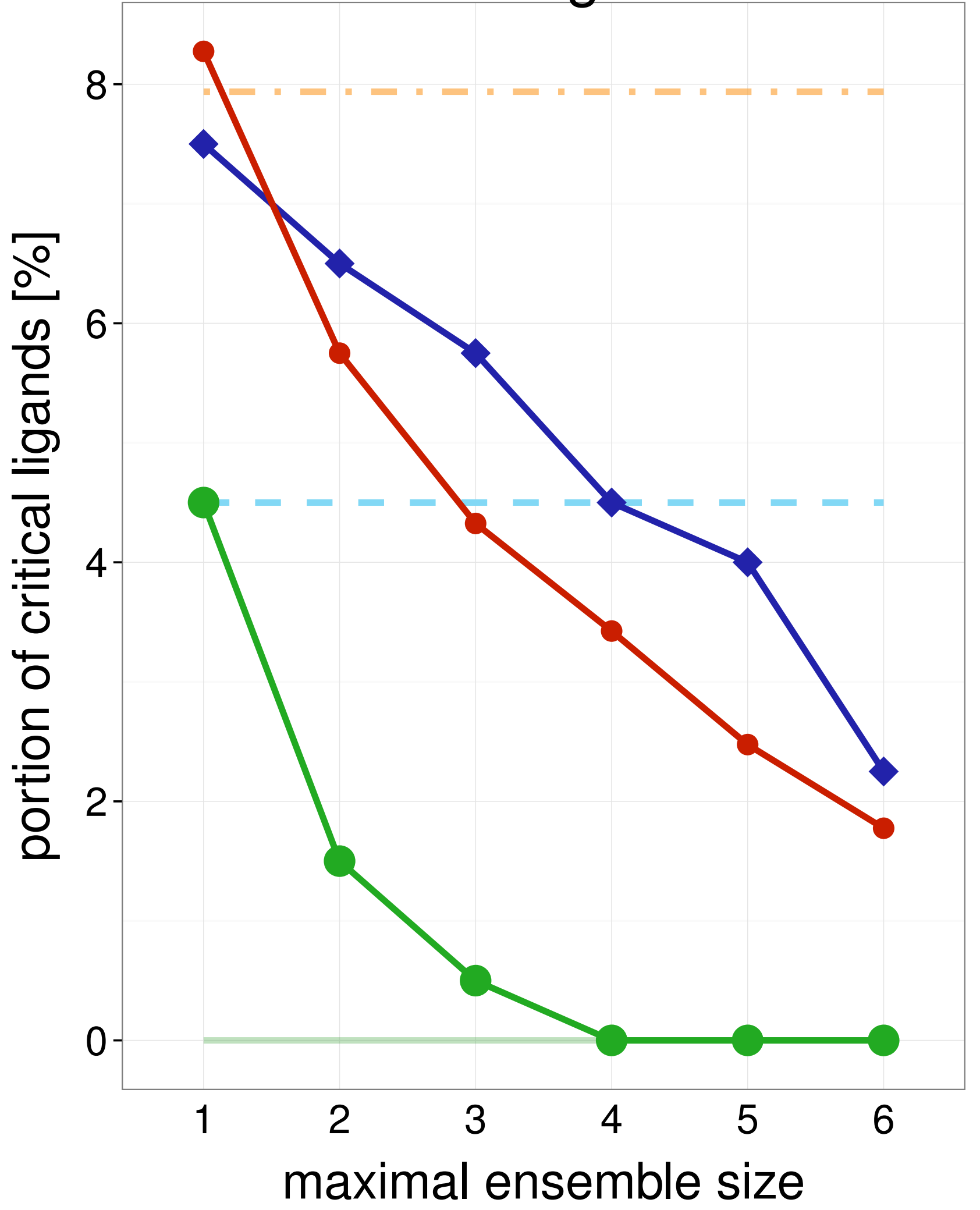

\section{Test set}

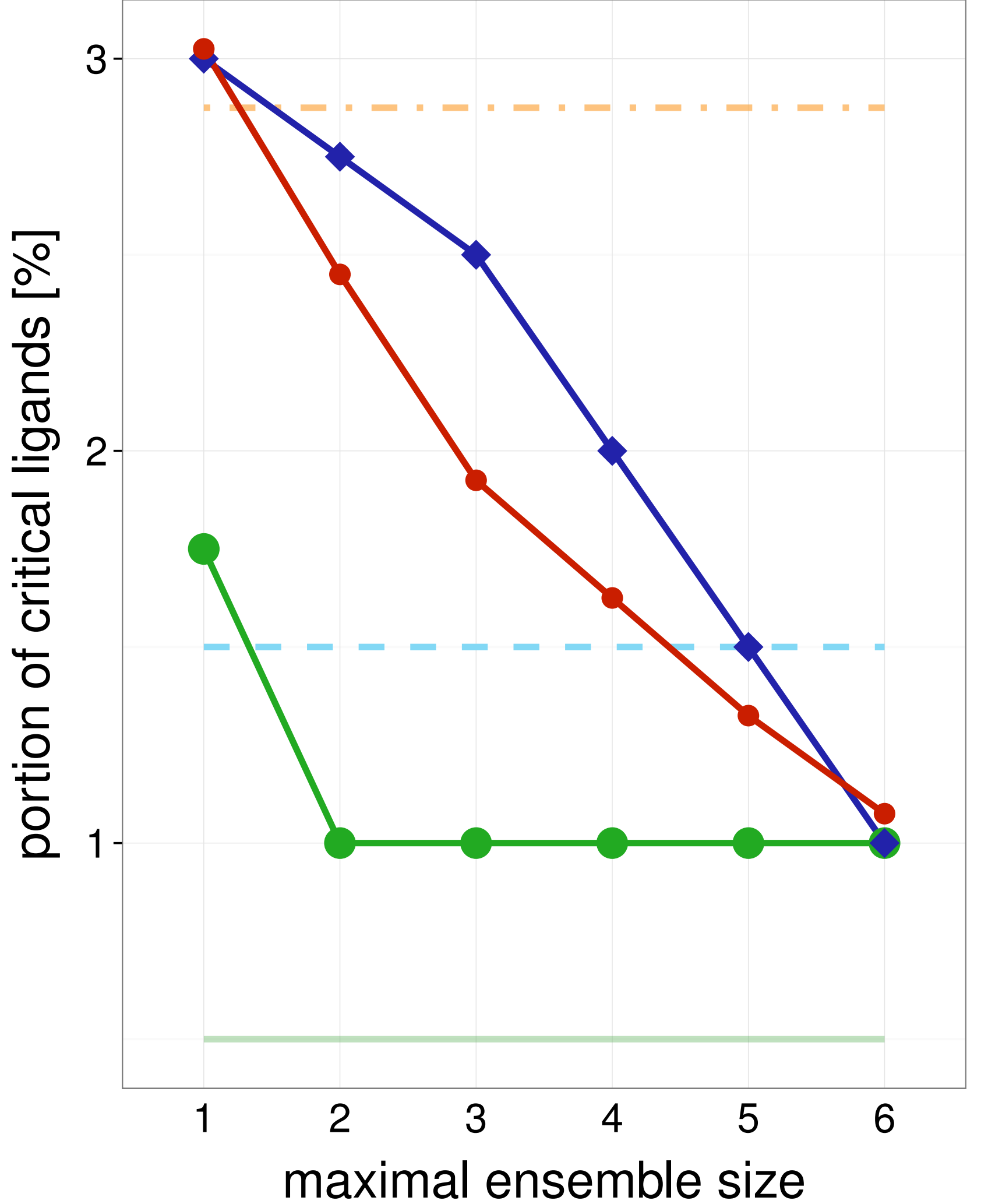

Average single structure

_ Best single structure

Whole ensemble

- SIENA

$\neg$ Clustering

$\multimap$ Random

Average single structure

_ _ Best single structure

Whole ensemble

- SIENA

$\neg$ Clustering

- Random
Training set

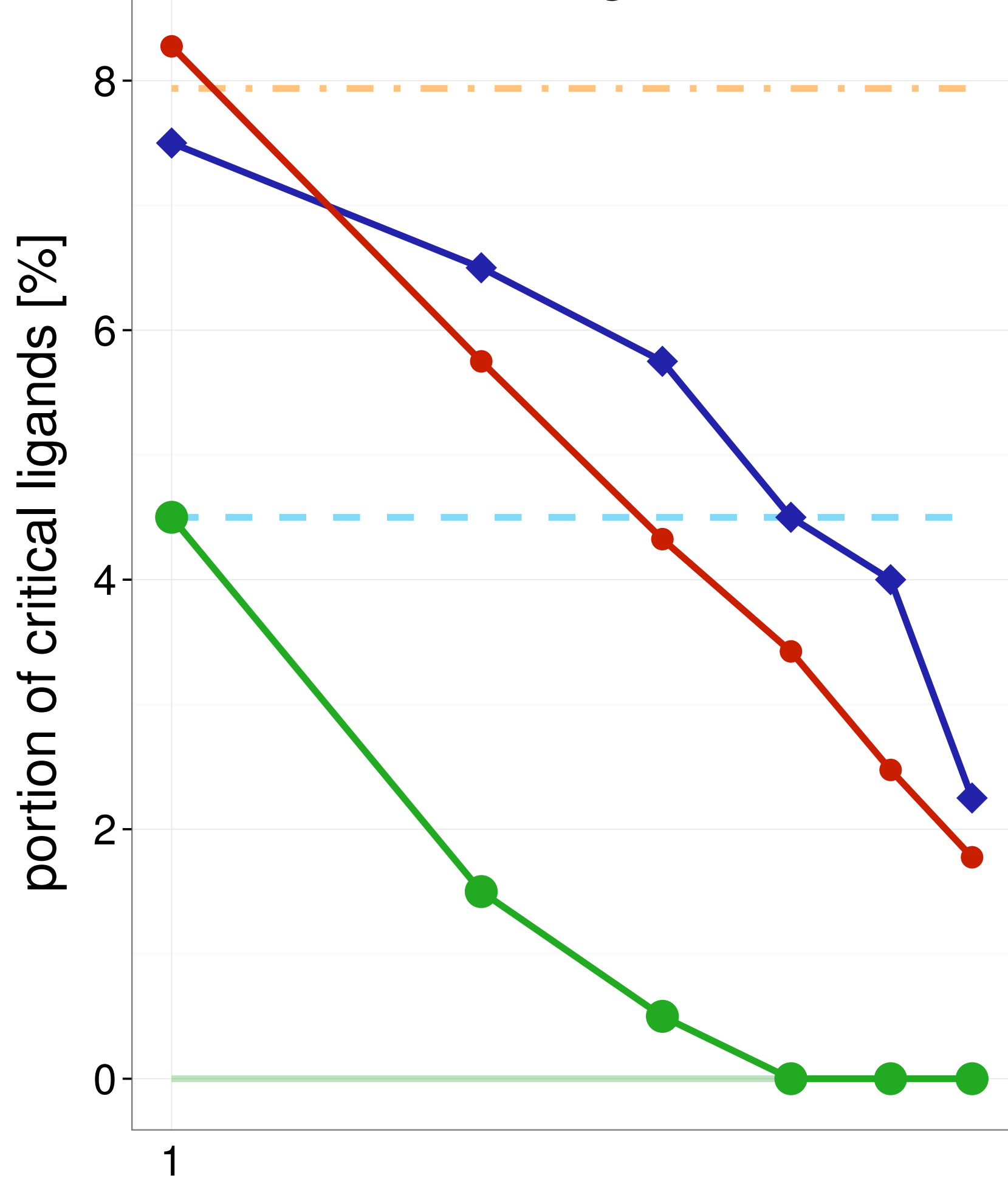

maximal ensemble size

Test set

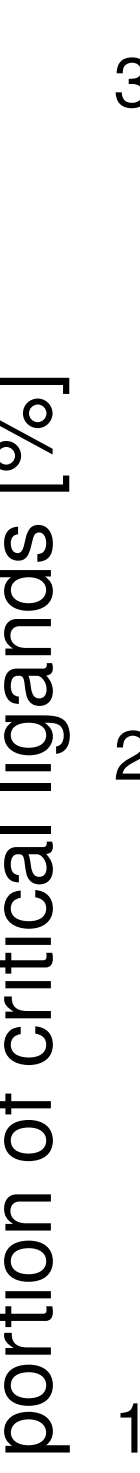

3-

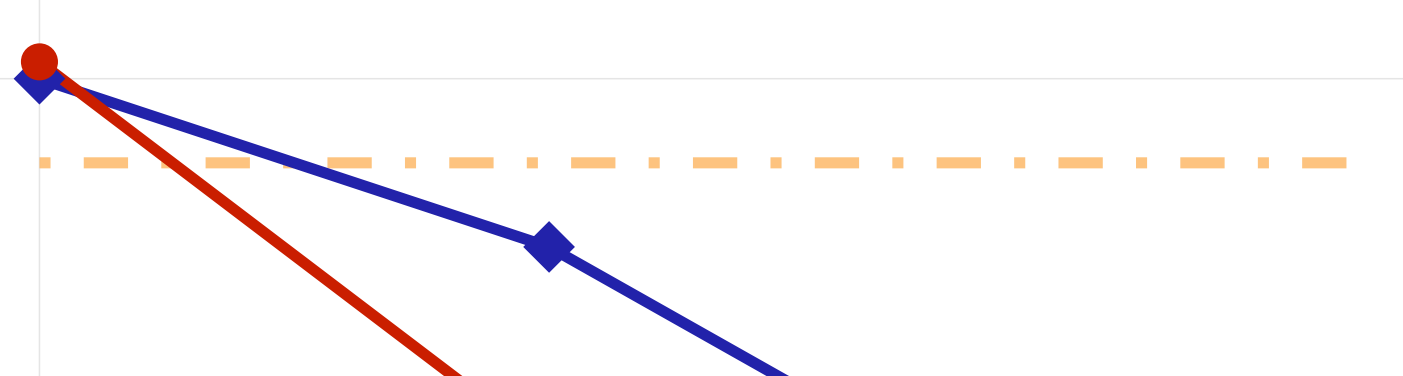

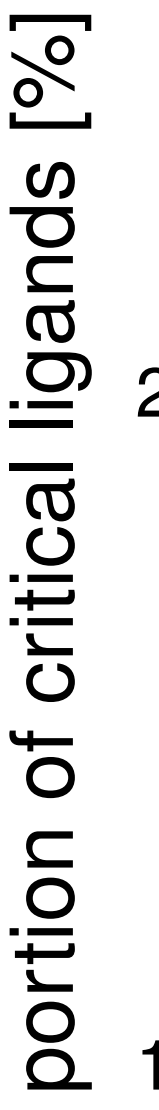

Average single structure

_ Best single structure

Whole ensemble

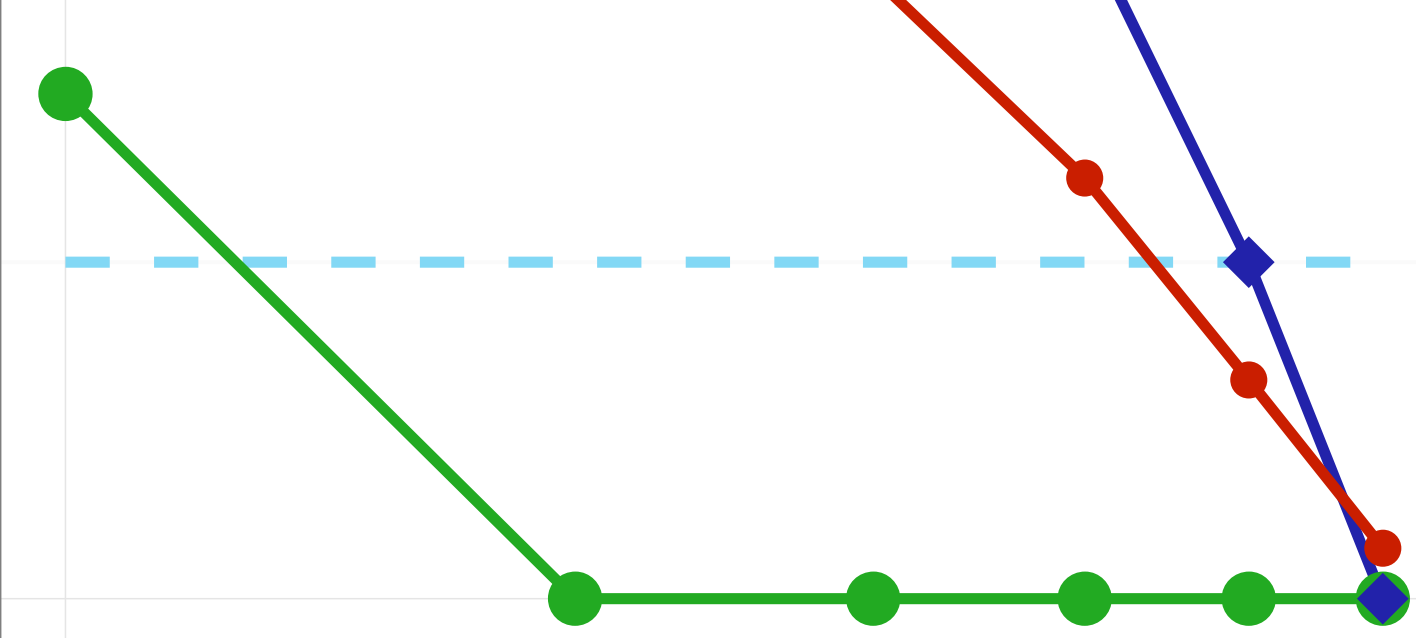

SIENA

$\checkmark$ Clustering

$\rightarrow$ Random 
Training set

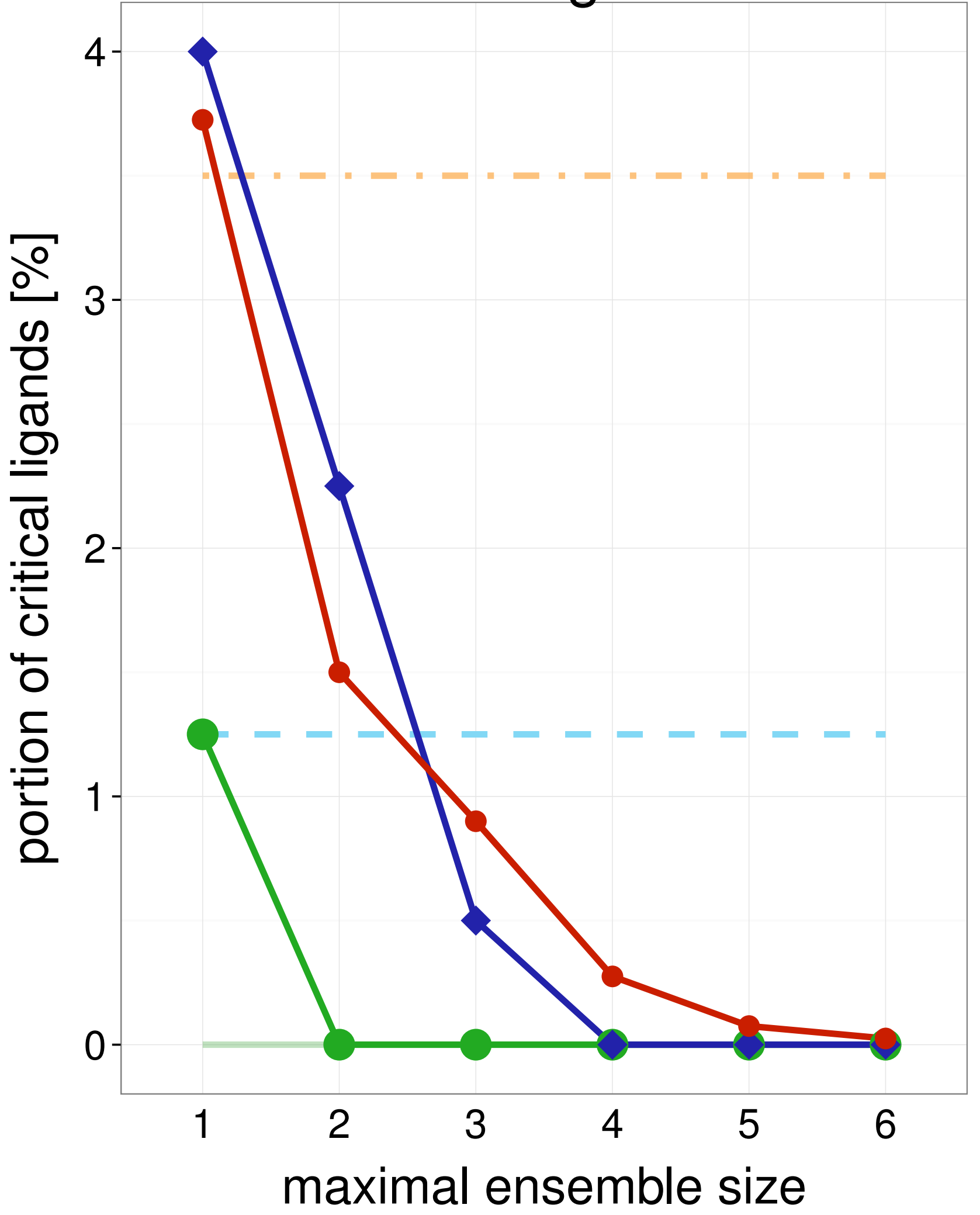

\section{Test set}

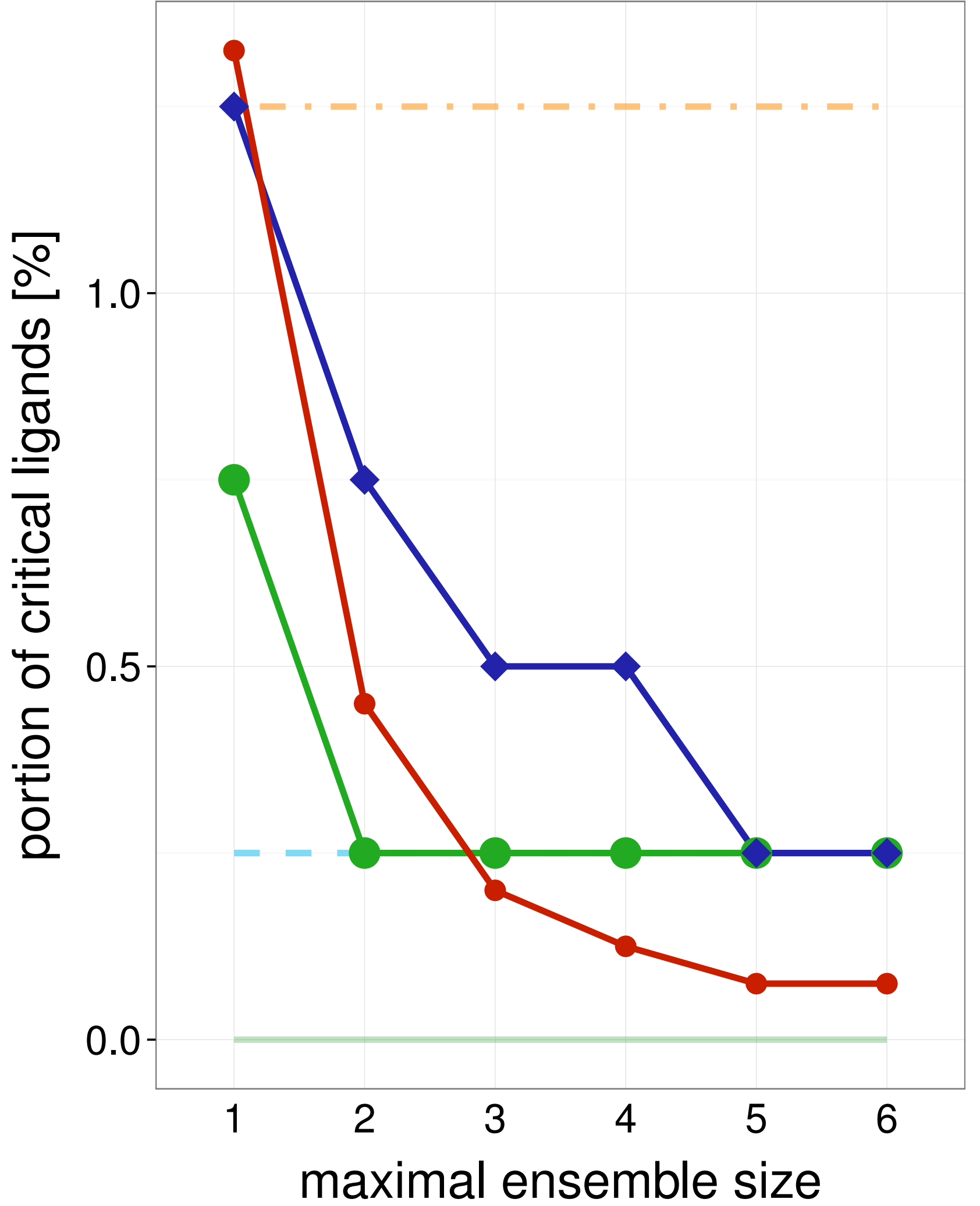

2JFF

Training set

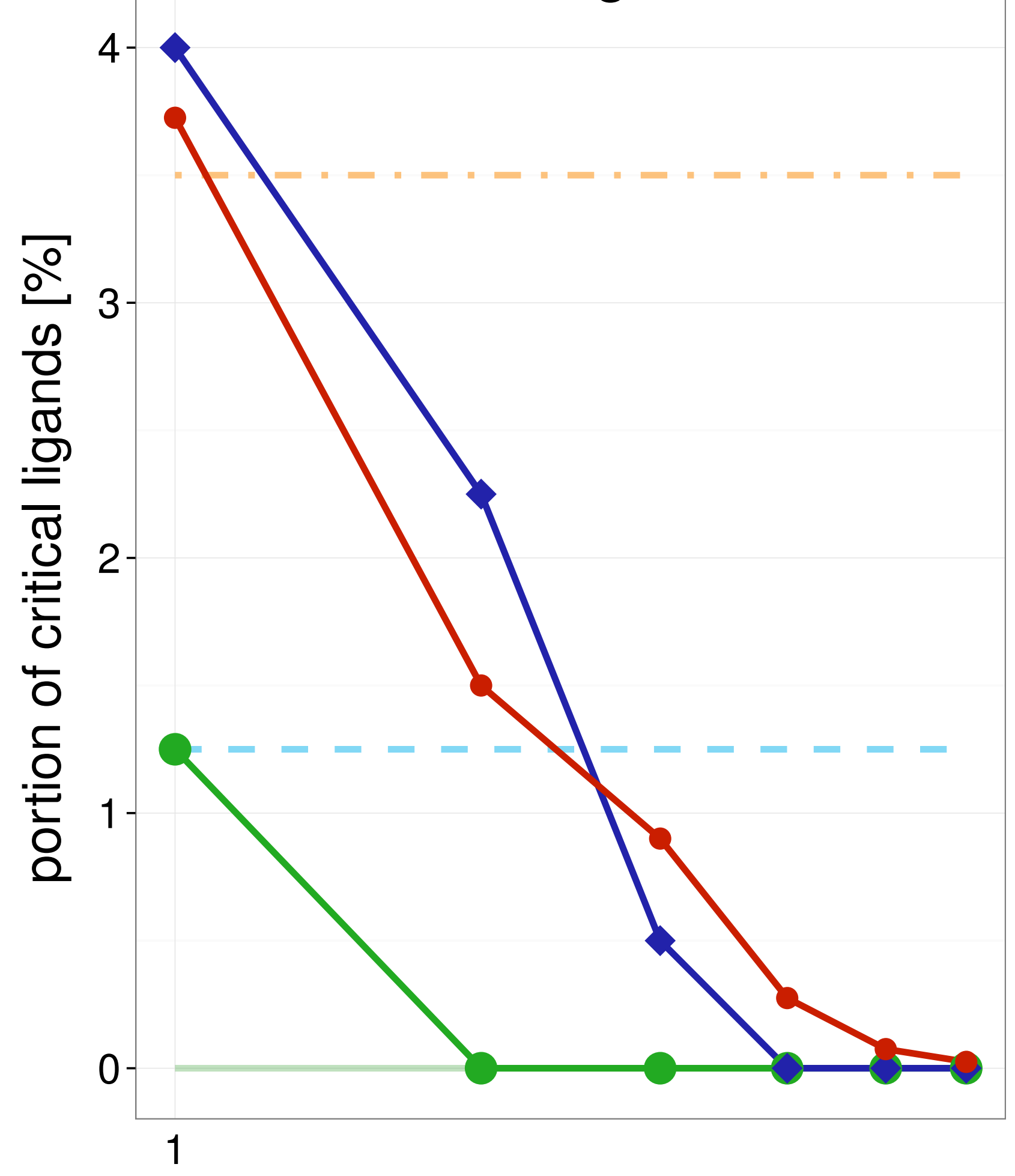

maximal ensemble size

Test set

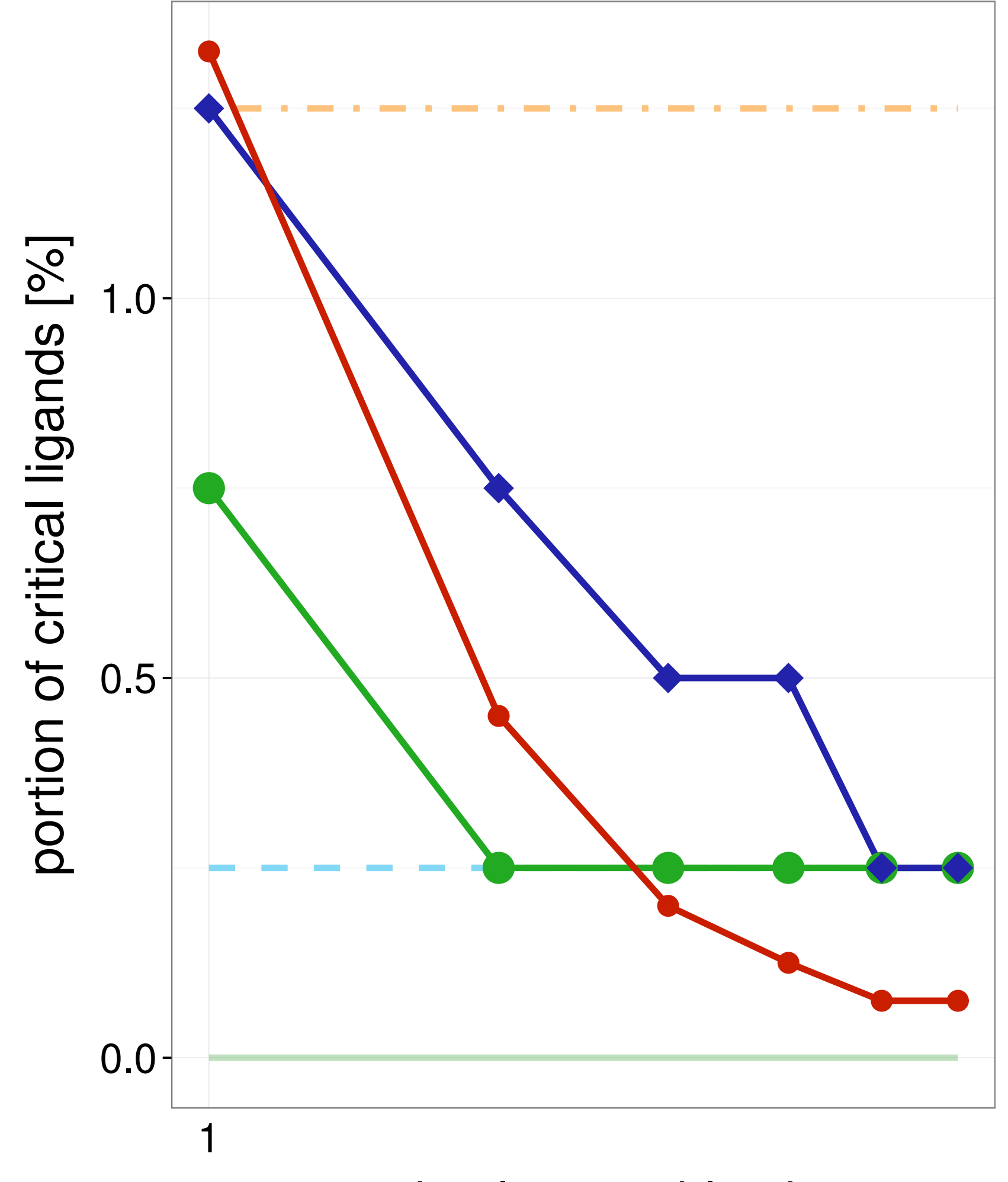

Average single structure

\section{_ Best single} structure

Whole ensemble

- SIENA

$\neg$ Clustering

$\because$ Random
Average single structure

Average single structure

Best single structure

Whole ensemble

- SIENA

$\checkmark$ Clustering

- Random

_ _ Best single structure

Whole ensemble

SIENA

$\neg$ Clustering

$\longrightarrow$ Random 
Training set

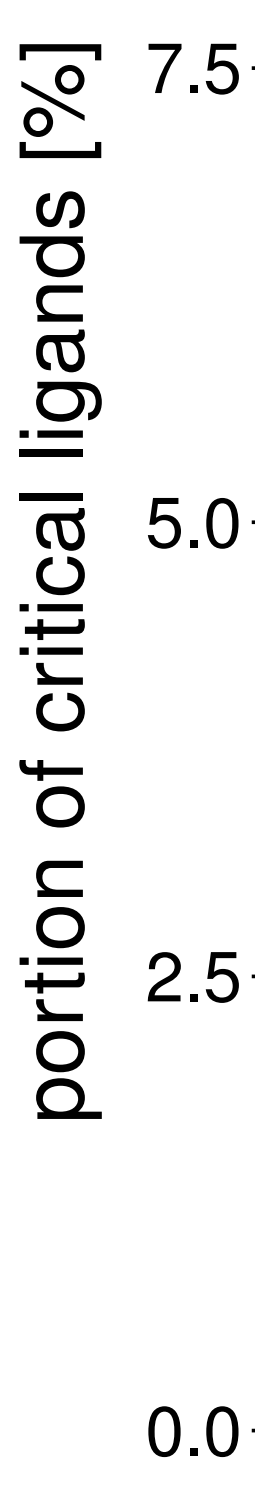

0.0

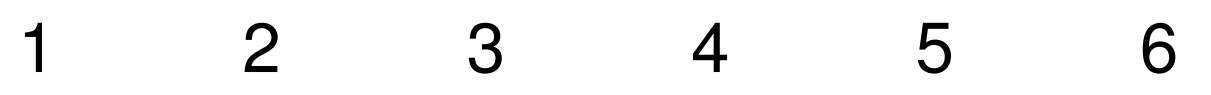

maximal ensemble size

Test set

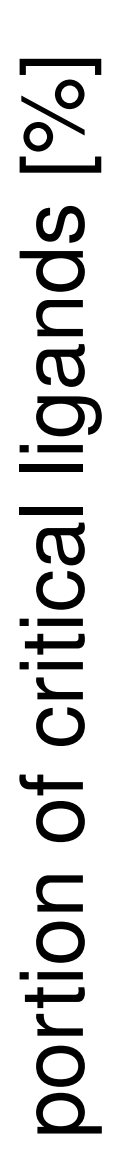

2.0

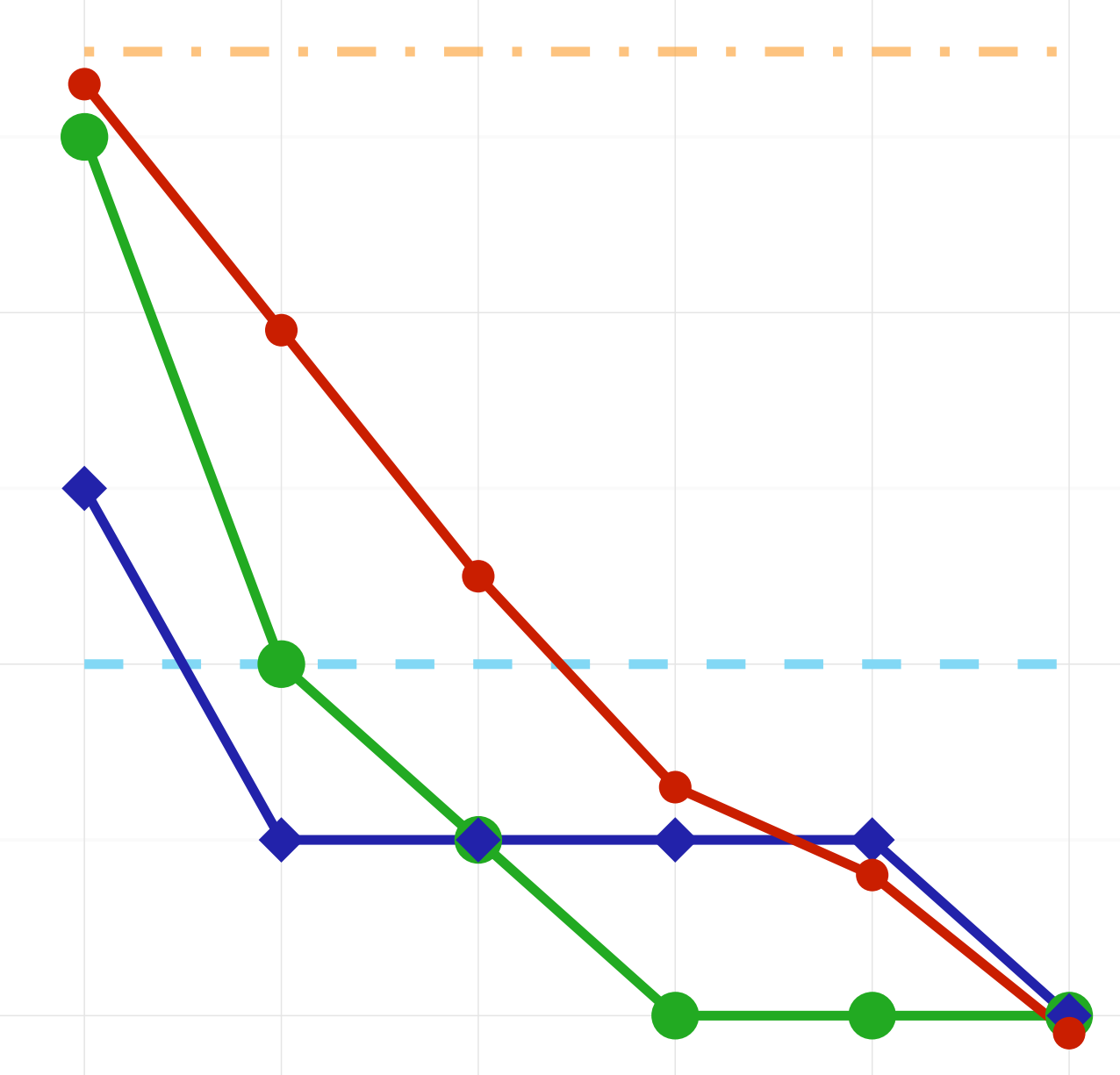

1.5

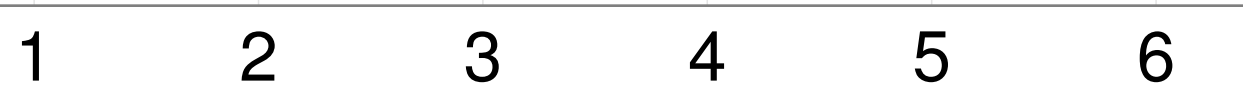

maximal ensemble size
Average single structure

- Best single structure

Whole ensemble

- SIENA

$\neg$ Clustering

$\multimap$ Random

Average single structure

_ Best single structure

Whole ensemble

- SIENA

$\neg$ Clustering

$\multimap$ Random

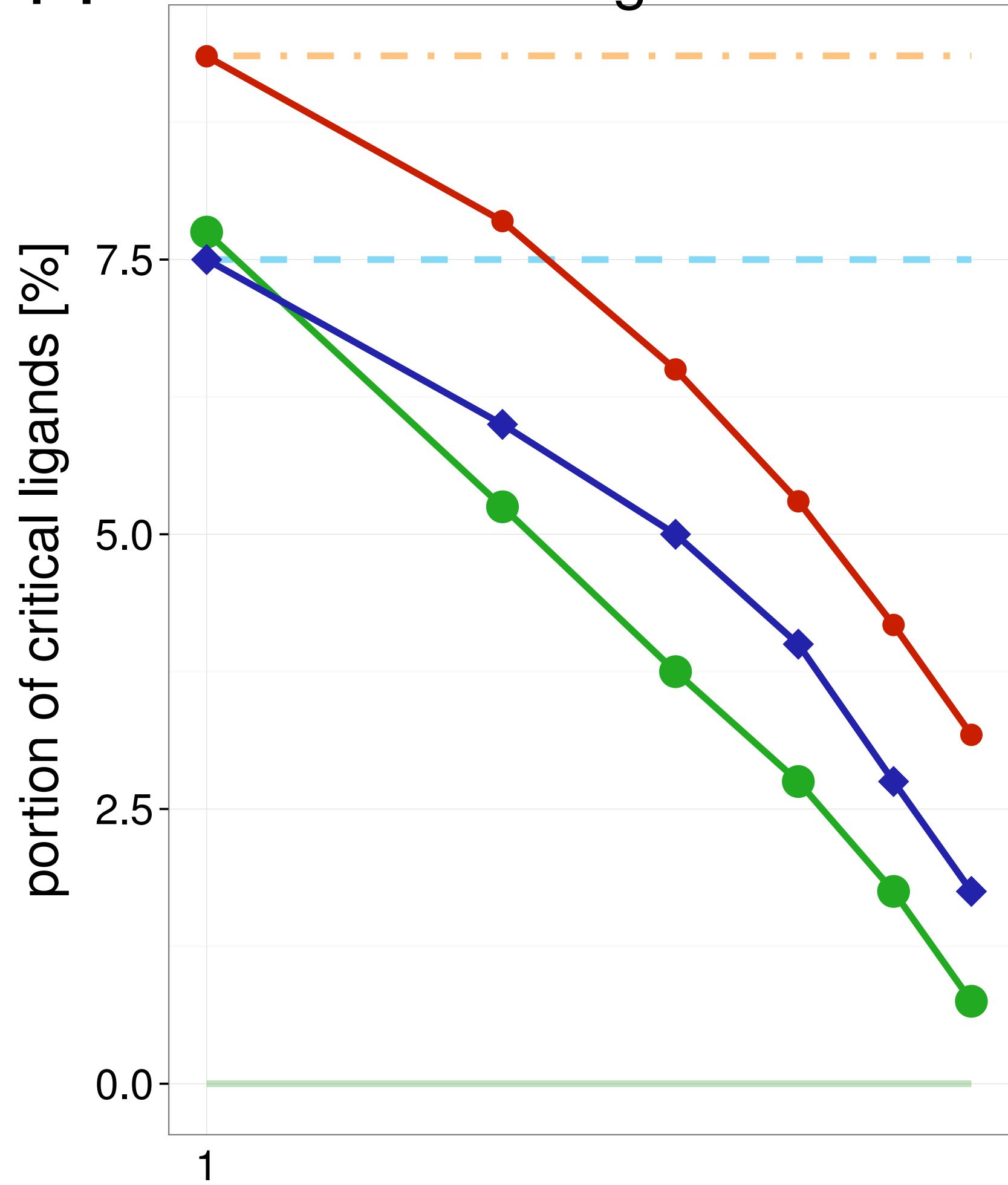

maximal ensemble size

Test set

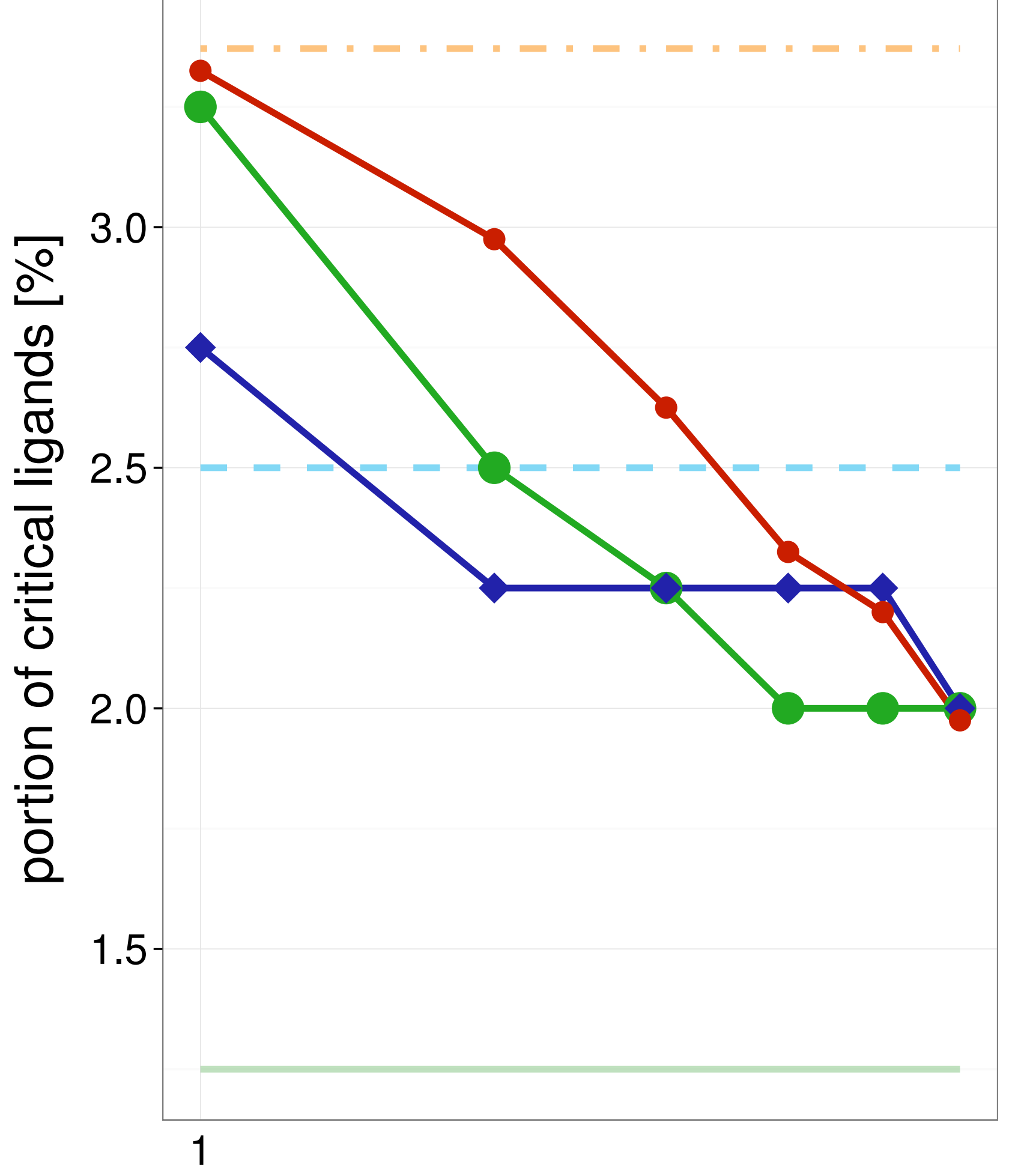

maximal ensemble size
Average single structure

_ Best single structure

Whole ensemble

SIENA

$\neg$ Clustering

$\multimap$ Random 


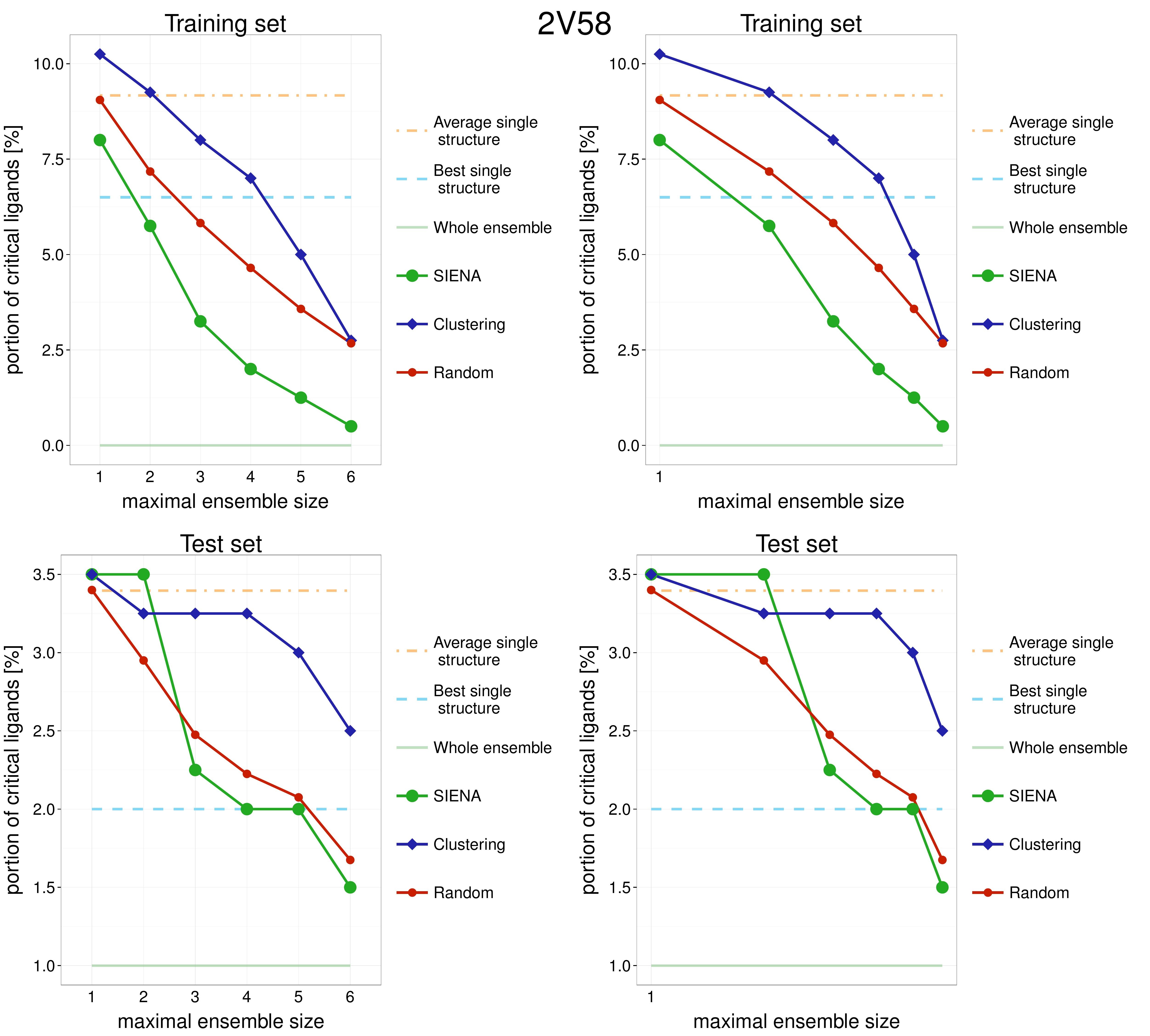


Training set

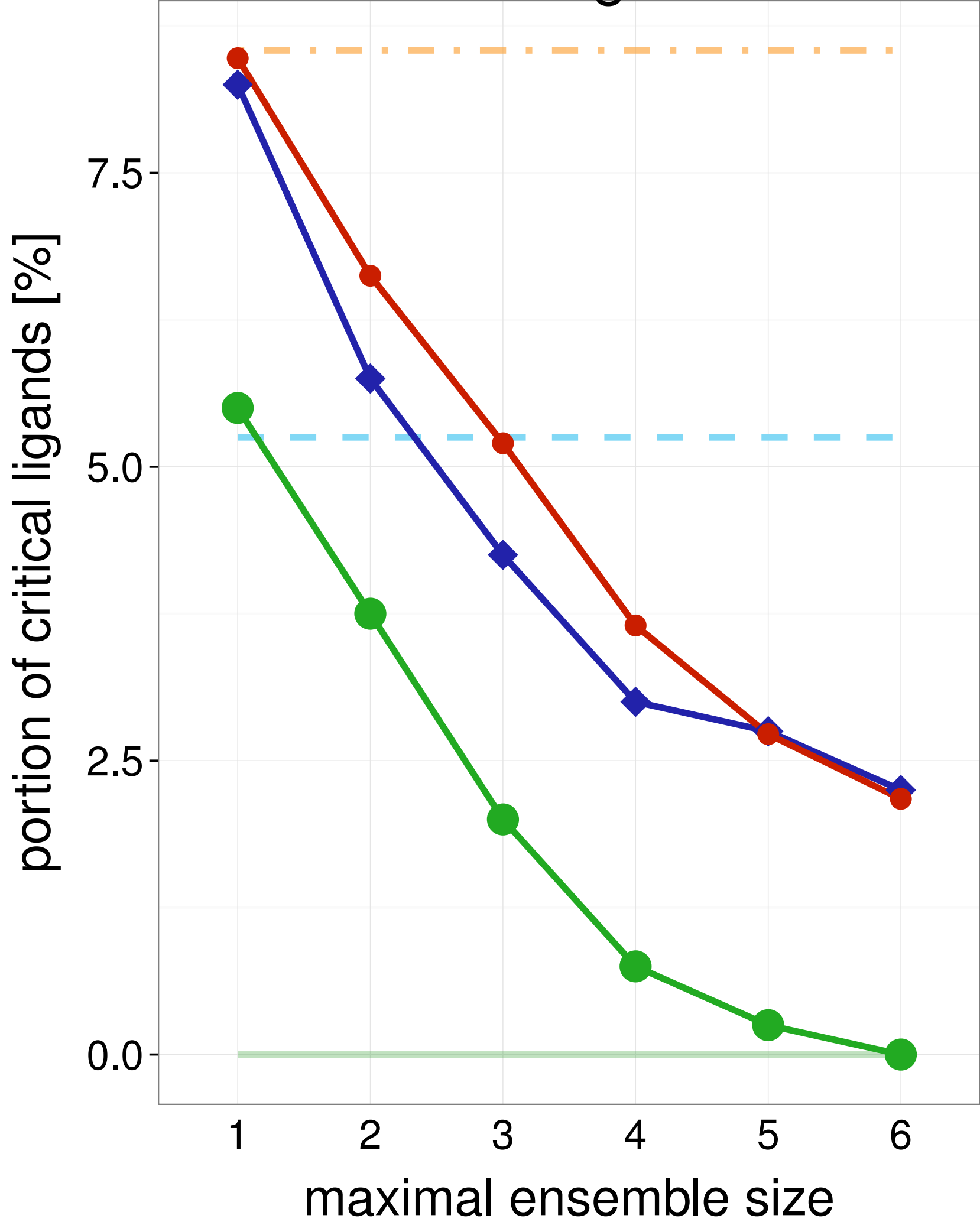

Test set

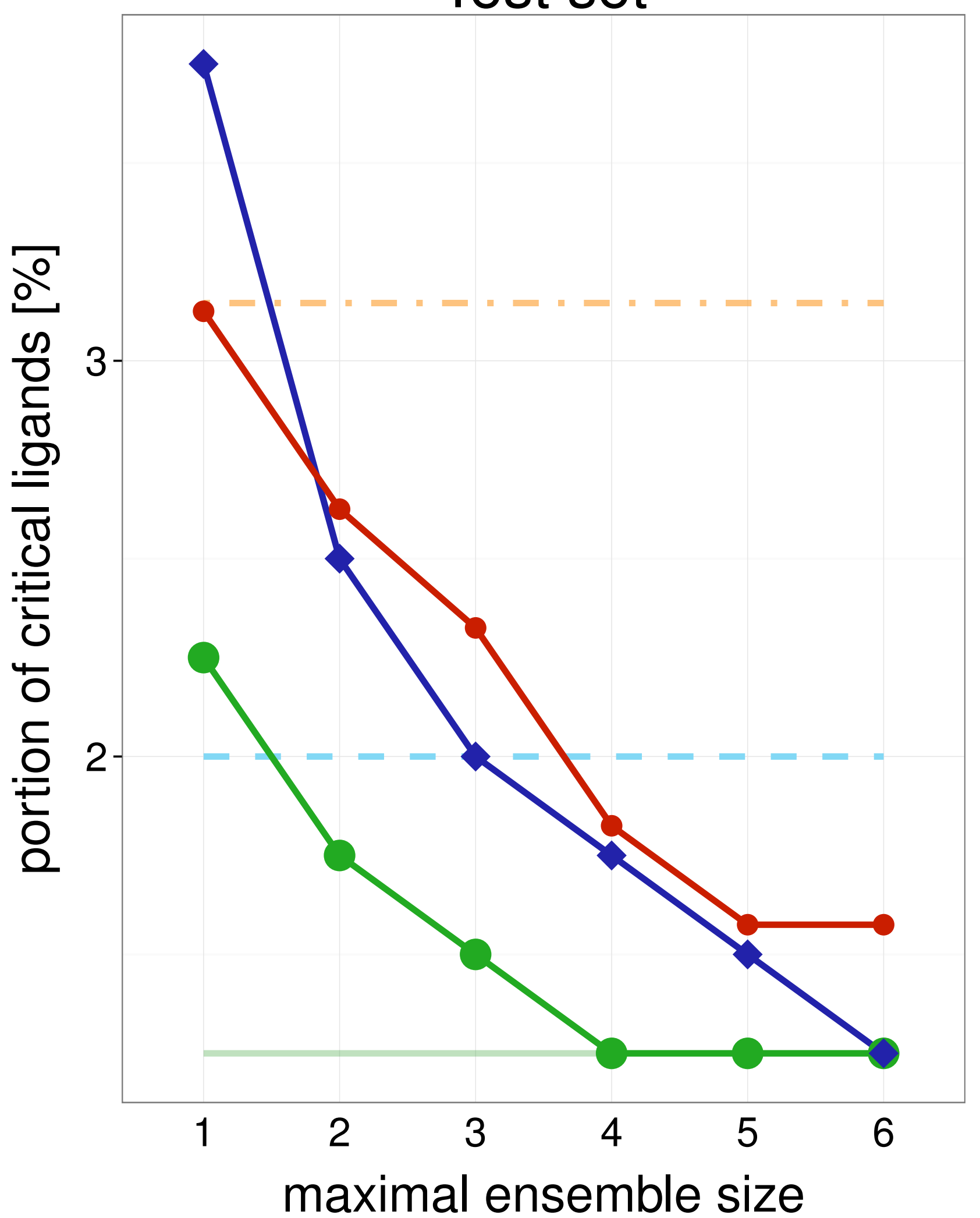

$3 A C X$

Training set

Average single structure

_ Best single structure

Whole ensemble

- SIENA

$\smile$ Clustering

$\multimap$ Random

Average single structure

_ Best single structure

Whole ensemble

- SIENA

$\neg$ Clustering

$\multimap$ Random

Test set

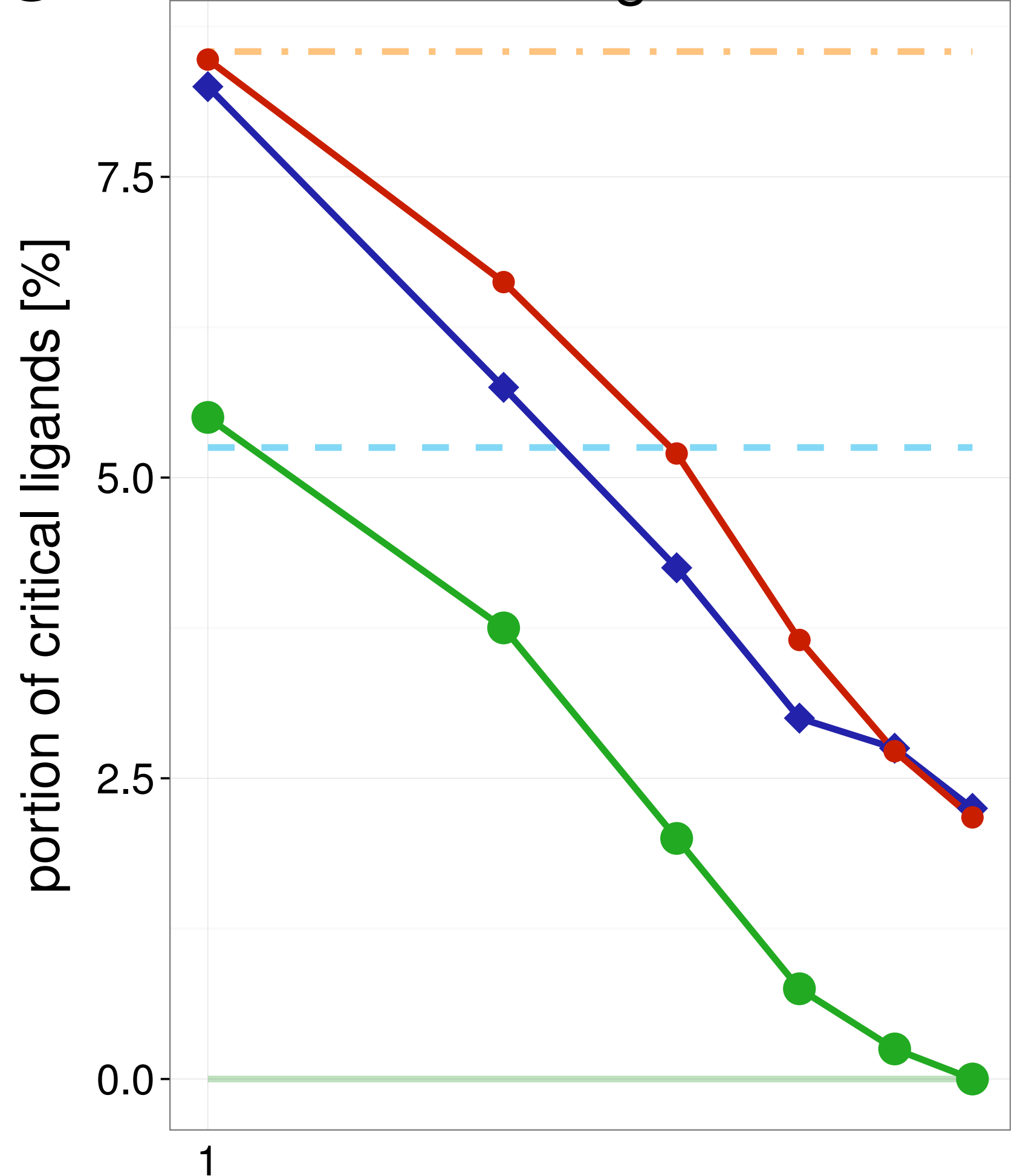

maximal ensemble size

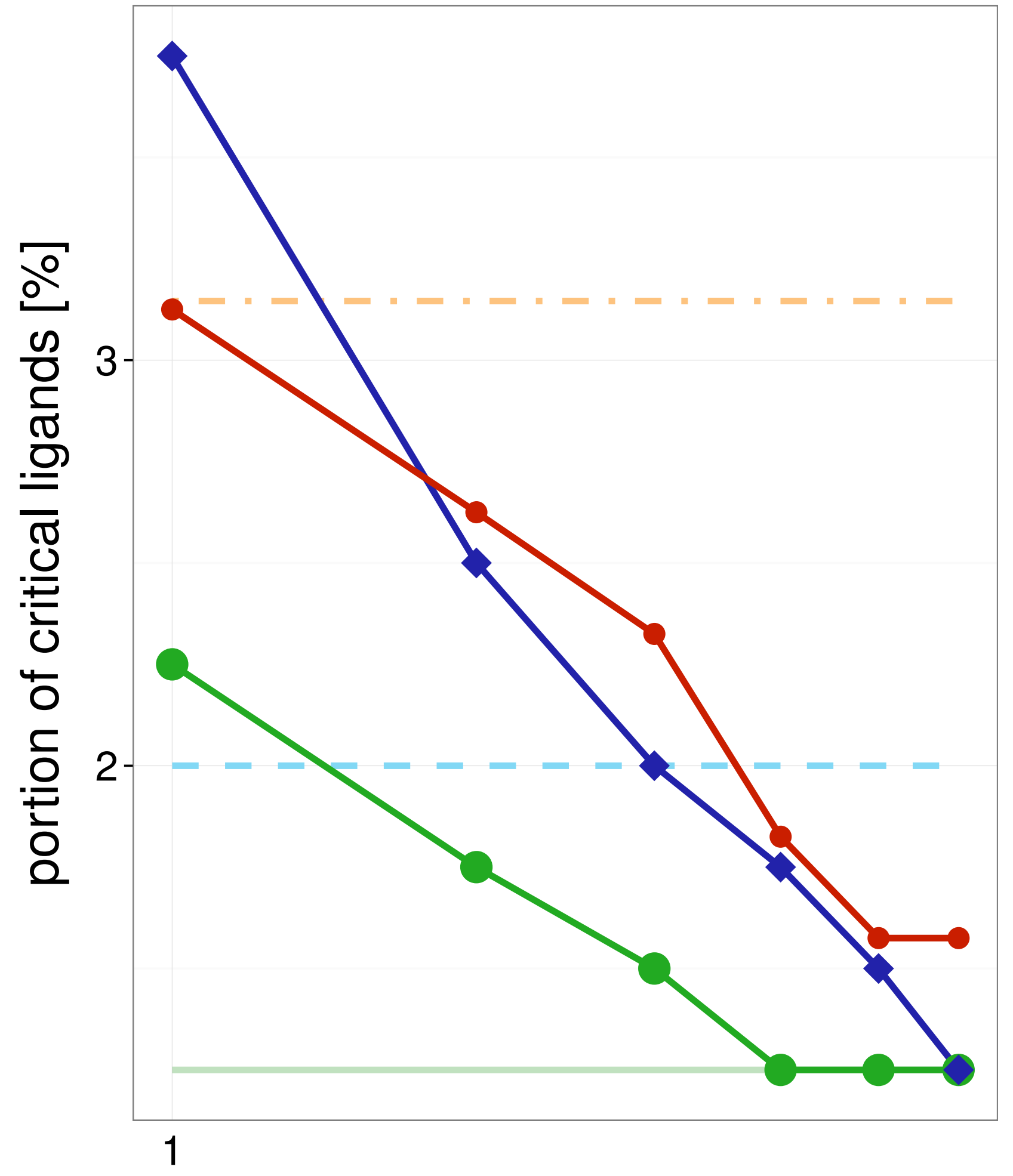

Average single structure

Best single structure

Whole ensemble

SIENA

$\neg$ Clustering

$\multimap$ Random 
Training set

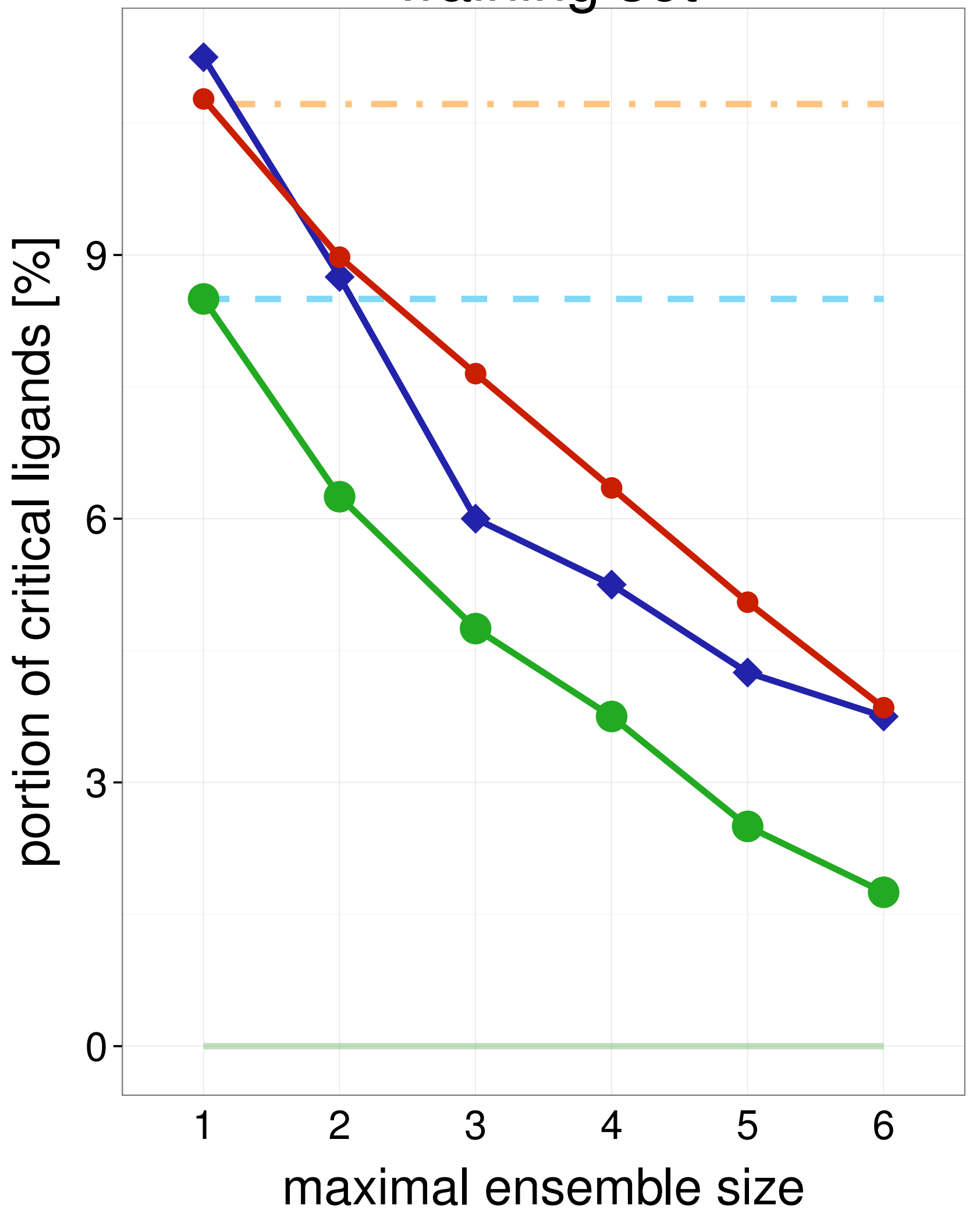

Test set

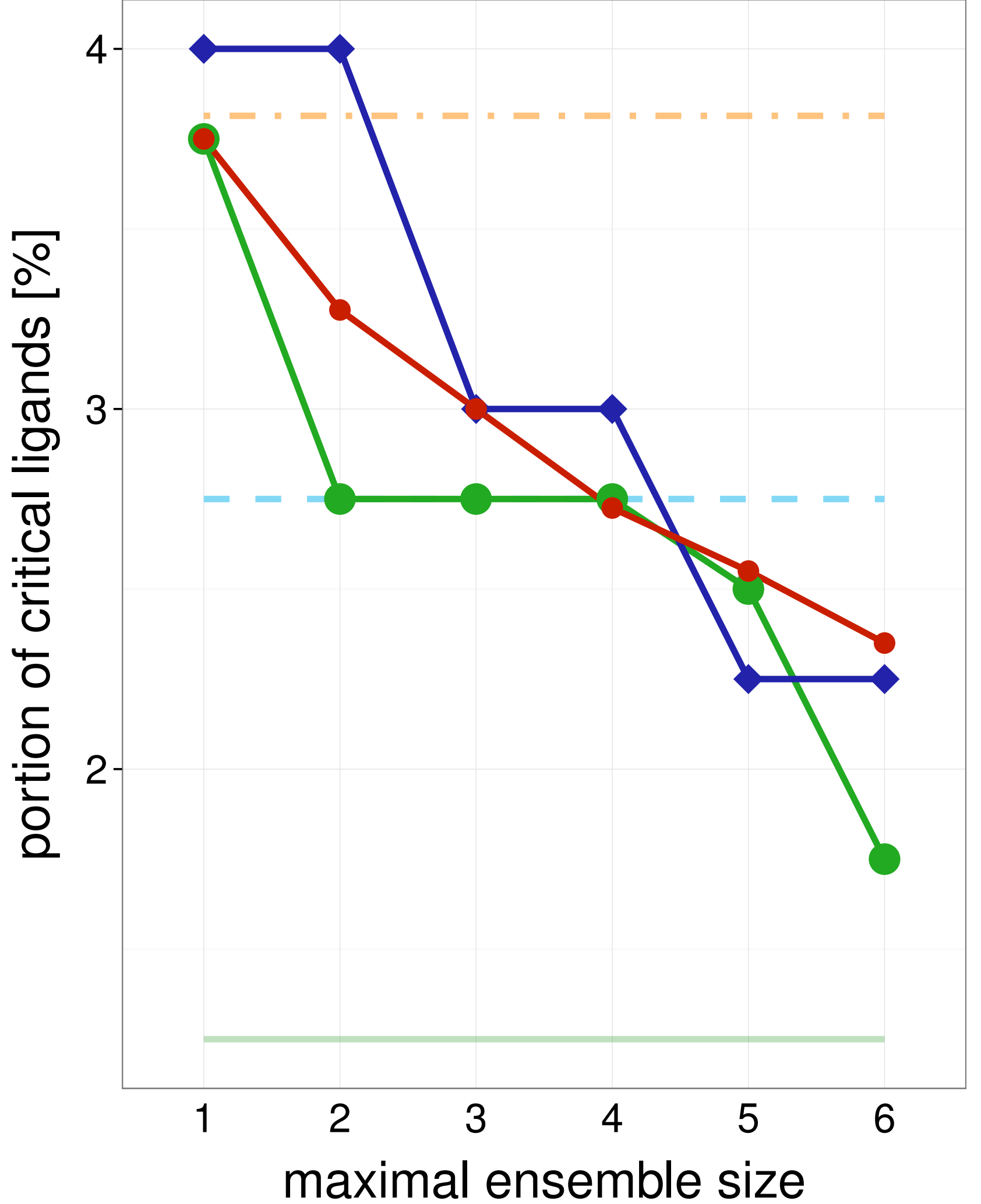

Average single structure

_ Best single structure

Whole ensemble

- - SIENA

$\prec$ Clustering

$\multimap$ Random

Average single structure

_ Best single structure

Whole ensemble

- SIENA

$\neg$ Clustering

$\multimap$ Random
Training set

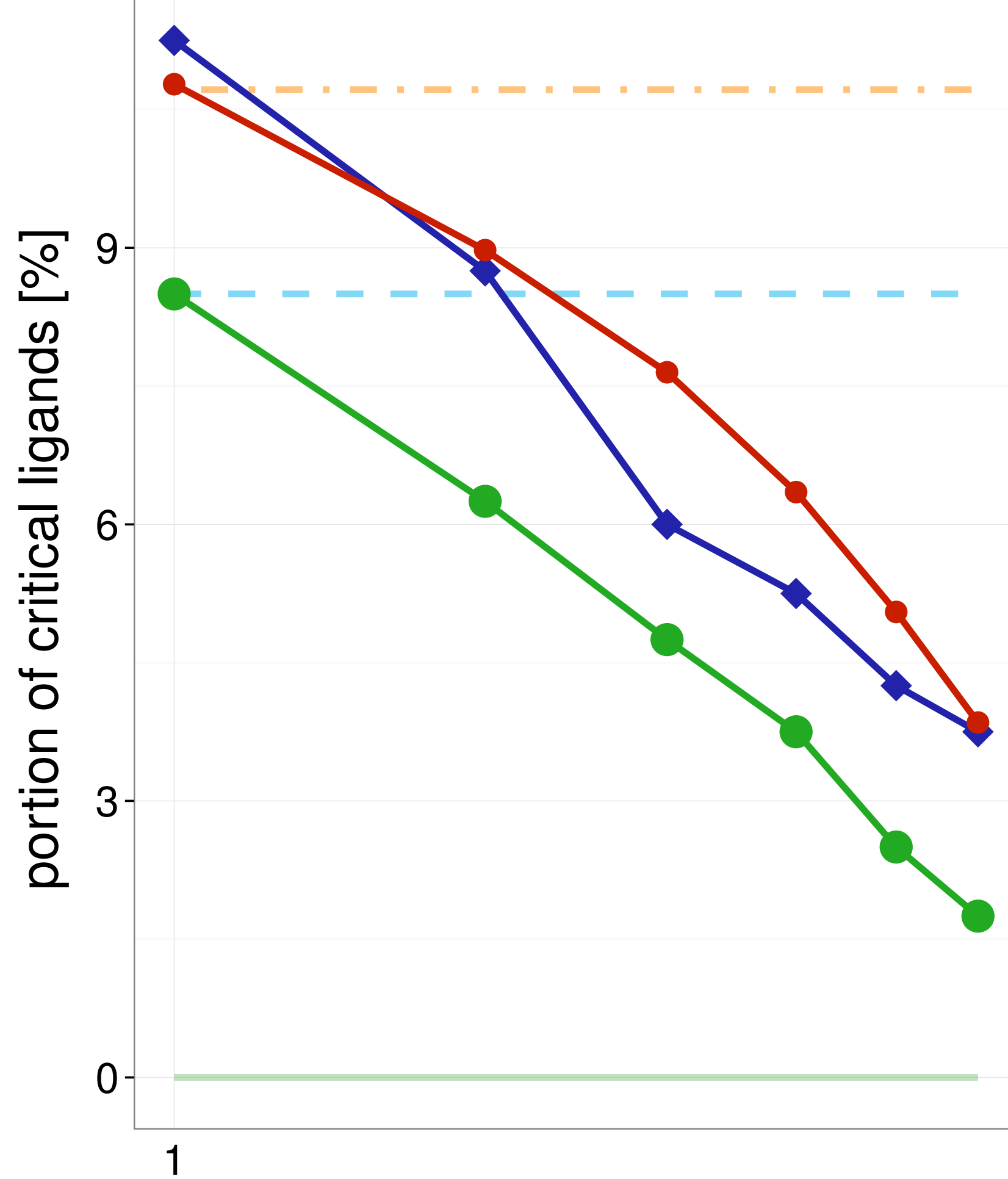

maximal ensemble size

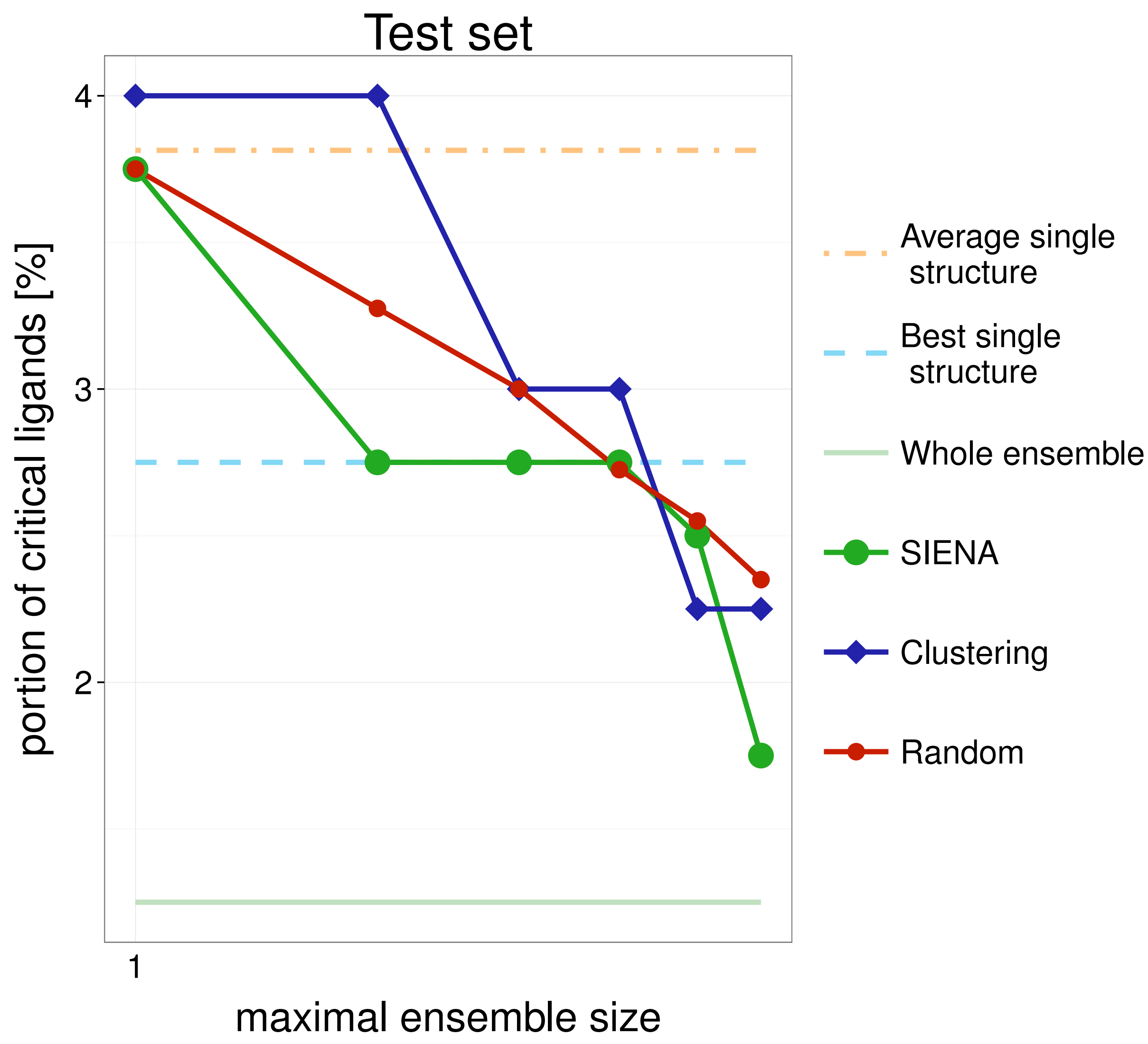


Training set

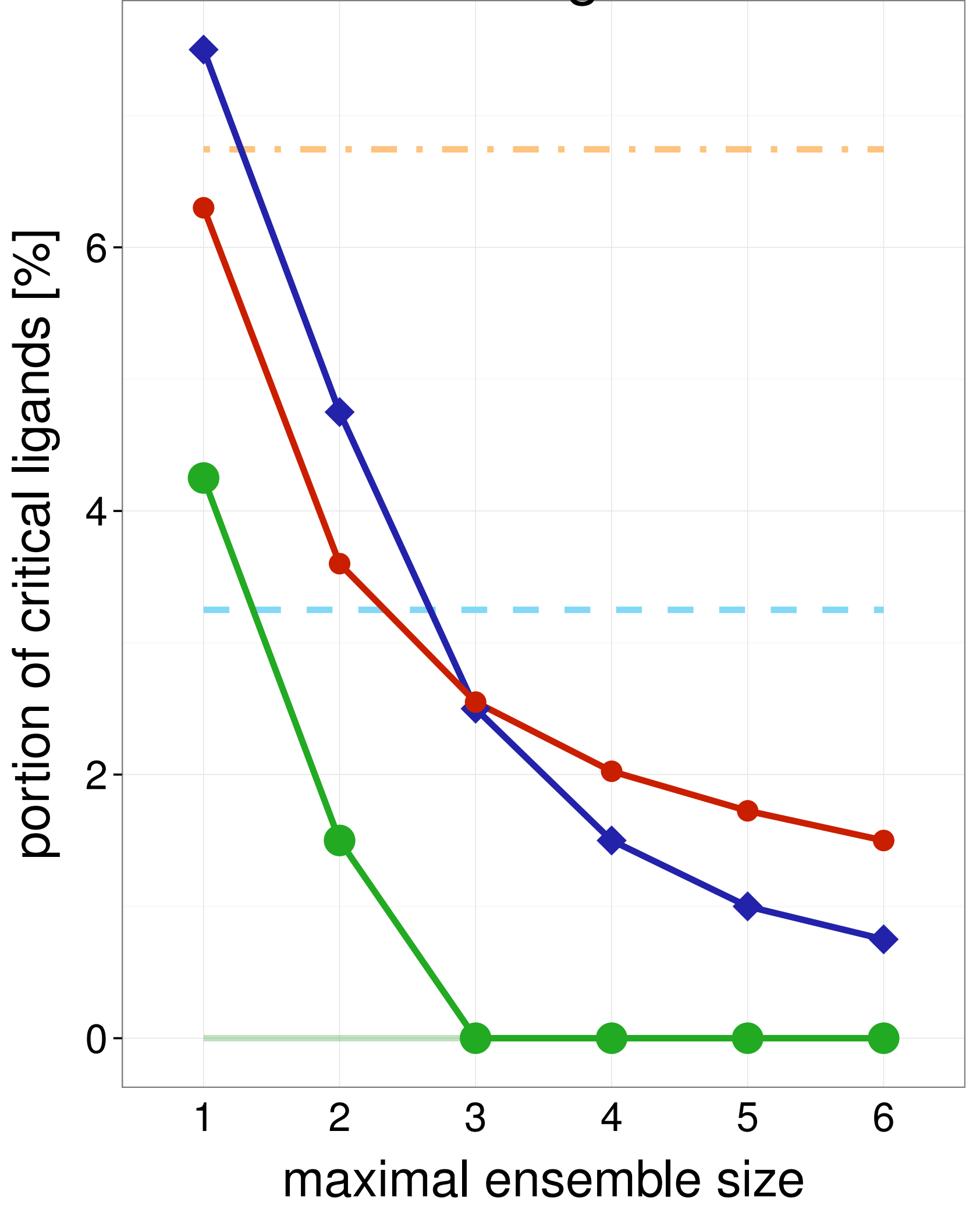

\section{Test set}

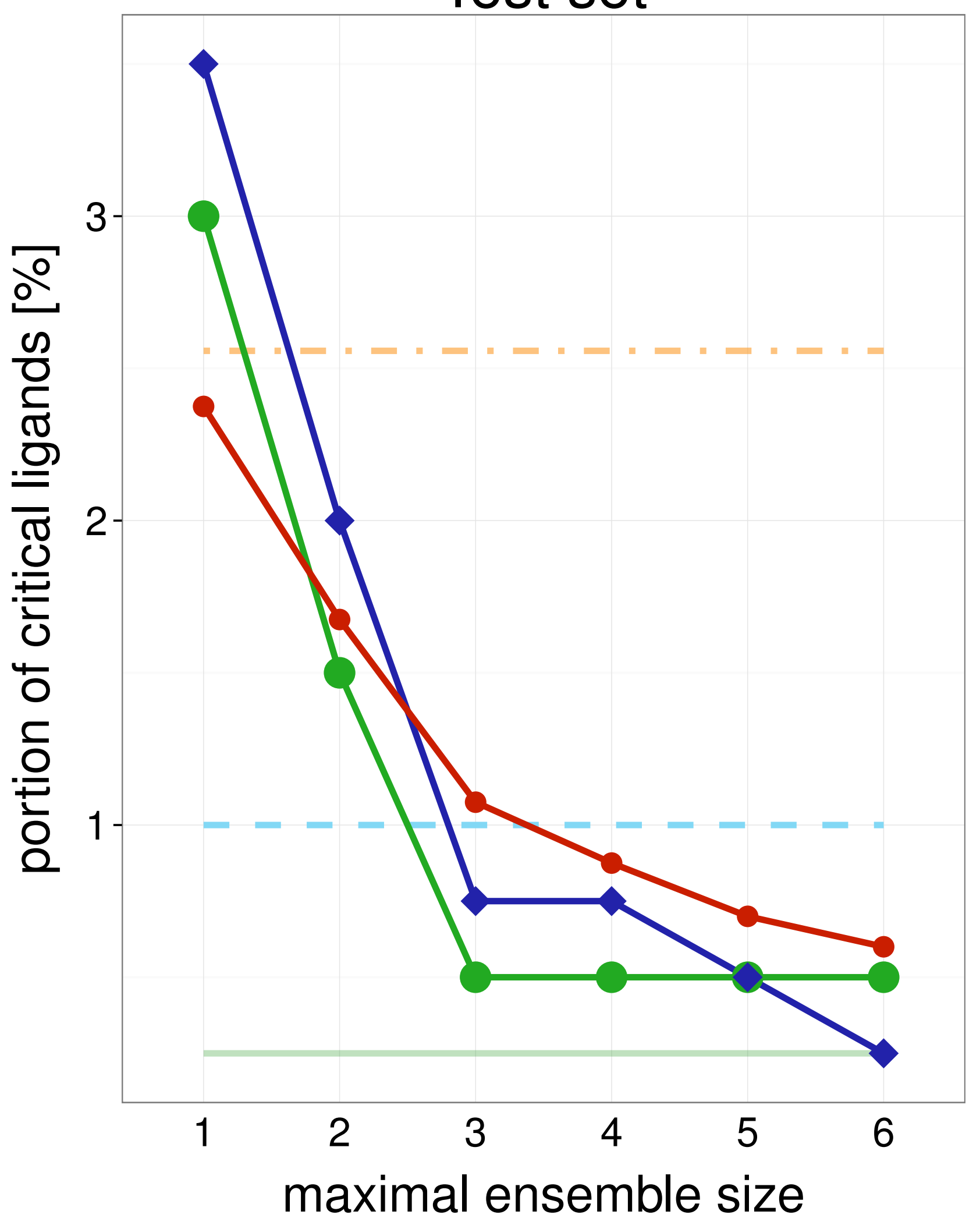

3DNG

Training set

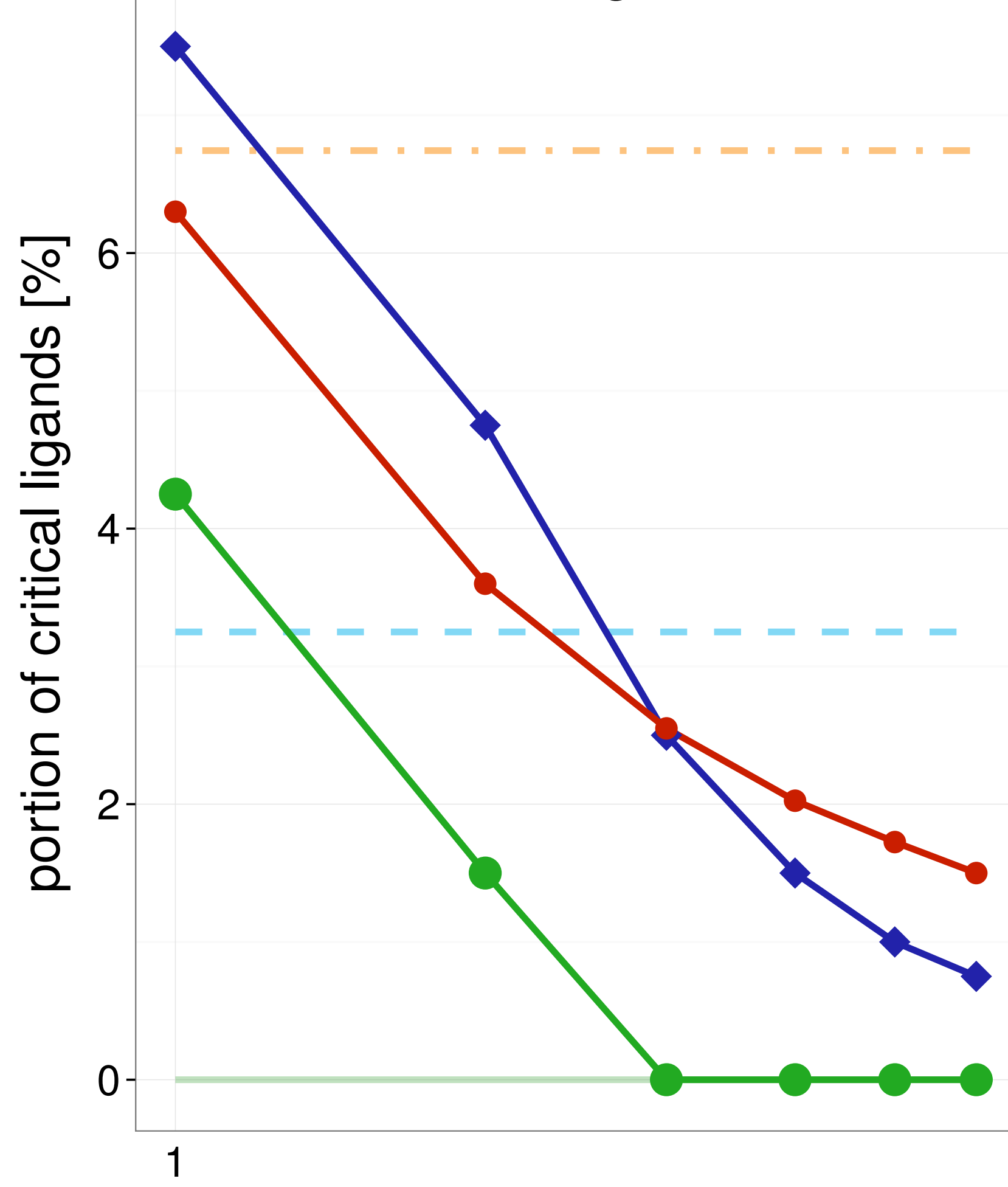

Average single structure

_ Best single structure

Whole ensemble

- SIENA

$\neg$ Clustering

$\multimap$ Random

Average single structure

_ _ Best single structure

Whole ensemble

- SIENA

$\neg$ Clustering

$\multimap$ Random maximal ensemble size

Test set

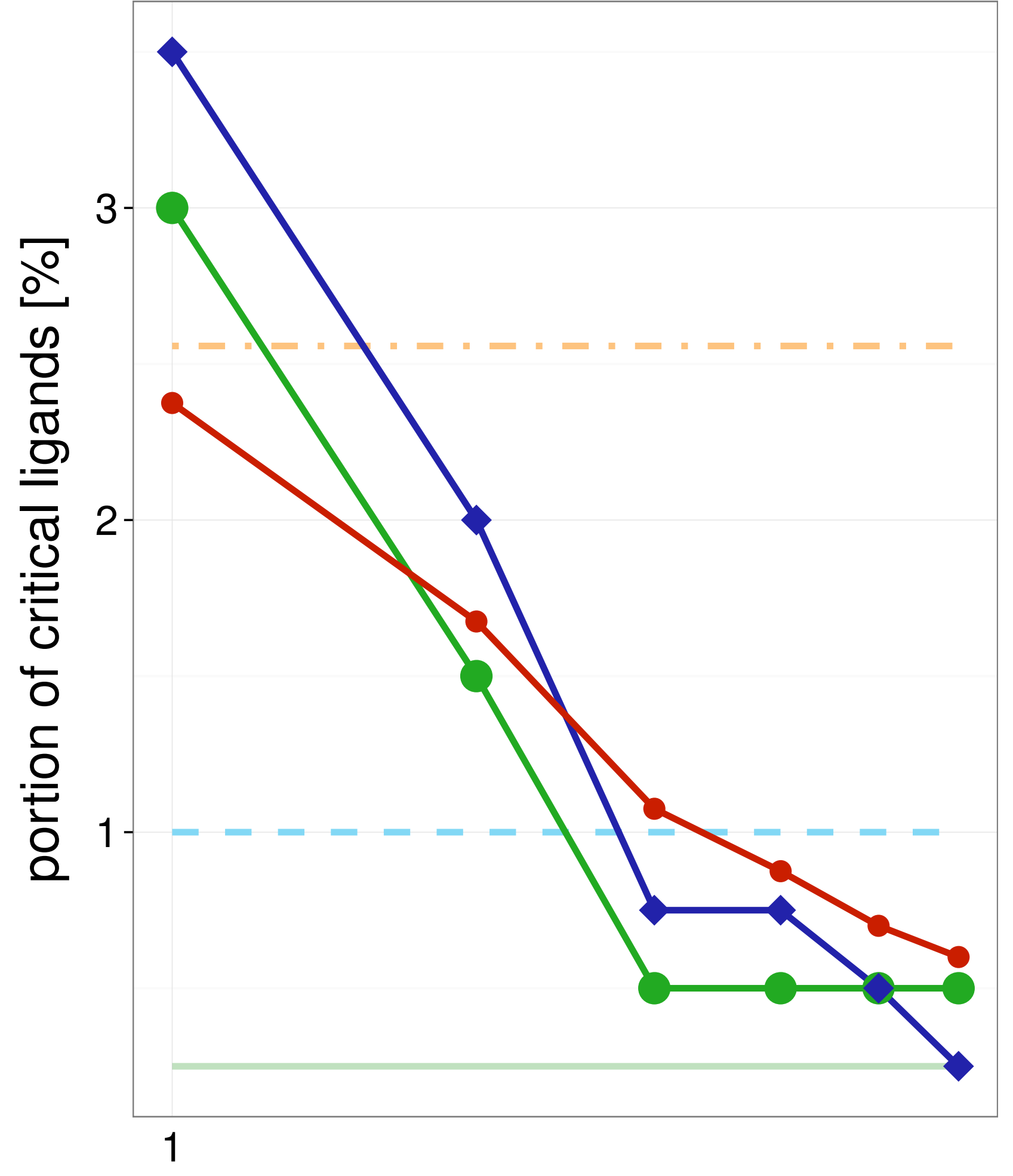

Average single structure

_ Best single structure

Whole ensemble

SIENA

$\sim$ Clustering

$\multimap$ Random 


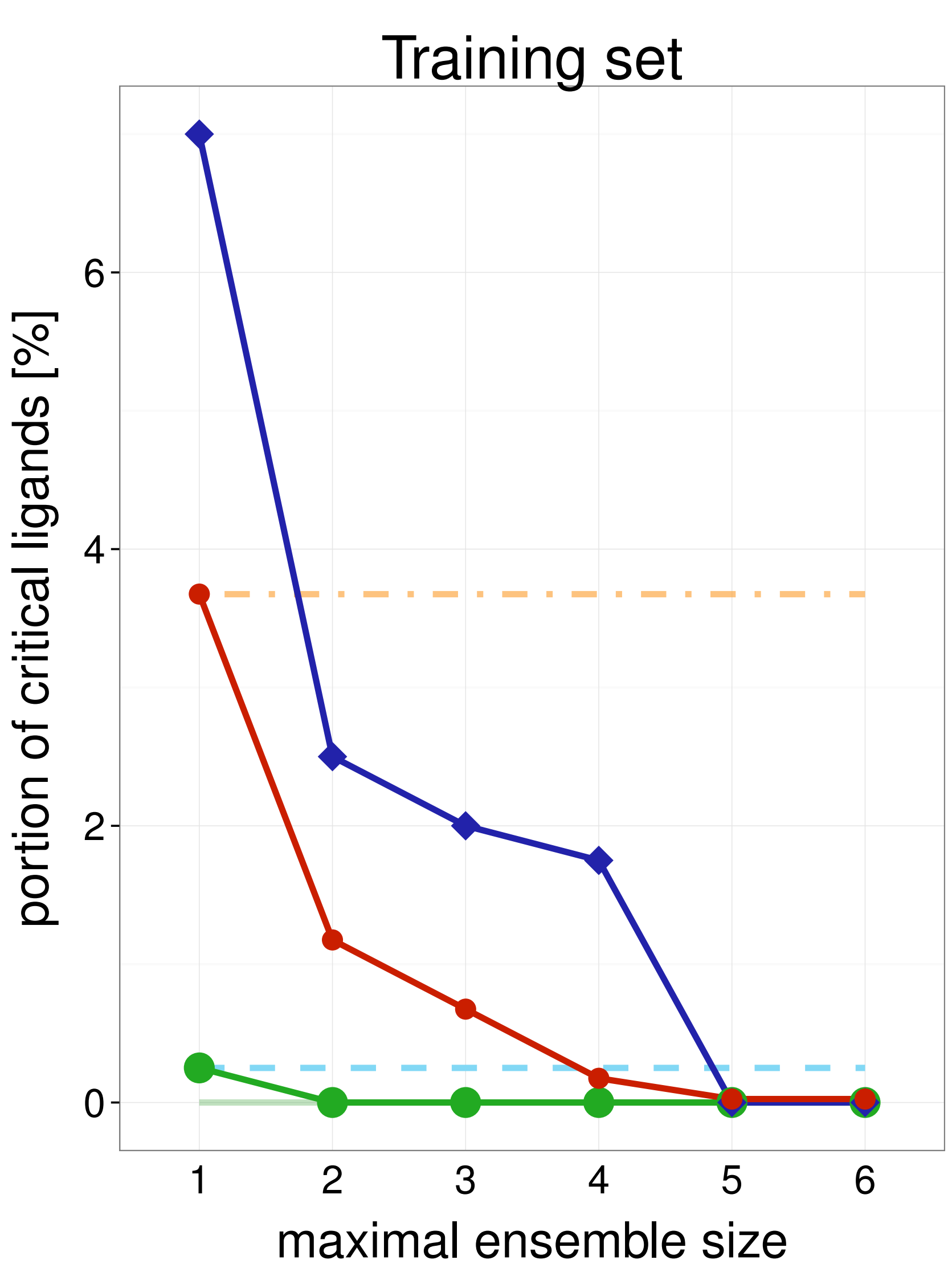

\section{Test set}

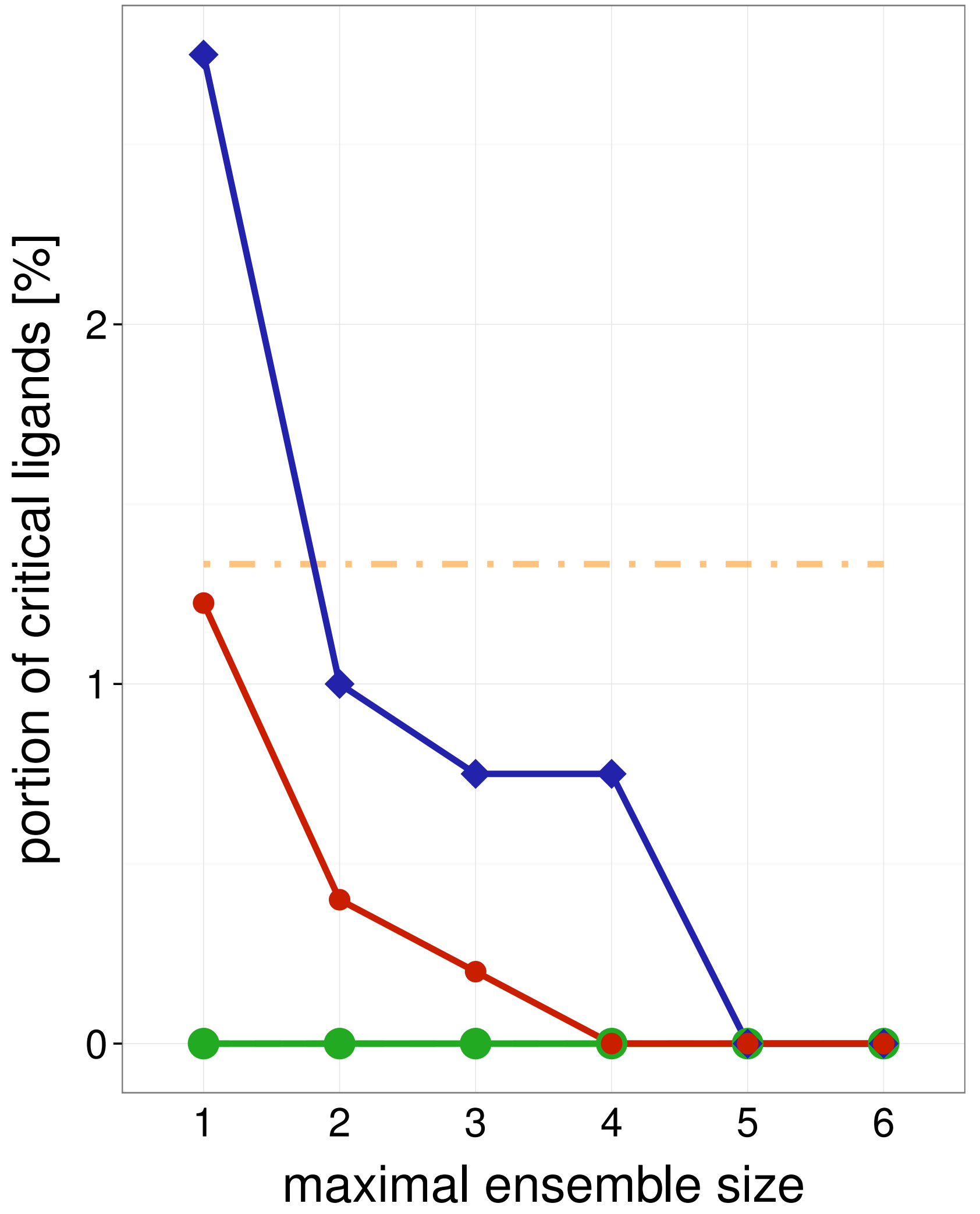

$3 G 2 Y$

Training set

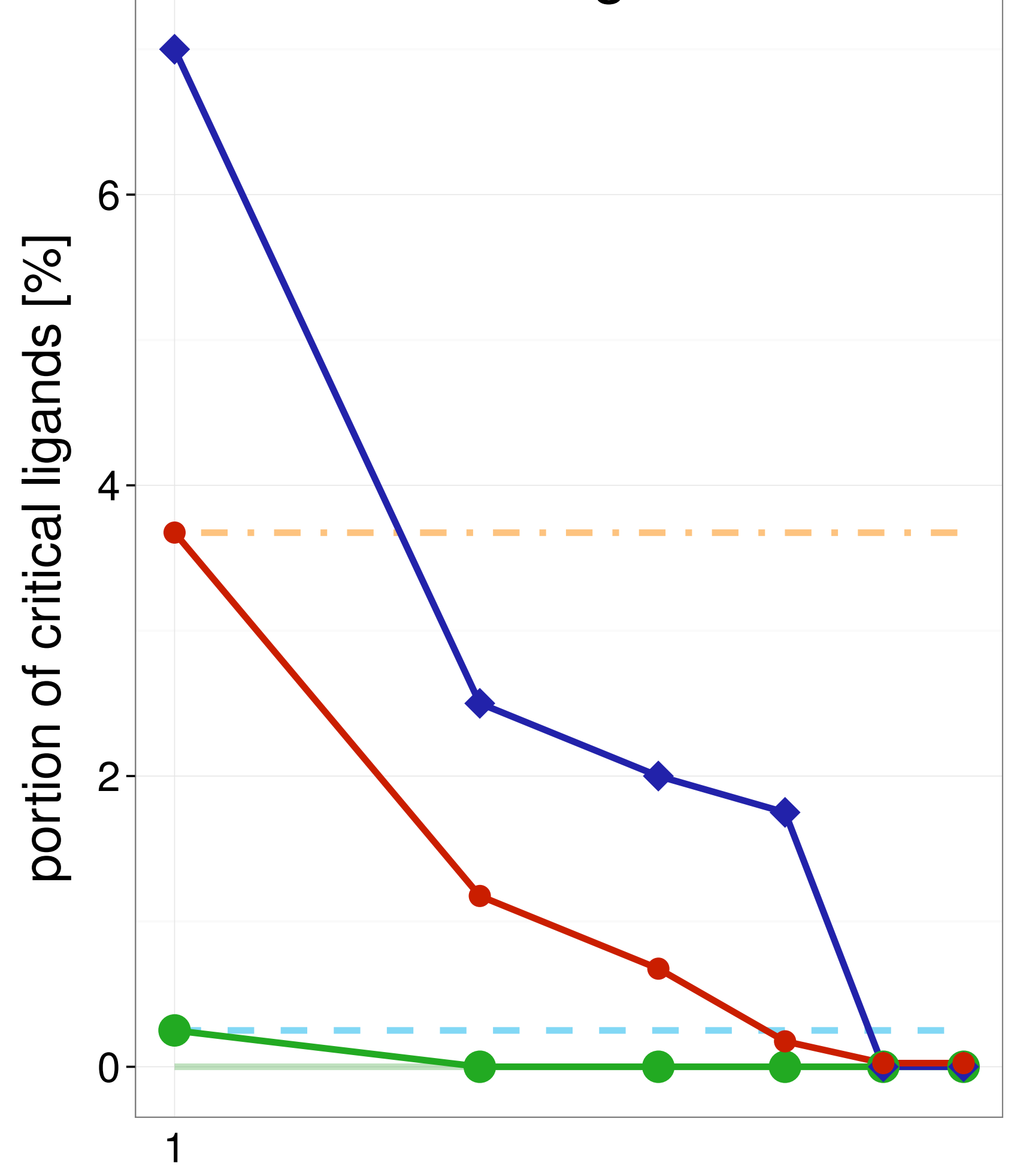

Average single structure

Best single structure

Whole ensemble

- SIENA

$\checkmark$ Clustering

$\multimap$ Random

Average single structure

_ Best single structure

Whole ensemble

- SIENA

$\neg$ Clustering

$\multimap$ Random maximal ensemble size

Test set

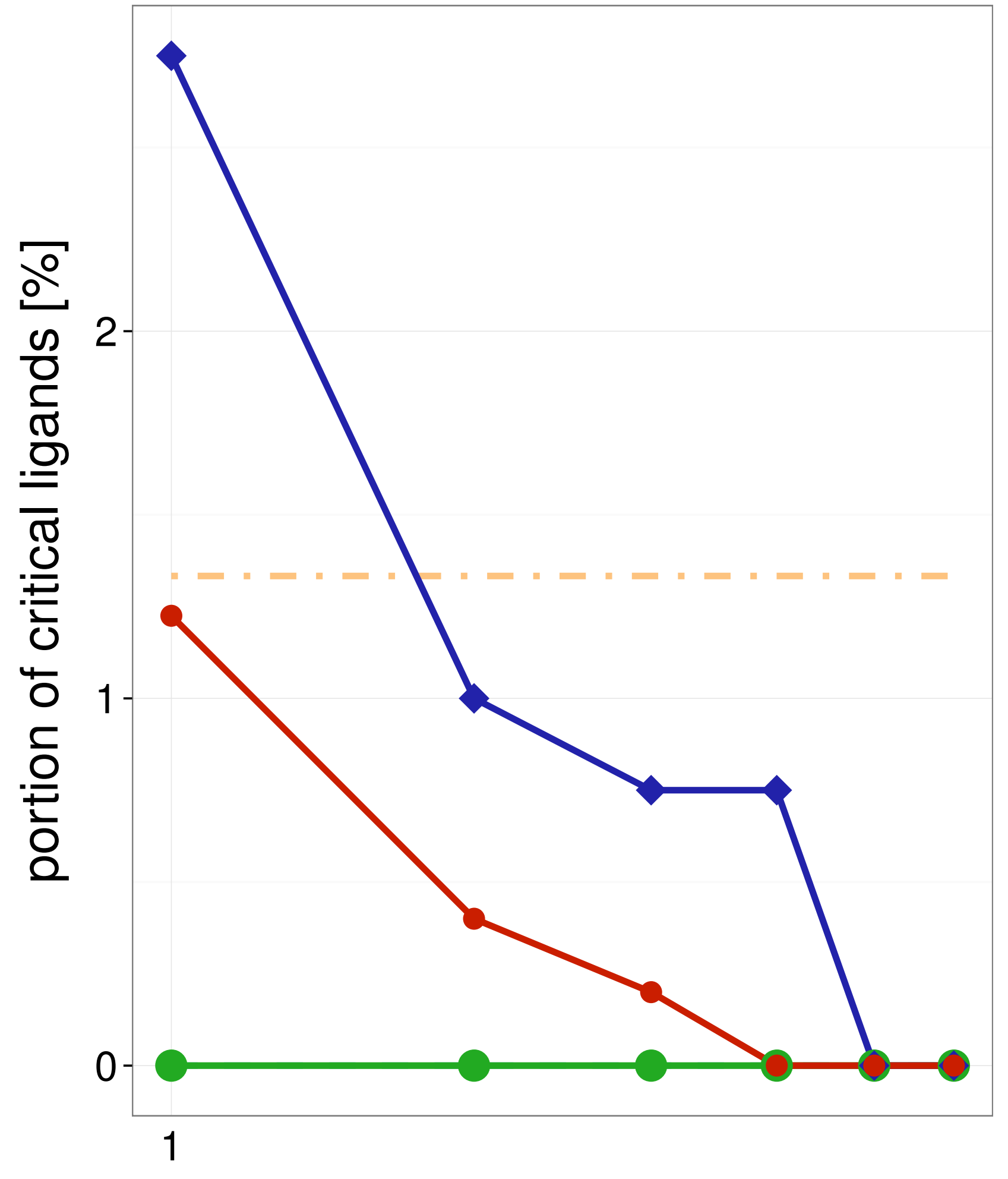

Average single structure

_ Best single structure

Whole ensemble

SIENA

$\neg$ Clustering

$\longrightarrow$ Random 


\section{Training set}

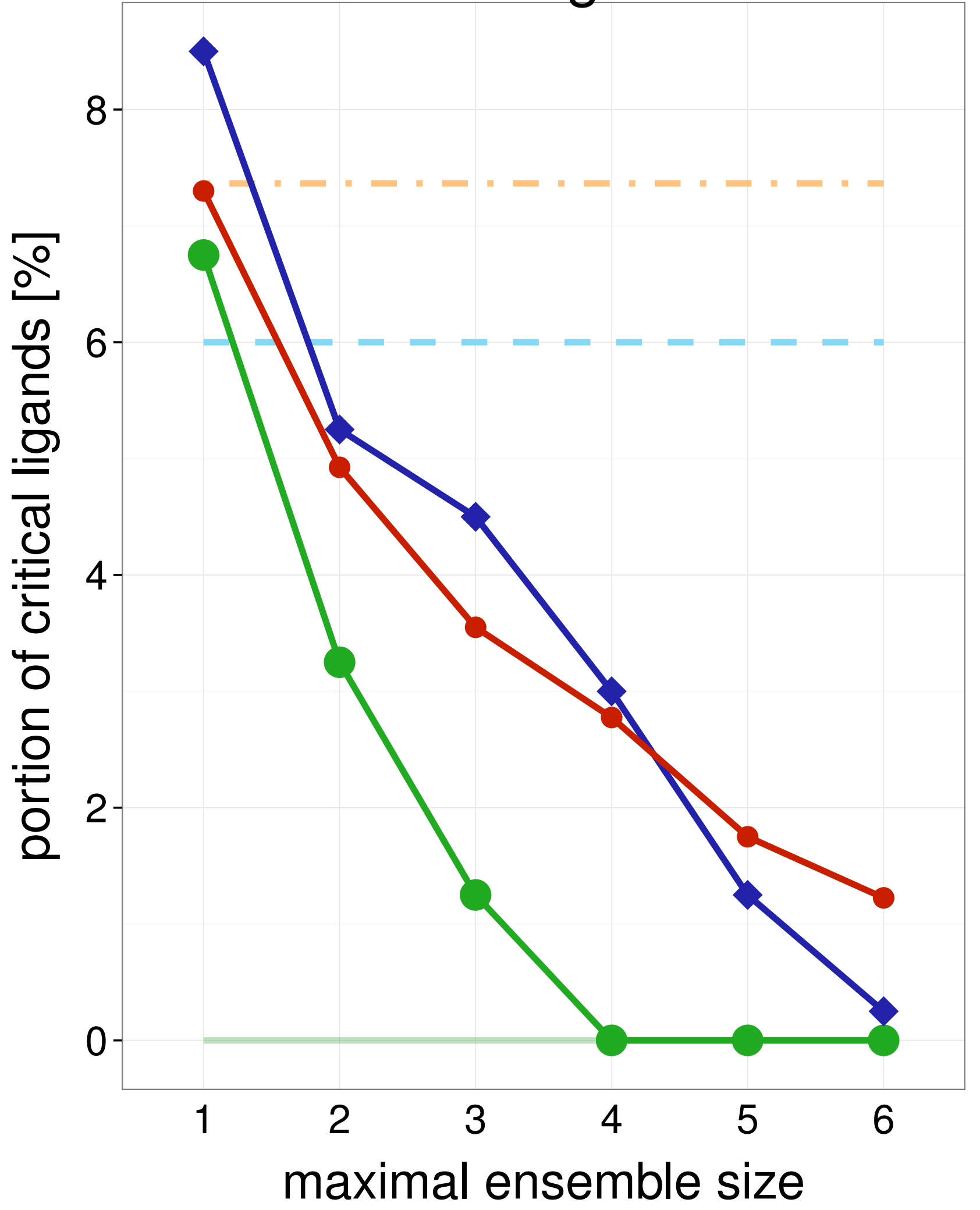

Test set

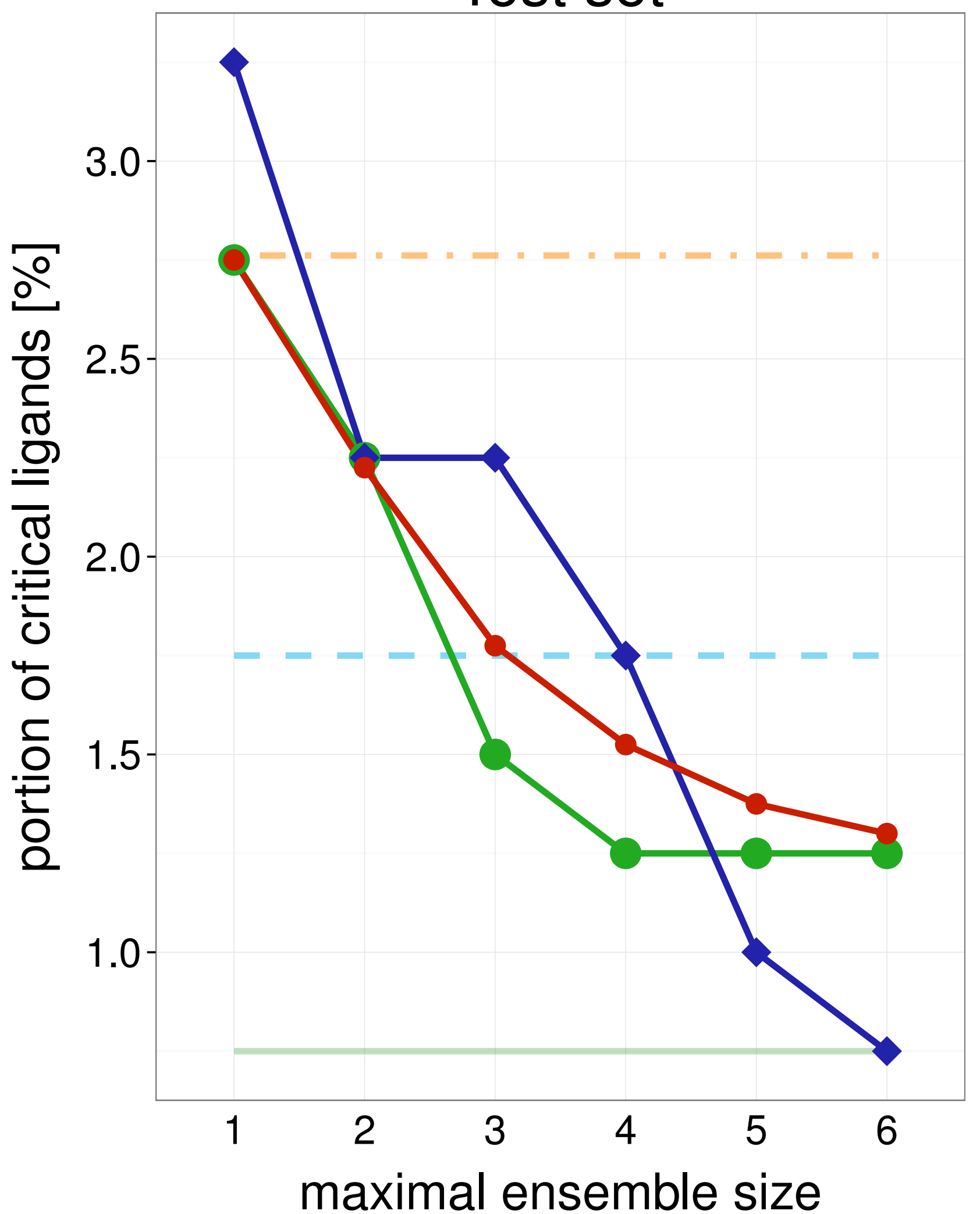

3Q30

Training set

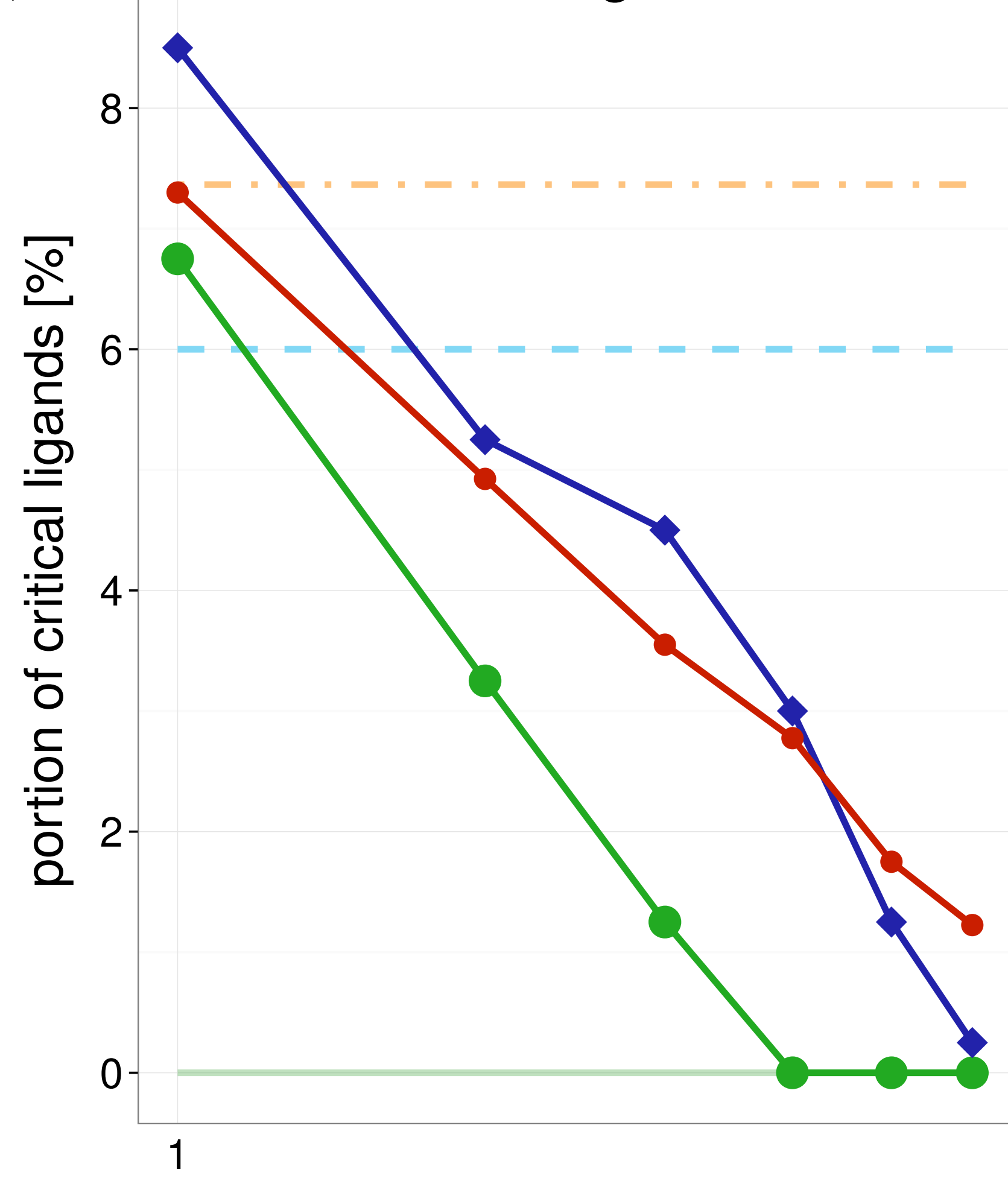

maximal ensemble size

Test set

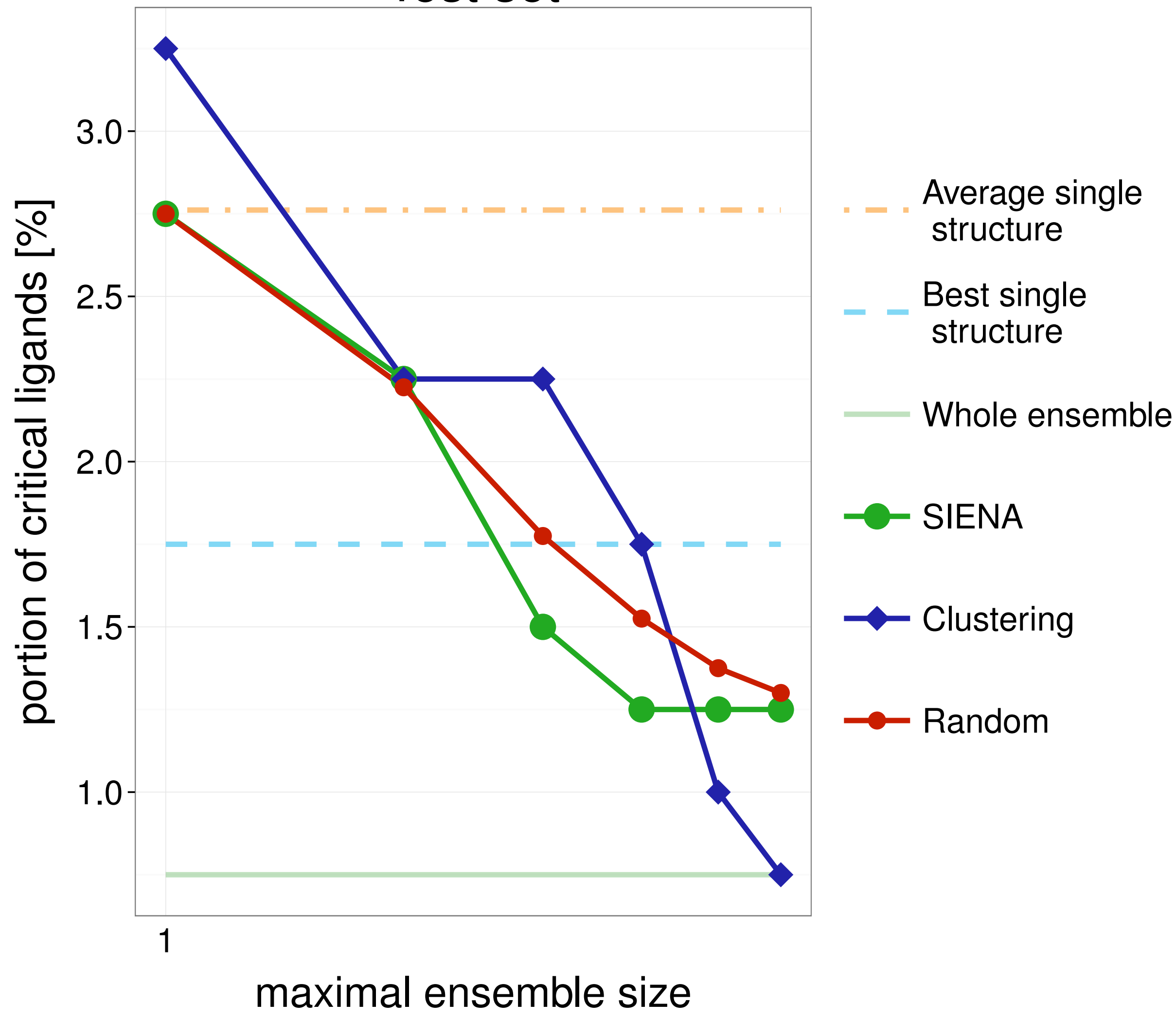


Training set

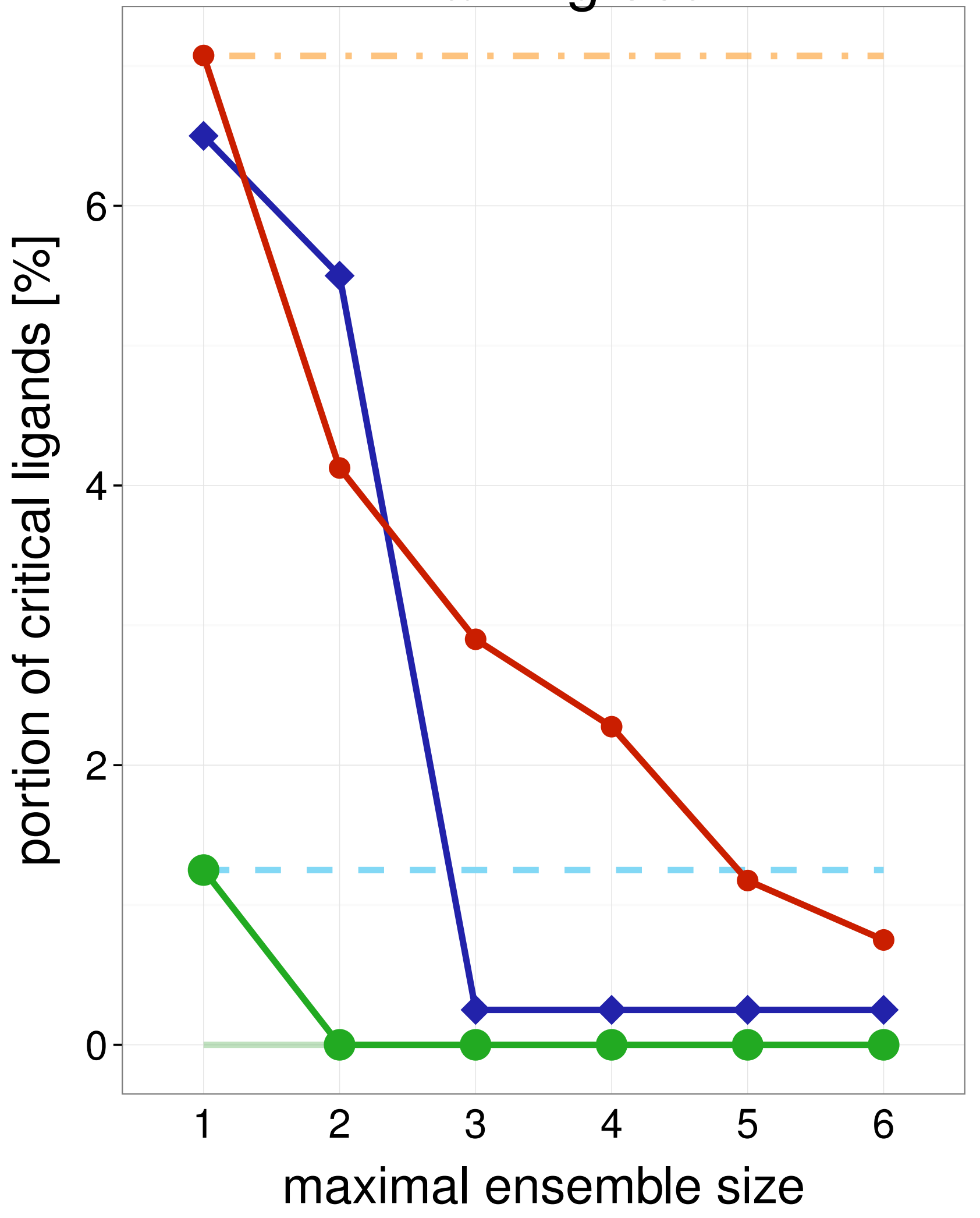

\section{Test set}

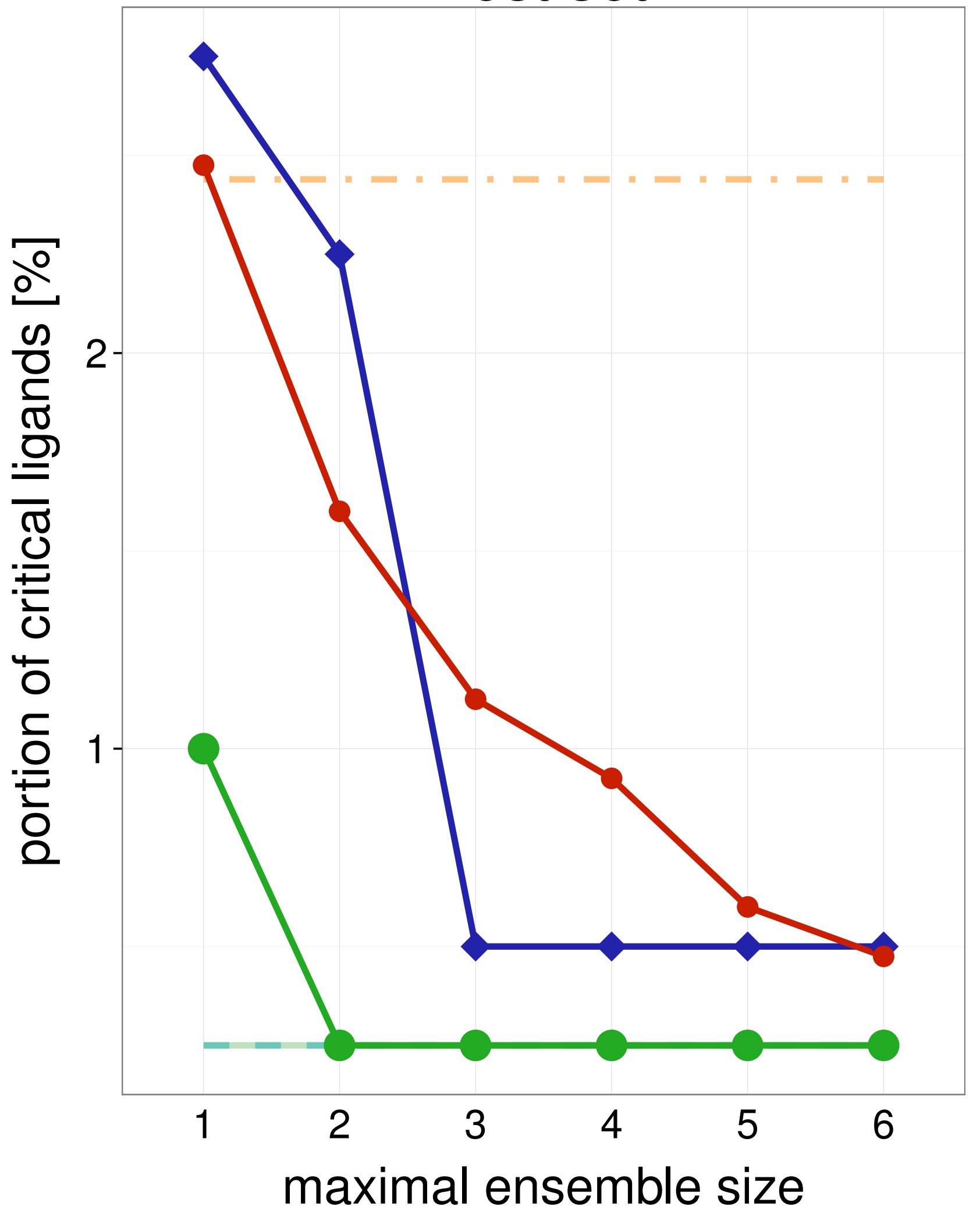

Average single structure

_ Best single structure

Whole ensemble

- - SIENA

$\neg$ Clustering

- Random

Average single structure

_ _ Best single structure

Whole ensemble

- SIENA

$\leadsto$ Clustering

$\multimap$ Random

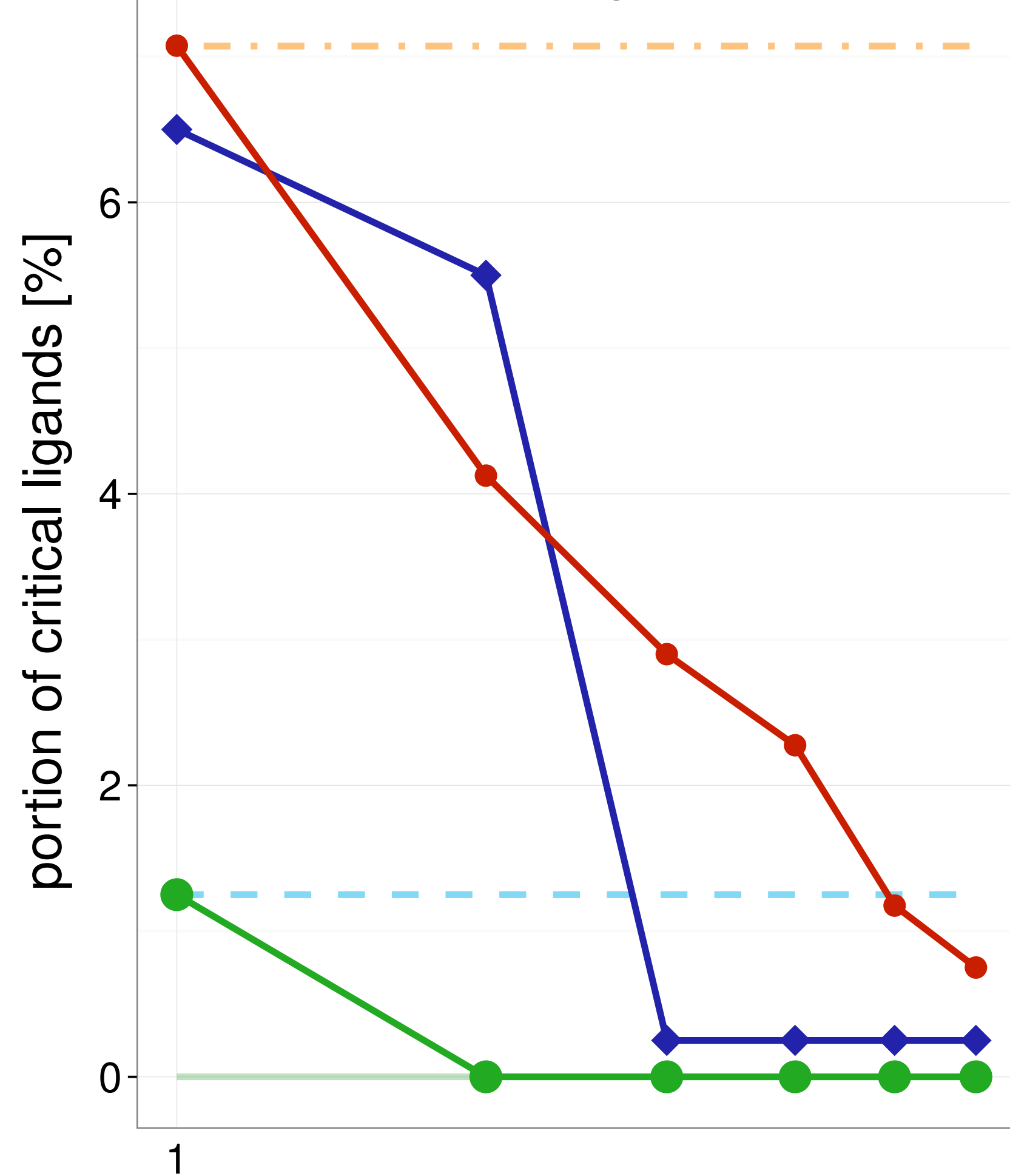

maximal ensemble size

Test set

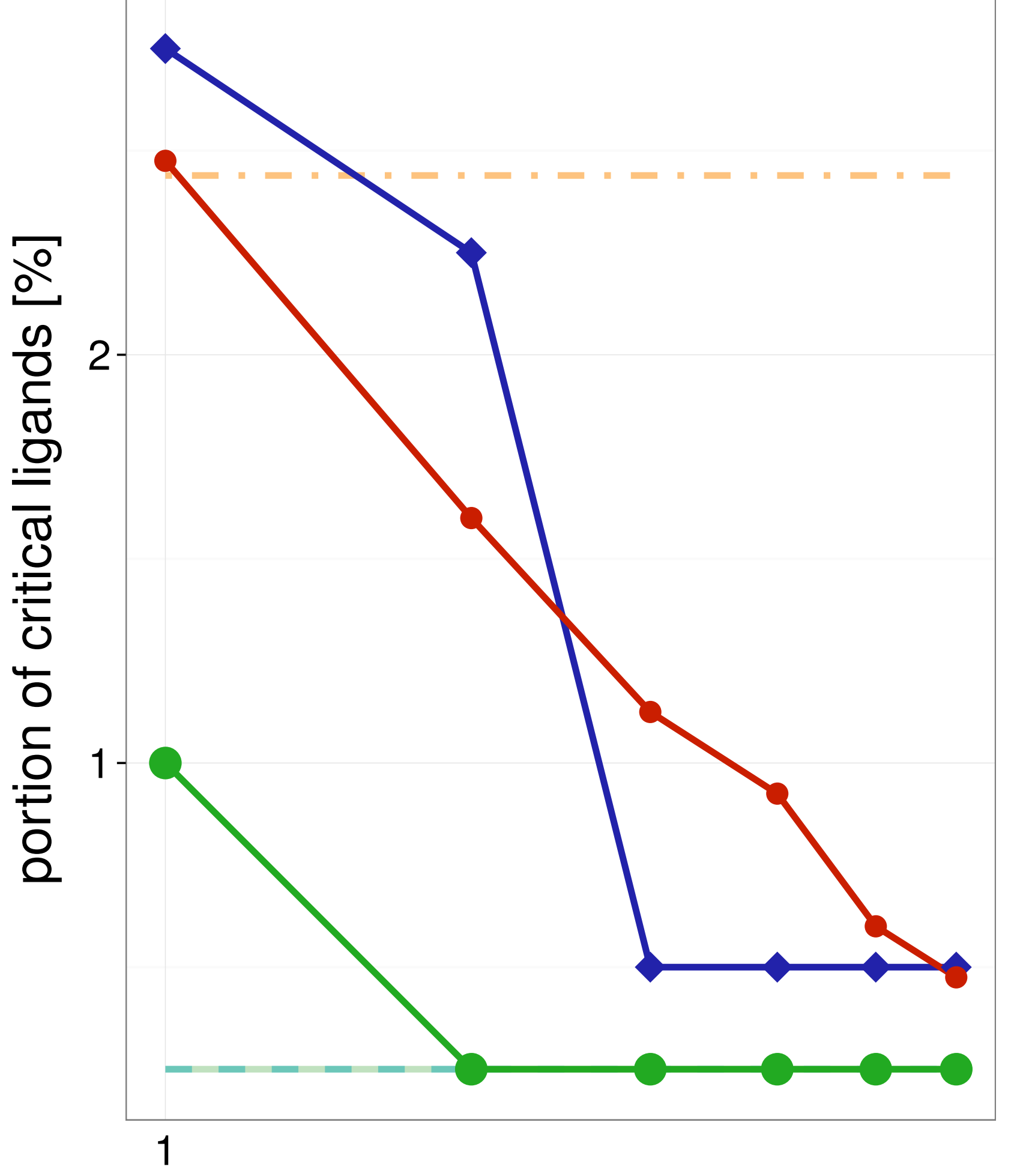

maximal ensemble size
Average single structure

_ Best single structure

Whole ensemble

SIENA

$\sim$ Clustering

$\longrightarrow$ Random 
Training set

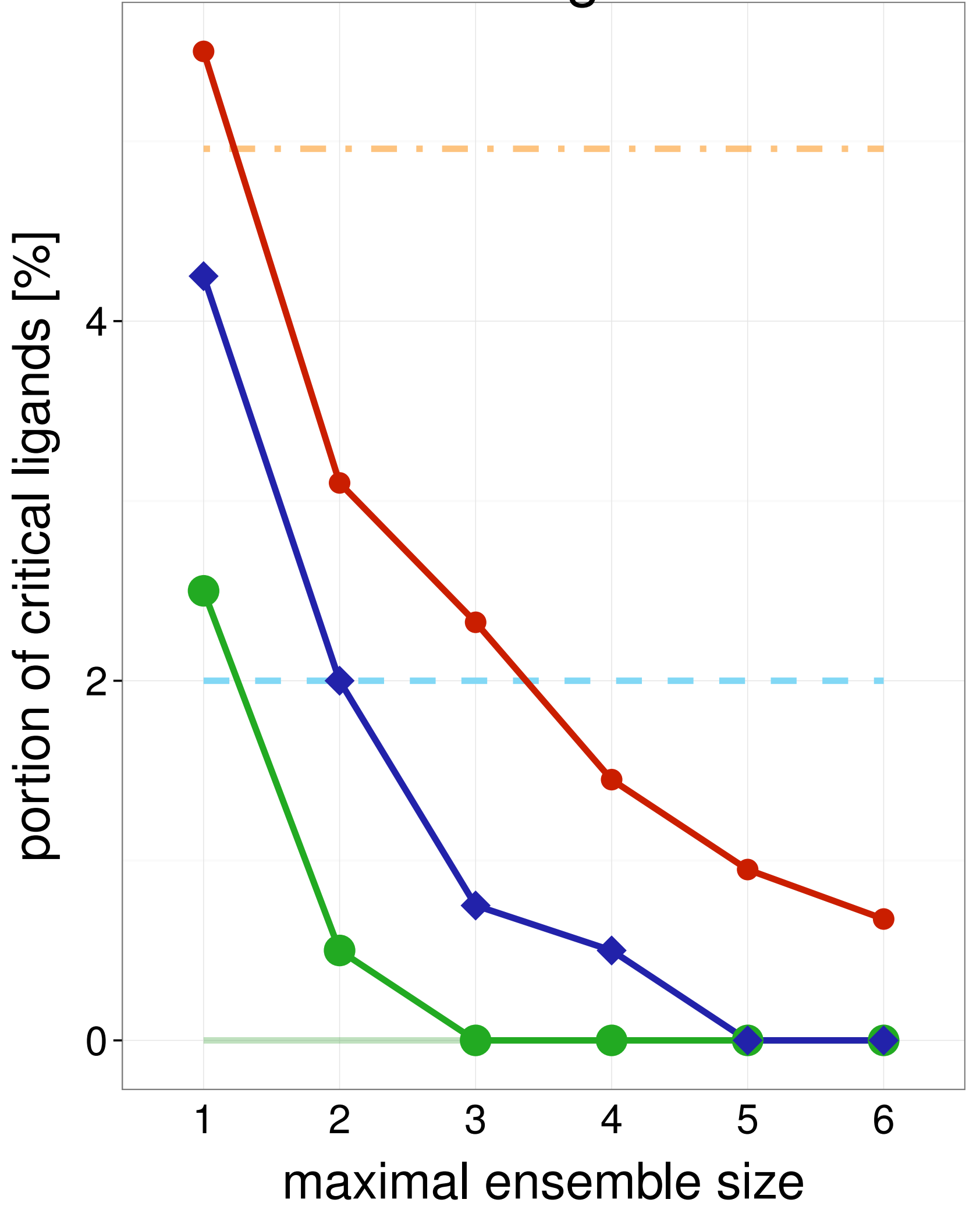

\section{Test set}

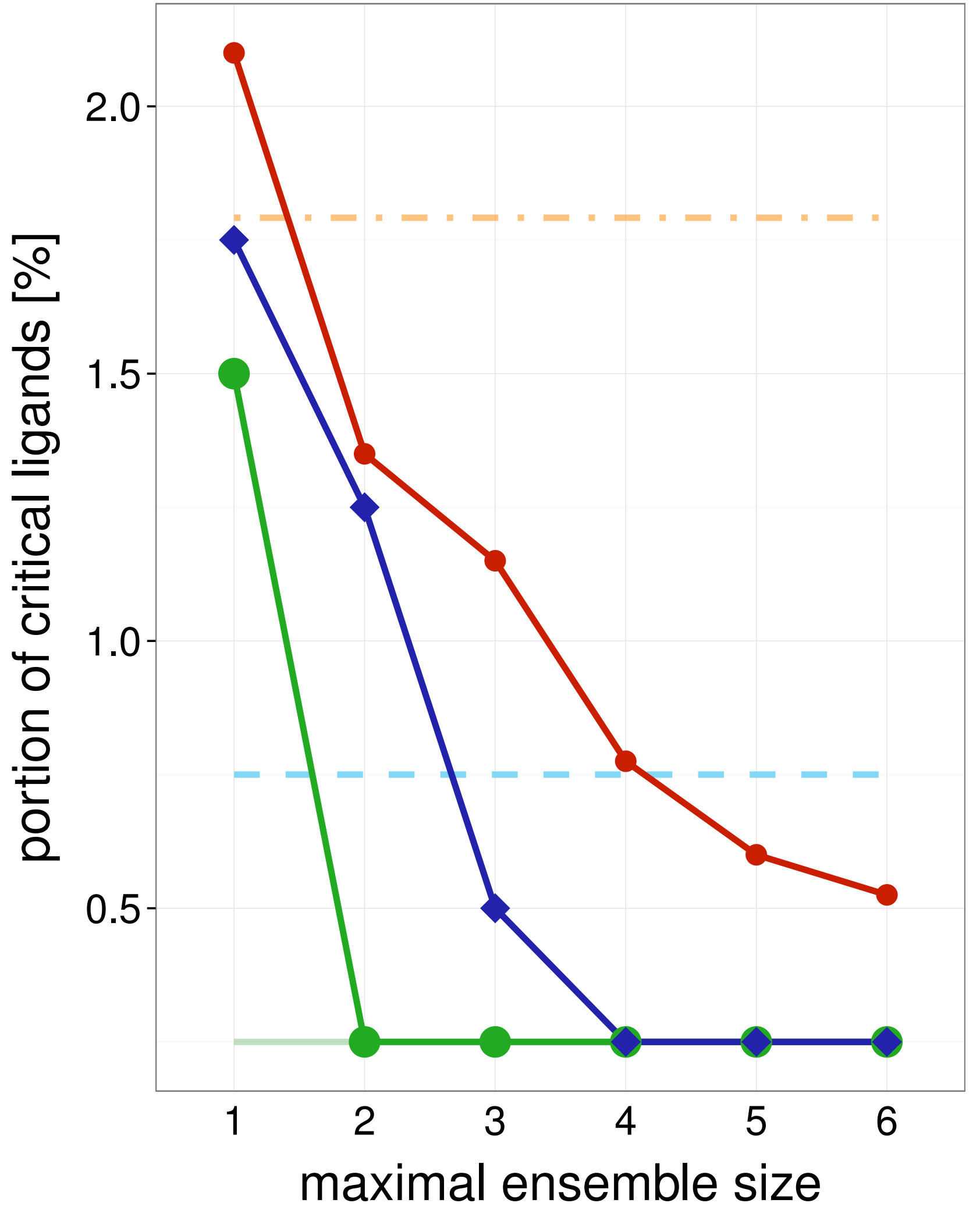

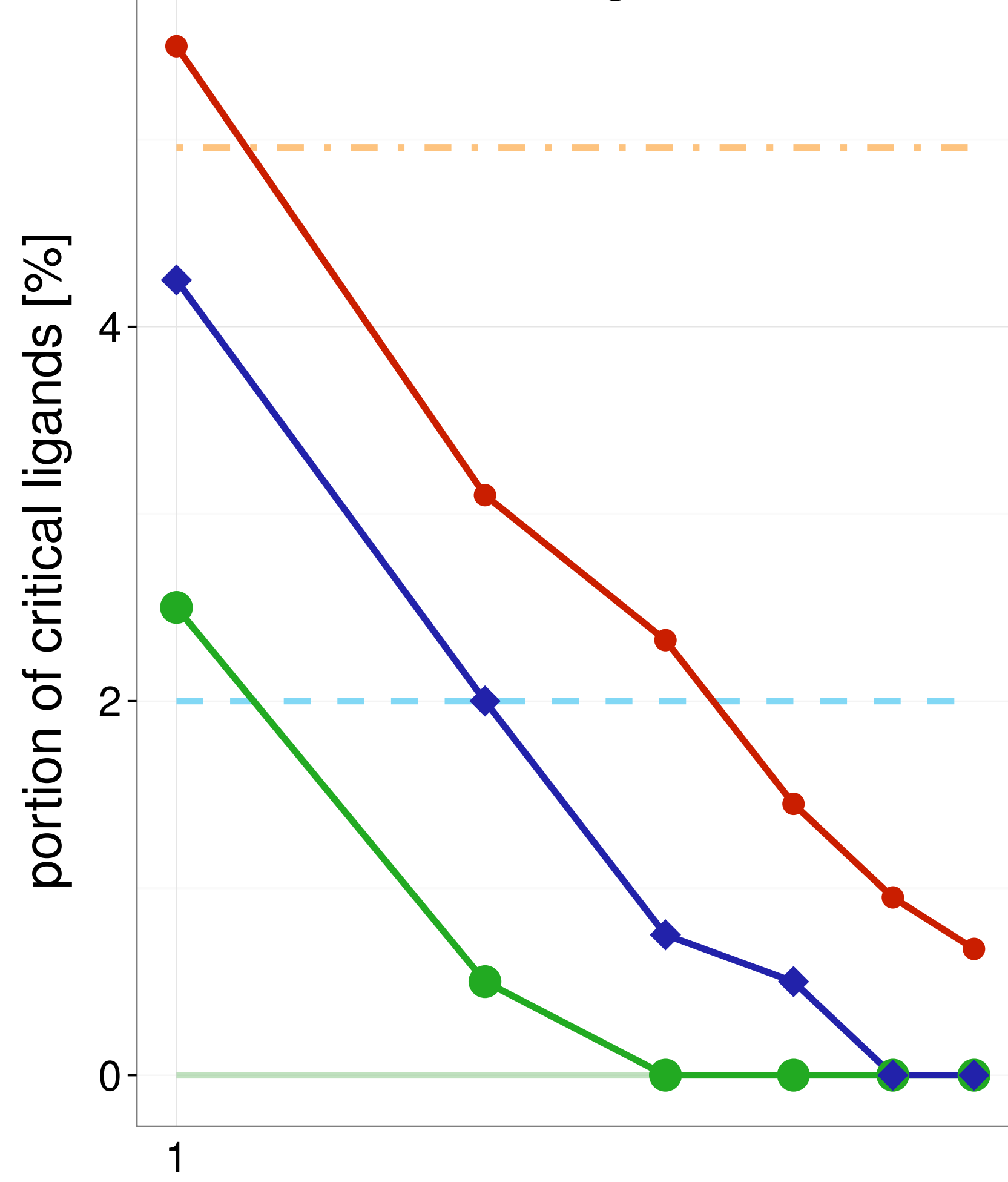

Average single structure

_ _ Best single structure

Whole ensemble

Whole ensemble

- SIENA

$\prec$ Clustering

$\because$ Random

Average single structure

_ _ Best single structure

Whole ensemble

- SIENA

$\smile$ Clustering

$\multimap$ Random

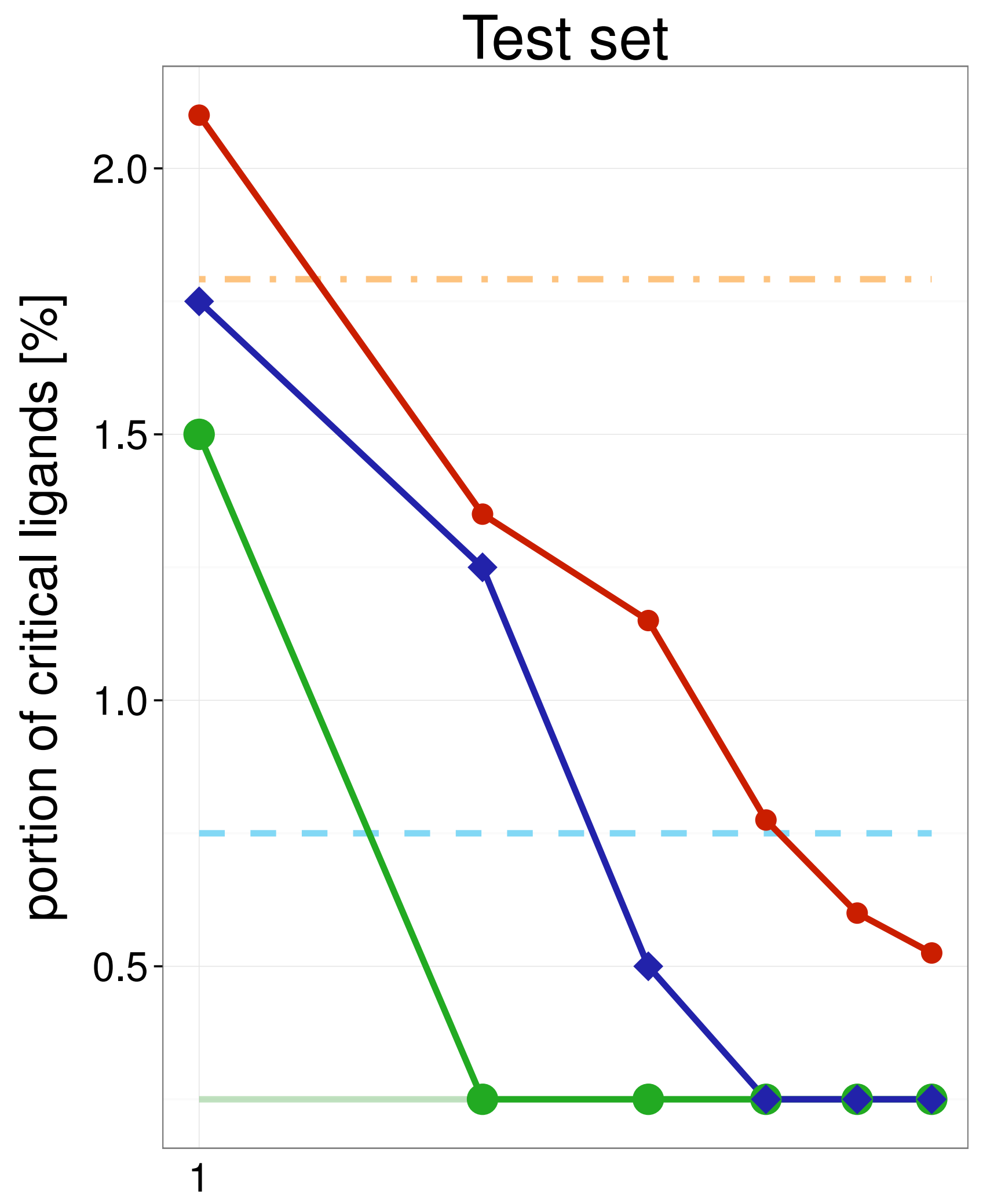

Average single structure

Best single structure

Whole ensemble

SIENA

$\neg$ Clustering

$\multimap$ Random 
Training set

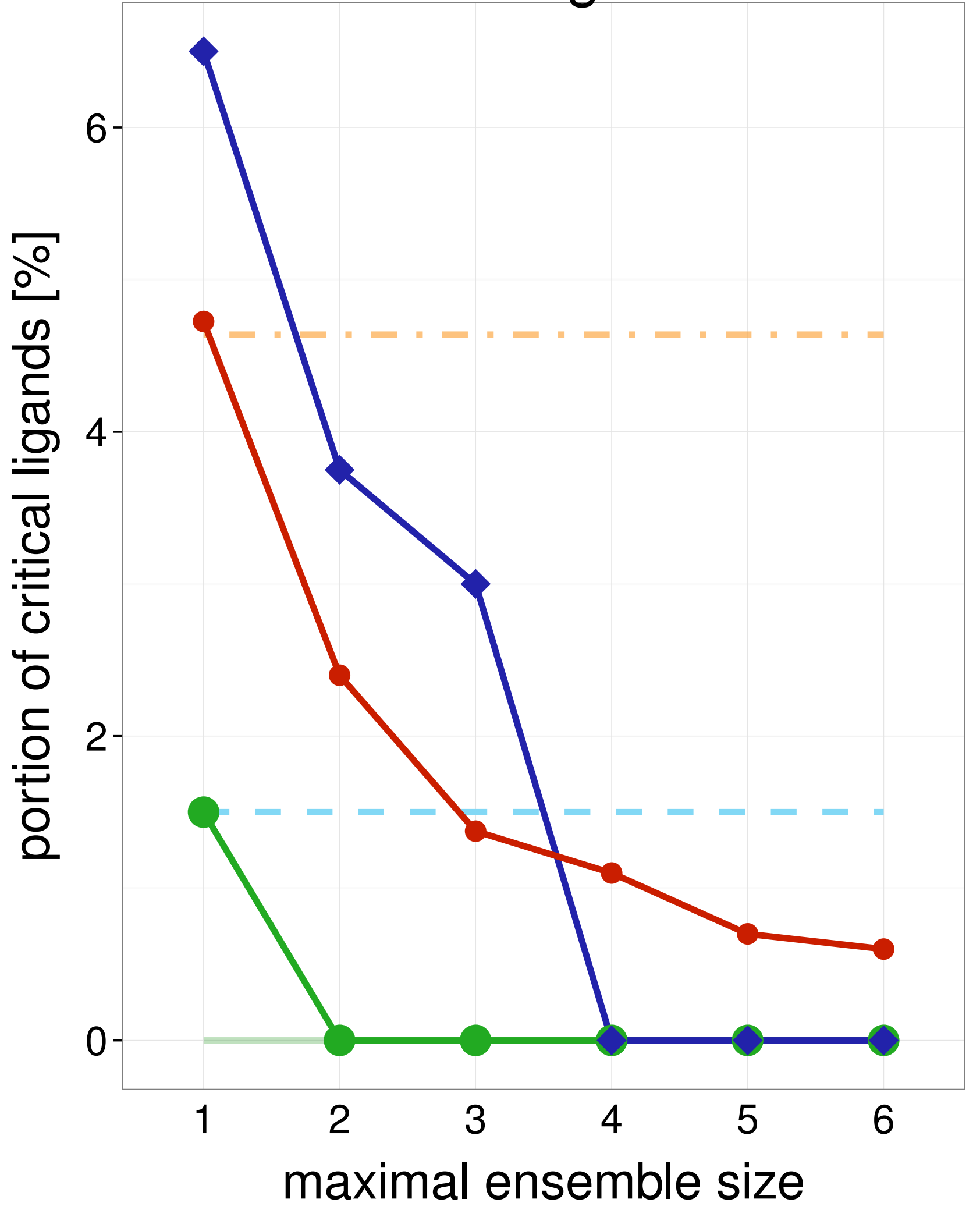

Test set

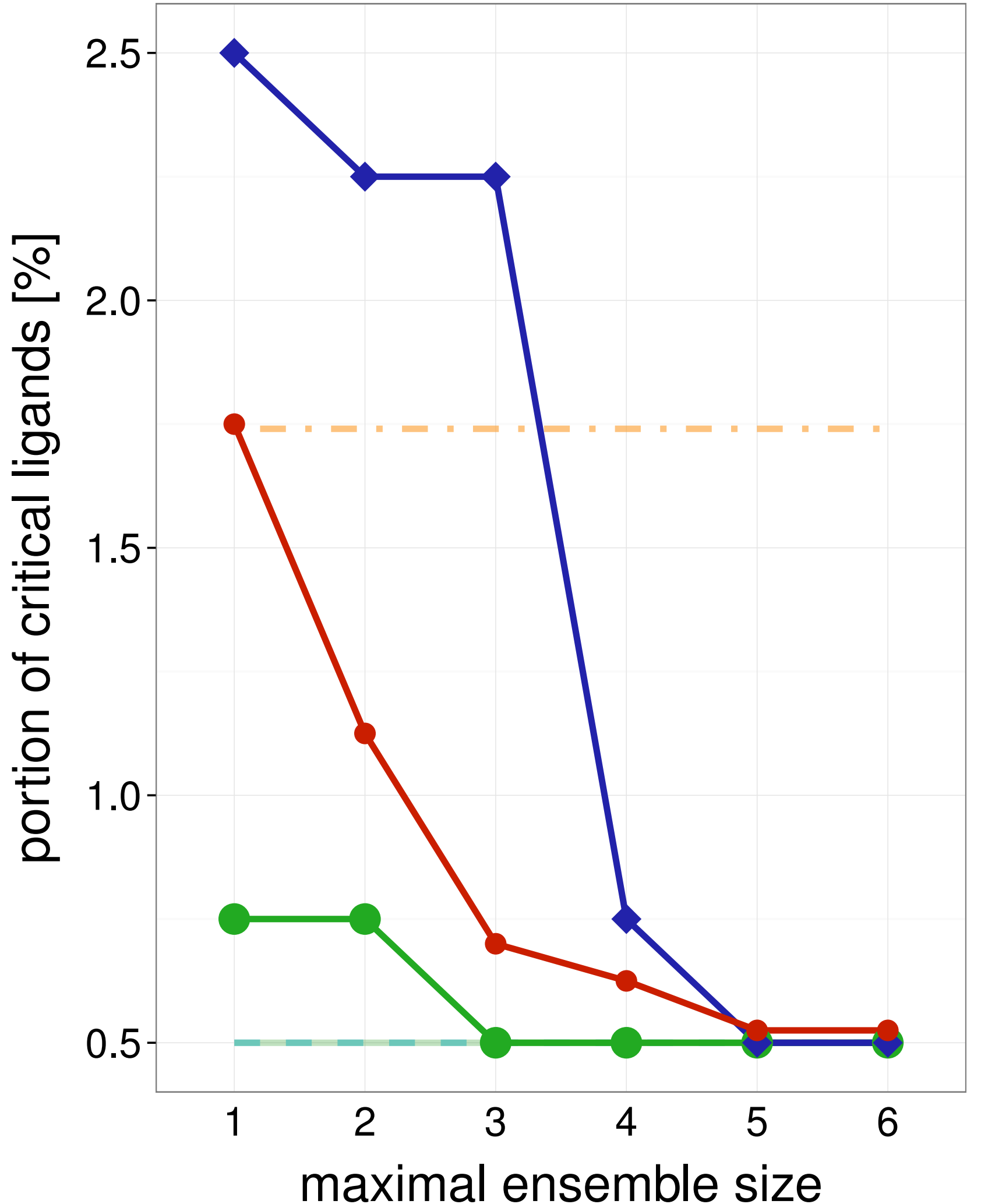

Average single structure

_ Best single structure

Whole ensemble

- SIENA

$\neg$ Clustering

$\multimap$ Random

Average single structure

_ _ Best single structure

Whole ensemble

- SIENA

$\neg$ Clustering

$\multimap$ Random

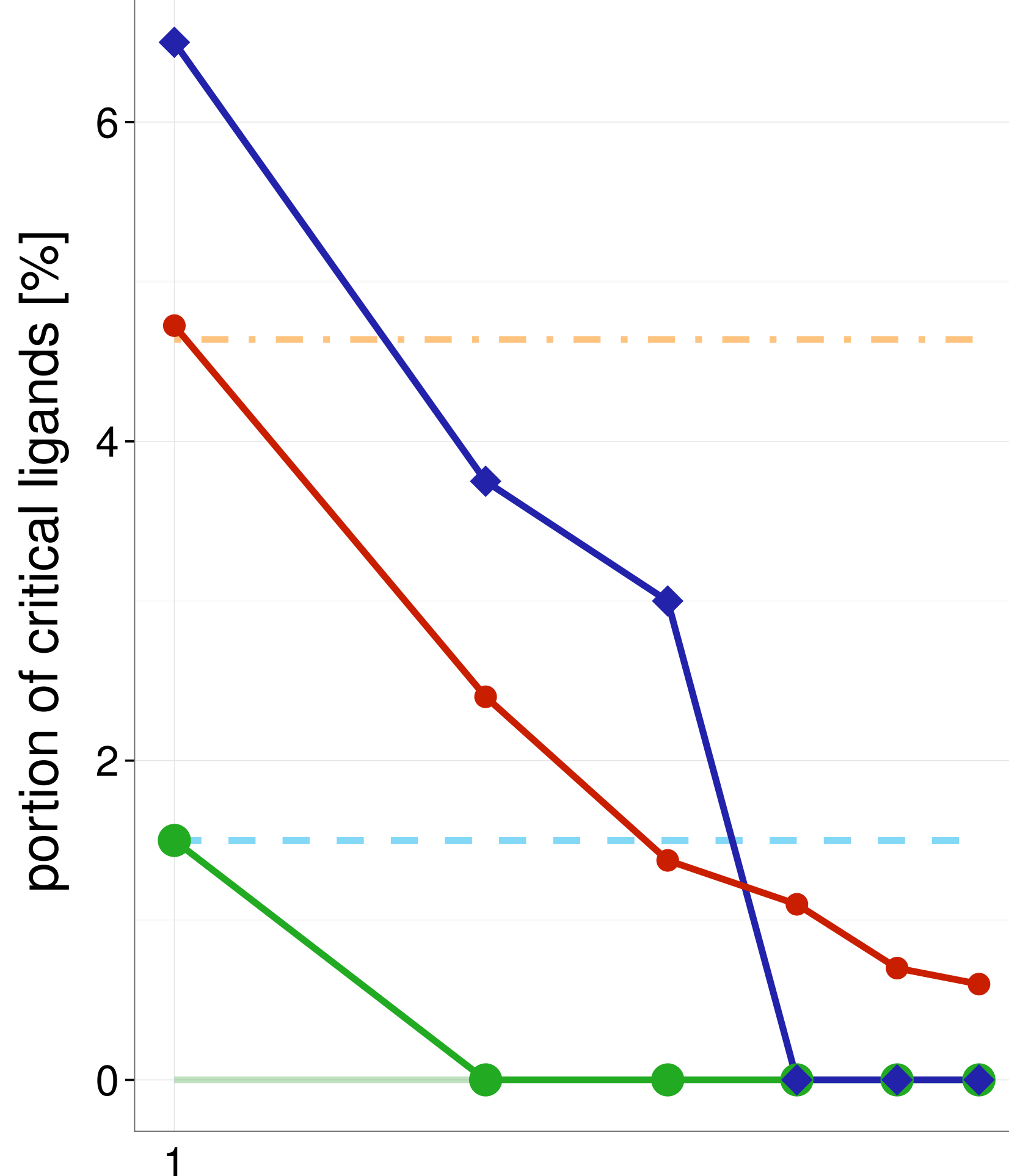

maximal ensemble size

Test set

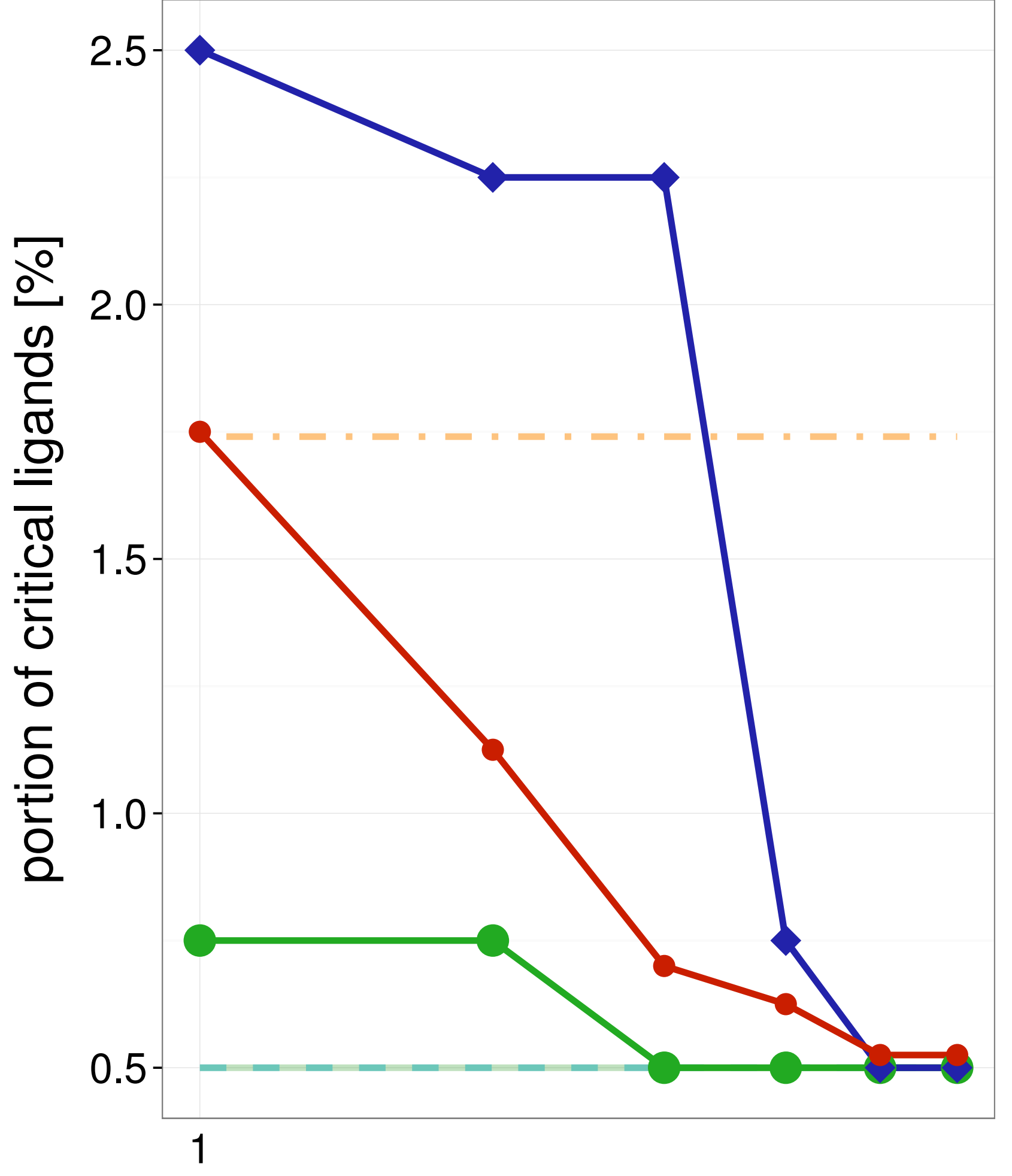

maximal ensemble size
Average single structure

_ Best single structure

Whole ensemble

SIENA

$\neg$ Clustering

$\multimap$ Random 
Training set

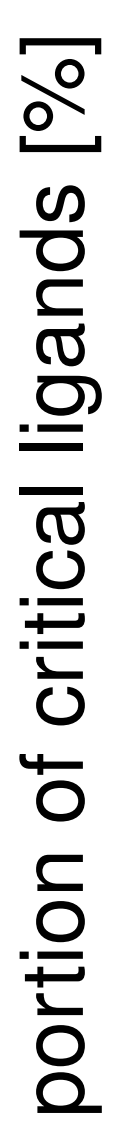

2.5

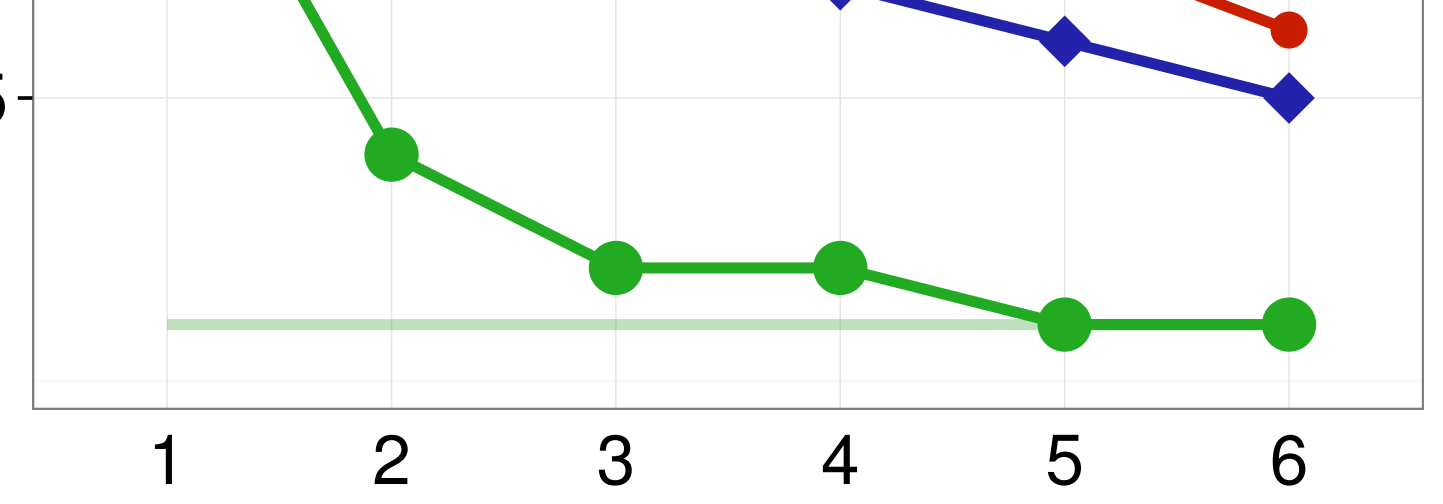

maximal ensemble size

Test set

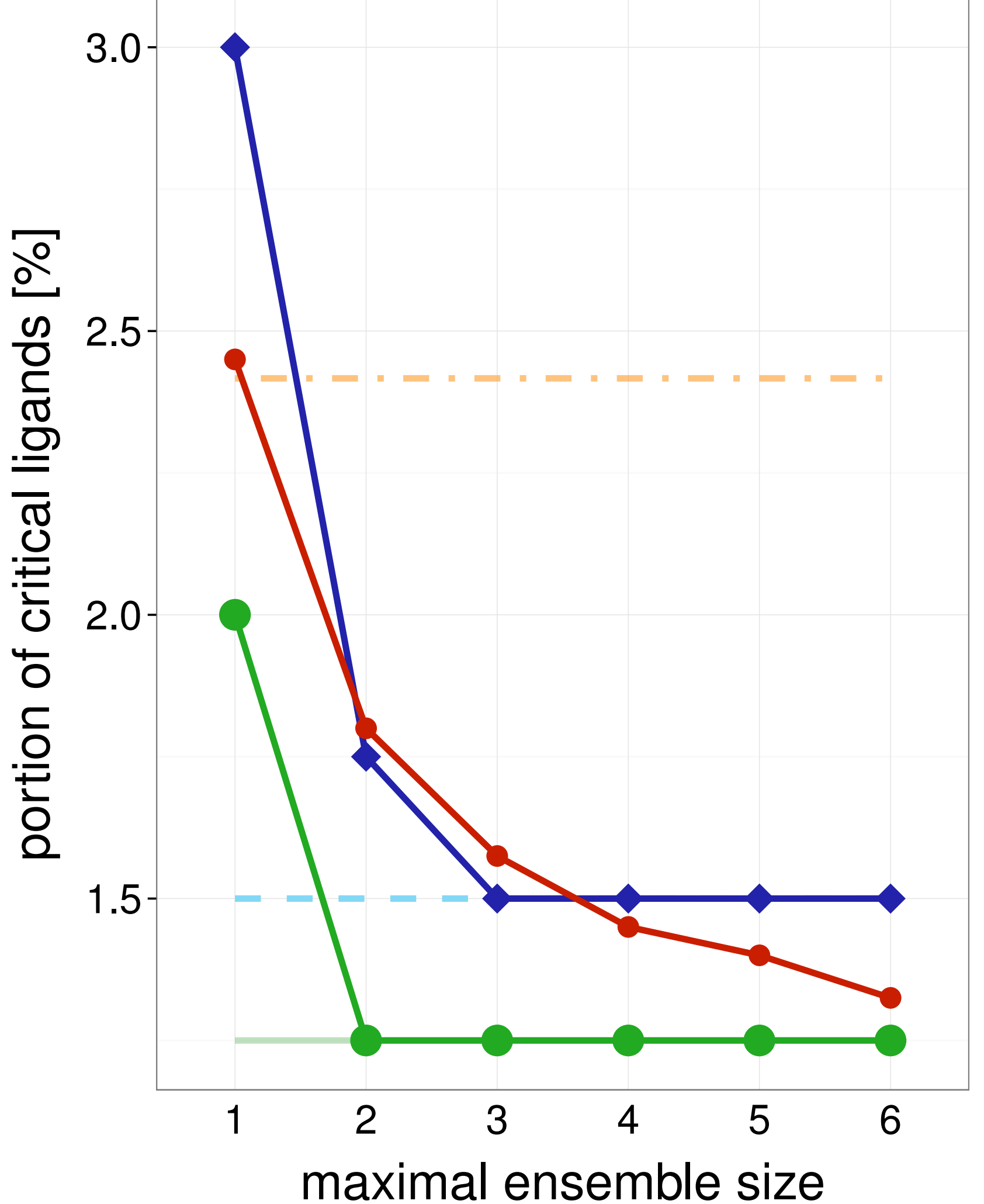

$4 \mathrm{M} \times 1$

Training set

Average single structure

_ $\quad$ Best single structure

Whole ensemble

- SIENA

$\neg$ Clustering

$\multimap$ Random

Average single structure

_ Best single structure

Whole ensemble

- SIENA

$\neg$ Clustering

$\multimap$ Random

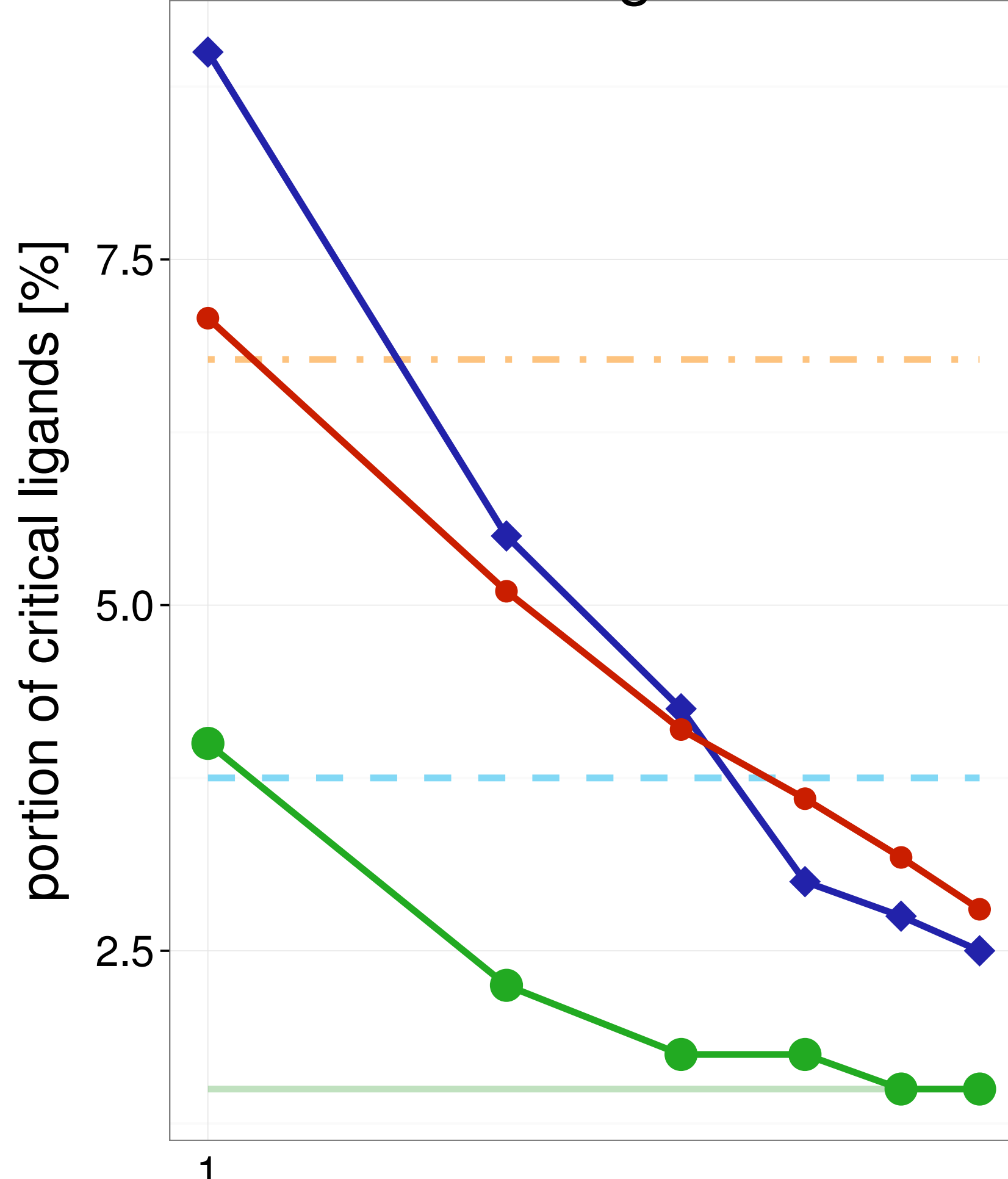

maximal ensemble size

Test set

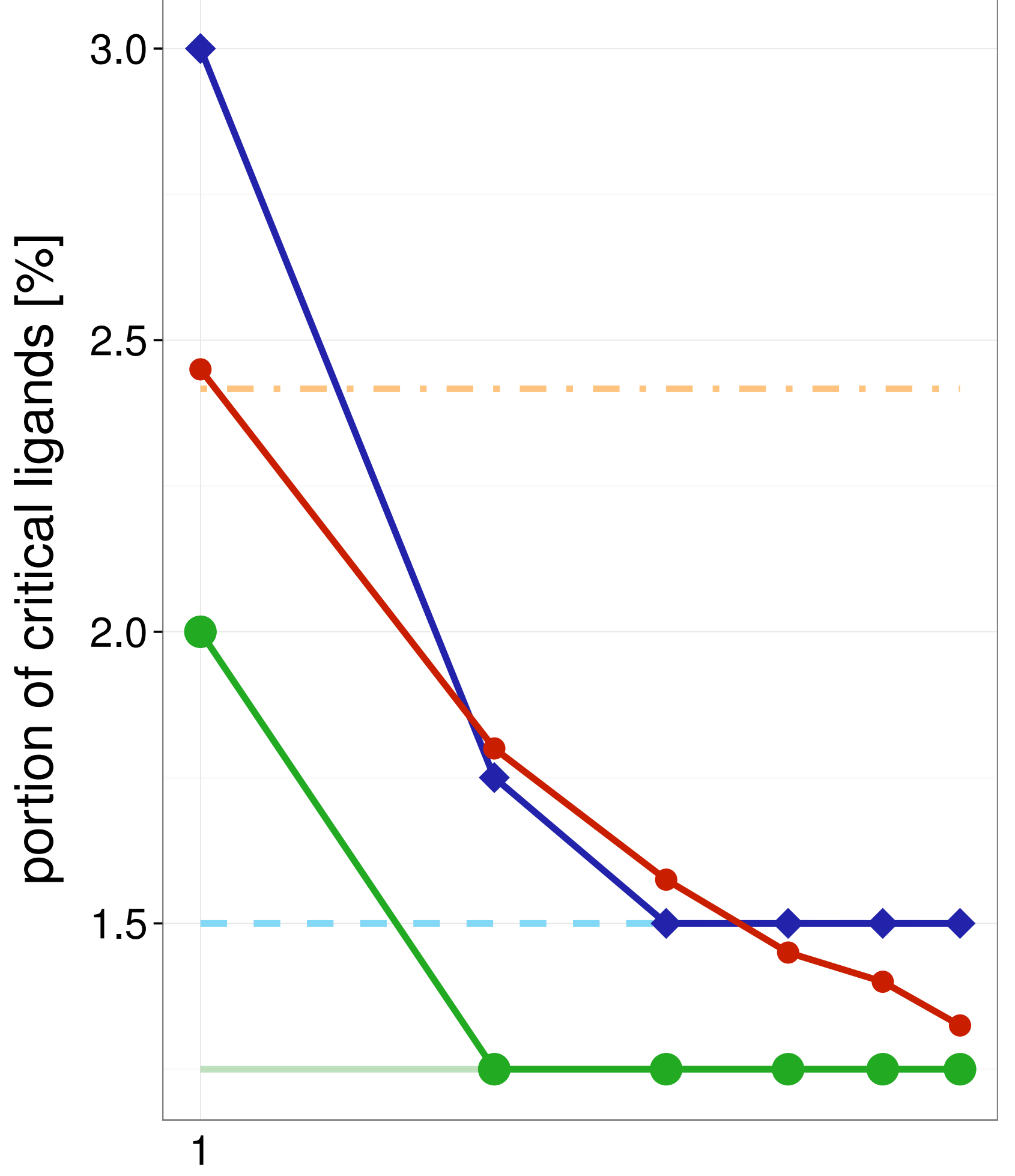

maximal ensemble size
Average single structure

_ Best single structure

Whole ensemble

SIENA

$\sim$ Clustering

$\multimap$ Random 
Training set

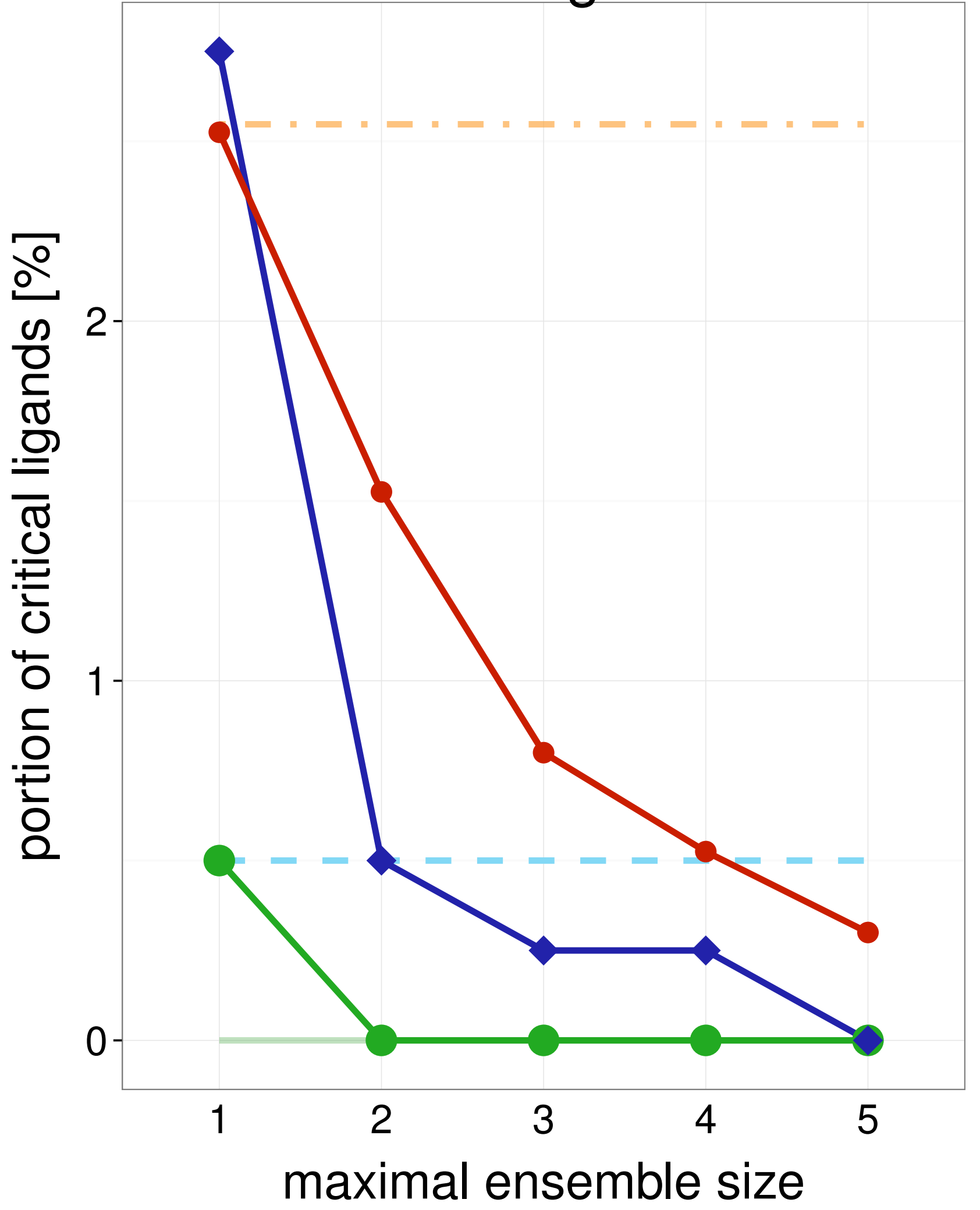

\section{Test set}

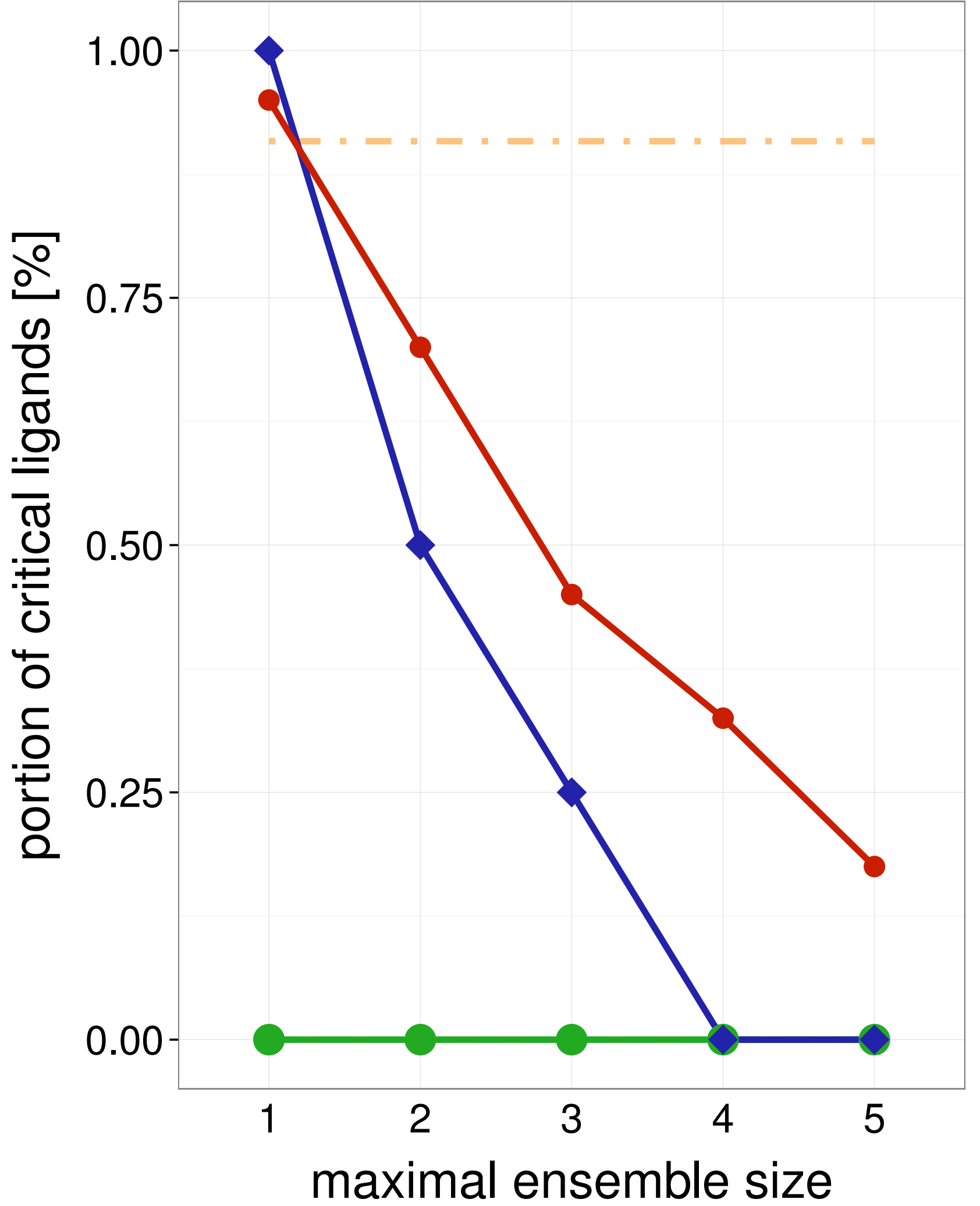

Average single structure

_ Best single structure

Whole ensemble

- SIENA

$\neg$ Clustering

$\multimap$ Random

Average single structure

_ _ Best single structure

Whole ensemble

- SIENA

$\neg$ Clustering

$\multimap$ Random
Training set

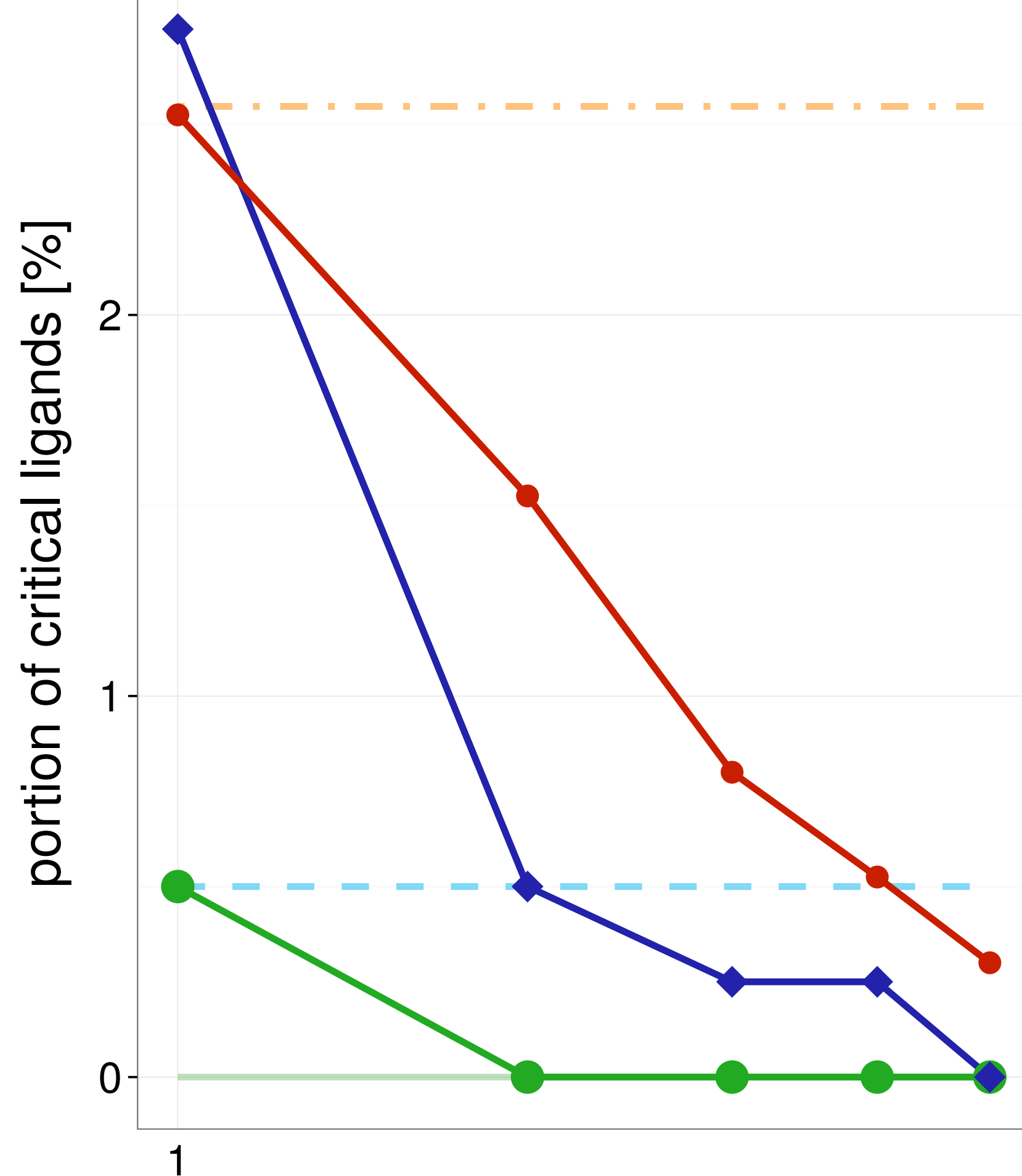

maximal ensemble size

Test set

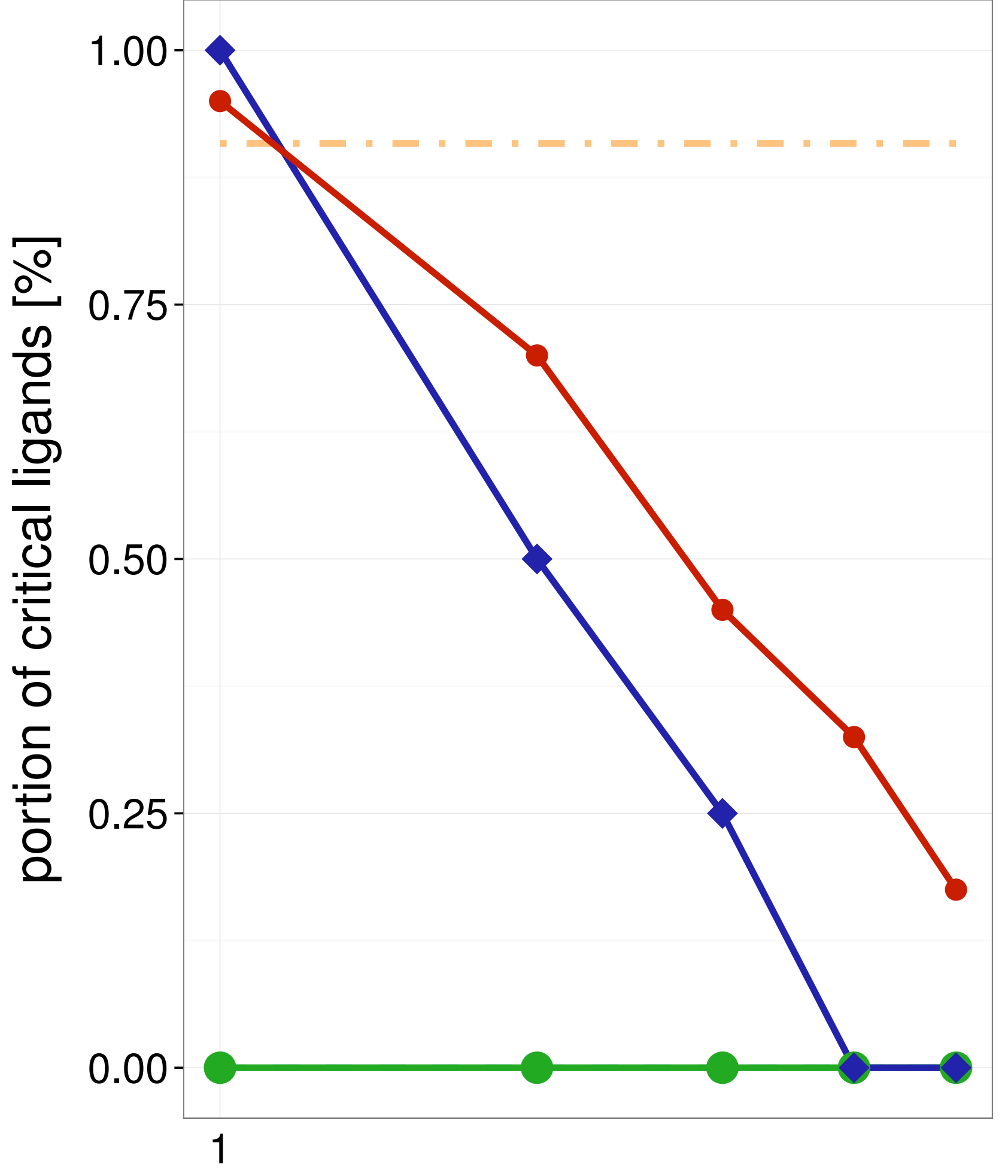

maximal ensemble size
Average single structure

_ Best single structure

Whole ensemble

SIENA

$\sim$ Clustering

$\multimap$ Random 
Training set

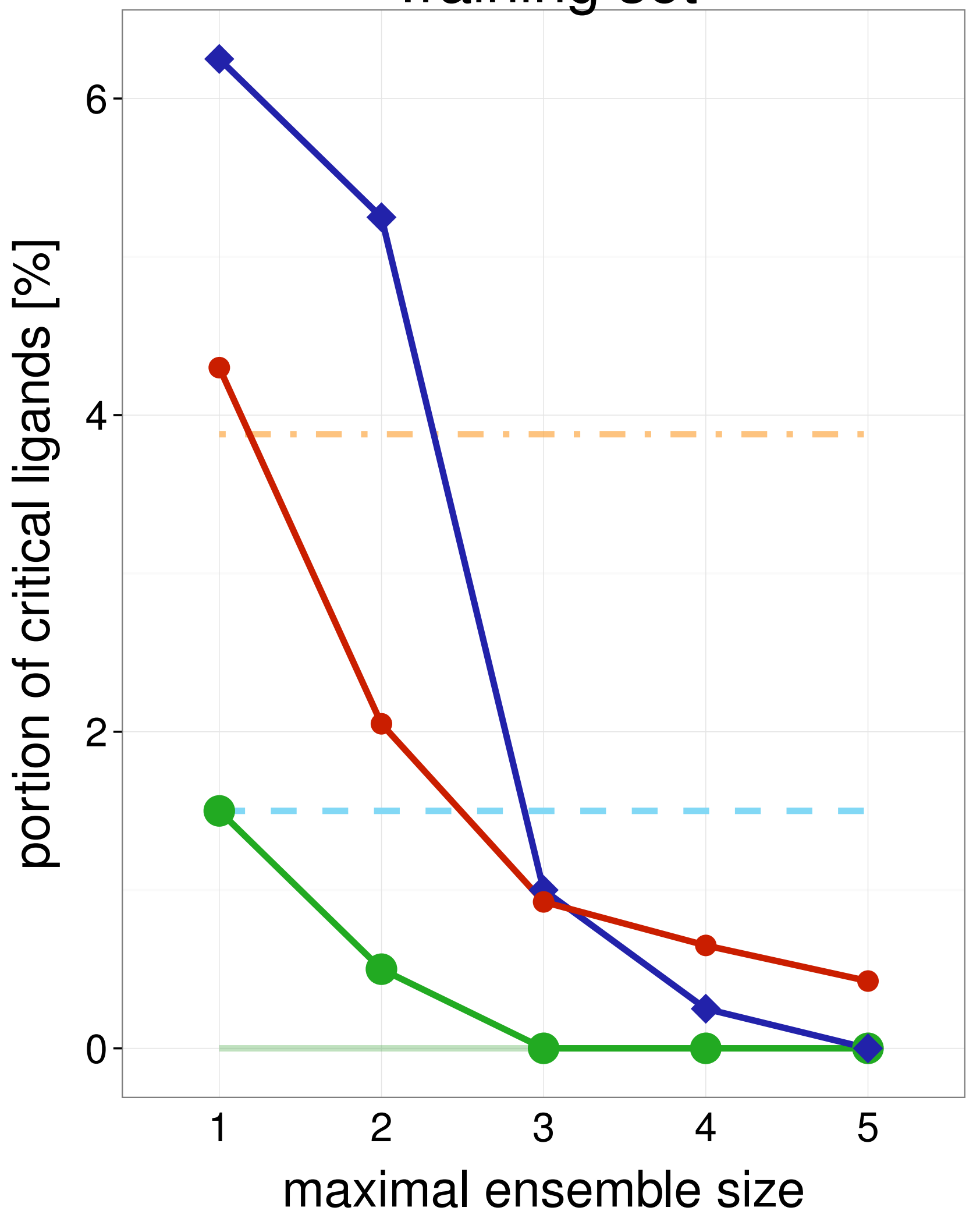

Test set

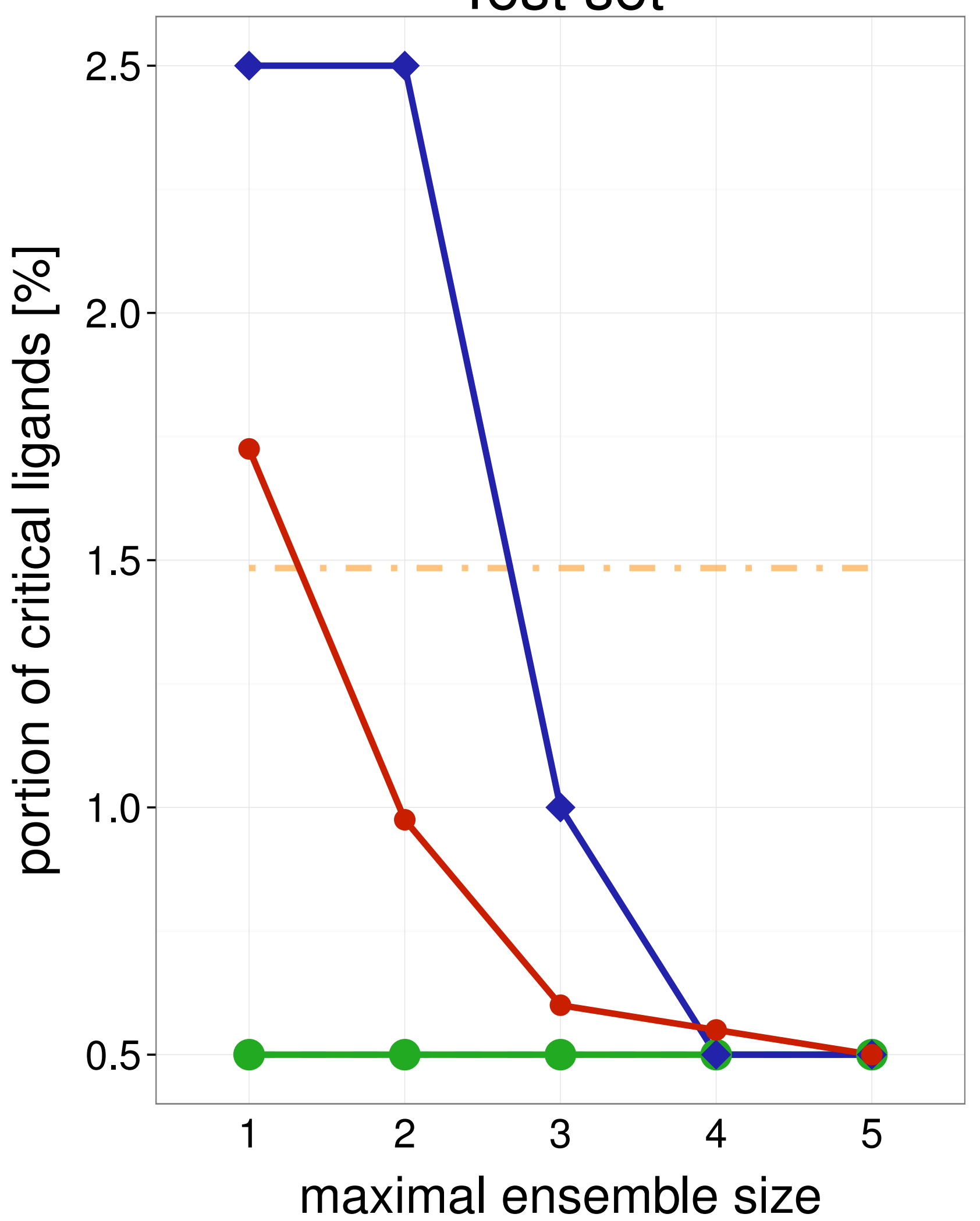

Average single structure

_ Best single structure

Whole ensemble

- SIENA

$\neg$ Clustering

$\rightarrow$ Random

Average single structure

_ _ Best single

structure

Whole ensemble

- SIENA

$\checkmark$ Clustering

$\because$ Random
Training set

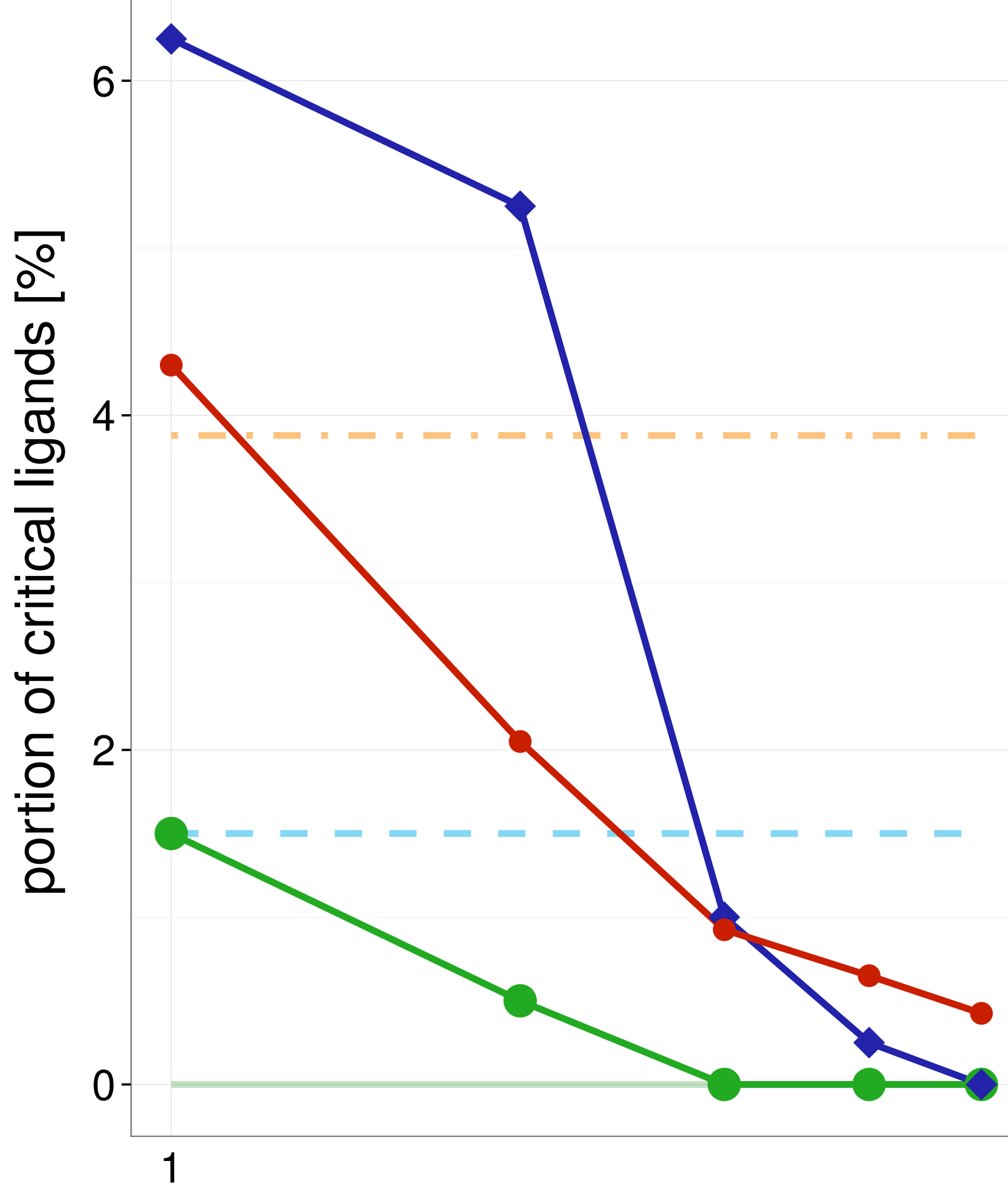

maximal ensemble size

\section{Test set}

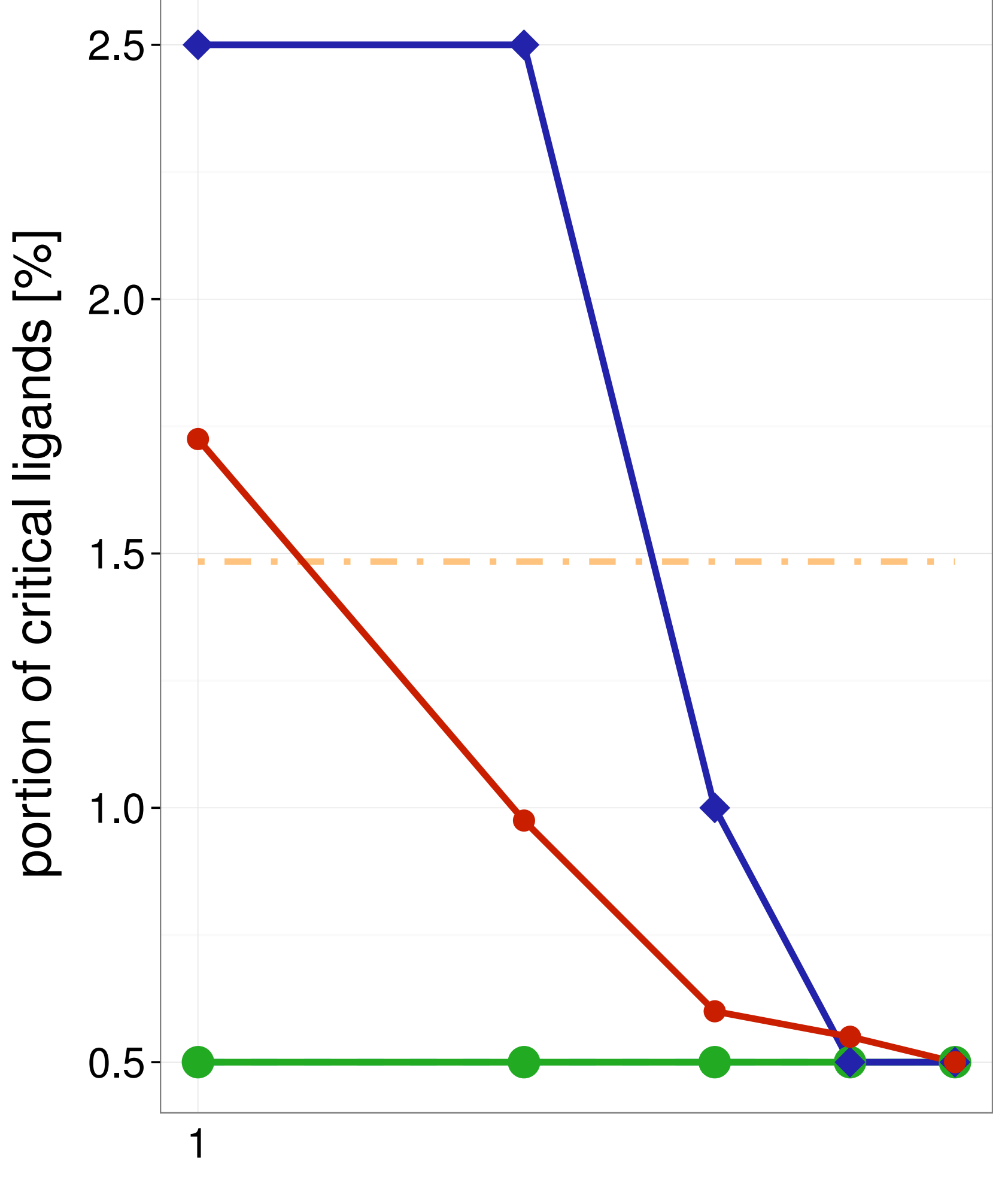

maximal ensemble size
Average single structure

_ Best single structure

Whole ensemble

SIENA

$\neg$ Clustering

$\multimap$ Random 
Training set

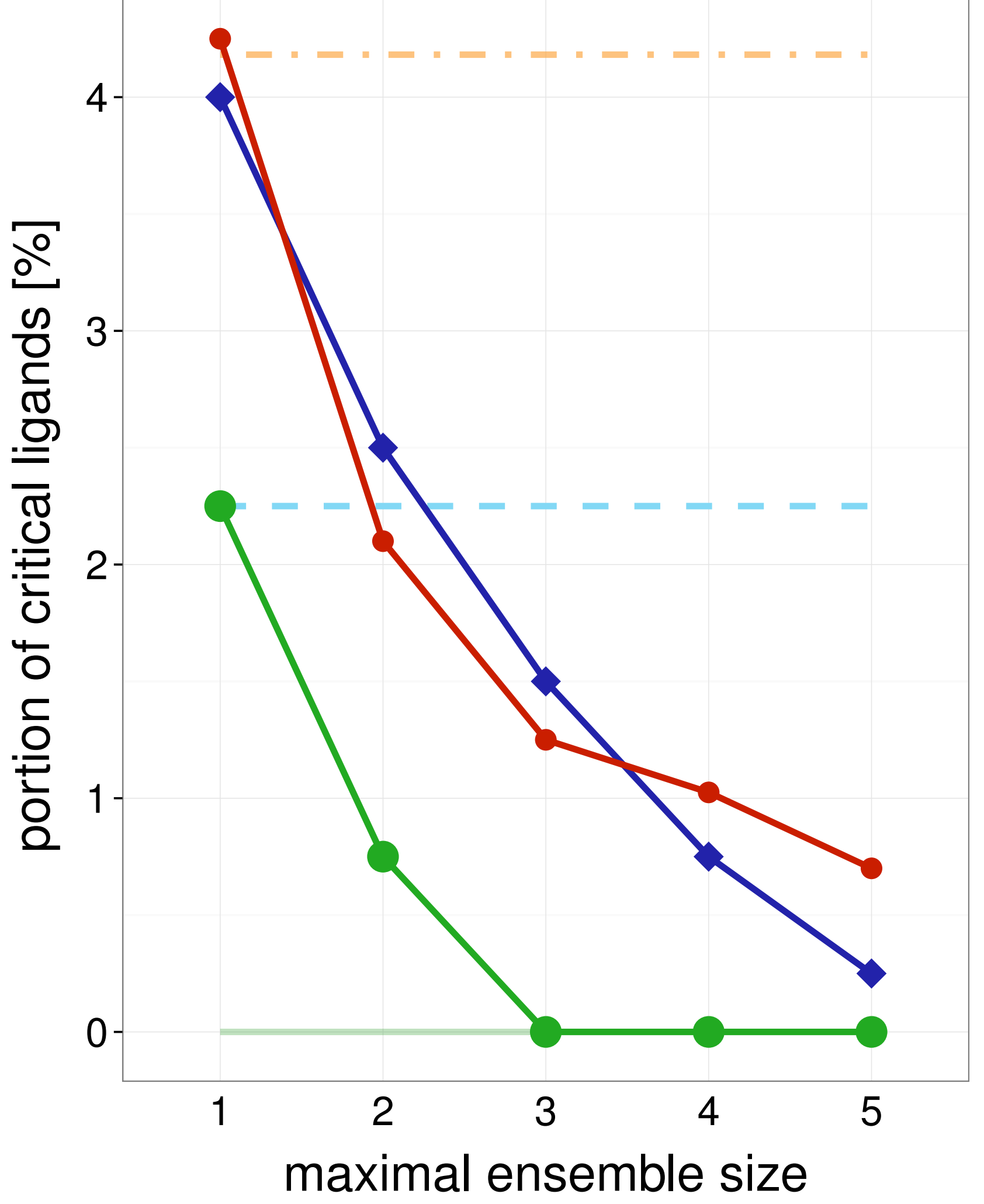

\section{Test set}

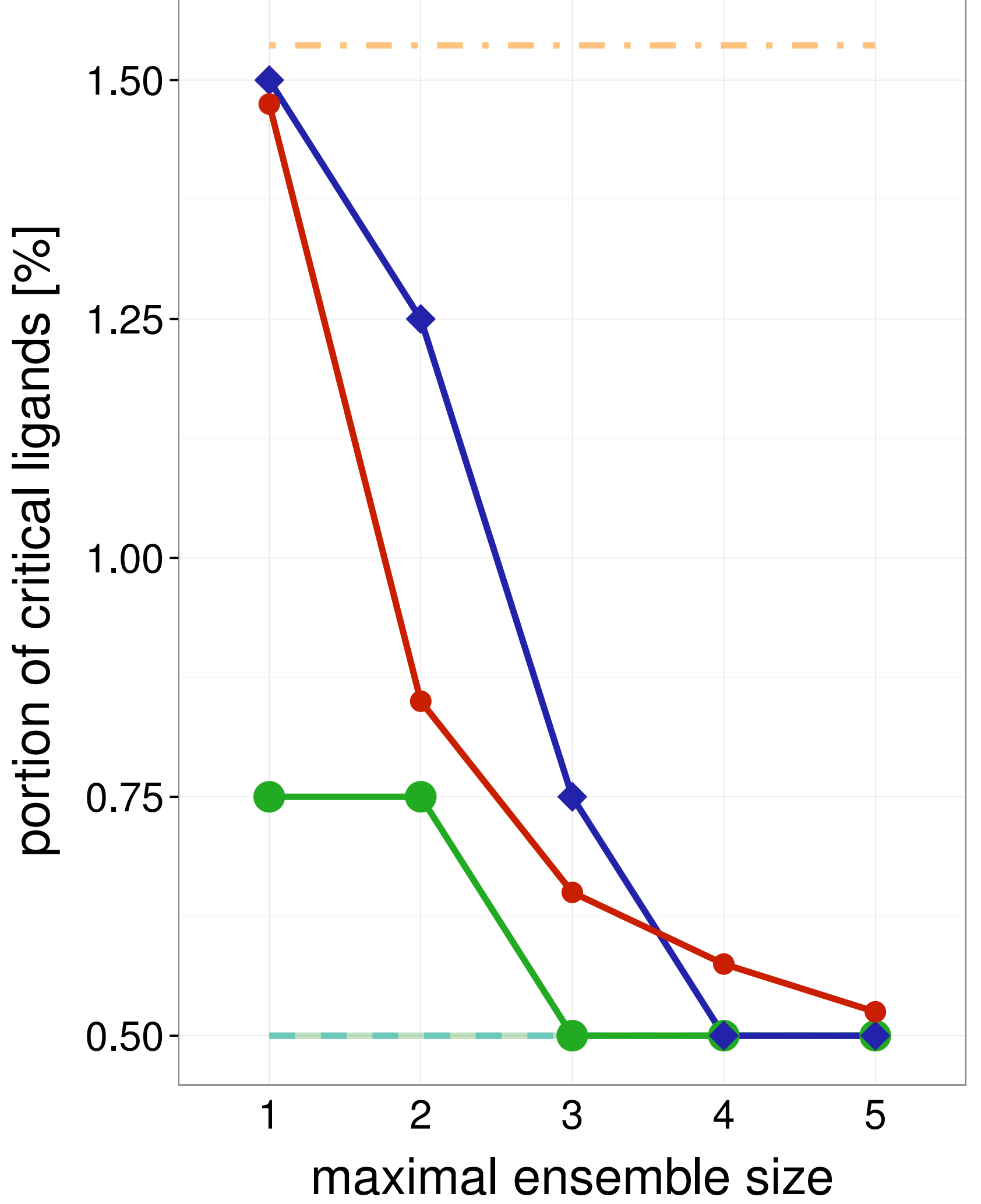

Training set

Average single structure

_ _ Best single structure

Whole ensemble

- SIENA

$\checkmark$ Clustering

$\longrightarrow$ Random

Average single structure

_ Best single structure

Whole ensemble

- SIENA

$\rightarrow$ Clustering

$\multimap$ Random

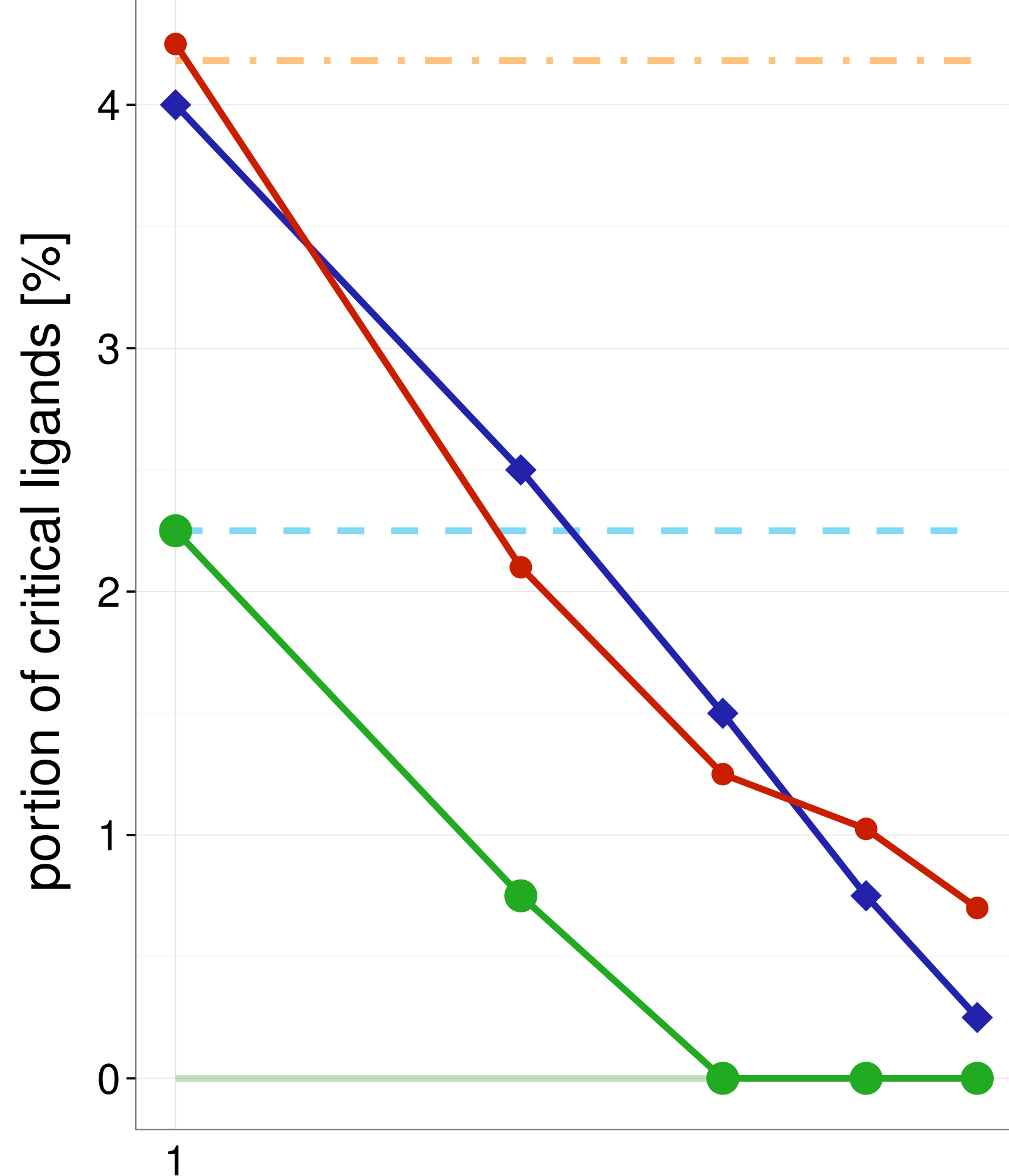

maximal ensemble size

Test set

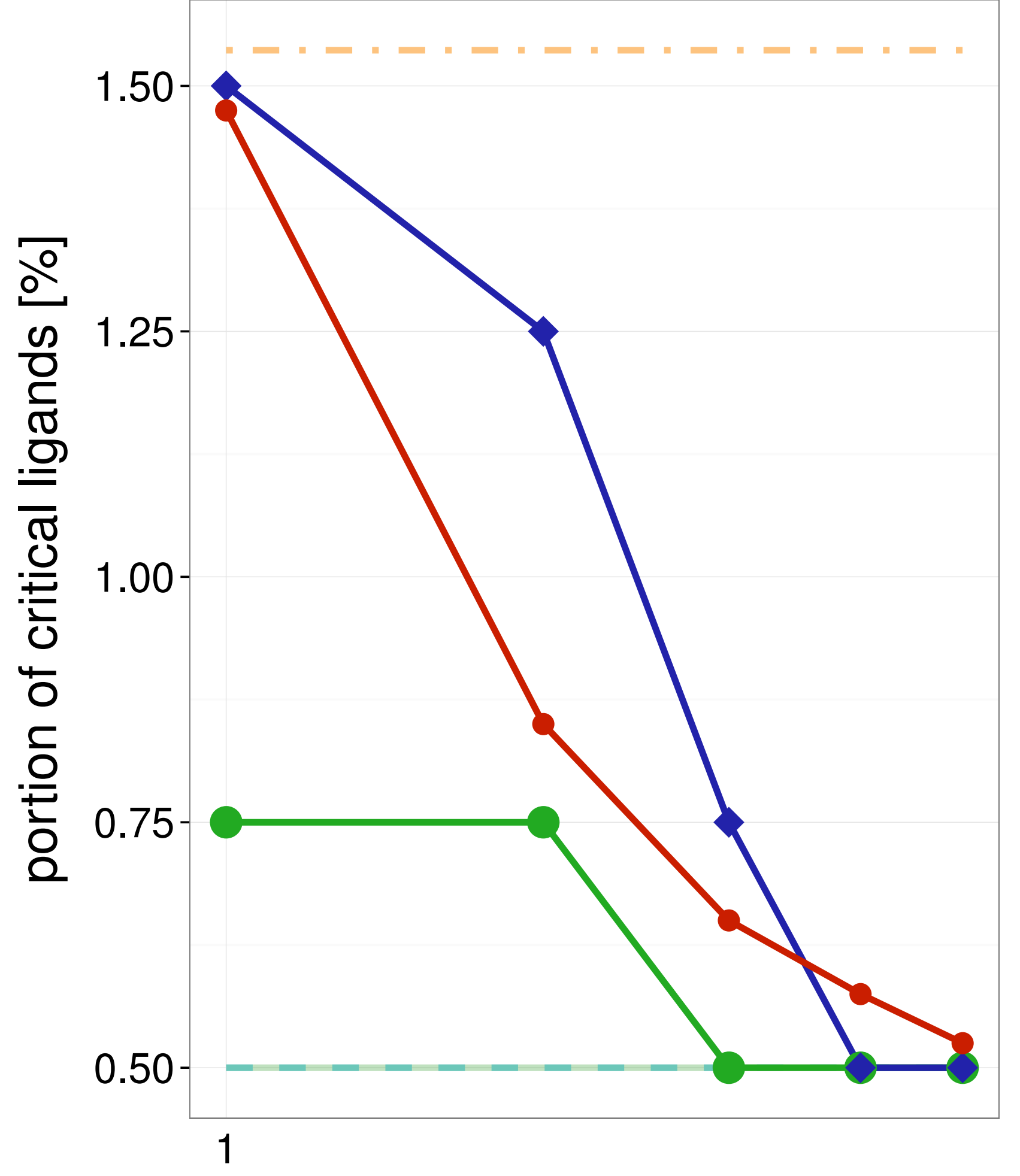

maximal ensemble size
Average single structure

_ Best single structure

Whole ensemble

SIENA

$\sim$ Clustering

$\longrightarrow$ Random 
Training set

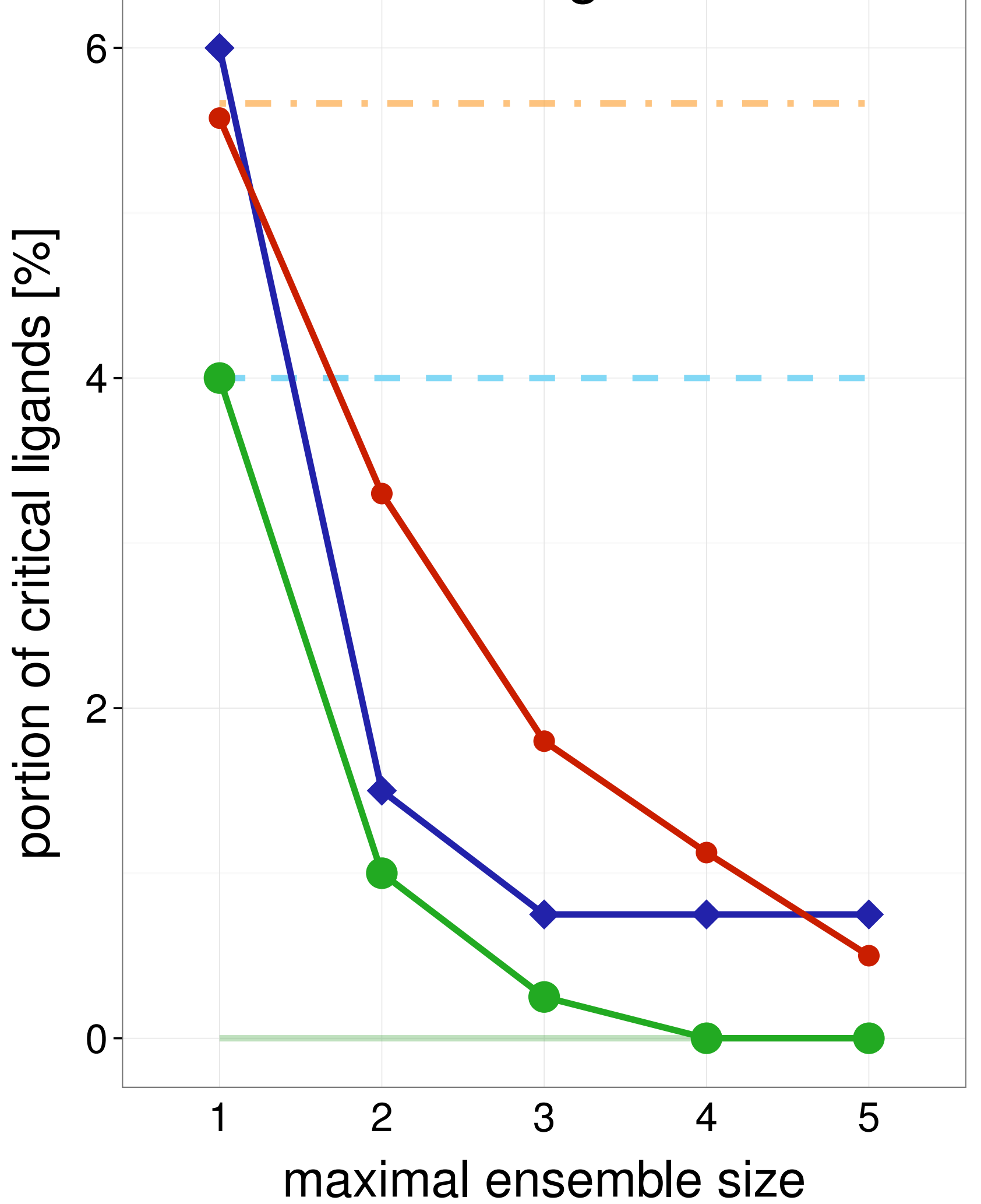

Test set

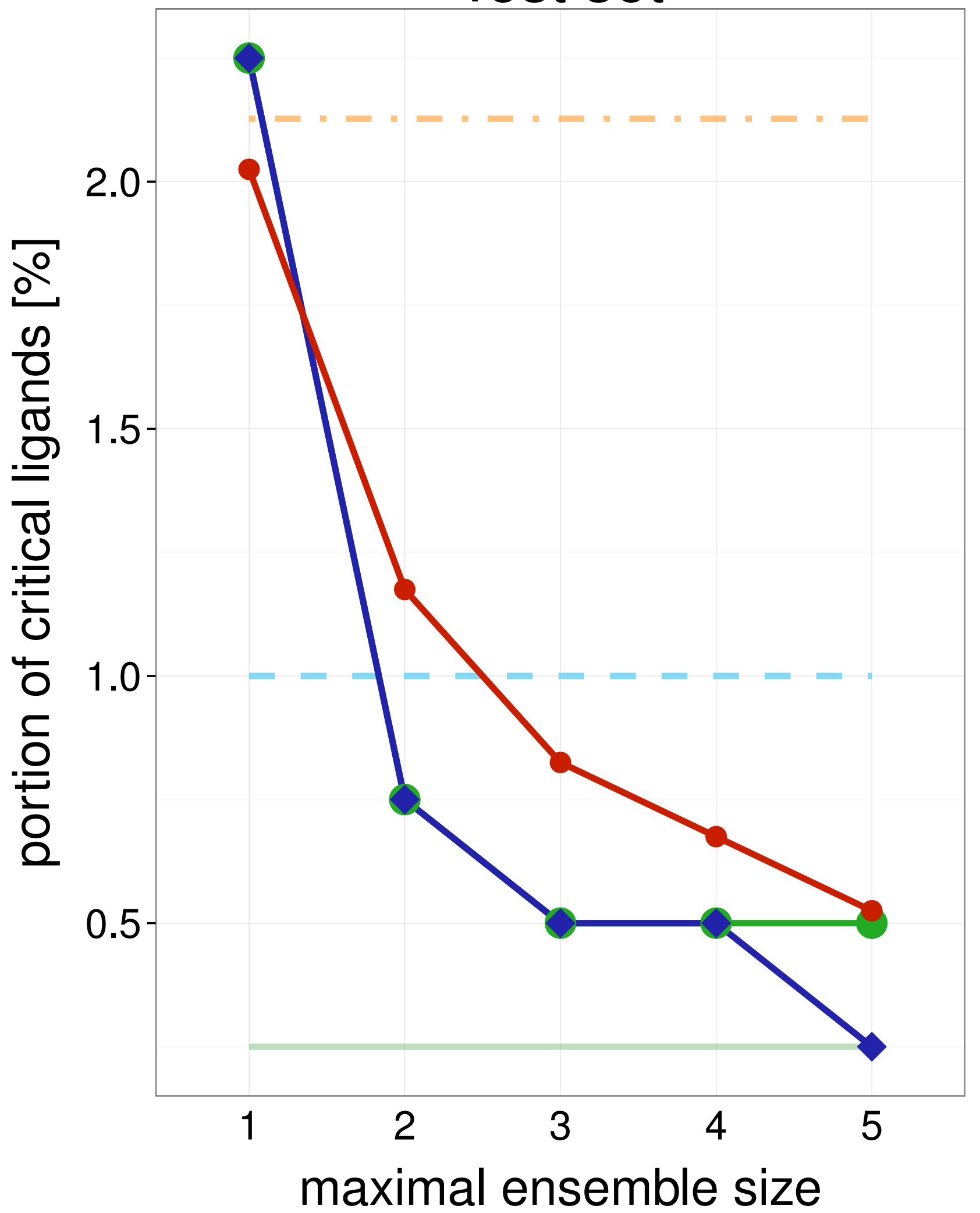

Average single structure

_ Best single structure

Whole ensemble

- SIENA

$\neg$ Clustering

$\multimap$ Random

Average single structure

_ Best single structure

Whole ensemble

- SIENA

$\neg$ Clustering

- Random
Training set

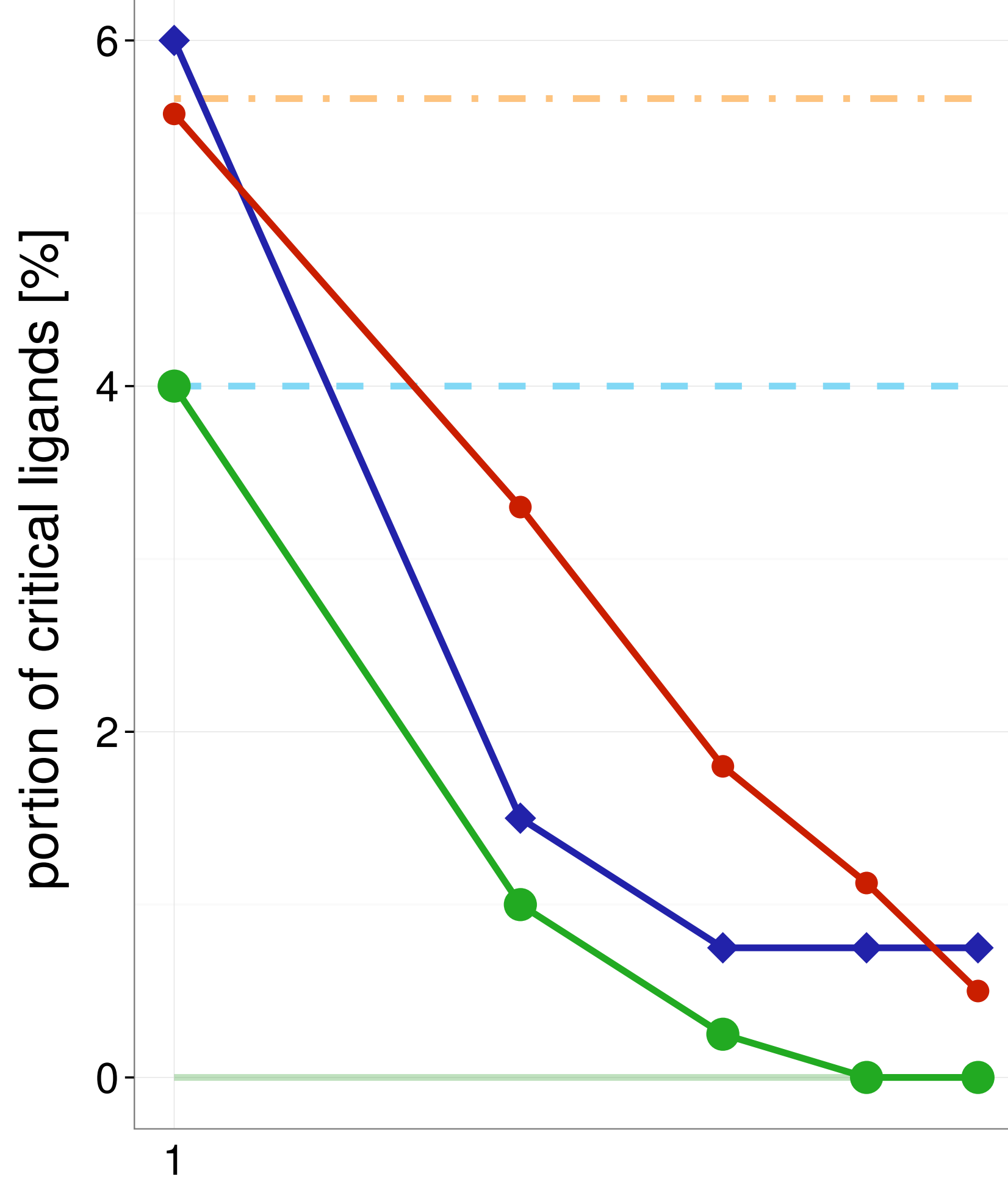

maximal ensemble size

Test set

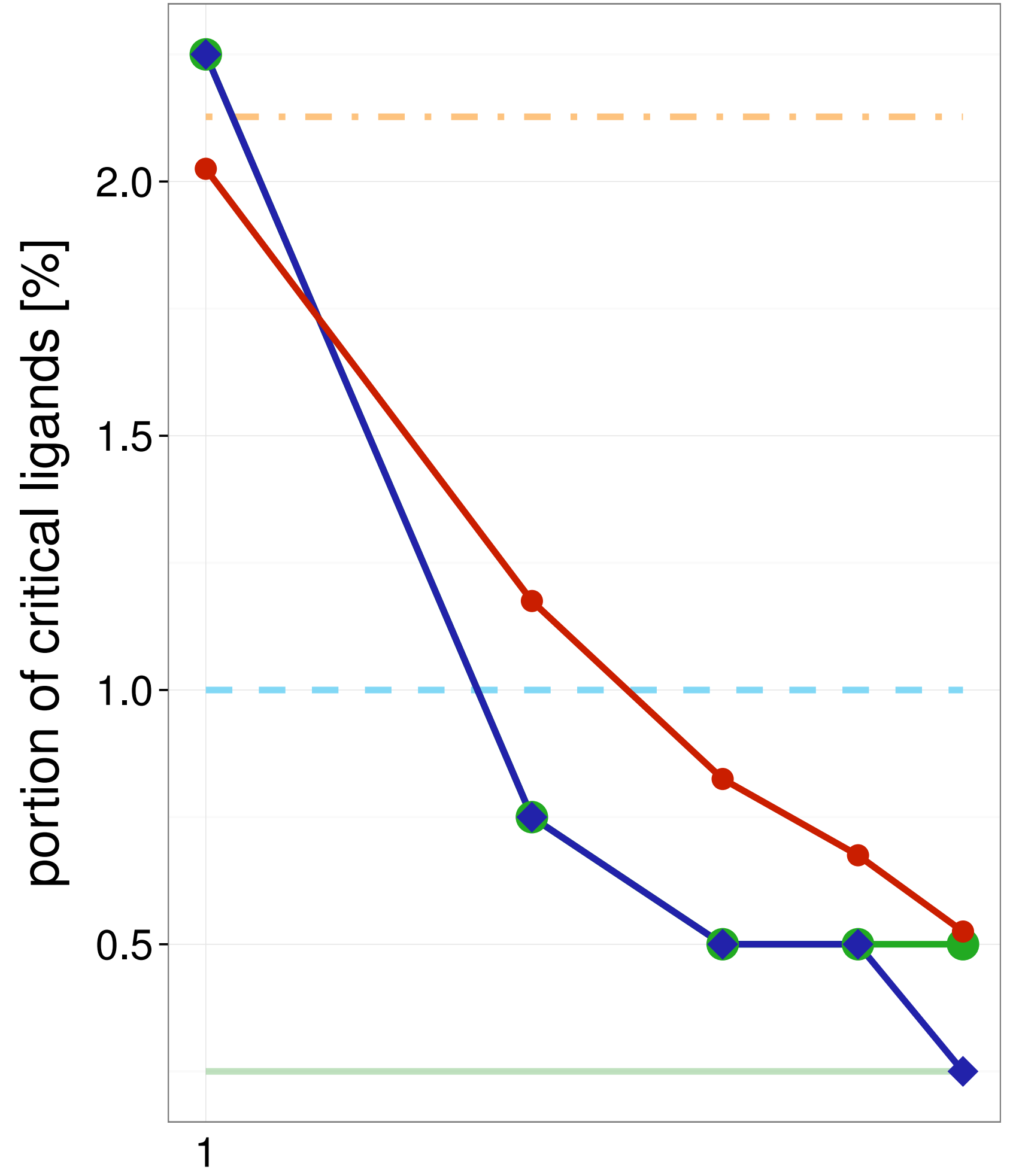

Average single structure

_ Best single structure

Whole ensemble

SIENA

$\leadsto$ Clustering

$\multimap$ Random 
Training set

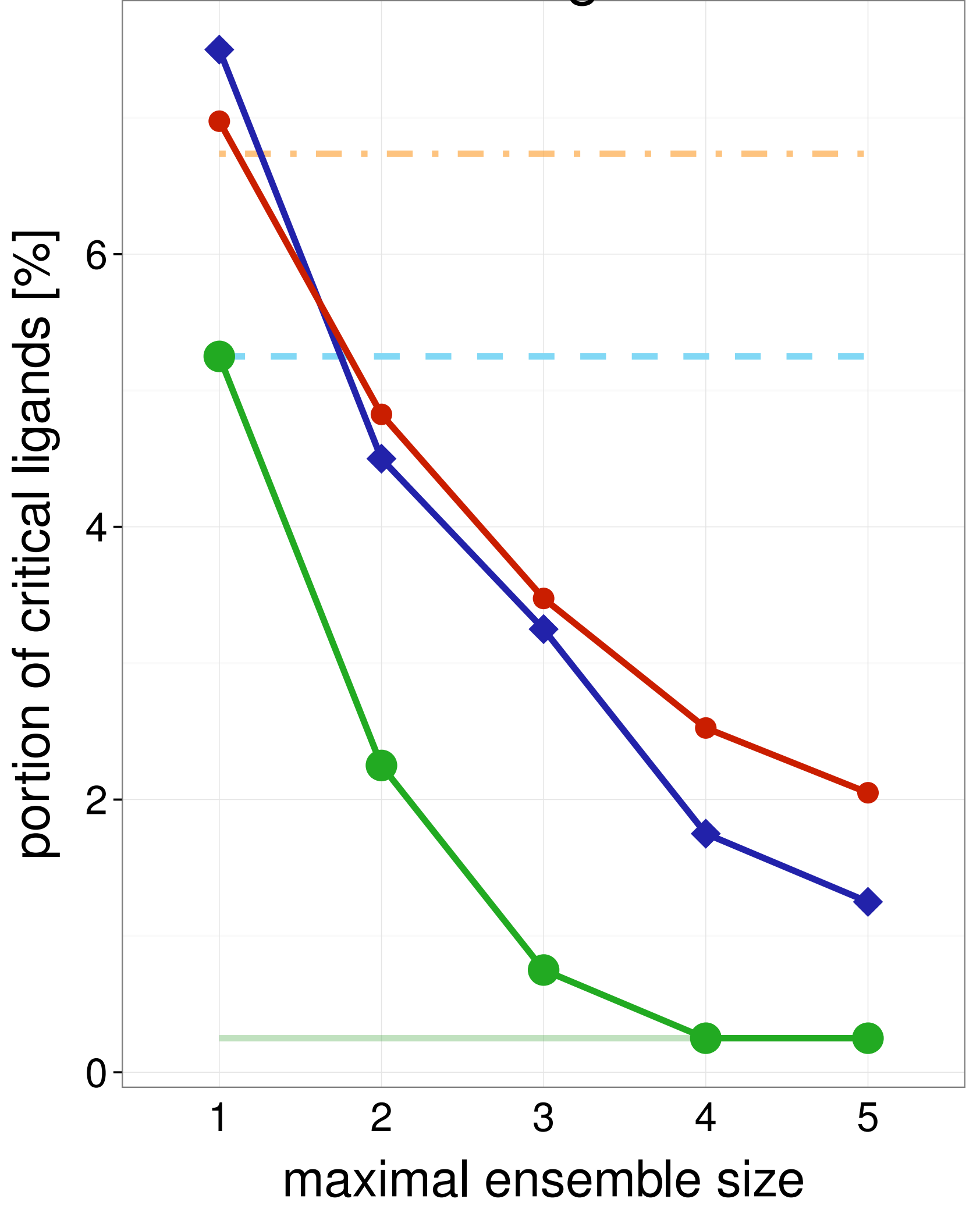

\section{Test set}

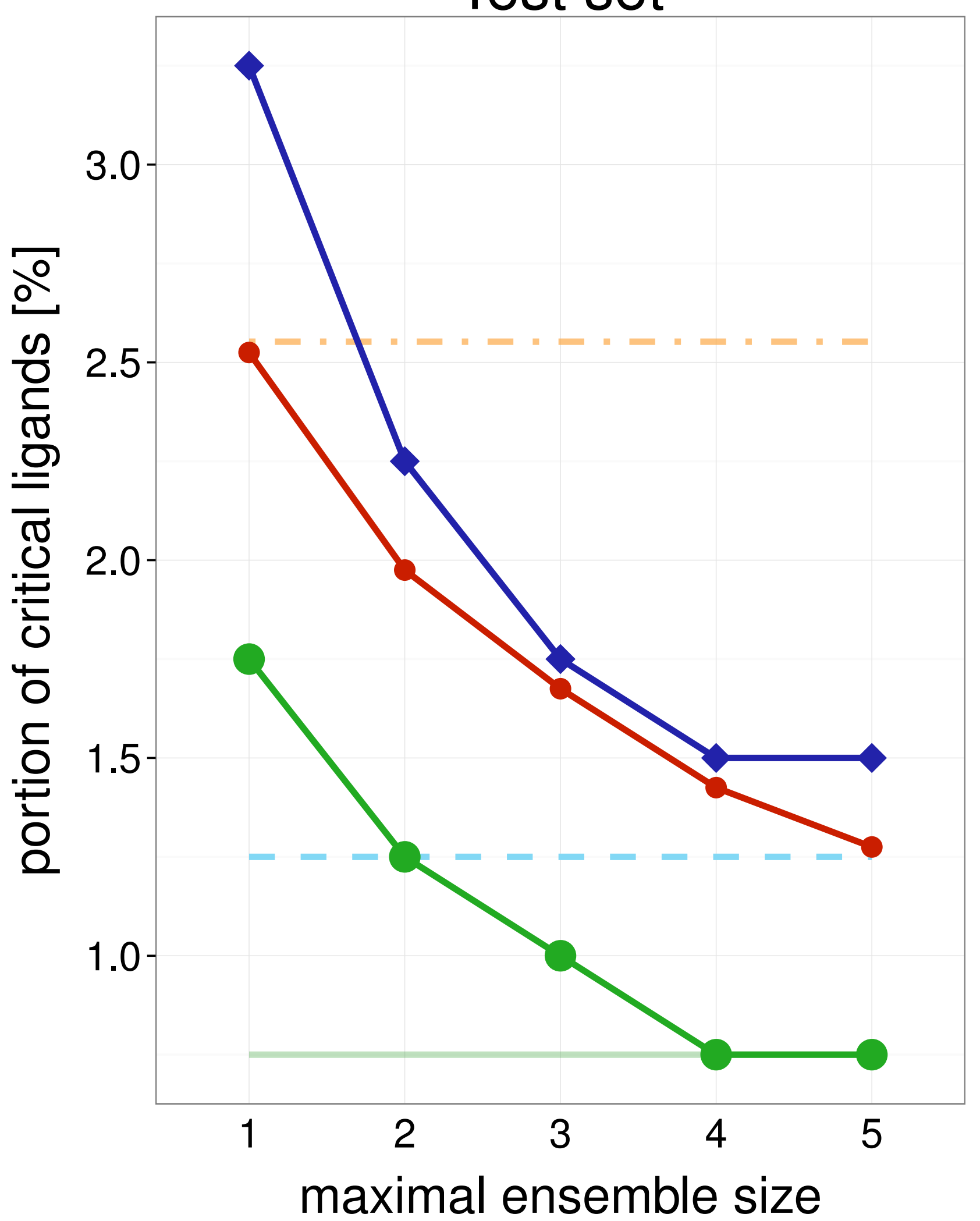

1061

Training set

Average single structure

_ _ Best single structure

Whole ensemble

$\multimap$ SIENA

$\neg$ Clustering

$\longrightarrow$ Random

Average single structure

_ Best single structure

Whole ensemble

- SIENA

$\leadsto$ Clustering

$\multimap$ Random

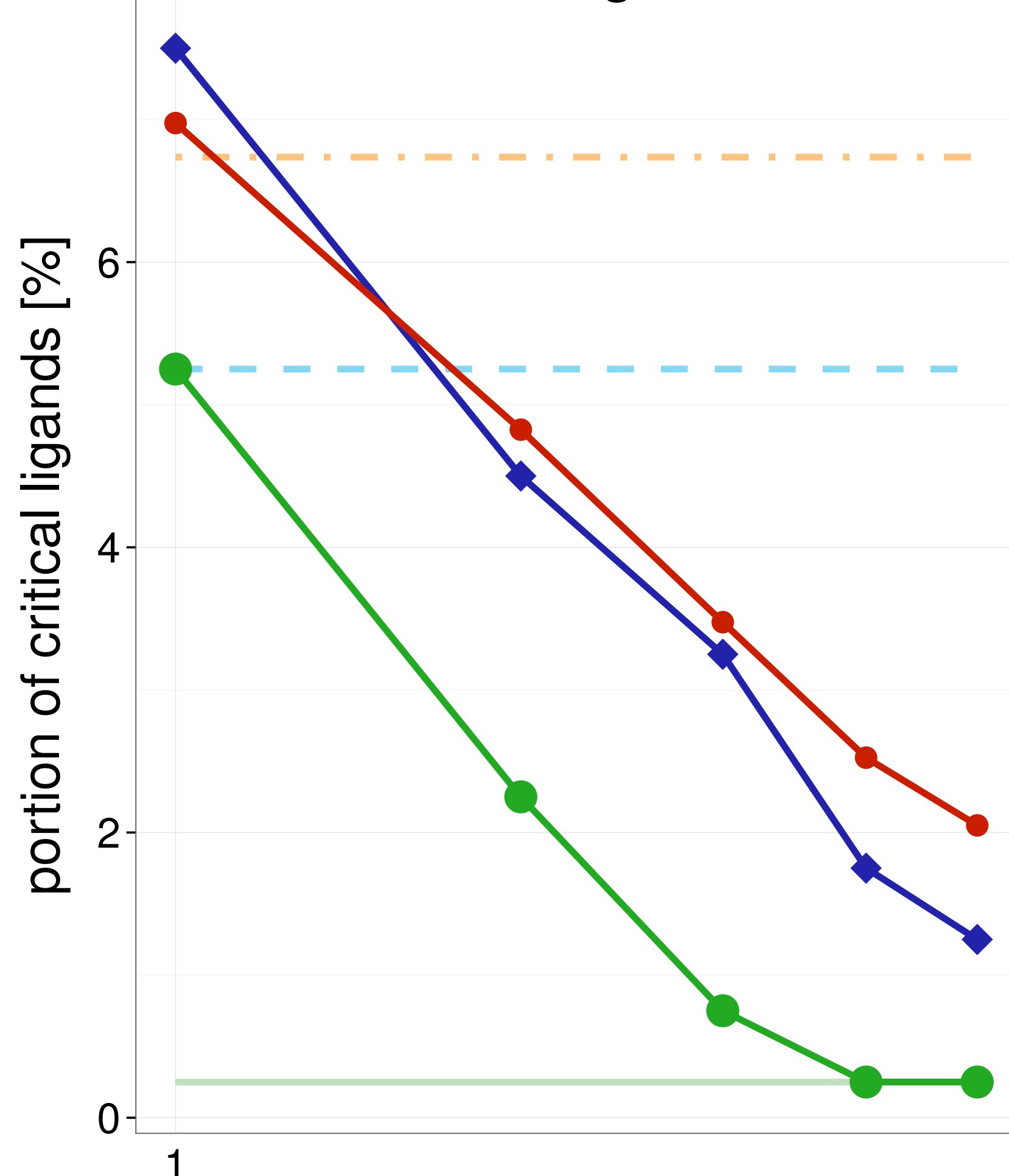

maximal ensemble size

Test set

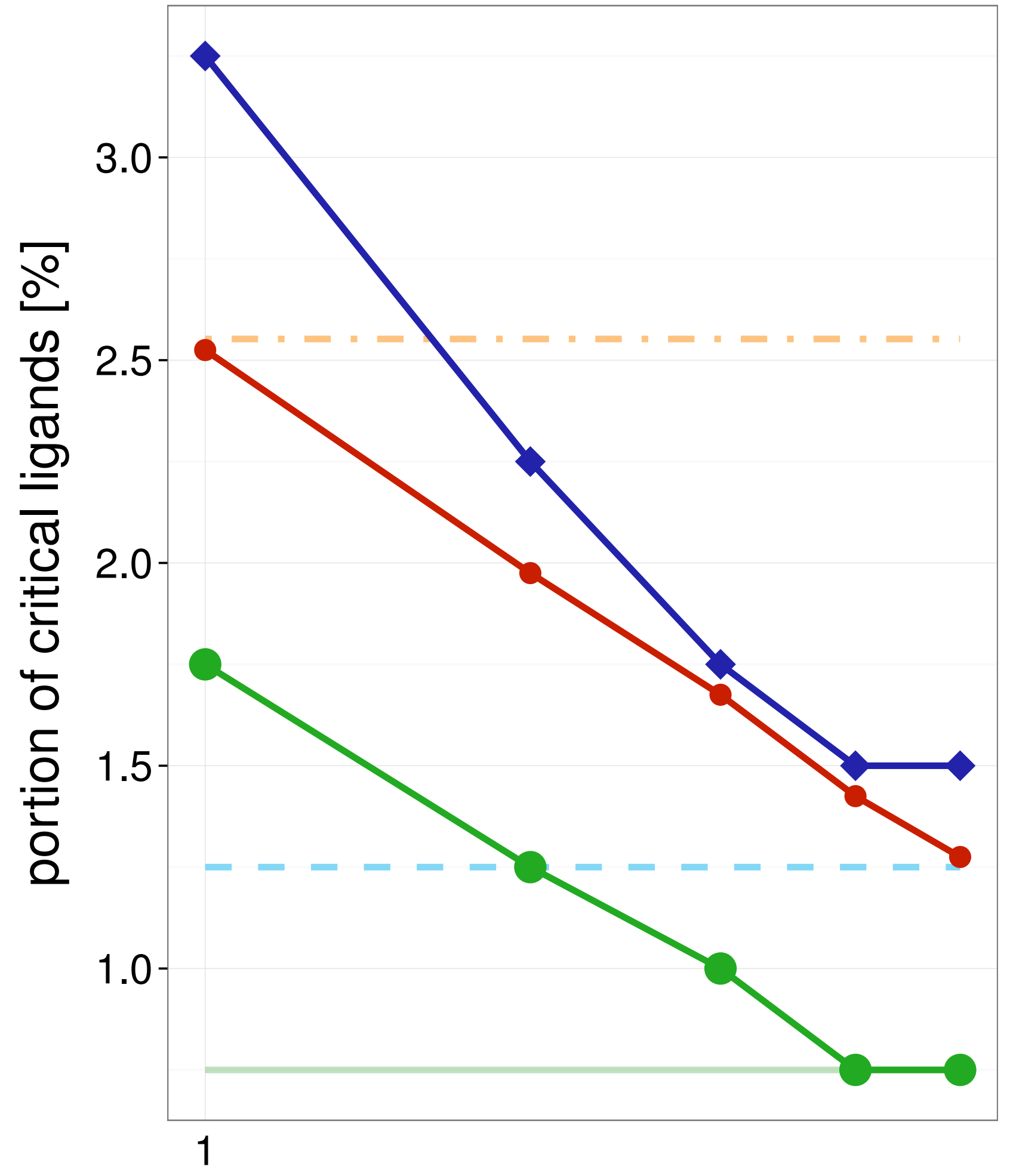

maximal ensemble size
Average single structure

_ Best single structure

Whole ensemble

SIENA

$\neg$ Clustering

$\multimap$ Random 


\section{Training set}
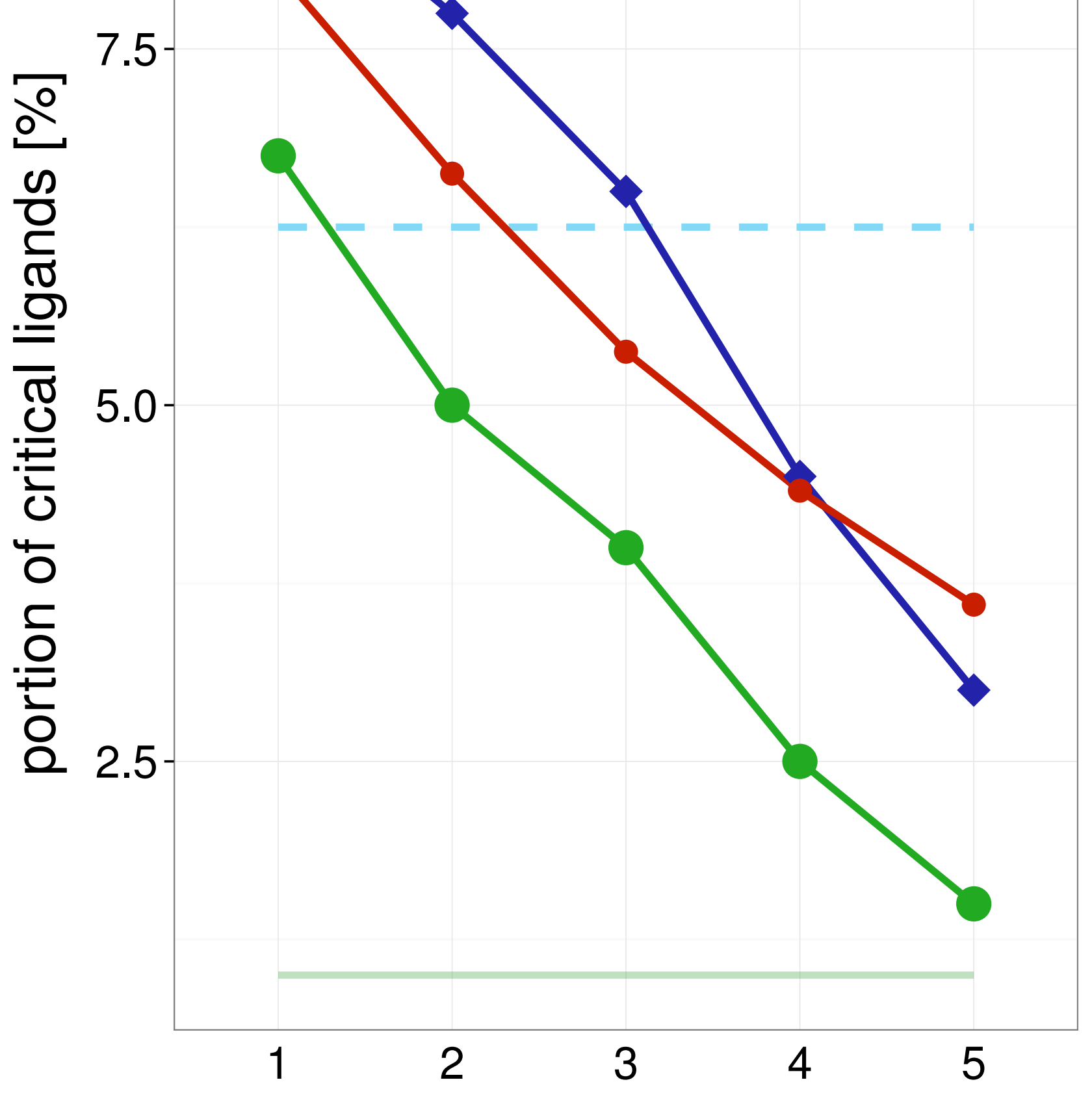

maximal ensemble size

Test set

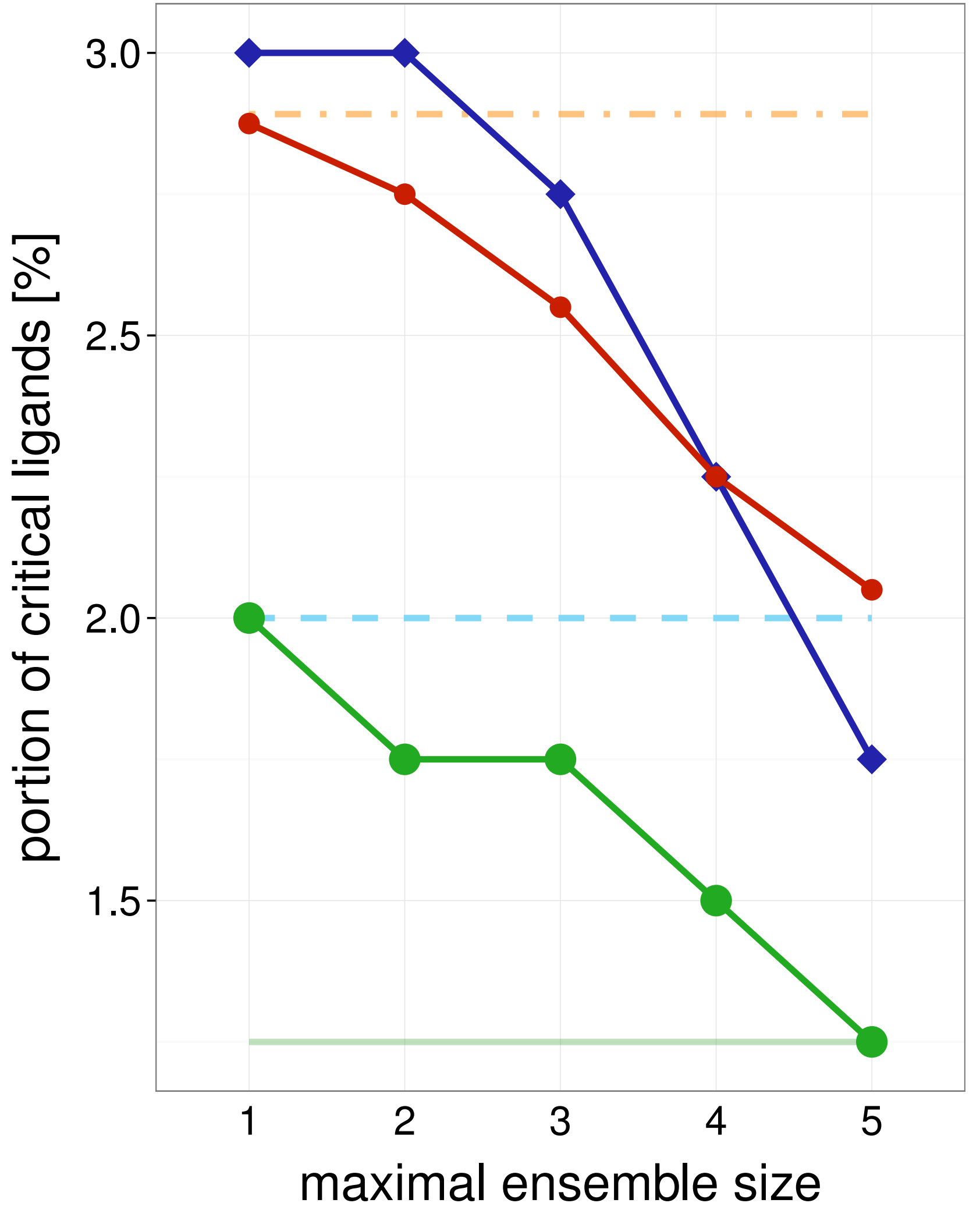

1RD4

Training set

Average single structure

_ Best single structure

Whole ensemble

- SIENA

$\neg$ Clustering

$\multimap$ Random

Average single structure

_ Best single structure

Whole ensemble

- SIENA

$\neg$ Clustering

$\multimap$ Random

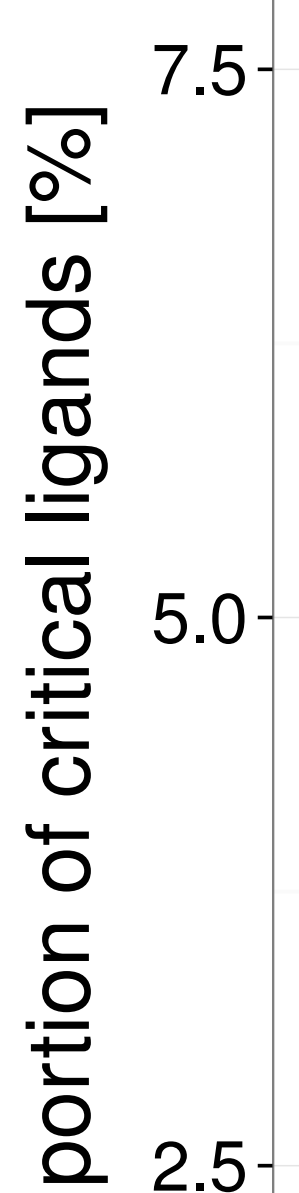$$
\text { . }
$$<smiles>Ic1ccccc1</smiles>

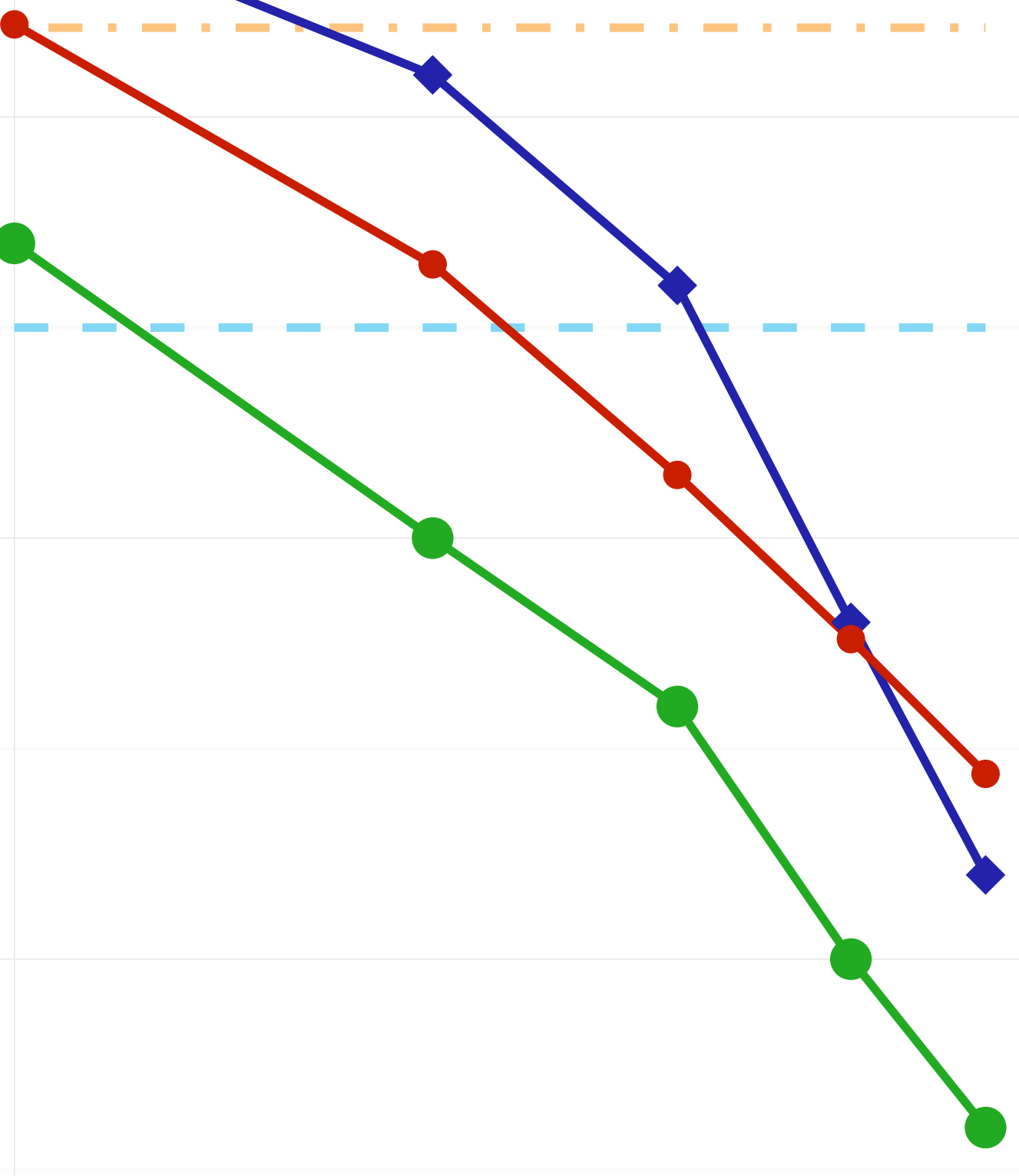

maximal ensemble size

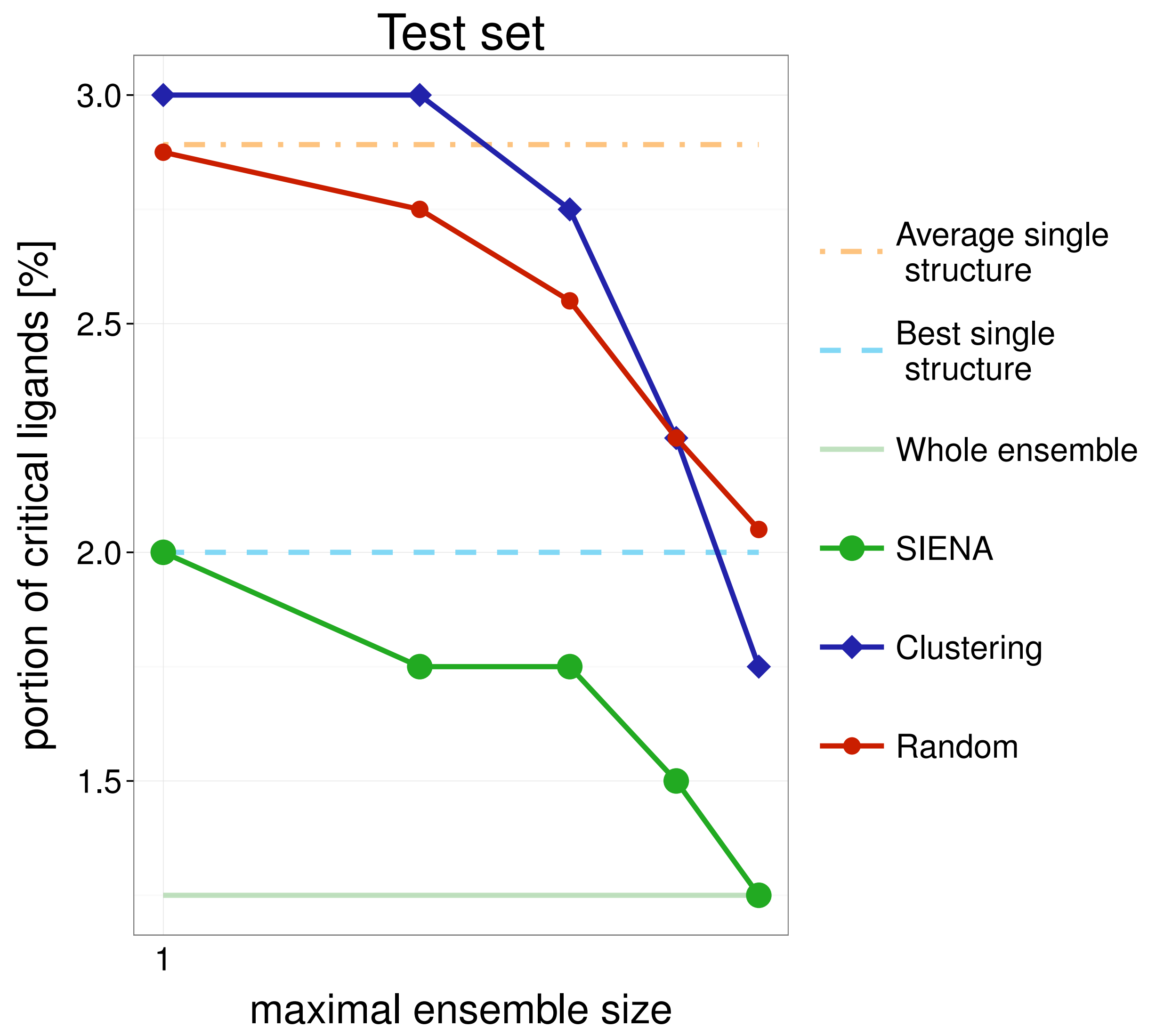




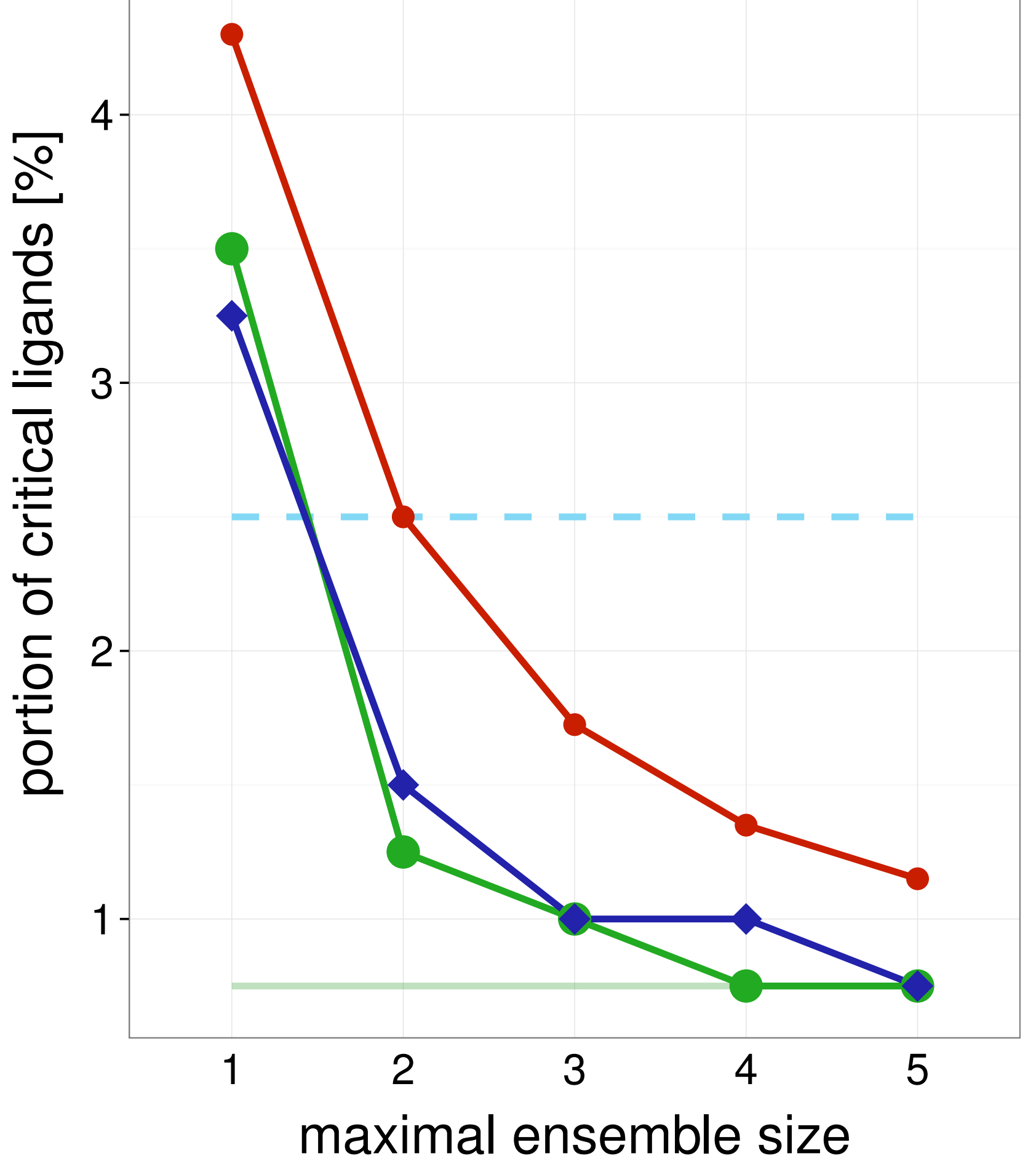

Test set

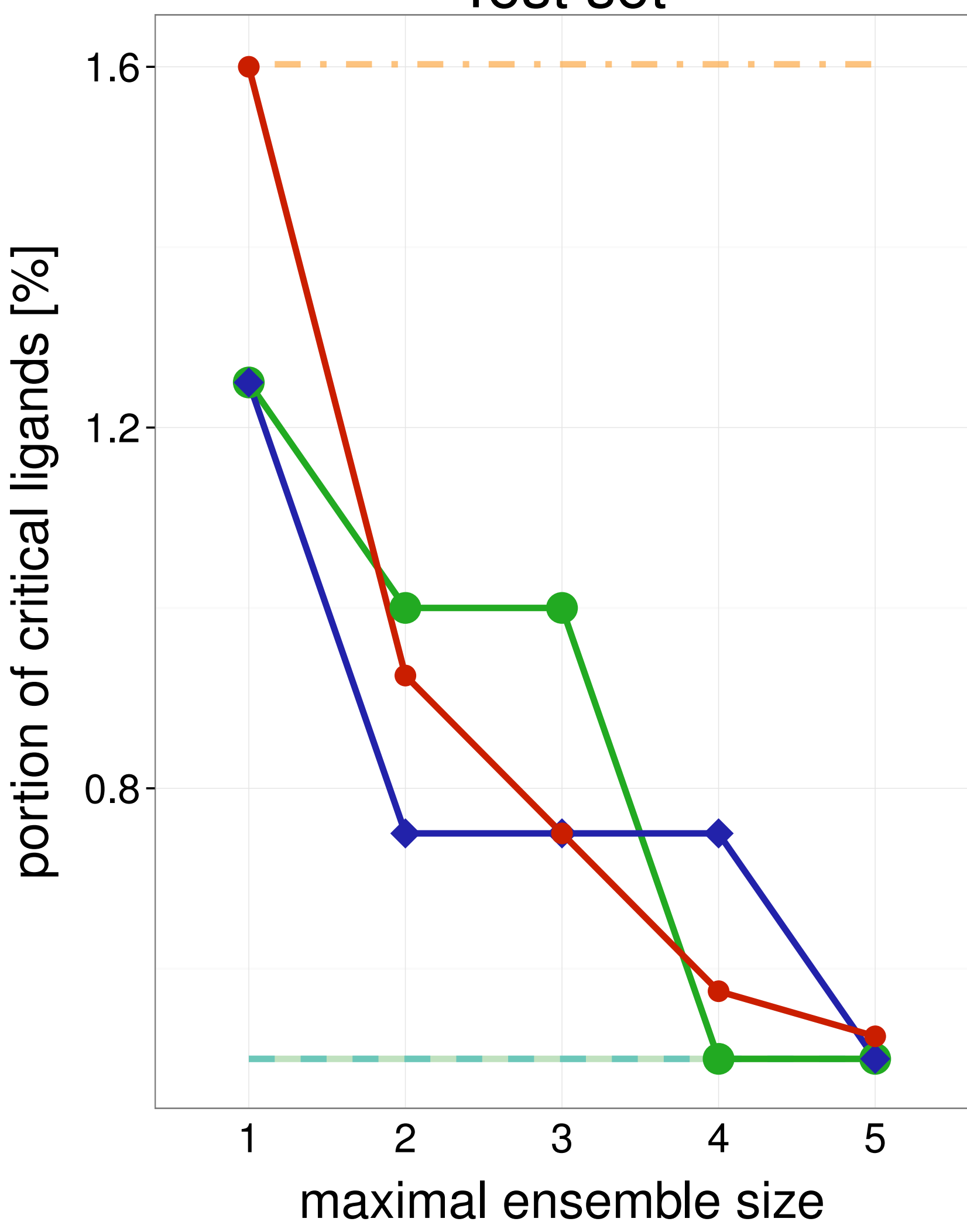

Average single structure

_ Best single structure

Whole ensemble

- SIENA

$\neg$ Clustering

$\multimap$ Random

Average single structure

_ Best single structure

Whole ensemble

- SIENA

$\neg$ Clustering

$\multimap$ Random

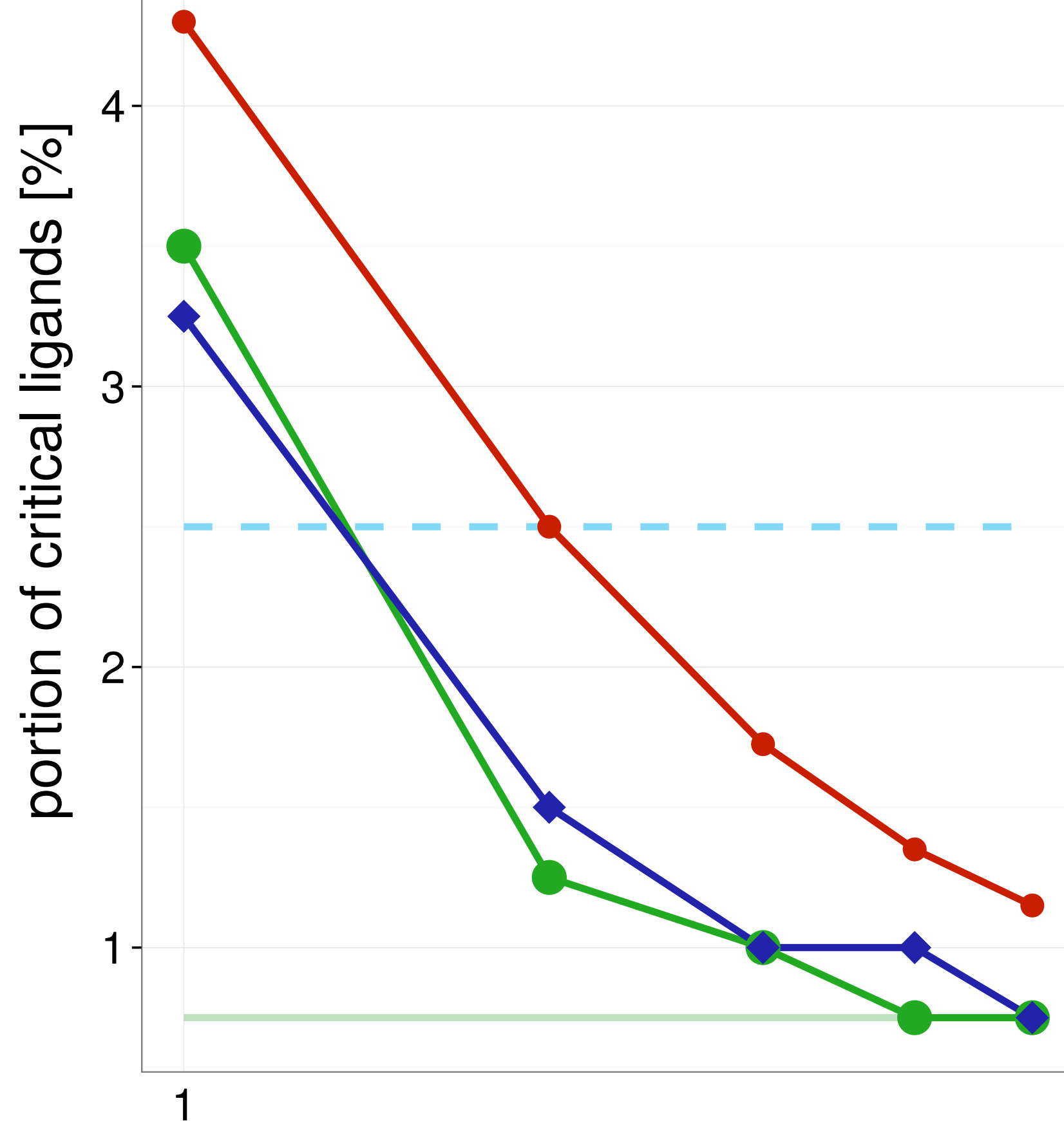

maximal ensemble size

Test set

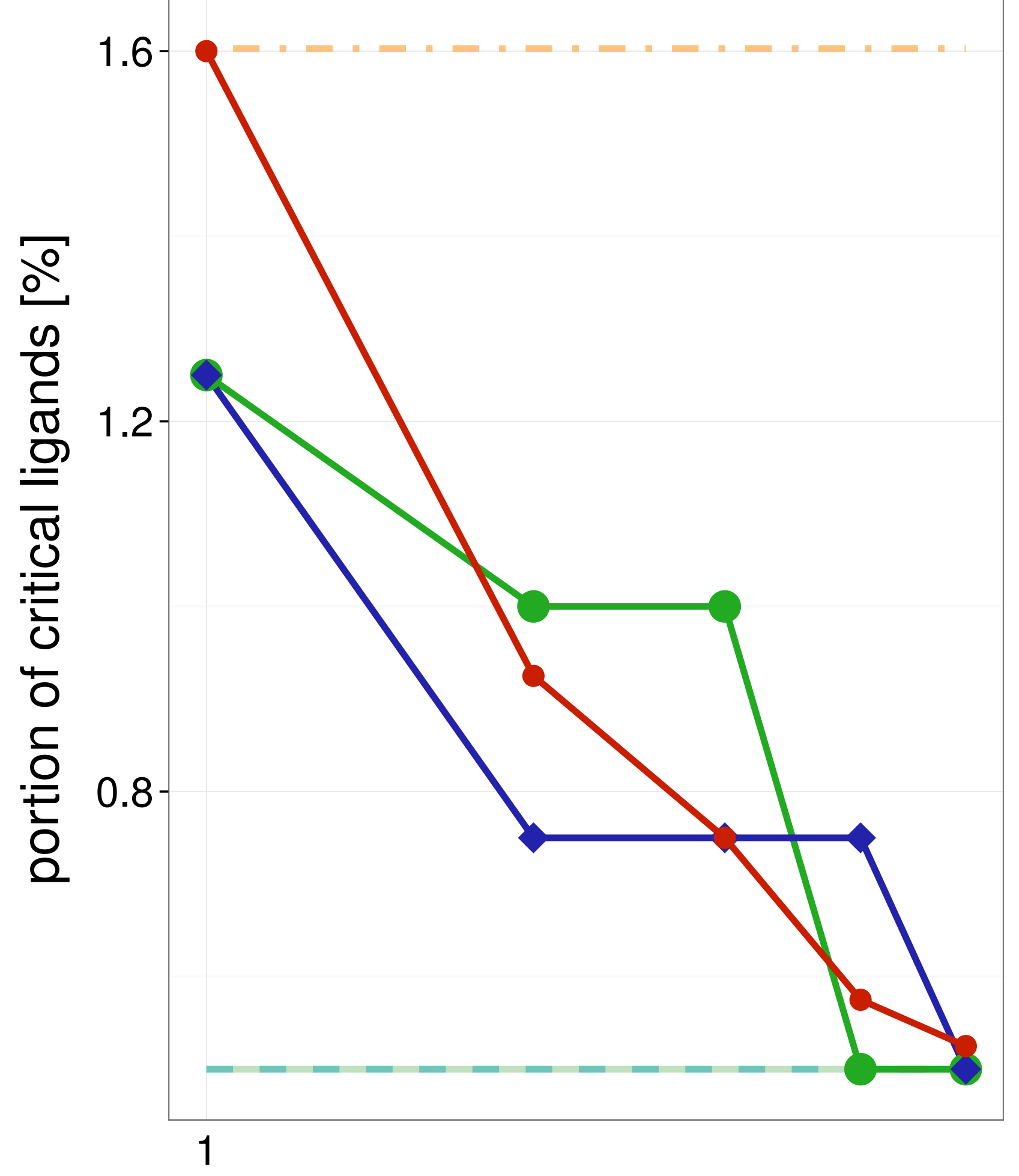

maximal ensemble size
Average single structure

_ Best single structure

Whole ensemble

SIENA

$\neg$ Clustering

$\multimap$ Random 
Training set

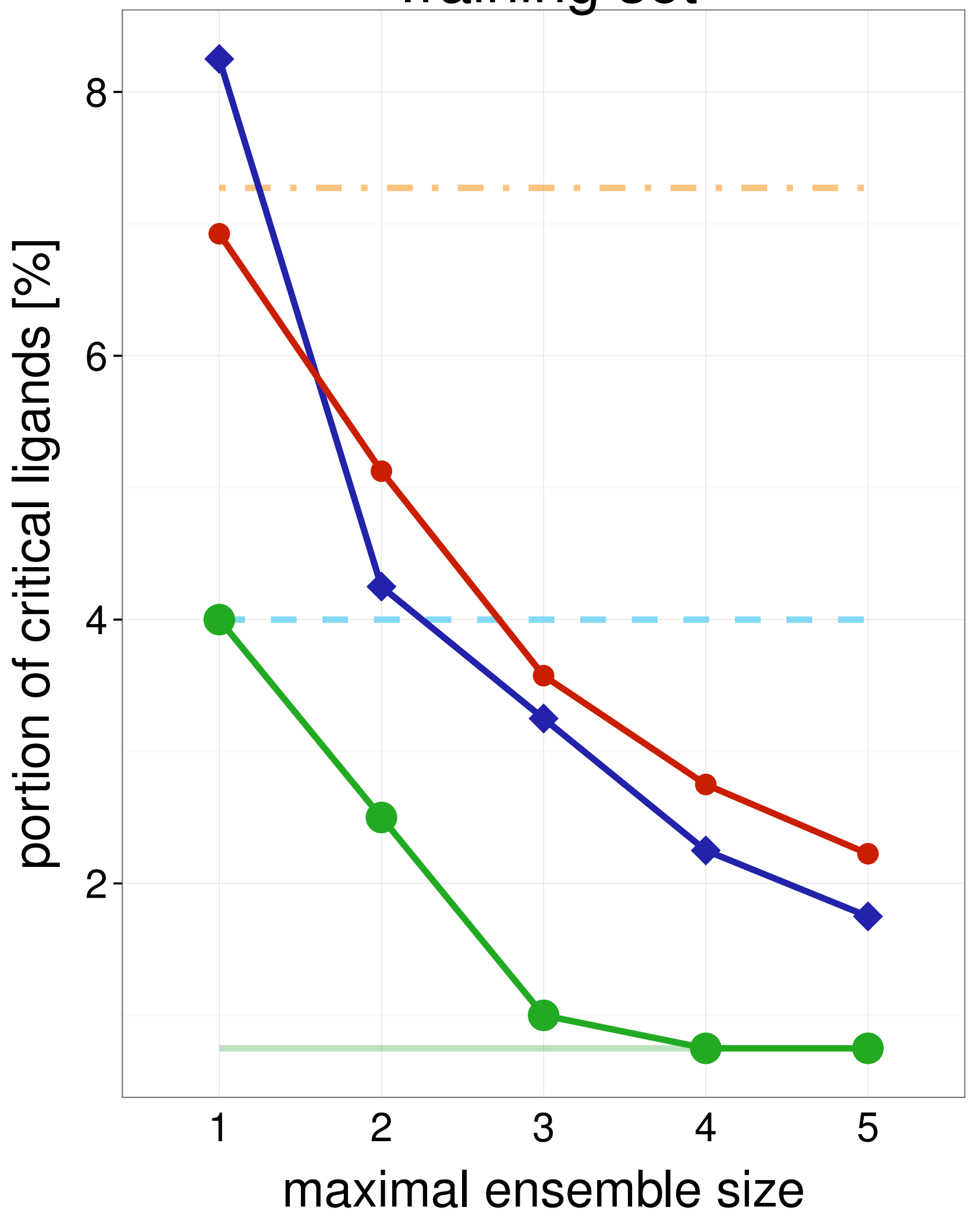

Test set

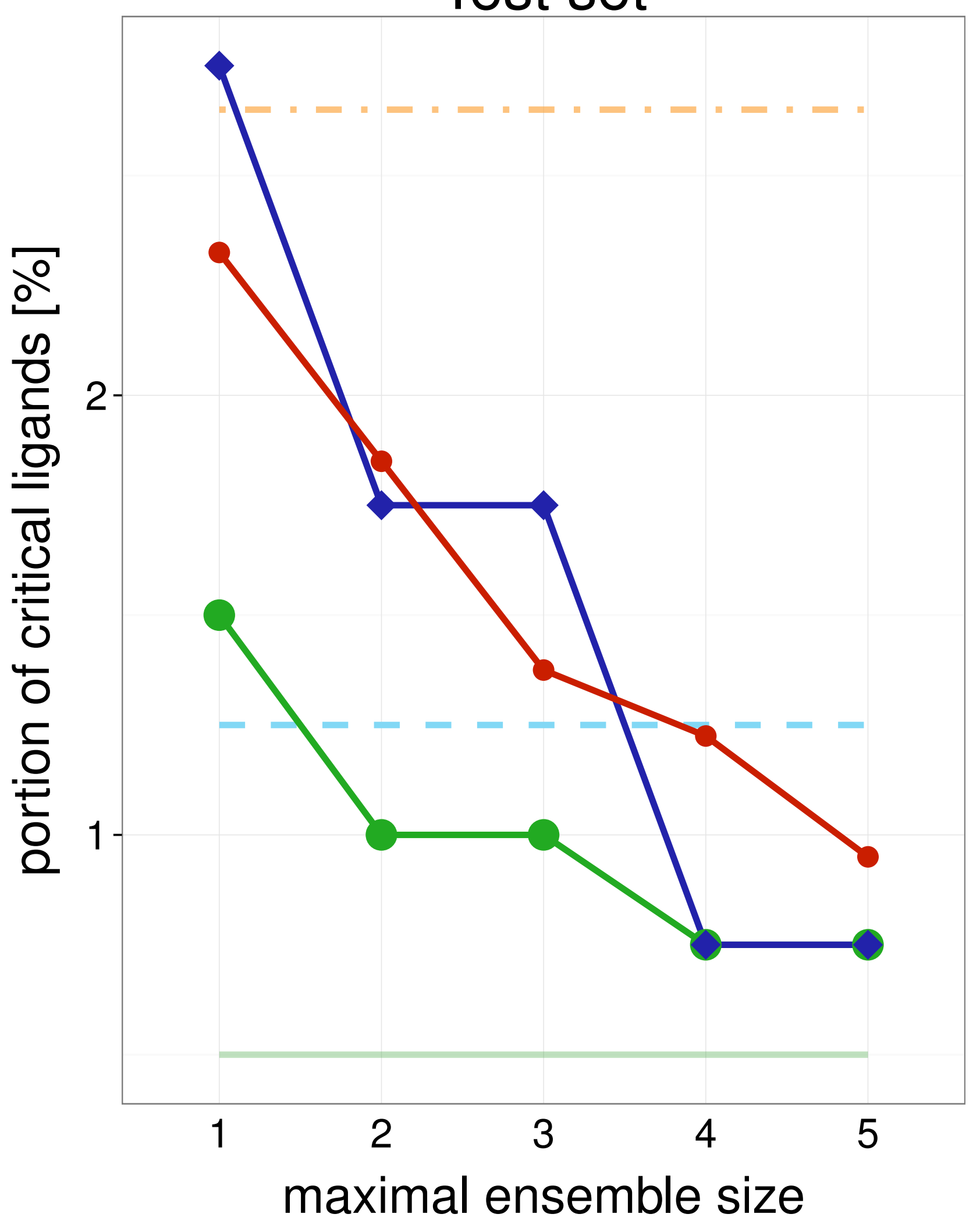

\section{QK8 $\quad$ Training set}

Average single structure

_ Best single structure

Whole ensemble

- SIENA

$\prec$ Clustering

$\multimap$ Random

Average single structure

_ _ Best single structure

Whole ensemble

- SIENA

$\neg$ Clustering

$\because$ Random

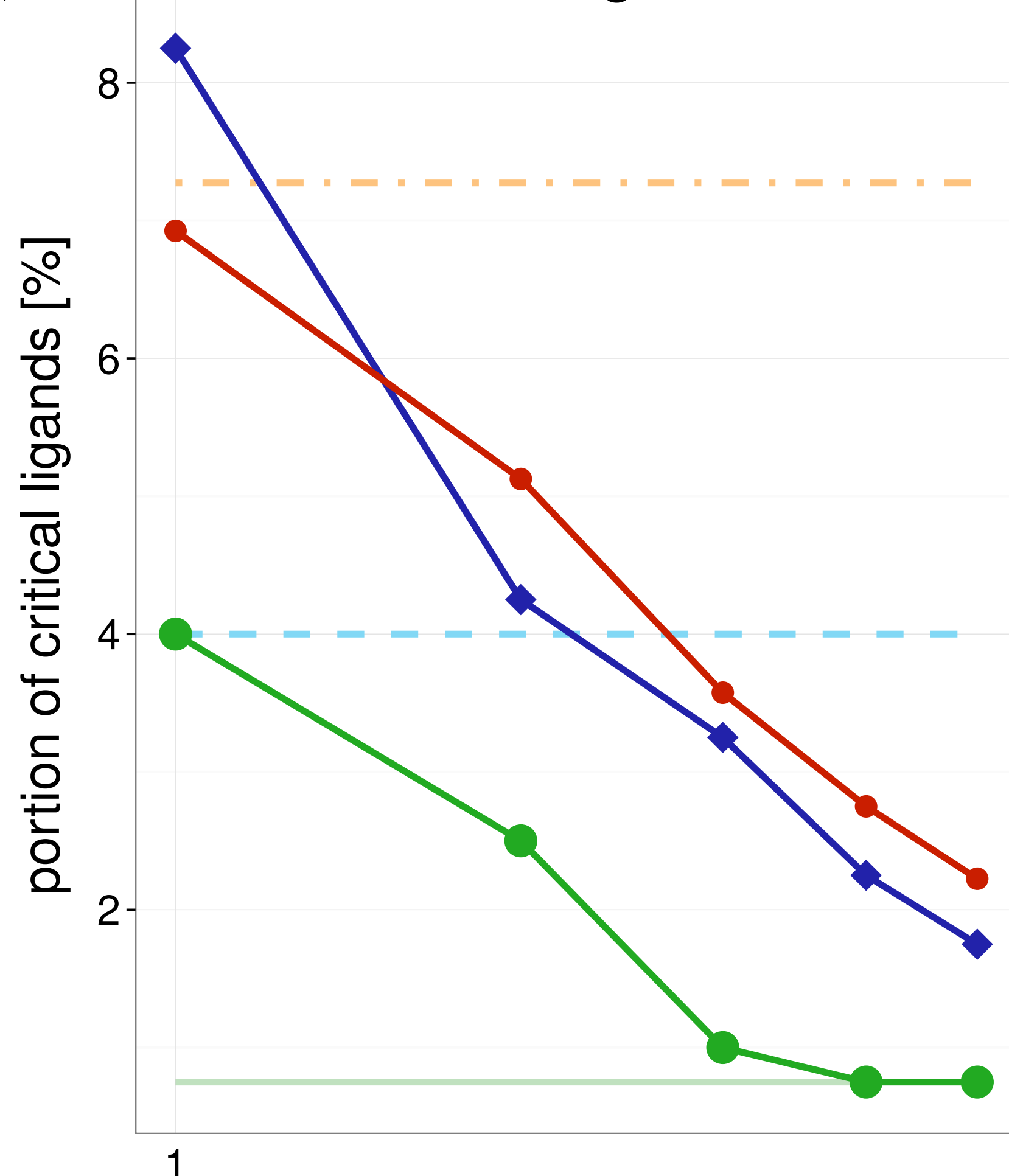

maximal ensemble size

Test set

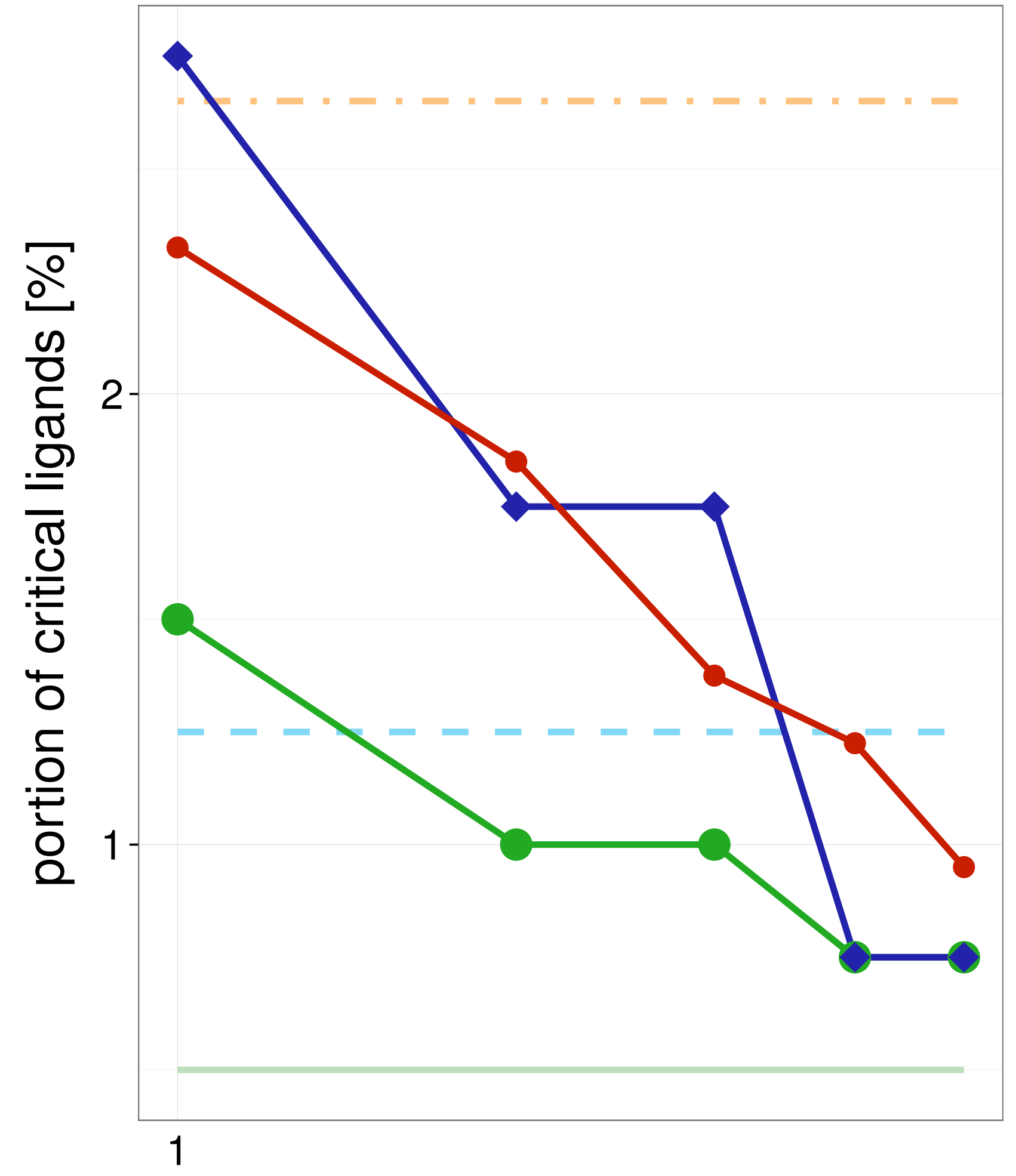

maximal ensemble size
Average single structure

_ _ Best single structure

Whole ensemble

SIENA

$\neg$ Clustering

$\multimap$ Random 
Training set

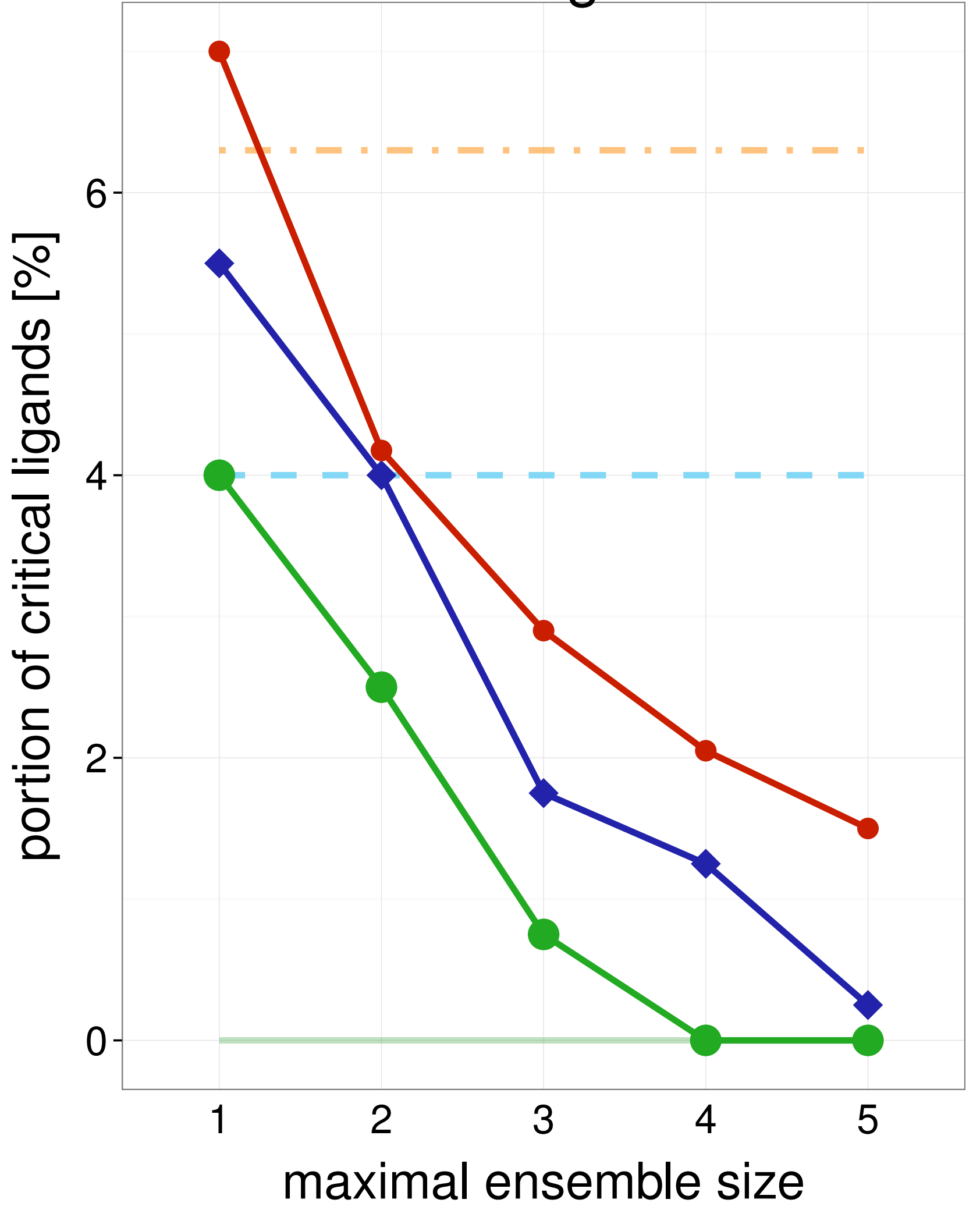

Test set

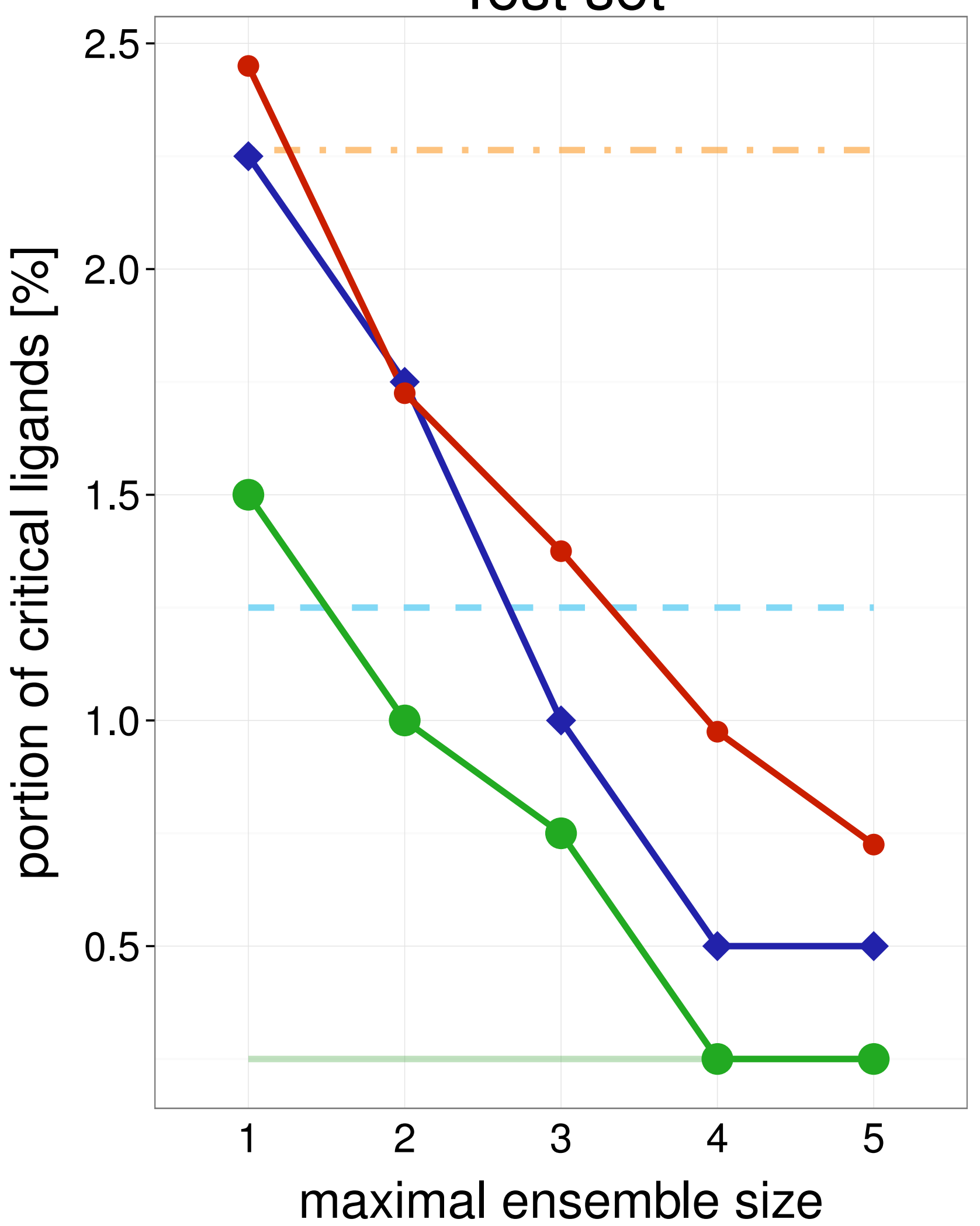

2VES

Training set

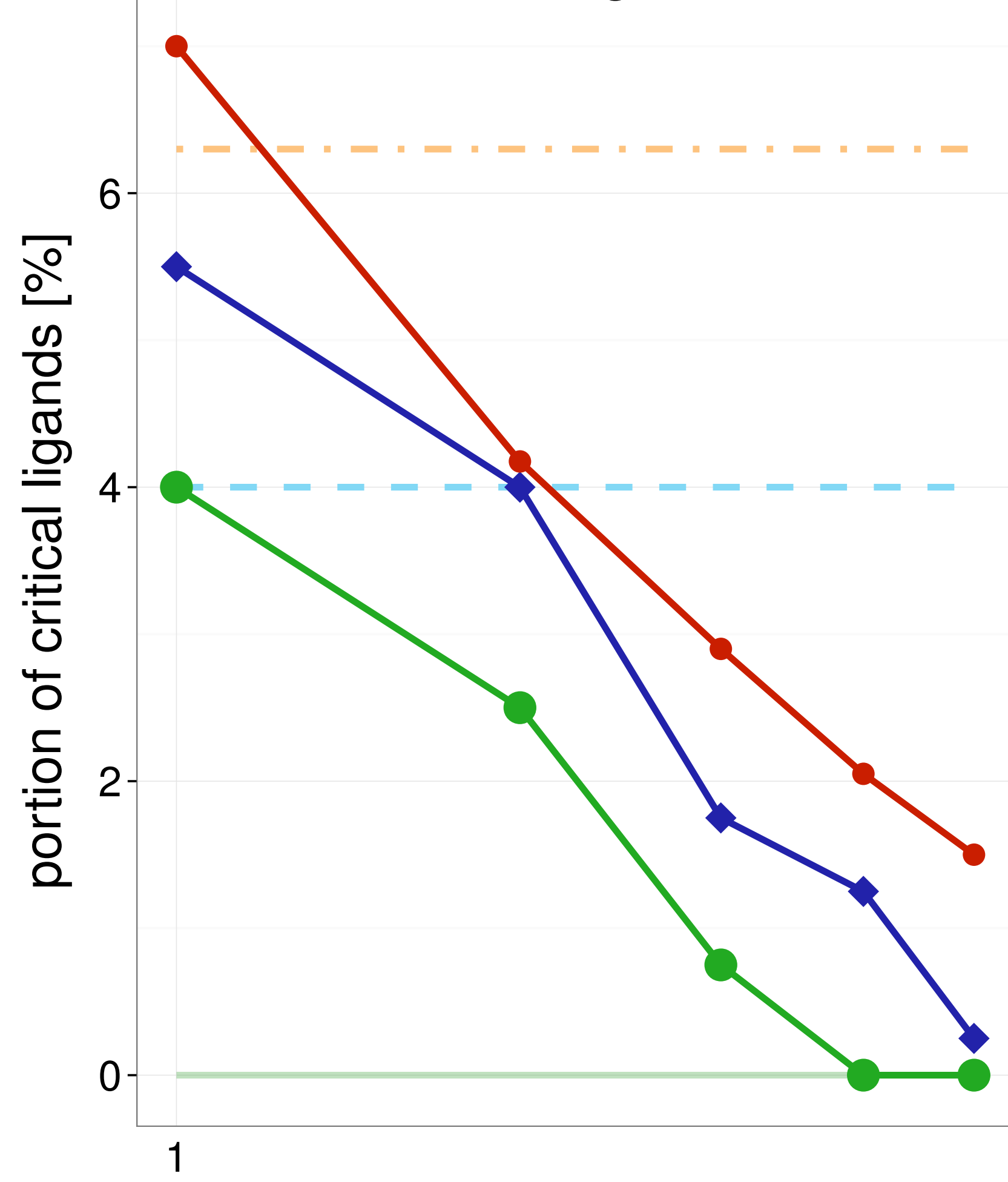

maximal ensemble size

\section{Test set}

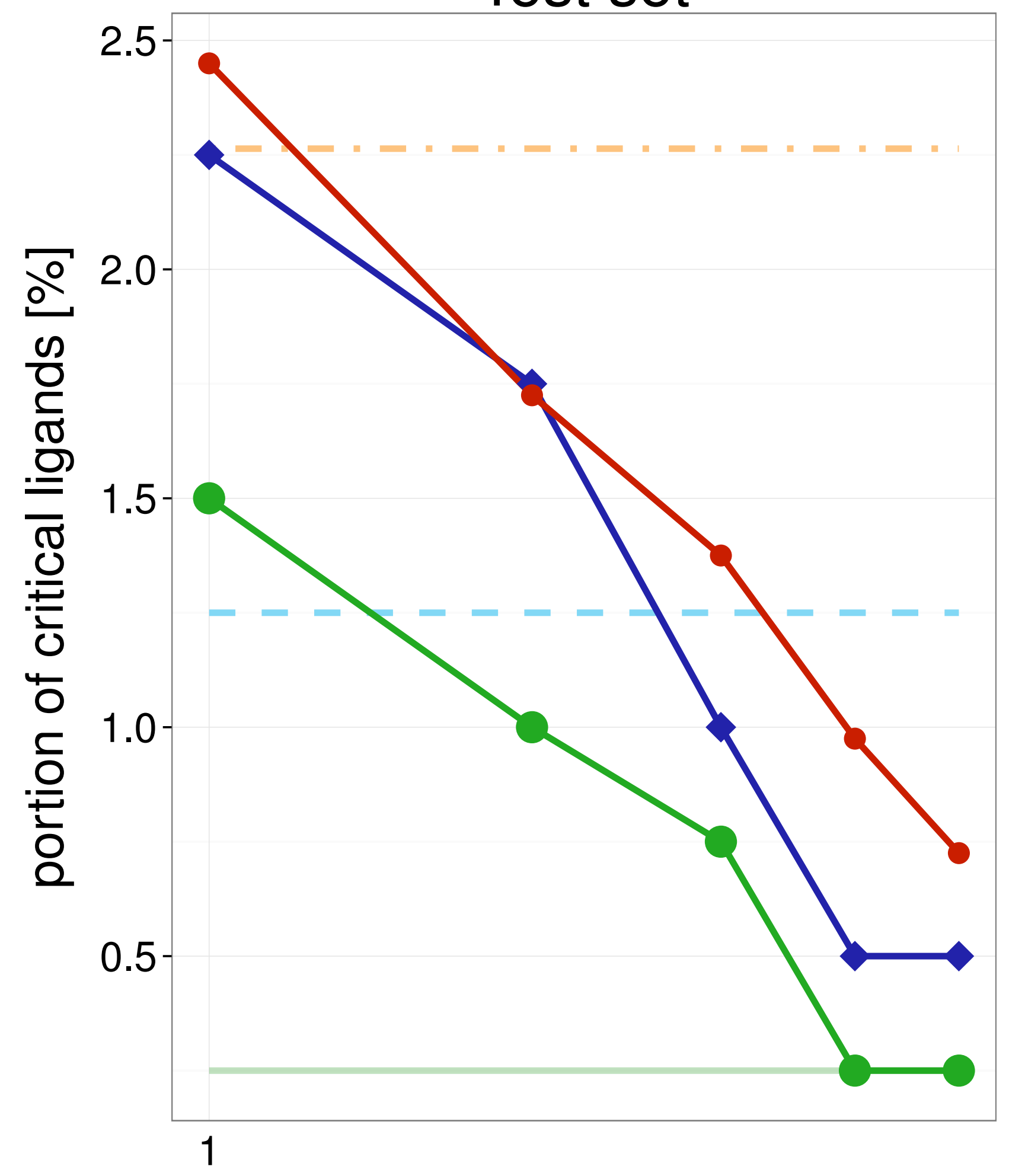

Average single structure

_ _ Best single structure

Whole ensemble

- SIENA

$\smile$ Clustering

$\multimap$ Random
Average single structure

Average single structure

Best single structure

Whole ensemble

- SIENA

$\checkmark$ Clustering

- Random

Best single structure

Whole ensemble

SIENA

$\neg$ Clustering

$\multimap$ Random 


\section{Training set}

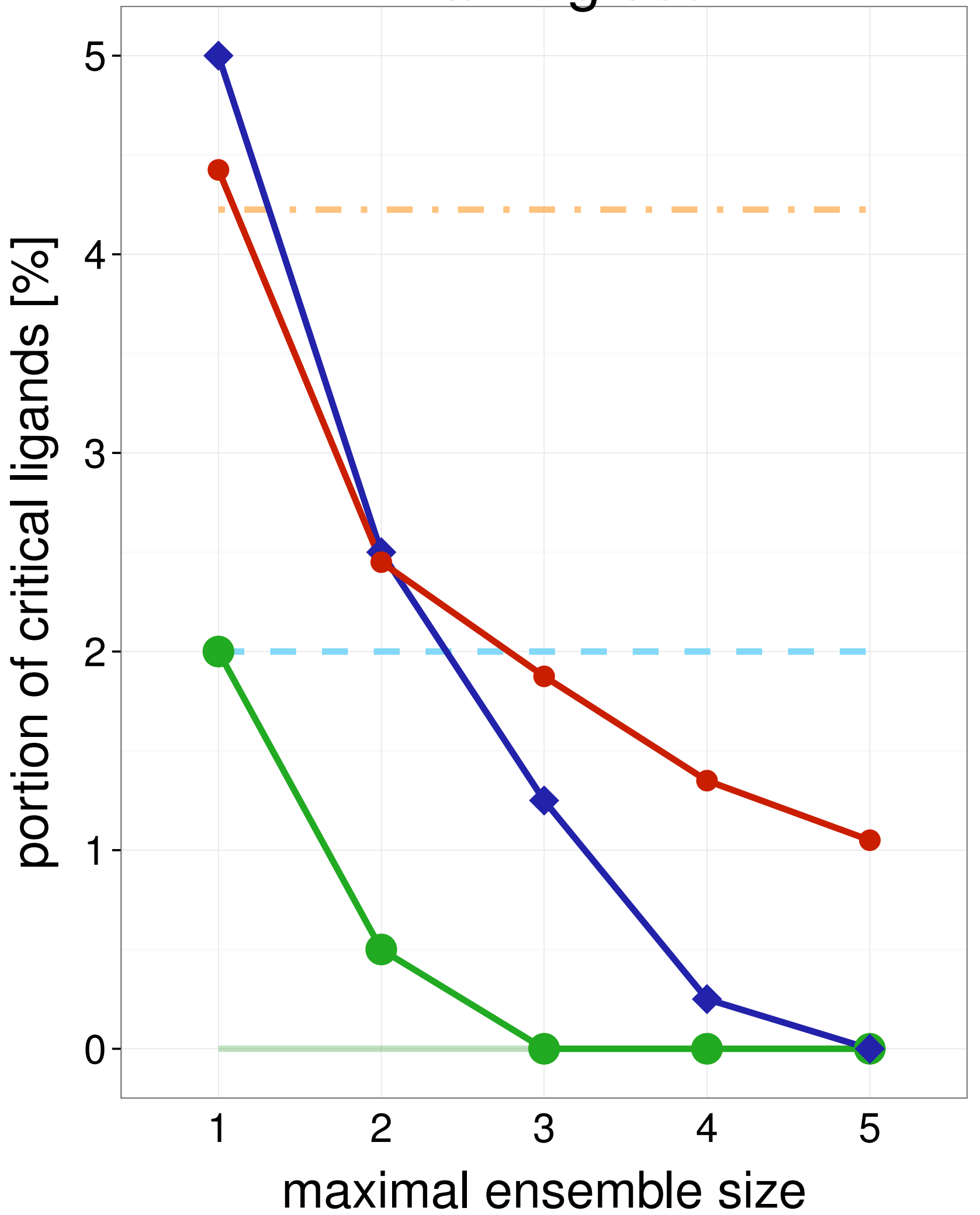

Test set

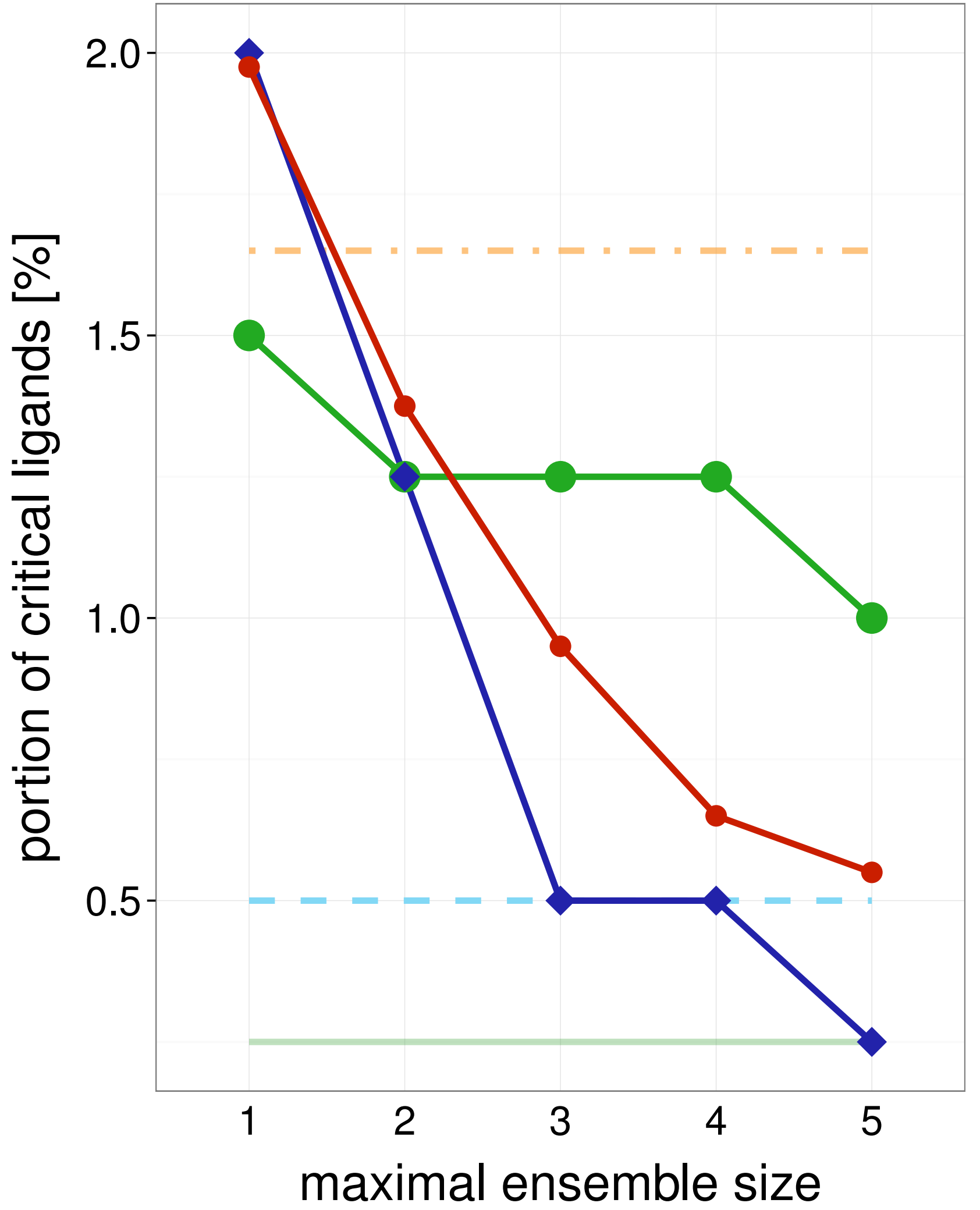

\section{VWU Training set}

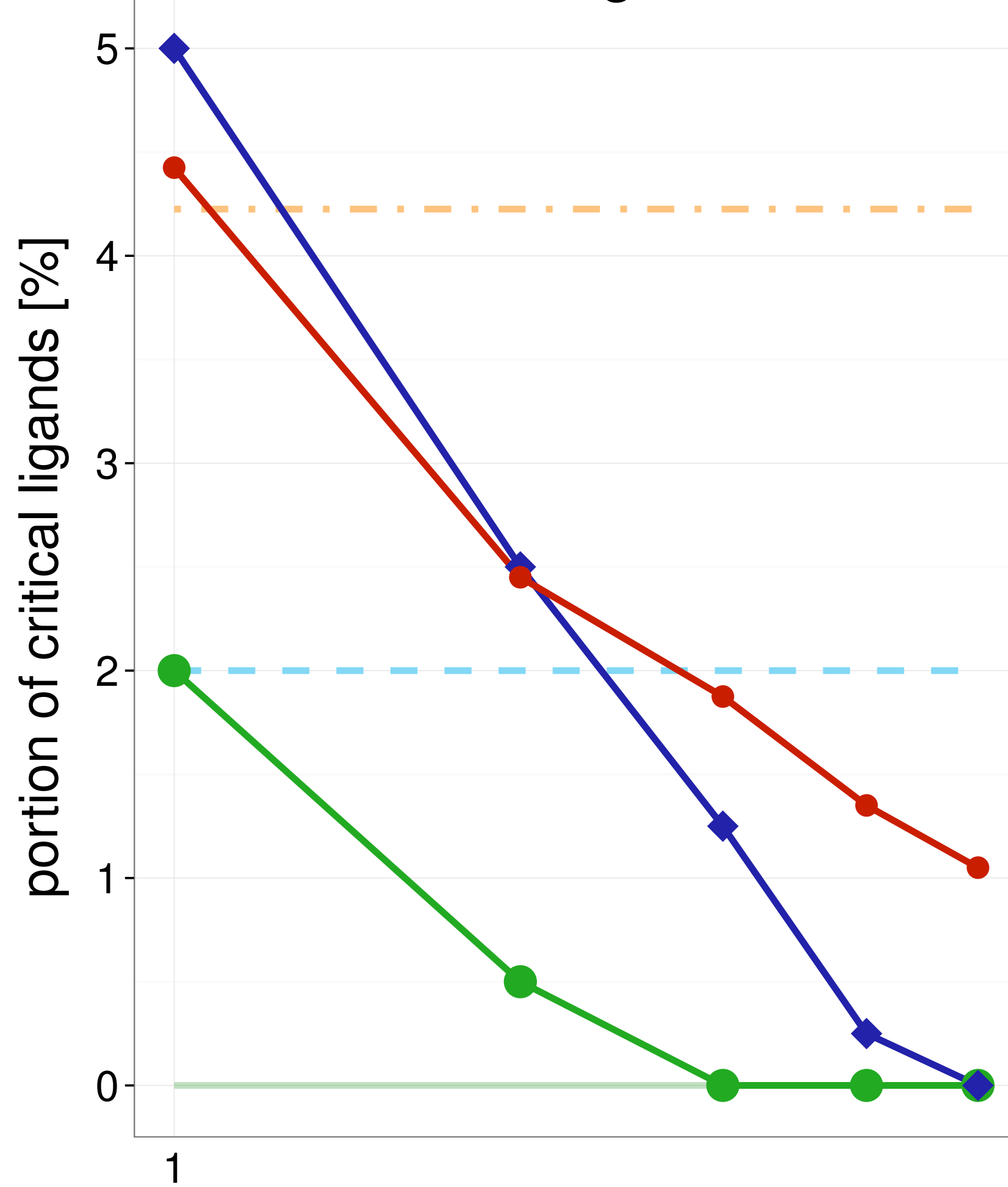

maximal ensemble size

\section{Test set}

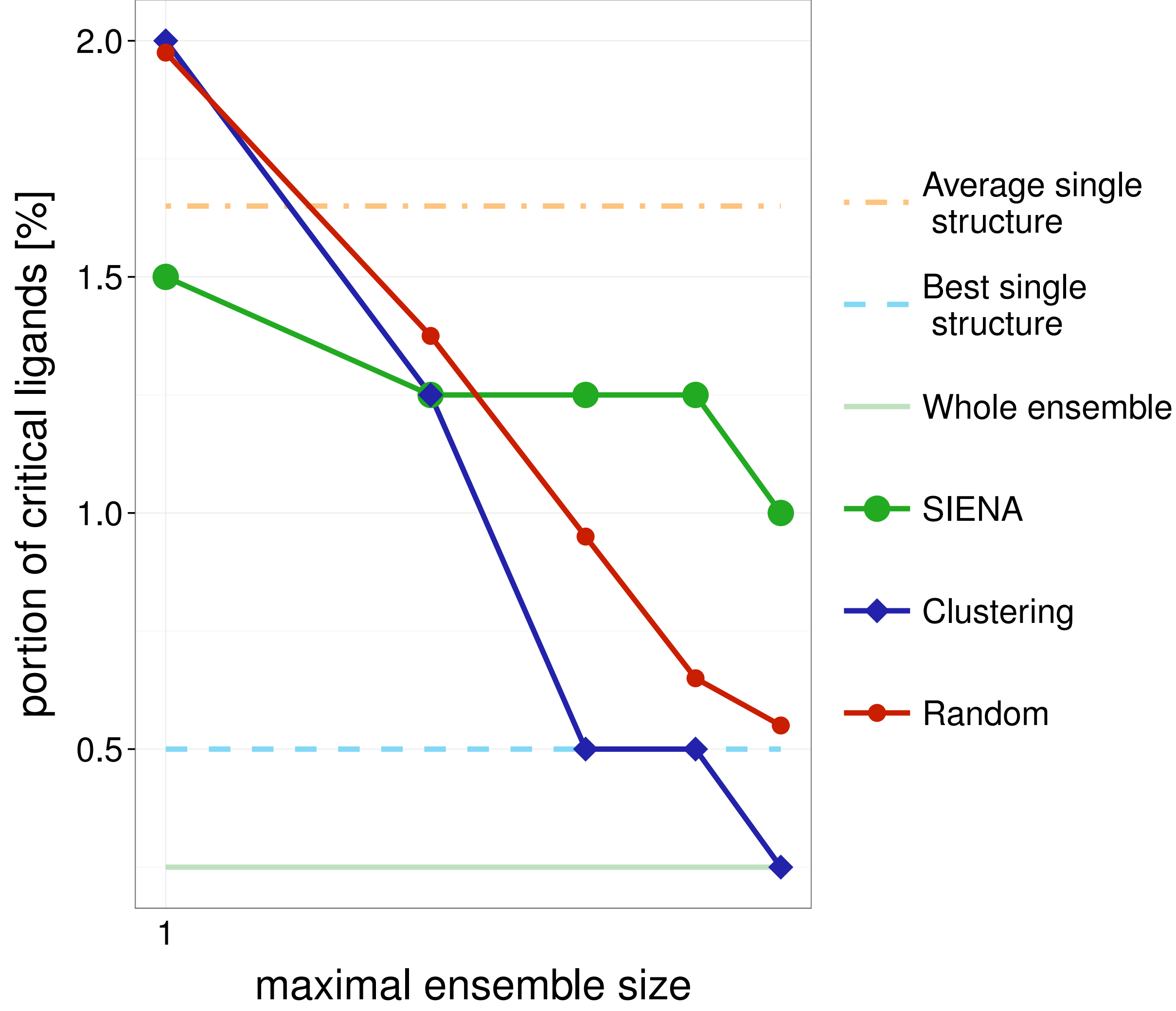


Training set

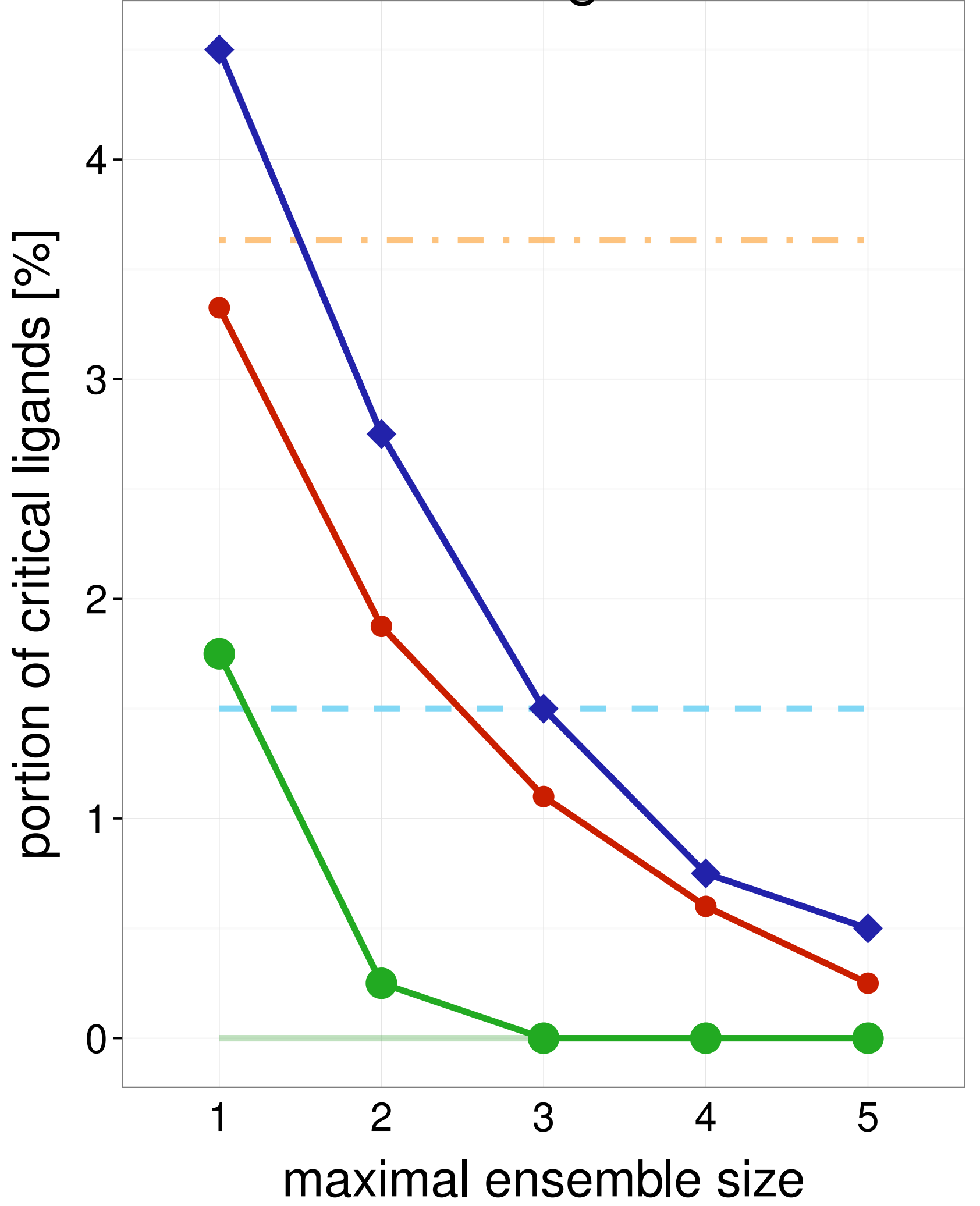

Test set

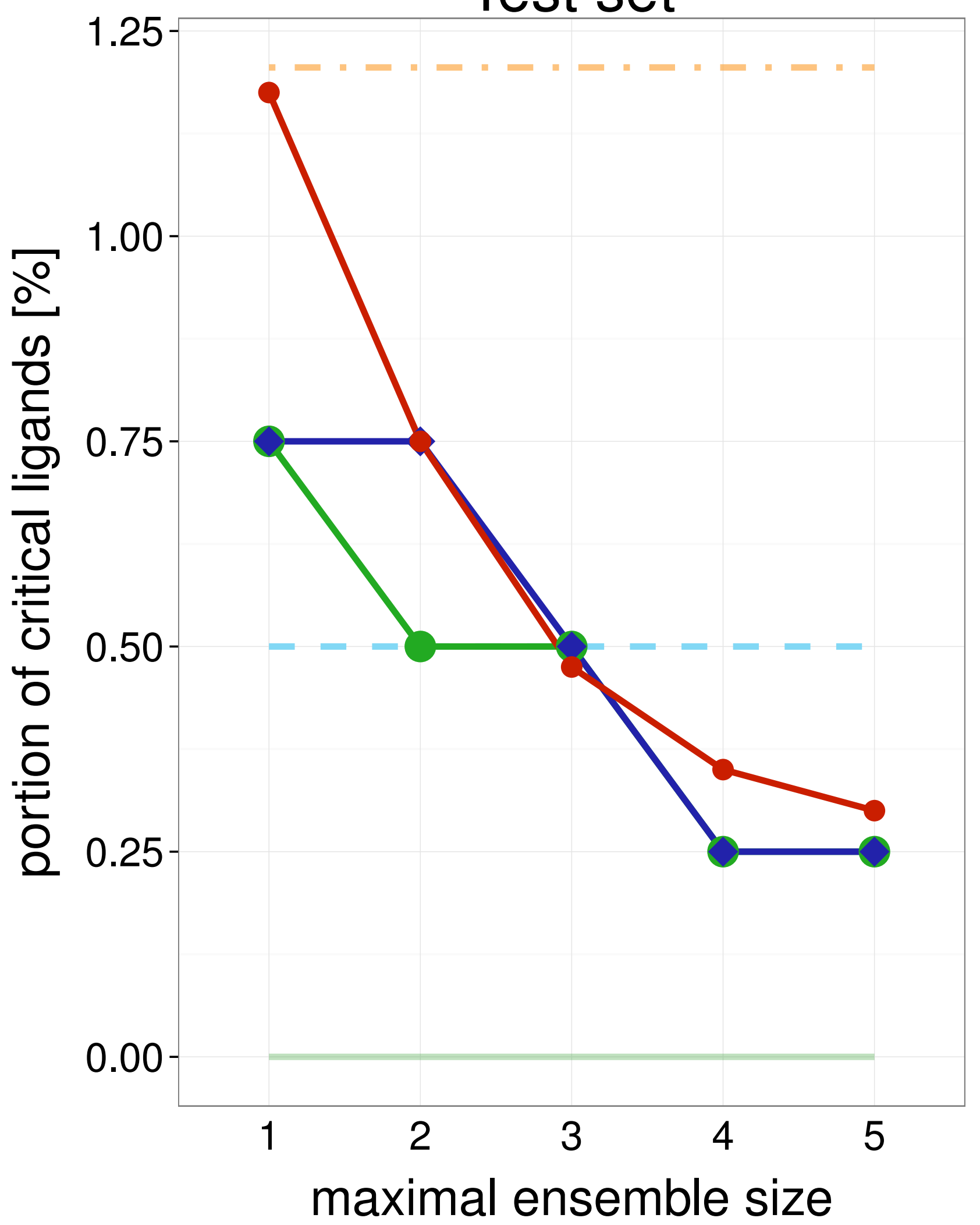

Average single structure

_ Best single structure

Whole ensemble

- SIENA

$\neg$ Clustering

$\multimap$ Random

Average single structure

_ Best single structure

Whole ensemble

- SIENA

$\smile$ Clustering

- Random
Training set

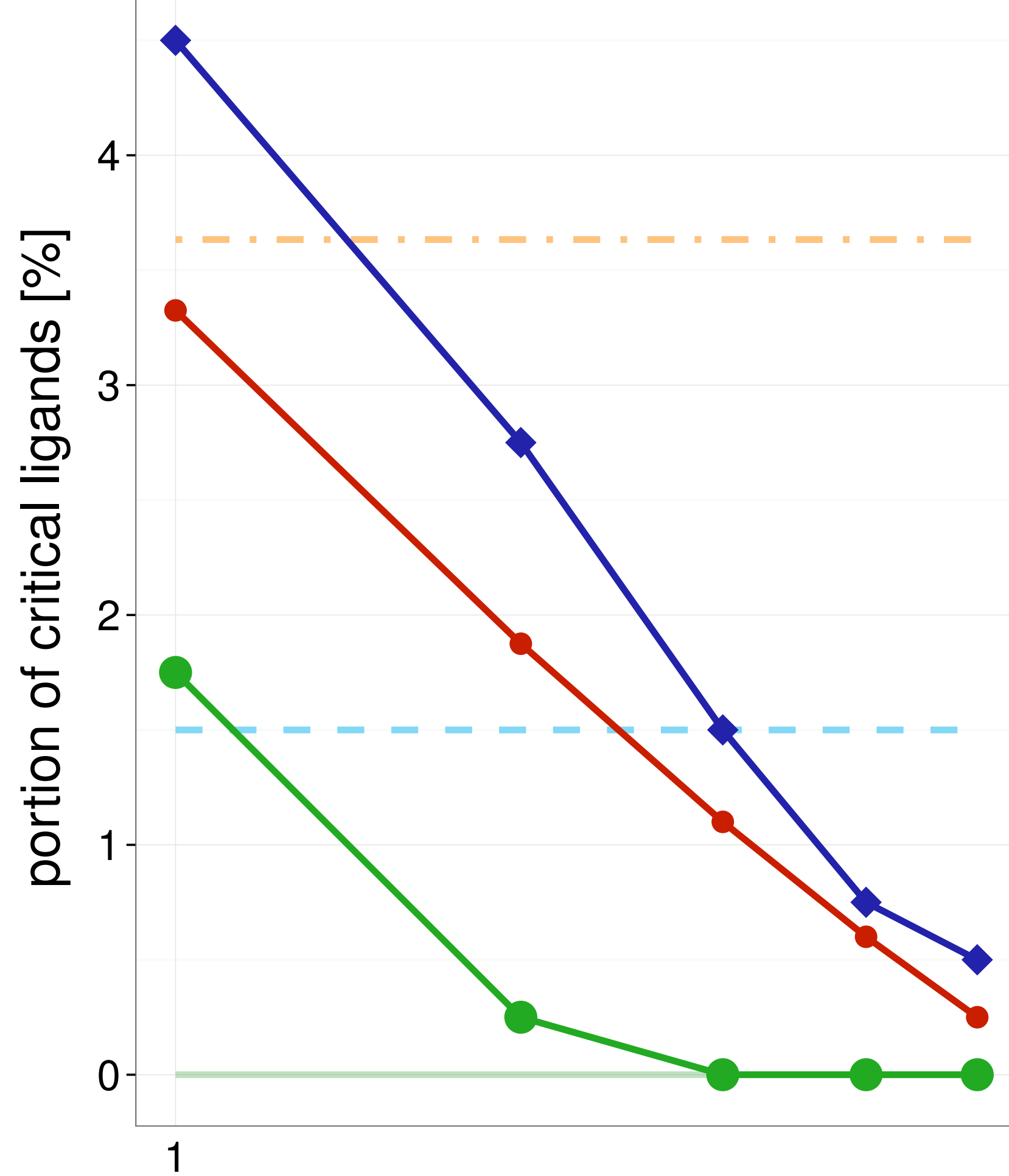

maximal ensemble size

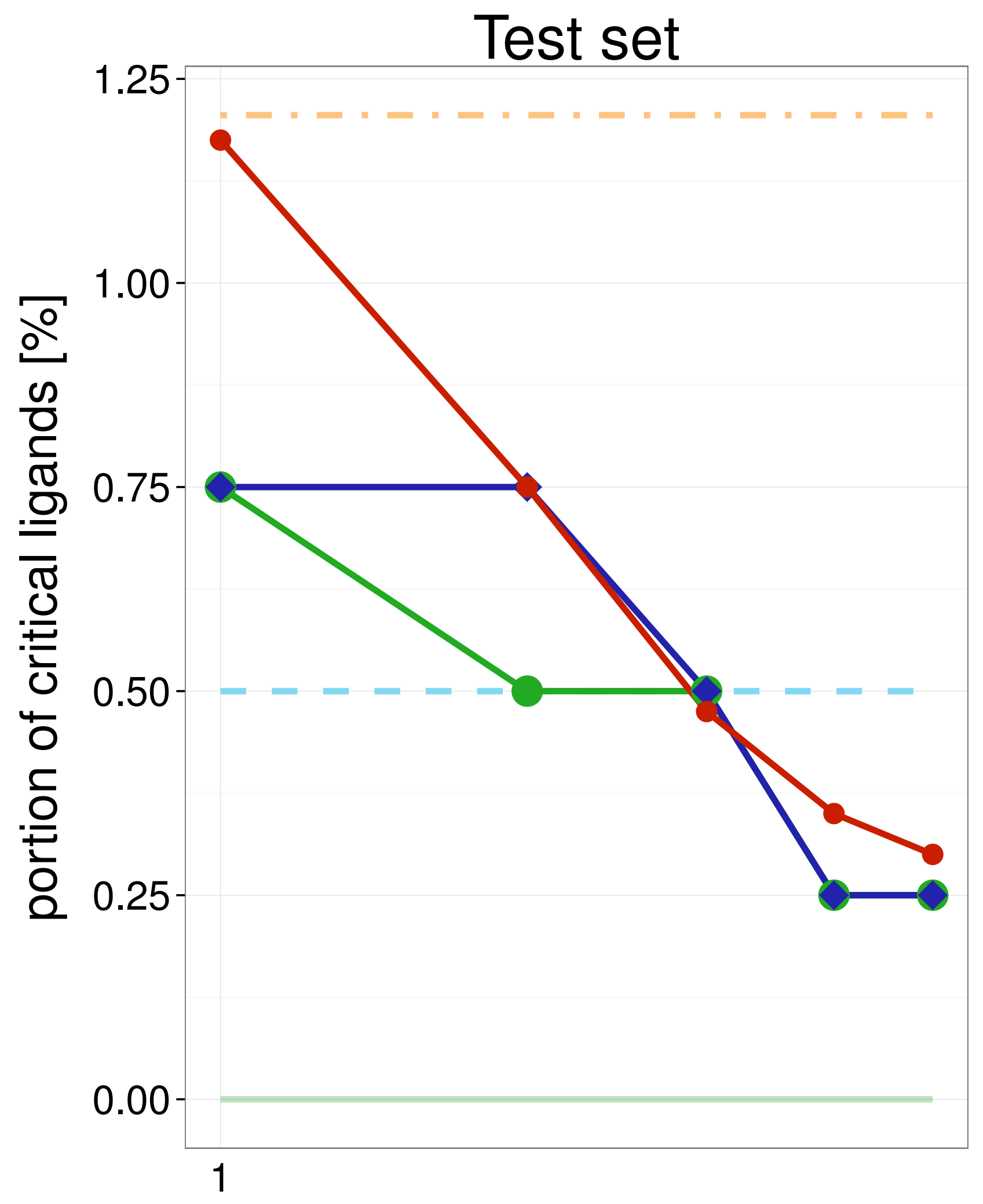

maximal ensemble size
Average single structure

_ Best single structure

Whole ensemble

SIENA

$\rightarrow$ Clustering

- Random 
Training set

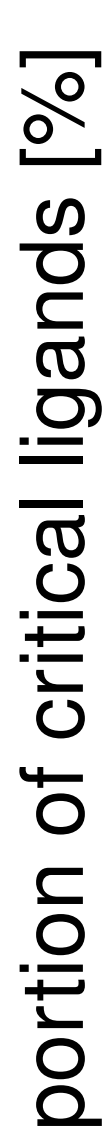

0.0

1.0

$$
1
$$

$\begin{array}{ccc}2 & 3 & 4 \\ \text { maximal ensemble size }\end{array}$

Test set

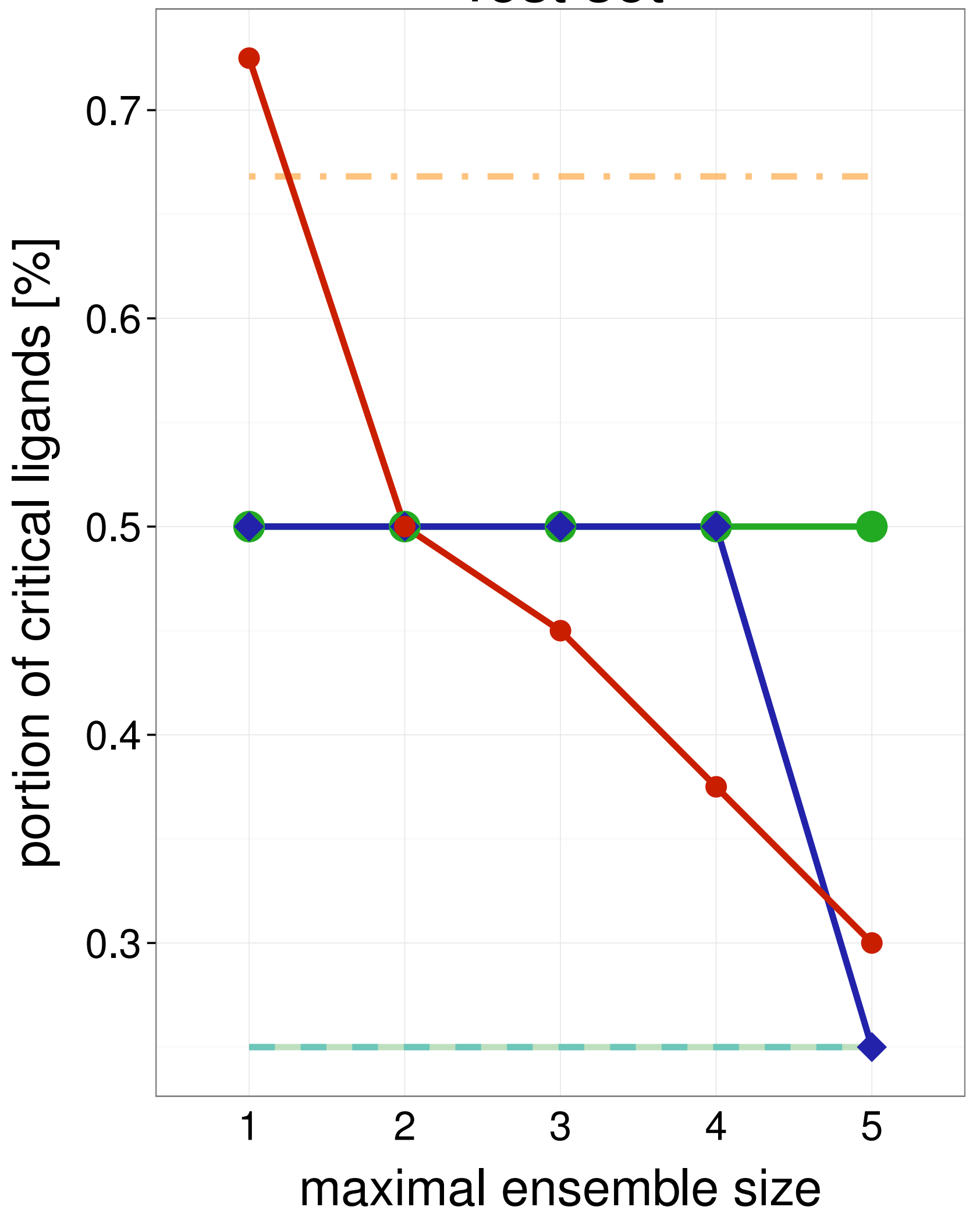

$2 \times 90$

Training set

Average single structure

_ Best single structure

Whole ensemble

- SIENA

$\neg$ Clustering

$\multimap$ Random

Average single structure

_ Best single structure

Whole ensemble

- SIENA

$\leadsto$ Clustering

$\multimap$ Random

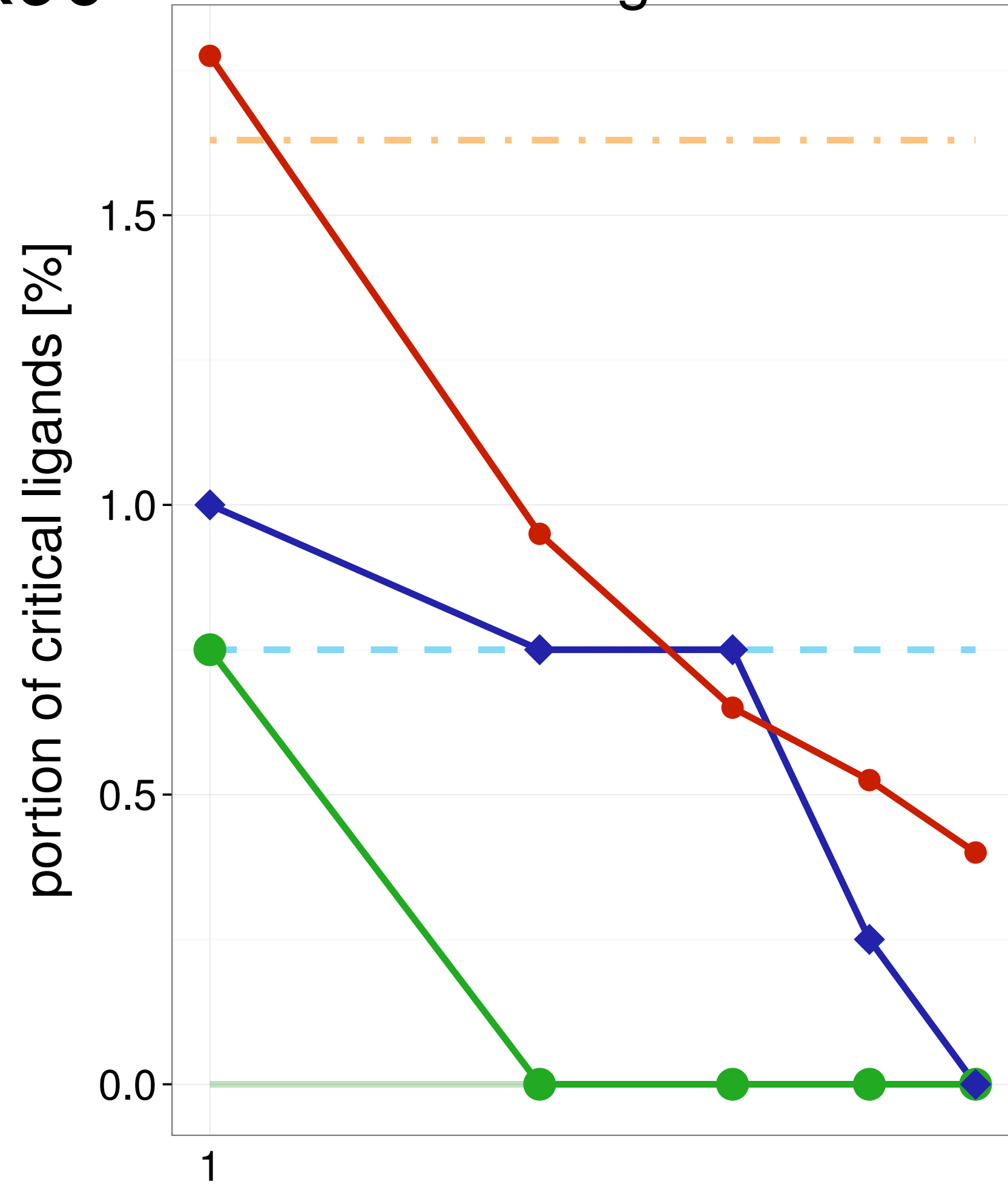

Average single structure

_ Best single structure

Whole ensemble

- SIENA

$\checkmark$ Clustering

$\longrightarrow$ Random

maximal ensemble size

Test set

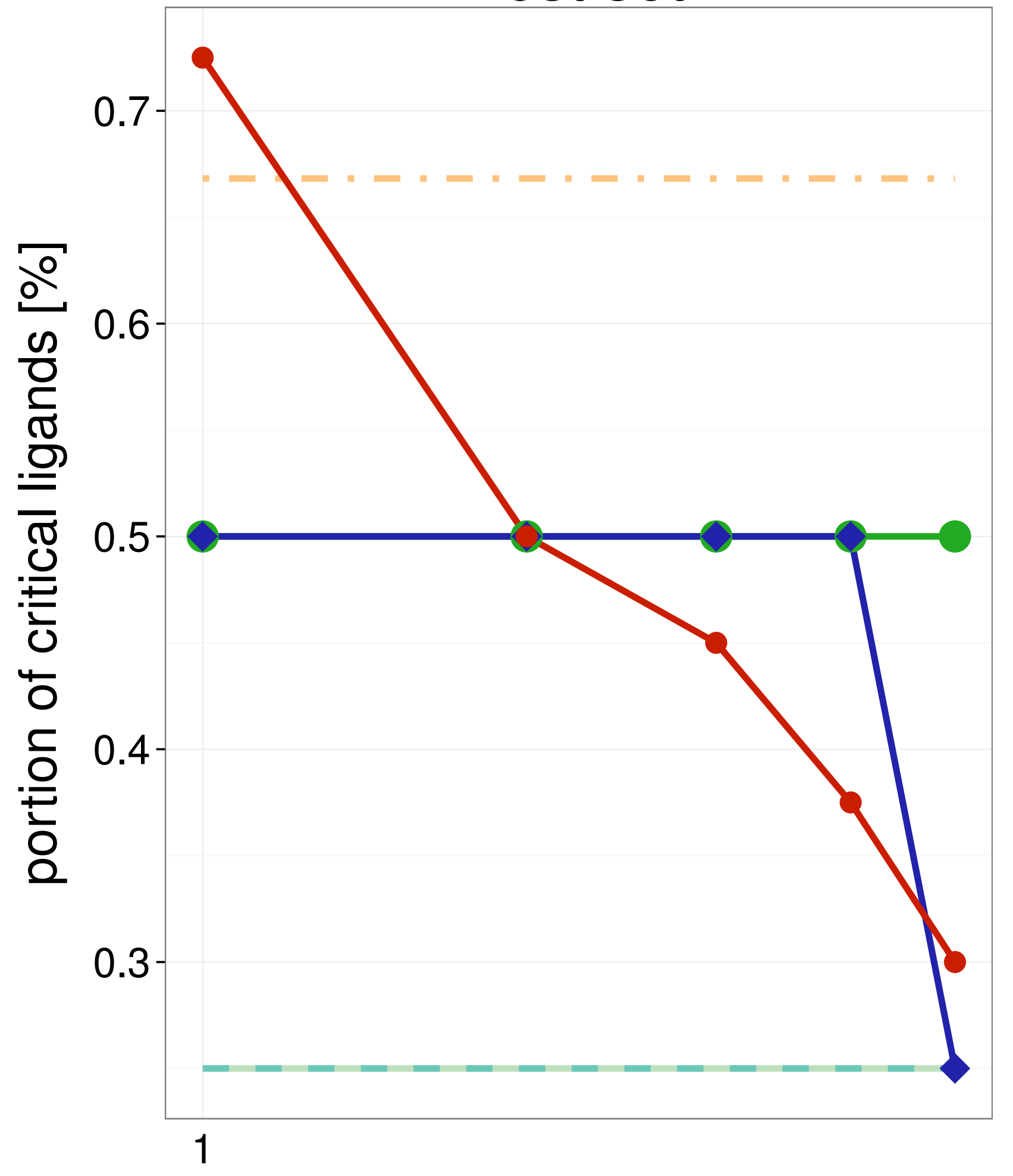

Average single structure

_ Best single structure

Whole ensemble

SIENA

$\neg$ Clustering

$\multimap$ Random 
Training set

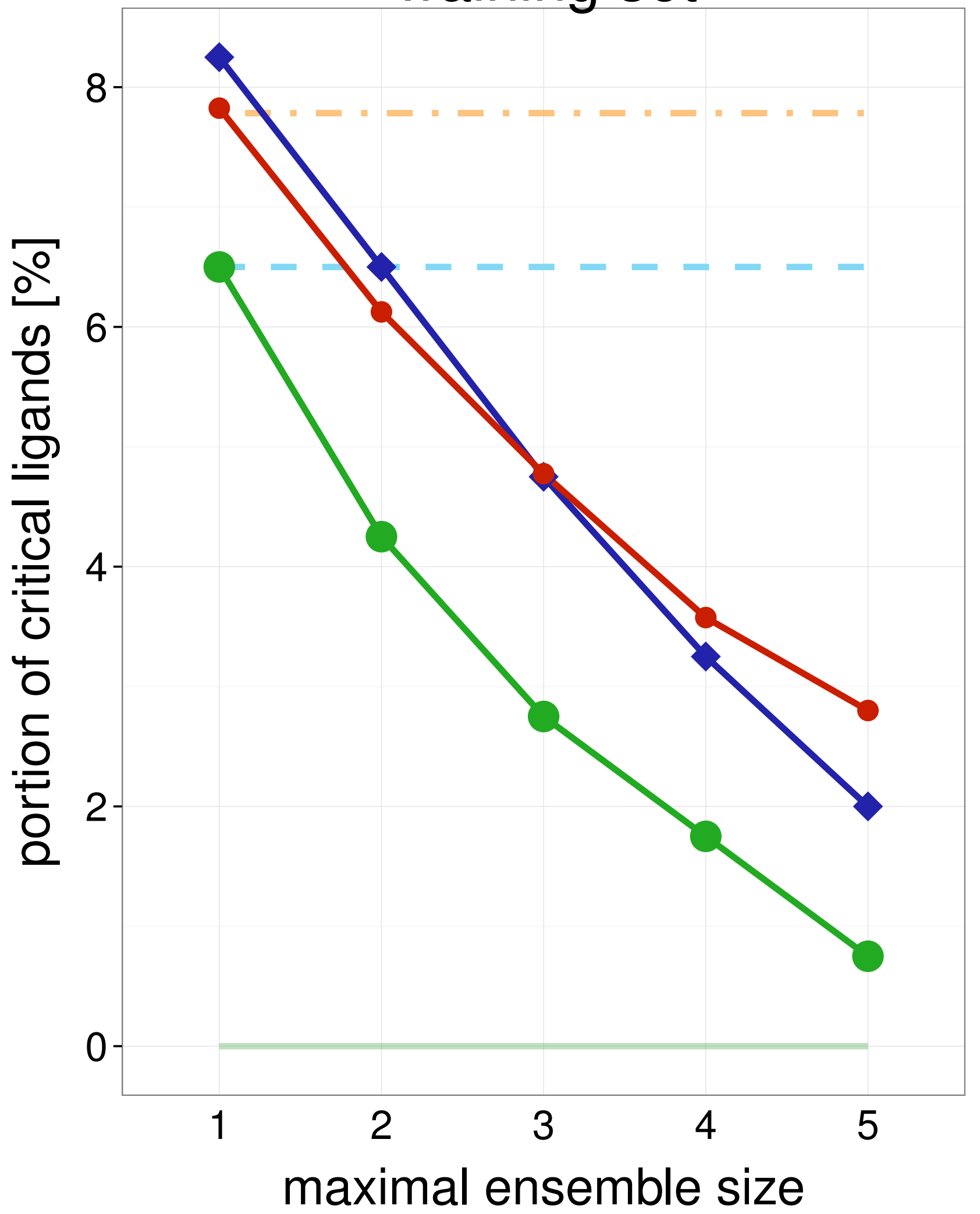

\section{Test set}

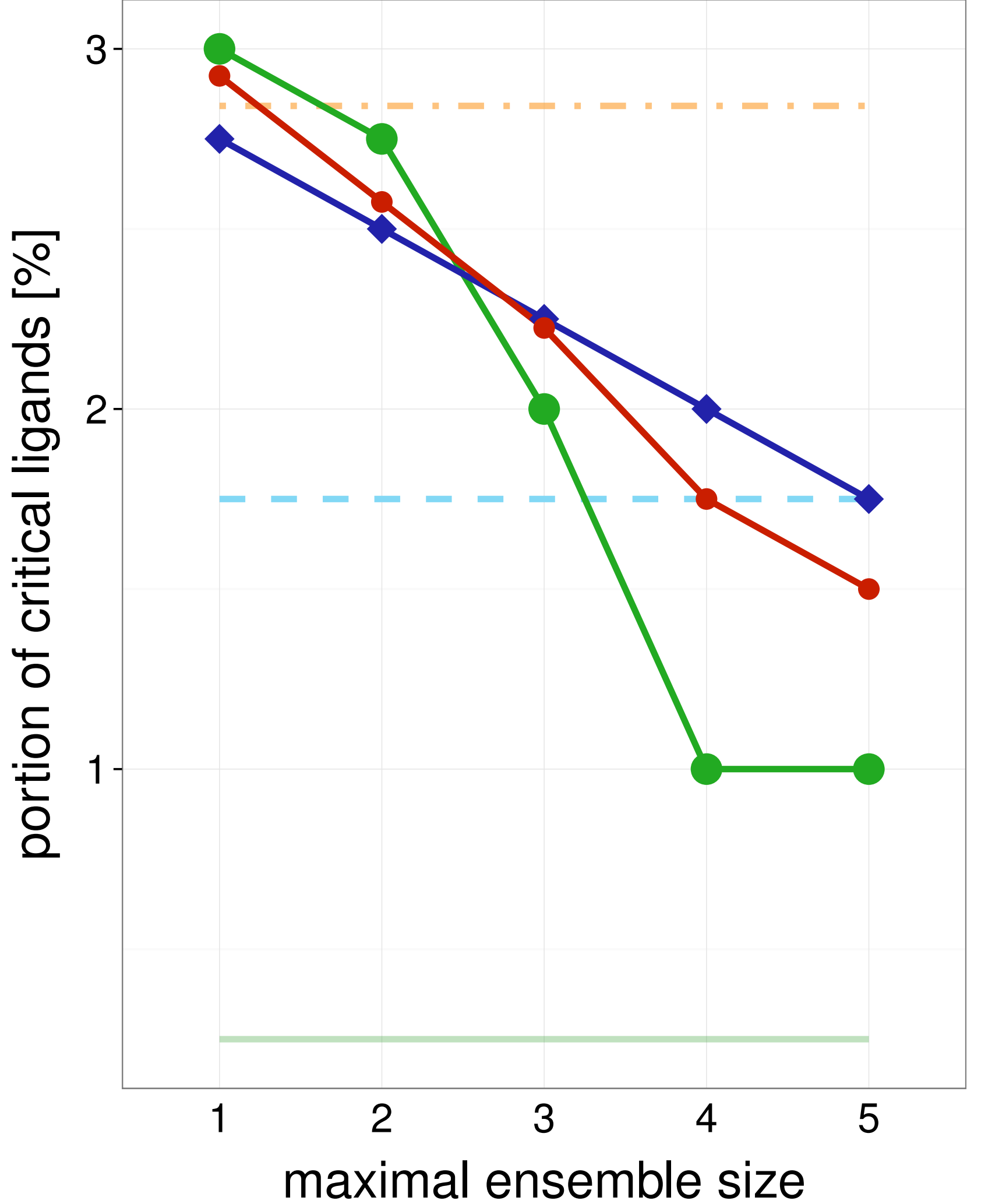

Average single structure

_ Best single structure

Whole ensemble

- SIENA

$\neg$ Clustering

$\multimap$ Random

Average single structure

_ _ Best single structure

Whole ensemble

- SIENA

$\checkmark$ Clustering

$\because$ Random
Training set

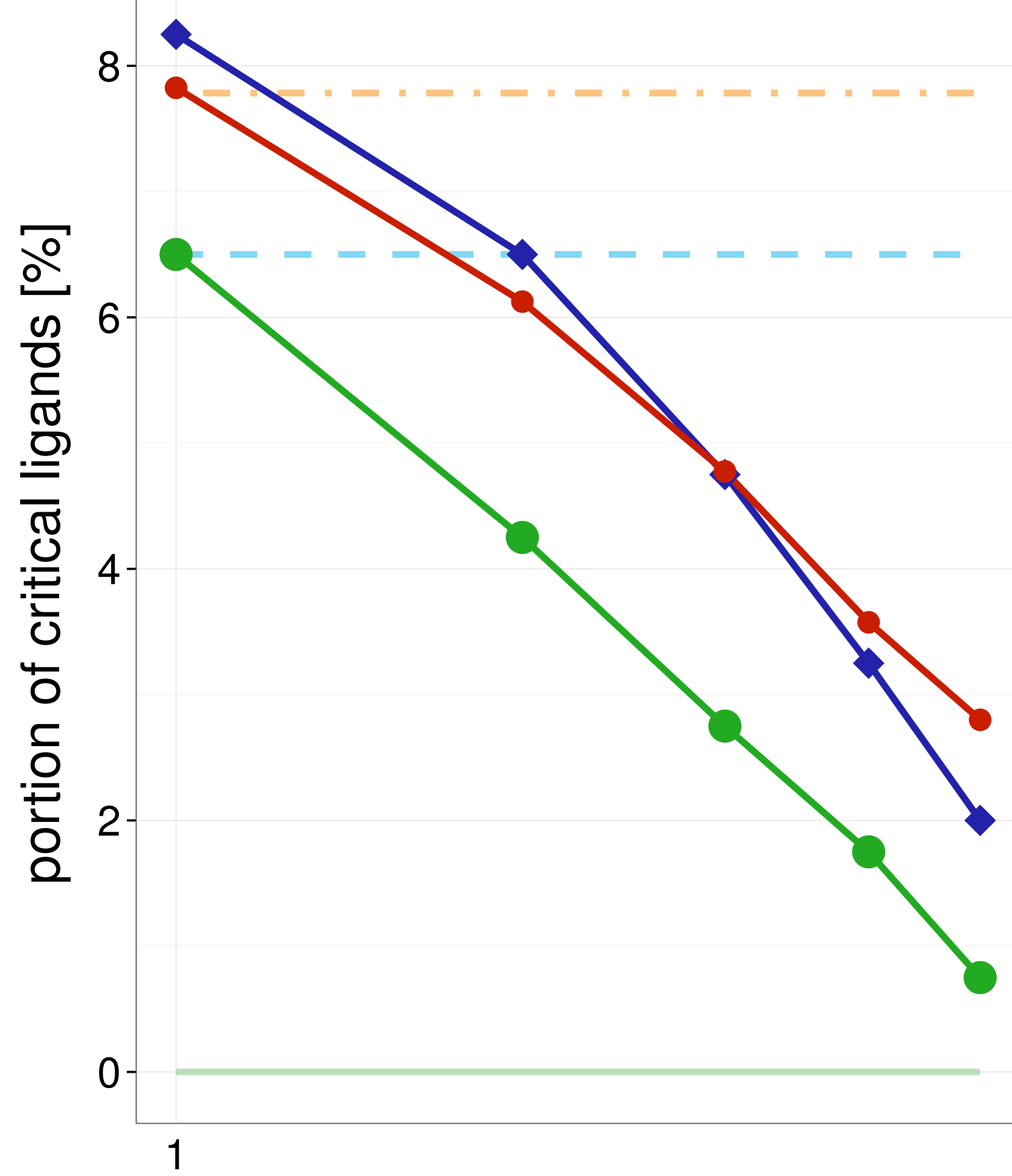

maximal ensemble size

Test set

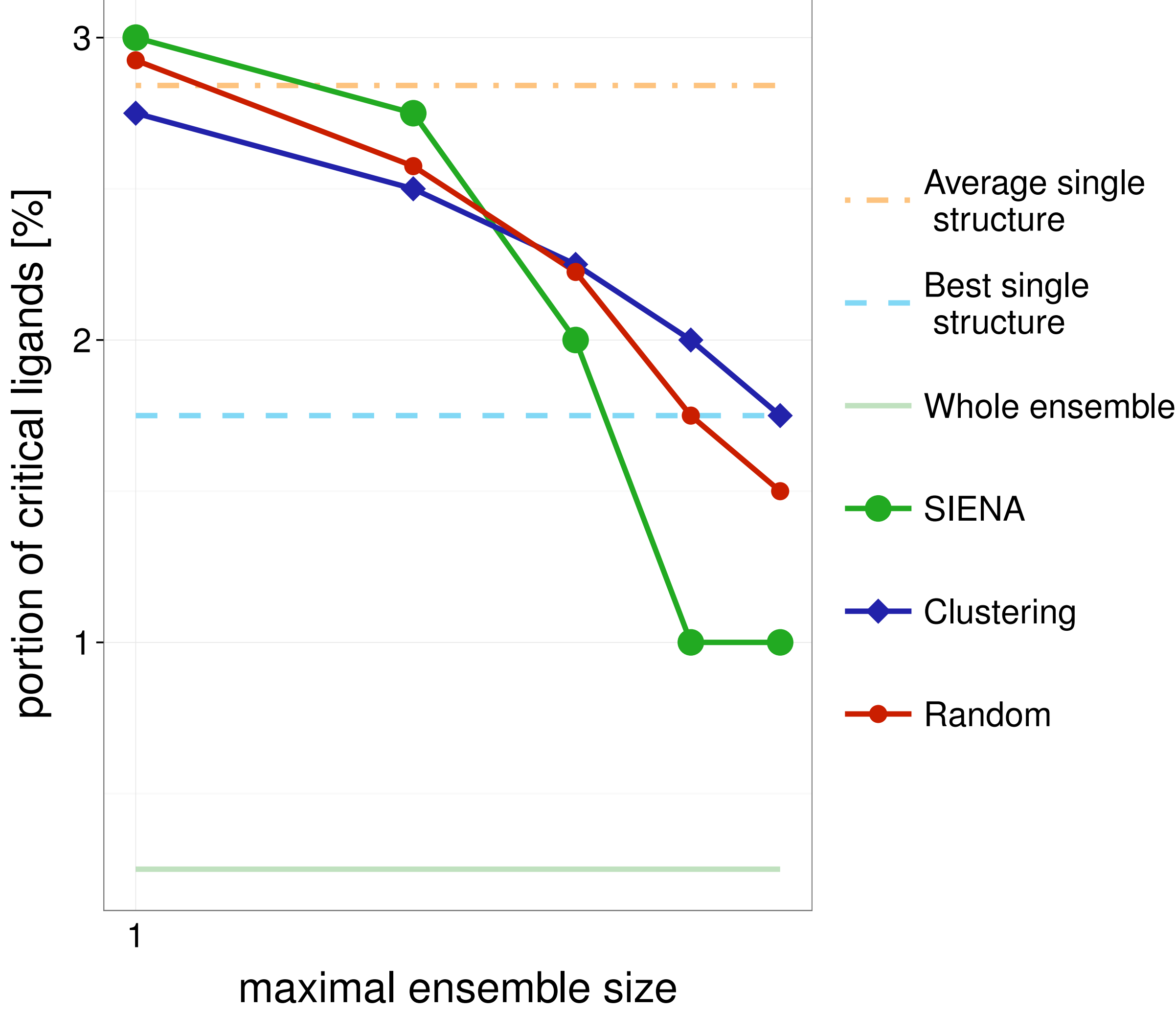


Training set

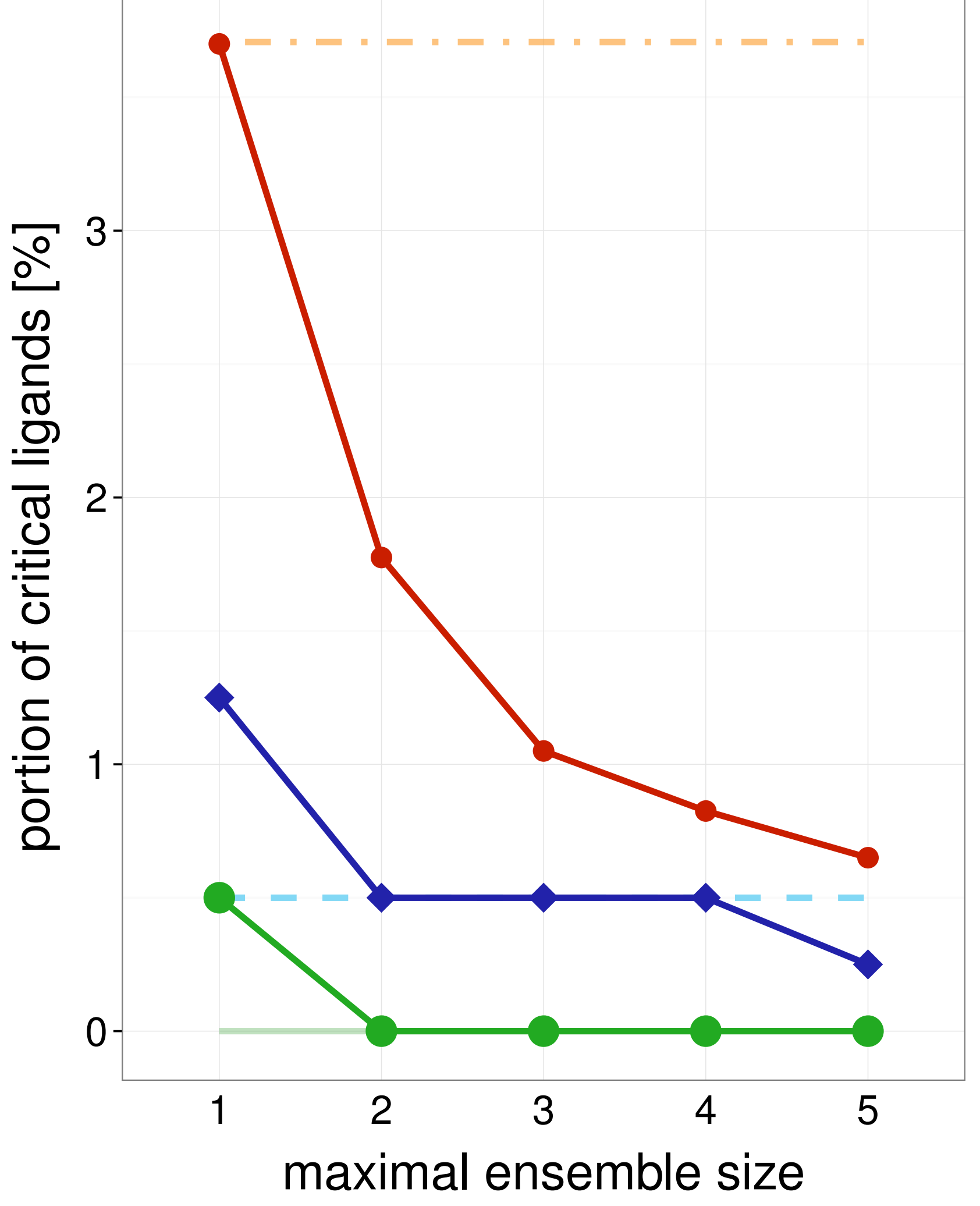

\section{Test set}

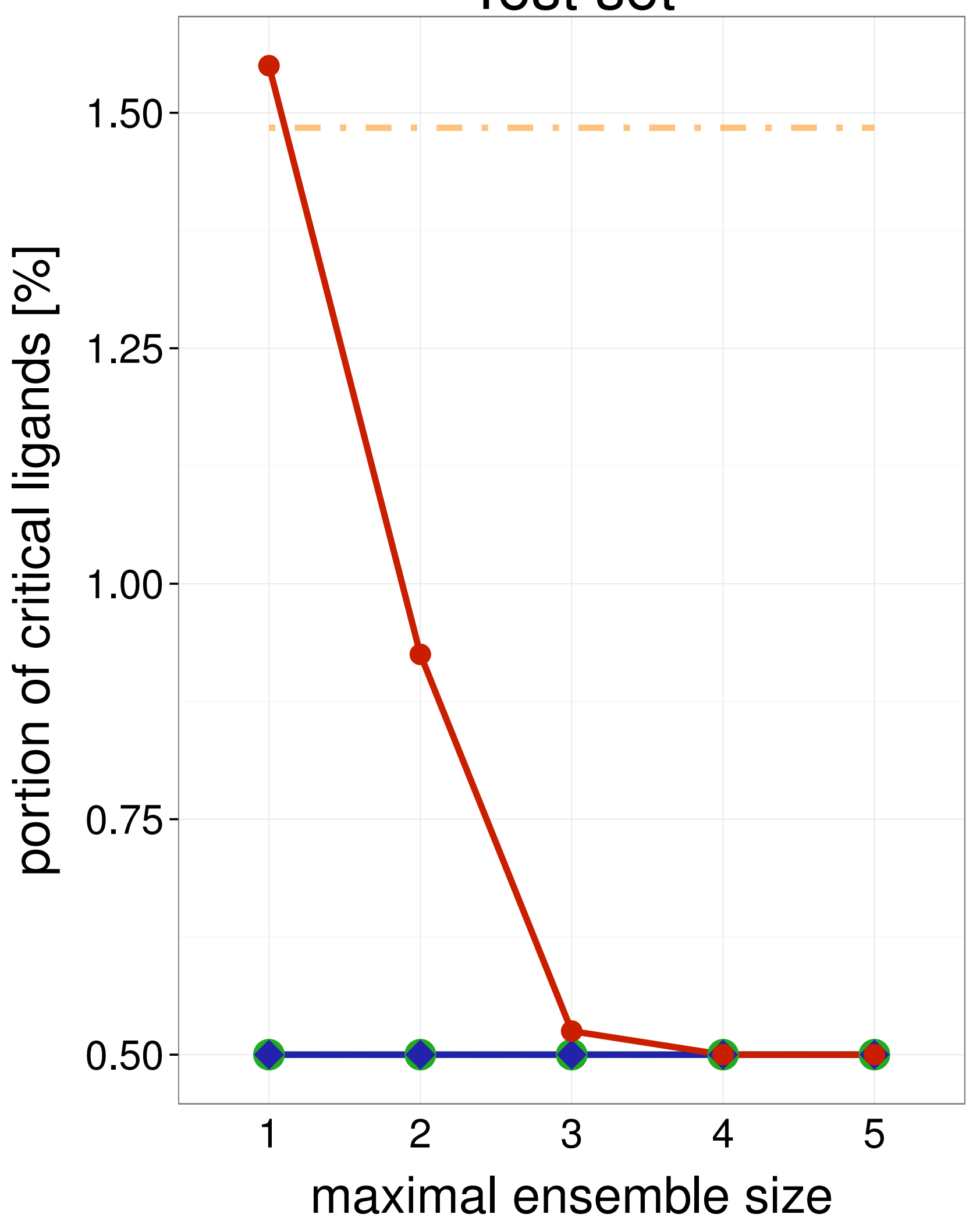

Average single structure

_ _ Best single structure

Whole ensemble

- SIENA

$\neg$ Clustering

$\multimap$ Random

Average single structure

_ Best single structure

Whole ensemble

- SIENA

$\neg$ Clustering

$\multimap$ Random

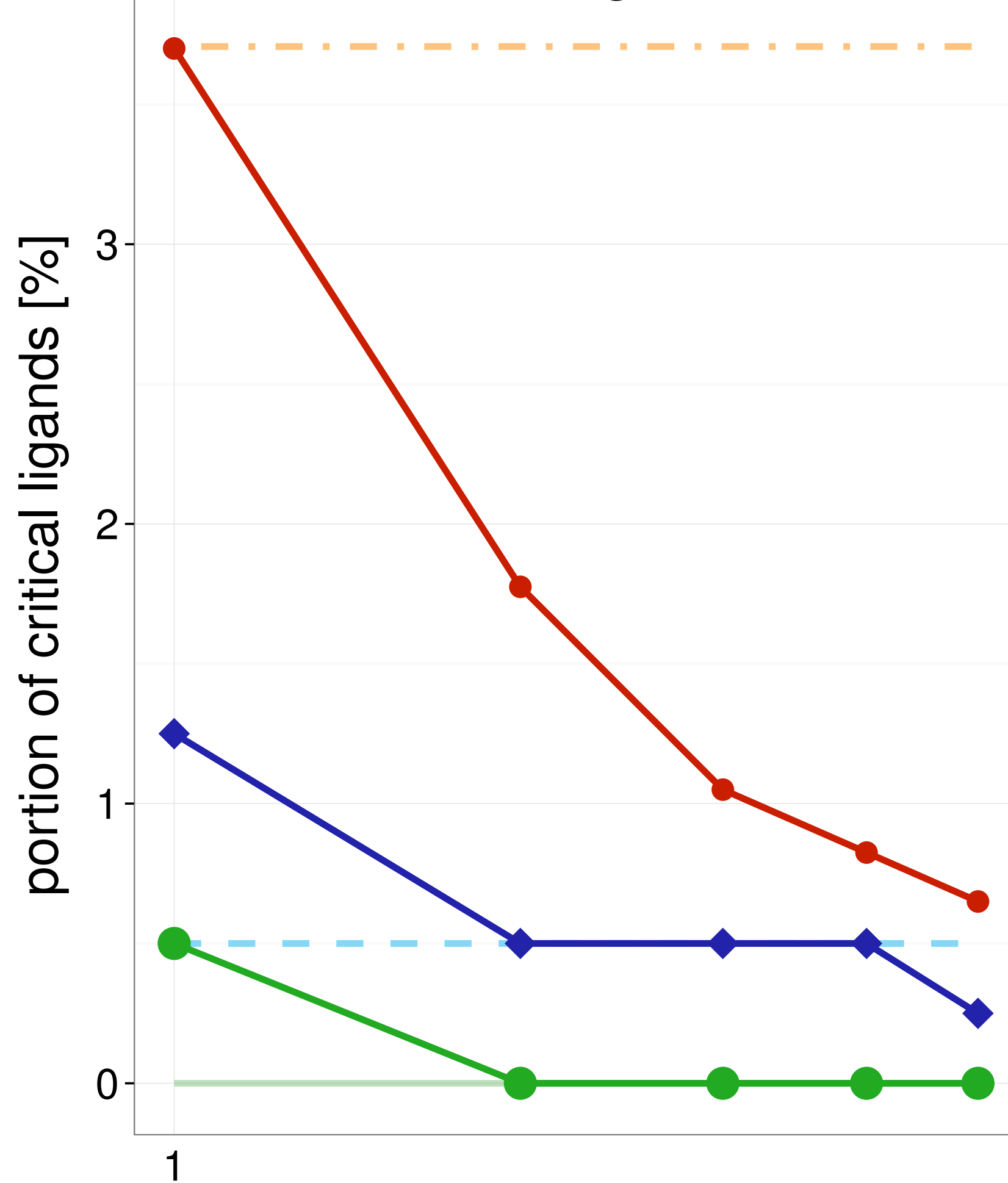

maximal ensemble size

Test set

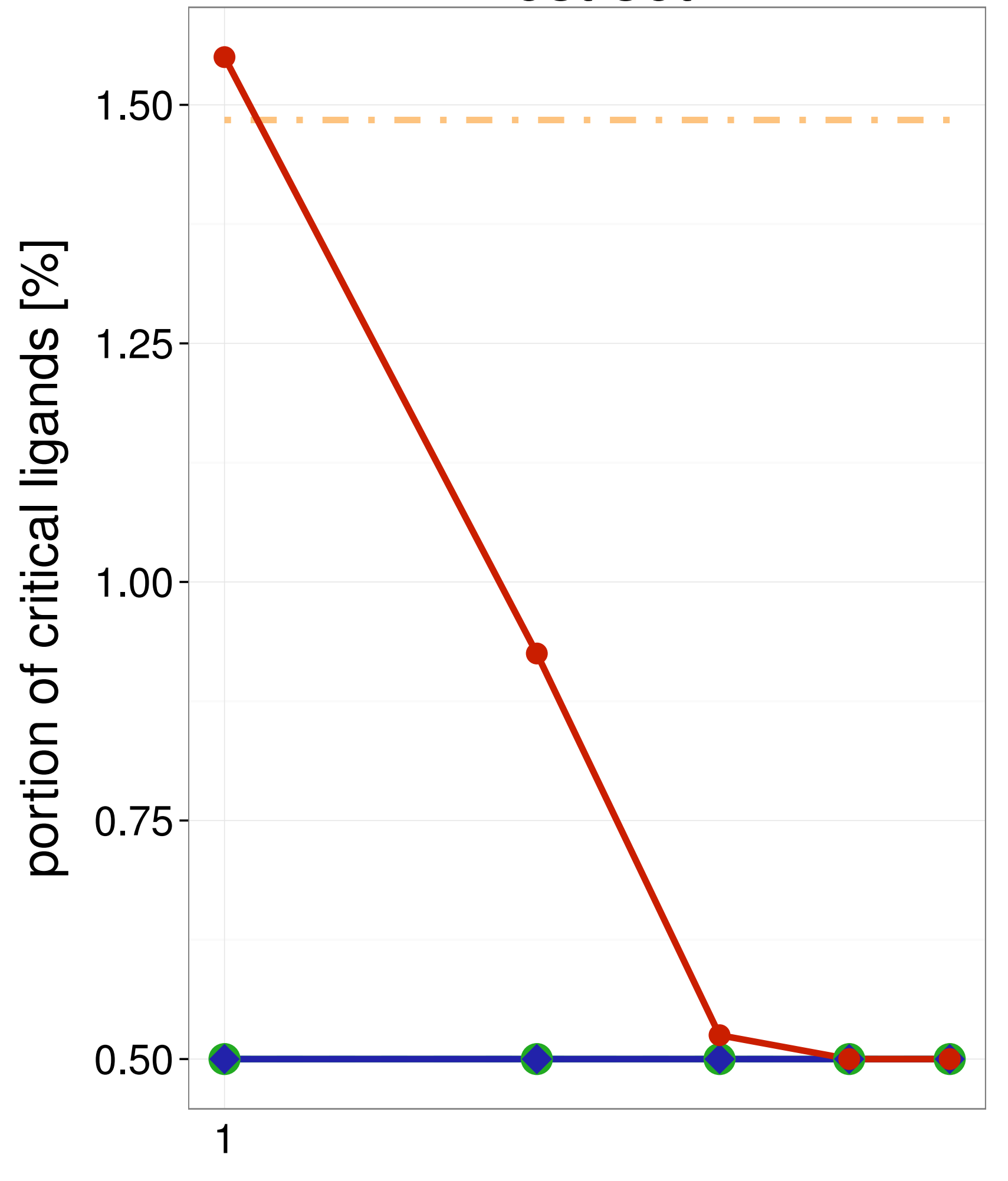

Average single structure

_ Best single structure

Whole ensemble

SIENA

$\sim$ Clustering

$\rightarrow$ Random 
Training set

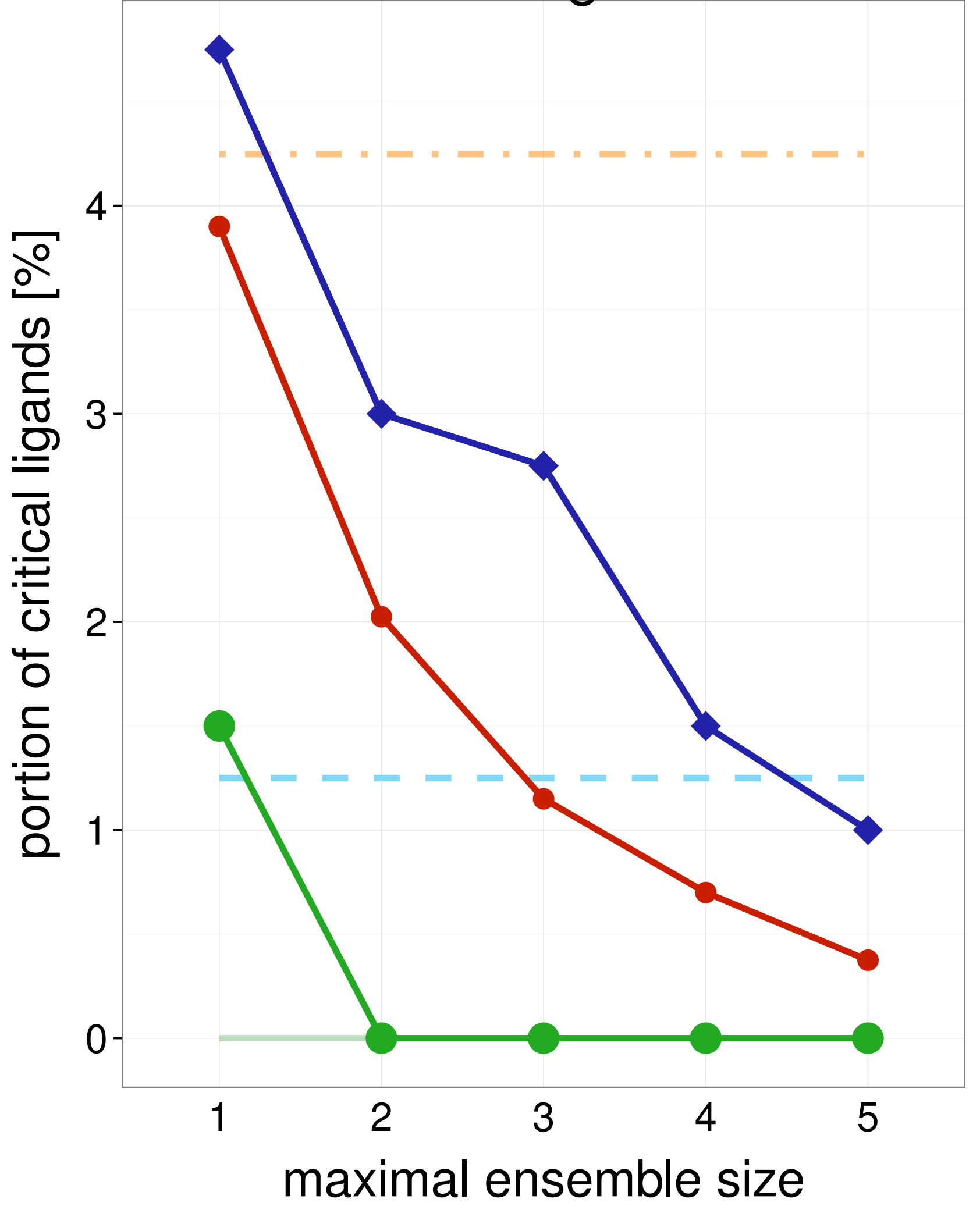

Test set

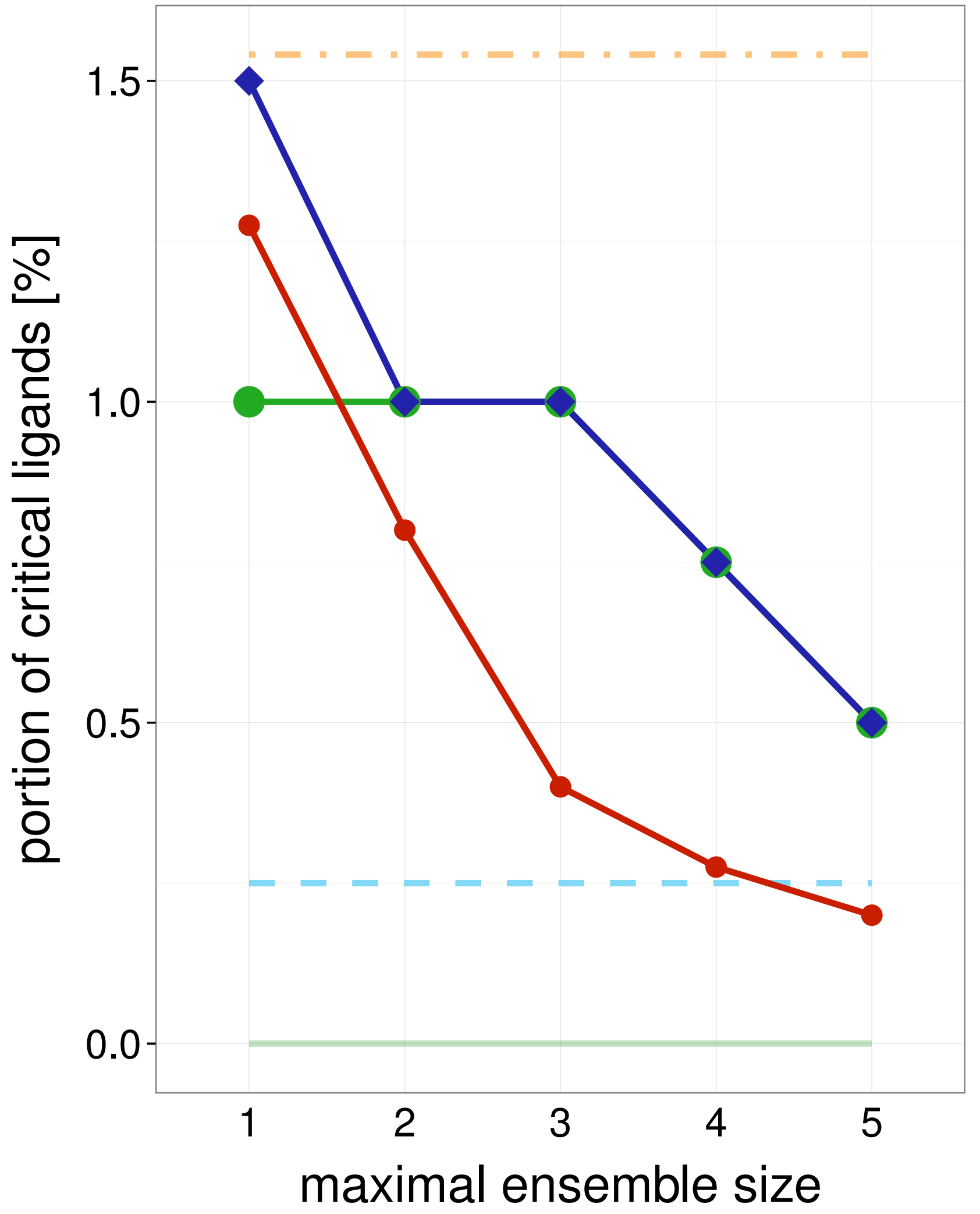

3EYG $\quad$ Training set

Average single structure

_ Best single structure

Whole ensemble

- SIENA

$\neg$ Clustering

$\multimap$ Random

Average single structure

_ _ Best single structure

Whole ensemble

SIENA

$\neg$ Clustering

$\multimap$ Random

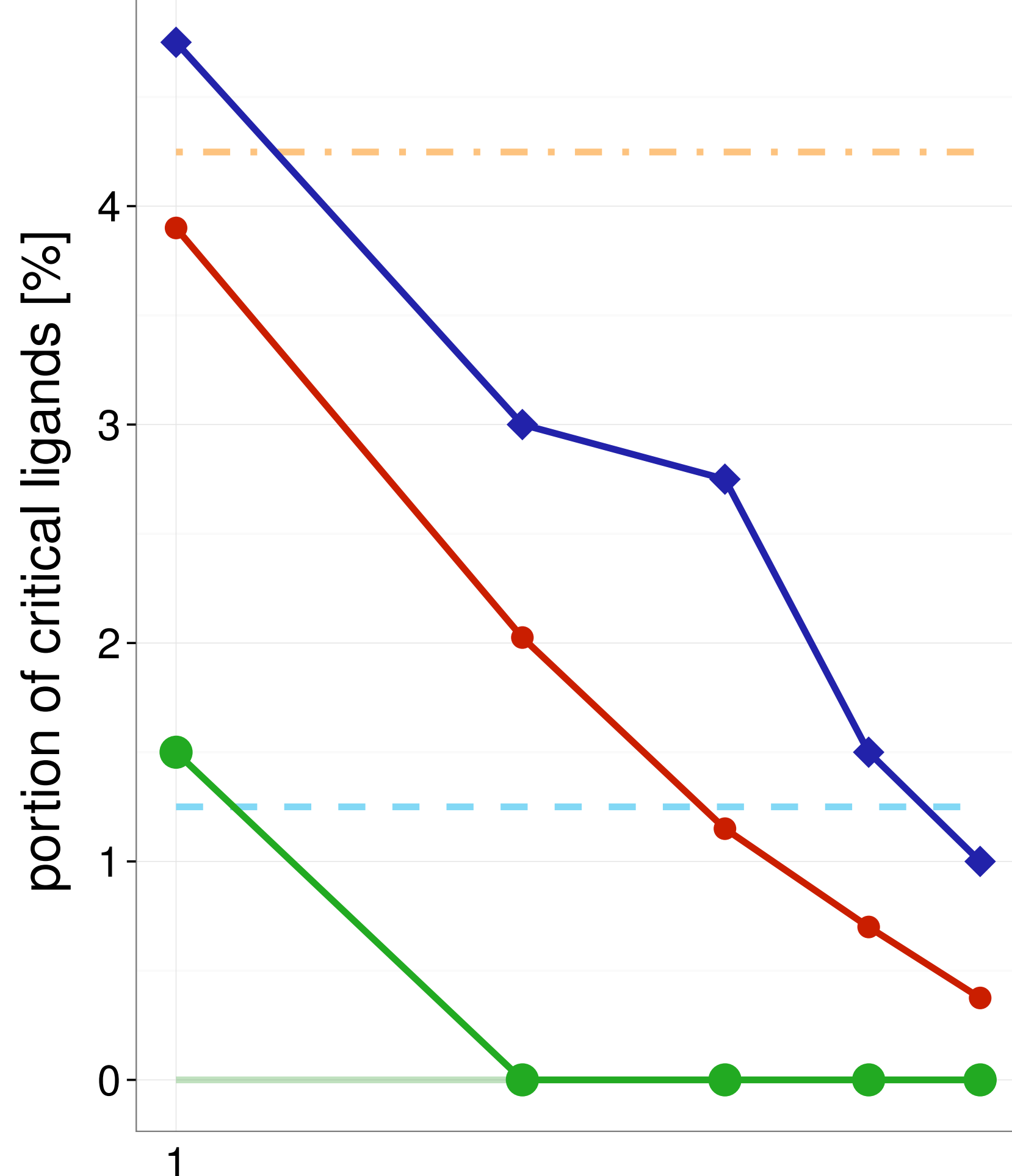

maximal ensemble size

Test set

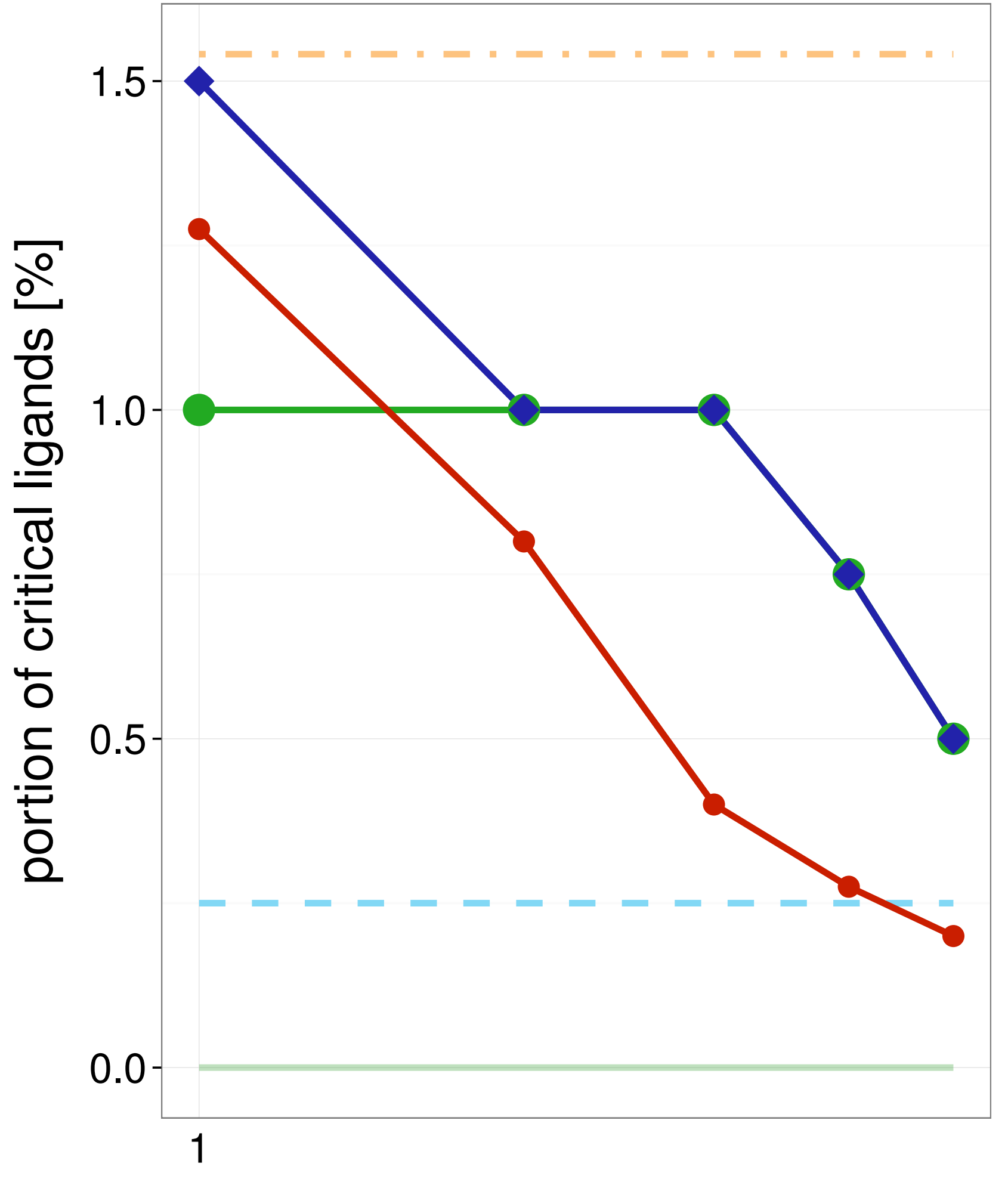

maximal ensemble size
Average single structure

_ Best single structure

Whole ensemble

SIENA

$\leadsto$ Clustering

$\rightarrow$ Random 
Training set

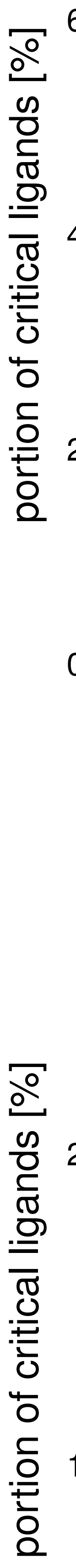

0

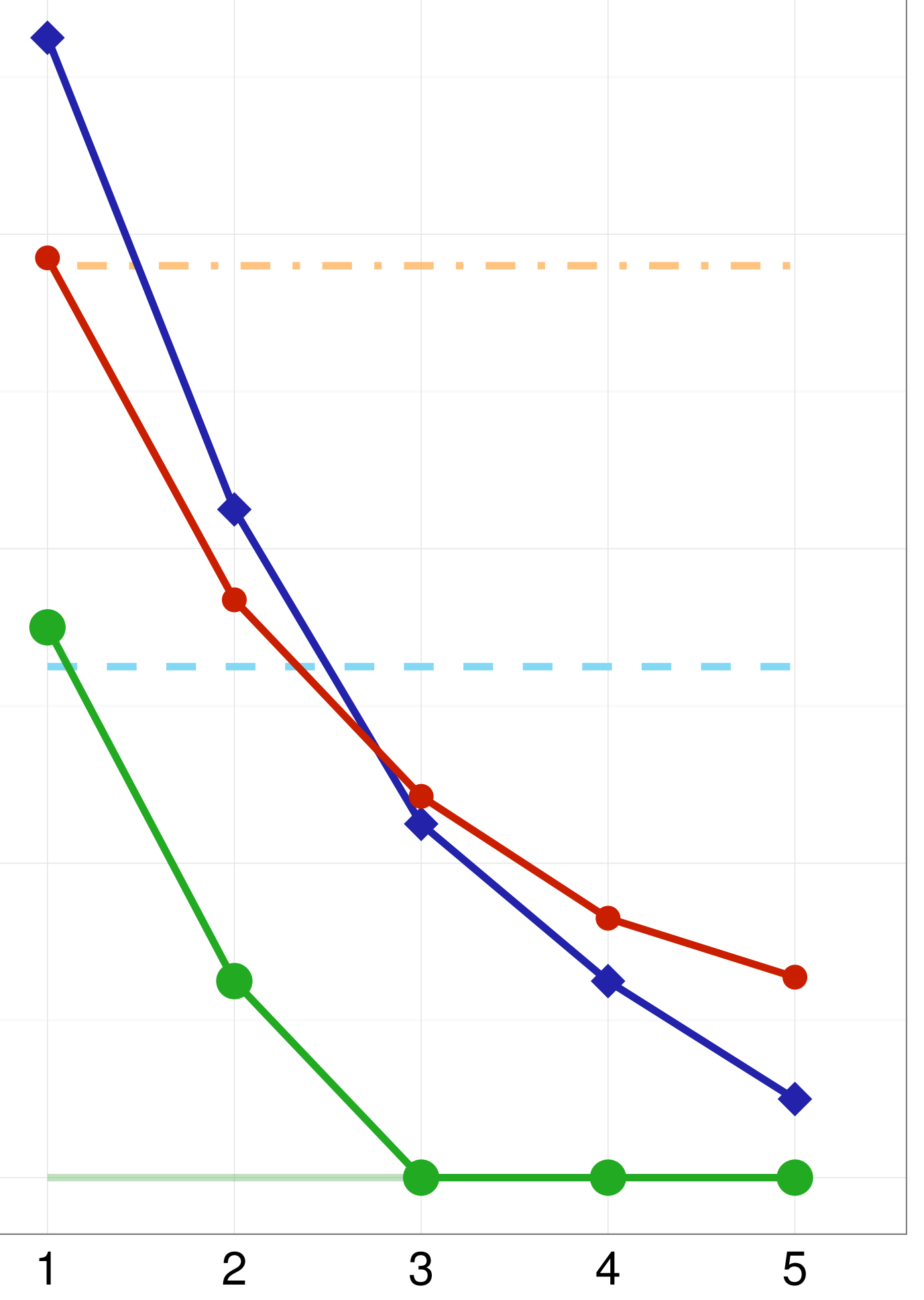

maximal ensemble size

Test set

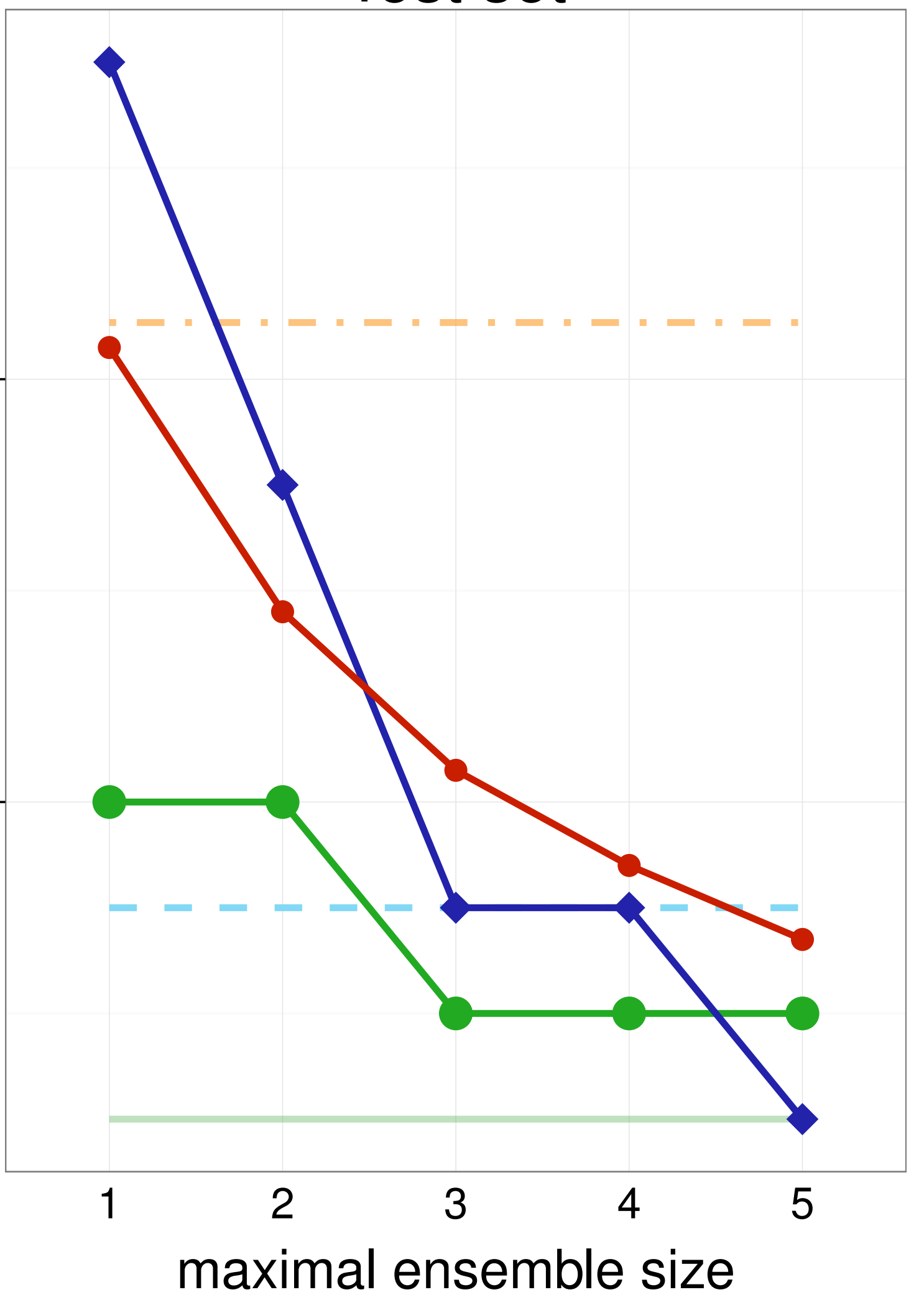

$3 \mathrm{HXE}$

Training set

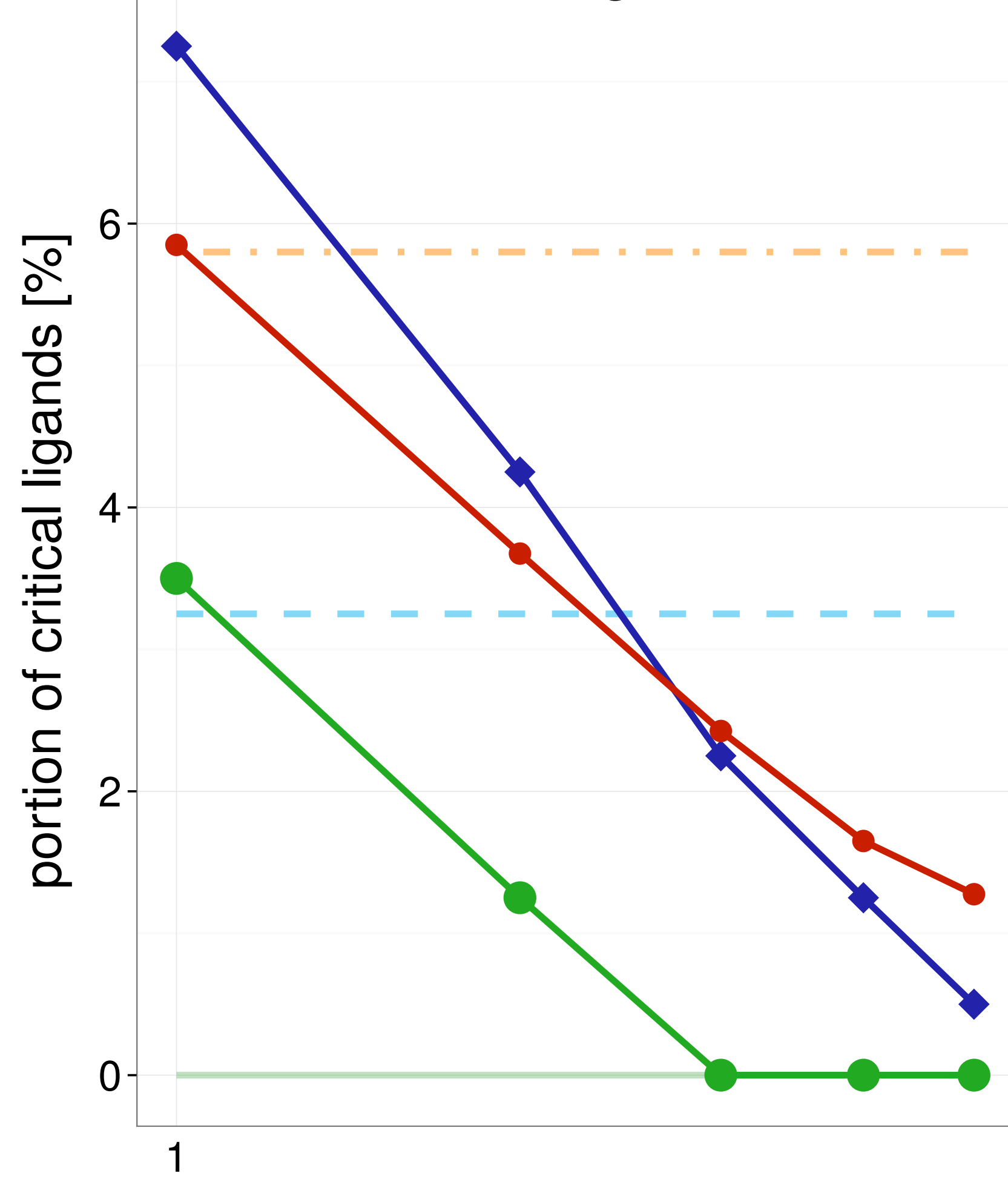

Average single structure

Best single structure

Whole ensemble

- SIENA

$\neg$ Clustering

$\longrightarrow$ Random

Average single structure

_ Best single structure

Whole ensemble

- SIENA

$\leadsto$ Clustering

$\multimap$ Random maximal ensemble size

Test set

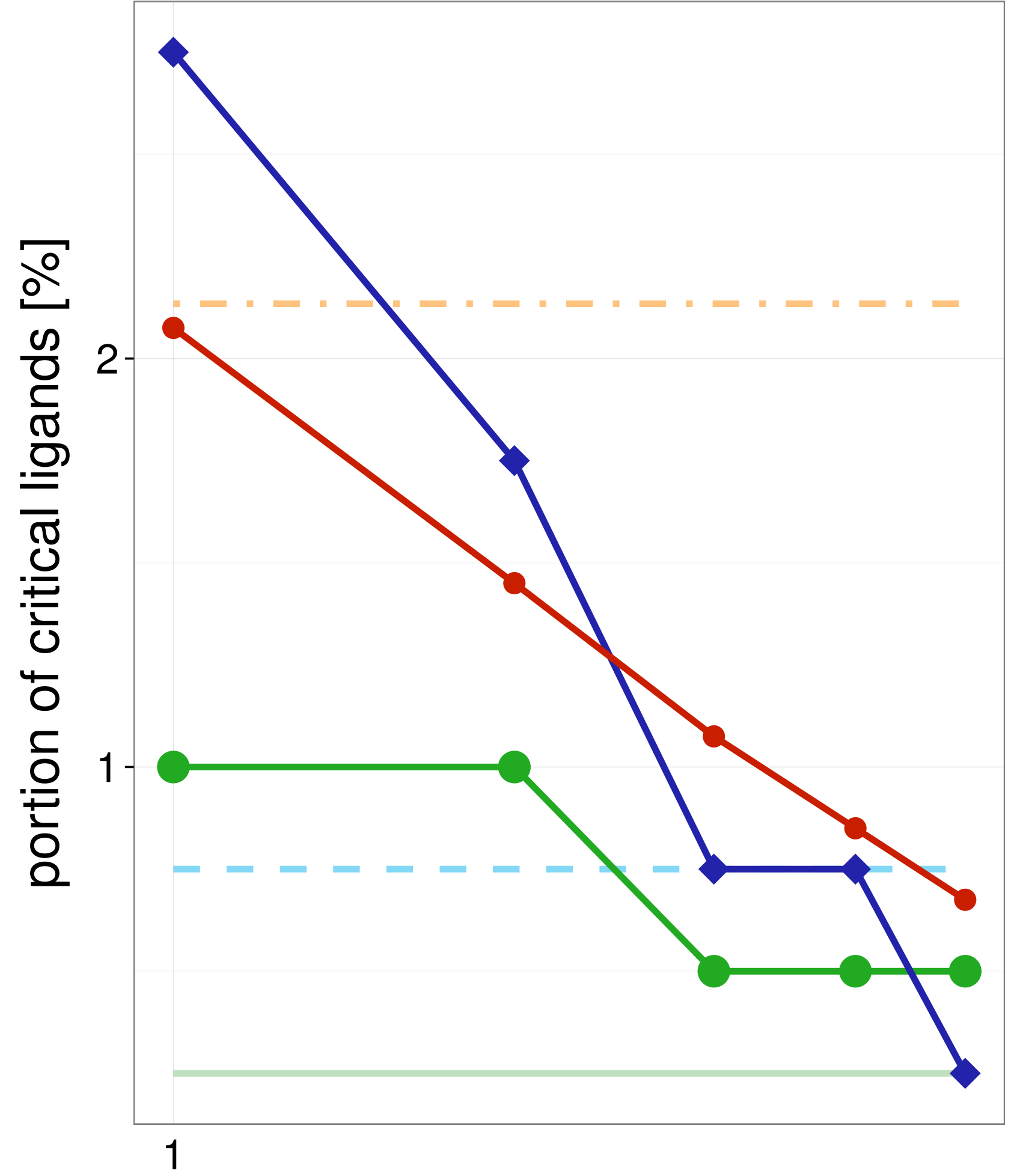

Average single structure

Best single structure

Whole ensemble

SIENA

$\leadsto$ Clustering $\multimap$ Random 
Training set

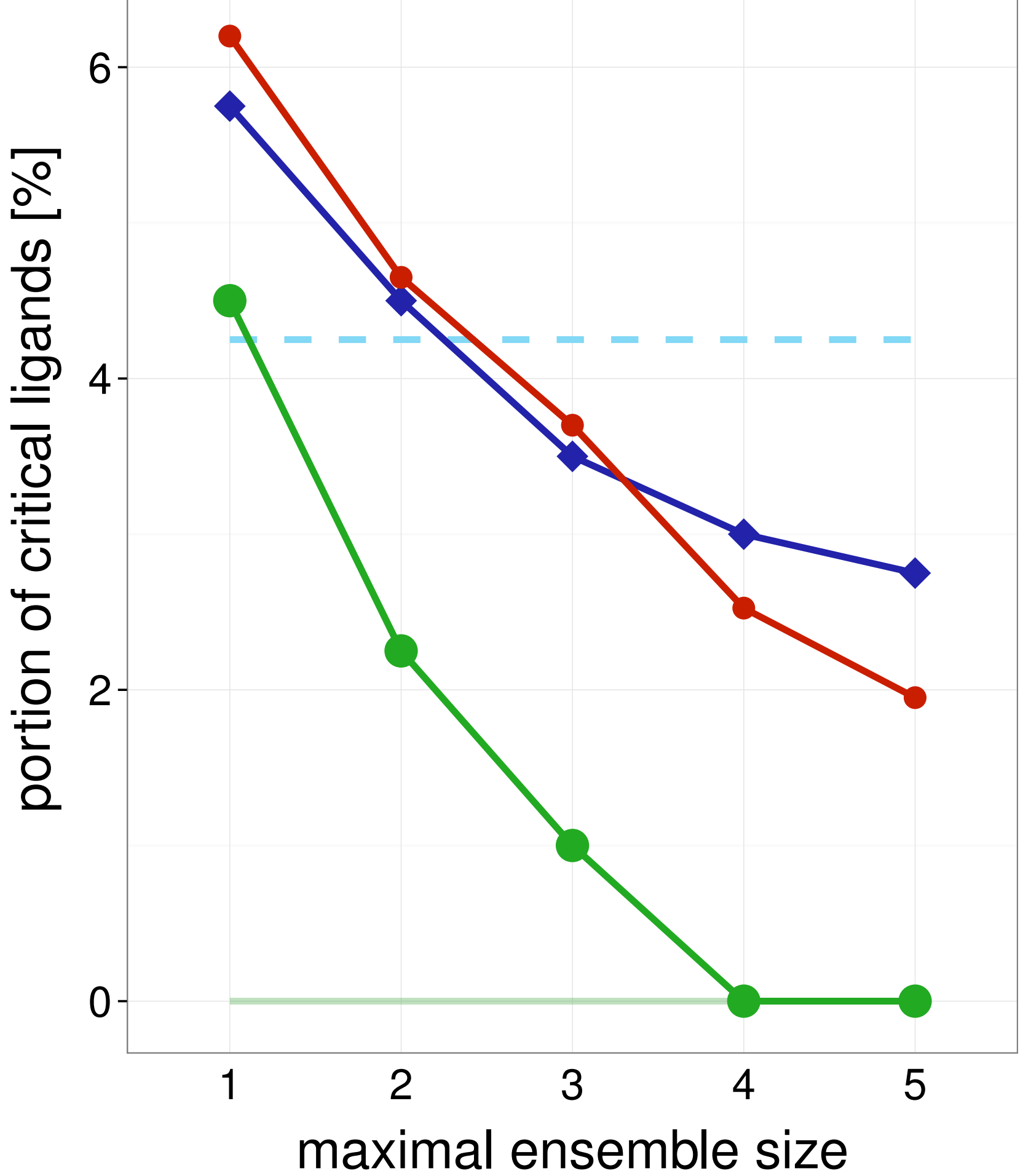

\section{Test set}

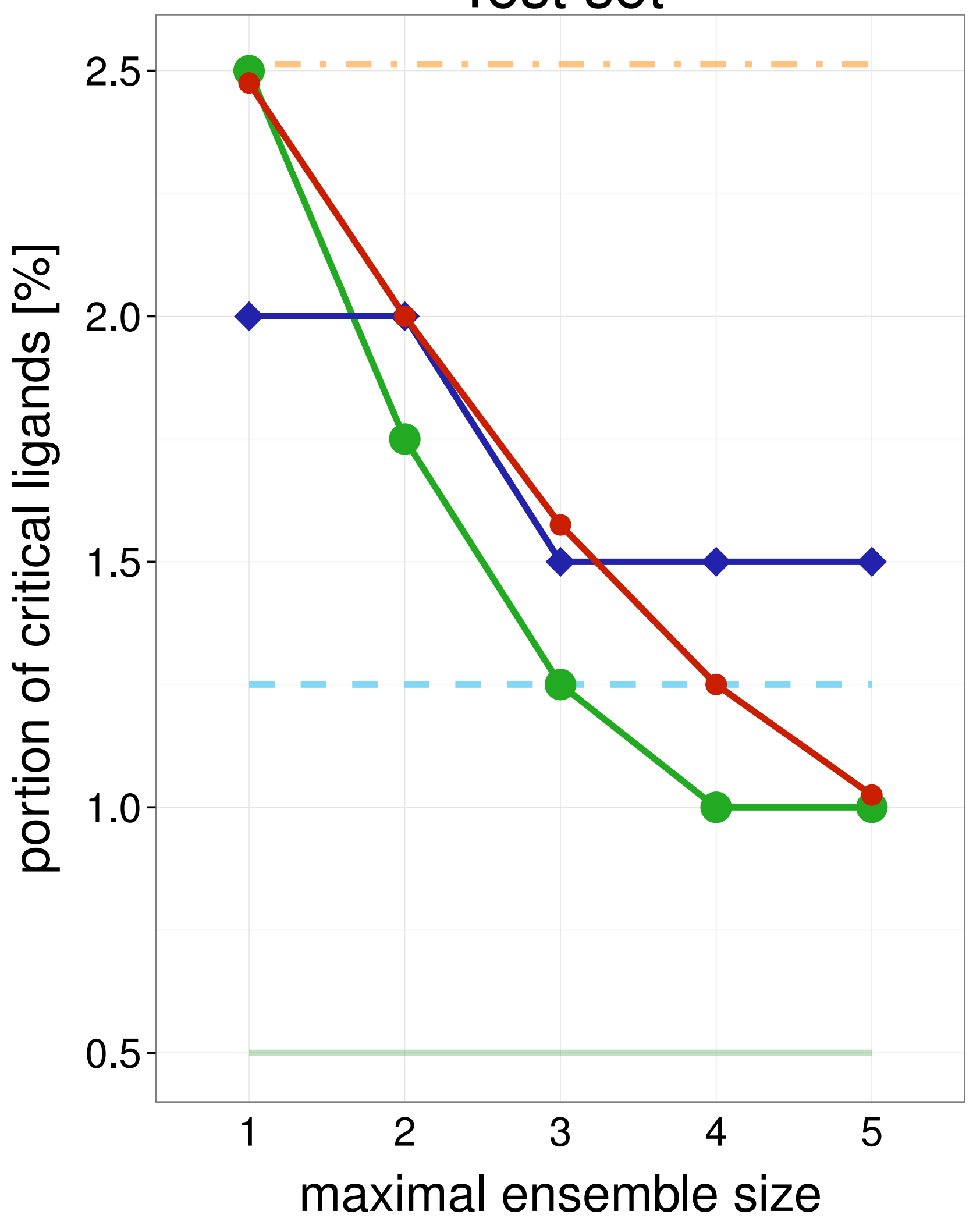

Average single structure

_ _ Best single structure

Whole ensemble

- SIENA

$\neg$ Clustering

$\multimap$ Random

Average single structure

_ _ Best single structure

Whole ensemble

- SIENA

$\neg$ Clustering

$\multimap$ Random

Test set

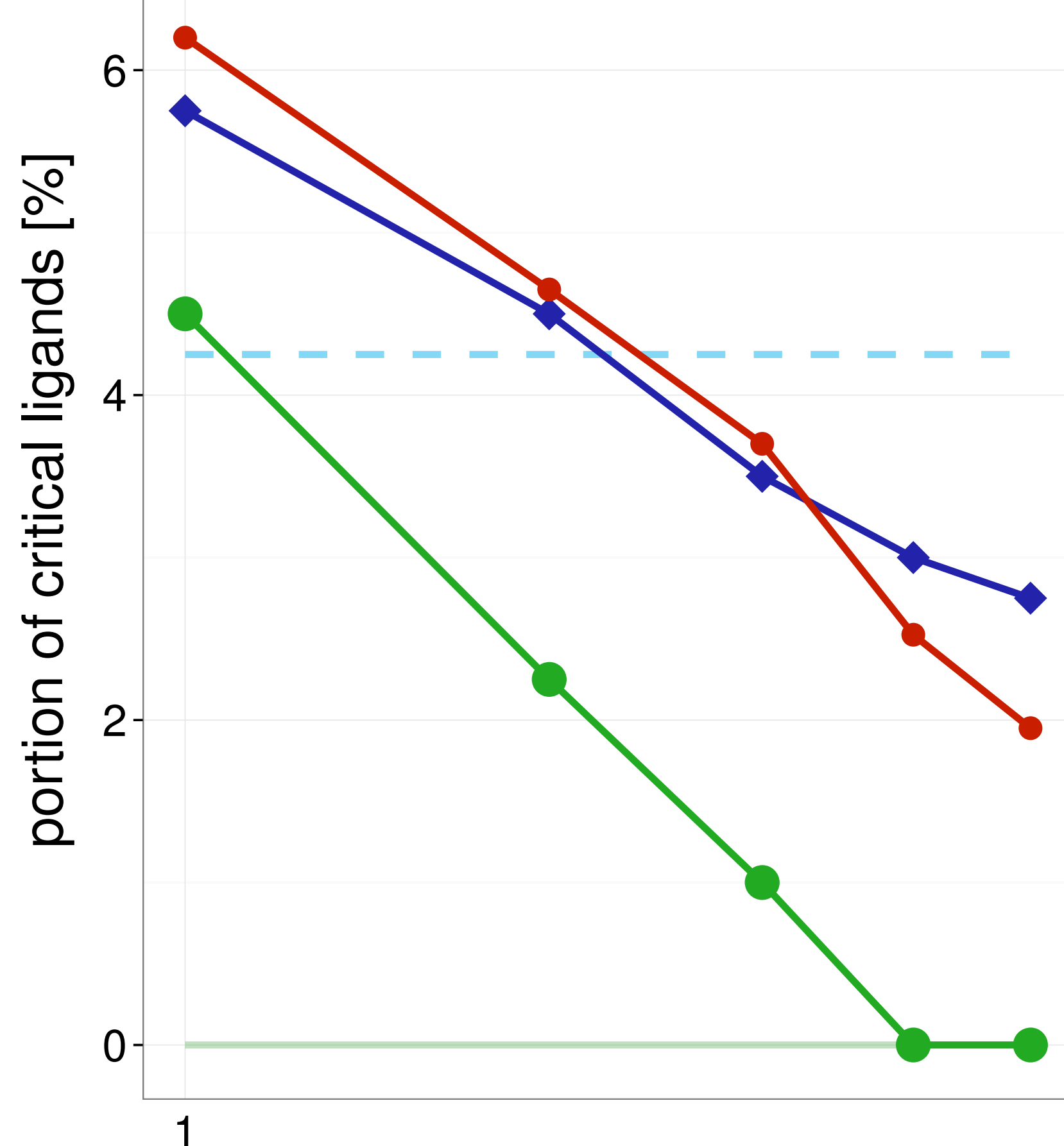

maximal ensemble size

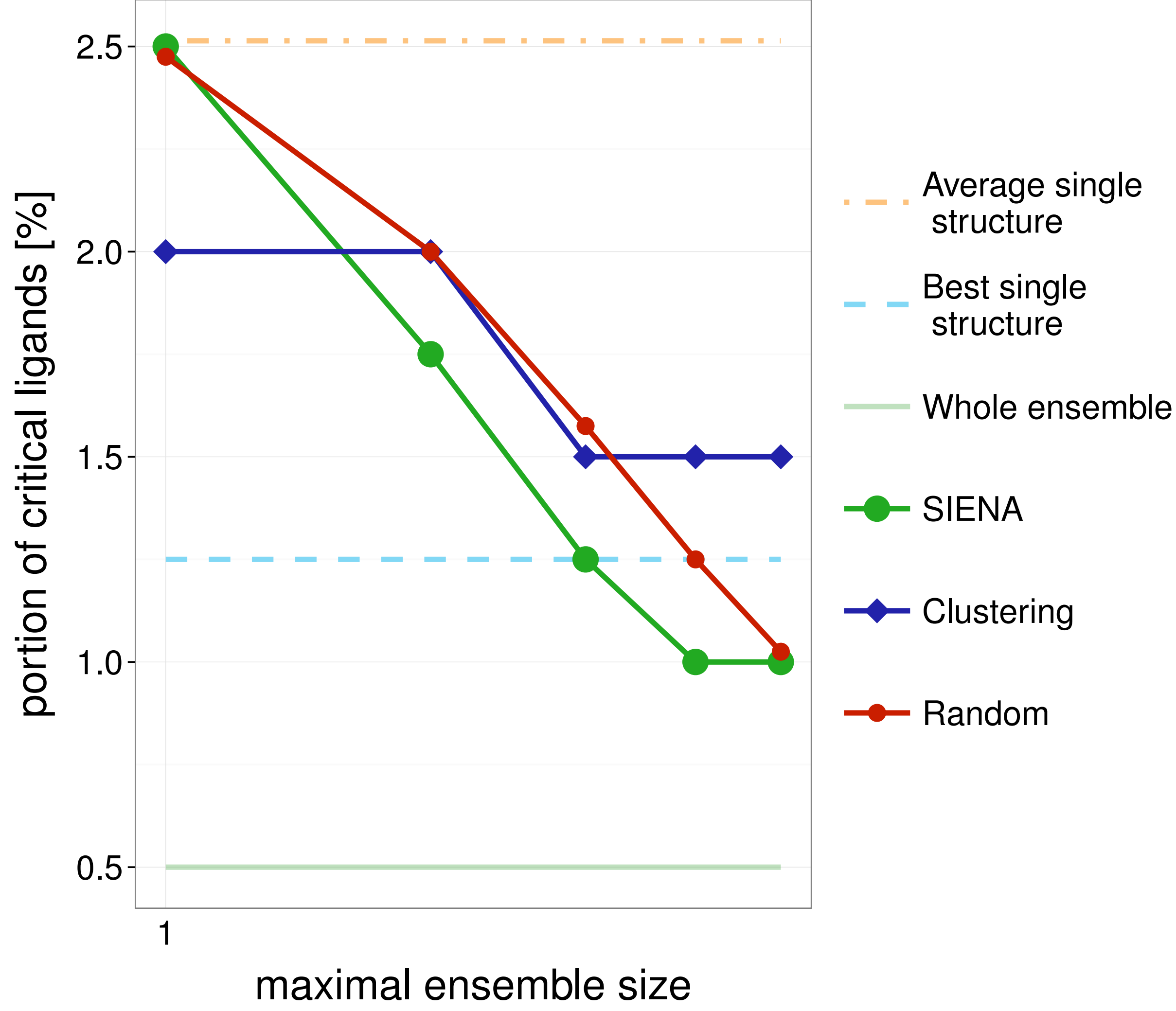




\section{Training set}

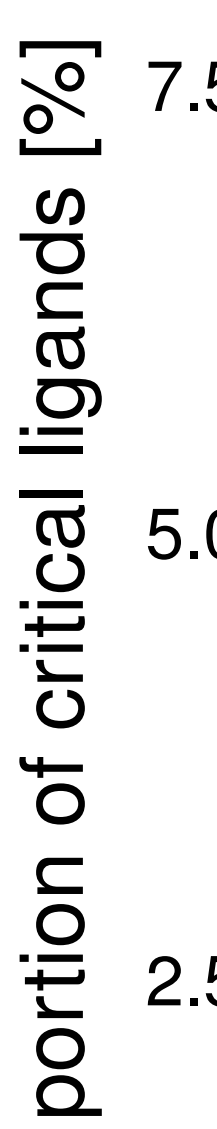

0.0 1

\section{maximal ensemble size}

Test set

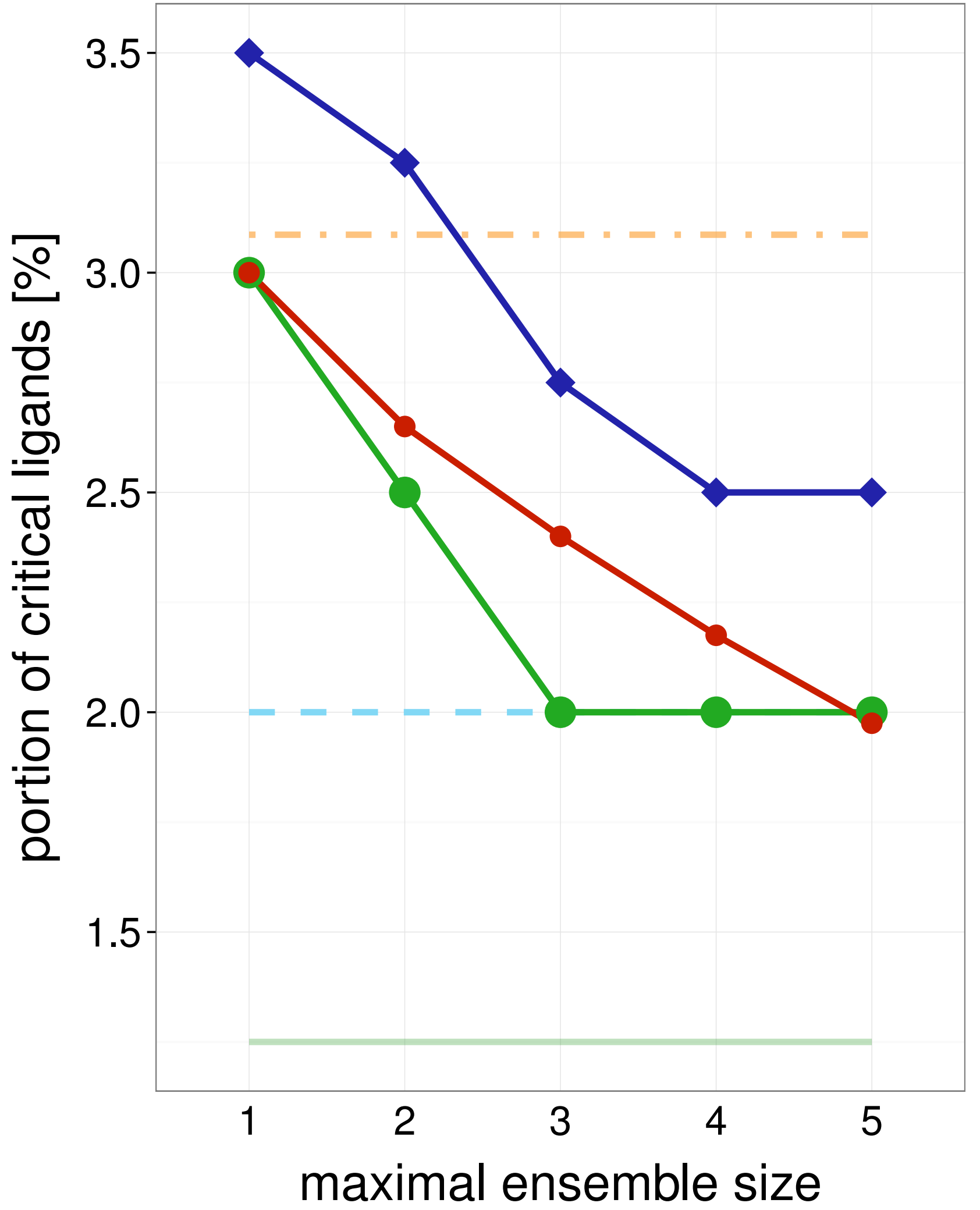

Average single structure

_ Best single structure

Whole ensemble

- SIENA

$\neg$ Clustering

$\multimap$ Random

Average single structure

_ Best single structure

Whole ensemble

- SIENA

$\checkmark$ Clustering

- Random
Training set

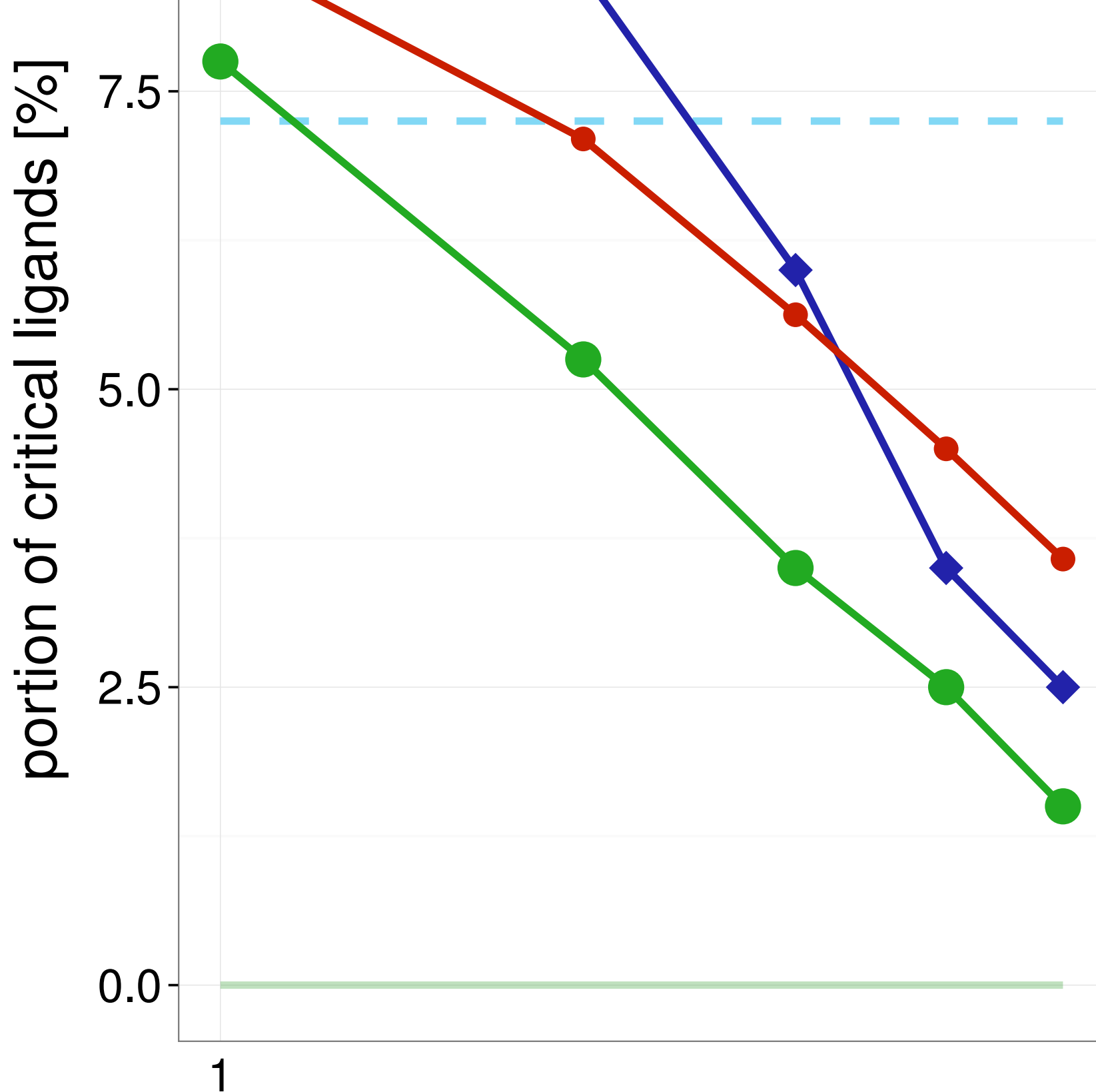

maximal ensemble size

Test set

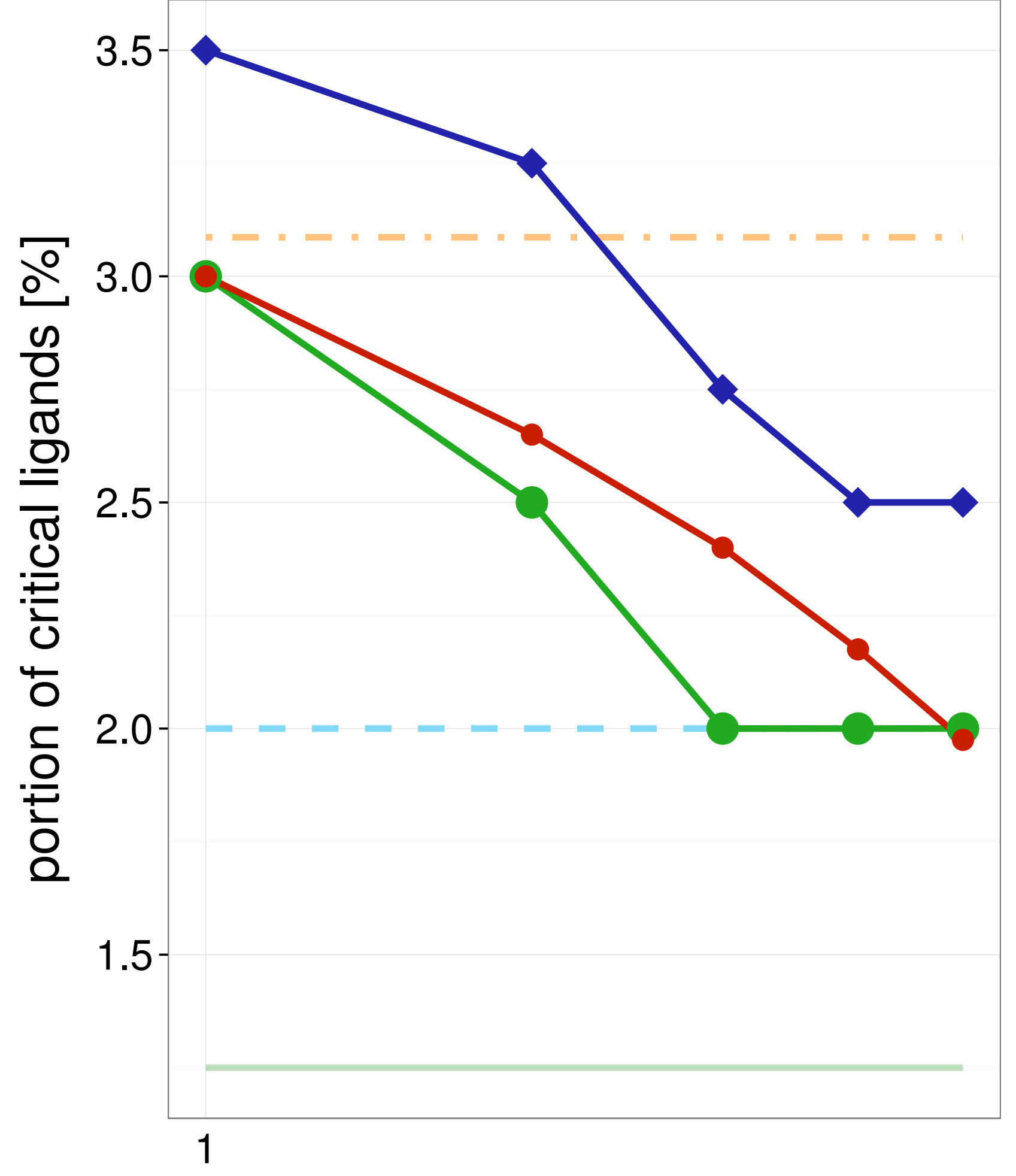

maximal ensemble size
Average single structure

_ Best single structure

Whole ensemble

SIENA

$\neg$ Clustering

$\multimap$ Random 
Training set

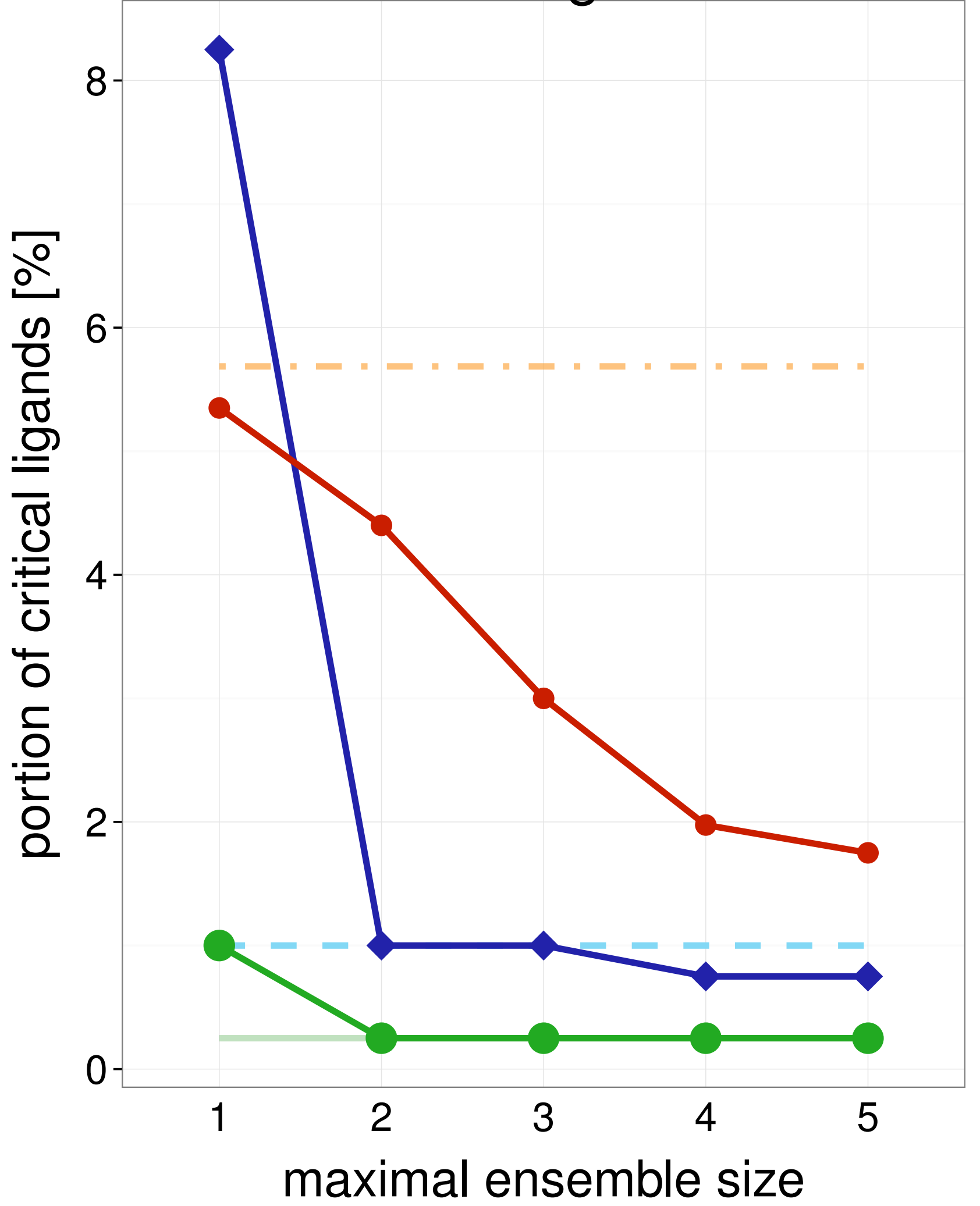

Test set

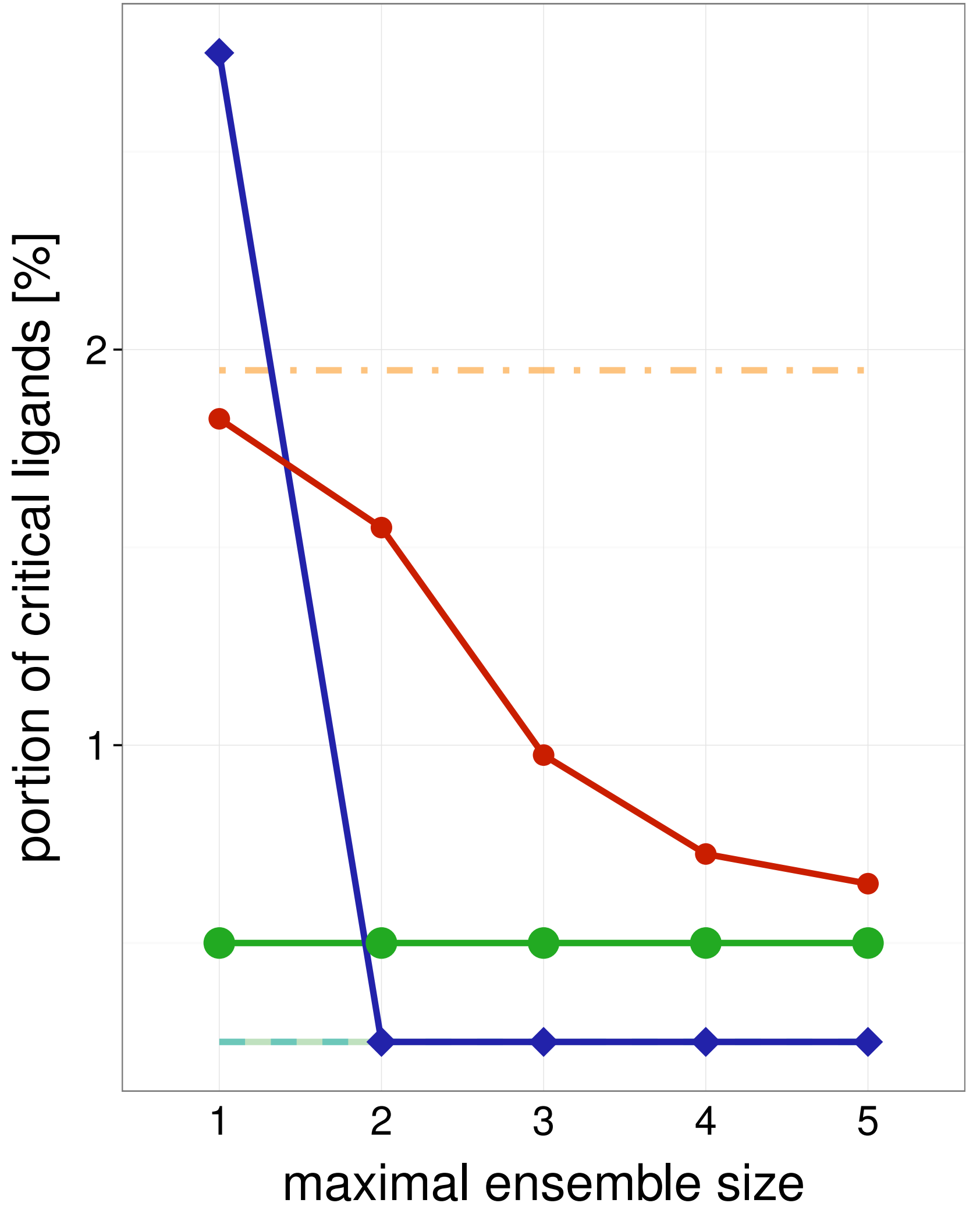

Average single structure

_ Best single structure

Whole ensemble

- SIENA

$\neg$ Clustering

$\multimap$ Random

Average single structure

_ _ Best single structure

Whole ensemble

- SIENA

$\smile$ Clustering

$\multimap$ Random
$3 \mathrm{PCl}$

Training set

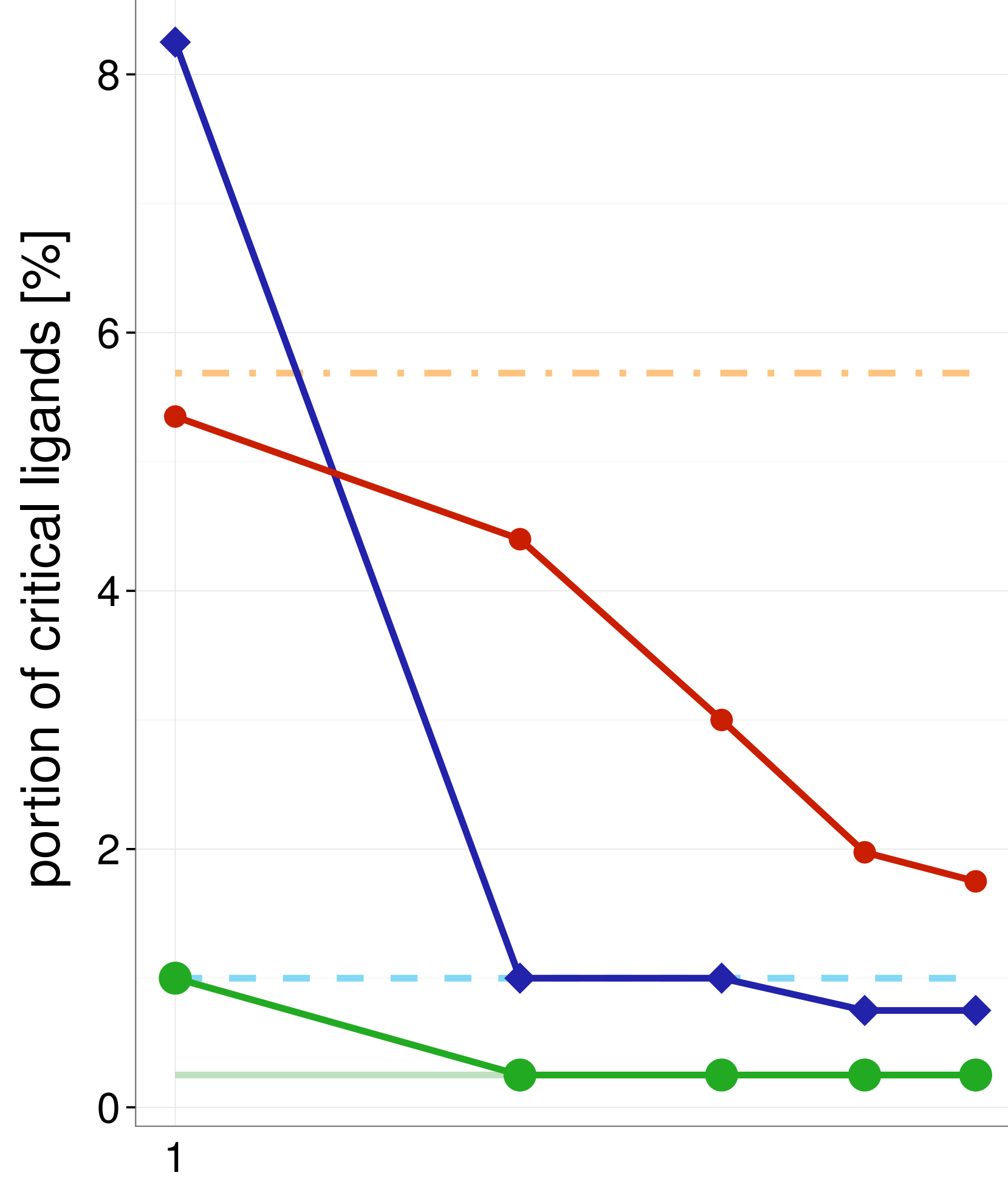

maximal ensemble size

Test set

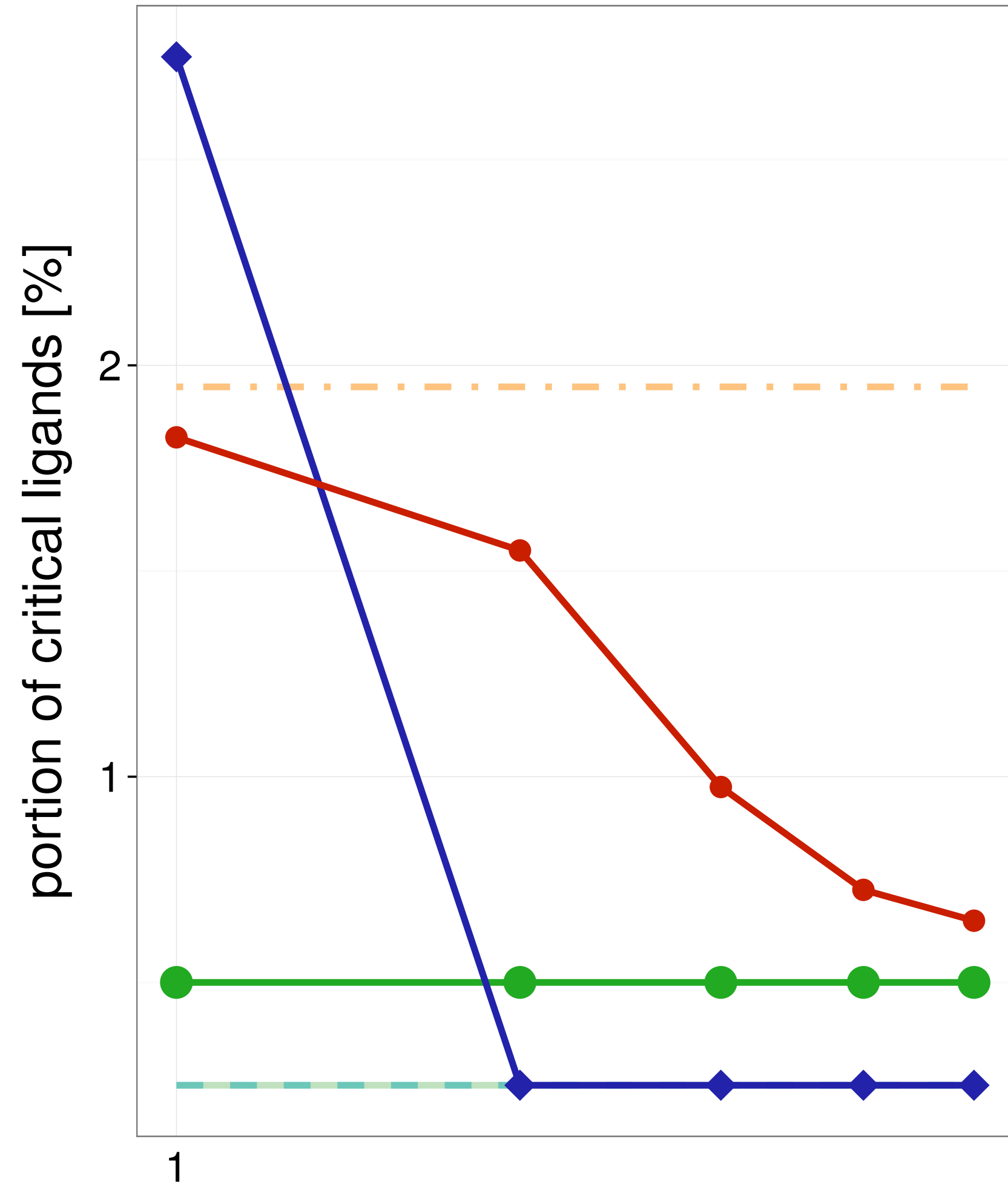

Average single structure

Best single structure

Whole ensemble

SIENA

$\leadsto$ Clustering

$\multimap$ Random 


\section{Training set}

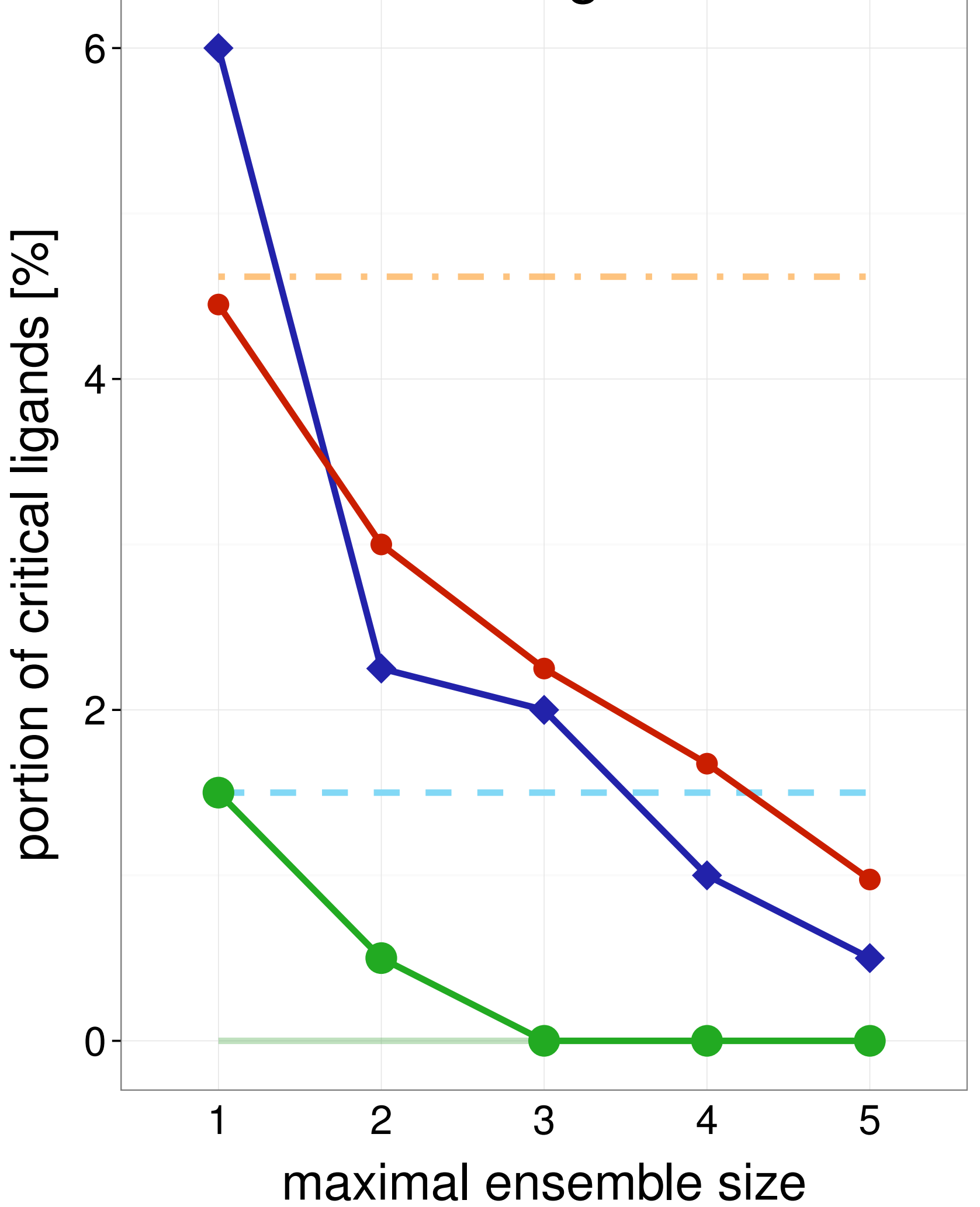

Test set

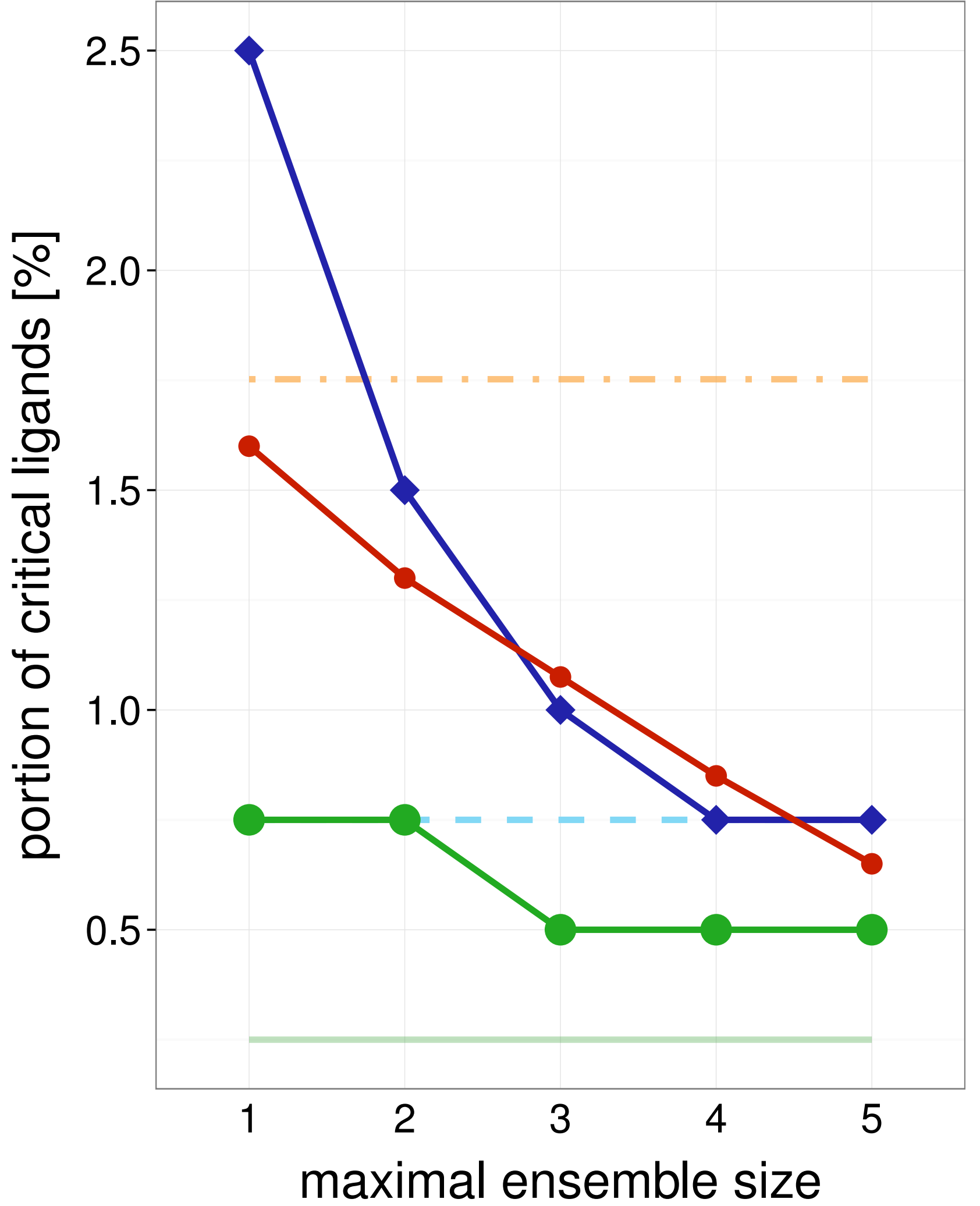

Average single structure

_ Best single structure

Whole ensemble

- SIENA

$\neg$ Clustering

$\multimap$ Random

Average single structure

_ _ Best single structure

Whole ensemble

- SIENA

$\smile$ Clustering

$\multimap$ Random
Training set

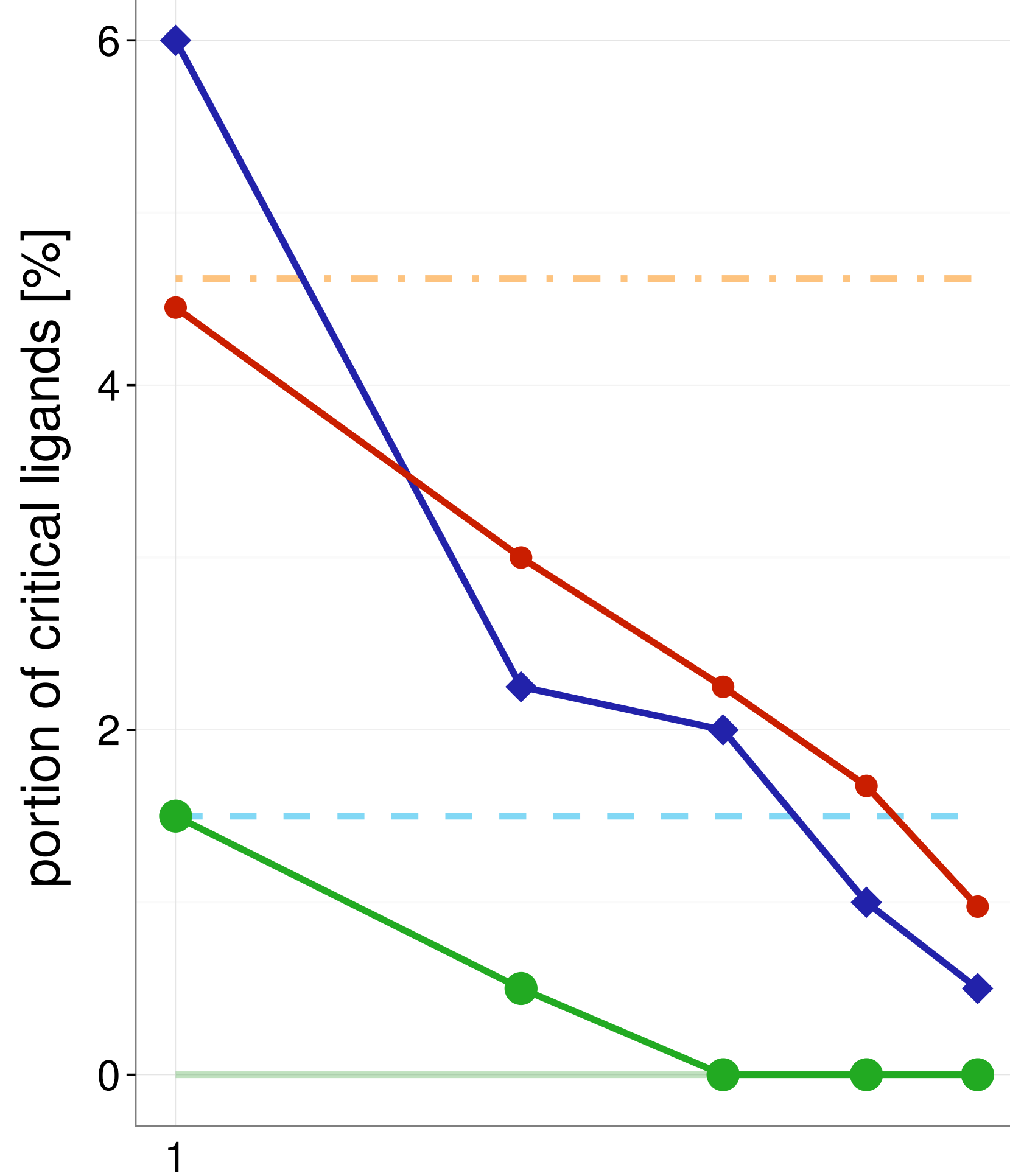

maximal ensemble size

Test set

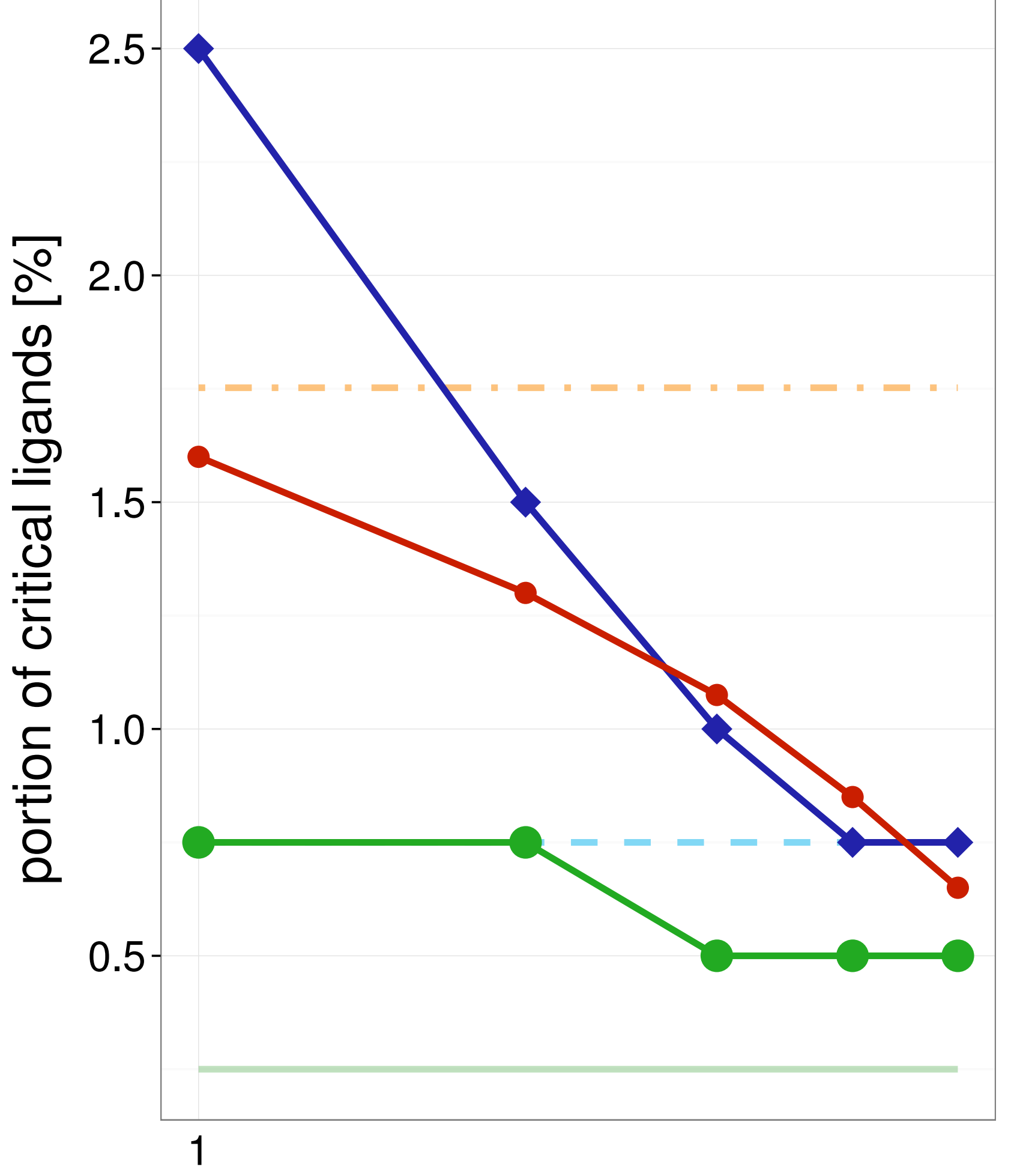

Average single structure

Best single structure

Whole ensemble

SIENA

$\sim$ Clustering

$\multimap$ Random 
Training set

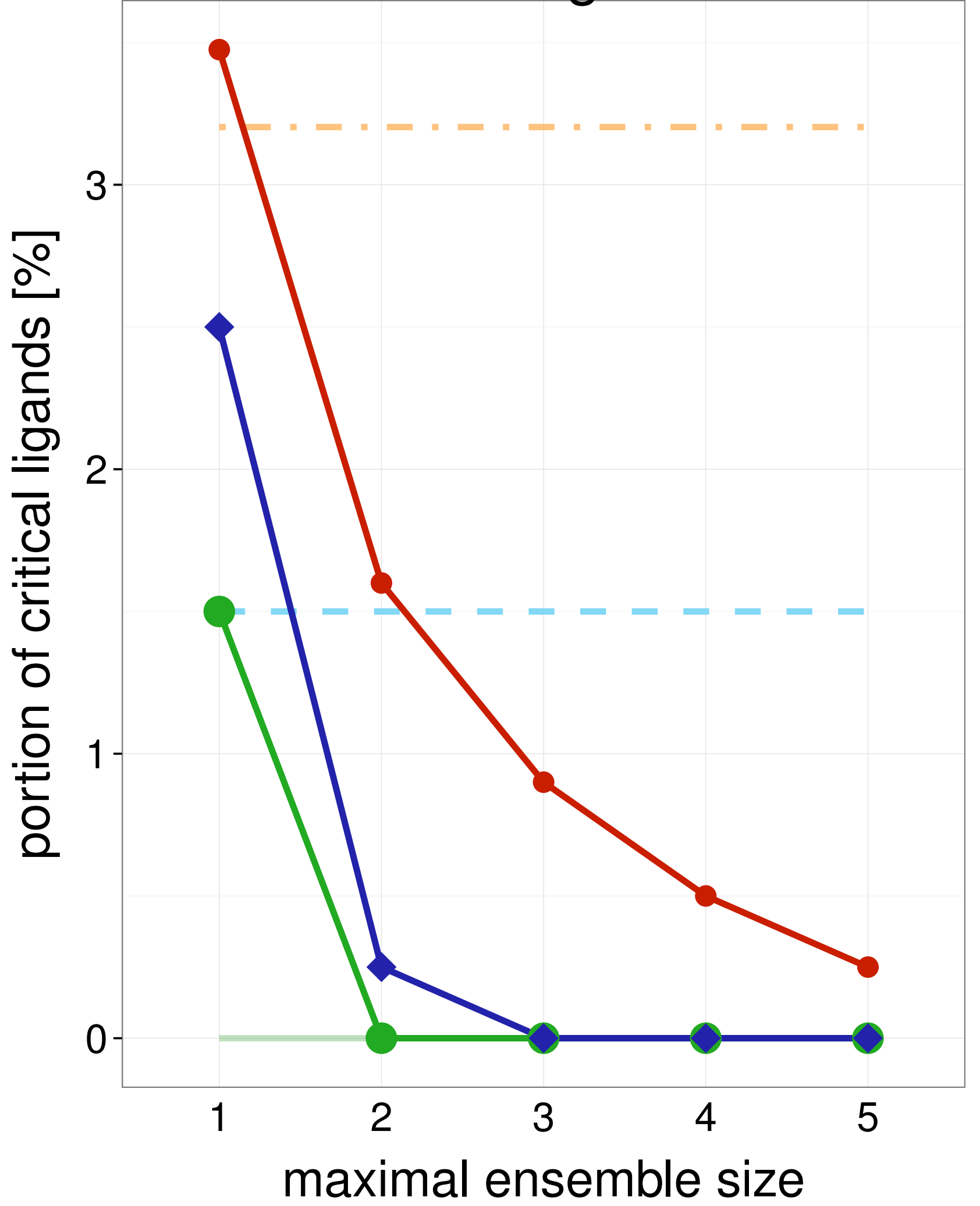

Test set

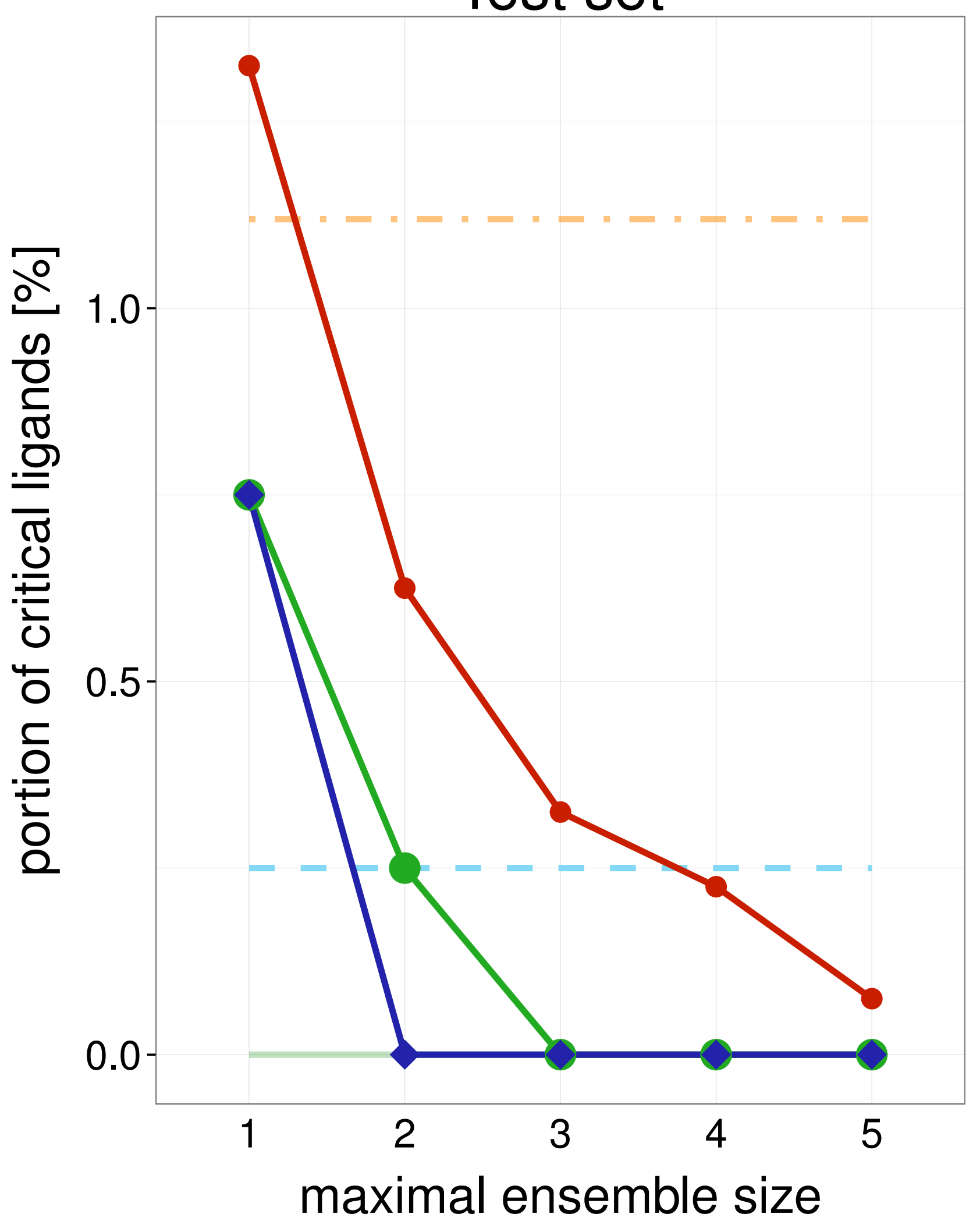

4A6L

Training set

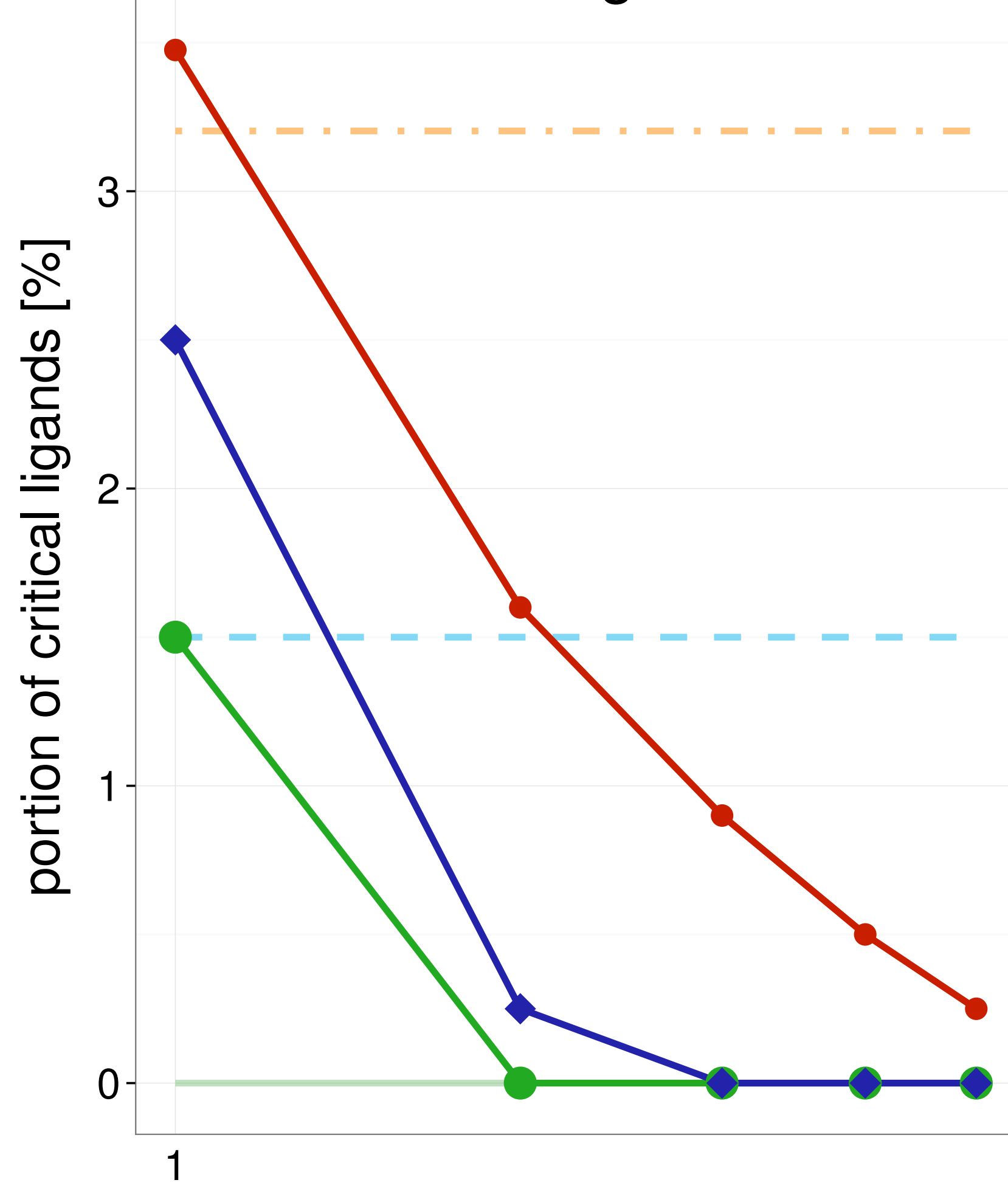

maximal ensemble size

Test set

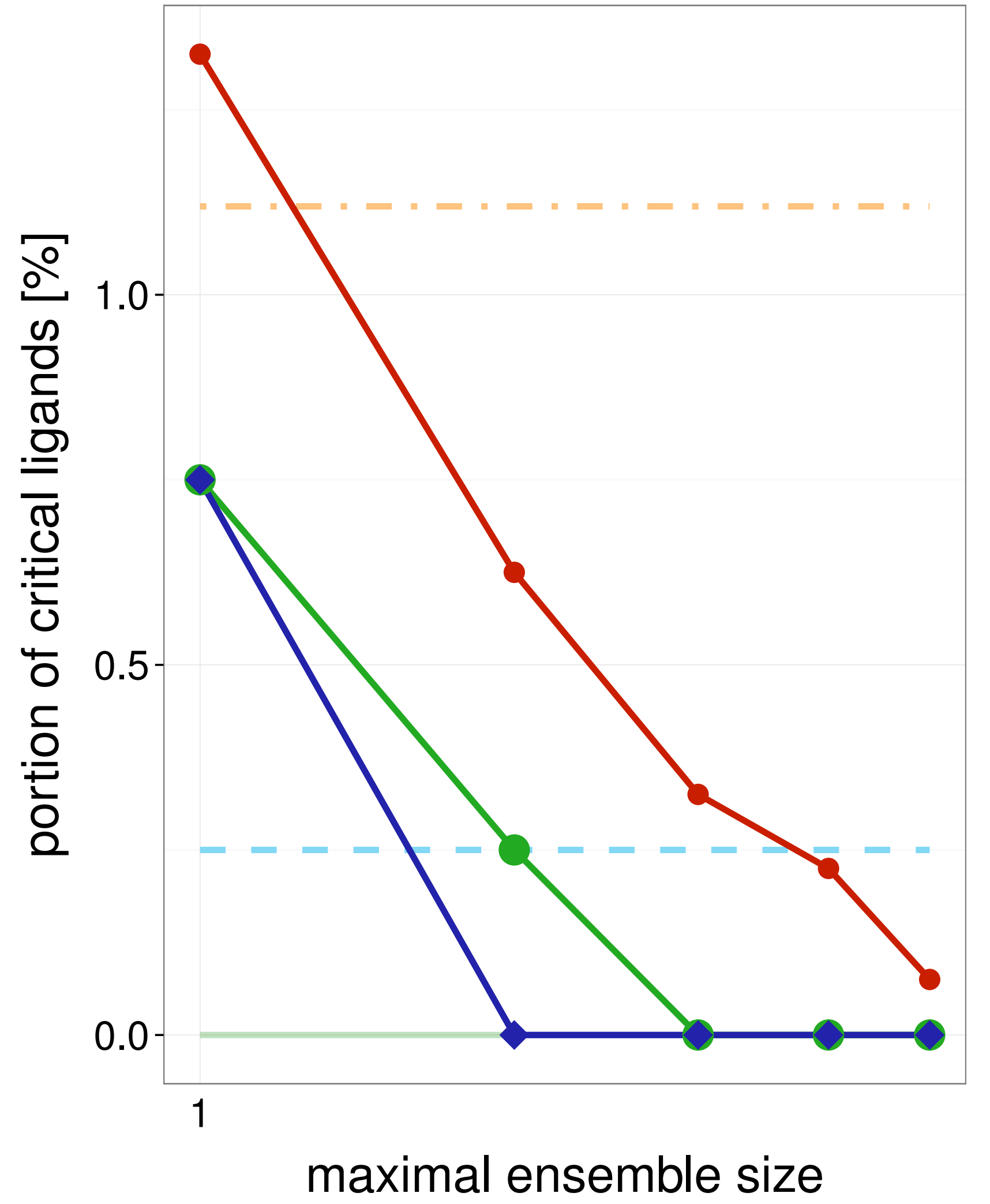

Average single structure

_ Best single structure

Whole ensemble

- SIENA

$\neg$ Clustering

$\multimap$ Random

Average single structure

Average single structure

_ _ Best single structure

Whole ensemble

- SIENA

$\smile$ Clustering

$\multimap$ Random
Best single structure

Whole ensemble

SIENA

$\neg$ Clustering

$\multimap$ Random 
Training set

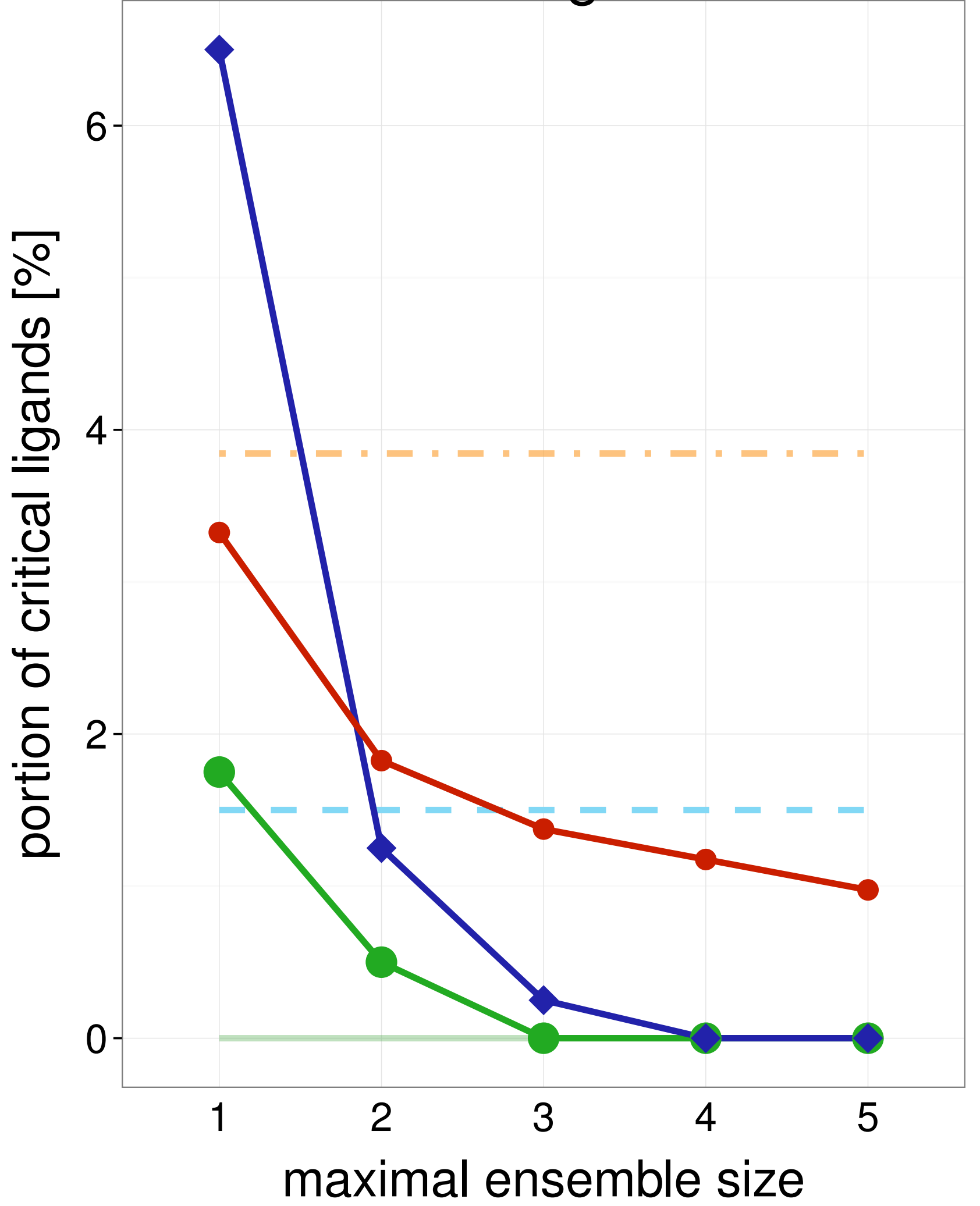

Test set

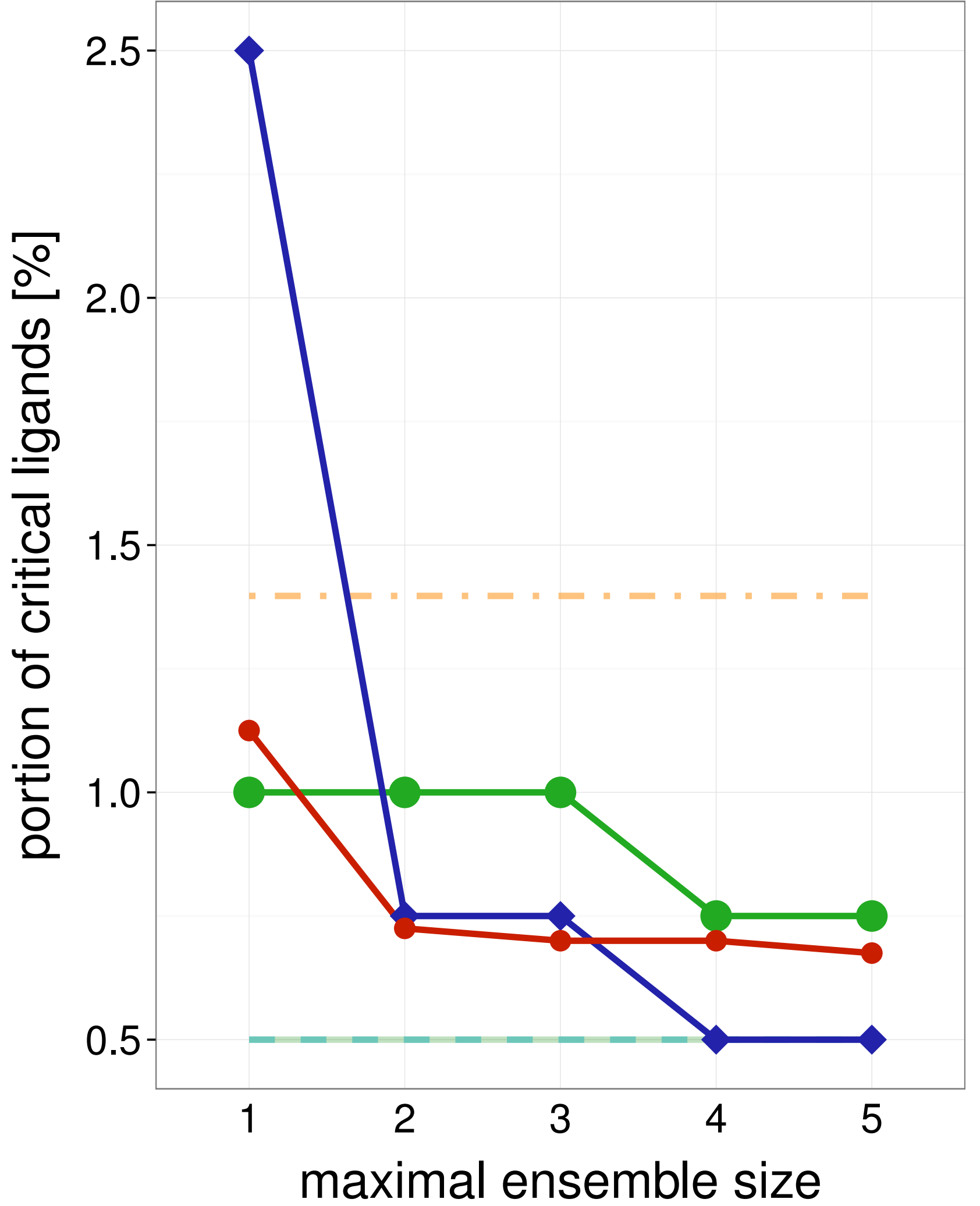

4AJN

Training set

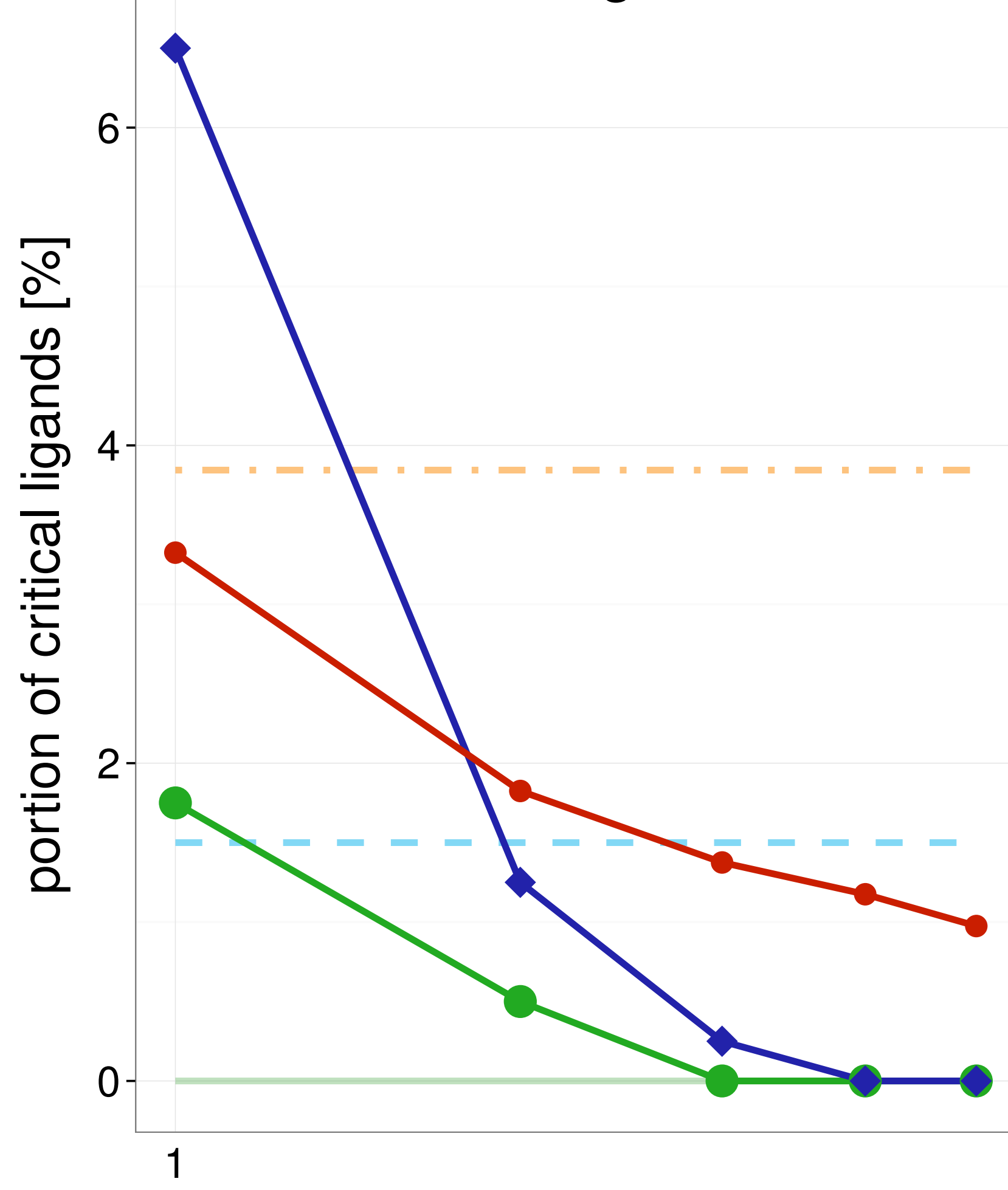

Average single structure

_ Best single structure

Whole ensemble

- SIENA

$\checkmark$ Clustering

$\neg$ Clustering

$\rightarrow$ Random

maximal ensemble size

Test set

Average single structure

_ _ Best single structure

Whole ensemble

- SIENA

$\neg$ Clustering

$\multimap$ Random

Average single structure

Best single structure

Whole ensemble

SIENA

Clustering

— Random

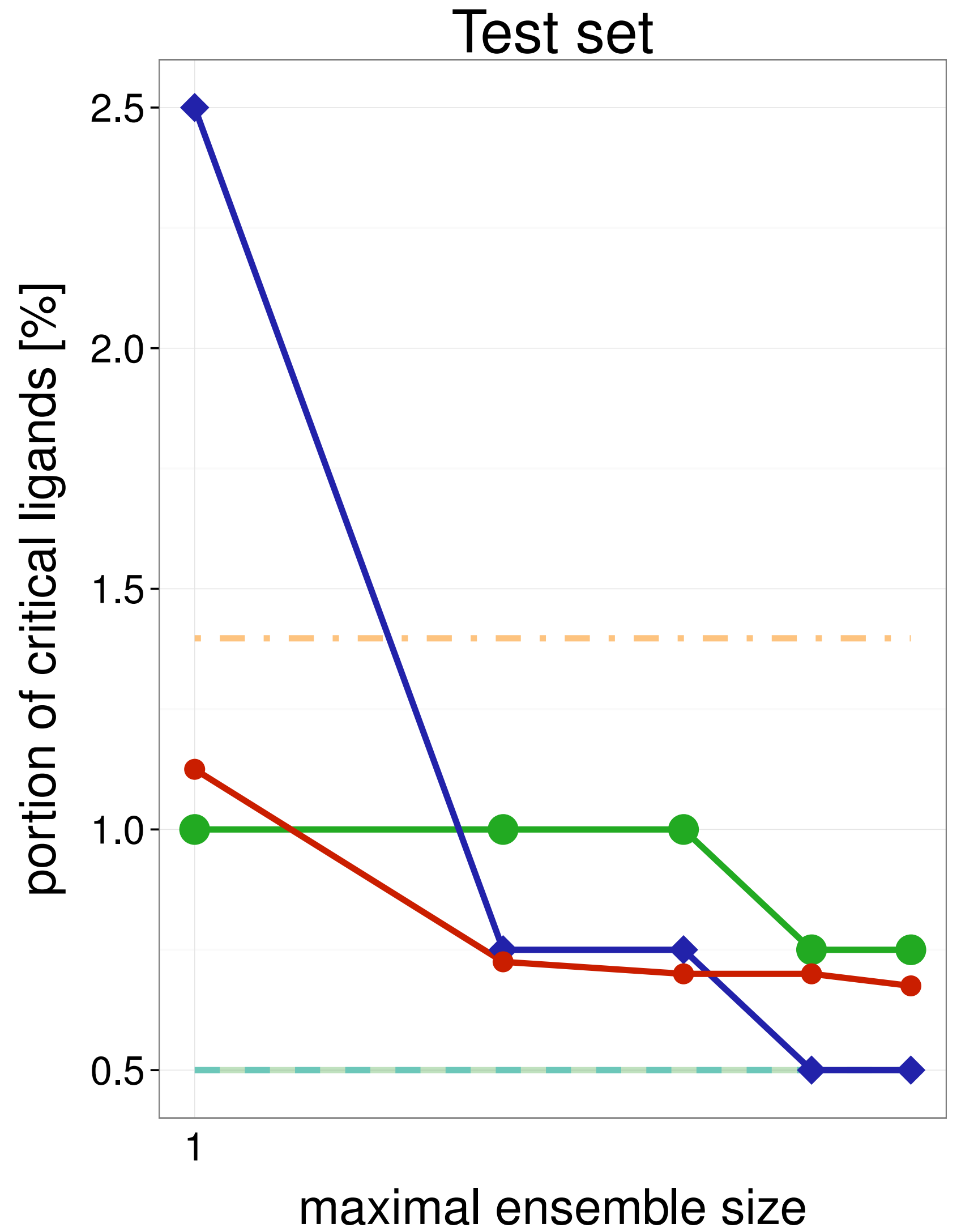


Training set

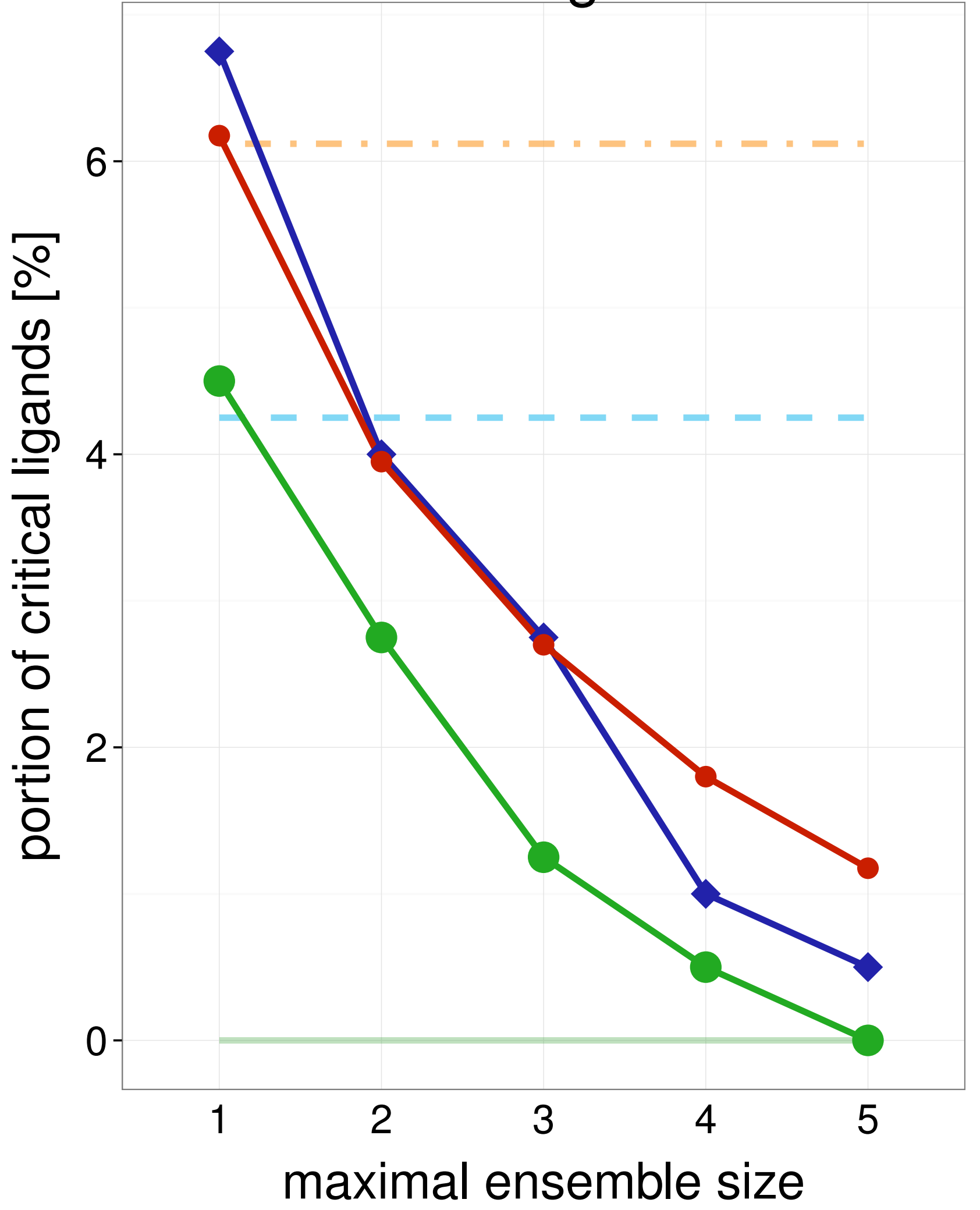

Test set

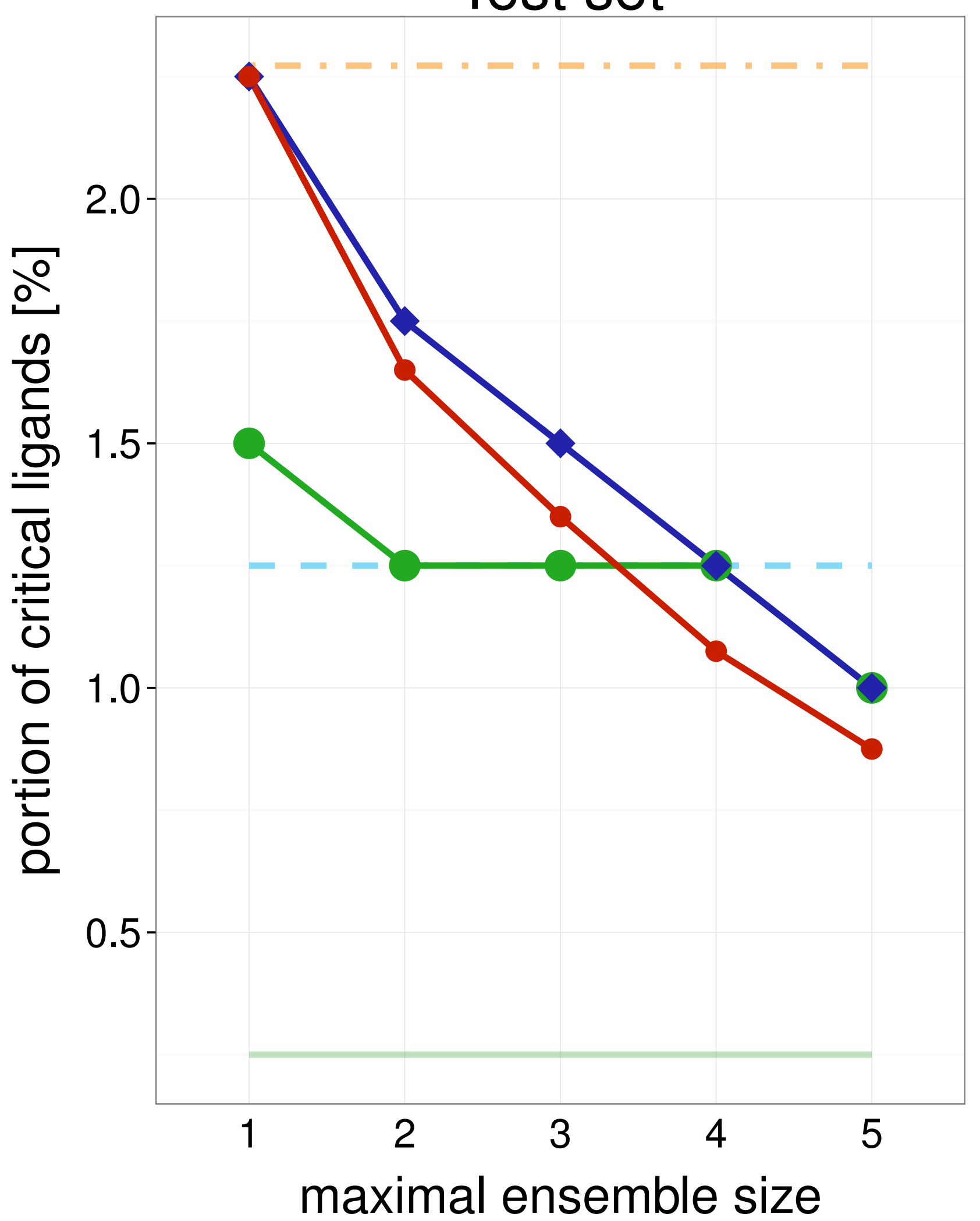

Training set

Average single structure

_ _ Best single structure

Whole ensemble

- SIENA

$\neg$ Clustering

$\multimap$ Random

Average single structure

_ _ Best single structure

Whole ensemble

- SIENA

$\neg$ Clustering

$\multimap$ Random

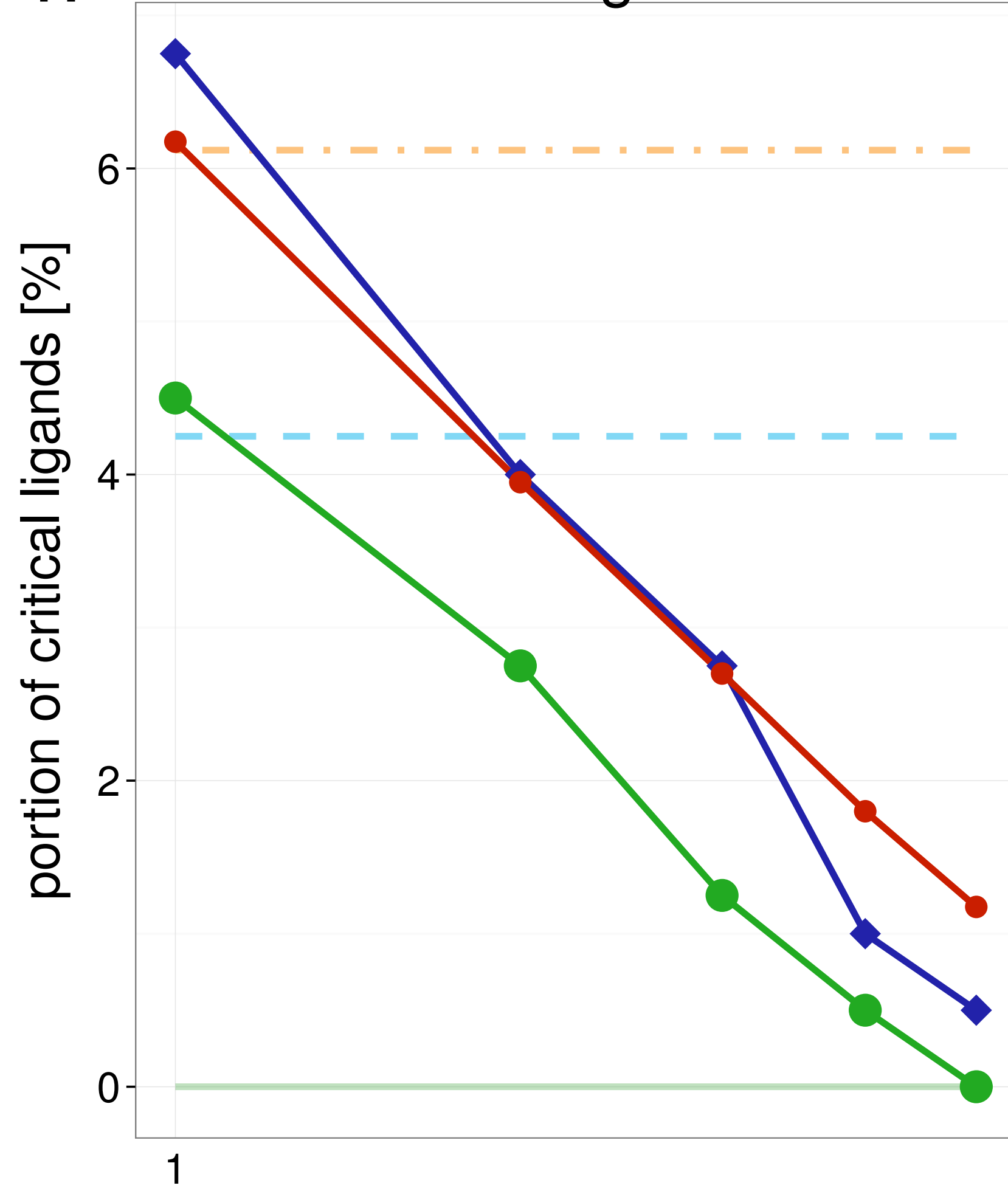

Average single structure

Best single structure

Whole ensemble

- SIENA

$\leadsto$ Clustering

maximal ensemble size

Test set

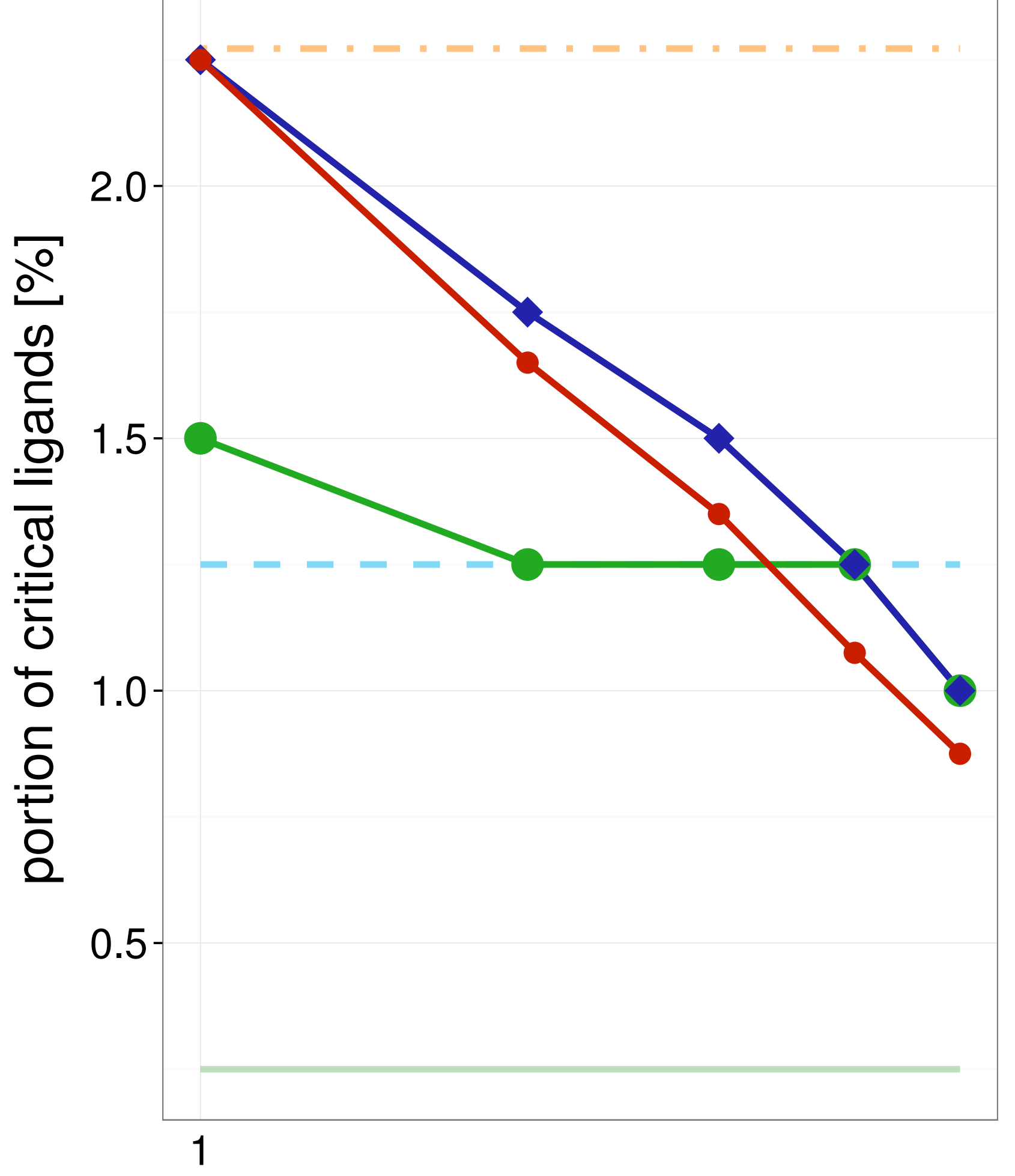

Average single structure

_ Best single structure

Whole ensemble

SIENA

$\sim$ Clustering

$\multimap$ Random 
Training set

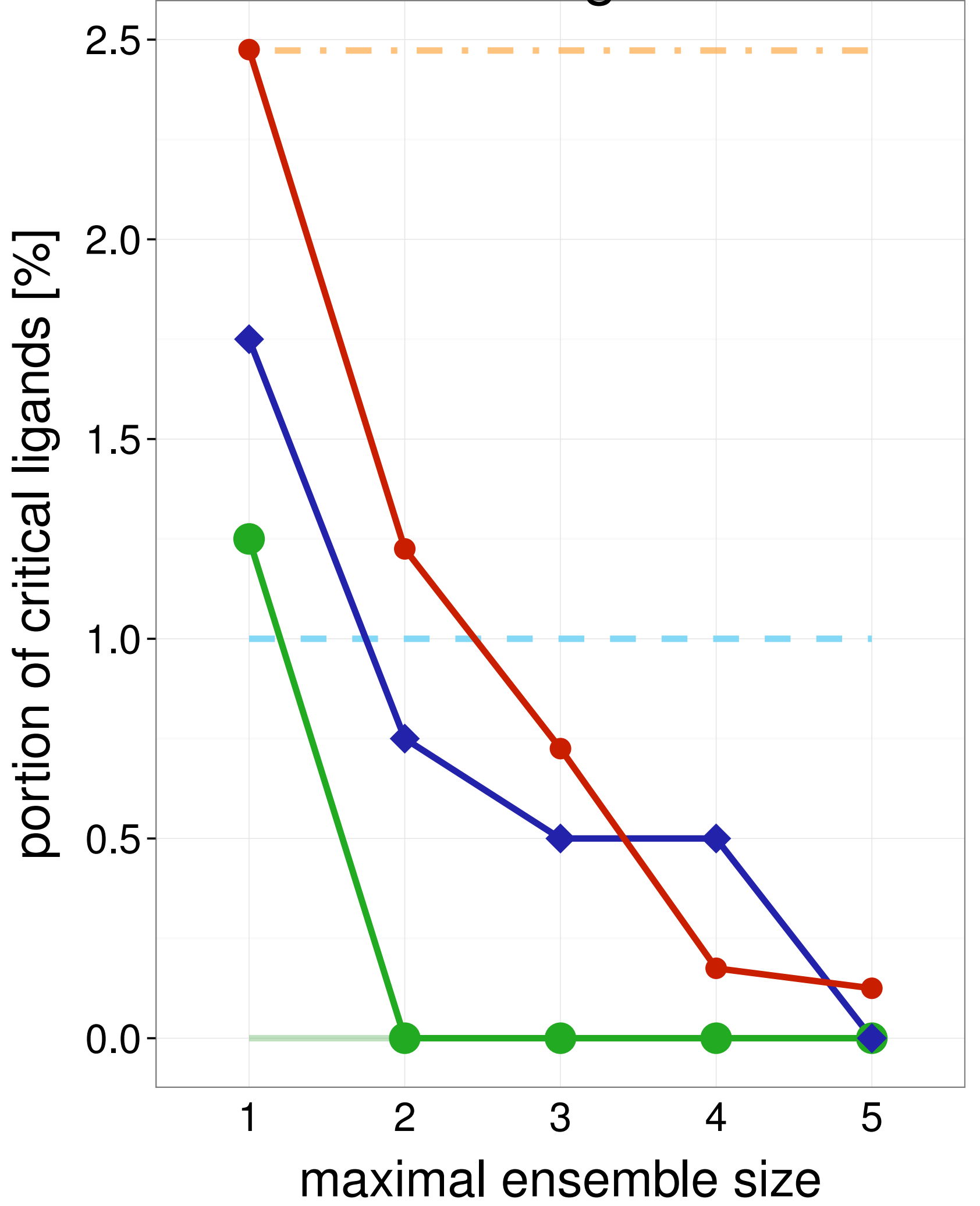

Test set

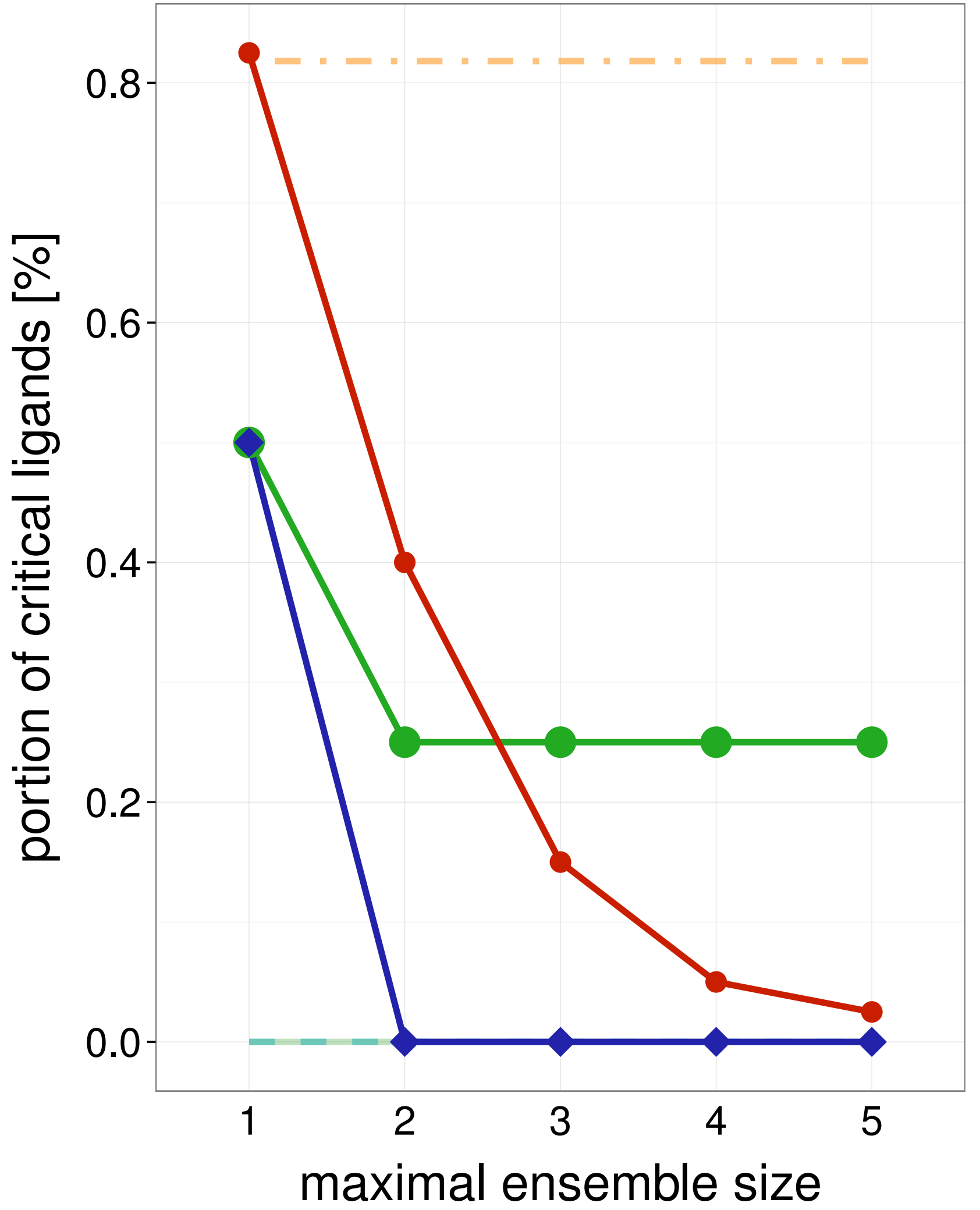

Training set

Average single structure

_ Best single structure

Whole ensemble

- SIENA

$\neg$ Clustering

$\multimap$ Random

Average single structure

_ _ Best single structure

Whole ensemble

- SIENA

$\neg$ Clustering

$\multimap$ Random

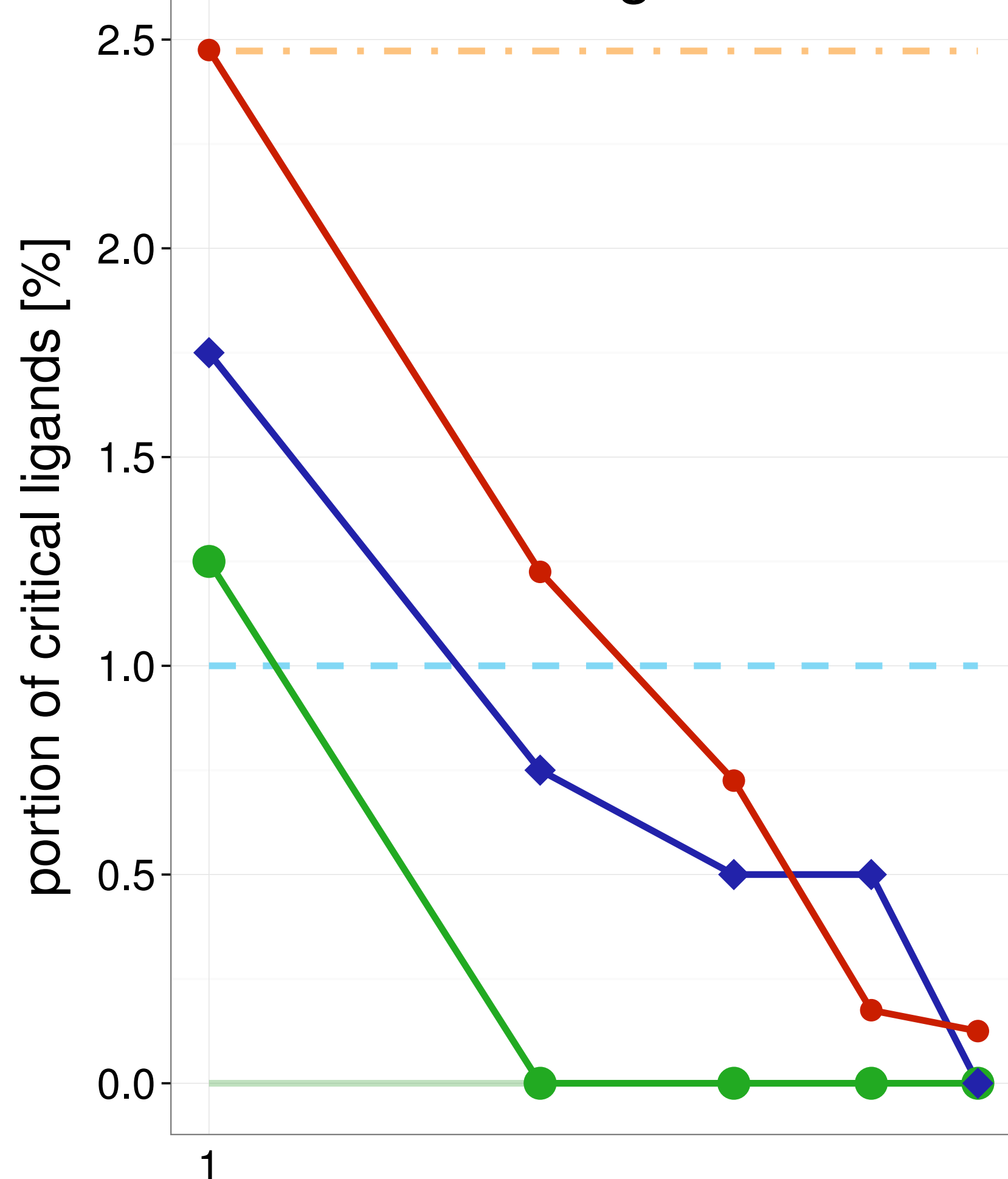

maximal ensemble size

Test set

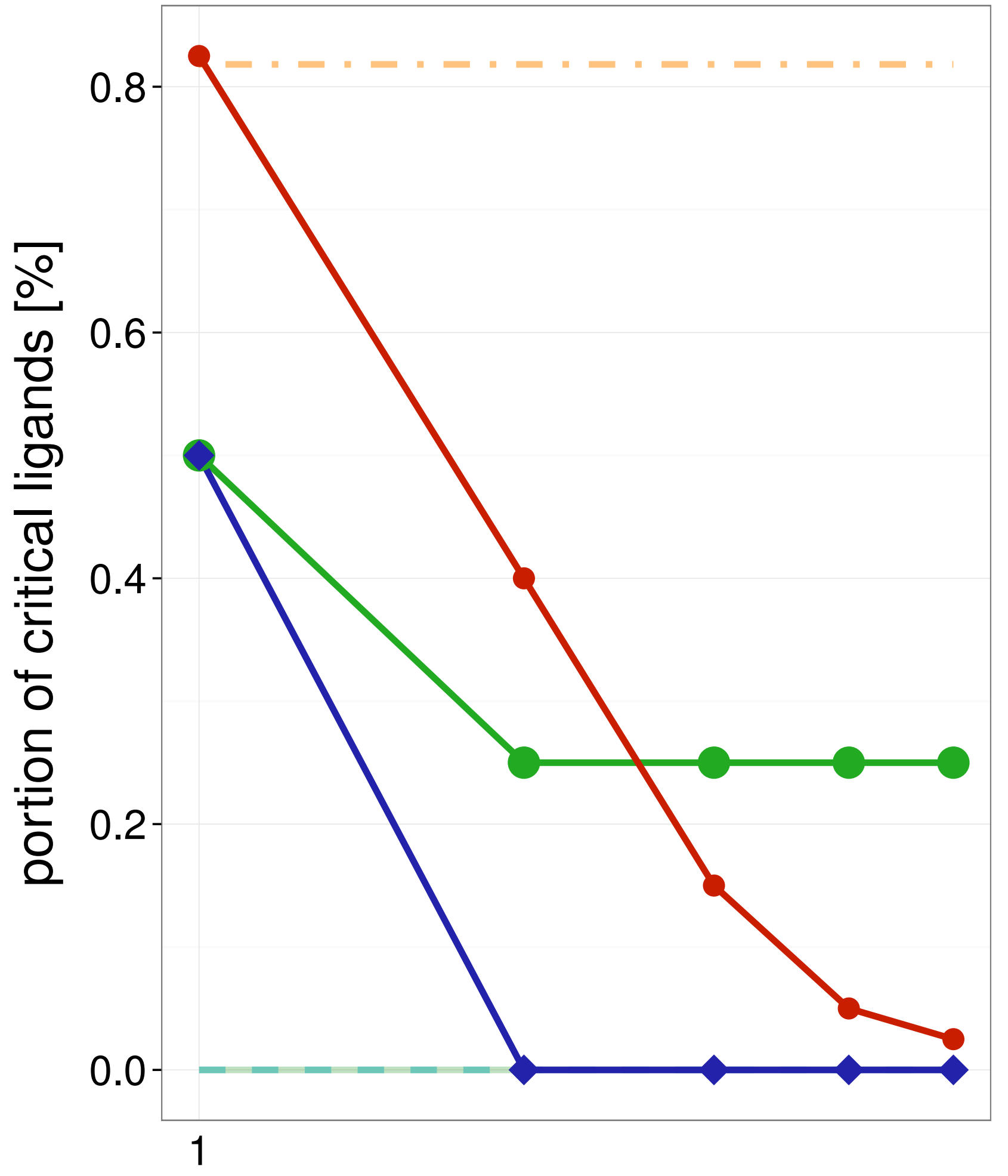

maximal ensemble size
Average single structure

_ _ Best single structure

Whole ensemble

SIENA

$\neg$ Clustering

$\multimap$ Random 
Training set

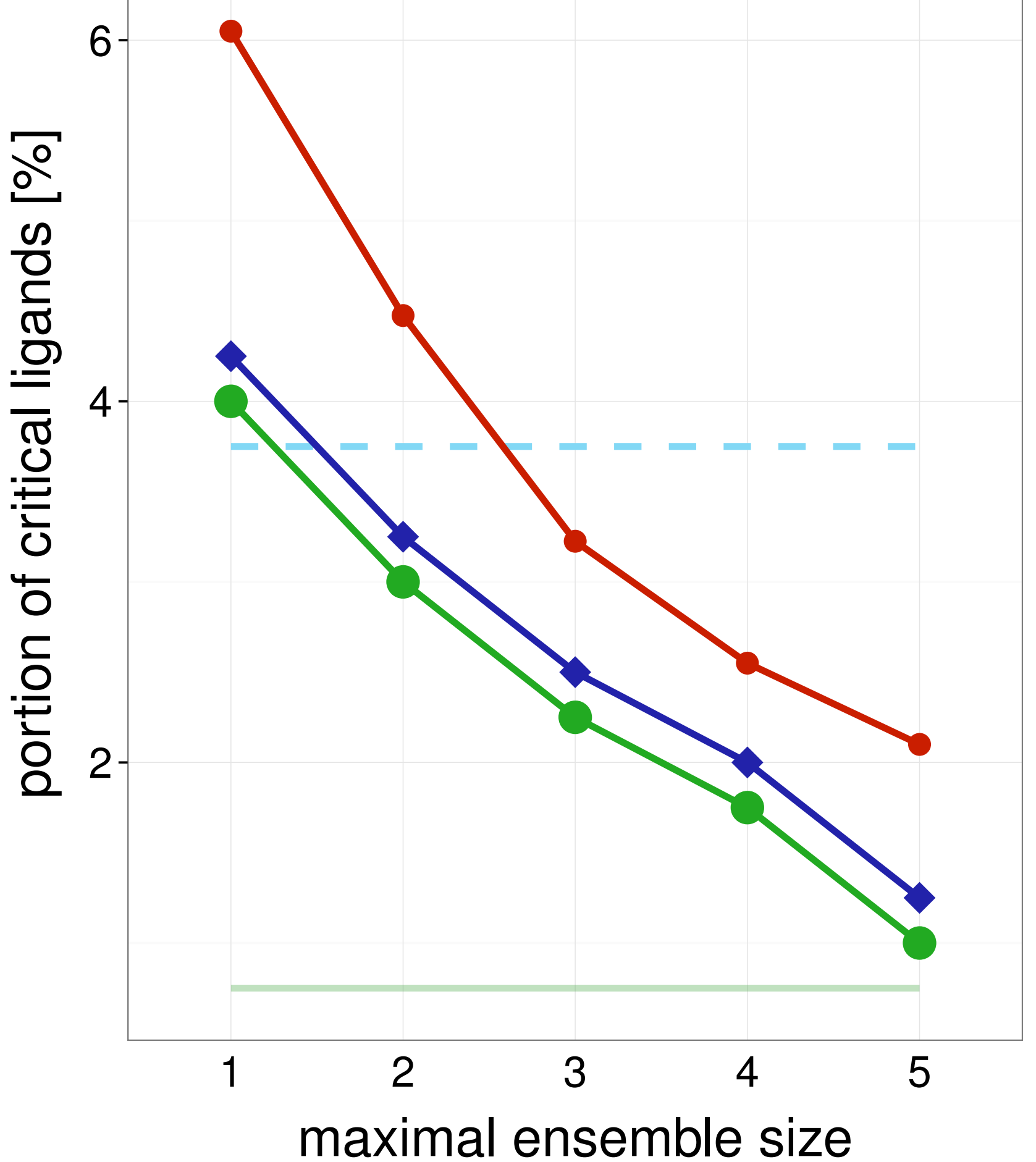

Test set

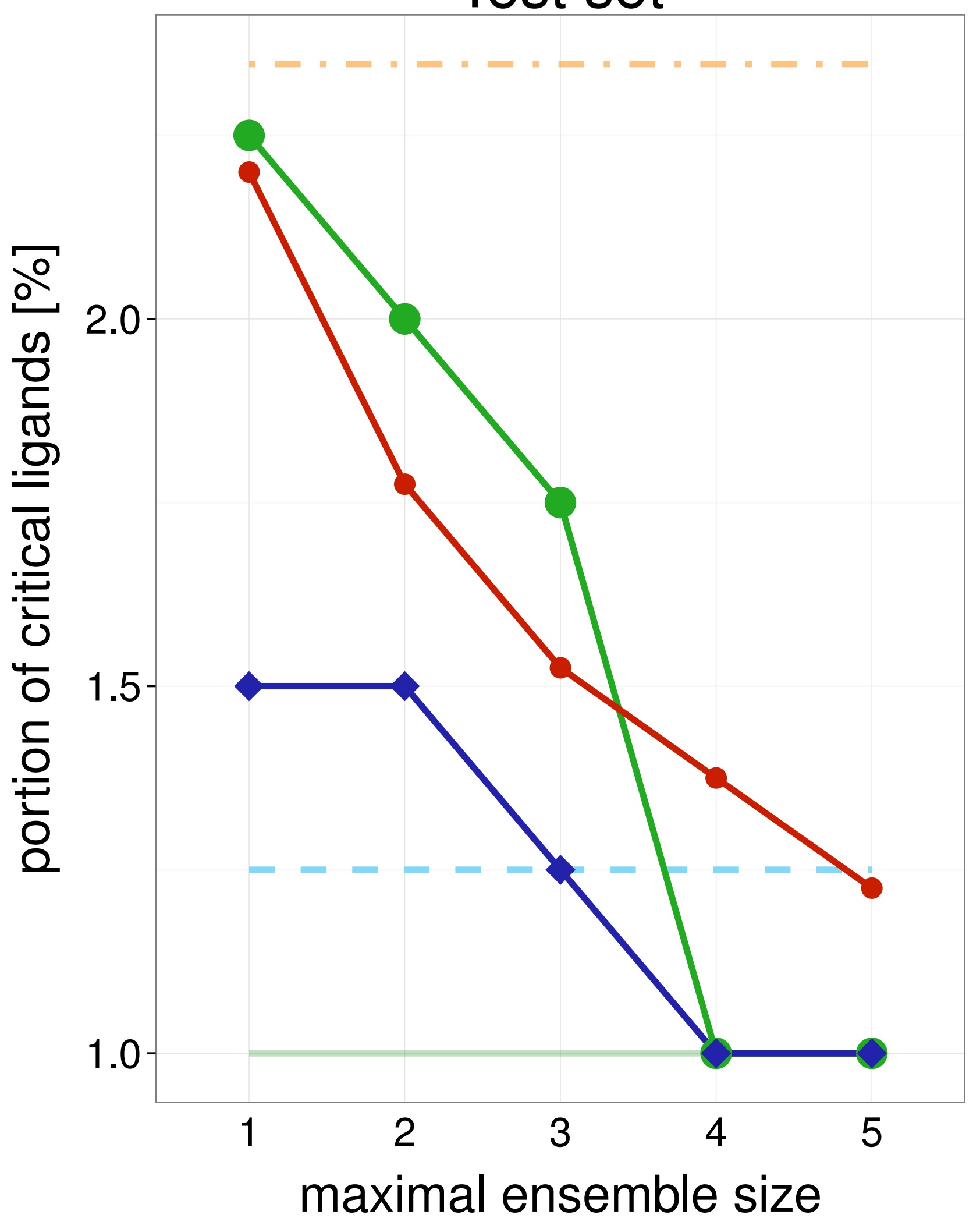

4HW2

Training set

Average single structure

_ Best single structure

Whole ensemble

- SIENA

$\prec$ Clustering

$\multimap$ Random

Average single structure

_ Best single structure

Whole ensemble

- SIENA

$\neg$ Clustering

$\multimap$ Random

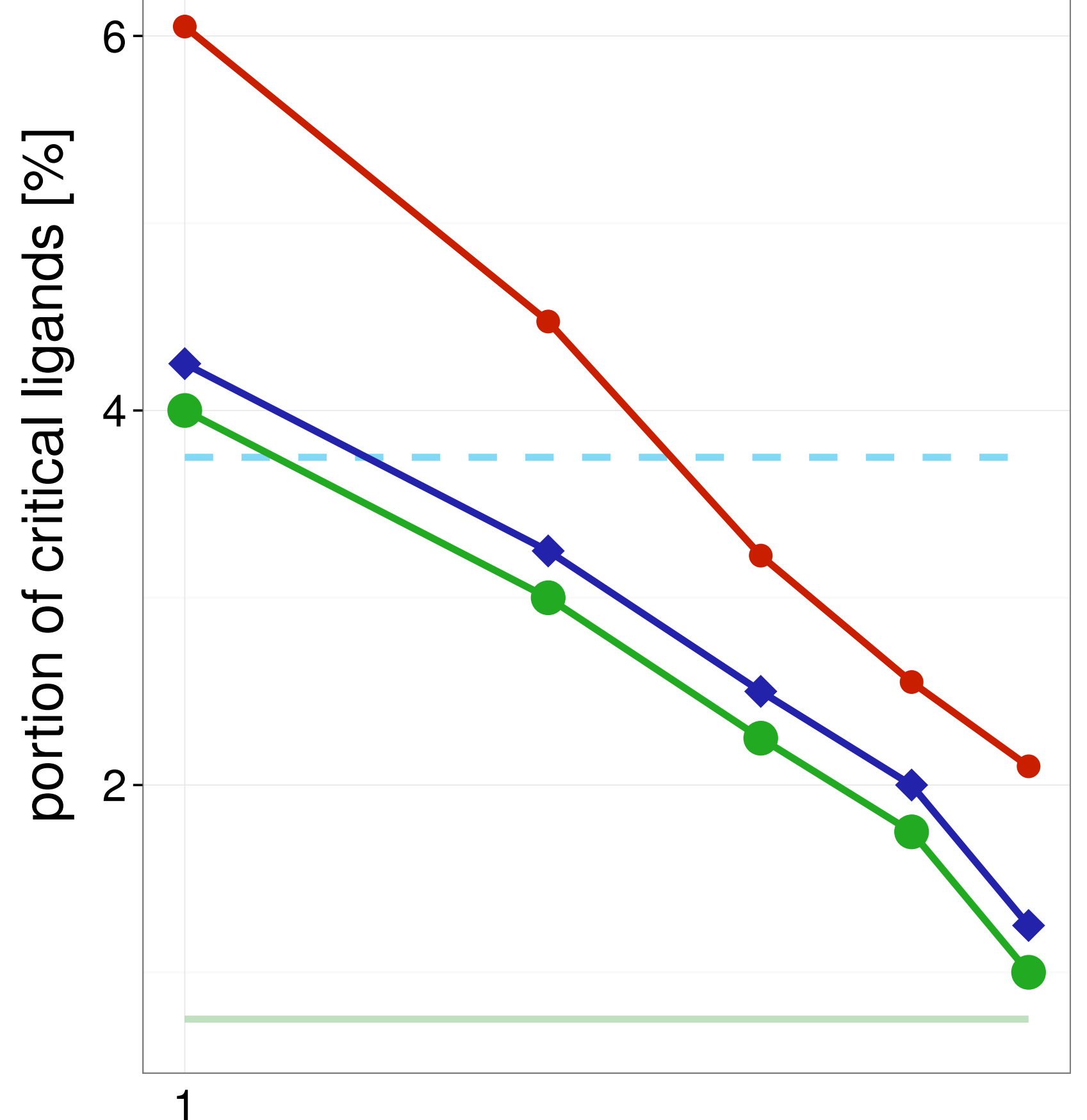

maximal ensemble size

Test set

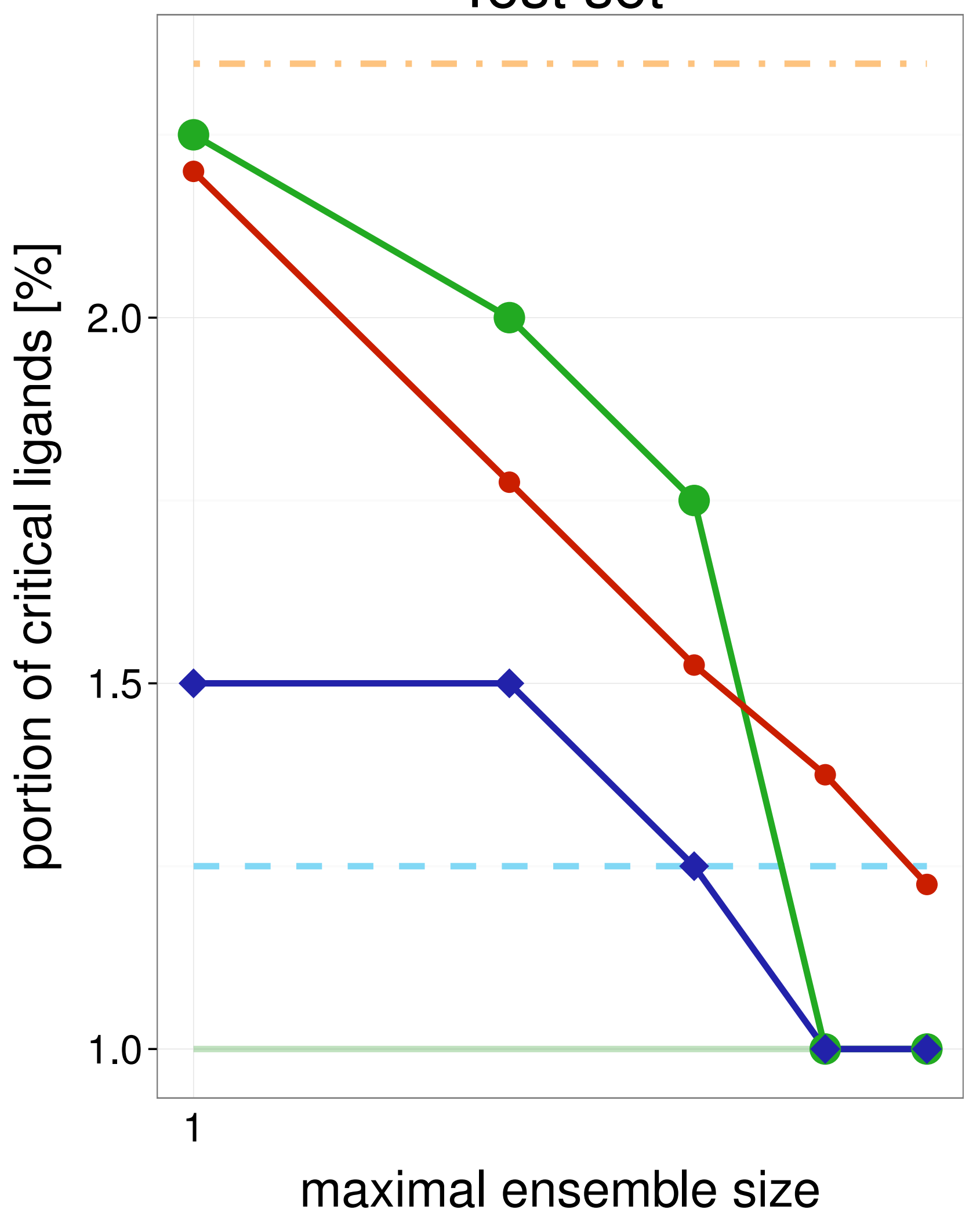

Average single structure

- Best single structure

Whole ensemble

- SIENA

$\checkmark$ Clustering

$\rightarrow$ Random

Average single structure

- Best single structure

Whole ensemble

SIENA

$\neg$ Clustering

$\multimap$ Random 
Training set

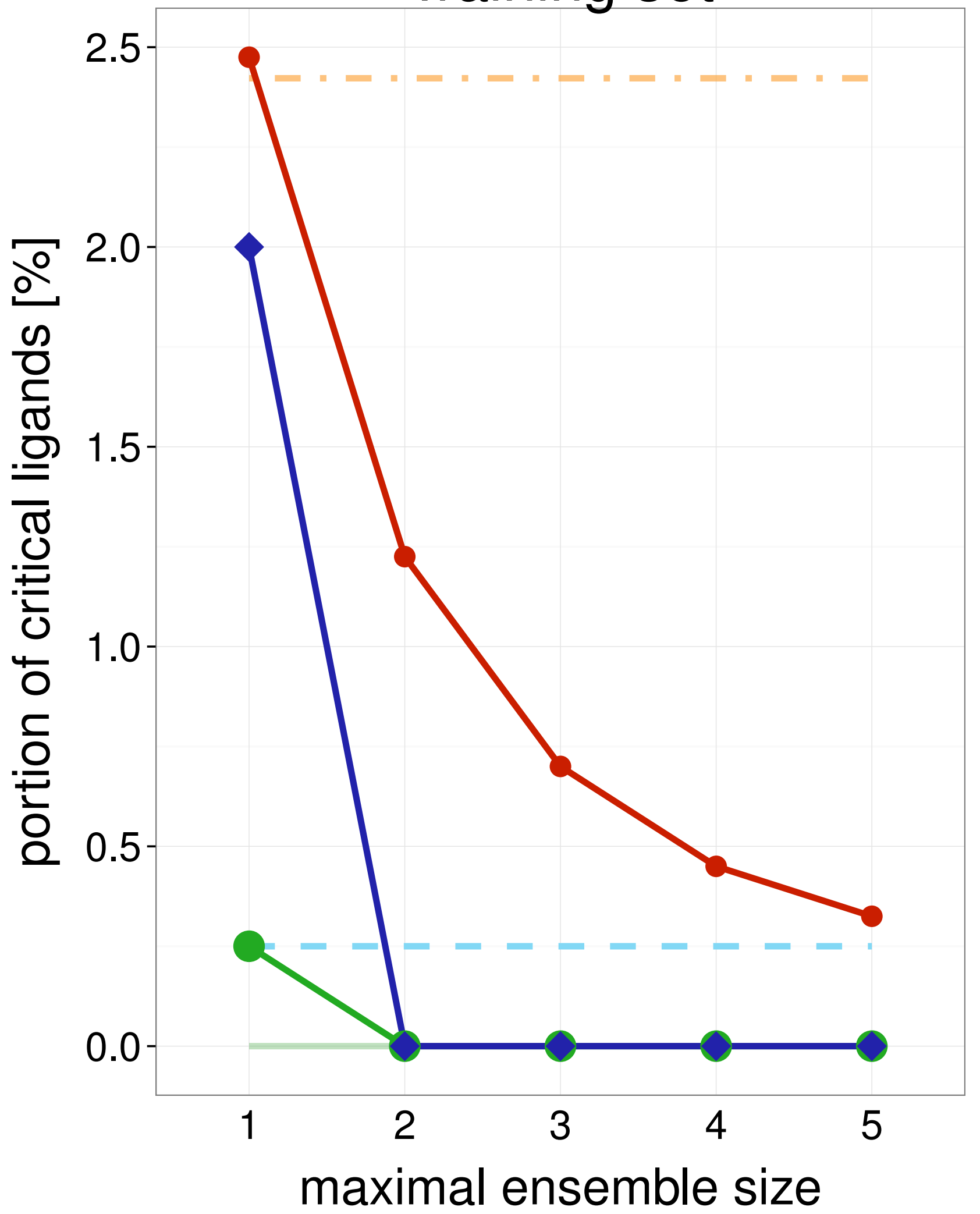

Test set

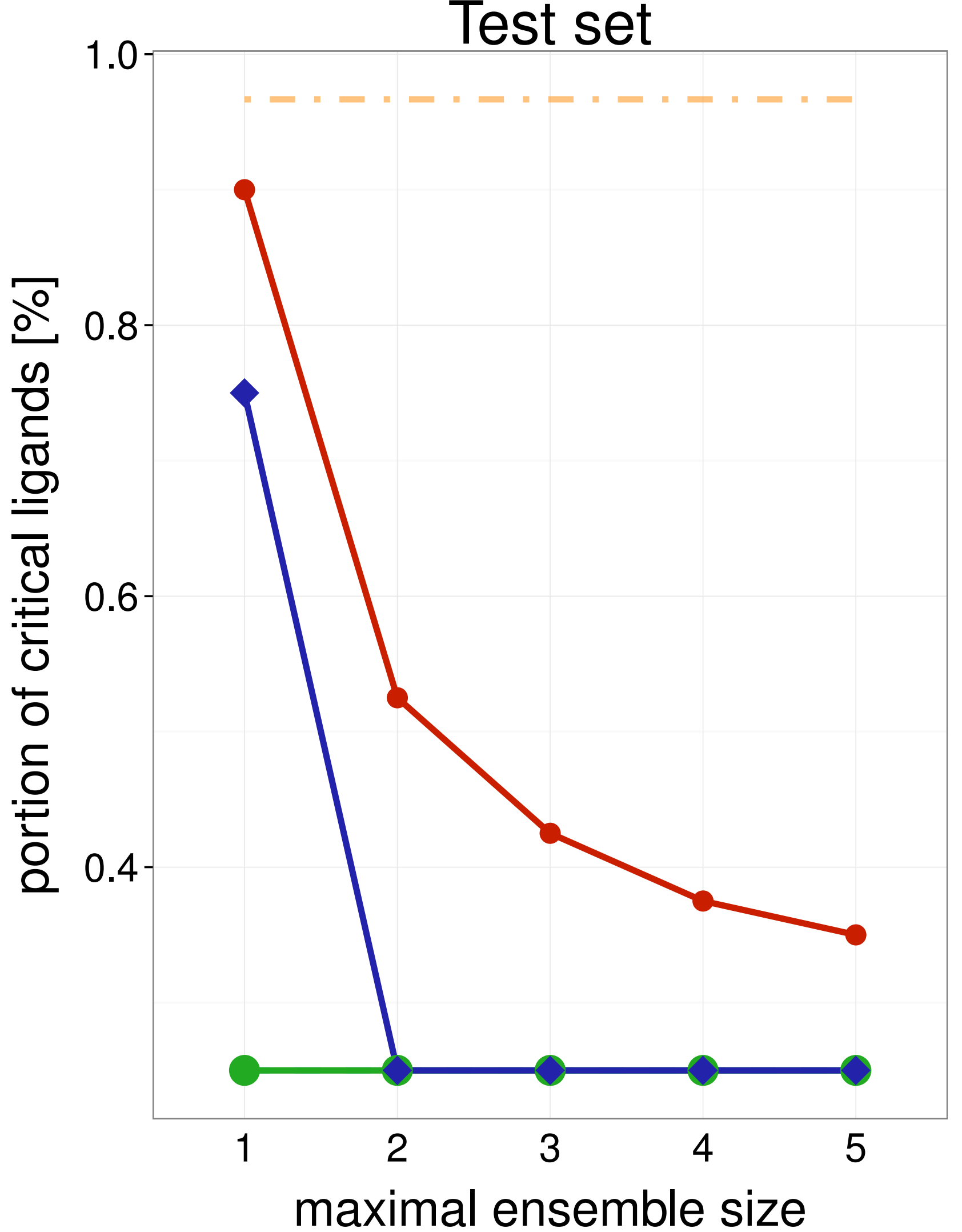

Training set

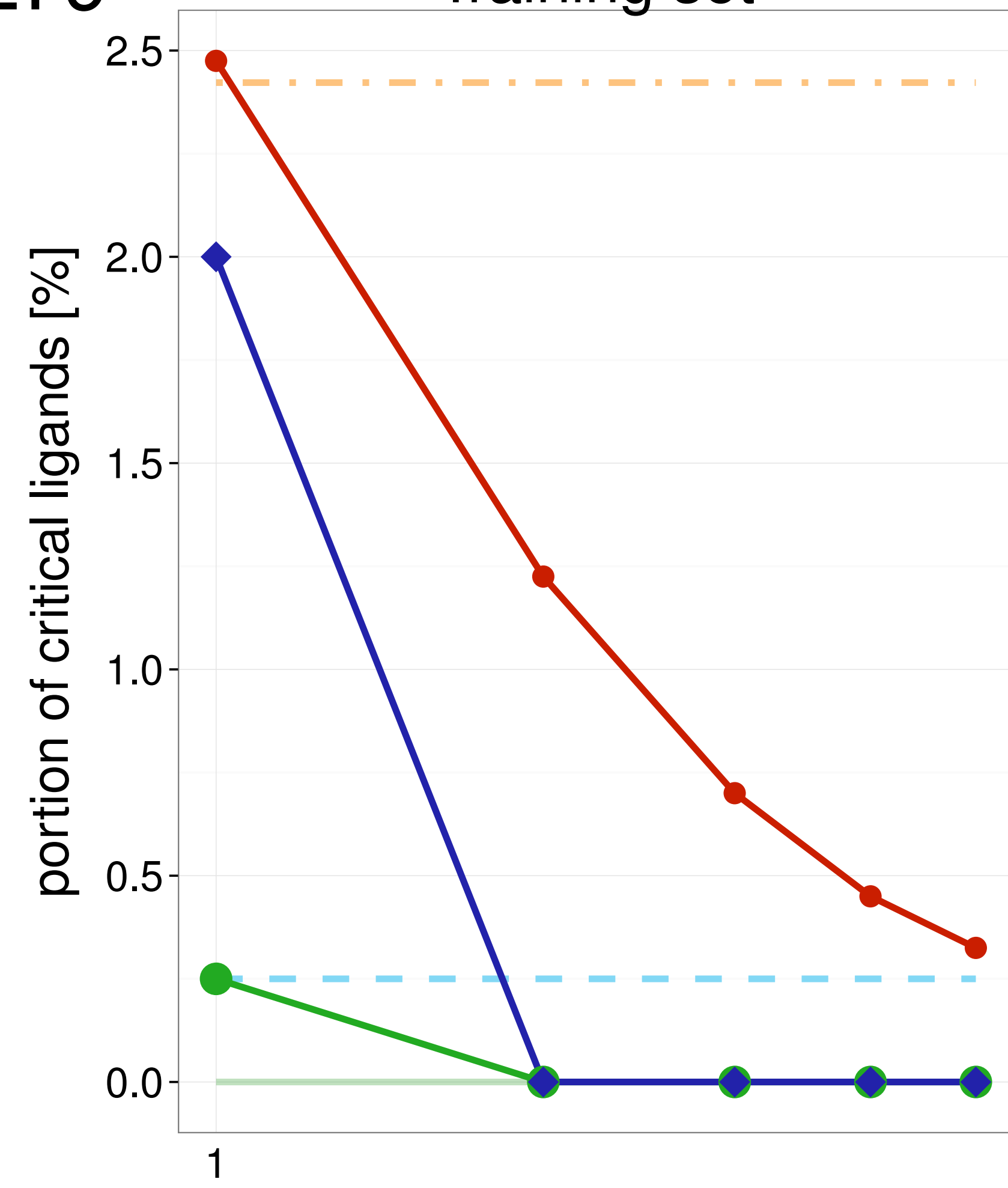

Average single structure

_ Best single structure

Whole ensemble

- SIENA

$\neg$ Clustering

$\because$ Random

Average single structure

_ _ Best single structure

Whole ensemble

- SIENA

$\neg$ Clustering

$\multimap$ Random
Average single structure

_ _ Best single structure

Whole ensemble

SIENA

$\sim$ Clustering

- Random maximal ensemble size

Test set

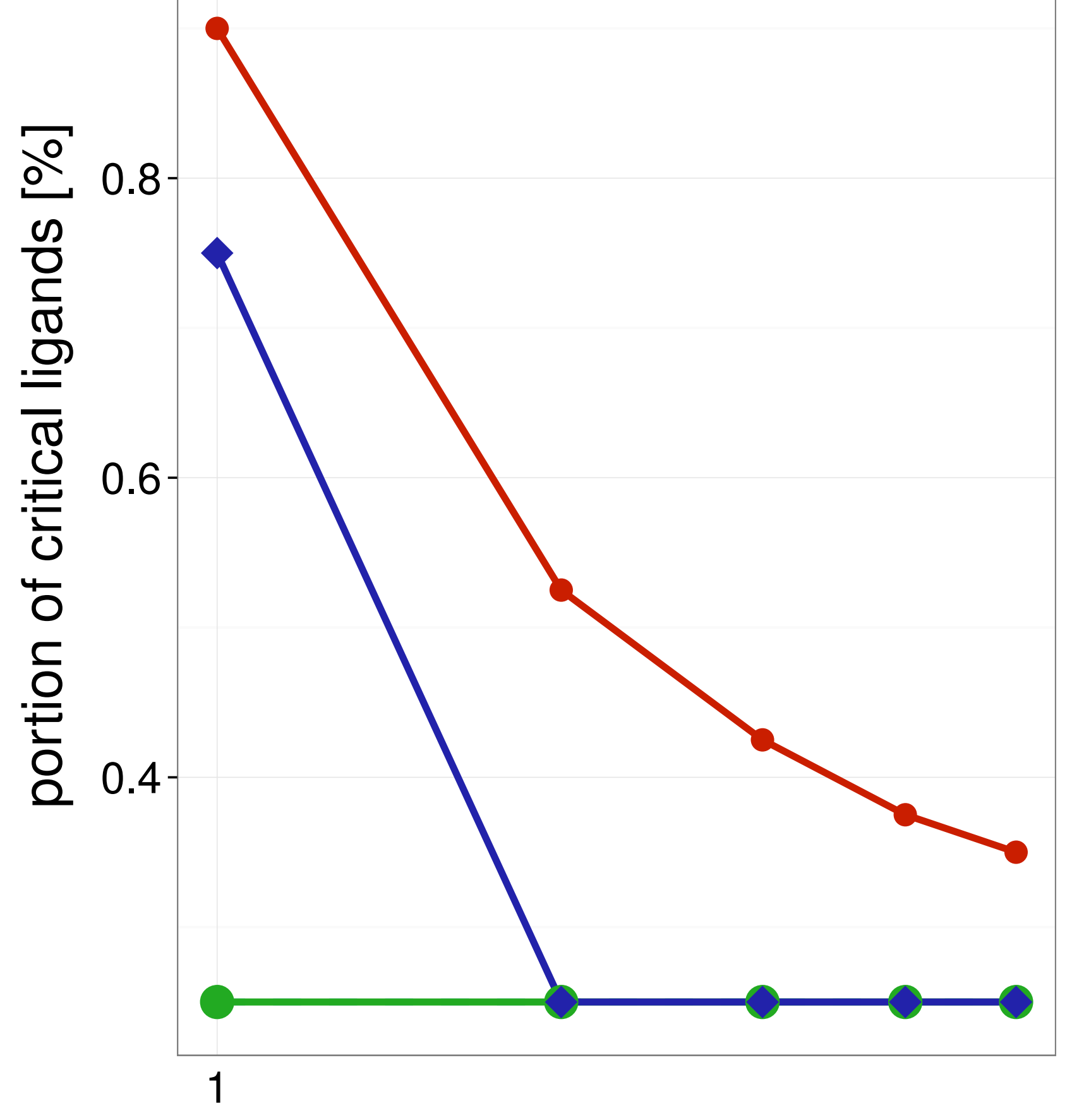

maximal ensemble size 
Training set

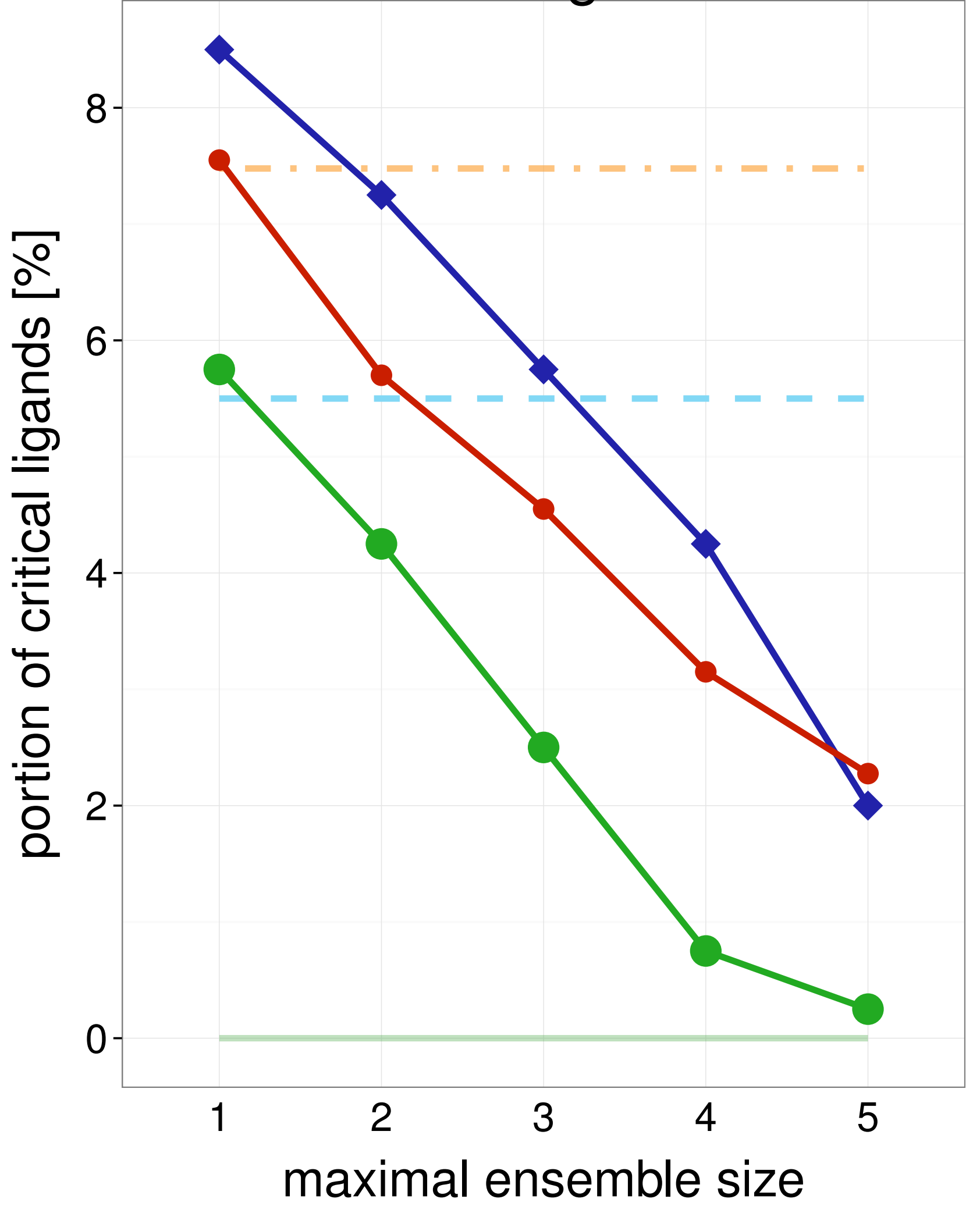

\section{Test set}

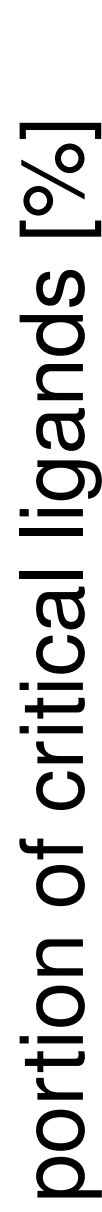

1.5

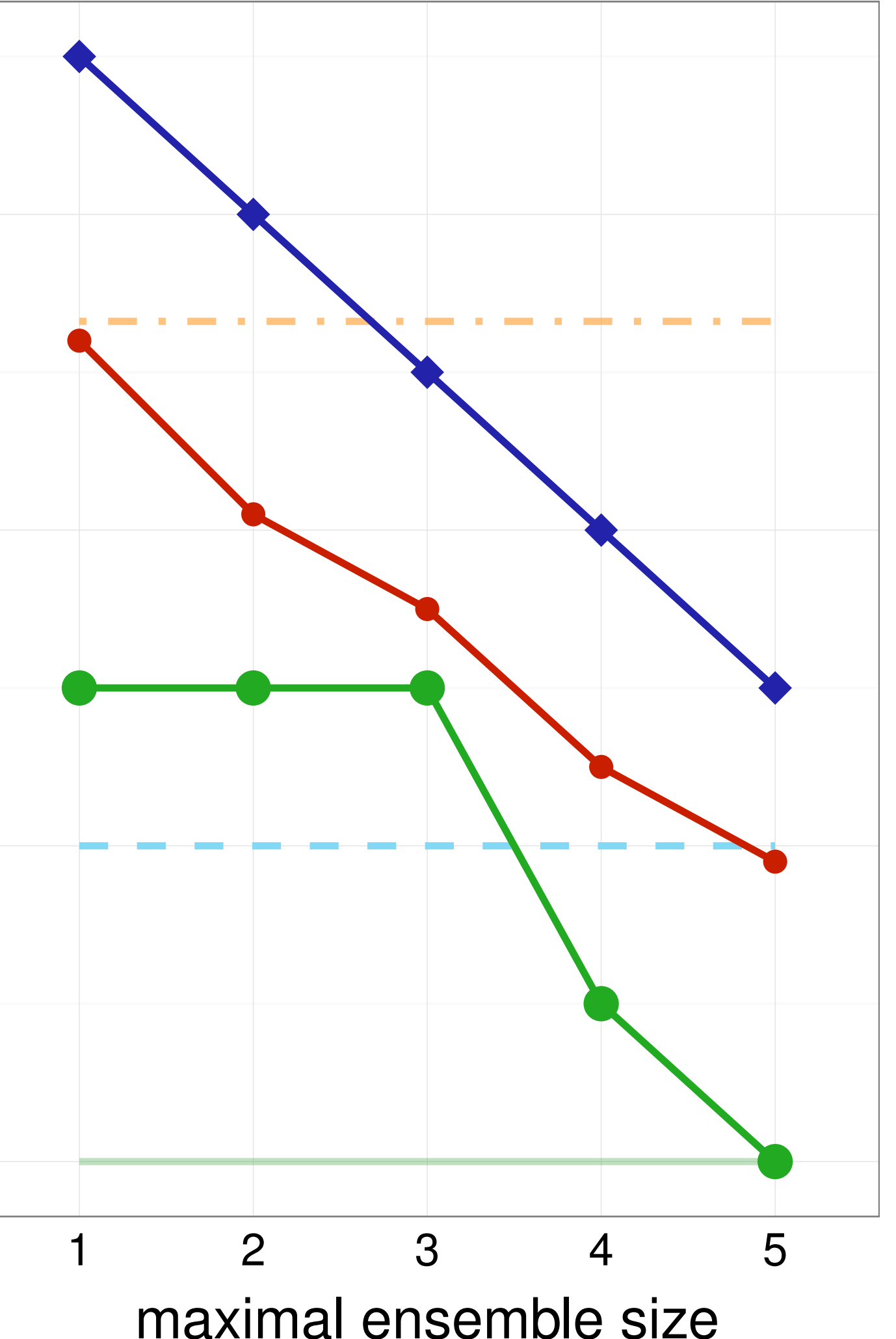

Average single structure

_ Best single structure

Whole ensemble

- SIENA

$\prec$ Clustering

$\multimap$ Random

Average single structure

_ Best single structure

Whole ensemble

- SIENA

$\neg$ Clustering

$\multimap$ Random
4NB6

Training set

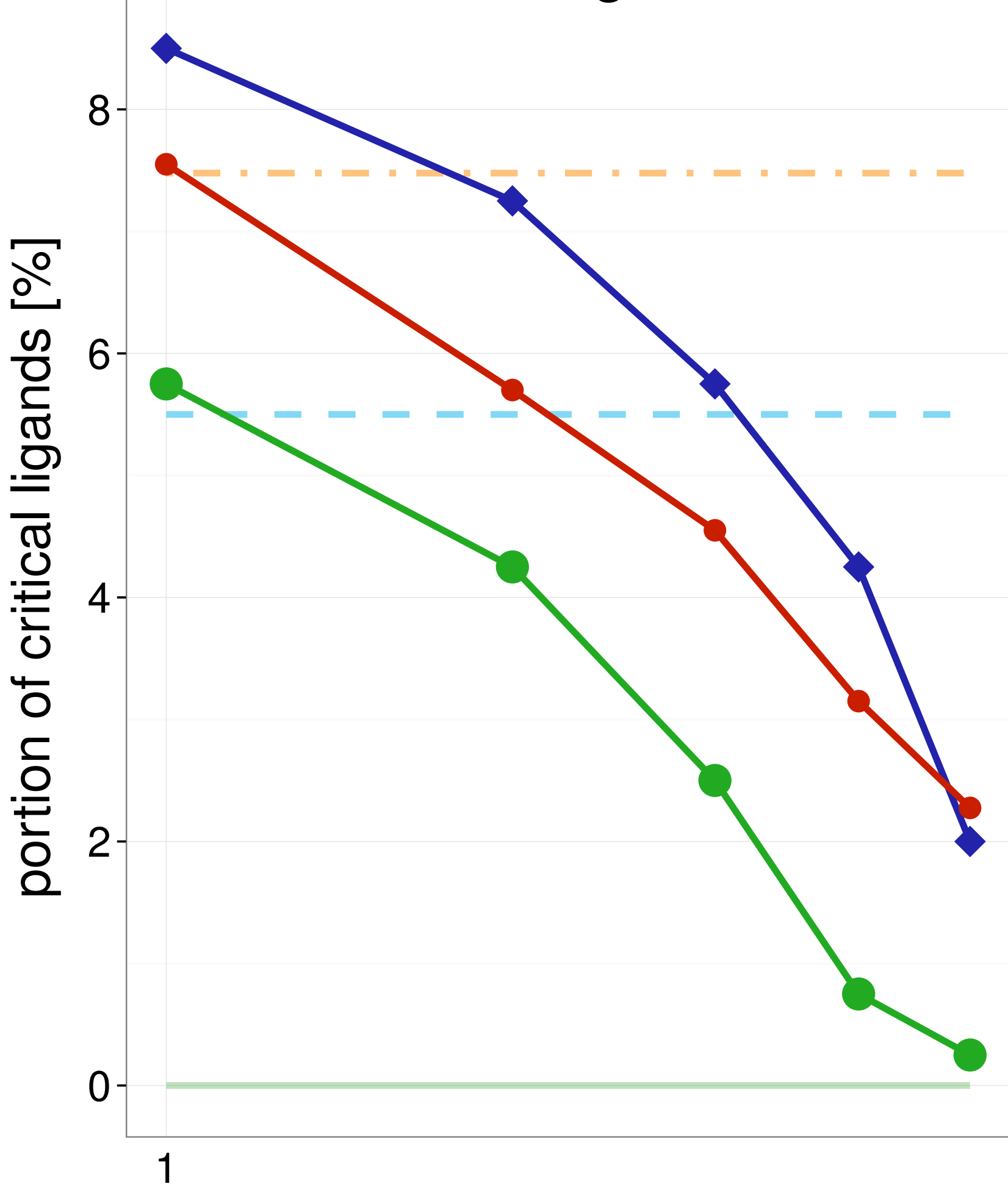

maximal ensemble size

Test set

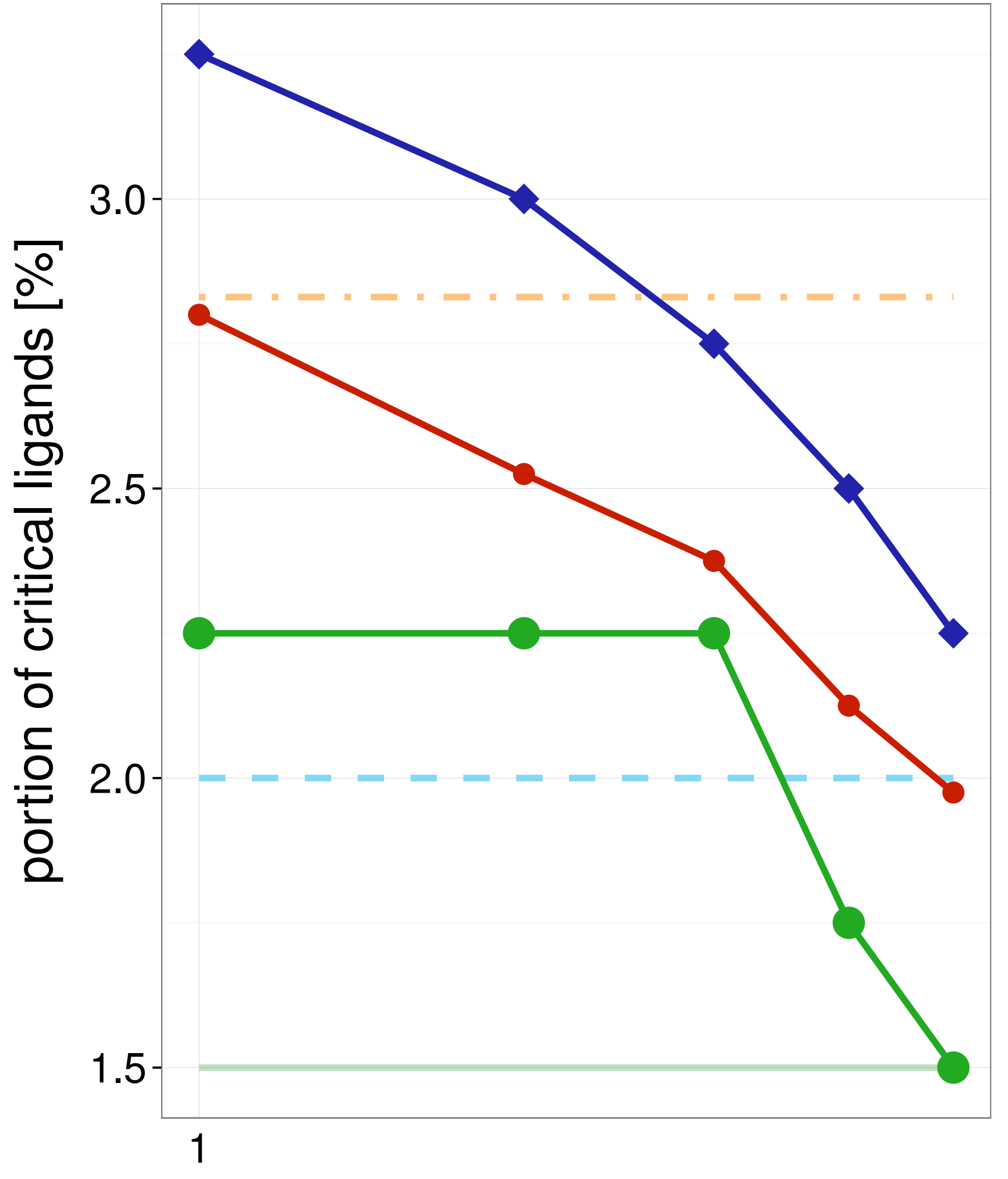

Average single structure

- Best single structure

Whole ensemble

SIENA

$\neg$ Clustering

$\multimap$ Random 